

\section{APPENDIX A}

\section{ESTIMATING DOE ANALYTICAL}

\section{LABORATORY CAPACITY AVAILABLE TO EM}

- MODEL DESCRIPTION 


\title{
ESTIMATING DOE
}

\section{ANALYTICAL LABORATORY}

\section{CAPACITY AVAILABLE}

\author{
TO EM
}

\section{MODEL \\ DESCRIPTION}

\section{Prepared By:}

Laboratory Management Division (EM-563)

Office of Technology Development

Environmental Restoration and Waste Management

U.S. Department of Energy

September 28, 1993 


\section{DISCLAIMER}

Portions of this document may be illegible in electronic image products. Images are produced from the best available original document. 
LIST OF ACRONYMS $\ldots \ldots \ldots \ldots \ldots \ldots \ldots \ldots \ldots$ ii

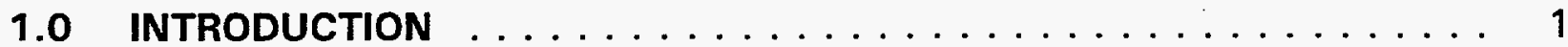

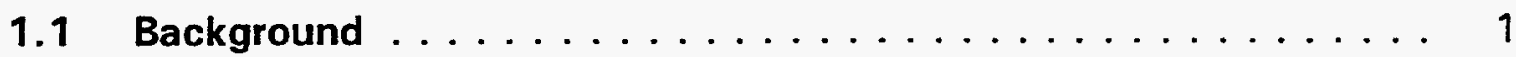

1.2 Objective of This Model . . . . . . . . . . . . . . 2

2.0 DEFINITIONS ...................... 3

2.1 Capability and Capacities . . . . . . . . . . . . 3

2.1.1 Annual Capacity Definitions . . . . . . . . . . . 3

2.2 Sample Analyses and Laboratory Operations . . . . . . . . . 4

2.3 Sample Characteristics ................... 6

2.4 Cycle Times and Operating Times .............. 7

3.0 BASIS FOR CAPACITY MODEL $\ldots \ldots \ldots \ldots \ldots \ldots \ldots \ldots \ldots$

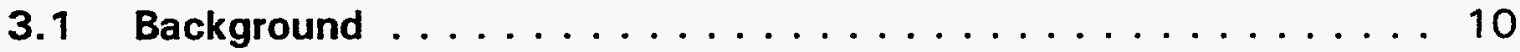

3.2 General Approach to Estimating Capacity . . . . . . . . . 10

3.3 Applicability of the Capacity Model . . . . . . . . . . . 11

3.4 Using the Model as a Lab Management Tool . . . . . . . . . 12

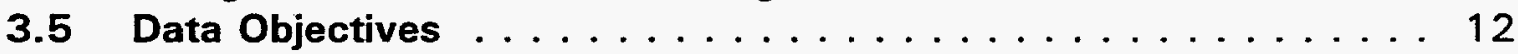

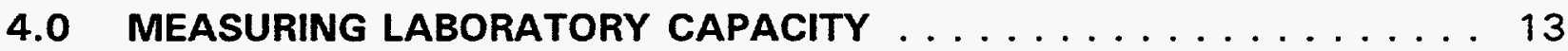

4.1 Background .................... 13

4.2 Theoretical Maximum Operational Cycles . . . . . . . . . 13

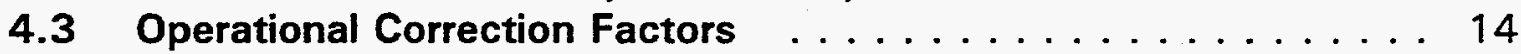

4.4 Definition of Operational Correction Factors . . . . . . . . 15

4.5 Calculation of Annual Capacity . . . . . . . . . . . . . 18

4.5.1 Calculation of Laboratory Operation Annual Capacity . . . 18

4.5.2 Calculation of Sample Analysis Annual Capacity . . . . . 19

4.5.3 Summary of Customer Sample Annual Capacity . . . . . 19

4.6 Implementation of Capacity Model . . . . . . . . . . . 19

4.6.1 Input of Data into Model . . . . . . . . . . . . . . 19

4.6.2 Calibration of the Annual Capacity Model . . . . . . . 20

4.6.3 Collection of Capacity Information by EM-563 . . . . . 20

Appendix A: Definitions of Terms Used in Estimating DOE Analytical Capacity

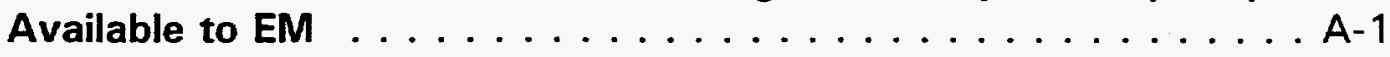

Table I: $\quad$ Sample Analyses $\ldots \ldots \ldots \ldots \ldots \ldots \ldots \ldots \ldots \ldots$ 


\section{LIST OF ACRONYMS}

\section{Acronym Description}

\begin{tabular}{ll} 
AA & Atomic Absorption Spectroscopy \\
AC & Annual Capacity \\
AES & Atomic Emission Spectroscopy \\
BF & Batch Factor \\
CT & Calibration Operating Time \\
CV & Cold Vapor \\
DOE & U.S. Department of Energy \\
EA & Equipment Availability \\
EC & Existing Capacity \\
EM & Environmental Restoration and Waste Management \\
EM-563 & Laboratory Management Division \\
EPA & Environmental Protection Agency \\
ERMC & Environmental Restoration Management Contractor \\
FA & Facility Availability \\
FAST & Future Analytical Support Team \\
GC & Gas Chromatography \\
GF & Graphite Furnace \\
IC & Instrument Calibration \\
ICP & Inductively Coupled Plasma \\
M\&O & Management and Operating \\
MC & Upgradable Capacity \\
ME & Matrix Effect \\
MMES & Martin Marietta Energy Systems \\
MS & Mass Spectrometry \\
N & Number of Available Units \\
OA & Operation Availability \\
OSM & DOE Office of Sample Management \\
PA & Personnel Availability \\
PCB & Polychlorinated Biphenyl \\
PE & Performance Evaluation \\
QC & Quality Control \\
QT & Quality Control Operating Time \\
R\&D & Research and Development \\
RCRA & Resource Conservation and Recovery Act \\
RCZ & Radiation Control Zone \\
ST & Customer Sample Operating Time \\
TCLP & Toxicity Characteristic Leaching Procedure \\
TMOC & Theoretical Maximum of Operational Cycles \\
UC & Utilized Capacity \\
VOA & Volatile Organic Analysis \\
& \\
\hline
\end{tabular}




\subsection{INTRODUCTION}

\section{$1.1 \quad$ Background}

The major mission of the Laboratory Management Division (EM-563) is to ensure the availability of cost-effective and high-quality chemical and radiochemical analyses needed to support the Department of Energy (DOE) Office of Environmental Restoration and Waste Management (EM) programs.

To accomplish this mission, EM-563 needs detailed, current information on the analytical capacity and capabilities of DOE Complex and commercial sector analytical laboratories. EM-563 lacks current information on existing and future analytical capacities and capabilities of site Management and Operating (M\&O) and Environmental Restoration Management Contractor (ERMC) laboratories. As EM programs have developed, and compliance agreements with State and Federal regulatory agencies have been implemented, the associated plans for site characterization and remediation have not clearly defined the sampling and analytical needs. Because of the current and future rapid growth in analytical needs, DOE site EM programs have struggled to define the analytical needs and to acquire sufficient analytical laboratory support. The particular characteristics of some DOE site samples, such as high radiation levels, the need for radiochemical analysis, and the need for unique organic compound determinations, have further complicated making laboratory capacity estimations. While the degree of concern varies from site to site, it is evident to both EM-563 and the analytical laboratories that a mechanism is needed to balance the program needs to analytical capacity.

Each DOE site may have one or more analytical laboratories in operation. These facilities were established to support site missions such as production, research and development, and personnel and environmental monitoring. With changing site missions and the DOE directives for environmental monitoring and clean-up, these laboratories are either devoting or planning to devote resources to support EM activities. The DOE site laboratories represent a considerable amount of capital investment and analytical capability, capacity, and expertise that can be applied to support the EM mission. This expertise ranges from providing cost-effective highvolume analytical laboratory services to being some of the most recognized analytical research and development centers. Several sites have already transitioned analytical capability from traditional production support to environmental monitoring and waste management support.

Measuring the capacity of a DOE site analytical laboratory can be a challenging opportunity. Some site laboratories are already focused on EM analyses and operate in a high-volume "production oriented" manner. For these laboratories, capacity measurement may be less challenging. Other DOE laboratories will be handling high radiation-level samples and capacity will be more difficult to estimate. For those laboratories that will be or are transitioning to an EM support role, capacity measurements may be more subjective. 


\subsection{Objective of This Model}

The objective of this model is to clearly define the method used to estimate the capabilities and capacities of the DOE analytical laboratory Complex to analyze EM samples. An accompanying instructions package* has been developed for DOE site contractor laboratories to estimate the existing and upgradable capacity and capability to analyze environmental and waste samples. While the focus of the survey will be on existing environmental and waste analytical capacity and capability, relevant capacity currently being devoted to other programs such as production support, $R \& D$, and site monitoring should be included in the capacity calculations (see Section 3.1). This ensures inclusion of all applicable analytical capacity that could become available for EM support as site missions change. The analytical capacity estimates will be compared with the results of a companion effort to estimate EM analytical needs.

This information will allow DOE EM-563 to assist headquarters management in focusing analytical laboratory priorities. Specifically, the information will help to:

- respond to requests from Congress and others for information on EM analytical needs, capacities, and capabilities;

- compare with EM program analytical needs projections;

- develop baseline information for justifying budgets;

- optimize the level and type of commercial laboratory participation;

- $\quad$ provide information needed by DOE Offices of Sample Management (OSMs) for shipment of samples for analysis; and

- provide a management tool for analytical laboratories supporting EM.

If a laboratory is to effectively utilize its available capacity and capability, there must be close communications between laboratory management and the sample submitting organizations (i.e. the local OSM and local EM project management). Of special note is that laboratory capacity must be managed as a "perishable commodity." A laboratory with a capacity to perform 2500 volatile organic analyses per year cannot do so if the samples are not scheduled to arrive on an even time distribution based on sample holding times and facility availability. There is some optimum minimum period, usually a few days to weeks, into which the annual capacity for a major laboratory analytical operation should be divided.

- Estimating DOE Analytical Capacity Available to EM - Reporting Formats and Instructions. 


\subsection{DEFINITIONS}

To determine analytical laboratory capacities in a consistent manner across the DOE Complex, clear definitions of relevant terms must be established. Appendix A includes a glossary of all the terms used in the model.

\subsection{Capability and Capacities}

This project addresses both analytical capability and analytical capacity. These terms are often used interchangeably but they have distinct meanings. These terms are defined below:

- Analytical capability describes the types of analytical measurements a laboratory is able to perform on a given sample type.

- Analytical capacity describes the number of analytical measurements a laboratory is able to complete in a given time.

The model described in this package is used to estimate the annual capacities (i.e. the numbers of analytical measurements a laboratory is able to complete in a year) of major analytical capabilities of a laboratory based on customer samples. (See Section 2.3 for the definition of a customer sample).

\subsubsection{Annual Capacity Definitions}

This model can be used to estimate three different kinds of laboratory annual capacities $(\mathrm{AC})$ to analyze customer samples. These are:

- Utilized Capacity (UC) -- UC is the capacity being utilized by the analytical laboratory to support the current work load. This value is useful to "calibrate" the model against work performed, and will indicate where capacity improvements can be made. The UC calculation is useful to develop a feel for the operational factors to be developed in calculating the EC and $M C$ values. UC operational factors will reflect current laboratory operations.

- Existing Capacity (EC) -- EC is the analytical capacity that is available with existing personnel levels, existing equipment, and sample loads optimized. This value is of interest to DOE because it represents the total analytical capacity available in the laboratory without expenditures for additional people or equipment. $E C$ will equal $U C$ if the laboratory is receiving samples at optimum rates. EC will be greater than UC if the laboratory is currently receiving fewer samples than it can analyze. EC can be less than UC if the laboratory is working significant amounts of overtime. Laboratory managers will be able to plan future work loads and staffing levels using the difference between EC and UC values. EC capacity estimates should include all relevant EM capacity that is now available or might be available when site missions change. 
- Upgradable Capacity (MC) -- $\mathrm{MC}$ is the capacity of the analytical laboratory when upgraded to the optimum number of staff for 7 days/week, 24 hours/day operation and when the optimum instrumentation is added. No additional lab space is constructed, but limited non-capital refurbishment of existing space is allowed. This value is of interest since it represents the maximum sample analyses capacity of a laboratory without the need for capital building expansion. MC capacity estimates should include all relevant EM capacity that is now available or might be available when site missions change.

\subsection{Sample Analyses and Laboratory Operations}

The capability of an analytical laboratory is determined in terms of the types of sample analyses that it is able to perform. A sample analysis is defined as a distinct set of major laboratory operations required to generate specific data for a customer. A given sample analysis can result in the determination of one or more analytes on a customer sample. A list of the major sample analyses typically expected to be performed on EM analytical samples is given in Table I. Only potentially rate-limiting sample analyses need to be counted in the model.

A sample analysis usually requires the completion of all three of the following laboratory operations:

- sample preparation;

- instrumental analysis; and

- data reporting.

Appendix A provides a description of each laboratory operation.

Work requested on a customer sample will require the completion of one or more sample analyses.

This model calculates the capacity of a laboratory to perform the major laboratory operations that are necessary to complete specific sample analyses. The model then uses this data to calculate sample analysis capacity. Capacities for minor sample analyses (such as $\mathrm{pH}$, conductivity, etc) are not included unless they are rate-limiting in the overall performance of the laboratory.

The capacity of a laboratory is determined in terms of the numbers of sample analysis cycles that it is able to perform. A sample analysis cycle is defined as the effort involved in performing a sample analysis on a single customer sample. Completion of a sample analysis cycle usually requires the completion of three laboratory operational cycles corresponding to sample preparation, instrumental analysis, and data reporting laboratory operations.

Laboratories are often structured so that several individual laboratory operations may be performed by the same group of people as a unit operation. For example, sample preparations, such as digestion of water and soil samples for metals determination by ICP or AA, are often considered part of the instrumental analysis operation since both 
Table I: $\quad$ Sample Analyses

\begin{tabular}{|c|c|}
\hline Sample Analysis & Description \\
\hline VOA-GC & Volatile organic analysis by gas chromatography \\
\hline VOA-GC/MS & $\begin{array}{l}\text { Volatile organic analysis by gas chromatography - } \\
\text { mass spectroscopy (GC/MS) }\end{array}$ \\
\hline SEMI-VOA & Semi-volatile organic analysis by GC/MS \\
\hline PEST & Pesticide analysis by $\mathrm{GC}$ \\
\hline HERB & Herbicide analysis by GC \\
\hline PCB & Polychlorinated biphenyl analysis by GC \\
\hline ICP-AES METALS & $\begin{array}{l}\text { Inductively Coupled Plasma - Atomic Emission } \\
\text { Spectroscopy (ICP-AES) measurement of a single } \\
\text { metal analyte or a suite of metal analytes }\end{array}$ \\
\hline ICP-MS METALS & $\begin{array}{l}\text { Inductively Coupled Plasma - Mass Spectrometry } \\
\text { (ICP-MS) measurement of a single metal analyte or a } \\
\text { suite of metal analytes }\end{array}$ \\
\hline GF-AA METALS & $\begin{array}{l}\text { Graphite Furnace Atomic Absorption (GF-AA) } \\
\text { measurement of individual metal analytes }\end{array}$ \\
\hline MERCURY & $\begin{array}{l}\text { Cold Vapor Atomic Absorption (CV-AA) } \\
\text { measurement of mercury }\end{array}$ \\
\hline CYANIDE & Measurement of cyanide \\
\hline ANIONS/CATIONS & $\begin{array}{l}\text { Anion/cation analysis, by ion chromatography or } \\
\text { wet chemistry (counted individually by ions, e.g., } \\
\text { nitrate, or ammonium for single analysis, or by ion } \\
\text { chromatography runs for simultaneous analyses) }\end{array}$ \\
\hline GROSS ALPHA/BETA & Gross alpha and beta counting \\
\hline $\begin{array}{l}\text { ALPHA SPEC } \\
\text { ISOTOPES (Specify) }\end{array}$ & $\begin{array}{l}\text { Spectrometric analysis for specified alpha-emitting } \\
\text { nuclides }\end{array}$ \\
\hline $\begin{array}{l}\text { GAMMA SPEC } \\
\text { ISOTOPES (Specify) }\end{array}$ & $\begin{array}{l}\text { Spectrometric analysis for specified gamma-emitting } \\
\text { nuclides }\end{array}$ \\
\hline TCLP EXTRACTION & Toxicity Characteristic Leaching Procedure (TCLP) \\
\hline OTHER (Specify) & Other sample analysis \\
\hline
\end{tabular}


operations are performed by the same group of people. However, to maintain consistency in this model, each laboratory operation for each sample analysis is considered separately.

The model will estimate the sample analysis capacity by examining the calculated capacity for each component laboratory operation. The sample analysis capacity equals the capacity of the limiting operation. The user will determine the sample analyses to be modeled based on their importance to the laboratory.

\subsection{Sample Characteristics}

Three types of analytical samples are processed in a laboratory:

- Customer samples - A customer sample is any sample sent to the laboratory to be analyzed separately. Customer samples may include field samples, field quality control (OC) samples, duplicate field samples, field blanks, field performance evaluation (PE) samples, sample phases and composite samples.

- Calibration samples - A calibration sample is any sample used in calibrating the analytical instrument. Calibration samples include initial calibration samples, calibration blanks, and standard additions. Calibration verification and check standards are considered to be QC samples.

- Quality Control samples - A quality control laboratory sample is any sample, other than a customer or calibration sample, analyzed by the instrument. $\mathrm{OC}$ samples include calibration verification, duplicates, laboratory blinds, doubleblinds (including laboratory PE samples), bench controls, and method-required QC samples (matrix spikes and duplicates).

Analytical samples are also categorized according to their handling requirements and matrix type. Five levels of requirements for handling samples are defined based on the radiation control zone ( $R C Z$ ) in which the analytical activity takes place:

$\begin{array}{ll}\text { - } & \text { R0 - No radiation containment required } \\ \text { - } & \mathbf{R 1} \text { - Bench top or lab hood in RCZ } \\ \text { - } & \mathbf{R} 2 \text { - Shielded hood in RCZ required } \\ \text { - } & \mathbf{R} 4 \text { - Hovebox in } R C Z \text { required } \\ \end{array}$

Note that no absolute ranges of sample radiation are given, but instead the level of radiation protection needed to handle the sample is given. Working with samples in radiation control zones will adversely impact the number of analytical operations that personnel can do in a given time. This project requires that capacities be estimated for major laboratory operations performed in radiation control zones. Site-specific activity levels are requested for each defined radiation control zone. 
Sample matrices must also be considered in determining analytical capacity, since the number of times that a major analytical operation may be repeated is a function of the difficulty that the sample matrix presents. This model requires capacities to be estimated for each of three levels of sample matrix difficulty:

- M1 - Customer sample matrix typically requiring only one laboratory operational cycle per customer sample processed, e.g., water.

- M2 - Customer sample matrix requiring on average two or less laboratory operational cycles per customer sample processed, e.g., soil, sediment, rock, air, biota.

- M3 - Customer sample matrix requiring on average more than two laboratory operational cycles per customer sample processed, e.g., sludge, waste, pure product, debris.

The user is required to input the estimated percentage of each sample matrix type analyzed in order for the model to calculate a "combined" capacity. This combined capacity represents the capacity for the laboratory based on the operational parameters input for the assumed mix of sample types.

\subsection{Cycle Times and Operating Times}

The amount of time spent performing specific operations in a laboratory is critical to making any estimate of laboratory capacity. Two classes of times are used in this model:

- Cycle Time - A cycle time is the time to complete one cycle (laboratory operation cycle, sample analysis cycle, or other cycle)

- Operating Time - An operating time is the total time spent during a specific time period performing a laboratory operation.

In this model weekly operating times are used. Weekly operating times can be related to cycle times by the following equation:

Weekly operating time $=$ Cycle time for operation $\times$ Number of times for an operation operation is performed per week

Specific cycle and operating times are used in this model as defined below:

- Operational Cycle Time - The operational cycle time is the time taken to complete one laboratory operation cycle on a customer or QC sample.

- Calibration Cycle Time - The calibration cycle time is the time taken to complete one calibration cycle of an instrument or process. This time is distinguished from other operational cycle times, since the time to run a calibration sample often differs from the time to run other samples. This cycle time may also include any time required in the calibration step for tuning an instrument or for rerunning any calibration samples. 
- Calibration Operating Time (CT) - Weekly operating time required to calibrate an instrument or system for a defined laboratory operation. CT is a function of instrument requirements and method-specific requirements. Calibration samples include initial calibration samples and calibration blanks only. Calibration verification and check standards are considered to be QC samples.

$\mathrm{CT}=$ average calibration cycle time $\mathrm{x}$ average \# of calibration samples per week

- Customer Sample Operating Time (ST) - Weekly operating time to run customer samples for a defined laboratory operation.

ST = average customer sample cycle time $\times$ average \# of customer samples per week

- Quality Control Operating Time (OT) - Weekly operating time required to run quality control samples for a defined laboratory operation. QC samples include calibration verification, duplicates, laboratory blinds, double-blinds (including PE samples), bench controls, and method required $O C$ samples (matrix spikes and duplicates).

OT = average $\mathrm{OC}$ cycle time $\mathrm{x}$ average \# of $\mathrm{OC}$ samples per week

- Average Operating Time - Average time an instrument operates or a process is performed per week (i.e. the average time per week that an instrument is actually analyzing samples or that a process is actually being performed). This time should not include instrument idle time (i.e. time that the instrument is on but not analyzing samples) nor should it include time required for warm-up and shut-down. This information should be available from run logs.

Note that an inaccurate overestimation of the average operating time is usually the largest source of error in making analytical capacity estimates. The model therefore allows calculation of an average operating time from calibration, customer sample and $\mathrm{OC}$ operating times that may be compared to the estimated value.

- Calculated Average Operating Time - Average operating time per instrument calculated from component operating times according to the equation below:

Calculated Average $=(C T+S T+Q T) /$ number of instruments Operating Time 
Note that for batch operations the calculated average operating time is equal to (CT $+\mathrm{ST}+\mathrm{QT}$ ) / ( number of batches $\mathrm{x}$ the number of samples per batch). (See Section 4). 


\subsection{BASIS FOR CAPACITY MODEL}

\subsection{Background}

This project addresses both analytical capability (the analyses a laboratory is able to perform on a given sample type) and the analytical capacity (how many of these measurements a laboratory is able to complete in a given time). Both capacity and capability considered in this model will include, as a minimum, the key sample analyses listed in Table I. The project will include analytical capability and capacity being used for EM programs as well as EM applicable analytical resources currently being used for other DOE programs. Previous estimates of capacity have traditionally been done using a "best estimate" based on existing sample throughput and extrapolating to full shift implementation. The first Complex-wide analytical needs assessment and capacity and capability determination was done by the DOE Future Analytical Support Team (FAST) in 1990 through surveying EM program managers and DOE analytical laboratories. The FAST study suffered from not having a clear definition of terms (i.e., what is a sample and what is an analysis) and inconsistent interpretations. Individual site determinations of analytical capability are frequently being performed and tend to suffer from the same inconsistencies.

The analytical capacity assessment will be a companion project to the DOE analytical needs assessment. Both projects use consistent definitions for sample, sample analysis, sample matrix, radiation containment requirements, etc. (see Section 2.0 and Appendix A). These projects will be ongoing and will require revision as EM programs change.

This model is considered to only estimate the analytical capacity of a laboratory. An ability to develop a model that will determine analytical lab capacity has not yet been realized. Application of this model, with a reasonable effort to collect the required input data, is expected to yield results to within $30-35$ percent of the actual lab capacity.

\subsection{General Approach to Estimating Capacity}

This approach to estimating the DOE Complex analytical capacity to analyze customer samples is based on a clear set of definitions and uses a Theoretical Maximum of Operational Cycles (TMOC) for each analytical operation as a bench mark. The TMOC value is then adjusted, using individual laboratory operations factors, to arrive at an actual annual capacity for specific laboratory operations.

This approach involves the following steps:

- Precisely defining all the terms used in the model so that there is consistency in the data collected for the different analytical laboratories in the Complex. 
- Defining the key sample analyses required in analyzing EM customer samples. These sample analyses will include as a minimum those in Table 1, but can include others considered as potentially rate-limiting by the user.

- Defining laboratory operating factors (both facility- and personnel-limited) that influence the number of sample analyses that can be performed.

- Determining the TMOC and the annual laboratory capacities for each key major lab operation.

- Determining annual capacities for each sample analysis.

The capacity of a laboratory to analyze various customer sample matrices using EPA and DOE-specified methodology is a function of the analytical needs at a given time. Many laboratories change capability as well as capacity as needs change. The analytical protocol (EPA, DOE and State-approved methods) used by a lab are a function of mission. In this project, laboratories will be asked to give the present distribution of work supporting the current site mission. Capacities for specific radiation control zones will be calculated for current operations and future maximized operations assuming a given mix of sample matrices and analytical protocol. The model assumes recalculation of capacity if the above factors change.

\subsection{Applicability of the Capacity Model}

This model has been piloted at two high-volume DOE laboratories that are focused on analyzing non-radioactive and low-level, contact-handled radioactive environmental and waste samples. Based on the results of these two pilots, the model demonstrated that sample analysis capacities can be estimated to within about 35 percent of actual numbers of sample analyses completed. This degree of confidence in predicting sample analysis capacity is quite acceptable, since the companion analytical needs assessment is expected to yield results with no greater confidence.

The following points regarding application of the model should be noted:

- Laboratory operation factors are time-averaged over one year for a defined sample matrix, radiation control zone, and analytical protocol. If the model is to be applied to lab operations that are not performed at a steady rate, then the user must develop operational parameters for an assumed laboratory operation. When laboratory operations are projected to change, the model must be rerun to determine changes in capacity.

- To project capacity resulting from future addition of resources (people and equipment), the model must be rerun with redefined operational factors.

- Calculating sample analysis capacities for samples that require special containment (R2-R4) levels may yield results with greater uncertainty. 
- Using the model to project capacity when there are shared resources involved (such as instruments, people or facilities) requires an up-front estimation of the distribution of these resources among the competing analytical needs.

- For a specific sample analysis, each operation is assumed to be a weighted average of all potential operation types. For example, if sample prep $A$ is used for $75 \%$ of sample analysis $Z$, and sample prep $B$ is used for $25 \%$ of sample analysis $Z$, then the input data used for the sample preparation laboratory operation for sample analysis $Z$ would be $0.75 \times$ (data for sample prep A) + $0.25 \times$ (data for sample prep B). The same principle applies to instrumental analysis and data reporting operations.

Future versions of this model may include resource loading factors that will account for the effects of shared resources on analytical capacities. The user will need to determine the applicability of these factors.

\subsection{Using the Model as a Lab Management Tool}

This capacity model can be adapted to be used as an effective management tool in the following decision-making processes:

- Determining if additional samples can be accepted, what kinds of analyses can be performed, and when the samples can be accepted.

- Determining when and where to add people and equipment to the laboratory.

- Determining what kind of instrumentation to procure and what personnel skills are needed.

- Estimating the equipment and staffing needs for new lab construction planning.

\subsection{Data Objectives}

Before the model is implemented, the user must clearly define the intended use of the data to be obtained. If the results are to be used to predict capacity for non-rad and contact-handled rad samples, the model as presented here will be adequate. The model works well for high-volume laboratories with enough resources to meet the analytical needs without a significant amount of shared resources.

If the objective of the model is to manage shared laboratory resources, then an additional level of detail beyond that given in the current model may be necessary. Other versions of the model are being developed to account for competing needs for personnel and equipment. 


\subsection{MEASURING LABORATORY CAPACITY}

\subsection{Background}

The first step in measuring analytical capacity is to determine the minimum scope and level of detail required that will meet the data objectives (see Section 3.5). A capacity model based on the definitions in Section 2 was developed to estimate the output of each major laboratory operation assuming a sample mix (matrix and analysis protocol) received by the lab. When determining UC, the sample mix received over the past year is assumed, and when determining $E C$ and $M C$, a sample mix that reflects the programs to be supported must be assumed. If this sample mix is expected to change, the factors in the model must reflect these changes. The laboratory operations making up the sample analyses shown in Table I contain both peoplelimited and facility-limited operations and either one can limit the number of samples that can be analyzed by the laboratory. This model assumes a fixed amount of lab space, but additional instruments and people can be added to the limits of existing lab space to achieve upgradable capacity (MC).

This model will estimate capacities based on current sample mix and analytical protocol in place. The model can be rerun for variations of sample mix and programs as lab missions change.

\subsection{Theoretical Maximum Operational Cycles}

The first parameter established in the capacity model is the theoretical maximum of operational cycles (TMOC) of each major analytical laboratory operation. For sample preparation and instrumental analysis operations, the TMOC is established by assuming continuous performance of the lab operation using optimal batch sizes. TMOC is the theoretical number of operational cycles (analytical, preparation, or other) that can be performed in one year. Under this idealized situation, all the instrument time and preparation time is spent performing the laboratory operation on customer samples and there is no lost time due to any reason such as maintenance, calibration, or personnel availability.

Major laboratory operations are either performed in series (e.g. introduction of samples to an instrument) or performed in parallel (e.g. preparation of batches of samples on a hot plate simultaneously). Generally, instrument-based laboratory operations and data reporting operations are serial, while sample preparations tend to be parallel.

The capacity of most laboratory operations is either limited by personnel availability or by the lack of facilities such as hoods, work benches, or instruments. Parallel operations such as sample preparation and data reporting are predominately peoplelimited, whereas serial operations such as GC/MS are predominately instrumentlimited. The model addresses these limitations based on the length of time that the operation is performed and the number of instruments available. 
For serial major laboratory operations, the TMOC is based on assuming processing of customer laboratory samples for $24 \mathrm{hrs} /$ day, 7 days/wk, 365 days/yr. TMOC for a given laboratory operation is then the total number of minutes in one year divided by the number of minutes required to complete one operational cycle, or:

TMOC $=\frac{525,960 \mathrm{~min} / \mathrm{yr}}{\text { \# of minutes/operational cycle }}$

For example, if an instrument cycle time for a VOA analysis operation by GC/MS is 45 minutes (i.e. the time required for the GC/MS to purge, trap, and analyze the sample and be ready for the next sample) then the TMOC is 11,688 VOA analytical cycles per year.

For parallel laboratory operations the TMOC is based on batch processing (most sample preparations will be batch processed). The batch size must first be determined for the process. The batch size may be, for example, 20 beakers on a hot plate, 10 cyanide stills, or 6 separatory funnels for semi-VOA extraction. The batch size is determined from actual laboratory experience in UC calculations, but is optimized based on facility and sample availability when EC or MC are calculated.

$\mathrm{TMOC}=\frac{525,960 \mathrm{~min} / \mathrm{yr} \times \mathrm{BF}}{\# \text { of minutes } / \text { operational cycle }}$

where: $\mathrm{BF}=$ total number of samples in the batch.

For example, if 6 semi-VOA extractions are performed simultaneously by a technician and the process requires five hours to finish the process through concentration, then the TMOC is 10,519 extractions/year.

TMOC values are universal and should be the same for identical procedures and instrumentation, independent of the laboratory being modelled except in UC estimates of parallel operations when the TMOC value will depend on the actual number of samples used in a batch.

\subsection{Operational Correction Factors}

A series of operational corrections are then applied to the TMOC to account for customer sample analysis time lost or gained due to the following factors:

- Number of Available Units

- Equipment Availability

- Facility Availability

- Personnel Availability

- Operation Availability

- Instrument Calibration

- Quality Control

- Matrix Effects 
These factors should address all the conditions that will increase or decrease the number of customer samples that a laboratory can analyze. These factors are laboratory-dependent and are based on current and planned laboratory operations (matrix, procedure, rad level, sample load, available personnel and instruments, etc). Values for the correction factors as defined in Section 4.4 are composites based on the current sample mix or the planned sample mix for future work.

To calculate utilized capacity $(U C)$ the factors must reflect current sample mix, sample load per analytical run, the number of calibration and $\mathrm{QC}$ samples per run, and the amount of time that instruments or preparation procedures are operated. On the other hand, existing capacity (EC) must be calculated by loading all major laboratory operations to the limits of existing staff and instruments. For example, EC calculations would assume that all batch sizes and existing instrument automation are optimized. Upgradable capacities (MC) must be calculated by loading the entire laboratory with maximum shifts, numbers of instruments, and sample loads that can be accommodated within the present laboratory buildings and yet maintain efficient operations. The operational factors must be computed for each of these capacity calculations. The only parameters that should vary significantly between the EC and MC scenarios are the amount of time the operation is performed and the number of instruments, preparation set-ups, and other equipment available.

Operational factors could be developed for all individual steps in the sample analysis process, e.g. for sample receipt, radiation screening, aliquoting, extraction, instrumental analysis, report preparation, and data review. In the present application of the model, however, all the steps in the sample analysis process are collected into the three laboratory operations: sample preparation, instrumental analysis, and data reporting. In the above example, the sample preparation operation would include sample receipt, radiation screening, aliquoting, and extraction, while the data reporting laboratory operation would include both report preparation and data review.

Operational factors could also be developed for each sample stream analyzed by a defined environmental protocol. While such detailed capacity estimates may be needed to meet some site-specific data objectives, the current model only requires an estimate of the operational factors based on the anticipated mix of work.

\subsection{Definition of Operational Correction Factors}

Once the TMOC values have been defined for each major laboratory operation, the next task is to identify and quantify the factors that reduce the TMOC to annual capacities. Development of the operational correction factors are significant to a meaningful calculation of laboratory capacities and should also be useful for lab managers to identify opportunities for capacity improvements. The model user is cautioned to verify all factors used in the capacity calculations. The following factors are calculated:

A. N: Number of Available Units -- For serial operations such as instrumental analyses, $\mathrm{N}$ will equal the number of instruments. For parallel major laboratory 
operations, such as semi-VOA extractions, $\mathrm{N}$ could be the number of batches that can be processed in one shift.

$\mathrm{N}=$ number of available units

- Note: For parallel operations, the number of sample aliquots processed in the batch is included in the batch factor (BF) used in the TMOC calculation.

B. FA: Facility Availability -- This factor accounts for the loss of time available for a major laboratory operation due to support facility failure (e.g. HVAC failure, hood failure, etc)

\section{$F A=1-$ total facility down time for previous year/days 365 days}

C. EA: Equipment Availability -- This factor accounts for equipment down time due to maintenance and equipment failure when the instrument is not available to analyze samples.

\section{$E A=1$ - equipment down time for previous vear/days 365 days}

D. PA: Personnel Availability -- This factor accounts for the loss of ability to perform a laboratory operation because personnel are not available due to time off for vacation, illness, personal reasons, training, and meetings, etc., and to time lost because of poor work efficiency, rework due to human error, and insufficient workload.

\section{$P A=1$ - hours/week lost due to personnel unavailability or inefficiency hours/week personnel are scheduled to work}

For example, if a technician performing an ICP operation works with overtime, on average, $44 \mathrm{hrs} /$ week, spends 6 hours/week in training and meetings, is off work an average of $4 \mathrm{hrs} / \mathrm{wk}$, and requires 1 hour per week for rework due to errors, then PA $=1-11 / 44$ or $P A=0.75$

E. OA: Operation Availability -- This factor reflects the fraction of time that a laboratory operation is performed.

For UC estimates, this is the fraction of time an instrument is actually analyzing samples or a process is actually being performed. For example, if for an 8-hour shift, an instrument is turned on 30 minutes into the shift, it requires one hour to warm up, it is idle for an average of two hours per day, and 30 minutes is required to shut down the instrument at the end of the shift, then the daily operations time is 4 hours/day $(8-1 / 2-1-2-1 / 2)$ or 20 hours/wk for a five- 
day week. One potential source of this information can be taken from an operator's run-log book.

For EC and MC estimates, this factor represents the fraction of time an instrument is capable of analyzing samples or a process is capable of being performed. Idle time should be minimized in such estimates.

$\mathrm{OA}=\frac{\text { average operating time in hrs } / \mathrm{wk}}{168 \mathrm{hrs} / \mathrm{wk}}$

This factor is most significant in determining the time that an instrument is operating or a preparation operation is being performed. This factor accounts for: (1) the amount of time the instrument is not operated because no personnel, samples, or equipment are available due to shift coverage or competition from other operations; $(2)$ the impact that instrument setup can have on operating efficiency; and (3) the gain in operating time when the instrument or process is operated by automation. Therefore, for OA equal to 0.24 for a 40 hour/wk work schedule implies that the instrument is capable to run sample analyses $100 \%$ of work schedule time (not withstanding any gain due to overtime). Any value equal to 0.24 should be closely evaluated.

The OA factor will accommodate situations when people or instruments must be shared between two or more laboratory operations. For example, if a GC instrument is used $25 \%$ of the time for pesticide determinations and $75 \%$ of the time for PCB determinations, then the OA factor for these two operations would reflect this resource sharing. The same type of OA factor calculation can be done for people sharing. Note, however, that the time personnel are not available due to absences, vacation, training, etc. is included in the PA factor.

The numerator of this factor must include only the time the instrument is lor planned to be) in actual operation analyzing samples (customer, calibration, or QC) either manually or automatically during each week.

F. IC: Instrument Calibration -- This factor represents the fraction of operating time applied to calibration samples.

$I C=\frac{C T}{C T+S T+Q T}$

The terms CT, ST, and QT are component operating times defined in Section 2.4. The sum of CT, ST, and $\mathrm{QT}$ equals the calculated average operating time (also defined in Section 2.4).

G. QC: Quality Control -- This factor represents the fraction of operating time applied to quality control samples. 
$\mathrm{OC}=\frac{\mathrm{QT}}{\mathrm{CT}+\mathrm{ST}+\mathrm{QT}}$

Note that the fraction of the calculated average operating time available for analysis of customer samples, on accounting for the time required for calibration and quality control, is equal to $1-I C-O C$.

H. ME: Matrix Effect -- This factor is inversely related to the average number of times (cycles) that a laboratory operation must be carried out in order to generate acceptable results for a sample of a given matrix type. For example, groundwater samples may yield satisfactory data after a single instrumental analytical operational cycle, but a set of waste samples may require an average of two dilutions and reanalyses per sample to achieve usable data. Note that instrument down-time due to contamination by high concentration samples should be accounted for under the EA factor, while the additional effort required for reanalysis of diluted samples should be reflected here.

$\mathrm{ME}=\frac{\text { number of customer samples processed }}{\text { total number of laboratory operational cycles required }}$

The number of operational cycles required includes all cycles for the original laboratory operation plus all cycles due to having to rerun the sample due to a QC failure. This includes any non-routine cycles needed to recalibrate the instrument. For example, if one of three soil or sediment samples usually needs rerunning the ME will equal 0.75 . Matrix effect factors can be generalized as to degree of difficulty and the model will calculate an annual capacity value for each of the three sample matrix types defined in Section 2.3.

\subsection{Calculation of Annual Capacity}

The accompanying reporting formats and instructions package provides a spreadsheet system for automatically calculating various $A C$ values. These calculations are described below:

\subsubsection{Calculation of Laboratory Operation Annual Capacity}

The annual capacity $(A C)$ for each major laboratory operation is calculated in the model by inserting the values for each of the applicable operational correction factors into the equation below:

$A C=T M O C \times N \times F A \times E A \times P A \times O A \times(1-I C-O C) \times M E$

ACs are calculated for each sample matrix (M1, M2, M3). Values for UC, EC or MC for current (UC) or projected (EC, MC) laboratory operations are then obtained by calculating a compounded $A C$ value based on the distribution of samples between the three matrix types. This is achieved by summing the products of the $A C$ value for each matrix type and the fraction of samples of that matrix type analyzed or projected 
to be analyzed. For example, if the $A C$ for a lab operation on $M 1$ samples by $A A$ is 9,100 and 8,600 for $M 2$, and the ratio of samples received by the lab is $3 M 1: 1 M 2$, then $6,825(9100 \times 3 / 4) \mathrm{M} 1$ laboratory operational cycles and 2,150 (8600 $\times 1 / 4)$ M2 laboratory operational cycles are the computed capacities for the current lab operations. The sum of these two numbers would be compared to actual samples analyzed for UC or would represent EC or MC for that projected mix of samples.

\subsubsection{Calculation of Sample Analysis Annual Capacity}

The capacity of a laboratory for a given sample analysis will be limited by the component laboratory operation with the least capacity. The model therefore determines the sample analysis capacity by finding the minimum capacity for the laboratory operations that make up the sample analysis. For example if a laboratory can perform 7200 semi-VOA instrumental customer sample operational cycles per year, and is capable of generating 12000 semi-VOA data reports per year, but can only perform 5400 semi-VOA extraction customer sample operational cycles per year, then the annual capacity for semi-VOA sample analyses is only 5400 customer sample analyses per year.

\subsubsection{Summary of Customer Sample Annual Capacity}

A laboratory's capacity to analyze customer samples is likewise limited by the capacity of the least efficient sample analysis. A final step in the capacity estimation process would therefore be to determine the customer sample capacity. This is accomplished by finding the minimum of the sample analysis capacities for the sample analyses required in analyzing the customer samples.

This model does not perform this calculation since it is not designed to predict actual number of customer samples that a laboratory can analyze. The model user will need to make this prediction through analysis of customer sample needs assessments and the sample analysis capacity estimates from the spreadsheets.

\subsection{Implementation of Capacity Model}

This model description package and the accompanying user guide are being distributed to the major DOE analytical laboratories of which EM-563 is aware. All these laboratories are requested to complete the model and return the data to EM-563 by the date specified in the user guide.

\subsubsection{Input of Data into Model}

Data should be provided for each of the three annual capacities (UC, EC, and MC) in each of the radiation control zones present in the laboratory.

The work distribution for a laboratory strongly reflects the site's mission, and the analytical protocol implemented will affect sample analysis capacity. Some DOE laboratories are currently devoting the majority of their effort to supporting 
environmental and waste management programs. Other labs have some site production, waste treatment, and R\&D programs to support. The model therefore requires input of the current mix (UC) or anticipated mix (EC and $M C$ ) of analytical protocols for each sample analysis, and the data entered into the model is divided into data pertaining to regulatory analytical protocols and data pertaining to non-regulatory analytical protocols.

\subsubsection{Calibration of the Annual Capacity Model}

The model for calculation of UC for major analytical operations can be "calibrated" by comparing the UC values for each major laboratory operation and sample analysis with the actual numbers of laboratory operations and sample analyses completed. If large discrepancies exist, the data entered into the model together with the operational correction factors computed should be carefully analyzed in order to resolve the differences. The UC factors will provide a basis for factors used in EC and MC calculations.

\subsubsection{Collection of Capacity Information by EM-563}

All the capacity information obtained at the DOE laboratories will be collected by EM 563 and will be stored in a database. The sample analysis capacity data will be sorted according to laboratory, capacity type, radiation control zone, and sample matrix type. DOE-wide analytical capacity available to EM may then be estimated, and unused capacity potentially available may be estimated from the difference between UC values and EC or MC values. Analysis of this data, along with data collected in a parallel project on EM analytical needs, will allow EM-563 to make the programmatic decisions necessary to make sampling and analysis activities considerably more effective within EM. Site laboratories may also wish to make the capacity information that is collected available to their site OSMs for use in assigning work or samples to their laboratory. 


\section{APPENDIX A}

Definitions of Terms Used in Estimating DOE Analytical Capacity Available to EM

\begin{tabular}{|l|l||}
\hline \multicolumn{1}{|c|}{ Term } & \multicolumn{1}{c|}{ Organization/Management } \\
\hline EM-563 & $\begin{array}{l}\text { Office of Environmental Restoration and Waste } \\
\text { Management's Laboratory Management Division }\end{array}$ \\
\hline $\begin{array}{l}\text { Commercial } \\
\text { Laboratory }\end{array}$ & $\begin{array}{l}\text { A privately or publicly owned laboratory operated for profit } \\
\text { and subject to NRC/State licensing when handling } \\
\text { radioactive materials }\end{array}$ \\
\hline DOE Laboratory & $\begin{array}{l}\text { DOE Complex laboratory operated by M\&O/ERMC } \\
\text { contractor and not subject to NRC/State licensing when } \\
\text { handling radioactive materials }\end{array}$ \\
\hline OSM & \begin{tabular}{l} 
Office of Sample Management or equivalent operation \\
\hline Capabilities and Capacities
\end{tabular} \\
\hline Capability & $\begin{array}{l}\text { The types of analytical measurements a laboratory is able } \\
\text { to perform on a given sample type. }\end{array}$ \\
\hline Capacity & $\begin{array}{l}\text { The number of analytical measurements a laboratory is } \\
\text { able to complete in a given time. }\end{array}$ \\
\hline $\begin{array}{l}\text { Utilized Capacity } \\
\text { (UC) }\end{array}$ & $\begin{array}{l}\text { The capacity being utilized by the analytical laboratory to } \\
\text { support the current work load. }\end{array}$ \\
\hline $\begin{array}{l}\text { Existing Capacity } \\
\text { (EC) }\end{array}$ & $\begin{array}{l}\text { The analytical capacity that exists with current personnel } \\
\text { levels, existing equipment and sample loads optimized. }\end{array}$ \\
\hline $\begin{array}{l}\text { Upgradable } \\
\text { Capacity (MC) }\end{array}$ & $\begin{array}{l}\text { The capacity of an analytical laboratory when upgraded to } \\
\text { the optimum number of staff for 7 day/wk, 24 hr/day } \\
\text { operation and the optimum level of instrumentation, } \\
\text { without construction of any additional laboratory space but } \\
\text { with limited non-capital refurbishment of existing space. }\end{array}$ \\
\hline Customer Sample & $\begin{array}{l}\text { Any sample sent to the laboratory to be analyzed } \\
\text { separately. These include field samples, field QA samples } \\
\text { (e.g., duplicate field samples, field blanks, PE samples) } \\
\text { sample phases and composite samples. }\end{array}$ \\
\hline Calibration Sample & $\begin{array}{l}\text { A sample that a laboratory analyzes to meet the } \\
\text { requirements of a method. }\end{array}$ \\
\hline A sample used for calibrating an instrument or process. \\
\hline \multicolumn{1}{|c|}{ Sample }
\end{tabular}




\begin{tabular}{|c|c|}
\hline Term & Definition \\
\hline EM Sample & A customer sample that is funded with EM money. \\
\hline RO & $\begin{array}{l}\text { Customer sample deemed non-radioactive and may be } \\
\text { handled out of any radiation control area }\end{array}$ \\
\hline R1 & $\begin{array}{l}\text { Customer sample that may be contact handled, but must } \\
\text { be in a radiation area }\end{array}$ \\
\hline $\mathbf{R 2}$ & Customer sample that must be handled in a shielded hood \\
\hline R3 & Customer sample that must be handled in a glove box \\
\hline R4 & Customer sample that must be handled in hot cell \\
\hline M1 & $\begin{array}{l}\text { Customer sample matrix typically requiring only one } \\
\text { laboratory operational cycle per customer sample } \\
\text { processed. Example: water }\end{array}$ \\
\hline M2 & $\begin{array}{l}\text { Customer sample matrix requiring on average two or less } \\
\text { laboratory operational cycles per customer sample } \\
\text { processed. Examples: soil, sediment, rock, air, biota }\end{array}$ \\
\hline M3 & $\begin{array}{l}\text { Customer sample matrix requiring on average more than } \\
\text { two laboratory operational cycles per customer sample } \\
\text { processed. Examples: sludge, waste, pure product, debris }\end{array}$ \\
\hline $\begin{array}{l}\text { Radiation Control } \\
\text { Zone }\end{array}$ & $\begin{array}{l}\text { The area of a laboratory in which radioactive samples are } \\
\text { required to be handled }\end{array}$ \\
\hline \multicolumn{2}{|r|}{ Sample Analyses and Laboratory Operations } \\
\hline Sample Analysis & $\begin{array}{l}\text { The distinct set of major laboratory operations required to } \\
\text { generate a specific set of data on a customer sample }\end{array}$ \\
\hline VOA-GC & Volatile organic analysis by gas chromatography (GC) \\
\hline VOA-GC/MS & $\begin{array}{l}\text { Volatile organic analysis by gas chromatography/mass } \\
\text { spectrometry (GC/MS) }\end{array}$ \\
\hline Semi-VOA & Semi-volatile organic analysis by GC/MS \\
\hline Pest & Pesticide analysis by GC \\
\hline Herb & Herbicide analysis by GC \\
\hline PCB & Polychlorinated biphenyl analysis by GC \\
\hline ICP-AES Metals & $\begin{array}{l}\text { Inductively Coupled Plasma - Atomic Emission } \\
\text { Spectroscopy (ICP-AES) measurement of a single metal } \\
\text { analyte or a suite of metal analytes }\end{array}$ \\
\hline
\end{tabular}




\begin{tabular}{|c|c|}
\hline Term & Definition \\
\hline ICP-MS Metals & $\begin{array}{l}\text { Inductively Coupled Plasma - Mass Spectrometry (ICP-MS) } \\
\text { measurement of a single metal analyte or a suite of metal } \\
\text { analytes }\end{array}$ \\
\hline GF-AA Metals & $\begin{array}{l}\text { Graphite Furnace Atomic Absorption (GF-AA) } \\
\text { measurement of individual metal analytes }\end{array}$ \\
\hline Mercury & $\begin{array}{l}\text { Cold Vapor Atomic Absorption (CV-AA) measurement of } \\
\text { mercury }\end{array}$ \\
\hline Cyanide & Measurement of cyanide \\
\hline Anions/Cations & $\begin{array}{l}\text { Anion/cation analysis, by ion chromatography or wet } \\
\text { chemistry (counted individually by ions, e.g., nitrate, or } \\
\text { ammonium for single analysis, or by ion chromatography } \\
\text { runs for simultaneous analyses) }\end{array}$ \\
\hline Gross Alpha/Beta & Gross alpha and beta counting \\
\hline $\begin{array}{l}\text { Alpha Spec } \\
\text { Isotopes }\end{array}$ & Spectrometric analysis of alpha-emitting nuclides \\
\hline $\begin{array}{l}\text { Gamma Spec } \\
\text { Isotopes }\end{array}$ & Spectrometric analysis of gamma-emitting nuclides \\
\hline TCLP Extraction & $\begin{array}{l}\text { Toxicity Characteristic Leaching Procedure (TCLP) - } \\
\text { regulatory method of sample extraction used to estimate } \\
\text { the leaching potential of contaminants from landfills - e.g., } \\
\text { Method } 1311\end{array}$ \\
\hline $\begin{array}{l}\text { Laboratory } \\
\text { Operation }\end{array}$ & $\begin{array}{l}\text { A potentially rate-limiting element of an individual sample } \\
\text { analysis (sample preparation, instrumental analysis, data } \\
\text { reporting) }\end{array}$ \\
\hline Parallel Operation & $\begin{array}{l}\text { Laboratory operation in which units are run concurrently, } \\
\text { such as sample preparation batch processing }\end{array}$ \\
\hline Serial Operation & $\begin{array}{l}\text { Laboratory operation in which units are run sequentially, } \\
\text { such as introduction of samples into an instrument }\end{array}$ \\
\hline $\begin{array}{l}\text { Sample } \\
\text { Preparation }\end{array}$ & $\begin{array}{l}\text { Preparation of sample aliquots for one or more laboratory } \\
\text { operation }\end{array}$ \\
\hline $\begin{array}{l}\text { Instrumental } \\
\text { Analysis }\end{array}$ & Generation of raw analytical data from samples \\
\hline Data Reporting & $\begin{array}{l}\text { Data review, reduction and report/deliverable preparation } \\
\text { required on average for reporting analytical results to a } \\
\text { customer }\end{array}$ \\
\hline
\end{tabular}




\begin{tabular}{|c|c|}
\hline Term & Definition \\
\hline \multicolumn{2}{|r|}{ Cycles, Cycle Times, and Operating Times } \\
\hline Cycle & Level of effort required to perform a single activity once \\
\hline $\begin{array}{l}\text { Sample Analysis } \\
\text { Cycle }\end{array}$ & $\begin{array}{l}\text { Effort required to perform a sample analysis on a single } \\
\text { customer sample }\end{array}$ \\
\hline $\begin{array}{l}\text { Laboratory } \\
\text { Operational Cycle }\end{array}$ & $\begin{array}{l}\text { Effort required to perform an individual laboratory } \\
\text { operation. }\end{array}$ \\
\hline Cycle Time & $\begin{array}{l}\text { Time required to complete one cycle (laboratory operation } \\
\text { cycle, sample analysis cycle, or other cycle) }\end{array}$ \\
\hline $\begin{array}{l}\text { Operational Cycle } \\
\text { Time }\end{array}$ & $\begin{array}{l}\text { Time required to complete one laboratory operation cycle } \\
\text { on a customer or } Q C \text { sample }\end{array}$ \\
\hline $\begin{array}{l}\text { Calibration Cycle } \\
\text { Time }\end{array}$ & $\begin{array}{l}\text { Time required to complete one calibration cycle of an } \\
\text { instrument or process. This time is distinguished from } \\
\text { other operational cycle times since the time to run a } \\
\text { calibration sample often differs from the time to run other } \\
\text { samples. This cycle time may also include any time } \\
\text { required for tuning an instrument or for rerunning any } \\
\text { calibration samples. }\end{array}$ \\
\hline Operating Time & $\begin{array}{l}\text { Total time spent during a specific time period performing a } \\
\text { laboratory operation. In this model weekly operating times } \\
\text { are used. }\end{array}$ \\
\hline $\begin{array}{l}\text { Calibration } \\
\text { Operating Time } \\
\text { (CT) }\end{array}$ & $\begin{array}{l}\text { Weekly operating time required to calibrate an instrument } \\
\text { or system for a defined laboratory operation. CT }= \\
\text { average calibration cycle time } \mathrm{x} \text { average } \# \text { of calibration } \\
\text { samples per week. }\end{array}$ \\
\hline $\begin{array}{l}\text { Customer Sample } \\
\text { Operating Time } \\
\text { (ST) }\end{array}$ & $\begin{array}{l}\text { Weekly operating time available to run customer samples } \\
\text { for a defined laboratory operation. ST = average } \\
\text { customer sample cycle time } x \text { average \# of customer } \\
\text { samples per week. }\end{array}$ \\
\hline $\begin{array}{l}\text { Quality Control } \\
\text { Operating Time } \\
\text { (QT) }\end{array}$ & $\begin{array}{l}\text { Weekly operating time required to run quality control } \\
\text { samples for a defined laboratory operation. OT }=\text { average } \\
O C \text { cycle time } x \text { average } \# \text { of } O C \text { samples per week. }\end{array}$ \\
\hline $\begin{array}{l}\text { Average Operating } \\
\text { Time }\end{array}$ & $\begin{array}{l}\text { Average time an instrument operates or a process is } \\
\text { performed per week (i.e. the average time per week that } \\
\text { an instrument is capable of analyzing samples or that a } \\
\text { process may be performed) }\end{array}$ \\
\hline
\end{tabular}




\begin{tabular}{|c|c|}
\hline Term & Definition \\
\hline $\begin{array}{l}\text { Calculated } \\
\text { Average Operating } \\
\text { Time }\end{array}$ & $\begin{array}{l}\text { Average operating time calculated by summing the } \\
\text { component operating times } \mathrm{CT}, \mathrm{ST} \text {, and } \mathrm{OT}\end{array}$ \\
\hline \multicolumn{2}{|r|}{ Capacity Correction Factors } \\
\hline $\begin{array}{l}\text { Theoretical } \\
\text { Maximum of } \\
\text { Operational Cycles } \\
\text { (TMOC) }\end{array}$ & $\begin{array}{l}\text { The theoretical number of operational cycles that can be } \\
\text { performed in one year }\end{array}$ \\
\hline Batch Factor (BF) & $\begin{array}{l}\text { The number of parallel laboratory operations that are or } \\
\text { may be performed simultaneously on a single instrument or } \\
\text { piece of equipment. This factor is determined from actual } \\
\text { laboratory experience when UC is calculated, but is } \\
\text { optimized based on facility and sample availability when } \\
\text { EC or MC are calculated. }\end{array}$ \\
\hline $\begin{array}{l}\text { Number of } \\
\text { Available Units (N) }\end{array}$ & $\begin{array}{l}\text { Number of instruments or other pieces of equipment } \\
\text { available for carrying out a specified laboratory operation }\end{array}$ \\
\hline $\begin{array}{l}\text { Facility Availability } \\
\text { (FA) }\end{array}$ & $\begin{array}{l}\text { Factor accounting for loss of time available for a major } \\
\text { laboratory operation due to support facility failure (e.g. } \\
\text { HVAC failure, hood failure, etc.) equal to } 1 \text { - (total facility } \\
\text { down time for previous year/days / } 365 \text { days) }\end{array}$ \\
\hline $\begin{array}{l}\text { Equipment } \\
\text { Availability (EA) }\end{array}$ & $\begin{array}{l}\text { Factor accounting for equipment down time due to } \\
\text { maintenance and equipment failure when the instrument is } \\
\text { not available to analyze samples equal to } 1 \text { - (equipment } \\
\text { down time for previous year/days / } 365 \text { days) }\end{array}$ \\
\hline $\begin{array}{l}\text { Personnel } \\
\text { Availability (PA) }\end{array}$ & $\begin{array}{l}\text { Factor accounting for the loss of ability to perform a } \\
\text { laboratory operation because personnel are not available } \\
\text { due to time off for vacation, illness, personal reasons, } \\
\text { training, and meetings, etc., and to time lost because of } \\
\text { poor work efficiency, rework due to human errors, and } \\
\text { insufficient workload equal to } 1 \text { - (hours/week lost due to } \\
\text { personnel unavailability or inefficiency / hours/week } \\
\text { personnel are scheduled to work) }\end{array}$ \\
\hline $\begin{array}{l}\text { Operation } \\
\text { Availability (OA) }\end{array}$ & $\begin{array}{l}\text { Factor reflecting the fraction of time that a laboratory } \\
\text { operation is operable (e.g. the time an instrument is } \\
\text { capable of analyzing samples) equal to the average } \\
\text { operating time in hrs/wk / } 168 \mathrm{hrs} / \mathrm{wk}\end{array}$ \\
\hline $\begin{array}{l}\text { Instrument } \\
\text { Calibration (IC) }\end{array}$ & $\begin{array}{l}\text { Factor representing the fraction of operating time applied } \\
\text { to calibration samples equal to } C T /(C T+S T+Q T)\end{array}$ \\
\hline
\end{tabular}




\begin{tabular}{|l|l|}
\hline \multicolumn{1}{|c|}{ Term } & \multicolumn{1}{c|}{ Definition } \\
\hline $\begin{array}{l}\text { Quality Control } \\
\text { (QC) }\end{array}$ & $\begin{array}{l}\text { Factor representing the fraction of operating time applied } \\
\text { to quality control samples equal to QT / (CT + ST + QT) }\end{array}$ \\
\hline Matrix Effect (ME) & $\begin{array}{l}\text { Factor inversely related to the average number of times } \\
\text { (cycles) that a laboratory operation must be carried out in } \\
\text { order to generate acceptable results for a sample of a } \\
\text { given matrix type equal to the number of customer } \\
\text { samples processed / the total number of laboratory } \\
\text { operational cycles required }\end{array}$ \\
\hline
\end{tabular}




\title{
APPENDIX B
}

\author{
ESTIMATING DOE ANALYTICAL
}

LABORATORY CAPACITY AVAILABLE TO EM

- REPORTING FORMATS AND INSTRUCTIONS 


\title{
ESTIMATING DOE
}

\section{ANALYTICAL LABORATORY}

\section{CAPACITY AVAILABLE}

\author{
TO EM
}

\section{REPORTING FORMATS \\ AND \\ INSTRUCTIONS}

\author{
Prepared By: \\ Laboratory Management Division (EM-563) \\ Office of Technology Development \\ Environmental Restoration and Waste Management \\ U.S. Department of Energy
}

September 28, 1993 
LIST OF APPENDICES $\ldots \ldots \ldots \ldots \ldots \ldots \ldots \ldots \ldots \ldots$ ii

LIST OF TABLES $\ldots \ldots \ldots \ldots \ldots \ldots \ldots \ldots \ldots \ldots \ldots$ iii

LIST OF FIGURES $\ldots \ldots \ldots \ldots \ldots \ldots \ldots \ldots \ldots \ldots \ldots$ iv

LIST OF ACRONYMS $\ldots \ldots \ldots \ldots \ldots \ldots \ldots \ldots \ldots \ldots \ldots$

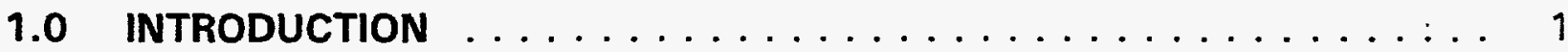

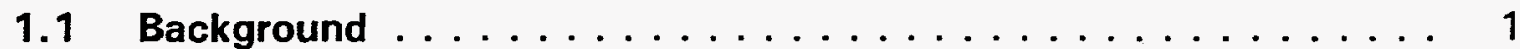

1.2 DOE Analytical Laboratory Capacity $\ldots \ldots \ldots \ldots \ldots \ldots \ldots$

1.3 Model Objective .................... 1

2.0 GENERAL INSTRUCTIONS $\ldots \ldots \ldots \ldots \ldots \ldots \ldots \ldots \ldots \ldots$

2.1 Deliverables ......................... 3

2.2 Submission of Deliverables $\ldots \ldots \ldots \ldots \ldots \ldots \ldots$

2.3 Conference Calls ..................... 3

2.4 Points of Contact $\ldots \ldots \ldots \ldots \ldots \ldots \ldots \ldots \ldots \ldots$

3.0 INSTRUCTIONS FOR COMPLETING CAPACITY CALCULATION

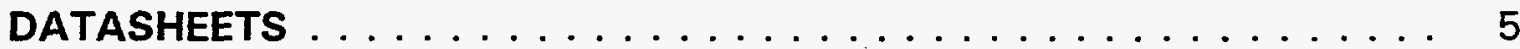

3.1 Description of the Report . . . . . . . . . . . . . . 5

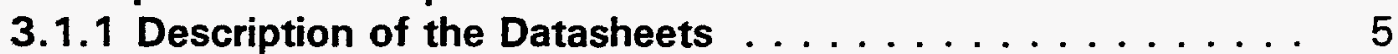

3.2 Instructions for Completing the Cover Sheet . . . . . . . 6

3.3 Instructions for Completing Datasheet $1 \ldots \ldots \ldots 6$

3.4 Instructions for Completing the Data Input Worksheets . . . . . 6 3.4.1 Header . . . . . . . . . . . . . . 7

3.4.2 Distribution of Customer Samples . . . . . . . . . . 8

3.4.3 Input Data for Regulatory-Driven Sample Analyses . . . . . 10 3.4.4 Input Data for Non-Regulatory-Driven Sample Analyses . . 11

3.5 Instructions for Completing the Summary Data Worksheets . . . 11

4.0 INSTRUCTIONS FOR USING THE LOTUS $1-2-3^{\circledR}$ ELECTRONIC FORMS . 13

4.1 The Diskette and the Files $\ldots \ldots \ldots \ldots \ldots \ldots$

4.2 Data Input Template . . . . . . . . . . . . . . . . 13

4.3 Summary Data Template . . . . . . . . . . . . . . . 13

4.3.1 Data Entry in the Summary Data Template . . . . . . . . 14

4.3.2 Calculations in the Summary Data Template . . . . . . . 14

4.3.3 Print Outs from the Summary Data Template . . . . . . . . 15

4.4 Saving the Spreadsheets $\ldots \ldots \ldots \ldots \ldots \ldots \ldots \ldots$

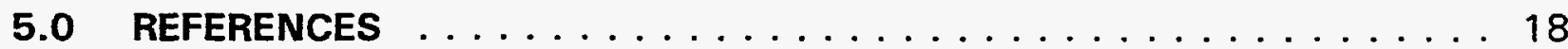




\section{LIST OF APPENDICES}

APPENDIX A: BLANK TRANSMITTAL AND DATA ENTRY FORMS FOR

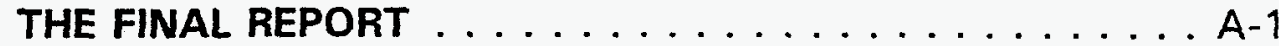

APPENDIX B: $\quad$ COMPLETED DATASHEET EXAMPLES $\ldots \ldots \ldots \ldots \ldots$ B-1

APPENDIX $C: \quad$ GLOSSARY OF TERMS $\ldots \ldots \ldots \ldots \ldots \ldots \ldots$. 1 


\section{LIST OF TABLES}

Table I: $\quad$ Sample Analyses $\ldots \ldots \ldots \ldots \ldots \ldots \ldots \ldots \ldots \ldots$

Table II: Printout Macros in the Summary Data Worksheet . . . . . . . . . . 15

Table III: Permitted Lotus Filenames for Data Input Spreadsheets . . . . . . 16

Table IV: Permitted Lotus Filenames for Summary Data Spreadsheets . . . . 17 


\section{LIST OF FIGURES}

Figure 1 : Data Input Sheet for the EM Analytical Capacity Determination Report ......................... 7

Figure 2 : Data Entry Section of a Blank Summary Data Worksheet for the EM Analytical Capacity Determination Report . . . . . . . . 12

Figure 3 : Layout of the Lotus 1-2-3 Template for the Summary Data Worksheet ....................... 14 


\section{LIST OF ACRONYMS}

Acronym Description
AA
$A C$
Atomic Absorption Spectroscopy
AES
Annual Capacity
CV
Atomic Emission Spectroscopy
DOE
Cold Vapor
EC
U.S. Department of Energy
EM
Existing Capacity
$E M-563$
Office of Environmental Restoration and Waste Management
GC
GF Laboratory Management Division
Gas Chromatography
Graphite Furnace
ICP
Inductively Coupled Plasma
$M C$
Upgradable Capacity
MMES
Martin Marietta Energy Systems
MS
Mass Spectrometry
OSM
DOE Office of Sample Management
PCB
Polychlorinalted Biphenyl
RCZ Radiation Control Zone
SAIC Science Applications International Corporation
TCLP Toxicity Characteristic Leaching Procedure
UC
Utilized Capacity
VOA
Volatile Organic Analysis 


\subsection{INTRODUCTION}

\subsection{Background}

The U.S. Department of Energy (DOE) has, as a major objective, to bring its sites into compliance with Federal and State environmental protection regulations. The DOE Office of Environmental Restoration and Waste Management (EM) is planning longterm strategies to support this priority. A fundamental element of EM programs is to establish and maintain analytical capabilities to effectively support these analytical needs. The major mission of the Laboratory Management Division (EM-563) is to ensure the availability of cost effective and high quality chemical and radiochemical analyses needed to support the EM programs. To meet this mission, EM-563 needs detailed information on current and future analytical needs and DOE analytical laboratory capacities. If the laboratory capacities exceed analytical needs, then a plan must be established for routing specific samples to existing laboratories. However, if analytical support needs exceed currently available analytical laboratory capacities, then programmatic decisions must be made to ensure that EM analytical needs are met.

\subsection{DOE Analytical Laboratory Capacity}

This model addresses both analytical capability (the analyses a laboratory is able to perform on a given sample type) and analytical capacity (how many of these measurements a laboratory is able to complete in a given time). Laboratory operations will be segregated into radioactive and non-radioactive operations. Radioactive operations will be classified into five subset radiation levels ( $R O$ - R4), and radioactive and non-radioactive operations will broken down into three different matrix types (M1 - M3). These radiation levels and matrix types are defined in Appendix A. For the sake of simplicity, capacity will be used to mean both capability and capacity, unless otherwise noted.

\subsection{Model Objective}

The objective in applying this model is to estimate the capacities of the DOE analytical laboratory Complex to analyze EM samples. The results of this report will be used in conjunction with an EM Analytical Needs Assessment report ${ }^{1}$ to develop a model across the complex as to what analytical capacities currently exist, what future DOE laboratory upgrades and/or construction will be necessary, and what size sample load will be assigned to commercial laboratories. A detailed explanation of the laboratory capability and capacity model, and its applications, is contained in a separate report ${ }^{2}$.

The information from application of this model will allow EM-563 to focus analytical laboratory priorities. Specifically, the information will help to:

- respond to requests from Congress and others for information on EM analytical needs, capacities and capabilities; 
- predict any shortfalls in capacity by comparing with EM analytical needs projections;

- develop baseline information for justifying budgets;

- optimize the level and type of commercial laboratory participation;

- $\quad$ provide information needed by DOE Offices of Sample Management (OSM's) for shipment of samples for analysis; and

- provide a management tool for analytical laboratories supporting EM. 


\title{
2.0 GENERAL INSTRUCTIONS
}

This section gives general instructions to participants in this estimation of DOE analytical capacity available to EM. Detailed instructions for completing the reporting forms are contained in Section 3.0.

\subsection{Deliverables}

At the completion of the reporting effort, all field participants, with the assistance of their EM-563 points of contact, should prepare a report consisting of the following deliverables:

1) A completed table providing the ranges of radiation levels of samples requiring specific radiation containment (See Datasheet 1, Appendix A);

2) Completed Analytical Laboratory Capacity Data Input Worksheets (See Datasheet 2, Appendix Al; and

3) Completed Analytical Laboratory Capacity Summary Data Worksheets (See Datasheet 3, Appendix A).

Deliverables 1) and 3) should be provided in both hardcopy and electronic form while deliverable 2) may be provided in electronic form only.

\subsection{Submission of Deliverables}

The report is due by November 30,1993 . All deliverables should be submitted to:

\author{
Mr. Robert Murray \\ US DOE, EM-563 \\ Laboratory Management Division \\ Trevion II \\ Washington, DC 20585-0002 \\ Tel: (301)-903-7644 \\ FAX: (301)-903-7613
}

\subsection{Conference Calls}

A series of conference telephone calls for all participants has been scheduled. These calls will allow exchange of views on the model, resolution of problems experienced in completing the datasheets, and receipt of any updated information concerning application of the model in your laboratory. The calls will take place on:

Tuesday, November 2, $1993 \quad 12: 00$ noon EST

Tuesday, November 9, 1993 12:00 noon EST

Tuesday, November 16, 1993 12:00 noon EST

Tuesday, November 23, 1993 12:00 noon EST 
The telephone number for the conference calls will be faxed to all participants in this exercise several days prior to each call.

\subsection{Points of Contact}

The following personnel have been designated the EM-563 points of contact to assist site personnel in the completion of the datasheets for this EM analytical laboratory capacity model.

Dr. Roy Morrow, MMES

Tel: (301)-601-1474

FAX: (301)-601-1452

(After December 1, 1993

Tel: (615)-576-0131

FAX: (615)-241-2280)

Dr. Steven B. Wyrick, SAIC

Tel: (301)-924-6168

FAX: (301)-924-4594

Dr. Richard Sassoon, SAIC

Tel: (301)-924-6126

FAX: (301)-924-4594 


\subsection{INSTRUCTIONS FOR COMPLETING CAPACITY CALCULATION DATASHEETS}

The DOE analytical laboratory capacity model can be used to determine three different types of laboratory annual capacities $(A C)$ for specific laboratory operations. These capacities are:

- Utilized Capacity (UC)

- Existing Capacity (EC)

- Upgradable Capacity (MC)

Definitions of these capacities are provided in Appendix $C$, which is a glossary of terms used in the model. All users of the model should read the model description report in detail before applying the model to their laboratories using this instructions package.

Participants in this excercise are requested to determine all of the above capacities for each sample analysis performed in every radiation control zone (RCZ) in their laboratory.

\subsection{Description of the Report}

The report that your organization will complete and submit to EM-563 consists of the following:

1. A cover sheet that provides information about the organization generating the report

2. The completed deliverables listed in section 2.1 .

The report should be submitted electronically by completing the spreadsheets in Lotus $1-2-3^{{ }^{*}}$ format provided on the enclosed diskette. Instructions and software requirements for using the Lotus $1-2-3^{\circledR}$ files appear in Section 4.0 . It is also requested that you submit this report in hardcopy by printing out the summary Lotus $1-2-3^{\circledR}$ spreadsheets.

\subsubsection{Description of the Datasheets}

The report will contain the following three types of datasheets, all of which are contained on the enclosed diskette, and hard copies of which appear in Appendix A:

1) A table depicting the ranges of radiation levels of samples requiring specific radiation containment (datasheet 1 )

2) Analytical Laboratory Capacity Data Input Worksheet - This is designed for collecting the required information for calculating the operational factors for a single sample analysis (datasheet 2 )

- Lotus $1-2-3^{\circledR}$ is a registered trademark of the Lotus Development Corporation. 
3) Analytical Laboratory Capacity Summary Data Worksheet - This is used to summarize in a single worksheet all the information collected on the data input worksheets and to calculate the required capacity for each sample analysis (datasheet 3)

\subsection{Instructions for Completing the Cover Sheet}

Complete the cover sheet provided in Appendix $A$ in hardcopy by entering the requested information. Be sure to enter the name and telephone number of the person responsible for determining the laboratory analytical capacity. This person will be the laboratory point of contact for EM-563.

\subsection{Instructions for Completing Datasheet 1}

Five types of radiation control zones (RCZs) have been defined for this model named RO through R4. (See model description package, section 2.3). Enter the ranges of radiation levels of samples which are used in each of the specified RCZs in your laboratory. Use units of $\mathrm{mR} / \mathrm{hr}$ on contact for beta/gamma radiation levels and use units of either $\mathrm{nCi} / \mathrm{g}, \mathrm{nCi} / \mathrm{mL}$, or $\mathrm{nCi} /$ container for total radiation levels. Enter the units used for alpha radiation levels in the aprropriate box in the table. Enter N/A for those $\mathrm{RCZs}$ that are not applicable to operations performed in your laboratory.

- Note: Some laboratories may choose not to distinguish between RO and R1 RCZs for the purposes of this model. In such cases rename the RO category RO, R1 and enter the data in this category.

\subsection{Instructions for Completing the Data Input Worksheets}

This section gives detailed instructions for completing the data input worksheets which are used for entering data from which calculations of your laboratory's capacity may be made. See section 4.0 for detailed instructions on using Lotus $1-2-3^{\circledR}$ for data input. A worksheet should be completed for each sample analysis performed in your laboratory, and this should be repeated for each RCZ in which the sample analysis is performed and for each of the three types of capacity being determined. Thus, if 12 sample analyses are performed in each of two RCZs, a total of 24 data input worksheets should be completed for each type of capacity to be determined; an overall total of 72 data input worksheets.

Only those operations performed in a RCZ are to be considered on the respective $R$ level worksheet. For example if a sample is digested for metals analysis in a radiation control zone but analyzed in a separate radiation control zone (due to dilution of the rad level) then the $A C$ for the preparation should, for example, be computed on the R1 spreadsheet and the instrumental operation on the RO spreadsheet.

Accurate numbers should be entered wherever possible. In some sections of the worksheet, however, only the best estimates of the laboratory manager are requested or may be available. 
- Note: When the number to be entered is zero, this may be indicated by either entering $O$ or by leaving that entry blank. All blank numerical entries will be assumed to be zero. If data is unavailable, enter N/A.

Please read all of the instructions and follow the examples given in Appendix $B$ before starting to complete the worksheets.

Definitions of key terms used in the datasheets and in this set of instructions are provided in the Glossary of Terms (Appendix C).

A blank data input worksheet is presented in Figure 1, and is provided in Appendix A. It is divided into four sections: Header, Distribution of Customer Samples, Input Data for Regulatory-Driven Sample Analyses, and Input Data for Non-Regulatory-Driven Sample Analyses. Instructions for completing each of the sections appear below.

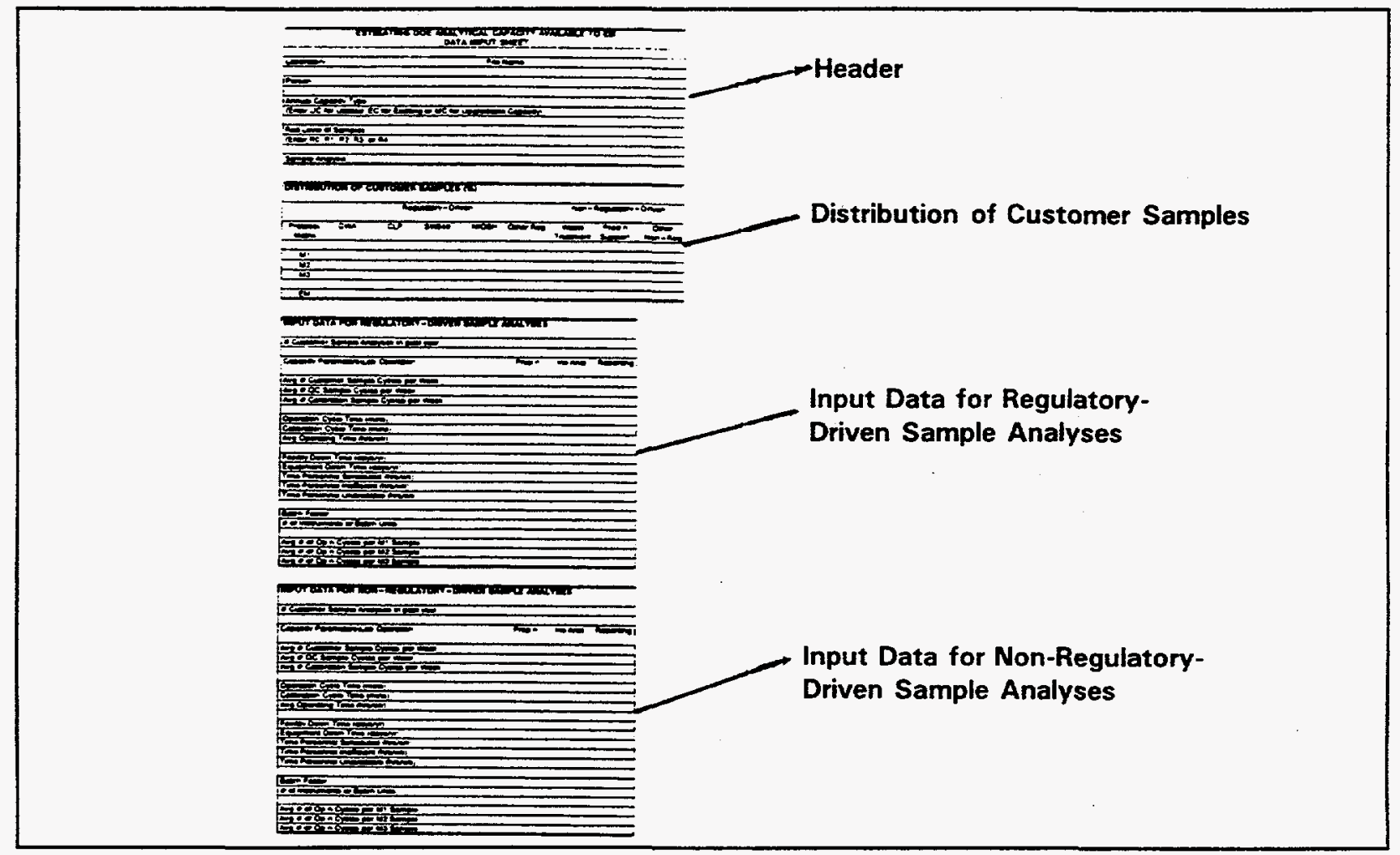

Figure 1: Data Input Sheet for the EM Analytical Capacity Determination Report

\subsubsection{Header}

Complete the Header section of the data input worksheet by making the following entries:

Filename:

The name to be given to this data input worksheet file. (See Section 4.4) 
Laboratory:

Person:

Annual Capacity Type: The type of analytical capacity for which the data is being

Rad Level of Samples: The radiation level of the samples (RO through R4, or

Sample Analysis: collected. The types of analytical capacity may be Utilized Capacity (UC), Existing Capacity (EC), and Upgradable Capacity (MC). $R O, R 1)$ corresponding to the radiation containment requirements given earlier in the report.

The name of the laboratory for which information is being provided.

The name of the person collecting the capacity information (laboratory supervisor or group leader).

The sample analysis, chosen from Table I, for which analytical capacity data is being collected. Enter this term in the datasheet exactly as it is designated in Table I.

- Note: Data input worksheets should be completed for each potentially rate limiting sample analysis performed in the laboratory. If sample analyses performed in your laboratory do not appear in Table I, but you choose to include them in the model, then data input worksheets should also be completed for them.

\subsubsection{Distribution of Customer Samples}

Complete the Distribution of Customer Samples section of the data input worksheet by making the following entries in the table in this section:

Complete the three rows of the table labelled $M 1, M 2$, and $M 3$ by entering the percentages of all customer samples (EM and others) analyzed in this sample analysis. Break down these percentages according to each of the protocols listed across the columns. (See Appendix $C$ for definitions of these matrix types). Because these are percentages of all customer samples, the sum of the percentages in this section of the table (M1/CWA through M3/Other Non-Reg) should equal 100\%. In the row of the table labelled EM, enter the percentages of total customer samples that are funded by EM for this sample analysis. Since not all customer samples are necessarily EMfunded, the sum of the percentages in this row of the table need not equal $100 \%$.

In the case of Utilized Capacity data, percentages should be taken from the numbers of sample analyses carried out in the appropriate $\mathrm{RCZ}$ during the previous year. For Existing and Upgradable Capacity data input, percentages should be the best estimates by the laboratory manager of the anticipated requirements for the sample analysis in the specified RCZ. 
Table 1: Sample Analyses

\begin{tabular}{|c|c|}
\hline Sample Analysis & Description \\
\hline VOA-GC & Volatile organic analysis by gas chromatography \\
\hline VOA-GC/MS & $\begin{array}{l}\text { Volatile organic analysis by gas chromatography - } \\
\text { mass spectroscopy (GC/MS) }\end{array}$ \\
\hline SEMI-VOA & Semi-volatile organic analysis by GC/MS \\
\hline PEST & Pesticide analysis by GC \\
\hline HERB & Herbicide analysis by GC \\
\hline PCB & Polychlorinated biphenyl analysis by GC \\
\hline ICP-AES METALS & $\begin{array}{l}\text { Inductively Coupled Plasma - Atomic Emission } \\
\text { Spectroscopy (ICP-AES) measurement of a single } \\
\text { metal analyte or a suite of metal analytes }\end{array}$ \\
\hline ICP-MS METALS & $\begin{array}{l}\text { Inductively Coupled Plasma - Mass Spectrometry } \\
\text { (ICP-MS) measurement of a single metal analyte or a } \\
\text { suite of metal analytes }\end{array}$ \\
\hline GF-AA METALS & $\begin{array}{l}\text { Graphite Furnace Atomic Absorption (GF-AA) } \\
\text { measurement of individual metal analytes }\end{array}$ \\
\hline MERCURY & $\begin{array}{l}\text { Cold Vapor Atomic Absorption (CV-AA) } \\
\text { measurement of mercury }\end{array}$ \\
\hline CYANIDE & Measurement of cyanide \\
\hline ANIONS/CATIONS & $\begin{array}{l}\text { Anion/cation analysis, by ion chromatography or } \\
\text { wet chemistry (counted individually by ions, e.g., } \\
\text { nitrate, or ammonium for single analysis, or by ion } \\
\text { chromatography runs for simultaneous analyses) }\end{array}$ \\
\hline GROSS ALPHA/BETA & Gross alpha and beta counting \\
\hline $\begin{array}{l}\text { ALPHA SPEC } \\
\text { ISOTOPES (Specify) }\end{array}$ & $\begin{array}{l}\text { Spectrometric analysis for specified alpha-emitting } \\
\text { nuclides }\end{array}$ \\
\hline $\begin{array}{l}\text { GAMMA SPEC } \\
\text { ISOTOPES (Specify) }\end{array}$ & $\begin{array}{l}\text { Spectrometric analysis for specified gamma-emitting } \\
\text { nuclides }\end{array}$ \\
\hline TCLP EXTRACTION & Toxicity Characteristic Leaching Procedure (TCLP) \\
\hline OTHER (Specify) & Other sample analysis \\
\hline
\end{tabular}




\subsubsection{Input Data for Regulatory-Driven Sample Analyses}

Complete the Input Data for Regulatory-Driven Sample Analyses section of the data input worksheet by filling in the table designated for this section. With the exception of the first entry, information should be provided for each of the three stages of the sample analysis, namely the preparation, instrumental, and data reporting stages. Use the distribution of customer samples provided in the table above for regulatory-driven sample analyses as assistance in estimating the input parameters when no accurate information is available.

Make the requested entries using the definitions in Appendix $C$ referred to below.

Entry

\# Customer Sample Analyses in past year:

Avg \# Customer Sample Cycles per Week:

Avg \# OC Sample Cycles per Week:

Avg \# Calibration Sample Cycles per Week:

Operation Cycle Time (mins):

Calibration Cycle Time (mins):

Avg Operating Time (hrs/wk):

Facility Down Time (days/yr):

Equipment Down Time (days/yr):

Time Personnel Scheduled

(hrs/wk):

Time Personnel Inefficient

(hrs/wk):

Time Personnel Unavailable (hrs/wk):

Batch Factor:

\# of Instruments or Batch Units:

Avg \# of Op'n Cycles per M1

Sample:

Avg \# of Op'n Cycles per M2 Sample:

\section{Related Terms Defined in Appendix C}

Customer Sample, Sample Analysis Cycle

Customer Sample, Laboratory Operational Cycle

Laboratory Quality Control Sample, Laboratory Operational Cycle

Calibration Sample, Laboratory Operational Cycle

Operational Cycle Time

Calibration Cycle Time

Average Operating Time

Facility Availability (FA)

Equipment Availability (EA)

Personnel Availability (PA)

Personnel Availability (PA)

Personnel Availability (PA)

Batch Factor (BF)

Number of Available Units (N)

Laboratory Operational Cycle, M1

Laboratory Operational Cycle, M2 
Entry

Avg \# of Op'n Cycles per M3 Sample:
Related Terms Defined in Appendix C

Laboratory Operational Cycle, M3

\subsubsection{Input Data for Non-Regulatory-Driven Sample Analyses}

Complete the Input Data for Non-Regulatory-Driven Sample Analyses section of the data input worksheet by filling in the table in this section in a similar fashion. As in the previous section, information should be provided for each of the three stages of the sample analysis, namely the preparation, actual analysis, and data reporting stages. Use the distribution of customer samples provided in the Distribution of Customer Samples table for non-regulatory-driven sample analyses as assistance in estimating the input parameters when no accurate information is available. Instructions for managing the data input worksheet files, on completing data entry, are given in Section 4.0 .

\subsection{Instructions for Completing the Summary Data Worksheets}

This section provides instructions for completing the summary data worksheets which are used for calculating your laboratory's capacity for all sample analyses from the data entered into the data input worksheets. A separate summary data worksheet should be used for each capacity within each RCZ in the laboratory. Thus if a laboratory contains two RCZs and each of the three types of capacity is determined then a total of 6 summary data worksheets are required. The data entry section of a blank summary data worksheet is presented in Figure 2.

Please read all of the instructions, especially those for using the Lotus $1-2-3^{\circledR}$ electronic forms, and follow the examples depicted in Appendix B before starting to complete these worksheets.

Enter the file name for the summary data worksheet (see Section 4.4) and the general information on the Operations Office, laboratory, date of completion of summary worksheet, and the name, telephone number, and FAX number of the person collecting the capacity data into the spreadsheet. Enter the type of capacity and the radiation level of the radiation control zone for which data is being collected.

Enter the remaining information concerning the data input sheets to be summarized into the worksheet. This information includes the number of data input sheets to be summarized, the name of the directory which contains the data input worksheets, and the names of each of the data input worksheet files. Enter the names of each of the data input worksheet files in the order corresponding to the order of sample analyses given in Table I. After entry of all the required information operate the macro associated with this worksheet by striking Alt A. Refer to Section 4.0 of this instructions package for more details concerning entry of this information into the worksheet and operation of the macro. 


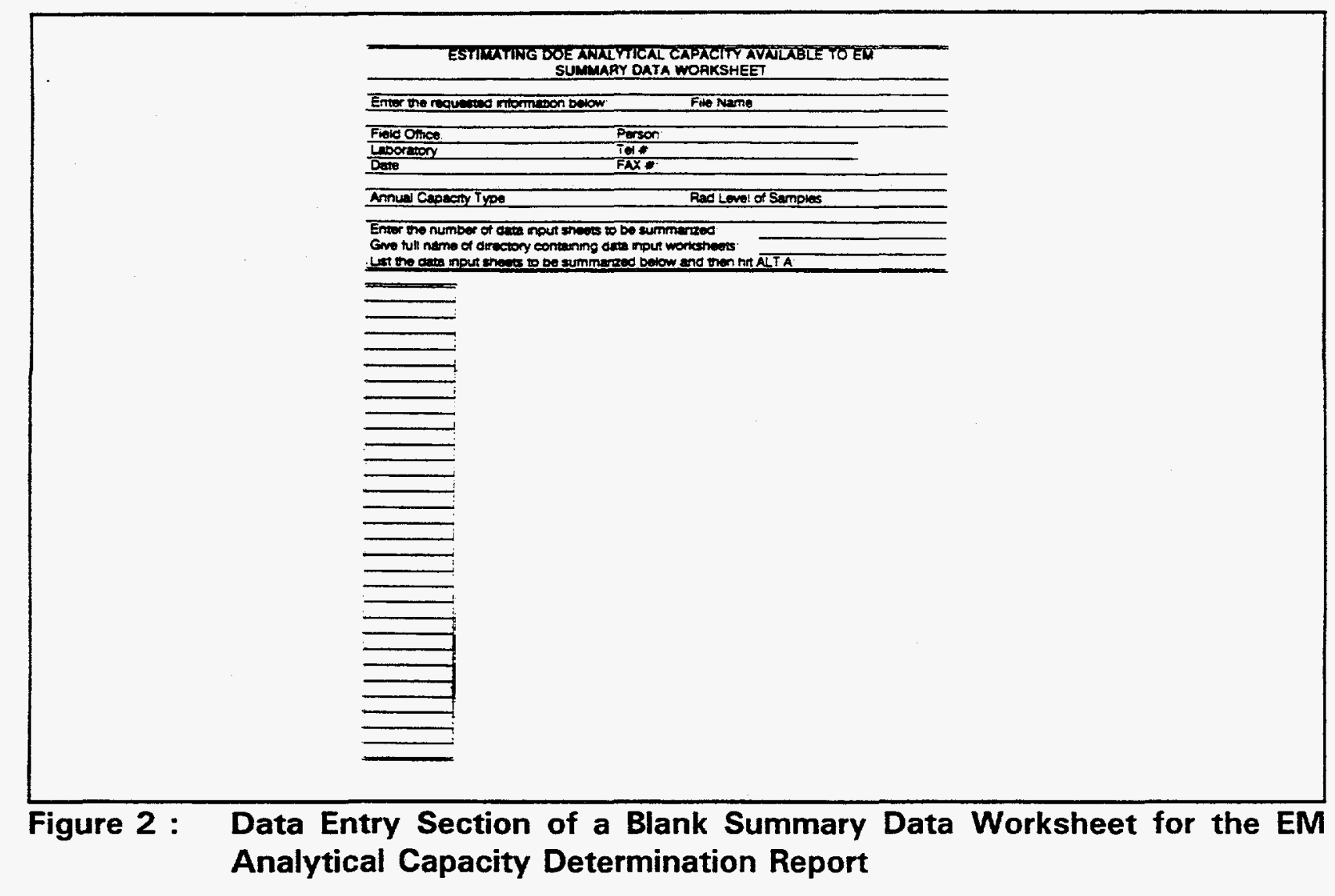




\subsection{INSTRUCTIONS FOR USING THE LOTUS $1-2-3^{\circledR}$ ELECTRONIC FORMS}

The data input and summary worksheets are provided on diskette in Lotus $1-2-3^{\circledR}$ format and data must be entered electronically in this effort. Lotus $1-2-3^{\circledR}$ release 2.4 or a higher version is required. The version must contain features such as Wysiwyg, and advanced format and print commands. It is assumed that users of these electronic forms possess a general working knowledge of Lotus $1-2-3^{\circledR}$ or similar software. For regular Lotus $1-2-3^{\circledR}$ commands and usage, the user should consult the Lotus $1-2-3^{\circledR}$ manual. Any specific questions related to the use of the Lotus $1-2-3^{\circledR}$ spreadsheets should be addressed to Richard Sassoon (Tel. 301-924-6126).

\subsection{The Diskette and the Files}

The diskette provided by the Laboratory Management Division (EM-563) should be backed up prior to use. The diskette is MS-DOS formatted and consists of the Lotus 1-2-3 ${ }^{\circledR}$.WK1 files named DATINPUT.WK1 and SUMDAT.WK1 for release 2.4, and the Lotus $1-2-3^{\circledR}$.WK3 files named DATINPUT.WK3 and SUMDAT.WK3 for releases 3.0 and higher. The files have read-only status (see below), protecting them against inadvertent erasure. This limit to write access can be removed by using the operating system ATTRIB command. The files can only be retrieved without a "file reservation" since they are read-only files. That is, any changes made or data entered into the files must be saved under different file names. (See Section 4.4 Saving the Spreadsheets).

\subsection{Data Input Template}

The template for the data input worksheet (see Figure 1 and Datasheet 2 in Appendix A) is found in DATINPUT.WK1 or DATINPUT.WK3. All cells are protected, except for the ranges that are used to accept data. Only labels should be entered into the Header section, and only values should be entered into the remainder of the worksheet. On completion of data entry, each worksheet should be saved according to the directions appearing in Section 4.4 .

\subsection{Summary Data Template}

The template for the summary data worksheet is found in SUMDAT.WK1 or SUMDAT.WK3. Again all cells are protected, except for the ranges that are used to accept data. The layout of the template is shown in Figure 3. Each of the areas of the template are shown together with the cell identifiers of their upper left- and lower right-hand corners. The user need only be concerned with the data entry area for entering information on the data input worksheets to be imported, and with the areas labelled "Summary of Input Data," "Capacity Correction Factors," and "Annual Capacities and Operating Times," which will contain the summarized data and which may be printed out in hardcopy. The user should, however, be cautioned not to enter data in unprotected cells in other areas of the spreadsheet. 


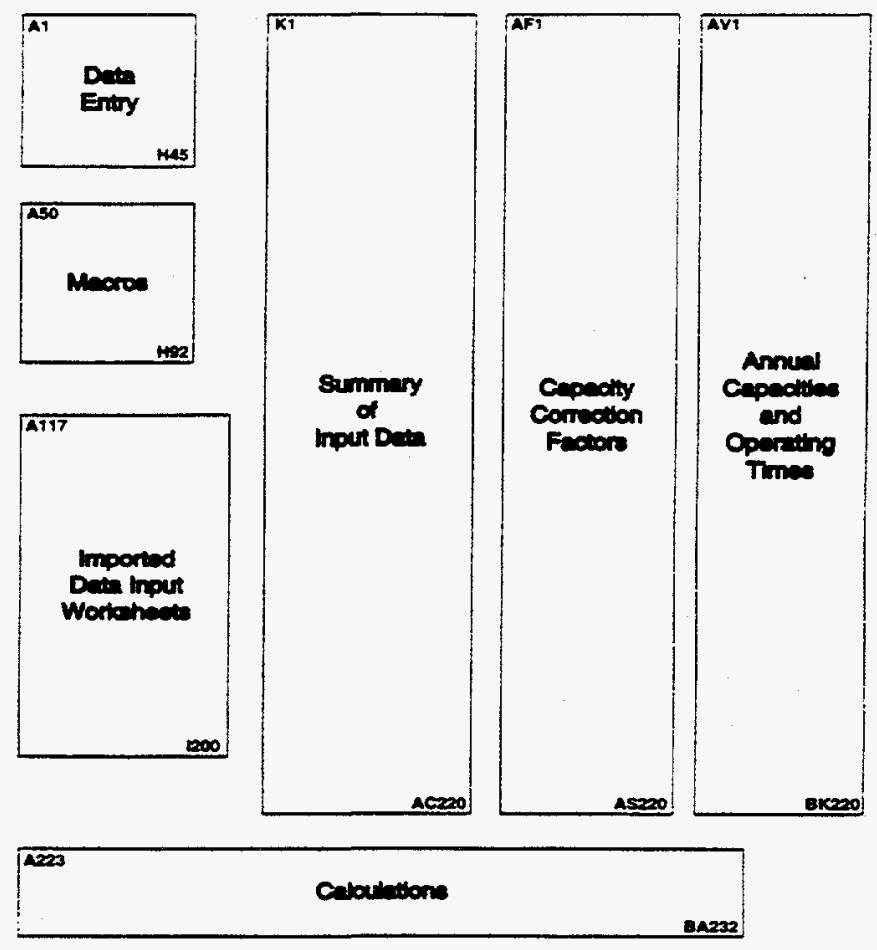

Figure 3 : Layout of the Lotus 1-2-3 Template for the Summary Data Worksheet

\subsubsection{Data Entry in the Summary Data Template}

On entering the requested data into the data entry section of the spreadsheet ensure that telephone and FAX numbers are entered as labels (i.e. enter an apostrophe (1) before the number). Enter the number of datasheets to be summarized in cell $G 12$ and enter the full name of the DOS directory containing the data input files in cell G13. Be sure to include a backslash at the end of the directory name le.g. $\mathrm{C}:(123$ \FILESICAP \). Enter the list of data input worksheet filenames beginning in cell $A 16$ and continuing down the $A$ column. A maximum of 30 data input sheets corresponding to 30 sample analyses may be summarized in a single summary data worksheet. If more than $\mathbf{3 0}$ sample analyses are to be summarized use more than one summary data worksheet. Entry of a .WK1 or .WK3 suffix at the end of the filename is optional.

\subsubsection{Calculations in the Summary Data Template}

Summaries of all the input data from the data input worksheets and results of calculations of all the correction factors and annual capacities are generated using the Lotus 1-2-3 ${ }^{\circledR}$ macro command Alt A. Operation of this command outputs the generated information in the areas labelled "Summary of Input Data," "Capacity Correction Factors," and "Annual Capacities and Operating Times." If an error is encountered in operating the macro hit CTRL BREAK to exit the macro and check that all data was entered correctly in the Data Entry section of the worksheet. 


\subsubsection{Print Outs from the Summary Data Template}

Hard copies of the summarized and calculated data from the spreadsheet may be obtained by invoking one of the macros given in Table II. If an error is encountered in operating any of the macros hit CTRL BREAK to exit the macro and check that all the print options on the spreadsheet are correctly set for your printer.

Table II: Printout Macros in the Summary Data Worksheet

\begin{tabular}{|l|l|}
\hline \hline MACRO NAME & MACRO DESCRIPTION \\
\hline \hline Alt B & Generates Hard Copy of Summary of Input Data \\
\hline Alt C & Generates Hard Copy of Capacity Correction Factors \\
\hline Alt D & $\begin{array}{l}\text { Generates Hard Copy of Annual Capacities and } \\
\text { Operating Times }\end{array}$ \\
\hline
\end{tabular}

\subsection{Saving the Spreadsheets}

The worksheets containing the data input and summary data templates must be saved with unique filenames. Tables III and IV describe the file-naming conventions required for data submission. 
Table III: Permitted Lotus Filenames for Data Input Spreadsheets

\begin{tabular}{|c|c|}
\hline $\begin{array}{l}\text { FILENAME } \\
\text { COMPONENTS }\end{array}$ & DESCRIPTION \\
\hline Laboratory Identifier & One alphanumeric character selected by the laboratory. \\
\hline $\begin{array}{l}\text { Sample Analysis } \\
\text { Identifier }\end{array}$ & $\begin{array}{l}\text { First } 3 \text { letters of the sample analysis or sample analysis } \\
\text { abbreviation. }\end{array}$ \\
\hline Radiation Control Zone & $\begin{array}{l}R C Z \text { identifier from } R O \text { through } R 4 \text {. Use the identifier } \\
01 \text { when data for } R O \text { and } R 1 \text { are combined. }\end{array}$ \\
\hline Capacity Type & Capacity Identifier - UC, EC, or MC \\
\hline File Extension & $\begin{array}{l}\text { WK1 for files saved in Lotus } 1-2-3 \text { Release } 2 \text { format. } \\
\text {.WK3 for files saved with Release } 3 \text {. (Refer to } 1-2-3 \\
\text { Manual) }\end{array}$ \\
\hline \multicolumn{2}{|c|}{ EXAMPLE OF VALID FILENAME } \\
\hline KVOAROEC.WK3 & $\begin{array}{l}\text { Data from K-25 laboratory on their existing capacity } \\
\text { for performing VOA sample analyses in a non-radiation } \\
\text { zone in a file saved in Lotus } 1-2-3 \text { Release } 3 \text { format }\end{array}$ \\
\hline
\end{tabular}


Table IV: Permitted Lotus Filenames for Summary Data Spreadsheets

\begin{tabular}{|l|l|}
\hline \hline $\begin{array}{l}\text { FILENAME } \\
\text { COMPONENTS }\end{array}$ & DESCRIPTION \\
\hline \hline Laboratory Identifier & $\begin{array}{l}\text { One-Four alphanumeric characters selected by the } \\
\text { laboratory to identify itself. }\end{array}$ \\
\hline Radiation Control Zone & $\begin{array}{l}\text { RCZ identifier from RO through R4. Use the identifier } \\
01 \text { when data from RO and R1 are combined. }\end{array}$ \\
\hline Capacity Type & Capacity Identifier - UC, EC, or MC \\
\hline File Extension & $\begin{array}{l}\text {.WK1 for files saved in Lotus 1-2-3 Release 2 format. } \\
\text { WK3 for files saved with Release 3. (Refer to 1-2-3 } \\
\text { Manual) }\end{array}$ \\
\hline \hline EXAMPLE OF VALID FILENAME \\
\hline \hline K_25ROEC.WK3 & $\begin{array}{l}\text { Summary data from K-25 laboratory on their existing } \\
\text { capacity for performing all sample analyses in non- } \\
\text { radiation zones in a file saved in Lotus 1-2-3 Release 3 } \\
\text { format }\end{array}$ \\
\hline
\end{tabular}




\subsection{REFERENCES}

1. Determination of EM Environmental Sampling and Analysis Needs for FY 92 -98.

2. Estimating DOE Analytical Laboratory Capacity Available to EM - Model Description. 
APPENDIX A

BLANK TRANSMITTAL AND DATA ENTRY FORMS

FOR THE FINAL REPORT

A-1 


\title{
ESTIMATING DOE ANALYTICAL
}

\author{
LABORATORY CAPACITY
}

AVAILABLE TO EM

FINAL REPORT

\begin{tabular}{||l|l|}
\hline \hline Field Office: & \\
\hline Site: & \\
\hline Laboratory: & \\
\hline Manager Responsible for Collection of & \\
Analytical Laboratory Capacity Data: & \\
\hline Telephone \#: & \\
\hline Final Report Date: & \\
\hline
\end{tabular}

Performed For:

Laboratory Management Division (EM-563)

Office of Technology Development

Environmental Restoration and Waste Management

U.S. Department of Energy 


\section{Datasheet 1:}

RADIATION LEVELS VERSUS RADIATION CONTAINMENT REQUIREMENTS

\begin{tabular}{|c|l|l|l|l|l||}
\hline $\begin{array}{l}\text { Rad } \\
\text { Level } \\
\text { R0- } \\
\text { R4 }\end{array}$ & \multirow{2}{*}{$\begin{array}{l}\text { Radiation Containment } \\
\text { Requirements }\end{array}$} & \multicolumn{2}{l|}{$\begin{array}{l}\text { Range of Rad } \\
\text { Levels } \\
\text { (mR/hr) on } \\
\text { Contact }\end{array}$} & \multicolumn{2}{l|}{$\begin{array}{l}\text { Range of Rad } \\
\text { Levels (Alpha) }\end{array}$} \\
\cline { 4 - 7 } & & From & To & From & To \\
\hline \hline R0 & $\begin{array}{l}\text { No radiation containment } \\
\text { required }\end{array}$ & & & & \\
\hline R1 & $\begin{array}{l}\text { Bench top or lab hood in } \\
\text { RCZ }\end{array}$ & & & & \\
\hline R2 & $\begin{array}{l}\text { Shielded hood in RCZ } \\
\text { required }\end{array}$ & & & & \\
\hline R3 & Glovebox in RCZ required & & & & \\
\hline R4 & Hot cell in RCZ required & & & & \\
\hline \hline
\end{tabular}


Datasheet 2:

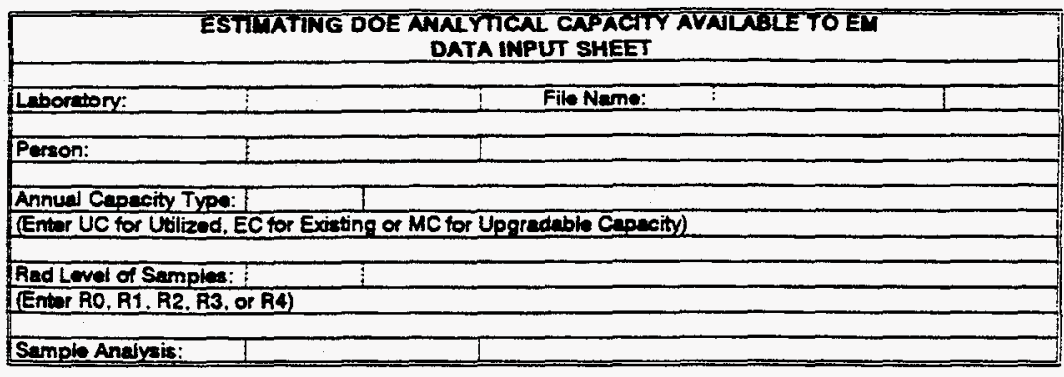

DIST RIBLTION OF CUSTOMER SAMPLES (X)

\begin{tabular}{|c|c|c|c|c|c|c|c|c|}
\hline & \multicolumn{5}{|c|}{ Reguintory-Driven } & \multicolumn{3}{|c|}{ Non-Reguletory-Driven } \\
\hline $\begin{array}{l}\text { Protocol/ } \\
\text { Metrix }\end{array}$ & CWA & CLP & SW846 & NIOSH & Other Rag & $\begin{array}{c}\text { Weat } \\
\text { Truetment }\end{array}$ & $\begin{array}{l}\text { Prod'n } \\
\text { Support }\end{array}$ & $\begin{array}{c}\text { Other } \\
\text { Non-Reg }\end{array}$ \\
\hline M1 & & & & & & & & \\
\hline M2 & & & & & & & & \\
\hline$M 3$ & & & & & & & & \\
\hline$F M$ & & & & & & & & \\
\hline
\end{tabular}

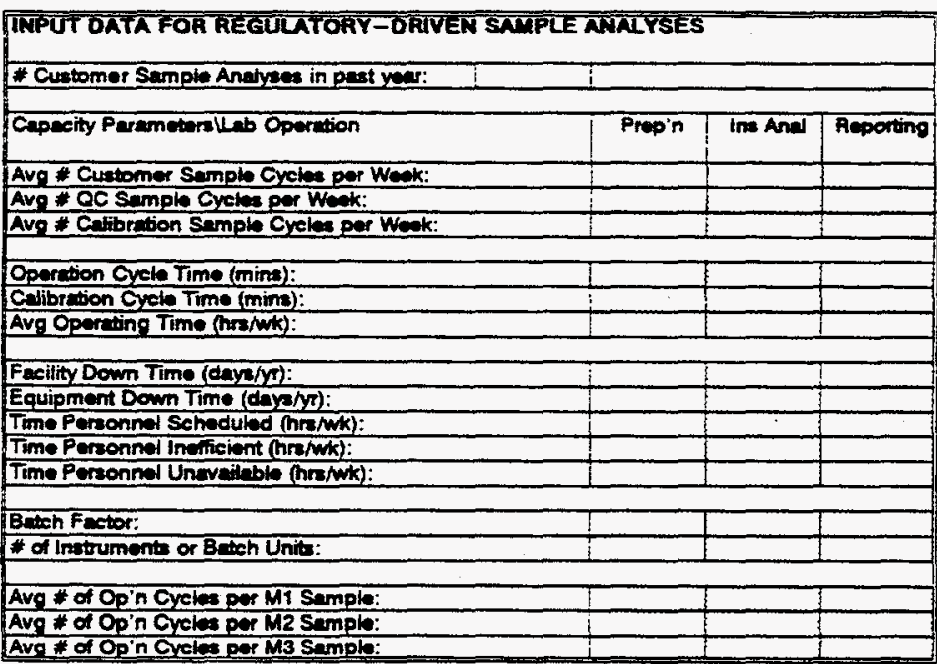

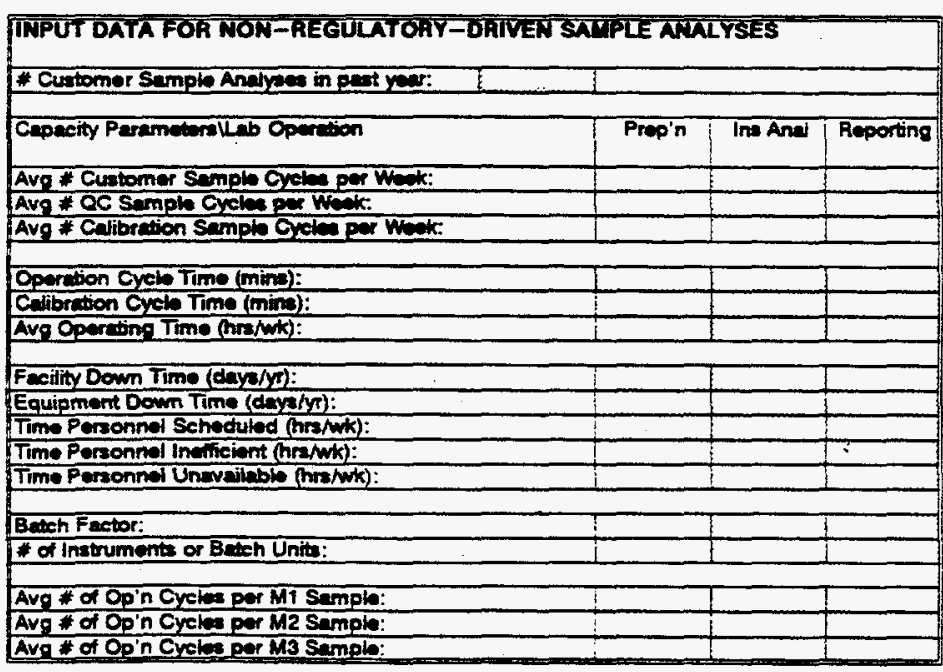




\section{Datasheet 3:}

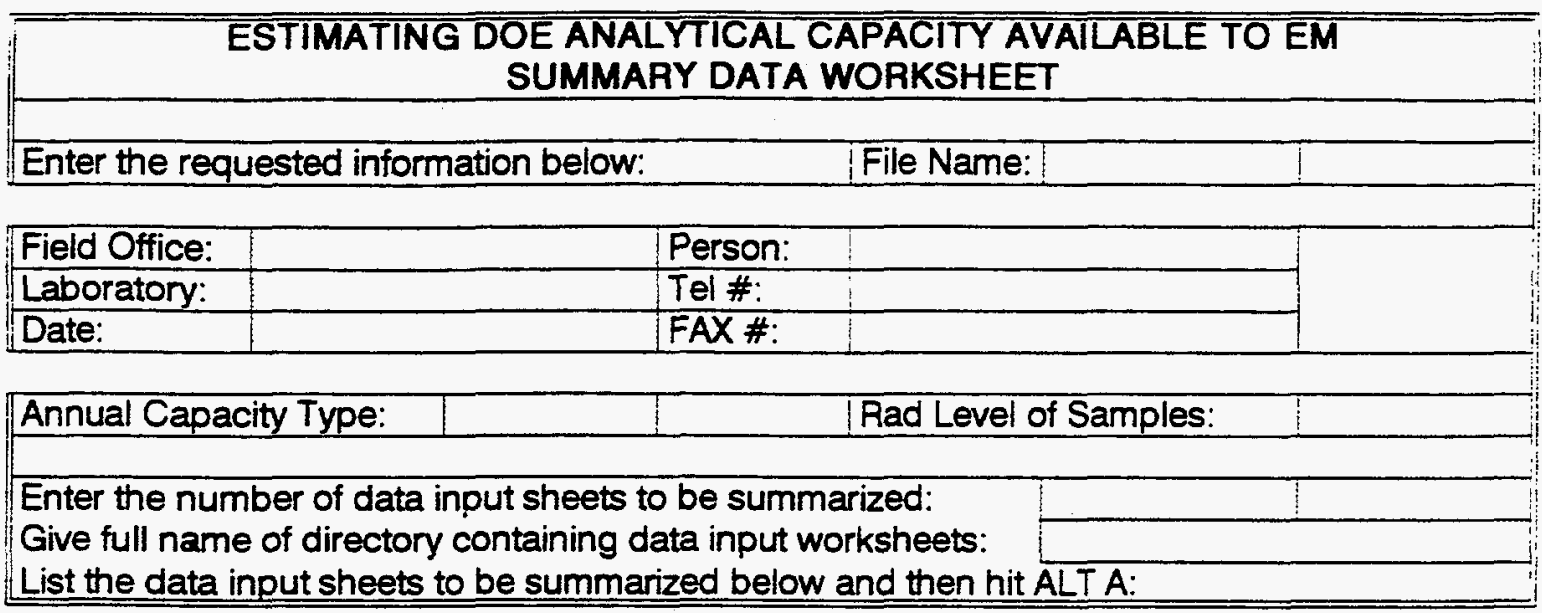

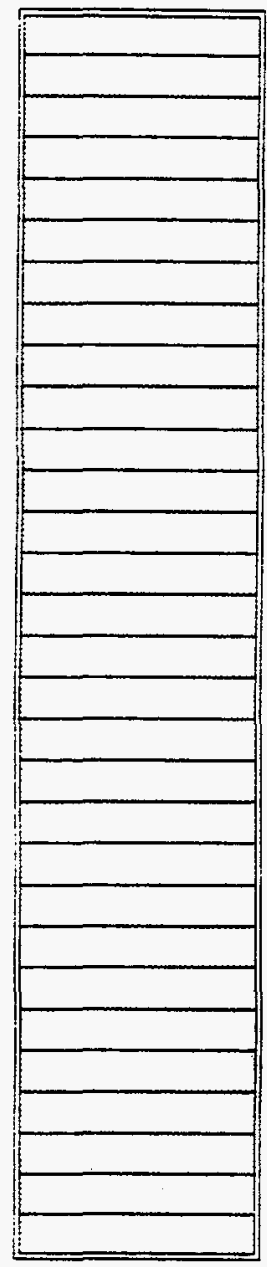




\section{APPENDIX B}

COMPLETED DATASHEET EXAMPLES 
On the following pages are a series of examples of completed datasheets. Pages B-3 through B-7 give examples of 5 completed data input worksheets for 5 sample analyses. The completed data entry section of the summary data worksheet, identifying the five data input worksheets to be summarized, is shown on page B-8. Printouts of the summarized data obtained by operating the macros Alt B, Alt C, and Alt $D$ are shown on pages $B-9$ through $B-11$. 


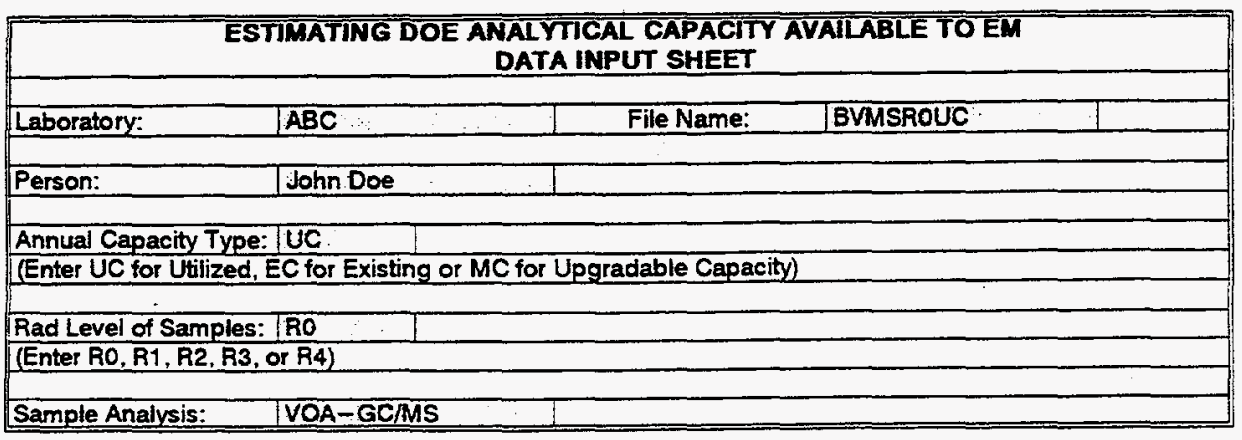

\section{DISTRIBUTION OF CUSTOMER SAMPLES (\%)}

\begin{tabular}{|c|c|c|c|c|c|c|c|c|}
\hline \multirow{2}{*}{$\frac{\text { Protocol/ }}{\text { Matrix }}$} & \multicolumn{4}{|c|}{ Regulatory - Driven } & & \multicolumn{3}{|c|}{ Non-Regulatory-Driven } \\
\hline & CWA & CLP & SW846 & $\mathrm{NIOSH}$ & Other Reg & $\begin{array}{c}\text { Waste } \\
\text { Treatment }\end{array}$ & $\begin{array}{l}\text { Prod'n } \\
\text { Support }\end{array}$ & $\begin{array}{c}\text { Other } \\
\text { Non-Reg }\end{array}$ \\
\hline$M 1$ & 10 & 15 & 13 & & & 30 & 10 & 6 \\
\hline M2 & & $\frac{5}{1}$ & 2 & & & $\frac{1}{7}$ & & 2 \\
\hline M3 & & & 7 & & & 3 & & \\
\hline & & & & & & & & \\
\hline$E M$ & 1.5 & 16 & 14 & & & 25 & 1 & 5 \\
\hline
\end{tabular}

\begin{tabular}{|c|c|c|c|}
\hline \multicolumn{4}{|c|}{ INPUT DATA FOR REGULATORY-DRIVEN SAMPLE ANALYSES } \\
\hline \# Customer Sample Analyses in past year: & 1496 & & \\
\hline Capacity Parameters|Lab Operation & Prep'n & Ins Anal & Reporting \\
\hline Avg \# Customer Sample Cycles per Week: & & 40 & 40 \\
\hline Avg \# OC Sample Cycles per Week: & & 10 & \\
\hline Avg \# Calibration Sample Cycles per Week: & & 10 & \\
\hline Operation Cycle Time (mins): & & 46 & 12.8 \\
\hline Calibration Cycle Time (mins): & & 50 & \\
\hline Avg Operating Time (hrs/wk): & & 49.5 & 8.2 \\
\hline Facility Down Time (days/yr): & & 15 & 15 \\
\hline Equipment Down Time (days/yr): & & 20 & 2 \\
\hline Time Personnel Scheduled (hrs/wk): & & 40 & 42 \\
\hline Time Personnel Inefficient (hrs/wk): & & 2.5 & 3 \\
\hline Time Personnel Unavailable (hrs/wk): & & 8.5 & 8.5 \\
\hline Batch Factor: & & T & 1 \\
\hline \# of Instruments or Batch Units: & & 1 & 1 \\
\hline Avg \# of Op'n Cycles per M1 Sample: & & 1.1 & \\
\hline Avg \# of Op'n Cycles per M2 Sample: & & 1.2 & \\
\hline Avg \# of Op'n Cycles per M3 Sample: & & 2 & 1 \\
\hline
\end{tabular}

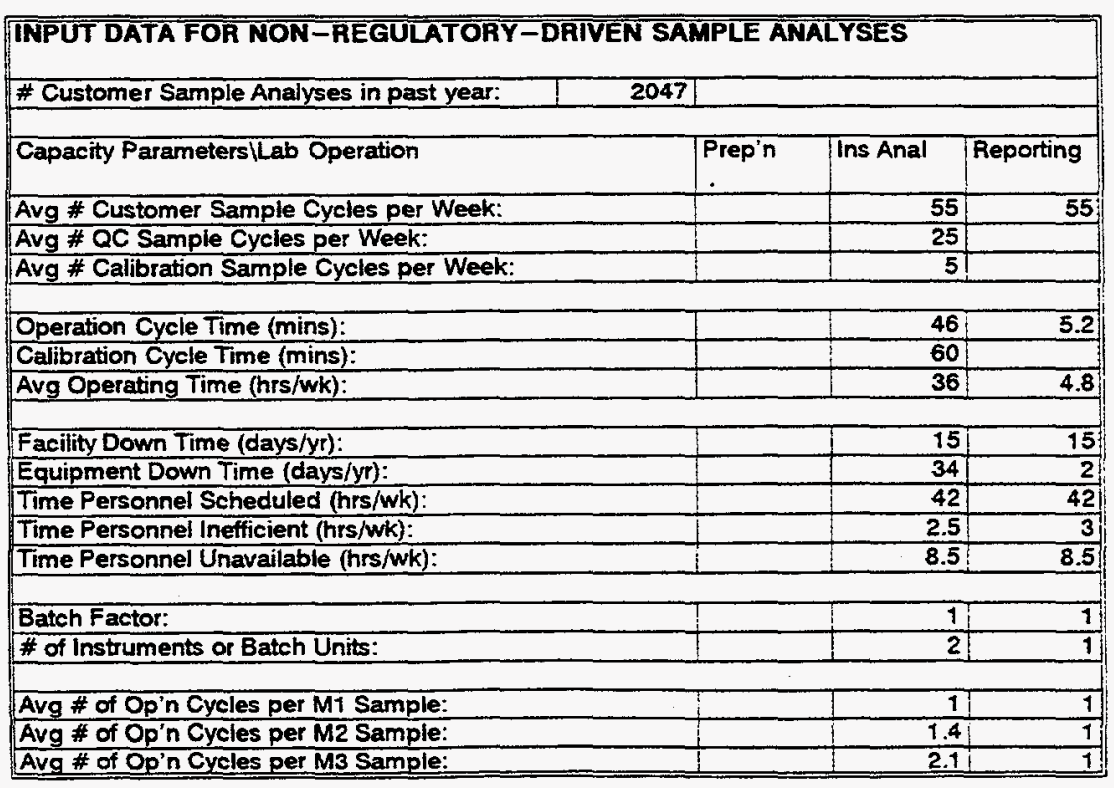




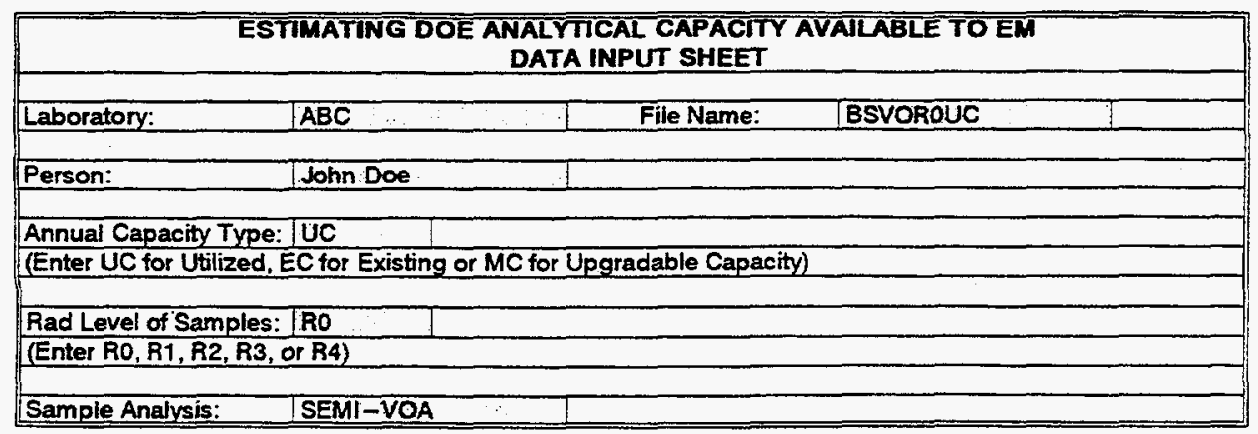

\begin{tabular}{|c|c|c|c|c|c|c|c|c|}
\hline \multicolumn{9}{|c|}{ DISTAIBUTION OF CUSTOMER SAMPLES (\%) } \\
\hline \multirow{2}{*}{$\frac{\text { Protocol/ }}{\text { Matrix }}$} & \multicolumn{4}{|c|}{ Regulatory-Driven } & & \multicolumn{3}{|c|}{ Non-Regulatory-Driven } \\
\hline & CWA & CLP & SW846 & NIOSH & Other Reg & $\begin{array}{c}\text { Waste } \\
\text { Treatment }\end{array}$ & $\begin{array}{l}\text { Prod'n } \\
\text { Support }\end{array}$ & $\begin{array}{c}\text { Other } \\
\text { Non-Reg }\end{array}$ \\
\hline 1 & 10 & 95 & 30 & & 4 & 3 & & \\
\hline$\frac{M 1}{M 2}$ & 10 & $\frac{15}{5}$ & $\frac{30}{5}$ & & 70 & 20 & & \\
\hline $\mathrm{M} 3$ & & 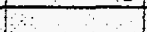 & 5 & & & & & \\
\hline & & & & & & & & \\
\hline$E M$ & 5 & 20 & 32 & & T6 & 15 & & \\
\hline
\end{tabular}

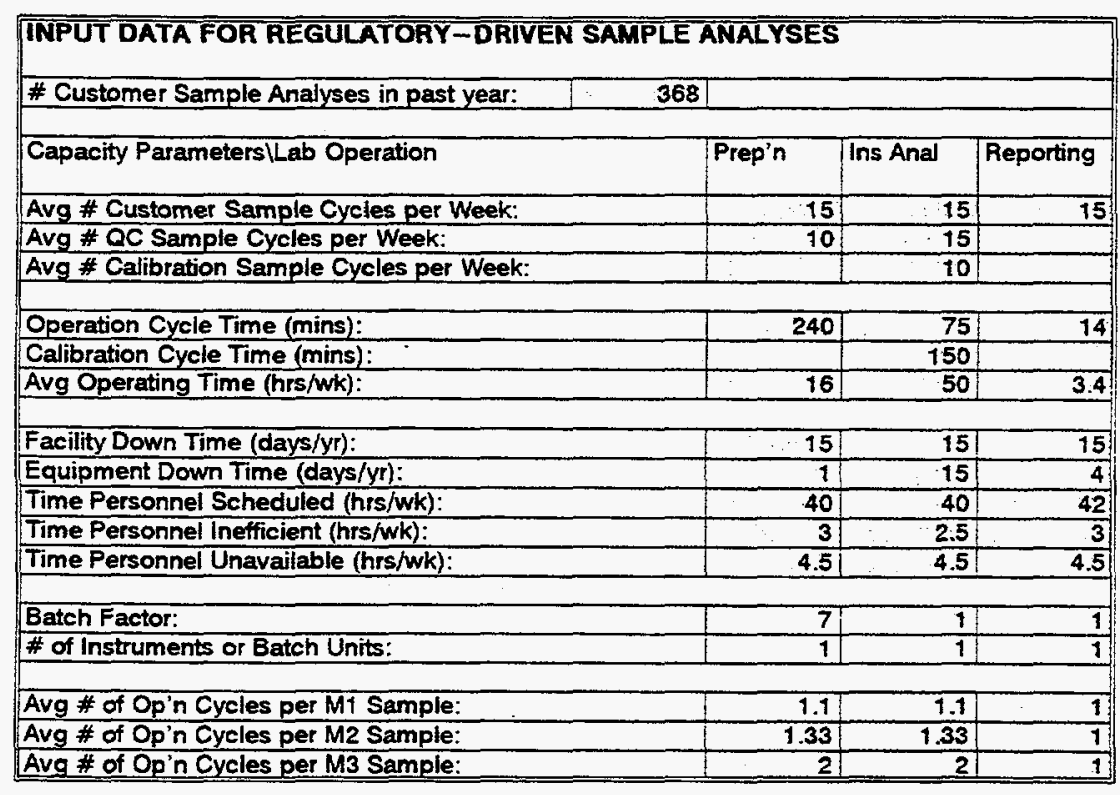

\begin{tabular}{|c|c|c|c|}
\hline \multicolumn{4}{|c|}{ INPUT DATA FOR NON-REGULATOAY-DRIVEN SAMPLE ANALYSES } \\
\hline \# Customer Sample Analyses in past year: & & & \\
\hline Capacity Parameters Lab Operation & Prep'n & Ins Anal & Reporting \\
\hline Avg \# Customer Sample Cycles per Week: & 1.75 & 1.75 & 1.75 \\
\hline Avg \# QC Sample Cycles per Week: & 2 & 4 & \\
\hline Avg \# Calibration Sample Cycles per Week: & & 11 & \\
\hline Operation Cycle Time (mins): & 480 & 75 & 10 \\
\hline Calibration Cycle Time (mins): & & 150 & \\
\hline Avg Operating Time (hrs/wk): & 7 & 111 & 0.4 \\
\hline Facility Down Time (days/yr): & 15 & 15 & 15 \\
\hline Equipment Down Time (days/yr): & 1 & 15 & \\
\hline Time Personnel Scheduled (hrs/wk): & 40 & 40 & 42 \\
\hline Time Personnel Inefficient (hrs/wk): & 3 & 2.5 & 3 \\
\hline Time Personnel Unavailable (hrs/wk): & 4.5 & 4.5 & 4.5 \\
\hline Batch Factor: & 6 & 1 & 1 \\
\hline \# of Instruments or Batch Units: & 1 & 11 & 1 \\
\hline Avg \# of Op'n Cycles per M1 S & 1.1 & 1.1. & 1 \\
\hline Avg \# of Op'n Cycles per M2 Sample: & 1.65 & 1.33 & \\
\hline Avg \# of Op'n Cycles per M3 Sample: & 2.3 & 21 & 1 \\
\hline
\end{tabular}




\begin{tabular}{|l|l|}
\hline \multicolumn{3}{|c|}{ ESTIMATING DOE ANALYTICAL CAPACTT AVAILABLE TO EM } \\
DATA INPUT SHEET
\end{tabular}

\begin{tabular}{|c|c|c|c|c|c|c|c|c|}
\hline \multicolumn{9}{|c|}{ DISTRIBUTION OF CUSTOMER SAMPLES (\%) } \\
\hline \multirow{2}{*}{$\begin{array}{l}\text { Protocol/ } \\
\text { Matrix }\end{array}$} & \multicolumn{4}{|c|}{ Regulatory-Driven } & & \multicolumn{3}{|c|}{ Non-Regulatory-Driven } \\
\hline & CWA & CLP & SW846 & NIOSH & Other Reg & $\begin{array}{c}\text { Waste } \\
\text { Treatment }\end{array}$ & $\begin{array}{l}\text { Prod'n } \\
\text { Support }\end{array}$ & $\begin{array}{c}\text { Other } \\
\text { Non-Reg }\end{array}$ \\
\hline & & & & & & & & \\
\hline M1 & 10 & 5 & 15 & & & 20 & 5 & 5 \\
\hline M2 & & 3 & 9 & 4 & & 10 & & \\
\hline M3 & & & 74 & & & & & \\
\hline & & & & & & & & \\
\hline$E M$ & 5 & 8 & 37 & 2 & & 20 & & 3 \\
\hline
\end{tabular}

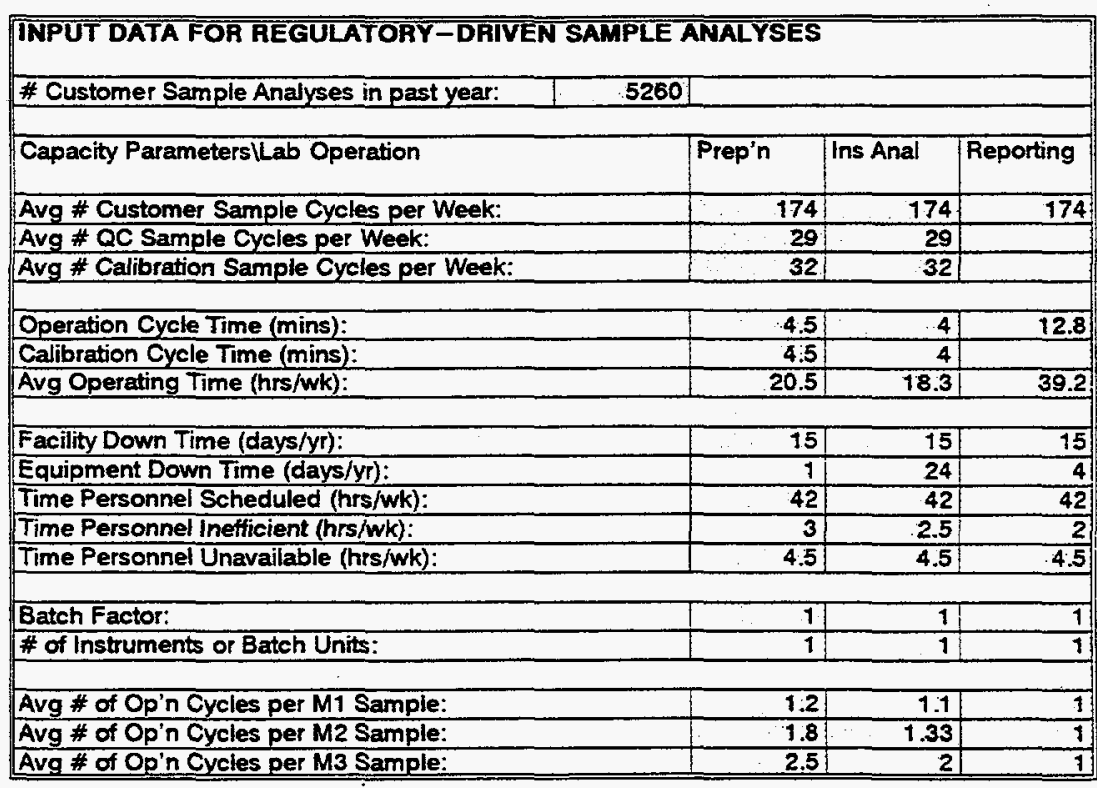

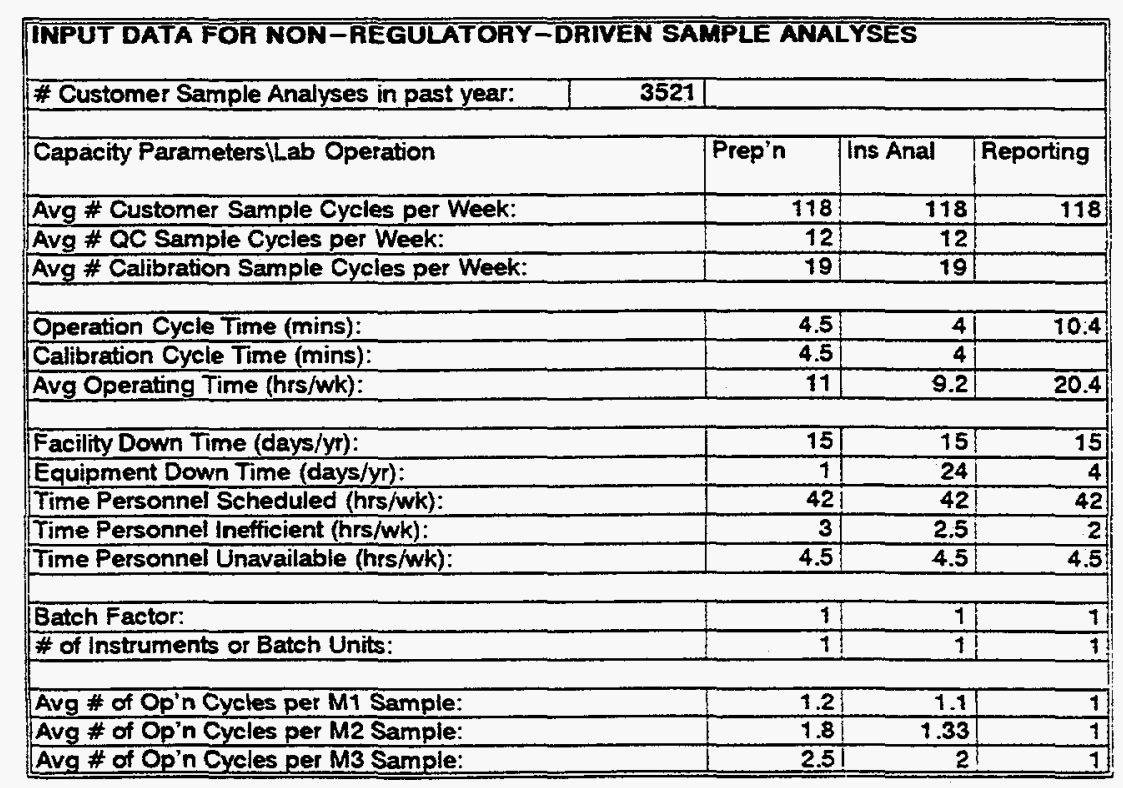

\section{B-5}




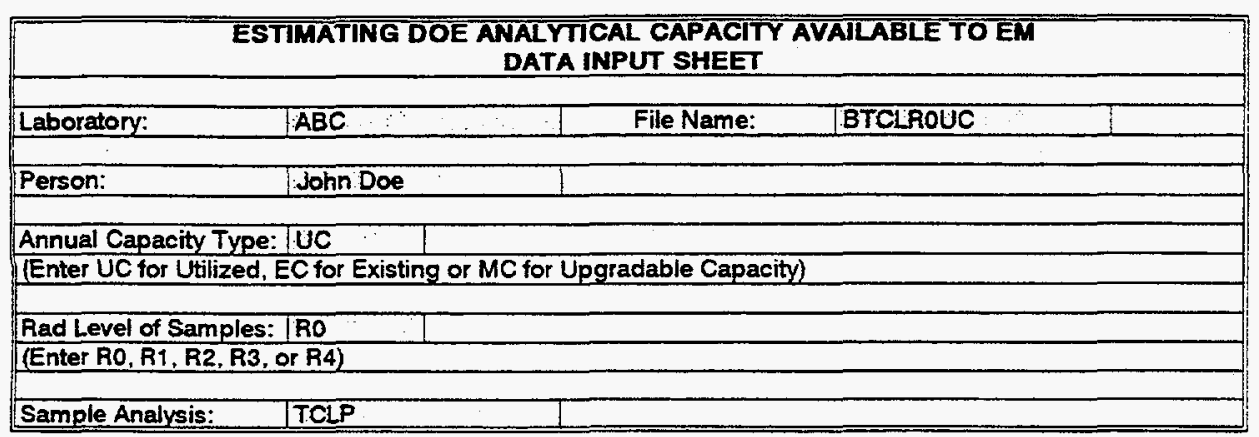

\begin{tabular}{|c|c|c|c|c|c|c|c|c|}
\hline \multicolumn{9}{|c|}{ DISTRIBUTION OF CUSTOMER SAMPLES (\%) } \\
\hline \multirow{2}{*}{$\frac{\text { Protocol/ }}{\text { Matrix }}$} & \multicolumn{4}{|c|}{ Regulatory-Driven } & & \multicolumn{3}{|c|}{ Non-Regulatory-Driven } \\
\hline & CWA & CLP & SW846 & NIOSH & Other Reg & \begin{tabular}{c|} 
Waste \\
Treatment \\
\end{tabular} & $\begin{array}{c}\text { Prod'n } \\
\text { Support } \\
\end{array}$ & $\begin{array}{c}\text { Other } \\
\text { Non-Reg }\end{array}$ \\
\hline M1 & & & & & & & & \\
\hline M2 & & & & & & & & \\
\hline M3 & & & 80 & & & 20 & & \\
\hline & & & & & & & & \\
\hline EM & & & 80 & & & 10 & & \\
\hline
\end{tabular}

\begin{tabular}{|c|c|c|c|}
\hline \multicolumn{4}{|c|}{ INPUT DATA FOA REGULATORY-DRIVEN SAMPLE ANALYSES } \\
\hline \# Customer Sample Analyses in past year: & \multicolumn{3}{|c|}{471} \\
\hline Capacity ParametersiLab Operation & Prep'n & ins Anal & Reporting \\
\hline Avg \# Customer Sample Cycles per Week: & 12 & & \\
\hline Avg \# QC Sample Cycles per Week: & 4 & & \\
\hline \multicolumn{4}{|l|}{ Avg \# Calibration Sample Cycles per Week: } \\
\hline Operation Cycle Time (mins): & 1200 & & \\
\hline \multicolumn{4}{|l|}{ Calibration Cycle Time (mins): } \\
\hline \multicolumn{4}{|l|}{ Avg Operating Time (hrs/wk): } \\
\hline Facility Down Time (days/yr): & 15 & & \\
\hline Equipment Down Time (days/yr): & 1 & & \\
\hline Time Personnel Scheduled (hrs/wk): & 31.5 & & \\
\hline Time Personnel Inefficient (hrs/wk): & 2.25 & & \\
\hline \multicolumn{4}{|l|}{ Time Personnel Unavailable (hrs/wk): } \\
\hline Batch Factor: & 8 & & \\
\hline \# of instruments or Batch Units: & 1 & & \\
\hline \multirow{2}{*}{\multicolumn{4}{|c|}{ Avg \# of Op'n Cycles per M1 Sample: }} \\
\hline & \multicolumn{3}{|c|}{ Avg \# of Op'n Cycles per M2 Sample: } \\
\hline Avg \# of Op'n Cycles per M3 Sample: & 1.05 & & \\
\hline
\end{tabular}

\begin{tabular}{|c|c|c|c|}
\hline \multicolumn{4}{|c|}{ INPUT DATA FOR NON-REGULATORY-DRIVEN SAMPLE ANALYSES } \\
\hline \# Customer Sample Analyses in past year: & \multicolumn{3}{|c|}{128} \\
\hline Capacity Parameters|Lab Operation & Prep'n & Ins Anal & Reporting \\
\hline Avg \# Customer Sample Cycles per Week: & 3.75 & & \\
\hline & 1 & & \\
\hline \multicolumn{4}{|l|}{ Avg \# Calibration Sample Cycles per Week: } \\
\hline \multicolumn{4}{|l|}{ Operation Cycle Time (mins): } \\
\hline \multicolumn{4}{|l|}{ Calibration Cycle Time (mins): } \\
\hline \multicolumn{4}{|l|}{ Avg Operating Time (hrs/wk): } \\
\hline \multicolumn{4}{|l|}{ Facility Down Time (days/yr): } \\
\hline Equipment Down Time (days/yr): & 1 & & \\
\hline Time Personnel Scheduled (hrs/wk): & 10.5 & & \\
\hline Time Personnel Inefficient (hrs/wk): & 0.75 & & \\
\hline \multicolumn{4}{|l|}{ Time Personnel Unavailable (hrs/wk): } \\
\hline Batch Factor: & 8 & & \\
\hline \multicolumn{4}{|l|}{ \# of Instruments or Batch Units: } \\
\hline \multicolumn{4}{|l|}{ Avg \# of Op'n Cycles per Mi Sample: } \\
\hline \multicolumn{4}{|l|}{ Avg \# of Op'n Cycles per M2 Sample: } \\
\hline Avg \# of Op'n Cycies per M3 Sample: & 1.05 & & \\
\hline
\end{tabular}




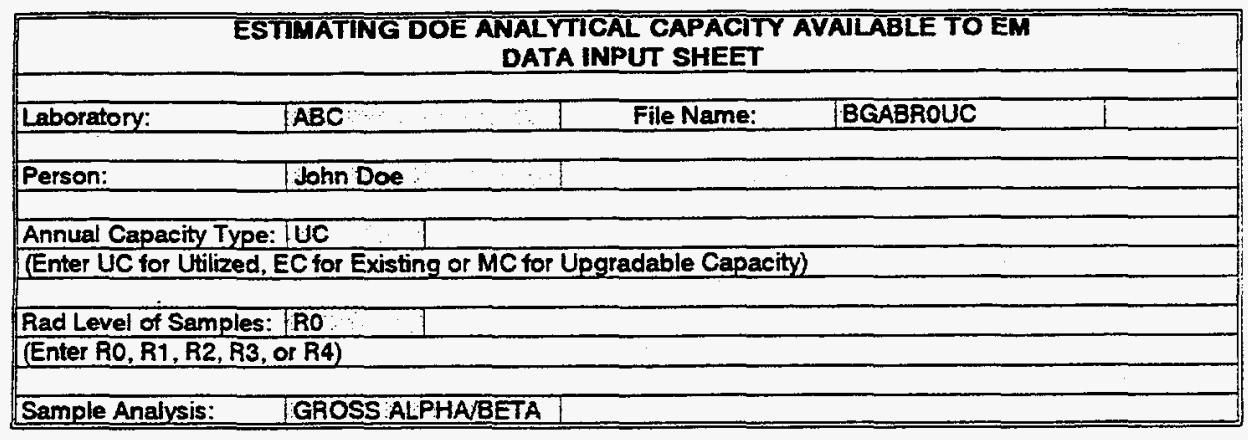

\begin{tabular}{|c|c|c|c|c|c|c|c|c|}
\hline \multicolumn{9}{|c|}{ DISTRIBUTION OF CUSTOMER SAMPLES (\%) } \\
\hline \multirow{2}{*}{\begin{tabular}{|l} 
Protocol/ \\
Matrix
\end{tabular}} & \multicolumn{4}{|c|}{ Regulatory-Driven } & & \multicolumn{3}{|c|}{ Non-Regulatory-Driven } \\
\hline & CWA & CLP & SW846 & NIOSH & Other Reg & \begin{tabular}{|c|} 
Waste \\
Treatment \\
\end{tabular} & $\begin{array}{c}\text { Prod'n } \\
\text { Support }\end{array}$ & $\begin{array}{c}\text { Other } \\
\text { Non-Reg }\end{array}$ \\
\hline M1 & & & & & & 5 & & 53 \\
\hline$M 2$ & & & & 10 & & & & 32 \\
\hline \multicolumn{9}{|l|}{$M 3$} \\
\hline & & & & & & & & \\
\hline$E M$ & & & & 10 & & 5 & & 65 \\
\hline
\end{tabular}

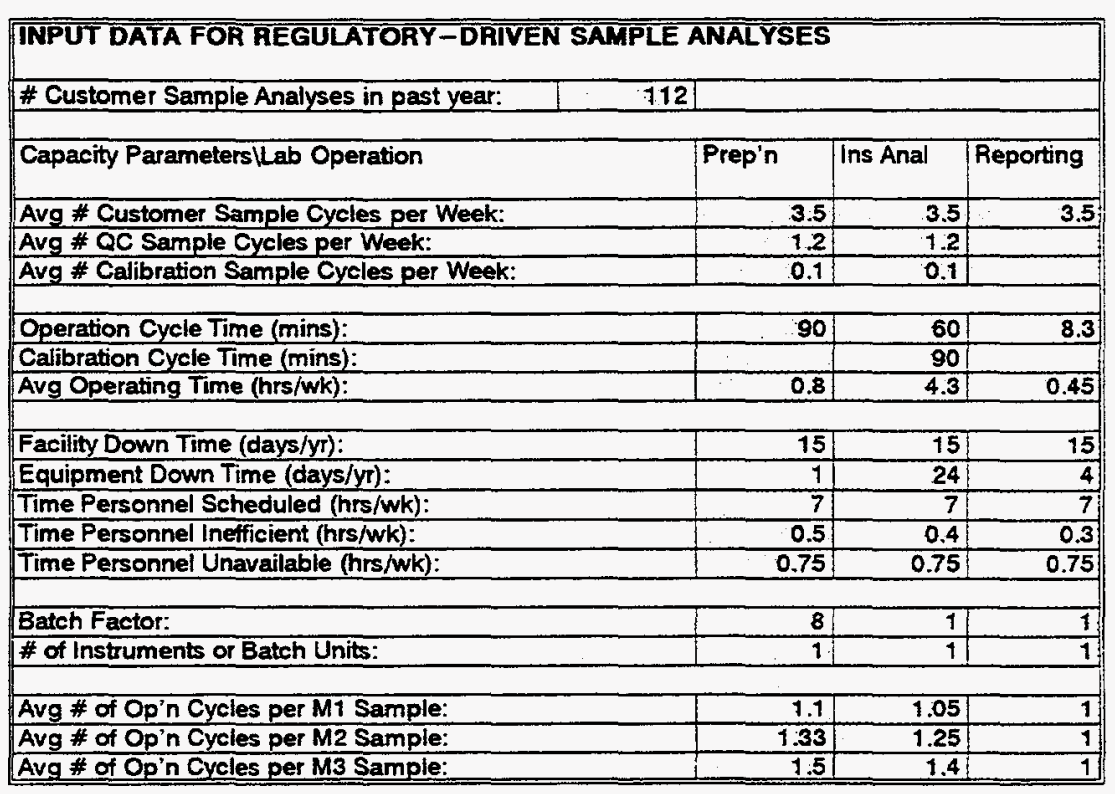

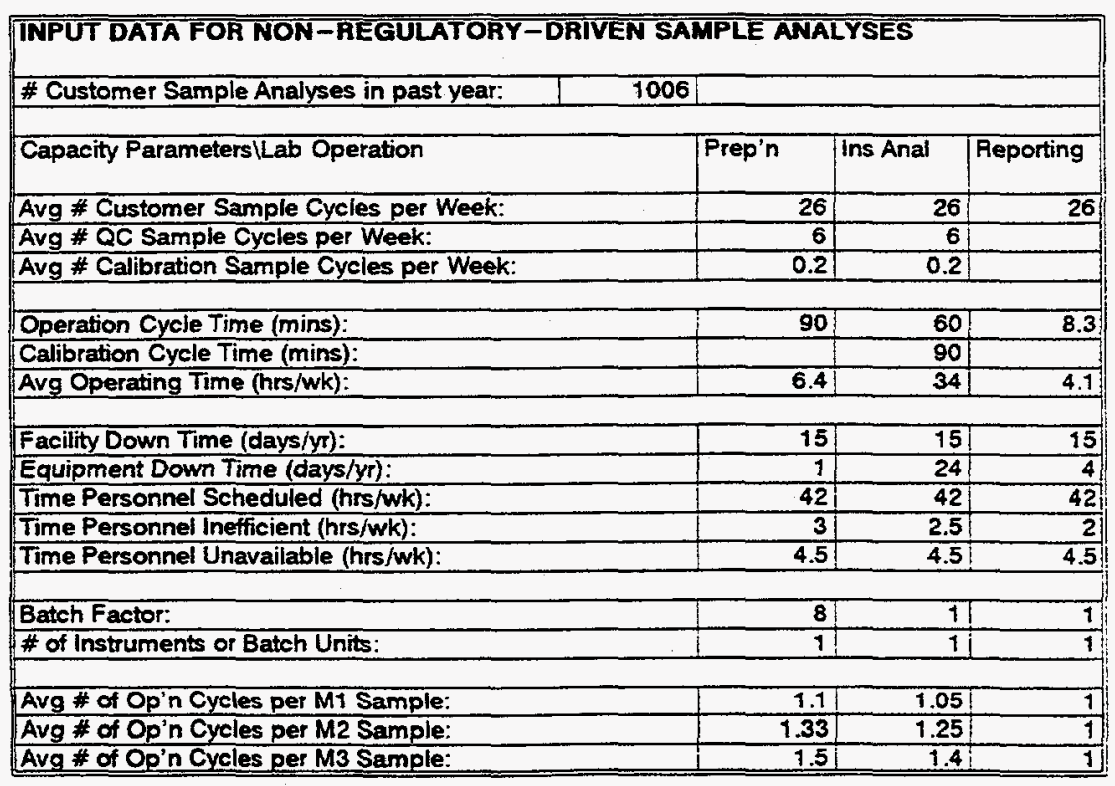




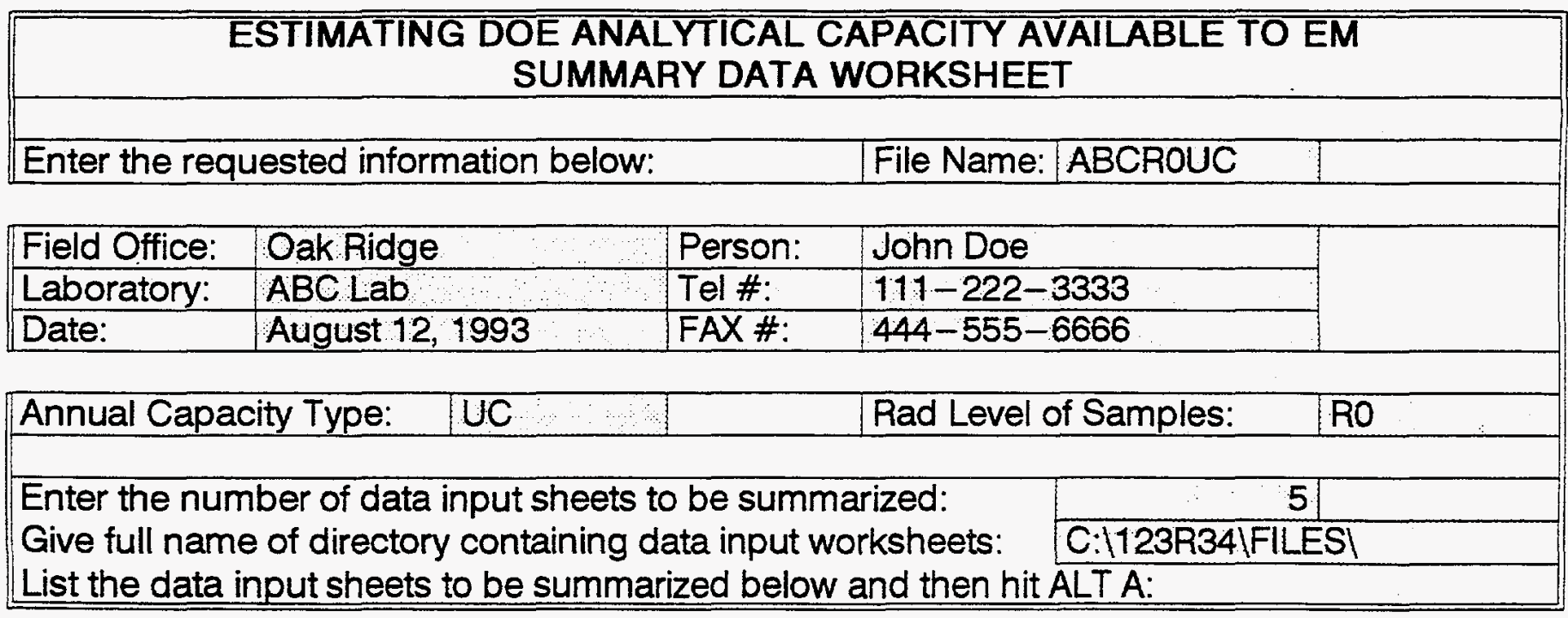

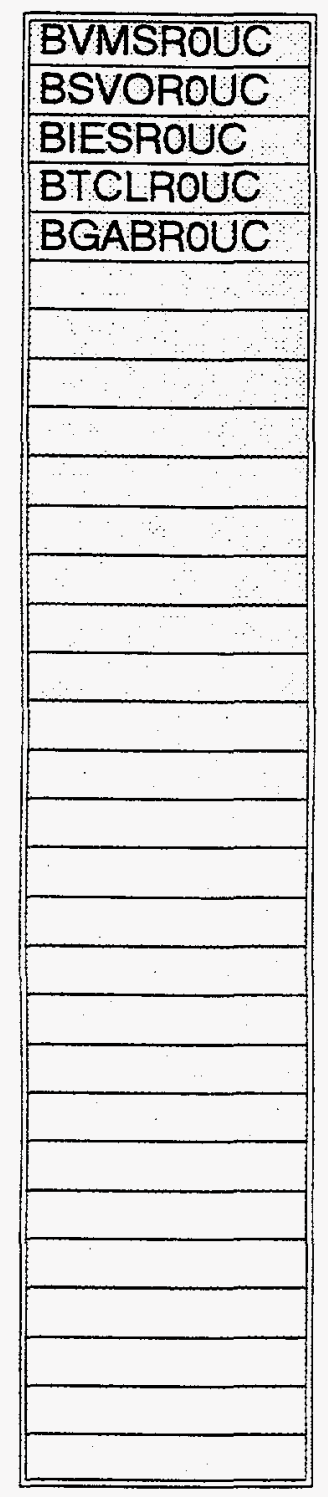


ESTIMATING DOE ANALYTICA CAPACITY AVALABLE TO EM

SUMMARY OF INPUT DATA

\begin{tabular}{|c|c|c|c|c|c|c|c|c|c|c|c|c|c|c|c|c|c|c|}
\hline \multirow{3}{*}{\multicolumn{2}{|c|}{$\begin{array}{l}\text { Field Office: } \\
\text { Laboratory: } \\
\text { Date: }\end{array}$}} & \multicolumn{2}{|c|}{ Oak Pidge } & Person: & \multicolumn{2}{|l|}{ John Doe } & \multicolumn{3}{|c|}{ Annual Capacity Type: } & \multicolumn{2}{|c|}{ UC } & & & & & & & \\
\hline & & \multicolumn{2}{|l|}{ ABCLab } & Tel \#: & \multicolumn{2}{|c|}{$111-222-3333$} & \multicolumn{3}{|c|}{ Rad Level of Samples: } & \multirow{2}{*}{\multicolumn{2}{|c|}{\begin{tabular}{|l} 
RO \\
$A B C R O U C$
\end{tabular}}} & & & & & & & \\
\hline & & \multicolumn{2}{|c|}{ August 12,1993} & FAX \#: & \multicolumn{2}{|c|}{$444-555-6666$} & \multicolumn{3}{|l|}{ File Name: } & & & & & & & & & \\
\hline \multicolumn{19}{|c|}{ INPUT DATA } \\
\hline $\begin{array}{l}\text { Sample } \\
\text { Analysis }\end{array}$ & $\begin{array}{c}\text { Reg/ } \\
\text { Non-Reg }\end{array}$ & $\begin{array}{l}\text { Lab } \\
\text { Op'n }\end{array}$ & $\begin{array}{l}\text { \#Cust } \\
\text { Cycles } \\
\text { per wk }\end{array}$ & $\begin{array}{l}\text { \#QC } \\
\text { Cycles } \\
\text { per wK }\end{array}$ & $\begin{array}{l}\text { \#Cal } \\
\text { Cycles } \\
\text { per wk }\end{array}$ & $\begin{array}{c}\text { Op Cycle } \\
\text { Time } \\
\text { (mins) }\end{array}$ & $\begin{array}{l}\text { Cal Cyc } \\
\text { Time } \\
\text { (mins) }\end{array}$ & $\begin{array}{l}\text { Op Time } \\
\text { (hrs/wk) }\end{array}$ & $\begin{array}{c}\text { Fac Down } \\
\text { Time } \\
\text { (days/yo) }\end{array}$ & $\begin{array}{c}\text { Ins Down } \\
\text { Time } \\
\text { (days/yr) }\end{array}$ & $\begin{array}{c}\text { Time } \\
\text { Per Schd } \\
\text { (hrsiwk) }\end{array}$ & $\begin{array}{l}\text { Time } \\
\text { Per Inef } \\
\text { (hrshwh) }\end{array}$ & $\begin{array}{c}\text { Time } \\
\text { Per Unav } \\
\text { (hrs/wk) }\end{array}$ & $\begin{array}{l}\text { Balch } \\
\text { Factor }\end{array}$ & $\begin{array}{c}\text { \# of Ins } \\
\text { or Batch } \\
\text { Units }\end{array}$ & $\begin{array}{l}\text { Avg \# of } \\
\text { Op Cycs } \\
\text { per M1 }\end{array}$ & $\begin{array}{l}\text { Avg \# of } \\
\text { Op Cycs } \\
\text { per M2 }\end{array}$ & $\begin{array}{l}\text { Avg \# of } \\
\text { Op Cycs } \\
\text { per M3 }\end{array}$ \\
\hline & & & & & & & & & & & & & & & & & & \\
\hline \multirow[t]{2}{*}{$\mathrm{VOA}-\mathrm{GC} / \mathrm{I}$} & Reg & Prep & 0.0 & 0.0 & 0.0 & 0.0 & 0.0 & 0.0 & 0.0 & 0.0 & 0.0 & 0.0 & 0.0 & of & 0 & 0.00 & 0.00 & $\frac{0.00}{200}$ \\
\hline & Reg & Anal & 40.0 & 10.0 & 10.0 & 46.0 & 50.0 & 49.5 & 15.0 & 20.0 & 40.0 & 2.5 & 8.5 & 1 & 1 & 1.10 & 1.20 & 2.00 \\
\hline \multirow{4}{*}{ VOA-GC/ } & Reg & Rep & 40.0 & 0.0 & 0.0 & 12.8 & 0.0 & 8.2 & 15.0 & 2.0 & 42.0 & 3.0 & 8.5 & 1 & 1 & 1.00 & 1.00 & 1.00 \\
\hline & Non-Reg & Prep & 0.0 & 0.0 & 0.0 & 0.0 & 0.0 & 0.0 & 0.0 & 0.0 & 0.0 & 0.0 & 0.0 & 0 & 0 & 0.00 & 0.00 & 0.00 \\
\hline & Non-Rөg & Anal & 55.0 & 25.0 & 5.0 & 46.0 & 60.0 & 36.0 & 15.0 & 34.0 & 42.0 & 2.5 & 8.5 & 1 & 2. & 1.00 & 1.40 & 2.10 \\
\hline & Non-Reg & Fep & 55.0 & 0.0 & 0.0 & 5.2 & 0.0 & 4.8 & 15.0 & 2.0 & 42.0 & 3.0 & 8.5 & 1 & 1 & 1.00 & 1.00 & 1.00 \\
\hline \multirow{3}{*}{ SEM-VOA } & Reg & Prep & 15.0 & 10.0 & 0.0 & 240.0 & $0.0]$ & 16.0 & 15.0 & 1.0 & 40.0 & 3.0 & 4.5 & 7 & 1 & 1.10 & 1.33 & 2.00 \\
\hline & Heg & Anal & 15.0 & 15.0 & 10.0 & 75.0 & 150.0 & 50.0 & 15.0 & 15.0 & 40.0 & 2.5 & 4.5 & 1 & 1 & 1.10 & 1.33 & 2.00 \\
\hline & Heg & Rep & 15.0 & 0.0 & 0.0 & 14.0 & 0.0 & 3.4 & 15.0 & 4.0 & 42.0 & 3.0 & 4.5 & 1 & 1 & 1.00 & 1.00 & 1.00 \\
\hline \multirow{3}{*}{ SEMI-VO } & Non-Reg & Prep & 1.8 & 2.0 & 0.0 & 480.0 & 0.0 & 7.0 & 15.0 & 1.0 & 40.0 & 3.0 & 4.5 & 6 & 1 & 1.10 & 1.65 & 2.30 \\
\hline & Non-Reg & Anal & 1.8 & 4.0 & 1.0 & 75.0 & 150.0 & 11.0 & 15.0 & 15.0 & 40.0 & 2.5 & 4.5 & 1 & 1 & 1.10 & 1.33 & -2.00 \\
\hline & Non-Aeg & Rep & 1.8 & 0.0 & 0.0 & 10.0 & 0.0 & 0.4 & 15.0 & 4.0 & 42.0 & 3.0 & 4.5 & 1 & 1 & 1.00 & 1.00 & 1.00 \\
\hline \multirow{2}{*}{ ICP-AES } & Reg & Prep & 174.0 & 29.0 & 32.0 & 4.5 & 4.5 & 20.5 & 15.0 & 1.0 & 42.0 & 3.0 & 4.5 & 1 & 1 & 1.20 & 1.80 & 2.50 \\
\hline & Reg & Rep & 174.0 & 0.0 & 0.0 & 12.8 & 0.0 & 39.2 & 15.0 & 4.0 & 42.0 & 2.0 & 4.5 & 1 & 1 & 1.00 & 1.00 & 1.00 \\
\hline \multirow[t]{3}{*}{ ICP-AES } & Non-Reg & Prep & 118.0 & 12.0 & 19.0 & 4.5 & 4.5 & 11.0 & 15.0 & 1.0 & 42.0 & 3.0 & 4.5 & 1 & 1 & 1.20 & 1.80 & 2.50 \\
\hline & Non-Reg & Anal & 118.0 & 12.0 & 19.0 & 4.0 & 4.0 & 9.2 & 15.0 & 24.0 & 42.0 & 2.5 & 4.5 & 1 & 1 & 1.10 & 1.33 & 2.00 \\
\hline & Non-Reg & TRep & 118.0 & 0.0 & 0.0 & 10.4 & 0.0 & 20.4 & 15.0 & 4.0 & 42.0 & 20 & 4.5 & 1 & 1 & 1.00 & 1.00 & 1.00 \\
\hline \multirow[t]{3}{*}{ TCLP } & Reg & Prep. & 12.0 & 4.0 & 0.0 & 1200.0 & 0.0 & 39.0 & 15.0 & 1.0 & 31.5 & 2.3 & 3.5 & 8 & 1 & 0.00 & 0.00 & 1.05 \\
\hline & Reg & Anal & 0.0 & 0.0 & 0.0 & 0.0 & 0.0 & 0.0 & 0.0 & 0.0 & 0.0 & 0.0 & 0.0 & 0 & $\underline{0}$ & 0.00 & 0.00 & 0.00 \\
\hline & Reg & Rep & 0.0 & 0.0 & 0.0 & 0.0 & 0.0 & 0.0 & 0.0 & 0.0 & 0.0 & 0.0 & 0.0 & 0 & 0 & 0.00 & 0.00 & 0.00 \\
\hline \multirow[t]{3}{*}{ TCLP } & Non-Feg & Prep & 3.8 & 1.0 & 0.0 & 1200.0 & 0.0 & 11.5 & 15.0 & 1.0 & 10.5 & 0.8 & 1.0 & 8 & 1 & 0.00 & 0.00 & 1.05 \\
\hline & Non-Reg & Anal & 0.0 & 0.0 & 0.0 & 0.0 & 0.0 & 0.0 & 0.0 & 0.0 & 0.0 & 0.0 & 0.0 & 0 & 0 & 0.00 & 0.00 & 0.00 \\
\hline & Non-Reg & Thep & 0.0 & 0.0 & 0.0 & 0.0 & 0.0 & 0.0 & 0.0 & 0.0 & 0.0 & 0.0 & 0.0 & 0 & 0 & 0.00 & 0.00 & 0.00 \\
\hline GROSS AL & Reg & TPrep & 3.5 & 1.2 & 0.1 & 80.0 & 0.0 & 0.8 & 15.0 & 1.0 & 7.0 & 0.5 & 0.8 & 8 & 1 & 1.10 & 1.33 & 1.50 \\
\hline & Reg & Anal & 3.5 & 1.2 & 0.1 & 60.0 & 90.0 & 4.3 & 15.0 & 24.0 & 7.0 & 0.4 & 0.8 & 1 & 1 & 1.05 & 1.25 & 1.40 \\
\hline & Reg & Rep & 3.5 & 0.0 & 0.0 & 8.3 & 0.0 & 0.5 & 15.0 & 4.0 & 7.0 & 0.3 & 0.8 & 1 & 1 & 1.00 & 1.00 & 1.00 \\
\hline GROSS AL & Non-Reg & Prep & 26.0 & 6.0 & 0.2 & 90.0 & 0.0 & 6.4 & 15.0 & 1.0 & 42.0 & 3.0 & 4.5 & 8 & 1 & 1.10 & 1.33 & 1.50 \\
\hline & Non-Reg & Anal & 26.0 & 6.0 & 0.2 & 60.0 & 90.0 & 34.0 & 15.0 & 24.0 & 42.0 & 2.5 & 4.5 & 1. & 1 & 1.05 & 1.25 & 1.40 \\
\hline & Non-Reg & |Rep & 26.0 & 0.0 & 0.0 & 8.3 & 0.0 & 4.1 & 15.0 & 4.0 & 42.0 & 2.0 & 4.5 & 1) & 1 & 1.00 & 1.00 & 1.00 \\
\hline
\end{tabular}


ESTIMATING DOE ANAL YTICA CAPACITY AVALABIE TO EM

SUMMARY OF CAPACTTY CORRECTON FACTORS

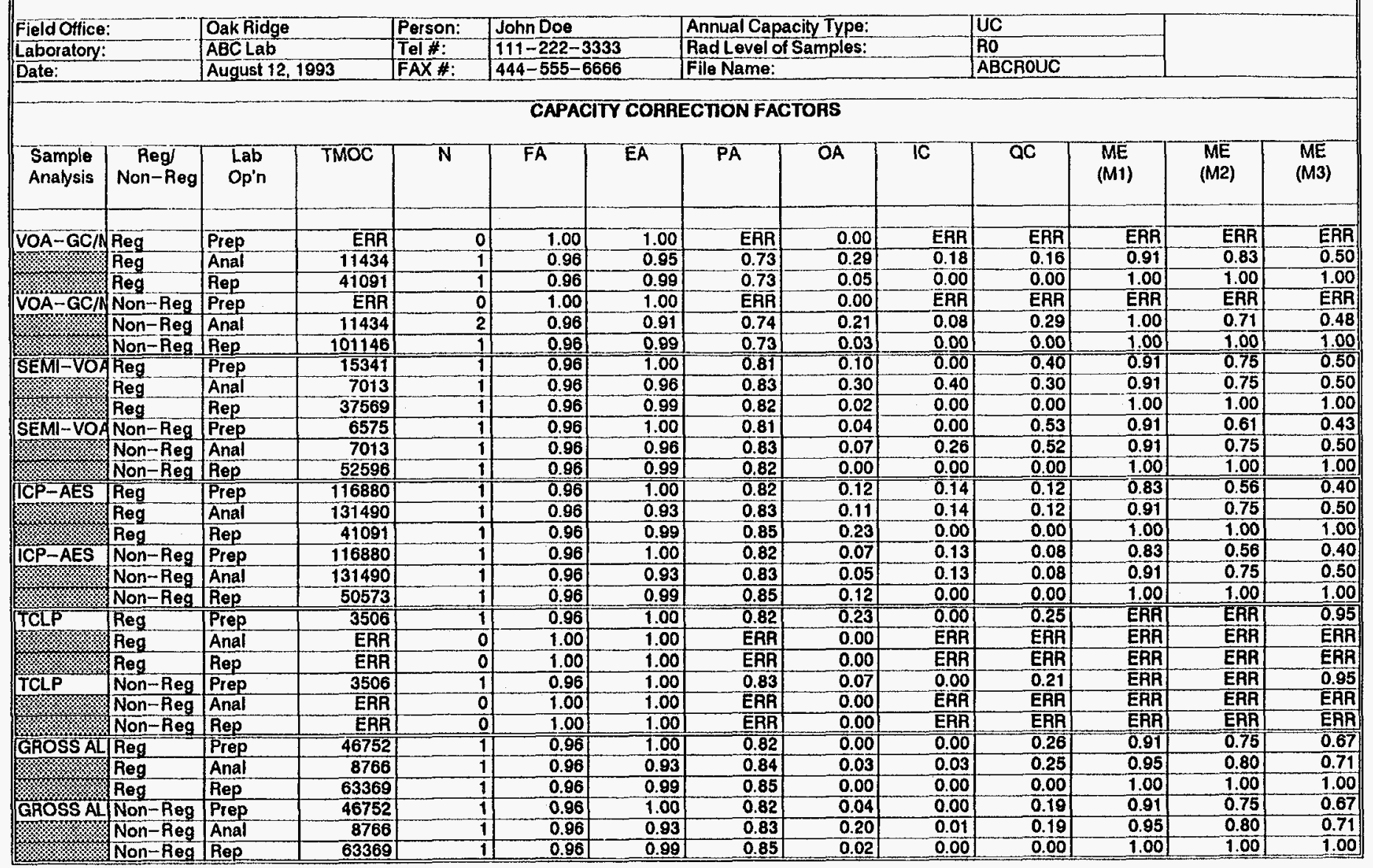




\begin{tabular}{|c|c|c|c|c|c|c|c|c|c|c|c|c|c|c|c|}
\hline \multicolumn{16}{|c|}{$\begin{array}{l}\text { ESTIMATNG DOE ANAIVTICAL CAPACITY AVAILABLE TOEM } \\
\text { SUMMAPY OF CALCULATED ANNUAL CAPACIMES AND OPERATING TIMES }\end{array}$} \\
\hline \multirow{2}{*}{\multicolumn{2}{|c|}{$\begin{array}{l}\text { Field Office: } \\
\text { Laboratory: } \\
\text { Date: }\end{array}$}} & \multirow{2}{*}{\multicolumn{2}{|c|}{\begin{tabular}{|l} 
Oak Ridge \\
ABCLab \\
August 12, 1993 \\
\end{tabular}}} & Person: & \multirow{2}{*}{\multicolumn{2}{|c|}{\begin{tabular}{|l} 
John Doe \\
$111-222-3333$ \\
$444-555-6666$ \\
\end{tabular}}} & \multirow{2}{*}{\multicolumn{2}{|c|}{$\begin{array}{l}\text { Annual Capacity Type: } \\
\text { Rad Level of Samples: } \\
\text { File Name: } \\
\end{array}$}} & & \multirow{2}{*}{\multicolumn{2}{|c|}{$\mid$\begin{tabular}{|l|}
$\mathrm{UC}$ \\
$\mathrm{RO}$ \\
$\mathrm{ABCAOUC}$ \\
\end{tabular}}} & & & & \multirow{2}{*}{ 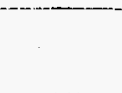 } \\
\hline & & & & $\begin{array}{l}\text { Tel\#: } \\
\text { FAXX\#: }\end{array}$ & & & & & & & & & & & \\
\hline \multicolumn{16}{|c|}{ CALCULATED ANNUAL CAPACITIES AND OPEAATINA TIMES } \\
\hline $\begin{array}{l}\text { Sample } \\
\text { Analysis }\end{array}$ & $\begin{array}{c}\text { Reg/ } \\
\text { Non-Reg }\end{array}$ & $\begin{array}{l}\text { Lab } \\
\text { Op'n }\end{array}$ & $\begin{array}{c}\text { Fraction } \\
\text { Mi }\end{array}$ & $\begin{array}{c}\text { Ann Cap } \\
\text { M1 } \\
\text { (Lab Ops) }\end{array}$ & $\begin{array}{l}\text { Fraction } \\
\text { M2 }\end{array}$ & $\begin{array}{c}\text { Ann Cap } \\
\text { M2 } \\
\text { (Lab Ops) }\end{array}$ & $\begin{array}{l}\text { Fraction } \\
M 3\end{array}$ & $\begin{array}{c}\text { Ann Cap } \\
\text { M3 } \\
\text { (Lab Ops) }\end{array}$ & $\begin{array}{c}\text { Overall } \\
\text { Ann Cap } \\
\text { (Lab Ops) }\end{array}$ & $\begin{array}{c}\text { Overall } \\
\text { Ann Cap } \\
\text { (Smp Ans) }\end{array}$ & $\begin{array}{c}\text { Actual } \\
\text { Ann Cap } \\
\text { (Smp Ans) }\end{array}$ & $\begin{array}{c}\text { \% Diff } \\
\text { Ann Cap } \\
\text { (Smp Ans) }\end{array}$ & $\begin{array}{c}\text { Fraction } \\
\text { EM }\end{array}$ & $\begin{array}{c}\text { Actual } \\
\text { Op Time } \\
\text { (hrswk) }\end{array}$ & $\begin{array}{l}\text { Calc } \\
\text { Op Time } \\
\text { (hrs } / w k)\end{array}$ \\
\hline \multirow{6}{*}{ 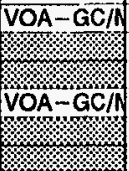 } & Reg & Prop & 0.90 & & 0.07 & $\overline{0}$ & 0.02 & & 0 & & & & & 0.0 & \\
\hline & Reg & Anal & 0.90 & 1323 & 0.07 & 1212 & 0.02 & 727 & 1301 & 1301 & 1496 & $-13 \%$ & $0.7 \overline{5}$ & $\frac{.0}{49.5}$ & $\frac{\mathrm{EHR}}{46.7}$ \\
\hline & Reg & Rep & 0.90 & 1389 & 0.07 & 1389 & 0.02 & 1389 & 1389 & & & & & 8.2 & 8.5 \\
\hline & Non-Reg & Prep & 0.79 & 0 & 0.16 & 0 & 0.05 & 0 & 0 & & & & & 0.0 & $E R A$ \\
\hline & Non-Reg & Anal & 0.79 & 2000 & 0.16 & 1428 & 0.05 & 952 & 1857 & 1857 & 2047 & $-9 \%$ & 0.53 & 36.0 & 332 \\
\hline & Non-Reg & Rep & 0.79 & 2001 & 0.16 & 2001 & 0.05 & 2001 & 2001 & & & & & 4.8 & 4.8 \\
\hline \multirow[t]{3}{*}{ SEMI-VOA } & Heg & Prep & 0.81 & 619 & 0.13 & 512 & 0.06 & 341 & 588 & & & & & 16.0 & 143 \\
\hline & Reg & Anal & 0.81 & 432 & 0.13 & 357 & 0.06 & 238 & 410 & $4 \overline{10}$ & 368 & $12 \%$ & 0.78 & 50.0 & 62.5 \\
\hline & Reg & Rep & 0.81 & 592 & 0.13 & 592 & 0.06 & 592 & 592 & & & & & 3.4 & 3.5 \\
\hline \multirow[t]{3}{*}{ SEMI-VOA } & Non-Reg & Prep & 1.00 & 80 & 0.00 & 60 & 0.00 & 43 & 90 & & & & & 7.0 & 5.0 \\
\hline & Non-Reg & Anal & 1.00 & $\overline{72}$ & 0.00 & 59 & 0.00 & 39 & 72 & 72 & 74 & $-3 \%$ & 0.75 & 11.0 & 9.7 \\
\hline & Non-Reg & Rep & 1.00 & 98 & 0.00 & 98 & 0.00 & 98 & 98 & & & & & 0.4 & 0.3 \\
\hline \multirow{3}{*}{ ICP-AES } & Reg & Trep & 0.50 & 6913 & 0.27 & 4609 & 0.23 & 3318 & 5460 & & & & & 20.5 & 17.6 \\
\hline & Reg & Anal & 0.50 & 7198 & 0.27 & 5953 & 0.23 & 3959 & 6110 & $5 \overline{460}$ & 5260 & $4 \%$ & 0.87 & 18 & 15.7 \\
\hline & Reg & Rep & 0.50 & 7686 & 0.27 & 7686 & 0.23 & 7686 & 7686 & & & & & 39.2 & 37.1 \\
\hline \multirow[t]{3}{*}{ ICP-AES } & Non-Reg & Prep & 0.75 & 3967 & 0.25 & 2645 & 0.00 & 1904 & 3637 & & & & & $\overline{11.0}$ & 11.2 \\
\hline & Non-Reg & Anal & 0.75 & 3870 & 0.25 & 3201 & 0.00 & 2129 & 3703 & 3637 & 3521 & $3 \%$ & 0.58 & 9.2 & 9.9 \\
\hline & Non-Reg & Rep & 0.75 & 4923 & 0.25 & 4923 & 0.00 & 4923 & 4923 & & & & & 204 & 20.5 \\
\hline \multirow{3}{*}{ TCLP } & Keg & Prep & 0.00 & 0 & 0.00 & 0 & 1.00 & 455 & 455 & & & & & $39 . \overline{0}$ & 40.0 \\
\hline & Reg & Anal & 0.00 & 0 & 0.00 & 0 & 1.00 & 0 & 0 & 455 & 471 & $-3 \%$ & 1.00 & 0.0 & ERA \\
\hline & Reg & Rep & 0.00 & 0 & 0.00 & 0 & 1.00 & 0 & 0 & & & & & 0.0 & $E$ ERA \\
\hline \multirow[t]{3}{*}{ TCLP } & Non-Reg & Prep & 0.00 & 0 & 0.00 & 0 & 1.00 & 144 & 144 & & & & & 11.5 & 11.9 \\
\hline & Non-Reg & Anal & 0.00 & 0 & 0.00 & 0 & 1.00 & 0 & 0 & 144 & 128 & $12 \%$ & 0.50 & 0.0 & ERP \\
\hline & Non-Reg & Rep & 0.00 & 0 & 0.00 & 0 & 1.00 & 0 & 0 & & & & & 0.0 & ERF \\
\hline \multirow[t]{3}{*}{ GROSS AL } & Theg & Prep & 0.00 & 118 & 1.00 & 98 & 0.00 & 87 & 98 & & & & & 0.8 & 0.9 \\
\hline & Reg & Anal & 0.00 & 115 & 1.00 & 97 & 0.00 & 87 & 97 & 97 & 112 & $-13 \%$ & 1.00 & 4.3 & 4.9 \\
\hline & Reg & Rep & 0.00 & 137 & 1.00 & 137 & 0.00 & 137 & 137 & & & & & 0.5 & 0.5 \\
\hline \multirow{3}{*}{ GROSS AL } & Non-Reg & Prep & 0.64 & 1033 & 0.36 & 855 & 0.00 & 758 & 970 & & & & & 6.4 & 6.0 \\
\hline & Non-Reg & Anal & 0.64 & 1015 & 0.36 & 853 & 0.00 & 762 & 958 & 958 & 1006 & $-5 \%$ & 0.78 & 34.0 & 32.3 \\
\hline & Non-Reg & Rep & 0.64 & 1240 & 0.36 & 1240 & 0.00 & 1240 & 1240 & & & & & 4.1 & 3.6 \\
\hline
\end{tabular}


APPENDIX C

GLOSSARY OF TERMS

C-1 


\section{INTRODUCTION}

This Glossary of Terms has been designed to capture and standardize terms used in the EM analytical capacity assessment. Efforts have been made to ensure that the terms are as consistent as possible with those used in the National Sample Tracking System (NSTS) and the EM analytical needs determination. 
Definitions of Terms Used in Estimating DOE Analytical Capacity Available to EM

\begin{tabular}{|c|c|}
\hline Term & Definition \\
\hline \multicolumn{2}{|r|}{ Organization/Management } \\
\hline EM-563 & $\begin{array}{l}\text { Office of Environmental Restoration and Waste } \\
\text { Management's Laboratory Management Division }\end{array}$ \\
\hline $\begin{array}{l}\text { Commercial } \\
\text { Laboratory }\end{array}$ & $\begin{array}{l}\text { A privately or publicly owned laboratory operated for profit } \\
\text { and subject to NRC/State licensing when handling } \\
\text { radioactive materials }\end{array}$ \\
\hline DOE Laboratory & $\begin{array}{l}\text { DOE Complex laboratory operated by M\&O/ERMC } \\
\text { contractor and not subject to NRC/State licensing when } \\
\text { handling radioactive materials }\end{array}$ \\
\hline osM & Office of Sample Management or equivalent operation \\
\hline \multicolumn{2}{|r|}{ Capabilities and Capacities } \\
\hline Capability & $\begin{array}{l}\text { The types of analytical measurements a laboratory is able } \\
\text { to perform on a given sample type. }\end{array}$ \\
\hline Capacity & $\begin{array}{l}\text { The number of analytical measurements a laboratory is } \\
\text { able to complete in a given time. }\end{array}$ \\
\hline $\begin{array}{l}\text { Utilized Capacity } \\
\text { (UC) }\end{array}$ & $\begin{array}{l}\text { The capacity being utilized by the analytical laboratory to } \\
\text { support the current work load. }\end{array}$ \\
\hline $\begin{array}{l}\text { Existing Capacity } \\
\text { (EC) }\end{array}$ & $\begin{array}{l}\text { The analytical capacity that exists with current personnel } \\
\text { levels, existing equipment and sample loads optimized. }\end{array}$ \\
\hline $\begin{array}{l}\text { Upgradable } \\
\text { Capacity (MC) }\end{array}$ & $\begin{array}{l}\text { The capacity of an analytical laboratory when upgraded to } \\
\text { the optimum number of staff for } 7 \text { day/wk, } 24 \mathrm{hr} / \mathrm{day} \\
\text { operation and the optimum level of instrumentation, } \\
\text { without construction of any additional laboratory space but } \\
\text { with limited non-capital refurbishment of existing space. }\end{array}$ \\
\hline \multicolumn{2}{|r|}{ Sample Characteristics } \\
\hline Customer Sample & $\begin{array}{l}\text { Any sample sent to the laboratory to be analyzed } \\
\text { separately. These include field samples, field } Q A \text { samples } \\
\text { (e.g., duplicate field samples, field blanks, PE samples) } \\
\text { sample phases and composite samples. }\end{array}$ \\
\hline $\begin{array}{l}\text { Laboratory QC } \\
\text { Sample }\end{array}$ & $\begin{array}{l}\text { A sample that a laboratory analyzes to meet the } \\
\text { requirements of a method. }\end{array}$ \\
\hline Calibration Sample & A sample used for calibrating an instrument or process. \\
\hline
\end{tabular}




\begin{tabular}{|l|l||}
\hline \multicolumn{1}{|c|}{ Term } & \multicolumn{1}{c|}{ Definition } \\
\hline EM Sample & A customer sample that is funded with EM money. \\
\hline R0 & $\begin{array}{l}\text { Customer sample deemed non-radioactive and may be } \\
\text { handled out of any radiation control area }\end{array}$ \\
\hline R1 & $\begin{array}{l}\text { Customer sample that may be contact handled, but must } \\
\text { be in a radiation area }\end{array}$ \\
\hline R2 & Customer sample that must be handled in a shielded hood \\
\hline R3 & Customer sample that must be handled in a glove box \\
\hline R4 & Customer sample that must be handled in hot cell \\
\hline M1 & $\begin{array}{l}\text { Customer sample matrix typically requiring only one } \\
\text { laboratory operational cycle per customer sample } \\
\text { processed. Example: water }\end{array}$ \\
\hline M2 & $\begin{array}{l}\text { Customer sample matrix requiring on average two or less } \\
\text { laboratory operational cycles per customer sample } \\
\text { processed. Examples: soil, sediment, rock, air, biota }\end{array}$ \\
\hline M3 & $\begin{array}{l}\text { Customer sample matrix requiring on average more than } \\
\text { two laboratory operational cycles per customer sample } \\
\text { processed. Examples: sludge, waste, pure product, debris }\end{array}$ \\
\hline Sample Analysis & $\begin{array}{l}\text { The area of a laboratory in which radioactive samples are } \\
\text { required to be handled }\end{array}$ \\
\hline Vodiation Control \\
gene distinct set of major laboratory operations required to \\
\hline goA-GC/MS & $\begin{array}{l}\text { Volatile organic analysis by gas chromatography (GC) } \\
\text { spectrometry (GC/MS) }\end{array}$ \\
\hline Semi-VOA & Semi-volatile organic analysis by GC/MS \\
\hline PCB & Pesticide analysis by GC \\
\hline ICP-AES Metals & $\begin{array}{l}\text { Inductively Coupled Plasma - Atomic Emission } \\
\text { Spectroscopy (ICP-AES) measurement of a single metal } \\
\text { analyte or a suite of metal analytes }\end{array}$ \\
\hline Herbicide analysis by GC \\
\hline Polychlorinated biphenyl analysis by GC
\end{tabular}




\begin{tabular}{|c|c|}
\hline Term & Definition \\
\hline ICP-MS Metals & $\begin{array}{l}\text { Inductively Coupled Plasma - Mass Spectrometry (ICP-MS) } \\
\text { measurement of a single metal analyte or a suite of metal } \\
\text { analytes }\end{array}$ \\
\hline GF-AA Metals & $\begin{array}{l}\text { Graphite Furnace Atomic Absorption (GF-AA) } \\
\text { measurement of individual metal analytes }\end{array}$ \\
\hline Mercury & $\begin{array}{l}\text { Cold Vapor Atomic Absorption (CV-AA) measurement of } \\
\text { mercury }\end{array}$ \\
\hline Cyanide & Measurement of cyanide \\
\hline Anions/Cations & $\begin{array}{l}\text { Anion/cation analysis, by ion chromatography or wet } \\
\text { chemistry (counted individually by ions, e.g., nitrate, or } \\
\text { ammonium for single analysis, or by ion chromatography } \\
\text { runs for simultaneous analyses) }\end{array}$ \\
\hline Gross Alpha/Beta & Gross alpha and beta counting \\
\hline $\begin{array}{l}\text { Alpha Spec } \\
\text { Isotopes }\end{array}$ & Spectrometric analysis of alpha-emitting nuclides \\
\hline $\begin{array}{l}\text { Gamma Spec } \\
\text { Isotopes }\end{array}$ & Spectrometric analysis of gamma-emitting nuclides \\
\hline TCLP Extraction & $\begin{array}{l}\text { Toxicity Characteristic Leaching Procedure (TCLP) - } \\
\text { regulatory method of sample extraction used to estimate } \\
\text { the leaching potential of contaminants from landfills - e.g., } \\
\text { Method } 1311\end{array}$ \\
\hline $\begin{array}{l}\text { Laboratory } \\
\text { Operation }\end{array}$ & $\begin{array}{l}\text { A potentially rate-limiting element of an individual sample } \\
\text { analysis (sample preparation, instrumental analysis, data } \\
\text { reporting) }\end{array}$ \\
\hline Parallel Operation & $\begin{array}{l}\text { Laboratory operation in which units are run concurrently, } \\
\text { such as sample preparation batch processing }\end{array}$ \\
\hline Serial Operation & $\begin{array}{l}\text { Laboratory operation in which units are run sequentially, } \\
\text { such as introduction of samples into an instrument }\end{array}$ \\
\hline $\begin{array}{l}\text { Sample } \\
\text { Preparation }\end{array}$ & $\begin{array}{l}\text { Preparation of sample aliquots for one or more laboratory } \\
\text { operation }\end{array}$ \\
\hline $\begin{array}{l}\text { Instrumental } \\
\text { Analysis }\end{array}$ & Generation of raw analytical data from samples \\
\hline Data Reporting & $\begin{array}{l}\text { Data review, reduction and report/deliverable preparation } \\
\text { required on average for reporting analytical results to a } \\
\text { customer }\end{array}$ \\
\hline
\end{tabular}




\begin{tabular}{|c|c|}
\hline Term & Definition \\
\hline \multicolumn{2}{|r|}{ Cycles, Cycle Times, and Operating Times } \\
\hline Cycle & Level of effort required to perform a single activity once \\
\hline $\begin{array}{l}\text { Sample Analysis } \\
\text { Cycle }\end{array}$ & $\begin{array}{l}\text { Effort required to perform a sample analysis on a single } \\
\text { customer sample }\end{array}$ \\
\hline $\begin{array}{l}\text { Laboratory } \\
\text { Operational Cycle }\end{array}$ & $\begin{array}{l}\text { Effort required to perform an individual laboratory } \\
\text { operation. }\end{array}$ \\
\hline Cycle Time & $\begin{array}{l}\text { Time required to complete one cycle (laboratory operation } \\
\text { cycle, sample analysis cycle, or other cycle) }\end{array}$ \\
\hline $\begin{array}{l}\text { Operational Cycle } \\
\text { Time }\end{array}$ & $\begin{array}{l}\text { Time required to complete one laboratory operation cycle } \\
\text { on a customer or QC sample }\end{array}$ \\
\hline $\begin{array}{l}\text { Calibration Cycle } \\
\text { Time }\end{array}$ & $\begin{array}{l}\text { Time required to complete one calibration cycle of an } \\
\text { instrument or process. This time is distinguished from } \\
\text { other operational cycle times since the time to run a } \\
\text { calibration sample often differs from the time to run other } \\
\text { samples. This cycle time may also include any time } \\
\text { required for tuning an instrument or for rerunning any } \\
\text { calibration samples. }\end{array}$ \\
\hline Operating Time & $\begin{array}{l}\text { Total time spent during a specific time period performing a } \\
\text { laboratory operation. In this model weekly operating times } \\
\text { are used. }\end{array}$ \\
\hline $\begin{array}{l}\text { Calibration } \\
\text { Operating Time } \\
\text { (CT) }\end{array}$ & $\begin{array}{l}\text { Weekly operating time required to calibrate an instrument } \\
\text { or system for a defined laboratory operation. CT = } \\
\text { average calibration cycle time } x \text { average \# of calibration } \\
\text { samples per week. }\end{array}$ \\
\hline $\begin{array}{l}\text { Customer Sample } \\
\text { Operating Time } \\
\text { (ST) }\end{array}$ & $\begin{array}{l}\text { Weekly operating time available to run customer samples } \\
\text { for a defined laboratory operation. ST = average } \\
\text { customer sample cycle time } x \text { average \# of customer } \\
\text { samples per week. }\end{array}$ \\
\hline $\begin{array}{l}\text { Quality Control } \\
\text { Operating Time } \\
\text { (OT) }\end{array}$ & $\begin{array}{l}\text { Weekly operating time required to run quality control } \\
\text { samples for a defined laboratory operation. OT = average } \\
\text { OC cycle time } x \text { average \# of OC samples per week. }\end{array}$ \\
\hline $\begin{array}{l}\text { Average Operating } \\
\text { Time }\end{array}$ & $\begin{array}{l}\text { Average time an instrument operates or a process is } \\
\text { performed per week (i.e. the average time per week that } \\
\text { an instrument is capable of analyzing samples or that a } \\
\text { process may be performed) }\end{array}$ \\
\hline
\end{tabular}




\begin{tabular}{|c|c|}
\hline Term & Definition \\
\hline $\begin{array}{l}\text { Calculated } \\
\text { Average Operating } \\
\text { Time }\end{array}$ & $\begin{array}{l}\text { Average operating time calculated by summing the } \\
\text { component operating times } \mathrm{CT}, \mathrm{ST} \text {, and } \mathrm{OT}\end{array}$ \\
\hline \multicolumn{2}{|r|}{ Capacity Correction Factors } \\
\hline $\begin{array}{l}\text { Theoretical } \\
\text { Maximum of } \\
\text { Operational Cycles } \\
\text { (TMOC) }\end{array}$ & $\begin{array}{l}\text { The theoretical number of operational cycles that can be } \\
\text { performed in one year }\end{array}$ \\
\hline Batch Factor (BF) & $\begin{array}{l}\text { The number of parallel laboratory operations that are or } \\
\text { may be performed simultaneously on a single instrument or } \\
\text { piece of equipment. This factor is determined from actual } \\
\text { laboratory experience when UC is calculated, but is } \\
\text { optimized based on facility and sample availability when } \\
\text { EC or MC are calculated. }\end{array}$ \\
\hline $\begin{array}{l}\text { Number of } \\
\text { Available Units (N) }\end{array}$ & $\begin{array}{l}\text { Number of instruments or other pieces of equipment } \\
\text { available for carrying out a specified laboratory operation }\end{array}$ \\
\hline $\begin{array}{l}\text { Facility Availability } \\
\text { (FA) }\end{array}$ & $\begin{array}{l}\text { Factor accounting for loss of time available for a major } \\
\text { laboratory operation due to support facility failure (e.g. } \\
\text { HVAC failure, hood failure, etc.) equal to } 1 \text { - (total facility } \\
\text { down time for previous year/days / } 365 \text { days) }\end{array}$ \\
\hline $\begin{array}{l}\text { Equipment } \\
\text { Availability (EA) }\end{array}$ & $\begin{array}{l}\text { Factor accounting for equipment down time due to } \\
\text { maintenance and equipment failure when the instrument is } \\
\text { not available to analyze samples equal to } 1 \text { - (equipment } \\
\text { down time for previous year/days / } 365 \text { days) }\end{array}$ \\
\hline $\begin{array}{l}\text { Personnel } \\
\text { Availability (PA) }\end{array}$ & $\begin{array}{l}\text { Factor accounting for the loss of ability to perform a } \\
\text { laboratory operation because personnel are not available } \\
\text { due to time off for vacation, illness, personal reasons, } \\
\text { training, and meetings, etc., and to time lost because of } \\
\text { poor work efficiency, rework due to human errors, and } \\
\text { insufficient workload equal to } 1 \text { - (hours/week lost due to } \\
\text { personnel unavailability or inefficiency / hours/week } \\
\text { personnel are scheduled to work) }\end{array}$ \\
\hline $\begin{array}{l}\text { Operation } \\
\text { Availability (OA) }\end{array}$ & $\begin{array}{l}\text { Factor reflecting the fraction of time that a laboratory } \\
\text { operation is operable (e.g. the time an instrument is } \\
\text { capable of analyzing samples) equal to the average } \\
\text { operating time in hrs/wk / } 168 \mathrm{hrs} / \mathrm{wk}\end{array}$ \\
\hline $\begin{array}{l}\text { Instrument } \\
\text { Calibration (IC) }\end{array}$ & $\begin{array}{l}\text { Factor representing the fraction of operating time applied } \\
\text { to calibration samples equal to } C T /(C T+S T+Q T)\end{array}$ \\
\hline
\end{tabular}




\begin{tabular}{||l|l||}
\hline \multicolumn{1}{|c|}{ Term } & \multicolumn{1}{c|}{ Definition } \\
\hline $\begin{array}{l}\text { Quality Control } \\
\text { (OC) }\end{array}$ & $\begin{array}{l}\text { Factor representing the fraction of operating time applied } \\
\text { to quality control samples equal to QT / (CT }+ \text { ST }+ \text { QT) }\end{array}$ \\
\hline Matrix Effect (ME) & $\begin{array}{l}\text { Factor inversely related to the average number of times } \\
\text { (cycles) that a laboratory operation must be carried out in } \\
\text { order to generate acceptable results for a sample of a } \\
\text { given matrix type equal to the number of customer } \\
\text { samples processed / the total number of laboratory } \\
\text { operational cycles required }\end{array}$ \\
\hline
\end{tabular}




\title{
APPENDIX C
}

\author{
ESTIMATING DOE ANALYTICAL
}

LABORATORY CAPACITY AVAILABLE TO EM

- OPERATION OF DATABASE SPREADSHEET SYSTEM 


\section{Operation of DOE Laboratory Capacity Spreadsheet System}

1. Each laboratory participant was requested to report its capacity data using Lotus $1-2-3^{\circledR}$ spreadsheets. Electronic data for individual sample analyses are collected at the laboratories in spreadsheet files, the format of which is given in the file named DATINPUT.WK3 which is located in directory C: 1 123R34IFILESICAPACITY. The completed DATINPUT worksheets are saved on diskettes using names that follow the naming convention given in the instructions package and then submitted to EM-263. These files are stored on the original diskettes submitted by the laboratory.

2. Capacity data for all sample analyses corresponding to a single capacity type and radiation control zone $(\mathrm{RCZ})$ at a laboratory are also collected by the laboratories in a spreadsheet file, the format of which is given in the file named SUMDAT.WK3 which is also located in directory $C: 1123 R 34$ IFILESICAPACITY 1. Operating macro ALT A imports data from the appropriate DATINPUT files into the spreadsheet and computes the capacities. Operating macros ALT B, ALT $C$, and ALT D generates a hard copy of the calculated data. These datasheets are saved both on the original diskettes submitted by the laboratory as well as in a directory for the laboratory named S:IRSASSOONICAPACITY Iabnamel. The original names assigned to the files by the laboratories are retained. These names should follow the naming convention given in the instructions package.

3. The data provided by the field in the SUMDAT electronic datasheets is reorganized into a number of databases. This is accomplished as described below.

4. The worksheet file CAPDB.WK3 is used to prepare a database of all the capacities for a particular laboratory. This worksheet in its blank form is located in directory $\mathrm{C}: \backslash 123 R 34 \mid$ FILESICAPACITY $\backslash$ and in a form completed with data is saved with the name CAPlab\#.WK3 in a directory named S:IRSASSOONICAPACITYVlabname. The following steps are performed in this worksheet:

(a) Header information and the number and names of summary datasheets, and the name of the directory containing the data input worksheets are entered into the data entry section of the worksheet.

(b) Operating macro ALT E creates the database which lists all the capacities for the laboratory according to the following format:

Ops Office Lab Rad Level Cap Type Samp Anal Reg/NR Lab Op'n Matrix Type Capacity

The database created for each laboratory is saved in a file named DBlab\#.WK3 in the directory S:IRSASSOONICAPACITY ICAPDATAD\. 
5. The worksheet file CAPSADB.WK3 is used to standardize all the sample analysis names in the database files created in the previous step. This worksheet is located in directory $\mathrm{C}: 1123 \mathrm{R} 34 \mid \mathrm{FILES} \backslash \mathrm{CAPACITY} \backslash$. The following steps are performed in this worksheet:

(a) Header information, and the number and names of the database generation directories (S:IRSASSOONICAPACITYVlabnamel), the database generation files (CAPlab\#.WK3), and the database files (DBlab\#.WK3) for all the laboratories are entered into the data entry section of the worksheet.

(b) Operating macro ALT G creates lists of all the sample analyses in the database files, operating ALT H prints out the lists, and operating ALT $\mathrm{I}$ inserts a blank column after each list.

(c) Standard sample analysis names are entered manually into the blank columns next to the sample analysis names listed from the DBlab\#.WK3 database files.

(d) Operating macro ALT A then creates a new set of database files named ABlab\#.WK3 in a directory S:IRSASSOONICAPACITY $\backslash C A P D A T A A \mid$ which are identical to the DBlab\#.WK3 database files but which contain standard sample analysis names.

6. The worksheet file CAPBB.WK3 is used to prepare another database of all the capacities for a particular laboratory. This worksheet in its blank form is located in directory $\mathrm{C}: \backslash 123 \mathrm{R} 34 \backslash \mathrm{FILES} \backslash \mathrm{CAPACITY} \backslash$ and in a form completed with data is saved with the name CAPBlab\#.WK3 in the directory named S:IRSASSOONICAPACITYlabname. The same steps as in CAPDB.WK3 are performed in this worksheet. The database created lists all the capacities for the laboratory according to the following format:

Ops Office Lab Rad Level Cap Type Samp Anal Reg Driver Matrix Type Capacity

The database created for each laboratory is saved in a file named BBlab\#.WK3 in the directory S: IRSASSOONICAPACITYICAPDATAB\. This database does not list capacities for laboratory operations but includes not just UC, EC, and MC sample analysis capacities, but also actual capacity (AC) sample analysis data. It also contains this information according to each regulatory or nonregulatory protocol which was not available in the DBlab\#.WK3 database files.

7. The worksheet file CAPSABB.WK3 is used to standardize all the sample analysis names in the BBlab\#.WK3 database files created in the previous step. This worksheet is located in directory C: $|123 R 34| F I L E S I C A P A C I T Y$. The same steps as in CAPSADB.WK3 are performed in this worksheet. The new set of database files created containing standard sample analysis names are named 
CBlab\#.WK3 and are located in a directory S:\RSASSOONICAPACITY CAPDATACl.

8. The worksheet file CAPEB.WK3 is used to prepare a set of minimized database files named EBlab\#.WK3 which are identical to CBlab\#.WK3 but which contain only records that have non-zero capacities. Searching through such databases is a lot faster. The file CAPEB.WK3 is located in the directory C: $\backslash 123 R 34 \backslash F I L E S \backslash C A P A C I T Y \backslash$ and the EBlab\#.WK3 database files generated are located in the directory named S:IRSASSOONICAPACITYICAPDATAE\. The following steps are performed in the worksheet CAPEB.WK3:

(a) Header information and the number, location, and names of the new database files to be generated are entered into the data entry section of the worksheet.

(b) Operating macro ALT F creates a database in EBlab\#.WK3 from the database in CBlab\#.WK3 with all the extraneous capacity records deleted.

(c) Operating macro ALT G creates a database file named EBCOMP.WK3 which contains all the records of all the EBlab\#.WK3 files and represents data from the whole DOE Complex. This file is also located in the directory S:IRSASSOONICAPACITYICAPDATAE $\backslash$.

9. The final tables of data presented in Appendix D of this report are prepared in worksheets with names corresponding to the table number of the table which appears in the final report. Figures in the report were prepared using the numbers collected in the tables in Appendix D.

10. A list of all the spreadsheets and the directories in which they are found is shown in the table below. 
SPAEADSHEETS FOR EM-263 ANALYTICAL LABORATORY CAPACITY MODEL

\begin{tabular}{|c|c|c|}
\hline Spreadsheet & Directory & Comments \\
\hline DATINPUT & C:|123R34|FILESICAPACITM & Blank data input sheets for field \\
\hline LSA\#R\#\#C & B:I & Completed data input sheets from field \\
\hline SUMDAT & C:1123R34|FILESICAPACITY & Blank summary data sheets for field \\
\hline Lab\#R\#\#C & S:IRSASSOOMICAPACITMlabnamel & Completed summary data sheets from field \\
\hline CAPDB & C:1123A34|FILESICAPACITM & Blank worksheet for generating database file \\
\hline CAPLab\# & S:IRSASSOOMCAPACITYlabnamel & Worksheet for generating database file for lab \\
\hline DBLab\# & S:IRSASSOOMICAPACITMCAPDATADV & Database file for lab \\
\hline CAPBB & C:I123R34IFILESICAPACITM & Blank worksheet for generating database file with $A C$ and reg drivers \\
\hline CAPBLab\# & S:IRSASSOONCAPACITMlabnamel & Worksheet for generating $A C$ database file for lab \\
\hline BBLab\# & S:IRSASSOOMICAPACITMCAPDATABI & Database file for lab with $A C$ and reg drivers \\
\hline CAPSADB & C:I123R34|FILESICAPACITM & Worksheet for standardizing capacity sample analyses in dbase files \\
\hline CAPSABB & C:|123R34|FILESICAPACITM & Worksheet for standardizing capacity sample analyses in $A C$ dbase files \\
\hline ABLab\# & S:IRSASSOOMCAPACITMCAPDATAAI & Database file for lab with std samp ans \\
\hline CBLab\# & S:IRSASSOOMCAPACITMCAPDATACI & Database file for lab with $A C$ and reg drivers with std samp ans \\
\hline CAPEB & C:I123R34IFILESICAPACITM & Blank worksheet for generating minimized database files with $\mathrm{AC}$ and reg drivers \\
\hline EBLab\# & S:IRSASSOOMCAPACITMCAPDATAE & Minimized database file for lab with $A C$ and reg drivers \\
\hline CAPTAB1 & S:IRSASSOOMCAPACITMTABLEII & Blank worksheet for generating Table \\
\hline CAPT1 & S:IRSASSOONICAPACITMTABLE1] & Completed Table I worksheet \\
\hline CAPTAB2 & S:IRSASSOOMCAPACIMTAABLE2 & Blank worksheet for generating Tables II \\
\hline CAPT2\# & S:IRSASSOOMCAPACIMTABLE2] & Completed Table II worksheets \\
\hline CAPTABB & S:IRSASSOONICAPACITYTABLE 3 & Blank worksheet for generating Table III \\
\hline CAPT3\# & S:IRSASSOONICAPACITYTABLES| & Completed Table III worksheets \\
\hline САРТАВ4 & S:IRSASSOONICAPACITMTABLE4| & Blank worksheet for generating Tables IV \\
\hline CAPT4\#\#\# & S:IRSASSOOMICAPACITMTABLE4| & Completed Table IV worksheets \\
\hline
\end{tabular}




\section{APPENDIX D}

ESTIMATING DOE ANALYTICAL

\section{LABORATORY CAPACITY AVAILABLE TO EM}

- DATA TABLES 


\section{List of Tables}

Table I:

Table Ila:

Table Ilb:

Table Ilc:

Table Illa:

Table Illb:

Table Illc:

Table IVa:
Total Sample Analyses by Capacity Type and Laboratory

UC Sample Analyses by Rad Level, Matrix Type, and Laboratory EC Sample Analyses by Rad Level, Matrix Type, and Laboratory MC Sample Analyses by Rad Level, Matrix Type, and Laboratory

UC Sample Analyses Complex-wide by Regulatory and EM Distribution

EC Sample Analyses Complex-wide by Regulatory and EM Distribution

MC Sample Analyses Complex-wide by Regulatory and EM Distribution

UC Sample Analyses Complex-wide by Rad Level and Matrix Type

(i) Complex-wide

(ii) Grand Junction

(iii) Los Alamos Nat'l Lab

(iv) Argonne East

(v) Argonne West

(vi) Brookhaven Nat'I Lab

(vii) Fernald

(viii) Idaho Nat'l Eng Lab

(ix) Idaho Nat'l Eng Lab -

(x) Idaho Nat'I Eng Lab - W

(xi) West Valley Nuclear $\mathrm{Se}$

(xii) Nevada Test Site

(xiii) Oak Ridge - K25

(xiv) Oak Ridge Nat'I Lab

(xv) Oak Ridge - Y12

(xvi) Paducah

(xvii) Portsmouth

(xviii) Hanford - WHC 222S

(xix) Hanford - PNL 325

(xx) Rocky Flats - 123 Lab

(xxi) Rocky Flats - GL881

(xxii) Rocky Flats - RF PA

(xxiii) WSRC-ALD-CLAB

(xxiv) WSRC-ALD-320M

(xxv) WSRC-ALD Water

(xxvi) WSRC-ALD-ETF

(xxvii) WSRC-ALD-Tritium

(xxviii)WSRC-ALD-ITP

(xxix) WSRC-SRTC-ADS

$(x x x)$ WSRC-SRTC-DWPT 
Table IVb:

EC Sample Analyses Complex-wide by Rad Level and Matrix Type

(i) Complex-wide

(ii) Grand Junction

(iii) Los Alamos Nat'l Lab

(iv) Argonne East

(v) Argonne West

(vi) Brookhaven Nat'I Lab

(vii) Fernald

(viii) Idaho Nat'l Eng Lab

(ix) Idaho Nat'l Eng Lab -

(x) Idaho Nat'l Eng Lab - W

(xi) West Valley Nuclear Se

(xii) Nevada Test Site

(xiii) Oak Ridge - K25

(xiv) Oak Ridge Nat'I Lab

(xv) Oak Ridge - Y12

(xvi) Paducah

(xvii) Portsmouth

(xviii) Hanford - WHC 222S

(xix) Hanford - PNL 325

(xx) Rocky Flats - 123 Lab

(xxi) Rocky Flats - GL881

(xxii) Rocky Flats - RF PA

(xxiii) WSRC-ALD-CLAB

(xxiv) WSRC-ALD-32OM

(xXv) WSRC-ALD Water

(xxvi) WSRC-ALD-ETF

(xxvii) WSRC-ALD-Tritium

(xxviii)WSRC-ALD-ITP

(xxix) WSRC-SRTC-ADS

$(x x x)$ WSRC-SRTC-DWPT

Table IVc:

MC Sample Analyses Complex-wide by Rad Level and Matrix Type

(i) Complex-wide

(ii) Grand Junction

(iii) Los Alamos Nat'l Lab

(iv) Argonne East

(v) Argonne West

(vi) Brookhaven Nat'l Lab

(vii) Fernald

(viii) Idaho Nat'l Eng Lab

(ix) Idaho Nat'l Eng Lab -

(x) Idaho Nat'l Eng Lab - W

(xi) West Valley Nuclear Se

(xii) Nevada Test Site

(xiii) Oak Ridge - K25 
(xiv) Oak Ridge Nat'l Lab

(xv) Oak Ridge - Y12

(xvi) Paducah

(xvii) Portsmouth

(xviii) Hanford - WHC 222S

(xix) Hanford - PNL 325

(xx) Rocky Flats - 123 Lab

(xxi) Rocky Flats - GL881

(xxii) Rocky Flats - RF PA

(xxiii) WSRC-ALD-CLAB

(xxiv) WSRC-ALD-32OM

(xxv) WSRC-ALD Water

(xxvi) WSRC-ALD-ETF

(xxvii) WSRC-ALD-Tritium

(xxviii)WSRC-ALD-ITP

(xxix) WSRC-SRTC-ADS

$(x x x)$ WSRC-SRTC-DWPT 
EM-263 SURVEY OF DOE ANALYTICAL CAPACITY AVAILABLE TO EM

Table I: Total Sample Analyses by Capacity Type and Laboratory

\begin{tabular}{|c|c|c|c|c|c|c|c|c|}
\hline Ops Office-Lab/Capacity & $\overline{\mathrm{UC}}$ & $\overline{E E C}$ & $\overline{\mathrm{MC}}$ & $\overline{E C-U C}$ & $\overline{\mathrm{MC}-\mathrm{UC}}$ & $\overline{M C}-\overline{E C}$ & $\overline{\mathrm{AC}}$ & $\begin{array}{l}U C-A C \\
\text { pet diff }\end{array}$ \\
\hline \multicolumn{9}{|l|}{ ALBUQUERQ̄UE } \\
\hline Grand Junction & $\overline{10064}$ & 53882 & 108921 & 43819 & 98857 & 55039 & 10253 & $-2 \%$ \\
\hline Los Alamos Nat'I Lab & 36710 & 34117 & 277283 & -2593 & 240572 & 243165 & 36380 & $1 \%$ \\
\hline TOTALAL & 46774 & 87999| & 386203 & 41225 & 339430 & 298204 & 46633 & $0 \%$ \\
\hline \multirow{2}{*}{\multicolumn{9}{|c|}{ CHICAGO }} \\
\hline & & & & & & & & \\
\hline \begin{tabular}{|l|} 
Argonne East \\
Argonne West
\end{tabular} & 4629 & 12610 & 69920 & 7981 & 65291 & 57310 & 4987 & $-7 \%$ \\
\hline Argonne West & 1860 & 0 & of & -1860 & -1860 & 0 & 3205 & $-42 \%$ \\
\hline Brookhaven Nat' I Lab & 11880 & of & 0 & -11880 & -11880 & 0 & 20290 & $-41 \%$ \\
\hline \multirow[t]{2}{*}{ TOTAL CH } & 18369 & 12610 & 69920 & -5759 & 51552 & 57310 & 28482 & $-36 \%$ \\
\hline & & & & & & & & \\
\hline \multicolumn{9}{|l|}{ FERNALD } \\
\hline Fernald & 38755 & 85157 & 254221 & 46401 & 215466 & 169065 & 44861 & $-14 \%$ \\
\hline \multirow[t]{3}{*}{ TOTALFS } & 38755 & 85157 & 254221 & 46401 & 215466 & 169065 & 44861 & $-14 \%$ \\
\hline & \multirow{2}{*}{\multicolumn{8}{|c|}{ IDAHO }} \\
\hline & & & & & & & & \\
\hline Idaho Nat'I Eng Lab - ACU & 407 & 1216 & 5130 & 809 & 4723 & 3914 & 416 & $-2 \%$ \\
\hline Idaho Nat'l Eng Lab - B\&W & 1460 & 10486 & 0 & 9026 & -1460 & -10486 & 1625 & $-10 \%$ \\
\hline Idaho Nat'I Eng Lab - WINCO & 0 & 24533 & 57741 & 24533 & 57741 & 33209 & 0 & ERR \\
\hline West Valley Nuclear Services & 4462 & 4481 & 4340 & 19 & -123 & -142 & 4480 & $-0 \%$ \\
\hline \multirow{2}{*}{ TOTALID } & 6329 & (40715 & 67211 & 34386 & 60882 & 26496 & 6521 & $-3 \%$ \\
\hline & & & & & & & & \\
\hline \multicolumn{9}{|l|}{ NEVADA } \\
\hline \multirow{3}{*}{$\begin{array}{l}\text { Nevada Test Site } \\
\text { TOTAL NV } \\
\end{array}$} & 29290 & 38800 & 71125 & 9509 & 41834 & 32325 & 37871 & $-23 \%$ \\
\hline & 29290 & 38800 & 71125] & 9509 & 41834 & 32325 & 37871 & $-23 \%$ \\
\hline \multirow{2}{*}{\multicolumn{9}{|c|}{ OAK RIDGE }} \\
\hline & & & & & & & & \\
\hline Oak Ridge - K25 & $4 \overline{41364}$ & 80361 & 324906 & 38997 & 283542 & 244545 & 45709 & $-10 \%$ \\
\hline Oak Ridge Nat'I Lab & 23204 & 28147 & 45391 & 4943 & 22187 & 17244 & 24091 & $-4 \%$ \\
\hline Oak Ridge - Y12 & 220573 & 311958 & 1031327 & 91385 & 810754 & 719369 & 226743 & $-3 \%$ \\
\hline Paducah & 59971 & 59926 & 152046 & -45 & 92075 & 92119 & 62899 & $-5 \%$ \\
\hline Portsmouth & 79564 & 86013 & 203463 & 6449 & 123899 & 117450 & 93563 & $-15 \%$ \\
\hline \multirow[t]{2}{*}{ TOTAL OR } & 424677 & 566405 & 1757132 & 141729 & 1332456 & 1190727 & 453005 & $-6 \%$ \\
\hline & & & & & & & & \\
\hline \multicolumn{9}{|l|}{ BICHLAND } \\
\hline Hanford - WHC 222S & 35012 & 631967 & 1452081 & 596955 & 1417069 & 820114 & 35813 & $-2 \%$ \\
\hline Hanford - PNL 325 & 7512 & 63037 & 273225 & 55524 & 265712 & 210188 & 7879 & $-5 \%$ \\
\hline \multirow[t]{2}{*}{ TOTALRL } & 42524 & 695003 & 1725305 & 652479 & 1682782 & 1030302 & 43692 & $-3 \%$ \\
\hline \multirow{2}{*}{\multicolumn{9}{|c|}{ ROCKY FLATS }} \\
\hline & & & & & & & & \\
\hline \multirow{4}{*}{$\begin{array}{l}\text { Rocky Flats - 123 Lab } \\
\text { Rocky Flats - GL881 } \\
\text { Rocky Flats - RF PA Lab } \\
\text { TOTAL RF } \\
\end{array}$} & 39168 & 225678 & 591129 & 186510 & 551961 & 365451 & 39331 & $-0 \%$ \\
\hline & 12611 & 14180 & 80173 & 1569 & 67562 & 65994 & 12334 & $2 \%$ \\
\hline & 5970 & 16674 & 95464 & 10704 & 89494 & 78790 & 6341 & $-6 \%$ \\
\hline & 57749 & 256532 & 766766 & 198783 & 709018 & 510235 & 58006 & $-0 \%$ \\
\hline & & & & & & & & \\
\hline \multicolumn{9}{|l|}{ SAVANNAH RIVER } \\
\hline WSRC-ALD-CLAB & 6341 & 20799 & 729214 & 14457 & 722873 & 708415 & 11847 & $-46 \%$ \\
\hline WSRC-ALD-320M & 0 & 1119 & 0 & 1119 & 0 & -1119 & 396 & $-100 \%$ \\
\hline WSRC-ALD Water & 6205 & 9054 & 22584 & 2849 & 16379 & 13530 & 29516 & $-79 \%$ \\
\hline WSRC-ALD-ETF & 1008 & 0 & 0 & -1008 & -1008 & 0 & 11760 & $-91 \%$ \\
\hline WSRC-ALD-Tritium & 1096 & 2566 & 2566 & 1470 & 1470 & 0 & 39024 & $-97 \%$ \\
\hline WSRC-ALD-ITP & 1070 & 0 & 0 & -1070 & -1070 & 0 & 1083 & $-1 \%$ \\
\hline WSRC-SRTC-ADS & 22935 & 41080 & 112468 & 18144 & 89533 & 71389 & 40342 & $-43 \%$ \\
\hline WSRC-SRTC-DWPT & 5033 & 8177 & 8177 & 3145 & 3145 & 0 & 12300 & $-59 \%$ \\
\hline TOTAL SR & 43688 & 82794 & 875010 & 39107 & 831322 & 792215 & 146268 & $-70 \%$ \\
\hline & & & & & & & & \\
\hline TOTAL COMPLEX & 708155 & 1866016 & 5972895 & 1157861 & 5264740 & 4106879 & 865339 & $-18 \%$ \\
\hline
\end{tabular}


Table lla: UC Sample Analyses by Rad Level, Matrix Type, and Laboratory

\begin{tabular}{|c|c|c|c|c|c|c|c|c|c|}
\hline Ops Office-Lab/Rad-Matrix & RO & RO/Rt & $\overline{\mathrm{R1}}$ & R2 & $\overline{R 3}$ & R4 & M1 & $\mathrm{M2}$ & M3 \\
\hline \multicolumn{10}{|l|}{ ALBUQUERQUE } \\
\hline Grand Junction & 0 & 10064 & 0 & $\overline{0}$ & 0 & 0 & 3457 & 6607 & 0 \\
\hline Los Alamos Nat'/ Lab & 34053 & 0 & 2657 & 0 & 0 & o & 20677 & 13909 & 2125 \\
\hline \multirow[t]{2}{*}{ TOTAL AL } & 34053 & 10064 & 2657 & - & 0 & 0 & 24133 & 20515 & 2125 \\
\hline & & & & 1 & $=$ & I & & & \\
\hline \multicolumn{10}{|l|}{ CHICAGO } \\
\hline Argonne East & 153 & 2823 & $15 \overline{21}$ & $\overline{0}$ & 132 & 0 & 1608 & 1284 & 1738 \\
\hline Argonne West & 0 & 0 & 1860 & 0 & 0 & 0 & 1305 & 443 & 112 \\
\hline Brookhaven Nat'l Lab & 0 & 11880 & 0 & 0 & 0 & D & 11880 & 0 & 0 \\
\hline \multirow[t]{2}{*}{ TOTAL CH } & 153 & 14703 & 3381 & 0 & 132 & 0 & 14792 & 1726 & 1850 \\
\hline & & & & & & & & & \\
\hline \multicolumn{10}{|l|}{ FEFNALD } \\
\hline Fernald & 0 & 38755 & 0 & 0 & 0 & o. & 22285 & 13858 & 2611 \\
\hline \multirow[t]{2}{*}{ TOTAL FS } & 0 & 38755 & 0 & 01 & 0 & 0 & 22285 & 13858 & 2611 \\
\hline & & & & & $=$ & & & & \\
\hline \multicolumn{10}{|l|}{ IDAHO } \\
\hline Idaho Nat'l Eng Lab - ACU & 407 & 01 & 0 & $\overline{01}$ & 0 & - & 325 & 73 & $\overline{8}$ \\
\hline Idaho Nat'l Eng Lab - B\&W & 0 & 1460 & 0 & 0 & 0 & - & 677 & 783 & 0 \\
\hline Idaho Nat'l Eng Lab - WINCO & 0 & 0 & 0 & 0 & 0 & - & 0 & 0 & 0 \\
\hline West Valley Nuclear Services & 0 & 0 & 4462 & 0 & 0 & 0 & 2411 & 1458 & 594 \\
\hline \multirow[t]{2}{*}{ TOTAL ID } & 407 & 1460 & 4462 & a) & 0 & ㅇ. & 3414 & 2313 & 602 \\
\hline & & & & 1 & 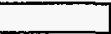 & ] & & & \\
\hline \multicolumn{10}{|l|}{ NEVADA } \\
\hline Nevada Test Site & 4833 & 01 & 24458 & 0 & 0 & Oा & 17569 & 7589 & 4133 \\
\hline \multirow[t]{2}{*}{ TOTAL NV } & 4833 & 0 & 24458 & - & 0 & 0 & 17569 & 7589 & 4133 \\
\hline & & & & & & & & & \\
\hline \multicolumn{10}{|l|}{$\overline{\overline{O A A K R I D G E}}$} \\
\hline Oak Ridge - K25 & 41364 & 0 & 0 & 0 & 0 & 인 & 26143 & 8732 & 6490 \\
\hline Oak Ridge Nat'I Lab & 23204 & 0 & 0 & 0 & 0 & 0 & 18586 & 2576 & 2043 \\
\hline Oak Ridge - Y12 & 32472 & 0 & 188101 & 0 & 0 & - & 40930 & 170348 & 9296 \\
\hline Paducah & 0 & 59971 & 0 & 0 & 0 & 0 & 41603 & 17207 & 1161 \\
\hline Portsmouth & 0 & 79564 & 0 & 0 & 0 & 0 & 61495 & 8817 & 9252 \\
\hline \multirow[t]{2}{*}{ TOTAL OR } & 97041 & 139535 & 188101 & 0 & 0 & 0 & 188755 & 207680 & 28242 \\
\hline & & & & & & & & & \\
\hline \multicolumn{10}{|l|}{ RICHLAND } \\
\hline Hanford - WHC $222 S$ & 0 & 22962 & 0 & 12017 & 0 & 32 & 19163 & 2302 & $135 \overline{47}$ \\
\hline Hanford - PNL 325 & 0 & 6777 & 0 & 618 & 0 & 117 & 2596 & 3715 & 1201 \\
\hline \multirow[t]{2}{*}{ TOTAL RL } & 0 & 29739 & 0 & 12635 & 0 & 150 & 21759 & 6017 & 14748 \\
\hline & & & & & & & & & \\
\hline \multicolumn{10}{|l|}{ ROCKY FLATS } \\
\hline Rocky Flats - $123 \mathrm{Lab}$ & 39168 & 0 & 0 & 0 & 0 & 0 & 39110 & 58 & $\overline{0}$ \\
\hline Rocky Flats - GL881 & 12611 & 0 & 0 & 0 & 0 & 0 & 7952 & 3228 & 1431 \\
\hline Rocky Flats - RF PA Lab & 0 & 0 & 0 & 0 & 5970 & 0 & 2935 & 1625 & 1410 \\
\hline TOTAL RF & 51779 & 01 & 0 & 0 & 5970 & (0) & 49997 & 4911 & 2841 \\
\hline & & & & & & & & & \\
\hline SAVANNAH RIVER & & & & & & & & & \\
\hline WSRC-ALD-CLAB & 0 & 01 & 2834 & 2674 & 189 & 644 & 6235 & 106 & $\overline{1}$ \\
\hline WSRC-ALD-32OM & 0 & 0 & 0 & 0 & 0 & 0 & 0 & 0 & 0 \\
\hline WSRC-ALD Water & 3121 & 0 & 3085 & 0. & 0 & 0 & 6205 & 0 & 0 \\
\hline WSRC-ALD-ETF & 0 & 0 & 1008 & 0 & 0 & 0 & 1008 & 0 & 0 \\
\hline WSRC-ALD-Tritium & 0 & 0 & 1096 & 0 & 0 & 0 & 1096 & 0 & 0 \\
\hline WSRC-ALD - ITP & 0 & 0 & 1070 & 0 & 0 & 0 & 535 & 0 & 535 \\
\hline WSRC-SRTC-ADS & 0 & 0 & 22857 & 0 & 0 & 78 & 12709 & 6898 & 3329 \\
\hline WSRC-SRTC-DWPT & 5033 & 0 & 0 & 0 & 0 & 0 & 1274 & 3016 & 743 \\
\hline TOTAL SR & 8153 & 0 & 31949 & 2674 & 189 & 722 & 29061 & 10020 & 4607 \\
\hline & & & & & & & & & \\
\hline TOTAL COMPLEX & 196419 & 234256 & 255009 & 15310 & 6290 & 872 & 371767 & 274630 & 61759 \\
\hline
\end{tabular}


Table Ilb: EC Sample Analyses by Rad Level, Matrix Type, and Laboratory

\begin{tabular}{|c|c|c|c|c|c|c|c|c|c|}
\hline Ops Office-Lab/Rad-Matrix & $\overline{\mathrm{RO}}$ & Ro/A1 & $\overline{\mathrm{R1}}$ & $\overline{R 2}$ & R3 & $\overline{\mathrm{R4}}$ & M1 & $\overline{\mathrm{M} 2}$ & $\overline{\mathrm{M} 3}$ \\
\hline \multicolumn{10}{|l|}{ ALBUQUERQUE } \\
\hline Grand Junction & 0 & 53882 & 0 & 0 & 0 & 0 & 19765 & 34117 & 0 \\
\hline Los Alamos Nat'I Lab & 34117 & 0 & 0 & 0 & 01 & $0:$ & 18751 & 13469 & 1898 \\
\hline \multirow[t]{2}{*}{ TOTAL AL } & 34117 & 53882 & 01 & 01 & 01 & O] & 38516 & 47586 & 1898 \\
\hline & & & & & & 77 & & & \\
\hline \multicolumn{10}{|l|}{ CHICAGO } \\
\hline Argonne East & 182 & 10295 & 1717 & 0 & 416 & of & 6188 & 2851 & 3572 \\
\hline Argonne West & 0 & 0 & 0 & 0 & 0) & 0 & 0 & 0 & 0 \\
\hline Brookhaven Nat'l Lab & 0 & 0 & 01 & 0 & 01 & 0.1 & 0 & 01 & 0 \\
\hline TOTAL CH & 182 & 10295 & 1717 & 0 & 416 & Q] & 6188 & 2851 & 3572 \\
\hline & & & & & & & & & \\
\hline \multicolumn{10}{|l|}{ FERNALD } \\
\hline Fernald & 0 & 85157 & 01 & 01 & 01 & 0] & 46430 & 34038 & 4688 \\
\hline TOTAL FS & 0 & 85157 & 0] & 01 & 0 (1) & Q] & 46430 & 34038 & 4688 \\
\hline & & & & & 1 & & & & \\
\hline \multicolumn{10}{|l|}{ IDAHO } \\
\hline Idaho Nat'l Eng Lab - ACU & 1216 & 0 & 01 & 0 & 0 & 0. & 972 & 219 & 24 \\
\hline Idaho Nat'l Eng Lab - B\&W & 0 & 10486 & 0 & 0 & 0 & 0 & 3559 & 4122 & 2805 \\
\hline Idaho Nat'I Eng Lab - WINCO & 0 & 0 & 17074 & 0 & 4046 & 3413 & 6982 & 11296 & 6255 \\
\hline West Valley Nuclear Services & 0 & 0 & 4481 & 01 & 0 & 0 & 2416 & 1469 & 596 \\
\hline \multirow[t]{2}{*}{ TOTAL ID } & 1216 & 10486 & 21555 & 0 & 4046 & 3413 & 13929 & 17106 & 9680 \\
\hline & & & & & & & & & \\
\hline \multicolumn{10}{|l|}{ NEVADA } \\
\hline Nevada Test Site & 4559 & 01 & 34241 & Q1] & 0I & 0 & 23140 & 9947 & 5713 \\
\hline \multirow[t]{2}{*}{ TOTAL NV } & 4559 & 01 & 34241 & 01 & 0 & $0]$ & 23140 & 9947 & 5713 \\
\hline & & & & & & & & & \\
\hline \multicolumn{10}{|l|}{ OAK RIDGE } \\
\hline Oak Ridge - K25 & 80361 & 0 & 0 & 01 & 0 & 0 & 55701 & 15348 & $\overline{9312}$ \\
\hline Oak Ridge Nat'l Lab & 28147 & 0 & 0 & 0 & 0 & 0 & 23177 & 2961 & 2009 \\
\hline Oak Ridge - Y12 & 68168 & 0 & 243790 & 0 & 0 & 0 & 82101 & 214341 & 15517 \\
\hline Paducah & 0 & 59926 & 0 & 0 & 0 & 0 & 45883 & 13475 & 569 \\
\hline Portsmouth & 0 & 86013 & 0 & 01 & O) & 이 & 66115 & 9596 & 10302 \\
\hline \multirow[t]{2}{*}{ TOTAL OR } & 176676 & 145939 & 243790 & 01 & 이 & 미 & 272976 & 255721 & 37708 \\
\hline & & & & & & & & & \\
\hline \multicolumn{10}{|l|}{ BICHLAND } \\
\hline Hanford - WHC $222 S$ & 0 & 219605 & 0 & 411652 & 0 & 709 & 367917 & 26248 & 237801 \\
\hline Hanford - PNL 325 & 0 & 48579 & 0 & 12661 & 0 & 1797 & 12308 & 33004 & 17724 \\
\hline \multirow[t]{2}{*}{ TOTAL RL } & $\overline{0}$ & 268184 & 01 & 424313 & OI & 2506 & 3802261 & 59252 & 255525 \\
\hline & & & & & & & & & \\
\hline \multicolumn{10}{|l|}{ ROCKY FLATS } \\
\hline Rocky flats - $123 \mathrm{Lab}$ & 225678 & 01 & 0 & 0 & 0 & 0 & 225563 & 116 & 0 \\
\hline Rocky Flats - GL881 & 14180 & 0 & 0 & 0 & 0 & 0 & 8266 & 4013 & 1901 \\
\hline Rocky Flats - RF PA Lab & 0 & 0 & 0 & 0 & 16674 & 0 & 6788 & 5527 & 4359 \\
\hline \multirow[t]{2}{*}{ TOTAL RF } & 239858 & 01 & 0 & 01 & 16674 & (0) & 240617 & 9655 & 6260 \\
\hline & & & & & & & & & \\
\hline \multicolumn{10}{|l|}{ SAVANNAH RIVER } \\
\hline WSRC-ALD-CLAB & 0 & 마 & 8259 & 6864 & 1890 & 3786 & 20423 & 374 & 2 \\
\hline WSRC-ALD-320M & 1119 & 이 & 0 & 0 & 0 & 0 & 1119 & 0 & 0 \\
\hline WSRC-ALD Water & 4773 & 0 & 4281 & 0 & 0 & 0 & 9054 & 0 & 0 \\
\hline WSAC-ALD-ETF & 0 & 0 & 0 & 0 & 0 & 0 & 0 & 0 & 0 \\
\hline WSRC-ALD-Tritium & 0 & 0 & 2566 & 0 & 0 & O & 2566 & 0 & 0 \\
\hline WSRC-ALD-ITP & 0 & 0 & 0 & 0 & 0 & 0 & 0 & 0 & 0 \\
\hline WSRC-SRTC-ADS & 0 & 0 & 40864 & 0 & 0 & 216 & 23624 & 12778 & 4678 \\
\hline WSRC-SRTC-DWPT & 8177 & 0 & 0 & O) & 0 & 0 & 1695 & 5131 & 1351 \\
\hline TOTAL SR & 14069 & 0 & 55970 & 6864 & 1890 & 4002 & 58482 & 18283 & 6030 \\
\hline & & & & & & & & & \\
\hline TOTAL COMPLEX & 470677 & 573943 & 357274 & 431176 & 23025 & 9921 & 1080503 & 454439 & 331074 \\
\hline
\end{tabular}


Table Ilc: MC Sample Analyses by Rad Level, Matrix Type, and Laboratory

\begin{tabular}{|c|c|c|c|c|c|c|c|c|c|}
\hline Ops Office-Lab/Rad-Matrix & RO & RO/A1 & $\mathrm{R} 1$ & R2 & $\overline{\mathrm{R} 3}$ & $\overline{\mathrm{R4}}$ & $\overline{\overline{M 1}}$ & $\overline{\mathrm{M} 2}$ & $\overline{\mathrm{M} 3}$ \\
\hline \multicolumn{10}{|l|}{ ALBUQUERQUE } \\
\hline Grand Junction & 0 & 108921 & 0 & 0 & 0 & 0 & 49166 & 59755 & $\overline{0}$ \\
\hline Los Alamos Nat' I Lab & 226746 & 0 & 50536 & 0 & 01 & 0 & 179775 & 84139 & 13368 \\
\hline TOTAL AL & 226746 & 108921 & 50536 & 0 & 01 & 0 & 228941 & 143894 & 13368 \\
\hline & & & & & & & & & \\
\hline \multicolumn{10}{|l|}{ CHICAGO } \\
\hline Argonne East & 1540 & 63099 & $52 \overline{47}$ & 0 & 35 & (0) & 32774 & 15031 & 22114 \\
\hline Argonne West & 0 & 0 & 0 & 0 & 0 & 0 & 0 & 0 & 0 \\
\hline Brookhaven Nat'l Lab & 0 & $\mathbf{0}$ & 0 & 0 & 0 & 0 & 0 & 0 & 0 \\
\hline TOTAL CH & 1540 & 63099 & 5247 & 0 & 351 & 0 & 32774 & 15031 & 22114 \\
\hline & & & & & 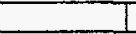 & & & & \\
\hline \multicolumn{10}{|l|}{ FERNALD } \\
\hline Fernald & 0 & 254221 & 0 & 0 & al & 0 & 132921 & 97248 & 24052 \\
\hline TOTAL FS & $\overline{0}$ & 254221 & 0 & 0 & 01 & 0 & 132921 & 97248 & 24052 \\
\hline \multirow{2}{*}{\multicolumn{10}{|c|}{ IDAHO }} \\
\hline & & & & & & & & & \\
\hline Idaho Nat'l Eng Lab - ACU & $5 \overline{130}$ & $\overline{0}$ & 0 & 0 & 01 & 0 & 4104 & 923 & 103 \\
\hline Idaho Nat'l Eng Lab - B\&W & 0 & 0 & 0 & 0 & 0 & 0 & 0 & 0 & 0 \\
\hline Idaho Nat'l Eng Lab - WINCO & 0 & 0 & 57741 & 0 & o: & 0 & 13650 & 33794 & 10297 \\
\hline West Valley Nuclear Services & 0 & 0 & 4340 & 0 & of & 0 & 2337 & 1423 & 579 \\
\hline \multirow[t]{2}{*}{ TOTAL ID } & $5 \overline{130}$ & 0 & 62081 & 0 & OI & 0 & 20091 & 36141 & 10979 \\
\hline & & & & & & & & & \\
\hline \multicolumn{10}{|l|}{ NEVADA } \\
\hline Nevade Test Site & $\overline{11654}$ & 0 & 59471 & 0 & 01 & 0. & 42786 & 18369 & 9970 \\
\hline TOTAL NV & 11654 & 0 & 59471 & 0 & 01 & 0 & 42786 & 18369 & 9970 \\
\hline & & & & & & 7 & & & \\
\hline \multicolumn{10}{|l|}{ OAK RIDGE } \\
\hline Oak Ridge - K25 & 324906 & 0 & $\overline{0}$ & 0 & 01 & 0 & 228828 & 61521 & 34557 \\
\hline Oak Ridge Nat'I Lab & 45391 & 0 & 0 & 0 & 0 & 0 & 36453 & 5228 & 3711 \\
\hline Oak Ridge - Y12 & 192779 & 0 & 838548 & 0 & 0 & 0 & 217882 & 762529 & 50916 \\
\hline Paducah & 0 & 152046 & 0 & 0 & 0 & 0 & 117285 & 32411 & 2350 \\
\hline Portsmouth & 0 & 203463 & 0 & 0 & of & 0 & 155723 & 21946 & 25794 \\
\hline TOTALOR & 563076 & $\mathbf{3 5 5 5 0 9}$ & 838548 & 0 & 01 & 0 & 756171 & 883634 & 117327 \\
\hline & & & & & & & & & \\
\hline \multicolumn{10}{|l|}{ FICHLAND } \\
\hline Hanford - WHC 222S & 0 & 453036 & 0 & 997626 & o1 & 1419 & 762329 & 66364 & $6 \overline{23388}$ \\
\hline Hanford - PNL 325 & 0 & 199327 & 0 & 53584 & of & 20313 & 51900 & 135074 & 86251 \\
\hline \multirow[t]{2}{*}{ TOTAL RL } & 0 & 652364 & $\overline{0}$ & 1051210 & 01 & 21732 & 814229 & 201438 & 709639 \\
\hline & & & & & & & & & \\
\hline \multicolumn{10}{|l|}{ ROCKY FLATS } \\
\hline Rocky Flats - $123 \mathrm{Lab}$ & 591129 & $\overline{0}$ & $\overline{0}$ & 0 & 01 & 0 & 590945 & 184 & $\overline{0}$ \\
\hline Rocky Flats - GL881 & 80173 & 0 & 0 & 0 & 0 & 0 & 44772 & 23006 & 12394 \\
\hline Rocky Flats - RF PA Lab & 0 & 0 & 0 & 0 & 95464 & 0 & 41373 & 30277 & 23814 \\
\hline TOTAL RF & 671302 & $\underline{\underline{0}}$ & $\underline{\underline{0}}$ & 0 & 95464 & 0 & 677091 & 53467 & 36208 \\
\hline & & & & & & & & & \\
\hline \multicolumn{10}{|l|}{ SAVANNAH RIVER } \\
\hline WSAC-ALD-CLAB & 0 & 0 & 512471 & 156780 & 6966 & 52997 & 728524 & $6 \overline{89}$ & $\overline{2}$ \\
\hline WSRC-ALD-320M & 0 & 0 & 0 & 0 & 01 & 0 & 0 & 0 & $\overline{0}$ \\
\hline WSRC-ALD Water & 3436 & 0 & 19148 & 0 & 0 & 0 & 22584 & 0 & 0 \\
\hline WSRC-ALD-ETF & 0 & 0 & 0 & 0 & 0 & 0 & 0 & 0 & 0 \\
\hline WSRC-ALD - Tritium & 0 & 0 & 2566 & 0 & 0 & 0 & 2566 & 0 & 0 \\
\hline WSRC-ALD-ITP & 0 & 0 & 0 & 0 & 0 & 0 & 0 & 0 & 0 \\
\hline WSAC-SRTC-ADS & 0 & 0 & 111151 & 0 & 0 & 1317 & 65107 & 35338 & 12023 \\
\hline WSRC-SRTC-DWPT & 8177 & 0 & 0 & 0 & 0 & 0 & 1695 & 5131 & 1351 \\
\hline \multirow[t]{2}{*}{ TOTAL SA } & 11613 & 0 & 645336 & 156780 & 6966 & 54314 & 820477 & 41158 & 13375 \\
\hline & & & & & & & & & \\
\hline TOTAL COMPLEX & 1491062 & 1434113 & 1661219 & 1207990 & 102465 & 76046 & 3525480 & 1490381 & 957034 \\
\hline
\end{tabular}


EM-263 SURVEY OF DOE ANALYTICAL CAPACITY AVAJLABLE TO EM

Table Illa: UC Sample Analyses Complex - wide by Regulatory and EM Distribution

\begin{tabular}{|c|c|c|c|c|c|c|c|c|c|c|}
\hline Reg-EM/ & & & egulatory & & & & n-Regulato & & Disti & Eution \\
\hline Samp Anal & CWA & CLP & SW846 & NIOSH & Other Reg & \begin{tabular}{c|} 
Waste \\
Treatment
\end{tabular} & $\begin{array}{l}\text { Prod'n } \\
\text { Support }\end{array}$ & $\begin{array}{c}\text { Other } \\
\text { Non-Reg }\end{array}$ & EM & Non-EM \\
\hline & & & & & & & & & & \\
\hline VOA-GC & 0 & 0 & 0 & 0 & 468 & 53 & 963 & 1285 & 978 & 1792 \\
\hline VOA-GCMS & 4923 & 3031 & 12994 & 0 & 2951 & 0 & 5 & 328 & 14815 & 9415 \\
\hline SEMI-VOA & 1155 & 166 & 5118 & 0 & 50 & 3 & 3 & 113 & 4828 & 1780 \\
\hline PEST & 125 & 27 & 681 & 0 & 0 & 5 & 0 & 352 & 667 & 523 \\
\hline HERB & 31 & 0 & 331 & 0 & 0 & 0 & 0 & 0 & 115 & 247 \\
\hline PCB & 859 & 36 & 10048 & 5465 & 4923 & 8 & of & 177 & 12348 & 9169 \\
\hline PCB/PEST & 0 & 37 & 261 & 0 & 0 & 0 & 0 & 0 & 261 & 37 \\
\hline OTHER-ORG & 668 & 0 & 939 & 0 & 0 & 0 & 524 & of & 1463 & 668 \\
\hline TOTAL ORGANCS & 7760 & 3298 & 30372 & 5465 & 8392 & 70 & 1494 & 2255 & 35476 & 23630 \\
\hline & & & & & & & & & & \\
\hline ICP-AESMETALS & 6863 & 381 & 24545 & 946 & 5276 & 14 & 1479 & 10803 & 34089 & 16218 \\
\hline İCP-MSMETALS & 2357 & 764 & 3006 & 0 & 3386 & 2074 & 509 & 2799 & 9808 & 5088 \\
\hline GF-AAMETALS & 8325 & 7 & 22190 & 3814 & 4430 & 0 & 439 & 1214 & 29307 & 11113 \\
\hline MERCUFY & 6233 & 153 & 13331 & 1937 & 697 & 8 & 17 & 3419 & 17640 & 8154 \\
\hline CYANIDE & 929 & 6 & 724 & 25 & 23 & 0 & 96 & 181 & 1250 & 735 \\
\hline ANIONS/CATIONS & 4447 & 191 & 6084 & 3158 & 887 & 402 & 4605 & 5647 & 17203 & 8219 \\
\hline WET CHEM & 166 & 0 & 316 & 0 & 268 & 0 & 62 & 0 & 813 & 0 \\
\hline OTHER-INORG & 3575 & 0 & 10669 & 2469 & 29713 & 4921 & 3817 & 11740 & 30686 & 36218 \\
\hline TOTALINORGANICS & 32896 & $15 \overline{03}$ & 80864 & 12348 & 44682 & 7420 & 11023 & 35803 & 140795 & 85745 \\
\hline & & & & & & & & & & \\
\hline GROSS ALPHAVBETA & 8760 & 318 & 17637 & 11969 & 194070 & 781 & 7254 & 5983 & 83419 & 163352 \\
\hline ALPHADET-Np237 & 0 & 0 & 325 & 0 & 0 & 0 & 0 & 0 & 325 & 0 \\
\hline ALPHA DET - Pu,Am & 571 & 0 & 1344 & 0 & 101 & 0 & 1342 & 0 & 3357 & 0 \\
\hline ALPHADET-Pu & 526 & 0 & 1314 & 0 & 5826 & 0 & 602 & 1499 & 2859 & 6908 \\
\hline ALPHA DET-U & 1284 & 0 & 1378 & 0 & 4657 & 0 & 2550 & 459 & 7853 & 2475 \\
\hline ALPHA DET-Am & 63 & $\overline{0}$ & 317 & 0 & 571 & 0 & of & 648 & 381 & 1219 \\
\hline ALPHA DET-Np & 448 & 0 & 1345 & $\overline{0}$ & 0 & 0 & 01 & 0 & 807 & 986 \\
\hline ALPHADET-Th & 333 & 0 & 1055 & $\overline{0}$ & 837 & o & 0 & 0 & 1461 & 763 \\
\hline ALPHADET-U,Th,Pu & 2760 & 0 & 552 & $\overline{0}$ & 0 & 0 & 0 & 197 & 7212 & -3704 \\
\hline ALPHA DET-U235 & of & 0 & 0 & $\overline{0}$ & 1470 & 0 & 1470 & 0 & 2941 & 0 \\
\hline ALPHA DET - Pu,U,Am & 1103 & 0 & 286 & 0 & 3544 & 0 & 0 & 0 & 4933 & 0 \\
\hline ALPHA DET-U,Np,Pu,Am & 0 & $\overline{0}$ & 0 & 0 & 0 & 0 & 0 & -0 & 0 & $\overline{0}$ \\
\hline ALPHA DET-Ra226 & ó & 0 & 0 & 0 & 413 & 0 & o & o: & 413 & 0 \\
\hline ALPHA DET-Pa228 & of & $\overline{0}$ & 0 & 0 & 286 & 0 & 0 & 0 & 286 & $\overline{0}$ \\
\hline ALPHA SPEC & 3953 & 0 & 2769 & 0 & 5656 & 33 & 297 & 1047 & 5181 & 8575 \\
\hline GPC-BETA-Pb210 & 0 & $\overline{0}$ & 0 & $\overline{0}$ & 0 & 0 & 01 & 0 & 0 & 0 \\
\hline GPC-BETA-Sr90 & 617 & 0 & 636 & 0 & 62 & 0 & 1209 & 412 & 2936 & $\overline{0}$ \\
\hline GPC-BETA-Pmi47 & of & 0 & 6 & 0 & 24 & 0 & o & 0 & 30 & 0 \\
\hline GPC-BETA-Se79 & o) & 0 & 38 & 0 & 0 & 0 & 0 & o & 38 & 0 \\
\hline LSC-BETA-H3 & 2357 & 0 & 3637 & 0 & 3620 & o & 224 & 11784 & 7955 & 13666 \\
\hline LSC-BETA-Rn222 & 0 & 0 & 206 & 0 & 0 & 0 & 0 & 0 & 0 & 206 \\
\hline LSC-BETA- 122 & o) & 0 & 197 & 0 & 0 & o) & 49 & 0 & 246 & 0 \\
\hline LSC-BETA-C14 & o & 0 & 247 & $\overline{0}$ & 0 & 0 & 297 & 0 & 543 & $\overline{0}$ \\
\hline LSC-BETA-TC99 & 927 & $\overline{0}$ & 4502 & 0 & 8637 & 0 & 191 & of & 4956 & 9301 \\
\hline LSC-BETA & 0 & 0 & 0 & 0 & 13 & 0 & 1096 & 129 & 0 & 1239 \\
\hline GAMMA SPEC & 4407 & 419 & 6970 & 0 & 14359 & 1342 & 3985 & 4987 & 21634 & 14834 \\
\hline OTHER-RAD & 2221 & 0 & 1480 & 0 & 2221 & 0 & 1898 & 814 & 5922 & 2712 \\
\hline TOTALAAD & 303301 & 737 & 46240 & 11909 & 246365 & $2 \sqrt{56}$ & 22464 & 27960 & 165688 & 222533 \\
\hline & & & & & & & & & & \\
\hline TCLP EXTRACTION & 0 & 6 & 3898 & 0 & 90 & 0 & 0 & 0] & 3341 & 652 \\
\hline OTHER-PREP & 632 & 110 & 2044 & 0 & 54 & 0 & 144 & 34 & 2386 & 632 \\
\hline OTHER-EXPLO & 0 & $\overline{0}$ & 0 & 1429 & 0 & 0 & 0 & 0. & $\overline{0}$ & 1429 \\
\hline OTHER-BIO & 979 & 0 & 0 & 0 & 176 & o & 0 & $51 \mid$ & 0 & 1206 \\
\hline OTHER-PHYS & 1883 & 0 & 1438 & 5059 & 5117 & o & 175 & 0 & 7625 & 6045 \\
\hline OTHER & 0 & 0 & 0 & 0 & 0 & 0 & 0 & 10842 & 7589 & 3252 \\
\hline TOTAL OTHEA & 3494 & 116 & $7 \overline{7379}$ & 6488 & 5436 & O) & 319 & 10927 & 20942 & 13216 \\
\hline & & & & & & & & & & \\
\hline TOTAL SAMPLEANALYSES & 74480 & $5 \overline{554}$ & 164856 & 36269 & 304875 & 964 & 35300 & 76945 & 362900 & $\overline{345124}$ \\
\hline
\end{tabular}


EM-263 SURVEY OF DOE ANALYTICAL CAPACITY AVAILABLE TO EM

Table IIlb: EC Sample Analyses Complex-wide by Regulatory and EM Distribution

\begin{tabular}{|c|c|c|c|c|c|c|c|c|c|c|}
\hline \multirow{2}{*}{$\begin{array}{l}\text { Reg-EM / } \\
\text { Samp Anal }\end{array}$} & \multicolumn{5}{|c|}{ Regulatory } & \multicolumn{3}{|c|}{ Non-Regulatory } & \multicolumn{2}{|c|}{ Distribution } \\
\hline & CWA & CLP & SW846 & $\mathrm{NIOSH}$ & Other Regl & $\begin{array}{c}\text { Waste } \\
\text { Treatment }\end{array}$ & $\begin{array}{c}\text { Prod'n } \\
\text { Support }\end{array}$ & $\begin{array}{c}\text { Other } \\
\text { Non-Reg }\end{array}$ & EM & Non-EM \\
\hline VOA-GC & 663 & 0 & 01 & 0 & 419 & 0 & 0 & 1614 & 1484 & \\
\hline VOA-GCMS & 6728 & 3590 & 17897 & 0 & 4176 & 116 & 192 & 817 & $\begin{array}{r}1404 \\
21958\end{array}$ & $\frac{1212}{11558}$ \\
\hline SEMI-VOA & 1311 & 260 & 6052 & 0 & 671 & 75 & 75 & 185 & 5873 & 2756 \\
\hline PEST & 127 & 77 & 1138 & o) & 635 & 83 & o & 354 & 1356 & 1059 \\
\hline HERB & 34 & 71 & 997 & 0 & 635 & 83 & 0 & 0 & 1512 & 308 \\
\hline PCB & 1288 & 116 & 13927 & 5481 & 5855 & 91 & 0 & 176 & 15986 & 10947 \\
\hline PCB/PEST & 0 & 37 & 459 & o| & o) & 0 & 0 & 0. & 459 & 37 \\
\hline OTHER-ORG & 1761 & 0 & 31957 & of & o) & 0 & 29779 & 0 & 60153 & 3344 \\
\hline TOTAL ORGANICS & 11913 & 4152 & 72427 & 5481 & 12391 & 444 & 30046 & 3146 & 108782 & 31221 \\
\hline & Oन्व & & & & 0107 & & & & al & \\
\hline $\begin{array}{l}\text { ICP-AESMETALS } \\
\text { ICP-MSMETALS }\end{array}$ & 9469 & 2840 & 50759 & 1389 & 9127 & 0 & 37017 & 24966 & 105756 & 29811 \\
\hline ICP-MS METALS & 6786 & 3042 & 11787 & 0 & 11797 & 2593 & 3491 & 4869 & 29247 & 15117 \\
\hline GF-AAMETALS & 12999 & 541 & 49630 & 4312 & 14896 & 0 & 512 & 10150 & 78553 & 14486 \\
\hline MERCURY & 10895 & 1238 & 52679 & 1850 & 1842 & 1696 & 72 & 6602 & 56488 & 20387 \\
\hline CYANIDE & 1199 & 800 & 33308 & 25 & 1711 & 0 & 24647 & 222 & 57925 & 3987 \\
\hline ANIONS/CATIONS & 8555 & 1955 & 3917 & 4930 & 5421 & 604 & 49424 & 14565 & 100581 & 24050 \\
\hline WET CHEM & 920 & 0 & 3522 & 0) & 1282 & 0 & 2824 & 0 & 8548 & 0 \\
\hline OTHER-INORG & 6310 & 50 & 46853 & 2582 & 46720 & 10586 & 42559 & 66953 & 191627 & 30986 \\
\hline TOTAL INORGANICS & 57132 & 10467 & 287716 & 15087 & 92796 & 15479 & 160546 & 128327 & 628726 & 138824 \\
\hline & & & & & & & & & & \\
\hline GROSS ALPHAVBETA & 17253 & 714 & 35834 & 11996 & 358236 & 1893 & 19251 & 10621 & 343774 & 112023 \\
\hline ALPHA DET-Np237 & 0 & 0 & 5477 & of & - & of & 0 & - & 5477 & - \\
\hline ALPHA DET-Pu,Am & 1701 & 0 & 4002 & 0 & 300 & of & 6039 & 0 & 12042 & 0 \\
\hline ALPHA DET -PU & 526 & 0 & 1314 & 0 & 8374 & of & 602 & 2515 & 5053 & 8279 \\
\hline ALPHA DET-U & 1362 & 0 & 1234 & 0 & 2529 & 3559 & 3559 & 459 & 6453 & 6250 \\
\hline ALPHADET-Am & 63 & 0 & 317 & 0 & 571 & 0 & 0 & 648 & 381 & 1219 \\
\hline ALPHADET-Np & 480 & 0 & 1440 & 0 & 0 & 0 & 0 & 0 & 864 & 1056 \\
\hline ALPHADET-Th & 304 & 0 & 961 & 0 & 1155 & 0 & of & - & 1724 & 696 \\
\hline ALPHADET-U,Th,PU & 3324 & 0 & 665 & 0 & 0 & of & 0 & 311 & 8686 & -4386 \\
\hline ALPHA DET-U235 & 0 & of & 0 & 0 & 1470 & 2321 & 2367 & 0 & 2321 & 2367 \\
\hline ALPHA DET - Pu,U,Am & 1527 & 0 & 286 & 0 & 9636 & 0 & 0 & D & 11449 & 0 \\
\hline ALPHA DET-U,Np,Pu,Am & 0 & of & 0 & O) & o & 0 & o) & 0 & 0 & o \\
\hline ALPHADET-Ra226 & 0 & 0 & 0 & 0 & 744 & 0 & 0 & 0 & 744 & 0] \\
\hline ALPHA DET-Ra228 & 0 & 0 & 0 & o) & 567 & 0 & 0 & D & 567 & a \\
\hline ALPHA SPEC & 5500 & 58 & 9767 & 0 & 6926 & 59 & 1163 & 2739 & 13150 & 13052 \\
\hline GPC-BETA-Pb210 & 0 & 0 & 609 & 01 & 0 & of & 0 & 0 & 609 & 0. \\
\hline GPC-BETA-Sr90 & 2586 & 0 & 4079 & 0) & 1317 & D & 3697 & 725 & 12404 & 0] \\
\hline GPC-BETA-Pm147 & 0 & o & 999 & o) & 3997 & 0 & o) & 0 & 4997 & 메 \\
\hline GPC-BETA-Se79 & 0 & of & 775 & of & 0 & of & वा & 0 & 775 & of \\
\hline LSC-BETA-H3 & 3918 & 0 & 11775 & 0 & 5057 & a: & 3990 & 3367 & 22830 & 5277 \\
\hline LSC-BETA-Rn222 & 0 & (a) & 421 & of & 0 & 0 & of & 0 & 421 & 0. \\
\hline LSC-BETA- 1129 & 0 & 0 & 506 & of & 0 & 0 & 516 & 0 & 1022 & 0) \\
\hline LSC-BETA-C14 & 0 & 0) & 821 & 0 & o & of & 820 & 0 & 1641 & o \\
\hline LSC-BETA-TC99 & 1726 & 0 & 6182 & 0 & 8995 & 0 & 2324 & 0 & 9541 & 9687 \\
\hline LSC-BETA & 0 & 0) & 0 & 0 & 13 & 0 & 2566 & 0 & 0 & 2566 \\
\hline GAMMA SPEC & 8507 & 602 & 30255 & 0 & 21170 & 3638 & 32176 & 7923 & 81115 & 23156 \\
\hline OTHER-AAD & 3661 & 0 & 2441 & 0 & 3661 & 0 & 2646 & 1134 & 9762 & 3780 \\
\hline TOTAL RAD & 52437 & 1374 & 120161 & 11996 & 434778 & 11471 & 81716 & 30443 & 557802 & 185031 \\
\hline & & & & & & & & & & \\
\hline TCLP EXTRACTION & 0 & 225 & 12385 & 0 & 528 & 0 & o & 0 & 9435 & 3702 \\
\hline OTHER-PREP & 1908 & 3968 & 41343 & 74 & 222 & o & 43328 & 94 & 87676 & 3260 \\
\hline OTHER-EXPLO & 0 & D) & 0 & 1319 & (0) & 0 & of & 0 & D & 1319 \\
\hline OTHER-BIO & 2378 & o & 0 & 0 & 198 & of & o) & 1772 & o & 4347 \\
\hline OTHER-PHYS & 4641 & 0 & 39784 & 6074 & 8126 & 0 & 35965 & 0 & 89325 & 5265 \\
\hline OTHER & 0 & 0 & 0 & 0 & 0 & 0 & 0 & 10842 & 7589 & 3252 \\
\hline TOTAL OTHER & 8927 & 4192 & $9 \overline{93511}$ & 7467 & $\overline{9073}$ & 0 & 79293 & 12708 & 194024 & 21146 \\
\hline & & & & & & & & & & \\
\hline TOTAL SAMPLE ANALYSES & 130409 & 20185 & 573815 & 40031 & 548979 & 27397 & 351601 & 174624 & 1489334 & 376223 \\
\hline
\end{tabular}


Table IIlc: MC Sample Analyses Complex-wide by Regulatory and EM Distribution

\begin{tabular}{|c|c|c|c|c|c|c|c|c|c|c|}
\hline Reg-EM/ & & & Regulatory & & & & n-Regulate & & Distri & oution \\
\hline Samp Anal & CWA & $C L P$ & SW846 & $\mathrm{NIOSH}$ & Other Reg & \begin{tabular}{c|} 
Waste \\
Treatment \\
\end{tabular} & $\begin{array}{c}\text { Prod'n } \\
\text { Support } \\
\end{array}$ & $\begin{array}{c}\text { Other } \\
\text { Non-Reg }\end{array}$ & $E M$ & Non-EM \\
\hline & & & & & & & & & & \\
\hline VOA-GC & 0 & 0 & 0 & 0 & 1258 & 0 & 0 & 2882 & 2787 & 1353 \\
\hline VOA-GCMS & 17218 & 14935 & 53676 & 0 & 14244 & 0 & 804 & 8954 & 73410 & 36422 \\
\hline SEMI-VOA & 3606 & 1167 & 26528 & 0 & 2490 & 450 & 450 & 6259 & 24306 & 16644 \\
\hline PEST & 234 & 320 & 2775 & 0 & 1904 & 348 & 0 & 702 & 4516 & 1768 \\
\hline HERB & 217 & 300 & 2779 & 0 & 1904 & 348 & 0 & 0 & 4517 & 1030 \\
\hline PCB & 2646 & 389 & 33978 & 11375 & 12381 & 4879 & 0 & 2265 & 43790 & 24124 \\
\hline PCB/PEST & 0 & 447 & 1805 & 0 & 0 & 0 & 0 & 0 & 1805 & 447 \\
\hline OTHER-ORG & 5486 & 0 & 84457 & 0 & 0 & 0 & 73488 & 0 & 159042 & 4389 \\
\hline TOTAL ORGANICS & 29407 & 17550 & 205998 & 11375 & 34180 & 6026 & $7 \overline{7442}$ & 21062 & 314173 & 86176 \\
\hline ICP-AESMETAIS & & & & & & & & & & \\
\hline $\begin{array}{l}\text { ICP-ALSMEIALS } \\
\text { ICP-MSMETALS }\end{array}$ & 27305 & 6566 & 124134 & 3149 & 31200 & 0 & 129851 & 85214 & 321356 & 86054 \\
\hline & 20189 & 5851 & 20092 & 0 & 38336 & 4088 & 14661 & 11110 & 70456 & 43871 \\
\hline & 50909 & 3301 & 206565 & 12073 & 52504 & 아 & 1334 & 39351 & 317436 & 48601 \\
\hline MERCURY & 40614 & 7274 & 332728 & 6300 & 9470 & 3544 & 0 & 22794 & 328325 & 94400 \\
\hline CYANIDE & 7249 & 3835 & 86840 & 75 & 10272 & 0 & 54772 & 3745 & 150532 & 16256 \\
\hline ANIONS/CATIONS & 33420 & 4827 & 60311 & 15819 & 12550 & 6889 & 61884 & 61519 & 181798 & 75419 \\
\hline WET CHEM & 2934 & 0 & 5642 & 0 & 5109 & 0 & 2824 & 0 & 16509 & 0 \\
\hline OTHER-INORG & 17095 & 9690 & 137221 & 13284 & 128815 & 22015 & 118266 & 202241 & 547125 & 101503 \\
\hline TOTAL INORGANICS & 199714 & 41345 & 973533 & 50700 & 288256 & 36536 & 383592 & 425974 & 1933537 & 466113 \\
\hline & & & & & & & & & & \\
\hline GROSS ALPHAVBTA & 33365 & 3396 & 56296 & 18226 & 1159529 & 93712 & 293278 & 30241 & 932288 & 755756 \\
\hline ALPHADET-Np237 & o & 0 & 10857 & 0 & 0 & 0 & 0 & 0 & 10857 & 0 \\
\hline ALPHADET-Pu,Am & 14209 & 0 & 33433 & 0 & 2507 & 0 & 45429 & 0 & 95579 & 0 \\
\hline ALPHADET-Pu & 1383 & 0 & 3458 & 0 & 19642 & 0 & 612 & 10685 & 15596 & 20184 \\
\hline ALPHAA DET-U & 4675 & 0 & 4253 & 0 & 10749 & 7897 & 7897 & 2250 & 19690 & 18032 \\
\hline ALPHA DET-Am & 436 & 0 & 2181 & 0 & 3926 & 0 & 0 & 7127 & 2618 & 11053 \\
\hline ALPHA DET-Np & 876 & 0 & 2628 & D. & 0 & 0 & 0 & 0 & 1577 & 1927 \\
\hline ALPHADET-Th & 960 & 0 & 3041 & 0 & 2374 & 0 & 0 & 0 & 4175 & 2201 \\
\hline ALPFA DET-U,Th,PU & 5429 & 0 & 1086 & 0 & 0 & 0 & 0 & 422 & 14187 & -7251 \\
\hline ALPHA DET-U235 & 0 & 0 & 0 & 0 & $\underline{0}$ & 3809 & 3809 & 0 & 3809 & 3809 \\
\hline ALPHA DET-Pu,U,Am & 3777 & 0 & 834 & 0 & 18362 & 0 & 0 & 0 & 22973 & 0 \\
\hline ALPHA DET-U,Np,Pu,Am & D) & 0 & 0 & 0 & 0 & 0 & 0 & 0 & 0 & 0 \\
\hline ALPHADET-Pa226 & D) & 0 & 0 & 0 & 1080 & 0. & $\overline{0}$ & 0 & 1080 & 0 \\
\hline ALPHADET-Ra228 & 0 & 0 & 0 & 0 & 706 & 0 & $\overline{0}$ & 0 & 706 & 0 \\
\hline ALPHA SPEC & 17016 & 0 & 22830 & 0 & 24678 & 5596 & 105916 & 4731 & 45655 & 135111 \\
\hline GPC-BETA-PB210 & 0 & 0 & 1927 & 0 & 0 & 0 & 0 & 0 & 1927 & 0 \\
\hline GPC-BETA-Sr9o & 5172 & 0 & 8483 & 0 & 5187 & 0 & 5642 & 2694 & 27179 & 0 \\
\hline GPC-BETA-Pm147 & D. & 0 & 999 & 0 & 3997 & 0 & $\mathbf{0}$ & 0 & 4997 & 0 \\
\hline GPC-BETA-Se79 & 0. & 0 & 2026 & 0 & $\mathbf{0}$ & 0 & 0 & 0 & 2026 & 0 \\
\hline LSC-BETA-H3 & 11319 & 0 & 24134 & 0 & 10408 & 0 & 5015 & 25827 & 47231 & 29472 \\
\hline LSC-BETA-Rn222 & 0 & 0 & 1238 & 0 & 0 & 0 & 0 & 0 & 1238 & 0 \\
\hline LSC-BETA-1129 & 0 & 0 & 3794 & 0 & 0 & 0 & 3794 & 0 & 7589 & 0 \\
\hline LSC-BETA-C14 & 0 & 0 & 1674 & 0 & 0 & 0 & 1714 & 0 & 3388 & 0 \\
\hline LSC-BETA-TC99 & 9597 & 0 & 24359 & 0 & 17779 & 0 & 8024 & 0 & 40612 & 19146 \\
\hline LSC-BETA & 0 & 0 & 0 & 0 & 0 & 0 & 2566 & 0 & 0 & 2566 \\
\hline GAMMASPEC & 13524 & 1044 & 62254 & 0 & 36933 & 86143 & 182075 & 20612 & 240303 & 162282 \\
\hline OTHER-RAD & 8202 & 0 & 5468 & 0 & 8202 & 0 & 12370 & 5302 & 21873 & 17672 \\
\hline TOTAL RAD & 129942 & 4441 & 277253 & 18226 & 1326061 & 197157 & 678141 & 109891 & 1569151 & 1171960 \\
\hline & & & & & & & & & & \\
\hline TCLP EXTRACTION & 0 & 943 & 32294 & 0 & 1237 & 0 & 0 & 0 & 29063 & 5411 \\
\hline OTHER-PREP & 6766 & 11735 & 69539 & 0 & 574 & 0 & 75892 & 1007 & 158747 & 6766 \\
\hline OTHER-EXPLO & 0 & 0 & 0 & 4084 & $\overline{0}$ & 0 & 0 & 0 & 0 & 4084 \\
\hline OTHER-BIO & 1006 & 0 & 0 & 0 & 2030 & 0 & $\overline{0}$ & 0 & 0 & 3036 \\
\hline OTHER-PHYS & 37795 & 0 & 67268 & 20602 & 35563 & 0 & 40918 & 0 & 167453 & 34694 \\
\hline OTHER & 0 & 0 & 0 & 0 & 0 & 0 & 0 & 21656 & 15159 & 6497 \\
\hline TOTAL OTHEA & 45567 & 12678 & 169101 & 24687 & 39404 & 0 & 1168009 & 22663 & 370422 & 60488 \\
\hline & & & & & & & & & & \\
\hline AMPLEAN & 404631 & 76022 & 1625885 & 104988 & 1687901 & 239719 & 1253284 & 9590 & 283 & 784 \\
\hline
\end{tabular}


EM-263 SURVEY OF DOE ANALYTICAL CAPACTY AVAILABLE TO EM

Table IIId: UC Sample Analyses by Regulatory and EM Distribution by Laboratory

\begin{tabular}{|c|c|c|c|c|c|c|c|c|c|c|}
\hline Reg-EMI & & & Kegulatory & & & & n-Regulato & & Distrik & bution \\
\hline Ops Office-Lab & CWA & CLP & SW846 & NIOSH & Other Reg & \begin{tabular}{c|} 
Waste \\
Treatment
\end{tabular} & $\begin{array}{l}\text { Prod'n } \\
\text { Support }\end{array}$ & $\begin{array}{c}\text { Other } \\
\text { Non-Reg }\end{array}$ & EM & Non-EM \\
\hline ALBUOUEROUE & & & & & & & & & & \\
\hline Grand Junction & ग) & 01 & 10064 & 0 & (0) & 01 & Dा & 0] & 2356 & 7708 \\
\hline Los Alamos Nat'l Lab & 8659 & O) & 14484 & 01 & 6781 & 0 & DI & 6787 & 24399 & 12311 \\
\hline TOTAL AL & 8659 & 01 & 24547 & वा & 6781 & 0 & DI & 6787 & 26755 & 20019 \\
\hline & & & & & & & & & & \\
\hline CHICAGO & & & & & & & & & & \\
\hline Argonne East & Oा & 156 & 1045 & 0 & 0. & 1911 & 339 & 2898 & 01 & 4629 \\
\hline Argonne West & 0 & 0 & 273 & 0 & 461 & 0 & 0 & 1126 & 0 & 1860 \\
\hline Brookhaven Nat' Lab & 0 & o. & 0 & 0 & o & 0 & 0 & 11880 & 6 & 11874 \\
\hline TOTAL CH & 01 & 156 & 1318 & 01 & 461 & 191 & 339 & 15903 & (6) & 18363 \\
\hline & & 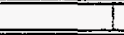 & & & & & & & & \\
\hline FERNALD & & & & & & & & & & \\
\hline Fernald & 6931 & 0 & 6366 & 01 & 25458 & 어 & D] & ㅁ] & 35477 & 3278 \\
\hline TOTALFS & 6931 & 0 & 6366 & 01 & 25458 & 01 & 이 & 0] & 35477 & 3278 \\
\hline & & & & $\square$ & & $=1$ & $=$ & $=$ & & \\
\hline IOAHO & & & & & & & & & & \\
\hline Idaho Nat'IEng Lab - ACU & 01 & 0 & (0) & 0 & 407. & 0 & D| & 0 & 407 & $\overline{0}$ \\
\hline Idaho Nat'I Eng Lab - B\&W & 0) & 0 & 783 & 0 & 0. & 0 & 0 & 677 & 0 & 1460 \\
\hline Idaho Nat'I Eng Lab - WINCO & of & 0 & 0 & 0 & o. & 0 & of & 0 & 0 & o \\
\hline West Valley Nuclear Services & O) & 0 & 0 & OI & D) & 0 & 3301 & 1161 & 4462 & 0 \\
\hline TOTAL ID & 01 & 01 & $783 \mid$ & DI & 407] & 01 & 3301 & 1839 & 48691 & 1460 \\
\hline & & & & & & & & $\square$ & & \\
\hline NEVADA & & & & & & & & & & \\
\hline Nevada Test Site & 775 & 0 & 2448 & 1610 & 24458 & 01 & 아 & [0] [ & 2840 & 26450 \\
\hline TOTALNV & 775 & $0 !$ & 2448 & 16101 & 24458 & 01 & 01 & 0] & 2840 & 26450 \\
\hline & & & & & & & & & & \\
\hline OAKRIDGE & & $=$ & & & & & & & & \\
\hline Oak Ridge - K25 & 4628 & $1642 !$ & 30282 & 905 & 1142 & 2074 & 可 & 691 & 23988 & 1737 \\
\hline Oak Ridge Nat'l Lab & 12909 & 0 & 3275 & 0 & 1729 & 340 & 476 & 4475 & 35747 & -12543 \\
\hline Oak Fidge - Y12 & 20592 & 1682 & 7812 & 6449 & 170990 & 5057 & 6236 & 1756 & 46159 & 174414 \\
\hline Paducah & 0 & 0 & 28501 & 8654 & 17820 & 0 & 3121 & 1876 & 40010 & 19961 \\
\hline Portsmouth & 9496 & o) & 33475 & 18652 & 17941 & 0 & 0 & 0 & 33475 & 46089 \\
\hline TOTALOA & 476251 & $3 \overline{34}$ & 103344 & 34659 & 209621 & 74711 & 9832 & 8798 & 179379 & 245298 \\
\hline & & & & & & & & & & \\
\hline FICHLAND & & & & & & & & & & \\
\hline Hanford - WHC 222S & 3413 & 94| & 19527 & 0 & 241 & 0 & 11736 & 0. & 35015 & -4 \\
\hline Hanford - PNL 325 & 4 & 346 & 172 & o & 3221 & 8 & 531 & 3231 & 6827 & 685 \\
\hline TOTALRL & 3417 & 440 & 19699 & 01 & 3462 & 8 & 12267 & 3231 & 41843 & 681 \\
\hline & & & & & & & & & & \\
\hline ROCKY FLATS & & & & & & & & & & \\
\hline Rocky Flats - 123 Lab & 966 & 可 & 마 & क्ष & 26260 & 0 & 0 & 11943 & 28254 & 10914 \\
\hline Rocky Flats - GL881 & 5692 & 1733 & 4703 & 0 & 483 & 0 & 0 & 0. & 12666 & -55 \\
\hline Rocky Flats - RF PA Lab & 0 & 0 & 1647 & 0 & 4322 & 0 & 0 & of & 8277 & -2307 \\
\hline TOTAL AF & 6658 & 1733 & 6350 & 이 & 31064 & Q1 & 01 & 11943 & 49197 & 8552 \\
\hline & & & & & & & & & & \\
\hline SAVANNAH RIVER & & & & & & & & & & \\
\hline WSRC-ALD-CLAB & 0 & 01 & वी & 0 & व) & 1895 & 4440 & 6] & 1824 & 4517 \\
\hline WSRC-ALD-32OM & 0 & 0 & 0 & 0 & 0 & 0 & 0 & o & 0 & 0 \\
\hline WSRC-ALD Water & 415 & of & of & of & 2706 & of & 2215 & 869 & 0 & 6205 \\
\hline WSRC-ALD-ETF & 0 & 01 & 01 & 0 & 0] & 42 & 955 & 10 & 0 & 1008 \\
\hline WSRC-ALD-Tritium & 0 & 0) & 0 & 0 & 요 & 0 & 1096 & 이 & 0 & 1096 \\
\hline WSRC-ALD-ITP & 0 & 0 & 0 & 0 & 0] & 53 & 963 & 53 & $\overline{0}$ & 1070 \\
\hline WSAC-SRTC-ADS & 0 & 0 & 0 & 0 & 457 & of & 0 & 22478 & 16070 & 6865 \\
\hline WSRC-SRTC-DWPT & 0 & 0 & 0 & 0 & o] & o & o & 5033 & 4656 & 377 \\
\hline TOTALSR & 415 & गा & 01 & 이 & 3162] & 1991 & 9669 & 28450 & 22550 & 21137 \\
\hline TOTAL COMPLEX & 74480 & 5654 & 164856 & 36260 & 304875 & 9661 & 35409 & 76951 & 362917 & 345238 \\
\hline
\end{tabular}




\section{EM-263 SURVEY OF DOE ANALYTICAL CAPACITY AVAILABLE TO EM}

Table llfe: EC Sample Analyses by Regulatory and EM Distribution by Laboratory

\begin{tabular}{|c|c|c|c|c|c|c|c|c|c|c|}
\hline \multirow{2}{*}{$\begin{array}{l}\text { Reg-EM/ } \\
\text { Ops Office-Lab }\end{array}$} & \multicolumn{5}{|c|}{ Regulatory } & \multicolumn{3}{|c|}{ Non-Regulatory } & \multicolumn{2}{|c|}{ Distribution } \\
\hline & CWA & CLP & SW846 & NIOSH & Other Reg & $\begin{array}{c}\text { Waste } \\
\text { Treatment }\end{array}$ & $\begin{array}{l}\text { Prod'n } \\
\text { Support }\end{array}$ & $\begin{array}{c}\text { Other } \\
\text { Non-Peg }\end{array}$ & $\mathrm{EM}$ & Non-EM \\
\hline ALBUQUERQUE & & & & & & & & & & \\
\hline Grand Junction & 0 & 0 & 53882 & 0 & 0 & 01 & 0 & 0 & 53882 & 0] \\
\hline Los Alamos Nat'l Lab & 7521 & 0 & 13215 & 0 & 6544 & 0 & 0 & 6837 & 21993 & 12124 \\
\hline TOTAL AL & 7521 & 01 & 67097 & $\overline{01}$ & 6544 & 01 & D) & 6837 & 75875 & 12124 \\
\hline & & & & & & & & & & \\
\hline CHICAGO & & & & & & & & & & \\
\hline Argonne East & of & 182 & 7039 & 0 & $\overline{0}$ & 219 & 831 & 4340 & 0 & 12610 \\
\hline Argonne West & 0 & 0 & 0 & 0 & 0 & 0 & 0 & 0. & 0 & D. \\
\hline Brookhaven Nat"l Lab & 0 & 0 & 0 & 0 & 0 & 01 & 0 & Q & 0 & D: \\
\hline TOTALCH & OI & 182 & 7039 & 0 & 0. & 219 & 831 & 4340 & $\overline{0}$ & 12610 \\
\hline & & & & & & & & & & \\
\hline FERNADD & & & & & & & & & & \\
\hline Fernald & 16804 & 01 & 20998 & 0 & 47354 & 01 & 01 & 0. & 80220 & 4937 \\
\hline TOTALFS & 16804 & 0 & 20998 & 0 & 47354 & 01 & 01 & 0. & 80220 & 4937 \\
\hline & & & & & & & & & & \\
\hline IDAFO & & & & & & & & & & \\
\hline Idaho Nat' Eng Lab - ACU & O & 0 & 0 & 0 & 1216 & 0 & 0 & 0 & 1216 & (0) \\
\hline Idaho Nat'l Eng Lab - B\&W & 0 & 0 & 5610 & 0 & 0 & 0 & 0 & 4875 & 0 & 10486 \\
\hline Idaho Nat'l Eng Lab - WINCO & 0 & 1600 & 14577 & 74 & 1873 & 116 & 624 & 5670 & 15 & 24518 \\
\hline West Valley Nuclear Services & O & $0 !$ & 0 & 0 & 0 & 0 & 3301 & 1180 & 4481 & 0 \\
\hline TOTAL D & 0 & 16001 & 20187 & 74 & 3088 & 116 & 3925 & 11725 & 5712 & 35004 \\
\hline & & & & & & & & & & \\
\hline NEVADA & & & & & & & & & & \\
\hline Nevada Test Site & 762 & 0 & 2307 & 1489 & 34241 & 0 & 0] & o. & 2676 & 36124 \\
\hline TOTALNV & 762 & 0 & 2307 & 1489 & 34241 & 01 & 01 & o. & 2676 & 36124 \\
\hline & & & & & & & & & & \\
\hline OAKRIDGE & & & & & & & & & & \\
\hline Oak Rudge - K25 & 8582 & 2470 & 61681 & 1837 & 2335 & 2593 & 01 & 864 & 52061 & 28301 \\
\hline Oak Ridge Nat'I Lab & 16134 & 0 & 3928 & 0 & 1695 & 524 & 706 & 5159 & 44807 & -16661 \\
\hline Oak Fidge - Y12 & 40535 & 6420 & 13247 & 8681 & 213209 & 16765 & 10714 & 2388 & 169176 & 142782 \\
\hline Paducah & 0 & 0 & 22596 & 8603 & 19866 & 0 & 5854 & 3007 & 36048 & 23878 \\
\hline Portsmouth & 10550 & 0 & 36670 & 19347 & 19445 & 0 & 0 & 0 & 36670 & 49343 \\
\hline TOIAL OR & 75801 & 8890 & 138123 & 38468 & 256549 & 19882 & 17275 & 11418 & 338762 & 227644 \\
\hline & & & & & & & 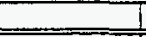 & & & \\
\hline RICHLAND & & & & & & & & & & \\
\hline Hanford-WHC222S & 18882 & 3814 & 297787 & 0 & 5348 & 0 & 306136 & $\overline{0}$ & 632245 & -279 \\
\hline Hanford - PNL 325 & 6 & 3967 & 5192 & 0 & 20698 & 324 & 4314 & 28536 & 52140 & 10896 \\
\hline TOTAL RI & 18888 & 7781 & 302979 & $\overline{0}$ & 26046 & 324 & 310449 & 28536 & 684386 & 10617 \\
\hline & & & & & & & & & & \\
\hline FOCKY FLAIS & & & & & & & & & & \\
\hline Rocky flats - 123 Lab & 2007 & 0 & 0 & 0 & 157114 & 0 & 0 & 66557 & 225678 & O. \\
\hline Rocky Flats - GL881 & 5692 & 1733 & 6272 & 0 & 483 & 0 & 0 & 0 & 14235 & -55 \\
\hline Rocky Flats - RF PA Lab & 0 & 0 & 8813 & 0 & 7861 & 0 & 0 & 0 & 19221 & -2547 \\
\hline TOTAL RF & 7700 & 17331 & 15085 & 0 & 165457 & 01 & 01 & 66557 & 259134 & -2602 \\
\hline & & & & 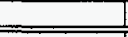 & & & & & & \\
\hline SAVANNAHRIVER & & & & & & & & & & \\
\hline WSAC-ALD-CLAB & 0 & 01 & $\overline{0}$ & 0 & 0 & 6914 & $138 \overline{3}$ & 22 & 5127 & 15672 \\
\hline WSRC-ALD-320M & 1119 & 0 & 0 & 0 & 0 & 0 & 0 & o. & 0 & 1119 \\
\hline WSRC-ALD Water & 1814 & 0 & 0 & 0 & 2959 & 0 & 3072 & 1209 & 0 & 9054 \\
\hline WSRC-ALD-ETF & 0 & 0 & 0 & 0 & 0 & 0 & 01 & 0 & $\overline{0}$ & 0 \\
\hline WSAC-ALD-Tritium & 0 & 0 & 0 & 0 & 0 & 0 & 2566 & 0: & 0 & 2566 \\
\hline WSRC-ALD-ITP & 0 & 0 & 0 & 0 & 0 & 0 & 0 & 욤 & $\overline{0}$ & $\overline{0}$ \\
\hline WSRC-SRTC-ADS & 0 & 0 & 0 & 0 & 5256 & 0 & 0 & 35823 & 30079 & 11001 \\
\hline WSAC-SRTC-DWPT & 0 & 0 & 0 & 0 & 0 & 0 & 0 & 8177 & 7424 & 753 \\
\hline TOTALSR & 2933 & 0) & $\overline{0}$ & 0 & 8215 & 6914 & 19501 & 45231 & 42630 & 40165 \\
\hline & & & & & & & & & & \\
\hline TOTAL COMPLEX & 130409 & 20185 & 573815 & 40031 & 547495 & 27454 & 351982 & 174645 & 1489394 & 376622 \\
\hline
\end{tabular}


EM-263 SURVEY OF DOE ANALYTICAL CAPACITY AVAILABLE TO EM

Table IIIf: MC Sample Analyses by Regulatory and EM Distribution by Laboratory

\begin{tabular}{|c|c|c|c|c|c|c|c|c|c|c|}
\hline Reg-EM/ & & & egulatory & & & & n-Regulato & & Distrit & sution \\
\hline Ops Office-Lab & CWA & CLP & SW846 & NIOSH & Other Reg & $\begin{array}{c}\text { Waste } \\
\text { Treatment }\end{array}$ & $\begin{array}{l}\text { Prod'n } \\
\text { Support }\end{array}$ & $\begin{array}{c}\text { Other } \\
\text { Non-Reg }\end{array}$ & EM & Non-EM \\
\hline ALBUQUEROUE & & & & & & & & & & \\
\hline Grand Junction & D] & 0 & 108921 & 0 & 0 & OI & 01 & 0] & 108921 & 0 \\
\hline Los Alamos Nat'I Lab & 68428 & of & 93101 & 0. & 28063 & of & 0) & 87691 & 166068 & 111215 \\
\hline TOTAL AL & 68428 & 01 & 202022 & 0 & 28063 & 01 & 01 & 87691[ & 274989 & 111215 \\
\hline CHICAGO & & & & & & & & & & \\
\hline Argonne East & 0 & 1792 & $430 \% 2$ & 0 & o & 1047 & 5091 & 23510 & $\frac{1}{01}$ & 69920 \\
\hline Argonne West & 0 & 0 & 0 & 0 & 0 & 0 & of & 0 & 0 & D \\
\hline Brookhaven Nat'I Lab & o & 0 & 0 & 0 & 0 & 0 & of & 0 & 0 & 0 \\
\hline TOTALCH & 0 & 1792 & 43062 & 01 & 0 - & 1047 & 509 & 23510 & 01 & 69920 \\
\hline & & & & & & & & & & \\
\hline FERNALD & & & & & & & & & & \\
\hline Fernald & 46389 & of & 56085 & 01 & 151747 & OI & a) & 0. & 2542211 & $\overline{0}$ \\
\hline TOTALFS & 46389 & 01 & 56085 & 0] & 151747 & 01 & (0) & $\overline{0}$ & 2542211 & $\overline{0}$ \\
\hline & & & & 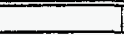 & & 1 & & & & \\
\hline IDAHO & & & & & & & & & & \\
\hline idaho Nat' Eng Lab - ACU & $\overline{0}$ & 0 & 0 & 0 & 5130 & 0 & 01 & (0) & 5130 & $\overline{0}$ \\
\hline Idaho Nat'I Eng Lab - B\&W & 0 & 0 & o & o & o. & 0 & of & o & of & $\overline{0}$ \\
\hline Idaho Nat'l Eng Lab - WINCO & 1163 & 11761 & 9331 & 0 & - & 0 & 2521 & 32966 & D) & 57741 \\
\hline West Valley Nuclear Services & 0 & 0 & 0 & 0 & o) & 0 & 3203 & 1137 & 4340 & 0 \\
\hline TOTALID & 1163 & 11761 & 9331 & 0 & 5130 & 0] & 5724] & 34103 & 94701 & 57741 \\
\hline & & & & & & & & & & \\
\hline NEVADA & & & & & & & & & & \\
\hline Nevada Test Site & 1524 & का & 5602 & 4528 & 59471 & 0 & 0 & 0. & 6563 & 64561 \\
\hline TOTALNV & 1524 & 01 & 5602 & 4528 & 5947] & OI & 01 & ㅇ․ & 6563 & 64561] \\
\hline & & & & & & & & & & \\
\hline OAKRIDGE & & & & & & & & & & \\
\hline Oak Ridge - k25 & 30687 & 9129 & 263439 & 7765 & 8437 & 4088 & 0 & 1363 & 218971 & 105935 \\
\hline Oak Ridge Nat'] Lab & 24056 & of & 6207 & 0 & 3077 & 825 & 1279 & 9948 & 67990 & -22599 \\
\hline Oak Ridge - Yi2 & 119901 & 16809 & 31292 & 25922 & 785873 & 33728 & 16889 & 914 & 447254 & 584072 \\
\hline Paducah & 0 & 0 & 60551 & 23646 & 41713 & 0 & 18201 & 7935 & 94132 & 57913 \\
\hline Portsmouth & 28818 & 0 & 87690 & 43126 & 43828 & 0 & 0 & 0 & 87690 & 115773 \\
\hline TOTALOR & 203461 & 25937 & 449179 & 100459 & 882927 & 38640 & 36369 & 20159 & 916038 & 841095 \\
\hline & & & & & & & & & & \\
\hline RICHLAND & & & & & & & & & & \\
\hline Hanford - WHC 222S & 50087 & 6148 & 747903 & 0 & 8524 & 0 & 639419 & 0. & 1452618 & -537 \\
\hline Hanford - PNL 325 & 24 & 22886 & 22547 & 0 & 81284 & 1495 & 25270 & 119719 & 227062 & 46163 \\
\hline TOTAL RL & 50111 & 29034 & 770450 & 0 & 89807 & 1495 & 664689 & 119719 & 1679580 & 45626 \\
\hline & & & & & & & & & & \\
\hline ROCKYFLATS & & & & & & & & & & \\
\hline Rocky flats - 123 Lab & $\overline{3419}$ & 0] & $\overline{0}$ & 0 & 403875 & 0] & o) & 183835 & 591129 & 0] \\
\hline Rocky Flats - GL881 & 30136 & 7498 & 40482 & 0 & 2058 & of & 0 & o & 80409 & -236 \\
\hline Rocky Flats - RF PA Lab & 0 & 0 & 49673 & 0 & 45791 & 0 & 0 & o & 100584 & -5120 \\
\hline TOTALRF & 33555 & 7498 & 90154 & 0 & 451724 & 0 & 0 & 183835 & 772122 & -5355 \\
\hline & & & & & & & & & & \\
\hline SAVANNAHRIVEA & & & & & & & & & & \\
\hline WSRC-ALD-CLAB & $\overline{0}$ & 0 & $\overline{0}$ & $\overline{0}$ & 0 & 198634 & 530533 & 47 & 183967 & 545247 \\
\hline WSRC-AL-320M & 0 & वा & 0 & 0 & 0 & 0 & 0 & 0 & 0 & 0 \\
\hline WSAC-ALDWater & 0 & of & 0 & 0 & 3436 & 0 & 13625 & 5523 & 0 & 22584 \\
\hline WSRC-ALD-ETF & 0 & o) & 0 & 0 & 0 & 0 & 0 & 마 & 0 & 0 \\
\hline WSAC-ALD-Tritium & $\overline{0}$ & 0 & 0 & 0 & $\overline{0}$ & 0 & 2566 & D & 0 & 2566 \\
\hline WSRC-ALD-ITP & 0 & 0 & 0 & 0 & 0 & 0 & 0 & 0 & o & 0 \\
\hline WSRC-SRTC-ADS & $\overline{0}$ & of & $\overline{0}$ & 0 & 15595 & 0 & 0 & 96873 & 82914 & 29554 \\
\hline WSAC-SRTC-DWPT & 0 & 0 & 0 & 0 & 0 & 0 & 0 & 8177 & 7424 & 753 \\
\hline TOTAL SR & 0 & 01 & 0 & 0 & 19032 & 198634 & 546725 & 110620 & 274305 & 600704 \\
\hline TOTAL COMPLEX & 404631 & 76022 & 1625885 & 104988 & 1687901 & 239817 & 1254015 & 579637 & 4187388 & 1785507 \\
\hline
\end{tabular}


EM-263 SURVEY OF DOE ANALYTICAL CAPACTIY AVAILABLE TO EM

Table IVa: UC Sample Analyses by Rad Level and Matrix Type (i) Complex-wide

\begin{tabular}{|c|c|c|c|c|c|c|c|c|c|c|}
\hline $\begin{array}{l}\text { Rad-Matrix } \\
\text { Samp Anal }\end{array}$ & Ro & RO/R1 & $\overline{\mathrm{Ri}}$ & R2 & $\mathrm{R3}$ & $\mathrm{R4}$ & MT & $\mathrm{M} 2$ & $\overline{\mathrm{M} 3}$ & Total \\
\hline & & & & & & & & & & \\
\hline VOA-GC & 1035 & 0 & 1734 & 0 & 0 & 0 & 1508 & 212 & 1050 & 2770 \\
\hline VOA-GCMS & 11621 & 9194 & 2530 & 136 & 750 & 0 & 18287 & 3800 & 2144 & 24231 \\
\hline SEMI-VOA & 3864 & 961 & 1691 & 93 & 0 & 0 & 4857 & 1164 & 587 & 6608 \\
\hline PEST & 674 & 485 & 0 & 31 & 0 & 0 & 590 & 437 & 164 & 1190 \\
\hline HERB & 218 & 144 & 0 & 0 & 0 & 0 & 256 & 77 & 29 & 362 \\
\hline PCB & 7909 & 12020 & 1587 & 0 & 0 & 0 & 16262 & 3066 & 2188 & 21516 \\
\hline PCB/PEST & 37 & 261 & 0 & 0 & 0 & 0 & 229 & 48 & 22 & 298 \\
\hline OTHER-ORG & 668 & 83 & 0 & 1380 & 0 & 0 & 1651 & 10 & 470 & 2131 \\
\hline TOTALORGANICS & 26026 & 23149 & 7542 & 1639 & 750 & 0 & 43640 & 8813 & 6653 & 59106 \\
\hline & & & & & & & & & & \\
\hline ICP-AES METALS & 18828 & 24314 & 6076 & 957 & 132 & 0 & 20422 & 23308 & 6576 & 50307 \\
\hline ICP-MS METALS & 9648 & 4692 & 463 & 93 & 0 & 0 & 8346 & 4983 & 1567 & 14897 \\
\hline GF-AAMETALS & 14400 & 23264 & 1720 & 1037 & 0 & 0 & 27346 & 9461 & 3614 & 40420 \\
\hline MERCURY & 15111 & 8292 & 1876 & 514 & 0 & 0 & 16757 & 5537 & 3499 & 25793 \\
\hline CYANIDE & 1304 & 303 & 0 & 378 & 0 & 0 & 1502 & 182 & 300 & 1985 \\
\hline ANIONS/CATIONS & 7228 & 10267 & 4351 & 3506 & 0 & 69 & 19098 & 3693 & 2631 & 25422 \\
\hline WET CHEM & 0 & 590 & 0 & 223 & 0 & 0 & 296 & 202 & 315 & 813 \\
\hline OTHER-INORG & 11668 & 31166 & 19996 & 1342 & 2703 & 29 & 41995 & 18776 & 6133 & 66904 \\
\hline TOTALINORGANICS & 78186 & 102889 & 34482 & 8051 & 2834 & 99 & 135763 & 66143 & 24634 & 226540 \\
\hline & & & & & & & & & & \\
\hline GROSS ALPHA/BETA & 37266 & 47237 & 158489 & 1063 & 2339 & 377 & 78943 & 162161 & 5667 & 246772 \\
\hline ALPHA DET - Np237 & 0 & 325 & D & $\overline{0}$ & 0 & 0 & 120 & 0 & 205 & 325 \\
\hline ALPHA DET - Pu,Am & 0 & 3357 & D & 0 & 0 & 0 & 1981 & 637 & 739 & 3357 \\
\hline ALPHA DET-PU & 5322 & 159 & 4286 & 0 & 0 & 0 & 2332 & 3559 & 3876 & 9767 \\
\hline ALPHA DET-U & 4403 & 824 & 5100 & 0 & 0 & 0 & 3810 & 5123 & 1395 & 10328 \\
\hline ALPHA DET-Am & 1600 & 0 & 0 & 0 & 0 & 0 & 642 & 894 & 63 & 1600 \\
\hline ALPHADET-Np & 1793 & वा & 0 & 0 & 0 & 0 & 90 & 807 & 897 & 1793 \\
\hline ALPHA DET -Th & 1388 & 837 & 0 & 0 & 0 & 0 & 683 & 723 & 819 & 2225 \\
\hline ALPHA DET $-U, T h, P u$ & 3509 & 0 & 0 & 0 & 0 & 0. & 3141 & 368 & 0) & 3509 \\
\hline ALPHA DET-U235 & 0 & o & 2941 & 0 & 0 & 0 & 1471 & 735 & 735 & 2941 \\
\hline ALPHA DET-PU,U,Am & 4933 & o & 0 & 0 & 0 & 0] & 4647 & 286 & 0 & 4933 \\
\hline ALPHA DET-U,Np,Pu,Am & 0 & 0 & 0 & 0 & 0 & 0 & 0 & 0 & 0 & 0 \\
\hline ALPHA DET-Ra226 & 0 & 413 & 0 & 0 & 0 & 0) & 310 & 83 & 21 & 413 \\
\hline ALPHA DET-Ra228 & 0 & 286 & 0 & 0 & 0 & 0 & 57 & 215 & 14 & 286 \\
\hline ALPHA SPEC & 4289 & 7493 & 1810 & 52 & 0 & 111 & 10329 & 1932 & 1494 & 13755 \\
\hline $\mathrm{GPC}-\mathrm{BETA}-\mathrm{Pb} 210$ & 0 & 0 & 0 & 0 & 0 & 0 & 0 & 0 & 0 & 0 \\
\hline GPC-BETA-Sr90 & 0 & 2203 & 733 & 0 & 0 & $\mathbf{0}$ & 1841 & 553 & 542 & 2936 \\
\hline GPC-BETA-Pm147 & 0 & 30 & 0 & 0 & 0 & 0 & 30 & 0 & 0 & 30 \\
\hline GPC-BETA-Se79 & 0 & 38 & 0 & 0 & 0 & 0 & 38 & 0 & 0 & 38 \\
\hline LSC-BETA-H3 & 7533 & 11177 & 2911 & 0 & 0 & $\mathbf{0}$ & 15915 & 5447 & 259 & 21621 \\
\hline LSC-BETA-Rin222 & 0 & 206 & 0 & 0 & 0 & 0 & 206 & 0 & 0 & 206 \\
\hline LSC-BETA-1129 & 0 & 246 & 0 & 0 & 0 & $\overline{0}$ & 246 & 0 & 0 & 246 \\
\hline LSC-BETA-C14 & 0 & 543 & 0 & 0 & 0 & 0 & 274 & 0 & 270 & 543 \\
\hline LSC-BETA-TC99 & 0 & 14258 & 0 & $\overline{0}$ & 0 & 0 & 11300 & 1329 & 1620 & 14258 \\
\hline LSC-BETA & 0 & of & 1239 & 0 & 0 & 0 & 1206 & 24 & 9 & 1239 \\
\hline GAMMA SPEC & 10279 & 8573 & 16287 & 1298 & 0 & 32 & 26836 & 5098 & 4534 & 36469 \\
\hline OTHER-RAD & 0 & 5922 & 2712 & 0 & 0 & 0 & 5080 & 2369 & 1184 & 8633 \\
\hline TOTAL RAD & 82314 & 104127 & 196508 & 2414 & 2339 & 520 & 171539 & 192339 & 24342 & 388221 \\
\hline & & & & & & & & & & \\
\hline TCLPEXTRACTION & 2570 & 1063 & 44 & 0 & 310 & 6 & 750 & 1881 & 1362 & 3993 \\
\hline OTHER-PREP & 1219 & 12 & 0 & 1591 & 0 & 195 & 1334 & 322 & 1362 & 3018 \\
\hline OTHER-EXPLO & 1429 & 0 & 0 & 0 & $\overline{0}$ & 0 & 1429 & 0 & 0 & 1429 \\
\hline OTHER-BIO & 1155 & 51 & 0 & 0 & 0 & 0 & 1206 & 0 & 0 & 1206 \\
\hline OTHER-PHYS & 3520 & 2965 & 5618 & 1568 & 0 & 0 & 10661 & 1773 & 1237 & 13671 \\
\hline OTHER & 0 & 0 & 10815 & 0 & 0 & 27 & 5421 & 3252 & 2168 & 10842 \\
\hline TOTAL OTHER & 9893 & 4092 & 16477 & 3159 & 310 & 228 & 20801 & 7228 & 6129 & 34158 \\
\hline & & & & & & & & & & \\
\hline TOTAL SAMPLE ANALYS & 196419 & 234256 & 255009 & 15262 & $6 \overline{233}$ & 846 & 371742 & 274524 & 61759 & 708024 \\
\hline
\end{tabular}


EM-263 SURVEY OF DOE ANALYTICAL CAPACITY AVAILABLE TO EM

Table IVa: UC Sample Analyses by Rad Level and Matrix Type (ii) Grand Junction

\begin{tabular}{|c|c|c|c|c|c|c|c|c|c|c|}
\hline $\begin{array}{l}\text { Fad-Matrix } \\
\text { Samp Anal }\end{array}$ & 70 & RO/R1 & Ri & 72 & $\overline{\mathrm{R} 3}$ & $\overline{\mathrm{RA4}}$ & $\overline{\mathrm{M} 1}$ & M2 & $\overline{M 3}$ & Total \\
\hline VOA-GC & 0 & 01 & 01 & 0 & 0 & $\pi$ & 0 & 0 & 0 & 0 \\
\hline VOA-GCMS & 0 & 561 & 0 & $\frac{1}{0}$ & o| & o & 382 & $\begin{array}{r}0 \\
180\end{array}$ & $\frac{0}{0}$ & $\frac{0}{561}$ \\
\hline SEMI-VOA & 0 & 220 & 0 & 0 & 0 & 0 & 171 & 48 & के & 220 \\
\hline PEST & 0 & 0 & 0 & 0 & 0| & 0 & 0 & 0 & 0] & 0 \\
\hline HERB & 0 & 0 & 0 & 0 & 0 & 0 & 0 & 0 & 0] & 0 \\
\hline PCB & 0 & 0 & 0 & 0 & 0) & 0. & $\overline{0}$ & 0 & 0. & $\overline{0}$ \\
\hline PCB/PEST & 0 & 261 & 0 & 0 & 이 & 0. & 214 & 47 & o. & 261 \\
\hline OTHER-ORG & 01 & 83 & 0] & 01 & 0] & $0]$ & 73 & 10 & 0 & 83 \\
\hline TOTAL ORGANICS & 01 & 1125 & 01 & (0) & 이 & 0] & 840 & 285 & 0 & 1125 \\
\hline & & & & & & & & & & \\
\hline ICP-AESMETALS & 0 & 1517 & 0 & 0 & 0 & 0 & 531 & 986 & 0 & 1517 \\
\hline ICP-MS METALS & 0 & 2344 & of & 0 & 0 & 0 & 727 & 1617 & 0 & 2344 \\
\hline GF-AAMETALS & 0 & 1310 & 0 & 이 & 0 & 0 & 472 & 838 & 0 & 1310 \\
\hline MERCURY & 0 & 643 & 0 & - & 0 & 0. & 309 & 334 & 0. & 643 \\
\hline CYANIDE & 0 & 0 & 0 & 0 & 0 & 0. & 0 & 0 & 0 . & 0 \\
\hline ANIONS/CATTONS & 0 & 517 & 0 & 0 & 0 & o & 367 & 150 & 0. & 517 \\
\hline WET CHEM & 0 & 0 & 이 & 0 & 0 & 0 & 0 & 01 & 0. & 0 \\
\hline OTHER-INORG & 0 & 0 - & of & 0 & 0 & 0 & 0 & 0 & o] & 0 \\
\hline TOTALINORGANICS & 01 & 6331 & 01 & 0 & 01 & 0 & 2405 & 3926 & 01 & 6331 \\
\hline & & & & & & 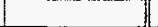 & & & 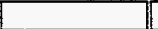 & \\
\hline GROSS ALPHAVETA & 0 & 1814 & 0 & 0 & 0 & 0 & 0 & $18+4$ & 0 & 1814 \\
\hline ALPHADET-Np237 & 0 & 0 & 0 & o & 0 & 0 & 0 & 0 & 0 & 0 \\
\hline ALPHA DET-PU,Am & 0 & 0 & 0 & O. & 0) & 0 & 0 & 0 & 0 & 0 \\
\hline ALPHADET-Pu & 01 & 0 & 0 & 0 & 0 & 0 & 0 & 0 & 요 & 0 \\
\hline ALPHADET-U & 0 & 0 & 0 & 0 & 0 & 0. & 0 & 0 & 0. & 0 \\
\hline ALPHA DET -Am & 0 & 0 & 0 & 01 & 0 & 0 & 0 & 0 & 0 & 0 \\
\hline ALPHADET $-\mathrm{Np}$ & o & 0 & 0 & 0 & 0 & 0 & 0 & 0 & a. & 0 \\
\hline ALPHADET -Th & 0 & 0 & 0 & 0 & 0 & 0 & 0 & 0 & 에 & 0 \\
\hline ALPHA DET-U,Th,Pu & 0 & 0 & o) & 0 & 0 & 0 & 0 & 0 & a & 0 \\
\hline ALPHADET-U235 & 0 & 0 & 이 & 0 & 0 & 으 & 0 & 0 & 이 & 0 \\
\hline ALPHA DET-Pu,U,Am & 0 & O) & 0) & o & 0 & 0 & 0 & 0 & 0. & 0 \\
\hline ALPHA DET - U,Np, Pu,Am & 0 & 0. & of & 0 & 0 & o. & 0 & o) & a & 0 \\
\hline ALPHA DET-Ra226 & 0 & of & 0 & 0 & 0 & 0. & 0 & 0 & o & 0 \\
\hline ALPHA DET-Ra228 & 0 & 0 & 0 & 0 & 0 & 0. & 0 & 0 & 0 & 0 \\
\hline ALPHA SPEC & 0 & of & 0 & 0 & 0 & 0. & of & of & 0. & 0 \\
\hline GPC-BETA-Pb210 & 0 & 0 & - & 0 & 0 & 0 & 01 & 0 & a & 0 \\
\hline GPC-BETA-Sr90 & 0 & 이 & 0 & 0 & 0 & 0 & 0 & 0 & 0 & 0 \\
\hline GPC-BETA-Pm147 & 0 & of & 0 & 0 & $\overline{0}$ & 0 & 0 & 0 & 0 & 0 \\
\hline GPC-BETA-Se79 & O) & 0) & 0. & 0 & 0 & a. & 0 & 0 & 0 & 0 \\
\hline LSC-BETA-H3 & O & 588 & 0 & 0 & 0 & o. & 6 & 582 & 0. & 588 \\
\hline LSC-BETA-Rn222 & 0 & 206 & 01 & 0 & 0 & a] & 206 & 0 & 의 & 206 \\
\hline LSC-BETA- 1129 & 의 & 0 & 0 & 0 & 0 & o. & 0 & of & 의 & 0 \\
\hline LSC-BETA-C14 & 0 & 0 & O) & 0 & D. & 0 & 0 & 0 & 0 & 0 \\
\hline LSC-BETA-TC99 & 0 & 0 & 0 & 0 & 0 & ㅇ. & 0 & 0 & o. & 0 \\
\hline LSC-BETA & 0 & 0 & O & 0 & 0 & 의 & 0 & of & 0. & 0 \\
\hline GAMMA SPEC & 0 & 0 & 0 & 0 & 0 & o. & 0 & of & of & 0 \\
\hline OTHER-RAD & 0 & of & of & 0 & 0 & (0) & 0 & of & 0] & 0 \\
\hline TOTAL RAD & 01 & 2607 & 01 & 0 & 0 & ] & 212 & 2396 & 0. & 2607 \\
\hline & & & & & & & & & & \\
\hline TCLP EXTRACTION & 0 & 0 & 0 & 0 & 으 & 0 & 0 & 0 & -0 & 0 \\
\hline OTHER-PREP & 0 & 0 & 0 & 0 & 0 & 0. & (0) & 0 & -0 & 0 \\
\hline OTHER-EXPLO & 0 & of & 0 & 0 & 0 & - & 0 & of & 0 & 0 \\
\hline OTHER-BIO & 0 & $\overline{0}$ & 0 & 0 & 0 & 0 & 0) & 0 & 0 & 0 \\
\hline OTHER-PHYS & 0 & 0 & a & 0 & 0 & 0 & 0 & 0 & 묘 & 0 \\
\hline OTHER & 0 & 0 & 0 & 0 & 0 & 0 & 0 & 0 & - & 0 \\
\hline TOTALOTHEA & 01 & 0) & 0 & 0 & 0 & 0 & 01 & 0 & 0 & 0 \\
\hline & & & & & & & & & & \\
\hline TOTAL SAMPLEANALYS & 0 & 10064 & 0 & 0 & 0 & 에 & 3457 & 6607 & 0 & 10064 \\
\hline
\end{tabular}


Table IVa: UC Sample Analyses by Rad Level and Matrix Type (iii) Los Alamos Nat'I Lab

\begin{tabular}{|c|c|c|c|c|c|c|c|c|c|c|}
\hline $\begin{array}{l}\text { Rad-Matrix } \\
\text { Samp Anal }\end{array}$ & Ro & RO/RT & R1 & $\mathrm{R2}$ & $\overline{R 3}$ & $\overline{\mathrm{RA}}$ & M1 & $\overline{\mathrm{M} 2}$ & $\overline{M 3}$ & Total \\
\hline & & & & & & & & & & \\
\hline VOA-GC & 0 & 0 & 0 & 0 & 0 & 0 & 0 & 0 & 0 & 0 \\
\hline VOA-GCMS & 1501 & 0 & 350 & 0 & 0 & of & 929 & 643 & 279 & 1851 \\
\hline SEMI-VOA & 1015 & 0 & 284 & 0 & 0 & o & 649 & 456 & 193 & 1299 \\
\hline PEST & 0 & 0 & 0 & 0 & 0 & of & 0 & 0. & o & 0 \\
\hline HERB & 0 & 0 & 0 & 0 & 0 & 0 & 0 & 0 & 0 & 0 \\
\hline$\overline{P C B}$ & 1453 & 0 & 0 & 0 & 0 & 0. & 467 & 913 & 74 & 1453 \\
\hline PCB/PEST & 0 & 0 & 0 & 0 & 0 & 0 & 0 & 0 & 0 & 0 \\
\hline OTHER-ORG & 0 & 0 & 0 & 0 & of & 0] & 01 & 0 & o & 0 \\
\hline TOTAL ORGANICS & 3969 & 01 & 634 & $\overline{0}$ & 01 & $0]$ & 2045 & 2012 & 546 & 4603 \\
\hline & & & & & & & & & & \\
\hline ICP-AES METALS & 2830 & 0 & 0 & 0 & 0 & o. & 1555 & 1135 & 140 & 2830 \\
\hline ICP-MSMETALS & 1278 & 0 & 321 & 0 & 0 & 마 & 1247 & 269 & 83 & 1599 \\
\hline GF-AAMETALS & 3455 & 0 & 1401 & 0 & 0 & of & 3674 & 932 & 249 & 4856 \\
\hline MERCUFY & 939 & 0 & 300 & $\overline{0}$ & 0 & o. & 924 & 240 & 76 & 1240 \\
\hline CYANIDE & 239 & D) & 0 & 0 & 0 & 0. & 216 & 23 & of & 239 \\
\hline ANIONS/CATIONS & 239 & 0 & 0 & 0 & 0 & ㅇ. & 216 & 23 & 0 & 239 \\
\hline WET CHEM & 0 & 0 & 0 & 0 & 0 & 0] & o & 0 & 0 & 0 \\
\hline OTHER-INORG & 754 & 0 & 0 & 0 & 0 & 0] & 378 & 3011 & 74 & 754 \\
\hline TOTAL INORGANICS & 9733 & $0 \mid$ & 2023 & 0 & 01 & 0] & 8210 & 2923 & 622 & 11756 \\
\hline & & & & & & & & & & \\
\hline GROSS ALPHA/BETA & 2347 & D & 0 & $\overline{0}$ & 0 & 0 & 1458 & 774 & 114 & 2347 \\
\hline ALPHA DET-Np237 & 0 & 0 & 0 & 0 & 0 & o & 0 & 0 & 0 & $\mathbf{0}$ \\
\hline ALPHA DET-Pu,Am & 0 & 0 & 0 & 0 & 0 & of & Dा & 0 & 0 & $\mathbf{0}$ \\
\hline ALPHADET-Pu & 5322 & of & 0 & $\overline{0}$ & 0 & 0 & 1872 & 3187 & 263 & 5322 \\
\hline ALPHADET-U & 1532 & 0 & 0 & 0 & 0 & 이 & 562 & 899 & 72 & 1532 \\
\hline ALPHADET-Am & 1600 & 0 & 0 & 0 & 0 & 매 & 642 & 894 & 63 & 1600 \\
\hline ALPHADET-NP & 0 & \begin{tabular}{l|l}
0 & \\
\end{tabular} & 0 & $\overline{0}$ & 0 & 0. & O) & O) & 0 & $\overline{0}$ \\
\hline ALPHA DET - Th & 0 & 0 & 0 & $\overline{0}$ & 0 & 0. & 0 & 0 & O. & $\mathbf{0}$ \\
\hline ALPHA DET - U,Th,Pu & 의 & 0 & 0 & 0 & 0 & 0. & D & of & 요 & 0 \\
\hline ALPHA DET-U235 & 0 & \begin{tabular}{l|l}
0 \\
\end{tabular} & 0 & $\overline{0}$ & 0 & o & 0 & 0 & - & $\overline{0}$ \\
\hline ALPHA DET-Pu,U,Am & of & 0 & 0 & 0 & 0 & 0. & 0 & O) & a & $\overline{0}$ \\
\hline ALPHA DET-U,Np,Pu,Am & 0 & 0 & 0 & 0 & 0 & o. & D) & 0 & - & $\overline{0}$ \\
\hline ALPHA DET-Ra226 & 0 & 0 & 0 & 0 & 可 & - & 0 & 0 & - & $\overline{0}$ \\
\hline ALPHADET-Ra228 & 0 & 0 & o| & 0 & 0 & o| & 0 & 0 & - & 0 \\
\hline ALPHA SPEC & 0 & 0 & O) & 0 & o & 0. & 0 & 0 & 0. & 0 \\
\hline GPC-BETA-Pb210 & 0 & 0 & 0 & 0 & of & 이 & 0 & of & 0 & 0 \\
\hline GPC-BETA-Sr90 & 0 & 0 & of & $\overline{0}$ & 0 & 0] & 0 & 0 & 0 & 0 \\
\hline GPC-BETA-Pm147 & 0 & 0 & 0 & 0 & of & 0 & 0 & 0 & 0 & 0 \\
\hline GPC-BETA-Se79 & of & 0 & D. & 0 & O & 0 & 0 & 0 & 요 & 0 \\
\hline LSC-BETA-H3 & 5605 & 0 & 0 & 0 & 0 & 0 & 3450 & 1896 & 259 & 5605 \\
\hline LSC-BETA-Rin222 & of & 0 & 0 & 0 & 0 & o. & of & 0 & 애 & 0 \\
\hline LSC-BETA-1129 & of & 0 & 0 & $\overline{0}$ & 0 & 0 & 0 & 0 & 0 & 0 \\
\hline LSC-BETA-C14 & 0 & 0 & 0 & 0 & 0 & 0 . & 0 & 0 & D. & 0 \\
\hline LSC-BETA-TC99 & 0 & 0 & 0 & 0 & 0 & 0 & 0 & 0 & 0 & 0 \\
\hline LSC-BETA & of & 0 & 0 & 0 & 0 & 0 & of & 0 & 0. & 0 \\
\hline GAMMASPEC & 3946 & 0 & 0 & 0 & of & 0 & 2437 & 1324 & 185 & 3946 \\
\hline OTHER-RAD & 01 & 0 & 0 & 0 & 0 & 0] & DI & 0 & (0) & 0 \\
\hline TOTAL RAD & 20351 & 0 & 0 & 0 & 0 & का & 10421 & 8973 & 956 & 20351 \\
\hline & & & & & & & & & & \\
\hline TCLP EXTRACTION & 0] & 0 & 0 & 0 & 0 & 0 & of & D) & D & 0 \\
\hline OTHER-PREP & 0 & 0 & 0 & 0 & 0 & o. & 0 & 0 & D. & $\overline{0}$ \\
\hline OTHER-EXPLO & 0 & 0 & 0 & 0 & 0 & 0 & 0 & 0 & - & 0 \\
\hline OTHER-BIO & 0 & of & 0 & 0 & 0 & oा & o & 0 & o. & 0 \\
\hline OTHER-PHYS & 0 & 0 & of & 0 & 0 & o. & o & D & 0 & 0 \\
\hline OTHER & of & 0 & 0 & 0 & of & o] & 0 & 0 & (0) & $\mathbf{0}$ \\
\hline TOTAL OTHEA & OI & 0 & O & 0 & 01 & 0 -1 & 0 & 0! & (0. & $\overline{0}$ \\
\hline & & & & & 1 & 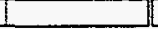 & & $=$ & & \\
\hline TOTAL SAMPLE ANAIYSES & 340531 & 01 & 2657 & 0 & 이 & $0][$ & 20677 & 13909 & 2125 & 36710 \\
\hline
\end{tabular}


EM-263 SURVEY OF DOE ANALYTICAL CAPACITY AVAILABLE TO EM

Table Na: UC Sample Analyses by Rad Level and Matrix Type (iv) Argonne East

\begin{tabular}{|c|c|c|c|c|c|c|c|c|c|c|}
\hline $\begin{array}{l}\text { Rad-Matrix } \\
\text { Samp Anal }\end{array}$ & RO & RO/RT & $\overline{\mathbf{R 1}}$ & $R 2$ & $\mathrm{R3}$ & $\overline{\mathrm{R4}}$ & $\overline{M 1}$ & $\sqrt{12}$ & $\mathrm{M3}$ & Total \\
\hline & & & & & & & & & & \\
\hline VOA-GC & 0 & 0 & 0 & 0 & 0 & 0 & $\overline{0}$ & 0 & 0 & 0 \\
\hline VOA-GCMS & 116 & 0 & 14 & 0 & 0 & 0 & 111 & 14 & 5 & 130 \\
\hline SEMI-VOA & 01 & 23 & 338 & 0 & 0 & 0 & 217 & 0 & 145 & 361 \\
\hline PEST & 0 & 0 & 0 & 0 & 0 & 0 & 0 & 0 & 0 & 0 \\
\hline HEFB & 0 & 0 & 0 & 0 & 0 & 0 & 0 & 0 & 0 & 0 \\
\hline $\mathrm{PCB}$ & 0 & 0 & 0 & 0 & 0 & 0 & 0 & 0 & o. & 0 \\
\hline PCB/PEST & 37 & 0 & 0 & 0 & 01 & 0 & 15 & 1 & 22 & 37 \\
\hline OTHER-ORG & 0 & 0 & 0 & 0 & 0 & 0 & 0 & 0 & 0 & 0 \\
\hline TOTAL ORGANICS & 153 & 23 & 352 & 01 & 01 & $\overline{0}$ & $34 \overline{3}$ & 15 & 171 & 528 \\
\hline & & & & & & & & & & \\
\hline ICP-AESMETALS & 0 & 2383 & 208 & 0 & 132 & 0 & 884 & 539 & 1299 & 2722 \\
\hline ICP-MS METALS & 0 & 0 & 0 & 0 & 0 & 0 & 0 & 0 & 0 & 0 \\
\hline GF-AAMETALS & 0 & 231 & 0 & 0 & 0 & 0 & 12 & 139 & 81 & $\overline{231}$ \\
\hline MERCUFY & D & 149 & 0 & 0 & 0 & 0 & 105 & 30 & 15 & 149 \\
\hline CYANIDE & 0 & 12 & 0 & 0 & 0 & 0 & 12 & 0 & 0 & 12 \\
\hline ANIONS/CATIONS & 0 & 0 & 0 & 0 & 0 & 0 & 0 & 0 & o) & 0 \\
\hline WET CHEM & 0 & 0 & 0 & 0 & 0 & 0 & $\overline{0}$ & 0 & 0 & 0 \\
\hline OTHER-INORG & 0 & 0 & 01 & D) & 0 & $\mathbf{0}$ & 0 & 0 & 0 & 0 \\
\hline TOTAL NOFGANICS & 01 & 27751 & 208 & D) & 132 & 0. & 1012 & 7081 & 1395 & 3114 \\
\hline & & & & & $=$ & $=$ & 7 & $=$ & & \\
\hline GROSS ALPHAVBETA & 0 & 0 & 315 & 0 & 0 & 0 & 126 & 95 & 95 & 315 \\
\hline ALPHA DET-Np237 & 0 & 0 & 0 & 0 & 0 & 0 & 0 & $\mathbf{0}$ & 0 & 0 \\
\hline ALPHA DET-Pu,Am & 0 & 0 & 0 & 0 & 0 & 0 & 0 & 0 & 0 & $\mathbf{0}$ \\
\hline ALPHA DET-PU & 0 & 0 & 0 & 0 & 0 & 0 & 0 & 0 & 0 & 0 \\
\hline ALPHADET-U & 0 & 0 & 0 & 0 & 0 & 0 & 0 & $\mathbf{0}$ & 0 & 0 \\
\hline ALPHA DET-Am & 0 & 0 & 0 & 0 & 0 & 0 & 0 & 0 & 0 & 0 \\
\hline ALPHADET-Np & 0 & 0 & 0 & 0 & 0 & 0 & 0 & $\mathbf{0}$ & 0 & 0 \\
\hline ALPHA DET-Th & 0 & 0 & 0 & 0 & 0 & 0 & 0 & 0 & o: & 0 \\
\hline ALPHA DET-U,Th,PU & 0 & 0 & 0 & 0 & 0 & 0. & 0 & 0 & 0 & 0 \\
\hline ALPHA DET-U235 & 0 & 0 & 0 & 0 & 0 & 0 & 0 & 0 & 0 & 0 \\
\hline ALPHA DET-Pu,U,Am & 0 & 0 & 0 & 0 & 0. & Q & 0 & 0 & 0 & 0 \\
\hline AlPHA DET-U,Np,Pu,Am & 0 & 0 & 0 & 0 & o) & 0 & 0 & 0 & 0 & 0 \\
\hline ALPHA DET-Ra226 & 0 & 0 & 0 & 0 & $\mathbf{0}$ & 0 & 0 & 0 & 0 & 0 \\
\hline ALPHAA DET-R2228 & 0 & 0 & 0 & 0 & 0 & 0 & 0 & 0 & 0 & 0 \\
\hline ALPHA SPEC & 0 & 0 & 389 & 0 & 0 & 0 & 0 & 389 & 0 & 389 \\
\hline GPC-BETA-Pb210 & 0 & 0 & D. & 0 & 0 & 0 & 0 & 0 & 0 & 0 \\
\hline GPC-BETA-Sr90 & 0 & 0 & 0 & 0 & 0 & 0 & 0 & $\mathbf{0}$ & 0 & 0 \\
\hline GPC-BETA-Pm147 & 0 & 0 & 01 & 0 & 0 & 0 & 0 & 0 & 0 & 0 \\
\hline GPC-BETA-Se79 & 0 & 0 & 0 & 0 & 0 & 0 & 0 & 0 & 0 & 0 \\
\hline LSC-BETA-H3 & 0 & 0 & 0 & 0 & 0 & 0. & 0 & 0 & a & 0 \\
\hline LSC-BETA-Pn222 & 0 & 0 & 0 & 0 & 0 & 0 & 0 & 0 & 0 & 0 \\
\hline LSC-BETA-I129 & 0 & 0 & 0 & 0 & 0 & 0. & 0 & 0 & 0 & 0 \\
\hline LSC-BETA-C14 & 0 & 0 & 0 & 0 & 0 & 0 & 0 & 0 & 0 & D \\
\hline LSC-BETA-TC99 & 0 & 0 & 0 & 0 & 0 & 0 & 0 & 0 & 0 & 0 \\
\hline LSC-BETA & 0 & 0 & 0 & 0 & 0 & 0 & 0 & 0 & 0 & 0 \\
\hline GAMMA SPEC & 0 & 0 & 257 & 0 & 0 & 0 & 103 & 77 & 77 & 257 \\
\hline OTHER-FAD & 0 & 0 & 01 & 0 & 0 & 0 & 0 & 0 & 0 & 0 \\
\hline TOIAL RAD & 0 & 01 & 962 & 0 & 01 & 0. & 229 & 5611 & 172 & 962 \\
\hline & & & & & & & & & & \\
\hline TCLPEXTRACTION & 0 & 25 & 0 & 0 & 0 & 0 & 25 & 0 & 0) & 25 \\
\hline OTHER-PREP & 0 & 0 & 0 & 0 & 0 & 0 & 0 & 0 & 0 & 0 \\
\hline OTHEA-EXPLO & 0 & 0 & 0 & 0 & 0 & D) & 0 & 0 & 0 & 0 \\
\hline OTHER-BIO & 0 & 0 & 0 & 0 & 0 & 0 & 0 & 0 & 0 & 0 \\
\hline OTHEA-PHYS & 0 & 0 & 0 & 0 & 0 & D. & 0 & 0 & D) & 0 \\
\hline OTHER & 0 & 0 & 0 & 0 & 0 & $\mathrm{O}$ & 0 & 0 & 0) & 0 \\
\hline TOIAL OTHER & 0 & 25 & $0]$ & $\overline{0}$ & 01 & 0 & 25 & 01 & 0. & 25 \\
\hline & & & & & & 3 & & & & \\
\hline TOTAL SAMPLEANALYSES & 153 & 2823 & 1521 & 0 & 132 & $\overline{0}$ & 1608 & 1284 & 1738 & 4629 \\
\hline
\end{tabular}


EM-263 SURVEY OF DOE ANALYTICAL CAPACITY AVAILABLE TO EM

Table IVa: UC Sample Analyses by Rad Level and Matrix Type (v) Argonne West

\begin{tabular}{|c|c|c|c|c|c|c|c|c|c|c|}
\hline $\begin{array}{l}\text { Rad-Matrix } \\
\text { Samp Anal }\end{array}$ & R0 & RO/R1 & $\overline{R 1}$ & $\mathrm{R} 2$ & $7 \overline{123}$ & $\overline{\mathrm{R} 4}$ & Mi & 7 M2 & $\overline{\mathrm{M} 3}$ & Total \\
\hline VOA-GC & of & 0 & 319 & 0 & 0 & 0 & 319 & & & 010 \\
\hline VOA-GCMMS & 0 & 0 & 0 & 0 & 0 & 0 & $\frac{519}{0 \mid}$ & $\frac{0}{0}$ & $\frac{0}{0}$ & $\frac{319}{0}$ \\
\hline SEMI-VOA & 0 & 0 & 0 & 0 & 0 & 0 & 0 & 0 & 0 & 0 \\
\hline PEST & 0 & 0 & 01 & 0 & 0 & o & a & 0 & 0 & 0 \\
\hline HERB & 0 & 0 & 0 & 0 & a & 0 & 0 & 0 & 0 & 0 \\
\hline PCB & 0 & 0 & 0 & 0 & 0 & of & 0 & 0 & 0 & 0 \\
\hline PCB/PEST & 0 & 0 & 0 & 0 & 0 & of & 0 & o) & 0 & 0 \\
\hline OTHER-ORG & 0 & 0 & 0 & 0 & 0 & 0 & 0 & 0 & 0 & 0 \\
\hline TOTAL OAGAVICS & 0 & 01 & 319 & 0 & 0 & 0] & 319 & 01 & 0 & 319 \\
\hline & & & & & & th & & & & \\
\hline ICP-AES METALS & 0 & 0 & 81 & 0 & 0 & o & 0 & 0 & 81 & 81 \\
\hline ICP-MS METALS & 0 & ot & 0 & 0 & 0 & 0 & 0 & 0 & 0 & 0 \\
\hline GF-AAMETALS & 0 & 0 & 40 & 0 & 0 & o & 0 & 40 & o & 40 \\
\hline MEACURY & 0 & 0 & 49 & 0 & 0 & 0 & 0 & 49 & 0 & 49 \\
\hline CYANDE & 0 & 0 & 0 & 0 & 0 & 0 & (0) & o) & 0 & 0 \\
\hline ANIONS/CATIONS & of & 0 & \begin{tabular}{l|l}
13 \\
\end{tabular} & 0 & 0 & 애 & 13 & 0 & o & 13 \\
\hline WET CHEM & 0 & 0 & 0 & 0 & 0 & 애 & 0 & $0 \mid$ & 0: & 0 \\
\hline OTHER-INORG & 0 & 0 & \begin{tabular}{|l|l|}
483 \\
\end{tabular} & 0 & 0 & ot & 289 & 194 & 0 & 483 \\
\hline TOTAL INORGANICS & 01 & 0 & (666 & 0 & 0 & 0] & 302 & 283 & 81 & 666 \\
\hline & & & & & & & & & -1 & \\
\hline GROSS ALPHA/BETA & 0 & 0 & 126 & 0 & 0 & ot & 55 & 69 & 3 & 126 \\
\hline ALPHA DET-Np237 & of & 01 & 0 & 0 & 0 & 0 & o) & 0 & $\overline{0}$ & 0 \\
\hline ALPHA DET - PU,Am & 0 & 0 & 0 & 0 & 0 & 0 & 0 & 0 & 0 & 0 \\
\hline ALPHA DET-PU & 0 & 0 & 0 & 0 & 0 & ; & o & 0 & 0 & 0 \\
\hline ALPHA DET-U & 0 & 0 & 0 & 0 & 0 & 0 & 0 & 0 & o & 0 \\
\hline ALPHA DET-Am & 0 & 0 & 0 & 0 & 0 & 0 & 0) & o & 0 & 0 \\
\hline ALPHADET-NP & 0 & 0 & 0 & 0 & 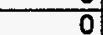 & 0 & o & 0 & 0 & 0 \\
\hline ALPHADET-Th & of & 0 & 0 & 0 & 0 & o & 0) & 0 & o & 0 \\
\hline ALPHA DET-U,Th,Pu & 0 & 0 & 0 & 0 & 0 & ot & 0 & 0 & 0 & 0 \\
\hline ALPHA DET-U235 & 0 & 0 & 0 & 0 & 0 & 0 & 0! & 0) & o & 0 \\
\hline ALPHA DET-PU,U,Am & 0 & 0 & 0 & 0 & 0 & oft & o & 0 & D & 0 \\
\hline ALPHADET-U,Np,Pu,Am & 0 & 0 & 0 & 0 & 0 & ô & of & 0 & 0] & 0 \\
\hline ALPHA DET-Ra226 & 0 & 0 & o & 0 & 0 & o & 0 & 0 & 0 & 0 \\
\hline ALPHADET-Ra228 & 0 : & 0 & 0 & 0 & 0 & 0 & 0) & 0 & D & 0 \\
\hline ALPHA SPEC & 0 & 0 & 0 & 0 & 0 & ot & 0 & 0 & 0 & 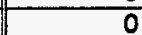 \\
\hline GPC-BETA-Pb210 & of & 0 & 0 & 0 & 0 & 0 & o) & 0 & o & 0 \\
\hline GPC-BETA-Sr 90 & 0 & 0 & 0 & 0 & 0 & o & 0 & 0 & 0 & 0 \\
\hline GPC-BETA-Pm147 & 0 & 0 & 0 & 0 & 0 & 0 & 0 & o & o & 0 \\
\hline GPC-BETA-Se79 & 0 & 0 & 0 & 0 & 0 & ot & 0 & 0 & 0 & 0 \\
\hline LSC-BETA-H3 & 0 & o & of & 0 & 0 & of & o & 0 & 0 & $\overline{0}$ \\
\hline LSC-BETA-Rn222 & 0 & 0 & 0 & 0 & 0 & o] & 0) & 0 & 0 & 0 \\
\hline LSC-BETA- 1129 & 0 & ot & of & $\frac{\pi}{0}$ & 0 & ot & 0! & 0 & o & 0 \\
\hline LSC-BETA-C14 & 0 & of & of & 0 & 0 & of & 0 & 0 & 0 & 0 \\
\hline LSC-BETA-Tc99 & ot & 0 & 0 & 0 & 0 & of & 0 & 0 & 0 & 0 \\
\hline LSC-BETA & 0 & 0 & 143 & 0 & 0 & o & 110 & 24 & 9 & $\overline{143}$ \\
\hline GAMMA SPEC & 0 & 0 & 586 & 0 & $\frac{\pi}{0}$ & of & 519 & 68 & 0 & 586 \\
\hline OTHER-RAD & 0 & 0 & 0 & 0 & 0 & 0 & 0 & 0 & 0 & 0 \\
\hline TOTAL RAD & 0 & $\overline{01}$ & 8551 & 0 & 0 & 0] & 684 & 160 & 11 & 855 \\
\hline & & & & & & & & & & \\
\hline TCLP EXTRACTION & of & 0 & 20 & 0 & 0 & ot & o & 0 & 20 & 20 \\
\hline OTHER-PREP & 0 & o & 0 & 0 & 0 & 0 & 0 & 0 & 0 & 0 \\
\hline OTHER-EXPLO & 0 & 0 & 0 & 0 & $\overline{0}$ & o & D & 0 & 요 & 0 \\
\hline OTHER-BIO & 0 & 0 & 0 & 0 & 0 & 0 & 0 & o & 0) & 0 \\
\hline OTHER-PHYS & 0 & 0 & 0 & 0 & 0 & 0 & o. & 0 & 0. & 0 \\
\hline OTHER & 0 & 0 & 0 & 0 & 0 & 0 & o & 0 & 0 & 0 \\
\hline TOTAL OTHER & 0 & 01 & 201 & $\overline{0}$ & 0 & 0 & O) & 0 & 20 & 20 \\
\hline & & & & & & & & & & \\
\hline TOTAL SAMPLE ANAIYSES & 01 & 01 & 18601 & $\overline{0}$ & $\overline{0}$ & 메 & 1305 & 443 & 112 & 1860 \\
\hline
\end{tabular}


EM-263 SURVEY OF DOE ANALYTICAL CAPACITY AVAILABLE TO EM

Table IVa: UC Sample Analyses by Rad Level and Matrix Type (vi) Brookhaven Nat'l Lab

\begin{tabular}{|c|c|c|c|c|c|c|c|c|c|c|}
\hline Samp Anal & Ro & RO/RI & R1 & $\mathrm{F} 2$ & R3 & $\overline{\mathrm{R4}}$ & M1 & $\overline{M L}$ & $\overline{\mathrm{M} 3}$ & Total \\
\hline $\mathrm{VOA}-\mathrm{GC}$ & 01 & & & & & & & & & \\
\hline VOA-GC/MS & 0 & 0 & $\begin{array}{l}0 \\
0\end{array}$ & $\frac{0}{0}$ & $\frac{0}{0}$ & $\frac{0}{0}$ & $\begin{array}{l}0 \\
0\end{array}$ & 0 & 애 & 0 \\
\hline SEMI-VOA & 0 & 0 & 0 & 0 & 0 & 0 & 0 & 0 & $\frac{0}{0}$ & 0 \\
\hline PEST & 0 & D & 0 & 0 & 이 & 0 & 0 & 0 & o: & 0 \\
\hline HERB & 0 & 0 & 0 & 0 & 0 & o & 0 & 0 & D & 0 \\
\hline$\overline{P C B}$ & 0 & 0 & 0 & 0 & 0 & D. & 0 & 0 & D. & 0 \\
\hline PCB/PEST & 0 & 0 & 0 & 0 & 0 & 0. & 0 & 0 & - & 0 \\
\hline OTHER-ORG & 0 & 0 & 0 & 0 & 0 & 0 & 0 & 0 & 0 & 0 \\
\hline TOTAL ORGANICS & 01 & 0 & 01 & 0 & 0 & 0] & 0 & 0 & 0 & 0 \\
\hline & & & & & & & & & & \\
\hline ICP-AES METALS & 0 & 0 & 0 & 0 & 0 & 0 & 0 & 0 & 0 & 0 \\
\hline ICP-MS METALS & 0 & 0 & 0 & 0 & 0 & 0 & 0 & 0 & 0 & 0 \\
\hline GF-AAMETALS & 0 & 0 & 0 & 0 & D & 0 & 0 & 0 & 0 & 0 \\
\hline MERCURY & 0) & 0 & 0 & o & 0 & 0. & 0 & 0 & 0 & 0 \\
\hline CYANIDE & 0 & 0. & a & 0 & 0 & 0. & 0 & 0 & 0. & 0 \\
\hline ANIONS/CATIONS & 0 & 요 & a & 0 & 0 & 요 & 0 & 0 & o & 0 \\
\hline WET CHEM & 0 & 0 & 0 & 0 & 0 & - $]$ & 0 & 0 & 0 & 0 \\
\hline OTHER-INORG & 0 & 0 & 0 & 0 & 0 & ㅁ․ & 0 & o & 0 & 0 \\
\hline TOTALINOAGANICS & 01 & 0] & 01 & OI & 0] & 0] [ & 0 & 0 & (0) & 0 \\
\hline & & & & & & & & & & \\
\hline GROSS ALPHAVBETA & 0 & 1260 & 0 & (0) & 0 & 0 & 1260 & 0 & (0) & 1260 \\
\hline ALPHA DET-Np237 & 0 & 0 & 0 & 0 & 0 & 0 & 0 & 0 & 0 & 0 \\
\hline ALPHIA DET-Pu,Am & 0 & 0 & o. & ग) & 0 & - & 0 & 0 & 요 & 0 \\
\hline ALPHA DET-PU & 0 & 0 & 0 & o. & 0 & 0] & 0 & 0 & - & 0 \\
\hline ALPHA DET-U & o & 0 & 0 & 0 & 0 & - & 0 & 0 & 으 & 0 \\
\hline ALPHA DET-Am & 0 & 0 & 0 & 0 & 0 & 0 & 0 & 0 & - & 0 \\
\hline ALPHADET-Np & 0 & 0 & 0 & 0 & 0 & ] & 0 & 0 & - & 0 \\
\hline ALPHA DET-Th & 0 & 0 & 0 & 0 & 0 & 0. & 0 & o & - & 0 \\
\hline ALPHA DET-U,Th,Pu & 0 & 0 & 0 & 0 & 0 & 요 & 0 & 0 & 애 & 0 \\
\hline ALPHA DET-U235 & 0 & 0 & 0 & 0 & 0 & 요 & of & 0 & 0 & 0 \\
\hline ALPHA DET-PU,U,Am & O) & 0 & o & 0 & 0 & 0 & 0 & 0 & 0 & 0 \\
\hline ALPHA DET-U,Np,Pu,Am & 0 & 0 & 0 & 0 & 0 & 요 & o & 0 & a & $\overline{0}$ \\
\hline ALPHA DET-Ra226 & D. & 0 & O & 0 & 0 & a & of & 0 & a & 0 \\
\hline ALPHADET-Ra228 & 0 & 0 & 0 & D) & 0 & 요 & 0 & D & 의 & 0 \\
\hline ALPHA SPEC & 0 & $\overline{0}$ & 0 & 0 & 0 & a & 0 & of & 0 & $\overline{0}$ \\
\hline GPC-BETA-Pb210 & 0 & 0 & 0 & 0 & 0 & a & D) & 0 & 0. & $\overline{0}$ \\
\hline GPC-BETA-Sr90 & 0 & 0 & 0 & 0 & 0 & a & D & 0 & 0. & 0 \\
\hline GPC-BETA-Pm 147 & 0 & 0 & 0 & 0 & 0 & a) & of & 0 & 0 & 0 \\
\hline GPC-BETA-Se79 & 0 & 0 & 0 & 0 & 0 & 요 & of & 0 & 0 & 0 \\
\hline LSC-BETA-H3 & 0 & 9554 & 0 & 0 & 0 & a & 9554 & of & 0 & 9554 \\
\hline LSC-BETA-Pn222 & o & 0 & 0 & D & 0 & of & 0) & 0 & 0 & 0 \\
\hline LSC-BETA- 1129 & 0 & $\overline{0}$ & o & 0 & 0 & of & 0) & 0. & 0. & 0 \\
\hline LSC-BETA-C14 & 0 & $\overline{0}$ & 0 & 0 & 0 & 0] & 0 & 0 & 0] & 0 \\
\hline LSC-BETA-TC99 & 0 & 0 & 0 & 0 & 0 & 0. & 0 & 0 & 0 & 0 \\
\hline LSC-BETA & 0 & 0 & 0 & 0 & 0 & 0. & 0 & 0 & (0) & 0 \\
\hline GAMMASPEC & 0 & 1065 & o & 0 & 0 & 이 & 1065 & of & 0 & 1065 \\
\hline OTHER-AAD & 0 & 0 & 0 & 0 & 0 & 0 & 0 & 0 & 0 & 0 \\
\hline TOTAL RAD & 0 & 11880 & 0 & 0 & $\overline{0}$ & 0 & 11880 & 01 & 0] & 11880 \\
\hline & & & & & & & & & & \\
\hline TCLP EXTRACTION & 0 & 0 & 0 & D & 0 & 0 & 0 & 0 & 의 & 0 \\
\hline OTHER-PREP & 0 & 0 & 0 & D & 0 & O. & 0 & 0 & 요 & $\overline{0}$ \\
\hline OTHER-EXPLO & 0 & 0 & 0 & 0 & 0 & 0 & 0 & 0 & 요 & 0 \\
\hline OTHER-BIO & 0 & 0 & 0 & 0 & 0 & 0 & 0 & 0 & - & 0 \\
\hline OTHER-PHYS & 0 & 0 & 0 & 0 & 0 & 0 & 0 & 0 & - & $\overline{0}$ \\
\hline OTHER & $\overline{0}$ & $\overline{0}$ & 0 & 0 & $\overline{0}$ & 0 & 0 & 0 & of & 0 \\
\hline TOTAL OTHER & 0 & 0 & $=\overline{0}$ & 0 & 0 & 0 & 0 & 미 & 0 & $\overline{0}$ \\
\hline & & & & & & & & & & \\
\hline TOTAL SAMPLE ANALYS & $\overline{0}$ & 11880 & $\overline{0}$ & 0 & 0 & 0 & 11880 & 01 & 0 & 11880 \\
\hline
\end{tabular}


EM-263 SURVEY OF DOE ANALYTICAL CAPACTY AVAILABLE TO EM

Table Ia: UC Sample Analyses by Rad Level and Matrix Type (vii) Fernald

\begin{tabular}{|c|c|c|c|c|c|c|c|c|c|c|}
\hline $\begin{array}{l}\text { Rad-Matrix } \\
\text { Samp Anal }\end{array}$ & $7 \mathrm{RO}^{\circ}$ & RO/RI & 74 & $\overline{\mathrm{R} 2}$ & $\overline{\mathrm{R}}$ & $\overline{R 4}$ & $\overline{\mathrm{M} 1}$ & M2 & 7 M3 & Total \\
\hline & & & & $\cdot$ & & & & & & \\
\hline VOA-GC & 0 & 0 & 0 & 0 & 0 & 0 & 0 & 0 & 0 & 0 \\
\hline VOA-GCMS & D. & 70 & 0 & 0 & 0 & 0 & 70 & 0 & - & 70 \\
\hline SEMI-VOA & 0 & 0 & 0. & 0 & 0 & 0 & o) & o & o & 0 \\
\hline PEST & Oा & 01 & 0 & 0 & 0 & 0 & 0 & 0 & 0 & 0 \\
\hline HERB & 0 & 0 & 0 & 0 & 0 & 0 & 0 & 0 & 0 & 0 \\
\hline PCB & 0 & 0 & 0 & 0 & 0 & 0 & ot & 0 & (0) & o \\
\hline PCB/PEST & 0 & 0 & 0 & 0 & 0 & 0 & 0 & 0 & 0 & 0 \\
\hline OTHER-ORG & 0 & 0 & 0 & 0 & $\overline{0}$ & 0. & 0 & 0 & o & 0 \\
\hline TOTAL ORGANICS & 0 & 701 & 01 & 01 & 01 & $0]$ & 70 & o| & (0] & 70 \\
\hline & & & & & & & & & & \\
\hline ICP-AES METALS & 0 & 1955 & 0 & 0 & 0 & 0 & 880 & 1075 & (0) & 1955 \\
\hline ICP-MS METALS & 0 & 1138 & 0 & 0 & 0 & 0 & 114 & 512 & 512 & 1138 \\
\hline GF-AAMETALS & 이 & 5633 & 0 & 0 & 0 & 0 & 3855 & 1778 & 요 & 5633 \\
\hline MERCURY & 0 & 838 & 0 & 0 & 0 & 0 & 503 & 335 & 0 & 838 \\
\hline CYANIDE & 0 & 0 & 0 & 0 & 0 & 0 & 0 & 0 & 0 & 0 \\
\hline ANIONS/CATIONS & 0 & 734 & 0 & 0 & 0 & 0 & 734 & 01 & 0 & 734 \\
\hline WET CHEM & 0 & 590 & 0 & 0 & 0 & 0 & 236 & 199 & 154 & 590 \\
\hline OTHER-INORG & 0 & 11390 & 0 & 0 & 0 & o & 10223 & 1167 & 0 & 11390 \\
\hline TOTAL INORGANICS & 01 & 22279 & 0. & 01 & 0 & 0 & 16545 & 5067 & 667 & 22279 \\
\hline & & & & & & & & & & \\
\hline GROSS ALPHA/BETA & 0 & 4855 & 0 & 0 & 0 & 0 & 555 & 4022 & 278 & 4855 \\
\hline ALPHADET-Np237 & o. & 0 & 0 & 0 & 0 & 0 & of & 0 & 0. & 0 \\
\hline ALPHA DET-PU,Am & 0 & 0 & 0 & 0 & 0 & 0. & 0 & 0 & 0 & 0 \\
\hline ALPHADET-Pu & 0 & 0 & 0 & 0 & 0 & 0 & of & 0 & o & 0 \\
\hline ALPHA DET-U & 0 & 824 & 0 & 0 & 0 & 0 & 824 & 0 & 0 & 824 \\
\hline ALPHA DET-Am & 0 & 0 & 0 & 0 & 0 & 0 & 0 & 0 & 0 & 0 \\
\hline ALPHA DET-Np & of & D) & 0 & of & 0 & 0] & 0 & 0 & 0] & $\overline{0}$ \\
\hline ALPHADET-Th & 0 & 837 & 0 & 0 & 이 & 0: & 627 & 167 & 42 & 837 \\
\hline ALPHA DET-U,Th,Pu & 0 & 0 & 0 & o| & 0 & 0. & of & 0 & 0 & o. \\
\hline ALPHA DET-U235 & 0 & 0) & 0 & 0 & 0 & of & 0 & 0 & 0] & $\overline{0}$ \\
\hline ALPHA DET-PU,U,Am & 0 & 0 & 0 & 0 & 0 & 0] & 0 & 0 & 0. & 0 \\
\hline ALPHA DET - U,Np,Pu,Am & 0 & of & 0 & 0 & 0 & o & 0 & 0 & 0. & 0 \\
\hline ALPHA DET-Ra226 & o & 413 & o & 0 & 0 & 0 & 310 & 83 & 21 & 413 \\
\hline ALPHA DET-Ra228 & 0 & 286 & 0 & of & 0 & 0] & 57 & 215 & 14 & 286 \\
\hline ALPHA SPEC & o & 0 & 0 & 0 & 0 & o & 0 & 0 & 0 & 0 \\
\hline GPC-BETA-Pb210 & 0 & 01 & 0 & 0 & 0 & 0 & 0 & 0 & 0 & $\overline{0}$ \\
\hline GPC-BETA-SP90 & 0 & 0 & 0 & 0 & 0 & o & 0 & o & 0 & 0 \\
\hline GPC-BETA-Pm147 & 0 & 0 & 0 & of & of & (0) & 0 & 0 & 0 & 0 \\
\hline GPC-BETA-Se79 & 0 & 0 & 0 & 0 & 0 & a & 0 & 0 & 0 & 0 \\
\hline LSC-BETA-H3 & o & 0 & 0 & 0 & 0 & o & 0 & 0 & 0 & 0 \\
\hline LSC-BETA-Rn222 & 0 & 0 & 0 & 0 & 0 & - & 0 & 0 & 에 & 0 \\
\hline LSC-BETA-1129 & 0 & 0 & 0 & 0 & 0 & a & o & 0 & 0. & 0 \\
\hline LSC-BETA-C14 & 0 & 0 & 0 & 0 & 0 & 0 & 0 & 0 & 0 & 0 \\
\hline LSC-BETA-TC99 & 0 & 0 & 0 & 0 & 0 & 0. & 0 & 0 & 0 & 0 \\
\hline LSC-BETA & 0 & 0 & 0 & 01 & 0 & 0 & o & 0 & 0 & 0 \\
\hline GAMMA SPEC & 0 & 0 & 0 & 0 & 0 & 0 & 0 & 0 & 0 & $\mathbf{0}$ \\
\hline OTHER-RAD & 0 & 5922 & 0 & 0 & 0 & o & 2369 & 2369 & 1184 & 5922 \\
\hline TOTAL RAD & 0 & 13136 & 0] & 0 & 0 & 0] & 4743 & 6855 & 1539 & 13136 \\
\hline & & & & & & 7 & & & 7 & \\
\hline TCLP EXTRACTION & 0 & 305 & 0 & 0 & 0 & o & 0 & 305 & 0 & 305 \\
\hline OTHER-PREP & 0 & 0 & 이 & 0 & 0 & 요 & 0 & 0 & 0 & $\mathbf{0}$ \\
\hline OTHER-EXPLO & 0 & 0 & 0 & 0 & 0 & 요 & 0 & o & 0: & 0 \\
\hline OTHER-BIO & 0 & 0 & 0 & 0 & 0 & 0 & 0 & 0 & 0 & 0 \\
\hline OTHER-PHYS & 0 & 2965 & 0 & 0 & 0 & 에 & 928 & 1631 & 406 & 2965 \\
\hline OTHER & 0 & 0 & 0 & 0 & 0 & 0 & 0 & 0 & 0 & 0 \\
\hline TOTAL OTHER & 0 & 3270 & 0 & 0 & 0 & 0 & 928 & 1936 & 406 & 3270 \\
\hline & & & & & & & & & & \\
\hline TOTAL SAMPLEANALYSES & 0 & 38755 & 0] & 01 & 0 & (0) & 22285 & 13858 & 2611 & 38755 \\
\hline
\end{tabular}


EM-263 SURVEY OF DOE ANALYTICAL CAPACITY AVAILABLE TO EM

Table IVa: UC Sample Analyses by Rad Level and Matrix Type (viii) Idaho Nat'l Eng Lab - ACU

\begin{tabular}{|c|c|c|c|c|c|c|c|c|c|c|}
\hline $\begin{array}{l}\text { Rad-Matrix } \\
\text { Samp Anal }\end{array}$ & $\overline{\mathrm{AOO}}$ & RO/A1 & Ri & $\overline{72}$ & $\overline{R 3}$ & $\overline{\mathrm{RH}}$ & $\overline{\mathrm{M1}}$ & $\overline{142}$ & M3 & Total \\
\hline & & & & & & & & & & \\
\hline VOA-GC & 0 & 0 & 이 & 0 & 0 & 0 & 0 & of & 0. & $\mathbf{0}$ \\
\hline VOA-GCMS & 407 & 0 & 0 & 0 & 0 & 0. & 325 & 73 & 8 & 407 \\
\hline SEMI-VOA & 0 & 0 & 0 & 0 & 0 & 0 & 0 & 0 & o & 0 \\
\hline PEST & 0 & 0 & 이 & 0 & 0 & 0 & DI & 0 & 0. & 0 \\
\hline HERB & of & 0 & 0 & 0 & 0 & 0: & 0 & of & o: & 0 \\
\hline PCB & 0 & D्f & DI & 0 & 0 & a & of & 0 & o & $\overline{0}$ \\
\hline PCB/PEST & 0 & 0 & 0 & 0 & 0 & 0 & 0 & of & 0 & 0 \\
\hline OTHER-ORG & 0 & 0 & 0 & 0 & 0 & 0 & 01 & 0 & a t & 0 \\
\hline TOTAL ORGANICS & 4071 & OI & 01 & 0 & 01 & 0] & 325 & 73 & 8] [] & 407 \\
\hline & & & & & & & & 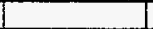 & $\square$ & \\
\hline ICP-AES METALS & 0 & 0 & 01 & 0 & 0 & D. & 0 & 0 & 0] & 0 \\
\hline ICP-MSMETALS & 0 & 可 & 0 & 0 & 0 & 0 & 0 & 0 & of & 0 \\
\hline GF-AAMETALS & 0 & of & 0 & 0 & 0 & 0: & 0 & 0 & o: & 0 \\
\hline MERCURY & 0 & 0 & 0 & o & 0 & a & o & 0 & (0) & $\overline{0}$ \\
\hline CYANIDE & 0 & 0 & 0 & 0 & 0 & a & 0 & 0 & 0 & 0 \\
\hline ANIONS/CATIONS & 0 & 0 & 0 & 0 & 0 & 0] & 0 & 0 & of & 0 \\
\hline WET CHEM & 0 & 0 & o) & 0 & 0 & a & 0 & 0 & 0 & 0 \\
\hline OTHER-INORG & 0 & 0 & 0 & 0 & 0 & 0] & 0 & 0 & 0 & 0 \\
\hline TOTAL INORGANICS & 0 & 0 & 01 & $0 !$ & 0 & O] & 0 & 01 & 0 & 0 \\
\hline & & & & & & & & & & \\
\hline GROSS ALPHA/BETA & 0 & 이 & $0]$ & 0 & 0 & 요 & 0 & 0 & 0 & 0 \\
\hline ALPHA DET-Np237 & 0 & o. & 0 & 0 & 0 & 0 & \begin{tabular}{l|l}
0 & \\
\end{tabular} & 0 & (0) & $\overline{0}$ \\
\hline ALPHA DET-Pu,Am & 0 & 0 & 0 & 0 & 0 & 0. & 0 & of & 0 & 0 \\
\hline ALPHADET-Pu & 0 & 0 & 0 & D. & 0 & 매 & 0 & o & 0 & 0 \\
\hline ALPHADET-U & 0 & 0 & 0 & a & 0 & 의 & 0 & O & 0 & 0 \\
\hline ALPHADET-Am & 0 & 0 & of & 0 & 0 & o & 0 & 0 & 0 & 0 \\
\hline ALPHA DET-Np & 0 & of & of & 0 & of & 0. & \begin{tabular}{l|l}
0 & \\
\end{tabular} & o & 0: & 0 \\
\hline ALPHADET-Th & 0 & 0 & of & 0 & of & - & 0 & of & - & 0 \\
\hline ALPHA DET - U,Th,Pu & 0 & 0 & 0 & 0 & 01 & ㅇ. & 0 & 0 & 0. & 0 \\
\hline ALPHADET-U235 & o) & o & of & 0 & of & 요 & 0 & 0 & o & 0 \\
\hline ALPHA DET-Pu,U,Am & 0 & 0 & of & 0 & 이 & O. & 0 & 0 & (0) & 0 \\
\hline ALPHADET-U,Np,Pu,Am & 0 & 0 & of & 0 & of & - & 0 & 0 & 0 & 0 \\
\hline ALPHADET-Ra226 & 0 & 우 & D) & 0 & 0 & 0 & 0 & 0 & 0. & 0 \\
\hline ALPHA DET-Ra228 & 0 & 0 & Dी & 0 & 0 & of & o & 0 & a. & 0 \\
\hline ALPHA SPEC & 0 & 0 & 이 & 0 & 0) & - & 0 & o. & 0 & 0 \\
\hline GPC-BETA-Pb210 & 0 & o. & of & 0 & 0] & o & 0 & 이 & 0 & 0 \\
\hline GPC-BETA-Sr90 & 0 & 0 & of & 0 & 0 & (0) & 0 & 0 & 요 & 0 \\
\hline GPC-BETA-Pm147 & 0 & 0 & of & 0 & 0) & - & 0 & 0 & (0) & 0 \\
\hline GPC-BETA-Se79 & 0 & 0 & 0 & 0 & 0 & 이 & 0 & 0 & 0 & 0 \\
\hline LSC-BETA-H3 & 0 & 0 & 0 & 0 & 0 & o & 01 & 0 & - & 0 \\
\hline LSC-BETA-Pin222 & 0 & 0 & $0 \mid$ & 0 & 0 & - & 01 & 0 & 0 & 0 \\
\hline LSC-BETA-1129 & 0 & 0 & 0 & 0 & 0 & - & 0 & 0 & 0: & 0 \\
\hline LSC-BETA-C 14 & 0 & 0 & 0 & 0 & 0 & 0 & 0 & 0 & 0 & 0 \\
\hline LSC-BETA-TC99 & 0 & o & 0 & 0 & 0 & 0 & o & Oा & 0 & 0 \\
\hline LSC-BETA & 0 & 0 & 0 & 0 & 0 & 0 & 0 & 0 & 0 & 0 \\
\hline GAMMASPEC & 0 & 0 & 0 & 0 & 0 & - & 0 & 0 & 0 & 0 \\
\hline OTHER-RAD & o & 0 & 0 & 0 & 0 & 0 & 0 & 0 & 0 & $\mathbf{0}$ \\
\hline TOTALRAD & 0 & 0 & 01 & Q & 0 & 0 & 01 & 이 & 이 & 0 \\
\hline & & & & & & & & & & \\
\hline TCLP EXTAACTION & 0 & 0 & 0 & 0 & 0 & 0 & 0 & 0 & 0 & 0 \\
\hline OTHER-PREP & 0 & 0 & of & 0 & o| & 의 & o & 0 & 0 & $\underline{0}$ \\
\hline OTHER-EXPLO & 0 & 0 & 0 & 0 & 0 & 0 & O) & 0 & 0 & 0 \\
\hline OTHER-BIO & 0 & 0 & 0 & 0 & 0 & 0. & \begin{tabular}{l|l}
0 &
\end{tabular} & 0 & 0. & 0 \\
\hline OTHER-PHYS & 0 & 0 & 0 & $\mathbf{0}$ & 0 & 0. & D. & 0 & 0 & 0 \\
\hline OTHER & $\mathbf{0}$ & 0 & 0 & 0 & 0 & a & D & 0 & (0) & 0 \\
\hline TOTAL OTHER & $\overline{0}$ & 0 & 0 & $\overline{0}$ & 01 & 0 & 01 & 01 & 0 & 0 \\
\hline & & & & & $T$ & $\square$ & 7 & 7 & 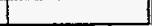 & 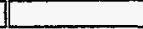 \\
\hline TOTAL SAMPLEANALYS & 407 & 0 & 01 & 0 & 0 & 0 & 325 & 731 & 8 & 407 \\
\hline
\end{tabular}


EM-263 SURVEY OF DOE ANALYTICAL CAPACTY AVAILABLE TO EM

Table IVa: UC Sample Analyses by Rad Level and Matrix Type (ix) Idaho Nat'I Eng Lab - B\&W

\begin{tabular}{|c|c|c|c|c|c|c|c|c|c|c|}
\hline $\begin{array}{l}\text { Rad-Matix } \\
\text { Samp Anal }\end{array}$ & $\overline{\mathrm{RO}}$ & RO/RT & $\overline{\mathrm{RI}}$ & $R 2$ & R3 & $\overline{R 4}$ & Mi & $\overline{M 2}$ & $\overline{M 3}$ & Total \\
\hline & & & & & & & & & & \\
\hline VOA-GC & 0 & 0 & 0 & 0 & 0 & 0: & 0 & 0 & ot & 0 \\
\hline VOA-GCMS & 0 & 0 & 0 & 0 & 0 & 0 & 0 & 0 & 0 & 0 \\
\hline SEMI-VOA & 0 & 0 & 0 & 0 & 0 & 0 & 0 & 0 & 0 & 0 \\
\hline PEST & 0 & 0 & 0 & 0 & 0 & o & 0 & 0 & 0 & 0 \\
\hline HERB & 0 & 0 & 0 & 0 & 0 & 0 & 0 & 0 & o & 0 \\
\hline PCB & 0 & 0 & 0 & 0 & 0 & 0 & 0 & 0 & 0 & 0 \\
\hline PCB/PEST & 0 & 0 & 0 & 0 & 0 & 0 & 0 & 0 & 0 & 0 \\
\hline OTHER-ORG & 0 & 0 & 0 & 0 & 0 & 0 & 0 & o) & 0. & 0 \\
\hline TOTAL ORGANICS & 01 & -0 & 0 & 0 & 0 & [0] & 0 & 01 & 0] & 0 \\
\hline & & & & & & & & & ] & \\
\hline ICP-AESMETALS & 0 & 0 & 0 & 0 & 0 & 0 & 0 & 0 & 0 & 0 \\
\hline ICP-MSMETALS & 0 & 0 & 0 & 0 & 0 & o & 0 & 0 & 0 & 0 \\
\hline GF-AAMETALS & 0 & 366 & 0 & 0 & 0 & 0] & 169 & 198 & 0 & 366 \\
\hline MERCURY & 0 & 196 & 0 & 0 & 0 & o. & 80 & 116 & 0 & 196 \\
\hline CYANIDE & 0 & 0 & 0 & 0 & 0 & 0 & 0 & 0 & o) & 0 \\
\hline ANIONS/CATIONS & 0 & 40 & 0 & 0 & 0 & 0 & 40 & 0 & 0 & 40 \\
\hline WET CHEM & 0 & 0 & 0 & 0 & 0 & 0 & 0 & 0 & o) & 0 \\
\hline OTHER-INORG & 0 & 746 & 0 & 0 & 0 & of & 337 & 409 & 0) & 746 \\
\hline TOTALINOAGANICS & $\overline{0}$ & 1349 & 0 & $\overline{01}$ & 01 & $0]$ & 626 & 722 & 0] & 1349 \\
\hline & & & & & & $\Rightarrow 1$ & 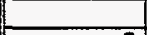 & $\ldots$ & 7 & \\
\hline GROSS ALPHABETA & 0 & 0 & 0 & 0 & 0 & 0 & 0 & 0 & 0 & 0 \\
\hline ALPHA DET-Np237 & 0 & o & 0 & 0 & 0 & 0 & 0 & 0 & o.: & o \\
\hline ALPHA DET-PU,Am & 0 & 0 & 0 & 0 & 0 & 0 & 0 & 0 & 0 & D: \\
\hline ALPHADET-PU & 0 & 0 & 0 & 0 & 0 & 0 & 0 & 0 & 0 & 0. \\
\hline ALPHADET-U & 0 & D) & 0 & 0 & 0 & 0 & 0 & 0 & 0 & 0) \\
\hline ALPHA DET-Am & 0 & 0 & 0 & 0 & 0 & 0 & 0 & 0 & 0 & 0) \\
\hline ALPHA DET-Np & 0 & 0 & 0 & 0 & 0 & 0 & 0 & 0 & 0. & 0 \\
\hline ALPHA DET-Th & 0 & of & 0 & 0 & 0 & 0 & 0 & 0 & 0 & 0 \\
\hline ALPHA DET-U,Th,PU & 0 & 0 & 0 & 0 & 0 & 0 & 0 & 0 & 0 & 0) \\
\hline ALPHA DET-U235 & 0 & 0) & 0 & 0 & 0 & 0. & 0 & 0 & 0 & D. \\
\hline ALPHA DET-PU,U,Am & 0 & 0 & 0 & 0 & 0 & 0 & 0 & 0 & 0 & 0 \\
\hline ALPHA DET-U,Np,Pu,Am & 0 & 0 & 0 & 0 & 0 & 0 & 0 & 0 & 0 & 0 \\
\hline ALPHA DET-R3226 & 0 & 0 & 0 & 0 & 0 & 0 & 0 & 0 & 0 & 0 \\
\hline ALPHA DET-Ra228 & 0 & 0 & 0 & 0 & 0 & 0 & 0 & 0 & 0 & 0 \\
\hline ALPHA SPEC & 0 & 0 & 0 & 0 & 0 & 0 & 0 & 0 & 0 & 0 \\
\hline GPC-BETA-Pb210 & 0 & 0 & 0 & 0 & 0 & 0 & 0 & 0 & 0 & 0 \\
\hline GPC-BETA-Sr90 & 0 & 0 & 0 & 0 & 0 & 0 & 0 & 0 & 0 & 0 \\
\hline GPC-BETA-Pm 147 & 0 & 0 & 0 & 0 & 0 & 0 & 0 & 01 & 0 & 0 \\
\hline GPC-BETA-Se79 & 0 & 0 & 0 & 0 & 0 & 0 & 0 & D) & 0] & 0 \\
\hline LSC-BETA-H3 & 0 & 0 & 0 & 0 & 0 & 0 & 0 & 0 & 0. & D: \\
\hline LSC-BETA-Rn222 & 0 & 0 & 0 & 0 & 0 & 0 & 0 & 0 & 0 & 0. \\
\hline LSC-BEIA-1129 & 0 & 0 & 0 & 0 & 0 & 0 & 0 & D) & 0 & 0 \\
\hline LSC-BETA-C14 & 0 & 0 & 0 & 0 & 0 & 0 & 0 & 0 & 0. & D. \\
\hline LSC-BETA-TC99 & 0 & 0 & 0 & 0 & 0 & 0 & 0 & 0 & 0 & 0. \\
\hline LSC-BETA & 0 & o & 0 & 0 & 0 & 0 & 0 & 0 & 0 & 0 \\
\hline GAMMA SPEC & 0 & 0 & 0 & 0 & 0 & 0 & 0 & 0 & o. & 0 \\
\hline OTHER-AAD & 0 & 0 & 0 & 0 & 0 & 0 & 0 & 0 & o. & 0 \\
\hline TOTAL BAD & 0 & O) & 0 & 01 & 01 & 0] & 0 & 01 & o] & 可 \\
\hline & & & & & & & & 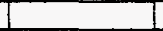 & 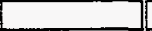 & \\
\hline TCLP EXIRACTION & 0 & 60 & 0 & 0 & 0 & $\mathbf{0}$ & 0 & 60 & 0 & 60 \\
\hline OTHER-PREP & 0 & 0 & 0 & 0 & 0 & 0 & 0 & 0 & 0] & 0 \\
\hline OTHER-EXPLO & 0 & 0 & 0 & 0 & 0 & 0. & 0 & 0 & of & $\overline{0}$ \\
\hline OTHER-BIO & 0 & 51 & 0 & 0 & 0 & 0 & 51 & 0 & 0 & 51 \\
\hline OTHER-PHYS & 0 & 0 & 0 & 0 & 0 & 0 & 0 & 0 & 0 & $\mathbf{0}$ \\
\hline OTHER & 0 & 0 & 0 & 0 & 0 & $0]$ & 0 & 0 & Q & 0 \\
\hline TOTAL OTHER & 01 & 111 & $\overline{0}$ & 01 & 01 & 0] & 51 & 60 & 0. & 111 \\
\hline & & & & & & & & & & \\
\hline TOTAL SAMPLEANALYSES & 0] & 1460 & $\overline{0}$ & 0 & 01 & O] & 677 & 783 & 01 & 1460 \\
\hline
\end{tabular}


EM-263 SURVEY OF DOE ANALYTICAL CAPACITY AVAILABLE TO EM

Table IVa: UC Sample Analyses by Rad Level and Matrix Type (x) Idaho Nat'l Eng Lab - WINCO

\begin{tabular}{|c|c|c|c|c|c|c|c|c|c|c|}
\hline Samp Anal & RO & RO/R1 & $\overline{R 1}$ & $\overline{7 R 2}$ & $\overline{\mathrm{R3}}$ & $\overline{R 4}$ & $\overline{\text { M1 }}$ & $\overline{\text { M2 }}$ & $\overline{M 3}$ & Total \\
\hline & & & & & & & & & & \\
\hline VOA-GC & 0 & 0 & 0 & 0 & 0 & 0 & 0 & 0 & 0 & 0 \\
\hline VOA-GCMS & 0 & 0 & 0 & 0 & 0 & 인 & 0 & 0 & 0 & 0 \\
\hline SEMI-VOA & 0 & 0 & 0 & of & 0 & a & o & 0 & 0 & $\overline{0}$ \\
\hline PEST & 0 & 0 & 0 & 0 & o & a & 0 & of & 0 & 0 \\
\hline HERB & 0 & 0 & 0 & o & 0 & a & 0 & 0 & o & $\overline{0}$ \\
\hline PCB & 0 & 0 & 0 & D & o & 므 & 0 & 0 & 0 & 0 \\
\hline PCB/PEST & 0 & 0 & 0 & D & 0 & 요 & 0 & 0 & 0 & $\overline{0}$ \\
\hline OTHER-ORG & 0 & 0 & 0 & 0 & 0 & o & 01 & of & $0]$ & 0 \\
\hline TOTAL ORGANICS & 01 & $\underline{\underline{0}}$ & 01 & $\overline{01}$ & 0 & 0 & 01 & 01 & $0]$ & $\overline{0}$ \\
\hline & & & & & & & & & & \\
\hline ICP-AES METALS & 0 & 0 & 0 & 0 & 이 & 0 & 0 & 0 & 0 & 0 \\
\hline ICP-MSMETALS & 0 & 0 & 0 & 0) & 0 & O & 0 & 0 & 0 & 0 \\
\hline GF-AAMETALS & 0 & 0 & 0 & 0 & of & o: & 0 & 0 & 0 & 0 \\
\hline MERCUAY & 0 & 0 & 0 & D) & 0 & o & 0 & 0 & 0 & 0 \\
\hline CYANIDE & o & 0 & 0 & 0 & 01 & 0 & 0 & 0 & - & 0 \\
\hline ANIONS/CATIONS & 0 & 0 & 0 & 0 & 이 & o & 0 & 0 & 0 & 0 \\
\hline WET CHEM & 0 & 0 & 0 & 0 & O & 요 & 0 & 0 & 0 & 0 \\
\hline OTHER-INORG & (0) & 0 & 01 & 0 & 0) & 0 & \begin{tabular}{l|l}
0 \\
\end{tabular} & of & 0] & 0 \\
\hline TOTAL INORGANICS & 0 & 0 & 0] & 01 & 이 & 0] & 01 & 01 & 0. & $\mathbf{0}$ \\
\hline & & & & & & & & & & \\
\hline GROSS ALPHAVBETA & 0 & 0 & 0 & 0 & 이 & - & 0 & 0 & 0 & 0 \\
\hline ALPHADET-Np237 & 0 & 0 & 0 & 0 & of & 0 & 0 & 0 & - & 0 \\
\hline ALPHA DET-Pu,Am & 0 & 0 & 0 & 0 & 0 & - & 0 & 0 & 0 & 0 \\
\hline ALPHADET-PU & 0 & 0 & 0 & 0 & 의 & of & 0 & 0 & - & $\mathbf{0}$ \\
\hline ALPHA DET-U & 0 & 0 & 01 & 0 & 0 & o & 0 & 0 & 요 & 0 \\
\hline ALPHADET-Am & 0 & 0 & 0 & 0 & 0 & 0 & 0 & 0 & 0: & 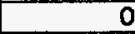 \\
\hline ALPHADET-Np & 0 & 0 & 0 & a & 0 & - & 0 & 0 & 0 & 0 \\
\hline ALPHA DET-Th & 0 & 0 & 0 & 0 & 0 & - & 0 & 0 & 요 & 0 \\
\hline ALPHA DET-U,Th,Pu & 0 & 0 & 0) & 0 & 0 & $\overline{0}$ & 0 & 0 & 0] & 0 \\
\hline ALPHADET-U235 & 0 & 0 & 0 & 0 & 요 & - & 0 & 0 & 0 & 0 \\
\hline ALPHA DET-PU,U,Am & 0 & 0 & 0 & 0 & of & 0 & 0 & of & - & $\overline{0}$ \\
\hline ALPHA DET-U,Np,Pu,Am & 0 & 0 & 0 & 0 & 0 & 의 & 01 & 0 & ㅇ․ & 0 \\
\hline ALPHA DET-Ra226 & of & 0 & 0 & 0 & 0 & D. & 0 & 0 & 0. & 0 \\
\hline ALPHADET-Ra228 & 0 & 0 & 0 & 0 & 0 & 의 & 0 & 0 & 요 & 0 \\
\hline ALPHA SPEC & of & 0 & 0 & 0 & 0 & o & 0 & 0 & 에 & $\overline{0}$ \\
\hline GPC-BETA-Pb210 & 0 & 0 & 0 & 0 & 0 & - & 0 & 0 & - & 0 \\
\hline GPC-BETA-Sr90 & 0 & 0 & 0 & o & 0 & o & 0 & 0 & ㅇ. & 0 \\
\hline GPC-BETA-Pm147 & 0 & 0 & 0 & 0 & 0 & 0 & 0 & 0 & 요 & 0 \\
\hline GPC-BETA-Se79 & o. & 0 & 0 & 0 & D. & 0 & - & O. & 요 & 0 \\
\hline LSC-BETA-H3 & 0 & 0 & 0 & 0 & 0 & - & 0 & 0 & 묘 & 0 \\
\hline LSC-BETA-An222 & 0 & 0 & 0 & 0 & D. & (0) & 0 & 0 & 므 & 0 \\
\hline LSC-BETA-1129 & 0 & 0 & 0 & 0 & 0 & 0 & 0 & 0 & 요 & 0 \\
\hline LSC-BETA-C14 & 0 & 0 & 0 & 0 & 0 & 0] & 0 & 0 & 0. & 0 \\
\hline LSC-BETA-TC99 & 0 & 0 & 0 & 0 & o & 0] & 0 & 0 & 0 & 0 \\
\hline LSC-BETA & 0 & 0 & 0 & 0 & 0 & 0 & 0 & 0 & 의 & 0 \\
\hline GAMMA SPEC & 0 & 0 & a) & 0 & 0 & 0 & 0 & of & 0 & 0 \\
\hline OTHER-RAD & 0 & 0 & 01 & 0 & 0 & 0 & 이 & 0 & 0 & 0 \\
\hline TOTAL RAD & 0 & $\underline{0}$ & 0] & 0 & 0 & -0. & 01 & 0 & 0 & $\overline{0}$ \\
\hline & & & & & & & & & 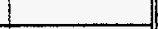 & \\
\hline TCLP EXTRACTION & 0 & 0 & 0 & 0 & 0 & 의 & 0 & 0 & 0 & 0 \\
\hline OTHER-PREP & 0 & 0 & 0 & 0 & 0 & 의 & 0 & 0 & 이 & 0 \\
\hline OTHER-EXPLO & 0 & 0 & 0 & 0 & 0 & 요 & O. & 0 & 0 & 0 \\
\hline OTHER-BIO & 0 & 0 & 0 & 0 & 0 & 요 & 0 & 0 & 의 & 0 \\
\hline OTHER-PHYS & D & 0 & 0 & 0 & 0 & 애 & 0 & 0 & 0 & $\overline{0}$ \\
\hline OTHEA & 0 & 0 & 0 & 0 & 0 & 0] & 0 & 0 & 0 & 0 \\
\hline TOTALOTHER & 0 & 0 & $0]$ & 0 & 0 & 에 & 0 & 0 & 0 & 0 \\
\hline & & & & & & & & & & \\
\hline TOTAL SAMPLEANALYSES & 0 & $\overline{0}$ & (0) & 0 & 0 & 0] & 0 & 0 & $\overline{0}$ & $\overline{0}$ \\
\hline
\end{tabular}


EM-263 SURVEY OF DOE ANALYTICAL CAPACITY AVAILABLE TO EM

Table Na: UC Sample Analyses by Rad Level and Matrix Type (xi) West Valley Nuclear Services

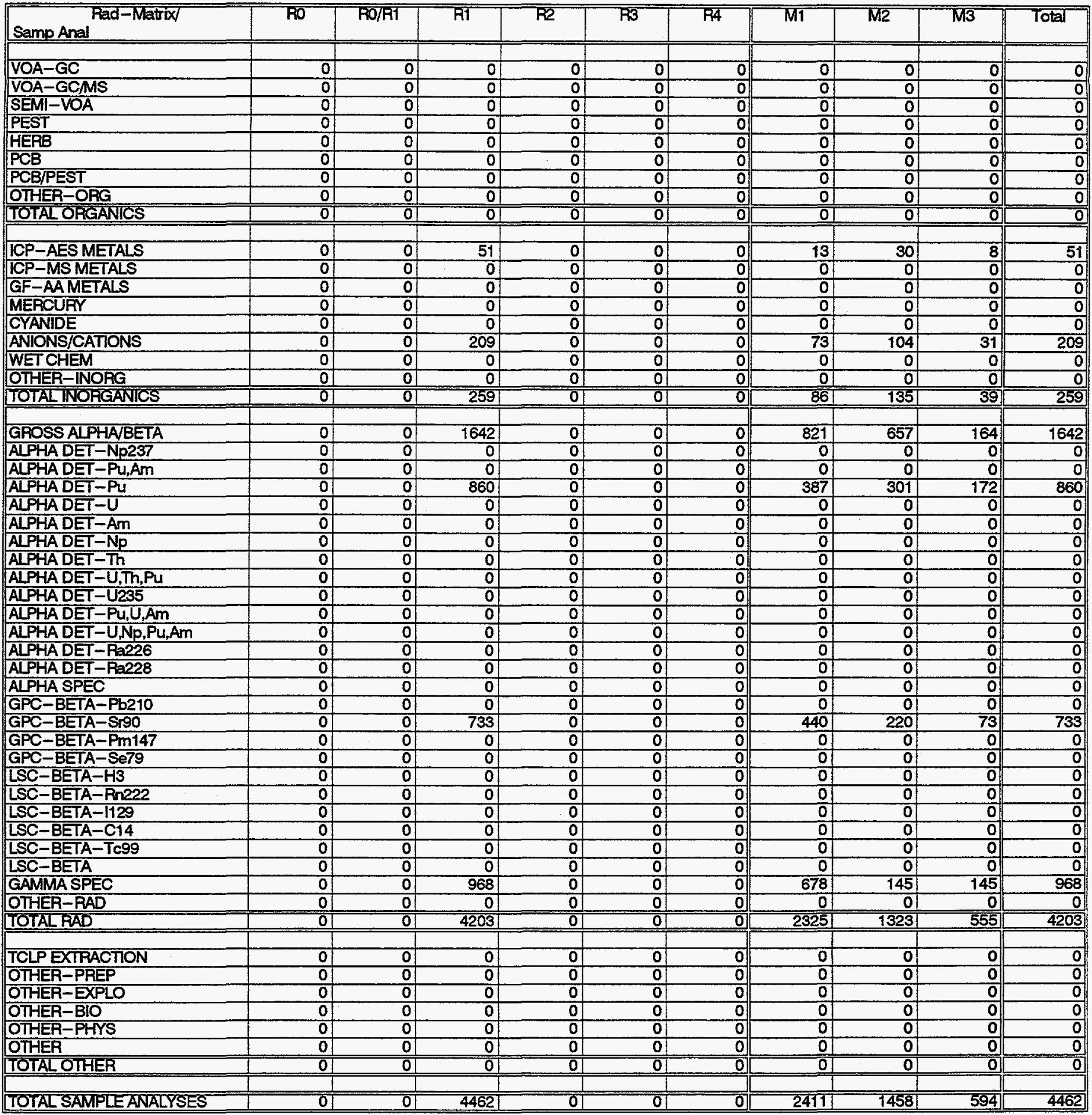


Table IV: UC Sample Analyses by Rad Level and Matrix Type (xii) Nevada Test Site

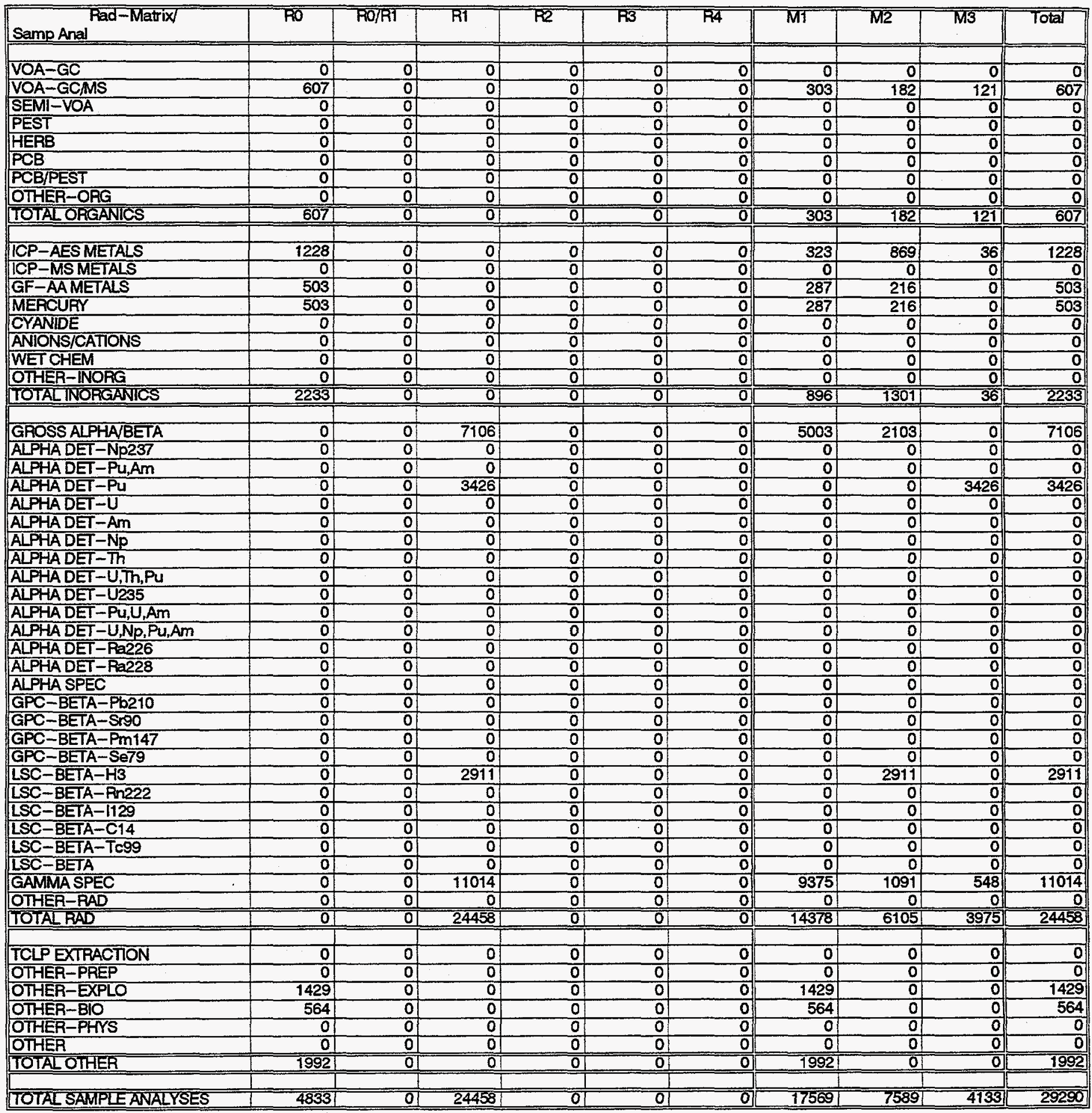


EM-263 SURVEY OF DOE ANALYTICAL CAPACTYY AVAILABLE TO EM

Table IVa: UC Sample Analyses by Rad Level and Matrix Type (xii) Oak Ridge - K25

\begin{tabular}{|c|c|c|c|c|c|c|c|c|c|c|}
\hline Samp Anal & RO & RO/R1 & R1 & $\mathrm{R} 2$ & $\overline{\mathrm{R} 3}$ & $\overline{\mathrm{RA}}$ & $\overline{\mathrm{M1}}$ & T12 & M3 & Total \\
\hline VOA-GC & 0 & 0 & 0 & 0 & of & 0 & 0 & & 0 & 0 \\
\hline VOA-GCMS & 3172 & 0 & 0 & 0 & o) & 0 & 2538 & 634 & 0 & $\frac{0}{3172}$ \\
\hline SEMI-VOA & 1106 & 0 & 0 & 0 & o) & 0 & 829 & 276 & 0 & 1106 \\
\hline PEST & 259 & 0 & 0 & 0 & 0 & o: & 239 & 21 & 0 & 259 \\
\hline HERB & 218 & 0 & 0 & 0 & 0) & 0 & 198 & 20 & 0 & 218 \\
\hline PCB & 3643 & 0 & o & 0 & 0 & of & 2368 & 911 & 364 & 3643 \\
\hline PCB/PEST & 0 & 0 & 0. & 0 & 0 & o & 0 & 0 & 0 & 0 \\
\hline OTHER-ORG & 0 & 0 & 0 & 0 & 01 & of & 0 & 0 & 0 & 0 \\
\hline TOTAL ORGANICS & $8398 \mid$ & OT & $\overline{0 .}$ & 01 & OI & 0 01 & 6172 & 1862 & 364 & 8398 \\
\hline & & & & & & & & & & \\
\hline ICP-AES METALS & 5829 & 0 & 0 & 0 & 0 & 0 & 4372 & 874 & 583 & 5829 \\
\hline ICP-MS METALS & 2766 & 0) & 0 & 0 & 0 & 0 & 2074 & 415 & 277 & 2766 \\
\hline GF-AAMETALS & 6458 & of & (0) & 0 & 0 & ôt & 4521 & 1292 & 646 & 6458 \\
\hline MERCURY & 4864 & 0 & 0! & 0 & 0 & o & 3648 & 730 & 486 & 4864 \\
\hline CYANIDE & 0 & 0 & 0 & 0 & 0 & 0 & 0 & 0 & 0 & 0 \\
\hline ANIONS/CATIONS & 1897 & O: & 0 & 0 & 0 & 0 & 1612 & 285 & 0 & 1897 \\
\hline WET CHEM & 0 & o. & 0 & 0 & 0 & 0 & 0 & 0 & 0 & 0 \\
\hline OTHER-INORG & 0 & O & 0 & 0 & 0 & 0 & 0 & 0 & 0 & 0 \\
\hline TOTAL INORGANICS & 21814 & 0 & 01 & & 01 & - & 16227 & 35951 & 1992 & 21814 \\
\hline & & & & & 1 & 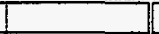 & & 1 & 7 & \\
\hline GROSS ALPHAVBETA & 2412 & 0 & 0 & 0 & 01 & 0 & 2412 & 0 & 0. & 2412 \\
\hline ALPHA DET-Np237 & o & 0 & 0 & 0 & 0 & 0 & 0 & 0 & 0 & 0 \\
\hline ALPHA DET-PU,Am & 0 & 0 & 0 & 0 & 0 & 0 & 0 & 0 & 0 & 0 \\
\hline ALPHA DET-PU & 0 & 0 & 0 & 0 & 0 & 0 & 0 & 0 & 0 & 은 \\
\hline ALPHA DET-U & 1594 & 0 & 0 & 0 & o: & 0 & 255 & 590 & 749 & 1594 \\
\hline ALPHA DET-Am & 0 & 0 & 0 & o & 0 & D] & 0 & of & 0 & 0 \\
\hline ALPHA DET-Np & 1793 & 0 & 0 & 0 & 0 & o & 90 & 807 & 897 & 1793 \\
\hline ALPHA DET-Th & 1388 & 0 & 0 & 0 & 이 & D. & 56 & 555 & 777 & 1388 \\
\hline ALPHA DET-U,Th, Pu & 0 & 0 & 0 & 0 & of & o & 0 & 0 & 0 & 0 \\
\hline ALPHA DET-U235 & 0 & 0 & 0 & 0 & of & D. & 0 & 0 & 0 & 0 \\
\hline ALPHA DET-PU,U,Am & of & 0 & 0 & o & 0 & o & 0 & 0 & 0 & $\overline{0}$ \\
\hline ALPHA DET-U,Np,Pu,Am & 0 & 0 & 0 & 0 & 0 & 0 & 0 & 0 & 0 & 0 \\
\hline ALPHA DET-R2226 & of & 0 & of & o & 0 & o. & 0 & 0 & 0 & $\overline{0}$ \\
\hline ALPHA DET-Ra228 & 0 & 이 & of & o & 0 & ㅇ. & 0 & 0 & 0 & 0 \\
\hline ALPHA SPEC & 0 & 0 & 0 & 0 & 0 & , & 0 & 0 & 0 & 0 \\
\hline GPC-BETA-Pb210 & o & o| & 0) & o & of & 0 & 0 & of & 0 & $\mathbf{0}$ \\
\hline GPC-BETA-Sr90 & 0 & 01 & 0 & 0 & 0 & o & $\overline{0}$ & 0 & 0 & 0 \\
\hline GPC-BETA-Pm 147 & 0 & 0 & 0 & 0 & 0 & 0 & 0 & 0 & 0 & $\mathbf{0}$ \\
\hline GPC-BETA-Se79 & 0 & D & 0 & 0 & 0 & 0 & \begin{tabular}{l|l}
0 \\
\end{tabular} & 0 & 0 & 0 \\
\hline LSC-BETA-H3 & 0 & 0 & 0 & 0 & o & 0 & 0 & 0 & 0 & 0 \\
\hline LSC-BETA-RI222 & D & D & 0 & 0 & o & 0 & $\mathbf{0}$ & 0 & 0 & 0 \\
\hline LSC-BETA-1129 & 0 & 0 & 0 & 0 & o & of & 0 & 0 & o & 0 \\
\hline LSC-BETA-C14 & D & of & 0 & 0 & 0 & o & 0 & 0 & 0 & D \\
\hline LSC-BETA-TC99 & 0 & 0 & 0 & 0 & 0 & 0 & 0 & 0 & o: & 0 \\
\hline LSC-BETA & of & 0 & 0 & 0 & o & 0 & $\mathbf{0}$ & o & o. & 0 \\
\hline GAMMA SPEC & 2043 & 0 & 0 & 0 & 0 & 0 & 307 & 204 & 1533 & 2043 \\
\hline OTHER-RAD & 0 & of & 0 & 0 & 0 & o. & 01 & O & 0 & 0 \\
\hline TOTAL RAD & 9231 & 0 & 0 & 0 & 0 & 0 & 3119 & 2156 & 3956 & 9231 \\
\hline & & & & & & & & & & \\
\hline TCLP EXTRACTION & 1187 & 0 & 0 & 0 & 0) & 0 & 0 & 1009 & 178 & 1187 \\
\hline OTHER-PREP & 734 & 0 & 0 & 0 & 0 & 0 & 624 & 110 & 0 & 734 \\
\hline OTHER-EXPLO & 0 & 0 & 0 & 0 & 0 & 0 & 0 & 0 & o & 0 \\
\hline OTHER-BIO & 0 & 0 & 0 & 0 & 0 & 0 & 0 & 0 & 0 & 0 \\
\hline OTHER-PHYS & 0 & 0 & 0 & 0 & 0 & 0 & 0 & 0 & of & 0 \\
\hline OTHER & 0 & 0 & 0 & 0 & 0 & 0] & 0 & 0 & 0 & 0 \\
\hline TOTALOTHER & 19211 & 0 & 0 & 0 & 0 & 0] & 624 & 1119 & 178 & 1921 \\
\hline & & & & & & & & & & \\
\hline TOTAL SAMPLE ANALYSES & 41364 & 0 & $\overline{0}$ & & 0 & क्षा & 26143 & 8732 & 6490 & 41364 \\
\hline
\end{tabular}


EM-263 SURVEY OF DOE ANALYTICAL CAPACTY AVAILABLE TO EM

Table IVa: UC Sample Analyses by Rad Level and Matrix Type (xiv) Oak Pidge Nat'l Lab

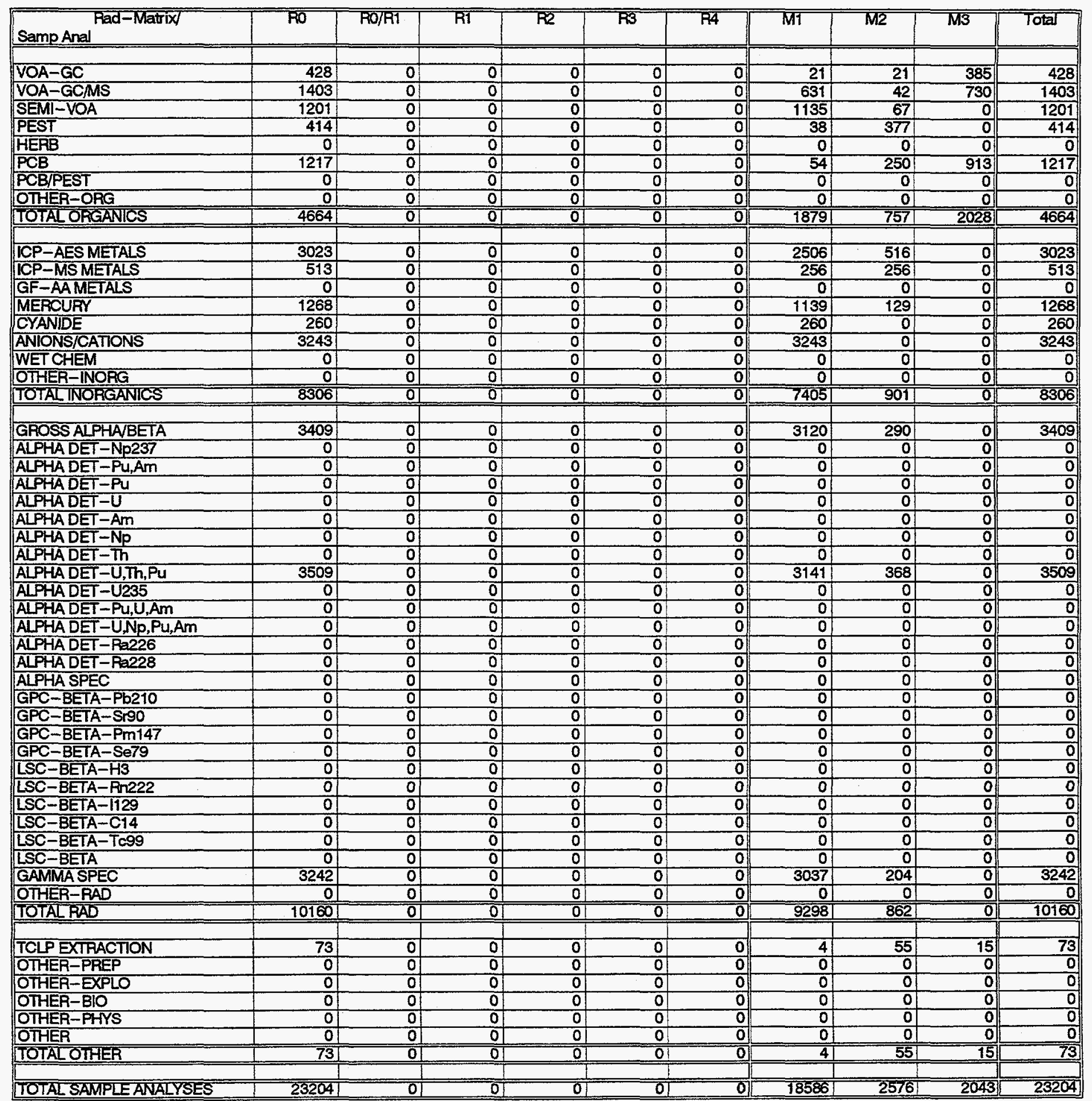




\section{EM-263 SUAVEY OF DOE ANALYTICAL CAPACITY AVAILABLE TO EM}

Table IVa: UC Sample Analyses by Rad Level and Matrix Type (xv) Oak Ridge - Y12

\begin{tabular}{|c|c|c|c|c|c|c|c|c|c|c|}
\hline $\begin{array}{l}\text { Rad-Matrix } \\
\text { Samp Anal }\end{array}$ & RPO & RO/R1 & $\overline{\mathrm{R} 1}$ & $\mathrm{~F} 2$ & $\overline{\mathrm{R} 3}$ & $\overline{\mathrm{R} 4}$ & TMT & M2 & M3 & Total \\
\hline & & & & & & & & & & \\
\hline VOA-GC & 0 & 0 & 0 & 0 & 0 & 0. & 0 & 0 & 0 & 0 \\
\hline VOA-GCMS & 2155 & 0 & 1820 & 0 & 0 & ㅇ․ & 3357 & 255 & 364 & 3976 \\
\hline SEMI-VOA & 252 & 0 & 1069 & 0 & 0 & 0 & 1296 & 13 & 13 & 1321 \\
\hline PEST & 0 & 0 & 0 & 0 & 0 & 0 & 0 & \begin{tabular}{l|l}
0 \\
\end{tabular} & 0 & 0 \\
\hline HERB & 0 & 0 & 0 & 0 & 0 & 0. & 0 & 0 & 0 & 0 \\
\hline PCB & 1595 & of & 1587 & 0 & 0 & (0) & 2376 & 440 & 365 & 3182 \\
\hline PCB/PEST & 0 & 0 & 0 & 0 & 0 & 인 & 0 & 0 & 0 & 0 \\
\hline OTHER-ORG & 668 & 0 & 0 & 0 & 0 & - & 668 & \begin{tabular}{l|l}
0 \\
\end{tabular} & 0 & 668 \\
\hline TOTAL ORGANICS & 4670 & OI & 4476 & 0 & 0 & 인 & 76971 & 708 & $\overline{742}$ & 9146 \\
\hline & & & & & & -1 & & & & \\
\hline ICP-AES METALS & 2800 & 0 & 1794 & o. & 0 & 0 & 2520 & 280 & 1794 & 4594 \\
\hline ICP-MSMETALS & 5092 & 0 & 0 & of & 0 & 0 & 3566 & 1170 & 356 & 5092 \\
\hline GF-AAMETALS & 2209 & 0 & 279 & o & 0 & 0 & 2488 & 0 & 0 & 2488 \\
\hline MERCURY & 6124 & 0 & 734 & 0 & 0 & o & 4329 & 1332 & 1197 & 6857 \\
\hline CYANIDE & 607 & 0 & 0 & 0 & 0| & o & 607 & 0 & 0 & 607 \\
\hline ANIONS/CATIONS & 594 & 0 & 1260 & o & 0 & 0 & 386 & 1453 & 14 & 1854 \\
\hline WET CHEM & 0 & 0 & 0 & 0 & o. & of & 0 & 0 & 0 & 0 \\
\hline OTHER-INORG & 0) & 0 & 19494 & 0 & 0 & al & 2801 & 14574 & 2119 & 19494 \\
\hline TOTAL INORGANICS & 17426 & 0 & 23560 & ग1 & 0 & $0:$ & 16696 & 18810 & 5480 & 40986 \\
\hline & & & & & & ] & & & & \\
\hline GROSSALPHA/BETA & 1590 & 0 & 145614 & 0 & 0 & 0 & 1113 & 145932 & 159 & 147204 \\
\hline ALPHA DET-Np237 & o) & 0 & 0 & 0 & 0 & 0 & 0 & o & 0 & 0 \\
\hline ALPHA DET-PU,Am & 0 & 0 & 0 & 0 & 0) & o & 0 & 0 & (0) & 0 \\
\hline ALPHADET-PU & 0 & of & 0 & 0 & 0) & o & 0 & \begin{tabular}{l|l}
0 \\
\end{tabular} & 0. & 0 \\
\hline ALPHADET-U & 1277 & 0 & 5100 & 0 & 0 & 0 & 2169 & 3634 & 574 & 6377 \\
\hline ALPHA DET-Am & 0 & 0 & 0 & 0 & 0 & 0 & 0 & 0 & 0. & 0 \\
\hline ALPHA DET-Np & of & 0 & 0 & 0 & 0 & 0 & 0 & 0 & 0 & 0 \\
\hline ALPHA DET-Th & 0 & 0 & 0 & 0 & 0 & 0 & 0 & 0 & 의 & 0 \\
\hline ALPHA DET - U,Th,Pu & 0 & 0 & 0 & 0 & 0 & 0 & 0 & 0 & 0 & 0 \\
\hline ALPHAA DET-U235 & 0 & 0 & 2941 & 0 & 0 & 0 & 1471 & 735 & 735 & 2941 \\
\hline ALPHA DET-Pu,U,Am & 0 & 0 & 0 & o & 0 & 0 & 0 & 0 & 0 & 0 \\
\hline ALPHA DET-U,Np,Pu,Am & 0 & 0 & 0 & 0 & 0 & 0 & 0 & 0 & 0 & 0 \\
\hline ALPHA DET-Ra226 & 0 & 0 & 0 & 0 & 0 & 0 & 0 & 0 & - & 0 \\
\hline ALPHA DET-Ra228 & 0 & 0 & 0 & 0 & 0 & - & 0 & 0 & ㅇ․ & 0 \\
\hline ALPHA SPEC & 4289 & 0 & 596 & 0 & 0 & 0 & 3860 & 214 & 811 & 4885 \\
\hline GPC-BETA-Pb210 & of & 0 & 0 & 0 & 0 & 0 & 0 & 0 & 0 & 0 \\
\hline GPC-BETA-Sr90 & 0 & 0 & 0 & 0 & 0 & 의 & 0 & 0 & 0 & $\overline{0}$ \\
\hline GPC-BETA-Pm147 & 0 & 0 & 0 & 0 & 0 & 0 & 0 & 0 & 0 & 0 \\
\hline GPC-BETA-Se79 & 0 & 0 & 0 & 0 & 0 & 0 & 0 & 0 & 0 & 0 \\
\hline LSC-BETA-H3 & 0 & 0 & 0 & 0 & 0 & 요 & 0 & 0] & 0 & 0 \\
\hline LSC-BETA-Pn222 & 0 & 0 & 0 & 0 & 0 & D. & 0 & 0 & 0 & $\mathbf{0}$ \\
\hline LSC-BETA-1129 & of & 0 & $\overline{0}$ & 0 & 0 & 0] & o & 0 & 0 & 0 \\
\hline LSC-BETA-C14 & 0 & 0 & 0 & 0 & 0 & 0 & 요 & 0 & 의 & 0 \\
\hline LSC-BETA-TC99 & 0 & 0 & 0 & 0 & 0 & 0. & o & 0 & 0 & 0 \\
\hline LSC-BETA & 0. & 0 & 0 & 0 & 0 & 0 & 0 & 0 & 으 & 0 \\
\hline GAMMA SPEC & 1048 & 0 & 195 & 0 & 0 & 0 & 795 & 140 & 307 & 1243 \\
\hline OTHER-RAD & 0 & 0 & 0 & 0 & 0 & 0 & 0 & 0 & 0 & 0 \\
\hline TOTAL RAD & 8203 & O) & 154447 & (]) & 0 & 0. & 9408 & 150656 & 2586 & 162650 \\
\hline & & & & & & & & & & \\
\hline TCLP EXTRACTION & 698 & 0 & 0 & 0 & 0 & 0 & 35 & 174 & 489 & 698 \\
\hline OTHER-PREP & 485 & 0 & 0 & 0 & 0 & o. & 485 & 0 & 0 & 485 \\
\hline OTHER-EXPLO & 0 & 0 & 0 & 0 & 0 & 0 & 0 & 0 & 0 & 0 \\
\hline OTHER-BIO & 0 & 0 & 0 & 0 & 0 & 0 & 0 & 0 & 0 & 0 \\
\hline OTHER-PHYS & 990 & 0 & 5618 & $\overline{0}$ & 0 & 0 & 6608 & 0 & 0 & 6608 \\
\hline OTHER & 0 & 0 & 0 & 0 & 0 & 0 & 0 & 01 & 0 & 0 \\
\hline TOTAL OTHER & 2173 & $\overline{\mathbf{0}}$ & 5618 & 0 & 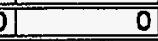 & 0 & 7128 & 174 & 489 & 7791 \\
\hline & & & & & & & & & & \\
\hline TOTAL SAMPLE ANALYSES & 32472 & 0 & 188101 & D) & $\overline{0}$ & 0 & 40930 & 170348 & 9296 & 220573 \\
\hline
\end{tabular}


EM-263 SURVEY OF DOE ANALYTICAL CAPACTY AVAILABLE TO EM

Table IVa: UC Sample Analyses by Rad Level and Matrix Type (xvi) Paducah

\begin{tabular}{|c|c|c|c|c|c|c|c|c|c|c|}
\hline $\begin{array}{l}\text { Rad-Matrix } \\
\text { Samp Anal }\end{array}$ & $\mathrm{RO}$ & RO/R1 & R1 & $\overline{\mathrm{F} 2}$ & R3 & $\overline{\mathrm{FA4}}$ & M1 & $\overline{\mathrm{M} 2}$ & M3 & Total \\
\hline VOA-GC & 0 & 0 & 0 & 0 & 0 & 0 & of $\rightarrow+2 \rightarrow$ & & o] & 0 \\
\hline VOA-GCMS & 0 & 4153 & of & 0 & 0 & 0 & 3945 & 208 & o. & $\frac{0}{4153}$ \\
\hline SEMI-VOA & 0 & 83 & 0 & 0 & 0 & 0 & 42 & 42 & 0 & 83 \\
\hline PEST & 0 & of & 01 & 01 & 0 & o & of & 0 & of & 0 \\
\hline HERB & 0 & 0 & 0 & 0 & 0 & 0 & 0 & 0 & 0 & 0 \\
\hline PCB & of & 10549 & of & of & 0) & 0 & 10232 & 316 & ot & 10549 \\
\hline PCB/PEST & of & 0 & 0 & 0 & 0 & a. & 0 & 0 & 0. & 0 \\
\hline OTHER-ORG & O) & 0) & of & 01 & 0 & 0 & 0 & 0 & of & 0] \\
\hline TOTAL ORGANICS & OI & 14784 & 01 & 01 & 0 & 0. & 14219 & 566 & $0]$ & 14784 \\
\hline ICP-AFSMETAIS & $0 !$ & 12089 & 01 & 01 & 0 & $\pi$ & 01 & 00 & OF & 10000 \\
\hline KCP-MSMETALS & o & 12003 & of & $\frac{4}{0}$ & $\frac{0}{0}$ & $\frac{0}{0}$ & 0 & 12005 & 욤 & 12083 \\
\hline GF-AAMETALS & ai & 10686 & 0 & 0 & $\frac{0}{0}$ & 에 & of & 0 & a. & 의 \\
\hline & & & & 0 & 0 & 의 & 6176 & 3432 & 1079 & 10686 \\
\hline & & 1903 & 01 & 0 & 0 & 의 & 1237 & 726 & 0] & 1963 \\
\hline CYANIDE & 0 & 17 & of & 0 & o & ㅇ․ & 14 & o & (3) & 17 \\
\hline ANIONS/CATIONS & 0 & 7253 & 0 & 0 & o & o) & 6907 & 266 & 80 & 7253 \\
\hline WET CHEM & 0 & 0 & \begin{tabular}{l|l}
0 & \\
\end{tabular} & 0 & 0 & 0 & 0 & 0 & 0 & 0 \\
\hline OTHER-INORG & of & 0 & 01 & 0 & O & 0 & O) & of & - & o. \\
\hline TOTAL INORGANICS & 01 & 32003 & 01 & OI & $0 !$ & o. & 143341 & 16508 & 1161 & 32003 \\
\hline & & & & & & & & 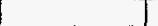 & + & \\
\hline GROSS ALPHAVBETA & 0 & 9147 & 0 & 0 & 0 & 0 & 9147 & 0 & of & 9147 \\
\hline ALPHA DET-Np237 & 0 & O) & 0 & 0 & 0 & D. & 0 & 0 & 요 & 요 \\
\hline ALPHADET-PU,Am & 0 & 0 & 0 & 0 & $\overline{0}$ & 0. & 0 & o & of & o \\
\hline ALPHADET-PU & 0 & 0 & of & 0 & 0 & 0 & 0. & 0 & of & 0 \\
\hline ALPHADET-U & 0 & 0 & 0 & O) & 0 & 요 & 0 & 0 & a & 0 \\
\hline ALPHADET-Am & 0 & 0 & 0 & 0 & 0 & 0 & 0 & 0 & (0) & 0 \\
\hline ALPHA DET-Np & 0 & 0 & 0] & 0 & 0 & 0 & 0 & 0 & 0. & 0 \\
\hline ALPHA DET-Th & 0 & o & of & 0 & 0 & o] & a & 0 & 0. & 0 \\
\hline ALPHADET-U,Th,PU & 0 & 0 & o) & 0 & 0 & - & 0 & 0 & a & (0) \\
\hline ALPHADET-U235 & D & 0 & 0 & 01 & 0 & o & 0 & 0 & 0 & 0. \\
\hline ALPHADET-Pu,U,Am & D. & 0 & 0 & 0 & 0 & 0 & 0 & 0 & 0 & D \\
\hline ALPHA DET-U,Np,Pu,Am & 0 & 0 & 0 & of & 0 & o. & 0 & 0 & 0 & 0 \\
\hline ALPHADET-Ra226 & 0 & 0 & o & 0 & 0 & 0 & 0 & 0 & 0 & D \\
\hline ALPHADET-Ra228 & $\overline{0}$ & o & of & 0 & 0 & 0 & 0 & of & 매 & D. \\
\hline ALPHA SPEC & 0 & 1339 & 0 & 0 & of & o & 1205 & 134 & 0. & 1339 \\
\hline GPC-BETA-Pb210 & 0 & 0 & 0 & 0 & 0 & 0 & 0 & 0 & of & 0 \\
\hline GPC-BETA-SROO & 0 & 0 & 0 & 0 & 0 & 0 & $\overline{0}$ & 0 & o. & 0 \\
\hline GPC-BETA-Pm147 & 0 & 0 & 0 & 0 & 0 & 0 & 0 & 0 & o & 0. \\
\hline GPC-BETA-Se79 & 0 & o! & 0 & 0 & 0 & o & 0 & 0 & o & o \\
\hline LSC-BETA-H3 & 0 & 0 & of & 0 & 0 & o. & 0 & of & o) & 의 \\
\hline LSC-BETA-Rn222 & 0 & 0 & 0 & 0 & 0 & o. & o & 0 & of & 0. \\
\hline LSC-BETA-1129 & 0 & 0 & 0 & D) & 0 & - & 0 & o) & (0) & 0 \\
\hline LSC-BETA-C14 & 0 & 0 & 0 & 0 & 0 & 요 & 0 & 0 & o. & 0 \\
\hline LSC-BETA-TC99 & 0 & of & 0 & 0 & 0 & 0 & 0 & 0 & 0. & 0 \\
\hline LSC-BETA & 0 & of & 0 & 0 & 0 & 0 & 0 & 0 & 0. & 0 \\
\hline GAMMA SPEC & 0 & 2025 & D) & 0 & 0 & 0 & 2025 & 0 & 0. & 2025 \\
\hline OTHER-RAD & 0 & 0 & 0 & 0 & 0 & 0 & 0 & 0 & 0 & 0 \\
\hline TOTAL RAD & $\overline{0}$ & 12511 & 01 & 01 & 01 & 의 & 12377 & 134 & 0 & 12511 \\
\hline & & & & & & & 1 & & & \\
\hline TCLP EXTRACTION & 0 & 673 & 0 & 0 & 0 & - & 673 & 0 & 0 & 673 \\
\hline OTHER-PREP & 0 & 0 & 0 & 0 & of & 0 & 0 & 0 & 0 & D. \\
\hline OTHER-EXPLO & $\overline{0}$ & o & 0) & 0 & 0 & 0 & 0 & of & 0 & 0 \\
\hline OTHER-BIO & $\overline{0}$ & o & 0 & 0 & of & o & 0 & 0 & 0 & 0 \\
\hline OTHER-PHYS & 0 & 0 & O) & 0 & O) & 0 & 0 & 0 & 0 & 0 \\
\hline OTHER & $\overline{0}$ & 0 & 0 & of & 01 & 0 & 0 & of & 0 & of \\
\hline TOTAL OTHER & 0 & 673 & $\overline{0}$ & 01 & 01 & 0 & 673 & 01 & 0 & 673 \\
\hline & & & & & 7 & 3 & I & & & \\
\hline TOTAL SAVPLF ANALYSES & $\overline{0}$ & 59971 & ] & 01 & 01 & ] & 41603 & 17207 & 1161 & 59971 \\
\hline
\end{tabular}


EM-263 SURVEY OF DOE ANALYTICAL CAPACITY AVAILABLE TO EM

Table IVa: UC Sample Analyses by Rad Level and Matrix Type (xvii) Portsmouth

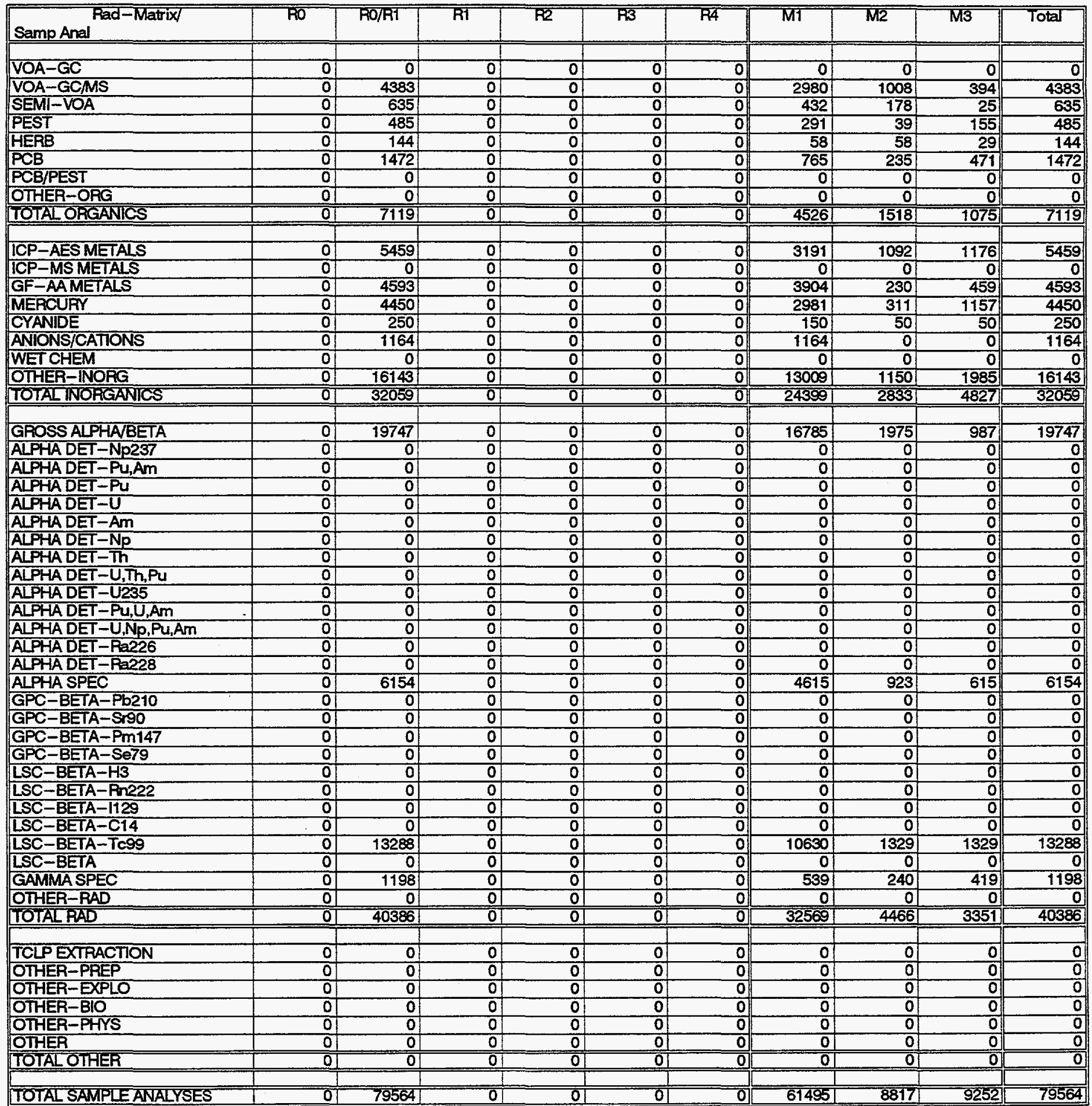


EM-263 SURVEY OF DOE ANALYTICAL CAPACITY AVAILABLE TO EM

Table IVa: UC Sample Analyses by Rad Level and Matrix Type (xviii) Hanford - WHC 222S

\begin{tabular}{|c|c|c|c|c|c|c|c|c|c|c|}
\hline $\begin{array}{l}\text { Rad-Matrix } \\
\text { Samp Anal }\end{array}$ & RO & Ro/R1 & $\overline{R 1}$ & $\mathrm{RE}$ & R3 & $\overline{R 4}$ & $\overline{M 1}$ & T12 & M3 & Total \\
\hline VOA-GC & 0 & 0 & 0 & 0 & 0 & (0) & 0 & 01 & 0 & 0 \\
\hline VOA-GCMS & 0 & 28 & 0 & 0 & 0 & o. & 28 & 0 & o. & 28 \\
\hline SEMI-VOA & 0 & 0 & 0 & 0 & 0 & 0) & 0 & 0 & o & 0 \\
\hline PEST & 0 & 0 & 0 & 0 & 0 & 0 & 0 & 0 & 0 & 0 \\
\hline HERB & 0 & 0 & 0 & 0 & 0 & ㅇ: & 0 & of & 0 & 0 \\
\hline PCB & 0 & 0 & 0 & 0 & 0 & 0 & 0 & 0 & 0 & 0 \\
\hline PCB/PEST & 0 & 0 & 0 & 0 & 0 & 0. & 0 & of & 0] & 0 \\
\hline OTHEA-ORG & 0 & 0 & 0 & 1380 & 0 & 0 & 910 & 0 & 470 & 1380 \\
\hline TOTALORGANICS & 0 & 28 & 0 & 1380 & 01 & (0] & 938 & 01 & 470 & 1408 \\
\hline & & & & & & ] & 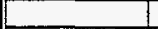 & 1 & & \\
\hline ICP-AES METALS & 0 & 0 & 0 & 751 & 0 & 0] & 413 & 15 & 323 & 751 \\
\hline ICP-MSMETALS & 0 & 0 & 0 & 0 & 0 & 0 & 0 & 0 & 0 & 0 \\
\hline GF-AAMETALS & 0 & 256 & 0 & 977 & 0 & 0] & 192 & 64 & 977 & 1234 \\
\hline MERCURY & 0 & 0 & 0 & 514 & 0 & 0 & 51 & 26 & 437 & 514 \\
\hline CYANIDE & 0 & 0 & 0 & 378 & 0 & o] & 152 & of & 226 & 378 \\
\hline ANIONS/CATIONS & 0 & 313 & 0 & 3505 & 0 & 0 & 1822 & 17 & 1979 & 3819 \\
\hline WET CHEM & 0 & 0 & 0 & 223 & 0 & 0] & 60 & 2 & 161 & 223 \\
\hline OTHER-INORG & 0 & 2887 & D & 1129 & 0 & ㅁ․ & 2543 & 197 & 1276 & 4016 \\
\hline TOTAL INORGANICS & 0 & 3457 & 0 & 7478 & 01 & 0 & 5234 & 321 & 5380 & 10935 \\
\hline GROSS ALPHAVBETA & 0 & 7686 & 0 & 0 & 0 & 0 & 5056 & 80 & 2550 & 7686 \\
\hline ALPHA DET-Np237 & 0 & 325 & 0 & 0 & 0 & 0 & 120 & 0 & 205 & 325 \\
\hline ALPHA DET-Pu,Am & 0 & 3357 & 0 & 0 & 0 & 0. & 1981 & 637 & 739 & 3357 \\
\hline ALPHADET-Pu & 0 & 0 & o & 0 & 0 & 0 & 0 & 0 & 0 & 0 \\
\hline ALPHADET $-U$ & 0 & 0 & 0 & 0 & 0 & D. & 0 & of & 0 & $\overline{0}$ \\
\hline ALPHADET-Am & 0 & o. & 0 & 0 & 0 & 0 & 0 & 0 & 0 & 0 \\
\hline ALPHADET-NP & 0 & 0 & 0 & 0 & 0 & 요 & 0 & 0 & 0 & 0 \\
\hline ALPHA DET-Th & 0 & 0 & 0 & 0 & 0 & 의 & 0 & 0 & 0 & 0 \\
\hline ALPHADET - U,Th,Pu & 0 & 0 & 0 & of & 0 & 이 & 0 & of & 0 & 0 \\
\hline ALPHADET-UZ35 & 0 & 0 & 0 & 0 & 0 & 0 & 0 & 0 & 0 & 0 \\
\hline ALPHADET - Pu,U,Am & 0 & 0 & 0 & 0 & 0 & 0 & 0 & o| & 0 & 0 \\
\hline ALPHA DET-U,Np,Pu,Am & 0 & 0 & 0 & 0. & 0 & Dof & 0 & 0 & 0 & 0 \\
\hline ALPHADET-Ra226 & 0 & 0 & 0 & 0 & 0 & D. & 0 & 0 & 0. & 0 \\
\hline ALPHADET-Ra228 & 0 & 0 & 0 & 0 & O & 요 & 0 & 0 & 0 & 0 \\
\hline ALPHA SPEC & $\overline{0}$ & 0 & 0 & 0 & 0 & - & 0 & 0 & 0 & 0 \\
\hline GPC-BETA-Pb210 & 0 & 0 & 0 & 아 & o & [0] & 0 & 0 & 0 & $\overline{0}$ \\
\hline GPC-BETA-Sr90 & 0 & 1875 & 0 & 0 & 0 & 요 & 1238 & 189 & 449 & 1875 \\
\hline GPC-BETA-Pm147 & 0 & 30 & 0 & 0 & o. & 의 & 30 & o & 0 & 30 \\
\hline GPC-BETA-Se79 & 0 & 38 & 0 & 0 & 0 & 0 & 38 & 0 & 0 & 38 \\
\hline LSC-BETA-H3 & 0 & 1035 & 0 & 0 & o. & 요 & 1035 & 0 & 0 & 1035 \\
\hline LSC-BETA-Rn222 & 0 & 0 & 0 & 0 & 0 & 0) & 0 & of & 0 & 0 \\
\hline LSC-BETA-1129 & 0 & 246 & 0 & 0 & 0 & 마 & 246 & o & 0 & 246 \\
\hline LSC-BETA-C14 & 0 & 543 & 0 & 0 & 0 & 0] & 274 & a) & 270 & 543 \\
\hline LSC-BETA-TC99 & 0 & 970 & 0 & 0 & 0 & 이 & 679 & 0 & 292 & 970 \\
\hline LSC-BETA & 0 & 0 & 0 & 0 & 0 & 0 & 0 & 0 & 0 & 0 \\
\hline GAMMA SPEC & 0 & 3359 & 0 & 0 & 0 & o & 1479 & 752 & 1128 & 3359 \\
\hline OTHER-RAD & 0 & 0 & 0 & 0 & 0 & - & 0 & 0 & 0 & 0 \\
\hline TOTAL RAD & 0 & 19466 & 0 & $0]$ & 0 & 0 & 12176 & 1658 & 5632 & 19466 \\
\hline & & & & & & & & & & \\
\hline TCLP EXTRACTION & 0 & 0 & 0 & 0 & 의 & 의 & 0 & 이 & 0 & 0 \\
\hline OTHER-PREP & 0 & 12 & 0 & 1591 & of & 32 & 220 & 181 & 1234 & 1636 \\
\hline OTHER-EXPLO & 0 & 0 & $\overline{0}$ & 0 & of & - & 0) & 0 & 0 & 0 \\
\hline OTHER-BIO & 0 & 0 & 0 & 0 & 0) & 0 & 0 & 아 & 0 & 0 \\
\hline OTHER-PHYS & 0 & 0 & 0 & 1568 & 0 & 0 & 595 & 142 & 831 & 1568 \\
\hline OTHER & 0 & 0 & 0 & 0 & of & a & 0 & 0 & 0 & 0 \\
\hline TOTAL OTHER & $\overline{0}$ & 12 & $\overline{0}$ & 3159 & 0 & 32 & 815 & 323 & 2065 & 3203 \\
\hline & & & & & & & & & & \\
\hline TOTAL SAMPLE ANALYSES & 0 & 22962 & 0 & 12017 & 01 & 32 & 19163 & 2302 & 13547 & 35012 \\
\hline
\end{tabular}


EM-263 SURVEY OF DOE ANALYTICAL CAPACITY AVAILABLE TO EM

Table IVa: UC Sample Analyses by Rad Level and Matrix Type (xix) Hanford - PNL 325

\begin{tabular}{|c|c|c|c|c|c|c|c|c|c|c|}
\hline $\begin{array}{l}\text { Rad-Matrix } \\
\text { Samp Anal }\end{array}$ & RiO & RO/R1 & R1 & $\overline{R 2}$ & $\overline{\text { R33 }}$ & $\overline{\mathrm{RA}}$ & $\overline{\mathrm{M1}}$ & $\overline{142}$ & $\overline{193}$ & Total \\
\hline & & & & & & & & & & \\
\hline VOA-GC & 0 & 0 & 0 & 0 & 0 & o & of & 0 & o & 0 \\
\hline VOA-GCMS & 0 & 0 & 0 & 136 & 0 & - & 36 & 17 & 83 & 136 \\
\hline SEMI-VOA & 0 & 0 & 0 & 93 & 0 & 0 & 10 & 11 & 72 & 93 \\
\hline PEST & 0 & 0 & 0 & 31 & of & 0 & 23 & 0 & 8 & 31 \\
\hline HERB & 0 & 0 & 0 & 0 & 0 & 0 & 0 & 0 & of & 0 \\
\hline$\overline{P C B}$ & 0 & 0 & 0 & 0 & 0 & 0 & 0 & 0 & 0 & 0 \\
\hline PCB/PEST & 0 & 0 & 0 & 0 & 0 & 0 & 0 & o & 0 & $\mathbf{0}$ \\
\hline OTHER-ORG & 0 & 0 & 0 & 0 & 0 & 0 & 0 & 0 & 0 & 0 \\
\hline TOTAL ORGANICS & 0 & 0 & 0 & 259 & 0 & $\overline{0}$ & 68 & 28 & 163 & 259 \\
\hline & & & & & & & & & & \\
\hline ICP-AES METALS & 0 & 917 & 0 & 206 & 0 & 0 & 94 & 860 & 168 & 1122 \\
\hline ICP-MSMETALS & 0 & 1210 & 0 & 93 & 0 & 0 & 292 & 700 & 311 & 1303 \\
\hline GF-AAMETALS & 0 & 188 & 0 & 60 & 0 & 0 & 0 & 179 & 69 & 248 \\
\hline MERCURY & 0 & 52 & 0 & 0 & 0 & 0 & \begin{tabular}{|c|c|}
13 \\
\end{tabular} & 25 & 14 & 52 \\
\hline CYANIDE & o & 25 & 0 & 0 & 0 & 0 & 0 & 11 & 14 & 25 \\
\hline ANIONS/CATIONS & 0 & 245 & 0 & 0 & 0 & 0 & 29 & 85 & 131 & 245 \\
\hline WET CHEM & 0 & 0 & 0 & 0 & 0 & 0 & 0 & 0 & 0 & 0 \\
\hline OTHER-INORG & 0 & 0 & 0 & 0 & of & 0 & 0 & 0 & o. & 0 \\
\hline TOTAL INORGANICS & वा & 2637 & $\overline{0}$ & 359 & 0 & 0 & 428 & 1860 & 707 & 2995 \\
\hline & & & & & & & & & & \\
\hline GROSS ALPHAVBETA & of & 2729 & 0 & 0 & 0 & 0 & 1401 & 1194 & 134 & 2729 \\
\hline ALPHA DET-Np237 & 0 & 0 & 0 & 0 & 0 & 0 & 0 & 0 & o. & 0 \\
\hline ALPHA DET-PU,Am & 0 & 0 & $\overline{0}$ & 0 & 0 & $\overline{0}$ & 0 & 0 & o. & 0 \\
\hline ALPHA DET-PU & 0) & 159 & $\overline{0}$ & 0 & 0 & 0 & 73 & 72 & 14 & 159 \\
\hline ALPHA DET-U & 0 & 0 & 0 & 0 & 0 & 0 & 0 & 0 & oा & $\overline{0}$ \\
\hline ALPHA DET-Am & 0 & 0 & 0 & 0 & of & 0 & 0 & 0 & of & 0 \\
\hline ALPHA DET-Np & 0 & 0 & 0 & 0 & 0 & 0 & 0 & 0 & o & 0 \\
\hline ALPHA DET-Th & 0 & 0 & 0 & 0 & 0 & (0) & 0 & 0 & ot & 0 \\
\hline ALPHA DET-U,Th,Pu & 0 & 0 & 0 & 0 & of & 0 & 0 & 0 & o. & 0 \\
\hline ALPHA DET-U235 & 0 & 0 & 0 & of & 0 & 0 & 0 & 0 & o & $\overline{0}$ \\
\hline ALPHA DET-Pu,U,Am & 0 & 0 & $\overline{0}$ & 0 & of & 0 & 0 & 0 & o. & $\overline{0}$ \\
\hline ALPHA DET-U,Np,Pu,Am & 0 & 0 & $\overline{0}$ & 0 & 0 & 0 & 0 & 0 & o. & 0 \\
\hline ALPHA DET-R 226 & 0 & of & $\overline{0}$ & 0 & 01 & 0 & 0 & 0 & 0. & 0 \\
\hline ALPHA DET-Ra228 & 0 & 0 & 0 & 0 & 0 & 0 & 0 & of & 0 & $\overline{0}$ \\
\hline ALPHA SPEC & $\overline{0}$ & 0 & 0 & 0 & 0 & 0 & 0 & o) & 0 & 0 \\
\hline GPC-BETA-Pb210 & 0 & 0 & $\overline{0}$ & 0 & 0 & 0 & 0 & 0 & o. & 0 \\
\hline GPC-BETA-Sr90 & 0 & 327 & 0 & 0 & 0 & 0 & 164 & 144 & 20 & 327 \\
\hline GPC-BETA-Pm147 & 0 & 0 & 0 & 0 & 0 & 0 & 0 & 0 & 0 & 0 \\
\hline GPC-BETA-Se79 & 0 & 0 & 0 & 0 & 0 & 0 & 0 & 0 & 0 & 0 \\
\hline LSC-BETA-H3 & 0 & 0 & 0 & 0 & 0 & 0 & 0 & 0 & 0 & $\overline{0}$ \\
\hline LSC-BETA-Rn222 & 0 & 0 & 0 & 0 & 0 & 0 & 0 & 0 & 0 & $\overline{0}$ \\
\hline LSC-BETA- 1129 & 0 & 0 & 0 & 0 & 0 & 0 & 0 & 0 & 0 & $\overline{0}$ \\
\hline LSC-BETA-C14 & 0 & 0 & 0 & 0 & 0 & 0 & 0 & 0 & 0 & $\overline{0}$ \\
\hline LSC-BETA-TC99 & 0 & 0 & 0 & 0 & 0 & 0. & 0 & 0 & 0 & $\overline{0}$ \\
\hline LSC-BETA & 0 & 0 & 0 & 0 & 0 & 0 & 0 & 0 & 0 & 0 \\
\hline GAMMA SPEC & 0 & 926 & 0 & 0 & 0 & 0 & 463 & 417 & 46 & 926 \\
\hline OTHER-RAD & 0 & 0 & 0 & 0 & 0 & 0 & 0 & 0 & 0 & 0 \\
\hline TOTALRAD & 0 & 4 & $\overline{0}$ & 01 & 0 & 0 & 2100 & 1826 & 214 & $\overline{4140}$ \\
\hline & & & & & & & & & & \\
\hline TCLP EXTRACTION & 0 & 0 & $\overline{0}$ & 0 & 0 & 6 & 0 & 0 & 6 & 6 \\
\hline OTHER-PREP & 0 & 0 & 0 & 0 & 0) & 112 & 0 & 0 & 112 & 112 \\
\hline OTHEA-EXPLO & 0 & 0 & 0 & 0) & 0 & 0 & of & of & 0 & 0 \\
\hline OTHER-BIO & 0 & 0 & $\overline{0}$ & 0 & 0 & 0 & o & 0 & o. & $\overline{0}$ \\
\hline OTHER-PHYS & 0 & 0 & 0 & 0 & 0 & 0 & 0 & 0 & 0 & $\overline{0}$ \\
\hline OTHER & 0 & 0 & 0 & 0 & 0 & 0 & 0 & 0 & 0 & 0 \\
\hline TOTAL OTHER & 0 & 0 & 0 & 0 & 0 & 117 & 01 & 0 & 117 & 1117 \\
\hline & & & & & & & & & & \\
\hline TOTAL SAMPLE ANAIYSES & 0 & 6777 & $\overline{\overline{0}}$ & 618 & 0 & 117 & 2596 & 3715 & 1201 & 7512 \\
\hline
\end{tabular}


EM-263 SUAVEY OF DOE ANALYTICAL CAPACITY AVAILABLE TO EM

Table IVa: UC Sample Analyses by Rad Level and Matrix Type (xx) Rocky Plats - 123 Lab

\begin{tabular}{|c|c|c|c|c|c|c|c|c|c|c|}
\hline $\begin{array}{l}\text { Rad-Matix } \\
\text { Samp Anal }\end{array}$ & Rio & RO/RI & R1 & R2 & $\mathrm{R3}$ & $\overline{\mathbf{P A}}$ & $\overline{\mathrm{Mi}}$ & $7 \sqrt{2}$ & $\overline{\mathrm{M3}}$ & Total \\
\hline VOA-GC & 01 & & & 0 & 01 & & & & & 0 \\
\hline VOA-GCMS & 0 & 0 & 0 & $\frac{0}{0}$ & o & $\frac{1}{0}$ & $\frac{0}{0}$ & \begin{tabular}{l|l}
0 \\
0
\end{tabular} & $\frac{0}{0}$ & $\frac{0}{0}$ \\
\hline SEMI-VOA & 0 & 0 & 0 & 0 & 0 & o & 0 & 0 & ö & 0 \\
\hline PEST & 0 & 0 & 0 & 0 & 0 & 0 & 0 & o & 아 & 0 \\
\hline HERB & 0 & 0 & 0 & 0 & 0 & 0 & 0 & 0 & 0 . & 0 \\
\hline PCB & 0 & 0 & 0 & 0 & 0 & (0) & 0 & 0 & 0 & 0 \\
\hline PCB/PEST & 0 & 0 & 0 & 0 & 0 & 0 & 0 & 0 & o & 0 \\
\hline OTHER-ORG & 0 & $\begin{array}{ll}0 \\
\end{array}$ & 0 & 0 & 0 & 0 & 0 & 0 & 0 & 0 \\
\hline TOTAL ORGANICS & 이 & $0 \mid$ & $\overline{0}$ & 0 & O| & [0] & $\overline{01}$ & 0 & $0]$ & 0 \\
\hline & & & & & & & & & & \\
\hline ICP-AES METALS & 0 & 0 & o & 0 & 0 & 0 & 0 & 0 & 0] & 0 \\
\hline ICP-MS METALS & 0 & 0 & 0 & 0 & 0 & 0 & 0 & 0 & 0 & 0 \\
\hline GF-AAMETALS & 0 & O & 0 & 0 & 0 & 0 & 0 & 0. & 0. & 0 \\
\hline MERCURY & 0 & D & 0 & 0 & 0 & - & 0 & 0 & o & 0 \\
\hline CYANDE & D. & 0 & 0 & O & 0 & 0. & 0 & 0 & 0 . & 0 \\
\hline ANIONS/CATIONS & 0 & o & 0 & 0 & 0 & D. & 0 & 0 & 0. & 0 \\
\hline WET CHEM & 0 & 0 & 0 & 0 & 0 & 요 & 0 & 0 & 0. & 0 \\
\hline OTHER-INOAG & 10914 & \begin{tabular}{l|l}
0 \\
\end{tabular} & 0 & 0 & 0 & 0. & 10914 & 0 & 01 & 10914 \\
\hline TOTALINORGANICS & 10914 & 0 & 01 & O] & 01 & D] & 10914 & 0 & 0] [ & 10914 \\
\hline & & & & & 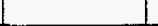 & 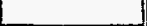 & & 1 & th & \\
\hline GROSS ALPHAVBTA & 22536 & 0 & 0 & 0 & 0 & D & 22536 & 0 & - & 22536 \\
\hline ALPHA DET-Np237 & 0 & 0 & 0 & 0 & 0 & 의 & 0 & 요 & 0. & 0 \\
\hline ALPHA DET-Pu,Am & 0 & 0) & a. & 0 & 0 & 요 & 0 & 0 & 0. & 0 \\
\hline ALPHA DET-Pu & 0 & 01 & 0 & 0 & 0 & 0 & 0 & 0 & D. & 0 \\
\hline ALPHA DET -U & 0 & 0 & 0 & 0 & 0 & 0 & 0 & o & 0 & 0 \\
\hline ALPHA DET-Am & 0. & 0 & 0 & 0 & 0 & 0 & 0 & 0 & of & 0 \\
\hline ALPHA DET-Np & 0 & 0 & 0 & 0 & 0 & 0 & 0 & D & 0 & 0 \\
\hline ALPHA DET-Th & 0 & 0 & 0 & 0 & 0 & 0 & 0 & D & 0 & 0 \\
\hline ALPHA DET - U,Th,Pu & 0 & 0 & 0 & 0 & 0 & 요 & 0 & 0 & 0 & 0 \\
\hline ALPHADET-U235 & 0 & 0 & of & 0 & 0 & 묘 & 0 & D. & a & 0 \\
\hline ALPHADET-PU,U,Am & 3790 & 0 & 0 & O & 0 & 으. & 3790 & 0 & 으. & 3790 \\
\hline ALPHA DET - U,Np,Pu,Am & 0 & D & 0 & 0 & 0 & 0 & 0 & o & 0. & 0 \\
\hline ALPHA DET-Ra226 & 0 & 01 & 0 & 0 & 0 & 요 & 0 & 0 & 의 & 0 \\
\hline ALPHA DET-Ra228 & 0 & of & 0 & 0 & 0 & 0 & 0 & 0 & 0. & 0 \\
\hline ALPHA SPEC & 0 & 0 & 0 & 0 & 0 & 0 & 0 & 0 & 0. & 0 \\
\hline GPC-BETA-Pb210 & 0 & O) & 0 & 0 & 0 & 이 & D. & 0 & D. & 0 \\
\hline GPC-BETA-Sr90 & 0 & (0) & 0 & 0 & 0 & 0 & 0 & o & ㅁ․ & 0 \\
\hline GPC-BETA-Pm147 & 0 & ot & 0 & 0 & 0 & 0 & 0 & $\overline{0}$ & 0 & 0 \\
\hline GPC-BETA-Se79 & 0 & 0 & of & 0 & 0 & 0 & 0 & D & 0. & 0 \\
\hline LSC-BETA-H3 & 1928 & 0 & 0 & 0 & 0 & 0 & 1870 & 58 & 0] & 1928 \\
\hline LSC-BETA-Rn222 & 0 & 0 & 0 & 0 & 0 & 0 & 0 & 0 & 0 & 0 \\
\hline LSC-BETA-1129 & 0 & 0 & 0 & 0 & $0 \mid$ & 0 & 0 & 0 & -0 & 0 \\
\hline LSC-BETA-C14 & 0 & O & 0 & 0 & 0 & 0 & 0 & 0 & 0 & $\overline{0}$ \\
\hline LSC-BETA-TC99 & 0 & 0 & 0 & 0 & 0 & 0 & 0 & 0 & 요 & 0 \\
\hline LSC-BETA & 0 & 0 & 0 & 0 & 0 & 0. & 0 & 0 & (0) & 0 \\
\hline GAMMA SPEC & 0 & 0 & 0 & 0 & 0 & 0 & 0 & 0 & 묘 & 0 \\
\hline OTHER-RAD & 0 & o & of & 0 & of & 0 & 01 & 0 & - & 0 \\
\hline TOTAL RAD & 28254 & 0 & 01 & 01 & 이 & 0 & 28196 & 58 & 0 & 28254 \\
\hline & & & & & & & & & & \\
\hline TCLP EXTRACTION & 0 & 0 & 0 & 0 & 0 & 0 & 0 & 0 & 0] & 0 \\
\hline OTHER-PAEP & O & 0 & 0 & 0 & of & 0 & o & 으 & 0 & 0 \\
\hline OTHER-EXPLO & 0 & D. & 요 & 0 & 이 & $\overline{0}$ & 0 & 0 & 0 & 0 \\
\hline OTHER-BIO & 0 & 0 & 0 & 0 & of & 0 & 0 & 0 & a & 0 \\
\hline OTHER-PHYS & 0 & 0 & 0 & 0 & 0 & 0 & 0 & D & 0 & 0 \\
\hline OTHER & 0 & 0 & 0 & 0 & 0 & 0 & 0 & 0 & 0 & 0 \\
\hline TOTALOTHEA & $\overline{0}$ & $\overline{01}$ & 0 & 0 & 01 & 0 & 0 & 0 & 0 & 0 \\
\hline & & & & & & & & & & \\
\hline TOTAL SAMPLE ANALYSES & 39168 & $\overline{01}$ & 0] & 0 & 이 & 0 & 39110 & 58 & 0 & 39168 \\
\hline
\end{tabular}


EM-263 SURVEY OF DOE ANALYTICAL CAPACTY AVAILABLE TO EM

Table IVa: UC Sample Analyses by Rad Level and Matrix Type (xxi) Rocky Fats - GL881

\begin{tabular}{|c|c|c|c|c|c|c|c|c|c|c|}
\hline $\begin{array}{l}\text { Rad-Matrix } \\
\text { Samp Anal }\end{array}$ & $\overline{R O}$ & RO/R1 & $\overline{\mathrm{R1}}$ & 182 & $\overline{R 3}$ & $\overline{R 4}$ & $\overline{M 1}$ & $\overline{\mathrm{M}} 2$ & $\overline{\mathrm{M} 3}$ & Total \\
\hline & & & & & & & & & & \\
\hline VOA-GC & 0] & 0 & of & 0 & 0 & 0 & 0 & 0 & 0] & 0 \\
\hline VOA-GCMS & 2260 & 0 & 0 & 0 & 0 & 0 & 1695 & 475 & 90 & 2260 \\
\hline SEMI-VOA & 290 & 0 & 0 & 0 & 0 & 0 & 77 & 73 & 140 & 290 \\
\hline PEST & 0 & 0 & 0 & 0 & \begin{tabular}{l|l}
0 \\
\end{tabular} & o & 0 & 0 & 0 & 0 \\
\hline HERB & 0 & 0 & 0 & 0 & 0 & 0 & 0 & 0 & -0 & 0 \\
\hline PCB & 0 & 0 & 0 & 0 & 0 & 0 & 0 & 0 & of & 0 \\
\hline PCB/PEST & 0 & 0 & 0 & 0 & 0 & o & 0 & 0 & 예 & 0 \\
\hline OTHER-ORG & 01 & 可 & 0 & 0 & 0 & 0 & 0 & 0 & 0 & 0 \\
\hline TOTAL ORGANICS & 2550 & 0 & 0 & DI & 01 & 0 & 1772 & 548 & 230 & 2550 \\
\hline & & & & & & & & & & \\
\hline ICP-AES METALS & 963 & 0 & 0 & 0 & 0 & 0 & 716 & 58 & 190 & 963 \\
\hline ICP-MSMETALS & 0 & 0 & of & 0 & o & 0 & 0 & 0 & 0 & 0 \\
\hline GF-AAMETALS & 1775 & 0 & 0 & 0 & 0 & 0. & 1597 & 124 & 53 & 1775 \\
\hline MERCURY & 398 & 0 & 0 & 0 & 0 & o. & 239 & 119 & 40 & 398 \\
\hline CYANIDE & 198 & 0 & 0 & 0 & 0 & 0 & 91 & 99 & 8 & 198 \\
\hline ANIONS/CATIONS & o & 0 & 0 & 0 & 0 & 0 & 0 & 0 & 0 & 0 \\
\hline WET CHEM & 0 & 0 & of & 0 & o & 0 & 0 & 0 & of & 0 \\
\hline OTHER-INORG & 0 & 0 & 0 & 0 & 0 & 0 & 0 & 0 & 0 & 0 \\
\hline TOTAL INORGANICS & 3334 & 0 & वा & 0 & 01 & (0) & 2643 & 4011 & $290]$ & $\overline{3334}$ \\
\hline & & & & & & & & & & \\
\hline GROSS ALPHAVBETA & 4972 & 0 & 0 & $0 \mid$ & 0 & 0 & 2680 & 1880 & 412. & 4972 \\
\hline ALPHA DET-Np237 & o) & (0) & 0 & 0 & 0 & (0) & 0 & 0 & o. & 0 \\
\hline ALPHA DET-PU,Am & 0 & 0 & 0 & 0 & 0 & 0 & 0 & 0 & 0 & $\overline{0}$ \\
\hline ALPHA DET-Pu & 0 & o: & of & 0 & of & 0 & 0 & 0 & o. & 0 \\
\hline ALPHA DET-U & 0 & o) & 0 & 0 & o & 0. & 0 & 0 & 0. & $\overline{0}$ \\
\hline ALPHADET-Am & 0 & 0 & 0 & 0 & of & 0 & 0 & 0 & 0. & 0 \\
\hline ALPHA DET-Np & 0 & 0 & 0 & 0 & 0 & 의 & 0 & 0 & 0. & 0 \\
\hline ALPHADET-Th & of & 0 & o) & 0 & of & 0. & 0 & of & o. & 0 \\
\hline ALPHADET-U,Th,PU & 0 & 0 & 0 & 0 & 0 & o. & o & 0 & o. & 0 \\
\hline ALPHA DET-U235 & 0 & of & 0 & 0 & 0 & 0 & o & 0 & o. & 0 \\
\hline ALPHA DET-PU,U,Am & 1143 & (0) & 0 & 0 & 0 & - & 857 & 286 & 0 & 1143 \\
\hline ALPHA DET-U,Np,Pu,Am & 0 & 0 & 0 & 0 & 0 & 0 & 0 & 0 & 0 & 0 \\
\hline ALPHA DET-Ra226 & 0 & 0 & 0 & 0 & 0 & 0 & o & of & 0 & $\overline{0}$ \\
\hline ALPHA DET-Ra228 & of & o & 0 & 0 & 0 & 0 & of & 0 & 0 & 0 \\
\hline ALPHA SPEC & 0 & of & 0 & \begin{tabular}{l|l}
0 & \\
\end{tabular} & 0 & 0 & 0 & 0 & 0 & 0 \\
\hline GPC-BETA-Pb210 & 0 & $\overline{0}$ & o. & 0 & o & 0 & 0 & 0 & 0 & 0 \\
\hline GPC-BETA-Sr90 & 0 & D्ञ & 0 & of & D & 0 & 0 & 0 & 0] & 0 \\
\hline GPC-BETA-Pm147 & o & $\overline{0}$ & 0 & 0 & 0 & 0. & 0 & 0 & 0 & 0 \\
\hline GPC-BETA-Se79 & 0 & D. & 0) & 0 & 0 & - & 0 & o) & o] & 0 \\
\hline LSC-BETA-H3 & 0 & of & 0 & 0 & 0 & O) & 0 & 0 & 0 & 0 \\
\hline LSC-BETA-Pn222 & 0) & 0 & 0 & 0 & 0 & 0 & 0 & 0 & 0. & $\overline{0}$ \\
\hline LSC-BETA- 1129 & of & 0 & 0 & 0 & o & 0 & 0 & 0 & 0 & 0 \\
\hline LSC-BETA-C14 & 0 & 0 & 0 & 0 & o & 0 & 0 & 0 & 0] & $\overline{0}$ \\
\hline LSC-BETA-TC99 & 0 & 0 & 0 & 0 & 0 & 0 & 0 & 0 & 0] & 0 \\
\hline LSC-BETA & 0 & ot & 0 & of & 0 & 0 & 0 & 0 & of & 0 \\
\hline GAMMA SPEC & 0 & D. & 0) & of & 0 & 0 & 0 & 0 & 0 ] & 0 \\
\hline OTHER-RAD & 0 & (0) & of & 0 & 0 & 0 & 0 & 0 & o] & 0 \\
\hline TOTAL AAD & 6115 & (0) & 01 & OI & 01 & of & 3537 & 2166 & $412]$ & 6115 \\
\hline & & & & & & & & & & \\
\hline TCLP EXTRACTION & 612 & 0 & 0 & 0 & o & 0 & of & 114 & 498 & 612 \\
\hline OTHER-PREP & 0 & o & 0 & 0 & 0 & 0 & 0 & 0 & of & $\overline{0}$ \\
\hline OTHER-EXPLO & 0 & ठ० & 0 & 0 & 0 & $\overline{0}$ & 0 & of & वी & 0 \\
\hline OTHEA-BIO & of & o| & 0 & 0 & 0 & 0 & 0 & 0 & of & 0 \\
\hline OTHER-PHYS & 0 & o & 0 & 0 & 0 & 0 & 0 & 0 & of & 0 \\
\hline OTHER & 0 & 0 & of & 0 & 0 & 0 & 0 & 0 & ㅁ] & 0 \\
\hline TOTAL OTHER & 612 & 0 & 01 & 01 & 0 & 0 & 0 & 114 & 498 & 612 \\
\hline & & & & 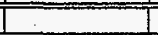 & 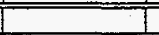 & 7 & & & & \\
\hline TOTAL SAMPLFANALYSES & 126111 & 01 & 01 & 01 & 0 & 0 & 7952 & 3228 & 1431] & 12611 \\
\hline
\end{tabular}


EM-263 SURVEY OF DOE ANALYTICAL CAPACITY AVAILABLE TO EM

Table IVa: UC Sample Analyses by Rad Level and Matrix Type (xoii) Rocky Flats - RF PA Lab

\begin{tabular}{|c|c|c|c|c|c|c|c|c|c|c|}
\hline $\begin{array}{l}\text { Rad-Matrix } \\
\text { Samp Anal }\end{array}$ & RO & ROO/R1 & R1 & $\overline{\mathrm{R} 2}$ & $\overline{R 3}$ & $\overline{R 4}$ & M1 & $\overline{\mathrm{M} 2}$ & $7 \overline{3}$ & Total \\
\hline VOA-GC & 0 & 0 & 0 & 0 & 0 & 0) & 0 & 0 & 0 & 0 \\
\hline VOA-GCMS & 0 & 0 & 0 & 0 & 750 & o & 750 & 0 & 0 & 750 \\
\hline SEMI-VOA & 0 & 0 & 0 & 0 & 0 & 0 & 0 & $0 \mid$ & 0 & 0 \\
\hline PEST & 0 & 01 & 요 & 0 & 0 & (0) & 0 & 0 & 0 & 0 \\
\hline HERB & 0 & 0 & 0 & 0 & 0 & o) & 0 & 0 & 0 & 0 \\
\hline PCB & 0 & 0 & O) & 01 & 0 & 0 & of & 0 & 0 & 0 \\
\hline PCB/PEST & 0 & 0 & 0 & 01 & 0 & o. & 0 & 0 & 0 & 0 \\
\hline OTHER-ORG & 0 & 0 & 0 & $0 \mid$ & 0 & o. & of & 0 & 0 & 0 \\
\hline TOTAL ORGANICS & 0 & 0 & 0) & 01 & 7501 & 이 & 750 & 01 & 0 & 750 \\
\hline & & & & & & & & & & \\
\hline ICP-AESMETALS & 0 & 0 & 0 & 0 & 0 & o. & 0 & 0 & 0 & 0 \\
\hline ICP-MS METALS & 0 & 0 & 0) & 0 & 0 & 0. & 0 & 0 & 0. & 0 \\
\hline GF-AAMETALS & 0 & 0 & 0 & 0 & 0 & 0 & 0 & 0 & of & 0 \\
\hline MERCURY & 0 & 0 & 0 & 0 & 0 & 0 & 0 & 0 & 0 & 0 \\
\hline CYANIDE & 0 & o & 0 & 0 & 0 & 0 & 0 & 0 & 0. & 0 \\
\hline ANIONS/CATIONS & 0 & 0 & 0 & 0 & 0 & 0 & 0 & 0 & 0 & 0 \\
\hline WET CHEM & 0 & 0 & 0 & 0 & 0 & (0) & 0 & 0 & o. & 0 \\
\hline OTHER-INORG & 0 & 0 & of & 0 & 2571 & 0. & 1109 & 784 & 678 & 2571 \\
\hline TOTAL INOAGANICS & 0 & 0 & 0 & 0 & 2571 & (0) & 1109 & 784 & 678 & 2571 \\
\hline & & & & & & & & & & \\
\hline GROSS ALPHAVBETA & 0 & 0 & 0 & 0 & 2339 & 0 & 1070 & 692 & 577 & 2339 \\
\hline ALPHA DET-Np237 & D & 0 & D: & 0 & 0 & 0 & 0 & 0 & 0 & 0 \\
\hline ALPHA DET-Pu,Am & 0 & 0 & 이 & 0 & of & 0 & 0 & 0 & 0. & 0 \\
\hline ALPHADET-Pu & 0 & 0 & 0 & 0 & 0 & of & 0 & 0 & of & 0 \\
\hline ALPHA DET $-U$ & 0 & of & 0 & 0 & 0 & 0 & 0 & 0 & o. & 0 \\
\hline ALPHA DET-Am & 0 & 0 & o) & 0 & 0 & 0 & 0 & 0 & o & $\mathbf{0}$ \\
\hline ALPHA DET-Np & 0 & 0 & 0 & 0 & 0 & 0 & 0 & 0 & of & 0 \\
\hline ALPHADET-Th & 0 & 0 & 0 & 0 & 0 & 0 & 01 & 0 & 0 & $\mathbf{0}$ \\
\hline Al PHA DET-U,Th, Pu & $\begin{array}{ll}0 \\
\end{array}$ & 0 & 0 & 0 & 0 & 0 & 0 & 0 & 0 & 0 \\
\hline ALPHA DET-U235 & 0 & 0 & 0 & o & 0 & 0. & o & 0 & 0 & 0 \\
\hline ALPHA DET-Pu,U,Am & 0 & 0) & 0 & 0 & 0 & 0 & 0 & 0 & 0. & 0 \\
\hline ALPHADET-U,Np,Pu,Am & \begin{tabular}{l|l}
0 \\
\end{tabular} & 0 & 0 & 0 & 0 & o. & 0 & 0 & 0 & 0 \\
\hline ALPHADET-Ra226 & \begin{tabular}{l|l}
0 & \\
\end{tabular} & 0 & O) & 0 & O & 0 & 0 & of & 0. & 0 \\
\hline ALPHADET-Ra228 & \begin{tabular}{l|l}
0 & \\
\end{tabular} & 0 & 0 & 0 & of & o & 0 & 0 & 0 & 0 \\
\hline ALPHA SPEC & \begin{tabular}{l|l}
0 & $-1-1-1$
\end{tabular} & 0 & a) & 0 & 0 & 0 & 0 & 0 & o & 0 \\
\hline GPC-BETA-Pb210 & 0 & 0 & 0 & 0 & 0 & 0. & 0 & 0 & 0 & 0 \\
\hline GPC-BETA-Sr90 & 0 & 0 & 0: & 0 & o & 0. & 0 & 0 & 0. & $\mathbf{0}$ \\
\hline GPC-BETA-Pm147 & 0 & 0 & 0 & 0 & 0 & (0. & 0 & of & 0 & 0 \\
\hline GPC-BETA-Se79 & o & 0 & 0. & 0 & o & 0 & 0 & 0 & 0 & 0 \\
\hline LSC-BETA-H3 & 0 & 0 & 0 & 0 & 0 & 0 & 0 & of & 0. & 0 \\
\hline LSC-BETA-Rn222 & 0 & 0 & 0 & 0 & 0 & o & 0 & 0 & 0. & 0 \\
\hline LSC-BETA-1129 & of & 0 & 0 & 0 & 0 & 0 & 0 & O) & 요 & 0 \\
\hline LSC-BETA-C14 & \begin{tabular}{l|l}
0 & \\
\end{tabular} & 0 & 0 & 0 . & 0 & 0 & 0 & 0 & 0 & 0 \\
\hline LSC-BETA-TC99 & 0 & $\overline{0}$ & 0 & 0 & 0 & 0 & 0 & 0 & 0 & 0 \\
\hline LSC-BETA & 0 & 0 & 0 & 0 & 0 & 0 & 0 & 0 & 0 & 0 \\
\hline GAMMA SPEC & 0 & 0 & 0 & 0 & 0 & 에 & 0 & 0 & 0 & 0 \\
\hline OTHER-PAD & 0 & 0 & O) & 01 & 0 & o] & 0 & 0 & 0] & 0 \\
\hline TOTAL RAD & 0 & $\overline{0}$ & 0. & 01 & 2339 & oil & 10701 & 692 & 577 & 2339 \\
\hline & & & & & & & & & & \\
\hline TCLP EXTRACTION & 0 & 0 & 0 & 0 & 310 & of & 6 & 149 & 155 & 310 \\
\hline OTHER-PREP & 0 & 0 & of & 0 & 0 & 0. & 0 & of & 0 & 0 \\
\hline OTHER-EXPLO & 0 & 0 & of & D) & 0 & 0 & 0 & 0 & 0 of & 0 \\
\hline OTHER-BIO & 0 & 0 & of & 0 & 0 & o & 0 & 0 & 0 & 0 \\
\hline OTHER-PHYS & 0 & 0 & 0 & 0 & 0 & o & 0 & 0 & 0 & 0 \\
\hline OTHER & of & $\overline{0}$ & 01 & 0 & 0 & 0 & 0 & of & 0. & 0 \\
\hline TOTAL OTHEA & 0 0I & 0 & 0 & 0 & 310 & 0 & (6) & 149 & 155 & 310 \\
\hline & & & & & & & & & & L \\
\hline TOTAL SAMPLE ANALYSES & 0 & 0 & 0 & 0 & 5970 & (0) & 2935] & 1625 & 1410 ] & 5970 \\
\hline
\end{tabular}




\section{EM-263 SURVEY OF DOE ANALYTICAL CAPACITY AVAILABLE TO EM}

Table IVa: UC Sample Analyses by Rad Level and Matrix Type (xxiii) WSAC-ALD-CLAB

\begin{tabular}{|c|c|c|c|c|c|c|c|c|c|c|}
\hline $\begin{array}{l}\text { Rad-Matix } \\
\text { Samp Anal }\end{array}$ & $\overline{\mathrm{RO}}$ & RO/RI & $\overline{\mathrm{R} 1}$ & $\overline{R 2}$ & $P 3$ & $\overline{R 4}$ & M1 & M2 & $\overline{\mathrm{MB}}$ & Total \\
\hline VOA-GC & 0 & 0 & 0 & 0 & 01 & of & 0 & 0 & 0 & 0. \\
\hline VOA-GCMS & 0 & 0 & 0 & 0 & 0 & 0 & 0 & 0 & 0 & 0 \\
\hline SEMI-VOA & 0 & 0 & of & 0 & 0 & 0 & 0 & o & 0 & 0 \\
\hline PEST & 0 & 0 & 0 & 0 & 0 & 0 & of & 0 & 0 & o \\
\hline HERB & 0 & 0 & of & 0 & 0 & 0 & 0 & 0 & 0 & 0 \\
\hline $\mathrm{PCB}$ & 0 & 0 & 0 & 0 & 0 & 0 & 0 & o & 요 & 0 \\
\hline PCB/PEST & 0 & 0 & 0 & 0 & of & of & o) & 0 & ot & 0 \\
\hline OTHER-ORG & 0 & 0 & 0 & 0 & of & a. & 01 & 0 & 0 & 0 \\
\hline TOTAL ORGANICS & 0 & 0 & 01 & 0 & 01 & $0]$ & $0 \mid$ & 01 & 0 - & (0) \\
\hline & & & & & & & & - & & \\
\hline ICP-AES METALS & of & 0 & 0 & 0 & 0 & a. & 0 & 0 & 0. & 0 \\
\hline ICP-MSMETALS & of & 0 & 0 & 0 & 0 & o. & 0 & 0 & 0 & 0 \\
\hline GF-AAMETALS & 0 & 0 & 0 & 0 & 0 & o. & 0 & 0 & 0 & 0 \\
\hline MERCURY & 0 & 0 & 0 & 0 & 0 & 0 & of & 0 & 0 & 0 \\
\hline CYANIDE & 0 & 0 & 0 & 0 & of & o) & 0 & 0 & 0 & 0 \\
\hline ANIONS/CATIONS & 0 & 0 & 0 & 1 & 0 & 69 & \begin{tabular}{l|l}
69 \\
\end{tabular} & 0 & 1 & 70 \\
\hline WET CHEM & 0 & 0 & 0 & 0 & 0 & o] & 0 & 0 & 0 & 0 \\
\hline OTHEA-INOAG & 0 & 0 & 19 & 213 & 131 & 29 & \begin{tabular}{l|l|}
392 \\
\end{tabular} & 0 & 0 & 392 \\
\hline TOTALINORGANICS & OI & 0 & 191 & 2141 & 1311 & 991 & 462 & 01 & II & 463 \\
\hline & & & & & 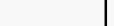 & If & & 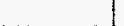 & 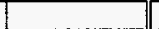 & \\
\hline GROSS ALPHAVBETA & 0 & 0 & 1226 & 1063 & of & 377 & 2666 & 0 & 0 & 2666 \\
\hline ALPHA DET-Np237 & 0 & 0 & 0 & 0 & of & 0. & 0 & 0 & 0 & 0 \\
\hline ALPHADET-PU,Am & 0 & 0 & 0 & 0 & of & 0 & of & 0 & o & 0 \\
\hline ALPHADET-Pu & 0 & 0 & 0 & 0 & 0 & D. & 0 & 0 & 0 & 0 \\
\hline ALPHADET-U & 0 & 0 & 0 & 0 & 0 & D. & 0 & 0 & 0 & 0 \\
\hline ALPHADET-Am & 0 & 0 & 0 & 0 & 0 & 0 & 0 & 0 & 0 & 0 \\
\hline ALPHADET-NP & 0 & 0 & 0 & 0 & 0 & o. & 0 & 0 & 0 & 0 \\
\hline ALPHA DET-Th & 0 & 0 & 0 & 0 & 0 & D & 0 & 0 & 0 & 0. \\
\hline ALPHA DET-U,Th,PU & 0 & 0 & 0 & 0 & 0 & of & 0 & 0 & 0 & 0 \\
\hline ALPHA DET-U235 & 0 & 0 & 0 & 0 & 0 & of & 0 & $\overline{0}$ & 0 & 요 \\
\hline ALPHA DET - Pu,U,Am & 0 & 0 & 0 & 0 & 0 & of & 0 & 0 & 0] & 0 \\
\hline ALPHA DET-U,Np,Pu,Am & 0 & $\mathbf{0}$ & 0 & 0 & 0 & 0 & 0 & 0 & 0 & 의 \\
\hline ALPHA DET-Ra226 & 0 & 0 & 0 & 0 & 0 & 0 & 0 & 0 & 0 . & 0 \\
\hline ALPHA DET-Ra228 & 0 & 0 & 0 & 0 & 0 & 0 & 0 & 0 & 0 & 으 \\
\hline ALPHA SPEC & 0 & 0 & 147 & 52 & 0 & 111 & 310 & 0 & 0 & 310 \\
\hline GPC-BETA-Pb210 & 0 & 0 & 0 & 0 & 0 & o & 0 & o) & 0 & 0. \\
\hline GPC-BETA-Sr90 & 0 & $\overline{0}$ & 0 & 0 & 0 & 0 & 01 & 0 & 0 & 0 \\
\hline GPC-BETA-Pm147 & 0 & 0 & 0 & 0 & o & 0 & 0 & of & 0 & 0 \\
\hline GPC-BETA-Se79 & 0 & $\mathbf{0}$ & 0 & 0 & D. & $\overline{0}$ & 0 & 0 & 0 & 0 \\
\hline LSC-BETA-H3 & 0 & 0 & 0 & 0 & 0 & o. & 0 & 0 & 0 & 0 \\
\hline LSC-BETA-Pin222 & 0 & 0 & 0 & $\overline{0}$ & o| & 0 & 0 & 0 & of & 0 \\
\hline LSC-BETA-1129 & 0 & 0 & 0 & 0 & \begin{tabular}{l|l}
0 & \\
\end{tabular} & 0 & of & 0 & 0. & 0 \\
\hline LSC-BETA-C14 & 0 & 0 & 0 & 0 & 0 & 0 & 0 & 0 & \begin{tabular}{l|l}
0 & \\
\end{tabular} & -0 \\
\hline LSC-BETA-TC99 & 0 & 0 & 0 & 0 & D) & 0 & 0 & 0 & 0 & 0 \\
\hline LSC-BETA & 0 & 0 & 0 & 0 & 0 & 0 & 0 & 0 & 0 & 0 \\
\hline GAMMA SPEC & 0 & 0 & 1442 & 1298 & 0 & 32 & 2772 & 0 & 0 & 2772 \\
\hline OTHEA-RAD & O & 0 & 0 & 0 & 0 & 0 ] & 0 & 0 & 0 & 0 \\
\hline TOTAL RAD & 01 & $\overline{0}$ & 2815 & 2414 & 01 & 520 & 5748 & 0I & $0]$ & 5748 \\
\hline & & & & & & & & & & \\
\hline TCLP EXTRACTION & 0 & 0 & 0 & 0 & 0 & 0 & 0 & 0 & 0 & 0 \\
\hline OTHER-PREP & 0 & 0 & 0 & 0 & 0 & 0 & 0 & 0 & 요 & 0 \\
\hline OTHER-EXPLO & 0 & 0 & 0 & $\overline{0}$ & 0 & 0 & 0 & 0 & 0 & 0 \\
\hline OTHER-BIO & 0 & 0 & 0 & 0 & 0 & 0 & 0 & 0 & o. & o \\
\hline OTHER-PHYS & 0 & 0 & 0 & 0 & 0 & 0 & 0 & 0 & 0 & 0 \\
\hline OTHER & 01 & 0 & 01 & 0 & 0 & 0] & 0 & 0 & 0] & 0 \\
\hline TOTAL OTHEA & 01 & 0 & OI & 01 & 01 & O] & 0 & 0] & 이민 & D \\
\hline & & & & & & & & 1 & I & \\
\hline TOTAL SAMPL ANALYSES & 0 & $\overline{0}$ & 2834 & 2627 & 131 & 618 & 6210 & 01 & TI & 6211 \\
\hline
\end{tabular}


EM-263 SURVEY OF DOE ANALYTICAL CAPACTY AVAILABLE TO EM

Table IVa: UC Sample Analyses by Rad Level and Matrix Type (xxiv) WSRC-ALD-320M

\begin{tabular}{|c|c|c|c|c|c|c|c|c|c|c|}
\hline $\begin{array}{l}\text { Rad-Matix } \\
\text { Samp Anal }\end{array}$ & RO & RO/HI & $\overline{\mathrm{Ri}}$ & Fi2 & $\overline{R 3}$ & R4 & M1 & $=\sqrt{2}$ & $\mathrm{M3}$ & Total \\
\hline & & & & & & & & & & \\
\hline VOA-GC & 0 & 0 & 0 & 0 & 0 & 0 & 0 & 0 & 0 & 0 \\
\hline VOA-GCMS & 0 & 0 & 0 & 0 & 0 & 0 & 0 & 0 & 0 & 0 \\
\hline SEMI-VOA & 0 & 0 & 0 & 0 & 0 & (0) & 0 & 0 & 0 & 0 \\
\hline PEST & 0 & 0 & 0 & 0 & 0 & 0 & 0 & 0 & 0 & 0 \\
\hline HERB & 0 & 0 & 0 & 0 & 0 & 0 & 0 & $\mathbf{0}$ & 0 & 0 \\
\hline$\overline{P C B}$ & 0 & $\overline{0}$ & 0 & 0 & 0 & 0 & 0 & 0 & 0 & 0 \\
\hline PCB/PEST & 0 & 0 & 0 & 0 & 0) & 0] & 0 & 0 & 0 & 0 \\
\hline OTHER-ORG & 0 & 0 & 0 & 0 & 0 & 0 & 0 & 0 & 0. & 0 \\
\hline TOTAL ORGANICS & 0 & 0 & 0 & O) & 0 & 0] & 0 & 01 & o) & $\overline{0}$ \\
\hline & & & & & $=$ & & & $=$ & $=7$ & \\
\hline ICP-AESMEIALS & 0 & D & 0 & 0 & 0 & 0 & 0 & 0 & 0 & 0 \\
\hline ICP-MSMETALS & 0 & 0 & 0 & 0 & 0 & 0 & 0 & 0 & 0 & 0 \\
\hline GF-AAMETALS & 0 & 0 & 0 & 0 & 0 & 0. & 0 & 0 & 0 & 0 \\
\hline MERCURY & 0 & 0 & 0 & 0 & 0 & 0 & 0 & 0 & 0 & 0 \\
\hline CYANIDE & 0 & 0 & 01 & 0 & 0 & 0 & 0 & 0 & 0 & 0 \\
\hline ANIONS/CATIONS & 0 & 0 & 0 & 0 & 0 & 0 & 0 & 0 & 0 & 0 \\
\hline WEICHEM & 0 & 0 & 0 & 0 & 0 & 0 & 0 & 0 & 0 & 0 \\
\hline OTHER-INORG & 0 & 0 & 01 & 0 & 01 & 0 . & 0 & 0 & 0 & 0 \\
\hline TOTAL INORGANICS & 0 & $\overline{0}$ & o] & 0 & 0 & O) & 0 & 0 & 0 & $\overline{0}$ \\
\hline & & & & & & & & & & \\
\hline GROSS ALPHAVEETA & 0 & 0 & 0 & 0 & 0 & 0. & 0 & 0 & 0 & 0 \\
\hline ALPHA DET-Np237 & 0 & 0 & 0 & 0 & 0 & 0. & 0 & 0 & 0 & 0 \\
\hline ALPHA DET-PU,Am & 0 & 0 & 0 & 0 & 0 & 0 & 0 & 0 & 0 & 0 \\
\hline ALPHA DET-PU & 0 & 0 & 0 & o) & 0 & o] & 0 & 0 & o & 0 \\
\hline ALPHADET-U & 0 & 0 & 0 & 0 & 0 & 0 & $\mathbf{0}$ & 0 & D. & 0 \\
\hline ALPHA DET-Am & 0 & 0 & 0 & 0 & 0 & 0 & 0 & 0 & 0 & 0 \\
\hline ALPHA DET $-N p$ & 0 & 0 & 0 & 0 & 0 & $\overline{0}$ & 0 & 0 & 0 & 0 \\
\hline ALPHADET-Th & 0 & 0 & 0 & 0 & 0 & 0 & 0 & 0 & 0 & 0 \\
\hline ALPHA DET - U,Th,PU & 0 & 0 & 0 & 0 & 0 & of & 0 & 0 & 0 & 0 \\
\hline ALPHA DET-U235 & 0 & 0 & 0 & 0 & 0 & 0 & 0 & 0 & 0 & 0 \\
\hline ALPHA DET-Pu,U,Am & 0 & 0 & 0 & 0 & of & O & 0 & 0 & 0 & 0 \\
\hline ALPHA DET-U,Np,PU,Am & 0 & 0 & 0 & 0 & 0 & 0 & 0 & 0 & 0 & 0 \\
\hline ALPHA DET-Ra226 & 0 & 0 & 0 & 0 & 0 & 0 & 0 & 0 & 0 & 0 \\
\hline ALPHA DET-Ra228 & 0 & 0 & 0 & 0 & 0 & 0 & 0 & 0 & 0 & 0 \\
\hline ALPHA SPEC & 0 & 0 & 0 & 0 & 0 & 0 & 0 & 0 & 0 & 0 \\
\hline GPC-BETA-Pb210 & 0 & 0 & 0 & 0 & 0 & 0 & 0 & 0 & 0 & 0 \\
\hline GPC-BETA-Sr90 & 0 & 0 & 0 & 0 & 0 & 0 & 0 & 0 & 0 & 0 \\
\hline GPC-BETA-Pm147 & 0 & 0 & 0 & 0 & 0 & 0 & 0 & 0 & 0 & 0 \\
\hline GPC-BETA-Se79 & 0 & 0 & 0 & O) & 0 & 0) & 0 & 0 & $\mathbf{0}$ & 0 \\
\hline LSC-BETA-H3 & 0 & 0 & 0 & 0 & 0 & 0. & 0 & 0 & 0 & 0 \\
\hline LSC-BETA-Pn222 & 0 & 0 & 0 & 0 & 0 & 0 & 0 & 0 & 0 & 0 \\
\hline LSC-BETA- 1129 & 0 & 0 & 0 & 0 & 0 & O) & 0 & 0 & 0 & 0 \\
\hline LSC-BETA-C14 & 0 & 0 & 0 & 0 & 0 & 0 & 0 & 0 & 0 & 0 \\
\hline LSC-BEIA-TC99 & 0 & 0 & 0 & 0 & 0 & 0 & 0 & 0 & 0 & 0 \\
\hline LSC-BETA & 0 & 0 & 0 & 0 & 0 & 0 & 0 & 0 & 0 . & 0 \\
\hline GAMMA SPEC & 0 & 0 & 0 & 0 & 0 & 0 & 0 & 0 & 0 & 0 \\
\hline OTHER-RAD & 0 & 0 & 0 & 0 & 0 & 0 & 0 & 0 & 0 & 0 \\
\hline TOIAL RAD & 0 & $\overline{0}$ & 0 & 0 & 0 & D. & 0 & 01 & 0 & $\underline{0}$ \\
\hline & & & & & & & & & & \\
\hline TCLP EXTRACTION & 0 & 0 & 0 & 0 & 0 & 0 & 0 & 0 & 0 & 0 \\
\hline OTHEA-PREP & 0 & 0 & 0 & 0 & 0 & 0 & 0 & 0 & 0 & 0 \\
\hline OTHER-EXPLO & 0 & 0 & 0 & 0 & 0 & 0 & 0 & 0 & o & 0 \\
\hline OTHER-BIO & 0 & 0 & 0 & 0 & 0 & 0 & 0 & $\mathbf{0}$ & 0 & 0 \\
\hline OTHER-PHYS & 0 & 0 & 0 & 0 & 0 & 0 & 0 & o & D. & 0 \\
\hline OTHEA & 0 & 0 & 0 & 0 & 0 & 0 & 0 & 0 & 0 & 0 \\
\hline TOTAL OTHEA & 0 & $\overline{0}$ & 0 & $\overline{0}$ & 0 & 0 & 0 & 0 & 0 & $\overline{\overline{0}}$ \\
\hline & & & & & I & 7 & 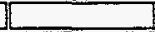 & 1 & 7 & \\
\hline TOTAL SAMPLE ANALYSES & $\overline{0}$ & 0 & D & 0 & 0 & 0 & $\overline{0}$ & DI & -0 & 1 \\
\hline
\end{tabular}


EM-263 SURVEY OF DOE ANALYTICAL CAPACITY AVAILABLE TO EM

Table IVa: UC Sample Analyses by Rad Level and Matrix Type (xxv) WSRC-ALD Water

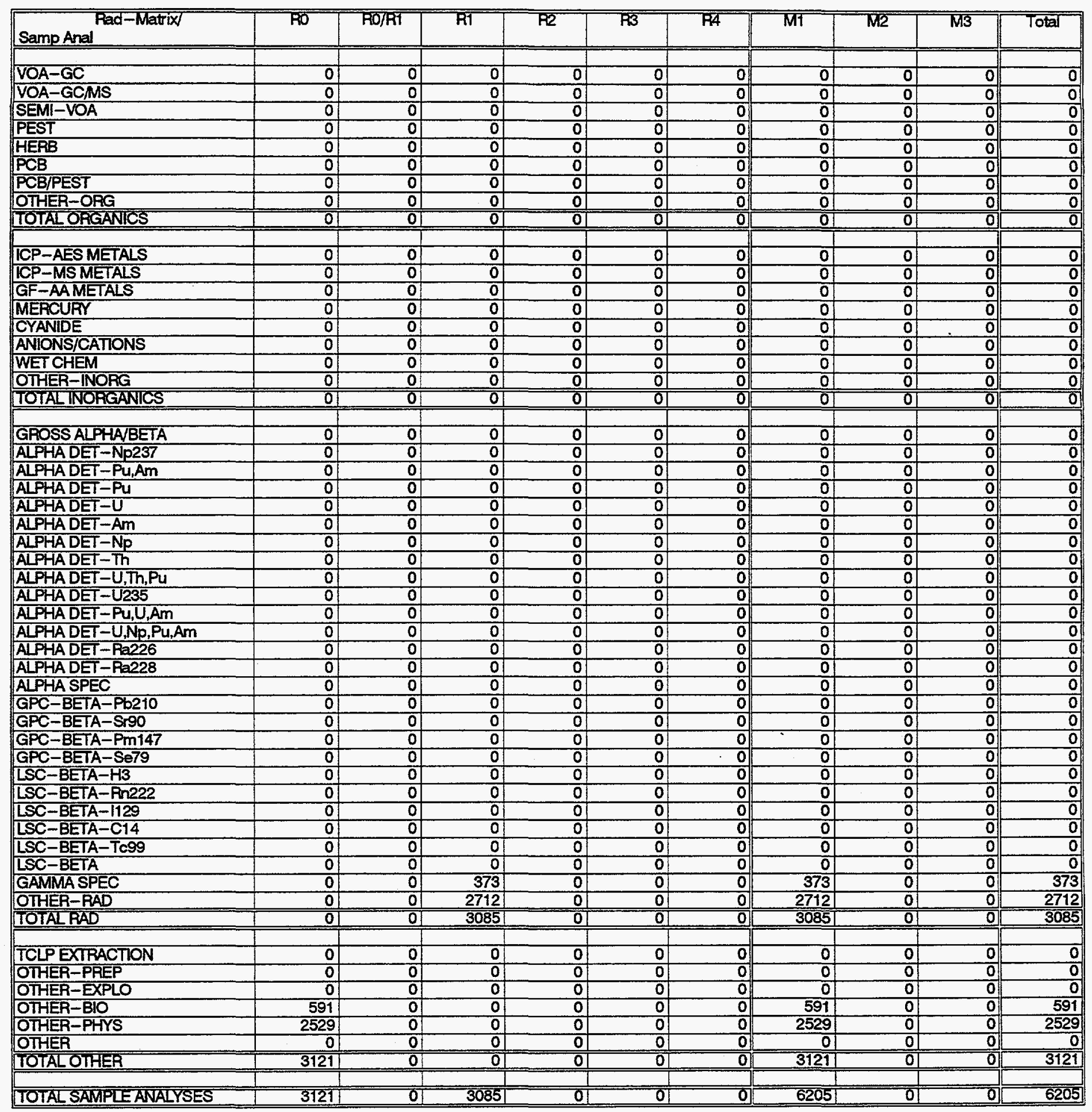


Table IVa: UC Sample Analyses by Rad Level and Matrix Type (xxvi) WSRC-ALD-ETF

\begin{tabular}{|c|c|c|c|c|c|c|c|c|c|c|}
\hline $\begin{array}{l}\text { Fad-Matrix } \\
\text { Samp Anal }\end{array}$ & RO & RO/R1 & R1 & $\mathrm{R} 2$ & 73 & $\overline{R 4}$ & M1 & M2 & $\mathrm{M3}$ & Total \\
\hline & & & & & & & & & & \\
\hline VOA-GC & 0 & 0 & O] & 0 & 0 & ㅇ. & 0 & 0 & 0 & 0 \\
\hline VOA-GCMS & 0 & 0] & o] & 0 & of & 0 & 0 & 0 & 0 & 요 \\
\hline SEMI-VOA & 0 & 0) & 0 & 0 & 0 & 0 & 0 & 0 & 0 & 0 \\
\hline PEST & 0 & 0 & 0 & 0 & 0 & 0 & 0 & 0 & 0 & 0 \\
\hline HERB & 0 & 0 & 0 & 0 & 0 & 0 & 0 & 0 & (0) & 0 \\
\hline PCB & 0 & 0 & 0] & 0 & 0 & 으 & 0 & 0 & 0] & 0 \\
\hline PCB/PEST & 0 & 0 & 0 & 0 & 0 & 0 & 0 & 0 & (0) & 0 \\
\hline OTHER-ORG & 0 & 0 & 01 & 01 & 0 & 0 & 0 & 0 & 0 & 0 \\
\hline TOTAL ORGANICS & 0 & 0 & OI & 01 & oI & 0 & $0 \mid$ & 01 & 0. & 0 \\
\hline & & & & & & & & $\rightarrow$ & & \\
\hline ICP-AES METALS & 0 & 0) & 476 & 0 & 0 & 0 & 476 & 이 & 0 & 476 \\
\hline ICP-MS METALS & 0 & 0 & 0 & 0 & 0 & 0 & 0 & 0 & 0 & 0 \\
\hline GF-AAMETALS & 0 & 0 & 0 & 0 & 0 & 0 & 0 & 0 & 0 & 0 \\
\hline MERCURY & 0 & 0 & 24 & 0 & 0 & 0) & 24 & 0 & 0 & 24 \\
\hline CYANIDE & 0 & 0 & 이 & 0 & 0 & 0 & 0 & 0 & 0 & 0 \\
\hline ANIONS/CATIONS & 0 & of & 0 & 0 & 0 & ㅁ․ & 0 & 0 & 0 & 0 \\
\hline WET CHEM & 0 & 0 & 0 & 0 & 0 & 0 & 0 & 0 & 으. & 0 \\
\hline OTHER-INORG & 0 & 0 & 0 & 0 & 0 & 0. & O) & 0 & 0 & 0 \\
\hline TOTAL INORGANICS & 0 & 01 & 5001 & 0 & 01 & 0 & 500 & 01 & 0 & 500 \\
\hline GROSS ALPHA/BETA & 0 & 0 & 508 & 0 & 0 & 0 & 508 & 0 & 0 & 508 \\
\hline ALPHA DET-Np237 & 0 & of & of & 0 & 0 & $\overline{0}$ & 0 & 0 & 0 & 0 \\
\hline ALPHA DET -PU,Am & 0 & 이 & 0 & 0 & 01 & 0 & 0 & 0 & 0 & 0 \\
\hline ALPHADET-Pu & 0 & OI & 0 & 0 & 0 & 0 & 0 & 0 & 0 & $\overline{0}$ \\
\hline ALPHADET-U & 0 & 0 & 0 & 0 & 0 & $\overline{0}$ & 0 & o & - & 0 \\
\hline ALPHA DET-Am & 0 & 0 & 0 & 0 & 0 & 0 & 0 & 0 & 0 & 0 \\
\hline ALPHA DET-Np & 0 & 0 & 0 & 0 & 이 & 0 & 0 & 0 & 0 & 0 \\
\hline ALPHADET - Th & 0 & 0 & 이 & 0 & 0 & 0 & 0 & O & D. & 0 \\
\hline ALPHA DET-U,Th,Pu & 0 & 0 & 0 & 0 & 0 & 으 & 아 & 0 & 0 & 0 \\
\hline ALPHADET-U235 & 0 & 0 & 0 & 0 & 0 & 0 & 0. & 0 & 0 & 0 \\
\hline ALPHA DET - Pu,U,Am & 0 & 이 & 0 & 0 & 0. & 0 & 0 & 0 & 0 & 0 \\
\hline ALPHA DET-U,No,Pu,Am & 01 & 0 & 0 & 0 & 0 & 0 & 0) & 0 & 0 & 0 \\
\hline ALPHADET-Ra226 & 0 & 0 & 0 & 0 & of & 0. & 이 & a) & $\mathbf{0}$ & 0 \\
\hline ALPHADET-Ra228 & 0 & of & 0 & 0 & 0 & 0. & of & 0 & 0 & 0 \\
\hline ALPHA SPEC & 0 & 0 & 01 & 0 & 이 & of & - & 0 & 0 & 0 \\
\hline GPC-BETA-Pb210 & 0 & 0. & 0 & of & 0 & 0 & 0 & 0 & 으. & 0 \\
\hline GPC-BETA-Sr90 & 0 & 아 & 0 & 0 & o & 0 & 0 & 0 & 0 & 0 \\
\hline GPC-BETA-Pm147 & 0 & 0 & 0 & 0 & 0 & 0 & 0 & 0 & 0 & 0 \\
\hline GPC-BETA-Se79 & 0 & o & 0 & 0 & 0 & 0 & 이 & 0 & 0. & 0 \\
\hline LSC-BETA-HB & 0 & 0 & 0 & 0 & 이 & 0 & 아 & ㅇ. & 의 & 0 \\
\hline LSC-BETA-Rn222 & 0 & 0 & 0 & 0 & 0 & 0 & 0 & 0 & 0 & 0 \\
\hline LSC-BETA- 1129 & 0 & 0 & 0 & 0 & 이 & 0 & 0 & o & 의 & 0 \\
\hline LSC-BETA-C14 & 0 & 0 & 0 & 0 & of & 0 & 0 & 0 & 0 & 0 \\
\hline LSC-BETA-TC99 & 0 & 0 & 0 & 0) & 0 & o & 이 & 0 & 0 & 0 \\
\hline LSC-BETA & 0 & 0 & 0 & of & 0 & 0 & 0 & 0 & 0 & 0 \\
\hline GAMMA SPEC & 0 & 0 & 0 & 01 & 0 & 0. & 0 & 아 & 0 & 0 \\
\hline OTHER-RAD & 0 & 0 & 0 & 01 & 0 & o] & 0 & 0 & ㅇ․ & 0 \\
\hline TOTAL RAD & 01 & 0 & 508 & 01 & 01 & 0] & 508 & 0 & 0 & 508 \\
\hline & & & & & & & & & & \\
\hline TCLP EXTRACTION & 0 & 0 & 이 & 0 & 의 & 에 & 0 & 이 & 여 & 0 \\
\hline OTHEA-PAEP & 0 & 0 & 0 & 0 & 0 & 0] & 0 & 0 & 0 & 0 \\
\hline OTHER-EXPLO & 0 & 0 & 0 & 0 & 0 & 0 & 0 & 0 & 요 & 0 \\
\hline OTHER-BIO & 0 & 0 & 0 & 0 & 0 & 0 & 0 & 0 & ㅁ․ & 0 \\
\hline OTHER-PHYS & 0 & 0 & 0 & 0 & 이 & ㅇ․ & 이 & 이 & 의 & 0 \\
\hline OTHER & 0 & 0 & 0 & 0 & 0 & 0 . & o & 0 & 0 & 0 \\
\hline TOTAL OIHER & 0 & 0 & 0 & 01 & 이 & 0: & 0 & 01 & 이 & 0 \\
\hline & & & 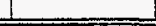 & & 1 & 县 & 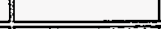 & $=$ & 1 & \\
\hline TOTAL SAMPLE ANALYSES & 0 & 0 & 1008 & 0 & 0 & 0] & 1008 & 0 & $0]$ & 1008 \\
\hline
\end{tabular}


EM-263 SURVEY OF DOE ANALYTICAL CAPACITY AVAILABLE TO EM

Table IVa: UC Sample Analyses by Rad Level and Matrix Type (xxvii) WSRC-ALD-Tritium

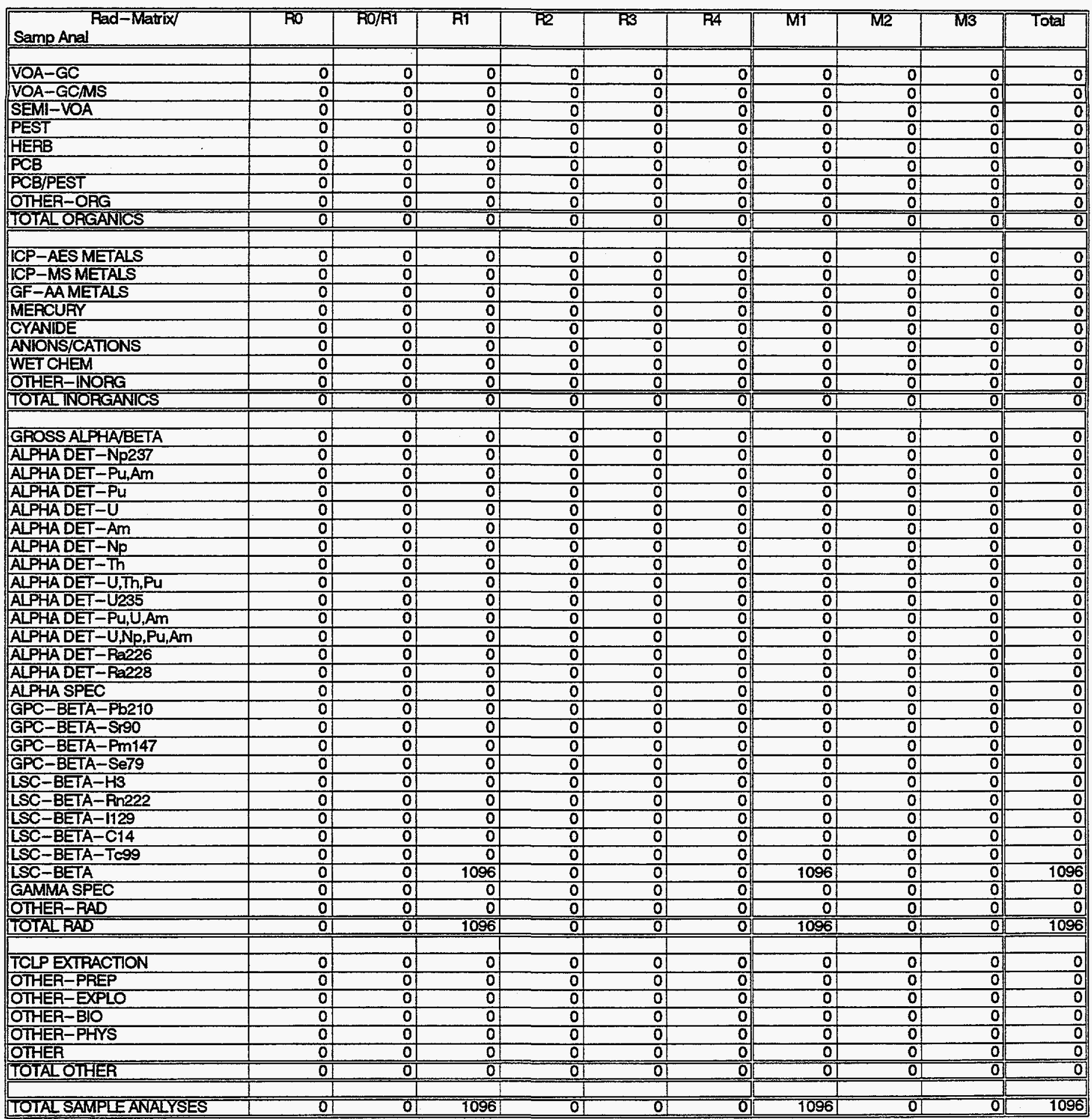


EM-263 SURVEY OF DOE ANALYTICAL CAPACITY AVAILABLE TO EM

Table IVa: UC Sample Analyses by Rad Level and Matrix Type (xoviii) WSRC-ALD-ITP

\begin{tabular}{|c|c|c|c|c|c|c|c|c|c|c|}
\hline $\begin{array}{l}\text { Rad-Matrix } \\
\text { Samp Anal }\end{array}$ & $\overline{\mathrm{RO}}$ & $\overline{\mathrm{Ro} / \mathrm{R1}}$ & $\overline{R T}$ & $\overline{R 2}$ & $\overline{\mathrm{R} 3}$ & $\overline{\mathrm{RA}}$ & $\overline{\mathrm{MIT}}$ & $M 2$ & V13 & Total \\
\hline & & & & & & & & & & \\
\hline VOA-GC & 0 & 0 & 1070 & o) & 0 & o. & 535 & 0 & 535 & 1070 \\
\hline VOA-GCMS & 0 & 0 & 0 & 0 & 0 & o) & 0 & 0 & 0 & 0 \\
\hline SEMI-VOA & 0 & 0 & 0 & 0 & 0 & 0 & 0 & 0 & 0 & O) \\
\hline PEST & 0 & 0 & 0 & 0 & 0 & 0 & o & 0 & 0 & 0] \\
\hline HERB & 0 & 0 & 0 & 0 & 0 & 0 & 0 & 0 & 0 & 0 \\
\hline $\mathrm{PCB}$ & 0 & 0 & 0 & 0 & 0) & 0 & 0 & 0 & o & 0 \\
\hline PCB/PEST & 0 & 0 & 0 & 0 & 0) & 0 & o & 0 & 0 & 0 \\
\hline OTHER-OAG & 0 & 0 & 01 & 0 & 01 & 0 & 0 & 01 & o. & 0 \\
\hline TOIAL ORGANICS & 01 & 0 & 1070 & 0 & 01 & 0] & 535 & 0] & 535 & 1070 \\
\hline & & & & & & 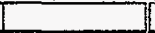 & 7 & 1 & i & \\
\hline ICP-AES METALS & 0 & 0 & 0 & 0 & 0 & 0 & 0 & 0 & 0 & 0. \\
\hline ICP-MSMETALS & 0 & 0 & 0 & 0 & 0 & o. & $\overline{0}$ & 0 & 0) & o \\
\hline GF-AAMETALS & 0 & 0 & 0 & 0 & 0 & 0 & 0 & 0 & o & 0 \\
\hline MERCUFY & 0 & 0 & 0 & 0 & 0 & o. & o & o & o) & 0 \\
\hline CYANIDE & 0 & 0 & 0 & 0 & 0 & 0 & 0 & 0 & 0 & 0] \\
\hline ANIONS/CATIONS & 0 & 0 & 01 & 0 & 0 & 0 & 0 & 0 & 0 & 0 \\
\hline WET CHEM & 0 & 0 & 0 & 0 & 0 & 0 & o & 0 & 0 & D. \\
\hline OTHER-INORG & 0 & 0 & 0 & 0 & 0 & o. & 0 & 0 ! & 0 & - \\
\hline TOTAL INOFGANICS & DI & 0 & 0 & 0 & of & ol] & DI & $\overline{01}$ & D. & [0] \\
\hline & & & & & & & & & 7 & \\
\hline GROSS ALPHAVBETA & 0 & 0 & 0 & 0 & 0 & of & 0 & 0 & 0. & 0 \\
\hline ALPHA DET-Np237 & 0 & 0 & 0 & 0 & 0 & 0 & 0 & o & o & 0 \\
\hline ALPHA DET-PU,Am & D & 0 & 0 & 0 & 0 & O) & 0 & 0 & 0 & 0 \\
\hline ALPHA DET-PU & 0 & 0 & 0 & 0 & 0 & O. & 0 & 0 & 0 & 0 \\
\hline ALPHADET-U & 0 & 0 & 0 & 0 & 0 & o. & 0 & 0 & 0 & 0 \\
\hline ALPHA DET-Am & 0 & 0 & 0 & 0 & 0 & of & 0 & 0 & 0 & 0 \\
\hline ALPHA DET-Np & 0 & 0 & 0 & 0 & 0 & 0. & 0 & 0 & 0 & D. \\
\hline ALPHADET-Th & 0 & 0 & 0 & 0 & 0 & of & o & 0 & 0. & $\underline{0}$ \\
\hline ALPHA DET-U,Th,PU & 0 & 0 & 0 & 0 & 0 & 0. & 0 & o & 0 & 0 \\
\hline ALPHA DET-U235 & 0 & 0 & 0 & 0 & 0 & of & 0 & 0 & 0. & 0] \\
\hline ALPHA DET-PU,U,Am & 0 & 0 & 0 & 0 & 0 & 0. & o) & 0 & 0. & 0. \\
\hline ALPHA DET-U,Np,Pu,Am & 0 & 0 & 0 & 0 & 0 & 0 & 0 & 0 & 0 & 0. \\
\hline ALPHA DET-Ra226 & 0 & 0 & 0 & 0 & 0 & oft & 0 & 0 & 0 & 0 \\
\hline ALPHA DET-Ra228 & 0 & 0) & 0 & 0 & 0 & 0 & 0 & of & 0 & 은 \\
\hline ALPHA SPEC & 0 & 0 & 0 & 0 & 0 & 0 & 0 & 0 & 0 & 0 \\
\hline GPC-BETA-Pb210 & 0 & 0 & 0 & 0 & 0 & o. & 0 & 0 & 0 & 0 \\
\hline GPC-BEIA-Sr90 & 0 & 0 & 0 & 0 & 0 & 0 & 0 & 0 & 0 & 0 \\
\hline GPC-BETA-Pm147 & 0 & 0 & 0 & 0 & 01 & 0 & 0 & 0 & 0 & 0 \\
\hline GPC-BETA-Se79 & 0 & 0 & 0 & 0 & 0 & 0 & o & 0 & 0 & 0 \\
\hline LSC-BETA-H3 & 0 & 0 & 0 & 0 & 0 & 0 & 0 & 0 & 0 & (0) \\
\hline LSC-BETA-Rin222 & 0 & 0 & 0 & 0 & 0 & 0 & 0 & 0 & 0 & 0. \\
\hline LSC-BETA- 1129 & 0 & 0 & 0 & 0 & 0 & 0 & 0 & 0 & 0 & 0] \\
\hline LSC-BETA-C14 & 0 & O & 0 & 0 & 0 & 0 & 0 & 0 & 0 & 문 \\
\hline LSC-BETA-TC99 & 0 & 0 & 0 & 0 & 0 & 0 & 0 & 0 & of & 0 \\
\hline LSC-BETA & 0 & 0 & 0 & 0 & 0 & 0) & 0 & 0 & 0 & 0. \\
\hline GAMMA SPEC & 0 & 0 & 0 & 0 & 0 & 0 & 0 & 0 & 0 & 0 \\
\hline OTHER-RAD & 0 & 0 & 0 & 0 & 0 & 0 & 0 & 0 & 0 & 0 \\
\hline TOIAL RAD & 0 & 0 & 0 & 0 & 0] & 0 & OI & 01 & 0] & 0. \\
\hline & & & & & & & & & & \\
\hline TCLPEXTRACTION & 0 & 0 & 0 & 0 & 0 & 0 & 0 & 0 & o & 0 \\
\hline OTHER-PREP & 0 & 0 & 0 & 0 & 0 & 0 & 0 & 0 & $\overline{0}$ & 0 \\
\hline OTHER-EXPLO & 0 & 0 & 0 & 0 & 0 & 0 & 0 & 0 & 0 & 0 \\
\hline OTHER-BIO & 0 & 0 & 0 & 0 & 0 & $\mathbf{0}$ & 0 & 0 & 0. & 0 \\
\hline OTHER-PHYS & 0 & 0 & 0 & 0 & 0 & 0 & 0 & 0 & 0 & a. \\
\hline OTHER & 0 & 0 & 0 & 0 & 0 & 0 & 0 & 0 & 0 & (0) \\
\hline TOTAL OTHEA & 0 & 0 & 0 & 0 & 01 & 0. & 0 & DI & 이 & 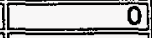 \\
\hline & & & & 7 & 1 & 7 & & 7 & & \\
\hline TOTAL SAMPLEANALYSES & 0 & - & 1070 & 0 & of & 0] & 535 & & 535] & 070 \\
\hline
\end{tabular}


EM-263 SURVEY OF DOE ANALYTICAL CAPACITY AVAILABLE TO EM

Table Na: UC Sample Analyses by Rad Level and Matrix Type (xxix) WSRC-SRTC-ADS

\begin{tabular}{|c|c|c|c|c|c|c|c|c|c|c|}
\hline \begin{tabular}{|l} 
Rad-Matrix \\
Samp Anal
\end{tabular} & RO & Fo/Ri & मा & $\mathrm{F2}$ & $\overline{\mathrm{R3}}$ & $\overline{\mathrm{R} 4}$ & TM1 & $\overline{M 2}$ & M3 & Total \\
\hline VOA-GC & 0 & 0 & 346 & 0 & 0 & 0 & 208 & 69 & 69 & 346 \\
\hline VOA-GCMS & 0 & 0 & 346 & 0 & 0 & 0 & 208 & 69 & 69 & 346 \\
\hline SEMI-VOA & of & $\overline{0}$ & 0 & 0 & 0 & 0 & 0 & 0 & 0 & 0 \\
\hline PEST & of & 0 & 0 & 0 & o) & 0 & 아 & 0 & 요 & 0 \\
\hline HERB & of & 0 & 0 & 0 & 0 & 0 & 0 & 0 & 0. & 0 \\
\hline PCB & of & 0 & 0 & 0 & 0 & 0 & of & 0 & a & $\overline{0}$ \\
\hline PCB/PEST & 0 & 0 & 0 & 0 & 0 & 0. & of & 요 & 묘 & $\mathbf{0}$ \\
\hline OTHER-ORG & 0 & 0 & 0 & 0 & 0 & 0 & of & 0 & 요 & 0 \\
\hline TOTAL OAGANICS & 0 & $\underline{0}$ & 692 & OI & O) & 0. & 415 & 138 & 138 & 692 \\
\hline & & & & & & & & & & \\
\hline ICP-AES METALS & 0 & 0 & 3466 & 0 & 0 & $\overline{0}$ & 1733 & 1387 & 347 & 3466 \\
\hline ICP-MSMETALS & 0 & 0 & 142 & 의 & 0 & (0) & 71 & 43 & 28 & 142 \\
\hline GF-AAMETALS & 0 & 0 & 0 & 0) & 0 & D: & 0 & 0 & 0 & 0 \\
\hline MERCURY & 0 & 0 & 769 & 0 & 0 & 0. & 381 & 311 & 78 & 769 \\
\hline CYANIDE & 0 & 0 & of & 0 & of & - & 0 & 0 & 0. & 0 \\
\hline ANIONS/CATIONS & 0 & 0 & 2870 & 0 & 0 & ㅁ․ & 2296 & 430 & 143 & 2870 \\
\hline WET CHEM & 0 & 0 & 0 & 0 & 0 & - & 0 & 0 & 0 & 0 \\
\hline OTHER-INORG & 0 & 0 & 0 & 0 & 0. & 이 & 0 & 0 & 0 & 0 \\
\hline TOTAL INOAGANICS & 01 & 0 & 7247 & 01 & 01 & 0] & 44811 & 2170 & 596 [ & 7247 \\
\hline & & & & & & & & & & \\
\hline GROSS ALPHA/BETA & 0 & 0 & 1952 & 0 & 0 & - & 1171 & 586 & 195 & 1952 \\
\hline ALPHADET-Np237 & 0 & a & 0 & 0 & 0 & -0. & a & 0 & 0 & 0 \\
\hline ALPHA DET-Pu,Am & 0 & 0 & 0 & 0 & 0 & 의 & of & 0 & ㅁ․ & 0 \\
\hline ALPHADET-Pu & 0 & 01 & 0 & 0 & 0 & 애 & 0 & 0 & 에 & 0 \\
\hline ALPHA DET-U & 0 & 0 & 01 & 0 & 0 & 요 & 0 & 0 & 0] & 0 \\
\hline ALPHADET-Am & 0 & 0 & 0 & 0 & o & 0 & 0 & 0 & 0. & 0 \\
\hline ALPHA DET-NP & 0 & 0 & 0 & 0 & 0 & 0 & o & 0 & ㅁ․ & 0 \\
\hline ALPHADET-Th & 0 & 0 & 0 & 0 & 0 & ol & 0 & 0 & 0 & 0 \\
\hline ALPHA DET - U,Th,Pu & o & 0 & 0 & 0 & 0 & 0. & 0 & 0 & 0 & o \\
\hline ALPHADET-U235 & 요 & 0 & 0 & 0 & of & ㅇ․ & 0 & 0 & o & 0 \\
\hline ALPHA DET-PU,U,Am & 0 & 0 & 0 & 0 & 0 & ㅁ․ & 0 & 0 & 0 & 0 \\
\hline ALPHA DET-U,Np,Pu,Am & 0 & 0 & 0 & 0 & of & ㅇ․ & 0 & 0 & 으. & 0 \\
\hline ALPHADET-Ra226 & 0 & of & 0 & 0 & 0 & 묘 & 0 & 0 & 0 & 0 \\
\hline ALPHA DET-Ra228 & 0 & 0 & 0 & 0 & 0 & 요 & 0 & of & 0 & 0 \\
\hline ALPHA SPEC & 0 & 0 & 67 & 0 & 0 & 애 & 339 & 271 & 68 & 677 \\
\hline GPC-BETA-Pb210 & o & 0 & 0 & 0 & 0 & 0 & 0 & of & 0 & 0 \\
\hline GPC-BETA-Sr90 & 0 & of & 0 & 0 & O & of & 0 & 0 & o. & 0 \\
\hline GPC-BETA-Pm147 & 0 & 0 & 0 & 0 & 0 & 의 & 0 & 의 & 요 & 0 \\
\hline GPC-BETA-Se79 & 0 & 0 & 0 & 0 & 0 & 0] & 0 & 0 & o] & 0 \\
\hline LSC-BETA-H3 & 0 & 可 & 0 & 0 & 0 & 요 & 0 & of & 요 & 0 \\
\hline LSC-BETA-Rn222 & 0 & 0 & 0 & 0 & 0 & 0. & 0 & 0 & 0 & 0 \\
\hline LSC-BETA- 1129 & 0 & 0 & 0 & 0 & O & 0] & 0 & 0 & 0] & 0 \\
\hline LSC-BETA-C14 & 0 & 0 & 0 & 0 & 0 & 0] & 0 & 0 & 0 & 0 \\
\hline LSC-BETA-TC99 & 0 & 0 & 0 & 0 & o & 0 & 0 & 0 & 0 & $\overline{0}$ \\
\hline LSC-BETA & 0 & ot & 0 & 0 & 0 & 0. & 0 & of & 0 & 0 \\
\hline GAMMASPEC & 0 & 0 & 1451 & 0 & of & o. & 870 & 435 & 145 & 1451 \\
\hline OTHER-AAD & 0 & 0 & 0 & 0 & 01 & 0] & \begin{tabular}{l|l}
0 & \\
\end{tabular} & 0 & o] & 0 \\
\hline TOTALRAD & 01 & 0 & 4080 & $\begin{array}{lll}0 & 1 \\
\end{array}$ & OI & 0 & 2380 & 1292 & $408]$ & 4080 \\
\hline & & & & & & & & & & \\
\hline TCLP EXTRACTION & 0 & 0 & 24 & 0 & 0 & 0 & 7 & 15 & 2 & 24 \\
\hline OTHER-PREP & 0 & 0 & 0 & 0 & 0) & 51 & 5 & 31 & 15. & 51 \\
\hline OTHER-EXPLO & का & 0 & 0 & 0 & of & 0 & 0 & 0 & ㅇ․ & 0 \\
\hline OTHER-BIO & 01 & 0 & 0 & 0 & 0 & ㅇ․ & 0 & 0 & 으 & 0 \\
\hline OTHER-PHYS & 01 & 0 & 0 & 0 & 0 & 묘 & 0 & 0 & 0. & 0 \\
\hline OTHER & 01 & 0 & 10815 & 0 & 0 & 27 & 5421 & 3252 & 2168 & 10842 \\
\hline TOTAL OTHER & 01 & 01 & 10839 & 0 & 이 & 78 & 5433 & 3298 & $2186]$ & 10917 \\
\hline & & & & & 4 & $\square$ & & & & \\
\hline TOTAL SAMPIE ANALYSE & 01 & 01 & 22857 & 01 & 01 & 78 & 12709 & 6898| & 3329 & 22935 \\
\hline
\end{tabular}


EM-263 SUAVEY OF DOE ANALYTICAL CAPACITY AVAILABLE TO EM

Table IVa: UC Sample Analyses by Rad Level and Matrix Type (xox) WSRC-SRTC-DWPT

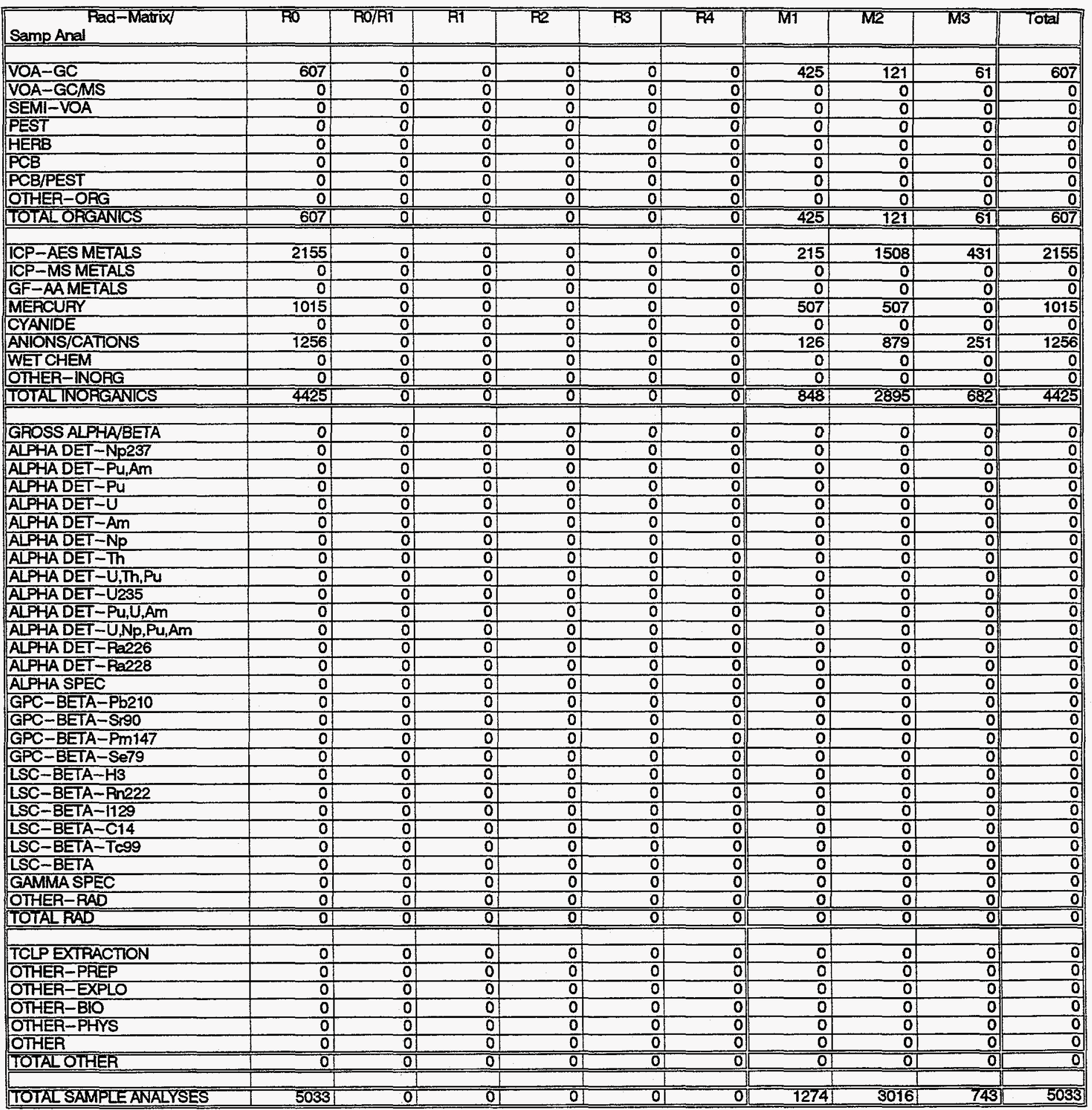


EM-263 SUAVEY OF DOE ANALYTICAL CAPACITY AVAILABLE TO EM

Table IVb: EC Sample Analyses by Pad Level and Matrix Type (i) Complex-wide

\begin{tabular}{|c|c|c|c|c|c|c|c|c|c|c|}
\hline $\begin{array}{l}\text { Rad-Matrix } \\
\text { Samp Anal }\end{array}$ & $\overline{\text { Ro }}$ & RO/R1 & R1 & $\overline{R 2}$ & $\overline{\mathrm{R} 3}$ & R4 & $\overline{\mathrm{M} 1}$ & $\overline{\mathrm{M} 2}$ & M3 & Total \\
\hline VOA-GC & 1858 & 0 & 838 & 0 & 0 & of & 1727 & 343 & 627 & 2697 \\
\hline VOA-GCMS & 14418 & 12750 & 3545 & 590 & 2115 & 98 & 24227 & 6476 & 2813 & 33517 \\
\hline SEMI-VOA & 4614 & 1232 & 2334 & 359 & 91 & o & 6305 & 1722 & 602 & 8629 \\
\hline PEST & 977 & 485 & 793 & 159 & 0 & o & 1339 & 812 & 263 & 2414 \\
\hline HERB & 546 & 466 & 649 & 159 & 이 & - & 1241 & 456 & 123 & 1820 \\
\hline $\mathrm{PCB}$ & 10607 & 12020 & 3942 & 159 & 205 & 이 & 19523 & 4418 & 2992 & 26933 \\
\hline PCB/PEST & 37 & 459 & 0 & 0 & 0 & 0) & 392 & 83 & 22 & 497 \\
\hline OTHER-ORG & 1761 & 253 & 1583 & 59900 & 0 & 0 & 43830 & 822 & 18845 & 63497 \\
\hline TOTALORGANICS & 34818 & 27665 & 13685 & 61326 & 2411 & 98 & 98584! & 15131 & 26288 & 140004 \\
\hline & & & & & & & & & & \\
\hline ICP-AES METALS & 27729 & 47391 & 10774 & 48583 & 1090 & 0 & 61020 & $\overline{42412}$ & 32135 & 135567 \\
\hline ICP-MSMETALS & 24337 & 14907 & 576 & 3408 & 1135 & 0 & 23669 & 15518 & 5177 & 44364 \\
\hline GF-AAMETALS & 22355 & 50388 & 2194 & 18102 & 0 & o. & 43662 & 27512 & 21866 & 93040 \\
\hline MEACURY & 32945 & 23630 & 3055 & 14575 & 972 & 1696 & 43619 & 14282 & 18973 & 76874 \\
\hline CYANIDE & 1615 & 8536 & 1798 & 49302 & 662 & 묘 & 33138 & 3262 & 25513 & 61913 \\
\hline ANIONS/CATIONS & 21051 & 53510 & 11201 & 35992 & 2101 & 777 & 82527 & 14726 & 27379 & 124631 \\
\hline WET CHEM & 0 & 2970 & 0 & 5578 & 0 & 0 & 3856 & 1097 & 3595 & 8548 \\
\hline OTHER-INORG & 65145 & 80849 & 34631 & 31941 & 9239 & 807 & 152362 & 37708 & 32542 & 222613 \\
\hline TOTAL INORGANICS & 195178 & 282181 & 64230 & 207482 & 15200 & 3280 & 443854 & 156517 & 167179 & 767550 \\
\hline GROSS ALPHABETA & 165855 & 83669 & 198985 & 2836 & 3004 & 1449 & 237610 & 197978 & 20209 & 455708 \\
\hline ALPHA DET -Np237 & 0 & 5477 & 0 & 0 & 0 & 0 & 2027 & 0 & 3451 & $\frac{400 / 50}{5477}$ \\
\hline ALPHA DET-PU,Am & 0 & 12042 & 0 & 0 & 0 & a & 6871 & 2868 & 2303 & 12042 \\
\hline ALPHADET-Pu & 5322 & 2353 & 5657 & 0 & 0 & 0 & 3377 & 4550 & 5404 & 13331 \\
\hline ALPHA DET-U & 4497 & 1087 & 7118 & 0 & 0. & 0 & 4728 & 6468 & 1507 & 12700 \\
\hline ALPHADET-Am & 1600 & 0 & 0 & 0 & 0 & 0 & 642 & 894 & 63 & 1600 \\
\hline ALPHADET-Np & 1921 & of & 0 & 0 & 0 & 0. & 96 & 864 & 960 & 1921 \\
\hline ALPHADET-Th & 1265 & 1155 & 0 & 0 & 0) & 0 & 917 & 737 & 766 & 2420 \\
\hline ALPHADET - U,Th,PU & 4300 & of & 0 & 0 & 0 & 0 & 3857 & 443 & 0 & 4300 \\
\hline ALPHADET-U235 & 0 & of & 4689 & 0 & 0 & 0 & 2321 & 1114 & 1253 & 4689 \\
\hline ALPHA DET-Pu,U,Am & 11449 & 0 & 0 & 0 & 0 & 0 & 11163 & 286 & 0 & 11449 \\
\hline ALPHA DET-U,Np,Pu,Am & 0 & 0 & 0 & $\overline{0}$ & 0 & 0 & 0 & 0 & (0) & 0 \\
\hline ALPHADET-Ra226 & 0 & 744 & 0 & 0 & 0 & 0 & 558 & 149 & 37 & 744 \\
\hline ALPHA DET-Ra228 & 0 & 567 & 0 & $\overline{0}$ & 0 & - & 113 & 426 & 28 & 567 \\
\hline ALPHA SPEC & 6097 & 14595 & 4466 & 199 & 0 & 855 & 16808 & 7009 & 2395 & 26212 \\
\hline GPC-BETA-Pb210 & 0 & 609 & 0 & 0 & 0 & 0 & 536 & 73 & 0 & 609 \\
\hline GPC-BETA-Sr90 & 0 & 11671 & 733 & 0 & 0 & 0 & 7077 & 3194 & 2133 & 12404 \\
\hline GPC-BETA-Pm147 & 0 & 4997 & 0 & 0 & o & 0 & 4997 & 0 & 0 & 4997 \\
\hline GPC-BETA-Se79 & 으. & 775 & 의 & 0 & o & 0. & 775 & 0 & 0 & 775 \\
\hline LSC-BETA-H3 & 9442 & 14588 & 4076 & 0 & 0 & 0 & 21171 & 6676 & 259 & 28106 \\
\hline LSC-BETA-Rin222 & 0 & 421 & 0 & 0 & 0 & 0 & 421 & 0 & 요 & 421 \\
\hline LSC-BETA- 1129 & 0 & 1022 & of & 0 & 0 & 0 & 1022 & 0 & 0 & 1022 \\
\hline LSC-BETA-C14 & 0 & 1641 & 0 & 0 & 0 & D & 896 & 0 & 745 & 1641 \\
\hline LSC-BETA-TC99 & 0 & 19228 & 0 & 0 & 0 & D & 15077 & 1384 & 2767 & 19228 \\
\hline LSC-BETA & 0 & 0 & 2566 & $\overline{0}$ & 0 & 0 & 2566 & Oा & 0 & 2566 \\
\hline GAMMMASPEC & 11268 & 61122 & 27545 & 3461 & 0 & 875 & 67718 & 19140 & 17413 & 104271 \\
\hline OTHER-PAD & 0 & 9762 & 3780 & 0 & 0 & 0 & 7685 & 3905 & 1952 & 13542 \\
\hline TOTAL RAD & 223015 & 247525 & 259615] & 6496 & 3004 & 3178 & 421029 & 258158 & 63646 & 742833 \\
\hline & & & & & & & & & & \\
\hline TCLP EXIRACTION & 3581 & 6920 & 535 & 0 & 1553 & 547 & 3196 & 6945 & 2997 & 13137 \\
\hline OTHER-PREP & 3950 & 1719 & 764 & 81150 & 666 & 2686 & 28445 & 5522 & 56968 & 90936 \\
\hline OTHER-EXPLO & 1319 & 0 & 0 & 0 & 0 & 0 & 1319 & 0 & - & 1319 \\
\hline OTHER-BIO & 2576 & 1772 & 0 & 0 & 0 & 0 & 4347 & 0 & 0 & 4347 \\
\hline OTHER-PHYS & 6240 & 6161 & 7631 & 74558 & 0 & 0 & 74223 & 8540 & 11827 & 94590 \\
\hline OTHER & 0 & 0 & 10815 & 0 & 0 & 27 & 5421 & 3252 & 2168 & 10842 \\
\hline TOTAL OTHER & 17666 & 16572 & 19745 & 155708 & 2220 & 3260 & 116951 & 24259 & 73960 & 215170 \\
\hline & & & & & & & & & & \\
\hline TOTAL SAMPLE ANALYS & 470677 & 573943 & 357274 & 431012 & 22835 & 9816 & 1080418 & 454065 & 331074 & 1865557 \\
\hline
\end{tabular}


EM-263 SURVEY OF DOE ANALYTICAL CAPACITY AVAILABLE TO EM

Table Mb: EC Sample Analyses by Rad Level and Matrix Type (ii) Grand Junction

\begin{tabular}{|c|c|c|c|c|c|c|c|c|c|c|}
\hline $\begin{array}{l}\text { Rad-Matrix } \\
\text { Samp Anal }\end{array}$ & RO & RO/R1 & R1 & R2 & $\overline{R 3}$ & $\overline{\mathbf{R 4}}$ & M1 & M2 & $\overline{143}$ & Total \\
\hline & & & & & & & & & & \\
\hline VOA-GC & 0 & 0 & 0 & 0 & 0 & 0 & 0 & 0 & o & $\overline{0}$ \\
\hline VOA-GCMS & D & 1297 & 0 & 0 & 0 & 0 & 882 & 415 & 0. & 1297 \\
\hline SEMI-VOA & 0 & 507 & 0 & 0 & 0 . & 0 & 395 & 112 & ôt & 507 \\
\hline PEST & 0 & 0 & 0 & 0 & 0 & 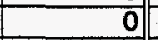 & 0 & 0 & o. & 0 \\
\hline HERB & 0 & 322 & 0 & 0 & 0 & 0 & 293 & 29 & 애 & 322 \\
\hline$\overline{P C B}$ & 0 & 0 & 0 & 0 & 0 & o & 0 & 0 & o & 0 \\
\hline PCB/PEST & 0 & 459 & 0 & 0 & 0 & 0 & 377 & 83 & 0 & 459 \\
\hline OTHER-ORG & 0 & 253 & 0 & 0 & 0 & 0 & 223 & 30 & 0 & 253 \\
\hline TOTAL ORGANICS & 0 & 2838 & 이 & 01 & 인 & 0] & 2169 & 668 & 0] & 2838 \\
\hline & & & & & & & & & & \\
\hline ICP-AESMETALS & 0 & 11486 & 0 & 0 & 0 & 0 & 4020 & 7466 & 0 & 11486 \\
\hline ICP-MSMETALS & 0 & 8846 & 0 & 0 & 0 & 0 & 2742 & 6104 & 0 & 8846 \\
\hline GF-AAMETALS & 0 & 5169 & 0 & 0 & 0 & 0 & 1861 & 3308 & o & 5169 \\
\hline MERCURY & 0 & 2297 & 0 & 0 & 0 & 0 & 1103 & 1195 & 0 & 2297 \\
\hline CYANIDE & 0 & 3101 & of & 0 & 0 & 0 & 2636 & 465 & of & 3101 \\
\hline ANIONS/CATIONS & 0 & 2286 & 0 & 0 & of & 0 & 734 & 1553 & of & 2286 \\
\hline WET CHEM & 0 & 0 & 0) & 0 & 0. & 요 & 0 & 0| & o] & 0 \\
\hline OTHER-INORG & 0 & 0 & of & 01 & 이 & 0 & 0 & 0 & o. & 0 \\
\hline TOTAL INORGANICS & $\overline{0}$ & 33185 & 01 & 0 & 01 & 0 & 13095 & 20090 & $0]$ & 33185 \\
\hline & & & & & & & & & 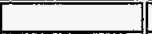 & \\
\hline GROSS ALPHA/BETA & 0 & 2825 & of & 0 & 0 & o. & 591 & 2234 & 0 & 2825 \\
\hline ALPHA DET-Np237 & 0 & 0 & ot & 0 & 0 & of & of & 0 & 0. & 0 \\
\hline ALPHA DET - Pu,Am & 0 & of & 0 & 0 & 0 & o. & 0 & 0 & 0 & 0 \\
\hline ALPHADET-PU & 0 & 0 & of & 0 & of & 0 & 0 & 0 & o. & 0 \\
\hline ALPHA DET -U & 0 & of & 0 & 0 & DI & o. & 0 & of & D. & 0 \\
\hline ALPHADET-Am & $\overline{0}$ & 0 & of & 0 & 0 & 0 & 0 & 0 & D & 0 \\
\hline ALPHADET-Np & 0 & 0 & 0 & 0 & 0 & 0. & 0 & 0 & (0) & 0 \\
\hline ALPHADET-Th & 0 & 0 & 0 & 0 & 0 & of & 0 & 0 & 0 & 0 \\
\hline ALPFIA DET-U,Th,PU & 0 & 0 & o. & 0 & 0 & 0] & of & 0 & 0 & 0 \\
\hline ALPHADET-U235 & 0 & 0 & o & 0 & 0 & o| & 0 & 0 & 0] & 0 \\
\hline ALPHADET-PU,U,Am & 0 & 0 & 0 & 0 & D. & 0] & 0 & 0 & o. & 0 \\
\hline ALPHA DET - U,Np,Pu,Am & 0 & 0 & 0 & 0 & 0 & 0. & 0 & 0 & 0. & 0 \\
\hline ALPHA DET-Ra226 & 0 & 0 & 0 & 0 & 01 & of & 0 & 0 & 0 & 0 \\
\hline ALPHA DET-Ra228 & 0 & 0) & 0 & 0 & 0 & ㅁ] & 0 & O & 0 & 0 \\
\hline ALPHA SPEC & 0 & 6912 & 0 & D. & 0 & (5) & 2765 & 4147 & o) & 6912 \\
\hline GPC-BETA-Pb210 & 0 & 609 & 0 & D. & 0 & [0] & 536 & 73 & 0 & 609 \\
\hline GPC-BETA-Sr90 & 0 & 1415 & 0 & 0 & 0 & 0 & 141 & 1273 & 0 & 1415 \\
\hline GPC-BETA-Pm147 & 0 & 0 & 0 & 01 & 0 & 0. & 0 & 0 & o & 0 \\
\hline GPC-BETA-Se79 & 0 & 0 & 0 & D) & o) & (0) & 0 & 0 & of & 0 \\
\hline LSC-BETA-H3 & 0 & 595 & 0 & 0) & 0 & 으, & 6 & 589 & 요 & 595 \\
\hline LSC-BETA-Rn222 & 0 & 421 & 0 & of & 0 & o: & 421 & 0 & o & 421 \\
\hline LSC-BETA-I129 & 0 & 0 & 0 & 0 & 0 & of & 0 & o) & 0 & $\overline{0}$ \\
\hline LSC-BETA-C14 & 0 & 0 & 0 & 0 & 0 & 0 & 0 & of & 0 & 0 \\
\hline LSC-BETA-TC99 & 0 & of & 0 & 0 & 0 & 0 & 0 & 0 & o & 0 \\
\hline LSC-BETA & $\overline{0}$ & D) & 0 & 0 & of & 0 & 0 & 0 & 매 & 0 \\
\hline GAMMA SPEC & $\overline{0}$ & 3988 & 0 & 0 & of & (0) & 40 & 3948 & - & 3988 \\
\hline OTHER-RAD & $\overline{0}$ & of & 0 & 0 & 0 & 0 & 0 & 0 & 0 & 0 \\
\hline TOTAL RAD & $\overline{0}$ & 16765 & 0 & 0 & 01 & [0] & 4501 & 12265 & 0 & 16765 \\
\hline & & & & & & & & & & \\
\hline TCLP EXTRACTION & $\overline{0}$ & 0 & 0 & 0 & 01 & 0 & 0 & 0 & o: & 0 \\
\hline OTHER-PREP & 0 & 0 & 0 & 0 & 0 & 0 & 0 & 0 & a & 0 \\
\hline OTHER-EXPLO & 0 & o & 0 & 0 & 0 & 요 & 0 & 0 & 므 & 0 \\
\hline OTHER-BIO & 0 & 0 & 0 & 0 & 0 & o] & 0 & 0 & o & 0 \\
\hline OTHER-PHYS & 0 & 1094 & 0 & 0 & DI & (0) & 0 & 1094 & 0 & 1094 \\
\hline OTHER & 0 & 0 & 0 & 0 & 0 & of & 0 & 0 & 0 & 0 \\
\hline TOTAL OTHER & $\overline{0}$ & 1094 & 0 & 0 & 01 & 0] & 0 & 1094 & 0 & 1094 \\
\hline & & & & & & & & & & \\
\hline TOTAL SAMPLEANALYSES & 0 & 53882 & 0 & 0 & 01 & 에 & 19765 & 34417 & o & 53882 \\
\hline
\end{tabular}




\section{EM-263 SURVEY OF DOE ANALYTICAL CAPACITY AVAILABLE TO EM}

Table IMb: EC Sample Analyses by Rad Level and Matrix Type (iii) Los Alamos Nat'l Lab

\begin{tabular}{|c|c|c|c|c|c|c|c|c|c|c|}
\hline Samp Anal & R0 & RO/R1 & R1 & R2 & $7 \overline{R 3}$ & $\overline{\mathrm{AA}}$ & $\overline{\mathrm{M} 1}$ & $\overline{\mathrm{M} 2}$ & $\overline{M 3}$ & Total \\
\hline & & & & & & & & & & \\
\hline VOA-GC & 0 & 0 & 0 & 0 & 0 & 의 & 0 & 0 & 0. & 0 \\
\hline VOA-GCMS & 1565 & of & 0 & 0 & 0 & 의 & 782 & 555 & 227 & 1565 \\
\hline SEMI-VOA & 1015 & 0 & 0 & 0 & 0 & 0 & 507 & 357 & 151 & 1015 \\
\hline PEST & 0 & 0 & o & 0 & 0 & 0 & 0 & 0 & o. & 0 \\
\hline HERB & 0 & 0 & 0 & 0 & 0 & 0. & 0 & 0 & 0 & $\overline{0}$ \\
\hline PCB & 1453 & 0 & 0 & 0 & 0 & 0. & 467 & 913 & 74 & 1453 \\
\hline PCB/PEST & 0 & of & 0 & 0 & 0 & 0 & 0 & 0 & 0. & 0 \\
\hline OTHER-ORG & 0 & 0 & 0 & 0, & 0 & 0 & 0 & 01 & 0. & 0 \\
\hline TOTALORGANICS & 4033 & D| & 0 & 0 & 0 & 0 & 1756 & 1824] & 453 & 4033 \\
\hline & & & & & & & & & & \\
\hline ICP-AES METALS & 2830 & 0 & 0 & 0 & (0) & 0. & 1555 & 1135 & 140 & 2830 \\
\hline ICP-MSMETALS & 1278 & 0 & a. & 0 & 0 & 0 & 973 & 237 & 67 & 1278 \\
\hline GF-AAMETALS & 3455 & 0 & 0 & 0 & 0 & 0 & 2536 & 757 & 162 & 3455 \\
\hline MERCURY & 939 & 0 & 0 & 0 & 0 & 0. & 699 & 195 & 46 & 939 \\
\hline CYANIDE & 239 & o & 0 & 0 & 0 & 0 & 216 & 23 & 0 & 239 \\
\hline ANIONS/CATIONS & 239 & 0 & 0 & 0 & 0 & 0 & 216 & 23 & 0. & 239 \\
\hline WET CHEM & 0 & 0 & 0 & 0 & 우 & 0 & 0 & 0 & 0 & 0 \\
\hline OTHER-INORG & 754 & 0 & 0 & 0 & 0 & 0 & 378 & 301 & 74 & 754 \\
\hline TOTAL INORGANICS & 9733 & 이 & 01 & 01 & 0 & 0 & 6573 & 26711 & 489 & 9733 \\
\hline & & & & & & & & & & \\
\hline GROSS ALPHAVBETA & 2347 & 0 & 아 & 01 & 0 & 의 & 1458 & 774 & 114 & 2347 \\
\hline ALPHA DET-Np237 & 0 & 0 & 0 & 0 & 0 & 0 & 0 & 0 & 0. & 0 \\
\hline ALPHA DET-PU,Am & 0 & 0 & 0 & 0 & 0 & 0 & 0 & 0 & 0 & 0 \\
\hline ALPHADET-Pu & 5322 & 0 & 0 & 0 & 0 & 0 & 1872 & 3187 & 263 & 5322 \\
\hline ALPHA DET-U & 1532 & 0 & of & 0 & 0 & 0 & 562 & 899 & 72 & 1532 \\
\hline ALPHA DET-Am & 1600 & 0 & 0 & 0 & 0 & 0 & 642 & 894 & 63 & 1600 \\
\hline ALPHADET-Np & 0 & o & 0 & 0 & 0 & 0 & 0 & 0 & o. & 0 \\
\hline ALPHADET-Th & 0 & 0 & o & 0 & 0 & 0 & 0 & 0 & 요 & 0 \\
\hline ALPHA DET-U,Th,Pu & 0 & 0 & 0 & of & 0 & 0 & 0 & 0 & (0) & 0 \\
\hline ALPHA DET-U235 & 0 & o & of & 0 & 0 & 0 & 0 & 이 & 묘 & 0 \\
\hline ALPHA DET-PU,U,Am & 0 & 0. & 0 & 0 & 0 & 0 & $\begin{array}{ll}0 & \\
\end{array}$ & O) & D. & 0 \\
\hline ALPHA DET - U,Np,Pu,Am & 0 & 0 & 0 & 0 & 0 & 0 & 0 & 요 & 요 & 0 \\
\hline ALPHA DET-Pa226 & 0 & 0 & 0 & 0 & 0 & 0 & 0 & 0 & D. & $\overline{0}$ \\
\hline ALPHA DET-Pa228 & 0 & 0 & 0 & of & 0 & 0 & 0 & 0 & 에 & 0 \\
\hline ALPHA SPEC & 0 & 0 & 이 & 0 & 0 & - & 0 & 0 & 의 & 0 \\
\hline GPC-BETA-Pb210 & 0 & 0 & 0 & 0 & 0 & D & 0 & 0 & 의 & 0 \\
\hline GPC-BETA-Sr90 & 0 & 0 & of & 0 & $\mathbf{0}$ & 0 & 0 & 0 & 0 & $\overline{0}$ \\
\hline GPC-BETA-Pm147 & 0 & D) & of & 0 & 0 & (0) & 0 & 0 & 의 & 0 \\
\hline GPC-BETA-Se79 & 0 & 이 & 0 & 01 & 0 & 0 & 0 & 0 & 요 & $\overline{0}$ \\
\hline LSC-BETA-H3 & 5605 & 0 & 0 & 0 & 0 & 0 & 3450 & 1896 & 259 & 5605 \\
\hline LSC-BETA-Rn222 & 0 & D. & 0 & 0 & 0 & 0 & 0 & 0 & 0 & $\underline{0}$ \\
\hline LSC-BETA-1129 & 0 & 0 & 요 & 0 & 0 & 0 & 0 & 0 & 0 & $\underline{\mathbf{0}}$ \\
\hline LSC-BETA-C14 & 0 & 0 & 0 & 0 & 0 & 0 & 0 & 0 & 으 & 0 \\
\hline LSC-BETA-TC99 & 0 & of & 0 & 0 & 0 & 0 & 0 & 0 & 0. & 0 \\
\hline LSC-BETA & 0 & 이 & 0 & 0 & 0 & 0 & 0 & 0 & 0 & $\overline{0}$ \\
\hline GAMMA SPEC & 3946 & 0 & of & 0 & 0 & $\overline{0}$ & 2437 & 1324 & 185 & 3946 \\
\hline OTHER-RAD & 0 & 0 & of & 01 & 0 & 0 & 0 & 0 & 0 & 0 \\
\hline TOTAL RAD & 20351 & 01 & of & 01 & $\overline{0}$ & 0 & 10421 & 8973 & 956 & 20351 \\
\hline & & & & & & & & & & \\
\hline TCLP EXTRACTION & 0 & 0 & O & 0 & 0 & 0 & 0 & 0 & 0 & 0 \\
\hline OTHER-PREP & 0 & 0 & 0 & 0 & 0 & 0 & 0 & 0 & 0 & 0 \\
\hline OTHER-EXPLO & 0 & 0 & 0 & 0 & 0 & 0 & 0 & 0 & 0 & 0 \\
\hline OTHER-BIO & 0 & o & 0 & 0 & 0 & 0 & 은 & 0 & 0 & 0 \\
\hline OTHER-PHYS & $\overline{0}$ & 0 & 0 & 0 & 0 & 0 & $\overline{0}$ & 0 & 0 & 0 \\
\hline OTHER & 0 & 0 & of & 0 & 0 & 0 & 0 & 0 & 0 & $\underline{0}$ \\
\hline TOTAL OTHER & 0 & 0 & 01 & 01 & 0 & 0 & 0 & 0 & 0 & $\underline{0}$ \\
\hline & & & & & & & & & & \\
\hline TOTAL SAMPLEANAL & $\overline{34117}$ & 0 & 0 & 0 & $\overline{0}$ & 0 & 18751 & 13469 & 1898 & 34117 \\
\hline
\end{tabular}


EM-263 SURVEY OF DOE ANALYTICAL CAPACITY AVAILABLE TO EM

Table IV: EC Sample Analyses by Rad Level and Matrix Type (iv) Argonne East

\begin{tabular}{|c|c|c|c|c|c|c|c|c|c|c|}
\hline $\begin{array}{l}\text { Rad-Matrix } \\
\text { Samp Anal }\end{array}$ & $\mathbf{F O}$ & Ro/R1 & Ri & $\mathrm{A2}$ & $\mathrm{RB}$ & $\overline{74}$ & $\overline{\mathrm{MT}}$ & $\overline{\mathrm{M} 2}$ & $\overline{\mathrm{MB}}$ & Total \\
\hline & & & & & & & & & & \\
\hline VOA-GC & 0 & 0 & 0 & 0 & 0 & 0 & 0 & 0 & 0 & 0 \\
\hline VOA-GCMS & 145 & 0 & 14 & 0 & 0 & 0 & 136 & 17 & 6 & 159 \\
\hline SEMI-VOA & 0 & 23 & 338 & 0 & 0 & 0 & 217 & 0 & 145 & 361 \\
\hline PEST & 0 & 0 & 0 & 0 & 0 & 0 & 0 & \begin{tabular}{l|l}
0 & \\
\end{tabular} & 0. & 0 \\
\hline HERB & 0 & 0 & 0 & 0 & 0 & 0 & 0 & 0 & 0 & 0 \\
\hline PCB & 0 & 0 & 0 & 0 & 0 & 0 & 0) & 0 & of & o \\
\hline PCB/PEST & 37 & 0 & 0 & 0 & 0 & 0 & 15 & 1 & 22 & 37 \\
\hline OTHER-ORG & 0 & 01 & 0 & 0 & 0 & O) & 0 & 0 & 0 & 0 \\
\hline TOTAL ORGANICS & 182 & 231 & 352 & 0 & 01 & 0] & 368 & 18 & $172]$ & 558 \\
\hline & & & & & & 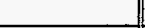 & & & & \\
\hline ICP-AESMETALS & 0 & 4511 & 416 & 0 & 416 & 0 & 1542 & 1234 & 2566 & 5342 \\
\hline ICP-MSMETALS & 0 & 0 & 0 & 0 & 0 & o & 0 & 0 & 0 & 0 \\
\hline GF-AAMEIALS & 0 & 1302 & 0 & 0 & 0 & o! & 65 & 781 & 456 & 1302 \\
\hline MERCURY & 0 & 1730 & 0 & 0 & 0 & 0 & 1211 & 346 & 173 & 1730 \\
\hline CYANIDE & 0 & 1952 & 0 & 0 & 0 & 0 & 1952 & 0 & 0 & 1952 \\
\hline ANIONS/CATIONS & 0 & 0 & 0 & 0 & o & 0] & 0 & 0 & 0 & 0 \\
\hline WET CHEM & 0 & 0 & 0 & 0 & 0 & 0 & 0 & 0 & 0 & 0 \\
\hline OTHER-INORG & 01 & 0 & 0 & $0 \mid$ & 0 & 0 & 0 & 0 & 0] & 0 \\
\hline TOTAL INORGANICS & 01 & 9496 & 416 & 01 & 416 & 0 & 4770 & 2362 & 3195 & 10327 \\
\hline & & & & & & & & & & \\
\hline GROSS ALPHAVBETA & 0 & o) & 444 & 0 & 0 & 0 & 177: & 133 & 133 & 444 \\
\hline ALPHA DET-Np237 & 0 & 0) & 0 & 0 & 0 & 0 & 0 & 0 & 0 & 0 \\
\hline ALPHA DET-PU,Am & 0 & 0 & 0 & 0 & 0 & 0 & 0 & 0 & 0 & 0 \\
\hline ALPHA DET-PU & 0 & 0 & 0 & 0 & 0 & 0 & 0 & 0 & 0 & 0 \\
\hline ALPHADET-U & 0 & $\mathbf{0}$ & 0 & 0 & 0 & 0 & 0 & 0 & o] & 0 \\
\hline ALPHADET-Am & 0 & 0 & 0 & 0 & 0 & 0 & 0 & 0 & 0 . & 0 \\
\hline ALPHA DET-Np & 0 & 0 & 0 & 0 & of & o & 0 & 0 & 0 & 0 \\
\hline ALPHA DET-Th & 0 & 0 & 0 & 0 & 0 & 0 & 0 & 0 & 0 & 0 \\
\hline ALPHADET-U,Th,Pu & 0 & 0 & 0 & 0 & 0 & 0 & 0 & 0) & 0 & 0 \\
\hline ALPHA DET-U235 & 0 & 0 & 0 & 0 & 0 & 0 & 0 & 0 & 0 & 0 \\
\hline ALPHA DET-PU,U,Am & 0 & 0 & 0 & 0 & 0 & 0 & 0 & 0 & $\overrightarrow{0}$ & 0 \\
\hline ALPHA DET-U,Np,Pu,Am & 0 & 0 & 0 & 0 & 0 & 0 & 0 & 0 & 0 & 0 \\
\hline ALPHA DET-Ra226 & 0 & 0 & 0 & 0 & 0 & o & 0 & 0 & 0. & 0 \\
\hline ALPHA DET-Ra228 & 0 & 0 & 0 & 0 & 0 & o & 0 & 0 & 0 & 0 \\
\hline ALPHA SPEC & 0 & 01 & 265 & 0 & 0 & 0 & 0 & 265 & D: & 265 \\
\hline GPC-BETA-Pb210 & 0 & 0 & 0 & 0 & 0 & o. & 0 & 0 & 0 & 0 \\
\hline GPC-BETA-Sr9O & 0 & 0 & 0 & 0 & 0 & o. & 0 & 0 & 0: & 0 \\
\hline GPC-BETA-Pm147 & 0 & 0 & 0 & 0 & 0 & 0 & \begin{tabular}{l|l}
0 \\
\end{tabular} & 0 & 0 & 0 \\
\hline GPC-BETA-Se79 & $0 !$ & 0 & 0 & 0 & 0 & o & 0 & 0 & 0. & 0 \\
\hline LSC-BETA-H3 & 01 & 0 & 0 & 0 & $\overline{01}$ & 0 & 0 & 0 & 0. & 0 \\
\hline LSC-BETA-Rr222 & 0 & 0 & 0 & 0 & 0 & 0. & 0 & 0 & 0. & 0 \\
\hline LSC-BETA- 1129 & 0 & 0 & 0 & 0 & 0 & 0 & 0 & 0 & 0 & 0 \\
\hline LSC-BETA-C14 & 0 & 0 & 0 & 0 & 0 & O) & 0 & 0 & 0 & 0 \\
\hline LSC-BETA-Tc99 & 0 & 0 & 0 & 0 & 0 & D. & 0 & 0 & 0 & 0 \\
\hline LSC-BETA & 0 & 0 & 0 & 0 & 0 & 0. & 0 & o & 0 & 0 \\
\hline GAMMA SPEC & 0 & 0 & 241 & 0 & 0 & O) & 96 & 72 & 72 & 241 \\
\hline OTHER-PAD & 0 & 0 & 01 & 0 & 0 & 0. & 0 & 0 & 0 & $\mathbf{0}$ \\
\hline TOTAL RAD & 0 & DI & 9501 & of & 01 & D. & 274 & 471 & 205 & 950 \\
\hline & & & & & & & & & & \\
\hline TCLPEXTRACTION & 0 & 776 & 0 & 0 & 0 & 0 & 776 & 0 & 0 & 776 \\
\hline OTHER-PREP & 0 & 0 & 0 & 0 & 0 & 0 & 01 & 0 & 0 & 0 \\
\hline OTHER-EXPLO & 0 & 0 & 0 & 0 & 0 & 0. & 0 & 0 & 0 & 0 \\
\hline OTHER-BIO & 0 & 0 & 0 & 0 & 0 & 0. & 0 & 0 & 0 & 0 \\
\hline OTHER-PHYS & 0 & 0 & 0 & 0 & 0 & D. & 01 & 0 & $\mathbf{0}$ & 0 \\
\hline OTHER & 0 & 0 & 0 & 0 & 0 & 0] & 0 & 01 & o. & 0 \\
\hline TOTAL OTHER & 0 & 776 & 0 & 01 & 01 & 0 & 776 & 0] & $\mathbf{0}$ & 776 \\
\hline & & & & 4 & & 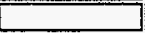 & & & & \\
\hline TOTAL SAMPLE ANALYSES & 182 & 10295 & 1717 & OI & 416 & $\mathbf{0}$ & 6188 & 2851 & 3572 & 12610 \\
\hline
\end{tabular}


EM-263 SURVEY OF DOE ANALYTICAL CAPACTY AVAILABLE TO EM

Table IVb: EC Sample Analyses by Rad Level and Matrix Type (v) Argonne West

\begin{tabular}{|c|c|c|c|c|c|c|c|c|c|c|}
\hline $\begin{array}{l}\text { Rad-Matrix } \\
\text { Samp Anal } \\
\end{array}$ & 190 & RojR1 & Fit & $\mathrm{R} 2$ & $\mathrm{R3} 3$ & $\overline{\mathrm{R4}}$ & $\mathrm{MI}$ & $\overline{M 2}$ & T13 & Total \\
\hline VOA-GC & & & & 0 & & & & & $\pi$ & \\
\hline VOA-GCMS & 0 & 0 & of & 0 & 0 & 0 & 0 & 0 & 0 & 0 \\
\hline SEMI-VOA & 0 & 0 & 0 & 0 & 0 & 0 & 0 & 0 & 0 & 0 \\
\hline PEST & 0 & 0 & 0 & 0 & 0 & 0 & o & 0 & of & 0 \\
\hline HERB & 0 & 0 & of & 0 & 0 & 0 & 0 & 0 & 0 & $\overline{0}$ \\
\hline PCB & 0 & o & of & 0 & 0 & D & 0 & 0 & 0. & 0 \\
\hline PCB/PEST & D & 0 & 0 & 0 & 0 & (0) & 0 & 0 & ㅇ․ & 0 \\
\hline OTHER-ORG & 0 & 0 & 0 & 0 & 0 & 0 & 0 & 0 & 0 & 0 \\
\hline TOTAL ORGANICS & 0 & $\overline{0}$ & 01 & 0 & 0 & $\overline{0}$ & 01 & 0 & 0 0] & 0 \\
\hline & & & & & & & & 1 & 7 & \\
\hline ICP-AES METALS & D & (0) & 0) & 0 & 0 & 0 & 0 & 0 & o. & $\mathbf{0}$ \\
\hline ICP-MSMETALS & 0 & 0 & 0 & 0 & 0 & 0 & 0 & 0 & ㅇ․ & 0 \\
\hline GF-AAMETALS & 0 & 0 & 0 & 0 & 0 & 0 & 0 & 0) & 요 & 0 \\
\hline MERCURY & 0 & a & 01 & 0 & 0 & - & 0 & 0 & (0) & $\overline{0}$ \\
\hline CYANIDE & 0 & 0 & of & 0 & 0 & 0 & 0 & 0 & 0 & $\overline{0}$ \\
\hline ANIONS/CATIONS & 0 & 0 & 0 & 0 & 0 & 0 & 0 & 0 & 0 & 0 \\
\hline WET CHEM & 0 & 0 & 0 & 0 & 0 & 0 & 0 & 0 & 0] & 0 \\
\hline OTHER-INORG & 0 & 0 & 0 & 0 & 0 & 0 & 0 & 0 & 0] & 0 \\
\hline TOTALINORGANICS & (0) & $\overline{01}$ & 01 & 01 & $\overline{0}$ & 0 & 01 & 01 & $0]$ & $\overline{0}$ \\
\hline & & & & & & & & & ] & 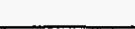 \\
\hline GROSS ALPHA/BETA & 0 & 0 & 0 & 0 & 0 & 0 & 0 & 0 & 에 & 0 \\
\hline ALPHA DET-Np237 & 0 & 0 & 0 & 0 & 0 & 0 & 0 & 0 & 요 & 0 \\
\hline ALPHADET-PU,Am & 0 & of & 0 & 0 & 0 & o & 0 & 0 & of & 0 \\
\hline ALPHADET-PU & 0 & 0 & 0 & 0 & 0 & o & 0 & 0 & of & 0 \\
\hline ALPHADET -U & 0 & 0 & 0 & 0 & 0 & 0 & 0 & 0 & of & $\mathbf{0}$ \\
\hline ALPHA DET-Am & 0 & 0 & 0 & of & 0 & 0 & 0 & 0 & - & 0 \\
\hline ALPHA DET-NP & 0 & 0) & 0 & 0 & 0 & 0 & 0 & 0 & o & 0 \\
\hline ALPHADET-Th & 0 & 0 & 0 & 0 & 0 & 0 & 0 & D. & 요 & 0 \\
\hline ALPHA DEI-U,Th,Pu & 0 & 0 & 0 & 0 & 0 & - & 0 & 0 & 에 & 0 \\
\hline ALPHAD DET-U235 & 0 & 0 & 0 & 0 & 0 & 0 & 0 & 0 & 요 & $\overline{0}$ \\
\hline ALPHADET-PU,U,Am & D & 0 & 0 & 0 & 0 & 0 & 0 & 0 & o. & 0 \\
\hline ALPHA DET-U,Np,Pu,Am & 0 & 0 & 0 & 0 & 0 & 0 & 0 & 0 & 0. & 0 \\
\hline ALPHADET-Ra226 & 0 & 0 & a & 0 & 0 & 0 & 0 & 0 & of & 0 \\
\hline ALPHA DET-Ra228 & $\mathbf{0}$ & 0 & o & 0 & 0 & 0 & 0 & 0 & 묘 & 0 \\
\hline ALPFAA SPEC & 0 & 0 & 0 & 0 & 0 & 의 & 0 & 0 & 에 & 0 \\
\hline GPC-BETA-Pb210 & 0 & 0) & 0 & 0 & 0 & 0 & 0 & 0 & (0) & 0 \\
\hline GPC-BETA-Sr90 & 0 & 0 & 0 & 0 & 0 & 0 & 0 & 0 & - & 0 \\
\hline GPC-BETA-Pm147 & 0 & 0 & $\mathbf{0}$ & o. & D & 0 & 0 & 0 & 0 & 0 \\
\hline GPC-BETA-Se79 & o & 0 & 0 & of & 0 & 0 & o & 0 & ㅁ. & 0 \\
\hline LSC-BETA-H3 & 0 & 0 & 0 & 0 & 0 & o & 0 & 0 & 이 & 0 \\
\hline LSC-BETA-Rn222 & 0 & 0 & 0 & 0 & 0 & 0 & 0 & 0 & - & 0 \\
\hline LSC-BETA-1129 & 0 & 0 & 0 & 0 & 0 & 0 & 0 & 0 & 의 & 0 \\
\hline LSC-BETA-C14 & $\overline{0}$ & 0 & a & 0 & 0 & 0 & 0 & 0 & 0 & 0 \\
\hline LSC-BETA-TC99 & $\overline{0}$ & 0 & 0 & $0 \mid$ & 0 & o: & $\overline{0}$ & 0 & 0 & 0 \\
\hline LSC-BETA & 0 & 0. & O & 0 & 0 & D & 0 & 0 & 0. & 0 \\
\hline GAMMA SPEC & 0 & 0 & 0 & of & 0 & o & 0 & 0 & 0 & 0 \\
\hline OTHER-RAD & 0 & 0 & 0 & 0 & 0 & 0] & 0 & 0 & 0] & 0 \\
\hline TOTAL RAD & 0 & $\overline{0}$ & 01 & 01 & 0 & 0] & 0 & 01 & 0. & 0 \\
\hline & & & & & & & & & & \\
\hline TCLP EXTRACTION & 0 & 0 & 0 & 0 & 0 & 0. & 0 & o. & (0) & 0 \\
\hline OTHER-PREP & 0 & 0 & 0 & of & 0 & o. & 0 & 0 & 0 & 0 \\
\hline OTHER-EXPLO & 0 & 0 & 0 & 0 & 0 & 0 & 0 & 0 & 0 & 0 \\
\hline OTHER-BIO & 0 & 0 & 0 & 0 & 0 & D. & $\overline{0}$ & 0 & 0. & 0 \\
\hline OTHER-PHYS & 0 & 0 & 0 & 0 & 0 & 0 & 0 & 0 & 0. & 0 \\
\hline OTHER & 0 & 0 & 0 & 0 & 0 & 0 & 0 & 0 & 0] & $\overline{0}$ \\
\hline TOTAL OTHEA & 0 & $\overline{0}$ & 0 & 01 & 0 & 0 & 0 & 0 & $0]$ & 0 \\
\hline & & & & & & & & & & \\
\hline TOTAL SAMPLEANALYSE & 0 & 0 & 0 & 01 & 0 & 0] & 0 & o & की & $\overline{0}$ \\
\hline
\end{tabular}


EM-263 SUAVEY OF DOE ANALYTICAL CAPACTY AVAILABLE TO EM

Table IV: EC Sample Analyses by Rad Level and Matrix Type (vi) Brookhaven Nat'I Lab

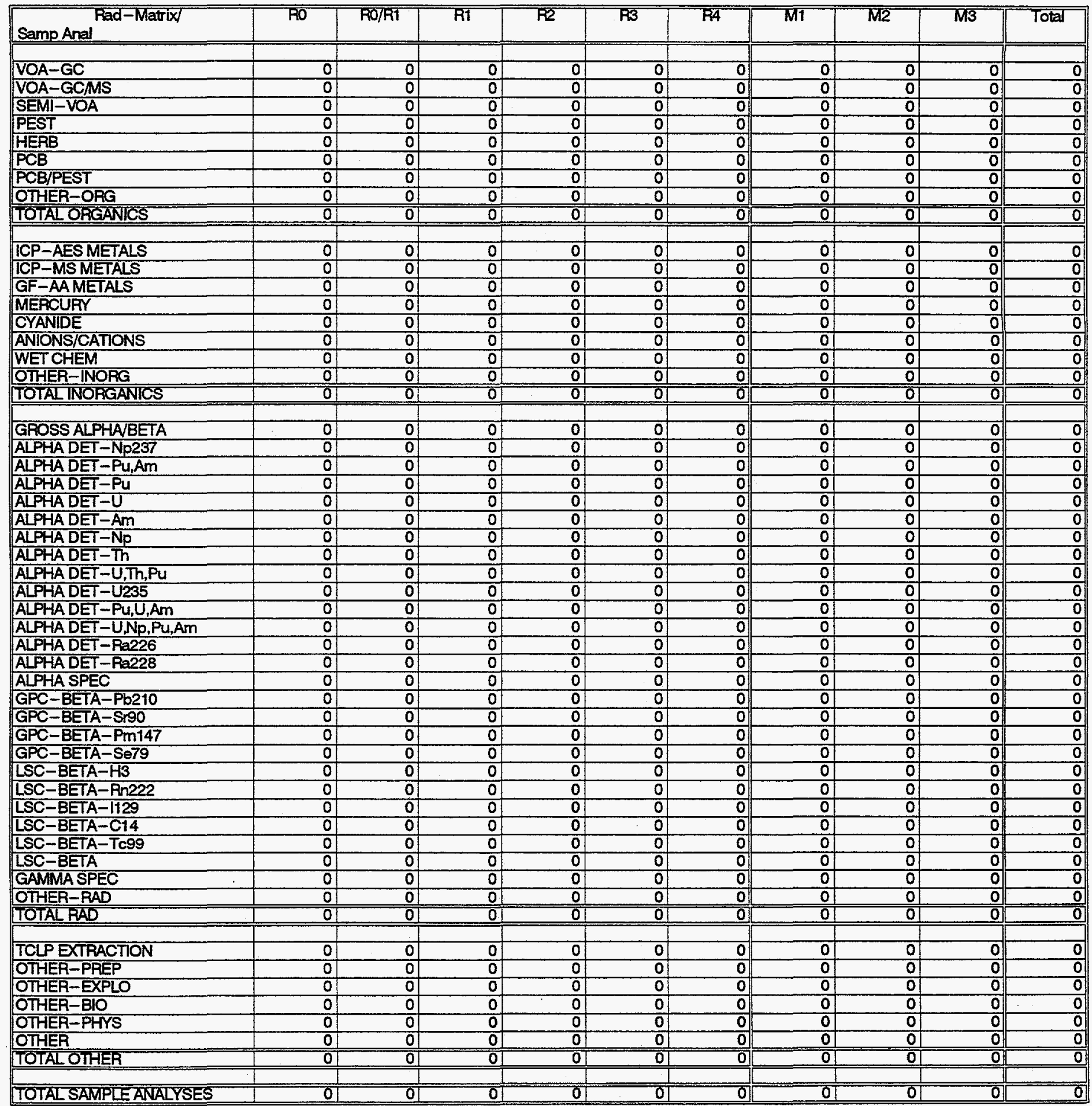


Table IVb: EC Sample Analyses by Rad Level and Matrix Type (vii) Fernald

\begin{tabular}{|c|c|c|c|c|c|c|c|c|c|c|}
\hline $\begin{array}{l}\text { Rad-Matrix } \\
\text { Samp Aral }\end{array}$ & RO & FO/RT & $\overline{\mathrm{R1}}$ & $\mathrm{R2}$ & R3 & $\mathrm{RA}$ & $\overline{\text { MT }}$ & $\mathrm{ML}$ & $\overline{\mathrm{M} 3}$ & Total \\
\hline & & & & & & & & & & \\
\hline VOA-GC & 0 & 0 & 0 & 0 & 0 & 0 & 0 & 0 & 0 & 0 \\
\hline VOA-GCAMS & 0 & 1745 & 0 & 0 & 0 & 0 & 872 & 872 & 0 & 1745 \\
\hline SEMI-VOA & 0 & 0 & 0 & 0 & 0 & 0 & 0 & 0 & 0 & 0 \\
\hline PEST & 0 & 0 & 0 & 0 & 0 & 0 & 0 & 01 & 0 & 0 \\
\hline HERB & 0 & o & 0 & 0 & 0 & 0 & 0 & 0 & 0 & 0 \\
\hline PCB & 0 & 0 & 0 & 0 & 0 & 0 & 0 & 0 & 0 & $\mathbf{0}$ \\
\hline PCB/PEST & 0 & 0 & 0 & 0 & 0 & 0 & 0 & 0 & 0 & 0 \\
\hline OTHER-ORG & 0 & 0 & 0 & 0 & 0 & 0 & 0 & 01 & o] & 0 \\
\hline IOIALORGANICS & $\overline{01}$ & 1745 & 0 & $0 \mid$ & 0 & D. & 872 & 872 & 0 & 1745 \\
\hline & & & & & & & & & & \\
\hline ICP-AESMETALS & 0 & 4937 & 0 & 0 & 0 & 0 & 2222 & 2715 & 0 & 4937 \\
\hline ICP-MSMETALS & 0 & 1677 & 0 & 0 & 0) & 0 & 168 & 755 & 755 & 1677 \\
\hline GF-AAMETALS & 0 & 16768 & 0 & 0 & 0 & 0 & 12405 & 4362 & 0 & 16768 \\
\hline MERCURY & 0 & 5989 & 0 & 0 & 0 & 0 & 3594 & 2396 & o) & 5989 \\
\hline CYANIDE & 0 & 0 & 0 & 0 & 0 & 0) & 0 & 0 & 0 & 0 \\
\hline ANIONS/CATIONS & 0 & 1563 & 0 & 0 & 0 & o & 1563 & 0 & o) & 1563 \\
\hline WET CHEM & 0 & 2970 & 0 & 0 & 0 & 0 & 1188 & 1059 & 723 & 2970 \\
\hline OTHER-INORG & 0 & 21964 & 0 & 0 & 0 & 0 & 16056 & 5908 & 0 & 21964 \\
\hline TOTALINORGANICS & 0 & 55868 & 0 & 01 & 01 & 0. & 37196 & 17194 & 1478 & 55868 \\
\hline & & & & & & $\rightarrow$ & & 1 & & \\
\hline GROSS ALPHAVBEIA & 0 & 5818 & 0 & 0 & 0 & 0 & 624 & 4881 & 312 & 5818 \\
\hline ALPHA DET-Np237 & 0 & 0 & 0 & 0 & 0 & 0 & 0 & 0 & of & 0 \\
\hline ALPHADET-Pu,Am & 0 & 0 & 0 & 0 & 0 & 0 & 0 & 0 & 0 & 0 \\
\hline ALPHADET-Pu & 0 & 0 & 0 & 0 & 0 & 0. & 0 & 0 & 0 & 0 \\
\hline ALPHA DET-U & 0 & 1087 & 0 & 0 & 0 & $\overline{0}$ & 1087 & 0 & 0 & 1087 \\
\hline ALPHA DET-Am & 0 & 0 & 0 & 0 & 0 & 0 & 0 & 0 & 0 & O \\
\hline ALPHA DET-Np & 0 & 0 & 0 & 0 & 0 & $\mathbf{0}$ & 0 & 0 & 0 & 0 \\
\hline ALPHADET-Th & 0 & 1155 & 0 & 0 & 0 & $\mathbf{0}$ & 866 & 231 & 58 & 1155 \\
\hline ALPHA DET -U,Th,Pu & 0 & 0 & 0 & 0 & 0 & $\mathbf{0}$ & 0 & 0 & of & 0 \\
\hline ALPHA DET-U235 & 0 & 0 & 0 & 0 & 0 & 0. & 0 & 0 & 0 & $\overline{0}$ \\
\hline ALPHADET-PU,U,Am & 0 & 0 & 0 & 0 & 0 & 0 & 0 & 0 & 0. & 0 \\
\hline ALPHA DET-U,Np,PU,Am & 0 & o & 0 & 0 & 0 & D. & 0 & 0 & 0 & 0 \\
\hline ALPHA DET-Ra226 & 0 & 744 & 0 & 0 & 0 & 0. & 558 & 149 & 37 & 744 \\
\hline ALPHA DET-Ra228 & 0) & 567 & 0 & 0 & 0 & D. & 113 & 426 & 28 & 567 \\
\hline ALPHA SPEC & 0 & 0 & 0 & 0 & 0 & 0 & 0 & 0 & 0.) & $\mathbf{0}$ \\
\hline GPC-BETA-Pb210 & 0 & 0 & 0 & 0 & 0 & 0 & 0 & 0 & 0) & 0 \\
\hline GPC-BETA-Sr90 & 0 & 0 & 0 & 0 & 0 & 0 & 0 & 0 & 0 & 0 \\
\hline GPC-BETA-Pm147 & 0 & 0 & 0 & 0 & 0 & 0 & 0 & 0 & 0 & 0 \\
\hline GPC-BETA-Se79 & 0 & 0 & 0 & 0 & 0 & D. & O: & 0 & (0) & D \\
\hline LSC-BETA-H3 & 0 & 0 & 0 & 0 & 0 & 0 & 0 & 0 & 0 & 0 \\
\hline LSC-BETA-Rn222 & 0 & 0 & 0 & 0 & 0 & 0 & 0 & 0 & 0 & 0 \\
\hline LSC-BETA-1129 & 0 & 0 & 0 & 0 & 0 & 0 & 0 & 0 & 0 & 0 \\
\hline LSC-BETA-C14 & 0 & 0! & 0 & 0 & 0 & 0 & o) & 0 & 0. & 0 \\
\hline LSC-BETA-TC99 & 0 & 0 & 0 & 0 & 0 & 0 & 0 & 0 & 0 & 0 \\
\hline LSC-BEIA & 0 & 0 & 0 & 0 & 0 & $\mathbf{0}$ & 0 & 0 & 0 & 0 \\
\hline GAMMA SPEC & 0 & 0 & 0 & 0 & 0 & 0 & 0 & 0 & 0) & 0 \\
\hline OTHER-RAD & 0 & 9762 & 0 & 0 & 0 & $\mathbf{0}$ & 3905 & 3905 & 1952 & 9762 \\
\hline TOTAL RAD & 0 & 19133 & $\overline{0}$ & 01 & 0 & 0] & 7154 & 9591 & 2388 & 19133 \\
\hline & & & & & & & & & -1 & \\
\hline TCLPEXTRACTION & 0 & 3344 & 0 & 0 & 0 & $\overline{0}$ & 0 & 3344 & 0 & 3344 \\
\hline OTHEA-PREP & 0 & 0 & 0 & 0 & 0 & 0 & 0 & 0 . & 0 & 0 \\
\hline OTHER-EXPLO & 0 & 0 & 0 & 0 & 0 & 0. & 0 & 0 & O) & - \\
\hline OTHER-BIO & 0 & 0 & 0 & 0 & 0 & 0 & 0 & 0 & 0 & $\underline{0}$ \\
\hline OTHER-PHYS & 0 & 5067 & 0 & 0 & 0 & o & 1208 & 3037 & 822 & 5067 \\
\hline OTHER & 0 & $\overline{0}$ & 0 & 0 & 0 & 0] & 0 & 0 & 0 & 0 \\
\hline TOTAL OTHER & 0 & 8411 & 0 & 01 & 0 & 0 & 1208 & 63811 & 822] & 8411 \\
\hline & & & & & & & & & & \\
\hline TOTAL SAMPLE ANALYSES & 0 & 85157 & 0 & 01 & 0 & 0 & 46430 & 34038 & 4688 & 85157 \\
\hline
\end{tabular}


EM-263 SURVEY OF DOE ANALYTICAL CAPACTY AVAILABLE TO EM

Table IVb: EC Sample Analyses by Rad Level and Matrix Type (viii) Idaho Nat'l Eng Lab - ACU

\begin{tabular}{|c|c|c|c|c|c|c|c|c|c|c|}
\hline $\begin{array}{l}\text { Rad-Matrix } \\
\text { Samp Anal }\end{array}$ & Ro & RO/RT & R1 & $\overline{\mathrm{R} 2}$ & R3 & $\overline{\mathrm{R} 4}$ & $\overline{M 1}$ & M12 & $\overline{M 3}$ & Total \\
\hline VOA-GC & 0 & & & & & & & & & \\
\hline$\frac{\text { VOA-GC }}{\text { VOA-GCMS }}$ & $\frac{0}{1216}$ & $\begin{array}{l}0 \\
0\end{array}$ & $\frac{0}{0}$ & 0 & 0. & 0 & 0 & 0 & 0 & 0 \\
\hline & $\frac{1<10}{0}$ & $\frac{0}{0}$ & \begin{tabular}{l|l}
0 \\
0
\end{tabular} & 0 & 0 & 0 & 972 & 219 & 24 & 1216 \\
\hline SEMI-VOA & 0 & 0 & 0 & 0 & 0 & 0 & 0 & 0 & 0 & 0 \\
\hline PEST & 0 & 0 & 0 & 0 & 0 & 0 & 0 & 0 & 0 & 0 \\
\hline HERB & 0 & of & 0 & 0 & 0 & 0 & 0 & 0 & o. & 0 \\
\hline PCB & 0 & 이 & 0 & 0 & 0 & 0 & 0 & 0 & 0 & 0 \\
\hline PCB/PEST & 0 & of & 0 & 0 & 0 & 0 & 0 & 0 & o. & 0 \\
\hline OTHER-ORG & 0 & 0 & 0 & 0 & 0 & 0 & 0 & of & o. & 0 \\
\hline TOTAL ORGANICS & 1216 & OI & 0 & 01 & 01 & $\overline{0}[$ & 972 & 219 & 24[ & 1216 \\
\hline & & & & & & & 1 & 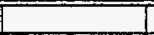 & $=1$ & \\
\hline ICP-AESMETALS & 0 & 0 & 0 & 0 & 0 & 0 & 0 & 0 & 0 & 0 \\
\hline ICP-MS METALS & 0 & 0 & 0 & 0 & 0 & 0 & 0 & 0. & 0. & 0 \\
\hline GF-AAMETALS & 0 & 0 & 0 & 0 & 0 & 0 & 0 & 0 & 0 & 0 \\
\hline MERCURY & 0 & 0 & 0 & 0 & 0 & 0 & 0 & 0 & 0. & 0 \\
\hline CYANIDE & 0 & 0] & 0 & 0 & 0 & 0 & 0 & 0 & 0. & 0 \\
\hline ANIONS/CATIONS & 0 & 01 & 0 & 0 & 0 & 0 & 0 & 0 & 0 & 0 \\
\hline WET CHEM & 0 & 0 & 0 & 0 & 0 & 0 & 0 & 0 & 0 & 0 \\
\hline OTHER-INORG & 0. & 01 & 0 & 0 & 0 & 0 & 0 & 0 & 0 & 0 \\
\hline TOTALINOAGANICS & 01 & 01 & 0 & 01 & 0 & $0]$ & ot & 0 & 0] & 0 \\
\hline & & & & & & & & & & \\
\hline GROSS ALPHA/BETA & 0 & 0 & 0 & 0 & 0 & 0 & 0 & 0 & 0. & 0 \\
\hline ALPHA DET-Np237 & 0 & 0 & 0 & 0 & 0 & 0 & 0 & 0 & 0. & 0 \\
\hline ALPHA DET-PU,Am & 0 & 0 & 0 & 0 & 0) & 0 & 0 & 0 & 0 & 0 \\
\hline ALPHA DET-Pu & a) & 0 & 0 & \begin{tabular}{l|l}
0 & \\
\end{tabular} & 0 & 0. & 0 & of & 0 & 0 \\
\hline ALPHA DET -U & of & 0 & 0 & 0 & 0 & 0 & 0 & 0 & 0 & 0 \\
\hline ALPHA DET-Am & 0 & 0 & 0 & 0 & 0 & 0 & 0 & 0 & 0. & 0 \\
\hline ALPHADET-NP & 0| & 0 & 0 & o & 0 & 0 & 0 & 0 & 0 . & 0 \\
\hline ALPHA DET-Th & 0 & 0 & 0 & 0 & of & 0 & 0 & 0 & 0 & 0 \\
\hline ALPHA DET-U,Th,Pu & 0 & 0 & 0 & 0 & of & 0 & 0 & 0 & 0 & 0 \\
\hline ALPHADET-U235 & 0 & 0 & 0 & 0 & 0 & 0 & 0 & 0 & 0 & 0 \\
\hline ALPHA DET-PU,U,Am & O) & 0 & 0 & 0 & of & 0. & 0 & 0 & 0) & 0 \\
\hline ALPHA DET-U,Np,PU,Am & o & 0 & 0 & 0 & 0 & 0. & 0 & 0 & 0 & 0 \\
\hline ALPHADET-Ra226 & 0 & 0 & a) & 0 & of & 0] & 0 & 0 & 0] & 0 \\
\hline ALPHA DET-Ra228 & 0 & 0 & of & 0 & of & o. & 0 & 0 & 0) & $\mathbf{0}$ \\
\hline ALPHA SPEC & 0 & O) & 0 & 0 & O| & 0] & 0 & 01 & 0 & 0 \\
\hline$G P C-B E T A-P b 210$ & 0 & 0 & of & 0 & of & 요 & 0 & of & of & 0 \\
\hline GPC-BETA-Sr90 & of & 0 & 0 & 0 & 0 & 0 & 0 & 0 & 0 & 0 \\
\hline GPC-BETA-Pm147 & 0 & 0 & 0 & 0 & of & 0 & 01 & 0 & 0 & 0 \\
\hline GPC-BETA-Se79 & 0 & 0 & 0 & 0 & 0 & 0 & of & 0 & 0. & 0 \\
\hline LSC-BETA-H3 & 0 & 0 & 0 & 0 & 0 & o. & of & 0 & of & 0 \\
\hline LSC-BETA-Pn222 & 0 & 0 & 0 & 0 & 0 & 0 & 0 & 0 & 0 & 0 \\
\hline LSC-BETA-1129 & 0 & 0 & 0 & o & 0 & (0) & 0 & 0 & 0 & 0 \\
\hline LSC-BETA-C14 & 0 & 0 & O) & o! & 0 & o & of & o & 0 & 0 \\
\hline LSC-BETA-TC99 & 0 & 0 & 0 & 0 & 0 & 0 & 0 & 0. & o & 0 \\
\hline LSC-BETA & 0 & 0 & 0 & o & 0 & 0 & 0 & 0 & 0 & 0 \\
\hline GAMMA SPEC & 0 & 0 & 0 & 0 & 0 & 0 & 0 & 의 & 으. & 0 \\
\hline OTHER-RAD & 0 & 0 & D. & 0 & 0 & 0 & 0 & of & 이 & 0 \\
\hline TOTAL RAD & 0 & 0 & 0 & 01 & 0 & 0 & 0 & 01 & 이 & 0 \\
\hline & & & & & & & & & 75 & \\
\hline TCLPEXTRACTION & 0 & 0 & 0 & 0 & 0 & 0. & 0 & 0 & 0 & 0 \\
\hline OTHER-PREP & 0 & 0 & 0 & 0 & 의 & 이 & 0 & 0 & 0. & 0 \\
\hline OTHER-EXPLO & $\mathbf{0}$ & 0 & 0 & 0 & 0 & 0] & 0 & 0 & D. & 0 \\
\hline OTHER-BIO & 0 & o) & o & 0 & 0 & 매 & 0 & 0 & 0. & 0 \\
\hline OTHER-PHYS & 0 & 0 & 0 & 0 & o & - & 0 & 0 & (0) & 0 \\
\hline OTHER & 0 & 0 & 0 & 0 & 이 & 요 & 0 & 0 & 0 & 0 \\
\hline TOTAL OTHER & 0 & 0 & 0 & 01 & 0 & 0] & 0 & 0 & 0 & 0 \\
\hline & & & & & 1 & ] & ] & 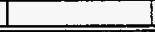 & $\sqrt{t}$ & $=$ \\
\hline TOTAL SAMPLEANALYSE & 1216 & 0] & $\overline{0}$ & 0 & (0) & 0 이 & 972 & 279 & 24 & 1216 \\
\hline
\end{tabular}


EM-263 SURVEY OF DOE ANALYTICAL CAPACTY AVAILABLE TO EM

Table IVb: EC Sample Analyses by Rad Level and Matrix Type (ix) Idaho Nat'l Eng Lab - B\&W

\begin{tabular}{|c|c|c|c|c|c|c|c|c|c|c|}
\hline $\begin{array}{l}\text { Pad-Matrix } \\
\text { Samp Anal }\end{array}$ & RO & RO/R1 & TH & $R^{2}$ & R3 & $\overline{744}$ & MT & M2 & $\mathrm{M3}$ & Total \\
\hline VOA-GC & 0 & 0 & 0 & 0 & & n & & & & \\
\hline VOA-GCMS & 0 & 0 & 0 & 0 & 0 & $\frac{0}{0}$ & $\frac{0}{0}$ & 花 & $\frac{0}{0}$ & $\frac{0}{0}$ \\
\hline SEMI-VOA & o & 0 & 0 & 0 & 0 & of & 0 & 0 & 0 & 0 \\
\hline PEST & 0 & 0 & 0 & 0 & 0 & ;. & 0 & 0 & 0 & 0 \\
\hline HERB & 0 & 0 & 0 & 0 & 0 & 0 & 0 & 0 & 0 & 0 \\
\hline PCB & 0 & 0 & 0 & D & 0 & 0 & $\overline{0}$ & 0 & 0 & 0 \\
\hline PCB/PEST & 0 & 0 & $\overline{0}$ & 0 & 0 & 0] & $\overline{0}$ & of & 0) & 0 \\
\hline OTHER-ORG & 0 & 0 & 0 & 0 & 0 & 0 & 0 & 0 & 0 & 0 \\
\hline TOTAL OAGANICS & 0 & 0] & $\overline{0}$ & DI & 0] & 0] & D. & 01 & 0 & 0 \\
\hline & & & & & & & & & & \\
\hline ICP-AESMETALS & 0 & 0 & $\overline{0}$ & 0 & 0 & 0 & 0 & 0 & 0 & $\overline{0}$ \\
\hline ICP-MSMETALS & 0 & 0 & 0 & 0 & 0 & of & o & 0 & 0 & 0 \\
\hline GF-AAMETALS & 0 & 1942 & 0 & 0 & 0 & of & 422 & 971 & 549 & 1942 \\
\hline MERCUAY & 0 & 1361 & 0 & 0 & 0 & o. & 234 & $\begin{array}{ll}680 \\
\end{array}$ & 446 & 1361 \\
\hline CYANDDE & 0 & 0 & 0 & 0 & a) & 0 & 0 & 0 & 0 & 0 \\
\hline ANIONS/CATIONS & 0 & 471 & 0 & 0 & 0 & of & 471 & 0 & $\overline{0}$ & 471 \\
\hline WET CHEM & 0 & 0 & 0 & 0 & 0 & of & 0 & 0 & 0 & 0 \\
\hline OTHER-INORG & 0 & 4161 & 0 & of & of & of & 661 & 2080 & 1420 & 4161 \\
\hline TOTAL INORGANICS & 0 & 7935 & 0 & 0 & 01 & 01 & 1787 & 3732 & $2 \overline{2416}$ & 7935 \\
\hline & & & & & & 7 & 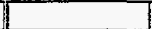 & 1 & 7 & \\
\hline GROSS ALPHAVETA & 0 & 0 & 0 & 0 & 0 & 0 & 0 & 0 & 0 & $\mathbf{0}$ \\
\hline ALPHA DET-Np237 & 0 & o & 0 & D. & 0 & o) & 0 & 0 & - & 0 \\
\hline ALPHA DET-Pu,Am & 0 & o. & 0 & 0 & 0 & a) & 0 & 0 & 0 & $\overline{0}$ \\
\hline ALPHADET-Pu & 0 & 0 & 0 & 0 & 0) & 0 - & 0 & 0 & 0. & 0 \\
\hline ALPHA DET-U & 0 & 0 & $\mathbf{0}$ & 0 & 0| & 0 & 0 & 0 & D. & 0 \\
\hline ALPHA DET-Am & 0 & 0 & 0 & 0 & 0 & o & 0 & 0 & D. & 0 \\
\hline ALPHA DET-Np & 0 & 0 & $\overline{0}$ & 0 & 0) & 0 - & 0 & 0 & 0. & 0 \\
\hline ALPHA DET-Th & 0 & 0 & 0 & 0 & 01 & a) & 0 & 의 & 므 & $\overline{0}$ \\
\hline ALPHA DET - U,Th,PU & 0 & 0 & $\overline{0}$ & 0 & 0 & 0 & 0 & 0 & (0) & 0 \\
\hline ALPHA DET-U235 & 0 & 0 & 0 & 0 & 0 & 0 & 0 & 0 & -0 & 0 \\
\hline ALPHA DET-Pu,U,Am & 0 & 0 & 0 & 0 & 0 & 0 & 0 & 0 & 0 & $\overline{0}$ \\
\hline ALPHA DET-U,Np,PU,Am & 0 & D) & $\overline{0}$ & D & 0) & o. & 0 & 0 & D. & $\overline{0}$ \\
\hline ALPHA DET-Ra226 & 0 & o. & 0 & o & 01 & o & of & 0 & o. & 0 \\
\hline ALPHADET-Ra228 & o & 0 & 0 & o. & 0 & 0. & o & 0 & 0 & 0 \\
\hline ALPHA SPEC & 0 & 0 & 0 & 0 & 0 & 0. & 0 & 0 & ot & 0 \\
\hline GPC-BETA-Pb210 & 0 & 0 & 0 & 0 & O & 요 & D. & 0 & D. & 0 \\
\hline GPC-BETA-Sr90 & 0 & $\overline{0}$ & 0 & o & 0 & of & 0 & 0 & $\overline{0}$ & $\overline{0}$ \\
\hline GPC-BETA-Pm147 & 0! & 0 & $\mathbf{0}$ & 0 & 0 & 0 - & D) & 0 & 0 & 0 \\
\hline GPC-BETA-Se79 & 0 & 0 & 0 & 0 & o & 0] & D) & 0 & 0 & 0 \\
\hline LSC-BETA-H3 & 0 & 0 & $\overline{0}$ & 0 & 0 & 0. & o & 0 & 0 & 0 \\
\hline LSC-BETA-Rn222 & 0 & o) & 0 & 0 & 0 & 0 & D & 0 & 0 & 0 \\
\hline LSC-BETA-1129 & 0 & 0 & 0 & 0 & of & o. & 0 & 0 & (0) & $\overline{0}$ \\
\hline LSC-BETA-C14 & 0 & 0 & 0 & 0 & 0 & 0. & 0 & 0 & 0 & 0 \\
\hline LSC-BETA-TC99 & 0 & o & 0 & 0 & 0 & of & D & 0 & 0. & 0 \\
\hline LSC-BETA & 0 & $\overline{0}$ & 0 & D. & 0 & o. & D & 0 & D. & 0 \\
\hline GAMMA SPEC & 0 & 0 & 0 & 0) & a) & o. & 0 & 0 & 0 & 0 \\
\hline OTHER-RAD & 0 & 0 & 0 & 0 & a & o] [ & 0 & $0 \mid$ & 0 & 0 \\
\hline TOTAL RAD & 0 & O) & 0 & 0 & 0 & oL & 0 & $0 \mid$ & 0 & 0 \\
\hline & & & & & & & & & & \\
\hline TCLP EXTRACTION & 0 & 779 & 0 & 0 & 0 & 0 & 0 & 390 & 390 & 779 \\
\hline OTHER-PREP & 0 & 0 & 0 & 0 & of & 0 & 0 & 0 & 0 & 0 \\
\hline OTHER-EXPLO & 0 & 0 & 0 & 0 & 0 & 0 & 0 & 0 & 0 & 0 \\
\hline OTHER-BIO & 0 & 1772 & 0 & o & 0 & o. & 1772 & 0 & 0 & 1772 \\
\hline OTHER-PHYS & 0 & 0 & 0 & 0 & 0 & o & 0 & 0 & 0. & $\overline{0}$ \\
\hline OTHER & 0 & 0 & 0 & 0 & 0 & 요 & 0 & 0 & 0. & $\overline{0}$ \\
\hline TOTAL OTHER & 01 & 2551 & 0 & 0 & 01 & o] [ & 1772 & 390 & 390 & 2551 \\
\hline & & & & & & & & & & \\
\hline TOTAL SAMPLE ANALYSE & 0] & 10486 & 0 & 0 & O) & O][ & 3559 & 41221 & 2805 & 10486 \\
\hline
\end{tabular}


EM-263 SURVEY OF DOE ANALYTICAL CAPACITY AVAILABLE TO EM

Table IVb: EC Sample Analyses by Rad Level and Matrix Type (x) Idaho Nat'I Eng Lab - WINCO

\begin{tabular}{|c|c|c|c|c|c|c|c|c|c|c|}
\hline Samp Anal & RO & RO/R1 & RI & $\overline{\mathrm{R} 2}$ & $\overline{\mathrm{R3}}$ & $\overline{R 4}$ & $\overline{\mathrm{MT}}$ & M2 & $1 \mathrm{M3}$ & Total \\
\hline VOA-GC & 0 & 0 & 0 & 0 & o. & 0 & 0 & 0 & 0 & 0 \\
\hline VOA-GCMS & 0 & 0 & 324 & 0 & 0 & 98 & 290 & 110 & 23 & 422 \\
\hline SEMI-VOA & 0 & 0 & 0 & 0 & 0 & 0 & 0 & of & 0 & 0 \\
\hline PEST & 0 & 0 & 159 & 0 & o) & 0 & 111 & 32 & 16 & 159 \\
\hline HERB & 0 & 0 & 15 & 0 & 0 & 0. & 10 & 3 & 1 & 15 \\
\hline PCB & 0 & 0 & 36 & 0 & 0 & - & 25 & 7 & 4 & 36 \\
\hline PCB/PEST & 0 & of & 0 & 0 & 0 & ㅁ. & 0 & 0 & D. & 0 \\
\hline OTHER-ORG & 0 & 0 & 1583 & 0 & 0 & 0. & 396 & 791 & 396 & 1583 \\
\hline TOTAL ORGANICS & 아 & 01 & 2116 & 0 & 01 & 98: & 832 & 943 & 439 & 2214 \\
\hline & & & & & & & & & & \\
\hline ICP-AES METALS & 0 & 0 & 1994 & 0 & 0! & 0 & 764 & 957 & 273 & 1994 \\
\hline ICP-MSMETALS & O. & 0 & 269 & 0 & o. & 0 & 54 & 188 & 27 & 269 \\
\hline GF-AAMETALS & D) & 0 & 165 & 0 & D्: & O & 104 & 61 & 0 & 165 \\
\hline MERCURY & O) & 0 & 909 & 0 & 0 & 0 & 461 & 424 & 24 & 909 \\
\hline CYANIDE & 0 & 0 & 1265 & 0 & o & o. & 316 & 633 & 316 & 1265 \\
\hline ANIONS/CATIONS & 0) & 0 & 2678 & 0 & 2101 & 690 & 1367 & 2735 & 1367 & 5469 \\
\hline WET CHEM & 0 & 0 & 0 & O) & of & 0 & 0 & 0 & 0 & 0 \\
\hline OTHEA-INOAG & 0 & 0 & 2548 & of & 861 & 686 & 723 & 2184 & 1188 & 4096 \\
\hline TOTAL INOAGANICS & (0) & 01 & 9829 & 01 & 2962 & 1376 & 3789 & 7182 & 3197] & 14167 \\
\hline GROSSALPHAVBTA & 0 & 0 & 1084 & 0 & 0 & 477 & 562 & 520 & 479 & 1561 \\
\hline ALPHA DET-Np237 & 0 & 0 & 0 & 0 & o & 0 & 0 & 0 & $\frac{410}{0}$ & $\frac{1001}{0}$ \\
\hline ALPHA DET - Pu,Am & 0 & 0 & 0 & 0 & o & 0 - & 0 & o & 0 & 0 \\
\hline ALPHADET-PU & 0 & 0 & 0 & D) & of & 0 & 0 & of & 0 & 0 \\
\hline ALPHADET-U & of & 0 & 0 & of & o) & 0 & 0 & 0 & 마 & a \\
\hline ALPHADET-Am & 0 & 0 & 0 & 0 & of & 0 & 0 & 0 & 0 & 0 \\
\hline ALPHA DET-Np & 0 & 0 & 0 & 0 & 0 & 0 & 0 & D) & 0. & 0 \\
\hline ALPHA DET-Th & 0 & 0 & 0 & 0 & 0 & 0. & 0 & of & 0 & 0 \\
\hline ALPHA DET - U,Th,Pu & of & 0 & 0 & o & 0 & 0 & of & 0 & 0. & 0 \\
\hline ALPHADET-U235 & D. & 0 & 0 & D. & 0 & 0 & D. & 0 & 0 . & 0 \\
\hline ALPHA DET-PU,U,Am & 0 & 0 & 0 & 0 & 0 & 0 & 0 & 0 & 0 & 0 \\
\hline ALPHA DET-U,Np,Pu,Am & 0 & 0 & 0 & 0 & 0 & 0] & 0) & o) & 0] & 0 \\
\hline ALPHADET-Ra226 & o & 0 & 0 & 0 & of & 0 & 0 & 0) & 0] & 0 \\
\hline ALPHA DET-Ra228 & 0 & 0 & 0 & D) & 0 & 0. & D & 0 & o. & 0 \\
\hline ALPHA SPEC & o & 0 & 1452 & 0 & 0 & 476 & 750 & 629 & 549 & 1927 \\
\hline GPC-BETA-Pb210 & 0 & 0 & 0 & 0 & of & 0 & 0 & 0 & 0. & 0 \\
\hline GPC-BETA-Sr90 & 0 & 0 & 0 & 0 & of & 0. & 0 & 0 & 0 & 0 \\
\hline GPC-BETA-Pm147 & 0 & 0 & 0 & 0 & 0 & 0. & 0 & 0 & of & 0 \\
\hline GPC-BETA-Se79 & 0 & 0 & 0 & 0 & 0 & 0 & 0 & 0 & o. & 0 \\
\hline LSC-BETA-H3 & 0 & 0 & 0 & 0 & o & 0 & 0 & 0 & 0. & 0 \\
\hline LSC-BETA-Rin222 & 0 & 0 & 0 & 0 & 0 & 0 & 0 & 0 & o. & 0 \\
\hline LSC-BETA- 1129 & 0 & 0 & 0 & 0 & 0] & 0 & 0 & 0 & 의 & 0 \\
\hline LSC-BETA-C14 & 0 & 0 & 0 & 0 & 0 & 요 & 0 & 0 & 0 . & 0 \\
\hline LSC-BETA-TC99 & 0 & 0 & 0 & 0 & o & -0. & 0 & of & 0 & 0 \\
\hline LSC-BETA & D) & 0 & 0 & 0 & 0 & 0 & 0 & 0 & 0 & 0 \\
\hline GAMMA SPEC & 0 & 0 & 1342 & 0 & 0 & 447. & 618 & 586 & 586 & 1790 \\
\hline OTHER-RAD & 0 & 0 & 0 & 0 & 0 & 0 & 0 & 0 & 0 & 0 \\
\hline TOTAL RAD & 0 & 01 & 3878 & 0 & 0 & 1401 & 1930 & 1736 & 1613 & 5279 \\
\hline & & & & & & & & & & \\
\hline TCLP EXTRACTION & 0 & 0 & 487 & 0 & 417 & 322 & 307 & 613 & 307 & 1227 \\
\hline OTHER-PREP & 0 & 0 & 764 & 0 & 666 & 215 & 124 & 823 & 699 & 1646 \\
\hline OTHER-EXPLO & 0 & 0 & 0 & 0 & 0 & 0 & 0 & 0 & 0 & 0 \\
\hline OTHER-BIO & 0 & 0 & 0 & 0 & 0 & o. & 0 & 0) & 0 & 0 \\
\hline OTHER-PHYS & 0 & 0 & 0 & 0 & 0 & 요 & 0 & 0 & 0 & 0 \\
\hline OTHER & 0 & 01 & 0 & 0 & 0 & 요 & 0 & 0 & o] & 0 \\
\hline TOTALOTHER & 0 & 01 & 1251 & 0 & 1084 & 538 & 431 & 1436 & 1006 & 2873 \\
\hline & & & & & & & & & & \\
\hline TOTAL SAMPLLE ANALYSE & 0 & 01 & 17074 & 0 & $4 \overline{046}$ & 3413 & 6982 & 11296 & 6255 & 24533 \\
\hline
\end{tabular}


EM-263 SURVEY OF DOE ANALYTICAL CAPACITY AVAILABLE TO EM

Table IVb: EC Sample Analyses by Rad Level and Matrix Type (xi) West Valley Nuclear Services

\begin{tabular}{|c|c|c|c|c|c|c|c|c|c|c|}
\hline $\begin{array}{l}\text { Rad-Matrix } \\
\text { Samp Anal }\end{array}$ & RO & RO/RT & RI & $\mathrm{F} 2$ & $7 \overline{73}$ & $\overline{\mathrm{RA}}$ & M1 & $\mathrm{M} 2$ & $\overline{\mathrm{M3}}$ & Total \\
\hline & & & & & & & & & & \\
\hline VOA-GC & 0 & 0 & 0 & 0 & 0 & 0 & 0 & 0 & 0 & 0 \\
\hline VOA-GCMS & 0 & 0 & 0 & 0 & 0 & 0 & 0 & 0 & 0 & 0 \\
\hline SEMI-VOA & 0 & 0 & 0 & 0 & 0 & 0 & 0 & 0 & 0 & 0 \\
\hline PEST & 0 & 0 & 0 & 0 & 0 & D) & 0 & 0 & 0 & 0 \\
\hline HERB & 0 & 0 & 0 & 0 & 0 & 0] & 0 & 0 & 0 & 0 \\
\hline PCB & 0 & 0 & 0 & 0 & 0 & 0 & 0 & 01 & 0 & 0 \\
\hline PCB/PEST & 0 & 0 & 0 & 0 & 0 & 0 & 0 & 0 & 0 & 0 \\
\hline OTHER-ORG & 01 & 0 & 0 & 0 & 0 & 0 & 0 & 0 & 0 & $\mathbf{0}$ \\
\hline TOTAL OAGANICS & 01 & 01 & 0 & 0 & 0 & 0 & 0 . & 01 & 0 & $\overline{0}$ \\
\hline & & & & & & 7 & - & & & 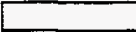 \\
\hline ICP-AES METALS & 0 & 0 & 70 & 0 & 0 & (0) & 17 & 42 & 10 & 70 \\
\hline ICP-MS METALS & 0 & 0 & 0 & 0 & 0 & $\overline{0}$ & 0 & 0 & $\overline{0}$ & 0 \\
\hline GF-AAMETALS & 0 & 0 & 0 & 0 & 0 & 0 & 0 & 0 & 0 & 0 \\
\hline MERCUFY & 0 & 0 & 0 & 0 & 0 & 0 & 0 & 0 & 0 & 0 \\
\hline CYANIDE & 0 & 0 & 0 & 0 & 0 & 0] & 0 & 0 & $\overline{0}$ & 0 \\
\hline ANIONS/CATIONS & 0 & 01 & 209 & 0 & 0 & 0] & 73 & 104 & 31 & 209 \\
\hline WET CHEM & 0 & 0 & 0 & 0 & 0 & 0 & 0 & 0 & 0 & 0 \\
\hline OTHER-INORG & 0 & 01 & 0 & 0 & 01 & 0] & 0 & 01 & 0 & 0 \\
\hline TOTAL INOHGANICS & 01 & 01 & 278 & 01 & 01 & 0] & 901 & 146 & 42 & 278 \\
\hline & & & & & & & & & & \\
\hline GROSS ALPHAVBETA & 0 & 0 & 1642 & 0 & 0 & 0 & 821 & 657 & 164 & 1642 \\
\hline ALPHA DET-Np237 & 0 & 0 & 0 & 0 & 0 & 0 & 0 & 0 & 0 & 0 \\
\hline ALPHA DET-Pu,Am & 0 & 0 & 0 & 0 & 0 & 0 & 0 & 0 & 0 & 0 \\
\hline ALPHADET-PU & 0 & 0 & 860 & 0 & 0 & 0 & 387 & 301 & 172 & 860 \\
\hline ALPHA DET-U & 0 & 0 & 0 & 0 & 0 & o. & 0 & 0 & 0 & 0 \\
\hline ALPHA DET-Am & 0 & 0 & 0 & 0 & 0 & 0] & 0 & 0 & 0. & 0 \\
\hline ALPHADET-Np & 0 & 0 & 0 & 0 & 0 & 0 & 0 & 0 & 0 & 0 \\
\hline ALPHA DET-Th & 0 & 0 & 0 & 0 & 0 & o. & 0 & 0 & O) & 0 \\
\hline ALPHA DET-U,Th,PU & 0 & 0 & 0 & 0 & 0 & 0 & 0 & 0 & 0 & $\overline{0}$ \\
\hline ALPHA DET-U235 & 0 & o & 0 & 0 & 0 & 0. & 0 & 0 & O. & 0 \\
\hline ALPHA DET-PU,U,Am & 0 & 0 & 0 & 0 & 0 & 0] & 0 & 0 & 0 & 0 \\
\hline ALPHA DET-U,Np,Pu,Am & 0 & 0 & 0 & 0 & o) & 0 & 0 & 0 & 0. & 0 \\
\hline ALPHA DET-Ra226 & 0 & 0 & 0 & 0 & 0 & $\mathbf{0}$ & 0 & 0 & 0 & 0 \\
\hline ALPHADET-Pa228 & 0 & 0 & 0 & 0 & 0 & $\mathbf{0}$ & 0 & 0 & 0 & 0 \\
\hline ALPHA SPEC & 0 & 0 & 0 & 0 & 0 & 0 & 0 & 0 & 0. & 0 \\
\hline GPC-BETA-PB210 & 0 & 0 & 0 & 0 & 0 & 0 & 0 & 0 & 0 & 0 \\
\hline GPC-BEIA-Sr90 & 01 & 0 & 733 & 0 & 0 & o. & 440 & 220 & 73 & 733 \\
\hline GPC-BETA-Pm147 & 0 & 0 & 0 & 0 & 0 & D & 0 & 0 & 0 & 0 \\
\hline GPC-BETA-Se79 & 0 & 0 & 0 & 0 & 0 & 0 & 0 & 0 & 0 & 0 \\
\hline LSC-BETA-HB & 0 & 0 & o & 0 & 01 & 0 & 0 & 0 & $\overline{0}$ & 0 \\
\hline LSC-BETA-Pin222 & 0 & 0 & 0 & 0 & 0 & 0 & 0 & 0 & 0 & 0 \\
\hline LSC-BETA-I129 & 0 & 0 & 0 & 0 & 0 & 0. & 0 & 0 & 0 & 0 \\
\hline LSC-BEIA-C14 & 0 & 0 & 0 & 0 & 0 & 0 & 0 & 0 & 0 & 0 \\
\hline LSC-BETA-TC99 & 0 & 0 & 0 & 0 & 0 & 0 & 0 & 0 & 0 & 0 \\
\hline LSC-BETA & 0 & 0 & 0 & 0 & 0 & 0 & 0 & 0 & 0 & $\overline{0}$ \\
\hline GAMMA SPEC & 0 & 0 & 968 & 0 & 0 & 0) & 678 & 145 & 145 & 968 \\
\hline OTHER-RAD & 01 & 0 & 0 & 0 & 0 & 0] & 0 & 0 & 0 & 0 \\
\hline TOTAL RAD & 01 & 01 & 4203 & 01 & 0 & 0] & 2325 & 1323 & 555 & $\overline{4203}$ \\
\hline & & & & & & & & & & \\
\hline TCLPEXTRACTION & 01 & 0 & 0 & 0 & 0 & 0] & 0 & 0 & 0 & $\overline{0}$ \\
\hline OTHER-PREP & 0) & 0 & 0 & 0 & 0 & 0. & 0 & 0 & 0 & 0 \\
\hline OTHER-EXPLO & $\mathbf{0}$ & 0 & 0 & 0 & 0 & 0) & 0 & 0 & 0 & 0 \\
\hline OTHEA-BIO & 0 & 0 & 0 & 0 & 0 & 0 & 0 & 0 & 0] & 0 \\
\hline OTHEA-PHYS & 0 & 0 & 0 & 0 & 0 & D. & 0 & 0 & 요 & 0 \\
\hline OTHER & $\mathbf{0}$ & 0 & 0 & of & 0 & 0 & 0 & 0 & $\overline{0}$ & 0 \\
\hline TOTAL OTHER & 0 & 0 & O & 01 & $0 !$ & Q] & 0 & D! & $\overline{0}$ & 0 \\
\hline & & & & & 1 & & & & & \\
\hline TOTAL SAMPLE ANALYSES & 0] & $\overline{01}$ & 44811 & DI & 0] & D. & 2416 & 1469 & 596 & 4481 \\
\hline
\end{tabular}


EM-263 SUAVEY OF DOE ANALYTICAL CAPACITY AVAILABLE TO EM

Table IVb: EC Sample Analyses by Rad Level and Matrix Type (xii) Nevada Test Site

\begin{tabular}{|c|c|c|c|c|c|c|c|c|c|c|}
\hline $\begin{array}{l}\text { Rad-Matrix } \\
\text { Samp Anal }\end{array}$ & RO & Fo/R1 & Rit & $\overline{R 2}$ & $\mathrm{R3}$ & $\overline{R 4}$ & TM1 & $\overline{M 2}$ & $\overline{M 3}$ & Total \\
\hline VOA-GC & 0 & of & 0 & 0 & 0 & 0 . & 0 & 0 & 0 & 에 \\
\hline VOA-GCMS & 569 & of & 0 & 0 & 0 & 0 & 284 & 171 & 114 & 569 \\
\hline SEMI-VOA & 0 & 0 & 0 & 0 & 0 & o. & 0 & 0 & 0 & 0 \\
\hline PEST & 0 & 0 & 0 & 0 & 0 & 0 & 0 & 0 & 0 & 0 \\
\hline HERB & 0 & 0 & 0 & of & 0 & 0. & 0 & 0 & 요 & 0 \\
\hline$\overline{P C B}$ & 0 & 0 & 0 & 0 & 0 & 0 & 0 & \begin{tabular}{l|l}
0 \\
\end{tabular} & 0 & - \\
\hline PCB/PEST & 아 & 이 & 0 & 0 & 0 & 0] & 0 & 0 & 0 & -0 \\
\hline OTHER-OAG & 0 & 0 & 0 & 0 & 0 & o. & 0 & 0 & (0) & 0 \\
\hline TOTAL ORGANICS & 569 & 이 & 0] & 01 & 01 & o] & 284 & 171 & 114 & 569 \\
\hline & & & & & & & & & & \\
\hline ICP-AES METALS & 1160 & 0. & 0 & Oा & of & o. & 304 & 822 & 34 & 1160 \\
\hline ICP-MSMETALS & 0 & 0 & 0 & of & 0 & 0 & 0 & 0 & 0 & 0 \\
\hline GF-AAMETALS & 474 & 0 & 0 & 0 & 0 & (0) & 270 & 204 & - & 474 \\
\hline MERCURY & 474 & 0 & 0 & of & 0] & 이 & 270 & 204 & 0 & 474 \\
\hline CYANIDE & 0 & 0 & o) & 0 & 이 & 요 & 0 & 0 & 요 & 0 \\
\hline ANIONS/CATIONS & 0 & 0 & 0 & 01 & of & 0 & 0 & 0 & o. & 0 \\
\hline WET CHEM & 0 & 0 & 0 & 이 & 0 & o. & 0 & 0 & 묘 & of \\
\hline OTHER-INORG & 0 & 0 & 01 & 0 & 0 & 0 & 0 & 0 & - & 0. \\
\hline TOTAL INORGANICS & 2108 & 01 & 01 & 01 & Of & $0]$ & 844 & 1229 & 34] & 2108 \\
\hline GROSS ALPHAVBETA & 0 & 0 & 9948 & of & 0 & 0 & 7004 & 2944 & 0 & 9948 \\
\hline ALPHA DET-NP237 & 0 & 0 & 0 & 0 & 01 & 0 & 0 & 0 & o & 0 \\
\hline ALPHA DET-Pu,Am & 0 & 0 & 0 & 0 & 0 & 0. & 0 & 0 & - & 0 \\
\hline ALPHA DET-Pu & 0 & 0 & 4797 & O) & 0 & 0 & 0 & 0 & 4797 & 4797 \\
\hline ALPHA DET-U & 0. & 0 & 0 & 0 & 0 & 0 & 0 & 0 & 0 & (0) \\
\hline ALPHA DET-Am & 0 & 0 & 0 & 0 & 0 & 0 & 0 & 0 & 0 & 0 \\
\hline ALPHA DET-Np & 0 & of & 0 & 0 & 0 & 0 & 0 & 0 & 0 & 0 \\
\hline ALPHA DET-Th & 요 & 0 & 0 & 0 & 0 & 0 & 0 & 0 & ㅇ. & 요 \\
\hline ALPHA DET-U,Th,PU & 0 & 0 & 0 & of & 0 & 0 & 0 & 0 & (0) & 0 \\
\hline ALPHA DET-U235 & 0 & 0 & 0 & 0 & of & 0 & 0 & 0 & 0. & 0 \\
\hline ALPHA DET-Pu,U,Am & 0 & 0 & 0 & DI & 0 & 0 & 0 & 0 & 요 & 0 \\
\hline ALPHA DET-U,Np,PU,Am & 0 & 이 & 0 & 0 & 0 & 요 & 0 & 0 & O & 0. \\
\hline ALPHA DET-Ra226 & 0 & 0 & 0 & 0 & o. & 0 & 0 & 0 & 0 & 0 \\
\hline ALPHA DET-Ra228 & 0 & 0 & 0 & 0 & 0 & 0 & 0 & 이 & 0 & 0 \\
\hline ALPHA SPEC & D & o & 0 & 0 & 0 & 0 & 0 & 0 & 은 & D \\
\hline GPC-BETA-Pb210 & 0 & 0 & - & 0 & 0 & 0 & 0 & 0 & 0 & 0 \\
\hline GPC-BETA-Sr90 & 0 & 0 & 0 & 0 & 0 & 0 & 0 & o & 0 & 0 \\
\hline GPC-BETA-Pm147 & 0 & 0 & 0 & 0 & 0 & 0 & 0 & 0 & 0 & 0 \\
\hline GPC-BETA-Se79 & 0 & 0 & 0 & 0 & 0 & - & 0 & 0! & 0 & 0 \\
\hline LSC-BETA-H3 & 0 & 0 & 4076 & 0 & 0 & 0 & 0 & 4076 & 0 & 4076 \\
\hline LSC-BETA-Rn222 & 0 & 0 & 0 & 0 & 0 & 0 & 0 & 이 & 0 & 으 \\
\hline LSC-BETA- 1129 & 0 & 0 & 0 & 0 & 0 & 0 & 0 & 0 & 0 & 0 \\
\hline LSC-BETA-C14 & 0 & 0 & 0 & 0 & 요 & 0 & 0 & 0 & 0. & 0 \\
\hline LSC-BETA-TC99 & 0 & 0 & 0 & 0 & 0 & (0) & 0 & 0 & 0 & 0 \\
\hline LSC-BETA & 0 & 0 & 0 & 0 & 0 & 이 & 0 & 0 & 0 & 0 \\
\hline GAMMA SPEC & 0 & 0 & 15420 & 0 & 0 & (0) & 13125 & 1528 & 768 & 15420 \\
\hline OTHER-RAD & 0 & 0 & 0 & 0 & 이 & 0. & 0 & o & 0 & 0 \\
\hline TOTALAAD & 0 & 0 & 34241 & 01 & 01 & 0 & 20129 & 8548 & 5565 & 34241 \\
\hline & & & & & & & & & & \\
\hline TCLP EXTRACTION & 0 & 0) & ㅇ. & 0 & 0) & 요 & 0 & ㅇ) & 으 & 의 \\
\hline OTHER-PREP & 0 & 0 & D. & 0 & 0 & 요 & 0 & of & 0 & 0 \\
\hline OTHER-EXPLO & 1319 & 0 & D) & 0 & 0 & 0 & 1319 & 0. & 0 & 1319 \\
\hline OTHER-BIO & 564 & 0 & 이 & 0 & 0 & 0 & 564 & 0 & ㅇ. & 564 \\
\hline OTHER-PHYS & 0 & 0 & (0) & 0 & 0 & 요 & 0 & 0 & 0 & 0 \\
\hline OTHER & 0 & 0 & 이 & 0 & 0 & $\overline{0}$ & 0 & 0 & ㅇ. & 0 \\
\hline TOTALOTHER & 1882 & 0 & OI & 0 & 0 & 0 & 1882 & 01 & 0] & 1882 \\
\hline & & & & & & 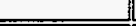 & & & & \\
\hline TOTAL SAMPLE ANAIYS & 4559 & 0 & 34241 & 0] & 0 & 0 & 23140 & 9947 & 5713 & 38800 \\
\hline
\end{tabular}


EM-263 SURVEY OF DOE ANALYTICAL CAPACITY AVAILABLE TO EM

Table Mb: EC Sample Analyses by Rad Level and Matrix Type (xii) Oak Ridge - K25

\begin{tabular}{|c|c|c|c|c|c|c|c|c|c|c|}
\hline $\begin{array}{l}\text { Rad-Matrix } \\
\text { Samp Anal }\end{array}$ & RO & RO/Ri & $\overline{\mathrm{RT}}$ & $\overline{R 2}$ & $\overline{\mathrm{R3}}$ & R4 & $\overline{M 1}$ & $\overline{1} 2$ & $\overline{\mathrm{MB}}$ & Total \\
\hline & & & & & & & & & & \\
\hline VOA-GC & 0 & 0 & a & 0 & 0 & 0 & 0 & o! & 0 & 0 \\
\hline VOA-GCMS & 3314 & 0 & 0 & 0 & 0 & 0 & 2652 & 663 & 0 & 3314 \\
\hline SEMI-VOA & 1693 & 0 & 0 & 0 & 0 & 0 & 1270 & 423 & o. & 1693 \\
\hline PEST & 561 & 0 & 0 & 0 & 0 & 0 & 516 & 45 & o. & 561 \\
\hline HEAB & 546 & 0 & 0 & 0 & 0 & 0 & 496 & 49 & o) & 546 \\
\hline$\overline{\mathrm{PCB}}$ & 4447 & 0 & 0 & 0 & 0 & 0 & 2891 & 1112 & 445 & 4447 \\
\hline PCB/PEST & 0 & 0 & 0 & 0 & 0 & 0 & 0 & 0 & 0 & 0 \\
\hline OTHEA-ORG & 01 & 0 & 0 & 0 & 0 & 0] & 0 & 0 & 0 & 0 \\
\hline TOIAL OAGANICS & 10561 & 01 & 0 & 01 & 0 & 0] & 7825 & 2292 & 445 & $105 \overline{61}$ \\
\hline & & & & & & & & & & \\
\hline KP-AES METALS & 11632 & 0 & 0 & 0 & 0 & 요 & 8724 & 1745 & 1163 & 11632 \\
\hline KCP-MSMETALS & 3457 & O) & 0 & 0 & 0 & o) & 2593 & 519 & 346 & 3457 \\
\hline GF-AAMETALS & 12445 & O & 0 & 0 & 0 & 요 & 8711 & 2489 & 1244 & 12445 \\
\hline MERCURY & 19778 & 0 & 0 & $\overline{0}$ & 0 & 0 & 14833 & 2967 & 1978 & 19778 \\
\hline CYANIDE & 0 & 0 & 0 & 0 & 0 & 0. & 0 & 0 & 요 & D. \\
\hline ANIONS/CATIONS & 8473 & 0 & 0 & 0 & 0 & 0] & 7202 & 1271 & o & 8473 \\
\hline WET CHEM & 0 & o & 0 & 0 & 0 & 0) & 0 & 0 & 0 & 0 \\
\hline OTHER-INORG & 0] & 0 & 0 & 01 & O) & 0) & 0 & 01 & O) & 0 \\
\hline TOTALL INORGANICS & 55785 & 01 & DI & $\overline{01}$ & 0 & 0 & 42064 & 89901 & 4731 & 55785 \\
\hline POnco AIDHAIPETA & 20501 & & & it & & T1 & 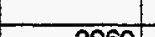 & 1 & 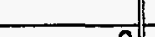 & \\
\hline GROSS ALPHAVBETA & 2969 & 0 & D) & 0 & 0 & 0. & 2969 & 01 & 0. & 2969 \\
\hline ALPHA DET-Np237 & 0 & 0 & D) & 0 & 0. & 0 & 0 & O) & 0 & 0 \\
\hline ALPHA DET-PU,Am & 0 & of & 0 & 0 & 0 & 0) & 0 & 0 & $\overline{0}$ & 0 \\
\hline ALPHA DET-PU & 0 & 0 & 0 & 0 & 0 & 0] & 01 & 0 & 0 & 0 \\
\hline ALPHA DET-U & 1369 & 0 & 0 & o & o) & 0. & 219 & 506 & 643 & 1369 \\
\hline ALPHA DET-AM & 0 & 0 & 0 & 0 & 0 & 0 & 0 & 0 & 0 & 0 \\
\hline ALFHA DET-Np & 1921 & 0 & 0 & 0 & 0 & 0. & 96 & 864 & 960 & 1921 \\
\hline ALPHADET-Th & 1265 & 0 & 0 & 0 & 0 & 0 & 51 & 506 & 708 & 1265 \\
\hline ALPHA DET-U,Th,PU & 0 & 0 & 0 & O) & 0 & 0. & 0 & 0 & 0) & $\overline{0}$ \\
\hline ALPHADET-U235 & D) & 0 & 0 & 0 & 0 & 0. & 0 & 0 & o & 0 \\
\hline ALPHA DET-Pu,U,Am & 0 & \begin{tabular}{l|l}
0 \\
\end{tabular} & 0 & 0 & 0 & 0: & 0 & 0 & 0 & 0 \\
\hline ALPHA DET-U,Np,Pu,Am & 0 & 0 & 0 & 0 & 0 & 0 & 0 & 0 & 0. & o. \\
\hline ALPHADET-Ra226 & 0 & 0 & 0 & 0 & 0 & 0 & 0 & 0 & 0. & 0 \\
\hline ALPHA DET-Ra228 & 0 & 0 & 0 & 0 & 0 & 0 & 0 & 0 & O & 0 \\
\hline ALPHA SPEC & 0 & 0 & 0 & 0 & 0 & 0 & 0 & 0 & O & $\overline{0}$ \\
\hline GPC-BETA-Pb210 & 0 & \begin{tabular}{l|l}
0 \\
\end{tabular} & 0 & 0 & 0 & 0 & 0 & 0 & 0 & 0 \\
\hline GPC-BETA-Sr90 & 0 & 0 & 0 & 0 & 0 & 0 & 0 & 0 & o. & D? \\
\hline GPC-BETA-Pm147 & D. & 0 & 0 & 0 & 0 & 0) & 0 & 0 & a) & 을 \\
\hline GPC-BETA-Se79 & 0 & 0 & 0 & 0 & 0 & 0 & 0 & 0 & o. & 0 \\
\hline LSC-BETA-H3 & 0) & 0 & O) & 01 & 0 & 0. & 0 & 0 & 0 & 0 \\
\hline LSC-BETA-Rn222 & 0 & o) & 0 & 0 & 0) & O & $\overline{0}$ & 0 & 0 & 0 \\
\hline LSC-BETA-1129 & 0. & 0 & 0 & 0 & 0 & 0] & 0 & 0 & ㅇ. & 0 \\
\hline LSC-BETA-C14 & 이 & 0) & 0 & 0 & 0 & 0. & 0 & 0 & o & 0 \\
\hline LSC-BETA-TC99 & 0 & 0 & O & 0 & 0 & 0 & 0 & 0 & of & D. \\
\hline LSC-BETA & 0 & 0 & 0 & 0 & 0 & 0 & 0 & 0 & o) & 0 \\
\hline GAMMA SPEC & 2055 & 0 & 0 & 0 & 0 & o: & 308 & 206 & 1542 & 2055 \\
\hline OTHER-AAD & 01 & 0 & 0 & a & 01 & 0. & 01 & 0 & o. & 0 \\
\hline TOIAL RAD & 9579 & 01 & DI & O! & 01 & (0] & 3643 & 2082 & 3853 & 9579 \\
\hline & & & & & & & & & & \\
\hline TCLPEXTRACTION & 1884 & 0 & 01 & 0 & 0 & 0 & 0 & 1601 & 283 & 1884 \\
\hline OTHER-PAEP & 2552 & 0 & 0 & 0 & o) & 0) & 2169 & 383 & 0 & 2552 \\
\hline OTHER-EXPLO & 0 & 0 & 0 & 0 & 0 & 0 & 0 & o. & 0 & D \\
\hline OTHER-BIO & 0 & 0 & 0 & 0 & 0 & 0 & 0 & 0 & o) & 0 \\
\hline OTHER-PHYS & 0 & 0 & 0 & 0 & 0 & 0) & 01 & 0 & 요 & 0 \\
\hline OTHEA & 01 & 0 & 0 & 0 & 01 & 요 & $0 !$ & $0]$ & 요 & 0 \\
\hline TOTALOTHER & 4436 & 0. & 0 & 0 & 01 & 0] [ & 2169 & 1984 & 2831 & 4436 \\
\hline & & & & & $T$ T & 1 & & & & 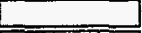 \\
\hline TOTAL SAMPLE ANALYSES & 803611 & 01 & 01 & 01 & 01 & (0) & 55701 & 15348 & 9312 & 80361 \\
\hline
\end{tabular}


EM-263 SURVEY OF DOE ANALYTICAL CAPACITY AVAILABLE TO EM

Table Mb: EC Sample Analyses by Rad Level and Matrix Type (xiv) Oak Ridge Nat'l Lab

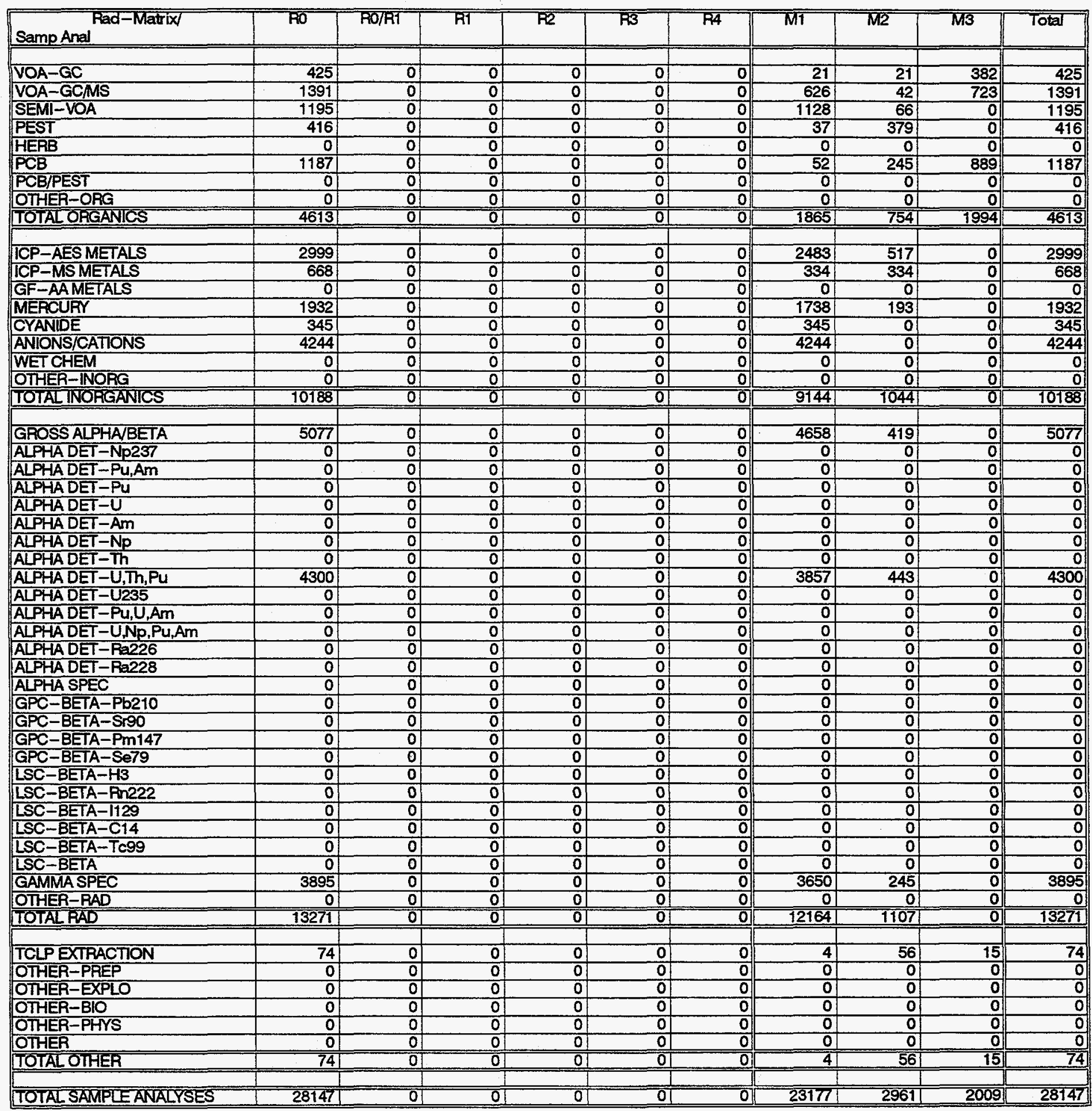


EM-263 SUAVEY OF DOE ANALYTICAL CAPACITY AVAILABLE TO EM

Table Mb: EC Sample Analyses by Rad Level and Matrix Type (xv) Oak Ridge - Y12

\begin{tabular}{|c|c|c|c|c|c|c|c|c|c|c|}
\hline $\begin{array}{l}\text { Rad-Matix } \\
\text { Samp Anal }\end{array}$ & Ro & RO/R1 & RI & $\mathrm{R2}$ & $\overline{R 3}$ & $\overline{\text { R44 }}$ & M1 & $\overline{\mathrm{M} 2}$ & TM3 & Total \\
\hline VOA-GC & & & & & & & & & & \\
\hline VOA-GCMS & 0 & t & 0 & 0 & 이 & 0. & 0 & 이 & 요 & 0 \\
\hline VOA-GCMS & 3959 & 0) & 2402 & 0 & 0) & 0] & 5149 & 732 & 480 & 6362 \\
\hline SEMI-VOA & 421 & 0 & 1375 & 0 & 0 & D. & 1796 & 0 & 0 & 1796 \\
\hline PEST & - & 0] & 0 & o & o & 0 & 0 & 0 & 0 & 0 \\
\hline HERB & 0 & 0 & 0 & of & 0 & 0 & o & 0 & 0 & 0 \\
\hline PCB & 3188 & of & 3272 & 0 & 0 & 0 & 4600 & 1003 & 857 & 6460 \\
\hline PCB/PEST & o & 0 & 0 & D & 0 & 0. & 0 & 0 & - & 0 \\
\hline OTHER-ORG & 1761 & 0 & 0 & 0 & 0 & ㅁ․ & 1467 & 0 & 293 & 1761 \\
\hline TOTAL ORGANICS & 9330 & (0) & 7049 & 0 & D. & 0] [ & 13012 & 1736 & 1631 & 16379 \\
\hline & & & & & & & & & & \\
\hline ICP-AESMETALS & 3992 & 0 & 3095 & 0 & 0 & (0) & 3592 & 399 & 3095 & 7087 \\
\hline ICP-MS METALS & 17697 & 0 & 0 & 0 & 0 & 0. & 14195 & 2728 & 774 & 17697 \\
\hline GF-AAMETALS & 4208 & 01 & 865 & 0 & 0) & 0 & 5072 & of & 0. & 5072 \\
\hline MERCUAY & 8386 & 0 & 1949 & of & 0 & 0 & 9215 & 374 & 747 & 10336 \\
\hline CYANIDE & 833 & 0 & 0 & O & o & -0 & 833 & 0 & -0. & 833 \\
\hline ANIONS/CATIONS & 5424 & 0 & 1922 & 0 & of & a & 3535 & 3781 & 29 & 7346 \\
\hline WET CHEM & $\mathbf{0}$ & 0 & 0 & 0 & 0 & (0) & 0 & 0 & 0 & 0 \\
\hline OTHER-INOAG & 0 & 01 & 31865 & 0 & 0 & 0] & 6022 & 21285 & 4557 & 31865 \\
\hline TOTAL INOAGANICS & 40539 & 0) & 39696 & $\overline{0}$ & 0 & 0] & 42465 & 28567 & 9203 & 80235 \\
\hline & & & & & & & & & & \\
\hline GROSS ALPHAVBETA & 3347 & 0 & 176388 & 0 & 0 & 0] & 2343 & 177057 & 335 & 179735 \\
\hline ALPHADET-Np237 & 0 & 01 & 0 & 0 & 0 & 0] & 0 & 요 & - & 요 \\
\hline ALPHA DET-Pu,Am & 0 & 0) & 0 & 0 & of & 0 & 0 & 0 & 0] & 0 \\
\hline ALPHADET-PU & 0 & 0 & 0 & (0) & D. & 요 & 0 & 0 & - & 요 \\
\hline ALPHADET-U & 1596 & 0 & 7118 & $\overline{0 !}$ & 0! & of & 2860 & 5063 & 792 & 8715 \\
\hline ALPHA DET-Am & 0. & 0 & 0 & $\overline{0}$ & D) & D. & 0 & o & - & 0 \\
\hline ALPHA DET-Np & 0 & 0) & 0 & 0 & 0 & 0] & 0 & D) & - & 0 \\
\hline ALPHA DET $-T h$ & 0 & 0) & 0 & 0 & 0 & 0] & 0 & 0 & 0] & - \\
\hline ALPHA DET $-U, T h, P U$ & 0 & 0 & 0 & D & 0 & 0] & 0 & 0 & 의 & 0 \\
\hline ALPHADET-U235 & 0 & 0 & 4689 & 0! & 0 & a & 2321 & 1114 & 1253 & 4689 \\
\hline ALPHA DET-Pu,U,Am & 0 & 0 & 0 & 0 & 0 & 0 & 0 & 0 & -0 & 0] \\
\hline ALPHA DET-U,Np,Pu,Am & 0 & 0 & 0 & o & 0 & 0] & 0 & o & - & 0 \\
\hline ALPHADET-Ra226 & 0 & 0 & 0 & 0. & 0 & 0 & 0 & 0 & 의 & 0 \\
\hline ALPHA DET-Ra228 & 0 & 0 & 0 & 0 & 0 & 매 & 요 & of & 에 & 0 \\
\hline ALPHA SPEC & 6097 & a & 763 & 0 & 0 & 0. & 5488 & 305 & 1068 & 6860 \\
\hline GPC-BETA-Pb210 & 0 & 0 & 01 & 0 & 0 & D. & 0 & 0 & 에 & 0 \\
\hline GPC-BETA-Sr90 & 0 & 0 & 01 & 0 & 0 & 의 & 0 & 0 & 에 & 0 \\
\hline GPC-BETA-Pm147 & 0 & 0 & of & o. & 0 & 0] & 0 & 0 & 0] & 0 \\
\hline GPC-BETA-Se79 & D. & 0 & 0) & 0 & 0 & 에 & 이 & of & 묘 & 0 \\
\hline LSC-BETA-H3 & 0 & 0 & 0 & 0 & 0 & 0] & of & O) & 0. & 0 \\
\hline LSC-BETA-Rin222 & 0 & 0 & 0 & 0 & 0 & 0] & 아 & 0 & (0) & 0 \\
\hline LSC-BETA-1129 & 0 & 0 & 0 & 0 & 0 & 0. & 0 & 0 & 0 & 0 \\
\hline LSC-BETA-C14 & 0 & 0 & 01 & 0 & 0 & (0) & 0 & 0 & (0) & 0 \\
\hline LSC-BETA-TC99 & 0 & 0 & 0 & 0 & of & 0] & O & 0 & 0. & 0 \\
\hline LSC-BETA & 0 & 0 & 0 & 0 & O) & ㅁ] & of & 0 & 0 & 0 \\
\hline GAMMA SPEC & 1372 & 0 & 457 & 으 & D) & 요 & 1054 & 246 & 528 & 1829 \\
\hline OTHER-RAD & 0 & 0 & 0 & 0 & 0 & 요 & of & 0 & 요 & 0 \\
\hline IOTAL RAD & 12412 & 0 & 189415 & 0 & (0) & 의 & 14066 & 183785 & 3976[ & 201827 \\
\hline & & & & & & & & & & \\
\hline TCLP EXTRACTION & $10+1$ & o) & a & 0 & 0 & ㅇ. & 51 & 253 & 708 & 1011 \\
\hline OTHER-PREP & 1397 & 0 & 0 & 0 & 0 & 0 & 1397 & 0 & D] & 1397 \\
\hline OTHER-EXPLO & 0 & 0 & 0 & 0 & 0) & (0) & 0 & 0) & 묘 & 0 \\
\hline OTHER-BIO & 0 & 0 & 0 & D & 0 & 0 & 0 & O) & - & 0 \\
\hline OTHER-PHYS & 3479 & 0 & 7631 & D & 0 & 의 & 11110 & D) & 0. & 11110 \\
\hline OTHER & 0 & O. & 0 & 0 & 0 & O. & 이 & 0 & $0]$ & ‥ \\
\hline TOTAL OTHEA & 5887 & 0 & 7631 & 0 & 0 & 0 & 12558 & 253 & 708 & 13518 \\
\hline & & & & & & & & & & \\
\hline TOTAL SAMIPLEANALY & 68168 & O) & 243790 & $\overline{0}$ & $\overline{0}$ & 0] & 82101 & 214341 & 5517 & 311958 \\
\hline
\end{tabular}


EM-263 SUAVEY OF DOE ANALYTICAL CAPACTYY AVAILABLE TO EM

Table IM: EC Sample Analyses by Rad Level and Matrix Type (xvi) Paducah

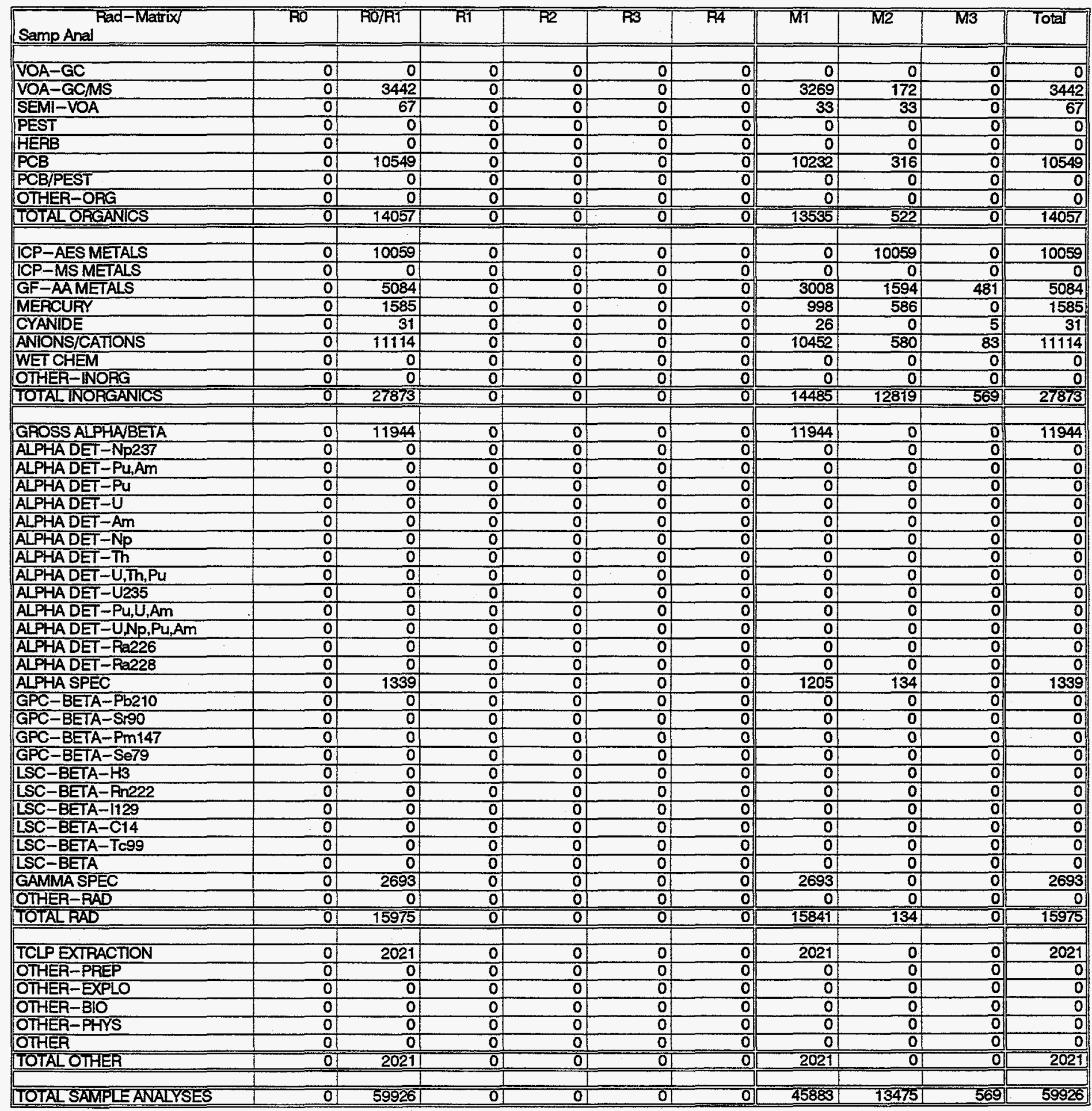


EM-263 SURVEY OF DOE ANALYTCAL CAPACITY AVAILABLE TO EM

Table Mb: EC Sample Analyses by Rad Level and Matrix Type (xvii) Portsmouth

\begin{tabular}{|c|c|c|c|c|c|c|c|c|c|c|}
\hline $\begin{array}{l}\text { Rad-Matix } \\
\text { Samp Anal } \\
\end{array}$ & $\mathrm{RO}$ & RO/R1 & R1 & $\mathrm{R2}$ & $\mathrm{R} \overline{3}$ & $\mathrm{RA}$ & MT & $\sqrt{12}$ & M13 & Total \\
\hline & & & & & & & & & & \\
\hline VOA-GC & D) & 0 & 0 & 0 & D & o. & 0 & 0 & D. & $\underline{\mathbf{0}}$ \\
\hline VOA-GCAMS & 0 & 4655 & 0 & 0 & 0 & 0 & 3165 & 1071 & 419 & 4655 \\
\hline SEMI-VOA & 0 & 635 & 0 & 0 & 0 & 0. & 432 & 178 & 25 & 635 \\
\hline PEST & 01 & 485 & 0 & 0 & 0 & o. & 291 & 39 & 155 & 485 \\
\hline HERB & 0 & 144 & 0 & 0 & 0 & 0 & 58 & 58 & 29 & 144 \\
\hline PCB & 0 & 1472 & 0 & 0 & 0 & 0 & 765 & 235 & 471 & 1472 \\
\hline PCB/PEST & 0 & 0 & 0 & D) & 0 & 0 & 0 & 0 & ol & 0 \\
\hline OTHER-ORG & 0 & 0 & 0 & of & 0] & $\mathbf{0}$ & 01 & 01 & D. & 0 \\
\hline TOIALORGANICS & 0 & 7390 & OI & O & 0 & 0 & 4711 & 1580 & 1099 & 7390 \\
\hline & & & & & & & & & & \\
\hline ICP-AES METALS & 0 & 7180 & 0 & 0 & 0 & 0. & 4196 & 1436 & 1548 & 7180 \\
\hline ICP-MSMETALS & 0 & 0 & 0 & 0 & 0 & 0 & 0 & 0 & 0 & 0 \\
\hline GF-AAMETALS & 0 & 5052 & 0 & 0 & 0 & 0 & 4294 & 253 & 505 & 5052 \\
\hline MERCURY & 0 & 5390 & 0 & D. & 0 & 0 & 3611 & 377 & 1401 & 5390 \\
\hline CYANIDE & 0 & 250 & of & D) & 0 & 0 & 150 & 50 & 50 & 250 \\
\hline ANIONS/CATIONS & 0 & 1164 & 0 & D) & 0) & 0 & 1164 & 0 & o. & 1164 \\
\hline WET CHEM & 0 & 0 & 0 & 0 & 0 & 0 & 0 & 0 & D. & 0 \\
\hline OTHER-INORG & 0 & 18450 & 0) & 0 & 0 & D. & 14836 & 1350 & 2273 & 18459 \\
\hline TOTAL INOFGANICS & 01 & 37485 & 01 & OI & 0] & 0 & 28251 & 34661 & 5777 & 37495 \\
\hline & & & & & & 7 & & & & \\
\hline GPOSS ALPHAVBETA & 0 & 19747 & 0 & 0 & of & 0 & 16785 & 1975 & 987 & 19747 \\
\hline ALPHA DET-Np237 & 0 & 0 & 0 & 0 & 0 & D & 0 & 0 & 0 & o \\
\hline ALPHADET-Pu,Am & 0 & 0 & 0 & 0 & 0 & o. & 0 & 0 & 0 & o \\
\hline ALPHADET-PU & 0 & 0 & 0 & 0 & 0 & 0 & 0 & 0 & 0 & D. \\
\hline ALPHA DET-U & 0 & 0 & 0 & 0 & 0 & 0 & 0 & 0 & 0. & 0 \\
\hline ALPHA DET-Am & 0 & 0 & 0 & 0 & 0 & $\overline{0}$ & 0 & 0 & 0 & 0. \\
\hline ALPHADET-Np & 0 & 0 & 0 & 0 & 0 & 0 & 0 & 0 & o: & O) \\
\hline ALPHA DET-Th & 0 & 0 & 0 & 0 & 0 & 0 & 0 & 0 & 0 & $\overline{0}$ \\
\hline AlPHA DET-U,Th,PU & 0 & 0 & 0 & 0 & 0 & 0. & 0 & 0 & D. & 0 \\
\hline ALPHA DET-U235 & 0 & 0 & 0 & D. & 0) & 0 & 0 & 0 & 0 & 0. \\
\hline ALPHA DET-PU,U,Am & 0 & 0 & 0 & D) & of & $\mathbf{0}$ & 0 & 0 & D. & D. \\
\hline ALPHA DET-U,Np,Pu,Am & 0 & 0 & D) & 0 & 0 & 0 & 0 & 0 & o. & 0 \\
\hline ALPHA DET-Ra226 & 0 & 0 & 0 & 0 & 0 & 0 & 0 & 0 & 0 & o \\
\hline ALPHA DET-Pa228 & 0 & 0 & 0 & 0 & 0 & 0 & 0 & 0 & o. & 0 \\
\hline ALPHA SPEC & 0 & 6344 & 0 & 0 & 0 & 0 & 4758 & 952 & 634 & 6344 \\
\hline GPC-BETA-Pb210 & 0 & 0 & 0 & 0 & 0 & 0 & 0 & 0 & 0 & 0 \\
\hline GPC-BETA-Sr90 & 0 & 0 & 0 & 0 & 0 & 0 & 0 & 0 & 0 & $\overline{0}$ \\
\hline GPC-BETA-Pm147 & 0 & 0 & 0 & 0 & 0 & o & o) & o) & 0. & D. \\
\hline GPC-BETA-Se79 & 0 & 0 & 0 & 0 & 0 & 0 & 0 & 0 & 0) & D. \\
\hline LSC-BETA-H3 & 0 & 0 & 0 & 0 & 0 & 0 & 0 & 0 & D) & 요 \\
\hline LSC-BETA-An222 & 0 & 0 & 0 & 0 & 0 & $\mathbf{0}$ & 0 & 0 & 0 & 0. \\
\hline LSC-BETA-1129 & 0 & 0 & 0 & 0 & 0 & 0 & 0 & o) & o. & 0 \\
\hline LSC-BETA-C14 & 0 & 0 & 0 & 0 & 0 & 0 & 0 & 0 & o & 0 \\
\hline LSC-BETA-TC99 & 0 & 13839 & 0 & 0 & 0 & o & 11071 & 1384 & 1384 & 13830 \\
\hline LSC-BETA & 0 & 0 & 0 & 0 & 0 & 0 & 0 & 0 & of & 0) \\
\hline GAMMA SPEC & 0 & 1198 & 0 & 0 & 0 & 0 & 539 & 240 & 419 & 1198 \\
\hline OTHER-RAD & 0 & 0 & 0 & 0 & 0 & o & 0 & 0 & o & 0 \\
\hline TOIAL AAD & 0 & 41128 & 01 & $0]$ & 0 & 0 & 33153 & 45501 & $3 \overline{425}$ & 41128 \\
\hline & & & & & & & & & & \\
\hline TCLPEXIRACTION & 0 & 0 & 0 & 0 & 0 & 0 & 0 & 0 & 0 & 0 \\
\hline OTHER-PREP & 0 & 0 & 0 & 0 & 0 & 0 & 0 & 0 & 0 & 0 \\
\hline OTHER-EXPLO & 0 & 0 & 0 & 0 & 0 & 0 & 0 & 0 & 0 & 요 \\
\hline OTHER-BIO & 0 & 0 & 0 & 0 & 0 & 0 & 01 & 0 & 0 & 으 \\
\hline OTHER-PHYS & 0 & 0 & 0 & 0 & 0 & 0) & 0 & 0 & 0 & 0 \\
\hline OTHER & 0 & O) & 01 & 0 & 0 & 0) & 0 & 0 & 0 & 0 \\
\hline TOTAL OTHEA & 01 & 0 & 01 & 0 & 0 & o] & 01 & 0 & o: & (0) \\
\hline & & & & & & & & & & \\
\hline TOTAL SAMPLE ANALYSES & 01 & 86013 & 01 & O) & D) & D] & 66115 & 9596 & 10302 & 86013 \\
\hline
\end{tabular}


Table IVb: EC Sample Analyses by Rad Level and Matrix Type (xviii) Hanford - WHC 222S

\begin{tabular}{|c|c|c|c|c|c|c|c|c|c|c|}
\hline $\begin{array}{l}\text { Rad-Matrix } \\
\text { Samp Anal }\end{array}$ & Ro & RO/R & $\overline{\mathrm{RI}}$ & F2 & $\overline{R 3}$ & $\overline{\mathrm{R4}}$ & $\overline{\mathrm{MI}}$ & M2 & $\overline{M B}$ & Total \\
\hline VOA-GC & 0] & 0 & 0 & 0 & 0 & 0 & 0 & 01 & 0 & \\
\hline VOA-GCMS & 이 & 1612 & 0 & 0 & 0 & 0 & 1612 & 0 & 0 & $\frac{0}{1612}$ \\
\hline SEMI-VOA & 0 & 0 & 0 & 0 & of & 0 & 0 & 0 & 0 & 0 \\
\hline PEST & 0 & 0 & 0 & 0 & 0 & 0 & 0 & o & 0 & $\overline{0}$ \\
\hline HERB & 0 & 0 & 0 & $\overline{0}$ & 0 & 0 & 0 & 0 & 0] & $\overline{0}$ \\
\hline PCB & o. & 0 & 0 & $\overline{0}$ & 0 & 0 & Of & 0 & 0 & $\overline{0}$ \\
\hline PCB/PEST & 0 & 0 & 0 & 0 & 0 & (0) & 0 & 0 & 0 & 0 \\
\hline OTHER-ORG & (0) & O) & 0 & 59900 & 0 & 0 & 41744 & 0 & 18156 & 59900 \\
\hline TOTAL ORGANICS & 0 & 1612 & 0 & 59900 & 0 & 0 & 43356 & 01 & 18156 & 61512 \\
\hline & & & & & & & & & & \\
\hline ICP-AES METALS & 0 & 0 & 0 & 44965 & 0 & 0. & 24705 & 381 & 19879 & 44965 \\
\hline ICP-MS METALS & 0 & 0 & 0 & 0 & 0 & 0 & 0 & 0 & 0 & 0 \\
\hline GF-AAMETALS & of & 3644 & 0 & 13894 & 0 & 0 & 2733 & 911 & 13894 & 17537 \\
\hline MERCURY & 0 & 0 & 0 & 14575 & 0 & 0 & 1457 & 729 & 12389 & 14575 \\
\hline CYANIDE & 0 & 0 & 0 & 49302 & 0 & 0 & 26292 & 0 & 23010 & 49302 \\
\hline ANIONS/CATIONS & of & 31544 & 0 & 35990 & 0 & 0 & 45240 & 79 & 22215 & 67534 \\
\hline WET CHEM & 0 & 0 & 0 & 5578 & 0 & 0 & 2668 & 39 & 2871 & 5578 \\
\hline OTHER-INORG & 0 & 36266 & 0 & 31739 & 0 & 0 & 43544 & 2921 & 21540 & 68005 \\
\hline TOTAL INORIGANICS & 01 & 71454 & 0 & 196043 & 01 & 0. & 146640 & 5059 & 115797 & 267497 \\
\hline & & & & & & & & & & \\
\hline GROSS ALPHA/BETA & 0 & 39693 & 0 & $\overline{0}$ & 0 & 0 & 23597 & 293 & 15804 & 39693 \\
\hline ALPHA DET-Np237 & O & 5477 & 0 & $\overline{0}$ & 0 & 0 & 2027 & 0 & 3451 & 5477 \\
\hline ALPHA DET - Pu,Am & 0 & 12042 & 0 & 0 & 0 & - & 6871 & 2868 & 2303 & 12042 \\
\hline ALPHADET-Pu & 0 & 0 & 0 & 0 & 0 & 0. & 0 & 0 & 0 & 0 \\
\hline ALPHADET-U & 0 & o & 0 & 0 & $0 \mid$ & 0 & $\overline{0}$ & 0 & 0. & 0 \\
\hline ALPHA DET-Am & 0 & O & 0 & 0 & 0 & 0 & $\overline{0}$ & 0 & 0 & 0 \\
\hline ALPHADET-Np & 0 & 0 & 0 & 0 & 0 & 0 & 0 & 0 & 0. & 0 \\
\hline ALPHADET-Th & of & of & 0 & 0 & 0 & 0 & 0 & 0 & 0 & 0 \\
\hline ALPHA DET - U,Th,PU & D. & 0 & 0 & $\overline{0}$ & 0 & 0 & 0 & $\overline{0}$ & 0. & 0 \\
\hline ALPHADET-U235 & 0 & 0 & 0 & $\overline{0}$ & 0 & 0. & $\overline{0}$ & 0 & 0 & 0 \\
\hline ALPHA DET-Pu,U,Am & O & 0 & 0 & 0 & 0 & 0 & 0 & 0 & 0 & 0 \\
\hline ALPHA DET-U,Np,PU,Am & 0 & 0 & 0 & $\overline{0}$ & 0 & 0 & of & 0 & 0 & 0 \\
\hline ALPHA DET-Ra226 & 0 & 0 & 0 & 0 & 0 & 0 & 0 & 01 & 0 & 0 \\
\hline ALPHA DET-Ra228 & 0 & 0 & 0 & 0 & 0 & 0 & 0 & 0 & 0 & 0 \\
\hline ALPHA SPEC & o) & 이 & 0 & 0 & of & - & 0 & 0 & 0 & 0 \\
\hline GPC-BETA-Pb210 & o. & 0 & 0 & 0 & of & 0 & 0 & 0 & 0 & 0 \\
\hline GPC-BETA-Sr90 & 0 & 8481 & 0 & 0 & 0 & 0 & 5608 & 991 & 1881 & 8481 \\
\hline GPC-BETA-Pm147 & 0 & 4997 & 0 & 0 & 0 & 0. & 4997 & 0 & 0 & 4997 \\
\hline GPC-BETA-Se79 & 0 & 775 & 0 & $\overline{0}$ & 0 & 0 & 775 & 0 & 0. & 775 \\
\hline LSC-BETA-H3 & 0) & 13993 & 0 & 0 & 0 & - & 13993 & 0 & 0 & 13993 \\
\hline LSC-BETA-Rn222 & 0 & 0 & 0 & 0 & 0 & 0 & 0 & 0 & 0 & 0 \\
\hline LSC-BETA-1129 & 0 & 1022 & 0 & 0 & of & 0 & 1022 & 0 & 0 & 1022 \\
\hline LSC-BETA-C14 & 0 & 1641 & 0 & 0 & 0 & of & 896 & 0 & 745 & 1641 \\
\hline LSC-BETA-TC99 & 0 & 5389 & 0 & 0 & 0 & 0 & 4006 & 0 & 1383 & 5389 \\
\hline LSC-BETA & 0 & 0 & 0 & $\overline{0}$ & 0 & 0 & 0 & 0 & 0 & 0 \\
\hline GAMMA SPEC & o & 51310 & 0 & 0 & 0) & (0) & 30250 & 8424 & 12636 & 51310 \\
\hline OTHER-RAD & 0 & 0 & 0 & 0 & of & 0 & 0 & 0 & 0] & 0 \\
\hline TOTAL RAD & 0 & 144820 & 0 & $\overline{0}$ & 01 & 0 & 940411 & 1257 & 38203 & 144820 \\
\hline & & & & & & & & & & \\
\hline TCLP EXTRACTION & o & 0 & 0 & 0 & 0 & 0 & 0 & 0 & 0 & 0 \\
\hline OTHER-PREP & o & 1719 & o) & 81150 & 0 & 709 & 24736 & 4203 & 54640 & 83579 \\
\hline OTHER-EXPLO & 0 & of & 0 & 0 & 0 & 0 & 0 & 0 & 0 & 0 \\
\hline OTHER-BIO & of & of & 0 & 0 & of & 0 & 0 & 0 & 0 & 0 \\
\hline OTHER-PHYS & 0 & of & 0 & 74558 & 0 & 0 & 59144 & 4409 & 11005 & 74558 \\
\hline OTHER & 0 & 0 & 0 & 0 & 0 & 0 & 0 & 0 & o. & 0 \\
\hline TOTAL OTHER & 01 & 1719 & 0 & 155708 & 0 & 709 & $83880 \mid$ & 8612 & 65645 & 158137 \\
\hline & & & & & & & & & & \\
\hline TOTAL SAMPLEANALY & $\overline{0}$ & 219605 & 0 & 411652 & 01 & 709 & 367977 & 26248 & 237801 & 631967 \\
\hline
\end{tabular}


EM-263 SURVEY OF DOE ANALYTICAL CAPACTY AVAILABLE TO EM

Table IVb: EC Sample Analyses by Rad Level and Matrix Type (xix) Hanford - PNL 325

\begin{tabular}{|c|c|c|c|c|c|c|c|c|c|c|}
\hline $\begin{array}{l}\text { Rad-Matrix } \\
\text { Samp Anal }\end{array}$ & RO & 7RO/RT & R1 & $\mathrm{R2}$ & $\mathbf{A B}$ & $\overline{R 4}$ & प1 & $\overline{\mathrm{M} 2}$ & $\overline{M 3}$ & Total \\
\hline & & & & & & & & & & \\
\hline VOA-GC & 0 & 0 & 0 & 0 & 0 & o. & 0 & 0 & D. & 0 \\
\hline VOA-GCMS & 0 & 0 & 0 & 590 & 0 & o. & 335 & 120 & 136 & 590 \\
\hline SEMI-VOA & 0 & 0 & 0 & 359 & 0 & 0 & 121 & 123 & 115 & 359 \\
\hline PEST & 0 & 0 & 0 & 159 & 0 & 0 & 67 & 0 & 92 & 159 \\
\hline HEFB & 0 & 0 & 0 & 159 & 0 & 0 & 67 & 0 & 92 & 159 \\
\hline$\overline{P C B}$ & 0 & 0 & 0 & 159 & 0 & 0 & 67 & 0 & 92 & 159 \\
\hline PCB/PEST & 0 & 0 & 0 & 0 & 0 & o. & 0 & 0 & 0 & 0 \\
\hline OTHER-ORG & 0 & 0 & 0 & 01 & 0 & 0 & 0 & 01 & 0. & 인 \\
\hline TOIAL ORGANICS & 01 & 0 & 0 & 1426 & 0. & $\overline{0}$ & 655 & 243 & 528 & 1426 \\
\hline & & & & & & & & & & \\
\hline ICP-AESMETALS & 0 & 9218 & 0 & 3618 & 0 & 0 & 2765 & 8329 & 1742 & 12836 \\
\hline ICP-MSMETALS & 0 & 4384 & 0 & 3408 & 0 & of & 1982 & 3375 & 2436 & 7792 \\
\hline GF-AAMETALS & 0 & 11427 & 0 & 4208 & 0 & 0 & 0 & 11114 & 4521 & 15635 \\
\hline MERCURY & 0 & 5278 & D & 0 & 0 & 0 & 1419 & 2436 & 1424 & 5278 \\
\hline CYANDE & 0 & 3201 & 0 & 0 & O) & 0 & 0 & 1409 & 1793 & 3201 \\
\hline ANIONS/CATIONS & 0 & 5367 & 0 & 0 & 0 & o. & 653 & 1884 & 2830 & 5367 \\
\hline WET CHEM & 0 & 0 & 0 & 0 & 0 & 0 & 0 & 0 & 0 & 0 \\
\hline OTHER-INORG & 0 & 0) & 0 & 0! & 0 & o. & 0 & 0 & D. & o. \\
\hline TOTAL INORGANICS & 0 & 38875 & 0 & 11234 & 0 & $0]$ & 6819 & 28547 & 14744 & 50110 \\
\hline & & & & & & $\Rightarrow$ & & & & \\
\hline GROSS AILHAVBETA & 0 & 3643 & 0 & 0 & 0 & 0 & 1863 & 1572 & 208 & 3643 \\
\hline ALPHA DET-Np237 & 0 & 0 & 0 & 0 & 0 & 0 & 0 & 0 & 0 & 0 \\
\hline ALPHA DET-PU,Am & 0 & 0 & 0 & 0 & 0 & 0 & 0 & 0 & o. & 0) \\
\hline ALPHADET-PU & 0 & 2353 & 0 & 0 & 0 & 0. & 1118 & 1063 & 172 & 2353 \\
\hline ALPHADET-U & 0 & 0 & 0 & 0 & 0 & 0 & 0 & 0 & 0 & 0 \\
\hline ALPHA DET-Am & 0 & 0 & 0 & 0 & 0 & 0. & 0 & 0 & 0 & 0 \\
\hline ALPHA DET-NP & 0 & 0 & 0 & 0 & 0 & 0 & 0 & 0 & 0) & 0 \\
\hline AIPHADET-Th & 0 & 0 & 0 & 0 & 0 & o) & 0 & 0 & 0 & $\overline{0}$ \\
\hline AlPHA DET-U,Th,Pu & 0 & 0 & 0 & 0 & 0 & 0 & 0 & 0 & 0 & 0 \\
\hline ALPHA DET-U235 & 0 & 0 & 0 & 0 & 0 & 0 & 0 & 0 & 0 & 0. \\
\hline ALPHA DET-PU,U,Am & 0 & 0 & 0 & 0 & 0 & of & 0 & 0 & o- & 0 \\
\hline ALPHA DET-U,Np,PU,Am & 0 & 0 & 0 & 0 & 0 & D. & 0 & 01 & 0. & ㅁ․ \\
\hline ALPHA DET-R2226 & $\mathbf{0}$ & 0 & 0 & 0 & 0 & 0. & 0 & 0 & 0 & 0. \\
\hline ALPHA DET-Ra228 & 0 & 0 & 0 & 0 & 0 & 0 & 0 & 0 & 요 & 0 \\
\hline ALPHA SPEC & 0 & 0 & 0 & 0 & 0 & o. & 0 & 0 & 0 & 0. \\
\hline GPC-BETA-Pb210 & 0 & 0 & 0 & 0 & 0 & 0 & 0 & 0 & 0 & 0 \\
\hline GPC-BETA-Sr90 & 0 & 1775 & 0 & 0 & 0 & 0 & 888 & 709 & 178 & 1775 \\
\hline GPC-BETA-Pm147 & 0 & 0 & 0 & 0 & 0 & 0 & 0 & 0 & o. & 0 \\
\hline GPC-BETA-Se79 & 0 & 0 & 0 & 0 & 0 & 0 & 0 & 0 & 0 & 0 \\
\hline LSC-BETA-H3 & 0 & 0 & 0 & 0 & 0 & 0 & 0 & 0 & 0 & 0. \\
\hline LSC-BETA-Rin222 & 0 & 0 & 0 & 0 & 0 & 0 & 0 & 0 & o. & a. \\
\hline LSC-BETA-I129 & 0 & 0 & 0 & 0 & 0 & 0 & 0 & 0 & D. & 뭉 \\
\hline LSC-BETA-C14 & 0 & 0 & 0 & 0 & 0 & 0 & 0 & 0 & 0 & 0 \\
\hline LSC-BETA-TC99 & 0 & 0 & 0 & 0 & 0 & 0 & 0 & 0 & 0 & o \\
\hline LSC-BETA & 0 & 0 & 0 & 0 & 0 & 0 & 0 & 0 & 0 & 음 \\
\hline GAMMA SPEC & 0 & 1933 & 0 & 0 & 0 & 0 & 967 & 870 & 96 & 1933 \\
\hline OTHER-RAD & 0 & 0 & 0 & 0 & 0 & o] & 0 & 01 & 0 & 0 \\
\hline TOTAL RAD & 0 & 9704 & 01 & 0 & 0 & 0] & 48351 & 42151 & 655 & 9704 \\
\hline & & & & & & & 7 & 7 & & \\
\hline TCLP EXTRACTION & 0 & 0 & 0 & 0 & 0 & 225 & 0 & 0 & 225 & 225 \\
\hline OTHER-PREP & 0 & 0 & 0 & 0 & 0 & 1572! & 0 & 0 & 1572 & 1572 \\
\hline OTHER-EXPLO & 0 & 0 & 0 & 0 & 0 & 0 & 0 & 0 & 0 & 0) \\
\hline OTHER-BIO & 0 & 0 & 0 & 0 & 0 & 0 & 0 & 0 & 0 & 0 \\
\hline OTHER-PHYS & 0 & $\mathbf{0}$ & 0 & 0 & 0 & 0 & 0 & 0 & 0 & 0 \\
\hline OTHER & 0 & 0 & 0 & 0 & 0 & 0 & 0 & 0 & 요 & a) \\
\hline TOTAL OTHER & O & 01 & 01 & O] & 0 & 1797 & 01 & 01 & 1797 & 1797 \\
\hline & & & & & & & & & & \\
\hline TOTAL SAMPLE ANALY & 0 & 48579 & 01 & 12661 & D) & 1797 & 123081 & 330041 & 17724 & 63037 \\
\hline
\end{tabular}


EM-263 SURVEY OF DOE ANALYTICAL CAPACITY AVAILABLE TO EM

Table IV: EC Sample Analyses by Rad Level and Matrix Type (xx) Rocky Flats - 123 Lab

\begin{tabular}{|c|c|c|c|c|c|c|c|c|c|c|}
\hline $\begin{array}{l}\text { Rad-Tatix } \\
\text { Samp Anal }\end{array}$ & RO & Ro/R1 & $\overline{\mathrm{R} 1}$ & R2 & $\overline{R 3}$ & $\overline{\overline{R 4}}$ & $\overline{\mathrm{Mi}}$ & M2 & M3 & Total \\
\hline & & & & & & & & & & \\
\hline VOA-GC & 0 & 0 & 0 & 0 & 0 & 0 & 0 & 0 & 0 & 0 \\
\hline VOA-GCMS & 0 & 0 & 0 & 0 & 0 & 0 & 0 & 0 & 0 & 0 \\
\hline SEMI-VOA & 0 & 0 & $\mathbf{0}$ & 0 & 0 & 0 & 0 & 0 & 0 & 0 \\
\hline PEST & 0 & 0 & 0 & 0 & 0 & 0 & 0 & 0 & 0 & 0 \\
\hline HEAB & $\overline{0}$ & 0 & 0 & 0 & 0 & D. & 0 & 0 & 0 & 0 \\
\hline $\mathrm{PCB}$ & 0 & 0 & 0 & 0 & 0 & 0 & 0 & 0 & 0 & 0 \\
\hline PCB/PEST & 0 & 0 & 0 & 0 & 0 & 0 & 0 & 0 & 0 & 0 \\
\hline OTHER-ORG & 0 & 0 & 0 & 0 & 0 & 0] & 0 & 0 & 0 & 0 \\
\hline TOTAL ORGANICS & 01 & OI & 0) & 01 & 01 & 01 & 01 & OLL & $\overline{0}$ & 0 \\
\hline & & & & & & & & 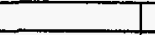 & - & \\
\hline ICP-AESMEIALS & 0 & 0 & 0 & 0 & 0 & 0 & 0 & 0 & 0 & 0 \\
\hline ICP-MSMETALS & 0 & 0 & 0 & 0 & 0 & 0 & 0 & 0 & 0 & 0 \\
\hline GF-AAMETALS & 0 & 0 & 0 & 0 & 0 & of & 0 & 0 & 0: & 0 \\
\hline MERCUAY & 0 & 0 & 0 & 0 & 0 & 0. & 0 & 0 & 0 & 0 \\
\hline CYANIDE & 0 & 0 & 0 & 0 & 0 & o. & 0 & 0 & o & 0 \\
\hline ANIONS/CATIONS & 0 & 0 & 0 & 0 & 0 & o. & 0 & 0 & 0 & 0 \\
\hline WET CHEM & 0 & 0 & 0 & 0 & 0 & 0 & 0 & 0 & 0 & 0 \\
\hline OTHER-INOFG & 64391 & 0 & 0 & 0 & 0 & 0] & 64391 & 0 & 0. & 64391 \\
\hline TOTAL INOFGANICS & 643911 & 0 & O] & 01 & 01 & O] & 64391 & 01 & o] & 64391 \\
\hline & & & & & & 7 & & 1 & & \\
\hline GROSSALPHA BETA & 147144 & 0 & 0 & 0 & 0 & 0 & 147144 & 0 & 0 & 147144 \\
\hline ALPHA DET-Np237 & 0) & 0 & 0 & 0 & 0 & of & 0 & 0 & 0 & 0 \\
\hline ALPHA DET-PU,Am & 0 & 0 & 0 & 0 & 0 & 0 & 0 & 0 & 0 & 0 \\
\hline ALPHA DET-PU & 0 & 0 & 0 & 0 & 0 & 0 & 0 & 0 & 0 & 0 \\
\hline ALPHADET-U & 0 & 0 & 0 & 0 & 0 & 0 & 0 & 0 & 0 & 0 \\
\hline ALPHADET-Am & 0 & 0 & 0) & 0 & 0 & 0 & 0 & 0 & 0 & 0 \\
\hline ALPHA DET-Np & 0 & 0 & 0 & 0 & 0 & 0 & 0 & 0 & 0 & 0 \\
\hline ALPHA DET-Th & 0 & 0 & 0 & 0 & 0 & 0 & 0 & 0 & 0. & 0 \\
\hline ALPHA DET-U,Th,Pu & 0 & 0 & 0 & 0 & 0 & 0 & 0 & 0 & o. & 0 \\
\hline ALPHADET-U235 & 0 & 0 & 0 & 0 & 0 & 0. & 0 & 0 & o & $\overline{0}$ \\
\hline ALPHA DET-PU,U,Am & 10306 & 0 & 0 & 0 & 0 & 0 & 10306 & 0 & 0 & 10306 \\
\hline ALPHA DET-U,Np,Pu,Am & 0 & 0 & 0 & 0 & 0 & D. & 0 & 0 & 0 & $\overline{0}$ \\
\hline ALPHA DET-Ra226 & 0 & 0 & 0 & 0 & 0 & 0 & 0 & 0 & 0 & 0 \\
\hline ALPHA DET-Ra228 & 0 & 0 & 0 & 0 & 0 & O- & 0 & 0 & 0 & 0 \\
\hline ALPHA SPEC & 0 & 0 & 0) & 0 & 0 & D. & 0 & 0 & 0 & 0 \\
\hline GPC-BETA-Pb210 & 0 & 0 & 0 & 0 & 0 & 0 & 0 & 0 & 0 & 0 \\
\hline GPC-BETA-Sr90 & 0 & 0 & 0 & 0 & 0 & 0 & 0 & 0 & 0 & 0 \\
\hline GPC-BETA-Pm147 & 0 & 0 & 0 & 0 & 0 & 0 & 0 & 0 & 0 & 0 \\
\hline GPC-BETA-Se79 & 0 & 0 & 0 & 0 & 0 & 0 & 0 & 0 & o. & 0 \\
\hline LSC-BETA-H3 & 3838 & 0 & o. & 0 & 0 & 0 & 3722 & 116 & 0 & 3838 \\
\hline LSC-BETA-Rn222 & 0 & 0 & 0 & 0 & 0 & 0 & 0 & 0 & 0 & $\overline{0}$ \\
\hline LSC-BETA-I129 & 0 & of & 0 & 0 & 0 & 0 & 0 & 0 & 0 & 0 \\
\hline LSC-BEIA-C14 & 0 & 0 & 0 & 0 & 0 & 0 & 0 & 0 & 0 & 0 \\
\hline LSC-BETA-TC99 & 0 & 0 & 0 & 0 & 0 & 0 & 0 & 0 & 0 & $\overline{0}$ \\
\hline LSC-BETA & 0 & 0 & 0 & 0 & 0 & 0 & 0 & 0 & o. & 0 \\
\hline GAMMA SPEC & 0 & 0 & 0 & 0 & 0 & 0 & 0 & 0 & 0 & 0 \\
\hline OTHER-PAD & 0 & 0 & 0 & 0 & 0 & 0 & 0 & 0 & 0 & 0 \\
\hline TOTAL RAD & 161287 & 01 & 0 & $\mathbf{0}$ & 01 & 0 & 161171 & 116 & 0 & 161287 \\
\hline & & & & & & & 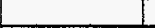 & 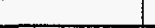 & - & \\
\hline TCLPEXTRACTION & 0 & 0 & 0 & 0 & 0 & 0 & 0 & 0 & 0. & 0 \\
\hline OTHER-PREP & 0 & 0 & 0 & 0 & 0 & 0 & 0 & 0 & 0 & 0 \\
\hline OTHEA-EXPLO & 0 & 0 & 0 & 0 & 0 & 0) & 0 & 0 & 0 & 0 \\
\hline OTHER-BLO & 0 & 0 & 0 & 0 & 0 & 0 & 0 & 0 & o & 0 \\
\hline OTHER-PHYS & 0 & 0 & 0 & 0 & 0 & 0 & 0 & 0 & o. & 0 \\
\hline OTHER & 0 & 0 & 0 & 0 & 0 & 0 & 0 & 0 & 0 & 0 \\
\hline TOTAL OIHEA & 0 & 01 & $\overline{0}$ & 01 & $0 \mid$ & 0 & 01 & 01 & D. & $\overline{0}$ \\
\hline & & & & & 1 & ل & & & & \\
\hline TOTAL SAMPLE ANALYSES & 225678 & 0 & 0 & $\mathrm{O}$ & 01 & O] & 225563 & 116 & 0] & 25678 \\
\hline
\end{tabular}


Table IVb: EC Sample Analyses by Rad Level and Matrix Type (xxi) Rocky Flats - GL881

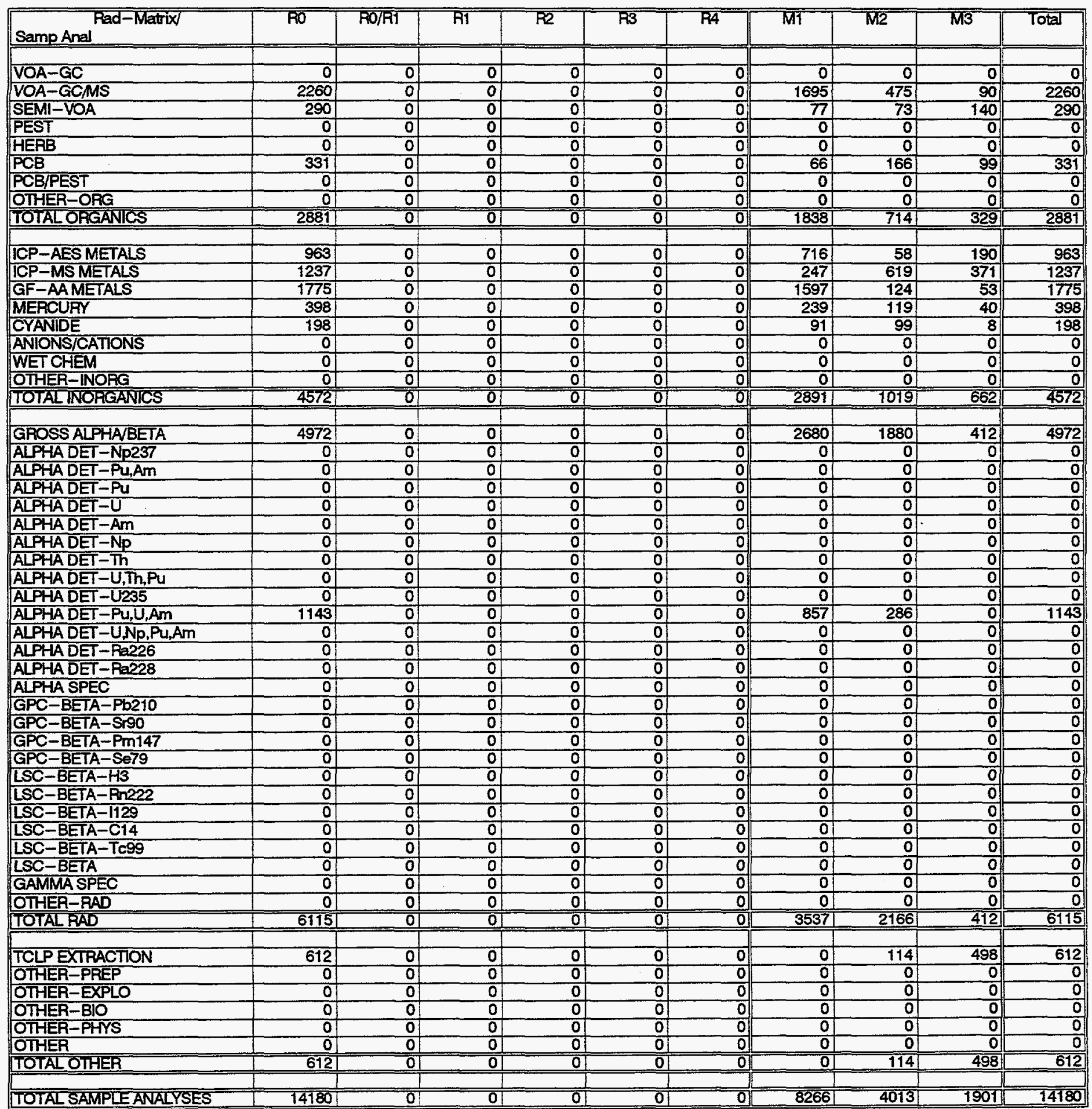


EM-263 SURVEY OF DOE ANALYTICAL CAPACITY AVAILABLE TO EM

Table IVb: EC Sample Analyses by Rad Level and Matrix Type (xxii) Rocky Flats - RF PA Lab

\begin{tabular}{|c|c|c|c|c|c|c|c|c|c|c|}
\hline $\begin{array}{l}\text { Rad-Matrix } \\
\text { Samp Anal }\end{array}$ & RO & RO/RT & R1 & $\mathrm{R} 2$ & R3 & R4 & $\overline{\mathrm{MT}}$ & $\overline{M 2}$ & $\mathrm{M} \mathrm{MB}$ & Total \\
\hline & & & & & & & & & & \\
\hline VOA-GC & 0 & 0 & 0 & 0 & 0 & 0 . & 01 & 0 & 0 & 0 \\
\hline VOA-GCMS & 0 & 0 & 0 & 0 & 2115 & 0 & 1023 & 683 & 410 & 2415 \\
\hline SEMI-VOA & 0 & 0 & 0 & 0 & 91 & 0 & 18 & 45 & 27 & 91 \\
\hline PEST & 0 & 0 & 0 & 0 & 0 & 0 & 0 & 0 & 0 & 0 \\
\hline HERB & 0 & $\overline{0}$ & 0 & 0 & 0 & 0. & 0 & 0 & 0 & 0 \\
\hline PCB & 0 & 0 & 0 & 0 & 205 & 0. & $4 t$ & 102 & 61 & 205 \\
\hline PCB/PEST & 0 & 0 & 0 & 0 & 0 & 0. & 0 & 0 & of & 0 \\
\hline OTHER-ORG & 0 & 0 & 0 & 0 & 0 & 0 & 0 & 0 & 0. & 0 \\
\hline TOTAL ORGANICS & 01 & 0 & 0 & 0 & $2 \overline{411}$ & $\overline{0}$ & 1082 & 831 & 498[ & 2411 \\
\hline & & & & & & & & & & \\
\hline ICP-AESMETALS & 0 & 0 & 0 & 0 & 675 & 0 & 135 & 337 & 202 & 675 \\
\hline ICP-MSMETALS & 0 & 0 & 0 & 0 & 1135 & 0) & 227 & 568 & 341 & 1135 \\
\hline GF-AAMETALS & 0 & 0 & 0 & 0 & 0 & 0 & 0 & 0 & 0. & 0 \\
\hline MERCUAY & 0 & 0 & 0 & 0 & 972 & 0 & 194 & 486 & 292 & 972 \\
\hline CYANIDE & 0 & 0 & 0 & 0 & 662 & 0) & 13 & 318 & 331 & 662 \\
\hline ANIONS/CATIONS & 0 & 0 & 0 & 0 & 0 & 0 & o & 0 & 0 & 0 \\
\hline WET CHEM & 0 & 0 & 0 & 0 & 0 & 0 & 0 & 0 & 0 & 0 \\
\hline OTHER-INOAG & 0 & 0 & 0 & 0 & 6679 & 0] & 3511 & 1678 & 1490 & 6679 \\
\hline TOTAL INORGANICS & o. & O & 01 & 0 & 10123 & o] & 4080 & 3387 & 2656 & 10123 \\
\hline & & & & & & & & & & \\
\hline GROSS ALPHAVBETA & 0 & 0 & 0 & 0 & 3004 & D. & 1603 & 764 & 637 & 3004 \\
\hline ALPHA DET-Np237 & 0 & 0 & 0 & 0 & o & o. & $\mathbf{0}$ & 0 & 0 & 0 \\
\hline ALPHA DET-Pu,Am & 0 & 0 & 0 & 0 & 0 & 0. & 0 & 0 & 0 & 0 \\
\hline ALPHA DEI-Pu & 0 & 0 & 0 & 0 & 0 & 0 & 0 & 0 & 0 & 0 \\
\hline ALPHA DET-U & 0 & 0 & 0 & 0 & 0 & 0 & 0 & 0 & 0 & 0 \\
\hline ALPHADET-Am & 0 & 0 & 0 & 0 & 0 & 0 & o & 0 & $\overline{0}$ & 0 \\
\hline ALPHA DET-Np & 0 & 0 & 0 & 0 & 0 & 0 & 0 & 0 & 0 & 0 \\
\hline ALPHAADET-Th & 0 & 0 & o & 0 & o & 0 & 0 & 0 & 0 & 0 \\
\hline ALPHA DET-U,Th,PU & 0 & 0 & 0 & 0 & 0 & 0 & 0 & 0 & 0 & 0 \\
\hline ALPHA DET-U235 & o & 0 & $\overline{0}$ & 0 & 0 & 0 & $\overline{0}$ & 0 & 0 & $\mathbf{0}$ \\
\hline ALPHA DET-PU,U,Am & 0 & 0. & 0 & 0 & 0 & 0 & 0 & D & 0 & 0 \\
\hline ALPHA DET-U,Np,Pu,Am & 0 & 0 & 0 & 0 & 0 & o & 0 & 0 & 0 & 0 \\
\hline ALPHA DET - Pa226 & 0 & 0 & $\overline{0}$ & 0 & 0 & o. & o & 0 & 0 & 0 \\
\hline ALPHADET-Ra228 & 0 & 0 & 0 & 0 & 0 & 0 & 0 & 0 & 0 & 0 \\
\hline ALPHA SPEC & 0 & 0 & 0 & 0 & 0 & 0 & o & 0 & 0 & 0 \\
\hline GPC-BETA-Pb210 & 0 & $\mathbf{0}$ & 0 & 0 & 0 & 0 & 0 & 0 & 0 & 0 \\
\hline GPC-BETA-Sr90 & 0 & $\mathbf{0}$ & 0 & 0 & 0 & 0 & D & 0 & 0 & 0 \\
\hline GPC-BETA-Pm147 & 0 & 0 & 0 & 0 & 0 & 0 & 0 & 0 & 0 & 0 \\
\hline GPC-BETA-Se79 & o & o & 0 & o & o) & 0 & o & 0 & 0 & 0 \\
\hline LSC-BETA-H3 & 0 & 0 & 0 & 0 & 0 & o. & 0 & 0 & 0 & 0 \\
\hline LSC-BETA-Pin222 & 0 & 0 & 0 & 0 & 0 & 0 & o & 0 & 0 & 0 \\
\hline LSC-BETA-1129 & 0 & 0 & 0 & 0 & 0 & o. & 0 & 0 & 0 & 0 \\
\hline LSC-BETA-C14 & 0 & 0 & 0 & 0 & 0 & 0 & 0 & 0 & $\overline{0}$ & 0 \\
\hline LSC-BETA-T099 & 0 & 0 & 0 & 0 & 0 & 0 & 0 & 0 & 0 & 0 \\
\hline LSC-BETA & 0 & 0 & 0 & 0 & 0 & o. & 0 & 0 & o & 0 \\
\hline GAMMA SPEC & 0 & a & 0 & 0 & 0 & 0 & o & 0 & 0 & 0 \\
\hline OTHER-RAD & 0 & 0 & 0 & 0 & 0 & o & $\mathbf{0}$ & 0 & 0 & 0 \\
\hline TOTAL BAD & 01 & 0] & 0 & 0 & 3004 & 0] & 16031 & 764 & 637 & 3004 \\
\hline & & & & & & & & & & \\
\hline TCLPEXTRACTION & 0 & 0 & 0 & 0 & 1136 & 0 & 23 & 545 & 568 & 1136 \\
\hline OTHER-PREP & 0 & 0 & 0 & 0 & 0 & 0 & 0 & 0 & 0 & 0 \\
\hline OTHER-EXPLO & o & 0 & 0 & 0 & 0 & 0 & 0 & 0 & 0 & 0 \\
\hline OTHER-BIO & 0 & 0 & 0 & 0 & 0 & o. & 0 & 0 & 0 & 0 \\
\hline OTHER-PHYS & 0 & 0 & 0 & 0 & 0 & 0 & 0 & 0 & 0 & 0 \\
\hline OTHER & 0 & 0 & 0 & 0 & 0 & 0. & o & 01 & 0 & 0 \\
\hline TOTAL OTHEA & $\mathbf{0}$ & 0 & 0] & 0 & 1136 & D. & 23 & 545 & 568 & 1136 \\
\hline & & & & & & . & & & & \\
\hline TOTAI SAMPLE ANALYSES & 0 & 0 & 0 & 01 & 16674 & 0] & 6788 & 5527 & 4359 & 16674 \\
\hline
\end{tabular}


Table IV: EC Sample Analyses by Rad Level and Matrix Type (xxiii) WSRC-ALD-CLAB

\begin{tabular}{|c|c|c|c|c|c|c|c|c|c|c|}
\hline $\begin{array}{l}\text { Rad-Matrix } \\
\text { Samp Anal }\end{array}$ & RO & RO/A1 & Ri & $\overline{P 2}$ & R3 & F4 & M1 & $\overline{M 2}$ & $\overline{M 3}$ & Total \\
\hline VOA-GC & 0 & 0 & 0 & 0 & 0 & 0 & o) & 0 & 0 & 0 \\
\hline VOA-GCMS & 0 & 0 & 0 & of & 0 & 0 & 0 & 0 & 0 & $\frac{1}{0}$ \\
\hline SEMI-VOA & 0 & 0 & 0 & 0 & 0 & (0) & 0 & 0 & 0 & $\overline{0}$ \\
\hline PEST & 0 & 0 & 0 & D) & 0 & D. & D & 0 & 0 & o \\
\hline HERB & of & 0 & 0 & 0 & 0 & 0 & 0 & 0 & (0) & 0 \\
\hline PCB & 0. & 0 & 0 & 0) & 0 & 0 & \begin{tabular}{l|l}
0 \\
\end{tabular} & 0 & 0 & 0 \\
\hline PCB/PEST & 0 & 0 & 0 & 0 & 0 & 0 & 0) & 0 & 0 & 0 \\
\hline OTHER-OAG & 0 & 0 & 0 & D & 0 & a & 0 & 0 & 0 & 0 \\
\hline TOTAL ORGANICS & 0 & 0 & $0]$ & 0] & 0 & 0 & 01 & 01 & 0. & 0 \\
\hline & & & & & & & & & 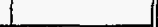 & \\
\hline ICP-AES METALS & 0 & o & 0 & 0 & 이 & 0] & 0 & 0 & 요 & 0 \\
\hline ICP-MSMETALS & 0 & 0 & 0 & 0 & of & 0 & 0 & 0 & (0) & $\overline{0}$ \\
\hline GF-AAMETALS & 0 & 0 & 0 & 0 & 0 & 0 & 0 & 0 & 0. & $\overline{0}$ \\
\hline MERCURY & 0 & 0 & 0 & 0 & 0 & 1696 & 1696 & 0 & - & 1696 \\
\hline CYANIDE & 0 & 0 & 0 & 0 & o & o & 0 & 0 & 0. & 0 \\
\hline ANIONS/CATIONS & D. & 0 & 0 & 2 & 0 & 87 & 87 & 0 & 2 & 89 \\
\hline WET CHEM & 0 & 0 & 0 & 0 & O & 0 & 0 & 0 & 0 & 0 \\
\hline OTHER-INORG & 0 & 0 & 218 & 202 & 1700 & 121 & 2241 & 0 & 인 & 2241 \\
\hline TOIAL INORGANICS & 0 & 0 & 218 & 204 & 1700 & 1904 & 40231 & 01 & 2 & 4025 \\
\hline & & & 0 & 000 & & 974 & 70001 & & $\pi$ & 700 \\
\hline ALPHA DET -Np237 & 0 & 0 & $\frac{0}{0}$ & -2 & $\frac{1}{0}$ & $=0$ & $\frac{10+0}{0}$ & $\frac{0}{0}$ & 0 & $\frac{1040}{0}$ \\
\hline ALPHADET-Pu,Am & 0 & 0 & 0 & 0 & of & o & 0 & $\overline{0}$ & 0 & $\frac{10}{0}$ \\
\hline ALPHADET-PU & 0 & 0 & $\overline{0}$ & o' & 0 & 0 & 0 & 0 & 0 & $\overline{0}$ \\
\hline ALPHADET-U & 0 & 이 & 0 & 0 & 0 & o. & 0 & 0 & ㅁ: & $\overline{0}$ \\
\hline ALPHA DET-Am & 0 & 0 & 0 & 0 & 0 & o. & 0 & 0 & 0) & 0 \\
\hline ALPHADET-Np & 0 & 0 & 0 & 0 & 0 & ㅇ. & 01 & 0 & 0 & 0 \\
\hline ALPHA DET-Th & O & 0 & 0 & 0 & 0 & o & 0 & 0 & 요 & 0 \\
\hline ALPHA DET-U,Th,Pu & 0 & 0 & 0 & 0 & 0 & 0 & 0 & 0 & 0. & $\overline{0}$ \\
\hline ALPHA DET-U235 & 0 & 0 & 0 & 0 & 0 & 의 & 0 & 0 & 의 & 0 \\
\hline ALPHA DET-PU,U,Am & o & 0 & O) & 0 & 0 & 0. & 0) & 0 & 의 & 0 \\
\hline ALPHA DET-U,Np,Pu,Am & 0 & 0 & 0 & 0 & O & 0. & 0 & 0 & of & D \\
\hline ALPHA DET-Ra226 & 0 & D. & 0 & 0 & of & 0 & 0 & 0 & 0 & 0 \\
\hline ALPHA DET-Ra228 & 0 & 0 & 0 & 0 & 0 & o. & 0 & 0 & 의 & 0 \\
\hline ALPHA SPEC & 0 & 0 & 544 & 199 & 0 & 379 & 1122 & 0 & 0 & 1122 \\
\hline GPC-BETA-Pb210 & 0 & 0 & 0 & 0 & 0 & 0 & 0 & 0) & (0) & 0 \\
\hline GPC-BETA-Sr90 & $\overline{0}$ & 0 & 0) & 0 & O & 0 & 0) & 0) & 요 & 0 \\
\hline GPC-BETA-Pm147 & o. & o & 0) & 0 & 0 & 0 & of & 01 & 0 & 0 \\
\hline GPC-BETA-Se79 & 0 & 0 & O) & o & 0 & 0 & 可 & o & 0 & $\overline{0}$ \\
\hline LSC-BETA-H3 & 0 & 0 & of & 0 & 0 & 0 & 0 & of & 0 & 0 \\
\hline LSC-BETA-Rin222 & 0 & 0 & O) & 0 & 0 & 0 & 0 & 0 & 0 & 0 \\
\hline LSC-BETA- 1129 & 0 & 0 & 0 & 0 & 0 & 0 & 0 & 0 & 0 & $\overline{0}$ \\
\hline LSC-BETA-C14 & 0 & 0 & 0 & 0 & 0 & 0 & 0 & 0 & 0 & $\overline{0}$ \\
\hline LSC-BETA-TC99 & 0 & 0 & 0 & 0 & 0 & 0 & 0 & 0 & 0 & 0 \\
\hline LSC-BETA & 0 & 0 & 0 & 0 & 0 & 0 & 0. & 0 & 0 & 0 \\
\hline GAMMA SPEC & 0 & 0 & 4263 & 3461 & 0 & 427 & 8152 & 0 & 0 & 8152 \\
\hline OTHER-RAD & 0 & 0 & (0) & O) & D. & 0 & 0 & 0 & 0 & 0 \\
\hline TOTAL RAD & 0 & 0 & 8041 & 6496) & 0 & 1778 & 16314 & 01 & 0 & 16314 \\
\hline & & & & & & & & & - & \\
\hline TCLP EXTRACTION & 0 & 0 & 0 & 0 & 0 & 0. & 0 & 0 & 0 & 0 \\
\hline OTHEA-PREP & 0 & 0 & 0 & 0 & 0 & 0 & a & 0) & 0 & 0 \\
\hline OTHER-EXPLO & 0 & 0 & 0 & 이 & 0 & 0 & 요 & 0 & 0 & 0 \\
\hline OTHER-BIO & 0 & 0 & 0 & 0. & 0 & 0 & 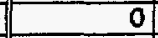 & 0 & 0 & 0 \\
\hline OTHER-PHYS & 0 & 0 & 0 & 0 & 0 & 0 & 0 & of & 0 & 0 \\
\hline OTHER & 0 & 0 & 0 & 0 & 0 & 0 & 0 & 0) & 0 & 0 \\
\hline TOTAL OTHER & 0 & 0 & 0 & 0 & $\overline{0}$ & $\overline{0}$ & 01 & 01 & 0 & 0 \\
\hline & & & & & & & - & 1 & $c_{-1}$ & \\
\hline TOTAL SAMPLE ANALYSES & 0 & 0 & 8259 & 6700 & 1700 & 3681 & 20338 & 01 & 2 & 20340 \\
\hline
\end{tabular}


EM-263 SURVEY OF DOE ANALYTICAL CAPACTY AVAILABLE TO EM

Table IVb: EC Sample Analyses by Rad Level and Matrix Type (xxiv) WSRC-ALD-320M

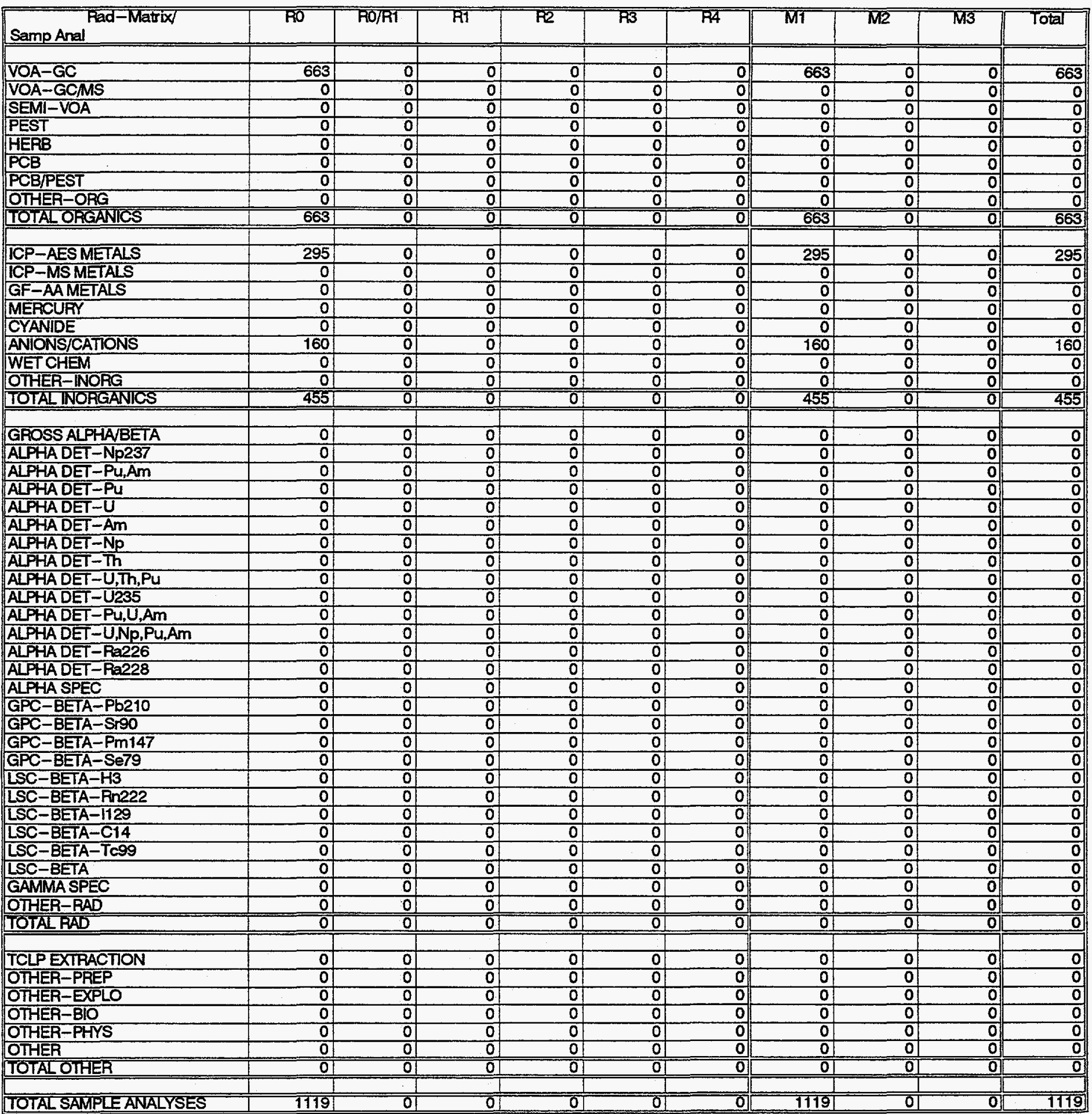


EM-263 SUAVEY OF DOE ANALYTICAL CAPACTYY AVAILABLE TO EM

Table IVb: EC Sample Analyses by Rad Level and Matrix Type (xxv) WSRC-ALD Water

\begin{tabular}{|c|c|c|c|c|c|c|c|c|c|c|}
\hline $\begin{array}{l}\text { Rad-Matrix } \\
\text { Samp Anal }\end{array}$ & Ro & RO/R1 & F1 & R2 & Ri3 & R4 & TVI & TM2 & 793 & Total \\
\hline VOA-GC & 0 & 0 & 0 & 0 & 0 & 0 & 0 & 0 & 0 & \\
\hline VOA-GCMS & 0 & 0 & 0 & 0 & 0 & 0 & 0 & 0 & 0 & 0 \\
\hline SEMI-VOA & 0 & 0 & 0 & 0 & 0 & 0 & 0 & 0 & (0) & 0 \\
\hline PEST & 0 & 0 & 0 & 0 & 0 & 0 & 0 & 0 & 0 & 0 \\
\hline HERB & 0 & 0 & 0 & 0 & 0 & 0 & 0 & 0 & 0 & 0 \\
\hline PCB & 0 & 0 & 0 & 0 & 0 & 0 & 0 & 0 & 마 & 0 \\
\hline PCB/PEST & 0 & 0 & 0 & 0 & 0 & -0 & 0 & 0 & 요 & 0 \\
\hline OTHER-ORG & 0 & 0 & 0 & 0 & 0 & $\overline{0}$ & 0 & a & 0 & 0 \\
\hline TOTAL OAGANICS & 이 & 0 & 01 & 0 & 0 & 0 & 0 & 0 & (0) & $\overline{0}$ \\
\hline & & & & & & & & & & \\
\hline ICP-AES METALS & O & 아 & 0 & 으 & 0 & 0 & 0 & 0 & -0 & 0 \\
\hline ICP-MSMETALS & 0) & of & 0 & 0 & 0 & (0) & 0 & 0 & 0 & 0 \\
\hline GF-AAMETALS & 이 & 요 & 으 & 0 & 0 & 0 & 0 & 0 & 의 & 0 \\
\hline MERCURY & 0 & 0] & 0 & 0 & 0 & 0 & 0 & D) & 무 & 0 \\
\hline CYANIDE & 0) & 0 & 0 & 0 & 0 & o & 0 & 0 & 0 & 0 \\
\hline ANIONS/CATIONS & 0 & D. & 0 & 0 & 0 & 0 & 0 & 0 & 0 & 0 \\
\hline WET CHEM & 이 & 0 & 0 & 0 & 0 & 0 & 0 & 0 & 0 & 0 \\
\hline OTHER-INORG & O) & 0. & 0 & 0 & 0 & 0 & 0 & 0 & 0 & 0 \\
\hline TOTAL INORGANICS & 01 & 01 & 01 & 0 & 0 & 0 & 0 & D) & 0 & 0 \\
\hline GROSS ALPHAVBETA & of & 0) & of & 0 & 0 & 0 & 0 & 0 & o & 0 \\
\hline ALPHA DET-Np237 & 0 & 0 & o & 0 & 0 & 0) & 0 & of & 0 & 0 \\
\hline ALPHA DET-Pu,Am & 0 & 0 & 0 & 0 & 0 & o & 0 & 0 & 0 & 0 \\
\hline ALPHADET-Pu & of & 0 & 0. & 0 & 0 & a & 0 & 0 & 무 & 0 \\
\hline ALPHA DET-U & 0 & 0 & 0 & o & 0 & 0 & 0 & 0 & 0. & 0 \\
\hline ALPHADET-Am & 0) & D. & (0) & 0 & $\overline{0}$ & 0 & 0 & 0 & (0) & 0 \\
\hline ALPHA DET-Np & 0 & 0 & 0 & o & 0 & 0 & o & 0 & 0 & 0 \\
\hline ALPHADET-Th & 0 & 0 & 0 & 0 & 0 & 의 & 0 & 0 & 의 & 0 \\
\hline ALPHADET-U,Th,PU & 0 & 0 & 0 & 0 & $\overline{0}$ & 0 & 0 & 0 & o. & 0 \\
\hline ALPHADET-U235 & 0 & 아 & 이 & o & 0 & 0 & 0 & 0 & 0 & 0 \\
\hline ALPHA DET-PU,U,Am & of & o) & 0 & 0 & 0 & 0 & 0 & D) & 마 & 0 \\
\hline ALPHADET - U,Np,Pu,Am & 0 & O) & D. & 0 & 0 & $\mathbf{0}$ & 0 & of & 0 & 0 \\
\hline ALPHA DET-Ra226 & 0 & O) & of & 0 & 0 & 0 & o & 0 & 0 & -0 \\
\hline ALPHA DET-Ra228 & 의 & 0 & D. & o & 0 & 0 & o & of & 0 & 0 \\
\hline ALPHA SPEC & O) & D. & D & 0 & $\overline{0}$ & 0 & 0 & D & 0 & 0 \\
\hline GPC-BETA-Pb210 & D) & 0 & 0 & 0 & 0 & 0) & 0 & of & -0. & 0. \\
\hline GPC-BETA-Sr90 & 0 & 0 & 0 & 0 & 0 & 0 & 0 & 0 & - & 0 \\
\hline GPC-BETA-Pm147 & o. & 0 & 0 & 0 & 0 & 0 & 0 & 이 & 0 & 0 \\
\hline GPC-BETA-Se79 & 0 & 0 & 0 & 0 & 0 & 0 & 0 & 0 & 므 & 0 \\
\hline LSC-BETA-H3 & 0 & 0 & 0 & 0 & 0 & 0 & 0 & 0 & 0 & 0 \\
\hline LSC-BETA-Rn222 & 0 & 0 & 0 & 0 & 0 & 0 & 0 & 아 & 0 & 0 \\
\hline LSC-BETA-1129 & O) & 므 & 可 & 으 & 0 & 0 & 으 & of & - & 0 \\
\hline LSC-BETA-C14 & o) & 0 & 0 & D & 0 & 0 & 0 & D. & 0] & 0 \\
\hline LSC-BETA-TC99 & 요 & of & 0 & 0 & 0 & 0 & 0 & 0 & 0 & 요 \\
\hline LSC-BETA & 01 & 요 & 0 & 아 & 0 & 0 & 0 & 0 & 0 & 0 \\
\hline GAMMA SPEC & O) & 0 & 501 & 0 & 0 & 0 & 501 & 0 & 0. & 501 \\
\hline OTHER-RAD & 0 & 0] & 3780 & 0 & 0 & a & 3780 & 0 & 0 & 3780 \\
\hline TOTAL RAD & 0 & 0 & 4281 & 0 & 0 & 0 & 4281 & 01 & 0. & 4281 \\
\hline & & & & & & & & & 1 & \\
\hline TCLP EXTRACTION & 0 & 0 & 0 & 0 & 0 & 0 & 0 & 0 & 며 & 의 \\
\hline OTHER-PREP & 0 & of & 0 & 0 & 0 & 0 & 0 & o & ㅇ. & 0 \\
\hline OTHER-EXPLO & 0) & D & 0 & 0 & 0 & 0 & 0 & O & (0) & 은 \\
\hline OTHER-BIO & 2012 & 0 & 0 & 0 & 0 & 0 & 2012 & 0 & 0 & 2012 \\
\hline OTHER-PHYS & 2761 & 0 & o & 0 & 0 & 0 & 2761 & 0 & 0. & 2761 \\
\hline OTHER & of & 0 & 0 & 0 & 0 & 0 & 0 & O) & 0 & 0 \\
\hline TOTAL OTHER & 4773 & 0 & 0] & 0 & 0 & 0 & 4773 & 01 & 可 & 4773 \\
\hline & & & & 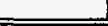 & & $z$ & & 1 & & \\
\hline TOTAL SAMPLE ANALYSES & 47731 & 0 & 4281 & 0 & $\underline{0}$ & (a) & 9054 & 01 & 0] & 9054 \\
\hline
\end{tabular}


EM-263 SURVEY OF DOE ANALYTICAL CAPACITY AVAILABLE TO EM

Table IVb: EC Sample Analyses by Rad Level and Matrix Type (xovi) WSRC-ALD-EIF

\begin{tabular}{|c|c|c|c|c|c|c|c|c|c|c|}
\hline $\begin{array}{l}\text { Rad-Matrix } \\
\text { Samp Anal }\end{array}$ & RO & RO/R1 & $\overline{\mathrm{R1}}$ & R2 & $\overline{\mathrm{F3}}$ & $\overline{\mathrm{FA}}$ & $\overline{\text { Mit }}$ & $\overline{M 2}$ & $\overline{M 3}$ & Total \\
\hline & & & & & & & & & & \\
\hline VOA-GC & 0 & 0 & 0 & 0 & 0 & 0 & 0 & 0 & 0 & D. \\
\hline VOA-GCMS & 0 & 0 & 0 & 0 & 0 & o & 0 & 0 & 0 & 0 \\
\hline SEMI-VOA & 0 & 0 & 0 & 0 & 0 & o. & 0 & 0 & 0 & 0 \\
\hline PEST & 0 & D: & 0 & 0 & 0 & 0 & 0 & 0 & 0 & 0 \\
\hline HERB & 0 & 0 & 0 & 01 & 0 & 0 & 0 & 0 & o. & 0 \\
\hline PCB & 0 & 0 & 0 & 0 & 0 & 0 & 0 & 0 & 0 & 0 \\
\hline FCB/PEST & 0 & 0 & 0 & 0 & 0 & 0 & 0 & 0 & 0 & 0 \\
\hline OTHER-ORG & 0 & 0 & 0 & 0 & 0 & o. & 0 & D & o. & D. \\
\hline TOTAL ORGANICS & 0 & $0]$ & $0]$ & OI & 01 & 01: & 0 & 0 & 0] & $\overline{0}$ \\
\hline & & & & & & & & & & \\
\hline ICP-AESMETALS & 0 & 0 & 0 & 0 & 0 & 0 & 0 & 0 & 0 & D \\
\hline ICP-MSMETALS & 0 & o & 0. & 0 & 0 & 0: & 0 & 0 & O) & D. \\
\hline GF-AAMETALS & 0 & 0 & of & 0 & 0 & 0) & 0 & 0 & 0 & D. \\
\hline MERCURY & 0 & 0 & 0) & 0 & 0 & 0 & 0 & 0 & o. & 0 \\
\hline CYANIDE & 0 & 0 & 0 & 0 & 0 & 0 & 0 & 0 & 0 & 0 \\
\hline ANIONS/CATIONS & 0 & 0 & 0 & 0 & 0 & 0 & 0 & 0 & 0 & $\overline{0}$ \\
\hline WET CHEM & 0 & 0 & 0 & 0 & 0 & 0 & 0 & 0 & 0 & $\mathbf{0}$ \\
\hline OTHER-INORG & 0 & 0 & $0 \mid$ & 0 & 0 & 0 & 0 & 0 & 0 & 0 \\
\hline TOTAL INORGANICS & 01 & DI & 01 & 01 & 01 & O] & 0 & OI & []] & $\overline{0}$ \\
\hline $\mathrm{ClC}$ & & & & & & 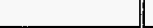 & - & 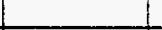 & 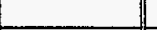 & \\
\hline GROSS ALPHAVBETA & 0 & 0 & 01 & 01 & 0 & 0 & 0 & 0 & 0 & $\overline{\mathbf{0}}$ \\
\hline ALPHADET-Np237 & 0 & 0 & 0 & 0 & 0 & o: & $\overline{0}$ & 0 & $\overline{0}$ & D \\
\hline ALPHA DET-Pu,Am & 0 & 0 & 0 & 0 & 0 & o) & 0 & 0 & 0. & 0 \\
\hline ALPHA DET-PU & 0 & 0 & 0 & 0 & 0 & o) & 0 & 0 & 0 & D. \\
\hline ALPHADET-U & 0 & 0 & 0 & 0 & 0 & 0 & 0 & O. & 0 & 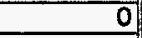 \\
\hline ALPHADET-Am & 0 & 0 & 0 & 0 & 0 & 0 & 0 & 0 & 0 & D \\
\hline ALPHADET-Np & 0 & 0 & 0 & 0 & 0 & 0 & $\overline{0}$ & 0 & 0 & 0 \\
\hline ALPHADET-Th & 0 & 0 & 0 & 0 & 0 & 0 & 0 & 0 & 0 & 0 \\
\hline ALPHA DET-U,Th,PU & 0 & 0 & 0 & 0 & 0 & 0 & 0 & 0 & 0 & 0 \\
\hline ALPHA DET-U235 & 0 & 0 & 0 & 0 & 0 & 0 & 0 & 0 & 0 & $\overline{0}$ \\
\hline ALPHADET-Pu,U,Am & 0 & 0 & 0 & 0 & 0 & 0 & 0 & 0 & 0 & 0 \\
\hline ALPHA DET-U,Np,PU,Am & 0 & 0 & 0 & 0 & 0 & 0 & 0 & 0 & 0 & 0 \\
\hline ALPHA DET-Ra226 & D & 0 & 0 & 0 & 0 & 0 & 0 & 0 & 0 & 0 \\
\hline ALPHADET-Ra228 & 0 & 0 & 0 & 0 & 0 & 0 & 0 & 0 & 0 & 0 \\
\hline ALPHA SPEC & 0 & 0 & 0 & 0 & 0 & $\mathbf{0}$ & 0 & 0 & 0 & 0 \\
\hline GPC-BETA-Pb210 & 0 & 0 & 0 & 0 & 0 & 0 & 0 & 0 & 0 & 0 \\
\hline GPC-BEIA-SROO & 0 & 0 & 0 & 0 & 0 & o & 0 & 0 & 0. & 0 \\
\hline GPC-BETA-Pm 147 & 0 & 0 & 0 & 0 & 0 & 0 & 0 & 0 & 0 & 웅 \\
\hline GPC-BETA-Se79 & 0 & 0 & 0 & 0 & 0 & 0 & 0 & 0 & 0 & 0 \\
\hline LSC-BETA-H3 & 0 & 0 & 0 & 0 & 0 & 0 & 0 & 0 & 0 & 0 \\
\hline LSC-BETA-Pn222 & 0 & 0 & 0 & 0 & 0 & 0 & 0 & 0 & 0 & 0 \\
\hline LSC-BETA-1129 & 0 & o) & 0 & 0 & 0 & $\overline{0}$ & 0 & 0 & 0 & 0 \\
\hline LSC-BETA-C14 & 0 & 0 & 0 & 0 & 0 & 0 & 0 & 0 & 0 & 요 \\
\hline LSC-BETA-TC99 & 0 & 0 & 0 & 0 & 0 & $\mathbf{0}$ & 0 & 0 & 0 & 0 \\
\hline LSC-BETA & o & o' & o & 0 & 0 & O) & 0 & 0 & 0 & 0 \\
\hline GAMMA SPEC & 0 & 0 & 0 & 0 & 0 & 0 & 0 & 0 & 0 & 요 \\
\hline OTHER-RAD & 0 & 0 & 0 & 0 & 01 & 0] & ol & 0 & 0 & ㅇ․ \\
\hline TOTAL RAD & 01 & 01 & 01 & 0 & OI & 0] & \begin{tabular}{l|l}
0 \\
\end{tabular} & 0 & 이 & (0) \\
\hline & & & & & & & & & & \\
\hline TCLPEXTRACTION & 0 & 0 & 0 & 0 & 0 & 0 & 0 & 0 & 0 & 0 \\
\hline OTHER-PREP & 0 & 0 & 0 & 0 & 0 & 0 & 0 & 0 & 0. & 0 \\
\hline OTHEA-EXPLO & 0 & 0 & 0 & 0 & 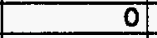 & 0 & 0 & 0 & o. & $\overline{0}$ \\
\hline OTHER-BIO & 0 & 0 & 0 & 0 & 0 & 0 & 0 & 0 & 0 & 0 \\
\hline OTHER-PHYS & 0 & 0 & 0 & 0 & 0 & 0 & 0 & 0 & 0 & D. \\
\hline OTHEA & 0 & o! & 0 & 0 & 0 & D. & 0 & 0 & o. & $\underline{0}$ \\
\hline TOTAL OTHER & 01 & $0 !$ & 0 & 01 & OI & 0. & 01 & 01 & Q]. & 0 \\
\hline & & & & & & 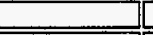 & $=0$ & & & \\
\hline TOTAL SAMPLE ANALYSES & 01 & 01 & 0 & 01 & 01 & 0][ & 0 & 01 & $\overline{0}[$ & $\underline{\underline{0}}$ \\
\hline
\end{tabular}


EM-263 SURVEY OF DOE ANALYTICAL CAPACTY AVAILABLE TO EM

Table Ib: EC Sample Analyses by Rad Level and Matrix Type (xxvii) WSRC-ALD-Tritium

\begin{tabular}{|c|c|c|c|c|c|c|c|c|c|c|}
\hline $\begin{array}{l}\text { Pad-Matix } \\
\text { Samp Anal }\end{array}$ & 70 & RO/R1 & Ri & $\overline{R 2}$ & R3 & $\overline{\mathrm{R} 4}$ & $\overline{\mathrm{MT}}$ & $\overline{\mathrm{T} 2}$ & $\overline{M 3}$ & Total \\
\hline VOA-GC & of & 0 & 0 & 0 & 0 & 0 & 0 & 0 & 0 & 0 \\
\hline VOA-GCMS & of & 0 & 0 & 0 & 0 & 0 & 0 & 0 & 0 & 0 \\
\hline SEMI-VOA & 0 & 0 & of & 0 & 0 & of & 0 & 0 & a & 0 \\
\hline PEST & 요 & 0 & 0 & 0 & 0 & 의 & 0 & 0 & o & 0 \\
\hline HERB & 0 & 0 & 0 & 0 & 0 & 0 & 0 & 0 & 0 & 0 \\
\hline PCB & 0 & 0 & 0 & 0 & 0 & D & 0 & 0 & 이 & 0 \\
\hline PCB/PEST & 0 & 0 & 0 & 0 & 0 & o & 0 & 0 & 이 & 0 \\
\hline OTHER-ORG & 0 & 0 & 0 & 0 & 0 & 0 & 0 & 0 & 이 & 0 \\
\hline TOTAL ORGANICS & 01 & 0 & 0 & 0 & 0 & - & 0 & 01 & 0] & 0 \\
\hline & & & & & & & & & $\|$ & \\
\hline ICP-AESMETALS & 0 & 0 & of & 0 & 0 & - & 0 & 0 & o. & 0 \\
\hline ICP-MS METALS & 0 & 0 & o & 0 & 0 & o & 0 & 0 & 0 & o \\
\hline GF-AAMETALS & 0 & 0 & 0 & 0 & 0 & 0 & 0 & 0 & 0 & 0 \\
\hline MERCURY & 0 & 0 & 0 & 0 & 0 & 0. & 0 & 0 & o & 0 \\
\hline CYANIDE & 0 & 0 & 0 & of & 0 & D. & 0 & 0 & 0 & 0 \\
\hline ANIONS/CATIONS & 0 & 0 & O) & 0 & 0 & 0 & 0 & 0 & 0 & 0 \\
\hline WET CHEM & of & 0 & o. & 0 & 0 & 0 & 0 & of & 0 & 0 \\
\hline OTHER-INOAG & 이 & 0 & 0 & 0 & 0 & (0) & 0 & of & 0 & 0 \\
\hline TOTAL INORGANICS & 01 & 0 & 01 & 0 & 01 & 0 - & 01 & 01 & 0 & 0 \\
\hline & & & & & & & + & 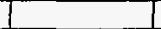 & 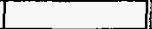 & \\
\hline GROSS ALPHABETA & 0 & 0 & D) & 0 & of & o. & of & 0 & 0 & 0 \\
\hline ALPHADET-Np237 & 0 & 0 & D. & o & 0 & o & o & 0 & 0 & 0 \\
\hline ALPHA DET-PU,Am & 0 & 0 & 0 & 0 & 0 & ㅁ & 0 & 0 & 0 & 0 \\
\hline ALPHA DET-PU & 0 & 0 & 0 & 0 & o & 0 & 0 & 0 & 0 & 0 \\
\hline ALPHA DET-U & 0 & 0 & 0 & 0 & 0 & 0 & 0 & 0 & 0 & 0 \\
\hline ALPHADET-Am & 0 & 0 & 0 & 0 & D. & 0 & 0 & 0 & (0) & 0 \\
\hline ALPHA DET-Np & of & 0 & 0 & 0 & 0 & 0 & 0 & 01 & - & 이 \\
\hline ALPHA DET-Th & 0 & 0 & 0 & 0 & 0 & (0) & o & of & 0 & 0 \\
\hline ALPHA DET-U,Th,PU & 0 & 0 & 0 & 0 & 0 & 요 & D & 0 & 0 & 0. \\
\hline ALPHADET-UZ235 & of & 0 & 0 & 0 & 0 & 0. & 0 & 0 & 0 & 0. \\
\hline ALPHA DET-PU,U,Am & 0 & 0 & 0 & 0 & 0 & of & 0 & 0 & 요 & 0 \\
\hline ALPHA DET-U,Np,Pu,Am & o. & 0 & D & 0 & 0 & 0 & 0 & 0 & 0 & 0 \\
\hline ALPHA DET-Ra226 & 0 & 0 & of & D & 0 & 0 & o & 0 & 0 & 0 \\
\hline ALPHA DET-Ra228 & .0 & 0 & D & 0 & 0 & 0 & 0 & 0 & 0. & 0 \\
\hline ALPHA SPEC & of & 0 & D) & 0 & 0 & 0 & of & 0 & 0. & 0 \\
\hline GPC-BETA-Pb210 & 0 & 0 & DI & 0 & 0 & 0 & 0 & 0 & 0 & 0 \\
\hline GPC-BETA-Sr90 & 0 & 0 & D) & 0 & 0 & 0) & 0 & 0 & a) & 0 \\
\hline GPC-BETA-Pm147 & of & 0 & 0 & 0 & 0 & 0. & of & 0 & 0 & 0 \\
\hline GPC-BETA-Se79 & 0 & 0 & 0 & 0 & 0 & 0 & 0 & 0 & 0 & 0 \\
\hline LSC-BETA-H3 & 0 & $\overline{0}$ & 0 & 0 & 0 & 0 & 0 & 0 & 0 & 0 \\
\hline LSC-BETA-Rn222 & 0 & $\overline{0}$ & Dा & 0 & 0 & 0 & 0 & 0 & 0 & 0 \\
\hline LSC-BETA- 1129 & 0 & 0 & 0 & 0 & 0 & 0 & 0 & 0 & 요 & 0. \\
\hline LSC-BETA-C14 & 0 & 0 & 0 & 0 & 0 & 0 & 0 & 0 & 의 & 0 \\
\hline LSC-BETA-TC99 & 0 & 0 & 0 & 0 & 0 & 0 & 0 & 0 & 이 & 0 \\
\hline LSC-BETA & 0 & 0 & 2566 & 0 & 0 & 0 & 2566 & 0 & 0 & 2566 \\
\hline GAMMA SPEC & 0 & 0 & 0 & 0 & 0 & 0 & 0 & 0 & 묘 & 0 \\
\hline OTHER-RAD & 0 & 0 & 0 & 0 & 0 & 0 & 0 & of & 0 & 0 \\
\hline TOTAL AAD & (0) & 0 & 2566 & 0 & 0 & 0. & 2566 & 01 & (0) & 2566 \\
\hline & & & & & & & & & & \\
\hline TCLP EXTRACTION & 0 & 0 & 0 & 0 & 0 & $\mathbf{0}$ & of & 0 & 0 & 0 \\
\hline OTHER-PREP & 0 & 0 & D & 0 & 0 & 0 & 0 & 0 & 0 & 0 \\
\hline OTHER-EXPLO & 0 & 0 & 0 & 0 & 0 & 0 & 0 & 0 & 0 & 0 \\
\hline OTHER-BIO & 0 & 0 & 0 & 0 & 0 & 0 & 0 & 0 & 0 & 0 \\
\hline OTHER-PHYS & 0 & 0 & 0 & 0 & 0 & 0 & 0 & 0 & 0 & 0 \\
\hline OTHER & 0 & $\overline{0}$ & 0 & 0 & 0 & 0 & 0 & 0 & 0 & 0 \\
\hline TOTAL OTHER & 0 & 0 & 0 & 0 & 0 & 이 & 0 & 0 & 0 & 0 \\
\hline & & & & & & & & & & \\
\hline TOTAL SAMMPLE ANALYSE & $0]$ & 0 & 2566 & 0 & 0 & 0] & 2566 & 0 & 0 & 2566 \\
\hline
\end{tabular}


EM-263 SURVEY OF DOE ANALYTICAL CAPACIY AVAILABLE TO EM

Table IV: EC Sample Analyses by Rad Level and Matrix Type (xoviii) WSRC-ALD-ITP

\begin{tabular}{|c|c|c|c|c|c|c|c|c|c|c|}
\hline $\begin{array}{l}\text { Pad-Matrix } \\
\text { Samp Anal }\end{array}$ & RO & Ro/R1 & $\overline{\text { R1 }}$ & $\overline{722}$ & R3 & $\overline{R 4}$ & $\overline{\text { M1 }}$ & $\overline{M 2}$ & M3 & Total \\
\hline VOA-GC & 0 & 0 & 0 & 0 & 0 & 0 : & 0 & 0 & of & 0 \\
\hline VOA-GCMS & 0 & 0 & 0 & 0 & 0 & 0 & 0 & 0 & o & 0 \\
\hline SEMI-VOA & 0 & 0 & o & 0 & 0 & (⿹ & 0 & 0 & o & $\overline{0}$ \\
\hline PEST & 0 & 0 & 0 & 0 & 0 & 0] & 0 & 0 & of & 0 \\
\hline HERB & 0 & 0 & 0 & 0 & 0 & 0 & 0 & 0 & on & 0 \\
\hline PCB & 0 & \begin{tabular}{l|l}
0 \\
\end{tabular} & 0 & 0 & o & 0 & 0 & 0 & of & 0 \\
\hline PCB/PEST & 0 & 0 & 0 & 0 & 0 & 0] & o & Oा & 0 & 0 \\
\hline OTHER-ORG & 0 & 0 & 0 & 0 & 0 & o] & 0 & 0 & 묘 & 0 \\
\hline TOTAL ORGANICS & 01 & 0 & 0 & 01 & 0 & 0 & 0 & 01 & of & (0] \\
\hline & & & & & & & & 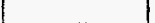 & & \\
\hline ICP-AESMETALS & 0 & 0 & 0 & 0 & 0 & - & 0 & 0 & 요 & 0 \\
\hline ICP-MS METALS & 0) & 0 & 0 & 0 & 0. & 요 & 0 & of & 0 & 0 \\
\hline GF-AAMETALS & 0 & 0 (1) & 0 & 0 & 0 & 요 & 0 & 0 & o. & 0 \\
\hline MERCURY & 0 & 0 & 0 & 0 & 0 & - & 0 & 0 & 0 & 0 \\
\hline CYANIDE & 0 & 0 & 0 & o & 0 & D. & o & 0 & 0. & 0 \\
\hline ANIONS/CATIONS & o) & 0 & 0 & 0 & 0 & - & 0 & of & 0. & 0 \\
\hline WET CHEM & 0 & 0 & 0 & 0 & 0 & 0 & 0 & o) & 요 & 0 \\
\hline OTHER-INORG & D) & $0 \mid$ & $0 \mid$ & 0 & 0. & o & of & 01 & 의 & 0 \\
\hline TOTAL INORGANICS & 0! & 01 & 01 & 0 & 0 & 0ा & 0 & 이 & 0] & $\overline{0}$ \\
\hline & & & & & & & & & & \\
\hline GROSS ALPHA/BETA & 0 & 0 & 0 & 0 & 0 & ㅁ. & 0 & 0 & 0. & 0 \\
\hline ALPHADET-Np237 & 0 & 0 & 0 & 0 & D. & o & o & 0 & of & 0 \\
\hline ALPHA DET-PU,Am & 0 & 0 & 0 & 0 & o. & 0 & 0 & 0 & 0 & 0 \\
\hline ALPHA DET -PU & 0 & 0 & 0 & 0 & 0 & 0 & 0 & of & o & 0 \\
\hline ALPHA DET-U & 0 & 0 & 0 & 0 & 0 & 0 & $\mathbf{0}$ & o & 0 & 0 \\
\hline ALPHA DET-Am & 0 & 0 & 0 & 0 & of & 이 & 0 & 0 & 의 & 0 \\
\hline ALPHA DET-NP & 0 & of & 0 & 0 & of & 이 & 0 & 0 & (0) & 0 \\
\hline ALPHADET-Th & 0 & 0 & 0 & 0 & 0 & - & $\mathbf{0}$ & 0 & (0) & 0 \\
\hline ALPF-IA DET-U,Th,Pu & 0 & 0 & 0 & 0 & of & 마 & 0 & 0 & 요 & 0 \\
\hline ALPHA DET-U235 & 0 & 0 & 0 & 0 & o & 0 & 0 & of & 0 & 0 \\
\hline ALPHA DET - PU,U,Am & 0 & o. & 0 & 0 & 0 & - & 0 & of & 0 & 0 \\
\hline ALPHA DET-U,Np,PU,Am & 0 & 0 & 0 & 0 & 0 & - & 0 & 0 & 0 & 0 \\
\hline ALPHADET-Ra226 & 0 & 0 & 0 & of & 0 & o. & 0 & 0 & - & 0 \\
\hline ALPHADET-Ra228 & 0 & 0 & 0 & 0 & 0 & - & (0) & 0 & 0 & 0 \\
\hline ALPHA SPEC & 0 & 0 & 0 & 0 & of & 0 & 0 & 0 & 0 & 0 \\
\hline GPC-BETA-Pb210 & 0 & 0 & 0 & 0 & 0 & 0 & 0 & 0 & 0. & 0 \\
\hline GPC-BETA-Sr90 & 0 & 0 & 0 & 0 & 0 & 0 & 0 & o & 0 & 0 \\
\hline GPC-BETA-Pm 147 & 0 & 0 & 0 & 0 & 0 & 0 & 0 & 0 & 0 & 0 \\
\hline GPC-BETA-Se79 & 0 & 0 & 0 & 0 & 0 & o & 0 & 0 & 0 & 0 \\
\hline LSC-BETA-H3 & 0 & 0 & 0 & 0 & 0 & 0 & 0 & of & 0 & 0 \\
\hline LSC-BETA-Rn222 & 0 & 0 & 0 & 0 & 0 & 0 & 0 & 0 & 이 & 0 \\
\hline LSC-BETA- 1129 & 0 & 0 & 0 & o & 0 & 0 & 0 & of & 0. & 0 \\
\hline LSC-BETA-C14 & 0 & 0 & 01 & 0 & 0 & 0 & 0 & 0 & 매 & 0 \\
\hline LSC-BETA-TC99 & 0 & 0 & 0 & 0 & 0 & 0 & 0 & 0 & a & 0 \\
\hline LSC-BETA & 0 & Oा & 0 & 0 & of & 0 & 0 & 0 & (0) & 0 \\
\hline GAMMA SPEC & 0 & 0 & 0 & 0 & 0 & 0 & 0 & 0 & 0 & 0 \\
\hline OTHER-RAD & 0 & 0 & 0 & 0 & 0 & 0 & 0 & 0 & 0. & 0 \\
\hline TOTAL RAD & $\overline{0}$ & 0.1 & 0. & 01 & 0 & 0 & 01 & 0 & 0 & 0 \\
\hline & & & & & 7 & I & $\square$ & & & \\
\hline TCLP EXTRACTION & 0 & 0 & 0 & 0 & 0 & 마 & 0 & 0 & o & 0 \\
\hline OTHER-PREP & 0 & 0 & of & 0 & 0 & 0 & 0 & 0 & 0 & 0 \\
\hline OTHER-EXPLO & 0 & of & 0 & 0 & 0 & 0 & 0 & 0 & 요 & 0 \\
\hline OTHER-BIO & $\overline{0}$ & 0 & 0 & 0 & 0 & 0 & 0 & 0 & 0 & 0 \\
\hline OTHER-PHYS & 0 & 0 & 으 & 0 & 0 & 0 & 0 & 0 & 0 & 0 \\
\hline OTHER & 0 & 0 & of & 0 & 0 & 0 & 0 & 0 & 0] & 0 \\
\hline TOTAL OTHER & 0 & 0 & 01 & 0 & 0 & 0 & 0 & 0 & 0 & 0 \\
\hline & & & & & 7 & ] & 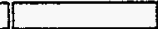 & t & & \\
\hline TOTAL SAMPLEANALYSE & $\overline{0}$ & 01 & 0 & 0 & 0 & 0 & 0 & 0 & 아 & 0 \\
\hline
\end{tabular}


Table Nb: EC Sample Analyses by Rad Level and Matrix Type (xxix) WSRC-SRTC-ADS

\begin{tabular}{|c|c|c|c|c|c|c|c|c|c|c|}
\hline $\begin{array}{l}\text { Aad-Matrix } \\
\text { Samp Anal }\end{array}$ & $\mathrm{RO}$ & Ro/R1 & $\overline{\mathrm{RI}}$ & $\overline{R 2}$ & $\overline{\mathrm{R} 3}$ & R4 & $\overline{M 1}$ & $\overline{\mathrm{M} 2}$ & TM3 & Tota \\
\hline & & & & & & & & & & \\
\hline VOA-GC & 0 & 0. & 838 & 0 & 0 & 0 & 503 & 168 & 168 & 838 \\
\hline VOA-GCMS & 0 & 0 & 805 & 0 & 0 & 0 & 483 & 161 & 161 & 805 \\
\hline SEMI-VOA & 0 & 0 & 621 & 0 & 0 & of & 311 & 311 & 0 & 621 \\
\hline PEST & 0 & 0 & 635 & 01 & 0 & 0 & 317 & 317 & $\overline{0}$ & 635 \\
\hline HERB & 0 & 0 & 635 & D. & 0 & 0 & 317 & 317 & 0 & 635 \\
\hline PCB & 0 & 0 & 635 & 0 & 0 & 0 & 317 & 317 & 0 & 635 \\
\hline PCB/PEST & 0 & 0 & 0 & 0 & 0 & 0 & 0 & 0 & 0 & 0 \\
\hline OTHER-ORG & 0 & 01 & 0 & 01 & 0 & 0 & 0 & $0 !$ & 묘 & O \\
\hline TOTALOAGANICS & 01 & 01 & 4168 & 01 & 01 & 0] & 2248 & 1591 & 329 & 4168 \\
\hline & & & & & & & & & & \\
\hline ICP-AES METALS & 0 & 0 & 5200 & 0 & 0 & o: & 2600 & 2080 & 520 & 5200 \\
\hline KCP-MSMETALS & 0 & 0 & 307 & 0 & 0 & 0 & 154 & 92 & 61 & 307 \\
\hline GF-AAMETALS & 0 & 0 & 1165 & 0 & 0 & o) & 582 & 582 & ㅇ. & 1165 \\
\hline MERCURY & 0 & 0 & 197 & 0 & 0 & a & 126 & 57 & 14 & 197 \\
\hline CYANIDE & 0 & 0 & 532 & 0 & 0 & $\overline{0}$ & 266 & 266 & 0 & 532 \\
\hline ANIONS/CATIONS & 0 & 0 & 6392 & 0 & 0 & 0 & 5114 & 959 & 320 & 6392 \\
\hline WET CHEM & 0 & 0 & 0 & 0 & 0 & o) & 0 & 0 & 0 & 0 \\
\hline OTHER-INORG & 0 & 0 & 0 & 0 & 0] & 0) & 0 & 0 & 0 & 0. \\
\hline TOTAL NOFGANICS & 01 & 01 & 13793 & 01 & O! & б] & 8842 & 4036 & 915 & 13793 \\
\hline & & & & & & . & & & & \\
\hline GROSS ALPHAVETA & 0 & D) & 6246 & 0 & 0 & 0 & 3748 & 1874 & 625 & 6246 \\
\hline ALPHA DET-Np237 & 0 & 0 & 0 & 0 & 0 & 0 & 0 & 0 & o) & $\mathbf{0}$ \\
\hline ALPHA DET-Pu,Am & 0 & 0 & 0 & 0 & 0 & 0 & 0 & 0 & 0 & 0 \\
\hline ALPHADET-PU & 0 & 0 & 0 & 0 & 0 & o & 0 & 0 & 0 & 0 \\
\hline ALPHA DET-U & 0 & 0 & 0 & 0 & 0 & 0 & 0 & 0 & 0 & 0 \\
\hline ALPHADET-Am & 0 & 0 & o & 0 & 0 & 0. & 0 & 0 & o) & 0 \\
\hline ALPHADET-Np & 0 & 0 & 0 & 0 & 0 & 0 & 0 & 0 & 0 & 0) \\
\hline ALPHA DET - Th & 0 & 0 & 0 & 0 & 0 & 0 & 0. & O & o. & O) \\
\hline ALPHA DET-U,Th,PU & 0 & 0 & 0 & 0 & 0 & 0. & 0 & 0 & o) & 0 \\
\hline ALPHA DET-U235 & 0 & 0 & 0 & 0 & 0 & O) & 0 & 0 & 0. & 0] \\
\hline ALPHA DET-Pu,U,Am & 0 & 0 & 0 & 0 & 0 & 0 & 0 & O & O) & D. \\
\hline ALPHA DET-U,Np,PU,Am & 0 & 0 & 0 & 0 & 0 & 0. & 0 & 0 & 0 & - \\
\hline ALPHA DET-Pa226 & 0 & 0 & 0 & 0 & 0 & O) & 0 & 0 & 요 & 0 \\
\hline ALPHADET-Pa228 & 0 & 0 & 0 & 0 & 0 & 0) & $\mathbf{0}$ & 0 & o & 0 \\
\hline ALPHA SPEC & 0 & 0 & 1441 & 0 & 0 & 0 & 721 & 577 & 144 & 1441 \\
\hline GPC-BETA-Pb210 & 0 & 0 & 0 & 0 & 0 & o. & 0 & 0 & 0 & 0 \\
\hline GPC-BETA-Sr90 & 0 & 0 & 0 & 0 & 0 & 0 & 0 & 0 & of & 인. \\
\hline GPC-BETA-Pm147 & 0 & 0 & 0 & 0 & 0 & of & 0 & 0 & 0 & 0. \\
\hline GPC-BETA-Se79 & 0 & 0 & 0 & 0 & 0 & 0 & 0 & 0 & 0 & 요 \\
\hline LSC-BETA-H3 & 0 & 0 & D) & 0 & 0 & 0 & 0 & 0 & of & (0) \\
\hline LSC-BETA-Rn222 & 0 & 0 & 0 & 0 & 0 & 0) & 0 & 0 & 에 & 요 \\
\hline LSC-BETA- 1129 & 0 & 0 & 01 & 0 & 0 & 0 & 0 & 0 & 0. & 욤 \\
\hline LSC-BETA-C14 & 0 & 0 & 0 & 0 & 0 & 0. & 01 & 0 & 0 & 욤 \\
\hline LSC-BETA-TC99 & 0 & 0 & 0 & 0 & 0 & 0 & 0 & 0 & 0 & 0 \\
\hline LSC-BETA & 0 & 0 & 0 & 0 & 0 & 0 & 0 & 0 & 0 & 0 \\
\hline GAMMA SPEC & 0 & 0 & 4352 & 0 & 0 & 0. & 2611 & 1306 & 435 & 4352 \\
\hline OTHEA-AAD & 0 & 0 & 0 & 0 & 01 & 0 & 0 & 01 & 0 & 0 \\
\hline TOIAL RAD & 01 & 01 & 12040 & 01 & 01 & 0] & 7080 & 3756 & 1204 & 12040 \\
\hline & & & & & & & & 1 & 4 & \\
\hline TCLP EXTPACTION & 0 & 0 & 48 & 0 & 0 & 0 & 15 & 29 & 5) & 48 \\
\hline OTHER-PREP & 0 & 0 & 0 & 0 & 0 & 189 & 19 & 113 & 57 & 189 \\
\hline OTHER-EXPLO & 0 & 0 & 0 & D. & 0 & 0 & 0 & 0 & 0 & 0 \\
\hline OTHER-BIO & 0 & 0 & 0 & 0 & 0 & 0 & 0 & \begin{tabular}{l|l}
0 \\
\end{tabular} & 0 & 0 \\
\hline OTHER-PHYS & 0 & 0 & 0 & 0 & 0 & 0 & 0 & 0 & 0 & 0 \\
\hline OTHER & 0 & 0 & 10815 & 01 & 0 & 27 & 5421 & 3252 & 2168 & 10842 \\
\hline TOTALOTHEA & 0 & 0 & 10863 & 01 & 01 & 216 & 5454 & 3395 & 2230 & 11079 \\
\hline & & & & & & & & & & \\
\hline TOTAL SAMPLE ANALYSES & 0 & 0 & 40864 & 0 & O) & $216]$ & 23624 & 12778 & 4678 & 41080 \\
\hline
\end{tabular}


EM-263 SURVEY OF DOE ANALYTICAL CAPACITY AVAILABLE TO EM

Table IV: EC Sample Analyses by Rad Level and Matrix Type (xox) WSRC-SRTC-DWPT

\begin{tabular}{|c|c|c|c|c|c|c|c|c|c|c|}
\hline $\begin{array}{l}\text { Fad-Matrix } \\
\text { Samp Anal }\end{array}$ & $\mathrm{RO}$ & RO/AT & Ri & $\mathrm{R} 2$ & $\mathrm{~F} 3$ & R4 & $\mathrm{MT}$ & $\mathrm{ML}$ & M3 & Total \\
\hline & & & & & & & & & & \\
\hline VOA-GC & 770 & 0 & 0 & 0 & 0 & 0 & 539 & 154 & 77 & 770 \\
\hline VOA-GCMS & 0 & 0 & 0. & 0 & 0 & 0 & O & 0 & 0 & 0 \\
\hline SEMI-VOA & 0 & 0 & 0 & 0 & 0 & 0 & 01 & 0 & 0 & 0 \\
\hline PEST & 0 & 0 & 0 & 0 & 0 & 0 & 0 & 0 & 0 & 0 \\
\hline HERB & 0 & 0 & 0 & 0 & 0 & 0 & 0 & 0 & 0 & 0 \\
\hline $\mathrm{PCB}$ & 0 & 0 & 0 & 0 & 0 & 0 & 0 & 0 & 0 & 0 \\
\hline PCB/PEST & 0 & 0 & 0 & 0 & 01 & 0 & D: & 0 & 0 & 0 \\
\hline OTHER-ORG & 0 & 0 & 0 & 0 & 01 & 0 & 0 & 0 & 0 & 0 \\
\hline TOTAL ORGANICS & 7701 & 01 & 0 & 0 & 01 & o] & 539 & 154 & 77 & 770 \\
\hline & & & & & & $\square$ & & & & \\
\hline ICP-AES METALS & 3858 & 0 & 0 & 0 & 0 & 0 & 386 & 2700 & 772 & 3858 \\
\hline ICP-MSMETALS & 0 & 0 & 0 & 0 & 0 & 0. & 0 & 0 & 0 & 0 \\
\hline GF-AAMETALS & 0 & 0 & 0 & 0 & 0 & 0 & 0 & 0 & $\mathbf{0}$ & 0 \\
\hline MERCURY & 1038 & 0 & 0 & 0 & 0 & 0 & 519 & 519 & 0. & 1038 \\
\hline CYANIDE & 0 & 0 & 0 & 0 & 0 & 0. & 01 & 0 & $\mathbf{0}$ & 0 \\
\hline ANIONS/CATIONS & 2511 & 0 & 0 & 0 & 0 & 0 & 251 & 1758 & 502 & 2511 \\
\hline WET CHEM & 0 & 0 & 0 & 0 & 0 & 0 & 0 & 0 & 0 & 0 \\
\hline OTHER-INORG & 01 & 0 & 0 & 0 & 01 & $0]$ & 0! & 01 & 0 & 0 \\
\hline LOTALINORGANICS & 7407 & 01 & 01 & $\overline{0}$ & 01 & D.] & 1156 & 4977 & 1274 & $\overline{7407}$ \\
\hline & & & & & & & & 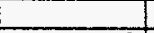 & & \\
\hline GROSS ALPHAVBETA & 0 & 0 & 0 & 0 & 0 & 0 & 0 & 0 & 0 & $\overline{0}$ \\
\hline ALPHADET-Np237 & 0 & 0 & 0 & 0 & 0 & 0 & O & 0 & $\mathbf{0}$ & 0 \\
\hline ALPHA DET-Pu,Am & 0 & 0 & 0 & 0 & 0 & 0 & D) & 0 & 0 & 0 \\
\hline ALPHA DET-PU & 0 & 0 & 0 & 0 & 0 & D. & 0 & 0 & 0 & $\overline{0}$ \\
\hline ALPHA DET-U & 0 & 0 & 0 & 0 & 0 & 0 & 0 & 0 & 0 & 0 \\
\hline ALPHADET-Am & 0 & 0 & 0 & 0 & 0 & 0. & 0 & 0 & 0 & 0 \\
\hline ALPHADET-Np & 0 & 0 & 0 & 0 & 0 & 0 & 0 & 0 & 0 & 0 \\
\hline ALPHA DET - Th & 0 & 0 & 0 & 0 & 0 & 0 & 01 & 0 & 0 & 0 \\
\hline ALPHA DET-U,Th,PU & 0 & 0 & 0 & 0 & $\mathbf{0}$ & 0. & 0 & 0 & 0 & 0 \\
\hline ALPHADET-U235 & 0 & 0 & 0 & 0 & 0 & 0. & 0) & 0 & 0 & 0 \\
\hline ALPHA DET-Pu,U,Am & 0 & 0 & 0 & 0 & 0 & 0. & 0 & 0 & of & 0 \\
\hline ALPHA DET-U,Np,Pu,Am & 0 & 0 & 0 & 0 & 0 & 0 & 0 & 0 & 0. & $\mathbf{0}$ \\
\hline ALPHADET-Pa226 & 0 & 0 & 0 & 0 & 0 & 0 & 0 & 0 & 욤 & 0 \\
\hline ALPHA DET-Pa228 & 0 & 0 & 0 & 0 & 0 & D. & 0 & 0 & D. & 0 \\
\hline ALPHA SPEC & 0 & 0 & 0 & 0 & 0 & o: & 0 & 0 & 0 & $\mathbf{0}$ \\
\hline GPC-BETA-PD210 & 0 & 0 & 0 & 0 & 0 & 0 & 01 & 0 & 0 & 0 \\
\hline GPC-BETA-Sr90 & 0 & 0 & 0 & 0 & 0 & 0. & 0 & 0 & 0 & $\overline{0}$ \\
\hline GPC-BETA-Pm147 & 0 & 0 & 0 & 0 & 0 & 0 & 0 & 0 & 0 & 0 \\
\hline GPC-BETA-Se79 & 0 & 0 & 0 & 0 & 0 & 0 & 0 & 0 & o & $\overline{0}$ \\
\hline LSC-BETA-H3 & 0 & 0 & 0 & 0 & 0 & 0 & 0 & 0) & 0 & 0 \\
\hline LSC-BETA-Rn222 & 0 & 0 & 0 & 0 & 0 & 0 & 0 & 0 & o. & 0 \\
\hline LSC-BETA-1129 & 0 & 0 & 0 & 0 & 0 & 0 & 0 & 0 & 0 & 0 \\
\hline LSC-BETA-C14 & 0 & 0 & 0 & 0 & 0 & 0 & 0 & 0 & 0. & 0 \\
\hline LSC-BETA-TC99 & 0 & 0 & 01 & 0 & 0 & 0 & 0 & 0 & 0 & 0 \\
\hline LSC-BETA & 0 & 0 & 0 & 0 & 0 & 0 & 0 & 0 & 0 & 0 \\
\hline GAMMASPEC & 0 & 0 & 0 & 0 & 0 & 0 & 0 & 0 & 0 & $\overline{0}$ \\
\hline OTHER-RAD & 0 & 0 & 0 & 0 & 01 & 0 & 0 & 0 & 0. & 0 \\
\hline TOTAL $\mathrm{AAD}$ & 01 & 0 & 01 & 0 & 01 & 0] & 01 & 01 & 0 & 0 \\
\hline & & & & & & & & & & \\
\hline TCLP EXTRACTION & 0 & o! & 0 & 0 & 0 & 0 & 0 & $\mathbf{0}$ & 0 & 0 \\
\hline OTHER-PREP & 0 & 0 & 0 & 0 & 0 & 0 & 0 & 0 & 0 & 0 \\
\hline OTHER-EXPLO & 0 & 0 & 0 & 0 & 0 & 0 & 0 & $\mathbf{0}$ & 0 & 0 \\
\hline OTHER-BIO & 0 & 0 & 0 & 0 & 0 & 0. & D) & 0 & 0 & 0 \\
\hline OTHER-PHYS & 0 & 0 & 0 & 0 & 0 & 0 & $\mathbf{0}$ & 0 & 0 & 0 \\
\hline OTHER & 0 & 0 & 0 & 0 & 0 & 0] & 0 & 0 & 0 & 0 \\
\hline TOIALOIAER & 0 & 0 & 0 & 0 & 0 & ol: & 01 & 01 & O] & $\underline{\underline{0}}$ \\
\hline & & & & & 1 & & & & & \\
\hline TOTAI SAVIPLE ANALYSES & 8177 & 01 & 0 & 0 & 01 & ㅇ] & 1695 & 5131 & 1351 & 8177 \\
\hline
\end{tabular}


EM-263 SURVEY OF DOE ANALYTICAL CAPACITY AVAILABLE TO EM

Table Nc: MC Sample Analyses by Rad Level and Matrix Type (i) Complex-wide

\begin{tabular}{|c|c|c|c|c|c|c|c|c|c|c|}
\hline Samp Anal & RO & FOO/RI & R1 & RE & R3 & $\overline{\mathrm{R4}}$ & $\overline{\overline{M 1}}$ & $\mathrm{M} 2$ & TM3 & Total \\
\hline VOA-GC & 1625 & $\overline{0}$ & 2515 & 0 & 0 & 0 & 2091 & 700 & 1349 & 4140 \\
\hline VOA-GCMS & 48349 & 35154 & 18242 & 2480 & 5608 & 0 & 77218 & 24026 & 8588 & 109832 \\
\hline SEMI-VOA & 20256 & 4253 & 13972 & 1916 & 553 & o & 25563 & 12027 & 3359 & 40950 \\
\hline PEST & 2897 & 815 & 1904 & 668 & of & o & 3699 & 1936 & 649 & 6284 \\
\hline HERB & 1486 & 1490 & 1904 & 668 & 0 & 0 & 3425 & 1533 & 590 & 5548 \\
\hline PCB & 30510 & 24369 & 10517 & 668 & 1849 & 0. & 45145 & 14076 & 8693 & 67914 \\
\hline PCB/PEST & 447 & 1805 & 0 & 0 & 0 & 요 & 1659 & 334 & 259 & 2252 \\
\hline OTHER-OAG & 5486 & 10126 & 0 & 147818 & 0 & 0 & 117410 & 1215 & 44805 & 163430 \\
\hline TOTAL ORGANICS & 111056 & 78012 & 49054 & 154218 & 8009 & 0. & 276211 & 55846 & 68292 & 400349 \\
\hline & & & & & & & & & & \\
\hline ICP-AES METALS & 77330 & 129356 & 34744 & 161097 & 4892 & 0 & 183097 & 111218 & 113105 & 407419 \\
\hline ICP-MS METALS & 54960 & 29953 & 13964 & 14315 & 1135 & 0 & 58429 & 36596 & 19303 & 114327 \\
\hline GF-AAMETALS & 84867 & 146490 & 27386 & 107294 & 0 & $\overline{0}$ & 155703 & 90177 & 120156 & 366037 \\
\hline MEACUAY & 185693 & 83965 & 21706 & 116600 & 11217 & 3544 & 215495 & 74931 & 132298 & 422724 \\
\hline CYANIDE & 12299 & 38907 & 1597 & 109544 & 4441 & 0 & 91915 & 12165 & 62708 & 166788 \\
\hline ANIONS/CATIONS & 87302 & 106829 & 25108 & 37644 & 0 & 336 & 184200 & 30544 & 42474 & 257218 \\
\hline WET CHEM & 0 & 10931 & 0 & 5578 & 0 & 0 & 7041 & 3634 & 5834 & 16509 \\
\hline OTHER-INORG & 189831 & 196100 & 79118 & 111032 & 61753 & 10793 & 462716 & 95930 & 89981 & 648628 \\
\hline TOTAL INOAGANICS & 692283 & 742529 & $20 \overline{3623}$ & 663104 & 83438 & 14672 & 1358595 & 455195 & 585860 & 2399650 \\
\hline & & & & & & & & & & \\
\hline GROSS ALPHAVBETA & 448289 & 119482 & 1020915 & 74111 & 7259 & 17988 & 911277 & 750693 & 26074 & 1688044 \\
\hline ALPHADET-Np237 & 0 & 10857 & 0 & 0 & of & 0 & 4017 & 0 & 6840 & 10857 \\
\hline ALPHA DET-PU,Am & 0 & 95579 & 0 & 0 & 0 & o. & 55012 & 21579 & 18988 & 95579 \\
\hline ALPHADET-PU & 16693 & 9882 & 9205 & 0 & 0 & o. & 11360 & 14499 & 9921 & 35780 \\
\hline ALPHA DET-U & 16440 & 5488 & 15794 & 0 & 0 & 0 & 16825 & 16651 & 4245 & 37721 \\
\hline ALPHA DET-Am & 13671 & 0 & 0 & 0 & 0 & 0 & 6021 & 7213 & 436 & 13671 \\
\hline ALPHA DET-Np & 3504 & $\overline{0}$ & 0 & 0 & 0 & 0 & 175 & 1577 & 1752 & 3504 \\
\hline ALPHADET-Th & 4002 & 2374 & 0 & 0 & 0 & 0 & 1941 & 2076 & 2360 & 6376 \\
\hline ALPHA DET-U,Th,Pu & 6936 & 0 & 0 & o & 0 & o & 6212 & 724 & 0 & 6936 \\
\hline ALPHADET-U235 & 0 & 0 & 7618 & 0 & 0 & 0 & 3809 & 1828 & 1981 & 7618 \\
\hline ALPHA DET-PU,U,Am & 22973 & 0 & 0 & 0 & 0 & o & 22139 & 834 & 0 & 22973 \\
\hline ALPHADET-U,Np,Pu,Am & 0 & o & 0 & 0 & 0 & 0. & 0 & 0 & 0 & 0 \\
\hline ALPHA DET-Pa226 & 0 & 1080 & 0 & 0 & a & 예 & 810 & 216 & 54 & 1080 \\
\hline ALPHA DET-Ra228 & 0 & 706 & 0 & 0 & O & 0. & 141 & 529 & $\overline{35}$ & 706 \\
\hline ALPHA SPEC & 19326 & 36224 & 125217 & 0 & 0 & 0 & 153116 & 16098 & 11552 & 180766 \\
\hline GPC-BETA-Pb210 & 0 & 1927 & $\mathbf{0}$ & 0 & 0 & 0 & 1695 & 231 & 0 & 1927 \\
\hline GPC-BETA-Sr90 & 0 & 26433 & 745 & 0 & 0 & 0 & 14936 & 7657 & 4586 & 27179 \\
\hline GPC-BETA-Pm147 & 0 & 4997 & 0 & O) & o & 0 & 4997 & 0 & 0 & 4997 \\
\hline GPC-BETA-Se79 & 0 & 2026 & $\overline{0}$ & 0 & 0 & 0 & 2026 & 0 & $\overline{0}$ & 2026 \\
\hline LSC-BETA-HB & 50897 & 18727 & 7079 & 0 & 0 & 0. & 49059 & 26327 & 1317 & 76703 \\
\hline LSC-BETA-Rn222 & 0 & 1238 & 0 & 0 & 0 & 0 & 1238 & 0 & 0 & 1238 \\
\hline LSC-BETA-1129 & 0 & 7589 & 0 & 0 & o & 이 & 7589 & 0 & 0 & 7589 \\
\hline LSC-BETA-C14 & 0 & 3388 & 0 & 0 & D. & (0) & 1830 & 0 & 1558 & 3388 \\
\hline LSC-BETA-TC99 & 0 & 59758 & 0 & 0 & o & 0. & 44855 & 2735 & 12168 & 59758 \\
\hline LSC-BETA & 0 & $\overline{0}$ & 2566 & 0 & 0) & 0 & 2566 & 0 & 0 & 2566 \\
\hline GAMMA SPEC & 16781 & 128484 & 155672 & 81411 & 0 & 20238 & 325985 & 42414 & 34186 & 402585 \\
\hline OTHER-PAD & 0 & 21873 & 17672 & 0 & 0 & 0 & 26421 & 8749 & 4375 & 39545 \\
\hline TOTALRAD & 619510 & 558110 & 1362484 & 155522 & 7259 & 38225 & $167 \overline{653}$ & 922630 & 142428 & 2741111 \\
\hline & & & & & & & & & & \\
\hline TCLPEXTRACTION & $188 \propto 2$ & 11145 & 182 & 0 & 3342 & 943 & 8143 & 15681 & 10650 & 34474 \\
\hline OTHER-PREP & 11420 & 1719 & 0 & 130295 & of & 22079 & 58002 & 8801 & 98650 & 165513 \\
\hline OTHEA-EXPLO & 4084 & $\overline{0}$ & 0 & 0 & 0. & 0 & 4084 & 0 & 0 & 4084 \\
\hline OTHER-BIO & 3036 & 0 & $\overline{0}$ & 0 & 0 & 0 & 3036 & 0 & $\overline{0}$ & 3036 \\
\hline OTHER-PHYS & 30811 & 42597 & 24248 & 104491 & 0 & 0 & 130280 & 25044 & 46823 & 202147 \\
\hline OTHER & 0 & 0 & 21629 & 0 & 0 & 27 & 10828 & 6497 & 4331 & 21656 \\
\hline TOTAL OTHER & 68213 & 5542 & 46058 & 234786 & 3342 & 23049 & 214434 & 56022 & 160454 & 430910 \\
\hline & & & & & & & & & & \\
\hline TOTAL SAMIPLEANAIYSES & 1491062 & 1434173 & 1661219 & 1207631 & 102049 & $75 \overline{947}$ & 3525293 & 1489692 & 957034 & $597 \overline{2020}$ \\
\hline
\end{tabular}


Table IV: MC Sample Analyses by Rad Level and Matrix Type (ii) Grand Junction

\begin{tabular}{|c|c|c|c|c|c|c|c|c|c|c|}
\hline $\begin{array}{l}\text { Rad-Matrix } \\
\text { Samp Anal }\end{array}$ & RO & RO/R1 & RT & R2 & $\overline{\mathrm{R} 3}$ & $\overline{\mathrm{R4}}$ & M1 & $\overline{M 2}$ & MB & Total \\
\hline VOA-GC & 0 & 0 & 0 & 0 & 0 & 0 & 01 & 8 & & 0 \\
\hline VOA-GCMS & o & 1297 & 0 & 0 & 0 & 0 & 882 & 415 & o & 1297 \\
\hline SEMI-VOA & 0 & 2003 & 0 & 0 & 0 & 0 & 1562 & 441 & o & 2003 \\
\hline PEST & 0 & o. & 0 & 0 & of & (⿹) & 0 & 0 & 0 & 0 \\
\hline HERB & 0 & 480 & 0 & 0 & 0 & 0 & 437 & 43 & o & 480 \\
\hline PCB & 0 & of & 0 & 0 & 0 & 0: & 0 & 0 & 0 & 0 \\
\hline PCB/PEST & 0 & 1805 & 0 & 0 & D & 0 & 1480 & 325 & (0) & 1805 \\
\hline OTHER-ORG & 0 & 10126 & 0 & 0 & 0 & [0] & 8911 & 1215 & 0 & 10126 \\
\hline TOTAL OAGANICS & $\overline{0}$ & 157111 & 0] & 01 & 01 & 0 & 13272 & 2439 & 0 & 15711 \\
\hline & & & & & & 7 & & & 7 & \\
\hline ICP-AESMETALS & 0 & 11682 & 0 & 0 & O & 0 & 4089 & 7594 & (0) & 11682 \\
\hline ICP-MS METALS & 0 & 8846 & 0 & 0 & 0 & 0 & 2742 & 6104 & 0 & 8846 \\
\hline GF-AAMETALS & 0 & 5921 & of & 0 & 0 & 0 & 2132 & 3789 & 0 & 5921 \\
\hline MERCURY & 0 & 5563 & o. & 0 & 0 & 0 & 2670 & 2893 & 0 & 5563 \\
\hline CYANIDE & 0 & 10720 & 0 & 0 & 0 & 0 & 9112 & 1608 & o & 10720 \\
\hline ANIONS/CATIONS & 0 & 8781 & o. & 0 & 0 & D & 2930 & 5851 & 0 & 8781 \\
\hline WET CHEM & 0 & o & 0 & 0 & 0 & D) & 0 & 0 & 0 & 0 \\
\hline OTHER-INORG & 0 & of & o. & 0 & 0 & o & 0 & 01 & 0] & 0 \\
\hline TOTAL INORGANICS & 0 & 51513 & OI & 01 & OL & $0]$ & 23675 & 27838 & 0 & 51513 \\
\hline & & & & & & & & & $\square$ & \\
\hline GROSS ALPHAVBETA & 0 & 4223 & 0 & 0 & 0 & 0 & 1836 & 2388 & 0 & 4223 \\
\hline ALPHA DET-Np237 & 0 & 0 & 0 & 0 & o & 0 & 0 & 0 & 0 & 0 \\
\hline ALPFA DET-Pu,Am & 0 & 0 & 0) & o & 0 & 0 & 0 & 0 & 0 & 0 \\
\hline ALPHADET-Pu & 0 & o) & 0 & 0 & of & 0 & 0 & 0 & 0 & 0 \\
\hline ALPHA DET-U & 0 & 0 & of & of & 요 & 0 & 0 & 0 & - & 0 \\
\hline ALPHADET-Am & 0 & 0 & of & 0 & 0 & 0. & 0 & 0 & - & $\overline{0}$ \\
\hline ALPHA DET-Np & 0 & 0 & 0 & 0 & 0. & 0] & 0 & of & -0 & $\mathbf{0}$ \\
\hline ALPHA DET-Th & 0 & 0 & of & o| & of & 0 & 0 & 0 & 0 & $\overline{0}$ \\
\hline ALPHA DET - U,Th,Pu & 0 & 0 & 0 & 0 & of & 0] & 0 & 0 & 요 & $\overline{0}$ \\
\hline ALPHA DET-U235 & 0 & o & o & o| & 0 & of & of & 0 & o & $\mathbf{0}$ \\
\hline ALPHA DET-PU,U,Am & 0 & 0 & O. & 0 & a & 0] & of & O) & (0) & 0 \\
\hline ALPHA DET-U,Np,Pu,Am & 0 & 0 & 0 & 0 & 0 & 0. & 0 & 0 & (0) & $\overline{0}$ \\
\hline ALPHA DET-Pa226 & 0 & 0 & 0 & 0 & 0 & D: & 0 & of & 0. & 0 \\
\hline ALPHA DET-Ra228 & 0 & 0 & 0 & 0 & 0 & 0 . & 0 & 0 & 0 & $\overline{0}$ \\
\hline ALPHA SPEC & 0 & 17524 & 0 & 0 & o| & 0. & 7009 & 10514 & 0 & 17524 \\
\hline GPC-BETA-Pb210 & 0 & 1927 & of & 0 & 0 & 0. & 1695 & 231 & - & 1927 \\
\hline GPC-BETA-Sr90 & 0 & 3154 & 0 & 0 & 0 & 요 & 315 & 2839 & - & 3154 \\
\hline GPC-BETA-Pm147 & 0 & 0 & of & 0 & 0 & 0 & 0 & 0 & 0 & 0 \\
\hline GPC-BETA-Se79 & 0 & 0 & of & 0 & 0 & 0 & 0 & 0 & 0 & 0 \\
\hline LSC-BETA-H3 & 0 & 1294 & 0 & 0 & 0 & 0 & 13 & 1281 & 0 & 1294 \\
\hline LSC-BETA-Rn222 & 0 & 1238 & 0 & 0 & 0 & of & 1238 & 0 & o & 1238 \\
\hline LSC-BETA- 1129 & 0 & 0 & 0 & 0 & 0 & 0 & 0 & 0 & 0 & 0 \\
\hline LSC-BETA-C14 & 0 & 0 & 0 & 0 & 0 & 0 & 0 & 0 & o & 0 \\
\hline LSC-BETA-TC99 & 0 & 0 & 0 & 0 & 0 & 0 & 0 & 0 & of & 0 \\
\hline LSC-BETA & 0 & 0 & of & 0 & 0 & 0 & \begin{tabular}{l|l}
0 \\
\end{tabular} & o & 0. & 0 \\
\hline GAMMA SPEC & 0 & 11243 & 0 & 0 & of & 0 & 112 & 11131 & - & 11243 \\
\hline OTHER-RAD & 0 & 0 & 0 & 0 & 0. & 0 [ & 01 & 0 & - & 0 \\
\hline TOTAL RAD & 0 & 40603 & 0] & 0 & 이 & 0 - & 12219 & 28384 & $\overline{0}$ & 40603 \\
\hline & & & & & & 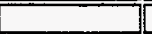 & & & $\square$ & \\
\hline TCLP EXTRACTION & 0 & 0 & - & 0 & 0 & 0] & 0 & D & - & 0 \\
\hline OTHER-PREP & 0 & 0 & 0 & 0 & 0 & ㅇ․ & O & 0 & - & 0 \\
\hline OTHER-EXPLO & 0 & 0 & 01 & 0 & o & 0] & 0 & 0 & - & 0 \\
\hline OTHER-BIO & 0 & o & O & 0 & 0 & 요 & 0 & 0 & 0 & 0 \\
\hline OTHER-PHYS & 0 & 1094 & of & 0 & 0 & ot & 01 & 1094 & $\overline{0}$ & 1094 \\
\hline OTHER & 0 & 0 & 0 & 0 & of & 요 & 0 & 0 & 0 & 0 \\
\hline TOTAL OTHER & 0 & 1094 & 01 & 0 & 0 & की [ & 01 & 1094 & 0 & 1094 \\
\hline & & & & & & & & & 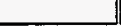 & \\
\hline TOTAL SAMPLE ANALY & 0 & 108921 & 0 & 0 & 0 & 0] & 49166 & 59755 & 0 & 108921 \\
\hline
\end{tabular}


EM-263 SURVEY OF DOE ANALYTICAL CAPACTTY AVAILABLE TO EM

Table IVc: MC Sample Analyses by Rad Level and Matrix Type (iii) Los Alamos Nat'l Lab

\begin{tabular}{|c|c|c|c|c|c|c|c|c|c|c|}
\hline $\begin{array}{l}\text { Pad-Matix } \\
\text { Samp Anal }\end{array}$ & RO & Ro/R1 & RT & $\mathrm{R} 2$ & $\overline{\mathrm{R} 3}$ & R4 & M1 & $\mathrm{M} 2$ & 143 & Total \\
\hline VOA-GC & 0 & & 01 & 0 & 01 & & 01 & & 0 & 0 \\
\hline VOA-GCMS & 10508 & 0 & 10508 & 0 & 0 & 0 & 10507 & 8162 & 2345 & $\frac{0}{21015}$ \\
\hline SEMI-VOA & 8545 & 0 & 8545 & of & 0 & D. & 8545 & 6665 & 1880 & 17089 \\
\hline PEST & 0 & 0 & 0 & \begin{tabular}{l|l}
0 & \\
\end{tabular} & 0 & 0] & 0 & 0 & 0 & 0 \\
\hline HERB & 0 & 0 & 01 & 0 & 0 & ㅇ. & 0 & 0 & 0 & 0 \\
\hline PCB & 3931 & 0 & 0 & 0 & 0 & 0 & 1613 & 2217 & 101 & 3931 \\
\hline PCB/PEST & 0 & 0 & 0 & 0 & 0 & 0 & 0 & 0 & (0) & $\overline{0}$ \\
\hline OTHER-OAG & 0 & 이 & 0 & 01 & 0 & 0 & 0 & 0 & 0] & $\overline{0}$ \\
\hline TOTALORGANICS & 22983 & 01 & 19052 & 01 & 01 & 0 & 20665 & 17044 & 4326 & 42035 \\
\hline & & & & & & & & & & \\
\hline ICP-AES METALS & 20216 & 0 & 0 & of & 0 & 0 & 11231 & 7862 & 1123 & 20216 \\
\hline ICP-MS METALS & 8228 & 0 & 8228 & 0 & 0 & 이 & 13370 & 2057 & 1028 & 16455 \\
\hline GF-AAMETALS & 12113 & 0 & 12113 & 0 & 0 & 0 & 19141 & 3679 & 1406 & 24226 \\
\hline MERCURY & 11144 & 0 & 11144 & 0 & 0 & 0 & 16858 & 4286 & 1143 & 22287 \\
\hline CYANIDE & 6890 & 0 & 0 & 0 & 0 & 0. & 6509 & 381 & 0 & 6890 \\
\hline ANIONS/CATIONS & 42888 & 0 & 0 & 0 & 0 & 요 & 40505 & 2383 & 0] & 42888 \\
\hline WET CHEM & 0 & 0 & 0 & o & 0 & 요 & 0 & 0 & 0. & 0 \\
\hline OTHER-INORG & 9430 & 01 & 01 & of & 0 & 0] & 4427 & 3772 & 1231 & 9430 \\
\hline TOTAL INORGANICS & 710908 & 01 & 31484 & 01 & 01 & 0] & 112041 & 24420 & 5931[ & 142392 \\
\hline & & & & & & & & & & \\
\hline GROSS ALPHAVBETA & 5862 & 可 & 0 & 0 & 0 & 0 & 3362 & 2327 & 172 & 5862 \\
\hline ALPHADET-Np237 & 0 & 0 & 0 & 0 & 0 & 0 & 0 & 0 & 0 & 0 \\
\hline ALPHA DET-Pu,Am & 0 & 可 & 마 & 0 & 0 & 요 & 0 & 0 & 0 & 0 \\
\hline ALPHADET-Pu & 16693 & 0 & 0 & 0 & 0 & 0 & 6272 & 9729 & 692 & 16693 \\
\hline ALPHA DET-U & 6383 & 0 & 0) & 0 & 0 & 0 & 2452 & 3655 & 275 & 6383 \\
\hline ALPHA DET-Am & 13671 & 0 & 0 & 0 & 0 & 0] & 6021 & 7213 & 436 & 13671 \\
\hline ALPHADET-Np & 0 & 0 & 01 & 이 & 0 & D. & 0 & 0 & 0 & 0 \\
\hline ALPHA DET-Th & 0 & 0 & 0 & 0 & 0 & a & 0 & 0 & 0 & $\overline{0}$ \\
\hline ALPHA DET-U,Th,PU & 0 & 0 & 0 & 0 & 01 & 0 & of & 0 & 0. & 0 \\
\hline ALPHADET-UZ35 & 0 & 0 & 0 & 0 & 0 & ㅇ․ & 이 & 0 & 0. & 0 \\
\hline ALPHA DET-PU,U,Am & $\begin{array}{lll}0 \\
\end{array}$ & 0 & 0 & 0 & 0 & - & 0 & 0 & 0 & 0 \\
\hline ALPHA DET - U,Np,Pu,Am & 0 & 0 & 0 & 0 & 0 & 0. & 0 & o & 0. & 0 \\
\hline ALPHADET-R2226 & 0 & 0 & 0 & 0 & 0 & 0 & 0 & 0 & 0] & $\overline{0}$ \\
\hline ALPHA DET-Ra228 & 0 & 0 & 0 & of & 0 & 요 & 0 & 0 & 0 & 0 \\
\hline ALPHA SPEC & 0 & 0 & 0 & 0 & o & 0] & 0 & 0 & 0 & $\overline{0}$ \\
\hline GPC-BETA-Pb210 & 0 & 0 & 0 & of & O) & 의 & of & of & 0] & 0 \\
\hline GPC-BETA-Sr90 & 0 & of & 0 & 0 & O- & 며 & of & 의 & 0 & 0 \\
\hline GPC-BETA-Pm147 & 0 & 0 & 0 & 0 & 이 & 0 & 0 & 0 & o. & 0 \\
\hline GPC-BETA-Se79 & 0 & 0 & 0 & 0 & of & 0] & 0 & 0 & 0 & $\begin{array}{l}\mathbf{0} \\
\end{array}$ \\
\hline LSC-BETA-H3 & 44786 & 0 & 0 & 0) & 0 & o. & 25686 & 17783 & 1317 & 44786 \\
\hline LSC-BETA-Rn222 & 0 & 0 & O) & O) & o) & 며 & 0 & 0 & 0 & 0 \\
\hline LSC-BETA-I129 & 0 & 0 & 0 & 0 & 0 & 묘 & D. & 0 & 0 & 0 \\
\hline LSC-BETA-C14 & 0 & 0 & 0 & 이 & 0 & ㅁ. & 이 & 0 & ㅇ. & $\underline{0}$ \\
\hline LSC-BETA-TC99 & 0 & 0 & 0 & O) & 0 & 0 & D. & 0 & 0 - & 0 \\
\hline LSC-BETA & 0 & 0 & 0 & 0 & 0 & 0 & Do & O) & of & 0 \\
\hline GAMMA SPEC & 5461 & 0 & 0 & 0 & 0 & 0 & 3276 & 1967 & 218 & 5461 \\
\hline OTHER-RAD & 0 & 0 & 0 & 0 & 01 & 0. & 0) & 0 & a] & 0 \\
\hline TOTAL RAD & 92855 & 이 & 0 & 01 & 01 & (⿹) & 47069 & 426751 & 31111 & 92855 \\
\hline & & & & & & & & & & \\
\hline TCLP EXTRACTION & 0 & 0 & 0 & 0 & 0 & 요 & 0 & 0 & 0. & 0 \\
\hline OTHER-PREP & 0 & 이 & 0 & 0 & 0 & 0 & 0 & 0 & 으 & 0 \\
\hline OTHER-EXPLO & 0 & 0 & 이 & 0 & O. & 요 & 0 & D & 요 & 0 \\
\hline OTHER-BIO & 0 & 이 & 0 & 0 & 이 & 0 & 0 & 0 & 요 & 0 \\
\hline OTHER-PHYS & 0 & $\overline{0}$ & 0 & 0 & of & 0 & 0 & 0 & ㅇ. & 0 \\
\hline OTHER & 0 & O & 0 & 0 & 0 & 0 & 0 & 01 & o. & 0 \\
\hline TOTAL OTHER & 0 & O) & O & 0 & 0 & 0 & 0 & 0 & 이 & 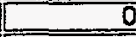 \\
\hline & & & & & & & & & & \\
\hline TOTAL SAMPLE ANALYSES & 226746 & 0 & 50536 & O) & 0 & 0 & 179775 & 84139 & $13368]$ & 277283 \\
\hline
\end{tabular}


EM-263 SUAVEY OF DOE ANALYTICAL CAPACITY AVAILABLE TO EM

Table Nc: MC Sample Analyses by Rad Level and Matrix Type (iv) Argonne East

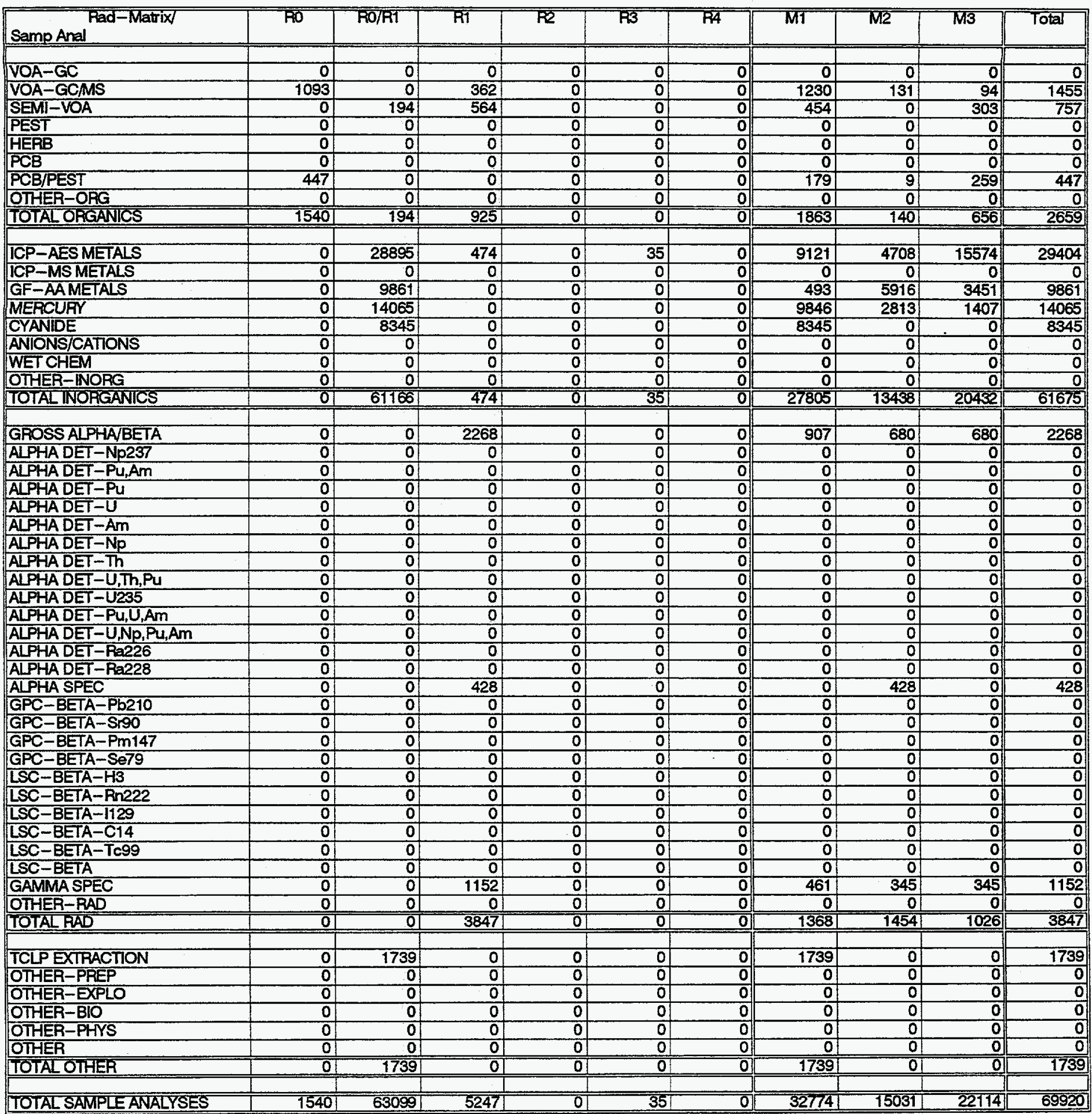


Table Nc: MC Sample Analyses by Rad Level and Matrix Type (v) Argonne West

\begin{tabular}{|c|c|c|c|c|c|c|c|c|c|c|}
\hline $\begin{array}{l}\text { Rad-Matrix } \\
\text { Samp Anal }\end{array}$ & RO & $\mathrm{RO} / \mathrm{R} 1$ & Ri & $\overline{\mathrm{R} 2}$ & $7 \overline{3}$ & $\overline{R 4}$ & $\overline{711}$ & $\sqrt{12}$ & $\mathrm{MB}$ & Total \\
\hline & & & & & & & & & & \\
\hline VOA-GC & 0 & 0 & 0 & 0 & 0 & 0. & 0 & 0 & 0 & 0 \\
\hline VOA-GCMS & 0 & 0 & 0 & 0 & 0 & 0 & 0 & 0 & 0 & o \\
\hline SEMI-VOA & 0 & 0 & 0 & 0 & 0 & (0) & 0 & 0 & 0 & 0 \\
\hline PEST & 0 & 0 & 0 & 0 & 0 & 0 & 0 & 0 & 0 & 0 \\
\hline HERB & 0 & 0 & 0 & 0 & 0 & 0) & 0 & 0 & 0 & 0 \\
\hline PCB & 0 & 0 & 0 & 0 & 0 & 0 & 0 & 0 & 0 & 0 \\
\hline PCB/PEST & 0 & 0 & 0 & 0 & 0 & 0. & 0 & 0 & 0 & 0 \\
\hline OTHER-ORG & 0 & 0 & 0 & 0 & 0 & 0. & 0 & 01 & 0 & 0 \\
\hline TOTAL ORGANICS & $\overline{0}$ & 0 & 0 & $\overline{0}$ & 01 & ol & 0 & 0 & 0 & 0 \\
\hline & & & & & & & . & & & \\
\hline ICP-AESMETALS & 0 & 0 & 0 & 0 & 0 & 0 & 0 & 0 & 0 & 0 \\
\hline ICP-MSMETALS & 0 & 0 & 0 & 0 & 0 & 0 & 0 & D) & 0 & 0 \\
\hline GF-AAMETALS & 0 & 0 & 0 & 0 & 0 & 0) & 0 & 0 & o) & 0 \\
\hline MERCURY & 0 & o & 0 & 0 & 0 & o & 0 & 0 & 0 & 0 \\
\hline CYANIDE & 0 & 0 & 0 & 0 & 0) & o & o) & 0 & 0 & 0 \\
\hline ANIONS/CATIONS & 0 & 0 & 0 & 0 & 0 & o. & का & 0 & 0 & 0 \\
\hline WET CHEM & 0 & 0 & 0 & 0 & 0 & D. & 0 & 0 & o. & 0 \\
\hline OTHEA-INORG & 0 & 0 & 0 & 0 & ö & 0 & 0 & 0 & o. & 0 \\
\hline TOTAL INORGANICS & 0 & 0 & 01 & 0 & 0 & o] & 0 & o] & $0]$ & D] \\
\hline & & & & & 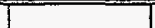 & . & - & . & . & \\
\hline GROSS ALPHAVBETA & 0 & 0 & 0 & 0 & 0 & 0 & 0 & 0 & 0 & 0 \\
\hline ALPHA DET-Np237 & 0 & 0 & 0 & 0 & 0 & 0 & 0 & o & 0 & 0 \\
\hline ALPHA DET-Pu,Am & 0 & 0 & 0 & 0 & o & 0 & 0 & 0 & D. & 0 \\
\hline ALPHA DET-PU & 0 & 0 & 0 & 0 & 0 & 0 & o) & 0 & 0 & $\mathbf{0}$ \\
\hline ALPHADET-U & 0 & 0 & 0 & 0 & 0 & 0 & 0) & o & 0 & 0 \\
\hline ALPHA DET-Am & 0 & 0 & 0 & 0 & 0 & 0 & 0 & 0 & 0 & 0 \\
\hline ALPHA DET-Np & 0 & 0 & 0 & $\overline{0}$ & 0 & 0. & 0 & 0 & o & 0 \\
\hline ALPHA DEI-Th & 0 & o & 0 & 0 & 0 & a) & 0 & 0 & 0 & 0 \\
\hline ALPHA DET-U,Th,PU & 0 & 0 & o & 0 & 0 & 0 & 0! & o) & 0 & 0 \\
\hline ALPHADET-U235 & 0 & o & 0 & 0 & 0 & 0 & 0 & 0 & o & 0 \\
\hline ALPHA DET-Pu,U,Am & 0 & 0 & 01 & 0 & 0 & 0. & 0 & o & 0 & $\mathbf{0}$ \\
\hline ALPHADET-U,Np,Pu,Am & 0 & 0 & 0 & 0 & 0 & 0 & 0 & 0) & 0 & $\overline{0}$ \\
\hline ALPHA DET-Ra226 & 0 & 0 & 0 & 0 & 0 & 0 & 0 & 0 & o. & 0 \\
\hline ALPHADET-Ra228 & 0 & 0 & 0 & 0 & 0 & 0 & 0 & o & o: & 0 \\
\hline ALPHA SPEC & 0 & 0 & 0 & 0 & 0 & 0 & 0 & 0 & 0 & $\overline{0}$ \\
\hline GPC-BETA-Pb210 & 0 & 0 & 0 & 0 & 0 & 0 & 0 & 0 & 0 & 0 \\
\hline GPC-BEIA-Sr90 & 0 & 0 & 0 & 0 & O) & 0 & 0 & 0 & o. & 0 \\
\hline GPC-BETA-Pm147 & 0 & 0 & 0 & 0 & 0 & 0 & 0 & 0 & 0 & 0 \\
\hline GPC-BETA-Se79 & 0 & o & 0 & 0 & o) & 0 & o & 0 & 0 & 0 \\
\hline LSC-BETA-H3 & 0 & 0 & 0 & 0 & 0 & 0 & 0 & 0 & 0 & 0 \\
\hline LSC-BETA-Rn222 & 0 & 0 & 0 & 0 & 0 & o) & 0 & 0 & 0 & $\mathbf{0}$ \\
\hline LSC-BEIA-1129 & 0 & 0 & 0 & 0 & 0 & 0] & 0 & 0 & 0 & 0 \\
\hline LSC-BETA-C14 & 0 & o) & 0) & 0 & 0 & 0 & 0 & 0 & 0 & 0 \\
\hline LSC-BETA-TC99 & 0 & 0 & 0 & 0 & 0 & 0 & 0 & 0 & 0 & 0 \\
\hline LSC-BETA & 0 & o! & 0 & 0 & 0 & 0 & 0) & 0 & 0 & 0 \\
\hline GAMMA SPEC & 0 & 0 & 0 & 0 & 0 & o & o) & 0 & 0 & 0 \\
\hline OTHER-PAD & 0 & 0 & 0 & 0 & 0 & 0 & 0 & 0 & 0 & 0 \\
\hline TOTAL RAD & 0 & 01 & 0 & 0] & 0 & 0 & 01 & 01 & 0] & 0 \\
\hline & & & & & & & & & & \\
\hline TCLPEXTRACTION & 0 & 0 & 0 & 0 & 0 & 0 & 0 & 0 & of & 0 \\
\hline OTHER-PREP & 0 & 0 & 0 & 0 & 0 & 0 & 0 & 0 & 0 & 0 \\
\hline OTHER-EXPLO & 0 & 0 & 0 & 0 & 0 & 0 & o & o & 0 & 0 \\
\hline OTHER-BIO & 0 & 0 & 0 & 0 & 0 & 0 & 0 & 0 & o) & 0 \\
\hline OTHER-PHYS & 0 & $\overline{0}$ & 0 & 0 & 0 & o & 0 & 0 & 0 & 0 \\
\hline OTHER & 0 & o & 0 & 0 & 0 & D) & 0 & 0 & 0 & 0 \\
\hline TOTALOTHER & $\overline{0}$ & 0 & 0 & $\overline{0}$ & 0 & D. & 0 & 0 & 0 & 0 \\
\hline & & & & & & 7 & the & $=$ & $=$ & 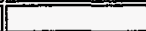 \\
\hline TOTAL SAMPLE ANAIYSES & D & o) & 0 & $\overline{0}$ & $\overline{0}$ & 0 & $\overline{0}$ & 01 & 01 & $\overline{0}$ \\
\hline
\end{tabular}


Table IVc: MC Sample Analyses by Rad Level and Matrix Type (vi) Brookhaven Nat'l Lab

\begin{tabular}{|c|c|c|c|c|c|c|c|c|c|c|}
\hline $\begin{array}{l}\text { Pad-Matrix } \\
\text { Samp Anal }\end{array}$ & 70 & RO/R1 & $\overline{\mathrm{R} 1}$ & $\overline{R 2}$ & $\overline{\mathrm{R3}}$ & R4 & पMT & $\overline{M 2}$ & $\bar{M} \overline{3}$ & Total \\
\hline VOA-GC & & & & & & & & & & \\
\hline & 0 & O & 0 & 0) & 0] & 예 & 0] & o) & 0 & 0 \\
\hline VOA-GCMS & 0 & 0 & 0 & 0 & 0 & 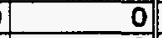 & 0 & 요 & 0 & $\underline{0}$ \\
\hline SEMI-VOA & 0 & 0 & 이 & 0 & 0] & ㅁ] & 01 & 0 & 0 & 0 \\
\hline PEST & 0 & 0 & of & 0 & 0 & a & 이 & 0 & 0 & $\overline{0}$ \\
\hline HERB & 0. & 0 & 0 & 0) & 0 & 0 & 0 & 0 & 0 & $\overline{0}$ \\
\hline PCB & 0 & 0 & 0 & of & 0 & 0] & 0 & 0 & 0 & $\overline{0}$ \\
\hline PCB/PEST & 0 & 0 & o- & of & 0 & 요 & o & 이 & 0 & 0 \\
\hline OTHER-ORG & 0 & 0 & 0 & of & 0 & ㅁ․ & 0 & 0 & 0 & $\overline{0}$ \\
\hline TOTAL ORGANICS & 0 & 01 & 이 & 01 & 01 & 요 & 01 & 01 & 0 & $\overline{0}$ \\
\hline & & & & & & & & & & \\
\hline ICP-AESMETALS & 0 & 0 & of & 0 & 0 & - & 0 & 0 & 0 & $\overline{0}$ \\
\hline ICP-MSMETALS & 0 & 0 & 이 & 0 & 0 & 요 & 0 & 0 & 0 & $\underline{0}$ \\
\hline GF-AAMETALS & 0 & 0 & of & 0 & 0 & 0 & 0 & 0 & (0) & $\overline{0}$ \\
\hline MERCURY & 0 & o & 01 & 0 & 0 & 이 & 0 & 0 & 0. & $\underline{0}$ \\
\hline CYANIDE & 0 & 0 & 0 & 0 & o & - & 0 & 0 & 0 & $\overline{0}$ \\
\hline ANIONS/CATIONS & 0 & 0 & 0) & 0 & 0 & 0 & 0 & 0 & 0 & 0 \\
\hline WET CHEM & o & (D) & of & 0 & 0 & of & 0 & 0 & (0) & $\mathbf{0}$ \\
\hline OTHER-INORG & 0 & 0 & of & 0 & 0 ] & of & 0 & O) & - & $\overline{0}$ \\
\hline TOTAL INORGANICS & 01 & 0 & OI & 01 & 이 & 0] & 01 & 01 & 0 & $\begin{array}{c}0 \\
\end{array}$ \\
\hline & & & & & & & & 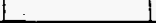 & 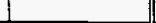 & \\
\hline GROSS ALPHAVBETA & 0 & 이 & of & 0 & 0 & 요 & 0 & 요 & 0 & 0 \\
\hline ALPHA DET-Np237 & 0 & 0 & of & 0 & 0 & 묘 & (0) & o & D. & $\overline{0}$ \\
\hline ALPHADET-Pu,Am & 0 & D. & of & 0 & 0 & - & o! & 0 & O & $\overline{\mathbf{0}}$ \\
\hline ALPHADET-Pu & 0 & D & 0 & 0 & 0 & 0] & 0 & 0 & 0 & $\overline{0}$ \\
\hline ALPHA DET-U & 0 & 0 & 0 & 0 & 0 & 0 & 01 & 0 & o & 0 \\
\hline ALPHA DET-Am & 0 & 0 & D. & 0 & 0 & 요 & of & O & 0 & 0 \\
\hline ALPHA DET-Np & 0 & 0 & 아 & 0 & 0 & 의 & a & 0 & o & $\overline{0}$ \\
\hline ALPHA DET-Th & 0 & 0 & of & 0 & 0 & o. & 이 & 可 & 0. & 0 \\
\hline ALPHA DET-U,Th,Pu & 0 & 0 & 0, & 0 & o & 0 & 0 & 0 & 0 & 0 \\
\hline ALPHADET-U235 & 0 & 0 & 이 & 0 & 0 & -0. & 0 & 0 & 0 & $\overline{0}$ \\
\hline ALPHA DET-Pu,U,Am & 0 & 0 & 01 & 0 & 0 & 아 & 0 & 0 & (0. & $\overline{0}$ \\
\hline ALPHA DET-U,Np,Pu,Am & 0 & 0 & 0.1 & 0 & 0 & 0. & 0 & 0 & $\overline{0}$ & $\begin{array}{l}0 \\
0\end{array}$ \\
\hline ALPHADET-Pa226 & 0 & 0 & of & 0 & $\begin{array}{ll}0 \\
\end{array}$ & 0. & 0 & 0 & 0] & 0 \\
\hline ALPHADET-Ra228 & $\overline{0}$ & D. & 0 & 0 & 0 & o. & 0 & 0 & 0 & $\overline{0}$ \\
\hline ALPHA SPEC & o & 0 & 0 & 0 & 0 & 0] & 0 & 0 & 0] & $\overline{0}$ \\
\hline GPC-BETA-Pb210 & 0 & 0 & 0 & 0 & 0 & of & 0 & 01 & - & 0 \\
\hline GPC-BETA-Sr90 & 0 & $\mathbf{0}$ & 0 & 0 & 0 & 0. & 이 & 0 & 0 & 0 \\
\hline GPC-BETA-Pm147 & 0 & o & 0 & 0 & 0 & (0) & of & 0 & 0. & $\overline{0}$ \\
\hline GPC-BETA-Se79 & 0 & 0 & 0 & 0 & 0 & 0 & 0 & 0 & ㅇ․ & $\underline{0}$ \\
\hline LSC-BETA-H3 & 0 & 0 & 0 & 0 & 0 & 0 & o) & of & 0 & $\overline{0}$ \\
\hline LSC-BETA-Pn222 & 0 & 0 & 0 & 0 & 0 & a & 0) & 0 & 요 & 0 \\
\hline LSC-BETA-1129 & $\overline{0}$ & 0 & 01 & 0 & 0 & o] & 0 & O & - & 0 \\
\hline LSC-BETA-C14 & 0 & o & 0 & 0 & 0 & 0 & 0 & 可 & 요 & 0 \\
\hline LSC-BETA-TC99 & 0 & 0 & 0 & $\overline{0}$ & 0 & 의 & o & of & 0] & $\overline{0}$ \\
\hline LSC-BETA & 0 & o & 0 & 0 & 0 & 0 & 0 & 0 & 매 & $\underline{0}$ \\
\hline GAMMA SPEC & 0 & 0 & 0 & 0 & 0 & 0. & 이 & 0 & 0 & $\underline{0}$ \\
\hline OTHER-RAD & 0 & 0 & 0 & \begin{tabular}{l|l}
0 \\
\end{tabular} & 0 & o: & of & of & 0] & $\overline{0}$ \\
\hline TOTAL RAD & 0 & 0 & 01 & 01 & 01 & ]. & 01 & O1 & 0] & $\underline{0}$ \\
\hline & & & & & & & & & & \\
\hline TCLP EXTRACTION & 0 & 0 & 0 & 0 & O) & 0] & 요 & 0] & 0 & 0 \\
\hline OTHER-PREP & 0 & 0 & 0 & 0 & O & 0. & of & of & 0 & $\overline{0}$ \\
\hline OTHER-EXPLO & 0 & 0 & 0 & 0 & of & 의 & 요 & 0 & 의 & 0 \\
\hline OTHER-BIO & 0 & 0 & O) & 0 & 0 & -0. & 0 & - & 0 & 0 \\
\hline OTHER-PHYS & 0 & 0 & 01 & 0 & 0 & 0. & 0 & 0) & D & $\underline{0}$ \\
\hline OTHER & 0 & 0 & 0 & 0 & 0 & (0) & 0 & 0 & o. & 0 \\
\hline TOTAL OTHER & 0 & 0 & O) & 01 & 0 & O. & $0]$ & 0 & 0 & 0 \\
\hline & & & & 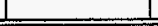 & 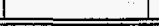 & 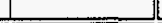 & $=$ & 1 & & \\
\hline TOTAL SAMPLE ANALYSES & 0 & 0 & 0 & 01 & 01 & of & 이 & 01 & 0. & $\underline{0}$ \\
\hline
\end{tabular}


Table IV: MC Sample Analyses by Rad Level and Matrix Type (vii) Fernald

\begin{tabular}{|c|c|c|c|c|c|c|c|c|c|c|}
\hline $\begin{array}{l}\text { Rad-Matrix/ } \\
\text { Samp Anal }\end{array}$ & ROO & RO/R1 & $\overline{\mathrm{R1}}$ & $\overline{P 2}$ & $\overline{\mathrm{R} 3}$ & $\overline{\mathrm{R4}}$ & 771 & $\overline{172}$ & $\overline{\mathrm{M} 3}$ & Total \\
\hline VOA-GC & 0 & o) & 0 & 0 & 0 & 0 & 0 & 0 & 0 & 0 \\
\hline VOA-GCMS & 0 & 4250 & 0 & 0 & 0 & 0 & 2125 & 2125 & 0 & 4250 \\
\hline SEMI-VOA & 0 & 0 & 0 & 0 & o & 0 & 0 & 0 & 0 & 0 \\
\hline PEST & 0 & 0 & 0 & 0 & 0 & 0 & 0 & of & o & 0 \\
\hline HERB & 0 & 이 & 0 & 0 & 0 & 0 & 0 & 0 & o & 0 \\
\hline PCB & 0 & 0 & 0 & 0 & 0 & 0 & 0 & 0 & 0 & 0 \\
\hline PCB/PEST & 0 & 0 & 0 & 0 & 0 & 0 & 0 & 0 & 0 & 0 \\
\hline OTHER-ORG & 0 & 0 & 0 & 0 & 0 & 0 & 0 & 0 & 0 & 0 \\
\hline TOTAL ORGANICS & 0 & 4250 & 0 & 0 & 0 & 0 & 2125 & $2125 \mid$ & 0 & 4250 \\
\hline$\overline{1}$ & & & & & & & & 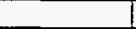 & 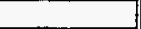 & \\
\hline ICP-AES METALS & 0 & 13771 & 0 & 0 & 0 & 0 & 6197 & 7574 & 0 & 13771 \\
\hline ICP-MS METALS & 0 & 2695 & 0 & 0 & 0 & 0 & 269 & 1213 & 1213 & 2695 \\
\hline GF-AAMETALS & 0 & 57744 & 0 & 0 & 0 & 0 & 44776 & 12968 & 0. & 57744 \\
\hline MERCUAY & 0 & 17038 & 0 & 0 & 0 & 0 & 10223 & 6815 & 0 & 17038 \\
\hline CYANIDE & 0 & 0 & 0 & 0 & 0 & 0 & 0 & 0 & 0 & 0 \\
\hline ANIONS/CATIONS & 0 & 1361 & 0 & 0 & 0 & 0 & 1361 & 0 & (0) & 1361 \\
\hline WET CHEM & 0 & 10931 & 0 & $\overline{0}$ & 0 & 0 & 4372 & 3595 & 2963 & 10931 \\
\hline OTHER-INORG & 0 & 54946 & 0 & 0 & 0 & 0 & 32464 & 22482 & 0 & 54946 \\
\hline TOTAL INORGANICS & 0 & 158485 & 0 & 0 & 0 & 0 & 99662 & 54648 & 4176 & 158485 \\
\hline GROSS ALPHA/BETA & 0 & 15118 & 0 & 0 & 0 & 0 & 5455 & 0351 & 312 & 15118 \\
\hline ALPHA DET-Np237 & 0 & 0 & 0 & 0 & 0 & 0 & 0 & $\frac{5001}{0}$ & 0 & 0 \\
\hline ALPHA DET - Pu,Am & 0 & 0 & 0 & 0 & 0 & 0 & 0 & 0 & o & 0 \\
\hline ALPHA DET-PU & 0 & 0 & 0 & 0 & 0 & 0 & 0 & 0 & 0 & 0 \\
\hline ALPHADET-U & 0 & 5488 & 0 & 0 & 0 & 0 & 5488 & 0 & 0 & 5488 \\
\hline ALPHADET-Am & 0 & 0 & 0 & 0 & 0 & 0 & 0 & 0 & 0 & 0 \\
\hline ALPHADET-Np & 0 & 0 & 0 & $\mathbf{0}$ & 0 & 0 & 0 & 0 & 0 & 0 \\
\hline ALPHADET-Th & 0 & 2374 & 0 & 0 & 0 & 0 & 1781 & 475 & 119 & 2374 \\
\hline ALPHA DET-U,Th,Pu & 0 & D. & $\overline{0}$ & 0 & 0 & 0 & 0 & 0 & 0 & $\overline{0}$ \\
\hline ALPHA DET-U235 & 0 & D & 0 & 0 & 0 & 0 & 0 & 0 & 0 & 0 \\
\hline ALPHA DET-PU,U,Am & 0 & 0 & 0 & 0 & 0 & 0 & 0 & 0 & 0 & 0 \\
\hline ALPHA DET-U,Np,Pu,Am & 0 & 0 & 0 & 0 & 0 & 0 & 0 & 0 & 0 & 0. \\
\hline ALPHA DET-Ra226 & 0 & 1080 & 0 & 0 & 0 & 0 & 810 & 216 & 54 & 1080 \\
\hline ALPHA DET-Ra228 & 0 & 706 & 0 & 0 & 0 & 0 & 141 & 529 & 35 & 706 \\
\hline ALPHA SPEC & 0 & 0 & 0 & 0 & 0 & 0 & 0 & 0 & 0 & 으 \\
\hline GPC-BETA-Pb210 & 0 & 0 & 0 & 0 & 0 & 0 & 0 & 0 & 0 & 은 \\
\hline GPC-BETA-Sr90 & $\overline{0}$ & 0 & $\overline{0}$ & 0 & 0 & $\overline{0}$ & $\overline{0}$ & 0 & a. & 0 \\
\hline GPC-BETA-Pm147 & 0 & 0] & 0 & 0 & 0 & 0 & 0 & 0 & 0 & 0 \\
\hline GPC-BETA-Se79 & 0 & 0 & 0 & 0 & 0 & 0 & 0 & 0 & 0 & 으 \\
\hline LSC-BETA-H3 & 0 & 0. & 0 & 0 & 0 & 0 & 0 & 0 & 0 & 요 \\
\hline LSC-BETA-Rr222 & 0 & 0 & 0 & 0 & 0 & 0 & 0 & 0 & 0 & 의 \\
\hline LSC-BETA-1129 & 0 & 0 & 0 & 0 & 0 & 0 & 0 & 0 & 0 & 0 \\
\hline LSC-BETA-C14 & 0 & 0 & 0 & $\overline{0}$ & 0 & 0 & 0 & 0 & 은 & 의 \\
\hline LSC-BETA-TC99 & 0 & 0 & 0 & 0 & 0 & 0 & 0 & 0 & 0 & 0 \\
\hline LSC-BETA & 0 & 0 & 0 & 0 & 0 & 0 & 0 & 0 & 0 & 0 \\
\hline GAMMA SPEC & $\mathbf{0}$ & 0 & 0 & 0 & 0 & 0 & 0 & 0 & 의 & 의 \\
\hline OTHER-RAD & 0 & 21873 & 0 & 0 & 0 & 0 & 8749 & 8749 & 4375 & 21873 \\
\hline TOTAL RAD & 0 & 46639 & 0 & $\overline{0}$ & 0 & $\overline{0}$ & 22424 & 19321 & 4895 & 46639 \\
\hline & & & & & & & & & & \\
\hline TCLP EXTRACTION & 0 & 3344 & 0 & 0 & 0 & 0 & 0 & 3344 & 0 & 3344 \\
\hline OTHER-PREP & 0 & 0 & 0 & 0 & 0 & 0 & 0 & 0 & 0 & 0 \\
\hline OTHER-EXPLO & 0 & 0 & 0 & 0 & 0 & 0 & 0 & 0 & 0 & 0 \\
\hline OTHER-BIO & 0 & 0 & 0 & 0 & $\mathbf{0}$ & 0 & 0 & 0 & 0 & 0 \\
\hline OTHER-PHYS & $\overline{0}$ & 41503 & 0 & 0 & 0 & $\overline{0}$ & 8710 & 17811 & 14982 & 41503 \\
\hline OTHER & 0 & 0 & 0 & 0 & 0 & $\overline{0}$ & 0 & 0 & 0 & 0 \\
\hline TOTALOTHER & $\overline{0}$ & $4 \overline{44847}$ & 0 & $\overline{0}$ & 0 & 0 & 8710 & 21155 & 14982 & 44847 \\
\hline & & & & & & & & & & \\
\hline TOTAL SAMPLE ANALY & 0 & 254221 & 0 & 0 & 0 & 0 & 132921 & 97248 & 24052 & 254221 \\
\hline
\end{tabular}


EM-263 SURVEY OF DOE ANALYTICAL CAPACITY AVAILABLE TO EM

Table IVc: MC Sample Analyses by Rad Level and Matrix Type (viii) Idaho Nat'l Eng Lab - ACU

\begin{tabular}{|c|c|c|c|c|c|c|c|c|c|c|}
\hline Samp Anal & RO & RO/Ri & $\overline{R 1}$ & R2 & $\mathrm{R3}$ & $\overline{\mathrm{P4}}$ & V1 & M2 & $\overline{\mathrm{M} 3}$ & Total \\
\hline VOA-GC & 0 & & & & & & & & & \\
\hline VOA-GCMS & 5130 & 0 & 0 & 01 & 이 & 옉 & 0 & 이 & 에 & 의 \\
\hline SEMI-VOA & & & 0 & 0 & 0 & 애 & 4104 & 923 & 103 & 5130 \\
\hline & & 0 & 0 & 01 & 0 & 앤 & 0 & 이 & 애 & 0. \\
\hline & & of & 0 & 0 & 0 & 0. & 0 & 0 & 0 & 요 \\
\hline HERB & 0 & 이 & 0 & 0 & 0 & 0 & 0 & 0 & 0. & ㅁ \\
\hline PCB & 0 & 우 & 0 & 0 & 0 & 0 & 0 & 0 & 묘 & ㅁ \\
\hline PCB/PEST & 0 & 0 & 0 & 0 & 0 & 0 & 0 & of & 요 & 요 \\
\hline OTHER-ORG & 01 & of & 0 & o. & 0 & 0] & 0 & 01 & 0] & 0] \\
\hline TOTAL ORGANICS & 5130 & 이 & 0 & 0 & 0 & 0] [ & 4104 & 9231 & 103 & 5130 \\
\hline & & & & & & & ? & & & \\
\hline ICP-AESMETALS & 0 & 0 & 0 & 0 & a) & 0 & D. & 0 & 의 & 0 \\
\hline ICP-MSMETALS & 0 & 0 & 0 & 0 & 0 & 0] & 0 & 0 & 0. & 0. \\
\hline GF-AAMETALS & 0 & 0) & 0 & 0 & 0 & 요 & D. & 0 & 0 & 에 \\
\hline MERCURY & 0 & 0 & 0 & 0 & 0 & 0. & 0 & 0. & 0 & [0. \\
\hline CYANIDE & 0 & 0 & 0 & 0 & 0 & 0. & 0 & of & 0 & 0. \\
\hline ANIONS/CATIONS & 0) & 0 & 0 & 0) & 0 & 의 & 0 & 이 & 0. & 에 \\
\hline WET CHEM & 0 & 0 & 0 & 0 & 0 & 요 & 0 & 0 & a. & 0. \\
\hline OTHER-INORG & 0. & 0 & 0 & 01 & 0! & 요 [ & 0 & 0 & 0 & 0] \\
\hline TOTAL INORGANICS & 01 & 0! & 01 & 01 & 0 & 의 [ & 01 & 0 & 0] & (0) \\
\hline & & & & & & & & & & -5 \\
\hline GROSS ALPHAVBETA & 0 & 0 & 0 & 아 & 0 & ㅁ․ & 0 & 0 & 의 & 요 \\
\hline ALPHA DET-Np237 & 0 & 0 & 0 & 0. & 0 & 요 & 0 & 0 & 요 & 0. \\
\hline ALPHA DET - Pu,Am & 0 & 0 & 0 & 0 & 0 & - & 0 & 0 & 0 & 애 \\
\hline ALPHA DET-PU & 0 & 0 & 0 & 0 & 0 & 으 & 0 & O) & 0 & 요 \\
\hline ALPHA DET-U & 0 & 0 & 0 & 0 & 0 & 의 & 0 & O) & 묘 & -0. \\
\hline ALPHA DET-Am & 0. & o & 0 & 0 & of & 요 & 0 & 0) & 요 & 요 \\
\hline ALPHA DET-Np & 0 & 0 & (0) & O & 0 & ㅁ․ & 0 & 요 & 므 & (0) \\
\hline ALPHA DET-Th & 0 & 0 & 0 & 0 & 요 & 0. & 0 & 아 & 으 & 0 \\
\hline ALPHADET-U,Th,Pu & 0 & 0 & $\overline{0}$ & 0 & of & 0 & 0 & 0) & 0 & 0 \\
\hline ALPHADET-U235 & 0 & 0 & 0 & 0 & 0 & 요 & 0 & 0 & 0] & 의 \\
\hline ALPHA DET - Pu,U,Am & 0 & O. & 0 & D. & 0 & 묘 & 0 & D) & 0. & 0 \\
\hline ALPHA DET-U,Np,Pu,Am & 0 & O. & 이 & D) & 0 & 0 & 0 & 0 & 0 & 0 \\
\hline ALPHADET-Ra226 & 0 & 0 & 0 & 01 & 0 & 0 & 0 & 0 & 0 & 0 \\
\hline ALPHADET-Ra228 & 0 & 0 & 0 & 0 & 이 & 0. & 0 & 0 & 0 & 의 \\
\hline ALPHA SPEC & 0 & 0 & 0 & 0 & 0 & 0 & 0 & 0 & 0 - & 으. \\
\hline GPC-BETA-PB210 & 0 & 0 & 0 & 0 & of & 0 & 0 & o & 0 & 0 \\
\hline GPC-BETA-Sr90 & of & 0 & of & 0 & of & 0 & 0 & 0 & 0 & 0 \\
\hline GPC-BETA-Pm147 & 0 & 0 & 0 & 0 & 0 & 0 & 0 & 0 & 0 & 0 \\
\hline GPC-BETA-Se79 & D & 0 & 0 & o| & 0 & $0]$ & 0 & 0 & 0 & 0 \\
\hline LSC-BETA-H3 & 0 & 0 & 0 & 0 & 0 & (0) & of & 0 & 요 & 으 \\
\hline LSC-BETA-Rin222 & 0 & 0 & 0 & 0 & 0] & 요 & 0 & 0 & 으 & 0 \\
\hline LSC-BETA-1129 & 0 & 0 & -0. & 0 & 0. & ㅇ․ & 0 & 0 & 0 & 0 \\
\hline LSC-BETA-C14 & 0 & 0 & 요 & 0 & 0 & 요 & 0 & 0 & 요 & 0 \\
\hline LSC-BETA-TC99 & 0 & 0 & 0 & 0 & 0) & 0] & 0 & 0 & 0. & 0 \\
\hline LSC-BETA & 0 & 0 & 0 & 0 & of & ㅁ] & 0 & 0 & 요 & 0 \\
\hline GAMMA SPEC & $\mathbf{0}$ & 0 & 0 & 0 & 0 & 요 & 0 & 0 & 요 & 0 \\
\hline OTHER-RAD & 0 & 0 & 01 & 0 & 0 & 0] & 0 & 0 & 0] & 0 \\
\hline TOTALRAD & 0 & $\overline{0}$ & 01 & $\overline{0 !}$ & 0 & D. & 01 & 0) & 0] [ & 0 \\
\hline & & & & & & II & & & & \\
\hline TCLP EXTRACTION & $\overline{0}$ & 0 & 0 & 0 & 이 & 0 & 0 & 0 & 의 & ㅇ. \\
\hline OTHER-PREP & 0 & 0 & 可 & 0 & 0 & 0] & O & 0 & 요 & 0 \\
\hline OTHER-EXPLO & 0 & 0 & 0 & 0 & 이 & 0 & 아 & 요 & 의 & 0 \\
\hline OTHER-BIO & 0 & 0 & 0 & 0 & 0 & 므: & of & 0 & 요 & 0 \\
\hline OTHER-PHYS & 0 & $\mathbf{0}$ & 0 & 0 & o. & 요 & 0 & 0 & 요 & 0 \\
\hline OTHER & 0 & 0 & of & 0 & $\overline{0 .}$ & -0. & 0 & 0 & 이 & 0 \\
\hline TOTAL OTHER & 0 & $\overline{0}$ & 0 & O & 01 & O. & 01 & 0 & of & 0 \\
\hline & & & & & & & & & & \\
\hline TOTAL SAMIPLE ANALYS & 5130 & 0 & D] & $\overline{0}$ & ग्र & 0 & 4104 & 923 & 103[ & 5130 \\
\hline
\end{tabular}


EM-263 SUAVEY OF DOE ANALYTICAL CAPACITY AVAILABLE TO EM

Table IVc: MC Sample Analyses by Rad Level and Matrix Type (ix) Idaho Nat'l Eng Lab - B\&W

\begin{tabular}{|c|c|c|c|c|c|c|c|c|c|c|}
\hline $\begin{array}{l}\text { Rad-Matrix } \\
\text { Samp Anal }\end{array}$ & RO & RO/Rt & R1 & $\overline{\mathrm{AP}}$ & $\overline{R 3}$ & $\overline{744}$ & $\overline{\mathrm{M} 1}$ & $\overline{\mathrm{M} 2}$ & "M3 & Total \\
\hline & & & & & & & & & & \\
\hline VOA-GC & 0 & $\overline{0}$ & 0 & 0 & 0 & 0 & 0 & 0 & 0 & 0 \\
\hline VOA-GCMS & 0 & 0 & 0 & 0 & 0 & 0 & 0 & 0 & 0 & 0 \\
\hline SEMI-VOA & 0 & 0 & 0 & 0 & 0 & o. & 0 & 0 & 0 & 0 \\
\hline PEST & 0 & o) & 0 & 0 & 0 & 0 & 0 & 0 & 0 & 0 \\
\hline HERB & 0 & 0 & 0 & 0 & 0 & 0 & 0 & 0 & 0 & 0 \\
\hline PCB & 0 & 0 & 0 & 0 & 0 & o) & 0 & 0 & o & 0 \\
\hline PCB/PEST & 0 & 0 & 0 & 0 & 0 & o) & 0 & 0 & 0 & 0 \\
\hline OTHER-ORG & 0 & 0 & 0 & 0 & o & o & 0 & 0 & o & 0 \\
\hline TOIAL ORGANICS & 01 & 01 & 0 & 0 & o] & 0] & o1 & 0I & 0. & 0 \\
\hline & & & & & & & & & & \\
\hline ICP-AESMETALS & 0 & 0 & 0 & 0 & 0 & 0 & 0 & 0 & 0 & 0 \\
\hline ICP-MSMETALS & 0 & 0 & 0 & 0 & 0 & o & 0 & 0 & 0 & 0 \\
\hline GF-AAMETALS & 0 & 0 & 0 & o) & 0 & o & 0 & 0 & 0 & 0 \\
\hline MERCUAY & 0 & 0 & 0 & 0 & 0 & oll & o & 0 & o & 0 \\
\hline CYANIDE & 0 & 0 & 0 & 0 & 0 & o. & 0 & 0 & 0 & 0 \\
\hline ANIONS/CATIONS & 0 & 0 & 0 & 0 & 0 & 0 & 0 & 0 & 0 & 0 \\
\hline WET CHEM & 0 & 0 & 0 & 0 & 0 & 0 & 0 & 0 & 0 & 0 \\
\hline OTHER-INORG & 01 & 0 & O) & 0 & 0 & of & 0 & 0 & 0 & 0 \\
\hline TOTAL INOAGANICS & 01 & 0 & 01 & 01 & O) & D] & 01 & 01 & D] & $\overline{0}$ \\
\hline 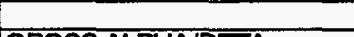 & & & t & SO & t 1 & $\rightarrow$ & 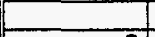 & 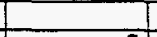 & $\square$ & \\
\hline GROSS ALPHAVBEIA & 0 & 0 & 0 & 0 & 0 & 0 & 0 & 0 & 0 & 0 \\
\hline ALPHA DET-Np237 & 01 & 0 & 0 & o & 0 & of & 0 & 0 & 0 & 0 \\
\hline ALPHA DET-Pu,Am & 0 & 0 & 0 & 0 & 0 & 0 & 0 & 0 & 0 & 0 \\
\hline ALPHADET-PU & 0 & 0 & 0 & 0 & 0 & 0 & 0 & 0 & 0 & 0 \\
\hline ALPHADET-U & 0 & 0 & 0 & 0 & 0 & 0 & 0 & 0 & 0 & 0 \\
\hline ALPHA DET-AM & 0 & 0 & 0 & 0 & 0 & 0 & 0 & 0 & 0 & 0 \\
\hline AIPHA DET-NP & 0 & 0 & o & O) & o & 0 & 0 & 0 & 0 & 0 \\
\hline ALPHA DET-Th & of & 0 & o' & 0 & o & o. & 0 & o & 0 & 0 \\
\hline ALPHA DET-U,Th,Pu & 0 & 0 & 0 & 0 & 0 & 0 & 0 & 0 & 0 & 0 \\
\hline ALPHA DET-U235 & 01 & 0 & 0 & 0 & o & of & 0 & 0 & 0 & 0 \\
\hline ALPHA DET-PU,U,Am & 0 & 0 & 0 & 0 & 0 & 0 & 0 & 0 & 0 & 0 \\
\hline ALPHA DET-UNp,Pu,Am & 0 & 0 & 0 & 0 & 0 & 0 & 0 & 0 & 0 & 0 \\
\hline ALPHA DET-Ra226 & 0 & 0 & 0 & 0 & 0 & 0 & 0 & 0 & 0 & 0 \\
\hline ALPHA DET-Ra228 & 01 & 0) & 0 & 0 & 0) & 0 & 0 & 0 & 0 & 0 \\
\hline ALPHA SPEC & 0 & 0 & 0 & 0 & 0 & ot & 0 & 0 & 0 & 0 \\
\hline GPC-BETA-Pb210 & 0 & 0 & 0 & 0 & 0 & 0 & 0 & 0 & 0 & 0 \\
\hline GPC-BETA-SROO & 0 & 0 & 0 & 0 & 0 & of & 0 & 0 & 0 & 0 \\
\hline GPC-BETA-Pm 147 & 0 & 0 & D & 0 & 0 & 0 & 0 & 0 & 0 & 0 \\
\hline GPC-BETA-Se79 & 0 & 0 & 0 & 0 & 0 & 0 & 0 & 0 & 0 & 0 \\
\hline LSC-BETA-H3 & 0 & 0 & 0 & 0 & 0 & 0. & 0 & 0 & 0 & 0 \\
\hline LSC-BETA-Pn222 & 0 & 0 & 0 & 0 & 0 & 0 & 0 & 0 & 0 & 0) \\
\hline LSC-BETA-1129 & 0 & 0 & 0 & 0 & 0 & of & 0 & 0 & 0 & 0 \\
\hline LSC-BETA-C14 & 0 & 0 & 01 & 0 & 0 & 0 & 0 & 0 & 0 & 0 \\
\hline LSC-BETA-TCS9 & 0 & 0 & 0) & 0 & 0 & 0 & 0 & 0 & 0 & 0 \\
\hline LSC-BETA & 01 & 0 & 0 & 0 & 0 & 0 & 0 & 0 & 0 & 0 \\
\hline GAMMA SPEC & 0 & 0 & 0 & 0 & 0 & 0 & 0 & 0 & 0 & 0 \\
\hline OTHER-RAD & 0 & 0 & 0 & 0 & 0 & 0 & 0 & 0 & D. & 0 \\
\hline TOTAL RAD & 0 & 0 & 01 & 0] & 0 & 0 & 01 & 0 & O. [ & 0 \\
\hline & & & & & & & & & & \\
\hline TCLPEXTRACTION & 0 & 0 & 0 & 0 & 0 & 0 & 0 & $\mathbf{0}$ & 0 & 0 \\
\hline OTHER-PREP & 0 & 0 & 0 & 0 & 0 & o. & 0 & 0 & 0 & 0 \\
\hline OTHER-EXPLO & 0 & 0 & 0 & 0 & 0 & 0 & 0) & 0 & 0 & 0 \\
\hline OTHER-BIO & 0 & 0 & 0 & 0 & 0) & 0 & 0 & 0 & 0 & 0 \\
\hline OTHER-PHYS & 0 & 0 & 0 & 0 & 0 & ol & 0 & 0 & 0 & 0 \\
\hline OTHER & 0 & 0 & 0 & 0 & 0 & o] & 0 & $\mathbf{0}$ & 0. & 0 \\
\hline TOIALOIHER & 01 & 01 & 0 & $\overline{0}$ & 0 & 0 & 0 & 0 & $\overline{0}$ & 0 \\
\hline & & & & & & & & & & \\
\hline TOTAL SAMPLE ANAIYSES & 01 & OI & का & 0 & 01 & $\overline{0]}[$ & 01 & 01 & $\overline{0}$ & 0 \\
\hline
\end{tabular}


EM-263 SURVEY OF DOE ANALYTICAL CAPACITY AVAILABLE TO EM

Table IVc: MC Sample Analyses by Rad Level and Matrix Type (x) Idaho Nat'I Eng Lab - WINCO

\begin{tabular}{|c|c|c|c|c|c|c|c|c|c|c|}
\hline $\begin{array}{l}\text { Rad-Matrix } \\
\text { Samp Anal }\end{array}$ & RO & RO/RT & $\overline{\mathrm{RI}}$ & $\overline{R 2}$ & R3 & $\overline{\mathrm{R4}}$ & $\overline{\mathrm{MI}}$ & $\overline{T / 2}$ & $\overline{\text { M3 }}$ & Total \\
\hline & & & & & & & & & & \\
\hline VOA-GC & 0 & 0 & 0 & 0 & 0 & 0 & 0 & 0 & 0 & 0 \\
\hline VOA-GCMS & 0 & 0 & 0 & 0 & 0 & 0 & 0 & 0 & 0 & 0 \\
\hline SEMI-VOA & 0 & 0 & 0 & 0 & 0 & 0) & 0 & 0 & 0 & 0 \\
\hline PEST & 0 & 0 & 0 & 0 & 0 & 0 & 0 & 0 & 0 & 0 \\
\hline HEPB & 0 & 0 & 0 & 0 & 0 & 0 & 0 & 0 & 0 & 0 \\
\hline PCB & 0 & 0 & 0 & 0 & 0 & 0 & 0 & $\mathbf{0}$ & 0 & 0 \\
\hline PCB/PEST & 0 & 0 & 0 & 0 & 0 & 0 & 0 & 0 & 0 & 0 \\
\hline OTHER-ORG & 0 & 0 & 01 & 0 & 0 & o. & 0 & 0 & 0 & 0 \\
\hline TOIALORGANICS & 01 & 01 & 01 & 01 & 0 & 0] & 0 & $0 \mid$ & 0] [ & 0 \\
\hline & & & & & & & & 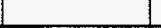 & & \\
\hline ICP-AESMETALS & 0 & 0 & 7329 & 0 & D) & 0 & 2311 & 4064 & 954 & 7329 \\
\hline ICP-MSMETALS & 0 & 0 & 4886 & 0 & 0 & D. & 913 & 3249 & 725 & 4886 \\
\hline GF-AAMETALS & 0 & 0 & 4886 & 0 & 0 & D. & 3059 & 1565 & 263 & 4886 \\
\hline MERCURY & \begin{tabular}{l|l}
0 \\
\end{tabular} & 0 & 8144 & 0 & 0 & 0) & 3926 & 3926 & 291 & 8144 \\
\hline CYANIDE & 0 & 0 & 0 & 0 & 0 & o) & 0 & o & 0 & 0 \\
\hline ANIONS/CATIONS & 0 & 0 & 0 & 0 & 0 & of & 0 & 0 & 0 & 0 \\
\hline WET CHEM & 0 & 0 & 0 & 0 & 0 & 0 & 0 & $\mathbf{0}$ & 0 & 0 \\
\hline OTHER-INORG & 0 & 0 & 32496 & 0 & 0 & 0) & 3441 & 20990 & 8065 & 32496 \\
\hline TOTAL INORGANICS & 0 & 01 & 57741 & 01 & 0 & 0] & 13650 & 33794 & 10297 & 57741 \\
\hline & & & & & & & & & & \\
\hline GROSS ALPHA/BETA & 0 & 0 & 0 & 0 & 0 & 0 & 0 & 0 & 0 & 0 \\
\hline ALPHA DET-Np237 & 0 & 0 & 0 & 0 & 0 & O) & 0 & $\overline{0}$ & 0 & 0 \\
\hline ALPHA DET-Pu,Am & 0 & 0 & 0) & 0 & 0 & 0 & 0 & 0 & 0 & 0 \\
\hline ALPHA DET-PU & 0 & 01 & 01 & 0 & 0 & 0 & 0 & 0) & 0 & 0 \\
\hline ALPHA DET-U & 0 & 0) & 0 & 0 & 0 & 0 & 0 & 0 & 0. & D. \\
\hline ALPHADET-Am & 0 & 0 & 0 & 0 & 0 & 0 & 0 & $\mathbf{0}$ & 0 & 0 \\
\hline ALPHA DET-Np & 0 & 0 & 0 & 0 & 0 & 0 & 0 & 0 & 0 & 0 \\
\hline ALPHA DET-Th & 0 & 0 & 0 & 0 & 0 & 0 & 0 & 0 & 0. & 0 \\
\hline ALPHA DET-U,Th,PU & 0 & 0 & 0 & 0 & 0 & o. & 0 & 0 & 0 & 0 \\
\hline ALPHA DET-U235 & 0 & 0 & 0 & 0 & 0 & 0. & 0 & 0 & 0 & $\overline{0}$ \\
\hline ALPHA DET-PU,U,Am & 0 & 0 & 0 & 0 & 0 & 0 & 0 & 0 & 0 & 0 \\
\hline ALPHA DET-UNp,PU,Am & 0 & 0 & 0 & 0 & 0 & 0 & 0 & 0 & o. & 0 \\
\hline ALPHADET-Ra226 & 0 & 0 & 0 & 0 & 0 & 0 & 0 & 0 & 0 & 0 \\
\hline ALPHADET-Ra228 & 0 & 0 & 0 & 0 & 0 & (0) & 0 & 0 & 0 & 0. \\
\hline ALPHA SPEC & 0 & 0 & 0 & 0 & 0 & o. & .0 & 0 & 0 & 0 \\
\hline GPC-BETA-Pb210 & O) & 0 & 0 & 0 & 0 & O & 0 & 0 & 0) & 0 \\
\hline GPC-BEIA-SROO & 0 & 0 & 0 & 0 & 0 & 0 & 0 & 0 & 0 & 0 \\
\hline GPC-BETA-Pm147 & 0 & 0 & 0 & 0 & 0 & 0. & 0 & 0 & 0 & 0 \\
\hline GPC-BETA-Se79 & 0) & 0 & 0 & 0 & 0 & 0 & 0 & 0 & 0 & 0 \\
\hline LSC-BETA-H3 & 0 & 0 & 0 & 0 & 0 & 0. & 0 & 0 & 0 & 0 \\
\hline LSC-BETA-Rn222 & 0 & 0 & 0 & 0 & 0 & 0 & 0 & 0 & 0 & 0 \\
\hline LSC-BETA-1129 & 0 & 0 & O) & 0 & 0 & 0 & 0 & 0 & 0 & 0 \\
\hline LSC-BETA-C14 & 0 & 0 & 0 & 0 & 0 & 0 & 0 & 0 & 0 & 0 \\
\hline LSC-BETA-TC99 & 0 & 0 & 0 & 0 & 0 & 0 & 0 & 0 & 0, & 0 \\
\hline LSC-BETA & o & 01 & 0 & 0 & o & 0 & 0 & 0 & o) & 0 \\
\hline GAMMA SPEC & 0 & 0 & 0 & 0 & 0 & 0 & 0 & 0 & 0. & 0 \\
\hline OTHER-RAD & 0 & 0 & 0 & 0 & 0 & 0 & 0 & 0 & 0 & 0 \\
\hline TOTAL RAD & 0 & 01 & 01 & Q1 & 0 & 0] & 01 & 01 & O] & (0] \\
\hline & & & & & & & & & 70 & \\
\hline TCLP EXTRACTION & 0 & 0 & 0 & 0 & 0 & ol & 0 & 0 & 0 & ㅇ. \\
\hline OTHER-PREP & 0 & 0 & 0 & 0 & 0 & 0 & 0 & 01 & 0 & 으 \\
\hline OTHER-EXPLO & 0 & 0 & 0 & 0 & 0 & 0 & 0 & 0 & o. & 0] \\
\hline OTHER-BIO & 0 & 0 & 0 & 0 & 0 & 0 & 0 & 0 & 0 & 0 \\
\hline OTHER-PHYS & 0 & 0 & 0 & 0 & 0 & 0 & 0 & $\mathbf{0}$ & 0 & O \\
\hline OTHER & 0 & 0 & 0 & 0 & 0 & 0 & 0 & 01 & o] & 0 \\
\hline TOTAL OIFER & 01 & 01 & 01 & 01 & (0) & D. & 01 & 01 & O] & 0 \\
\hline & & & & & & & & & & \\
\hline TOTAL SAMPLE ANALYSES & O] & 01 & 57741 & 01 & O. & D] & 13650 & 33794 & 10297 & 57741 \\
\hline
\end{tabular}


EM-263 SURVEY OF DOE ANALYTCAL CAPACITY AVAILABLE TO EM

Table IV: MC Sample Analyses by Rad Level and Matrix Type (xi) West Valley Nuclear Services

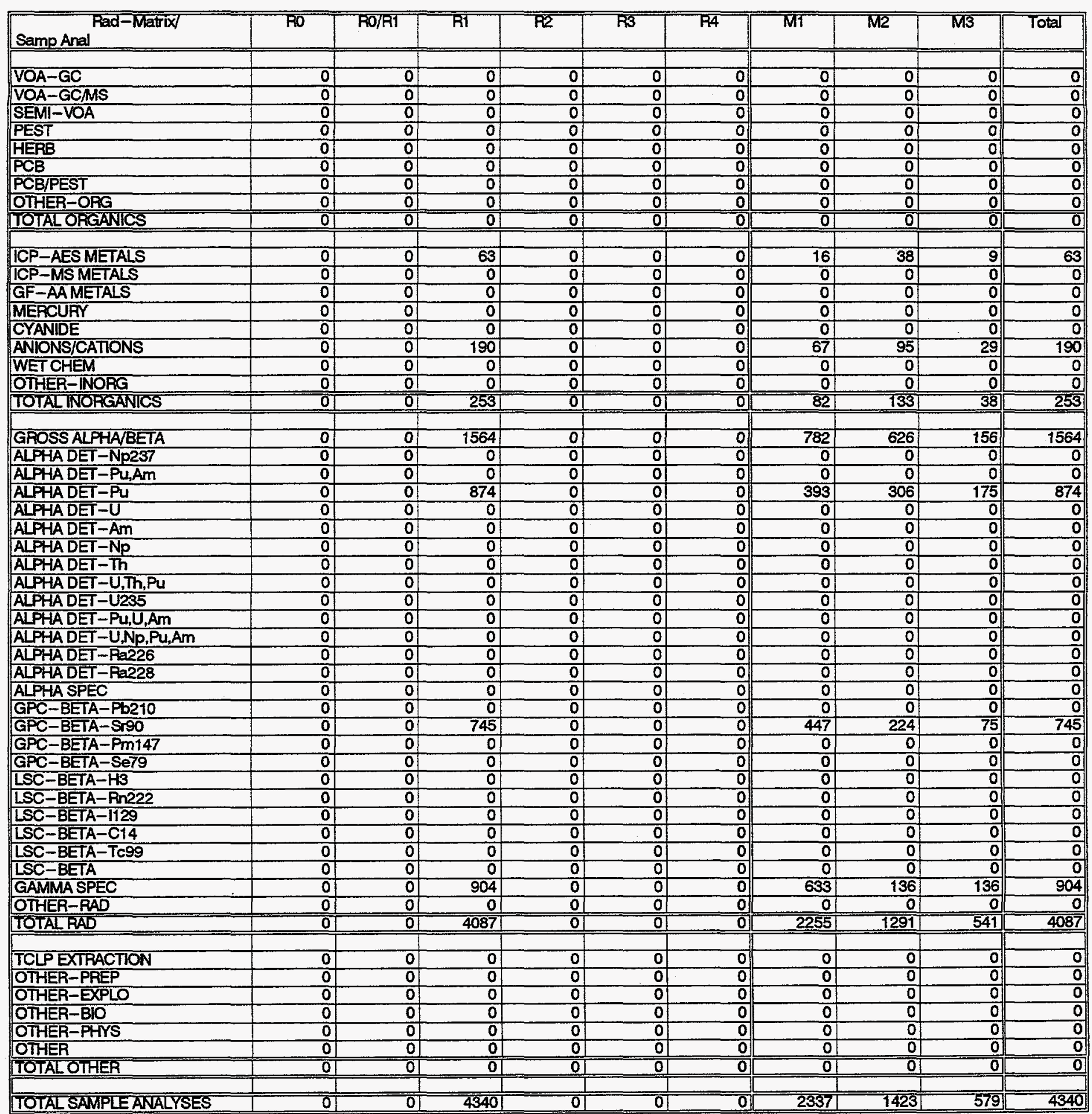


EM-263 SURVEY OF DOE ANALYTICAL CAPACITY AVAILABLE TO EM

Table IVc: MC Sample Analyses by Rad Level and Matrix Type (xii) Nevada Test Site

\begin{tabular}{|c|c|c|c|c|c|c|c|c|c|c|}
\hline $\begin{array}{l}\text { Fad-Matrix } \\
\text { Samp Anal }\end{array}$ & RO & Ro/R1 & R1 & R2 & $\overline{83}$ & $\overline{\mathrm{R4}}$ & MI & M2 & $\overline{743}$ & Total \\
\hline & & & & & & & & & & \\
\hline VOA-GC & 0 & 0 & 0 & 0 & 0 & 0 & 0 & 0 & 0 & 0 \\
\hline VOA-GCMS & 1076 & 0 & 0 & 0 & 0 & 0 & 538 & 323 & 215 & 1076 \\
\hline SEMI-VOA & 0 & 0 & 0 & of & 0 & o & 0 & 0 & 0 & 0 \\
\hline PEST & 0 & 0 & 0 & 0 & 0 & 0 & 0 & 0 & 0 & 0 \\
\hline HERB & 0 & 0 & 0 & 0 & 0 & 0 & 0 & of & 0 & 0 \\
\hline PCB & 0 & 0 & 0 & 0 & 0 & 0 & 0 & 0 & 0] & 0 \\
\hline PCB/PEST & 01 & 0 & 0 & 0 & \begin{tabular}{l|l}
0 \\
\end{tabular} & 0 & 0 & 0 & (0) & 0 \\
\hline OTHER-ORG & 0 & 0 & D) & 0 & 0 & (0) & 0 & 0 & 0 & 0 \\
\hline TOTALORGANICS & 1076 & 01 & 0.1 & 01 & OL & 0 & 538 & 323 & 215 & 1076 \\
\hline & & & & & & & & & & \\
\hline ICP-AESMETALS & 3021 & 0 & 0 & 0 & 0 & $\overline{0}$ & 792 & 2140 & 89 & 3021 \\
\hline ICP-MSMETALS & 01 & 0 & 0 & 0 & 0 & 0 & o & 0 & 0 & 0 \\
\hline GF-AAMETALS & 1233 & 0 & 0 & 0 & 0 & o. & 703 & 530 & 0 & 1233 \\
\hline MERCUAY & 1233 & 0 & 0 & 0 & 0 & D & 703 & 5 & 0 & 1233 \\
\hline CYANIDE & 0 & 0 & 0 & 0 & 0 & $\overline{0}$ & 0 & 0 & 0 & 0 \\
\hline ANIONS/CATIONS & 0 & 0: & 0 & 0 & 0 & - & 0 & 0 & of & 0 \\
\hline WET CHEM & 0 & of & 0 & 0 & 0 & 0. & 0 & 0 & 0. & 0 \\
\hline OTHEA-INOAG & of & of & 0 & 0 & of & : & 0 & 0 & a. & 0 \\
\hline TOTAL INORGANICS & 5488 & 01 & 0 & 01 & OI & 0 & 2198 & 3200 & 89[ & 5488 \\
\hline & & & & & & & & & & \\
\hline GROSS ALPHAVBTA & 0 & 0 & 17278 & 0 & 01 & 0 & 12165 & 5113 & o. & 17278 \\
\hline ALPHADET-NP237 & 0 & 0 & 0 & 0 & 0 & 0 & D) & 0 & a & 0 \\
\hline ALPHA DET - Pu,Am & 0 & 0 & 0 & 0 & 0 & 0 & 0 & 0 & 0 & 0 \\
\hline ALPHADET-PU & 0 & 0 & 8332 & 0 & 0 & 0 & 0 & 0 & 8332 & 8332 \\
\hline ALPHADET-U & 0 & 0 & 0 & 0 & 0 & 0 & 0 & 0 & 0 & 0 \\
\hline ALPHADET-Am & 0 & 0 & 0 & 0 & 0 & 0 & 0 & 0 & 0 & 0 \\
\hline ALPHA DET-NP & 0 & 0 & 0 & 0 & 0 & 0 & 0 & 0 & 0 & 0 \\
\hline ALPHA DET-Th & 0 & 0 & 0 & 0 & 0 & 0 & 0 & 0 & 0 & 0 \\
\hline ALPHADET-U,Th,Pu & 0 & 0 & 0 & 0 & 0 & 0 & 0 & 0 & 0 & 0 \\
\hline ALPHADET-U235 & 0 & 0 & 0 & 0 & 0 & 0 & 0 & of & 0 & 0 \\
\hline ALPHA DET - Pu,U,Am & o & 0 & 0 & 0 & 0 & 0 & 0 & 0 & 0 & 0 \\
\hline ALPHADET-U,Np,Pu,Am & 0 & 0 & 0 & 0 & 0 & 0 & 0 & 0 & 0 & 0 \\
\hline ALPHA DET-Ra226 & 0 & 0 & 0 & 0 & 0 & 0 & $\mathbf{0}$ & of & 0 & 0 \\
\hline ALPHADET-Ra228 & 0 & 0 & 0 & 0 & 0 & 0 & o & o & 0 & 0 \\
\hline ALPHA SPEC & 0 & 0 & o & 0 & 0 & 0 & 0 & 0 & 0 & 0 \\
\hline GPC-BETA-Pb210 & 0 & 0 & 0 & of & of & 0 & \begin{tabular}{l|l}
0 & \\
\end{tabular} & 0 & 0] & 0 \\
\hline GPC-BETA-Sr 90 & 0 & of & 0 & 0 & 0 & 0 & a & 0 & 0 & 0 \\
\hline GPC-BETA-Pm147 & 0 & 0 & 0 & o & 0 & 0 & 0 & 0 & 0 & 0 \\
\hline GPC-BETA-Se79 & 0 & 0 & 0 & 0 & 0 & 0 & 0 & 0 & 0 & 0 \\
\hline LSC-BETA-H3 & 0 & 0 & 7079 & 0 & of & 0 & 0 & 7079 & - & 7079 \\
\hline LSC-BETA-Rn222 & 0 & 0 & 0 & 0 & 0 & 0 & 0 & 0 & - & 0 \\
\hline LSC-BETA- 1129 & 0 & 0 & 0 & 0 & 0 & 0 & 0 & 0 & o & 0 \\
\hline LSC-BETA-C14 & 0 & 0 & 0 & 0 & 0 & 0 & 0 & 0 & 0 & 0 \\
\hline LSC-BETA-TC99 & 0 & 0 & o & of & 0 & 0 & 0 & 0 & o & 0 \\
\hline LSC-BETA & of & 0 & o & of & of & 0 & 0 & 0 & o & 0 \\
\hline GAMMA SPEC & 0 & 0 & 26782 & o) & 0 & 0 & 22795 & 2654 & 1334 & 26782 \\
\hline OTHER-RAD & 0 & 0 & 0 & 0 & 0 & 0 & \begin{tabular}{l|l}
0 \\
\end{tabular} & 0 & 0. & 0 \\
\hline TOTAL RAD & OI & ०ण & 59471 & 0 & 0 & 0 & 34960 & 14846 & 9665 & 59471 \\
\hline & & & & & & & & & & \\
\hline TCLP EXTRACTION & $0 \mid$ & o & D & 0 & 0 & 0 & 0 & 0 & 0 & 0 \\
\hline OTHER-PREP & 0 & 0 & 0 & 0 & 0 & 0 & 0 & 0 & of & 0 \\
\hline OTHEA-EXPLO & 4084 & o & 0 & 0 & o & 0 & 4084 & 0 & a] & 4084 \\
\hline OTHEA-BIO & 1006 & 0 & 0 & 0 & 0 & 0 & 1006 & 0 & 0 & 1006 \\
\hline OTHER-PHYS & 0 & 0 & $\overline{0}$ & 0 & 0 & 0 & of & of & 에 & 0 \\
\hline OTHEA & 0 & o) & 0 & 0 & 이 & 0 & of & 0 & 0 & 0 \\
\hline TOTAL OTHER & 5091 & 0 & 0 & $\overline{0]}$ & 01 & 0 & 50911 & 0 & 0 & 5091 \\
\hline & & & & & & & & & & \\
\hline TOTAL SAMPLEANALYSE & 11654 & $\overline{0}$ & 59471 & $\overline{0}$ & 이 & $\overline{0}$ & 42786 & 18369 & 9970 & 71125 \\
\hline
\end{tabular}


EM-263 SURVEY OF DOE ANALYTICAL CAPACTY AVAILABLE TO EM

Table IVc: MC Sample Analyses by Rad Level and Matrix Type (xii) Oak Ridge - K25

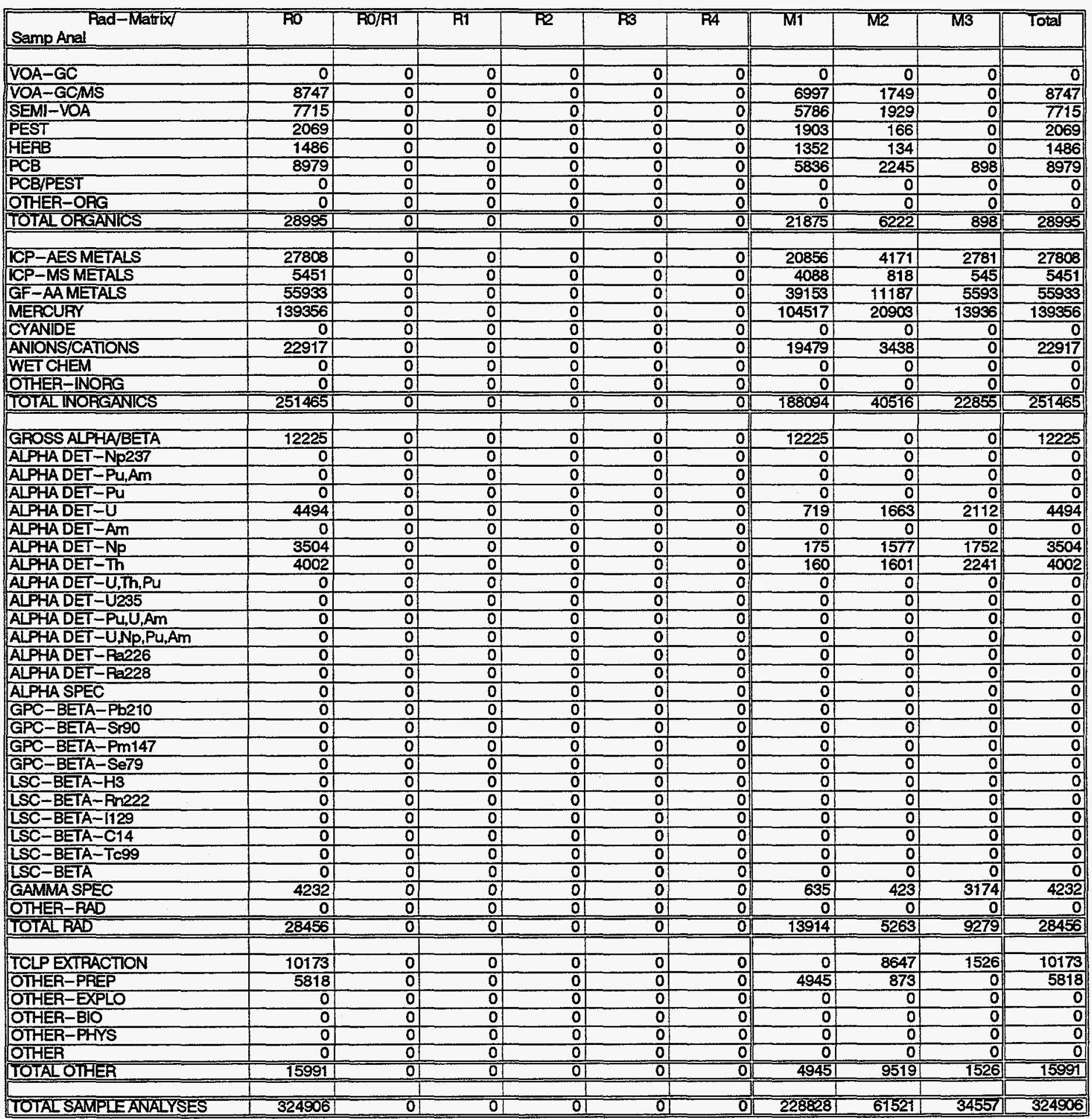


EM-263 SURVEY OF DOE ANALYTICAL CAPACITY AVAILABLE TO EM

Table IVc: MC Sample Analyses by Rad Level and Matrix Type (xiv) Oak Pidge Nat'l Lab

\begin{tabular}{|c|c|c|c|c|c|c|c|c|c|c|}
\hline $\begin{array}{l}\text { Pad-Matrix } \\
\text { Samp Anal }\end{array}$ & RO & RO/R1 & $\overline{\mathrm{R} 1}$ & $\overline{R 2}$ & $\overline{\mathrm{R3}}$ & $\overline{R 4}$ & M1 & M2 & $\overline{M 3}$ & Total \\
\hline & & & & & & & & & & \\
\hline VOA-GC & 855 & 0 & of & D. & 0 & 0 & 43 & 43 & 769 & 855 \\
\hline VOA-GCMS & 2318 & 0 & 01 & 0 & 0 & 0 & 1043 & 70 & 1206 & 2318 \\
\hline SEMI-VOA & 2376 & 0 & 0 & 0 & 0 & - & 2244 & 132 & 0 & 2376 \\
\hline PEST & 829 & 0 & 0 & 0 & 0 & 0 & 76 & 753 & 0 & 829 \\
\hline HERB & 0 & 0 & 0 & 0 & 0 & 0 & 0 & 0 & 0 & 0 \\
\hline$\overline{P C B}$ & 2265 & 0 & 0 & \begin{tabular}{l|l}
0 \\
\end{tabular} & 0 & 으 & 100 & 461 & 1704 & 2265 \\
\hline PCB/PEST & 0] & 0 & 0 & \begin{tabular}{l|l}
0 \\
\end{tabular} & 0 & 0 & 0 & 0 & 요 & 0. \\
\hline OTHER-OAG & 0) & 0 & 0 & 0 & 0 & 0 & \begin{tabular}{l|l}
0 \\
\end{tabular} & 0 & ㅇ] & 0 \\
\hline TOTAL ORGANICS & 8643 & 0] & o1 & 01 & 0। & 0 & 3506 & 1458 & 3679 & 8643 \\
\hline & & & & & & & & & & \\
\hline ICP-AES METALS & 6390 & 0 & 0 & 0 & \begin{tabular}{l|l}
0 & \\
\end{tabular} & 0 & 5371 & 1019 & 0. & 6390 \\
\hline ICP-MSMETALS & 1037 & 0 & of & 0 & 0 & 0 & 518 & 518 & 0. & 1037 \\
\hline GF-AAMETALS & 이 & 0 & 0 & 0 & a & 요 & 0 & 0 & 0. & (0) \\
\hline MERCURY & 3364 & 0 & 0| & 0 & 0 & 0] & 3026 & 337 & 0 & 3364 \\
\hline CYANIDE & 504 & 0 & of & 0 & 0 & 0 & 504 & 0 & o] & 504 \\
\hline ANIONS/CATIONS & 6836 & 0 & 0 & 0 & 0 & o & 6836 & 0 & 으 & 6836 \\
\hline WET CHEM & of & 0 & 0 & 0 & 0 & 0 & 0 & 0 & 0. & 0 \\
\hline OTHER-INORG & of & a & of & \begin{tabular}{l|l}
0 & \\
\end{tabular} & 0] & 0] & of & of & 0. & 0 \\
\hline TOTAL INORGANICS & 18131 & 01 & 01 & O) & $0 !$ & 0 & 16256 & 1875 & 이 & 18131] \\
\hline & & & & & & & & & & \\
\hline GROSS ALPHAVBETA & 7046 & 이 & 인 & 0 & O) & 0. & 6278 & 768 & 요 & 7046 \\
\hline ALPHA DET-Np237 & 0) & 0. & 어 & 0 & 요 & 0 . & 0 & 0 & 요 & 의 \\
\hline ALPHA DET-Pu,Am & 0 & 0 & 0 & \begin{tabular}{l|l}
0 \\
\end{tabular} & 0 & 0 & 0 & 0 & 0 & 0] \\
\hline ALPHADET-Pu & 0 & 0 & 0 & 0 & D & -0 & 01 & 0 & 0. & a \\
\hline ALPHA DET-U & 0 & 0. & 0 & 0 & D & 0 & 0 & 이 & (]. & 0 \\
\hline ALPHA DET-Am & 0 & 0 & of & \begin{tabular}{l|l}
0 & \\
\end{tabular} & 0 & 0 & 0 & 0 & 0 . & 0 \\
\hline ALPHADET-Np & 0 & D) & 0 & 0 & 0 & 0. & 0] & 0 & 0] & a \\
\hline ALPHA DET-Th & 0 & O) & o & 0 & D. & 0. & 0 & $\overline{0}$ & -0: & 0 \\
\hline ALPHADET-U,Th,PU & 6936 & 0 & 0 & 0 & 0 & 0. & 6212 & 724 & 0 & 6936 \\
\hline ALPHA DET-U235 & 0 & 0 & 0 & 0 & 0 & (0) & 0 & 0 & 0] & 0 \\
\hline ALPHA DET-Pu,U,Am & 0 & 0 & 0 & 0 & 0 & ㅁ: & 0 & 0 & 0. & O \\
\hline ALPHA DET - U,Np,Pu,Am & 0 & 0 & 0 & 0 & 0 & 0 & 0 & 0 & 0 & D. \\
\hline ALPHA DET-Ra226 & 0 & 0 & 0 & 0 & D & 0] & 0 & 0 & [0. & 0 \\
\hline ALPHA DET-Ra228 & 0 & 0 & 0 & 0 & 0 & 0. & 0 & 0 & 0] & 은 \\
\hline ALPHA SPEC & 0 & 0 & 0 & 0 & 0 & - & 0 & 0 & 0 & 0 \\
\hline GPC-BETA-Pb210 & 0 & of & 0 & 0 & 0 & 미 & 0 & 0 & 0 & 0 \\
\hline GPC-BETA-Sr90 & 0 & of & 0 & 0 & 0 & ㅁ․ & 0 & o & -0. & 0 \\
\hline GPC-BETA-Pm147 & 0 & 0 & 0 & 0 & 0 & 0 & 0 & 0 & 0 & 0 \\
\hline GPC-BETA-Se79 & 0 & D. & 01 & 0 & 0 & 0 & 0 & 0 & 0 & 0 \\
\hline LSC-BETA-H3 & 0 & 0 & 0 & 0 & 0 & 0 & 0 & 0 & 므: & 0 \\
\hline LSC-BETA-Rn222 & 0 & 0 & 0 & 0 & 0 & 요 & 0 & 0 & 0 & 0 \\
\hline LSC-BETA- 1129 & 0 & O) & 0 & 0 & 0 & 이 & of & 0 & 0] & 0 \\
\hline LSC-BETA-C14 & 0 & 0 & 0 & 01 & 0 & 에 & 0 & 0 & ㅁ․ & 0 \\
\hline LSC-BETA-TC99 & 0 & 0 & 0 & 0 & 0 & 0] & 0 & 으 & 의 & 0 \\
\hline LSC-BETA & 0 & 여 & 0. & 0 & 0 & 이 & 0 & 0 & 요 & 0 \\
\hline GAMMASPEC & 4477 & 0) & 0 & 0 & 0 & 의 & 4193 & 284 & 요 & 4477 \\
\hline OTHEA-RAD & 0 & 0 & 0 & 0 & 0 & (0) & 0 & 0 & o] & 0 \\
\hline TOTAL RAD & 18459 & 01 & 01 & 0 & 0 & ㅇ] [ & 16683 & 1776 & 이 & 18459 \\
\hline & & & & & & & & & & \\
\hline TCLP EXTRACTION & 158 & 0 & 0 & 0 & 0 & ㅇ․ & 8 & 119 & 32) & 158 \\
\hline OTHER-PREP & 0 & o & 0 & of & 0 & ㅇ. & 0 & 0 & 에 & 인 \\
\hline OTHER-EXPLO & 0 & 0 & 0 & 0 & 0 & ㅇ. & O & 0 & 매 & 0 \\
\hline OTHER-BIO & 0 & 0 & 01 & 01 & 0 & o] & 이 & 0 & 요 & 0 \\
\hline OTHER-PHYS & 0 & 可 & of & 이 & 0 & 0 & 0 & 0 & o & 0 \\
\hline OTHEA & 0 & 이 & 01 & 이 & 0 & 0. & 0 & 0 & - & 0 \\
\hline TOTAL OTHEA & 158 & 01 & 01 & 01 & 0 & 0 & 81 & 119 & 32 & 158 \\
\hline & & & & & & & & & & \\
\hline TOTAL SAMPLE ANALYSES & 45391 & 0] & 01 & 01 & $\overline{0}$ & 0] & 36453 & 5228 & 3711 & 4539 \\
\hline
\end{tabular}




\section{EM-263 SURVEY OF DOE ANALYTICAL CAPACTY AVAILABLE TO EM}

Table IVc: MC Sample Analyses by Rad Level and Matrix Type (xv) Oak Ridge - Y12

\begin{tabular}{|c|c|c|c|c|c|c|c|c|c|c|}
\hline $\begin{array}{l}\text { Rad-Matix } \\
\text { Samp Anal }\end{array}$ & $\overline{R O}$ & RO/RI & $\overline{\mathbf{R 1}}$ & $\overline{R 2}$ & $\overline{\mathrm{R3}}$ & $\overline{R 4}$ & MT & T12 & $\overline{\mathrm{M} 3}$ & Total \\
\hline & & & & & & & & & & \\
\hline YOA-GC & 0 & 0 & 0 & 0 & 의 & 0 & 0 & o. & 매 & 0 \\
\hline VOA-GCMS & 9649 & 0 & 4958 & 0 & 0 & 0 & 11956 & 1659 & 992 & 14607 \\
\hline SEMI-VOA & 673 & of & 3001 & 0 & of & D] & 3606 & 67 & o. & 3674 \\
\hline PEST & 0 & 0 & 0 & 0 & 0 & 0. & 0 & 0 & - & 0 \\
\hline HERB & of & 0 & $\overline{0}$ & 0 & 0 & o. & 0 & 0 & 0 & $\overline{0}$ \\
\hline PCB & 7298 & 0 & 8613 & 0 & 0 & - ] & 11809 & 2220 & 1882 & 15911 \\
\hline FCB/PEST & O. & 0 & 0 & 0 & 0 & 0] & 0 & 0 & 애 & $\overline{0}$ \\
\hline OTHER-ORG & 5486 & 0 & 0 & 0 & 0 & 매 & 5486 & 0 & 이 & 5486 \\
\hline TOTAL ORGANICS & 23106 & 0 & 16572 & (01 & DI & 0] & 32857 & 3947 & 2874: & 39678 \\
\hline & & & & & & 7 & & & & \\
\hline İCP-AESMETALS & 7940 & 0? & 11279 & 0 & 0 & 에 & 7146 & 794 & 11279 & 19219 \\
\hline ICP-MS METALS & 39008 & 0 & 0 & 0 & of & of & 27306 & 7021 & 4681 & 39008 \\
\hline GF-AAMETALS & 10508 & 0] & 6893 & $0 \mid$ & 0 & o] & 17402 & 0 & 0. & 17402 \\
\hline MERCURY & 12123 & 0 & 2419 & 0 & 0 & 0| & 12860 & 560 & 1121 & 14541 \\
\hline CYANIDE & 4212 & 0 & 0 & 0 & 0 & o] & 4212 & 0 & 0 & 4212 \\
\hline ANIONS/CATIONS & 12150 & 0 & 5742 & 0 & o & 애 & 7922 & 4199 & 5771 & 17892 \\
\hline WET CHEM & 0 & 0 & 0 & 0 & D) & 0. & 0 & 이 & o] & 0 \\
\hline OTHER-INORG & O) & 0. & 37161 & 0 & 0 & o] & 6388 & 25938 & 4834 & 3716 \\
\hline TOTAL INORGANICS & 85940 & 01 & 63493 & 0] & 01 & 요 & 83235 & 38513 & 27686 & 149434 \\
\hline & & & & & & & & & & \\
\hline GROSS ALPHAVBETA & 16980 & 이 & 701036 & O) & 0 & 이 & 11886 & 704432 & 1698 & 718017 \\
\hline ALPHADET-Np237 & 0 & 0 & 0 & 0 & 0 & 의 & 0 & 0 & 0 & 0 \\
\hline ALPHADET-Pu,Am & 0 & 0 & 0 & 0 & 0 & 0] & 0 & 0 & 요 & $\overline{0}$ \\
\hline ALPHADET-PU & O) & 0 & 0 & 0 & of & 에 & 0 & D) & 0 & 0 \\
\hline ALPHADET-U & 5563 & 0 & 15794 & 0. & 0. & - & 8166 & 11334 & 1858 & 21357 \\
\hline ALPHADET-Am & 0 & 0 & 0 & $\overline{0}$ & 0| & 0] & 0 & of & O्: & $\overline{0}$ \\
\hline ALPHADET-NP & O) & 0 & 0 & 0 & 0 & a & 0 & 0 & - & 0 \\
\hline ALPHADET-Th & 0 & 0 & 0 & O & 0) & 요 & of & 0 & 요 & 0 \\
\hline ALPHA DET-U,Th,PU & 0 & o & 0 & 0 & 0 & o. & 0 & 0 & 요 & 0 \\
\hline ALPHA DET-U235 & 0 & 0 & 7618 & o) & 0 & 0 & 3809 & 1828 & 1981 & 7618 \\
\hline ALPHA DET-PU,U,Am & 0 & (0) & 0 & of & 0 & 요 & of & O) & 0. & 0 \\
\hline ALPHA DET-U,Np,Pu,Am & 0 & 0 & 0 & 0) & 0| & a. & 0 & 0 & 요 & $\mathbf{0}$ \\
\hline ALPHA DET-Pa226 & 0 & 0 & 0 & 0 & of & (0) & 0 & o & 이 & 0 \\
\hline ALPHADET-Ra228 & 0 & 0 & 0 & 0 & 0 & 0] & of & o & D. & 0 \\
\hline ALPHA SPEC & 19326 & 0 & 8975 & 0 & 0 & 0] & 17393 & 966 & 9941 & 28300 \\
\hline GPC-BETA-Pb210 & 0 & 0 & 0 & 0 & 0 & 요 & 0 & 0 & - & 0 \\
\hline GPC-BETA-Sr9O & 0 & 0 & 0 & 0 & 0 & - & 0 & o. & 0 & 0 \\
\hline GPC-BETA-Pm147 & 0 & 0 & 0 & 0 & 이 & 요 & 0 & 0 & 0] & 0 \\
\hline GPC-BETA-Se79 & 0 & 0 & 0 & 0 & 0 & 요 & 0 & D & - & 0 \\
\hline LSC-BETA-H3 & 0. & 0 & 0 & 0 & 0 & ㅇ․ & 0 & 0 & 요 & 0 \\
\hline LSC-BETA-Rin222 & 0 & 0 & 0 & o & 0 & 0 & of & of & - & 0 \\
\hline LSC-BETA-1129 & 0 & 0 & 0 & 0 & 0 & 0. & 이 & 0 & 의 & 0 \\
\hline LSC-BETA-C14 & o & D) & 0 & 0 & 0! & 0 & 0 & of & 0. & 0 \\
\hline LSC-BETA-TC99 & 0 & 0 & 0 & 0 & 0 & -0. & 0 & 0 & 요 & 0 \\
\hline LSC-BETA & o & 0 & 0 & 0 & 0 & a & 0 & 0 & 0 & 0 \\
\hline GAMMA SPEC & 2611 & 0 & 813 & D & O) & D. & 2004 & 447| & 974 & 3424 \\
\hline OTHER-RAD & 0 & 0 & 0 & 0 & 0 & 0 & 0 & of & 0 & 0 \\
\hline TOTAL RAD & 44481 & 0 & 7342351 & 0 & OI & 0 & 43258 & 719007 & 16451 & 778716 \\
\hline & & & & & & & & & & \\
\hline TCLP EXTAACTION & 4246 & 0 & 可 & 0 & 0 & 0 & 212 & 1061 & 2972 & 4246 \\
\hline OTHER-PREP & 5602 & 0 & 0 & 0 & 0 & 0 & 4669 & 0 & 934 & 5602 \\
\hline OTHER-EXPLO & 0 & 0 & 0 & 0 & 0) & 0 & 0 & 0 & 0 & $\overline{0}$ \\
\hline OTHER-BIO & 0 & 0 & 0 & 0 & 이 & 0 & D! & 0 & 0. & $\overline{0}$ \\
\hline OTHER-PHYS & 29404 & 0 & 24248 & 0 & 0 & 0. & 53652 & 0. & o & 53652 \\
\hline OTHER & 0 & 0 & 0 & 0 & 0 & 0. & 0 & 01 & a & 0 \\
\hline TOTAL OTHER & 39252 & 0 & 24248 & D & O) & 0 & 58533 & 1061 & 3906 & 63500 \\
\hline & & & & & & 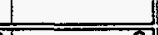 & & & & \\
\hline TOTAL SAMPLE ANALYS & 192779 & 0 & 838548 & 0 & 01 & 0] & 217882 & 762529 & 50916 & 1031327 \\
\hline
\end{tabular}


EM-263 SURVEY OF DOE ANALYTICAL CAPACITY AVAILABLE TO EM

Table Nc: MC Sample Analyses by Rad Level and Matrix Type (xvi) Paducah

\begin{tabular}{|c|c|c|c|c|c|c|c|c|c|c|}
\hline $\begin{array}{l}\text { Fad-Matrix } \\
\text { Samp Anal }\end{array}$ & 780 & PRO/R1 & $\overline{\mathrm{R} 1}$ & R2 & R33 & $\overline{R 4}$ & $\overline{\mathrm{MT}}$ & $\overline{\mathrm{M} 2}$ & $\overline{M 3}$ & Total \\
\hline & & & & & & & & & & \\
\hline VOA-GC & 0 & 0) & 0 & 0 & 0 & 0 & o & 0 & 0 & 0 \\
\hline VOA-GCMS & 0 & 10513 & 0 & 0 & 0 & 0 & 9987 & 526 & 0 & 10513 \\
\hline SEMI-VOA & 0 & 215 & 0 & 0 & 0 & 0 & 107 & 107 & 0 & 215 \\
\hline PEST & 0 & 0 & 0 & 0 & 0 & 0 & 0 & 0 & 0 & 0 \\
\hline HERB & 0 & 0 & 0 & 0 & 0 & 0 & 0 & 0 & 0 & 0 \\
\hline$\overline{\mathrm{PCB}}$ & 0 & 22014 & 0 & 0 & 0 & 0 & 21354 & 660 & 0! & 22014 \\
\hline PCB/PEST & 0 & 0 & 0 & 0 & 0 & 0 & 0 & 0 & 0 & 0 \\
\hline OTHER-ORG & 0 & 0 & 0 & 0 & 0 & 0 & 0 & 0 & 0 & 0 \\
\hline TOTAL ORGANICS & 01 & 32742 & 01 & o] & 0 & 0] & 31449 & 1293 & 0] & 32742 \\
\hline & & & & & & & & & $=$ & \\
\hline ICP-AESMETALS & 0 & 20443 & 0 & 0 & 0 & 0 & 0 & 20443 & 0 & 20443 \\
\hline ICP-MSMETALS & 0 & 0 & 0 & 0 & 0 & 0 & 0! & 0 & 0 & 0 \\
\hline GF-AAMETALS & 0 & 14989 & 0 & 0 & 0 & 0 & 8829 & 4723 & 1437 & 14989 \\
\hline MERCUFY & 0 & 8962 & 0 & 0 & 0 & 0 & 5646 & 3316 & 0 & 8962 \\
\hline CYANIDE & 0 & 3752 & 0 & 0 & 0 & of & 3152 & 0 & 600 & 3752 \\
\hline ANIONS/CATIONS & 0 & 39108 & 0 & 0 & o & 0 & 36851 & 1945 & 312 & 39108 \\
\hline WET CHEM & 0 & 0 & 0 & 0 & 0 & 0 & 0 & 0 & 0 & 0 \\
\hline OTHER-INORG & 0 & 0 & 0 & 0 & o & o] & 0 & 0 & o & 0 \\
\hline TOTALINORGANICS & 0 & 87255 & 0 & 0 & 01 & O] & 54478 & 30427 & 2350 & 87255 \\
\hline & & & & & & & & $\Omega$ & 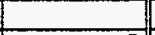 & \\
\hline GROSS ALPHAVBETA & 0 & 15791 & 0 & 0 & 0 & 0 & 15791 & 0 & o. & 15791 \\
\hline ALPHA DET-Np237 & 0 & 0 & 0 & 0 & 0 & 0 & 0 & 0 & 0 & 0 \\
\hline ALPHA DET-Pu,Am & 0 & 0 & 0 & 0 & a & 0 & 0 & 0 & 0 & 0 \\
\hline ALPHA DET-Pu & 0 & 0 & 0 & 0 & 0 & 0 & 0 & 0 & 0 & 0 \\
\hline ALPHA DET $-U$ & 0 & 0 & 0 & 0 & 0 & 0 & 0 & 0 & 0 & 0 \\
\hline ALPHA DET-Am & 0 & 0 & 0 & 0 & 0 & 0 & 0 & 0 & 0 & 0 \\
\hline ALPHADET-Np & 0 & 0 & 0 & 0 & 0 & 0 & 0 & 0 & of & 0 \\
\hline ALPHA DET -Th & 0 & 0 & 0 & 0 & 0 & 0 & 0 & 0 & ol & 0 \\
\hline ALPHA DET $-U, T h, P U$ & 0 & 0 & 0 & 0 & 0 & 0. & 0 & 0 & 0 & 0 \\
\hline ALPHADET-U235 & 0 & 0 & 0 & 0 & 0 & 0 & 0 & 0 & 0 & 0 \\
\hline ALPHA DET-PU,U,Am & 0 & 0 & 0 & 0 & 0 & 0 & 0 & 0 & $\overline{0}$ & 0 \\
\hline ALPHADET-U,Np,Pu,Am & 0 & 0 & 0 & 0 & 0 & $\overline{0}$ & 0 & 0 & 0 & 0 \\
\hline ALPHA DET-Ra226 & O) & 0 & 0 & 0 & 0 & 0 & 0 & 0 & 0 & 0 \\
\hline ALPHA DET-Ra228 & 0 & 0 & 0 & 0 & 0 & 0 & 0 & 0 & 0 & 0 \\
\hline ALPHA SPEC & 0) & 6909 & 0 & 0 & 0 & 0 & 6218 & 691 & 0 & 6909 \\
\hline GPC-BETA-Pb210 & 0 & 0 & 0 & 0 & 0 & o) & 0 & 0 & o & 0 \\
\hline GPC-BETA-Sr90 & 0 & 0 & 0 & 0 & 0 & 0 & 0 & 0 & 0 & 0 \\
\hline GPC-BETA-Pm147 & 0 & 0 & 0 & 0 & 0 & 0 & 0 & 0 & 0 & 0 \\
\hline GPC-BETA-Se79 & 0 & .0 & 0 & 0 & 0 & 0 & 0 & 0 & 0 & 0 \\
\hline LSC-BEIA-H3 & 0 & 01 & 0 & 0 & 0 & 0 & 0 & 0 & 0 & $\overline{0}$ \\
\hline LSC-BETA-Rn222 & 0 & 0 & 0 & 0 & 0 & 0 & 01 & 0 & 0 & 0 \\
\hline LSC-BETA- 1129 & 0 & 0 & 0 & 01 & 0 & 0 & 0 & 0 & 0 & 0 \\
\hline LSC-BEIA-C14 & 0 & 0 & 0 & 0 & 0 & 0 & 0 & 0 & of & 0 \\
\hline LSC-BETA-TC99 & 0 & 0 & 0 & 0 & 0 & 0 & 0 & 0 & 0 & 0 \\
\hline LSC-BETA & 0 & 0 & 0 & 0 & 0 & 0 & 0 & 0 & 0 & 0 \\
\hline GAMMA SPEC & 0 & 3286 & 0 & 01 & 0 & 0 & 3286 & 0 & 0 & 3286 \\
\hline OTHER-RAD & 0 & 0 & 0 & 0 & 0 & 0. & 0 & 01 & 0 & 0 \\
\hline TOIAL RAD & 0 & 25986 & 01 & 01 & 01 & 0. & 25295 & 6911 & 0 & 25986 \\
\hline & & & & & & & & & & \\
\hline TCLPEXTRACTION & 0 & 6063 & 0 & 0 & 0 & 0 & 6063 & 0 & 0 & 6063 \\
\hline OTHER-PREP & 0 & 0 & 0 & 0 & 0 & 0 & 0 & 0 & 0 & 0 \\
\hline OTHER-EXPLO & 0 & 0 & 0 & 0 & 0 & 0 & 0 & 0 & 0. & 0 \\
\hline OTHER-BIO & 0 & 0 & 0 & 0 & 0 & 0 & 0 & 0 & 0 & 0 \\
\hline ÖTHER-PHYS & 0 & 0 & 0 & 0 & 0 & 0 & 0 & 0 & 0 & 0 \\
\hline OTHER & $\overline{0}$ & 0 & 0 & 0 & 0 & 0 & 0 & 0 & 0. & 0 \\
\hline TOIAL OTHER & $\overline{0}$ & 6063 & $\overline{0}$ & 01 & 0 & 0] & 6063 & O] & 0 & 6063 \\
\hline & & & & & & - & & & & \\
\hline TOTAL SAVIPLE ANALYSES & o & 152046 & $\overline{0}$ & 01 & (0) & 0] & 117285 & 32411। & 2350 & 152046 \\
\hline
\end{tabular}


EM-263 SURVEY OF DOE ANALYTICAL CAPACITY AVAILABLE TO EM

Table Nc: MC Sample Analyses by Rad Level and Matrix Type (xvii) Portsmouth

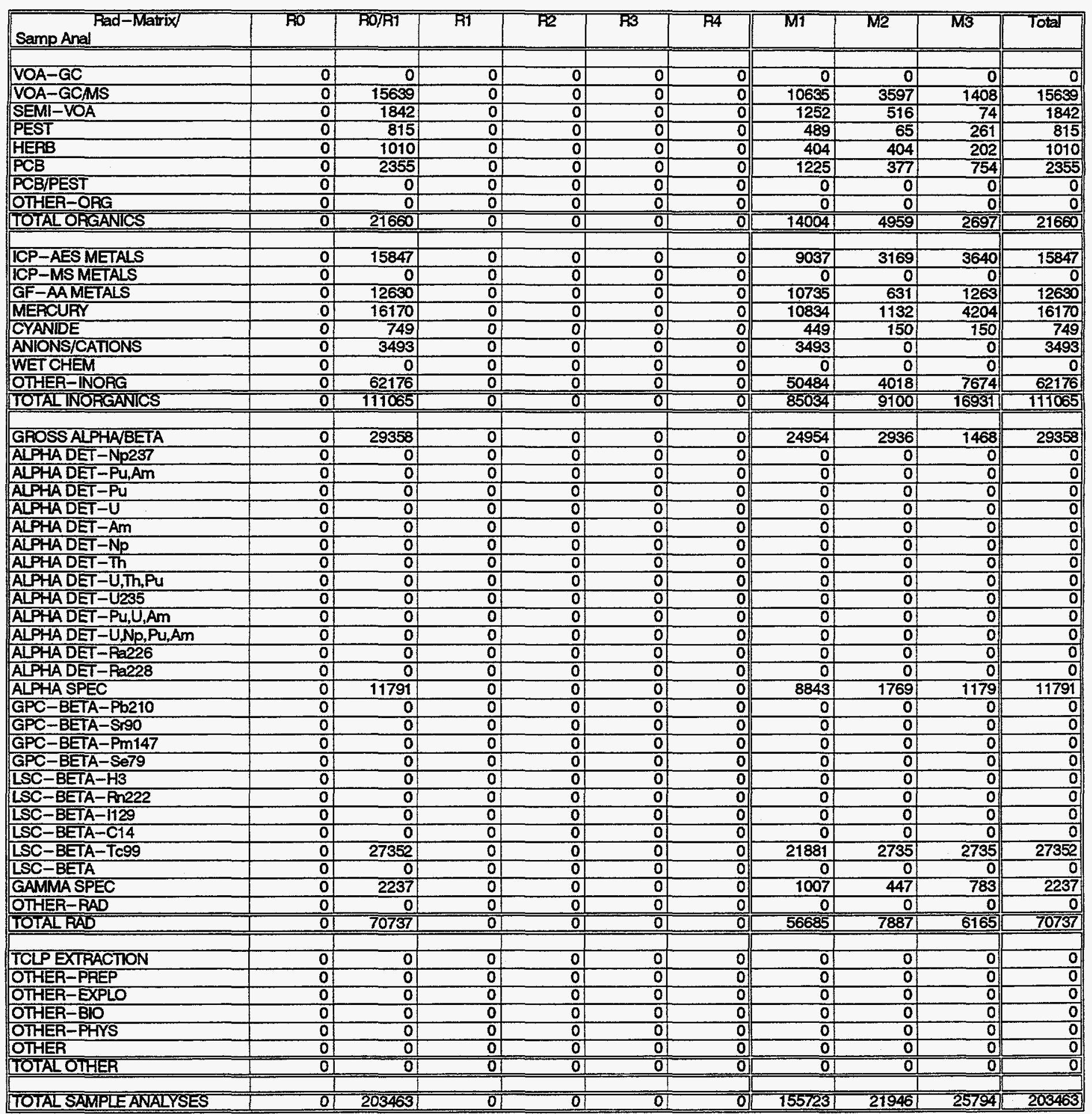


EM-263 SURVEY OF DOE ANALYTICAL CAPACITY AVAILABLE TO EM

Table IVc: MC Sample Analyses by Rad Level and Matrix Type (xviii) Hanford - WHC 222S

\begin{tabular}{|c|c|c|c|c|c|c|c|c|c|c|}
\hline $\begin{array}{l}\text { Rad-Matrix } \\
\text { Samp Anal }\end{array}$ & $\overline{\text { RO }}$ & Fo/R1. & $\overline{\mathrm{RI}}$ & $\mathrm{R2}$ & $\overline{73}$ & $\overline{\overline{R 4}}$ & $\overline{\mathrm{MH}}$ & $\overline{\mathrm{M} 2}$ & 193 & Total \\
\hline VOA-GC & 0 & of & of & 0 & 0 & 0 & 0! & 0 & o. & 0 \\
\hline VOA-GCMS & 0 & 3455 & 0 & 0 & 0 & 0 & 3455 & 0 & 0 & 3455 \\
\hline SEMI-VOA & 0 & of & 0 & 0 & 0 & 0 & 0 & 0 & 0 & 0 \\
\hline PEST & 0 & D) & 0 & 0 & 0 & 0 & 0 & 0 & 0 & 0 \\
\hline HERB & 0 & 0 & 0 & 0 & 0 & 0. & 0 & 0 & 0 & 0 \\
\hline PCB & 0 & 0 & o & 0 & 0 & 0 & 0 & o & 0 & 0 \\
\hline PCB/PEST & O) & of & of & o & of & 0 & 0 & 0 & 0 & 0 \\
\hline OTHER-ORG & 01 & 이 & 01 & 147818 & 0 & 0 & 103014 & 0 & 44805 & 147818 \\
\hline TOTAL ORGANICS & O) & 3455] & 01 & 147818 & - & [0] & 106468 & DI & 44805 & 151273 \\
\hline & & & & & & & & & & \\
\hline ICP-AESMETALS & 0 & 요 & of & 145903 & 0 & 0 & 80143 & 805 & 64955 & 145903 \\
\hline ICP-MSMETALS & O) & 0 & 0 & 0 & 0 & 0 & 0 & 0 & 0 & 0 \\
\hline GF-AAMETALS & 0 & 3951 & 0 & 89619 & 0 & 0. & 2963 & 988 & 89619 & 93570 \\
\hline MERCURY & 0 & 0 & 0 & 116600 & 0 & 0 & 11600 & 5830 & 99110 & 116600 \\
\hline CYANIDE & 0 & of & of & 109544 & 0 & o & 58423 & 0 & 51120 & 109544 \\
\hline ANIONS/CATIONS & 0 & 31544 & 0 & 37642 & 0 & 0 & 46084 & 86 & 23016 & 69186 \\
\hline WET CHEM & 0 & 0) & 0 & 5578 & 0 & 0. & 2668 & 39 & 2871 & 5578 \\
\hline OTHER-INORG & 0 & 78978 & of & 110136 & 0 & 0] & 128091 & 4827 & 56196 & 189114 \\
\hline TOTAL INORGANICS & 01 & 114473 & 이 & 615021 & 0 & 0 & 330033 & 12575 & 386887 & 729495 \\
\hline & & & & & & & & & & \\
\hline GROSS ALPHAVBETA & 0 & 39693 & of & 0 & 0 & 0 & 23597 & 293 & 15804 & 39693 \\
\hline ALPHA DET-Np237 & 0 & 10857 & 0 & 0 & 0 & 0 & 4017 & 0 & 6840 & 10857 \\
\hline ALPHA DET-Pu,Am & 0 & 95579 & 0 & 0 & 0 & 0 & 55012 & 21579 & 18988 & 95579 \\
\hline ALPHA DET-Pu & 0 & 0 & 0 & 0 & 0 & 0 & 0 & 0 & of & 0 \\
\hline ALPHADET-U & 0 & 0 & 0 & D & 0 & - & 0 & 0 & 0] & 요 \\
\hline ALPHA DET-Am & 0 & 0 & 0 & o & 0 & 0 & 0 & 0 & of & 0 \\
\hline ALPHA DET-NP & 0 & 0 & 0 & 0. & 0 & 0 & 0 & 0 & 0. & 0 \\
\hline ALPHA DET-Th & 0 & 0 & 0 & 0) & 0 & 0 & 0 & 0 & 0 & 0 \\
\hline ALPHA DET-U,Th,PU & 0 & 0 & 0 & 0 & 0 & 0 & 0 & 0 & 0 & 0. \\
\hline ALPHADET-U235 & 0 & 0 & of & 0 & 0 & 0 & 0 & 0 & of & 0 \\
\hline ALPHADET-PU,U,Am & 0 & 0 & 0 & 0 & (0) & 0. & 0 & 0 & 0 & 0 \\
\hline ALPHA DET-U,Np,Pu,Am & 0 & 0 & 0 & 0 & 0 & 0 & 0 & 0 & 0. & 0 \\
\hline ALPHADET-Ra226 & 0 & 0 & 0 & 0 & 0 & 0 & 0 & 0 & 0. & 0 \\
\hline ALPHA DET-Ra228 & 0 & 0 & 0 & 0) & O) & 0 & 0 & 0 & 0 & 0 \\
\hline ALPHA SPEC & 0 & 0 & 0 & 0 & 0 & 0 & 0 & 0 & 0 & 0 \\
\hline GPC-BETA-PB210 & 0 & 0 & 0 & 0 & 0 & 0 & 0 & 0 & 0 & 요 \\
\hline GPC-BETA-Sr90 & 0 & 15823 & 0 & 0 & o) & 0 & 10446 & 1616 & 3762 & 15823 \\
\hline GPC-BETA-Pm147 & 0 & 4997 & 0 & 0 & 0 & 0 & 4997 & 0 & (0. & 4997 \\
\hline GPC-BETA-Se79 & 0 & 2026 & 0 & 0 & 0 & 0 & 2026 & 0 & 0 [- & 2026 \\
\hline LSC-BETA-H3 & 0 & 17433 & of & 0 & 0 & 0 & 17433 & 0 & 0 & 17433 \\
\hline LSC-BETA-Rn222 & 0 & 0 & 0 & 0 & 0 & 0 & 0 & 0 & ㅇ․ & 의 \\
\hline LSC-BETA- 1129 & 0 & 7589 & 0 & 0 & D & 0 & 7589 & 0 & ㅁ. & 7589 \\
\hline LSC-BETA-C14 & 0 & 3388 & 0 & 0 & o! & 0] & 1830 & 0 & 1558 & 3388 \\
\hline LSC-BETA-TC99 & 0 & 32406 & 0 & 0 & 0 & - & 22974 & 0 & 9433 & 32406 \\
\hline LSC-BETA & 0 & o & of & 0 & 요 & 0 & 0 & of & 0. & 의 \\
\hline GAMMA SPEC & 0 & 103598 & 0 & 0 & 0 & 0 & 61077 & 17000 & 25513 & 103598 \\
\hline OTHER-RAD & 0 & 0) & 0 & 0 & 0! & 0] & of & of & 0. [ & 0 \\
\hline TOTAL RAD & 0 & 333389 & 01 & 0] & 0] & (0) & 210996 & 40496 & 81897[ & 333389 \\
\hline & & & & & & & & & & \\
\hline TCLP EXTRACTION & 0 & 0 & 0 & 0 & 으 & 요 & of & 0 & - & 이 \\
\hline OTHER-PREP & 0 & 1719 & 0 & 130295 & D & 1419 & 48319 & 7154 & 77959 & 133432 \\
\hline OTHER-EXPLO & 0 & 0 & 0 & 0 & o & 0 & 0 & 0 & of & 0 \\
\hline OTHER-BIO & 0 & 0 & o & 0 & o & 0 & 0) & 0 & ㅇ. & 0 \\
\hline OTHEA-PHYS & 0 & 0 & 0 & 104491 & o & 0 & 66512 & 6139 & 31841 & 104491 \\
\hline OTHER & 0 & 0 & 0 & 0 & 0 & 0 & 0 & 0 & of & 0 \\
\hline TOTAL OTHEA & 0 & 1719 & 0 & 234786 & 0 & 1419 & 114832 & 13293 & 109799 & 237924 \\
\hline & & & & & & & & & & \\
\hline TOTAL SAMPLE ANALYSES & 0 & 453036 & 0 & 997626 & 0 & 1419 & 762329 & 66364 & 623388 & 1452081 \\
\hline
\end{tabular}


EM-263 SUAVEY OF DOE ANALYTICAL CAPACITY AVAILABLE TO EM

Table IVc: MC Sample Analyses by Rad Level and Matrix Type (xix) Hanford - PNL 325

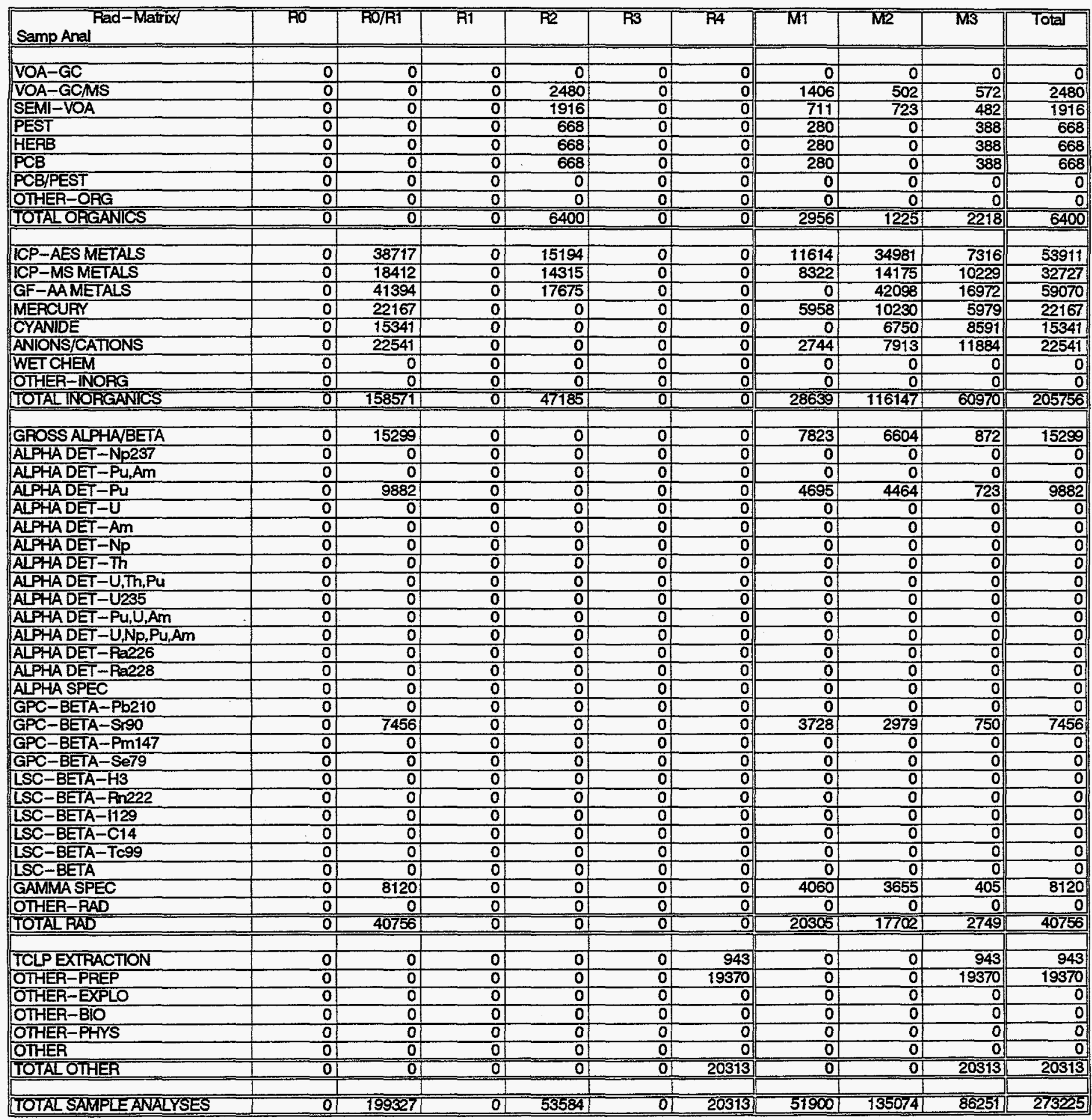


EM-263 SURVEY OF DOE ANALYTICAL CAPACITY AVAILABLE TO EM

Table IVc: MC Sample Analyses by Rad Level and Matrix Type (xx) Rocky Flats - 123 Lab

\begin{tabular}{|c|c|c|c|c|c|c|c|c|c|c|}
\hline $\begin{array}{l}\text { Rad-Matrix/ } \\
\text { Samp Anal }\end{array}$ & RO & RO/R1 & R1 & $\bar{R} 2$ & R63 & R4 & Th1 & $M 2$ & M3 & Total \\
\hline VOA-GC & 0 & 0 & 0 & 0 & 0 & 0 & 0 & 0 & 이 & 0 \\
\hline VOA-GCMS & 0 & 0 & 0 & 0 & 0 & 0 & 0 & 0 & 0 & 0 \\
\hline SEMI-VOA & 0 & 0 & of & $\overline{0}$ & o & 0 & 0 & 0 & 0 & 0 \\
\hline PEST & 0 & 0) & 0 & o & 0 & 0 & 0 & 0 & 0. & 0 \\
\hline HERB & 0 & of & 0 & 0 & 0 & 0 . & 0 & 0 & 0 & 0 \\
\hline PCB & 0 & 0) & 0 & 0 & 0 & 0 & 0 & 0 & 0 & 0 \\
\hline PCB/PEST & 0 & 0 & 0 & 0 & D & 0. & o. & 0 & 0 & 0 \\
\hline OTHER-ORG & 0 & 0 & 0 & 0 & 0 & 0. & 0 & 0 & 0 & 0 \\
\hline TOTAL ORGANICS & 01 & 01 & 0 & (0) & 0 & $0]$ & (0! & 0 & 0 & 0 \\
\hline & & & & & & 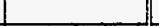 & & & & \\
\hline ICP-AES METALS & 0 & 0 & 0 & 0 & 0 & 0 & 0 & $\mathbf{0}$ & 0 & 0 \\
\hline ICP-MSMETALS & 0 & 0 & 0 & 0 & 0 & 0. & 0 & 0 & 요 & 0 \\
\hline GF-AAMETALS & 이 & 0 & 0 & 0 & 0 & 0 & 0 & 0 & 요 & 0 \\
\hline MERCUPY & 0 & 0 & 0 & 0 & 0 & 0 & 0 & 0 & ㅇ. & 0 \\
\hline CYANIDE & 0 & 0 & 0 & 0 & 0 & 0 & 0 & 0 & 0 & 0 \\
\hline ANIONS/CATIONS & O & 0 & 0 & 0 & 0 & 0 & 0 & 0 & ㅇ. & 0 \\
\hline WET CHEM & 0 & 0 & 0 & 0 & 0 & 0 & 0 & 0 & 0. & 0 \\
\hline OTHER-INORG & 180401 & - & of & of & O) & $0]$ & 180401 & 0 & 0] & 180401 \\
\hline TOTALINOAGANICS & 180401 & 0 & 01 & 0 & - & $0]$ & 180401 & 0 & 0] & 180401 \\
\hline GROSS AIPHARBA & & & & & & & 301070 & & th & 001070 \\
\hline ALPHADET-Np237 & & 0 & of & $\frac{0}{0}$ & $\frac{0}{0}$ & $\frac{0}{n}$ & 304910 & $\frac{0}{0}$ & a. & 304910 \\
\hline ALPHA DET $-P u, A m$ & 0 & 0 & 0 & 0 & 0 & 0 & 0 & 0 & of & $\frac{0}{0}$ \\
\hline ALPHA DET-Pu & 0 & 0 & 0 & 0 & 0 & 0 & 0 & 0 & 0 & 0 \\
\hline ALPHADET-U & 0 & 0 & 0 & 0 & 0 & 0 & 0 & 0 & 0 & 0 \\
\hline ALPHA DET-Am & 0 & 0 & 0 & 0 & 0 & 0 & 0 & 0 & (0) & 0 \\
\hline ALPHA DET-NP & 0 & of & of & 0 & 0 & 0 & 0 & 0 & 0 & 0 \\
\hline ALPHADET-Th & 요 & 0 & 0 & 0 & 아 & 0. & 0 & 0 & 0. & 0 \\
\hline ALPHA DET $-U, T h, P u$ & 0 & 이 & o & 0 & 이 & 0. & 이 & 0 & o. & 0 \\
\hline ALPHADET-U235 & 0 & 0 & of & 0 & 0 & 0 & 0 & 0 & 이 & 0 \\
\hline ALPHA DET-Pu,U,Am & 19638 & 0 & O) & 0 & 이 & 0 & 19638 & 0 & ㅁ․ & 19638 \\
\hline ALPHA DET-U,Np,Pu,Am & of & 0 & of & \begin{tabular}{l|l}
0 & \\
\end{tabular} & 0) & 0 & 0 & 0 & 0 & 0 \\
\hline ALPHADET-Ra226 & 0 & 0 & 0 & 0 & 0 & o. & of & O & 0 & 0 \\
\hline ALPHA DET-R2228 & O) & 0 & 0 & 0 & 0 & 0. & of & 0 & 요 & 0 \\
\hline ALPHA SPEC & D & O) & 0 & 0 & 0 & 묘 & 0 & 0 & 0] & 0 \\
\hline GPC-BETA-Pb210 & 0 & 0 & 0 & 0 & 0 & 0 & 0 & 0 & a] & 0 \\
\hline GPC-BETA-Sr90 & 0 & 0 & 0 & 0 & 0 & 0. & 0 & 0 & 묘 & 0 \\
\hline GPC-BETA-Pm147 & 0 & 0 & 01 & 0 & 0 & 0] & 0) & 0 & 에 & 0 \\
\hline GPC-BETA-Se79 & 0 & of & 0 & 01 & 0 & 요 & 0 & 0 & 0 & 0 \\
\hline LSC-BETA-H3 & 6112 & 0 & 0 & 0 & 0 & 0. & 5927 & 184 & 의 & 6112 \\
\hline LSC-BETA-Rn222 & 0 & 0 & 0 & 0 & 0 & o. & 0 & 0 & 요 & 0 \\
\hline LSC-BETA- 1129 & 0. & 0 & 0 & 01 & 0 & 의 & 0 & 0 & 이 & 0 \\
\hline LSC-BETA-C14 & 0 & 0 & 0 & 0 & 0 & of & $\overline{0}$ & 0 & 0 & 0 \\
\hline LSC-BETA-TC99 & 0. & 0 & 0 & 0 & 0 & 0] & 0 & 0. & 0] & 0 \\
\hline LSC-BETA & 0 & 0 & 0 & 0 & 0 & 0 & of & of & 요 & 0 \\
\hline GAMMA SPEC & 0 & 0 & 0 & 0 & 0 & 0 & 0 & 0 & 의 & 0 \\
\hline OTHER-RAD & 0 & 0 & 0 & 0 & 0 & 0 & $0 \mid$ & 0 & 0. & 0 \\
\hline TOTAL $A \bar{~}$ & 410728 & 0 & 01 & 01 & 0 & 요 & 410544 & 184 & $0]$ & 410728 \\
\hline 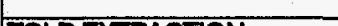 & & & & & & & & & & \\
\hline TCLP EXTRACTION & 0 & 0 & 0 & 0 & 0 & 이 & 0 & 0 & 의 & 0 \\
\hline OTHER-PREP & 0 & 0 & 0 & 0 & 0 & 으. & 0 & 0 & 0 & $\underline{0}$ \\
\hline OTHER-EXPLO & of & 0 & o & 0 & 0 & - & 0 & 0 & 으 & 0 \\
\hline OTHER-BIO & 0 & 0 & 0 & 0 & 0 & o & 0 & 0 & o & 0 \\
\hline OTHER-PHYS & 0 & 0 & 0 & 0 & 0 & 으. & 0 & 0 & 0 & 0 \\
\hline OTHEA & 0 & 0 & 0 & 0 & 0 & 0. & 0 & 01 & (0) & 0 \\
\hline TOTALOTHER & 0 & 0 & 01 & 01 & 0 & 0 & 0 & 01 & 0 & $\overline{0}$ \\
\hline & & & & & & & & & & \\
\hline TOTAL SAMPLE ANALYS & 591129 & 0 & 01 & 01 & 0 & 0] & 590945 & 184 & $0]$ & 591129 \\
\hline
\end{tabular}


EM-263 SUAVEY OF DOE ANALYTICAL CAPACITY AVAILABLE TO EM

Table Nc: MC Sample Analyses by Rad Level and Matrix Type (xxi) Rocky Flats - GL881

\begin{tabular}{|c|c|c|c|c|c|c|c|c|c|c|}
\hline $\begin{array}{l}\text { Rad-Matrix } \\
\text { Samp Anal }\end{array}$ & RO & RO/RI & RT & R2 & $7 \mathrm{R3}$ & $\overline{\mathrm{RA} 4}$ & MI & M2 & $\overline{M B}$ & Total \\
\hline & & & & & & & & & & \\
\hline VOA-GCMS & $\frac{0}{9829}$ & 0 & $\frac{0}{0}$ & $\frac{0}{0}$ & $\frac{0}{0}$ & 0 & $\frac{0}{7372}$ & $\frac{0}{2064}$ & 0: & $\frac{0}{9829}$ \\
\hline SEMI-VOA & 947 & 0 & 0 & 0 & 0 & 0 & 252 & 240 & 455 & 947 \\
\hline PEST & 0 & 0 & 0 & 0 & 0 & 0 & 0 & 0 & 0 & 0 \\
\hline HERB & 0 & 0) & o & 0 & 0 & 0 & \begin{tabular}{l|l}
0 & \\
\end{tabular} & 0 & 0 & 0 \\
\hline PCB & 8037 & D & 0 & 0 & 0 & 0 & 1607 & 4019 & 2411 & 8037 \\
\hline PCB/PEST & 0 & o| & 0 & 0 & 0 & o & 0 & 0 & 0 & 0 \\
\hline OTHER-ORG & 0 & 0. & 0 & 0 & 0 & 0 & of & 0 & a & 0 \\
\hline TOTAL ORGANICS & 18813 & 0 & 01 & 0 & 0 & 0] & 92311 & 6322 & 3260 [ & 18813 \\
\hline & & & & & & & & & & \\
\hline ICP-AES METALS & 8097 & 0 & 0 & 0 & 0 & 0 & 6016 & 486 & 1595 & 8097 \\
\hline ICP-MSMETALS & 1237 & 0 & o! & 0 & 0 & (0) & 247 & 619 & 371 & 1237 \\
\hline GF-AAMETALS & 5079 & 0 & 0 & 0 & 0 & - & 4571 & 356 & 152 & 5079 \\
\hline MERCURY & 17436 & o| & 0 & 0 & 0 & 0] & 1046 & 5231 & 1744 & 17456 \\
\hline CYANIDE & 693 & 0 & 0 & 0 & 0 & 0 & 320 & 347 & 26 & 693 \\
\hline ANIONS/CATIONS & 0 & o & 0 & 0 & 0 & 0 & D & 0 & 0 & 0 \\
\hline WET CHEM & 0 & o & 0 & 0 & 0 & 0 & 0 & 0 & o & 0 \\
\hline OTHER-INORG & 0 & O) & DI & 0 & 0 & 의 & 0 & 0 & 0 & 0 \\
\hline TOTAL INORGANICS & 32542 & 0! & 01 & 0) & 0 & - & 21616 & 7037 & 3889 & 32542 \\
\hline & & & & & & & & & & \\
\hline GROSS AL PHAVBETA & 21197 & 0 & 0 & 0 & 0 & 0 & 11424 & 8016 & 1758 & 21197 \\
\hline ALPHADET-NP237 & 0 & 0) & 0 & 0 & 0 & 묘 & 0 & 0 & 0 & 0 \\
\hline ALPHADET-Pu,Am & 0 & 0 & 0 & 0 & 0 & - & 0 & 0 & 0 & 0 \\
\hline ALPHA DET-Pu & 0. & 0 & 0 & 0 & 0 & 0] & 0 & 0 & (0) & 0 \\
\hline ALPHADET $-U$ & D. & 0 & (0) & 0 & 0 & (0) & 0 & 0 & 0 & $\overline{0}$ \\
\hline ALPHADET-Am & 0 & 0 & 0 & 0 & 0 & 므 & 0 & 0 & (0) & 0 \\
\hline ALPHADET-Np & 0 & 0 & 0 & 0 & 0 & (0) & 0 & 0 & 0. & 0 \\
\hline ALPHADET-Th & 0 & 0 & 0 & 0 & 0 & (0) & D & of & a & (0) \\
\hline ALPHA DET-U,Th,Pu & 0 & 0 & 0 & 0 & 0 & 0 & o & 0 & 묘 & 0 \\
\hline ALPFIA DET-U235 & 0 & 0 & 0) & 0 & 0 & (0) & of & 0 & 0 & 0. \\
\hline ALPHA DET-Pu,U,Am & 3335 & 0) & 0 & 0 & 0 & 0 & 2501 & 834 & 의 & 3335 \\
\hline ALPHA DET-U,Np,Pu,Am & 0 & 0 & 0 & 0 & 0 & 0 & 0 & 0 & 0 & 0 \\
\hline ALPHA DET-Ra226 & of & 0 & 0 & 0 & 0 & 의 & of & 0 & 0 & o \\
\hline ALPHA DET-Ra228 & 0 & 0 & 0| & 0 & 0 & 0. & D) & 0 & 0 & - \\
\hline ALPHA SPEC & 0 & 0 & 0 & 0 & 0 & 예 & 0 & 0 & 0 & 0 \\
\hline GPC-BETA-Pb210 & D. & 0 & 0 & 0 & $\overline{0}$ & a & O) & 0 & 0 & 0 \\
\hline GPC-BETA-SPO0 & 0 & 0 & 0 & O & 0 & a & 0 & 0 & (0) & 0 \\
\hline GPC-BETA-Pm147 & 0) & 0 & D. & 0 & 0 & (0) & 0 & $\overline{0}$ & 0. & 0 \\
\hline GPC-BETA-Se79 & 0 & 0] & 0 & 0 & 0 & a) & 0 & 0 & 0 & 0 \\
\hline LSC-BETA-H3 & 0 & o & 0 & 0 & 0 & 요 & D & 0 & a) & 으 \\
\hline LSC-BETA-Rin222 & 0 & 0 & 0 & 0 & 0 & o & 0 & 0 & a & 0 \\
\hline LSC-BETA- 1129 & 0 & of & 0) & 0 & 0 & - & 0 & 0 & 0. & 0 \\
\hline LSC-BETA-C14 & 0 & 0. & 0 & 0 & 0 & 0 & 0 & 0 & 0 & 0 \\
\hline LSC-BETA-TC99 & 0 & 0 & 0 & 0 & 0 & 0 & 0 & 0 & 0 & 0 \\
\hline LSC-BETA & 0 & 0 & 0 & 0 & 0 & 0 & 01 & 0 & 0 & 이 \\
\hline GAMMA SPEC & D. & 0 & D. & 0 & 0 & 의 & o & of & 0 & 의 \\
\hline OTHER-RAD & 0 & 0 & 0 & 0 & D & - & 0 & 0 & 0 & 0 \\
\hline TOTALAAD & 24532 & (01 & Dा & 0 & $\overline{0}$ & 이 & 13925 & 8850 & 1758 & 24532 \\
\hline & & & & & Z & I & & & & \\
\hline TCLP EXTRACTION & 4286 & 이 & 0 & ㅇ. & 0 & 요 & 0 & 797 & 3489 & 4286 \\
\hline OTHER-PREP & 0 & o & 0 & 0 & 0 & 0 & 0 & 0 & 0 & 0 \\
\hline OTHER-EXPLO & 0 & o & 0 & 0 & 0 & 의 & o & 0 & 의 & 으 \\
\hline OTHER-BIO & 0 & 0 & (0) & 0 & 0 & 0 & 0 & 0 & 요 & 0 \\
\hline OTHER-PHYS & 0 & 0 & 아 & 0 & 0 & 의 & 0 & 0 & of & ㅁ. \\
\hline OTHER & 0 & 0 & 이 & 0 & 0 & 0 & 0 & 01 & 0 & 익 \\
\hline TOTALOTHER & $4 \overline{286}$ & 01 & 01 & 0 & 0 & 0 & 01 & 797 & 3489 & 4286 \\
\hline & & & & & & & & & & \\
\hline TOTAL SAMPLE ANALY & 80173 & ग्र & 01 & $\overline{0}$ & $\overline{0}$ & 0] & 447721 & 3006 & $12394]$ & 80173 \\
\hline
\end{tabular}


EM-263 SURVEY OF DOE ANALYTICAL CAPACTY AVAILABLE TO EM

Table IVc: MC Sample Analyses by Rad Level and Matrix Type (xxi) Rocky Flats - RF PA Lab

\begin{tabular}{|c|c|c|c|c|c|c|c|c|c|c|}
\hline $\begin{array}{l}\text { Rad-Matrix } \\
\text { Samp Anal }\end{array}$ & RO & RO/HI & Ri & $\overline{R 2}$ & $\overline{R B}$ & $\overline{\mathrm{RA}}$ & TMT & $\overline{\mathrm{M} 2}$ & $\overline{\mathrm{MB}}$ & Total \\
\hline & & & & & & & & & & \\
\hline VOA-GC & 0 & 0) & 01 & 0 & 0 & 0) & 0 & 0 & 0 & 0 \\
\hline VOA-GCMS & 0 & 01 & 0 & 0 & 5608 & 0. & 3532 & 1297 & 778 & 5608 \\
\hline SEMI-VOA & 0 & 01 & 0 & 0 & 553 & 0 & 111 & 276 & 166 & 553 \\
\hline PEST & 0 & 0 & 01 & 0 & 0 & o. & 0 & 0 & 0 & 0 \\
\hline HERB & 0 & 0 & 0 & 0 & 0 & 0 & 0 & 0 & 0 & 0 \\
\hline PCB & 0 & 0 & 0 & 0 & 1849 & 0. & 370 & 925 & 555 & 1849 \\
\hline PCB/PEST & 0 & 0 & 0 & 0 & 0 & 0 & 0 & 0 & 0 & 0 \\
\hline OTHER-ORG & 0 & 0 & 01 & 0 & 0 & 0 & 0 & 0 & 0 & 0 \\
\hline TOTAL ORGANICS & 01 & 01 & 01 & 01 & 8009 & O] & 4013 & 2498 & 1499 & 8009 \\
\hline & & & & & & & & & & \\
\hline ICP-AES METALS & 0 & 0 & 01 & 0 & 4857 & 0 & 971 & 2429 & 1457 & 4857 \\
\hline ICP-MSMETALS & 0 & 0 & 01 & 0 & 1135 & 0 & 227 & 568 & 341 & 1135 \\
\hline GF-AAMETALS & 0 & 0 & 0 & 0 & 0 & 0 & 0 & 0 & $\mathbf{0}$ & 0 \\
\hline MERCURY & 0 & 0 & 0 & 0 & 11217 & 0 & 2243 & 5609 & 3365 & 11217 \\
\hline CYANIDE & 0 & 0 & 0 & 0 & 4441 & 0 & 89 & 2132 & 2220 & 4441 \\
\hline ANIONS/CATIONS & 0 & 0 & 01 & 0 & 0 & 0 & 0 & 0 & 0 & 0 \\
\hline WET CHEM & 0 & 0 & 0 & 0 & 0 & 0 & 0 & 0 & - & 으. \\
\hline OTHER-INORG & 01 & 0 & 0 & 0 & 55203 & 0 & 29320 & 13903 & 11981 & 55203 \\
\hline TOTAL INORGANICS & 01 & $0]$ & OI & 0 & 76854 & -0] & 32850 & 24639 & 19364 & 76854 \\
\hline & & & & & & & & & & \\
\hline GROSS ALPHAVBETA & 0 & 0 & 0 & 0 & 7259 & 0 & 4443 & 1536 & 1280 & 7259 \\
\hline ALPHA DET-Np237 & 0 & 0 & 0 & 0 & 0 & 0 & o & 0 & 0 & 0 \\
\hline ALPHADET-Pu,Am & 0 & 0 & 0 & 0 & 0 & 0 & 0 & 0 & 0 & 0 \\
\hline ALPHADET-Pu & 0 & 0 & 0 & 0 & 0 & 0 & 0 & 0 & 0 & 0 \\
\hline ALPHA DET-U & 0 & 0 & 0 & 0 & 0 & 0 & 0 & 0 & of & 0 \\
\hline ALPHADET-Am & 0 & 0 & 0 & 0 & 0 & 0 & 0 & 0 & 0) & 0 \\
\hline ALPHADET-Np & 0 & 0 & 0 & 0 & 0 & 0 & 0 & 0 & 0. & 0 \\
\hline ALPHADET-Th & 0 & 0 & 0 & 0 & 0 & 0 & 0 & 0 & 0 & 0 \\
\hline ALPHA DET $-U, T h, P U$ & 0 & 0 & 0 & 0 & 0 & 0 & 0 & 0 & 0. & $\mathbf{0}$ \\
\hline ALPHADET-U235 & 0 & 0 & 0 & 0 & 0 & 0. & 0 & 0 & 0 & 0 \\
\hline ALPHADET-Pu,U,Am & 0 & 0 & 0 & 0 & 0 & 0. & 0 & 0 & 0 & 0 \\
\hline ALPHA DET-U,Np,Pu,Am & 0 & 0 & 0 & 0 & 0 & 0 & 0 & 0 & 0 & O. \\
\hline ALPHA DET-Ra226 & 0 & 0 & 0 & 0 & 0 & 0 & 0 & 0 & o) & 0] \\
\hline ALPHA DET-Ra228 & 0 & 0 & 0 & 0 & 0 & 0] & 0 & 0 & 0 & $\mathbf{0}$ \\
\hline ALPHA SPEC & 0 & 0 & 0 & 0 & 0 & 0. & 0 & 0 & 0 & 0 \\
\hline GPC-BETA-Pb210 & 0 & 0 & 0 & 0 & 0 & 0 & 0 & 0 & 0 & 0. \\
\hline GPC-BETA-Sr9O & 0 & 0 & 0 & 0 & 0 & 0 & 0 & 0 & 0 & 0] \\
\hline GPC-BETA-Pm147 & 0 & 0 & 0 & 0 & 0 & 0 & 0 & 0 & 0 & 0 \\
\hline GPC-BETA-Se79 & 0 & 0 & 0 & 0 & 0 & 0 & 0 & 0 & 0 & 0 \\
\hline LSC-BEIA-H3 & 0 & 0 & 0 & 0 & $\mathbf{0}$ & 0 & 0 & 0 & 0 & 0] \\
\hline LSC-BETA-Rn222 & 0 & 0 & 0 & 0 & 0 & 0 & 0 & 0 & 0 & 0 \\
\hline LSC-BEIA-I129 & 0 & 0 & 0 & 0 & 0 & 0 & 0 & 0 & 0 & 0] \\
\hline LSC-BETA-C14 & 0 & 0 & 0 & 0 & 0 & 0 & 0 & 0 & 0 & 0) \\
\hline LSC-BETA-TC99 & 0 & 0 & 0 & 0 & 0 & 0 & 0 & 0 & 0 & 0 \\
\hline LSC-BETA & 0 & 0 & 0 & 0 & 0 & 0 & 0 & 0 & 0 & 0 \\
\hline GAMMA SPEC & 0 & 0 & 0 & 0 & 0 & 요 & 0 & 0 & 0 & 0 \\
\hline OTHER-RAD & 0 & 0 & 0 & 0 & 0 & 0 & 0 & 0 & 0 & 0] \\
\hline TOTAL RAD & DI & 0 & 01 & 0 & 7259 & 0] [ & $4 \overline{4431}$ & 1536 & 1280: & 7259 \\
\hline & & & & & & & & & & \\
\hline TCLP EXIRACTION & 0 & 0 & 0 & 0 & 3342 & 0 & 67 & 1604 & 1671 & 3342 \\
\hline OTHER-PREP & 0 & 0 & 0 & 0 & 0 & ㅇ. & 0 & 0 & 0. & 0 \\
\hline OTHER-EXPLO & 0 & D & 0 & 0 & 0 & 0 & 0 & 0 & 0. & 0 \\
\hline OTHER-BIO & 0 & 0 & 0 & 0 & 0 & 0 & 0 & 0 & 0 & 0 \\
\hline OTHER-PHYS & 0 & 0 & 0 & 0 & 0 & 0 & 0 & O & 0. & 0 \\
\hline OTHER & 0 & 0 & 0 & 0 & 0 & 0. & 0 & 0 & 0] & 0 \\
\hline TOTALOTHER & 0 & 0 & Q] & $\underline{0}$ & 3342 & 0. & 671 & 1604 & 1671 & $3 \overline{342}$ \\
\hline & & & & & & & & & & \\
\hline TOTAL SAMPLE ANALYSES & 0 & 0 & 0 & 0 & 95464 & $0]$ & 41373 & 30277 & $23814]$ & 64 \\
\hline
\end{tabular}


EM-263 SURVEY OF DOE ANALYTICAL CAPACTY AVAILABLE TO EM

Table IVc: MC Sample Analyses by Rad Level and Matrix Type (xoiii) WSRC-ALD-CLAB

\begin{tabular}{|c|c|c|c|c|c|c|c|c|c|c|}
\hline $\begin{array}{l}\text { Rad-Matro } \\
\text { Samp Anal }\end{array}$ & $\mathrm{AD}$ & RD/AI & $\overline{\mathrm{BT}}$ & $\mathrm{R2}$ & $\mathrm{AB}$ & RA & M1 & M12 & $\overline{M 3}$ & Total \\
\hline & & & & & & & & & & \\
\hline VOA-GC & 0 & 0 & 0 & 0 & 0 & 0 & 0 & 0 & 0 & 0 \\
\hline VOA-GCMS & 0 & $\overline{0}$ & 0 & 0 & 0 & 0 & 0 & 0 & 0 & 0 \\
\hline SEMI-VOA & 0 & 0 & 0 & 0 & 0 & 0 & 0 & 0 & $\mathbf{0}$ & $\mathbf{0}$ \\
\hline PEST & 0 & 0 & 0 & 0 & 0 & 0. & 0 & 0 & 0 & $\overline{0}$ \\
\hline HEAB & 0 & 0 & 0 & 0 & 0 & 0 & 0 & 0 & $\mathbf{0}$ & 0 \\
\hline PCB & 0 & 0 & 0 & 0 & 0 & 0 & 0 & 0 & 0 & 0 \\
\hline PCB/PEST & 0 & 0 & 0 & 0 & 0 & 0 & 0 & 0 & 0 & 0 \\
\hline OTHER-ORG & 01 & 0 & 0 & 0 & 0 & a) & 0 & 0 & 0 & 0 \\
\hline TOTAL OHGANICS & 01 & 0 & 0 & 0 & 0 & a] & $\overline{0}$ & 01 & 0 & $\overline{0}$ \\
\hline & & & & & & & . & - & 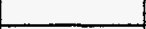 & \\
\hline ICP-AESMETALS & 0 & 0 & 0 & 0 & 0 & 0 & 0 & 0 & 0 & 0 \\
\hline ICP-MSMETALS & 0 & $\overline{0}$ & 0 & 0 & 0 & a. & 0 & 0 & 0 & 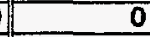 \\
\hline GF-AAMETALS & $\mathbf{0}$ & 0 & 0 & 0 & 0 & 0. & 0 & 0 & 0 & $\mathbf{0}$ \\
\hline MERCURY & 0 & 0 & 0 & 0 & 0 & 3544 & 3544 & 0 & 0 & 3544 \\
\hline CYANIDE & 0 & 0 & 0 & 0 & 0 & 0 & 0 & 0 & 0 & 0 \\
\hline ANIONS/CATIONS & 0 & 0 & 0 & 2 & 0 & 336 & 336 & 0 & 2 & 338 \\
\hline WET CHEM & 0 & 0 & 0 & 0 & 0 & 0 & 0 & 0 & $\mathbf{0}$ & 0 \\
\hline OTHER-INORG & 0 & 0 & 9461 & 896 & 6550 & 10793 & 27700 & 0 & 0 & 27700 \\
\hline TOTAL INORGANICS & 01 & 0 & 9461 & 898 & 6550 & 14672 & 31579 & 01 & 2 & 31581 \\
\hline & & & & & & & & & & \\
\hline GROSS ALPHAVBETA & 0 & 0 & 280030 & 74111 & 0 & 17988 & 372128 & 0 & 0 & 372128 \\
\hline ALPHA DET-Np237 & 0 & 0 & 0 & 0 & 0 & 0 & 0 & 0 & $\overrightarrow{0}$ & 0 \\
\hline ALPHA DET-Pu,Am & 0 & 0 & 0 & 0 & 0 & o] & 0 & 0 & 0 & 0 \\
\hline ALPHADET $-P U$ & 01 & 0 & 0 & 0 & 0 & 0 & 0 & 0 & D. & 0 \\
\hline ALPHADET-U & 0 & 0 & 0 & 0 & 0 & 0. & 0 & 0 & 0 & 0 \\
\hline ALPHA DET-Am & 0 & 0 & 0 & 0 & 0 & 0) & 0 & 0 & 0 & 0 \\
\hline ALPHA DET -Np & 0 & 0 & 0 & 0 & 0 & o. & 0 & 0 & 0 & 0 \\
\hline ALPHADET-Th & 0 & 0 & 0 & 0 & 0 & 0. & 0 & of & D) & 0 \\
\hline ALPHA DET-U,Th,Pu & 0 & 0 & 0 & 01 & 0 & 0. & 0 & 0 & 0 & 0 \\
\hline ALPHADET-U235 & 0 & 0 & 0 & 0 & 0 & 0] & 0 & 0 & 0 & 0 \\
\hline ALPHA DET-Pu,U,Am & 0 & 0 & 0 & 0 & 0 & 0 & 0 & 0 & 0 & 0 \\
\hline ALPHA DET-U,Np,Pu,Am & 0 & 0 & 0 & 0) & 0 & 0 & 0 & D) & $\mathbf{0}$ & 0 \\
\hline ALPHA DET-Ra226 & 0 & 0 & 0 & 0 & 0 & 0] & o) & 0 & 0 & 0 \\
\hline ALPHA DET-Ra228 & 0 & 0 & 0 & 0 & 0 & 0] & 0 & 0 & $\overline{0}$ & $\overline{0}$ \\
\hline ALPHA SPEC & 0 & 0 & 111490 & 0 & 0 & 0] & 111490 & 0 & 0 & 111490 \\
\hline GPC-BETA-Pb210 & 0 & 0 & 0 & 0 & 0 & 0 & 0 & 0 & $\mathbf{0}$ & 0 \\
\hline GPC-BEIA-Sr90 & 0 & 0 & 0 & 0 & 0 & a) & 0 & 0 & 0 & 0 \\
\hline GPC-BETA-Pm147 & 0 & 0 & 0 & 0 & 0 & 0 & 0 & 0 & 0 & 0 \\
\hline GPC-BETA-Se79 & 0 & 0 & 0 & 0 & 0 & 0. & 0 & 0 & 0 & 0 \\
\hline LSC-BETA-H3 & 0 & 0 & 0 & 0 & 0 & 0 & 0 & 0 & 0 & 0 \\
\hline LSC-BETA-Rin222 & 0 & 0 & 0 & 0 & 0 & 0) & 0 & 0 & 0 & 0 \\
\hline LSC-BETA-1129 & 0 & 0 & 0 & 0 & 0 & 0 & 0 & 0 & 0 & 0 \\
\hline LSC-BETA-C14 & 0 & 0 & 0 & 0 & 0 & 0] & O) & 0 & 0 & 0 \\
\hline LSC-BEIA-TC99 & 0 & 0 & 0 & 0 & 0 & 0 & 0 & 0 & 0 & 0 \\
\hline LSC-BETA & 0 & $\mathbf{0}$ & 0 & 01 & 0 & a) & 0 & 0 & 0 & 0 \\
\hline GAMMA SPEC & 0 & 0 & 111490 & 81411 & 0 & 20238 & 213139 & 0 & 0 & 213139 \\
\hline OTHER-RAD & 0 & 0 & 0 & 0 & 0 & 0 & 0 & 01 & 0 & 0 \\
\hline TOTAL RAD & o] & 0 & 503010 & 155522 & 01 & 38225 & 696757 & 01 & $\overline{0}$ & 696757 \\
\hline & & & & & & & & & & \\
\hline TCLP EXTRACTION & 0 & 0 & 0 & 0 & 0 & 0 & 0 & $\mathbf{0}$ & 0 & 0 \\
\hline OTHER-PREP & 0 & 0 & 0 & 0 & 0 & 0 & 0 & 0 & 0 & $\mathbf{0}$ \\
\hline OTHER-EXPLO & 0 & 0 & 0 & 0 & 0 & 0 & 0 & 0 & 0 & 0 \\
\hline OTHER-BIO & 0 & 0 & 0 & 0 & 0 & 0 & 0 & 01 & 0 & $\overline{\mathbf{0}}$ \\
\hline OTHER-PHYS & 01 & 0 & 0 & 0 & 0 & 0 & 0 & 0 & 0 & $\mathbf{0}$ \\
\hline OTHEA & 0 & 0 & 0 & 0 & 0 & 0 & 0 & 01 & 0 & $\underline{0}$ \\
\hline TOTAL OTHER & 0 & 0 & 0 & 01 & 0 & Q0 & 0] & 0 & 0 & $\overline{0}$ \\
\hline & & & & & & & & 1 & 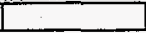 & \\
\hline TOTAL SAMPLEANALYSES & $\overline{0}$ & $\overline{0}$ & 512471 & 156420 & 6550 & 52898 & 728337 & OI & 2 & 28338 \\
\hline
\end{tabular}


EM-263 SUAVEY OF DOE ANALYTICAL CAPACITY AVAILABLE TO EM

Table IVc: MC Sample Analyses by Rad Level and Matrix Type (xxiv) WSRC-ALD-320M

\begin{tabular}{|c|c|c|c|c|c|c|c|c|c|c|}
\hline $\begin{array}{l}\text { Rad-Matrix } \\
\text { Samp Anal }\end{array}$ & RO & RO/R1 & $\overline{\text { R1 }}$ & $\overline{R 2}$ & $\overline{\mathrm{F} \times \mathbf{3}}$ & $\overline{R 4}$ & $\overline{M T}$ & $\overline{\mathrm{ML}}$ & $\overline{\mathrm{M} 3}$ & Total \\
\hline VOA-GC & 0 & 0 & 0 & 0 & 0 & 0 & 01 & 01 & & \\
\hline VOA-GCMS & 0 & 0 & 0 & 0 & 0 & 0 & 0 & 0 & $\frac{0}{0}$ & $\frac{0}{0}$ \\
\hline SEMI-VOA & 0 & 0 & 0 & 0 & 01 & 0 & of & 0 & ôt & 0 \\
\hline PEST & 0 & 0 & 0 & 0 & 0 & 0 & 0 & 0 & 0 & $\overline{0}$ \\
\hline HERB & (0) & 0 & 0 & 0 & 0 & 에 & 0 & 0 & (0) & 0 \\
\hline PCB & 0 & $\overline{0}$ & 0 & 0 & 0 & 0] & 0 & 0 & - & $\overline{0}$ \\
\hline PCB/PEST & 0 & 0 & 0 & 0 & 0 & 0] & 0 & 0 & o] & 0 \\
\hline OTHER-ORG & 0 & 0 & 0 & 0 & 0 & 0 & 0 & o) & -0] & 0 \\
\hline TOTAL ORGANICS & 0 & 0 & 0 & 0 & 이 & 0 & 01 & 01 & 0] & $\overline{0}$ \\
\hline & & & & & & & 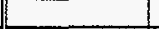 & 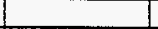 & & \\
\hline ICP-AES METALS & 0 & 0 & 0 & D. & of & (0) & 0 & 0 & 0 & $\overline{0}$ \\
\hline ICP-MSMETALS & 0 & 0 & O & o & 0 & 0. & 0 & 0 & o. & 0 \\
\hline GF-AAMETALS & 0 & 0 & O) & 0 & 0 & 0] & 0 & 0 & 0 & 0 \\
\hline MERCURY & 0 & 0 & 0 & of & 0 & o & 0 & 0 & o. & 0 \\
\hline CYANIDE & 0 & 0 & O & 0 & 0 & 0. & 0 & of & D] & 0 \\
\hline ANIONS/CATIONS & 0 & 0 & 0 & 0 & 0 & 0. & 0 & 0 & D. & $\overline{0}$ \\
\hline WET CHEM & 0 & 0 & 0 & 0 & 0 & 요 & 0 & 0 & 0. & $\overline{0}$ \\
\hline OTHER-INORG & 0 & 0 & 0 & O) & 0. & 0. & of & 0 & 0] & 0 \\
\hline TOTALINOAGANICS & 0 & 0 & 0 & 01 & 01 & 0[ & 0 & 01 & 0] [ & $\overline{0}$ \\
\hline & & & & & & & 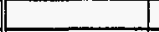 & & & \\
\hline GROSS ALPHA/BETA & 0 & 0 & 0 & 0 & 이 & 묘 & 0 & of & 0] & 0 \\
\hline ALPHA DET-Np237 & $\overline{0}$ & 0 & 0 & 0 & 0 & 0. & 0 & 0 & 0] & 0 \\
\hline ALPHA DET-Pu,Am & 0 & 0 & 0 & 0 & 아 & 0. & 0 & 0 & 0] & 0 \\
\hline ALPHADET-Pu & 0 & 0 & 0 & (0) & 01 & 0. & 0 & 0| & 0] & 0 \\
\hline ALPHA DET - U & 0 & 0 & 0 & 0 & 0) & 여 & 0 & 0 & 0] & $\overline{0}$ \\
\hline ALPHADET-Am & 0 & 0 & 0 & o. & 0 & 0 & \begin{tabular}{l|l}
0 & \\
\end{tabular} & 0 & 0 & 0 \\
\hline ALPHA DET-NP & 0 & 0 & o. & 0 & of & 0 & 0 & 0 & 0 & $\overline{0}$ \\
\hline ALPHA DET-Th & 0 & 0 & 0 & 0 & 0) & 0. & \begin{tabular}{l|l}
0 & \\
\end{tabular} & 0 & 0 & 0 \\
\hline ALPHADET-U,Th,PU & 0 & 0 & 0. & of & 0 & 0 & 0 & 0 & 0] & $\overline{0}$ \\
\hline ALPHADET-U235 & 0 & 0 & 0 & of & of & 0. & 0 & 0 & 0] & $\overline{0}$ \\
\hline ALPHA DET - Pu,U,Am & 0 & 0 & $\overline{0}$ & 0 & 0 & 0] & 0 & 0 & o. & $\overline{0}$ \\
\hline ALPHA DET - U,Np,Pu,Am & 0 & 0 & 0 & of & of & 0 & 0 & 0 & 묘 & $\overline{0}$ \\
\hline ALPHA DET-Ra226 & 0 & 0 & 0 & 0 & 0 & o & 0 & 0 & 미 & $\overline{0}$ \\
\hline ALPHA DET-Ra228 & 0 & 0 & o. & 0 & O) & 묘 & 0 & 0 & 묘 & $\overline{0}$ \\
\hline ALPHA SPEC & 0 & $\overline{0}$ & 0 & 0 & 0 & 0 & 0 & 0 & o & $\overline{0}$ \\
\hline GPC-BETA-Pb210 & 0 & $\overline{0}$ & 0 & 0 & 0 & ㅇ․ & 0 & 0 & 매 & $\mathbf{0}$ \\
\hline GPC-BETA-Sr90 & 0 & $\overline{0}$ & 0 & 0 & O & 요 & 0 & 0| & 애 & 0 \\
\hline GPC-BETA-Pm147 & 0 & $\mathbf{0}$ & 0 & 0 & 0 & 0 & 0 & 0 & 0 & 0 \\
\hline GPC-BETA-Se79 & 0 & $\overline{0}$ & 0 & 0 & 0 & 0 & 0 & 0 & 0 & $\overline{0}$ \\
\hline LSC-BETA-H3 & 0 & $\mathbf{0}$ & 0 & 0 & 0 & 0 & 0 & 0 & o & $\overline{0}$ \\
\hline LSC-BETA-Rn 222 & 0 & $\overline{0}$ & o & 0 & 0 & 요 & 0 & 0 & 으. & 0 \\
\hline LSC-8ETA-1129 & o & 0 & o & 0 & 0 & 0) & 0 & 0 & 요 & $\overline{0}$ \\
\hline LSC-BETA-C14 & $\overline{0}$ & 0 & 0 & 0 & 0 & 0 & 0 & 0 & 의 & 0 \\
\hline LSC-BETA-TC99 & 0 & 0 & 0 & 0 & 0 & 이 & 0 & 0 & 요 & 0 \\
\hline LSC-BETA & 0 & 0 & 0 & 0 & 0 & 이 & 0 & 0 & 0. & 0 \\
\hline GAMMA SPEC & 0 & 0 & 0 & 0 & 0 & 0 & 0 & 0 & 0 & $\overline{0}$ \\
\hline OTHER-RAD & 0 & 0 & 0 & 0 & 0 & 0 & $0 \mid$ & 0 & (0) & 0 \\
\hline TOTAL RAD & o & $\overline{0}$ & 0] & 01 & 01 & 0] & 01 & 01 & 이 & 0 \\
\hline & & & & & & & & & & \\
\hline TCLP EXIRACTION & 0 & 0 & 0 & 0 & 0 & 0 & 0 & 0) & 의 & 0 \\
\hline OTHER-PREP & $\overline{0}$ & 0 & 0 & 0 & 0 & 0 & 0 & 0 & 므 & 0 \\
\hline OTHER-EXPLO & $\overline{0}$ & 0 & 0 & 0 & 0 & 0 & 0 & 0 & 요 & 0 \\
\hline OTHER-BIO & $\overline{0}$ & 0 & 0 & 0 & 0 & 0 & 0 & 이 & 요 & 0 \\
\hline OTHER-PHYS & $\overline{0}$ & $\overline{0}$ & 0 & 0 & 0 & 0 & 0 & 0 & 요 & $\overline{0}$ \\
\hline OTHER & $\overline{0}$ & 0 & 0 & 0 & 0 & 의 & 0 & 0 & - & 0 \\
\hline TOTAL OTHER & 0 & 0 & 0 & 01 & 0) & 0 & 01 & 0 & 0 & $\underline{0}$ \\
\hline & & & _ & I & 1 & $\square$ & 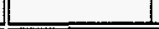 & ] & 1 & 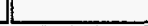 \\
\hline TOTAL SAMPLE ANALYSES & 0 & 0 & 0 & 01 & 01 & 0 & 01 & 0 & 0 & 0 \\
\hline
\end{tabular}


EM-263 SURVEY OF DOE ANALYTICAL CAPACTY AVAILABLE TO EM

Table IVc: MC Sample Analyses by Rad Level and Matrix Type (xxv) WSRC-ALD Water

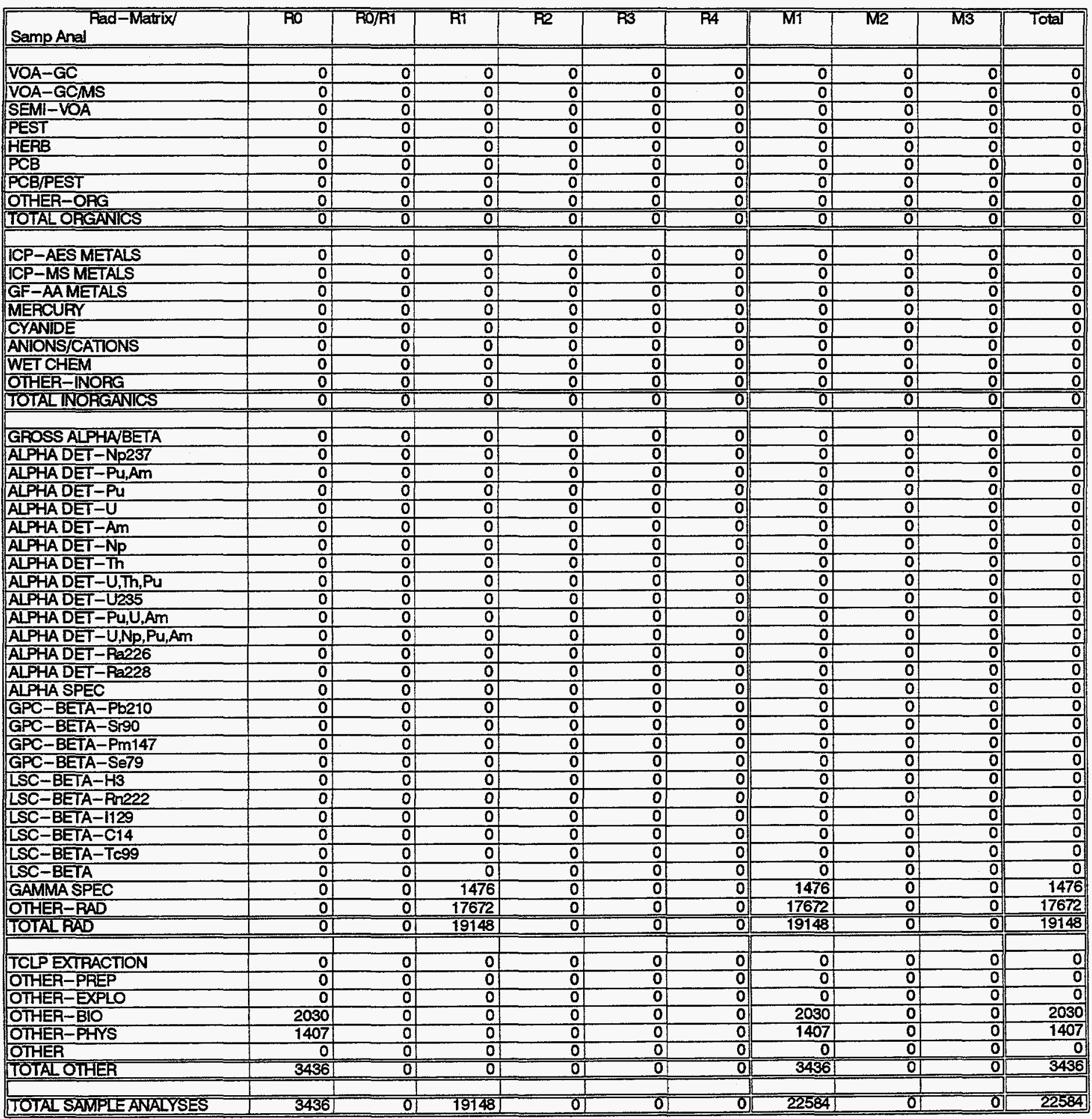


EM-263 SURVEY OF DOE ANALYTICAL CAPACITY AVAILABLE TO EM

Table IVc: MC Sample Analyses by Rad Level and Matrix Type (xovi) WSRC-ALD-EIF

\begin{tabular}{|c|c|c|c|c|c|c|c|c|c|c|}
\hline $\begin{array}{l}\text { Rad-Matrix } \\
\text { Samp Anal }\end{array}$ & 70 & Ro/RI & R1 & 72 & $\mathrm{R3}$ & 74 & $\mathrm{MI}$ & $\mathrm{M} 2$ & $\overline{M 3}$ & Total \\
\hline & & & & & & & & & & \\
\hline VOA-GC & 0 & 0 & 0 & 0 & 0 & 0 & 0 & 0 & 0 & 0 \\
\hline VOA-GCMS & 0 & 0 & 0 & 0 & 0 & 0 & 0 & 0 & 0 & 0 \\
\hline SEMI-VOA & 0 & 0 & 0 & 0 & 0 & 0 & 0 & 0 & $\mathbf{0}$ & 0 \\
\hline PEST & 0 & 0 & 0 & 0 & 0 & 0 & 0 & 0 & 0 & 0 \\
\hline HERB & 0 & 0 & 0 & 0 & 0 & 0 & 0 & 0 & 0 & 0 \\
\hline PCB & 0 & 0 & 0 & o & 0 & 0 & 0 & 0 & 0 & 0 \\
\hline PCB/PEST & 0 & 0 & 0 & 0 & 0 & 0 & 0 & 0 & 0 & 0 \\
\hline OTHER-ORG & 0 & 01 & 0 & 0 & 0 & 0 & 0 & 0 & 0 & 0 \\
\hline TOTAL ORGANICS & 0 & OI & 0 & 01 & 0 & 0] & 01 & 01 & o] [ & $\overline{0}$ \\
\hline & & & & & & 7 & . & 7 & 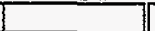 & \\
\hline ICP-AES METALS & 0 & 0 & 0 & 0 & 0 & 0 & 0 & 0 & 0 & 0 \\
\hline ICP-MSMETALS & 0 & 0 & 0 & 0 & 0 & 0 & 0 & 0 & 0 & 0 \\
\hline GF-AAMETALS & 0 & 0 & 0 & 0 & 0 & 0 & 0 & 0 & 0 & 0 \\
\hline MERCURY & 0 & 0 & 0 & 0 & 0 & o. & 0 & 0 & 0 & 0 \\
\hline CYANIDE & 0 & 0 & 0 & 0 & 0 & 0. & 0 & 0 & 0 & 0 \\
\hline ANIONS/CATIONS & 0 & 0 & 0 & 0 & 0 & 0 & 0 & 0 & 0 & 0 \\
\hline WET CHEM & 0 & 0 & 0 & 0 & 0 & 0 & 0 & 0 & 0 & 0 \\
\hline OTHER-INORG & 0 & 0 & 0 & 0 & 0 & o. & 0 & 0 & 0 & 0 \\
\hline TOTAL INORGANICS & 01 & O) & 0 & 0 & 0 & 0. & 01 & ठ & 0] & 0 \\
\hline & & & & & & & & & & \\
\hline GROSS ALPHA/BETA & 0 & 0 & 0 & 0 & 0 & o & 0 & 0 & 0 & 0 \\
\hline ALPHA DET-Np237 & 0 & 0! & 0 & 0 & 0 & 0 & 0 & 0 & 0 & 0 \\
\hline ALPHA DET-PU,Am & 0 & 0 & $\mathbf{0}$ & 0 & 0 & 0 & 0 & 0 & 0 & 0 \\
\hline ALPHA DET-PU & 0 & 0 & 0 & 0 & 0 & 0 & 0 & 0 & 0 & 0 \\
\hline ALPHADET-U & 0 & 0 & 0 & 0 & 0 & 0 & 0 & 0 & 0 & 0 \\
\hline ALPHA DET-Am & 0 & 0 & 0 & 0 & 0 & 0 & 0 & 0 & 0 & 0 \\
\hline ALPHADET-NP & $\mathbf{0}$ & 0 & 0 & 0 & 0 & o) & 0 & 0 & 0. & 0 \\
\hline ALPHADET-Th & 0 & 0 & 01 & 0 & 0 & 0. & 0 & 0 & 0 & 0 \\
\hline ALPHA DET-U,Th,PU & 0 & 0 & 0 & 0 & 0 & 0 & 0 & 0 & 0 & 0 \\
\hline ALPHA DET-U235 & 0 & 0 & 0 & 0 & 0 & 0 & 0 & 0 & 0 & 0 \\
\hline ALPHA DET-PU,U,Am & 0 & 0 & 0 & 0 & 0 & 0 & 0 & 0 & 0 & 0 \\
\hline ALPHA DET-U,Np,Pu,Am & 0 & 0 & 0 & 0 & 0 & 0 & 0 & 0 & 0. & 0 \\
\hline ALPHA DET-Ra226 & 0 & o & 0 & 0 & 0 & 0. & 0 & 0 & o & 0 \\
\hline ALPHA DET-Ra228 & 0 & 0 & 0 & 0 & 0) & 0 & 0 & 0 & 0 & 0 \\
\hline ALPHA SPEC & 0 & 0 & 0 & 0 & 0 & 0. & 0 & 0 & 0 & 0 \\
\hline GPC-BETA-Pb210 & 0 & 0 & 0 & 0 & 0 & 0 & 0 & 0 & o. & 0 \\
\hline GPC-BETA-Sr90 & 0 & 0 & 0 & 0 & 0 & 0 & 0 & 0 & 0 & 0 \\
\hline GPC-BETA-Pm147 & 0 & 0 & 0 & 0 & 0 & 0 & 0 & 0 & 0. & 0 \\
\hline GPC-BETA-Se79 & 0 & 0 & 0 & 0 & 0 & 0. & 0 & 0 & 0. & 0 \\
\hline LSC-BETA-H3 & 0 & 0 & 0 & 0 & 0 & o. & 0 & 0 & 0 & 0 \\
\hline LSC-BETA-Pr222 & 0 & 0 & 0 & 0 & 0 & 0 & 0 & 0 & 0. & 0 \\
\hline LSC-BETA- 1129 & 0 & 0 & 0 & 0 & 0 & 0 & 0 & 0 & o: & 0 \\
\hline LSC-BETA-C14 & 0 & 0 & 0 & 0 & 0 & 0 & 0 & 0 & 0 & 0 \\
\hline LSC-BETA-Tc99 & 0 & 0 & 0 & 0 & 0 & 0 & 0 & 0 & 0 & 0 \\
\hline LSC-BETA & 0 & 0 & 0 & 0 & 0 & 0 & 0 & 0 & 0 & 0 \\
\hline GAMMA SPEC & 0 & 0 & 0 & 0 & 0 & 0 & 0 & 0 & 0 & 0 \\
\hline OTHER-RAD & 0 & 0 & 0 & 0 & 0 & 0 & 0 & 0 & 0 & 0 \\
\hline TOTALRAO & 0 & 01 & 0 & $\overline{0}$ & 0 & 0 & 0 & 0 & 0 & 0 \\
\hline & & & & & & & $\overline{-1}$ & & 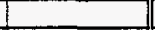 & \\
\hline TCLP EXTPACTION & 0 & 0 & 0 & 0 & 0 & 0 & 0 & 0 & 0 & 0 \\
\hline OTHER-PREP & 0 & 0 & 0 & 0 & 0 & 0 & 0 & 0 & 0 & 0 \\
\hline OTHEA-EXPLO & 0 & 0 & 0 & 0 & 0 & 0 & 0 & 0 & 0. & 0 \\
\hline OTHER-BIO & 0 & 0 & 0 & 0 & 0 & 0 & 0 & 0 & 0 & 0 \\
\hline OTHER-PHYS & 0 & 0 & 0 & 0 & 0 & D & 0 & 0 & 0 & 0 \\
\hline OTHER & 0 & 0 & 0 & 0 & 0 & 0 & 0 & 0 & 0 & 0 \\
\hline TOTALOTHER & 0 & 0 & 0 & 0 & 0 & 0 & 0 & 0 & 0 & 0 \\
\hline$=$ & & & & & $=$ & 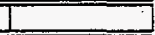 & 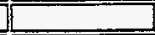 & 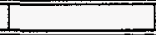 & -1 & \\
\hline TOTAL SAMPLE ANALYSES & $\overline{0}$ & 0 & 0 & 0 & 0 & 0 & 0 & 0 & 0 & 0 \\
\hline
\end{tabular}


EM-263 SURVEY OF DOE ANALYTICAL CAPACTTY AVAILABLE TO EM

Table IV: MC Sample Analyses by Rad Level and Matrix Type (xxvii) WSRC-ALD-Tritium

\begin{tabular}{|c|c|c|c|c|c|c|c|c|c|c|}
\hline $\begin{array}{l}\text { Rad-Matrix } \\
\text { Samp Anal }\end{array}$ & Ro & RO/RI & R1 & $R 2$ & $\overline{\mathrm{R} 3}$ & $\overline{R 4}$ & TM1 & $\overline{\mathrm{M} 2}$ & M3 & Total \\
\hline & & & & & & & & & & \\
\hline $\begin{array}{l}\text { VOA-GC } \\
\text { VOA-GCMS }\end{array}$ & 0 & 0 & 이 & 0 & 이 & 0 & 0 & 0 & 의 & 0 \\
\hline VOA-GCMS & 0 & of & 0 & 0 & 이 & 0 & 0 & 0 & o. & 0 \\
\hline SEMI-VOA & 0 & 0 & 0 & 0 & 0 & 0 & 0 & 0 & 0 & 0 \\
\hline PEST & 0 & 우 & 0 & 0 & of & 0. & 0 & of & 의 & 0 \\
\hline HERB & 0 & 可 & 0 & of & 0 & 0 & 0 & 0 & 이 & 0 \\
\hline PCB & 0 & 0 & 0. & 0 & 01 & 0 & 0 & 0 & D. & 0 \\
\hline PCB/PEST & 0 & 0 & 0 & 0 & 0 & 0 & 0 & Of & 의 & 0 \\
\hline OTHER-ORG & 0 & 0 & DI & 0 & 0 & 0 & 0 & 0 & 0 & 0 \\
\hline TOTAL ORGANICS & 0 & 0 & 0 & 0 & (0) & 0. & $\overline{0}$ & 0 & 0 & $\overline{0}$ \\
\hline & & & & & & & & I & & \\
\hline ICP-AES METALS & 0 & 0 & 0 & 0 & 0 & 0. & 0 & 0 & 0. & $\overline{0}$ \\
\hline ICP-MS METALS & 0 & of & 0 & 0 & 0 & 0. & 0 & 0 & 0 & 0 \\
\hline GF-AAMETALS & 0 & of & O & 0 & 0 & 0] & 0 & 0 & 의 & 0 \\
\hline MERCURY & 0 & 0 & 0 & 0 & 01 & 0 & 0 & 0 & 0. & 0 \\
\hline CYANIDE & 0 & D & 0 & 0 & 0 & 0 & 0 & o) & 0. & 0 \\
\hline ANIONS/CATIONS & 0 & 0) & 이 & 0 & 0 & 0 - & 0 & 0 & 이 & 0 \\
\hline WET CHEM & 0 & 0 & 0 & 0 & 0 & 0. & 0 & O) & 0 & 0 \\
\hline OTHER-INORG & 0 & 0 & 0 & 0 & 0 & 0] & 0 & 0 & a & 0 \\
\hline TOTAL INORGANICS & 0 & 01 & 01 & 0 & 01 & 0] & 0 & 0] & (0) & 0 \\
\hline & & & & & & & & & & \\
\hline GROSS ALPHAVBTA & 0 & 요 & 이 & 0 & 0 & 0. & 0 & 0 & 0 & 0 \\
\hline ALPHA DET-Np237 & 0 & o) & 0 & 0 & 0 & 0 & 0 & 0 & 0 & 0 \\
\hline ALPHA DET - Pu,Am & 0 & o & 0 & 0 & 0 & 0 & 0 & 0 & 0 & 0 \\
\hline ALPHADET-Pu & 0 & o) & 0 & 0 & 0 & 0 & $\overline{0}$ & 0 & 0 & $\mathbf{0}$ \\
\hline ALPHA DET - U & 0 & 0. & 0 & 0 & 0 & 0 & 0 & 0 & 0 & 0 \\
\hline ALPHA DET-Am & 0 & 0 & 0 & 0 & 이 & 0 & 0 & 0 & 0 & 0 \\
\hline ALPHA DET-Np & 0 & O & 0 & 0 & 0 & 0. & 0 & 0 & 0 & 0 \\
\hline ALPHA DET-Th & 0 & O & O & 0 & of & 0 . & 0 & 0 & 0 & 0 \\
\hline ALPHA DET-U,Th,PU & 0 & of & 0 & 0 & 0 & 0 & 0 & 0 & of & 0 \\
\hline ALPHA DET-U235 & 0 & 0 & 0 & 0 & of & 0. & 0 & 0 & 요 & 0 \\
\hline ALPHA DET-Pu,U,Am & 0 & 의 & O. & 0 & 0 & o. & 0 & 이 & 은 & 0 \\
\hline ALPHA DET-U,No,Pu,Am & 0 & 0 & 0 & 0 & 0 & 0 & 0 & 0 & 0. & $\overline{0}$ \\
\hline ALPHA DET-Ra226 & 0 & 0 & 0 & 0 & 0 & 0. & 0 & 0 & $\overline{0}$ & $\overline{0}$ \\
\hline ALPHA DET-Ra228 & 0 & of & 0 & 0 & 0 & 0] & 0 & 0 & 0 & 0 \\
\hline ALPHA SPEC & 0 & 0 & 이 & 0 & 0 & 0 & 0 & 0 & 0 & 0 \\
\hline GPC-BETA-Pb210 & 0 & O) & 0 & 0 & 0 & 0 & 0 & 0 & o & 0 \\
\hline GPC-BETA-Sr90 & 0 & of & 0 & 0 & 0 & 0 & 0 & 0 & 0 & 0 \\
\hline GPC-BETA-Pm147 & 0 & 0 & 0 & 0 & 0 & 0. & 0 & 0 & of & 0 \\
\hline GPC-BETA-Se79 & 0 & 0 & 이 & 0 & 0 & 0 & 0 & 0 & 0 & 0 \\
\hline LSC-BETA-H3 & 0 & 0 & of & 0 & 0 & 0 & 0 & 0 & 0 & 0 \\
\hline LSC-BETA-PIn222 & 0 & 0 & 0 & 0 & 0 & 0 & 0 & 0 & 0 & 0 \\
\hline LSC-BETA- 1129 & 0 & 0 & 0 & 0 & 0 & 0 & 0 & 0 & ㅇ. & 0 \\
\hline LSC-BETA-C14 & 0 & 0) & of & 0 & 0 & 0 & 0 & 0 & (0) & 0 \\
\hline LSC-BETA-TC99 & 0 & 0. & 0 & 0 & 0 & 0 & 0 & 0 & 0 & 0 \\
\hline LSC-BETA & 0 & 0 & 2566 & 0 & of & 0 & 2566 & 0 & 0 & 2566 \\
\hline GAMMA SPEC & 0 & 0 & 0 & 0. & 0. & 0 & 0 & 0 & 0 & 0 \\
\hline OTHER-RAD & 0 & 0 & of & 0 & of & O. & 0 & 0 & 0 & 0 \\
\hline TOTAL PAD & 0 & 01 & 2566 & 01 & 01 & 0] & 2566 & 0] & 0 & 2566 \\
\hline & & & & & & & & 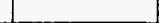 & & \\
\hline TCLP EXTRACTION & 0 & 0 & o) & 0 & 0 & 0 & 0 & 0 & 0 & 0 \\
\hline OTHER-PREP & 0 & 이 & 0 & 0 & 이 & 0 & D & 0 & 0 & 0 \\
\hline OTHER-EXPLO & 0 & O) & 0 & 0 & 0 & o. & 0 & 0 & 0 & 0 \\
\hline OTHEA-BIO & 0 & 0 & 0 & 0 & 0 & 0. & 0 & 0 & 0 & 0 \\
\hline OTHER-PHYS & 0 & 0) & 0 & 0 & 0 & 0 & 0 & 0 & 요 & 0 \\
\hline OTHEA & 0 & 0 & 0 & 0 & 0 & 0 & 0 & 0 & 0 & 0 \\
\hline TOTAL OTHER & 0 & 0 & 0 & 0 & DI & 0] & 0 & 0 & 이 & 0 \\
\hline & & & & & & & & & & IL \\
\hline TOTAL SAMPLE ANAL & 0 & 0 & 2566 & 0 & 0 & O] & 2566 & 0 & 0 & 2566 \\
\hline
\end{tabular}


EM-263 SURVEY OF DOE ANALYTICAL CAPACTY AVAILABLE TO EM

Table IVc: MC Sample Analyses by Rad Level and Matrix Type (xxvii) WSRC-ALD-ITP

\begin{tabular}{|c|c|c|c|c|c|c|c|c|c|c|}
\hline $\begin{array}{l}\text { Rad-Matrix } \\
\text { Samp Anal }\end{array}$ & FO & RO/RI & RI & 72 & $\overline{R 3}$ & $\overline{\mathrm{R4}}$ & M1 & $\overline{M 2}$ & $\overline{1 M 3}$ & Total \\
\hline & & & & & & & & & & \\
\hline VOA-GC & 0 & 0 & 0 & 0 & 0 & 0 & 0 & o & o & 0 \\
\hline VOA-GCMS & 0 & 0 & 0 & 0 & 0 & 0 & 0 & 0 & 0 & 0 \\
\hline SEMI-VOA & 0 & 0 & 0 & 0 & 0 & 0 & 0 & 0 & 0 & 0 \\
\hline PEST & 0 & 0 & 0 & 0 & 0 & o & 0 & 0 & 0 & 0 \\
\hline HERB & 0 & 0 & 0 & 0 & 0 & o & 0 & 0 & 0 & 0 \\
\hline$\overline{\mathrm{CB} B}$ & 0 & $\mathbf{0}$ & 0 & 0 & 0 & 0 & 0 & 0 & 0 & 0 \\
\hline PCB/PEST & 0 & 0 & 0 & 0 & 0 & 0 & 0 & 0 & 0 & 0 \\
\hline OTHER-ORG & 0 & 0 & 0 & 0 & 0 & 0 & 01 & 0 & 0 & 0 \\
\hline TOTALORGANICS & 0 & 01 & 01 & 0 & 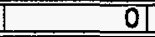 & 0 & 01 & 01 & 0 & 0 \\
\hline & & & 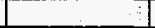 & & & & & & 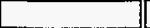 & \\
\hline ICP-AES MEIALS & 0 & 0 & 0 & 0 & 0 & 0 & 0 & 0 & 0 & 0 \\
\hline ICP-MSMETALS & 0 & D & 0 & 0 & 0 & o. & 0 & 0 & 0 & 0 \\
\hline GF-AAMETALS & 0 & 0 & 0 & 0 & 0 & 0 & 0 & 0 & o & 0 \\
\hline MERCURY & 0 & 0 & 0 & 0 & 0 & 0 & 0 & 0 & 0. & 0 \\
\hline CYANIDE & 0 & o) & 0 & 0 & 0 & 0 & 0 & 0 & 0 & 0 \\
\hline ANIONS/CATIONS & 0 & 0 & 0 & 0 & 0 & 0 & 0 & 0 & 0 & 0 \\
\hline WET CHEM & 0 & 0 & 0 & 0 & 0 & 0 & 0 & 0 & 0 & 0 \\
\hline OTHER-INORG & 0 & 01 & 01 & 0 & 0 & of & 0 & 0 & 0 & 0 \\
\hline TOTALINOHGANCS & D & OI & Oा & 01 & 01 & Ol & OI & 01 & O] & 0 \\
\hline & & & & & & & & & & \\
\hline GROSS ALPHABBETA & 0 & 0 & 0 & 0 & 0 & 0 & 0 & 0 & 0) & O \\
\hline ALPHA DET-Np237 & 0 & 0 & 0 & 0 & 0 & 0 & 0 & 0 & o] & 0 \\
\hline ALPHA DET-Pu,Am & 0 & 0 & 0 & 0 & 0 & 0 & 0 & 0 & 0 & 0 \\
\hline ALPHA DET-PU & 0 & 0 & 0 & 0 & 0) & 0 & 0 & 0 & o: & 0 \\
\hline ALPHA DET-U & 0 & 0 & 0 & 0 & 0 & 0 & 0 & 0 & o] & 0 \\
\hline ALPHA DET-AM & 0 & 0 & 0 & 0 & 0 & 0 & 0 & 0 & 0 & 0 \\
\hline ALPHADET-NP & 0 & $\mathbf{0}$ & 0. & 0 & 0 & 0 & 0 & 0 & o & 0 \\
\hline ALPHA DET-Th & 0 & 0 & 0 & 0 & 0 & 0 & 0 & 0 & 0 & 0 \\
\hline ALPHA DET-U,Th,PU & 0 & 0 & 0 & 0 & 0 & 0 & 0 & 0 & 0 & 0 \\
\hline ALPHA DET-U235 & 0 & 0 & 0 & 0 & 0 & 0 & 0 & 0 & 0 & 0 \\
\hline ALPHA DET-PU,U,Am & 0 & 0 & 0 & 0 & 0 & 0 & 0 & 0 & 0 & 0 \\
\hline ALPHA DET-U,Np,Pu,Am & $\mathbf{0}$ & 0 & 0 & 0 & 0 & 0 & 0 & 0 & o. & 0 \\
\hline ALPHADET-Ra226 & 0 & 0 & 0 & 0 & 0 & 0 & 0 & 0 & 0 & $\mathbf{0}$ \\
\hline ALPHA DET-Ra228 & 0 & 0 & 01 & 0 & 0 & 0 & 0 & 0 & 0 & 0 \\
\hline ALPHA SPEC & 0 & 0 & 0 & 0 & 0 & 0 & 0 & 0 & D. & 0 \\
\hline GPC-BETA-Pb210 & 0 & 0 & 0 & 0 & 0 & 0 & 0 & 0 & 0 & 0 \\
\hline GPC-BETA-Sr90 & 0 & 0 & 0 & 0 . & 0 & 0 & 0 & 0 & 0 & 0 \\
\hline GPC-BETA-Pm147 & 0 & 0) & 0 & 0 & 0 & 0 & 0 & 0 & o. & 0 \\
\hline GPC-BETA-Se79 & 0 & 0 & 0 & 0 & 0 & 0 & 0 & 0 & 0 & 0 \\
\hline LSC-BETA-H3 & 0 & 0 & 0 & 0 & 0 & 0 & 0 & 0 & 0 & 0 \\
\hline LSC-BETA-Rn222 & 0 & 0 & 0 & 0 & 0 & 0 & 0 & 0 & 0 & 0 \\
\hline LSC-BETA- 1129 & 0 & 0 & 0 & 0 & 0 & 0 & 0 & 0 & 0 & 0 \\
\hline LSC-BETA-C14 & 0 & 0 & 0 & 0 & 0 & 0 & 0 & 0 & 0 & 0 \\
\hline LSC-BETA-TC99 & 0 & 0 & 0 & 0 & 0 & 0 & 0 & 0 & 0 & 0 \\
\hline LSC-BETA & 0 & 0 & 0 & 01 & 0 & 0 & 0 & 0 & o. & 0 \\
\hline GAMMA SPEC & 0 & 0 & 0 & 0 & 0 & $\mathbf{0}$ & 0 & 0 & 0 & 0 \\
\hline OTHER-AAD & 0 & 0 & 0 & 01 & 0 & 0 & 0 & 0 & o & 0 \\
\hline TOTAL AAD & 0 & 01 & 01 & 01 & 0 & 0 & 01 & 01 & 0 & 0 \\
\hline & & & & & & & & & & \\
\hline TCLP EXTRACTION & 0 & 0 & 0 & 0 & 0 & 0 & 0 & 0 & D. & 0 \\
\hline OTHER-PREP & 0 & 0 & 0 & 0 & 0 & 0 & 0 & 0 & 0 & 0 \\
\hline OTHER-EXPLO & 0 & 0 & 0 & 0 & 0 & 0 & 0 & 0 & 0 & 0 \\
\hline OTHER-BIO & 0 & 0 & 0 & 0 & 0 & 0 & 0 & 0 & o & 0 \\
\hline OTHER-PHYS & 0 & 0 & 0 & 0 & 0 & 0. & 0 & 0 & 0 & 0 \\
\hline OTHER & 0 & 0 & 0 & 01 & 0 & 0. & 0 & 0 & 0 & 0 \\
\hline TOTAL OTHEA & $\overline{0}$ & 0 & $\overline{0}$ & 0 & 0 & 0. & 0 & $\mathbf{0}$ & 0 & 0 \\
\hline & & & & & & & & & & \\
\hline TOTAL SAMPLE ANALYSES & $\overline{0}$ & D] & 0 & 01 & 0 & 0] & 0 & 01 & 0 & 0 \\
\hline
\end{tabular}


Table IV: MC Sample Analyses by Rad Level and Matrix Type (xxix) WSAC-SRTC-ADS

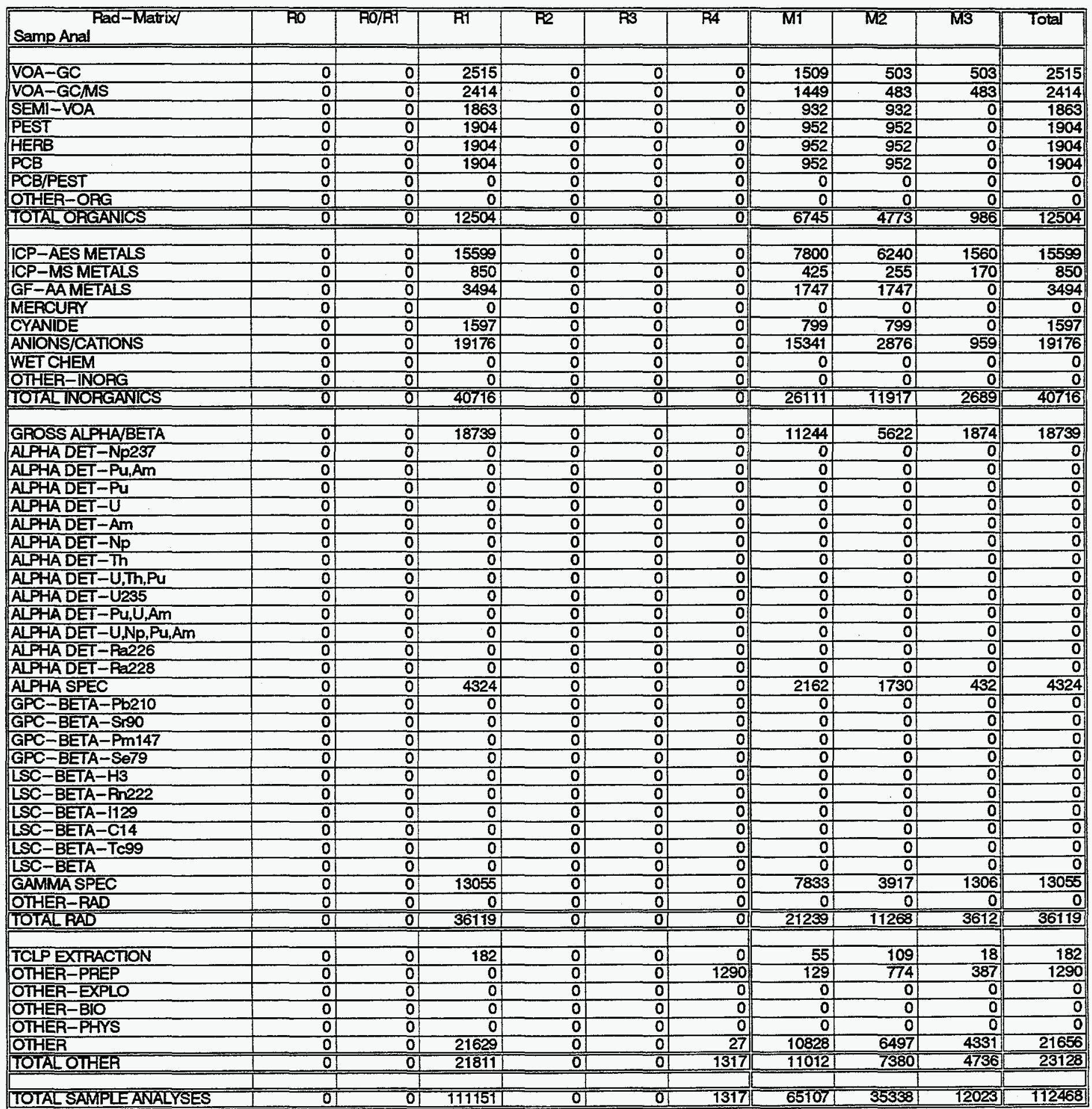


EM-263 SURVEY OF DOE ANALYTICAL CAPACTY AVAILABLE TO EM

Table IVc: MC Sample Analyses by Rad Level and Matrix Type (xox) WSRC-SRTC-DWPT

\begin{tabular}{|c|c|c|c|c|c|c|c|c|c|c|}
\hline $\begin{array}{l}\text { Rad-Matrix } \\
\text { Samp Anal }\end{array}$ & RिO & RO/RI & R1 & R2 & $\overline{\mathrm{RB}}$ & PA4 & $\overline{\mathrm{MT}}$ & M2 & $\overline{\mathrm{M} 33}$ & Total \\
\hline VOA-GC & 770 & & & & & & & & & \\
\hline VOA-GCMS & 0 & $\frac{0}{0}$ & 0 & 0 & 이 & 이 & 539 & 154 & 77 & 770 \\
\hline SEMI-VOA & & $\frac{0}{0}$ & 인 & 0 & 이 & 0 & 0. & 이 & 0. & 0 \\
\hline PEST & & 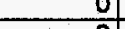 & ㅇ. & 0 & 이 & 요 & 01 & 이 & 0 & 0 \\
\hline & & 의 & 0 & 0 & 0 & 이 & 0 & 의 & o 0 & 므 \\
\hline$H E R B$ & 0 & 이 & 0 & 0 & 이 & 0 & 0 & 이 & 0 & 0 \\
\hline PCB & 0 & O & 0 & 0 & 0 & 0 & 0 & 0 & 0 & 0 \\
\hline PCB/PEST & 0 & 0 & 0 & 0 & 0 & 0 & 0 & 0 & (0) & 0 \\
\hline OTHER-ORG & 0 & 0 & 0 & 0 & 이 & 0 & 0 & 0 & 0 & 0 \\
\hline TOTAL ORGANICS & 770 & 01 & 0 & 0 & 01 & 0 & 5391 & 154] & 77 & 770 \\
\hline & & & & & & 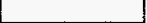 & & & & \\
\hline ICP-AESMETALS & 3858 & o & 0 & 0 & 0 & 0 & 386 & 2700 & 772 & 3858 \\
\hline ICP-MS METALS & 0 & 0 & Of & 0 & 0 & 0 & 0 & 0 & 0 & 0 \\
\hline GF-AAMETALS & 0 & 0 & 0 & 0 & 0 & 0 & 0 & 01 & 0 & 0 \\
\hline MERCURY & 1038 & 0 & 0 & 0 & 01 & 0 & 519 & 519 & 0 & 1038 \\
\hline CYANIDE & 0 & 0 & 0 & 0 & 0 & 0 & 0 & 0 & 0 & 0 \\
\hline ANIONS/CATIONS & 2511 & 0 & O & 0 & 0 & 0 & 251 & 1758 & 502 & 2511 \\
\hline WET CHEM & 0 & 0 & of & 0 & 0 & 0 & 0 & 0 & 0 & 0 \\
\hline OTHER-INOAG & 0 & 0 & of & 0 & 이 & 0 & 0 & 0 & (0) & 0 \\
\hline TOTAL INOAGANICS & 7407 & 0 & 01 & 01 & 01 & 0 & 1156 & 4977 & 1274 & 7407 \\
\hline & & & & & & & & & & \\
\hline GROSS ALPHABBTA & 0 & 의 & 0 & 0 & of & 0 & 0 & 0 & 0 & 0 \\
\hline ALPHA DET-Np237 & 0 & 0 & 0 & 0 & of & 0 & 0 & 0 & 0 & 0 \\
\hline ALPHA DET-PU,Am & D & 0 & 0 & 0 & 01 & 0 & 0 & 0 & 0 & 0 \\
\hline ALPHA DET-PU & 0 & 0 & 0 & 0 & 0 & 0 & 0 & 0 & 0. & 0 \\
\hline ALPHADET-U & o & 0 & of & 0 & 0 & 0 & 0 & 0 & ㅇ. & 0 \\
\hline ALPHA DET-Am & D & 0 & o. & 0 & 0 & 0 & 0 & 0 & 0 & 0 \\
\hline ALPHADET-Np & D & 0 & 0 & 0 & of & 0) & 0 & 0 & 0. & 0 \\
\hline ALPHA DET-Th & D & 0 & 0 & 0 & 0 & 0 & 0 & 0 & 0. & 0 \\
\hline ALPHA DET-U,Th,PU & $\overline{0}$ & 0 & o. & 0 & of & 0 & 0 & 0 & o & 0 \\
\hline ALPHA DET-U235 & 0 & 0 & 0 & 0 & 0 & 0. & of & 0 & 0 & 0 \\
\hline ALPHA DET-Pu,U,Am & 0 & 0 & o. & 0 & of & 0 & 0 & 0 & 0. & o \\
\hline ALPHA DET-UNp,Pu,Am & $\mathbf{0}$ & 0 & of & 0 & o & 0 & 0 & 0 & 0. & 0 \\
\hline ALPHA DET-Ra226 & $\mathbf{0}$ & of & 0 & 0 & 0 & 0 & 0 & 0 & 애 & 0 \\
\hline ALPHADET-Ra228 & 0 & 0 & 0 & 0 & of & 0 & 0 & 0 & (0) & 0 \\
\hline ALPHA SPEC & 0 & 0 & 0 & 0 & 0 & 0 & 0 & 0 & of & 0 \\
\hline GPC-BETA-Pb210 & 0 & 0 & o) & 0 & 0 & 0 & 0 & 0 & o & 0 \\
\hline GPC-BETA-Sr90 & 0 & of & 0 & 0 & 0 & 0 & \begin{tabular}{l|l}
0 \\
\end{tabular} & 0 & 0 & 0 \\
\hline GPC-BETA-Pm 147 & 0 & o| & 0 & 0 & ol & 0 & 0 & 0 & 0 & 0 \\
\hline GPC-BETA-Se79 & 0 & 0 & 0 & 0 & 01 & 0 & 0 & 0 & o & 아 \\
\hline LSC-BETA-H3 & 0 & 0 & 0 & 0 & of & 0 & 0 & $\overline{0}$ & o & 0 \\
\hline LSC-BETA-Rn222 & 0 & 0 & 0 & 0 & 0. & 0 & 0 & 0 & 0 & 0 \\
\hline LSC-BETA- 1129 & 0 & 0 & DI & 0 & 0) & 0 & 0 & 0 & 의 & 0 \\
\hline LSC-BETA-C14 & D & of & 0 & 0 & D) & a & \begin{tabular}{l|l}
0 \\
\end{tabular} & 0 & 0 & 0 \\
\hline LSC-BETA-TC99 & 0 & 0 & 0 & 0 & 0 & 0 & 0 & 0 & o & 0 \\
\hline LSC-BETA & 0 & 0) & 0 & 0 & $0 \mid$ & 0 & \begin{tabular}{l|l}
0 \\
\end{tabular} & 0 & 0) & 0 \\
\hline GAMMA SPEC & 0 & o & 0 & 0 & 0 & 0 & 0 & 0 & 의 & 0 \\
\hline OTHER-RAD & $\overline{0}$ & 0 & 0 & 0 & 0 & 0 & 0 & o & 0 & 0 \\
\hline TOTAL RAD & $\overline{0}$ & 01 & 01 & 01 & 01 & $\overline{0}$ & 01 & प] & 이 & 0 \\
\hline & & & & & & & & & & \\
\hline TCLP EXTRACTION & 0 & (0) & 0 & 0 & 0 & 0 & 0 & 0 & 0 & 0 \\
\hline OTHER-PREP & 0 & 0 & 0 & 0 & 0 & 0 & 0 & 0 & 0 & $\overline{0}$ \\
\hline OTHER-EXPLO & $\mathbf{0}$ & 0 & 0 & of & 0 & 0 & 0 & 0 & 0 & 0 \\
\hline OTHER-BIO & $\overline{0}$ & o & 0 & 0 & 0 & 0 & 0 & 0 & 0 & 0 \\
\hline OTHER-PHYS & $\overline{0}$ & 0 & 0 & 0 & 0 & 0 & 0 & 0 & 0 & 0 \\
\hline OTHER & 0 & $\overline{0}$ & 0 & O) & 0 & 0 & o & O & 0 & $\overline{0}$ \\
\hline TOTAL OTHER & $\overline{0}$ & 01 & OI & 01 & 0 & 0 & 01 & 0 & 0 & $\mathbf{0}$ \\
\hline & & & . & & & t & 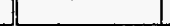 & & & \\
\hline TOTAL SAVIPLE ANALYSE & 8177 & 0 & 01 & 01 & 01 & 0 & 1695 & 51311 & 1351 & 8177 \\
\hline
\end{tabular}




\title{
APPENDIX E
}

\section{DETERMINATION OF EM ENVIRONMENTAL}

\author{
SAMPLING AND ANALYSIS NEEDS
}

FOR FY 93-99

- REPORTING FORMATS AND INSTRUCTIONS 


\title{
766L l Rueniqa]
}

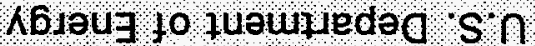

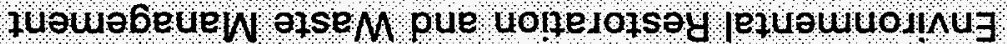

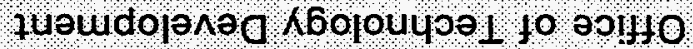

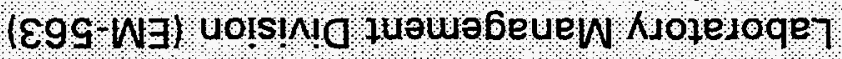

Ag pasedald

\author{
SNOLLOMUISNI \\ ONV

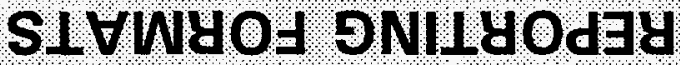

66 - ع6 ג」 시

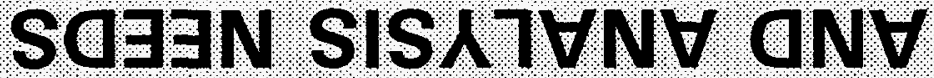

\section{כNITdW $7 \forall \perp N \exists W N O Y I \wedge N \exists$}

\section{Wヨ $\unlhd O$ NOI $\forall N I W Y \exists \perp \exists O$}




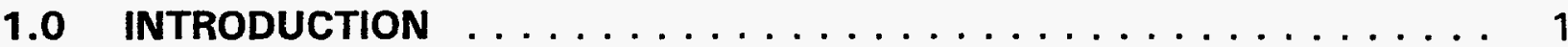

1.1 Background .................... 1

1.2 Previous EM Analytical Needs Projections $\ldots \ldots \ldots \ldots \ldots \ldots$

1.3 Current EM Analytical Needs Projections . . . . . . . . . . . 1

2.0 GENERAL INSTRUCTIONS $\ldots \ldots \ldots \ldots \ldots \ldots \ldots \ldots \ldots \ldots \ldots$

2.1 Deliverables ..................... 3

2.2 Submission of Deliverables $\ldots \ldots \ldots \ldots \ldots \ldots \ldots \ldots$

2.3 Training Session . . . . . . . . . . . . . . 3

2.4 EM-563 Points of Contact . . . . . . . . . . . . 3

2.5 Strategy for Collecting Analytical Needs Data . . . . . . . . . . 4

3.0 INSTRUCTIONS FOR COMPLETING THE EM ANALYTICAL NEEDS

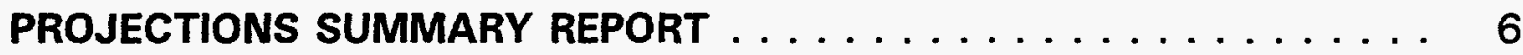

3.1 Description of the Summary Report . . . . . . . . . . . 6

3.1.1 Description of the Datasheets . . . . . . . . . . 6

3.2 Instructions for Completing the Cover Sheet . . . . . . . . 6

3.3 Instructions for Completing the ADS Summary Sheets . . . . . . 6

3.3.1 Part 1: Summary of ADS Sampling and Analysis Requirements ................ 6

3.3.2 Part 2: Summary of ADS Activities $\ldots \ldots \ldots \ldots \ldots$

4.0 INSTRUCTIONS FOR COMPLETING THE EM ANALYTICAL NEEDS PROJECTIONS FINAL REPORT $\ldots \ldots \ldots \ldots \ldots \ldots \ldots \ldots$

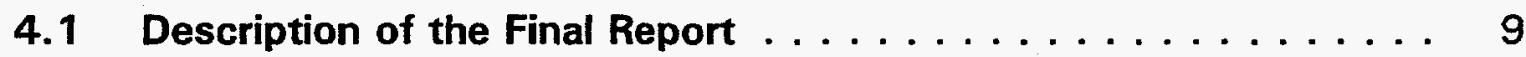

4.1.1 Description of the Datasheets . . . . . . . . . 9

4.2 Instructions for Completing the Cover Sheet .......... 9

4.3 Instructions for Completing the Datasheets on Each ADS . . . . . 10

4.3.1 Header Information . . . . . . . . . . . . 10

4.3.2 Part 3: General Description of Activity . . . . . . . . . 11

4.3.3 Part 4: Activity Sampling and Analysis . . . . . . . 13

4.3.4 Part 5: Activity Documentation ... . . . . . . . 15

5.0 INSTRUCTIONS FOR USING THE LOTUS 1-2-3 ELECTRONIC FORMS - 17

5.1 The Diskette and the Files $\ldots \ldots \ldots \ldots \ldots \ldots \ldots \ldots$

5.2 Macro Commands ..................... 17

5.3 Entering Data into the Parts 3, 4, and 5 Templates . . . . . . 18

5.4 Saving the Spreadsheets $\ldots \ldots \ldots \ldots \ldots \ldots \ldots$ 


\section{LIST OF APPENDICES}

APPENDIX A: SITE TRANSMITTAL AND DATA ENTRY FORMS FOR THE SUMMARY REPORT . . . . . . . . . . . . A-1 APPENDIX B: $\quad$ BLANK TRANSMITTAL AND DATA ENTRY FORMS FOR THE FINAL REPORT . . . . . . . . . . . . B-1 APPENDIX C: $\quad$ COMPLETED DATASHEET EXAMPLES . . . . . . . C 1

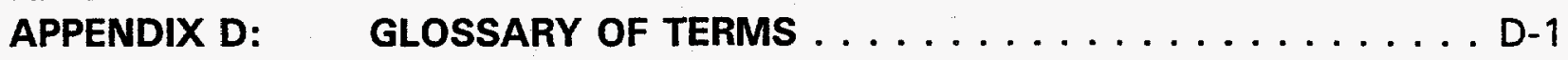




\section{LIST OF TABLES}

Table I: $\quad$ Activity Codes . . . . . . . . . . . . . . . 12

Table II: $\quad$ Regulatory Drivers $\ldots \ldots \ldots \ldots \ldots \ldots \ldots \ldots$

Table III: $\quad$ Alt P User Options in PART345.WK1 $\ldots \ldots \ldots \ldots \ldots \ldots \ldots 18$

Table IV: Permitted Lotus Filenames . . . . . . . . . . . . . . . . . . . . 19 


\section{LIST OF FIGURES}

Figure 1: A Blank Datasheet for Part 1 of the EM Analytical Needs Determination Report ................. 7

Figure 2: A Blank Datasheet for Part 2 of the EM Analytical Needs Determination Report . . . . . . . . . . . . . 8

Figure 3 : Datasheet for Part 3 of the EM Analytical Needs Determination Report . . . . . . . . . . . . . . . . . . . . 11

Figure 4 : Top of Datasheet for Part 4 of the EM Analytical Needs Determination Report .................... 14

Figure 5 : Datasheet for Part 5 of the EM Analytical Needs Determination Report . . . . . . . . . . . . . . . . . 16 


\section{LIST OF ACRONYMS}

$\begin{array}{ll}\text { ADS } & \text { Activity Data Sheet } \\ \text { DOE } & \text { U.S. Department of Energy } \\ \text { EM } & \text { Office of Environmental Restoration and Waste Management } \\ \text { EM-30 } & \text { Office of Waste Management } \\ \text { EM-40 } & \text { Office of Environmental Restoration } \\ \text { EM-50 } & \text { Office of Technology Development } \\ \text { EM-563 } & \text { Laboratory Management Division } \\ \text { ICP } & \text { Inductively Coupled Plasma } \\ \text { NSTS } & \text { National Sample Tracking System } \\ \text { OSM } & \text { Office of Sample Management } \\ \text { OU } & \text { Operable Unit } \\ \text { PTS } & \text { Progress Tracking System } \\ \text { SAIC } & \text { Science Applications International Corporation } \\ \text { SWMU } & \text { Solid Waste Management Unit } \\ \text { TCLP } & \text { Toxicity Characteristics Leaching Procedure } \\ \text { WAG } & \text { Waste Area Group } \\ \text { WPI } & \text { Waste Policy Institute }\end{array}$




\subsection{Background}

The Department of Energy's (DOE's) Office of Environmental Restoration and Waste Management (EM) has been tasked with addressing all environmental contamination and waste problems facing the Department. A key element of any waste management or environmental restoration program is the organization of physical and chemical data. An effective and efficient sampling and analysis program is required to generate credible environmental data. The Laboratory Management Division (EM563) of the Office of Technology Development (EM-50) was established to provide the programmatic direction needed to establish and operate such an EM-wide analytical services program.

\subsection{Previous EM Analytical Needs Projections}

In very large sampling and analysis operations, problems arise when trying to balance supply and demand, set priorities, and maintain sample flow from the field to the laboratory. EM-563 has therefore initiated a multi-faceted program to address these problems. One of the key tools being developed is the National Sample Tracking System (NSTS), through which EM-563 will collect, store, and disseminate information regarding the numbers, costs, and timeliness of sample analyses performed for the EM Complex. A pilot test of the NSTS was run in February-March 1993 and full implementation of the system began in early FY 94.

A second effort made preliminary forecasts of EM analytical needs at nine of the major sites within the DOE Complex. This was based on the Activity Data Sheet (ADS) fiveyear planning documents. A model was constructed in which each ADS was assigned to one of 15 categories of environmental activity (e.g. high level waste management), and a set of factors was developed to estimate the number of key major laboratory operations expected for each activity. These numbers were then collected to provide overall projections of the numbers of samples and sample analyses required in the planning period covered by the ADSs. A draft report summarizing the model and its preliminary projections was sent to the field in September 1992.

A third effort, being undertaken by EM-563, involves assessment of the laboratory capacity available for handling and analyzing EM environmental samples. A survey of DOE analytical laboratory managers was made to determine the available DOE laboratory capacity based on a model developed by EM-563. The collected data is currently being summarized.

\subsection{Current EM Analytical Needs Projections}

In the current study, EM-563 is attempting to obtain more reliable projections of EM analytical needs by refining the model described in the draft September 1992 report. The essential elements of the refined model are given below: 
- $\quad$ Projections of EM analytical needs are made for the period FY 94 - FY 99;

- Actual numbers of environmental samples collected and sample analyses performed in FY 93 are used to calibrate the model;

- Sample and sample analysis information is collected only for those ADSs funded by EM;

- A set of environmental activities is assigned to each EM ADS, and projections of the numbers of environmental samples to be collected and sample analyses to be performed for each activity are made; and

- Numbers will be collected from major DOE EM installations and projections will be made for the whole of EM from these numbers.

This package contains instructions for field participation in this study. Specifically, you are requested to provide the following:

- Information concerning the activities taking place within each ADS at your site; and

- Actual numbers and types of environmental samples collected and sample analyses performed in FY 93 at your site for each activity.

This request will be made only once, since reports from the field about their sample analysis operations will be provided on a monthly basis when the NSTS develops into a fully working system, and this will yield the required information for making updated needs projections. 


\title{
2.0 GENERAL INSTRUCTIONS
}

This section gives general instructions to participants in this determination of EM analytical needs projections. Detailed instructions for completing the reporting forms are contained in Section 3.0.

\subsection{Deliverables}

At the completion of the reporting effort, all field participants, with the assistance of their EM-563 points of contact, should prepare the following deliverables for EM-563:

1) A summary report - this report contains the requested data summarizing ADS activities and sample and analysis requirements in hard copy format; and

2) A final report - this report contains the requested detailed sample and analysis data for FY 93 in either hard copy or electronic format.

Both reports should be accompanied by cover letters that should include any comments on the contents of the reports and explanations of any deviations from the data entry protocol.

\subsection{Submission of Deliverables}

The summary report is due by March 25, 1994, and the final report is due by April 29, 1994. All deliverables should be submitted to:

\author{
Mr. Robert Murray, \\ US DOE, EM-563 \\ Laboratory Management Division \\ Trevion II \\ Washington, DC 20585-0002
}

Tel: 301-903-7644

FAX: 301-903-7613

\subsection{Training Session}

A training session for field participants will be given by representatives of EM-563 at the Third Office of Sample Management Workshop in Denver on March 10, 1994. Please contact Mr. Robert Murray if you would like to attend.

\section{$2.4 \quad$ EM-563 Points of Contact}

The following personnel have been designated the EM-563 points of contact to assist site personnel in completing this EM analytical needs projections report: 
Mr. Kelvin Wright, WPI

Tel: 301-990-3015

FAX: 301-990-4889

Dr. Richard Sassoon, SAIC

Tel: 301-924-6126

FAX: 301-924-4594

Dr. Steve Wyrick, SAIC

Tel: 301-924-6168

FAX: 301-924-4594

Ms. Farideh Moghadami, SAIC

Tel: 301-924-6124

FAX: 301-924-4594

They have been assigned to work with the following sites:

$\begin{array}{ll}\text { Site } & \text { Lead } \\ \text { INEL } & \text { Wright } \\ \text { Rocky Flats } & \text { Sassoon } \\ \text { Fernald } & \text { Wright } \\ \text { ORNL, K-25, Y-12 } & \text { Moghadami } \\ \text { LANL } & \text { Wright } \\ \text { Hanford } & \text { Sassoon } \\ \text { NTS } & \text { Sassoon } \\ \text { Savannah River } & \text { Wyrick }\end{array}$

Please contact your site lead if you have any questions or concerns regarding completion of this package.

\subsection{Strategy for Collecting Analytical Needs Data}

Collection of the data necessary to complete these reports involves input of information from many sources at each site. Because of the different organizational structures at the different Operations Offices, no single strategy is optimum for every site. Some recommendations and suggestions are made below, however, which may assist in the efficient and effective collection of the required data:

- Appoint a single coordinator at each site who is responsible for maintaining contact with the headquarters site lead, managing the personnel collecting the data, and preparing the reports.

- If there is a Central Planning Office at your site, it may be a good source of information about the local work breakdown structure, future budgets and planned activities, and which projects have analytical needs. 
- It may be useful to delegate the work according to EM organizations, i.e. Office of Waste Management (EM-30), Office of Environmental Restoration (EM-40), etc., and/or contractors and subcontractors.

- If projects involving sampling and analysis can be clearly identified, survey each project manager to obtain the required information.

- Many sites now have Offices of Sample Management (OSMs) that are required to report sampling and analysis activities to EM-563 via the NSTS. For those sites that have functioning OSMs with fully operating information systems, most of the FY 93 sampling and analysis data should be available from the OSMs. Note, however, that many OSMs currently do not handle every sample from their sites.

- Additional information about activities conducted under each ADS should be available through the EM Progress Tracking System (PTS).

It is also recognized that organization of EM activities may differ markedly under each Operations Office, and even at different sites under the same Operations Office. For this reason, the analytical needs model is based around the ADS system, which is standard across EM. It may, however, be more convenient for individual sites to collect the appropriate analytical needs data at a lower level in the work breakdown structure (e.g. according to work packages, projects, etc.). Such work units may correspond more closely with the categories of activity provided later in these instructions. Participants may enter data into their reports according to these work units rather than ADSs. If participants choose to provide data sorted in this manner, the following conditions must be fulfilled:

1. Every reference to an ADS in the report should be replaced by a reference to the selected work unit.

2. An additional table should be submitted with both the summary and final reports that provides a crosswalk between the work units and ADSs.

3. Each work unit must be funded under only one ADS.

4. Full explanations of the data entry protocol used should be provided in cover letters accompanying the two reports. 


\subsection{INSTRUCTIONS FOR COMPLETING THE EM ANALYTICAL NEEDS PROJECTIONS SUMMARY REPORT}

\subsection{Description of the Summary Report}

The summary report that your organization will complete and submit to EM-563 consists of two sections:

1. A cover sheet that provides information about the organization generating the report; and

2. Two ADS summary datasheets, identifying ADSs that include environmental sampling and analysis activities.

This report should be submitted in hard copy by completing the forms and matrices as they appear in Appendix A.

\subsubsection{Description of the Datasheets}

The two summary datasheets are numbered Part 1 and Part 2. These datasheets have been partially completed for your site by EM-563. The remainder of these datasheets should be completed as described in Section 3.3. The datasheets are used to report the following information:

Part 1: Summary of ADS Sampling and Analysis Requirements: Site confirmation of EM-563 determination of whether sampling and analysis is required within each ADS; and

Part 2: Summary of ADS Activities: Activity list, summary activity information, and contacts for ADS activities requiring sampling and analysis.

\subsection{Instructions for Completing the Cover Sheet}

Complete the cover sheet provided in Appendix $A$ by entering the requested information. Be sure to enter the name and telephone number of the person responsible for determining the site-wide analytical needs.

\subsection{Instructions for Completing the ADS Summary Sheets}

Parts 1 and 2 of the report consist of ADS summary sheets, which should be completed as described below.

\subsubsection{Part 1: Summary of ADS Sampling and Analysis Requirements}

Part 1 has been partially completed by EM-563 and is found in Appendix A. A blank version of Part 1 is shown in Figure 1. A complete list of the current ADSs for your site is given together with EM-563's projection of whether any sampling and analysis 
needs exist for FY 93 and FY 94-99. Complete the last two columns of the summary report by entering a $\mathrm{Y}$ (yes) or $\mathrm{N}(\mathrm{no})$ in these columns, indicating whether sampling and analysis actually took place in FY 93, or is planned for any time in FY 94-99 for each ADS.

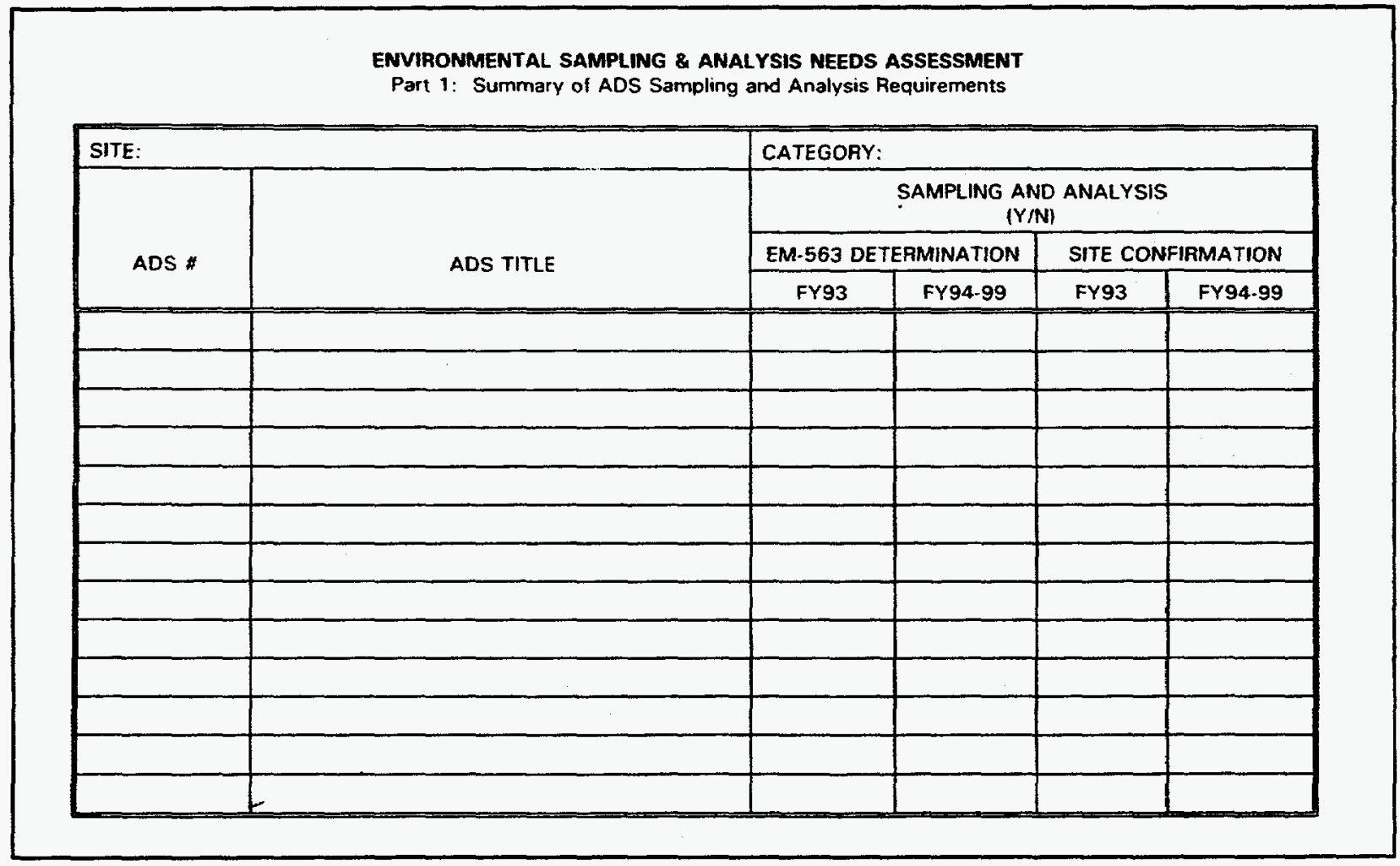

Figure 1: A Blank Datasheet for Part 1 of the EM Analytical Needs Determination Report

\subsubsection{Part 2: Summary of ADS Activities}

Part 2 has also been partially completed by EM-563 and is found in Appendix A. A blank version of Part 2 is shown in Figure 2. The first three columns of the datasheet contain a list of current ADSs for your site, together with the activities identified by EM-563, based on review of the ADSs, to involve sampling and analysis during FY 9399. Complete the last five columns of the summary report by entering the number, type, and average size of units addressed in each activity, an activity contact name, and the contact's telephone number. Unit types could be, for example, solid waste management units (SWMUs), operable units (OUs), waste area groups (WAGs), tanks, drums, buildings, or pits. Give the average size of the unit in terms of volume or area. If any $A D S s$ or activities requiring sampling and analysis were not listed on the datasheet, enter them, with the appropriate activity information, in the blank area at the bottom of the datasheet. Make any needed corrections in the first three columns.

- Note: Every ADS identified by the site in Part 1 as having sampling and analysis needs at any time in FY 93-99 should also be entered in Part 2. For those ADSs for which more than one activity involving sampling and 


\begin{tabular}{|c|c|c|c|c|c|c|c|}
\hline \multicolumn{8}{|c|}{$\begin{array}{l}\text { ENVIRONMENTAL SAMPLING \& ANALYSIS MEEDS ASSESSMENT } \\
\text { Part 2: Summary of ADS Activities }\end{array}$} \\
\hline \multicolumn{3}{|l|}{ SIZE: } & \multicolumn{5}{|c|}{ CATEGORY: } \\
\hline \multirow[b]{2}{*}{ ADS \# } & \multicolumn{2}{|c|}{ ACTIVITY } & \multicolumn{3}{|c|}{ UNITS } & \multirow{2}{*}{$\begin{array}{l}\text { ACTIVITY } \\
\text { CONTACT }\end{array}$} & \multirow[b]{2}{*}{ TELEPHONE } \\
\hline & CODE & DESCRIPTION & $\#$ & TYPE & $\begin{array}{c}\text { AVERAGE } \\
\text { SIZE }\end{array}$ & & \\
\hline & & & & & & & \\
\hline & & & & & & & \\
\hline & & & & & & & \\
\hline & & & & & & & \\
\hline & & & & & & 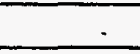 & \\
\hline & & & & & & & \\
\hline & & & & & & & \\
\hline & & & & & & & \\
\hline & & & & & & & \\
\hline & & & & & & & \\
\hline & & & & & & & \\
\hline & & & & & & & \\
\hline & & & & & & & \\
\hline & & & & & & & \\
\hline & & & & & & & \\
\hline
\end{tabular}

Figure 2: A Blank Datasheet for Part 2 of the EM Analytical Needs Determination Report

analysis is identified, an entry must be made in Part 2 for each activity.

- Note: The activities entered in column 2 are from the list of activities provided in Section 4.3.2 of this set of instructions. 


\subsection{INSTRUCTIONS FOR COMPLETING THE EM ANALYTICAL NEEDS PROJECTIONS FINAL REPORT}

\subsection{Description of the Final Report}

The final report that your organization will complete and submit to EM-563 consists of two sections:

1. A cover sheet that provides information about the organization generating the report; and

2. A set of completed datasheets that provides specific sample and analysis information for activities conducted during FY 93.

The report can be submitted either in hard copy, by filling out the forms and matrices as they appear in Appendix B, or in electronic version, by filling out the spreadsheets in Lotus $1-2-3^{1}$ format provided on the enclosed diskette. Instructions for using the Lotus 1-2-3 files appear in Section 5.0. If you choose to provide a hard copy of the reports, you will need to make multiple copies of the forms before you begin. Our recommendation is that deliverables be submitted electronically, since the Lotus 1-2-3 files include several time-saving and error-avoidance features.

\subsubsection{Description of the Datasheets}

The three sets of datasheets are numbered Part 3, Part 4, and Part 5. These datasheets should be completed for each activity covered in each ADS identified in Parts 1 and 2 of the summary report as requiring environmental sampling and analysis. The datasheets are used to report the following information:

Part 3: General Description of Activity: Activity description, including regulatory driver, number of units addressed, activity schedule, and activity costs;

Part 4: Activity Sampling and Analysis: Schedule, average batch size, types and total numbers of customer samples sent, and sample analyses requested during the reporting period;

Part 5: Activity Documentation: Planning documents, project reports, budgetary documents, and regulatory documents associated with the activity.

\subsection{Instructions for Completing the Cover Sheet}

Complete the Cover Sheet provided in Appendix B by entering the requested information. Be sure to enter the name and telephone number of the person responsible for determining the site-wide analytical needs.

\footnotetext{
${ }^{1}$ Lotus $1-2-3$ is a registered trademark of the Lotus Development Corporation.
} 


\subsection{Instructions for Completing the Datasheets on Each ADS}

This section gives detailed instructions for completing the datasheets for Parts 3-5 of the EM analytical needs projections report. Datasheets for Parts 3,4 , and 5 of the report should be completed for each activity performed under each ADS. If the same activity is performed to support several projects under the same ADS, it should only be entered once. If a project covers more than one activity, however, then each activity within the project must be reported on a separate set of datasheets.

Accurate numbers should be entered wherever possible. In some situations, only best estimates may be available. In such cases, enter these numbers into the datasheets and describe the reliability of the numbers in the comments section of the questionnaire which accompanies this document.

- Note: When the number to be entered is zero, this may be indicated by either entering $O$ or by leaving that entry blank. All blank numerical entries will be assumed to be zero. If data is unavailable, enter N/A.

Dates should be entered using the format MM-DD-YY, where $M M$ is a 2-digit number from 01 to 12 representing the month, DD is a 2-digit number from 01 to 31 representing the day, and $Y Y$ is a 2-digit number representing the year.

Please read all of the instructions and follow the examples given in Appendix $\mathrm{C}$ before starting to complete the datasheets.

Definitions of key terms used in the datasheets and in this set of instructions are provided in the Glossary of Terms (Appendix D).

\subsubsection{Header Information}

The header information required for the datasheets for Parts 3,4 and 5 of the report contains common data elements. For an example of a Header section, see Figure 3 , which shows the datasheet for Part 3.

Make the following entries in the headers:

Site:

The name of the site for which information is being provided.

ADS \#: $\quad$ The activity data sheet number for which information on the specified activity is being supplied.

- Note: A full ADS number should be entered into the header, including all dashes (RL-5037-EB, for example).

Activity Type: Alphanumeric code from the Table I. 


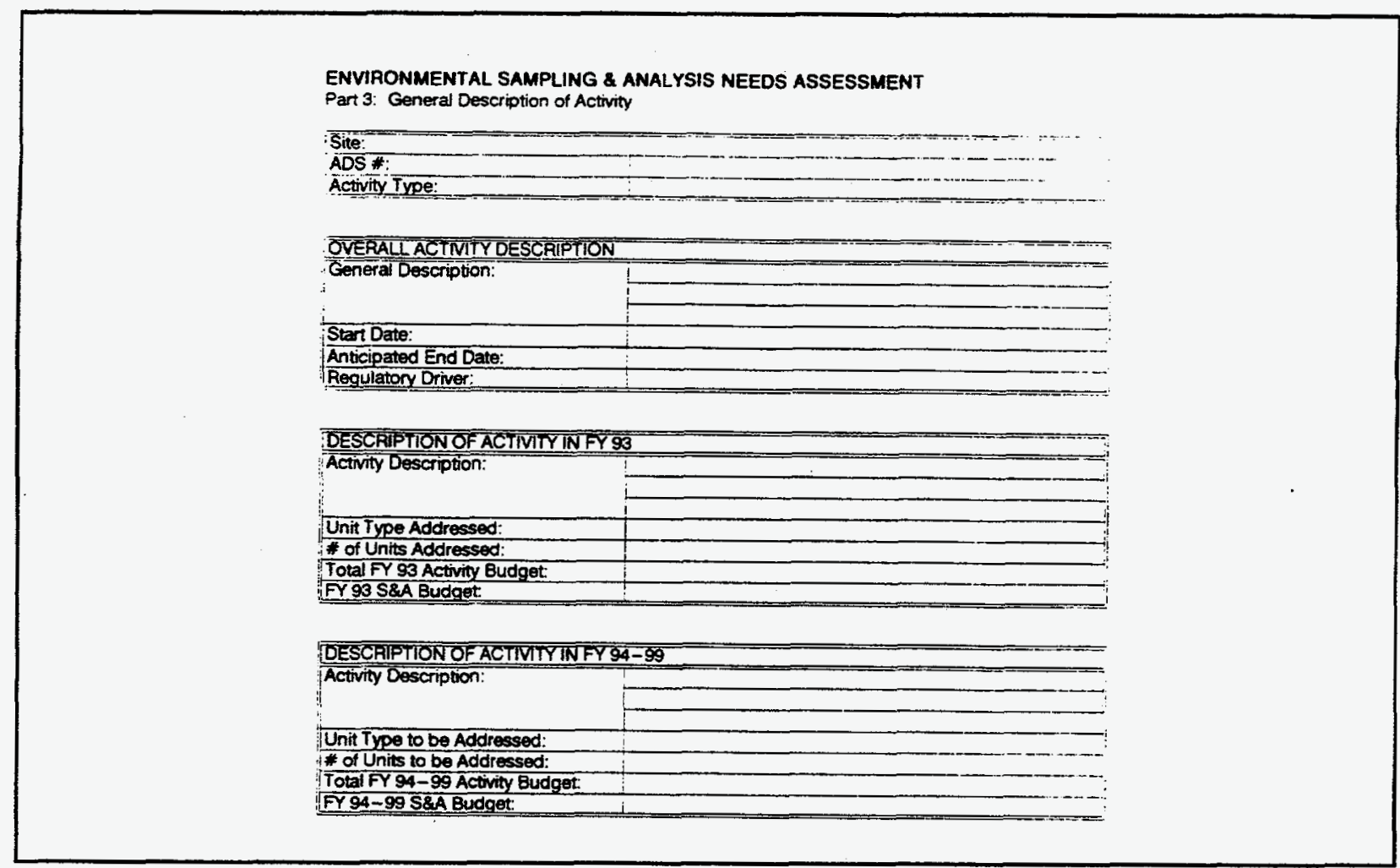

Figure 3 : Datasheet for Part 3 of the EM Analytical Needs Determination Report

- Note: Only one code may be entered into the field labelled ACTIVITY TYPE. If more than one activity is performed under a single ADS, a separate set of datasheets should be completed for each activity under that ADS for Parts 3,4 and 5.

\subsubsection{Part 3: General Description of Activity}

The datasheet for Part 3, presented in Figure 3, is divided into four sections: Header, Overall Activity Description, Description of Activity in FY 93, and Description of Activity in FY 94-99.

Header: Complete the Header section according to the instructions given in Section 4.3.1.

Overall Activity Description: Enter the requested information in the Overall Activity Description section of the datasheet. Give a brief description of the work performed and to be performed during the lifetime of the activity. Enter the start and end dates for the activity in the format specified earlier in Section 4.3.

- Note: The end date entered should be the date on which the activity is realistically expected to end. 
Table I: Activity Codes

\begin{tabular}{|c|c|}
\hline Activity Type & Code \\
\hline $\begin{array}{l}\text { Preliminary Assessment/Site Inspection or } \\
\text { RCRA Facility Assessment }\end{array}$ & $\mathrm{PA} / \mathrm{SI}$ \\
\hline $\begin{array}{c}\text { Remedial Investigation/Feasibility Study or } \\
\text { RCRA Facility Investigation/Corrective } \\
\text { Measures Study }\end{array}$ & $\mathrm{RI} / \mathrm{FS}$ \\
\hline $\begin{array}{l}\text { Remedial Design/Remedial Action } \\
\text { or Corrective Measure/Corrective Action }\end{array}$ & $\mathrm{RD} / \mathrm{RA}$ \\
\hline RCRA Facility Closure & RFC \\
\hline Decontamination and Decommissioning & $D \& D$ \\
\hline Groundwater Monitoring & GW MON \\
\hline Effluent Water Monitoring & EFF MON \\
\hline Transuranic Waste Management/Disposal & TRU \\
\hline Hazardous Waste Management/Disposal & HAZ \\
\hline $\begin{array}{l}\text { Mixed/Low Level Waste } \\
\text { Management/Disposal }\end{array}$ & MIX/LLW \\
\hline High Level Waste Management & HLW \\
\hline $\begin{array}{l}\text { Environmental Monitoring (this activity } \\
\text { includes routine high volume analyses such } \\
\text { as those performed on air stacks) }\end{array}$ & ENV MON \\
\hline $\begin{array}{c}\text { Underground Storage Tanks containing } \\
\text { nonradioactive waste }\end{array}$ & UST \\
\hline Miscellaneous RCRA/CERCLA Activity & MISC R/C \\
\hline Other (specify full name of activity) & \\
\hline
\end{tabular}

Enter the primary regulatory driver from the list given in Table II.

- Note: Only one regulatory driver should be entered on the datasheet. This should be the primary driver for taking and analyzing samples and should be a Federal or State act rather than any specific agreement. If this is not applicable, enter additional drivers and explain.

Description of Activity in FY 93 and FY 94-99: Enter the requested information in each of the two sections in the datasheet for the activity. For each period, give a 
Table II: Regulatory Drivers

\begin{tabular}{||c|c|}
\hline Regulatory Driver & Code \\
\hline \hline Resource Conservation and Recovery Act & RCRA \\
\hline $\begin{array}{c}\text { Comprehensive Environmental Response, } \\
\text { Compensation and Liability Act }\end{array}$ & CERCLA \\
\hline National Environmental Policy Act & NEPA \\
\hline Clean Air Act & CAA \\
\hline Clean Water Act & CWA \\
\hline National Pollutant Discharge Elimination \\
System & NPDES \\
\hline Toxic Substances Control Act & TSCA \\
\hline State & State (specify) \\
\hline Other & Other (specify) \\
\hline
\end{tabular}

description of the activity carried out or to be carried out, the type and number. of units addressed, and the budgets for the overall activity and for sampling and analysis. Leave a section blank if the activity was not performed in the period covered by that section.

See Example \#1 in Appendix C, which illustrates a typical completed datasheet for Part 3.

\subsubsection{Part 4: Activity Sampling and Analysis}

The datasheet for Part 4, the top of which is presented in Figure 4, is divided into three sections: Header, General Sampling and Analysis Description, and Sampling and Analysis Activity in FY 93.

Header: Complete the Header section according to the instructions given in Section 4.3.1.

General Sampling and Analysis Description: Enter the information requested in the section where appropriate. Give start and end dates in the format specified in Section 4.3. Also give the requested information concerning sampling and analysis activities prior to FY 93 (i.e. in FY 89 - FY 92) and after FY 93 (i.e. in FY 94 and beyond) if any sampling and analysis activity takes place during these periods. Data for FY 93 are entered into the section described below. Also enter here the average size of a batch of customer samples sent to a laboratory for analysis during this activity. 


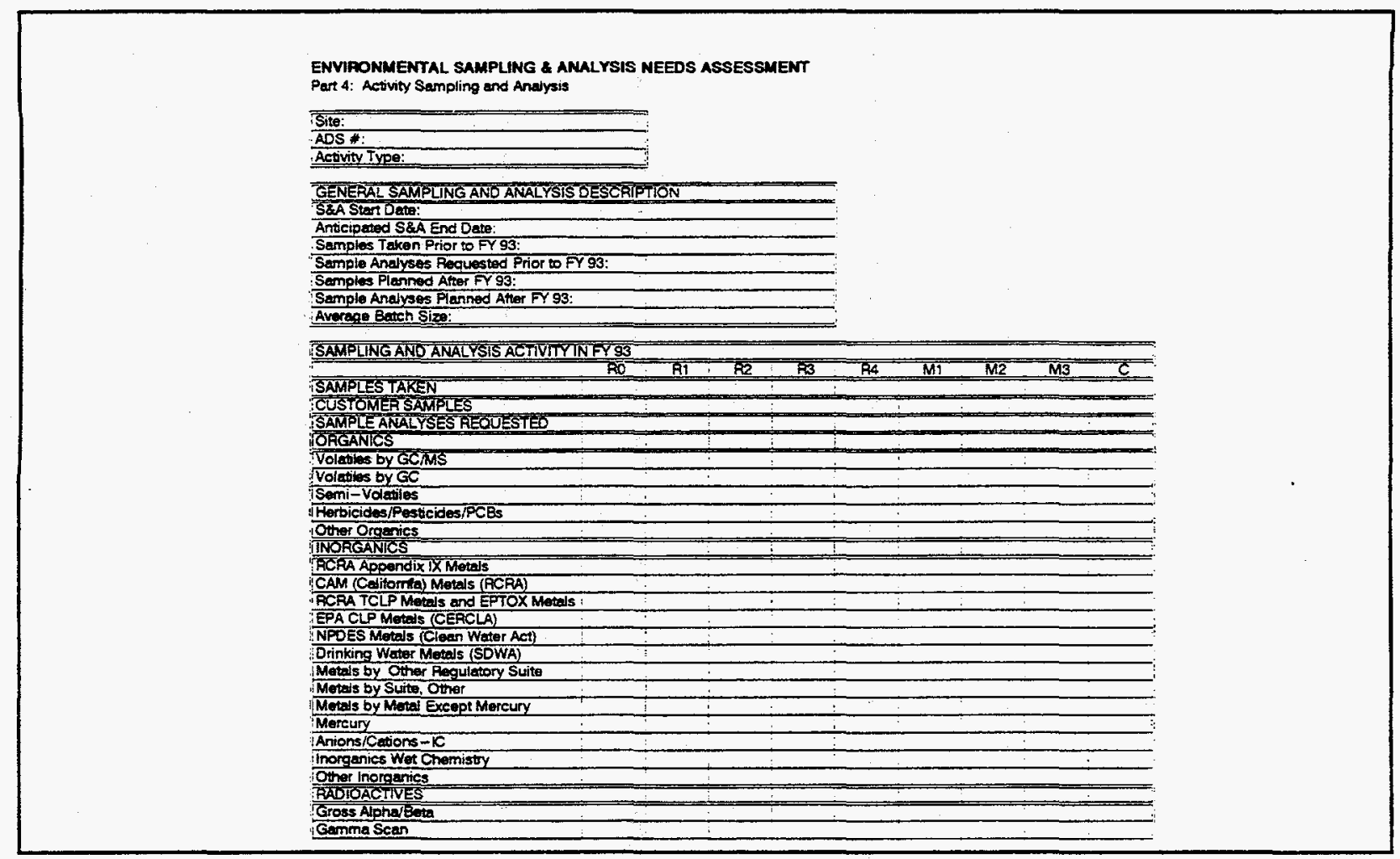

Figure 4 : Top of Datasheet for Part 4 of the EM Analytical Needs Determination Report

Sampling and Analysis Activity in FY 93: Complete this section by entering the appropriate information into the table. The table is subdivided into two sub-sections: Samples Taken and Sample Analyses Requested.

Samples Taken: The Samples Taken section of the table will be completed with the numbers of customer samples sent for analysis.

Enter the number of customer samples sent for analysis in the data entry row of the Samples Taken section of the table. Each sample will be entered at least twice in the table. It will be entered once according to its activity level, once according to its matrix type, and if it is a classified sample, it will be entered a third time. Five designations (RO, R1, R2, R3, and R4) are used to describe the activity levels of the samples, and three designations ( $M 1, M 2$, and $M 3$ ) are used to describe the matrix types of the samples. The designation " $\mathrm{C}$ " is used to identify classified samples. These designations are defined in the Glossary of Terms (Appendix D). The definition of a customer sample is also given in the Glossary of Terms.

- Note: Customer samples include duplicate field samples, trip blanks, field blanks, performance evaluation samples, composite samples, and separate phases of a field sample if each phase is analyzed separately. Customer samples do not include any peripheral samples, such as duplicates, quality control samples, or blanks added by the laboratory. 
Sample Analyses Requested: The Sample Analyses Requested section will be completed with the numbers of sample analyses requested to be performed on the samples. These numbers are required to be broken down according to sample activity level and matrix type.

Complete the Sample Analyses Requested section of the table by entering the numbers of each type of sample analysis requested. These numbers are reported by sample activity level, matrix type, and classification. Thus, as with the samples taken, each sample analysis is entered at least twice into the table, once according to sample activity level and once according to sample matrix type. If a sample analysis is performed on a classified sample it will be entered a third time. The total number of each type of sample analysis entered in the RO through R4 columns should equal the total number entered in the $M 1$ through $M 3$ columns, and should be greater than or equal to the number entered in the $C$ column. It is useful to perform this check after completing a table to ensure that numbers have been entered correctly.

Several points should be noted concerning entry of sample analysis data into the Sample Analyses Requested section of the datasheet:

- The numbers of any group of sample analyses requested may be larger than the actual number of samples sent to the laboratory, for one of several reasons. For example, if a laboratory was requested to perform separate inductively coupled plasma (ICP) runs for a suite of 5 metals on each customer sample, then the numbers entered in the row labelled METALS BY SUITE, OTHER will be five times as large as the numbers entered in the row labelled CusTOMER SAMPLES.

- If repeat analyses are requested on samples already sent to a laboratory, then the numbers of sample analyses requested will be entered in the Sample Analyses Requested section of the datasheet, but no entries will be made in the Samples Taken section of the datasheet related to field analytical samples sent.

- In the case of Toxicity Characteristic Leaching Procedure (TCLP) extractions, only the number of extractions themselves are entered in the row labelled TCLP EXTRACTION in the category OTHER. Any sample analyses that need to be performed on the extracts are entered in other rows of the datasheet such as RCRA TCLP AND EPTOX METALS in the category INORGANICS.

See Example \#2 in Appendix C, which illustrates a typical completed datasheet for Part 4.

\subsubsection{Part 5: Activity Documentation}

The datasheet for Part 5, presented in Figure 5, is divided into two sections: Header and Activity Documents. 


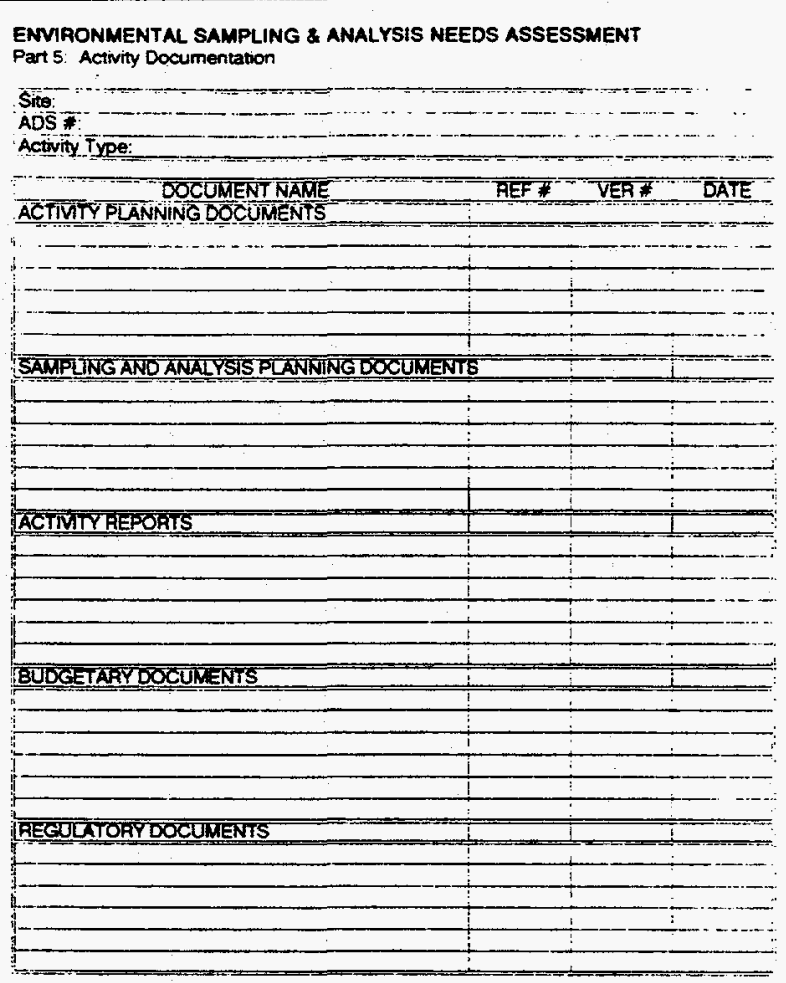

Figure 5 : Datasheet for Part 5 of the EM Analytical Needs Determination Report

Header: Complete the Header section according to the instructions given in Section 4.3.1.

Activity Documents: Enter the names, reference numbers, version numbers, and dates of issue of any activity-related documents that may be of assistance in assessing analytical needs. Such documents may provide actual sample and sample analysis numbers, or may just give general information on the scope of the activity. 


\subsection{INSTRUCTIONS FOR USING THE LOTUS 1-2-3 ELECTRONIC FORMS}

The datasheets for Parts 3, 4, and 5 in the final report have been implemented in Lotus $1-2-3$ as a data entry option. Lotus $1-2-3$ release 2.4 or a higher version is required. It is assumed that users of these electronic forms possess a general working knowledge of Lotus 1-2-3 or similar software. For regular Lotus 1-2-3 commands and usage, the user should consult the Lotus 1-2-3 manual. Any specific questions related to the use of the Lotus 1-2-3 spreadsheets should be addressed to Richard Sassoon (Tel. 301-924-6126).

\subsection{The Diskette and the Files}

The diskette provided by the Laboratory Management Division (EM-563) should be backed up prior to use. The diskette is MS-DOS formatted and consists of the Lotus 1-2-3 .WK1 file named PART345.WK1 for release 2.4 and the Lotus 1-2-3 .WK3 file named PART345.WK3 for releases 3.0 and higher. The files have read-only status (see below), protecting them against inadvertent erasure. This limit to write access can be removed by using the operating system ATTRIB command.

Both files contain the templates for the Parts 3,4 and 5 datasheets. The files can be loaded directly by using regular Lotus 1-2-3 commands; however, they can only be retrieved without a "file reservation" since they are read-only files. That is, any changes made or data entered into PART345.WK1 must be saved under a different file name. (See Section 5.4).

\subsection{Macro Commands}

Lotus 1-2-3 macros have been added to facilitate data entry. These macros are located in the range starting at cell V1 of files PART345.WK1 and PART345.WK3. To exit any menu or macro, press the ESC key twice. The available macro commands are:

Alt F - allows the user to freeze or unfreeze the column and row headings. This command works as a toggle switch. When a spreadsheet is first loaded, the Alt- $F$ macro may need to be pressed twice.

Alt P - invokes the custom menu that allows the user to select the template corresponding to the datasheets for Parts 3,4 , and 5 in PART345.WK1 or PART345.WK3. The custom menu also provides options to print the portions of the template corresponding to the datasheets for Parts 3, 4, and 5, and to save the file using the same or a new filename.

Full descriptions of the user options available with the Alt $P$ macro in the spreadsheets are given in Table III. These macros should not be altered, but the user may add other macros to ease operations. If an error is encountered in printing with the Alt $\mathrm{P}$ macro, check that all the print options on the spreadsheet are correctly set for your printer. 
Table III: $\quad$ Alt P User Options in PART345.WK1

\begin{tabular}{|l|l|}
\hline MENU OPTION & OPTION DESCRIPTION \\
\hline \hline Part_3 & Complete Part 3 of the spreadsheet \\
\hline Part_4 & Complete Part 4 of the spreadsheet \\
\hline Part_5 & Complete Part 5 of the spreadsheet \\
\hline Print_3 & Print Part 3 of the spreadsheet \\
\hline Print_4 & Print Part 4 of the spreadsheet \\
\hline Print_5 & Print Part 5 of the spreadsheet \\
\hline Save_New & $\begin{array}{l}\text { Save the current spreadsheet file as a new user } \\
\text { supplied filename }\end{array}$ \\
\hline Resave & Save the current spreadsheet file as the current name \\
\hline
\end{tabular}

\subsection{Entering Data into the Parts 3, 4, and 5 Templates}

The templates for the Parts 3, 4, and 5 datasheets are found in PART345.WK1 and PART345.WK3. Each template starts with a section for identification information; the information entered in Part 3 will be copied automatically to Parts 4 and 5 . All cells are protected, except for the ranges that are used to accept data. The cost data cells in Part 3 have a format of CO (Currency, 0 decimal place), since the only units permitted for reporting costs are dollars.

\subsection{Saving the Spreadsheets}

The worksheet containing the datasheets for Parts 3,4 and 5 must be saved with a unique filename. The filename should consist of three components: the activity type, ADS \#, and the file extension. The following table describes the file-naming conventions required for data submission. 
Table IV: Permitted Lotus Filenames

\begin{tabular}{|c|c|}
\hline $\begin{array}{l}\text { FILENAME } \\
\text { COMPONENTS }\end{array}$ & DESCRIPTION \\
\hline Activity Type & $\begin{array}{l}\text { A for } P A / S I \\
B \text { for } R I / F S \\
C \text { for RD/RA } \\
D \text { for RFC } \\
\text { E for D\&D } \\
F \text { for GW MON } \\
G \text { for EFF MON } \\
H \text { for TRU } \\
\text { I for HAZ } \\
J \text { for MIX/LLW } \\
K \text { for HLW } \\
L \text { for ENV MON } \\
M \text { for UST } \\
N \text { for MISC R/C } \\
O \text { for OTHER } \\
\end{array}$ \\
\hline ADS \# & $\begin{array}{l}\text { First } 7 \text { letters and/or numbers of the ADS number } \\
\text { excluding the } 2 \text { - or } 3 \text {-character Operations Office } \\
\text { identifier and excluding any dashes. }\end{array}$ \\
\hline File Extension & $\begin{array}{l}\text { WK1 for files saved in Lotus } 1-2-3 \text { Release } 2 \text { format. } \\
\text {.WK3 for files saved with Release } 3 \text {. (Refer to } 1-2-3 \\
\text { Manual) }\end{array}$ \\
\hline \multicolumn{2}{|c|}{ EXAMPLES OF VALID FILENAMES } \\
\hline B46B2.WK1 & $\begin{array}{l}\text { Data for Parts } 3,4 \text {, and } 5 \text { for the RI/FS activity } \\
\text { performed under ADS number FS- } 46-B 2 \text { in a file saved } \\
\text { in Lotus } 1-2-3 \text { Release } 2 \text { format }\end{array}$ \\
\hline $\mathrm{H} 1002 \mathrm{C} 1$. WK3 & $\begin{array}{l}\text { Data for Parts } 3,4 \text {, and } 5 \text { for Hazardous Waste } \\
\text { Management activity performed under ADS number ID- } \\
100.2-C 1 \text { in a file saved in Lotus } 1-2-3 \text { Release } 3 \\
\text { format }\end{array}$ \\
\hline
\end{tabular}


APPENDIX A

\section{SITE TRANSMITTAL AND DATA ENTRY FORMS}

FOR THE SUMMARY REPORT

A-1 


\title{
DETERMINATION OF EM ENVIRONMENTAL
}

\author{
SAMPLING AND ANALYSIS
}

NEEDS FOR FY 93 - 99

\section{SUMMARY REPORT}

\begin{tabular}{|l|l|}
\hline Field Office: & \\
\hline Site: & \\
\hline $\begin{array}{l}\text { Manager Responsible for Site-Wide } \\
\text { Collection of Analytical Needs Data: }\end{array}$ & \\
\hline Telephone \#: & \\
\hline Summary Report Date: & \\
\hline
\end{tabular}

Performed For:

Laboratory Management Division (EM-563)

Office of Technology Development

Environmental Restoration and Waste Management

U.S. Department of Energy 
ENVIRONMENTAL SAMPLING \& ANALYSIS NEEDS ASSESSMENT

Part 2: Summary of ADS Activities

\begin{tabular}{|c|c|c|c|c|c|c|c|}
\hline \multicolumn{3}{|l|}{ SIZE: } & \multicolumn{5}{|c|}{ CATEGORY: } \\
\hline \multirow[b]{2}{*}{ ADS \# } & \multicolumn{2}{|c|}{ ACTIVITY } & \multicolumn{3}{|c|}{ UNITS } & \multirow{2}{*}{$\begin{array}{l}\text { ACTIVITY } \\
\text { CONTACT }\end{array}$} & \multirow[b]{2}{*}{ TELEPHONE } \\
\hline & CODE & DESCRIPTION & $\#$ & TYPE & $\begin{array}{l}\text { AVERAGE } \\
\text { SIZE }\end{array}$ & & \\
\hline & & & & & & & \\
\hline & & & & & & & \\
\hline & & & & & & & \\
\hline & & & & & & & \\
\hline & & & & & & & \\
\hline & & & & & & & \\
\hline & & & & & & & \\
\hline & & & & & & & \\
\hline & & & & & & & \\
\hline & & & & & & & \\
\hline & & & & & & & \\
\hline & & & & & & & \\
\hline & & & & & & & \\
\hline & & & & & & & \\
\hline & & & & & & & \\
\hline
\end{tabular}


ENVIRONMENTAL SAMPLING \& ANALYSIS NEEDS ASSESSMENT

Part 1: Summary of ADS Sampling and Analysis Requirements

\begin{tabular}{|c|c|c|c|c|c|}
\hline \multicolumn{2}{|c|}{ SITE: } & \multicolumn{4}{|c|}{ CATEGORY: } \\
\hline \multirow{3}{*}{ ADS \# } & \multirow{3}{*}{ ADS TITLE } & \multicolumn{4}{|c|}{$\begin{array}{l}\text { SAMPLING AND ANALYSIS } \\
(Y / N)\end{array}$} \\
\hline & & \multicolumn{2}{|c|}{ EM-563 DETERMINATION } & \multicolumn{2}{|c|}{ SITE CONFIRMATION } \\
\hline & & FY93 & FY94-99 & FY93 & FY94-99 \\
\hline & & & & & \\
\hline & & & & & \\
\hline & & & & & \\
\hline & & & & & \\
\hline & & & & & \\
\hline & & & & & \\
\hline & & & & & \\
\hline & & & & & \\
\hline & & & & & \\
\hline & & & & & \\
\hline & & & & & \\
\hline & & & & & \\
\hline & & & & & \\
\hline
\end{tabular}


APPENDIX B

BLANK TRANSMITTAL AND DATA ENTRY FORMS

FOR THE FINAL REPORT

B-1 


\title{
DETERMINATION OF EM ENVIRONMENTAL
}

\author{
SAMPLING AND ANALYSIS
}

NEEDS FOR FY 93 - 99

FINAL REPORT

\begin{tabular}{||l|l||}
\hline Field Office: & \\
\hline Site: & \\
\hline Manager Responsible for Site-Wide \\
Collection of Analytical Needs Data: & \\
\hline Telephone \#: & \\
\hline Final Report Date: & \\
\hline
\end{tabular}

Performed For:

Laboratory Management Division (EM-563)

Office of Technology Development

Environmental Restoration and Waste Management

U.S. Department of Energy 
ENVIRONMENTAL SAMPLING \& ANALYSIS NEEDS ASSESSMENT

Part 3: General Description of Activity

\begin{tabular}{|l|l|}
\hline Site: & \\
\hline ADS \#: & \\
\hline Activity Type: & \\
\hline
\end{tabular}

\begin{tabular}{|l|l|}
\hline \hline OVERALL ACTIVITY DESCRIPTION & \\
\hline General Description: & \\
\cline { 2 - 2 } & \\
\hline Start Date: & \\
\hline Anticipated End Date: & \\
\hline Regulatory Driver: & \\
\hline
\end{tabular}

\begin{tabular}{|l|l|}
\hline DESCRIPTION OF ACTIVITY IN FY 93 \\
\hline Activity Description: & \\
\cline { 2 - 2 } & \\
\hline Unit Type Addressed: & \\
\hline \# of Units Addressed: & \\
\hline Total FY 93 Activity Budget: & \\
\hline FY 93 S\&A Budget: & \\
\hline
\end{tabular}

\begin{tabular}{|l|l|}
\hline DESCRIPTION OF ACTIVITY IN FY 94-99 \\
\hline Activity Description: & \\
\cline { 2 - 3 } & \\
\hline Unit Type to be Addressed: & \\
\hline \# of Units to be Addressed: & \\
\hline Total FY 94-99 Activity Budget: & \\
\hline FY 94-99 S\&A Budget: & \\
\hline
\end{tabular}


ENVIRONMENTAL SAMPLING \& ANALYSIS NEEDS ASSESSMENT

Part 4: Activity Sampling and Analysis

Site:

ADS \#:

Activity Type:

GENERAL SAMPLING AND ANALYSIS DESCRIPTION

S\&A Start Date:

Anticipated S\&A End Date:

Samples Taken Prior to FY 93:

Sample Analyses Requested Prior to FY 93:

Samples Planned After FY 93:

Sample Anaiyses Planned After FY 93:

Average Batch Size:

SAMPLING AND ANALYSIS ACTIVITYINFY 93

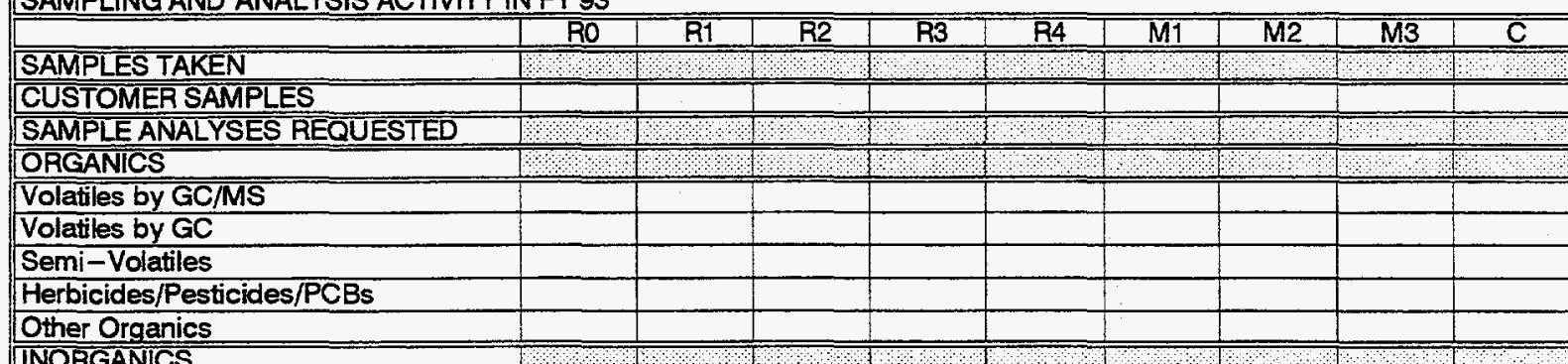

INO RG

RCRA Appendix IX Metals

CAM (California) Metals (RCRA)

RCRA TCLP Metals and EPTOX Metals

EPA CLP Metals (CERCLA)

NPDES Metals (Clean Water Act)

Drinking Water Metals (SDWA)

Metals by Other Regulatory Suite

Metals by Suite, Other

Metáls by Metal Except Mercury

Mercury

Anions/Cations - IC

Inorganics Wet Chemistry

Other Inorganics

RADIOACTIVES

Gross Alpha/Beta

Gamma Scan

Transuranic

Plutonium Isotopes

Uranium Isotopes

Curium Isotopes

Thorium Isotopes

Radium Isotopes

Americium-241

Neptunium-237

Polonium-210

Tritium

Radon-222

Lead-210

Carbon-14

Nickel-63

Selenium -79

Technetium-99

Strontium Isotopes

Strontium-89

Strontium-90

lodine-129

Nickel-59

Total Uranium

Total Thorium

Total Strontium

Other RAD

OTHER

Other Wet Chemistry

TCLP Extraction

Asbestos

Industrial Hygiene, NOS

Physical Properties

Other, Not Otherwise Specified (NOS)

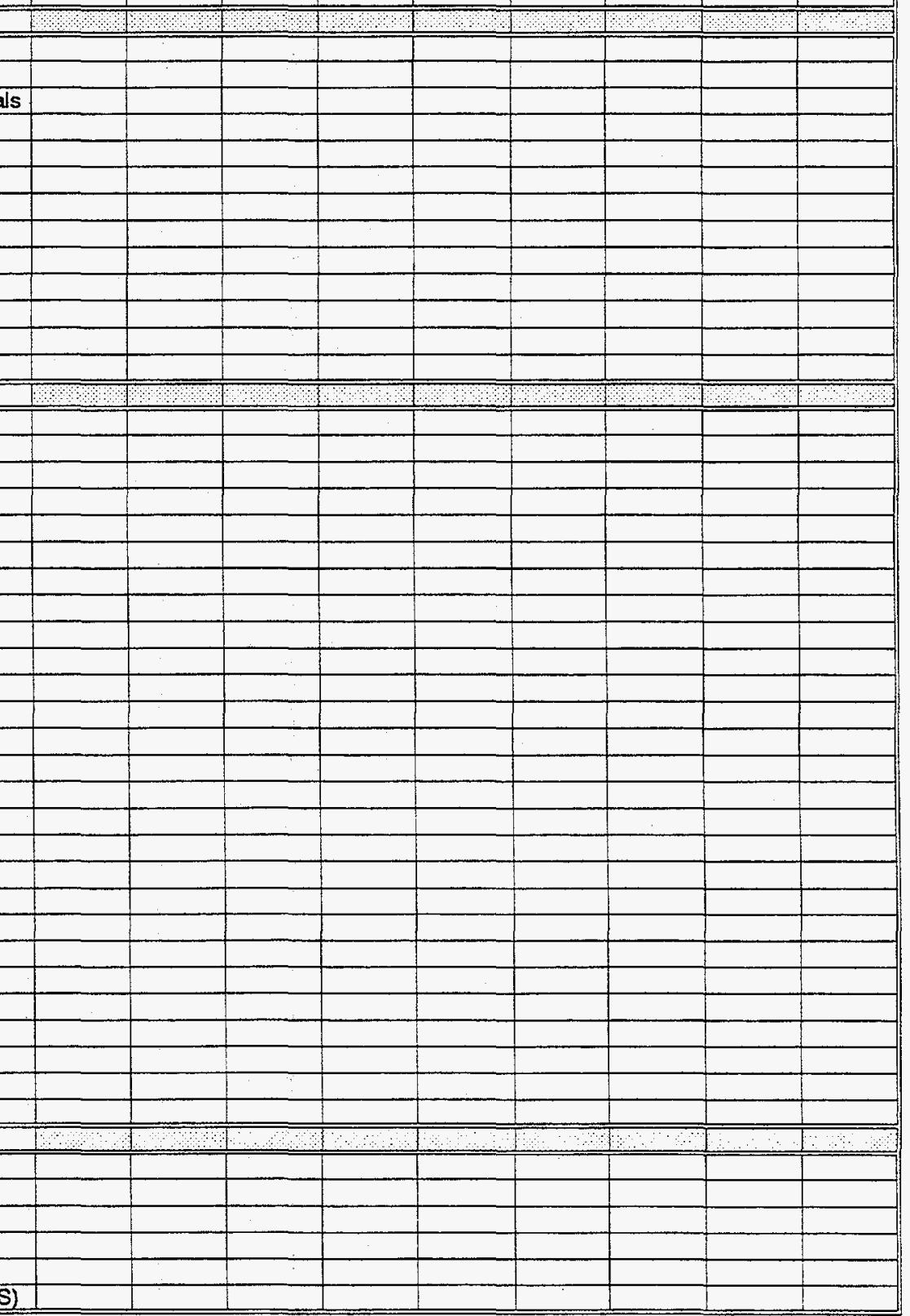


ENVIRONMENTAL SAMPLING \& ANALYSIS NEEDS ASSESSMENT

Part 5: Activity Documentation

\section{Site:}

ADS \#:

Activity Type:

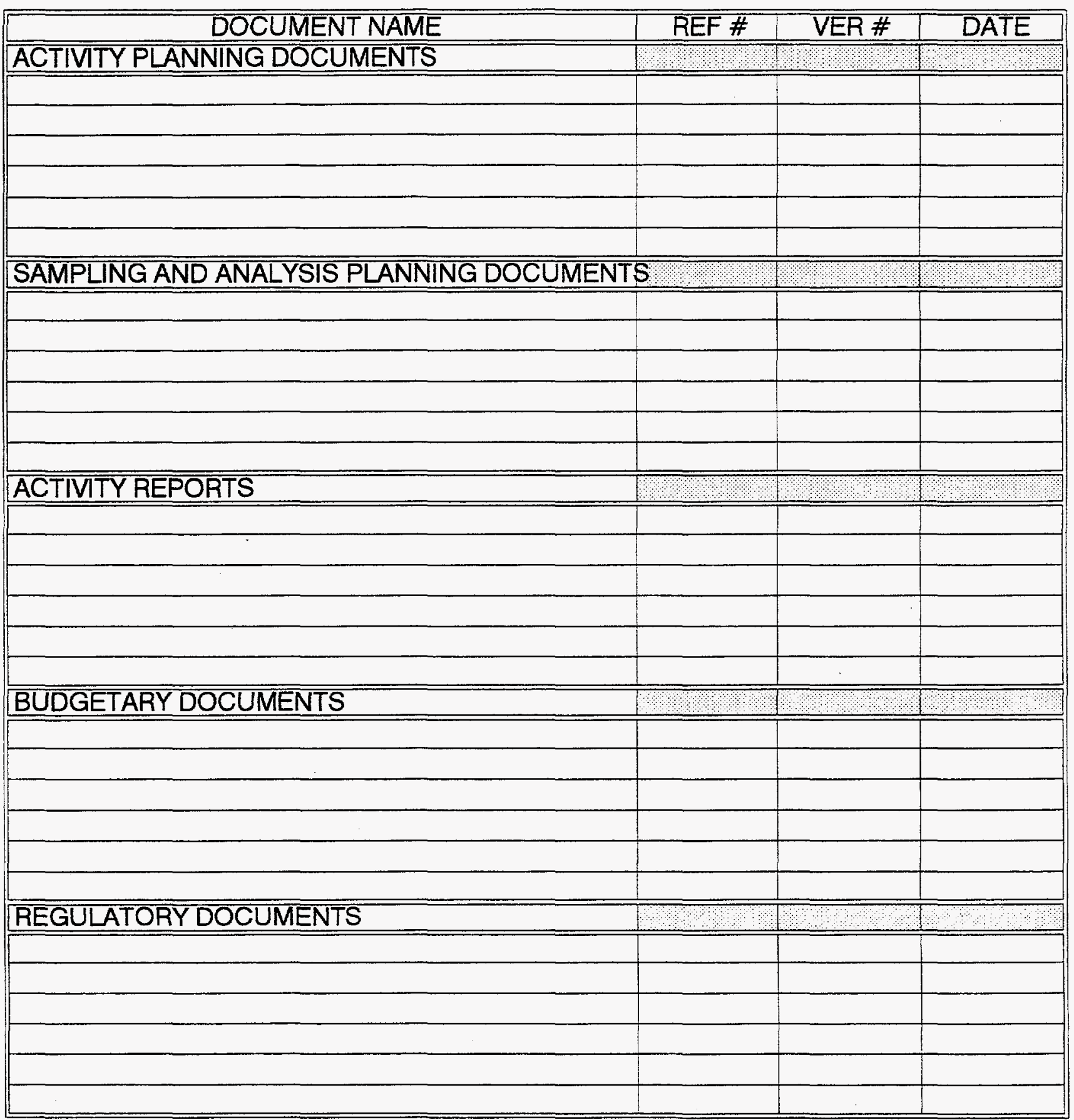


APPENDIX C

COMPLETED DATASHEET EXAMPLES

C-1 


\section{EXAMPLE \# 1: PART 3 OF NEEDS ASSESSMENT}

Waste Area Group (WAG) 1 at Idaho National Engineering Laboratory (INEL) includes 71 potential release sites in 10 Operable Units (OU). An assessment (PA/SI) will be conducted for each of the 71 potential release sites. These assessments will determine which sites require additional investigation as part of the RI/FS. One comprehensive RI/FS will be performed for WAG 1, with field activities scheduled to start in FY96.

For this example, field sampling activities will be conducted at 24 of the 71 sites in FY 93. $27 \mathrm{PA} / \mathrm{Sl}$ s were conducted prior to FY 93. The total budget for PA/SI activities in $F Y 93$ is $\$ 4,002,000$. The sampling and analysis budget for $F Y 93$ $\mathrm{PA} / \mathrm{SIs}$ is $\$ 1,200,000$. The remaining 20 sites will be investigated during FY 94 and FY 95. The total budget for the PA/Sls is $\$ 12,000,000$, the sampling and analysis budget is $\$ 2,800,000$. The other activities associated with this $A D S$, the comprehensive Rl/FS and any remedial actions (RD/RA), will have separate datasheets (Parts 3, 4, and 5) for the Needs Assessment. 
ENVIRONMENTAL SAMPLING \& ANALYSIS NEEDS ASSESSMENT

Part 3: General Description of Activity

\begin{tabular}{|l|l|}
\hline Site: & Idaho Nat Eng Lab \\
\hline ADS \#: & ID-28-EG \\
\hline Activity Type: & PASI \\
\hline
\end{tabular}

\section{OVEAALI ACTIVITY DESCAIPTION}

General Description:

Start Date:

Anticipated End Date:

Regulatory Driver:

\begin{tabular}{l} 
Assessment of Test Area North for 71 potential \\
release sites in 10 OUs under WAG 1 \\
\hline $02-18-91$ \\
$08-01-95$ \\
CERCLA
\end{tabular}

\section{DESCRIPTION OF ACTIVITYIN FY 93}

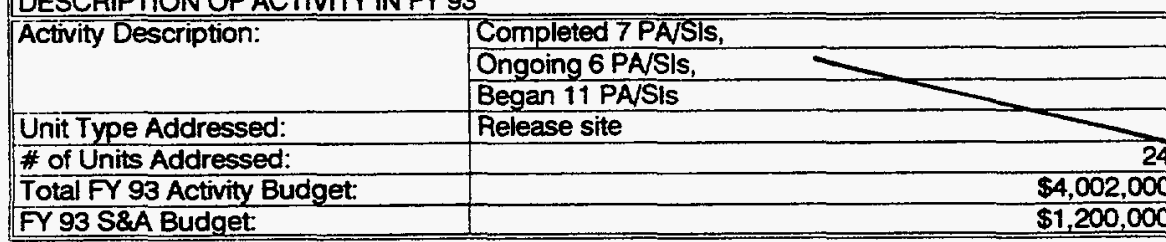

DESCAIPTION OF ACTIVITY IN FY 94-99 Activity Description:

Complete all PA/SIs including remaining 20

Unit Type to be Addressed:

\# of Units to be Addressed:

Total FY 94-99 Activity Budget:

FY 94-99 S\&A Budget:
Release site

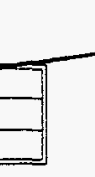

Use activity code from Table 1.

Enter only one regulatory driver.

Enter only one type of activity (e.g. PA/SI) for each set of datasheets (Parts 3, 4, and 5). Another set of datasheets to be completed for the RI/FS. 
The PA/Sls conducted in FY 93 resulted in the collection of 2023 customer samples; 150 groundwater samples, 1850 soil samples, and 23 sludge samples. The radioactivity level of each of the samples was measured and resulted in the following distribution:

7 samples of medium activity were handled in a shielded hood 112 samples of very low activity were handled in a radiation area 1904 samples were non-radioactive

Nonradioactive samples were not analyzed for radioactive components. Seventy samples were collected for characterizing investigation derived waste (IDW) for disposal purposes to determine if the wastes met LDR requirements. These samples were first prepared using the TCLP extraction procedure prior to sample analysis.

Because of the various materials reported to be disposed of in the 24 potential release sites, a different suite of analyses were required for each customer sample. The PA/SI work plans and the laboratory analytical reports confirmed the total quantity of samples analyzed by matrix, by activity level, and by analytical method. The example datasheet for Part 4 is filled in with the hypothetical analyses requested. 
Cells in header section are

automatically copied from Part

3 , when using Lotus $1-2-3$.

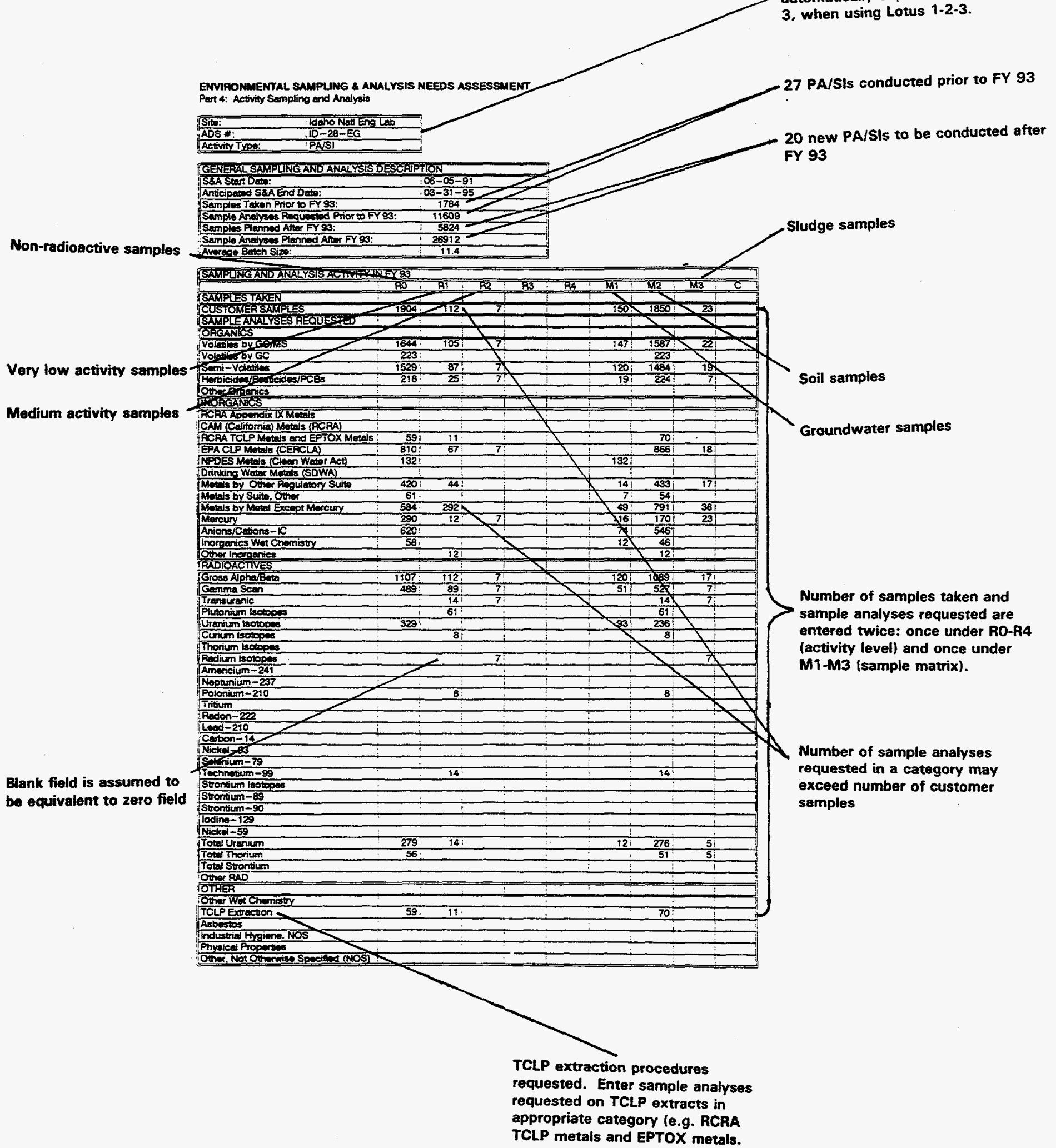


APPENDIX D

GLOSSARY OF TERMS

D-1 


\section{INTRODUCTION}

This glossary of terms has been designed to capture and standardize terms used in the EM analytical needs assessment. Efforts have been made to ensure that the terms are as consistent as possible with those used in the National Sample Tracking System (NSTS) and the EM analytical capacity determination. The Glossary of Terms is organized into the following five sections:

- Organization/Management

- Key Types of Environmental Activities

- Sampling and Analysis

- Customer Sample Characteristics

- Sample Analyses 


\begin{tabular}{|c|c|}
\hline TERM & DEFINITION \\
\hline \multicolumn{2}{|r|}{ Organization / Management } \\
\hline ADS & $\begin{array}{l}\text { Activity Data Sheet -- a document used to describe } \\
\text { the scope of work, requirements, and budget of } \\
\text { specific projects in DOE's Office of Environmental } \\
\text { Restoration and Waste Management (EM). }\end{array}$ \\
\hline Site & $\begin{array}{l}\text { Land, installation, and/or facility for which DOE has or } \\
\text { shares responsibility for EM activities. }\end{array}$ \\
\hline Activity Type & $\begin{array}{l}\text { A series of operations performed to conform to } \\
\text { specific regulatory drivers. Key environmental activity } \\
\text { types are given in the following section. }\end{array}$ \\
\hline Unit & $\begin{array}{l}\text { A discrete portion of the site or waste undergoing } \\
\text { investigation or cleanup. Examples of unit types } \\
\text { include solid waste management units (SWMUs), } \\
\text { operable units (OUs), waste area groups (WAGs), } \\
\text { tanks, drums, buildings, or pits. }\end{array}$ \\
\hline \multicolumn{2}{|r|}{ Key Types of Environmental Activities } \\
\hline $\begin{array}{l}\text { Preliminary } \\
\text { Assessment/ } \\
\text { Site Inspection } \\
\text { (PA/SI) }\end{array}$ & $\begin{array}{l}\text { Preliminary Assessment/Site Inspection or RCRA } \\
\text { Facility Assessment (RFA) performed under CERCLA } \\
\text { or RCRA, respectively. A PA is a review of existing } \\
\text { information and an off-site and/or on-site } \\
\text { reconnaissance to determine if a release may require } \\
\text { additional investigation or action. An SI is an on-site } \\
\text { investigation to determine whether there is a release } \\
\text { or potental release and the nature of the associated } \\
\text { threats. An RFA is the RCRA version of the PA/SI, } \\
\text { and involves a review of existing information and } \\
\text { potentially some sample collection and analysis. } \\
\text { These activities include sampling and analysis (S\&A) } \\
\text { of environmental media, and investigation-derived } \\
\text { wastes (IDW) for disposal purposes. PA/SIs and RFAs } \\
\text { normally require a minimal level of S\&A. }\end{array}$ \\
\hline
\end{tabular}




\begin{tabular}{|c|c|}
\hline TERM & DEFINITION \\
\hline $\begin{array}{l}\text { Remedial Investigation/ } \\
\text { Feasibility Study } \\
\text { (RI/FS) }\end{array}$ & $\begin{array}{l}\text { Remedial investigation (including risk assessment) and } \\
\text { feasibility study (including treatability study) or RCRA } \\
\text { Facility Investigation (RFI) and Corrective Measures } \\
\text { Study (CMS) performed under CERCLA or RCRA, } \\
\text { respectively. An RI is a process to determine the } \\
\text { nature and extent of the problem presented by the } \\
\text { release, and emphasizes data collection and site } \\
\text { characterization. An FS is a study to develop and } \\
\text { evaluate options for remedial action and emphasizes } \\
\text { data analysis. The RI and FS are generally performed } \\
\text { concurrently. The RFI and CMS are the RCRA } \\
\text { equivalents of the RI/FS. The RFI characterizes the } \\
\text { nature and extent of the contamination, and the CMS } \\
\text { identifies and evaluates corrective remedies. These } \\
\text { activities include S\&A of environmental media, } \\
\text { treatment residuals, and IDW. Sampling and analysis } \\
\text { needs are dependent on the size of the operable unit } \\
\text { and the number of environmental matrices impacted } \\
\text { by past waste management practices. }\end{array}$ \\
\hline $\begin{array}{l}\text { Remedial Design/ } \\
\text { Remedial Action } \\
\text { (RD/RA) }\end{array}$ & $\begin{array}{l}\text { Remedial design and remedial action or corrective } \\
\text { measure/action performed under CERCLA or RCRA, } \\
\text { respectively, including interim removal actions or } \\
\text { corrective measures. RD is the technical analysis and } \\
\text { preparation of the plans and specifications for the } \\
\text { selected remedy. RA is the action(s) taken in the } \\
\text { event of a release or threatened release. The } \\
\text { corrective measure/action is the RCRA version of the } \\
\text { RD/RA, and consists of the same types of actions. } \\
\text { These activities include S\&A of environmental media, } \\
\text { IDW, treatment residuals, and confirmatory S\&A. The } \\
\text { sampling needs for RD/RA activities are dependent on } \\
\text { the chosen remedial option. Excavation and disposal, } \\
\text { groundwater pump-and-treat, soil vapor extraction, } \\
\text { and biotreatment each have different S\&A needs, } \\
\text { which are dependent on both the size of the operable } \\
\text { unit and the length of time needed to achieve the } \\
\text { cleanup standards. S\&A needs for RD/RA activities } \\
\text { are evaluated for each operable unit. }\end{array}$ \\
\hline
\end{tabular}




\begin{tabular}{|c|c|}
\hline TERM & DEFINITION \\
\hline $\begin{array}{l}\text { RCRA Facility Closure } \\
\text { (RFC) }\end{array}$ & $\begin{array}{l}\text { Closure of interim status waste management units } \\
\text { (units for which a Part A permit application has been } \\
\text { filed) and RCRA-permitted waste management units. } \\
\text { Closures control, minimize, or eliminate the escape of } \\
\text { hazardous constituents to the environment by } \\
\text { removing or capping the contaminants. RCRA } \\
\text { closures include confirmatory S\&A for clean closure. }\end{array}$ \\
\hline $\begin{array}{l}\text { Decontamination and } \\
\text { Decommissioning } \\
\text { (D\&D) }\end{array}$ & $\begin{array}{l}\text { Decontamination and decommissioning of buildings, } \\
\text { structures, processing equipment, hot cells, } \\
\text { experimental reactors, vehicles, etc. prior to salvage, } \\
\text { demolition, disposal, and/or recommissioning. D\&D } \\
\text { typically involves S\&A of D\&D-derived waste (e.g., } \\
\text { sandblasting residue) for treatment/disposal and } \\
\text { confirmatory S\&A. }\end{array}$ \\
\hline $\begin{array}{l}\text { Groundwater } \\
\text { Monitoring } \\
\text { (GW MON) }\end{array}$ & $\begin{array}{l}\text { Monitoring of groundwater as specified in any } \\
\text { compliance monitoring program. This includes RCRA } \\
\text { interim status groundwater monitoring ( } \$ 265 \text { Subpart } \\
\text { F); detection, compliance, and corrective action } \\
\text { effectiveness monitoring for RCRA-permitted units } \\
\text { (\$264 Subpart F); and long-term monitoring of } \\
\text { CERCLA operable units. }\end{array}$ \\
\hline $\begin{array}{l}\text { Effluent Water } \\
\text { Monitoring } \\
\text { (EFF MON) }\end{array}$ & $\begin{array}{l}\text { Monitoring of aqueous discharges regulated by an } \\
\text { existing NPDES, SPDES, or storm water permit, or } \\
\text { discharges to a publicly owned treatment works } \\
\text { (POTW). }\end{array}$ \\
\hline $\begin{array}{l}\text { Transuranic Waste } \\
\text { Management/Disposal } \\
\text { (TRU) }\end{array}$ & $\begin{array}{l}\text { Management of DOE's TRU. Involves S\&A for storage } \\
\text { (e.g., screening and repackaging), treatment, and } \\
\text { ultimate disposal in a geologic repository (such as } \\
\text { WIPP). S\&A needs are evaluated for each site } \\
\text { managing TRU. }\end{array}$ \\
\hline
\end{tabular}




\begin{tabular}{|c|c|}
\hline TERM & DEFINITION \\
\hline $\begin{array}{l}\text { Hazardous Waste } \\
\text { Management/Disposal } \\
\text { (HAZ) }\end{array}$ & $\begin{array}{l}\text { Sampling and analysis of wastes generated from } \\
\text { routine, day-to-day activities as required for } \\
\text { management and disposal purposes. This includes } \\
\text { routine site functions such as operational data for } \\
\text { waste treatment units, waste storage, and disposal. } \\
\text { This category also includes TCLP extractions, and } \\
\text { subsequent analyses, to determine whether the } \\
\text { wastes meet land disposal restrictions }(\$ 268) \text {, } \\
\text { delisting of wastes ( } \$ 260.20 \text { and } \$ 260.22) \text {, and any } \\
\text { special analytical requirements of commercial facility } \\
\text { accepting the waste. }\end{array}$ \\
\hline $\begin{array}{l}\text { Mixed Waste/Low- } \\
\text { Level Waste } \\
\text { Management/Disposal } \\
\text { (MIX/LLW) }\end{array}$ & $\begin{array}{l}\text { Sampling and analysis of mixed and low-level wastes } \\
\text { as required for management and disposal purposes. } \\
\text { Mixed and low-level waste activities are similar to } \\
\text { hazardous waste management and disposal activities. }\end{array}$ \\
\hline $\begin{array}{l}\text { High-Level Waste } \\
\text { Management } \\
\text { (HLW) }\end{array}$ & $\begin{array}{l}\text { Management of DOE's HLW. Involves S\&A for } \\
\text { storage (e.g., tank safety monitoring), treatment (e.g., } \\
\text { grouting, vitrification), and ultimate disposal in a } \\
\text { geologic repository. S\&A needs are evaluated for } \\
\text { each site managing HLW. }\end{array}$ \\
\hline $\begin{array}{l}\text { Environmental } \\
\text { Monitoring } \\
\text { (ENV MON) }\end{array}$ & $\begin{array}{l}\text { Long-term monitoring and surveillance of other } \\
\text { environmental media. This includes room/air stack } \\
\text { analyses, bioassays, and quantitative environmental } \\
\text { assessments (such as benthic and microorganism } \\
\text { populations). S\&A needs for environmental } \\
\text { monitoring are evaluated for each site. }\end{array}$ \\
\hline $\begin{array}{l}\text { Underground Storage } \\
\text { Tanks } \\
\text { (UST) }\end{array}$ & $\begin{array}{l}\text { Sampling and analysis of environmental media as a } \\
\text { result of a suspected leaking underground storage } \\
\text { tank (non-radioactive). This includes confirmatory } \\
\text { S\&A after tank removal/replacement. }\end{array}$ \\
\hline $\begin{array}{l}\text { Miscellaneous } \\
\text { RCRA/CERCLA } \\
\text { (MISC R/C) }\end{array}$ & $\begin{array}{l}\text { Other RCRA or CERCLA activities not described by } \\
\text { previously-defined activity types. }\end{array}$ \\
\hline \multicolumn{2}{|r|}{ Sampling \& Analysis } \\
\hline Sample & $\begin{array}{l}\text { A portion of material considered representative of the } \\
\text { whole. }\end{array}$ \\
\hline Field Sample & $\begin{array}{l}\text { Material representing a single spacial and temporal } \\
\text { point. }\end{array}$ \\
\hline
\end{tabular}




\begin{tabular}{|c|c|}
\hline TERM & DEFINITION \\
\hline Sample Phase & $\begin{array}{l}\text { One physical phase of a field sample (field samples } \\
\text { may consist of one or more physical phases) -- } \\
\text { multiple phases may or may not be analyzed } \\
\text { separately. }\end{array}$ \\
\hline Composite Sample & $\begin{array}{l}\text { A combination of two or more field samples and/or } \\
\text { sample phases to be analyzed as a single customer } \\
\text { sample. }\end{array}$ \\
\hline Customer Sample & $\begin{array}{l}\text { Any sample sent to the laboratory to be analyzed } \\
\text { separately. This includes field samples, field OA } \\
\text { samples (e.g., duplicate field sample, field blank), } \\
\text { sample phases, and composite samples. It does not, } \\
\text { however, include laboratory-generated samples such } \\
\text { as laboratory OC samples. }\end{array}$ \\
\hline Laboratory Sample & Any sample analyzed in the laboratory. \\
\hline Laboratory QC Sample & $\begin{array}{l}\text { Any sample the laboratory has to analyze that is not a } \\
\text { customer sample. This includes laboratory duplicates, } \\
\text { laboratory blanks, and laboratory calibration check } \\
\text { standards. }\end{array}$ \\
\hline Sample Analysis & $\begin{array}{l}\text { The set of laboratory operations required to generate } \\
\text { data acceptable to the customer for a distinct } \\
\text { requested measurement (e.g., volatile organics by } \\
\text { GC/MS). }\end{array}$ \\
\hline Determination & Measurement of an analyte. \\
\hline Analyte & $\begin{array}{l}\text { A single physical or chemical characteristic (i.e., it is } \\
\text { represented by a single datum point). }\end{array}$ \\
\hline \multicolumn{2}{|r|}{ Customer Sample Characteristics } \\
\hline RO & $\begin{array}{l}\text { Customer sample deemed non-radioactive, no } \\
\text { NRC/State license required, sample may be handled } \\
\text { out of radiation control zone. }\end{array}$ \\
\hline R1 & $\begin{array}{l}\text { License required, customer sample must be handled in } \\
\text { a radiation control zone. (For the purposes of DOE's } \\
\text { Analytical Needs Assessment, the activity of a sample } \\
\text { in this category is estimated to be }<10 \mathrm{mR} / \mathrm{hr} \text { on } \\
\text { contact, }<100 \mathrm{nCi} \text { alpha/g, or }<1.00 \mathrm{nCi} \text { alpha/ml.) }\end{array}$ \\
\hline
\end{tabular}




\begin{tabular}{|c|c|}
\hline TERM & DEFINITION \\
\hline $\mathbf{R} 2$ & $\begin{array}{l}\text { License required, customer sample must be handled in } \\
\text { a shielded hood. (For the purposes of DOE's } \\
\text { Analytical Needs Assessment, the activity of a sample } \\
\text { in this category is estimated to be } 10-200 \mathrm{mR} / \mathrm{hr} \text { on } \\
\text { contact, }<100 \mathrm{nCi} \text { alpha/g, or }<100 \mathrm{nCi} \text { alpha/ml.) }\end{array}$ \\
\hline R3 & $\begin{array}{l}\text { License required, customer sample must be handled in } \\
\text { glove box. (For the purposes of DOE's Analytical } \\
\text { Needs Assessment, the activity of a sample in this } \\
\text { category is estimated to be }<200 \mathrm{mR} / \mathrm{hr} \text { on contact, } \\
>100 \mathrm{nCi} \text { alpha/g, or }>100 \mathrm{nCi} \text { alpha/ml.) }\end{array}$ \\
\hline R4 & $\begin{array}{l}\text { License required, customer sample must be handled in } \\
\text { hot cell. (For the purposes of DOE's Analytical Needs } \\
\text { Assessment, the activity of a sample in this category } \\
\text { is estimated to be }>200 \mathrm{mR} / \mathrm{hr} \text { on contact.) }\end{array}$ \\
\hline M1 & $\begin{array}{l}\text { Relatively easy customer sample matrix for analytical } \\
\text { operations. Includes: water. }\end{array}$ \\
\hline M2 & $\begin{array}{l}\text { More difficult customer sample matrix for analytical } \\
\text { operations. Includes: soil, sediment, rock, air, biota. }\end{array}$ \\
\hline M3 & $\begin{array}{l}\text { Most difficult customer sample matrix for analytical } \\
\text { operations. Includes: sludge, waste, pure product, } \\
\text { debris. }\end{array}$ \\
\hline \multicolumn{2}{|r|}{ Key Sample Analyses ${ }^{2}$} \\
\hline Americium-241 & Analysis for americium- 241 . \\
\hline Anions/Cations-IC & $\begin{array}{l}\text { Analysis by lon Chromatography runs for simultaneous } \\
\text { analyses. }\end{array}$ \\
\hline Asbestos & Analysis for asbestos. \\
\hline $\begin{array}{l}\text { CAM (California) } \\
\text { Metals (RCRA) }\end{array}$ & $\begin{array}{l}\text { Metals analysis as specified under the California list } \\
\text { for RCRA. }\end{array}$ \\
\hline Carbon-14 & Analysis for carbon- 14 . \\
\hline
\end{tabular}

2 Each of the key sample analyses listed here may, for some applications, be performed using different methods for a specific sample matrix or sample radioactivity. For example, a particular laboratory might perform the VOA analysis by GC/MS using two distinct sample analyses: EPA method 524.2 on non-rad waters (RO, M1), and EPA method 624 on low level soils (R1, M2). 


\begin{tabular}{|c|c|}
\hline TERM & DEFINITION \\
\hline Curium Isotopes & $\begin{array}{l}\text { Analysis by alpha spectroscopy for isotopes of curium. } \\
\text { For reporting purposes, each set of such analyses is to } \\
\text { be reported as one curium isotope analysis. }\end{array}$ \\
\hline $\begin{array}{l}\text { Drinking Water } \\
\text { Metals - Safe Drinking } \\
\text { Water Act (SDWA) }\end{array}$ & $\begin{array}{l}\text { Metals analysis for metals specified for SDWA } \\
\text { compliance. }\end{array}$ \\
\hline $\begin{array}{l}\text { EPA Contract } \\
\text { Laboratory Program } \\
\text { Metals (CERCLA) }\end{array}$ & $\begin{array}{l}\text { Metals analysis for the list of } 23 \text { metals as specified } \\
\text { under the CERCLA Target Compound List for the } \\
\text { Contract Laboratory Program Inorganics Statement of } \\
\text { Work. }\end{array}$ \\
\hline Gamma Scan & Gamma spectroscopic analysis of radionuclides. \\
\hline Gross Alpha/Beta & $\begin{array}{l}\text { Gross alpha and/or gross beta analysis. If ordered } \\
\text { separately, each gross alpha or gross beta analysis is } \\
\text { counted as one gross alpha/beta analysis. }\end{array}$ \\
\hline $\begin{array}{l}\text { Herbicides/Pesticides/ } \\
\text { PCBs }\end{array}$ & $\begin{array}{l}\text { Herbicide, pesticide, or PCB analysis. For example, } \\
\text { this includes Methods } 508,608,615,8080,8150, \\
8280 \text {, and dioxins/furans. }\end{array}$ \\
\hline $\begin{array}{l}\text { Industrial Hygiene, } \\
\text { NOS }\end{array}$ & $\begin{array}{l}\text { Sample analyses not otherwise specified, which are } \\
\text { performed for human health monitoring purposes, (e.g. } \\
\text { fecal coliform). }\end{array}$ \\
\hline $\begin{array}{l}\text { Inorganic Wet } \\
\text { Chemistry }\end{array}$ & $\begin{array}{l}\text { Analyses for inorganic elements or compounds using } \\
\text { wet chemistry. Includes } \mathrm{CN} \text {, nitrate, sulfide, } \mathrm{F}, \mathrm{Cl} \text {, } \\
\text { ALK, sulfate, } \mathrm{NO}_{3}, \mathrm{NO}_{2}, \mathrm{NH}_{3}, \mathrm{P}, \mathrm{PO}_{4} \text {, total phosphate, } \\
\text { total organic nitrogen, total and partial organic carbon, } \\
\text { and bromide as examples. }\end{array}$ \\
\hline Inorganics & $\begin{array}{l}\text { Analyses of samples for the presence and/or quantity } \\
\text { of elements and inorganic compounds. }\end{array}$ \\
\hline lodine-129 & Analysis for iodine-129. \\
\hline Lead-210 & Analysis for lead-210. \\
\hline Mercury & $\begin{array}{l}\text { Analysis for mercury when not ordered as part of a } \\
\text { regulatory suite. }\end{array}$ \\
\hline
\end{tabular}




\begin{tabular}{|c|c|}
\hline TERM & DEFINITION \\
\hline $\begin{array}{l}\text { Metals by Metal } \\
\text { Except Mercury }\end{array}$ & $\begin{array}{l}\text { One or more metals (other than mercury) ordered for } \\
\text { analysis on a particular sample which are not part of a } \\
\text { regulatory suite, and which cannot be analyzed using } \\
\text { the same sample preparation. For reporting purposes, } \\
\text { each individual metal so ordered counts as one sample } \\
\text { analysis. }\end{array}$ \\
\hline Metals by Suite, Other & $\begin{array}{l}\text { A group of metals ordered together for analysis on a } \\
\text { particular sample, which can be analyzed using the } \\
\text { same sample preparation. For reporting purposes, } \\
\text { each such group counts as one sample analysis. }\end{array}$ \\
\hline $\begin{array}{l}\text { Metals by Other } \\
\text { Regulatory Suite }\end{array}$ & $\begin{array}{l}\text { A group of metals ordered for analysis on a particular } \\
\text { sample, which group's constituents and methods of } \\
\text { analysis are defined by Federal or state regulations } \\
\text { other than those otherwise specified (i.e., other than } \\
\text { RCRA Appendix IX, CAM, TCLP, CLP, NPDES, or } \\
\text { SDWA). For reporting purposes, each such group } \\
\text { counts as one sample analysis. }\end{array}$ \\
\hline Neptunium-237 & Analysis for neptunium-237. \\
\hline Nickel-59 & Analysis for nickel-59. \\
\hline Nickel-63 & Analysis for nickel- 63 . \\
\hline $\begin{array}{l}\text { NPDES Metals (Clean } \\
\text { Water Act) }\end{array}$ & $\begin{array}{l}\text { Metals analysis for metals specified for NPDES } \\
\text { compliance. }\end{array}$ \\
\hline Organics & $\begin{array}{l}\text { Organic analyses of samples, which may include VOA, } \\
\text { semi-volatile organic analysis, pesticide/ } \\
\text { polychlorinated biphenyl/herbicide analysis, etc. }\end{array}$ \\
\hline Other Inorganics & $\begin{array}{l}\text { Other inorganic analyses. Includes } \mathrm{x} \text {-ray fluorescence, } \\
\mathrm{x} \text {-ray metals, analysis in the field by photovac, argon, } \\
\text { halogens, and silica as examples. }\end{array}$ \\
\hline Other Analyses, NOS & $\begin{array}{l}\text { All analyses which do not fall within the definitions for } \\
\text { organics, inorganics, or RAD analyses, and which do } \\
\text { not fall within any other analysis type specified. } \\
\text { Includes chemical dynamic processes such as } \\
\text { Langelier Saturation Index. }\end{array}$ \\
\hline Other An & $\begin{array}{l}\text { Sample analyses which do not fall within the } \\
\text { definitions for organics, inorganics, or RAD analyses. }\end{array}$ \\
\hline
\end{tabular}




\begin{tabular}{|c|c|}
\hline TERM & DEFINITION \\
\hline Other Organics & $\begin{array}{l}\text { Other organic analyses. Includes atmospheric gases, } \\
\text { additive fingerprint, high explosive compounds, HPLC } \\
\text { analyses, acrylonitrile, and surfactants. }\end{array}$ \\
\hline Other RAD & $\begin{array}{l}\text { Other radiochemical/radiological analyses. Includes } \\
\text { radiological screening, chlorine isotopes, cobalt- } 58 \text {, } \\
\text { and cobalt- } 60 \text {. }\end{array}$ \\
\hline Other Wet Chemistry & $\begin{array}{l}\text { Analyses using wet chemistry which are not otherwise } \\
\text { specified. Includes BOD, COD, TPH, TOX, oil and } \\
\text { grease, TOC, TSS, TDS, residue, orthophosphate, and } \\
\text { TKN. }\end{array}$ \\
\hline Physical Properties & $\begin{array}{l}\text { Analyses for non-chemical properties. Includes soil } \\
\text { moisture content, grain size, RCRA characteristics } \\
\text { (excluding TCLP), permeability, pH, hardness, cation } \\
\text { exchange capacity, temperature, field soil vapor, and } \\
\text { specific conductance. }\end{array}$ \\
\hline Plutonium Isotopes & $\begin{array}{l}\text { Analysis by alpha spectroscopy for isotopes of } \\
\text { plutonium. For reporting purposes, each set of such } \\
\text { analyses is to be reported as one plutonium isotope } \\
\text { analysis. }\end{array}$ \\
\hline Polonium-210 & Analysis for polonium-210. \\
\hline Radioactives & $\begin{array}{l}\text { Radiochemical/radiological analyses of samples which } \\
\text { may include gross alpha, gross beta, gamma scan, } \\
\text { isotopic analysis, etc. }\end{array}$ \\
\hline Radium Isotopes & $\begin{array}{l}\text { Analysis by alpha spectroscopy for isotopes of radium. } \\
\text { For reporting purposes, each set of such analyses is to } \\
\text { be reported as one radium isotope analysis. }\end{array}$ \\
\hline Radon-222 & Analysis for radon-222. \\
\hline $\begin{array}{l}\text { RCRA TCLP Metals } \\
\text { and EPTOX Metals }\end{array}$ & $\begin{array}{l}\text { Metals analysis for the list of eight metals as specified } \\
\text { in RCRA in conjunction with the TCLP extraction. }\end{array}$ \\
\hline $\begin{array}{l}\text { RCRA Appendix IX } \\
\text { Priority Pollutant } \\
\text { Metals }\end{array}$ & $\begin{array}{l}\text { Metals analysis for the list of } 25 \text { metals as specified } \\
\text { within RCRA Appendix IX (or a subset of the list) } \\
\text { which are commonly requested for groundwater } \\
\text { contaminant analysis. }\end{array}$ \\
\hline Selenium-79 & Analysis for selenium-79. \\
\hline
\end{tabular}




\begin{tabular}{|c|c|}
\hline TERM & DEFINITION \\
\hline Semi-Volatiles & $\begin{array}{l}\text { Semi-volatile organic analysis (e.g., Methods } 525 \text {, } \\
625,8270 \text {, packed column, and capillary column). }\end{array}$ \\
\hline Strontium Isotopes & $\begin{array}{l}\text { A single sample analysis for determining both } \\
\text { strontium- } 89 \text { and strontium } 90 \text {. }\end{array}$ \\
\hline Strontium-89 & Analysis for strontium-89 only. \\
\hline Strontium-90 & Analysis for strontium-90 only. \\
\hline TCLP Extraction & $\begin{array}{l}\text { Toxicity Characteristic Leaching Procedure; extraction } \\
\text { procedure only (e.g., Method 1311). }\end{array}$ \\
\hline Technetium-99 & Analysis for technetium- 99 . \\
\hline Thorium Isotopes & $\begin{array}{l}\text { Analysis by alpha spectroscopy for isotopes of } \\
\text { thorium. For reporting purposes, each set of such } \\
\text { analyses is to be reported as one thorium isotope } \\
\text { analysis. }\end{array}$ \\
\hline Total Strontium & $\begin{array}{l}\text { Analysis for total of all isotopes (radioactive and non- } \\
\text { radioactive) of strontium. }\end{array}$ \\
\hline Total Thorium & $\begin{array}{l}\text { Analysis for total of all isotopes (radioactive and non- } \\
\text { radioactive) of thorium. }\end{array}$ \\
\hline Total Uranium & $\begin{array}{l}\text { Analysis for total of all isotopes (radioactive and non- } \\
\text { radioactive) of uranium. }\end{array}$ \\
\hline Transuranic & $\begin{array}{l}\text { Analysis by alpha spectroscopy for isotopes of } \\
\text { plutonium, neptunium-237, americium- } 241 \text {, curium- } \\
242 \text {, and curium- } 243 / 244 \text {. For reporting purposes, } \\
\text { each order for analysis of this group of transuranic } \\
\text { isotopes for a particular customer sample counts as } \\
\text { one transuranic analysis. Orders for subsets and } \\
\text { supersets of this group are to be reported separately } \\
\text { under individual categories, and not under this } \\
\text { category. }\end{array}$ \\
\hline Tritium & Analysis for tritium. \\
\hline Uranium Isotopes & $\begin{array}{l}\text { Analysis by alpha spectroscopy for isotopes of } \\
\text { uranium. For reporting purposes, each set of such } \\
\text { analyses is to be reported as one uranium isotope } \\
\text { analysis. }\end{array}$ \\
\hline
\end{tabular}




\begin{tabular}{|c|c|}
\hline TERM & DEFINITION \\
\hline Volatiles by GC/MS & $\begin{array}{l}\text { Volatile organic analysis performed on GC/MS } \\
\text { instruments, (e.g., Methods } 524,624,8240,8260 \text {, } \\
\text { head space analysis, purge and trap). }\end{array}$ \\
\hline Volatiles by GC & $\begin{array}{l}\text { Volatile organic analysis performed on GC } \\
\text { instruments. For example, volatiles by GC includes } \\
\text { purgeable aromatics, purgeable hydrocarbons, EPA } \\
\text { methods } 502,504,601,602,8010,8015,8020 \text {, } \\
8021,6120 \text {, and trihalomethanes. }\end{array}$ \\
\hline
\end{tabular}




\section{APPENDIX F}

\section{DETERMINATION OF EM ENVIRONMENTAL}

SAMPLING AND ANALYSIS NEEDS

FOR FY 93-99

- OPERATION OF DATABASE SPREADSHEET SYSTEM 


\section{Operation of EM Analytical Needs Spreadsheet System}

1. Each site participant was requested to report data in Lotus 1-2-3 ${ }^{\circledR}$ spreadsheets for Parts 3, 4 and 5 of their final report. The format of the spreadsheet is given in a file named PART345.WK3 which is located in directory C:1123R34IFILESINEEDS\. Completed spreadsheets were submitted to EM-263 either on diskette or via electronic mail.

2. Each set of electronic data is saved in a directory for an individual site named S:IRSASSOONINEEDSIsitenamel. The original names assigned to the files by the sites are retained. These names should follow the naming convention given in the instructions package.

3. In each site directory a list of data files in that directory is also prepared in a file named siteLIST.WK3. The number of files in the file list is also noted.

4. Basic information on the data files is collected in a worksheet file named SUM1NEED.WK3. This worksheet in its blank form is located in directory C:I123R34IFILESINEEDS $\backslash$ and in a form completed with data in directory $S: I R S A S S O O N I N E E D S I$. This file is used to prepare a database of all the files so that files may be sorted according to EM office, activity type, unit type, regulatory driver, etc. The following steps are performed in this worksheet:

(a) Header information and the names of site directories, filelist names, and the number of files associated with each file list are entered into the data entry section of the worksheet.

(b) Operating macro ALT A creates the database which lists all the needs files according to the following format:

Directory Filename EM Office Site ADS\# Activity Unit Feg Diver

(c) The EM office with which each ADS is associated was not requested in the original data call, and therefore must be entered manually.

(d) Operating macro ALT B prints out the database.

This database is revised to SUM1NEER.WK3 in which standard site, ADS\#, and activity names are entered. It is then revised further to SUM1NEES.WK3 when standardized regulatory drivers are also added.

5. Summary of all the data collected from the field is performed in a worksheet entitled SUM2NEED.WK3 which in its blank form is located in directory C: 1123R34|FILESINEEDS\. Completed worksheets are saved with a name corresponding to the activity type, unit type, and regulatory driver in the directory S:IRSASSOONINEEDSISUM2l. This file is used for determining the 
number of unit-years, on average, an activity requires, and the sampling and analysis profile for one unit-year. The following steps are performed in this worksheet:

(a) Header information which includes the activity, unit, and regulatory driver are entered into the data entry section of the worksheet. (For more specific sorting of data the EM Office and site may be entered here).

(b) Operating macro ALT A extracts the file names from the database prepared in SUM1NEED.WK3 that meet the criteria in the header listing directories and file names. It then imports each file from the list and provides a summary of facts on the activity taking place in FY 93 and planned to take place in FY 94-99.

(c) Data is then entered into the summary area manually on the number of unit-years completed for each activity identified based on the duration and number of projects taking place, and on review of the originally submitted data. Again this summary can be printed out using the macro ALT Q.

(d) Operating macro ALT B creates a database of each of the activities in the datasheet and the number of unit-years of the activity completed in each fiscal year from FY 93 through FY 99.

(e) Operating macro ALT C prepares activity sampling and analysis profiles for a unit-year and for a unit. These profiles are given in terms of the sample analysis list given in the needs package as well as in a form common with the list of sample analyses used in the capacity study. The protocol used in converting the needs/NSTS list to the list common with the capacity sample analyses is given in the attached tables.

A total for all sampling and analysis needs for any fiscal year for this activity can then be determined by multiplying the total number of unityears completed or anticipated to be completed in the fiscal year by the sampling and analysis profile for a unit-year.

The following print-outs can be obtained with the following macros:

\begin{tabular}{|c|l|}
\hline Macro & \multicolumn{1}{|c|}{ Print-out } \\
\hline \hline ALT P & List of files imported into spreadsheet \\
\hline ALT Q & Summary of activities in FY 93-99 \\
\hline ALT R & $\begin{array}{l}\text { Database of unit-years of activity per fiscal } \\
\text { year }\end{array}$ \\
\hline
\end{tabular}




\begin{tabular}{|c|l||}
\hline ALTS & $\begin{array}{l}\text { Activity sampling and analysis profile per } \\
\text { unit-year in terms of needs/NSTS sample } \\
\text { analysis list }\end{array}$ \\
\hline ALT T & $\begin{array}{l}\text { Activity sampling and analysis profile per } \\
\text { unit in terms of needs/NSTS sample } \\
\text { analysis list }\end{array}$ \\
\hline ALT U & $\begin{array}{l}\text { Activity sampling and analysis profile per } \\
\text { unit-year in terms of capacity sample } \\
\text { analysis list }\end{array}$ \\
\hline ALT V & $\begin{array}{l}\text { Activity sampling and analysis profile per } \\
\text { unit in terms of capacity sample analysis } \\
\text { list }\end{array}$ \\
\hline
\end{tabular}

6. The databases of activities prepared in SUM2NEED.WK3, containing information on the scope of activities in terms of number of unit-years, are collected together for a single fiscal year in the worksheet SUM3NEED.WK3 which in its blank form is located in directory C:1123R34IFILESINEEDSI. Completed worksheets containing the database of all activities completed in a fiscal year are saved in the directory S:IRSASSOONINEEDSISUM3!.

7. The final tables of data presented in Appendix $G$ of this report are prepared in worksheets with names corresponding to the table number of the table which appears in the final report. Figures in the report were prepared using the numbers collected in the tables in Appendix G.

8. A list of all the spreadsheets and the directories in which they are found is shown in the table below. 
SPREADSHEETS FOR EM-263 ANALYTICAL NEEDS MODEL

\begin{tabular}{|c|c|c|}
\hline Spreadsheet & Directory & Comments \\
\hline PART345 & C: $|123 R 34| F|L E S| N E E D S \mid$ & Blank data entry file for field \\
\hline \#ADS\#\#\#\# & S:IRSASSOONINEEDS|sitenamel & Completed data files from field \\
\hline SiteLIST & S:IRSASSOONINEEDSISitenameI & List of data files from sites \\
\hline SUMINEED & C:1123R34|FILESINEEDSI & Blank worksheet for creating database of all data files from field \\
\hline SUMINEED & S:|RSASSOONINEEDSISUM1| & Completed worksheet containing database of all data files from field \\
\hline SUM2NEED & C: $(123 R 34|F I L E S| N E E D S \mid$ & Blank worksheet for creating database and analysis profile for each activity \\
\hline SUM2NEE\# & S:IRSASSOONNEEDSISUM2I & Completed worksheet containing database and analysis profile for each activity \\
\hline SUMBNEED & C: $|123 R 34|$ FILESINEEDS $\mid$ & Blank worksheet for creating database of activities completed by fiscal year \\
\hline SUMBNEE\# & S:IRSASSOONINEEDSISUMB! & Completed worksheet containing database of activities completed in a fiscal year \\
\hline NEEDTAB1 & S:|RSASSOONINEEDSITABLE1] & Blank worksheet for generating Table \\
\hline NEEDT1\# & S:IRSASSOONINEEDSITABLE1| & Completed Table I worksheets \\
\hline NEEDTAB2 & S:IRSASSOONINEEDSITABLE2| & Blank worksheet for generating Tables II \\
\hline NEEDT2\# & S:IRSASSOONINEEDSITABLE2| & Completed Table II worksheets \\
\hline NEEDTAB3 & S.IRSASSOONLNEEDSITABIE3I & Blank worksheet for aeneratina Table III \\
\hline NEEDT3\#\# & S:|RSASSOONINEEDSITABLEB| & Completed Table III worksheets \\
\hline & & \\
\hline NEEDTAB4 & S:|RSASSOONINEEDSITABLE4| & Blank worksheet for generating Tables IV \\
\hline NEEDT4\# & S:IRSASSOONINEEDS|TABLE4| & Completed Table IV worksheets \\
\hline NEEDTAB5 & S:|RSASSOONINEEDSITABLE5] & Blank worksheet for generating Tables V \\
\hline NEET15\#\# & S:IRSASSOONINEEDSITABLESI & Completed Table V worksheets \\
\hline NEEDTAB6 & S:IRSASSOONINEEDSITABLEG| & Blank worksheet for generating Tables VI \\
\hline NEEDT6 & S:IRSASSOONINEEDSITABLE6| & Completed Table VI worksheets \\
\hline
\end{tabular}




\title{
APPENDIX G
}

\section{DETERMINATION OF EM ENVIRONMENTAL}

\author{
SAMPLING AND ANALYSIS NEEDS
}

FOR FY 93-99

- DATA TABLES 


\section{List of Tables}

Table la:

Table lb:

Table Ila:

Table Ilb:

Table Ilc:

Table Ild:

Table Ile:

Table IIf:

Table IIg:

Table Ilh:

Table Ili:

Table Ilj:

Table Ilk:

Table III:

Table IIm:

Table IIn:

Table Illa:

Table IIlb:

Table IIlc:

Table IIId:

Table Ille:

Table IIIf:

Table Illg:

Table IVa:

Table IVb:

Table IVc:

Table IVd:

Table IVe:

Table IVf:

Table IVg:

Table Va:
Total Sample Analyses by Fiscal Year

Total Samples by Fiscal Year

FY 93 Sample Analyses by Rad Level and Matrix Type FY 94 Sample Analyses by Rad Level and Matrix Type FY 95 Sample Analyses by Rad Level and Matrix Type FY 96 Sample Analyses by Rad Level and Matrix Type FY 97 Sample Analyses by Rad Level and Matrix Type FY 98 Sample Analyses by Rad Level and Matrix Type FY 99 Sample Analyses by Rad Level and Matrix Type FY 93 Samples by Rad Level and Matrix Type FY 94 Samples by Rad Level and Matrix Type FY 95 Samples by Rad Level and Matrix Type FY 96 Samples by Rad Level and Matrix Type FY 97 Samples by Rad Level and Matrix Type FY 98 Samples by Rad Level and Matrix Type FY 99 Samples by Rad Level and Matrix Type

FY 93 Samples and Sample Analyses Complex-wide by Activity FY 94 Samples and Sample Analyses Complex-wide by Activity FY 95 Samples and Sample Analyses Complex-wide by Activity FY 96 Samples and Sample Analyses Complex-wide by Activity FY 97 Samples and Sample Analyses Complex-wide by Activity FY 98 Samples and Sample Analyses Complex-wide by Activity FY 99 Samples and Sample Analyses Complex-wide by Activity

FY 93 Samples and Sample Analyses Complex-wide by Regulatory Driver

FY 94 Samples and Sample Analyses Complex-wide by Regulatory Driver

FY 95 Samples and Sample Analyses Complex-wide by Regulatory Driver

FY 96 Samples and Sample Analyses Complex-wide by Regulatory Driver

FY 97 Samples and Sample Analyses Complex-wide by Regulatory Driver

FY 98 Samples and Sample Analyses Complex-wide by Regulatory Driver

FY 99 Samples and Sample Analyses Complex-wide by Regulatory Driver

FY 93 Samples and Sample Analyses by Rad Level and Matrix Type

(i) Complex-wide 

(ii) $\mathrm{EM}-30$
(iii) EM-40
(iv) EM-60
(v) Fernald
(vi) INEL
(vii) NTS
(viii) Oak Ridge K-25
(ix) Oak Ridge $X-10$
(x) Oak Ridge $Y-12$
(xi) Rocky Flats
(xii) Savannah River

Table Vb:

FY 94 Samples and Sample Analyses by Rad Level and Matrix Type

(i) Complex-wide

(ii) EM-30

(iii) EM-40

(iv) EM-60

(v) Fernald

(vi) INEL

(vii) NTS

(viii) Oak Ridge K-25

(ix) Oak Ridge $X-10$

(x) Oak Ridge $Y-12$

(xi) Rocky Flats

(xii) Savannah River

Table Vc: $\quad$ FY 95 Samples and Sample Analyses by Rad Level and Matrix Type

(i) Complex-wide

(ii) $\quad E M-30$

(iii) EM-40

(iv) EM-60

(v) Fernald

(vi) INEL

(vii) NTS

(viii) Oak Ridge K-25

(ix) Oak Ridge $X-10$

(x) Oak Ridge $Y-12$

(xi) Rocky Flats

(xii) Savannah River

Table Vd: $\quad$ FY 96 Samples and Sample Analyses by Rad Level and Matrix Type

(i) Complex-wide

(ii) $\mathrm{EM}-30$ 

(iii) EM-40
(iv) EM-60
(v) Fernald
(vi) INEL
(vii) NTS
(viii) Oak Ridge K-25
(ix) Oak Ridge $X-10$
(x) Oak Ridge Y-12
(xi) Rocky Flats
(xii) Savannah River

Table Ve:

FY 97 Samples and Sample Analyses by Rad Level and Matrix Type

(i) Complex-wide

(ii) EM-30

(iii) EM-40

(iv) $\mathrm{EM}-60$

(v) Fernald

(vi) INEL

(vii) NTS

(viii) Oak Ridge K-25

(ix) Oak Ridge $X-10$

(x) Oak Ridge $Y-12$

(xi) Rocky Flats

(xii) Savannah River

Table Vf: $\quad$ FY 98 Samples and Sample Analyses by Rad Level and Matrix Type

(i) Complex-wide

(ii) EM-30

(iii) EM-40

(iv) EM-60

(v) Fernald

(vi) INEL

(vii) NTS

(viii) Oak Ridge K-25

(ix) Oak Ridge $X-10$

(x) Oak Ridge $Y-12$

(xi) Rocky Flats

(xii) Savannah River

Table Vg: $\quad$ FY 99 Samples and Sample Analyses by Rad Level and Matrix Type

(i) Complex-wide

(ii) EM-30

(iii) $\mathrm{EM}-40$ 


$\begin{array}{ll}\text { (iv) } & \text { EM-60 } \\ \text { (v) } & \text { Fernald } \\ \text { (vi) } & \text { INEL } \\ \text { (vii) } & \text { NTS } \\ \text { (viii) } & \text { Oak Ridge K-25 } \\ \text { (ix) } & \text { Oak Ridge X-10 } \\ \text { (x) } & \text { Oak Ridge Y-12 } \\ \text { (xi) } & \text { Rocky Flats } \\ \text { (xii) } & \text { Savannah River }\end{array}$

Table VI:

Total Sample Analyses by Fiscal Year

Activity Sampling and Analysis Profiles for One Unit-Year for:

Preliminary Assessment/Site Inspection (PA/SI);

Remedial Investigation/Feasibility Study (RI/FS);

Remedial Design/ Remedial Action (RD/RA);

RCRA Facility Closure (RFC);

Decontamination and Decommissioning (D\&D);

Groundwater Monitoring (GW MON);

Effluent Monitoring (EFF MON);

Transuranic Waste Disposal (TRU);

Hazardous Waste Management/Disposal (HAZ);

Mixed Waste/Low Level Waste Management/Disposal (MIX/LLW);

High Level Waste Management (HLW);

Environmental Monitoring (ENV MON);

Underground Storage Tanks (UST);

Miscellaneous RCRA/CERCLA (MISC R/C);

Other (OTHER).

Budget Figures by Site and Fiscal Year 
EM-263 ASSESSMENT OF EM SAMPLING AND ANALYSIS NEEDS

Table la: Total Sample Analyses by Fiscal Year

\begin{tabular}{|c|c|c|c|c|c|c|c|}
\hline $\begin{array}{c}\text { Site-EM Office/ } \\
\text { Fiscal Year }\end{array}$ & $\overline{993}$ & $\overline{94}$ & $\overline{95}$ & $\overline{966}$ & $\overline{97}$ & $\overline{988}$ & $\overline{99}$ \\
\hline & & & & & & & \\
\hline \multicolumn{8}{|l|}{ Fernald } \\
\hline$E M-30$ & 0 & 0 & 0 & 0 & 0 & 0 & 0 \\
\hline$E M-40$ & 69717 & 111208 & 110300 & 10727 & 10076 & 16216 & 9735 \\
\hline$E M-60$ & 0 & 0 & 0 & 0 & 0 & 0 & 0 \\
\hline \multirow[t]{2}{*}{ TOTAL Fernald } & 69717 & 111208 & 110300 & 10727 & 10076 & 16216 & 9735 \\
\hline & & & & & & & \\
\hline \multicolumn{8}{|l|}{ INEL } \\
\hline$E \bar{M}-30$ & $\overline{13534}$ & 13937 & 13937 & 13937 & 14927 & 14854 & 14854 \\
\hline$E M-40$ & 16213 & 17562 & 22395 & 20448 & 15988 & 21036 & 21036 \\
\hline$E M-60$ & 4346 & 4346 & 4346 & 4346 & 4346 & 4346 & 4346 \\
\hline TOTAL INEL & 34093 & 35846 & 40678 & 38731 & 35261 & 40236 & 40236 \\
\hline & & & & & & & \\
\hline \multicolumn{8}{|l|}{ Nevada Test Site } \\
\hline$E M-30$ & 1113 & 113 & $11 \overline{11}$ & 113 & 113 & 113 & 1113 \\
\hline$E M-40$ & 1265 & 31373 & 48116 & 48424 & 48424 & 49014 & 48326 \\
\hline$E M-60$ & 0 & 0 & 0 & 0 & 0 & 0 & 0 \\
\hline TOTAL NTS & 1378 & 31486 & 48229 & 48536 & 48536 & $4 \overline{49127}$ & 48439 \\
\hline \multirow{2}{*}{\multicolumn{8}{|c|}{ Oak Ridge K-25 }} \\
\hline & & & & & & & \\
\hline EM-30 & 11895 & 14112 & 14112 & 11895 & 11895 & 9677 & 9677 \\
\hline$E M-40$ & 4257 & 336051 & 335997 & 23235 & 22113 & 22113 & 22625 \\
\hline$E M-60$ & 0 & 0 & 0 & 0 & 0 & 0 & 0 \\
\hline TOTAL ORK-25 & 16151 & 350163 & 350109 & 35130 & 34008 & 31791 & 32303 \\
\hline & & & & & & & \\
\hline \multicolumn{8}{|l|}{ Oak Ridge X-10 } \\
\hline$E M-30$ & $\overline{7413}$ & 8778 & 8733 & 8733 & $8 \overline{8733}$ & $\overline{8733}$ & 8733 \\
\hline$E M-40$ & 7937 & 15675 & 13133 & 7325 & 6820 & 6021 & 5964 \\
\hline$E M-60$ & 0 & 0 & 0 & 0 & 0 & 0 & 0 \\
\hline TOTAL OR $X-10$ & 15349 & 24453 & 21866 & 16058 & 15553 & 14754 & 14697 \\
\hline & & & & & & & \\
\hline \multicolumn{8}{|l|}{ Oak Ridge $Y-12$} \\
\hline$\overline{E M-30}$ & $\overline{442}$ & 531 & $\overline{442}$ & 442 & 442 & 442 & 442 \\
\hline$E M-40$ & 3954 & 4516 & 4098 & 4247 & 4040 & 4040 & 2679 \\
\hline$E M-60$ & 0 & 0 & 0 & 0 & 0 & 0 & 0 \\
\hline TOTAL ORY -12 & 4396 & 5047 & 4540 & 4689 & $4 \overline{4482}$ & 4482 & 3121 \\
\hline & & & & & & & \\
\hline \multicolumn{8}{|l|}{ Rocky Flats } \\
\hline$E M-30$ & $\overline{660}$ & 13607 & 14927 & $\overline{6057}$ & $\overline{6057}$ & $\overline{6057}$ & $4 \overline{4077}$ \\
\hline EM-40 & 11491 & 115758 & 52227 & 52424 & 50608 & 50346 & 50346 \\
\hline$E M-60$ & 45916 & 45916 & 45916 & 45916 & 45916 & 45916 & 45916 \\
\hline TOTALRF & 58067 & 175280 & 113070 & 104398 & 102581 & 102319 & 100339 \\
\hline & & & & & & & \\
\hline \multicolumn{8}{|l|}{ Savannah River } \\
\hline$E M-30$ & 66657 & 69361 & 69292 & 68984 & $\overline{70048}$ & $6 \overline{9752}$ & 69752 \\
\hline EM-40 & 0 & 0 & 0 & 0 & 0 & 0 & 0 \\
\hline$E M-60$ & 0 & 0 & 0 & 0 & 0 & 0 & 0 \\
\hline \multirow[t]{2}{*}{ TOTAL SR } & 66657 & 69361 & 69292 & 68984 & 70048 & $69 \overline{9752}$ & 69752 \\
\hline & & & & & & & \\
\hline \multicolumn{8}{|l|}{$E \overline{E M}$} \\
\hline$E M-30$ & 263866 & 325185 & 323336 & 293028 & 298490 & 291610 & $2 \overline{86344}$ \\
\hline$E M-40$ & 275599 & 1757359 & 1670860 & 473800 & 442593 & 472600 & 448384 \\
\hline$E M-60$ & 113090 & 143247 & 126158 & 126158 & 126660 & 126660 & 126660 \\
\hline TOTAL EM & 691100 & 2231908 & 2183282 & 9939219 & 913553 & 936726 & 904884 \\
\hline
\end{tabular}


EM-263 ASSESSMENT OF EM SAMPLING AND ANALYSIS NEEDS

Table Ib: Total Samples by Fiscal Year

\begin{tabular}{|c|c|c|c|c|c|c|c|}
\hline $\begin{array}{l}\text { Site-EM Office/ } \\
\text { Fiscal Year }\end{array}$ & 93 & $\overline{\overline{94}}$ & 95 & $\overline{96}$ & $\overline{\overline{97}}$ & $\overline{\overline{98}}$ & $\overline{\overline{99}}$ \\
\hline \multirow{2}{*}{\multicolumn{8}{|c|}{ Fernald }} \\
\hline & & & & & & & \\
\hline EM-30 & 0 & 0 & \begin{tabular}{l|l}
0 \\
\end{tabular} & (01 & 0 & 0 & 0 \\
\hline$E M-40$ & 8089 & 13467 & 13899 & 1898 & 1826 & 2730 & 1992 \\
\hline$E M-60$ & 0 & 0 & 0 & 0 & 0 & 0 & 0 \\
\hline TOTAL Fernald & 8089 & 13467 & 13899 & 1898 & 1826 & 2730 & 1992 \\
\hline & & & & & & & \\
\hline \multicolumn{8}{|l|}{ IINEL } \\
\hline$E M-30$ & 5197 & 5353 & 5353 & 5353 & 5795 & 5787 & 5787 \\
\hline$E M-40$ & 4890 & 5382 & 7474 & 8723 & 6365 & 8573 & 8573 \\
\hline$E M-60$ & 442 & 442 & 442 & 442 & 442 & 442 & 442 \\
\hline TOTAL INEL & 10528 & 111177 & 13268 & 14517 & 12602 & 14802 & 14802 \\
\hline \multicolumn{8}{|l|}{ Nevada Test Site } \\
\hline EM-30 & $\overline{111}$ & $\overline{11 \mid}$ & $\frac{1}{11}$ & 111 & 11 & $\frac{1}{111}$ & 11 \\
\hline$E M-40$ & 473 & 2708 & 9866 & 9915 & 9915 & 9938 & 9911 \\
\hline$E M-60$ & 0 & 0 & 0 & 0 & 0 & 0 & 0 \\
\hline TOTALNTS & 484 & 2719 & 9878 & 9926 & 9926 & 9949 & 9922 \\
\hline & & & & & & & \\
\hline \multicolumn{8}{|l|}{ Oak Ridge K-25 } \\
\hline$E M-30$ & 721 & 850 & 850 & 721 & 721 & 591 & 591 \\
\hline$E M-40$ & 531 & 27643 & 27607 & 9202 & 9030 & 9030 & 9262 \\
\hline$E M-60$ & 0 & 0 & 0 & 0 & 0 & 0 & 0 \\
\hline TOTAL ORK -25 & 1252 & 28493 & 28457 & 9922 & 9750 & 9621 & 9853 \\
\hline & & & & & & & \\
\hline \multicolumn{8}{|l|}{ Oak Ridge X-10 } \\
\hline$E M-30$ & 3580 & $\overline{4173}$ & 4170 & $\overline{44170}$ & 4170 & 4170 & 4170 \\
\hline$E M-40$ & 2171 & 4725 & 4442 & 2149 & 2093 & 2043 & 2037 \\
\hline$E M-60$ & 0 & 0 & 0 & 0 & 0 & 0 & 0 \\
\hline TOTAL OR X -10 & 5751 & 8899 & 8612 & 6319 & 6263 & 6212 & 6206 \\
\hline & & & & & & & \\
\hline \multicolumn{8}{|l|}{ Oak Ridge $Y-12$} \\
\hline EM-30 & 50 & $\overline{98}$ & 50 & 50 & 50 & 50 & $\overline{50}$ \\
\hline$E M-40$ & 446 & 1025 & 971 & 1050 & 1045 & 1045 & 831 \\
\hline$E M-60$ & 0 & 0 & 01 & 0 & 0 & 0 & 0 \\
\hline TOTAL OR Y-12 & 496 & 1123 & 1021 & 1101 & 1095 & 1095 & 881 \\
\hline & & & & & & & \\
\hline \multicolumn{8}{|l|}{ Rocky Flats } \\
\hline EM-30 & 295 & 4498 & 2088 & 1569 & 1569 & 1569 & $\overline{684}$ \\
\hline$E M-40$ & 832 & 12170 & 5454 & 5461 & 5266 & 5246 & 5246 \\
\hline$E M-60$ & 707 & 707 & 707 & 707 & 707 & 707 & 707 \\
\hline TOTAL RF & 1833 & 14375 & 8248 & 7737 & 7542 & 7522 & 6637 \\
\hline & & & & & & & \\
\hline \multicolumn{8}{|l|}{ Savannah River } \\
\hline EM-30 & 27735 & 28019 & 28011 & 27888 & 28249 & 28237 & 28237 \\
\hline EM-40 & 0 & 0 & 0 & 0 & 0 & 0 & 0 \\
\hline$E M-60$ & $\mathbf{0}$ & 0 & 0 & 0 & 0 & 0 & 0 \\
\hline TOTAL SR & 27735 & 28019 & 28011 & 27888 & 28249 & 28237 & 28237 \\
\hline \multicolumn{8}{|l|}{$\overline{E M}$} \\
\hline$E M-\overline{30}$ & $\overline{98483}$ & 108006 & 107817 & $\overline{105768}$ & 107903 & 107505 & 105151 \\
\hline$E M-40$ & 41836 & 186592 & 198682 & 109052 & 99511 & 108093 & 105608 \\
\hline$E M-60$ & 2584 & 3273 & 2883 & 2883 & 2894 & 2894 & 2894 \\
\hline TOTALEM & 146040 & 300991 & 320815 & 227619 & 220172 & 228481 & 223029 \\
\hline
\end{tabular}


EM-263 ASSESSMENT OF EM SAMPLING AND ANALYSIS NEEDS

Table lla: FY 93 Sample Analyses by Rad Level and Matrix Type

\begin{tabular}{|c|c|c|c|c|c|c|c|c|}
\hline \begin{tabular}{|l|} 
Site-EM Office/ \\
Rad Lvl-Matrix Type
\end{tabular} & $\overline{\mathrm{RO}}$ & $\overline{\mathrm{R} 1}$ & $\overline{\mathrm{R} 2}$ & $\overline{\mathrm{R} 3}$ & $\overline{\overline{\mathrm{R} 4}}$ & $\overline{\mathrm{M} 1}$ & $\overline{\mathrm{M} 2}$ & $\overline{\mathrm{M} 3}$ \\
\hline & & & & & & & & \\
\hline \multicolumn{9}{|l|}{ Fernald } \\
\hline$E M-30$ & 0 & 0 & 0 & 0 & $\overline{0}$ & 0 & 0 & $\overline{0}$ \\
\hline$E M-40$ & 45026 & 22462 & 3110 & 67 & 0 & 16959 & 22979 & 28830 \\
\hline$E M-60$ & 0 & 0 & 0 & 0 & 0 & 0 & 0 & 0 \\
\hline TOTAL Fernald & 45026 & 22462 & 3110 & 67 & 0 & 16959 & 22979 & 28830 \\
\hline & & & & +7 & - & & & \\
\hline \multicolumn{9}{|l|}{ INEL } \\
\hline$E M-30$ & $5 \overline{5861}$ & 10126 & $\mid 123$ & 6 & $\overline{0}$ & 8851 & 1065 & 1035 \\
\hline$E M-40$ & 6051 & 9800 & 366 & 149 & 0 & 3614 & 11022 & 1424 \\
\hline$E M-60$ & 4357 & 65 & 21 & 0 & 0 & 3203 & 1046 & 0 \\
\hline \multirow[t]{2}{*}{ TOTAL INEL } & 16269 & 19990 & 511 & 155 & 0 & 15668 & 13133 & 2460 \\
\hline & & & & T1 & - & & & \\
\hline \multicolumn{9}{|l|}{ Nevada Test Site } \\
\hline$E M-30$ & 113 & 2 & 1 & 0 & $\overline{0}$ & 83 & $\overline{27}$ & 0 \\
\hline$E M-40$ & 580 & 659 & 24 & 0 & 0 & 186 & 753 & 328 \\
\hline$E M-60$ & 0 & 0 & 0 & 0 & 0 & 0 & 0 & 0 \\
\hline \multirow[t]{2}{*}{ TOTAL NTS } & 693 & 661 & 24 & 01 & 0 & 269 & 780 & 328 \\
\hline & & & & & & & & \\
\hline \multicolumn{9}{|l|}{ Oak Ridge K-25 } \\
\hline$E M-30$ & 304 & 9092 & 2473 & 0 & 0 & 5889 & 1746 & 4286 \\
\hline$\overline{E M-40}$ & 3014 & 1186 & 65 & 0 & 0 & 1760 & 2404 & 84 \\
\hline$E M-60$ & 0 & 0 & 0 & 0 & 0 & 0 & 0 & 0 \\
\hline \multirow[t]{2}{*}{ TOTAL OR K-25 } & 3318 & 10278 & 2538 & 01 & 0 & 7649 & 4150 & 4369 \\
\hline & & & & $=$ & - & & & \\
\hline \multicolumn{9}{|l|}{ Oak Ridge X-10 } \\
\hline$E M-30$ & 2144 & 7109 & 14 & 5 & $\overline{0}$ & 5305 & $\overline{166}$ & 82 \\
\hline$E M-40$ & 2936 & 4956 & 60 & 0 & 0 & 1934 & 5079 & 909 \\
\hline$E M-60$ & 0 & 0 & 0 & 0 & 0 & 0 & 0 & 0 \\
\hline \multirow[t]{2}{*}{ TOTAL OR X-10 } & 5081 & 12065 & 74 & 5 & 0 & 7239 & 5244 & 991 \\
\hline & & & & & & & & \\
\hline \multicolumn{9}{|l|}{ Oak Ridge Y-12 } \\
\hline EM-30 & 267 & 155 & 29 & 01 & 0 & 37 & 117 & 280 \\
\hline$E M-40$ & 2808 & 1074 & 72 & 0 & 0 & 1522 & 2377 & 56 \\
\hline$E M-60$ & 0 & 0 & 0 & 0 & $\overline{0}$ & 0 & 0 & 0 \\
\hline \multirow[t]{2}{*}{ TOTAL OR Y -12} & 3075 & 1228 & 101 & 01 & 0 & 1558 & 2493 & 336 \\
\hline & & & & & & & & \\
\hline \multicolumn{9}{|l|}{ Rocky Flats } \\
\hline EM-30 & $\overline{0}$ & о & 0 & 660 & $\overline{0}$ & $\overline{0}$ & $\overline{0}$ & 660 \\
\hline$E M-40$ & 9321 & 2032 & 137 & 0 & 0 & 5514 & 5881 & 97 \\
\hline$E M-60$ & 45553 & 571 & 23 & 0 & 0 & 30527 & 15128 & 30 \\
\hline \multirow[t]{2}{*}{ TOTAL RF } & 54874 & 2603 & 160 & 660 & 0 & 36041 & 21009 & 787 \\
\hline & & & & & & & & \\
\hline \multicolumn{9}{|l|}{ Savannah River } \\
\hline EM-30 & 38418 & 36343 & 270 & 157 & 1550 & 45267 & 1581 & 9727 \\
\hline$E M-40$ & 0 & 0 & 0 & 0 & 0 & 0 & 0 & 0 \\
\hline$E M-60$ & 0 & 0 & 0 & 0 & 0 & 0 & 0 & 0 \\
\hline \multirow[t]{2}{*}{ TOTAL SR } & 38418 & 36343 & 270 & $157 \mid$ & 1550 & 45267 & 1581 & 9727 \\
\hline & & & & & & & & \\
\hline \multicolumn{9}{|l|}{ EM } \\
\hline EM-30 & 123423 & 164604 & 7622 & 2169 & $\overline{4061}$ & 171431 & 12318 & 42105 \\
\hline$E M-40$ & 167366 & 101206 & 9201 & 518 & 0 & 75573 & 121187 & 76148 \\
\hline$E M-60$ & 112298 & 1429 & 99 & 1 & 0 & 75894 & 36391 & 67 \\
\hline TOTALEM & 433561 & 274638 & 17646 & 2714 & 4030 & 339693 & 185562 & 124355 \\
\hline
\end{tabular}


EM-263 ASSESSMENT OF EM SAMPLING AND ANALYSIS NEEDS

Table Ilb: FY 94 Sample Analyses by Rad Level and Matrix Type

\begin{tabular}{|c|c|c|c|c|c|c|c|c|}
\hline $\begin{array}{l}\text { Site-EM Office/ } \\
\text { Rad LVl-Matrix Type }\end{array}$ & $\overline{\mathrm{RO}}$ & $\overline{\mathrm{R} 1}$ & $\overline{\mathrm{R} 2}$ & $\overline{R 3}$ & $\overline{\mathrm{R4}}$ & $\overline{\mathrm{M} 1}$ & $\overline{\mathrm{M} 2}$ & $\overline{\mathrm{M} 3}$ \\
\hline \multirow{2}{*}{\multicolumn{9}{|c|}{ Fernald }} \\
\hline & & & & & & & & \\
\hline EM-30 & 0 & 0 & 0 & 0 & $\overline{0}$ & 0 & $\overline{\overline{0}}$ & $\overline{0}$ \\
\hline$E M-40$ & 67566 & 39260 & 6372 & 1 & 0 & 14856 & 31713 & 62648 \\
\hline$E M-60$ & 0 & 0 & 0 & 0 & 0 & 0 & 0 & 0 \\
\hline TOTAL Fernald & 67566 & 39260 & 6372 & 1 & 0 & 14856 & 31713 & 62648 \\
\hline & & & & & - & & & \\
\hline \multicolumn{9}{|l|}{ INEL } \\
\hline$E \overline{E M}-30$ & 5906 & 10151 & 128 & 336 & $\overline{0}$ & 8857 & 1084 & 1412 \\
\hline$E M-40$ & 6401 & 10444 & 384 & 479 & 0 & 3909 & 11523 & 1986 \\
\hline$E M-60$ & 4357 & 65 & 21 & 0 & 0 & 3203 & 1046 & 0 \\
\hline TOTAL INEL & 16664 & 20660 & 534 & 815 & 0 & 15969 & 13653 & 3397 \\
\hline & & & & & & & & \\
\hline \multicolumn{9}{|l|}{ Nevada Test Site } \\
\hline$E M-30$ & 113 & 2 & $\overline{11}$ & 0 & $\overline{0}$ & 83 & |27| & $\overline{0}$ \\
\hline$E M-40$ & 24129 & 6838 & 395 & 0 & 0 & 14049 & 16795 & 538 \\
\hline$E M-60$ & 0 & 0 & 0 & 0 & 0 & 0 & 0 & 0 \\
\hline TOTAL NTS & 24243 & 6840 & 396 & 0 & 0 & 14132 & 16823 & 538 \\
\hline & & & & & & & & \\
\hline \multicolumn{9}{|l|}{ Oak Ridge K-25 } \\
\hline EM-30 & 304 & 10828 & 2947 & 0 & 0 & 6985 & 2065 & 5096 \\
\hline$E M-40$ & 3236 & 265370 & 66404 & 0 & 0 & 155469 & 64473 & 117150 \\
\hline$E M-60$ & 0 & 0 & 0 & 0 & 0 & 0 & 0 & 0 \\
\hline TOTAL OR K-25 & 3540 & 276198 & 69350 & 0 & 0 & 162454 & 66538 & 122246 \\
\hline \multirow{2}{*}{\multicolumn{9}{|c|}{ Oak Ridge $X-10$}} \\
\hline & & & & & & & & \\
\hline$\overline{E M}-30$ & 2183 & 7115 & $\overline{14}$ & 1325 & $\overline{\overline{0}}$ & 5335 & 180 & $\overline{1404}$ \\
\hline$E M-40$ & 4647 & 10939 & 102 & 0 & 0 & 2926 & 10860 & 1875 \\
\hline$E M-60$ & 0 & 0 & 0 & 0 & 0 & 0 & 0 & 0 \\
\hline TOTAL OR $X-10$ & 6831 & 18055 & 116 & 1325 & $\overline{0}$ & 8261 & 11040 & 3279 \\
\hline & & & & & & & & \\
\hline \multicolumn{9}{|l|}{ Oak Ridge $Y-12$} \\
\hline$E M-30$ & 294 & 216 & 29 & 0 & 0 & (65 & 168 & 291 \\
\hline$E M-40$ & 1841 & 2633 & 39 & 0 & 0 & 1153 & 3023 & 344 \\
\hline$E M-60$ & 0 & 0 & 0 & 0 & 0 & 0 & 0 & 0 \\
\hline \multirow[t]{2}{*}{ TOTAL OR Y -12 } & 2134 & 2850 & 68 & 0 & 0 & 1217 & 3191 & 635 \\
\hline & & & & & 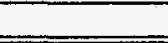 & & & \\
\hline \multicolumn{9}{|l|}{ Rocky Flats } \\
\hline EM-30 & $\overline{2068}$ & 8139 & 2116 & 1320 & $\overline{0}$ & 4 & 2180 & $\overline{6724}$ \\
\hline$E M-40$ & 84006 & 29735 & 2002 & 0 & 0 & 46312 & 68022 & 1439 \\
\hline$E M-60$ & 45553 & 571 & 23 & 0 & 0 & 30527 & 15128 & 30 \\
\hline \multirow[t]{2}{*}{ TOTAL RF } & 131628 & 38445 & 4141 & 1320 & 0 & $81504 \mid$ & 85329 & 8193 \\
\hline & & & & & & & & \\
\hline \multicolumn{9}{|l|}{ Savannah River } \\
\hline EM-30 & 40501 & 36890 & $\overline{371}$ & 157 & $\overline{1556}$ & 46500 & 1994| & 10753 \\
\hline$E M-40$ & 0 & 0 & 0 & 0 & 0 & 0 & 0 & 0 \\
\hline$E M-60$ & 0 & 0 & 0 & 0 & 0 & 0 & 0 & $\overline{0}$ \\
\hline TOTAL SR & 40501 & 36890 & 371 & 157 & 1556 & 46500 & 1994| & 10753 \\
\hline & & & & & & & & \\
\hline \multicolumn{9}{|l|}{ EM } \\
\hline EM-30 & $\overline{138694}$ & 198023 & $1 \overline{15133}$ & $\overline{8473}$ & $\overline{4200}$ & 195723 & 20786 & 69338 \\
\hline$E M-40$ & 533278 & 1015313 & 210438 & 1332 & 0 & 663513 & 573817 & 517026 \\
\hline$E M-60$ & 142244 & 1810 & 126 & 1 & 0 & 96133 & 46095 & 85 \\
\hline TOTALEM & 814833 & 1220969 & 226143 & 10057 & 4325 & 958806 & 640183 & 588501 \\
\hline
\end{tabular}


EM-263 ASSESSMENT OF EM SAMPLING AND ANALYSIS NEEDS

Table llc: FY 95 Sample Analyses by Rad Level and Matrix Type

\begin{tabular}{|c|c|c|c|c|c|c|c|c|}
\hline $\begin{array}{l}\text { Site-EM Office/ } \\
\text { Rad Lvl-Matrix Type }\end{array}$ & Ro & $\overline{\mathrm{R} 1}$ & $\overline{\overline{\mathrm{R} 2}}$ & R3 & $\overline{\overline{R 4}}$ & $\overline{\mathrm{M1}}$ & M2 & M3 \\
\hline \multicolumn{9}{|l|}{ Fernald } \\
\hline$E M-30$ & 0 & 0 & 0 & 0 & 0 & $\overline{0}$ & 1 & 0] \\
\hline$E M-40$ & 66732 & 39199 & 6341 & 1 & 0 & 14577 & 30969 & 62781 \\
\hline$E M-60$ & 0 & 0 & 0 & 0 & 0 & 0 & 0 & 0 \\
\hline TOTAL Fernald & 66732 & 39199 & 6341 & 1 & 01 & 14577 & $30 \overline{969}$ & 62781 \\
\hline \multicolumn{8}{|l|}{ INEL } & \\
\hline$E M-30$ & 5906 & 10151 & 128 & 336 & 0 & 8857 & $\overline{1084}$ & 1412 \\
\hline$E M-40$ & 6609 & 15059 & 384 & 479 & 0 & 4146 & 15316 & 2796 \\
\hline$E M-60$ & 4357 & 65 & 21 & 0 & 0 & 3203 & 1046 & 0 \\
\hline TOTAL INEL & 16872 & 25275 & 534 & 815 & 0 & 16206 & 17447 & 4208 \\
\hline & & & & & 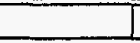 & & & \\
\hline \multicolumn{9}{|l|}{ Nevada Test Site } \\
\hline$E M-30$ & 113 & 2 & 1 & 0 & 0 & 83 & 27 & 0 \\
\hline$E M-40$ & 24296 & 23418 & 395 & 0 & 0 & 14267 & 30363 & 3493 \\
\hline$E M-60$ & 0 & 0 & 0 & 0 & 0 & 0 & 0 & 0 \\
\hline TOTAL NTS & 24409 & 23419 & \begin{tabular}{l|l}
396 \\
\end{tabular} & 0 & 0 & 14350 & 30390 & 3493 \\
\hline \multirow{2}{*}{\multicolumn{9}{|c|}{ Oak Ridge K-25 }} \\
\hline & & & & & & & & \\
\hline$E M-30$ & 304 & 10828 & 2947 & 0 & 0 & 6985 & 2065 & $50 \overline{96}$ \\
\hline$E M-40$ & 3206 & 265348 & 66402 & 0 & 0 & 155465 & 64450 & 117123 \\
\hline EM-60 & 0 & 0 & \begin{tabular}{l|l}
0 & \\
\end{tabular} & 0 & 0 & 0 & 0 & 0 \\
\hline TOTAL OR K-25 & 3509 & 276176 & 69349| & 0 & 0 & 162450 & 66515 & 122219 \\
\hline \multirow{2}{*}{\multicolumn{9}{|c|}{ Oak Ridge X-10 }} \\
\hline & & & & & & & & \\
\hline$E M-30$ & 2144 & 7109 & 14 & 1325 & 0 & 5305 & 166 & 1402 \\
\hline$E M-40$ & 2848 & 10244 & 54 & 0 & 0 & 1958 & 9318 & 1845 \\
\hline$E M-60$ & 0 & 0 & 0 & 0 & 0 & 0 & 0 & 0 \\
\hline TOTAL OR $X-10$ & 4992 & 17353 & 68 & 1325 & 0 & 7263 & 9484 & 3247 \\
\hline & & & & & & & & \\
\hline \multicolumn{9}{|l|}{ Oak Ridge $Y-12$} \\
\hline$E M-30$ & 267 & 155 & 29 & 0 & (0| & 37 & 117 & 280 \\
\hline$E M-40$ & 1546 & 2518 & 31 & 0 & 0 & 996 & 2774 & 332 \\
\hline$E M-60$ & 0 & 0 & 0 & 0 & 0 & 0 & 0 & 0 \\
\hline TOTAL OR Y -12 & 1813! & 2673 & 601 & 0 & 0 & 1032 & 28911 & 612 \\
\hline & & & & & $\square$ & & & \\
\hline \multicolumn{9}{|l|}{ Rocky Flats } \\
\hline$E M-30$ & 2068 & 8139 & 2116 & 2640 & वा & 4665 & 2180 & $8 \overline{8044}$ \\
\hline$E M-40$ & 38010 & 13313 & 896 & 0 & 0 & 21013 & 30545 & 678 \\
\hline$E M-60$ & 45553 & 571 & 23 & 0 & 0 & 30527 & 15128 & 30 \\
\hline TOTAL RF & 85631 & 22023 & 3035 & 2640 & 0 & 56206 & 47853 & 8752 \\
\hline & & & & & & & & \\
\hline \multicolumn{9}{|l|}{ Savannah River } \\
\hline$E M-30$ & $4 \overline{40459}$ & $36 \overline{866}$ & 366 & 157 & 1556 & 46495 & 1976 & $\overline{10709}$ \\
\hline$E M-40$ & 0 & 0 & 0 & 0 & 0 & 0 & 0 & 0 \\
\hline$E M-60$ & 0 & 0 & 0 & 0 & 0 & 0 & 0 & 0 \\
\hline TOTAL SR & 40459 & 36866 & 366 & 157 & 1556 & 46495 & 1976 & 10709 \\
\hline & & & & & & & & \\
\hline \multicolumn{9}{|l|}{ EM } \\
\hline$E M-30$ & 136355 & $\overline{194845}$ & 14897 & $\overline{11858}$ & 4138 & 192654 & 20257 & 71669 \\
\hline$E M-40$ & 408254 & 1051931 & 212338 & 1366 & 0 & 605401 & 523645 & 538786 \\
\hline$E M-60$ & 125275 & 1594 & 111 & 1 & 0 & 84664 & 40596 & 75 \\
\hline TOTAL EM & 703925 & 1275794 & 230830 & 14220 & 4480 & 917505 & 597669 & 622140 \\
\hline
\end{tabular}


EM-263 ASSESSMENT OF EM SAMPLING AND ANALYSIS NEEDS

Table IId: FY 96 Sample Analyses by Rad Level and Matrix Type

\begin{tabular}{|c|c|c|c|c|c|c|c|c|}
\hline $\begin{array}{l}\text { Site-EM Office/ } \\
\text { Rad LvI-Matrix Type }\end{array}$ & $\overline{\mathrm{RO}}$ & $\overline{\mathrm{R} 1}$ & $\overline{\mathrm{R} 2}$ & $\overline{\mathrm{R3}}$ & $\overline{\mathrm{R} 4}$ & $\overline{\mathrm{M1}}$ & $\overline{\mathrm{M} 2}$ & $\overline{M 3}$ \\
\hline & & & & & & & & \\
\hline \multicolumn{9}{|l|}{ Fernald } \\
\hline$E M-30$ & 0 & 01 & 0 & 0 & 0 & 0 & 0 & 0 \\
\hline$E M-40$ & 6962 & 3845 & 14 & 1 & 0 & 5948 & 4173 & 513 \\
\hline$E M-60$ & 0 & 0 & 0 & 0 & 0 & 0 & 0 & 0 \\
\hline TOTAL Fernald & 6962 & 3845 & 14 & 11 & $0]$ & 5948 & 4173 & 513 \\
\hline & & & & & & & & \\
\hline \multicolumn{9}{|l|}{$\sqrt{\text { INEL }}$} \\
\hline$E M-30$ & 5906 & 10151 & 128 & 336 & (0) & 8857 & 1084 & 1412 \\
\hline$E M-40$ & 2848 & 17011 & 207 & 434 & 0 & 2407 & 14579 & 3410 \\
\hline$E M-60$ & 4357 & 65 & 21 & 0 & 0 & 3203 & 1046 & 0 \\
\hline TOTAL INEL & 13110 & 27227 & 356 & 771 & 0 & 14467 & 16709 & 4822 \\
\hline & & & & & & & & \\
\hline \multicolumn{9}{|l|}{ Nevada Test Site } \\
\hline EM-30 & 113 & 2 & 1 & 0 & 0 & $\overline{83}$ & 27 & $\overline{0}$ \\
\hline$E M-40$ & 24649 & 23364 & 404 & 0 & 0 & 14449 & 30480 & 3500 \\
\hline$E M-60$ & 0 & 0 & 0 & 0 & 0 & 0 & 0 & 0 \\
\hline TOTAL NTS & 24762 & 23366 & 405 & 01 & 0 & 14532 & 30508 & 3500 \\
\hline \multirow{2}{*}{\multicolumn{9}{|c|}{ Oak Ridge K-25 }} \\
\hline & & & & & & & & \\
\hline$E M-30$ & 304 & 9092 & 2473 & 0 & 0 & 5889 & 1746 & 4286 \\
\hline$E M-40$ & 1544 & 21679 & 21 & 0 & 0 & 1155 & 18351 & 3721 \\
\hline$E M-60$ & 01 & 0 & 0 & 0 & 0 & 0 & 0 & 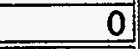 \\
\hline TOTAL OR K-25 & 1848 & 30771 & 2494 & 01 & $\underline{\underline{0.0}}$ & 7044 & 20096 & 8007 \\
\hline & & & & & & & & \\
\hline \multicolumn{9}{|l|}{ Oak Ridge X-10 } \\
\hline$E M-30$ & 2144 & 7109 & 14 & 1325 & 0 & 5305 & 166 & 1402 \\
\hline$E M-40$ & 2462 & 4830 & 46 & 0 & 0 & 1703 & 4694 & 915 \\
\hline$E M-60$ & 0 & 0 & 0 & 0 & 0 & 0 & 0 & 0 \\
\hline TOTAL OR X-10 & 4606 & 11940 & 60 & 1325 & 0 & 7008 & 4860 & 2317 \\
\hline & & & & & & & & \\
\hline \multicolumn{9}{|l|}{ Oak Ridge $Y-12$} \\
\hline EM-30 & 267 & 155 & $\overline{29}$ & $\overline{0}$ & $\overline{0}$ & $\overline{37}$ & 1177 & 280 \\
\hline$E M-40$ & 1589 & 2621 & 31 & 0 & 0 & 1043 & 2859 & 351 \\
\hline$E M-60$ & 0 & 0 & 0 & 0 & 0 & 0 & 0 & 0 \\
\hline TOTAL OR Y-12 & 1857 & 2775 & 60 & 이 & 0 & 1079 & 2976 & 631 \\
\hline & & & & & & & & \\
\hline \multicolumn{9}{|l|}{ Rocky Flats } \\
\hline$E M-30$ & 2068 & 1196 & 221 & 2640 & 0 & 282 & 903 & 4805 \\
\hline$E M-40$ & 38016 & 13502 & 896 & 0 & 0 & 21026 & 30731 & 678 \\
\hline$E M-60$ & 45553 & 571 & 23 & 0 & 0 & 30527 & 15128 & 30 \\
\hline TOTALRF & 85637 & 15268 & 1140 & 2640 & $\overline{0}$ & 51835 & 46761 & 5513 \\
\hline \multirow{2}{*}{\multicolumn{9}{|c|}{ Savannah River }} \\
\hline & & & & & & & & \\
\hline EM-30 & 40424 & 36637 & $\overline{313}$ & 124 & 1556 & 46487 & 1752 & 10676 \\
\hline EM-40 & 0 & 0 & 0 & 0 & 0 & 0 & 0 & 0 \\
\hline$E M-60$ & 0 & 0 & 0 & 0 & 0 & $\overline{0}$ & 0 & 0 \\
\hline TOTAL SR & 40424 & 36637 & $\overline{313}$ & 124 & 1556 & 46487 & 1752 & 10676 \\
\hline & & & & & & & & \\
\hline \multicolumn{9}{|l|}{ EM } \\
\hline$E M-30$ & 136262 & 171148 & 8453 & 11770 & 4138 & 178058 & 15415 & 60811 \\
\hline EM-40 & 221718 & 246661 & 4599 & 1235 & 0 & 135556 & 300660 & 37170 \\
\hline$E M-60$ & 125275 & 1594 & 111 & 1 & 0 & 84664 & 40596 & 75 \\
\hline TOTAL EM & 514322 & 435750 & $1 \overline{3894}$ & 13948 & 4465 & 425911 & 366887 & 103261 \\
\hline
\end{tabular}


EM-263 ASSESSMENT OF EM SAMPLING AND ANALYSIS NEEDS

Table lle: FY 97 Sample Analyses by Rad Level and Matrix Type

\begin{tabular}{|c|c|c|c|c|c|c|c|c|}
\hline $\begin{array}{l}\text { Site-EM Office/ } \\
\text { Rad Lvl-Matrix Type }\end{array}$ & RO & $\overline{\mathrm{R} 1}$ & $\overline{\mathrm{R} 2}$ & $\overline{\mathrm{R} 3}$ & $\overline{R 4}$ & $\overline{\mathrm{M1}}$ & $\overline{\mathrm{M} 2}$ & $\overline{\mathrm{M3}}$ \\
\hline & & & & & & & & \\
\hline \multicolumn{9}{|l|}{ Fernald } \\
\hline EM-30 & $\overline{0}$ & $\overline{0}$ & (0) & 0 & $\overline{0}$ & $\overline{0}$ & 01 & $\overline{0}$ \\
\hline$E M-40$ & 6501 & 3666 & 2 & 1 & 0 & 5701 & 3776 & 505 \\
\hline$E M-60$ & 0 & 0 & 0 & 0 & 0 & 0 & 0 & 0 \\
\hline TOTAL Fernaid & 6501 & 3666 & 2 & 1 & 0 & 5701 & 3776 & 505 \\
\hline \multirow{2}{*}{\multicolumn{9}{|c|}{ INEL }} \\
\hline & & & & & & & & \\
\hline EM-30 & $5 \overline{5906}$ & $\overline{10151}$ & 128 & 1326 & $\overline{0}$ & (8857 & 1084 & 2402 \\
\hline$E M-40$ & 1637 & 14160 & 207 & 104 & 0 & 1095 & 12223 & 2551 \\
\hline$E M-60$ & 4357 & 65 & 21 & 0 & 0 & 3203 & 1046 & 0 \\
\hline TOTAL INEL & 11899 & 24376 & $35 \overline{1}$ & $14 \overline{141}$ & $\overline{0}$ & $1 \overline{13155}$ & 14354 & 49952 \\
\hline & & & & & & & & \\
\hline \multicolumn{9}{|l|}{ Nevada Test Site } \\
\hline$E M-30$ & 113 & 2 & 1 & 0 & 0 & 83 & 27 & $\overline{0}$ \\
\hline$E M-40$ & 24649 & 23364 & 404 & 0 & 0 & 14449 & 30480 & 3500 \\
\hline$E M-60$ & 0 & 0 & 0 & 0 & 0 & 0 & 0 & $\overline{0}$ \\
\hline TOTAL NTS & 24762 & 23366 & 405 & 0 & 0 & 14532 & 30508 & 3500 \\
\hline & & & & 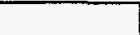 & 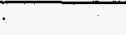 & & & \\
\hline \multicolumn{9}{|l|}{ Oak Ridge K-25 } \\
\hline$E M-30$ & 304 & 9092 & 2473 & 0 & $\overline{0}$ & 5889 & 1746 & 4286 \\
\hline$E M-40$ & 793 & 21329 & 3 & 0 & 0 & 729 & 17679 & 3694 \\
\hline$E M-60$ & 0 & 0 & 0 & 0 & 0 & 0 & 0 & 0 \\
\hline TOTAL OR K-25 & 1097 & 30420 & 2476 & 0 & 0 & 6619 & 19425 & 7980 \\
\hline & & & & & & & & \\
\hline \multicolumn{9}{|l|}{ Oak Ridge X-10 } \\
\hline EM-30 & 2144 & 7109 & 14 & 1325 & $\overline{0}$ & $\overline{\overline{5305}}$ & 166 & 1402 \\
\hline$E M-40$ & 2103 & 4693 & 37 & 0 & 0 & 1508 & 4390 & 909 \\
\hline$E M-60$ & 0 & 0 & 0 & 0 & 0 & 0 & 0 & 0 \\
\hline TOTAL OR X-10 & 4247 & 11803 & 51 & 1325 & 0 & 6813 & 4556 & 2311 \\
\hline & & & & & & & & \\
\hline \multicolumn{9}{|l|}{ Oak Ridge $Y-12$} \\
\hline EM-30 & 267 & $\overline{155}$ & 29 & $\overline{0}$ & $\overline{0}$ & $\overline{37}$ & 1117 & 280 \\
\hline$E M-40$ & 1668 & 2337 & 31 & 0 & 0 & 1111 & 2581 & 351 \\
\hline$E M-60$ & 0 & 0 & 0 & 0 & 0 & 0 & 0 & 0 \\
\hline TOTAL OR Y -12 & 1936 & 2492 & 601 & 0 & 0 & 1148 & 2698 & 631 \\
\hline & & & & & & & & \\
\hline \multicolumn{9}{|l|}{ Rocky Flats } \\
\hline EM-30 & 2068 & 1196 & 221 & 2640 & $\overline{0}$ & 282 & 903 & 4805 \\
\hline$E M-40$ & 36799 & 12935 & 864 & 0 & 0 & 20369 & 29591 & 656 \\
\hline$E M-60$ & 45553 & 571 & 23 & 0 & 0 & 30527 & 15128 & 30 \\
\hline TOTAL RF & 84420 & 14701 & 1108 & 2640 & 0 & $5 \overline{51179}$ & 45622 & 5491 \\
\hline \multirow{2}{*}{\multicolumn{9}{|c|}{ Savannah River }} \\
\hline & & & & & & & & \\
\hline EM-30 & $\overline{40445}$ & 37553 & 4 & 223 & $\overline{1556}$ & $4 \overline{46517}$ & 2661 & 10676 \\
\hline$E M-40$ & 0 & 0 & 0 & 0 & 0 & $\begin{array}{l}0 \\
0\end{array}$ & 0 & $\overline{0}$ \\
\hline$E M-60$ & 0 & 0 & 0 & 0 & 0 & 0 & 0 & 0 \\
\hline TOTAL SR & 40445 & 37553 & 463 & 223 & 1556 & $4 \overline{46517}$ & 2661 & 10676 \\
\hline \multirow{2}{*}{\multicolumn{9}{|c|}{ EM }} \\
\hline & & & & & & & & \\
\hline EM-30 & 136317 & 173585 & $88 \overline{3}$ & 14668 & $4 \overline{4138}$ & $\overline{178140}$ & 17832 & 63445 \\
\hline$E M-40$ & 207620 & 230957 & 4334 & 294 & 0 & 125898 & 282019 & 34063 \\
\hline$E M-60$ & 125774 & 1600 & 111 & 1 & 0 & 85001 & 40758 & 76 \\
\hline TOTAL EM & 499626 & 422876 & 14023 & 16016 & 4434 & 415143 & 352256 & $\overline{102733}$ \\
\hline
\end{tabular}


EM-263 ASSESSMENT OF EM SAMPLING AND ANALYSIS NEEDS

Table If: FY 98 Sample Analyses by Rad Level and Matrix Type

\begin{tabular}{|c|c|c|c|c|c|c|c|c|}
\hline $\begin{array}{l}\text { Site-EM Office/ } \\
\text { Rad LvI-Matrix Type }\end{array}$ & RO & $\overline{\mathrm{R} 1}$ & R2 & $\overline{\mathrm{R} 3}$ & $\overline{\mathrm{R} 4}$ & M1 & M2 & $\overline{\mathrm{M3}}$ \\
\hline & & & & & & & & \\
\hline \multicolumn{9}{|l|}{ Fernald } \\
\hline EM-30 & 0 & 0 & 0 & 0 & $\overline{01}$ & $\overline{0 \mid}$ & 0 & $\overline{0}$ \\
\hline$E M-40$ & 10553 & 5460 & 420 & 1 & 0 & 6338 & 4999 & 4660 \\
\hline$E M-60$ & 0 & 0 & 0 & 01 & 0 & 0 & 0 & 0 \\
\hline TOTAL Fernald & 10553 & 5460 & 420 & 11 & 0 & 6338 & 4999 & 4660 \\
\hline & & & & & & & & \\
\hline \multicolumn{9}{|l|}{ INEL } \\
\hline$E M-30$ & 58611 & 10126 & 123 & 1326 & 0 & 8851 & 1065 & 2355 \\
\hline$E M-40$ & 1514 & 19344 & 187 & 104 & 0 & 1146 & 16490 & 3287 \\
\hline$E M-60$ & 4357 & 65 & 21 & 0 & 0 & 3203 & 1046 & 0 \\
\hline TOTAL INEL & 11732 & 29535 & 332 & $143 \pi$ & 01 & 13200 & 18600 & 5643 \\
\hline \multirow{2}{*}{\multicolumn{9}{|c|}{ Nevada Test Site }} \\
\hline & & & & & & & & \\
\hline$E M-30$ & 113 & 2 & 11 & 01 & 0 & 83 & 27 & 0] \\
\hline$E M-40$ & 24667 & 23932 & 404 & 0 & 0 & 14488 & 31037 & 3500 \\
\hline$E M-60$ & 0 & 0 & 0 & 0 & 0 & 0 & D & 0 \\
\hline TOTAL NTS & 24780 & 23934 & 4051 & 01 & 0 & 14571 & 31064 & 3500 \\
\hline & & & & 10 & $=$ & & & \\
\hline \multicolumn{9}{|l|}{ Oak Ridge $\mathrm{K}-25$} \\
\hline$E M-30$ & 304 & 7356 & 1999 & 01 & 0 & 4793 & 1427 & 3476 \\
\hline$E M-40$ & 793 & 21329 & 3 & 0 & 0 & 729 & 17679 & 3694 \\
\hline$E M-60$ & 0 & 0 & 0 & 0 & 0 & 0 & 0 & 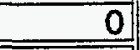 \\
\hline TOTAL OR K-25 & 1097 & 28684 & 2002 & 이 & 0 & 5523 & 19106 & 7170 \\
\hline & & & & & & & & \\
\hline \multicolumn{9}{|l|}{ Oak Ridge $X-10$} \\
\hline$E M-30$ & 2144 & 7109 & 14 & 1325 & (0 & 5305 & 166 & 1402 \\
\hline$E M-40$ & 1498 & 4514 & 20 & 0 & 0 & 1194 & 3906 & 909 \\
\hline $\mathrm{EM}-60$ & 0 & 0] & 0 & 01 & 0 & 01 & 0 & 0) \\
\hline TOTAL OR $X-10$ & 3642 & 11624 & 341 & 1325 & 0 & 6498 & 4072 & 2311 \\
\hline & & & & & & & & \\
\hline \multicolumn{9}{|l|}{ Oak Ridge $Y-12$} \\
\hline$E M-30$ & 267 & 155 & 29 & 0 & $\overline{0}$ & 37 & 117 & 280 \\
\hline$E M-40$ & 1668 & 2337 & 31 & 0 & 0 & 1111 & 2581 & 351 \\
\hline$E M-60$ & 0 & 01 & 0 & 0 & 0 & 0 & 0 & 0 \\
\hline TOTAL OR Y -12 & 1936 & 2492 & 60 & O) & 0 & 1148 & 2698 & 631 \\
\hline & & & & & & & & \\
\hline \multicolumn{9}{|l|}{ Rocky Flats } \\
\hline$E M-30$ & 2068 & 1196 & 221 & 2640 & 0 & 282 & 903 & 4805 \\
\hline$E M-40$ & 36673 & 12804 & 860 & 0 & 0 & 20297 & 29452 & 605 \\
\hline$E M-60$ & 45553 & 571 & 23 & 0 & 0 & 30527 & 15128 & 30 \\
\hline TOTAL RF & 84294 & 14570 & 1103 & 2640 & 0 & 51107 & 45482 & 5440 \\
\hline \multirow{2}{*}{\multicolumn{9}{|c|}{ Savannah River }} \\
\hline & & & & & & & & \\
\hline$E M-30$ & 40436 & 37269 & 463 & 223 & 1556 & 46498 & 2383 & 10676 \\
\hline$E M-40$ & 0 & 0 & 0 & 0 & 0 & 0 & 0 & 0 \\
\hline$E M-60$ & 0 & 0 & 0 & 0 & 0 & 0 & 0 & 0 \\
\hline TOTAL SR & 40436 & 37269 & 463 & 223 & 1556 & 46498 & 2383 & 10676 \\
\hline \multirow{2}{*}{\multicolumn{9}{|c|}{$\overline{E M}$}} \\
\hline & & & & & & & & \\
\hline EM-30 & 136174 & 168144 & 7580] & 14668 & 4138 & 175158 & 16192 & 61166 \\
\hline$E M-40$ & 216626 & 251218 & 5395 & 294 & 0 & 126851 & 297199 & 47617 \\
\hline$E M-60$ & 125774 & 1600 & 111 & 1 & 0 & 85001 & 40758 & 76 \\
\hline TOTAL EM & 508638 & 437669 & 13738 & 16016 & 4434 & 412918 & 365950 & 114089 \\
\hline
\end{tabular}


EM-263 ASSESSMENT OF EM SAMPLING AND ANALYSIS NEEDS

Table Ilg: FY 99 Sample Analyses by Rad Level and Matrix Type

\begin{tabular}{|c|c|c|c|c|c|c|c|c|}
\hline $\begin{array}{l}\text { Site-EM Office/ } \\
\text { Rad Lvl-Matrix Type }\end{array}$ & $\overline{\mathrm{RO}}$ & R1 & R2 & R3 & $\overline{\mathrm{R} 4}$ & $\overline{\mathrm{M} 1}$ & $\overline{\mathrm{M} 2}$ & $\overline{\mathrm{M3}}$ \\
\hline \multirow{2}{*}{\multicolumn{9}{|c|}{ Fernald }} \\
\hline & & & & & & & & \\
\hline$E \overline{E M}-30$ & 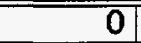 & 0 & 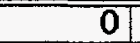 & 0 & 0 & 0 & 0 & 0 \\
\hline$E M-40$ & 6631 & 3193 & 2 & 1 & 0 & 5803 & 3286 & 554 \\
\hline$E M-60$ & 0 & 0 & 0 & 0 & 0 & 0 & 0 & 0 \\
\hline TOTAL Fernald & 6631 & 3193 & 21 & 11 & 01 & 5803 & 3286 & $\overline{554}$ \\
\hline & & & & & $=$ & & & \\
\hline \multicolumn{9}{|l|}{ INEL } \\
\hline$E M-30$ & 5861 & 10126 & 123 & 1326 & 0 & 8851 & 1065 & 2355 \\
\hline$E M-40$ & 1514 & 19344 & 187 & 104 & 0 & 1146 & 16490 & 3287 \\
\hline$E M-60$ & 4357 & 65 & 21 & 0 & 0 & 3203 & 1046 & 0 \\
\hline TOTAL INEL & 11732 & $2 \overline{9535}$ & 332 & 1431 & 0 & 13200 & 18600 & 5643 \\
\hline & & & & & & & & \\
\hline \multicolumn{9}{|l|}{ Nevada Test Site } \\
\hline$\overline{E M}-30$ & $\mid 113$ & 2 & 11 & 0 & 0 & 83) & 27 & $\overline{0}$ \\
\hline$E M-40$ & 24646 & 23271 & 404 & 0 & 0 & 14443 & 30389 & 3500 \\
\hline$E M-60$ & 0 & 0 & 0 & 0 & 0 & 0 & 0 & 0 \\
\hline TOTAL NTS & 24759 & 23272 & 405 & 0 & 01 & 14526 & 30416 & 3500 \\
\hline & & & & & 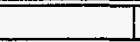 & & & \\
\hline \multicolumn{9}{|l|}{ Oak Ridge K-25 } \\
\hline$E M-30$ & 304 & 7356 & 1999 & 0 & 0 & |4793| & 1427 & 3476 \\
\hline$E M-40$ & 801 & 21750 & 103 & 66 & 0 & 737 & 18099 & 3694 \\
\hline$E M-60$ & 0 & 0 & 0 & \begin{tabular}{l|l}
0 \\
\end{tabular} & 0 & 0 & 0 & 0 \\
\hline TOTAL OR K-25 & 1105 & 29106 & 2102 & 66 & 0 & 5530 & 19526 & 7170 \\
\hline \multirow{2}{*}{\multicolumn{9}{|c|}{ Oak Ridge $X-10$}} \\
\hline & & & & & & & & \\
\hline$E M-30$ & 2144 & 7109 & 14 & 1325 & 0 & 5305 & 166 & 1402 \\
\hline EM-40 & 1441 & 4513 & 20 & 0 & 0 & 1152 & 3893 & 909 \\
\hline$E M-60$ & 0 & 0 & 0 & 0 & 0 & 0 & 0 & 0 \\
\hline TOTAL OR X-10 & 3586 & 11623 & 34 & 1325 & 01 & 6457 & 4059 & 2311 \\
\hline \multirow{2}{*}{\multicolumn{9}{|c|}{ Oak Ridge Y-12 }} \\
\hline & & & & & & & & \\
\hline EM-30 & 267 & 155 & 29 & 0 & 0 & 37. & 1117 & 280 \\
\hline$E M-40$ & 763 & 1906 & 9 & 0 & 0 & 596 & 1766 & 316 \\
\hline$E M-60$ & 0 & 0 & 0 & 0 & 0 & 0 & 0 & 0 \\
\hline TOTAL OR Y-12 & 1030 & 2060 & 38 & 01 & 01 & 633 & 1883 & 596 \\
\hline & & & & & $=$ & & & \\
\hline \multicolumn{9}{|l|}{ Rocky Flats } \\
\hline EM-30 & 2068 & 1196 & 221 & 660 & 0 & 282 & 903 & 2825 \\
\hline$E M-40$ & 36673 & 12804 & 860 & 0 & 0 & 20297 & 29452 & 605 \\
\hline$E M-60$ & 45553 & 571 & 23 & 0 & 0 & 30527 & 15128 & 30 \\
\hline TOTALRF & 84294 & 14570 & 1103 & 6601 & 01 & 51107 & 45482 & 3460 \\
\hline & & & & & & & & \\
\hline \multicolumn{9}{|l|}{ Savannah River } \\
\hline EM-30 & 40436 & 37269 & 463 & 223 & 1556 & 46498 & $2 \overline{2383}$ & 10676 \\
\hline$E M-40$ & 0 & 0 & 0 & 0 & 0 & 0 & 0 & 0 \\
\hline$E M-60$ & 0 & 0 & 0 & 0 & 0 & 0 & 0 & 0 \\
\hline TOTAL SR & 40436 & 37269 & 463 & 223 & 1556 & 46498 & 2383 & 10676 \\
\hline & & & & & & & & \\
\hline \multicolumn{9}{|l|}{ EM } \\
\hline $\mathrm{EM}-30$ & 136174 & 168144 & $7 \overline{7580}$ & $9 \overline{9402}$ & 4138 & 175158 & 16192 & 55900 \\
\hline$E M-40$ & 202190 & 242120 & 4425 & 478 & 0 & 123246 & 288414 & 35894 \\
\hline$E M-60$ & 125774 & 1600 & 111 & 1 & 0 & 85001 & 40758 & 76 \\
\hline TOTALEM & 492947 & 427785 & 12723 & 10525 & 4418 & 408261 & 356803 & 96305 \\
\hline
\end{tabular}


EM-263 ASSESSMENT OF EM SAMPLING AND ANALYSIS NEEDS

Table Ilh: FY 93 Samples by Rad Level and Matrix Type

\begin{tabular}{|c|c|c|c|c|c|c|c|c|}
\hline $\begin{array}{l}\text { Site-EM Office/ } \\
\text { Rad Lvl-Matrix Type }\end{array}$ & Ro & R1 & $\mathrm{R2}$ & $\overline{\mathrm{R} 3}$ & R4 & $\overline{M 1}$ & $\mathrm{M} 2$ & $\overline{M 3}$ \\
\hline \multicolumn{9}{|l|}{ Fernald } \\
\hline$E M-30$ & 01 & $\overline{0}$ & 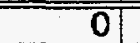 & 0 & $\overline{0}$ & \begin{tabular}{l|l}
0 \\
\end{tabular} & 0 & 0 \\
\hline$E M-40$ & 1542 & 6481 & 350 & 20 & 0 & 1731 & 5304 & 751 \\
\hline$E M-60$ & 0 & 0 & 0 & 0 & 01 & 0 & 0 & 0 \\
\hline TOTAL Fernald & 1542 & 6481 & 350 & 20 & 미 & 1731 & 5304 & 751 \\
\hline & & & & & $=$ & & & \\
\hline \multicolumn{9}{|l|}{ INEL } \\
\hline$E M-30$ & 438 & 5362 & 17 & 6 & \begin{tabular}{l|l}
0 \\
\end{tabular} & 4230 & 269 & 71 \\
\hline$E M-40$ & 1256 & 3532 & 276 & 43 & 3 & 698 & 2965 & 1006 \\
\hline$E M-60$ & 426 & 14 & 2 & 0 & 0 & 325 & 116 & 0] \\
\hline TOTAL INEL & 2120 & 8909 & 295 & 49 & 31 & 5254 & 3350 & 1077 \\
\hline & & & & & $=$ & & & \\
\hline \multicolumn{9}{|l|}{ Nevada Test Site } \\
\hline EM-30 & 11 & 0 & $\overline{0}$ & \begin{tabular}{l|l}
0 \\
\end{tabular} & $\overline{0}$ & 8 & \begin{tabular}{l|l}
3 \\
\end{tabular} & 0 \\
\hline$E M-40$ & 344 & 117 & 20 & 0 & 8 & 31 & 121 & 304 \\
\hline$E M-60$ & 0 & 0 & 0 & 0 & 0 & 0 & 0 & 0 \\
\hline TOTAL NTS & 355 & 118 & 20 & 0 & 8 & 40 & 124 & 304 \\
\hline \multirow{2}{*}{\multicolumn{9}{|c|}{ Oak Ridge K-25 }} \\
\hline & & & & & & & & \\
\hline$E M-30$ & 23 & 510 & 176 & 0 & 0 & 217 & 101 & 414 \\
\hline$E M-40$ & 242 & 362 & 27 & 0 & 1. & 156 & 240 & 36 \\
\hline$E M-60$ & 0 & 0 & 0 & \begin{tabular}{l|l}
0 \\
\end{tabular} & 0 & 01 & 0 & 0 \\
\hline TOTAL OR K-25 & 265 & 872 & 203 & 01 & 11 & 373 & 341 & 450 \\
\hline & & & & & 1 & & & \\
\hline \multicolumn{9}{|l|}{ Oak Ridge $X-10$} \\
\hline EM-30 & 178 & 3854 & 7 & 5 & 0 & 3000 & 74 & 41 \\
\hline$E M-40$ & 177 & 2045 & 19 & 0 & 0 & 165 & 1299 & 637 \\
\hline$E M-60$ & 0 & 0 & 0 & 0 & 01 & $0 \mid$ & 0 & $\underline{0}$ \\
\hline TOTAL OR X-10 & 356 & 5898 & 26 & 51 & 01 & 3164 & 1373 & 678 \\
\hline \multirow{2}{*}{\multicolumn{9}{|c|}{ Oak Ridge $Y-12$}} \\
\hline & & & & & & & & \\
\hline$\overline{E M-30}$ & 11 & 47 & 2 & o) & 0 & $\overline{8 \mid}$ & 36 & 6 \\
\hline$E M-40$ & 139 & 394 & 30 & 0 & 0 & 101 & 214 & 14 \\
\hline$E M-60$ & 01 & 01 & 0 & 0 & 01 & 0 & 0 & 0 \\
\hline TOTAL ORY Y-12 & 140| & 441 & 32 & 01 & 01 & 108 & 251 & 20 \\
\hline & & & & & & & & \\
\hline \multicolumn{9}{|l|}{ Rocky Flats } \\
\hline$E M-30$ & 0 & 0 & 0 & 295 & 01 & 0 & 0 & 295 \\
\hline$E M-40$ & 252 & 746 & 56 & 0 & 0 & 191 & 404 & 14 \\
\hline$E M-60$ & 449 & 288 & 2 & 0 & 01 & 547 & 124 & 3 \\
\hline TOTAL RF & 701 & 1034 & 59 & 295 & 01 & 738 & 528 & 312 \\
\hline & & & & & & & & \\
\hline \multicolumn{9}{|l|}{ Savannah River } \\
\hline$E M-30$ & 10161 & 19488 & 227 & 62 & 210 & 21299 & 808 & 3216 \\
\hline$E M-40$ & 0 & 0 & 0 & 0 & 0 & 0 & 0 & 0 \\
\hline$E M-60$ & 0 & 0 & 0 & 0 & $0]$ & 0 & 01 & 0 \\
\hline TOTAL SA & 10161 & 19488 & 227 & 62 & 210 & 21299 & 808 & 3216 \\
\hline \multicolumn{9}{|l|}{ EM } \\
\hline$E M-30$ & 28326 & 76666 & 1123 & (965 & 550 & 75357| & 3384 & 10595 \\
\hline$E M-40$ & 9484 & 32823 & 1871 & 150 & 29 & 7375 & 25313 & 6630 \\
\hline$E M-60$ & 1968 & 680 & 9 & $1]$ & 0 & 1962 & 542 & 6 \\
\hline TOTAL EM & 40659 & 112425 & 3151 & 1121 & 577 & 85039 & 31406 & 17703 \\
\hline
\end{tabular}


EM-263 ASSESSMENT OF EM SAMPLING AND ANALYSIS NEEDS

Table Ili: FY 94 Samples by Rad Level and Matrix Type

\begin{tabular}{|c|c|c|c|c|c|c|c|c|}
\hline $\begin{array}{l}\text { Site-EM Office/ } \\
\text { Rad LvI-Matrix Type }\end{array}$ & RO & R1 & $\overline{\mathrm{R} 2}$ & $\overline{\mathrm{R} 3}$ & $\overline{\mathrm{R} 4}$ & M1 & $\overline{\mathrm{M} 2}$ & M3 \\
\hline & & & & & & & & \\
\hline \multicolumn{9}{|l|}{ Fernald } \\
\hline$\overline{E M}-\mathbf{3 0}$ & $\overline{0}$ & 0 & (0) & 0 & $\overline{0}$ & $\overline{0}$ & 0 & $\overline{0}$ \\
\hline$E M-40$ & 1546 & 11603 & 440 & 1 & 0 & 2679 & 9138 & 1527 \\
\hline$E M-60$ & 0 & 0 & 0 & 0 & 0 & 0 & 0 & 0 \\
\hline TOTAL Fernald & 1546 & 11603 & 440 & 1 & $\overline{0}$ & 2679 & 9138 & 1527 \\
\hline & & & & & & & & \\
\hline \multicolumn{9}{|l|}{ IINEL } \\
\hline$E M-30$ & 438 & 5370 & 17 & 154 & $\overline{0}$ & 42231 & 275 & 220 \\
\hline$E M-40$ & 1479 & 3660 & 271 & 190 & 0 & 893 & 3206 & 1065 \\
\hline$E M-60$ & 426 & 14 & 2 & 0 & 0 & 325 & 116 & 0 \\
\hline TOTAL INEL & 2343 & 9044 & 289 & 344 & 0 & 5450 & 3597 & 1285 \\
\hline & & & & & 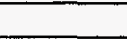 & & & \\
\hline \multicolumn{9}{|l|}{ Nevada Test Site } \\
\hline EM-30 & 11 & 0 & $\overline{0}$ & (0 & $\overline{\overline{0}}$ & $\overline{88}$ & 3 & $\overline{0}$ \\
\hline$E M-40$ & 985 & 2169 & 172 & 0 & 7 & 558 & 1232 & 293 \\
\hline$E M-60$ & 0 & 0 & 0 & 0 & 0 & 0 & 0 & 0 \\
\hline TOTAL NTS & 996 & 2170 & 172 & 0 & $\overline{7}$ & 567 & 1235 & 293 \\
\hline \multirow{2}{*}{\multicolumn{9}{|c|}{ Oak Ridge K-25 }} \\
\hline & & & & & & & & \\
\hline$E M-30$ & $\overline{23}$ & 604 & 209 & (0 & $\overline{\overline{0}}$ & 254 & $\overline{117}$ & $\overline{4993}$ \\
\hline$E M-40$ & 286 & 22367 & 4739 & 0 & 1 & 5391 & 8229 & 14273 \\
\hline$E M-60$ & 0 & 0 & 0 & 0 & 0 & 0 & 0 & 0 \\
\hline TOTAL ORK-25 & 309 & 22971 & 4948 & 0 & 1 & 5645 & 8346 & 14767 \\
\hline \multirow{2}{*}{\multicolumn{9}{|c|}{ Oak Ridge X-10 }} \\
\hline & & & & & & & & \\
\hline$E M-30$ & 181 & 3854 & 7 & 595 & 0 & 3000 & 77 & $\overline{632}$ \\
\hline$E M-40$ & 310 & 4517 & 36 & 0 & 0 & 278 & 2878 & 1432 \\
\hline$E M-60$ & 0 & 0 & 0 & 0 & $\overline{0}$ & 0 & 0 & 0 \\
\hline TOTAL OR X-10 & 491 & 8371 & 44 & 595 & 0 & 3278 & 2955 & 2063 \\
\hline & & & & & & & & \\
\hline \multicolumn{9}{|l|}{ Oak Ridge Y-12 } \\
\hline$E M-30$ & 37 & 59 & 2 & 0 & $\overline{0}$ & 27 & (211 & 10 \\
\hline$E M-40$ & 105 & 966 & 16 & o & 0 & 86 & 603 & 273 \\
\hline$E M-60$ & 0 & 0 & 0 & 0 & 0 & 0 & 0 & 0 \\
\hline TOTAL OR Y -12 & 142 & 1025 & 18 & 0 & 0 & 113 & 664 & 282 \\
\hline \multirow{2}{*}{\multicolumn{9}{|c|}{ Rocky flats }} \\
\hline & & & & & & & & \\
\hline EM-30 & $\overline{7}$ & 741 & 149 & 590 & $\overline{\overline{0}}$ & 205 & $3 \overline{347}$ & $\overline{956}$ \\
\hline$E M-40$ & 3689 & 10906 & 825 & 0 & 0 & 2794 & 5915 & 210 \\
\hline$E M-60$ & 449 & 288 & 2 & 0 & 0 & 547 & 124 & 3 \\
\hline \multirow[t]{2}{*}{ TOTAL RF } & 4146 & 11936 & 977 & 590 & $\overline{0}$ & 3545 & 6386 & $\overline{1169}$ \\
\hline & & & & & & & & \\
\hline \multicolumn{9}{|l|}{ Savannah River } \\
\hline EM-30 & 10269 & 19656 & 233 & 62 & 211 & 21420 & 937 & $\overline{3249}$ \\
\hline$E M-40$ & 0 & 0 & 0 & 0 & 0 & 0 & 0 & 0 \\
\hline$E M-60$ & 0 & 0 & 0 & 0 & 0 & 0 & 0 & 0 \\
\hline TOTAL SR & 10269 & 19656 & 233 & 62 & 211 & 21420 & 937 & 3249 \\
\hline & & & & & & & & \\
\hline \multicolumn{9}{|l|}{$\overline{E M}$} \\
\hline EM-30 & 29609 & 81769 & 1670 & 3782 & 569 & 78695 & 4905 & 15012 \\
\hline$E M-40$ & 23353 & 156205 & 18069 & 530 & 20 & 35247 & 86737 & 53022 \\
\hline$E M-60$ & 2493 & 862 & 12 & 1 & 0 & 2486 & 686 & 8 \\
\hline TOTALEM & 56271 & 241238 & 19800 & 4426 & 607 & 118698 & 92457 & 68486 \\
\hline
\end{tabular}


Table Ilj: FY 95 Samples by Rad Level and Matrix Type

\begin{tabular}{|c|c|c|c|c|c|c|c|c|}
\hline $\begin{array}{l}\text { Site-EM Office/ } \\
\text { Rad Lvl-Matrix Type }\end{array}$ & RO & $\overline{\mathrm{R} 1}$ & R2 & $\overline{\mathrm{R} 3}$ & $\overline{\mathrm{R} 4}$ & $\overline{\mathrm{M} 1}$ & M2 & $\overline{\mathrm{M} 3}$ \\
\hline \multicolumn{9}{|l|}{ Fernald } \\
\hline$E M-30$ & 0 & 01 & 0 & 0 & 0 & 0 & o| & 0 \\
\hline$E M-40$ & 1964 & 11579 & 428 & 1 & 0 & 2893 & 9366 & 1567 \\
\hline$E M-60$ & 0 & 0 & 0 & 0 & 0 & \begin{tabular}{l|l}
0 \\
\end{tabular} & 0 & 0 \\
\hline TOTAL Fernald & 1964 & 11579 & 428 & 11 & 0 & 2893 & 9366 & 1567 \\
\hline & & & & & 4 & & & \\
\hline \multicolumn{9}{|l|}{ INEL } \\
\hline$E M-30$ & $4 \overline{438}$ & $5 \overline{5370}$ & 17 & 154 & 0 & 42311 & 275 & 220 \\
\hline$E M-40$ & 1713 & 5518 & 271 & 190 & 0 & 1040 & 4502 & 1715 \\
\hline$E M-60$ & 426 & 14 & 2 & 0 & 0 & 325 & 116 & 0 \\
\hline TOTAL INEL & 2577 & 10902 & 289 & 344 & 01 & 5596 & 4893 & 1935 \\
\hline & & & & & & & & \\
\hline \multicolumn{9}{|l|}{ Nevada Test Site } \\
\hline$E M-30$ & 11 & 0 & 0 & 0 & 0 & $\overline{8}$ & 3 & -0) \\
\hline$E M-40$ & 1017 & 9296 & 172 & 0 & 7 & 651 & 5781 & 2809 \\
\hline$E M-60$ & 0 & 0 & 0 & 0 & 0 & 0 & 0 & 0 \\
\hline TOTAL NTS & 1028 & (9296 & 172 & 01 & 71 & 659 & 5784 & 2809 \\
\hline & & & & & 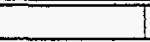 & & & \\
\hline \multicolumn{9}{|l|}{ Oak Ridge K-25 } \\
\hline EM-30 & 23 & 604 & 209 & 0 & 0 & 254 & 117 & (493 \\
\hline$E M-40$ & 259 & 22361 & 4737 & 0 & 0 & 5389 & 8221 & 14248 \\
\hline$E M-60$ & 0 & 0 & 0 & 0 & 0 & \begin{tabular}{l|l}
0 \\
\end{tabular} & 0 & 0] \\
\hline TOTAL OR K-25 & 282 & 22965 & 4946 & $0 \mid$ & 0 & 5643 & 8338 & 14741 \\
\hline & & & & & & & & \\
\hline \multicolumn{9}{|l|}{ Oak Ridge X-10 } \\
\hline$E M-30$ & 178 & 3854 & 7 & 595 & 0 & 3000 & 74 & 631 \\
\hline$E M-40$ & 226 & 4260 & 17 & 0 & 0 & 213 & 2742 & 1427 \\
\hline EM-60 & 0 & 0 & 0 & 0 & 0 & 0 & 0 & 0 \\
\hline TOTAL OR X-10 & 405 & 8114 & 241 & 595 & 0 & 3212 & 2816 & 2058 \\
\hline & & & & & & & & \\
\hline \multicolumn{9}{|l|}{ Oak Ridge $Y-12$} \\
\hline$\overline{E M-30}$ & $\overline{11}$ & 47 & 2 & $\overline{01}$ & 0 & 8 & 36 & 6 \\
\hline$E M-40$ & 85 & 924 & 13 & 0 & 0 & 75 & 579 & 266 \\
\hline$E M-60$ & 0 & 0 & 0 & 0 & 0 & 0 & 0 & 0 \\
\hline \multirow[t]{2}{*}{ TOTAL OR Y -12} & 86 & 972 & 151 & $\overline{01}$ & 01 & 83 & 615 & 272 \\
\hline & & & & & & & & \\
\hline \multicolumn{9}{|l|}{ Rocky Flats } \\
\hline$E M-30$ & 7 & 741 & 149 & 1180 & 0 & 205 & 347 & $\overline{1546}$ \\
\hline$E M-40$ & 1660 & 4876 & 369 & 0 & 0 & 1251 & 2656 & 95 \\
\hline$E M-60$ & 449 & 288 & 2) & 0 & 0 & 547 & 124 & 3 \\
\hline TOTAL RF & 2116 & 5906 & 520 & 1180 & 0 & 2003 & 3126 & 1644 \\
\hline & & & & & & & & \\
\hline \multicolumn{9}{|l|}{ Savannah River } \\
\hline$E M-30$ & 10269 & 19648 & 233 & 62 & 211 & 21419 & 931 & 3248 \\
\hline$E M-40$ & 0 & 0 & 0 & 0 & 0 & 0 & 0 & (0) \\
\hline$E M-60$ & 0 & 0 & 0 & 0 & 0 & 0 & 0 & 0 \\
\hline TOTAL SR & 10269 & 19648 & 233 & 62 & 211 & 21419 & 931 & 3248 \\
\hline & & & & & & & & \\
\hline \multicolumn{9}{|l|}{ EM } \\
\hline$E M-30$ & 29066 & 80504 & 1645 & 5296 & 5611 & 77471 & (4744| & $\overline{16347}$ \\
\hline$E M-40$ & 19734 & 167620 & 17116 & 544 & 19 & 32808 & 96461 & 63063 \\
\hline$E M-60$ & 2196 & 759 & 10 & 1 & 0 & 2189 & 604 & 7 \\
\hline TOTAL EM & 53931 & 257417 & 19089 & 6284 & 626 & 119544 & 103306 & 81433 \\
\hline
\end{tabular}


EM-263 ASSESSMENT OF EM SAMPLING AND ANALYSIS NEEDS

Table Ilk: FY 96 Samples by Rad Level and Matrix Type

\begin{tabular}{|c|c|c|c|c|c|c|c|c|}
\hline $\begin{array}{l}\text { Site-EM Office/ } \\
\text { Rad Lvl-Matrix Type }\end{array}$ & Ro & $\overline{\mathrm{R} 1}$ & $\overline{\mathrm{R} 2}$ & R3 & R4 & M1 & M2 & M3 \\
\hline \multicolumn{9}{|l|}{ Fernald } \\
\hline$E M-30$ & $\overline{0}$ & 0 & 0 & 0 & $\overline{0}$ & 0 & 0 & 0 \\
\hline$E M-40$ & 1120 & 845 & 6 & 1 & 0 & 861 & 868 & 96 \\
\hline$E M-60$ & 0 & 0 & 0 & 0 & 0 & 0 & 0 & 0 \\
\hline TOTAL Fernald & 1120 & 845 & 6 & 1 & $\overline{0}$ & 861 & 868 & 96 \\
\hline & & & & & & & & \\
\hline \multicolumn{9}{|l|}{ INEL } \\
\hline EM-30 & 438 & 5370 & 17 & 154 & $\overline{0}$ & 4231 & 275 & 220 \\
\hline$E M-40$ & 2233 & 6189 & 161 & 177 & 0 & 1291 & 5233 & 2162 \\
\hline$E M-60$ & 426 & 14 & 2 & 0 & 0 & 325 & 116 & 0 \\
\hline TOTAL INEL & 3097 & 11573 & 180 & 331 & $\overline{0}$ & 5848 & 5624 & 2382 \\
\hline & & & & & & & & \\
\hline \multicolumn{9}{|l|}{ Nevada Test Site } \\
\hline$E M-30$ & $\overline{11}$ & 0 & 0 & $\overline{0 \mid}$ & $\overline{0}$ & 8 & $\overline{23}$ & $\overline{0}$ \\
\hline$E M-40$ & 1032 & 9340 & 176 & 0 & 7 & 661 & 5803 & 2810 \\
\hline$E M-60$ & 0 & 0 & 0 & 0 & 0 & 0 & 0 & 0 \\
\hline TOTAL NTS & 1043 & 9340 & 176 & 01 & 7 & 670 & 5806 & 2810 \\
\hline & & & & 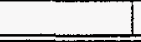 & - & 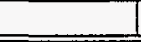 & 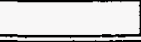 & \\
\hline \multicolumn{9}{|l|}{ Oak Ridge K-25 } \\
\hline$E M-30$ & $2 \overline{3}$ & 510 & 176 & 0 & $\overline{0}$ & 217 & 101 & 414 \\
\hline$E M-40$ & 180 & 9044 & 8 & 0 & 0 & 214 & 5806 & 3151 \\
\hline$E M-60$ & 0 & 0 & 0 & 0 & 0 & 0 & 0 & 0 \\
\hline TOTAL OR K-25 & 203 & 9554 & 183 & 01 & 0 & 432 & 5907 & 3565 \\
\hline & & & & & 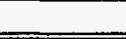 & & & \\
\hline \multicolumn{9}{|l|}{ Oak Ridge X-10 } \\
\hline$E M-30$ & 178 & 3854 & 7 & 595 & $\overline{0}$ & 3000 & 74 & 631 \\
\hline$E M-40$ & 200 & 1984 & 13 & 0 & 0 & 171 & 1292 & 640 \\
\hline$E M-60$ & 0 & 0 & 0 & 0 & 0 & 0 & 0 & 0 \\
\hline TOTAL OR X-10 & 378 & 5838 & 21 & 595 & 0 & 3170 & 1366 & 1271 \\
\hline & & & & & & & & \\
\hline \multicolumn{9}{|l|}{ Oak Ridge $Y-12$} \\
\hline$E M-30$ & 1 & 47 & $\overline{2}$ & 0 & $\overline{0}$ & 8 & 36 & $\overline{\overline{6}}$ \\
\hline EM-40 & 145 & 944 & 13 & 0 & 0 & 108 & 620 & 271 \\
\hline$E M-60$ & 0 & 0 & 0 & 0 & 0 & 0 & 0 & 0 \\
\hline TOTAL OR Y-12 & 146 & 991 & 15 & 0 & 0 & 116 & 657 & 278 \\
\hline & & & & & $=$ & & & \\
\hline \multicolumn{9}{|l|}{ Rocky Flats } \\
\hline$E M-30$ & 7 & 367 & 15 & 1180 & 0 & 59 & 281 & 1229 \\
\hline$E M-40$ & 1662 & 4882 & 369 & 0 & 0 & 1253 & 2661 & 95 \\
\hline$E M-60$ & 449 & 288 & 2 & 0 & 0 & 547 & 124 & 3 \\
\hline \multirow[t]{2}{*}{ TOTALRF } & 2118 & 5538 & 386 & 1180 & $\overline{0}$ & 1859 & 3067 & 1327 \\
\hline & & & & & & & & \\
\hline \multicolumn{9}{|l|}{ Savannah River } \\
\hline$E M-30$ & $\overline{10267}$ & 19585 & 185 & 53 & $\overline{211}$ & 21416 & 813 & 3248 \\
\hline$E M-40$ & 0 & 0 & 0 & 0 & 0 & 0 & 0 & 0 \\
\hline$E M-60$ & 0 & 0 & 0 & 0 & 0 & 0 & 0 & 0 \\
\hline TOTAL SR & 10267 & 19585 & 185 & 53 & 211 & 21416 & 813 & 3248 \\
\hline \multirow{2}{*}{\multicolumn{9}{|c|}{ EM }} \\
\hline & & & & & & & & \\
\hline EM-30 & 29061 & 79094 & 1069 & 5270 & 561 & 76978 & 4210 & 15291 \\
\hline$E M-40$ & 18661 & 94366 & 2118 & 506 & 19 & 12949 & 63284 & 26202 \\
\hline$E M-60$ & 2196 & 759 & 10 & 1 & 0 & 2189 & 604 & 7 \\
\hline TOTAL EM & 52724 & 181569 & 3306 & 6198 & $\overline{624}$ & 98644 & 69187 & 42985 \\
\hline
\end{tabular}


Table III: FY 97 Samples by Rad Level and Matrix Type

\begin{tabular}{|c|c|c|c|c|c|c|c|c|}
\hline $\begin{array}{l}\text { Site-EM Office/ } \\
\text { Rad LvI-Matrix Type }\end{array}$ & RO & $\overline{\mathrm{R} 1}$ & $\overline{\mathrm{R} 2}$ & $\overline{\mathrm{R} 3}$ & R4 & M1 & $\overline{\mathrm{M} 2}$ & $\overline{\mathrm{M3}}$ \\
\hline & & & & & & & & \\
\hline \multicolumn{9}{|l|}{ Fernald } \\
\hline$E M-30$ & 0 & 01 & 01 & 0 & 0 & 0 & 0 & 0 \\
\hline$E M-40$ & 1098 & 779 & 1 & 1 & 0 & 844 & 833 & 95 \\
\hline$E M-60$ & 0 & 0 & 0 & 0 & 0 & 0 & 0 & 0 \\
\hline TOTAL Fernald & 1098 & 779 & 11 & 11 & 0 & 844 & 833 & 95 \\
\hline & & & & & $\longrightarrow$ & & & \\
\hline \multicolumn{9}{|l|}{ IINEL } \\
\hline$E M-30$ & 438 & 5370| & (17| & 596 & 0 & 4231 & 275 & 662 \\
\hline$E M-40$ & 568 & 5644 & 161 & 30 & 0 & 382 & 4082 & 1863 \\
\hline$E M-60$ & 426 & 14 & 2 & 0 & 0 & 325 & 116 & 0 \\
\hline TOTAL INEL & 1431 & 11028 & 180 & 626 & 0 & 4939 & 4473 & 2526 \\
\hline & & & & & & & & \\
\hline \multicolumn{9}{|l|}{ Nevada Test Site } \\
\hline$E M-30$ & 11 & 0 & 0 & 0 & 0 & 8 & 3 & 0 \\
\hline$E M-40$ & 1032 & 9340 & 176 & 0 & 7 & 661 & 5803 & 2810 \\
\hline$E M-60$ & 0 & 0 & 0 & 0 & 0 & 0 & 0 & 0 \\
\hline TOTAL NTS & 1043 & 9340 & 176 & 0 & 7 & 670 & 5806 & 2810 \\
\hline & & & & & 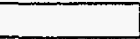 & & & \\
\hline \multicolumn{9}{|l|}{ Oak Ridge K-25 } \\
\hline$E M-30$ & 23 & 510 & 176 & $\overline{\overline{0}}$ & $\overline{0}$ & 217 & 101 & $\overline{4414}$ \\
\hline$E M-40$ & 101 & 8928 & 0 & 0 & 0 & 164 & 5721 & 3145 \\
\hline$E M-60$ & 0 & 0 & 01 & 0 & 0 & \begin{tabular}{l|l}
0 \\
\end{tabular} & 0 & 0 \\
\hline TOTAL OR K-25 & 124 & 9439 & 176 & 0 & 0 & 381 & 5822 & 3559 \\
\hline & & & & & & & & \\
\hline \multicolumn{9}{|l|}{ Oak Ridge X -10} \\
\hline$E M-30$ & 178 & 3854 & 7 & 595 & 0 & 3000 & $\overline{74 \mid}$ & 631 \\
\hline$E M-40$ & 183 & 1934 & 9 & 0 & 0 & 158 & 1264 & 639 \\
\hline$E M-60$ & 0 & 0 & 0 & 0 & 0 & 0 & 0 & 0 \\
\hline TOTAL OR X $\bar{X}-10$ & 361 & 5787 & 17 & 595 & $\overline{0}$ & 3157 & 1339 & 1270 \\
\hline & & & & & 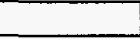 & & & \\
\hline \multicolumn{9}{|l|}{ Oak Ridge $Y-12$} \\
\hline EM-30 & $\overline{71}$ & 47 & 2 & (01 & $\overline{0}$ & 8 & 36 & 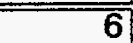 \\
\hline$E M-40$ & 148 & 935 & 13 & 0 & 0 & 111 & 612 & 271 \\
\hline$E M-60$ & 0 & 0 & 0 & 0 & 0 & 0 & 0 & 0 \\
\hline \multirow[t]{2}{*}{ TOTAL ORY -12} & 149 & 982 & 15 & 0 & 0 & 119 & 648 & 278 \\
\hline & & & & & & & & \\
\hline \multicolumn{9}{|l|}{ Rocky Flats } \\
\hline$E M-30$ & $\overline{7}$ & 367 & 15 & 1180 & $\overline{0}$ & 59 & 281 & 1229 \\
\hline$E M-40$ & 1604 & 4706 & 356 & 0 & 0 & 1209 & 2565 & 92 \\
\hline$E M-60$ & 449 & 288 & 2 & 0 & 0 & 547 & 124 & 3 \\
\hline \multirow[t]{2}{*}{ TOTAL RF } & 2060 & 5362 & 373 & 1180 & 0 & 1815 & 2970 & 1324 \\
\hline & & & & & & & & \\
\hline \multicolumn{9}{|l|}{ Savannah River } \\
\hline EM-30 & $\overline{10275}$ & |19766 & 328 & $\overline{81}$ & 211 & 21424 & 1165 & 3248 \\
\hline$E M-40$ & 0 & 0 & 0 & 0 & 0 & 0 & 0 & 0 \\
\hline$E M-60$ & 0 & 0 & 0 & 0 & 0 & 0 & 0 & 0 \\
\hline TOTAL SR & 10275 & 19766 & 328 & 81 & $\overline{211}$ & 21424 & 1165 & 3248 \\
\hline & & & & & & & & \\
\hline \multicolumn{9}{|l|}{$E \bar{M}$} \\
\hline EM-30 & 29082 & 79575 & 1450 & 6523 & 561 & $\mid 77000$ & 5147 & 16468 \\
\hline$E M-40$ & 13255 & 90345 & 2005 & 86 & 18 & 9885 & 58464 & 24964 \\
\hline$E M-60$ & 2204 & 762 & 10 & 1 & 0 & 2198 & 607 & 7 \\
\hline TOTAL EM & $\overline{47144}$ & 178079 & 3607 & 7077 & 620 & 95047 & 65709 & 43062 \\
\hline
\end{tabular}


EM-263 ASSESSMENT OF EM SAMPLING AND ANALYSIS NEEDS

Table IIm: FY 98 Samples by Rad Level and Matrix Type

\begin{tabular}{|c|c|c|c|c|c|c|c|c|}
\hline $\begin{array}{l}\text { Site-EM Office/ } \\
\text { Rad Lvl-Matrix Type }\end{array}$ & $\overline{\mathrm{RO}}$ & $\overline{\mathrm{R} 1}$ & $\overline{\mathrm{R} 2}$ & $\overline{\overline{\mathrm{R} 3}}$ & $\overline{\mathrm{R4}}$ & $\overline{\mathrm{M} 1}$ & $\overline{\mathrm{M} 2}$ & $\overline{\mathrm{M}} \overline{3}$ \\
\hline & & & & & & & & \\
\hline \multicolumn{9}{|l|}{ Fernald } \\
\hline$E M-30$ & 0 & 0 & \begin{tabular}{l|l}
0 \\
\end{tabular} & 0 & 0 & 0 & 0 & $\overline{0}$ \\
\hline$E M-40$ & 1253 & 1501 & 29 & 1 & 0 & 1028 & 1448 & 202 \\
\hline$E M-60$ & 0 & 0 & 0 & 0 & 0 & 0 & 0 & 0 \\
\hline \multirow[t]{2}{*}{ TOTAL Fernald } & 1253 & 1501 & 29 & 1 & 0 & 1028 & 1448 & 202 \\
\hline & & & & & - & & & \\
\hline \multicolumn{9}{|l|}{ INEL } \\
\hline$E M-30$ & $43 \overline{8}$ & $\overline{\mid 5362}$ & 17 & 596 & $\overline{0}$ & 4230 & 269 & $\overline{661}$ \\
\hline$E M-40$ & 578 & 7843 & 160 & 30 & 0 & 407 & 5483 & 2645 \\
\hline$E M-60$ & 426 & 14 & 2 & 0 & 0 & 325 & 116 & 0 \\
\hline \multirow[t]{2}{*}{ TOTAL INEL } & 1442 & 13219 & 179 & 626 & 0 & 4963 & 5868 & 3307 \\
\hline & & & & & & & & \\
\hline \multicolumn{9}{|l|}{ Nevada Test Site } \\
\hline$E M-30$ & 11 & 0 & 0 & 0 & 0 & 8 & 3 & 0 \\
\hline$E M-40$ & 1038 & 9358 & 176 & 0 & 7 & 667 & 5820 & 2810 \\
\hline$E M-60$ & 0 & 0 & 0 & 0 & 0 & 0 & 0 & 0 \\
\hline \multirow[t]{2}{*}{ TOTAL NTS } & 1049 & 9358 & 176 & $\overline{0}$ & 7 & 676 & 5823 & 2810 \\
\hline & & & & $\pi$ & 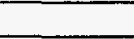 & & & \\
\hline \multicolumn{9}{|l|}{ Oak Ridge K-25 } \\
\hline$E M-30$ & 23 & 417 & 142 & 0 & 0 & 181 & 85 & 335 \\
\hline$E M-40$ & 101 & 8928 & 0 & 0 & 0 & 164 & 5721 & 3145 \\
\hline$E M-60$ & 0 & 0 & 0 & 0 & 0 & 0 & 0 & 0 \\
\hline \multirow[t]{2}{*}{ TOTAL OR K-25 } & 124 & 9345 & 142 & 0 & 0 & $3 \overline{345}$ & 5805 & $\overline{3480}$ \\
\hline & & & 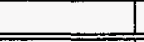 & 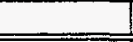 & 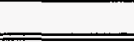 & & & \\
\hline \multicolumn{9}{|l|}{ Oak Ridge X-10 } \\
\hline$E M-30$ & 178 & (3854 & $\overline{77}$ & 595 & $\overline{0}$ & 3000 & $\overline{74}$ & $\overline{631}$ \\
\hline$E M-40$ & 189 & 1857 & 3 & 0 & 0 & 155 & 1241 & 640 \\
\hline$E M-60$ & 0 & 0 & 0 & 0 & 0 & 0 & 0 & 0 \\
\hline \multirow[t]{2}{*}{ TOTAL OR $X-10$} & 367 & 5711 & 10 & 595 & 0 & 3154 & 1316 & 1272 \\
\hline & & & & & 7 & & & \\
\hline \multicolumn{9}{|l|}{ Oak Ridge $Y-12$} \\
\hline EM-30 & 1 & 47 & 2 & 0 & $\overline{0}$ & 8 & 36 & 6 \\
\hline$E M-40$ & 148 & 935 & 13 & 0 & $\overline{0}$ & 111 & 612 & 271 \\
\hline$E M-60$ & 0 & 0 & 0 & 0 & 0 & 0 & 0 & 0 \\
\hline \multirow[t]{2}{*}{ TOTAL OR Y-12 } & 149 & 982 & 15 & 0 & 0 & 119 & 648 & 278 \\
\hline & & & & & & & & \\
\hline \multicolumn{9}{|l|}{ Rocky Flats } \\
\hline$E M-30$ & $\overline{71}$ & 367 & 15! & 1180 & $\overline{\overline{0}}$ & 59 & $\overline{281}$ & 1229 \\
\hline$E M-40$ & 1597 & 4694 & 355 & 0 & 0 & 1206 & 2550 & 91 \\
\hline$E M-60$ & 449 & 288 & 2 & 0 & 0 & 547 & 124 & 3 \\
\hline \multirow[t]{2}{*}{ TOTALRF } & 2053 & 5349 & 372 & 1180 & 0 & 1811 & 2956 & $\overline{1323}$ \\
\hline & & & & & & & & \\
\hline \multicolumn{9}{|l|}{ Savannah River } \\
\hline EM-30 & 10272 & 19757 & 328 & 81 & $\overline{211}$ & 21421 & $\overline{1156}$ & $\overline{3248}$ \\
\hline$E M-40$ & 0 & 0 & 0 & 0 & 0 & 0 & 0 & 0 \\
\hline$E M-60$ & 0 & 0 & 0 & 0 & 0 & 0 & 0 & 0 \\
\hline \multirow[t]{2}{*}{ TOTAL SR } & 10272 & 19757 & 328 & 81 & 211 & 21421 & 1156 & $\overline{3248}$ \\
\hline & & & & & & & & \\
\hline \multicolumn{9}{|l|}{$E M$} \\
\hline$E M-30$ & 29074 & 79282 & 1360 & 6523 & 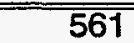 & 76891 & $5 \overline{5065}$ & 16255 \\
\hline$E M-40$ & 13730 & 98323 & 2060 & 86 & 18 & 10466 & 64049 & 27453 \\
\hline$E M-60$ & 2204 & 762 & 10 & 1 & 0 & 2198 & 607 & 7 \\
\hline TOTALEM & 47619 & 185885 & 3566 & 7077 & 620 & 95523 & 71306 & 45367 \\
\hline
\end{tabular}


EM-263 ASSESSMENT OF EM SAMPLING AND ANALYSIS NEEDS

Table IIn: FY 99 Samples by Rad Level and Matrix Type

\begin{tabular}{|c|c|c|c|c|c|c|c|c|}
\hline $\begin{array}{l}\text { Site-EM Office/ } \\
\text { Rad LVl-Matrix Type }\end{array}$ & RO & $\overline{\mathrm{R} 1}$ & $\overline{\mathrm{R} 2}$ & $\overline{\mathrm{R} 3}$ & $\overline{\overline{R 4}}$ & $\overline{\mathrm{M1}}$ & $\overline{\mathrm{M} 2}$ & M3 \\
\hline & & & & & & & & \\
\hline \multicolumn{9}{|l|}{ Fernald } \\
\hline EM-30 & $\overline{\overline{0}}$ & 0 & 0 & (0) & $\overline{0}$ & 0 & $\overline{0}$ & $\overline{\overline{0}}$ \\
\hline$E M-40$ & 1239 & 804 & 1 & 1 & 0 & 916 & 914 & 109 \\
\hline$E M-60$ & 0 & 0 & 0 & 0 & 0 & 0 & 0 & $\mathbf{0}$ \\
\hline TOTAL Fernald & 1239 & 804 & 11 & 1 & 0 & 916 & 914 & 109 \\
\hline & & & & & & & & \\
\hline \multicolumn{9}{|l|}{ INEL } \\
\hline$E M-30$ & 438 & 5362 & 17 & 596 & 0 & 4230 & 269 & $\overline{661}$ \\
\hline$E M-40$ & 578 & 7843 & 160 & 30 & 0 & 407 & 5483 & 2645 \\
\hline$E M-60$ & 426 & 14 & 2 & 0 & 0 & 325 & 116 & 0 \\
\hline \multirow[t]{2}{*}{ TOTAL INEL } & 1442 & 13219 & 179 & 626 & $\overline{0}$ & 4963 & 5868 & 3307 \\
\hline & & & & 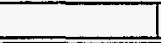 & - & & -1 & \\
\hline \multicolumn{9}{|l|}{ Nevada Test Site } \\
\hline$E M-30$ & 11 & 0 & 0 & $\overline{0}$ & 0 & 8 & 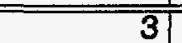 & $\overline{0}$ \\
\hline$E M-40$ & 1031 & 9337 & 176 & 0 & 7 & 660 & 5800 & 2810 \\
\hline$E M-60$ & 0 & 0 & 0 & 0 & 0 & 0 & 0 & $\overline{0}$ \\
\hline TOTALNTS & 1042 & 9337 & 176 & 0 & 7 & 669 & 5803 & 2810 \\
\hline & & & & & & & & \\
\hline \multicolumn{9}{|l|}{ Oak Ridge K-25 } \\
\hline$E M-30$ & $\overline{23}$ & 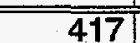 & 142 & 0 & 0 & 181 & 85 & $\overline{335}$ \\
\hline$E M-40$ & 105 & 9043 & 96 & 19 & 0 & 167 & 5950 & 3145 \\
\hline$E M-60$ & 0 & 0 & 0 & 0 & 0 & 0 & 0 & 0 \\
\hline \multirow[t]{2}{*}{ TOTAL OR K-25 } & 128 & 9460 & 238 & 19 & 0 & 348 & 6034 & 3480 \\
\hline & & & & & 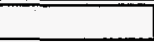 & & & \\
\hline \multicolumn{9}{|l|}{ Oak Ridge $\bar{X}-10$} \\
\hline$E M-30$ & $17 \overline{178}$ & 3854 & 7 & 595 & $\overline{0}$ & 3000 & 74 & $\overline{631}$ \\
\hline$E M-40$ & 183 & 1857 & 3 & 0 & 0 & 151 & 1240 & 640 \\
\hline$E M-60$ & 0 & 0 & 0 & 0 & 0 & 0 & 0 & 0 \\
\hline TOTAL OR $\overline{X-10}$ & $\overline{362}$ & 5710 & 10 & 595 & $\overline{0}$ & 3150 & 1314 & $\overline{1272}$ \\
\hline \multirow{2}{*}{\multicolumn{9}{|c|}{ Oak Ridge $Y-12$}} \\
\hline & & & & & & & & \\
\hline$E M-30$ & 1 & $\overline{487}$ & 2 & 01 & $\overline{0}$ & 8 & 36 & $\overline{\overline{6}}$ \\
\hline$E M-40$ & 47 & 795 & 4 & 0 & 0 & 47 & 505 & 263 \\
\hline$E M-60$ & 0 & 0 & 01 & 0 & 0 & 0 & 0 & 0 \\
\hline TOTAL OR Y -12 & 48 & 842 & 6 & 0 & 0 & 55 & 541 & 270 \\
\hline \multirow{2}{*}{\multicolumn{9}{|c|}{ Rocky Flats }} \\
\hline & & & & & & & & \\
\hline$E M-30$ & 7 & 367 & 15 & 295 & 0 & 59 & 281 & 344 \\
\hline$E M-40$ & 1597 & 4694 & 355 & 0 & 0 & 1206 & 2550 & 91 \\
\hline$E M-60$ & 449 & 288 & 2 & 0 & 0 & 547 & 124 & $\overline{3}$ \\
\hline TOTALRF & 2053 & 5349 & 372 & 295 & 0 & 1811 & 2956 & 438 \\
\hline & & & & & & & & \\
\hline \multicolumn{9}{|l|}{ Savannah River } \\
\hline EM-30 & $1027 \overline{2}$ & 19757 & 328 & 81 & 211 & 21421 & 1156 & $\overline{3248}$ \\
\hline$E M-40$ & 0 & 0 & 0 & 0 & 0 & 0 & 0 & 0 \\
\hline$E M-60$ & 0 & 0 & 0 & 0 & 0 & 0 & 0 & $\underline{0}$ \\
\hline \multirow[t]{2}{*}{ TOTAL SR } & 10272 & 19757 & 328 & 81 & 211 & 21421 & 1156 & 3248 \\
\hline & & & & & & & & \\
\hline \multicolumn{9}{|l|}{$\overline{E M}$} \\
\hline EM-30 & $\overline{290 \overline{74}}$ & 79282 & 1360 & 4169 & $\overline{561}$ & 76891 & 5065 & $1 \overline{13901}$ \\
\hline$E M-40$ & 13338 & 95899 & 2216 & 138 & 18 & 9919 & 62614 & 27073 \\
\hline$E M-60$ & 2204 & 762 & 10 & 1 & 0 & 2198 & 607 & 7 \\
\hline TOTALEM & 47103 & 183122 & 3719 & $4 \overline{4593}$ & 617 & 94668 & 69828 & 42407 \\
\hline
\end{tabular}


Table llia: FY 03 Samples and Sample Analyses Complex-wide by Activity

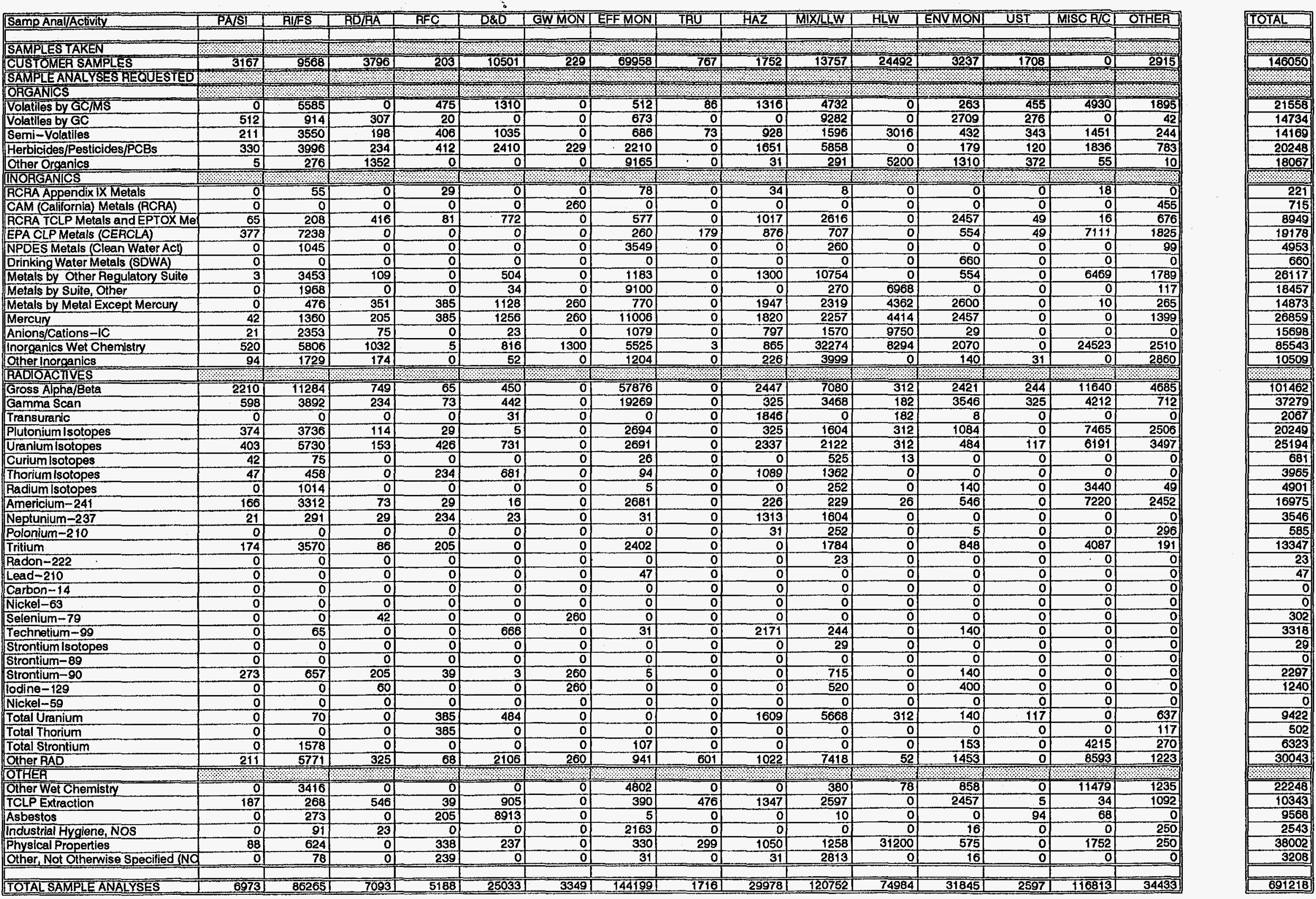


Table IIIb: FY 94 Samples and Sample Analyses Complex-wide by Activity

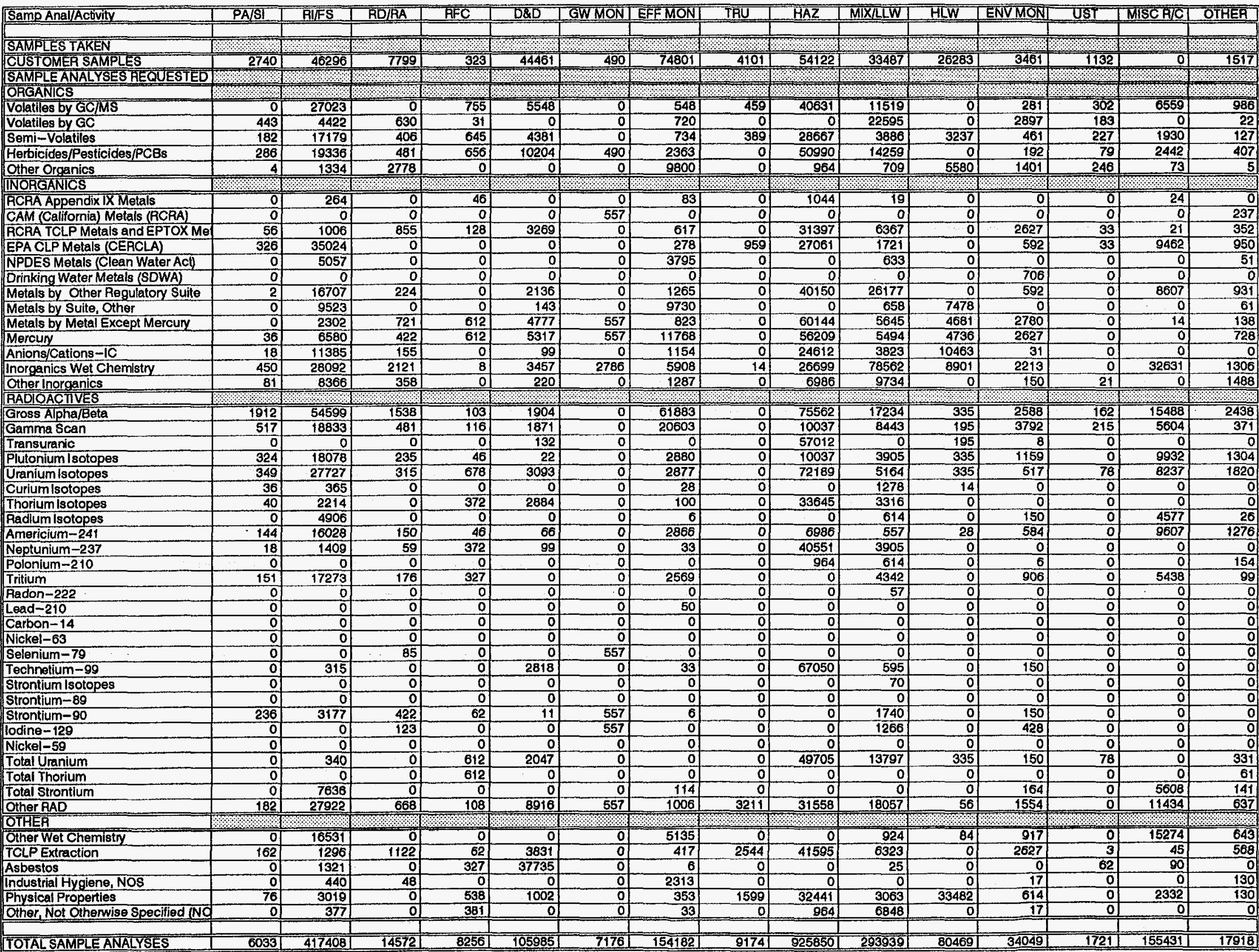


Table IIIC: FY 95 Samples and Sample Analyses Complex-wide by Activity

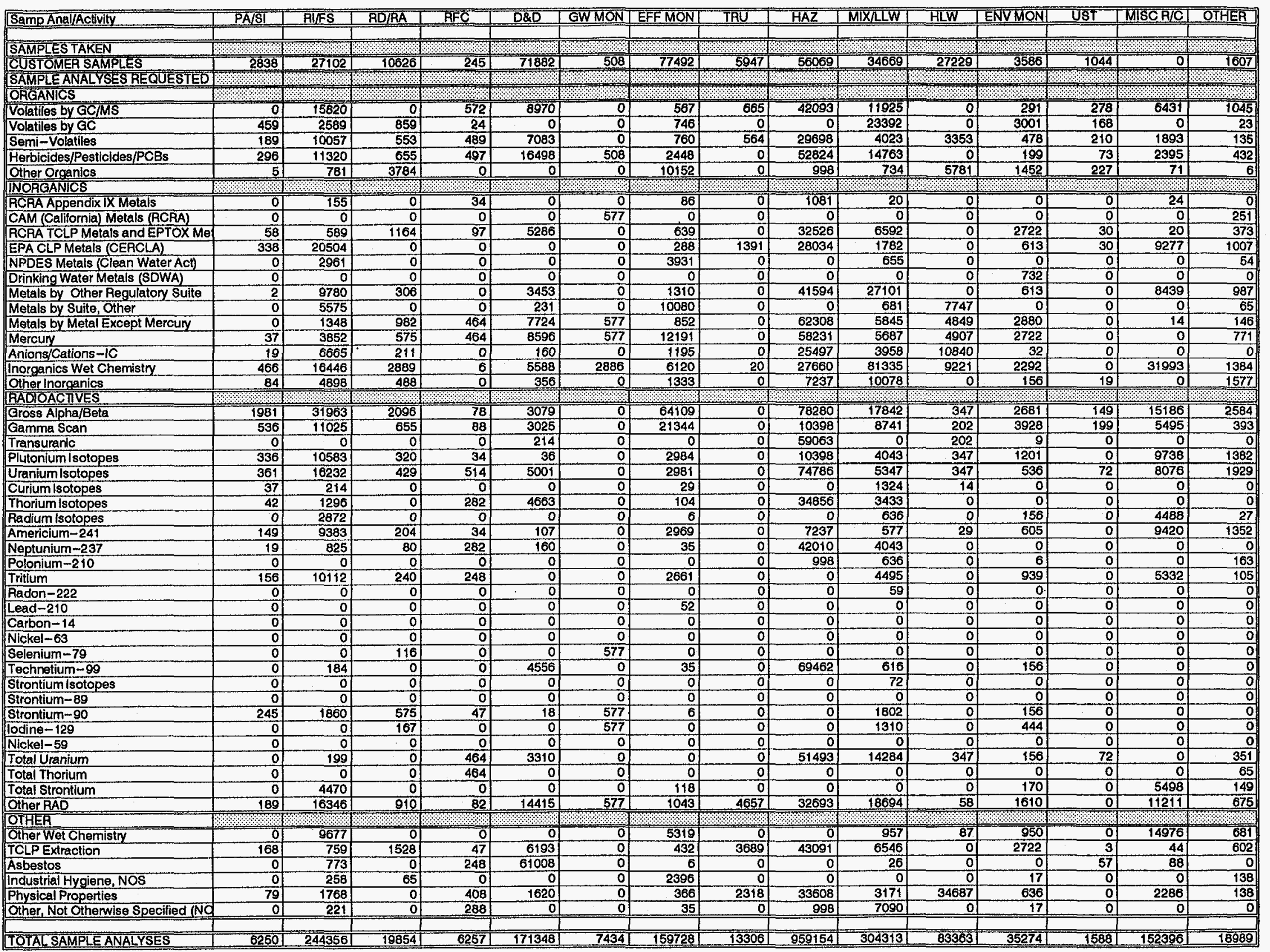


EM-283 ASSESSMENT OF EM SAMPUNG AND ANALYSIS NEEDS

Table IIId: FY 96 Samples and Sample Analyses Complex-wide by Activity

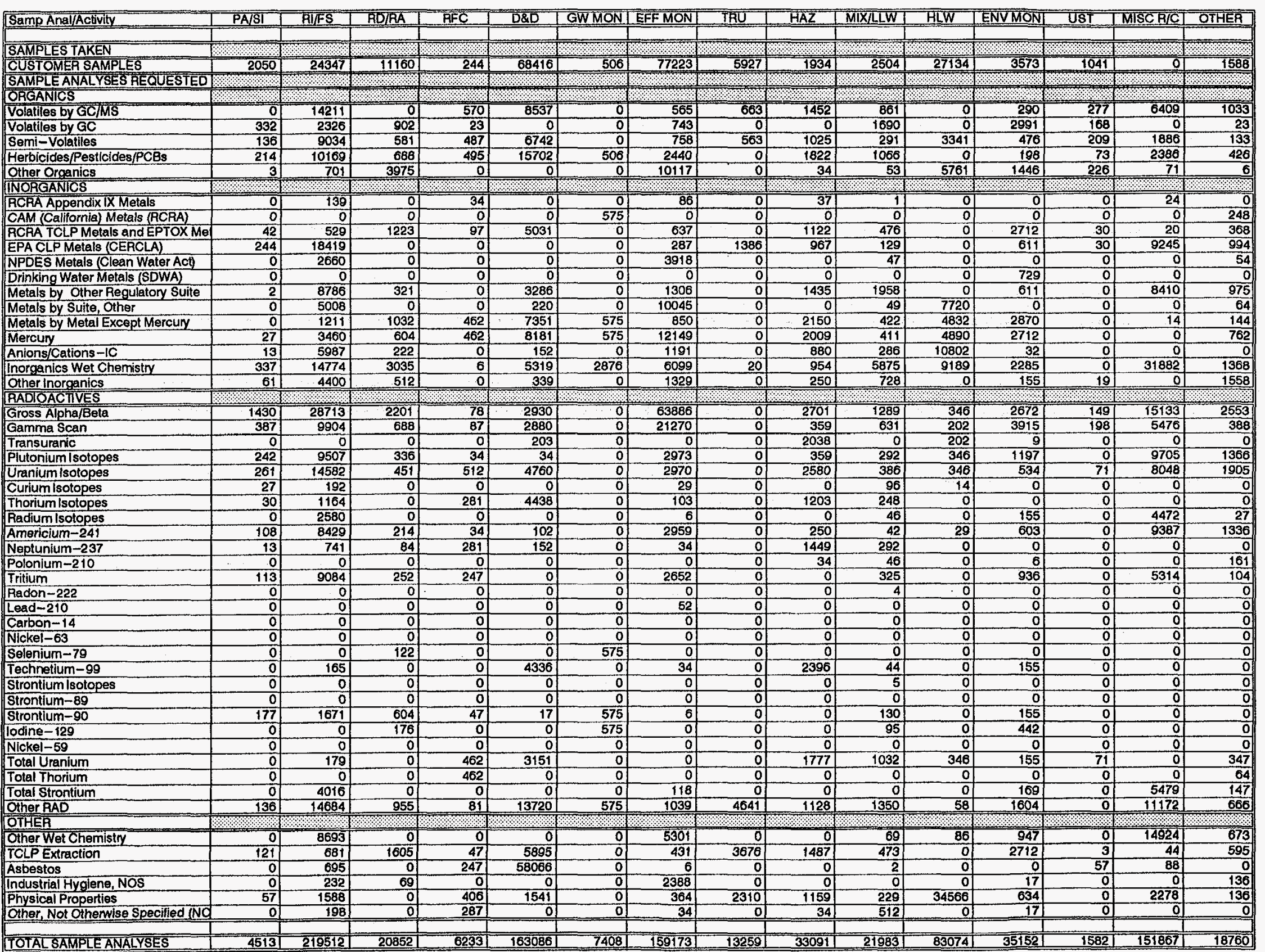


Table Hile: FY 97 Samples and Sample Analyses Complex-wide by Activity

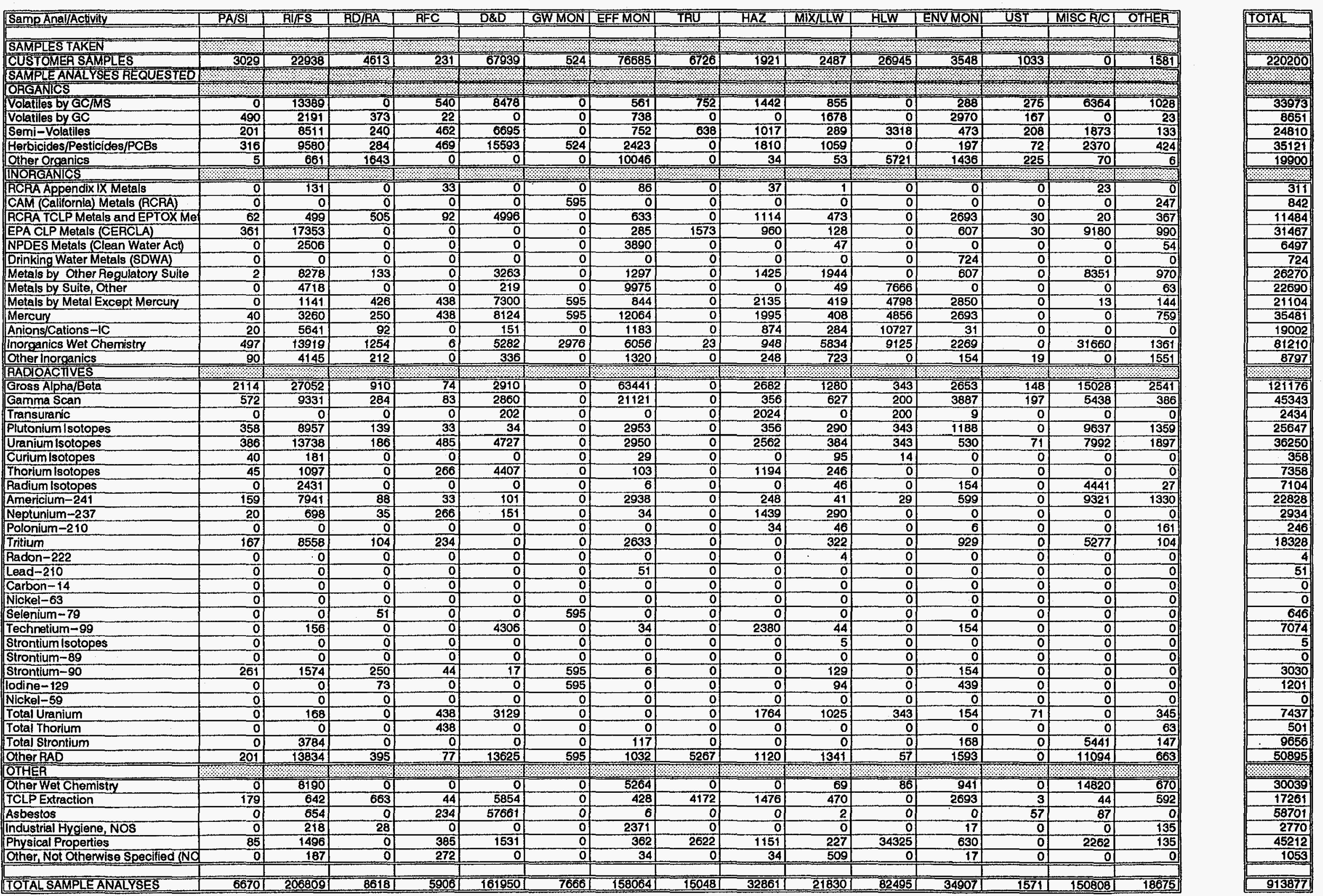


Table IIIf: FY 98 Samples and Sample Analyses Complex-wide by Activity

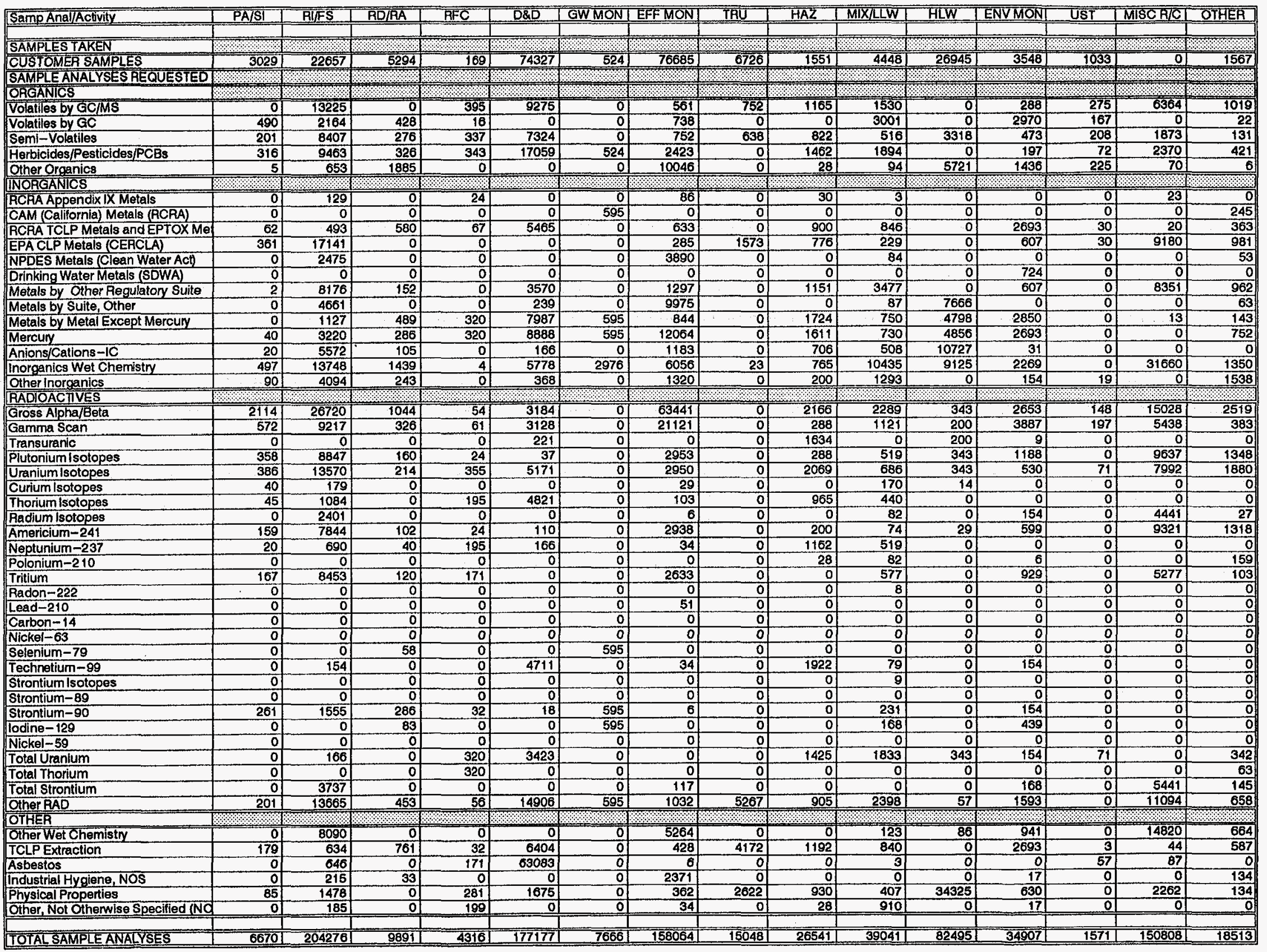


Table IIIg: FY 99 Samples and Sample Analyses Complex-wide by Activity

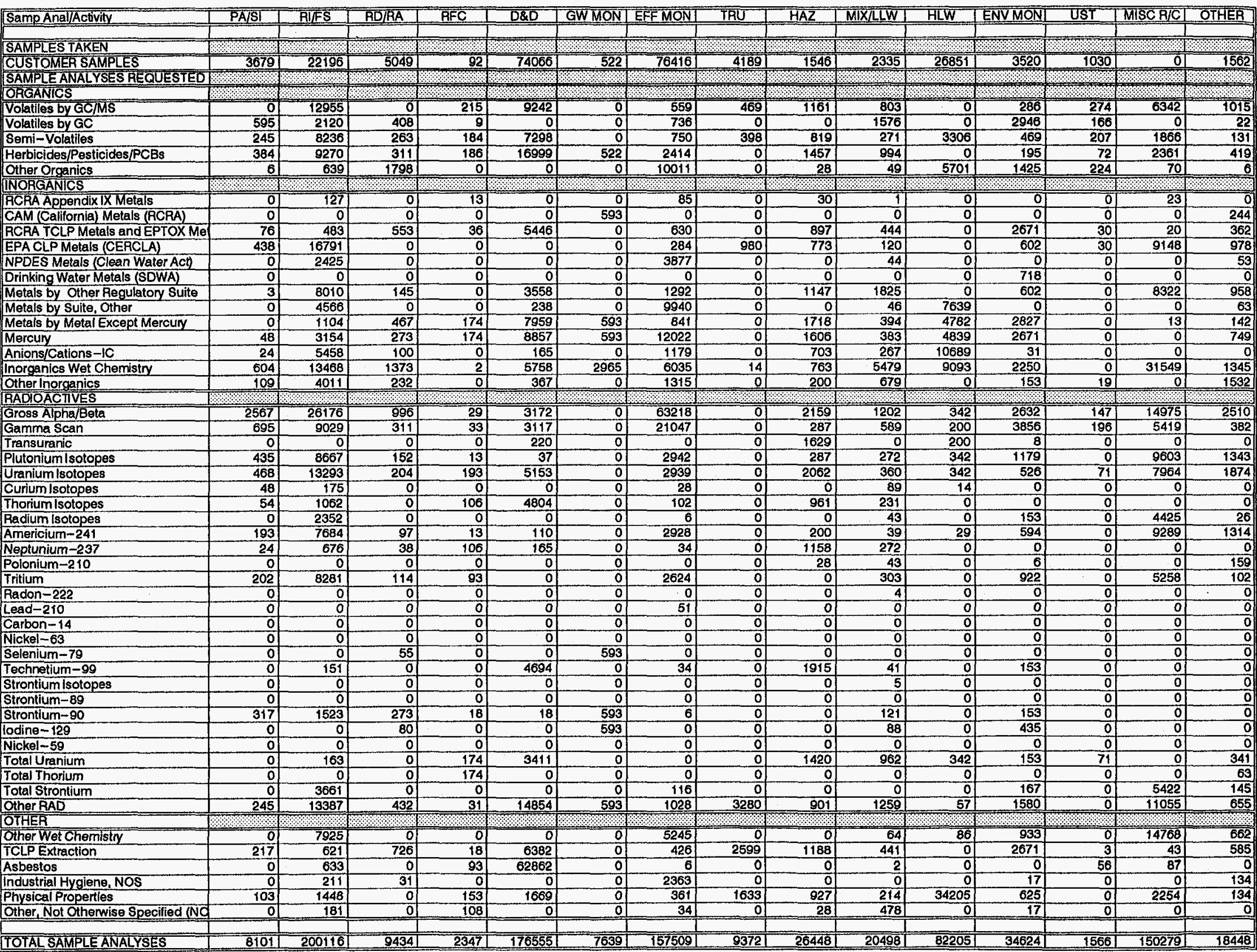


Table IVa: FY 93 Samples and Sample Analyses Complex-wide by Regulatory Driver

\begin{tabular}{|c|c|c|c|c|c|c|c|c|c|}
\hline Samp Anal/Reg Driver & ACRA & CERCLA & NEPA & $\overline{C A A}$ & CWA & NPDES & TSCA & STATE & OTHER \\
\hline & & & & & & & & & \\
\hline \multicolumn{10}{|l|}{ SAMPLES TAKEN } \\
\hline CUSTOMER SAMPLES & 24293 & 13450 & 601 & 832 & 11704 & 560 & 1428 & 47076 & 46647 \\
\hline \multicolumn{10}{|l|}{ SAMPLE ANALYSES AEQUESTED } \\
\hline \multicolumn{10}{|l|}{ ORGANICS } \\
\hline Volatiles by GC/MS & 7097 & 4672 & 5| & 66 & 243 & 8 & 1092 & 340 & 8036 \\
\hline Volatiles by GC & 1080 & 1481 & 50 & 674 & 1680 & 5 & 1 & 447 & 9315 \\
\hline Semi-Volatiles & 3376 & 3197 & 8 & 108 & 351 & 6 & 775 & 537 & 5811 \\
\hline Herbicides/Pesticides/PCBs & 5462 & 3767 & 3 & 45 & 421 & 19 & 1359 & 1466 & 7703 \\
\hline Other Organics & 1771 & 1645 & 24 & 330 & 2049 & 73 & 25 & 6221 & 5930 \\
\hline \multicolumn{10}{|l|}{ INORGANICS } \\
\hline RCRA Appendix IX Metals & 55 & 43 & 0 & $\overline{0}$ & 11 & 1 & 29 & 52 & 30 \\
\hline CAM (California) Metals (RCRA) & 145 & 116 & 0 & 0 & 4 & 1 & 0 & 0 & 448 \\
\hline RCRA TCLP Metals and EPTOX Me & 1237 & 756 & 45 & 612 & 1525 & 6 & 826 & 383 & 3558 \\
\hline EPA CLP Metals (CERCLA) & 6963 & 6347 & 10 & 138 & 377 & 6 & 708 & 172 & 4457 \\
\hline NPDES Metals (Clean Water Act) & 519 & 809 & 0 & 1 & 497 & 28 & 0 & 2354 & 744 \\
\hline Drinking Water Metals (SDWA) & 30 & 37 & 12 & 164 & 387 & 0 & 0 & 0 & 30 \\
\hline Metals by Other Regulatory Suite & 7200 & 3251 & 10 & 138 & 506 & 13 & 1050 & 785 & 13163 \\
\hline Metals by Suite, Other & 1343 & 1524 & 0 & 3 & 1274 & 72 & 0 & 6226 & 8015 \\
\hline Metals by Metal Except Mercury & 1971 & 980 & 48 & 647 & 1632 & 7 & 1597 & 629 & 7363 \\
\hline Mercury & 3548 & 1539 & 45 & 616 & 2990 & 90 & 1494 & 7421 & 9116 \\
\hline Anions/Cations-IC & 264 & 1911 & 1 & 8 & 168 & 9 & 644 & 981 & 11713 \\
\hline Inorganics Wet Chemistry & 26877 & 8180 & 38 & 517 & 2007 & 49 & 699 & 3891 & 43286 \\
\hline Other Inorganics & 469 & 1588 & 3 & 35 & 277 & 16 & 183 & 799 & 7140 \\
\hline \multicolumn{10}{|l|}{ RADIOACTIVES } \\
\hline Gross Alpha/Beta & 21101 & 11775 & 45 & 624 & 9553 & 468 & 1980 & 38403 & 17513 \\
\hline Gamma Scan & 8028 & 4096 & 65 & 890 & 4777 & 154 & 267 & 12788 & 6214 \\
\hline Transuranic & 32 & 0 & of & 2 & 5 & 0 & 1491 & 5 & 532 \\
\hline Plutonium Isotopes & 7662 & 3804 & 20 & 271 & 1034 & 27 & 264 & 1795 & 5372 \\
\hline Uranium isotopes & 7697 & 5289 & 9 & 121 & 691 & 29 & 1914 & 1794 & 7649 \\
\hline Curium Isotopes & 42 & 84 & 0 & 0 & 4 & 0 & 0 & 18 & 534 \\
\hline Thorium Isotopes & 991 & 383 & 0 & 0 & 13 & 1 & 894 & 62 & 1621 \\
\hline Radium Isotopes & 3234 & 1022 & 3 & 35 & 83 & 0 & 0 & 3 & 520 \\
\hline Americium-241 & 7279 & 3259 & 10 & 137 & 717 & 26 & 184 & 1779 & 3583 \\
\hline Neptunium-237 & 323 & 267 & 0 & 0 & 4 & 0 & 1075 & 21 & 1856 \\
\hline Polonium-210 & 11 & 1 & 0 & 1 & 6 & 1. & 25 & 0 & 540 \\
\hline Tritium & 4548 & 3277 & 16 & 212 & 834 & 19 & 13 & 1594 & 2835 \\
\hline Radon-222 & 1 & 0 & 0 & 0 & 0 & 0 & 0 & 0 & 22 \\
\hline Lead-210 & 7 & 0 & 0 & 0 & 7 & 0 & 0 & 31 & 2 \\
\hline Carbon-14 & 0 & 0 & 0 & 0 & 0 & 0 & 0 & 0 & 0 \\
\hline Nickel-63 & 0 & 0 & 0 & 0 & 0 & 0 & 0 & 0 & 0 \\
\hline Selenium-79 & 145 & 156 & 0 & 0 & 0 & 0 & 0 & 0 & 0 \\
\hline Technetium-99 & 687 & 58 & 3 & 35 & 87 & 0 & 1754 & 21 & 674 \\
\hline Strontium Isotopes & 1 & 0 & of & 0 & 0 & o & 0 & 0 & 27 \\
\hline Strontium-89 & $\mathbf{0}$ & 0 & 0 & 0 & 0 & 0. & 0 & 0 & 0 \\
\hline Strontium-90 & 329 & 1005 & 3 & 35 & 83 & 0 & 2 & 3 & 837 \\
\hline lodine -129 & 185 & 197 & 7 & 100 & 234 & 0 & 0 & 0 & 517 \\
\hline Nickel-59 & 0 & 0 & 0 & 0 & 0 & 0 & 0 & 0 & 0 \\
\hline Total Uranium & 1195 & 64 & 3 & 35 & 88 & 1 & 1324 & 8 & 6704 \\
\hline Total Thorium & 361 & 0 & 0 & 0 & 1 & 0 & 24 & 0 & 115 \\
\hline Total Strontium & 3965 & 1512 & 3 & 38 & 107 & 1 & 0 & 71 & 625 \\
\hline Other RAD & 10956 & 5694 & 27 & 362 & 993 & 10 & 830 & 626 & 10545 \\
\hline \multicolumn{10}{|l|}{ OTHER } \\
\hline Other Wet Chemistry & 11469 & 3461 & 16 & 215 & 1185 & 41 & 0 & 3188 & 2673 \\
\hline TCLP Extraction & 1326 & 1011 & 45 & 612 & 1503 & 5 & 1090 & 259 & 4492 \\
\hline Asbestos & 9259 & 216 & 0 & 0 & 1 & 0 & 13 & 3 & 76 \\
\hline Industrial Hygiene, NOS & 307 & 96 & 0 & 5 & 314 & 18 & 0 & 1435 & 369 \\
\hline Physical Properties & 2352 & 687 & 11 & 143 & 385 & 3 & 869 & 1068 & 32484 \\
\hline \multirow{2}{*}{\multicolumn{10}{|c|}{ Other, Not Otherwise Specified (NO }} \\
\hline & & & & & & & & & \\
\hline TOTAL SAMPLE ANALYSES & 173248 & 89321 & 587 & 7979 & 39118 & 1215 & 24535 & 97701 & 257514 \\
\hline
\end{tabular}


Table IVb: FY 94 Samples and Sample Analyses Complex-wide by Regulatory Driver

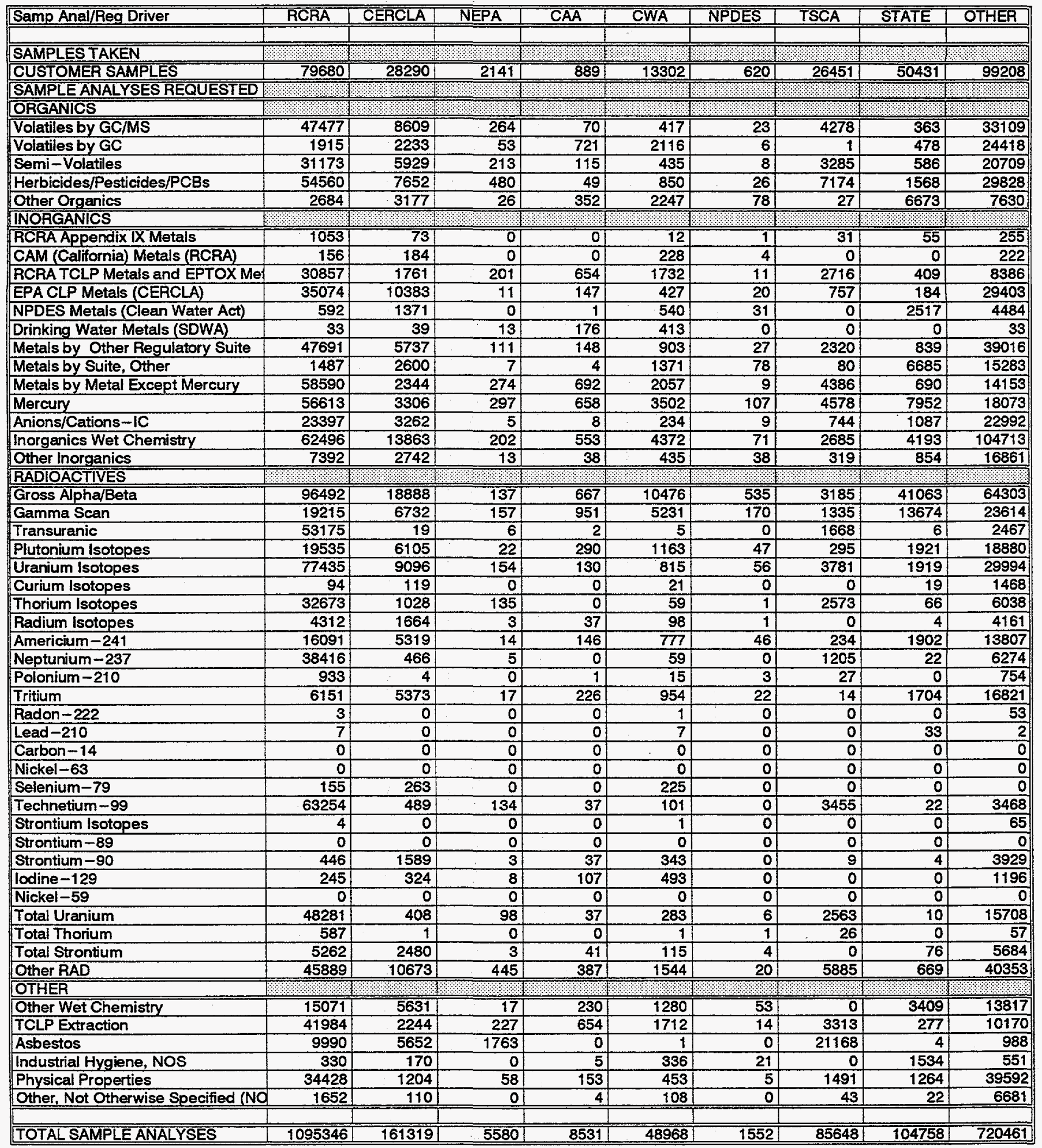


EM-263 ASSESSMENT OF EM SAMPLING AND ANALYSIS NEEDS

Table IVc: FY 95 Samples and Sample Analyses Complex-wide by Regulatory Driver

\begin{tabular}{|c|c|c|c|c|c|c|c|c|c|}
\hline Samp Anal/Reg Driver & RCRA & CERCLA & NEPA & $\overline{\mathrm{CAA}}$ & $\overline{\overline{C W A}}$ & NPDES & TSCA & STATE & OTHER \\
\hline & & & & & & & & & \\
\hline \multicolumn{10}{|l|}{ SAMPLES TAKEN } \\
\hline CUSTOMER SAMPLES & 97910 & 30518 & 2218 & 921 & 13632 & 631 & 27403 & 52245 & 95363 \\
\hline SAMPLE ANALYSES REQUESTED & & & & & २० & 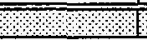 & & & \\
\hline \multicolumn{10}{|l|}{ ORGANICS } \\
\hline Volatiles by GC/MS & 50897 & 7696 & 274 & 73 & 428 & 16 & 4432 & 376 & 24466 \\
\hline Volatiles by GC & 1963 & 2388 & 55 & 747 & 2174 & 6 & 1 & 495 & 23430 \\
\hline Semi-Volatiles & 33634 & 5622 & 221 & 119 & 442 & 8 & 3403 & 607 & 15428 \\
\hline Herbicides/Pesticides/PCBs & 59889 & 7339 & 498 & 50 & 868 & 24 & 7432 & 1624 & 25183 \\
\hline Other Organics & 2774 & 4200 & 27 & 365 & 2279 & 80 & 28 & 6913 & 7324 \\
\hline \multicolumn{10}{|l|}{ INORGANICS } \\
\hline RCRA Appendi IX Metals & 1078 & 66 & 0 & 0 & 12 & 1 & 32 & 57 & 154 \\
\hline CAM (California) Metals (RCRA) & 161 & 194 & 0 & 0 & 236 & 3 & 0 & 0 & 234 \\
\hline RCRA TCLP Metals and EPTOX Me & 33067 & 2090 & 208 & 678 & 1777 & 9 & 2814 & 424 & 9030 \\
\hline EPA CLP Metals (CERCLA) & 36334 & 9114 & 11 & 153 & 442 & 14 & 784 & 191 & 16222 \\
\hline NPDES Metals (Clean Water Act) & 613 & 1257 & 0 & 1 & 560 & 32 & 0 & 2608 & 2530 \\
\hline Drinking Water Metals (SDWA) & 34 & 40 & 13 & 182 & 428 & 0 & 0 & 0 & 34 \\
\hline Metals by Other Regulatory Suite & 50140 & 5016 & 115 & 153 & 923 & 21 & 2403 & 869 & 33944 \\
\hline Metals by Suite, Other & 1590 & 2387 & 7 & 4 & 1420 & 81 & 83 & 6925 & 11883 \\
\hline Metals by Metal Except Mercury & 62190 & 2603 & 284 & 717 & 2117 & 8 & 4543 & 715 & 14812 \\
\hline Mercury & 60329 & 3367 & 308 & 682 & 3619 & 105 & 4743 & 8238 & 17220 \\
\hline Anions/Cations $-1 \mathrm{C}$ & 24272 & 3066 & 5 & 8 & 239 & 9 & 771 & 1126 & 19080 \\
\hline Inorganics Wet Chemistry & 65916 & 12391 & 210 & 573 & 4468 & 64 & 2781 & 4344 & 97561 \\
\hline Other Inorganics & 7731 & 2713 & 14 & 39 & 441 & 28 & 330 & 885 & 14044 \\
\hline \multicolumn{10}{|l|}{ RADIOACTIVES } \\
\hline Gross Alpha/Beta & 100588 & 17508 & 142 & 691 & 10821 & 536 & 3299 & 42540 & 44249 \\
\hline Gamma Scan & 20518 & 6226 & 163 & 986 & 5408 & 173 & 1383 & 14166 & 17007 \\
\hline Transuranic & 55133 & 20 & 7 & 2 & 5 & 0 & 1728 & 6 & 2587 \\
\hline Plutonium Isotopes & 20231 & 5288 & 23 & 300 & 1200 & 39 & 306 & 1990 & 12026 \\
\hline Uranium Isotopes & 81106 & 8203 & 160 & 134 & 837 & 45 & 3917 & 1988 & 20219 \\
\hline Curium Isotopes & 97 & 112 & 0 & 0 & 22 & 0 & 0 & 20 & 1368 \\
\hline Thorium Isotopes & 34748 & 994 & 140 & 0 & 60 & 1 & 2666 & 69 & 5999 \\
\hline Radium Isotopes & 4467 & 1312 & 3 & 39 & 101 & 0 & 0 & 4 & 2258 \\
\hline Americium-241 & 16680 & 4528 & 14 & 152 & 802 & 39 & 243 & 1971 & 7638 \\
\hline Neptunium - 237 & 39727 & 458 & 5 & 0 & 59 & 0 & 1248 & 23 & 5933 \\
\hline Polonium-210 & 966 & 7 & 0 & 1 & 15 & 2 & 28 & 0 & 784 \\
\hline Tritium & 6280 & 4769 & 17 & 235 & 984 & 22 & 14 & 1765 & 10202 \\
\hline Radon-222 & 3 & 0 & 0 & 0 & 1 & 0 & 0 & 0 & 55 \\
\hline Lead-210 & 7 & 0 & 0 & 0 & 7 & 0 & 0 & 34 & 2 \\
\hline Carbon-14 & 0 & 0 & 0 & 0 & 0 & 0 & 0 & 0 & 0 \\
\hline Nickel-63 & 0 & 0 & 0 & 0 & 0 & 0 & 0 & 0 & 0 \\
\hline Selenium-79 & 161 & 302 & 0 & 0 & 231 & 0 & 0 & 0 & 0 \\
\hline Technetium - 99 & 66511 & 496 & 139 & 39 & 104 & 0 & 3579 & 23 & 4116 \\
\hline Strontium Isotopes & 4 & 0 & 0 & 0 & 1 & 0 & 0 & 0 & 67 \\
\hline Strontium - 89 & 0 & 0 & 0 & a & 0 & 0 & 0 & 0 & 0 \\
\hline Strontium -90 & 448 & 1689 & 3 & 39 & 347 & 0 & 9 & 4 & 2744 \\
\hline lodine-129 & 254 & 378 & 8 & 110 & 508 & 0 & 0 & 0 & 1239 \\
\hline Nickel-59 & 0 & 0 & 0 & 0 & 0 & 0 & 0 & 0 & 0 \\
\hline Total Uranium & 50554 & 416 & 102 & 39 & 289 & 4 & 2655 & 11 & 16606 \\
\hline Total Thorium & 438 & 2 & 0 & 0 & 1 & 1 & 26 & 0 & 60 \\
\hline Total Strontium & 5451 & 2013 & 3 & 42 & 119 & 3 & 0 & 78 & 2696 \\
\hline Other RAD & 50610 & 9759 & 461 & 401 & 1582 & 16 & 6097 & 693 & 33538 \\
\hline \multicolumn{10}{|l|}{ OTHER } \\
\hline Other Wet Chemistry & 15612 & 4461 & 18 & 239 & 1325 & 50 & 01 & 3532 & 7411 \\
\hline TCLP Extraction & 44809 & 2673 & 236 & 677 & 1752 & 10 & 3432 & 287 & 11948 \\
\hline Asbestos & 23406 & 5808 & 1827 & 0 & 1 & 0 & 21929 & 4 & 9232 \\
\hline Industrial Hygiene, NOS & 342 & 180 & 0 & 5 & 348 & 21 & 0 & 1590 & 389 \\
\hline Physical Properties & 35865 & 1023 & 60 & 159 & 469 & 4 & 1545 & 1309 & 40651 \\
\hline Other, Not Otherwise Specified (NO & 1603 & 102 & 0 & 4 & 110 & 0 & 44 & 23 & 6763 \\
\hline & & & & & & & & & \\
\hline TOTAL SAMPLE ANALYSES & 1169233 & 150277 & 5780 & 8838 & 50383 & 1477 & 88728 & 108526 & 600367 \\
\hline
\end{tabular}


Table IVe: FY 97 Samples and Sample Analyses Complex-wide by Regulatory Driver

\begin{tabular}{|c|c|c|c|c|c|c|c|c|c|}
\hline Samp AnallReg Driver & RCRA & CERCLA & NEPA & $\overline{\mathrm{CAA}}$ & CWA & NPDES & TSCA & STATE & OTHER \\
\hline & & & & & & & & & \\
\hline \multicolumn{10}{|l|}{ SAMPLES TAKEN } \\
\hline CUSTOMER SAMPLES & 49665 & 13985 & 2195 & 912 & 13478 & 625 & 27117 & 51701 & 60361 \\
\hline \multicolumn{10}{|l|}{ SAMPLE ANALYSES REQUESTED } \\
\hline \multicolumn{10}{|l|}{ ORGANICS } \\
\hline Volatiles by GC/MS & 11892 & 4775 & 271 & 72 & 420 & 16 & 4386 & 372 & 11676 \\
\hline Volatiles by GC & 2027 & 1481 & 55 & 739 & 2143 & 6 & 1 & 490 & 1693 \\
\hline Semi-Volatiles & 6236 & 3301 & 219 & 118 & 436 & 7 & 3367 & 601 & 10466 \\
\hline Herbicides/Pesticides/PCBs & 11241 & 3943 & 492 & 50 & 854 & 24 & 7354 & 1607 & 9488 \\
\hline Other Organics & 1820 & 1953 & 26 & 361 & 2255 & 80 & 28 & 6841 & 6532 \\
\hline \multicolumn{10}{|l|}{ INORGANICS } \\
\hline RCRA Appendix IX Metals & 67 & 45 & 01 & 0 & 12 & 1 & 32 & 57 & 96 \\
\hline CAM (California) Metals (RCRA) & 164 & 210 & 0 & 0 & 233 & 3 & 0 & 0 & 232 \\
\hline RCRA TCLP Metals and EPTOX Me & 2959 & 865 & 206 & 670 & 1757 & 9 & 2784 & 420 & 1810 \\
\hline EPA CLP Metals (CERCLA) & 10226 & 6320 & 11 & 151 & 436 & 13 & 776 & 189 & 13223 \\
\hline NPDES Metals (Clean Water Act) & 586 & 863 & 0 & 1 & 553 & 31 & 0 & 2581 & 1864 \\
\hline Drinking Water Metals (SDWA) & 33 & 40 & 13 & 180 & 424 & 0 & 0 & 0 & 33 \\
\hline Metals by Other Regulatory Suite & 11332 & 3222 & 113 & 152 & 904 & 21 & 2378 & 860 & 7230 \\
\hline Metals by Suite, Other & 1544 & 1623 & 7 & 4 & 1405 & 80 & 82 & 6853 & 11061 \\
\hline Metals by Metal Except Mercury & 4386 & 1189 & 281 & 710 & 2093 & 8 & 4496 & 707 & 7225 \\
\hline Mercury & 6334 & 1763 & 304 & 675 & 3580 & 104 & 4693 & 8153 & 9854 \\
\hline Anions/Cations-IC & 465 & 2041 & 5 & 8 & 235 & 9 & 762 & 1114 & 14322 \\
\hline Inorganics Wet Chemistry & 39969 & 8059 & 207 & 567 & 4394 & 63 & 2752 & 4298 & 20803 \\
\hline Other Inorganics & 971 & 1731 & 13 & 39 & 433 & 28 & 327 & 875 & 4350 \\
\hline RADIOACTIVES & & & & & & & & & \\
\hline Gross Alpha/Beta & 27699 & 11449 & 140 & 684 & 10702 & 531 & 3265 & 42097 & 24420 \\
\hline Gamma Scan & 10889 & 4022 & 161 & 975 & 5349 & 172 & 1368 & 14018 & 8323 \\
\hline Transuranic & 90 & 0 & 6 & 2 & 5 & 0 & 1710 & 6 & 614 \\
\hline Plutonium Isotopes & 10429 & 3630 & 23 & 297 & 1186 & 39 & 302 & 1969 & 7710 \\
\hline Uranium Isotopes & 11444 & 5278 & 158 & 133 & 827 & 45 & 3876 & 1967 & 12426 \\
\hline Curium Isotopes & 103 & 77 & 0 & $\overline{0}$ & 21 & 0 & 0 & 19 & 137 \\
\hline Thorium Isotopes & 2430 & 395 & 138 & 0 & 59 & 1 & 2638 & 68 & 1621 \\
\hline Radium Isotopes & 4400 & 928 & 3 & 38 & 100 & 0 & 0 & 4 & 1614 \\
\hline Americium - 241 & 9800 & 3119 & 14 & 150 & 793 & 38 & 240 & 1950 & 6667 \\
\hline Neptunium-237 & 556 & 285 & 5 & 0 & 57 & 0 & 1235 & 23 & 768 \\
\hline Polonium-210 & 35 & 6 & D) & 1 & 15 & 2 & 28 & 0 & 160 \\
\hline Tritium & 6167 & 3258 & 17 & 232 & 972 & 22 & 14 & 1747 & 5839 \\
\hline Radon-222 & 3 & 0 & 0 & 0 & 1 & 0 & 0 & 0 & 0 \\
\hline Lead-210 & 7 & 0 & 0 & 0 & 7 & 0 & 0 & 34 & 2 \\
\hline Carbon-14 & $\overline{0}$ & 0 & 0 & 0 & 0 & 0 & 0) & $\overline{0}$ & $\overline{0}$ \\
\hline Nickel-63 & 0 & 0 & 0 & 0 & 0 & 0 & 0 & 0 & 0 \\
\hline Selenium-79 & 164 & 253 & 0 & 0 & 229 & 0 & 0 & 0 & 0 \\
\hline Technetium-99 & 1949 & 63 & 138 & 38 & 103 & 0 & 3542 & 23 & 1217 \\
\hline Strontium Isotopes & 4 & 0 & 0 & $\overline{0}$ & 1 & $\mathbf{0}$ & 0 & 0 & 0 \\
\hline Strontium-89 & 0 & 0 & 0 & 0 & $\mathbf{0}$ & 0 & 0 & 0 & 0 \\
\hline Strontium -90 & 491 & 1093 & 3 & 38 & 343 & 0 & 9 & 4 & 1038 \\
\hline lodine-129 & 256 & 301 & 81 & 109 & 503 & 0 & 0 & 0 & 24 \\
\hline Nickel-59 & 0 & 0 & 0 & 0 & 0 & 0 & 0 & 0 & 0 \\
\hline Total Uranium & 2653 & 91 & 101 & 38 & 281 & 4 & 2628 & 11 & 1630 \\
\hline Total Thorium & 412 & 2 & 0 & 0 & 1 & 1 & 26 & 0 & 60 \\
\hline Total Strontium & 5363 & 1415 & 3 & 42 & 117 & 3 & 0 & 78 & 2609 \\
\hline Other RAD & 21152 & 5752 & 456 & 397 & 1559 & 16 & 6034 & 686 & 14747 \\
\hline \multicolumn{10}{|l|}{ OTHER } \\
\hline Other Wet Chemistry & 15380 & 3165 & 17 & 236 & $13 \overline{1311}$ & 49 & 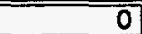 & 3495 & 6328 \\
\hline TCLP Extraction & 5422 & 1120 & 233 & 670 & 1731 & 10 & 3397 & 284 & 4389 \\
\hline Asbestos & 25856 & 226 & 1808 & 0 & 1 & 0 & 21701 & 4 & 9101 \\
\hline Industrial Hygiene, NOS & 336 & 108 & 0 & 5 & 344 & 20 & o & 1573 & 381 \\
\hline Physical Properties & 4878 & 627 & 60 & 157 & .463 & 4 & 1529 & 1295 & 36188 \\
\hline Other, Not Otherwise Specified (NO & 649 & 72 & 0 & 4 & 106 & 0 & 44 & 23 & 153 \\
\hline & & & & & & & & & \\
\hline TOTAL SAMPLE ANALYSES & 281272 & 90160 & 5720 & $\overline{8746}$ & 49753 & 1461 & $87 \overline{87804}$ & 107396 & 280125 \\
\hline
\end{tabular}


Table IVf: FY 98 Samples and Sample Analyses Complex-wide by Regulatory Driver

\begin{tabular}{|c|c|c|c|c|c|c|c|c|c|}
\hline Samp Anal/Reg Driver & RCAA & CERCLA & NEPA & $\overline{\mathrm{CAA}}$ & CWA & NPDES & TSCA & STATE & OTHEA \\
\hline & & & & & & & & & \\
\hline \multicolumn{10}{|l|}{ SAMPLES TAKEN } \\
\hline CUSTOMER SAMPLES & 55861 & 16503 & 2195 & 912 & 13478 & 625 & 27103 & 51701 & 59964 \\
\hline SAMPLEANALYSES REQUESTED & & & & & & & & & \\
\hline \multicolumn{10}{|l|}{ ORGANICS } \\
\hline Volatiles by GC/MS & 12527 & 5344 & 271 & 72 & 420 & 16 & 4353 & 372 & 11380 \\
\hline Volatiles by GC & 1926 & 2929 & 55 & 739 & 2143 & 6 & 0 & 490 & 1693 \\
\hline Semi-Volatiles & 6752 & 3477 & 219 & 118 & 436 & 7 & 3340 & 601 & 10268 \\
\hline Herbicides/Pesticides/PCBs & 12548 & 4767 & 492 & 50 & 854 & 24 & 7326 & 1607 & 9133 \\
\hline Other Organics & 1817 & 2232 & 26 & 361 & 2255 & 80 & 28 & 6841 & 6525 \\
\hline INORGANICS & 1 & & 8 & & & 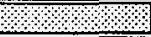 & 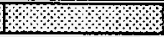 & & \\
\hline ACRA Appendix IX Metals & 60 & 45 & 0 & 0 & 12 & 1 & 30 & 57 & 89 \\
\hline CAM (California) Metals (RCAA) & 164 & 212 & 0 & $\mathbf{0}$ & 233 & 3 & 0 & 0 & 228 \\
\hline RCRA TCLP Metals and EPTOX Mel & 3382 & 1337 & 206 & 670 & 1757 & 9 & 2779 & 420 & 1589 \\
\hline EPA CLP Metals (CERCLA) & 10219 & 6224 & 11 & 151 & 436 & 13 & 776 & 189 & 13022 \\
\hline NPDES Metals (Clean Water Act) & 583 & 872 & 0 & 1 & 553 & 31 & 0 & 2581 & 1863 \\
\hline Drinking Water Metals (SDWA) & 33 & 40 & 13 & 180 & 424 & 0 & $\overline{0}$ & 0 & 33 \\
\hline Metals by Other Regulatory Suite & 11527 & 4794 & 113 & 152 & 904 & 21 & 2378 & 860 & 6939 \\
\hline Metals by Suite, Other & 1562 & 1607 & 7 & 4 & 1405 & 80 & 82 & 6853 & 11060 \\
\hline Metals by Metal Except Mercury & 4957 & 1594 & 281 & 710 & 2093 & 8 & 4470 & 707 & 6812 \\
\hline Mercury & 6983 & 2111 & 304 & 675 & 3580 & 104 & 4667 & 8153 & 9457 \\
\hline Anions/Cations-IC & 463 & 2225 & 5 & 8 & 235 & 9 & 762 & 1114 & 14154 \\
\hline Inorganics Wet Chemistry & 40128 & 13023 & 207 & 567 & 4394 & 63 & 2752 & 4298 & 20597 \\
\hline Other Inorganics & 961 & 2337 & 13 & 39 & 433 & 28 & 327 & 875 & 4276 \\
\hline RADIOACTIVES & $\sqrt{3+1}$ & की & +ि & & & 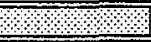 & & & \\
\hline Gross Alpha/Beta & 27884 & 12357 & 140 & 684 & 10702 & 531 & 3261 & 42097 & 23860 \\
\hline Gamma Scan & 11104 & 4484 & 161 & 975 & 5349 & 172 & 1363 & 14018 & 8248 \\
\hline Transuranic & 109 & 0 & 6 & 2 & 5 & 0 & 1710 & 6 & 225 \\
\hline Plutonium Isotopes & 10409 & 3798 & 23 & 297 & 1186 & 39 & 300 & 1969 & 7618 \\
\hline Uranium Isotopes & 11765 & 5479 & 158 & 133 & 827 & 45 & 3847 & 1967 & 11900 \\
\hline Curium Isotopes & 97 & 155 & 0 & 0 & 21 & 0 & 0 & 19 & 137 \\
\hline Thorium lsotopes & 2775 & 590 & 138 & 0 & 59 & 1 & 2622 & 68 & 1391 \\
\hline Radium Isotopes & 4397 & 937 & 3 & 38 & 100 & 0 & 0 & 4 & 1614 \\
\hline Americium - 241 & 9800 & 3082 & 14 & 150 & 793 & 38 & 238 & 1950 & 6596 \\
\hline Neptunium - 237 & 498 & 527 & 5 & 0 & 57 & 0 & 1219 & 23 & 491 \\
\hline Polonium-210 & 32 & 46 & 0 & 1 & 15 & 2 & 28 & 0 & 151 \\
\hline Tritium & 6100 & 3442 & 17 & 232 & 972 & 22 & 0 & 1747 & 5837 \\
\hline Radon-222 & 3 & 4 & 0 & 0 & 1 & 0 & 0 & 0 & 0 \\
\hline Lead-210 & 7 & 0 & 0 & 0 & 7 & 0 & 0 & 34 & 2 \\
\hline Carbon-14 & 0 & 0 & 0 & 0 & 0 & 0 & 0 & 0 & 0 \\
\hline Nickel-63 & 0 & 0 & 0 & 0 & 0 & 0 & 0 & 0 & 0 \\
\hline Selenium-79 & 164 & 261 & 0 & 0 & 229 & 0 & 0 & 0 & 0 \\
\hline Technetium-99 & 2352 & 98 & 138 & 38 & 103 & 0 & 3542 & 23 & 760 \\
\hline Strontium Isotopes & 4 & 4 & 0 & 0 & 1 & 0 & 0 & 0 & 0 \\
\hline Strontium - 89 & 0 & $\underline{0}$ & 0 & 0 & 0 & 0 & 0 & 0 & 0 \\
\hline Strontium - 90 & 476 & 1219 & 3 & 38 & 343 & 0 & 6 & 4 & 1038 \\
\hline lodine-129 & 250 & 391 & 8 & 109 & 503 & 0 & 0 & 0 & 24 \\
\hline Nickel-59 & 0 & 0 & 0 & 0 & 0 & 0 & 0 & 0 & 0 \\
\hline Total Uranium & 2797 & 959 & 101 & 38 & 281 & 4 & 2602 & 11 & 1285 \\
\hline Total Thorium & 320 & 2 & 0 & 0 & 1 & 1 & 0 & 0 & 59 \\
\hline Total Strontium & 5363 & 1370 & 3 & 42 & 117 & 3 & 0 & 78 & 2607 \\
\hline Other RAD & 22339 & 6781 & 456 & 397 & 1559 & 16 & 6029 & 686 & 14520 \\
\hline OTHER & & & & & & 3 & & & \\
\hline Other Wet Chemistry & 15376 & 3128 & 17 & 236 & 1311 & 49 & $\overline{0}$ & 3495 & 6317 \\
\hline TCLP Extraction & 5936 & 1612 & 233 & 670 & 1731 & 10 & 3394 & 284 & 4095 \\
\hline Asbestos & 31229 & 220 & 1808 & 0 & 1 & 0 & 21687 & 4 & 9101 \\
\hline Industrial Hygiene, NOS & 336 & 111 & 0 & 5 & 344 & 20 & 0 & 1573 & 379 \\
\hline Physical Properties & 4929 & 803 & 60 & 157 & 463 & 4 & 1506 & 1295 & 35964 \\
\hline Other, Not Otherwise Specified (NO & 563 & 500 & 0 & 4 & 106 & 0 & $2 \overline{8}$ & 23 & 146 \\
\hline & & & & & & & & & \\
\hline TOTAL SAMPLE ANALYSES & 294003 & 107533 & 5720 & 8746 & 49753 & 1461 & 87451 & 107396 & 273481 \\
\hline
\end{tabular}


Table IVg: FY 99 Samples and Sample Analyses Complex-wide by Regulatory Driver

\begin{tabular}{|c|c|c|c|c|c|c|c|c|c|}
\hline Samp Anal/Reg Driver & RCRA & CERCLA & NEPA & $\overline{\mathrm{CAA}}$ & CWA & NPDES & TSCA & STATE & OTHER \\
\hline & & & & & & & & & \\
\hline \multicolumn{10}{|l|}{ SAMPLES TAKEN } \\
\hline CUSTOMER SAMPLES & 55589 & 14384 & 2187 & 909 & 13431 & 623 & 27008 & 51520 & 57241 \\
\hline \multicolumn{10}{|l|}{ SAMPLE ANALYSES REQUESTED } \\
\hline \multicolumn{10}{|l|}{ ORGANICS } \\
\hline Volatiles by GC/MS & 12304 & 43801 & 270 & 72 & 418 & 16 & 4338 & 371 & 11059 \\
\hline Volatiles by GC & 1912 & 1542 & 55 & 737 & 2136 & 6 & 0 & 488 & 1687 \\
\hline Semi-Volatiles & 6575 & 3110 & 218 & 118 & 435 & 7 & 3328 & 599 & 9993 \\
\hline Herbicides/Pesticides/PCBs & 12349 & 3752 & 491 & 50 & 851 & 24 & 7300 & 1601 & 9101 \\
\hline Other Organics & 1811 & 2083 & 26 & 360 & 2247 & 79 & 28 & 6817 & 6502 \\
\hline \multicolumn{10}{|l|}{ INORGANICS } \\
\hline RCRA Appendix IX Metals & 49 & 41 & D) & 0 & 12 & 11 & 30 & 57 & 89 \\
\hline CAM (California) Metals (RCRA) & 163 & 212 & 0 & 0 & 233 & 3 & 0 & 0 & 227 \\
\hline RCRA TCLP Metals and EPTOX Me & 3340 & 901 & 206 & 668 & 1750 & 9 & 2769 & 418 & 1583 \\
\hline EPA CLP Metals (CERCLA) & 10183 & 5882 & 11 & 151 & 435 & 13 & 773 & 188 & 12388 \\
\hline NPDES Metals (Clean Water Act) & 581 & 788 & 0 & 1 & 552 & 31 & 0 & 2572 & 1856 \\
\hline Drinking Water Metals (SDWA) & 33 & 37 & 13 & 180 & 422 & 0 & 0 & 0 & 33 \\
\hline Metals by Other Regulatory Suite & 11486 & 2991 & 113 & 151 & 901 & 21 & 2370 & 857 & 6914 \\
\hline Metals by Suite, Other & 1556 & 1481 & 7 & 4 & 1400 & 79 & 82 & 6829 & 11021 \\
\hline Metals by Metal Except Mercury & 4795 & 1182 & 280 & 707 & 2086 & 8 & 4454 & 705 & 6789 \\
\hline Mercury & 6813 & 1690 & 303 & 672 & 3567 & 104 & 4651 & 8124 & 9424 \\
\hline Anions/Cations-IC & 461 & 1884 & 5 & 8 & 234 & 9 & 760 & 1111 & 14105 \\
\hline Inorganics Wet Chemistry & 39985 & 7862 & 207 & 565 & 4378 & 63 & 2742 & 4283 & 20516 \\
\hline Other Inorganics & 958 & 1658 & 13 & 39 & 432 & 28 & 326 & 872 & 4261 \\
\hline \multicolumn{10}{|l|}{ RADIOACTIVES } \\
\hline Gross Alpha/Beta & 27761 & 11188 & 140 & 682 & 10664 & 529 & 3249 & 41950 & 23776 \\
\hline Gamma Scan & 11038 & 3877 & 161 & 972 & 5330 & 171 & 1358 & 13969 & 8219 \\
\hline Transuranic & 108 & 0 & 6 & 2 & 5 & 0 & 1704 & 6 & 224 \\
\hline Plutonium Isotopes & 10362 & 3457 & 23 & 296 & 1182 & 39 & 299 & 1962 & 7591 \\
\hline Uranium Isotopes & 11563 & 4980 & 157 & 133 & 824 & 45 & 3834 & 1961 & 11858 \\
\hline Curium lsotopes & 97 & 80 & 0 & 0 & 21 & o & 0 & 19 & 136 \\
\hline Thorium Isotopes & 2677 & 372 & 138 & 0 & 58 & 1 & 2613 & 68 & 1387 \\
\hline Radium Isotopes & 4382 & 854 & 3 & 38 & 99 & 0 & 0 & 4 & 1608 \\
\hline Americium-241 & 9755 & 2932 & 14 & 150 & 790 & 38 & 237 & 1943 & 6573 \\
\hline Neptunium -237 & 408 & 272 & 5 & 0 & 57 & 0 & 1215 & 23 & 489 \\
\hline Polonium-210 & 32 & 7 & 0 & 1 & 14 & 2 & 28 & 0 & 150 \\
\hline Tritium & 6001 & 3043 & 17 & 231 & 969 & 22 & 0 & 1741 & 5817 \\
\hline Radon-222 & 3 & o & 0 & 0 & 1 & (0) & 0 & 0 & 0 \\
\hline Lead-210 & 7 & 0 & 0 & 0 & 7 & 0 & 0 & 34 & 2 \\
\hline Carbon-14 & 0 & 0 & 0 & 0 & 0 & 0 & $\overline{0}$ & 0 & 0 \\
\hline Nickel-63 & 0 & 0 & 0 & 0 & 0 & 0 & 0 & 0 & 0 \\
\hline Selenium-79 & 163 & 257 & 0 & 0 & 228 & 0 & 0 & 0 & $\overline{0}$ \\
\hline Technetium -99 & 2343 & 57 & 137 & 38 & 102 & 0 & 3529 & 23 & 757 \\
\hline Strontium Isotopes & 4 & 0 & 0 & 0 & 1 & 0 & 0 & 0 & $\overline{0}$ \\
\hline Strontium -89 & 0 & (0) & 0 & 0 & 0 & 0 & $\overline{0}$ & 0 & $\overline{0}$ \\
\hline Strontium -90 & 459 & 1124 & 3 & 38 & 342 & 0 & 6 & 4 & 1035 \\
\hline lodine-129 & 249 & 305 & 8 & 109 & 501 & 0 & 0 & 0 & $\overline{24}$ \\
\hline Nickel-59 & 0 & 0 & 0 & 0 & 0 & 0 & $\mathbf{0}$ & 0 & $\overline{0}$ \\
\hline Total Uranium & 2642 & 88 & 101 & 38 & 280 & 4 & 2592 & $\overline{11}$ & 1280 \\
\hline Total Thorium & 174 & 2 & 0 & 0 & 1 & 1 & 0 & 0 & 58 \\
\hline Total Strontium & 5344 & 1302 & 3 & 42 & 117 & 3 & 0 & 77 & 2598 \\
\hline Other RAD & 22235 & 5414 & 455 & 395 & 1554 & 16 & 6008 & 684 & 12501 \\
\hline \multicolumn{10}{|l|}{ OTHEA } \\
\hline Other Wet Chemistry & 15322 & 2919 & 17 & 235 & 1307 & 49 & 0 & 3482 & 6294 \\
\hline TCLP Extraction & 5901 & 1194 & 232 & 668 & 1725 & 10 & 3382 & 283 & 2522 \\
\hline Asbestos & 31042 & 207 & 1801 & 0 & 1 & 0 & 21610 & 4 & 9069 \\
\hline Industrial Hygiene, NOS & 335 & 106 & 0 & 5 & 343 & 20 & $\overline{0}$ & 1568 & 378 \\
\hline Physical Properties & 4784 & 599 & 59 & 156 & 461 & 4 & 1500 & 1291 & 34858 \\
\hline Other, Not Otherwise Specified (ND & 471 & 66 & 0 & 4 & 106 & 0 & 28 & 23 & 146 \\
\hline & & & & & & & & & \\
\hline TOTAL SAMPLE ANALYSES & 291018 & 86178 & 5700 & 8715 & 49578 & 1456 & $\overline{87144}$ & 107019 & 266899 \\
\hline
\end{tabular}


Table Va: FY 93 Samples and Sample Analyses by Rad Level and Matrix Type (i) Complex-wide

\begin{tabular}{|c|c|c|c|c|c|c|c|c|c|}
\hline $\begin{array}{c}\text { Rad-Matrix } \\
\text { Sample-Sample Analysis }\end{array}$ & $\overline{\mathrm{RO}}$ & $\overline{\mathrm{R} 1}$ & $\overline{\mathrm{R} 2}$ & $\overline{\mathrm{R} 3}$ & $\overline{\mathrm{R} 4}$ & $\overline{\mathrm{M1}}$ & $\bar{M} 2$ & $\overline{M 3}$ & TOTAL \\
\hline CUSTOMER SAMPLES & 40667 & 112427 & 3151 & $1121 \mid$ & 577 & 85041 & 31413 & 17703 & 146050 \\
\hline & & & & & & & & & \\
\hline \multicolumn{10}{|l|}{ SAMPLE ANALYSES } \\
\hline Volatiles by GC/MS & 16268 & 4729 & 465 & 86 & 0 & 854 & 6861 & 6159 & 21558 \\
\hline Volatiles by GC & 5712 & 8453 & 650 & 52 & o. & 3481 & 9797 & 1323 & 14734 \\
\hline Semi-Volatiles & 8793 & 4823 & 455 & 86 & 0 & 5473 & 6627 & 2080 & 14169 \\
\hline Herbicides/Pesticides/PCBs & 12332 & 7818 & 52 & 26 & 0 & 6172 & 7179 & 6916 & 20248 \\
\hline Other Organics & 7925 & 19211 & 31 & 0 & 0 & 6204 & 1706 & 1058 & 18067 \\
\hline TOTAL ORGANICS & 51030 & 45035 & 1654 & 250 & 0 & 29877 & 32170 & 17537 & 88776 \\
\hline & & & & & & & & & \\
\hline RCRA Appendix IX Metals & 88 & 99 & 0. & 0 & 0. & 96 & 86 & 73 & 221 \\
\hline CAM (California) Metals (RCRA) & 260 & 260 & 0 & 0 & 0 & 520 & 325 & 65 & 715 \\
\hline RCRA TCLP Metals and EPTOX Me & 4589 & 3578 & 1074 & 10 & 0 & 2681 & 4134 & 1833 & 8949 \\
\hline EPA CLP Metals (CERCLA) & 14204 & 4168 & 575 & 231 & 0 & 8151 & 9235 & 1791 & 19178 \\
\hline NPDES Metals (Clean Water Act) & 1479 & 3474 & 0 & o. & 요 & 4953 & 0 & 0 & 4953 \\
\hline Drinking Water Metals (SDWA) & 660 & 0 & 0 & 01 & 0 & 660 & 0 & 0 & 660 \\
\hline Metals by Other Regulatory Suite & 24079 & 1687 & 234 & 0 & 0 & 9214 & 5873 & 11146 & 26117 \\
\hline Metals by Suite, Other & 6968 & 20639 & 81 & 0 & 0 & 5052 & 668 & 3507 & 18457 \\
\hline Metals by Metal Except Mercury & 8466 & 6763 & 260 & 0 & 273 & 6991 & 3897 & 3097 & 14873 \\
\hline Mercury & 10062 & 25139 & 68 & 5 & 273 & 11021 & 4529 & 2621 & 26859 \\
\hline Anions/Cations-IC & 13107 & 2709 & 109 & 0 & 546 & 11198 & 351 & 3375 & 15698 \\
\hline Inorganics Wet Chemistry & 70257 & 9269 & 4919 & 107 & 0 & 45999 & 15171 & 25363 & 85543 \\
\hline Other Inorganics & 6682 & 3827 & 01 & 0 & 0 & 5153 & 3422 & 1934 & 10509 \\
\hline TOTAL INORGANICS & 160901 & 81611 & $7 \overline{319}$ & 354 & 1092 & 111691 & 47692 & 54805 & 232733 \\
\hline & & & & & & & & & \\
\hline Gross Apha/Beta & 38098 & 73107 & 902 & 325 & 546 & 65387 & 21159 & 3401 & 101462 \\
\hline Gamma Scan & 13801 & 22836 & 330 & 130 & 286 & 23603 & 10382 & 3190 & 37279 \\
\hline Transuranic & 0 & 949 & 936 & 0) & 286 & 1248 & 637 & 78 & 2067 \\
\hline Plutonium Isotopes & 18140 & 1604 & 166 & 26 & 546 & 9116 & 8988 & 1911 & 20249 \\
\hline Uranium Isotopes & 17446 & 6573 & 840 & 23 & 546 & 11034 & 10894 & 3032 & 25194 \\
\hline Curium Isotopes & 0 & 645 & 16 & 8 & 26 & 198 & 166 & 304 & 681 \\
\hline Thorium Isotopes & 1362 & 2413 & 88 & 101 & o. & 902 & 1279 & 1784 & 3965 \\
\hline Radium Isotopes & 4649 & 252 & 0 & 0 & 0 & 3614 & 1037 & 250 & 4901 \\
\hline Americium-241 & 15933 & 936 & 68 & 13 & 52 & 8913 & 7670 & 367 & 16975 \\
\hline Neptunium-237 & 1362 & 1524 & 660 & 0 & 0 & 697 & 884 & 1966 & 3546 \\
\hline Polonium-210 & 296 & 257 & 31 & 0 & 0 & 5 & 296 & 283 & 585 \\
\hline Tritium & 9357 & 4243 & 819 & 26 & 0 & 7506 & 4667 & 75 & 13347 \\
\hline Radon-222 & 23 & 0 & 0 & 0 & 0 & 23 & 0 & 0 & 23 \\
\hline Lead-210 & 0 & 47 & 0 & 0 & 0 & 0 & 0 & 47 & 47 \\
\hline Carbon-14 & 0 & 0 & 0 & 0 & 0 & 0 & 0 & 0 & 0 \\
\hline Nickel-63 & 0 & 0 & 0 & 0 & 0 & o & 0 & 0 & 0 \\
\hline Selenium-79 & 260 & 42 & 0 & 0 & 0 & 283 & 10 & 8 & 302 \\
\hline Technetium-99 & 140 & 2197 & 980 & 0 & 0 & 1404 & 1316 & 598 & 3318 \\
\hline Strontium Isotopes & 0 & 29 & 0 & 0 & 0 & 0 & 13 & 16 & 29 \\
\hline Strontium-89 & 0 & o) & 0 & 0] & 0. & ㅇ] & 0 & 0] & 0 \\
\hline Strontium -90 & 400 & 1258 & 611 & 26 & 0 & 577 & 1695 & 26 & 2297 \\
\hline lodine-129 & 660 & 580 & 0 & 0 & 0 & 1206 & 21 & 13 & 1240 \\
\hline Nickel-59 & 0 & 0 & 0 & 0 & 0 & 0 & 0 & 0 & 0 \\
\hline Total Uranium & 5619 & 2577 & 915 & 0 & 546 & 900 & 1633 & 6656 & 9422 \\
\hline Total Thorium & 0 & 502 & 0 & 0 & 0 & 117 & 385 & 0 & 502 \\
\hline Total Strontium & 6276 & 47 & 아 & 0 & 0 & 3526 & 2701 & 96 & 6323 \\
\hline Other RAD & 21746 & 6924 & 759 & 627 & 26 & 9552 & 13172 & 7280 & 30043 \\
\hline TOTAL RAD & 155571 & 129540 & 8122 & 1305 & 2860 & 149812 & 89006 & 31379 & 283798 \\
\hline & & & & & & & & & \\
\hline Other Wet Chemistry & 21884 & 286 & 01 & 0 & 78 & 19893 & 2278 & 78 & 22248 \\
\hline TCLP Extraction & 4358 & 5164 & 465 & 497 & 0 & 2920 & 4248 & 3034 & 10343 \\
\hline Asbestos & 161 & 9402 & 0 & 0 & 0 & 3 & 7254 & 2317 & 9568 \\
\hline Industrial Hygiene, NOS & 2519 & 23 & 0 & 0 & 0 . & 2441 & 99 & 3 & 2543 \\
\hline Physical Properties & 34453 & 3185 & 55 & 309 & 0 & 22784 & 2829 & 12389 & 38002 \\
\hline Other, Not Otherwise Specified (NO & 2785 & 408 & 31 & $\underline{0}$ & o. & 351 & 23 & 2818 & 3208 \\
\hline TOTAL OTHER & 66160 & 18468 & 551 & 806 & 78 & 48391 & 16731 & 20639 & 85912 \\
\hline TOTAL SAMPLE ANALYSES & 433662 & 274654 & 17646 & 2714 & 4030 & 339771 & 185598 & 124361 & 691218 \\
\hline
\end{tabular}


Table Va: FY 93 Samples and Sample Analyses by Rad Level and Matrix Type (ii) EM-30

\begin{tabular}{|c|c|c|c|c|c|c|c|c|c|}
\hline $\begin{array}{c}\text { Rad-Matrix/ } \\
\text { Sample-Sample Analysis }\end{array}$ & $\overline{\mathrm{RO}}$ & R1 & $\overline{\text { R2 }}$ & $\overline{\mathrm{R3}}$ & $\overline{\mathrm{R4}}$ & $\overline{\mathrm{M1}}$ & $\overline{\mathrm{M} 2}$ & $\overline{\mathrm{M} 3}$ & TOTAL \\
\hline CUSTOMER SAMPLES & 28326 & 76666 & 1123 & 965 & 550 & 75357 & 3384 & 10595 & 98483 \\
\hline & & & & & & & & & \\
\hline SAMPLE ANALYSES & & & & & & & & & \\
\hline Volatiles by GC/MS & 741 & 1077 & 281 & 86 & - & 558 & 413 & 1215 & 2185 \\
\hline Volatiles by GC & 918 & 805 & 68 & 20 & 0 & 1003 & 690 & 69 & 1787 \\
\hline Semi-Volatiles & 3603 & 1010 & 269 & 78 & 0. & 2906 & 650 & 1403 & 4960 \\
\hline Herbicides/Pesticides/PCBs & 2253 & 2004 & 20 & 10 & 0 & 3473 & 237 & 577 & 4287 \\
\hline Other Organics & 5492 & 17970 & 31 & 01 & 0 & 4822 & 5 & 776 & 14548 \\
\hline TOTAL ORGANICS & 13007 & 22867 & 669 & 195 & 0 & 12763 & 1995 & 4040 & 27767 \\
\hline 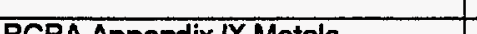 & & & & & & & & & \\
\hline RCAA Appendix IX Metals & 41 & 36 & 0 & 0 & o. & 72 & 0 & 73 & 111 \\
\hline CAM (Calfifornia) Metals (RCRA) & 32 & 1 & 0 & 0 & ㅇ. & 32 & 1 & 0 & 33 \\
\hline RCAA TCLP Metals and EPTOX Me & 769 & 873 & 550 & 4 & 0] & 838 & 258 & 1114 & 2203 \\
\hline EPA CLP Metals (CERCLA) & 370 & 996 & 40 & 201 & (0. & 364 & 156 & 1088 & 1608 \\
\hline NPDES Metals (Clean Water Act) & 330 & 3170 & 0 & ol & 0. & 3500 & 0 & 0 & 3500 \\
\hline Drinking Water Metals (SDWA) & 126 & 0 & 0 & 01 & 0] & 126 & o & 0 & 126 \\
\hline Metals by Other Regulatory Suite & 1733 & 1091 & 236 & 0 & (0) & 2109 & 230 & 720 & 3060 \\
\hline Metals by Suite, Other & 7022 & 17903 & 0 & 0 & 0 & 3722 & 0 & 3311 . & 15979 \\
\hline Metals by Metal Except Mercury & 5401 & 2607 & 241 & 이 & 275 & 4380 & 234 & 2350 & 7744 \\
\hline Mercury & 6118 & 19700 & 40 & 2 & 275 & 6466 & 462 & 2376 & 17720 \\
\hline Anions/Cations-IC & 10325 & 1565 & 110 & 0 & 550 & 8655 & 238 & 2099 & 11771 \\
\hline Inorganics Wet Chemistry & 15068 & 839 & 318 & 43 & (0) & 11951 & 1142 & 4333 & 16847 \\
\hline Other Inorganics & 1024 & 637) & 0 & 0 & 0 & 1060 & 201 & 400 & 1661 \\
\hline TOTAL INORGANICS & 48358 & 49419 & 1535 & 249 & 1100 & 43277 & 2922 & 17864 & 82362 \\
\hline & & & & & & & & & \\
\hline Gross Alpha/Beta & $\frac{9127}{1013}$ & $\frac{61040}{19148}$ & $\frac{144}{121}$ & 125 & $\frac{550}{289}$ & 49135 & $\frac{1674}{1020}$ & 832 & 61314 \\
\hline Transuranic & 0 & 918 & 943 & 0 & $\frac{288}{288}$ & $\frac{18513}{1258}$ & $\frac{1222}{604}$ & $\frac{675}{79}$ & $\frac{20516}{2045}$ \\
\hline Plutonium Isotopes & 2884 & 421 & 90 & 10 & 550 & 2732 & 246 & 505 & 3720 \\
\hline Uranium Isotopes & 2778 & 1770 & 798 & 9 & 550 & 4252 & 394 & 787 & 5669 \\
\hline Curium isotopes & 0 & 56 & 6 & 3 & 26 & 7 & 20 & 38 & 78 \\
\hline Thorium Isotopes & 60 & 1076 & 36 & 95 & 0 & 770 & 18 & 480 & 1268 \\
\hline Radium Isotopes & 32 & 11 & 0 & 0 & 0. & 32 & 0 & 11 & 43 \\
\hline Americium-241 & 2723 & 313 & 9 & 5 & 52 & 2703 & 92 & 255 & 3076 \\
\hline Neptunium - 237 & 60 & 708 & 665 & 0 & 0 & 655 & 349 & 429 & 1433 \\
\hline Polonium-210 & 1 & 12 & 31 & 0 & 0 & 1 & 11 & 43 & 45 \\
\hline Tritium & 2437 & 193 & 81 & 10 & 0 & 2383 & 209 & 26 & 2669 \\
\hline Radon-222 & 1 & 0 & 0 & 0 & 0. & 1 & 0 & 0 & 1 \\
\hline Lead-210 & 0 & 46 & 0 & 0 & 0 & 0 & 0 & 46 & 46 \\
\hline Carbon-14 & 0 & 0 & 0 & 0 & 0 & 0 & 0 & o & $\overline{0}$ \\
\hline Nickel-63 & 0 & 0 & 0 & 0 & 0 & 0 & 0 & 0 & 0 \\
\hline Selenium-79 & 32 & 0 & 0 & 0 & 0 & 32 & 0 & 0 & 32 \\
\hline Technetium-99 & 27 & 1255 & 975 & 0 & 0 & 1284 & 603 & 369 & 2256 \\
\hline Strontium Isotopes & 0 & 1 & 0 & 0 & 0 & 0 & 1 & 1 & 1 \\
\hline Strontium-89 & 0 & 0 & 0 & 0 & 0 & 0 & 0 & D. & 0 \\
\hline Strontium -90 & 58 & 79 & 53 & 10 & 0 & 63 & 137 & ㅁ & 200 \\
\hline lodine-129 & 108 & 23 & 0 & 0 & 0 & 131 & 0 & o & 131 \\
\hline Nickel-59 & 0 & 0 & 0 & 0 & 으 & 0 & 0 & 0 & 0 \\
\hline Total Uranium & 265 & 714 & 922 & 0 & 550 & 353 & 1049 & 578 & 2216 \\
\hline Total Thorium & 0 & 0 & 0 & 0 & 0 & 0 & 0 & o & 0 \\
\hline Total Strontium & 89 & 46 & 0 & 0 & 0 & 91 & 13 & 31 & 135 \\
\hline Other RAD & 862 & 1788 & 79 & 615 & 26 & 1624 & 299 & 1372 & 3333 \\
\hline TOTAL RAD & 22557 & 89618 & 4955 & 932 & 2882 & 86022 & 6930 & 6554 & 110225 \\
\hline & & & & & 烈 & & & & \\
\hline Other Wet Chemistry & 4868 & 37 & 0 & 0 & 79 & 4905 & का & 799| & 4984 \\
\hline TCLP Extraction & 506 & 1490 & 421 & 487 & 0 & 655 & 415 & 1782 & 2878 \\
\hline Asbestos & 1 & 0 & 0 & 0 & 0. & 0 & 12 & 0] & 6 \\
\hline Industrial Hygiene, NOS & 2130 & 0 & 0 & 0 & 0 & 2128 & 2 & 요 & 2130 \\
\hline Physical Properties & 31874 & 1133 & 11 & 305 & 0 & 21650 & 42 & 11632 & 33324 \\
\hline Other, Not Otherwise Specified (NO & 122 & 39 & 31 & 0 & 0 & 32 & of & 154 & 189 \\
\hline TOTAL OTHER & 39501 & 2700 & 463 & 793 & 79 & 29369 & (471 & 13647 & 43512 \\
\hline & & & & & & & & & \\
\hline TOTAL SAMPLE ANALYSES & 123423 & 164604 & 7622 & 2169 & 4061 & 171431 & 12318 & 42105 & 263866 \\
\hline
\end{tabular}


Table Va: FY 93 Samples and Sample Analyses by Rad Level and Matrix Type (iii) EM-40

\begin{tabular}{|c|c|c|c|c|c|c|c|c|c|}
\hline $\begin{array}{c}\text { Rad-Matrix/ } \\
\text { Sample-Sample Analysis }\end{array}$ & RO & $\overline{\mathrm{R} 1}$ & $\overline{R 2}$ & R3 & $\overline{\mathrm{R} 4}$ & M1 & $\overline{\mathrm{M} 2}$ & $\overline{\mathrm{M3}}$ & TOTAL \\
\hline CUSTOMER SAMPLES & 9484 & 32823 & 1871 & 150 & 29 & 7375 & 25313 & $6630]$ & 41836 \\
\hline \multicolumn{10}{|l|}{ SAMPLE ANALYSES } \\
\hline Volatiles by GC/MS & 10008 & 3379 & 173 & 0 & $0]$ & 4139 & 4864 & 4573 & 13568 \\
\hline Volatiles by GC & 2607 & 7017 & 521 & 30 & 0 & 735 & 8253 & 1158 & 10160 \\
\hline Semi-Volatiles & 3305 & 3525 & 174 & 7 & 0 & 1655 & 4743 & 634 & 7022 \\
\hline Herbicides/Pesticides/PCBs & 7639 & 5377 & 30 & 15 & 0 & 1909 & 5334 & 5853 & 13078 \\
\hline Other Organics & 1368 & 1114 & o & D) & 0] & 437 & 1526 & 266] & 2356 \\
\hline TOTAL ORGANICS & 24927 & 20411 & 896 & 52 & O] & 8875 & 24721 & 12485[ & 46184 \\
\hline \multicolumn{10}{|l|}{ 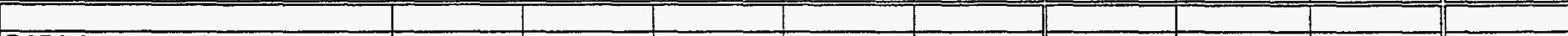 } \\
\hline RCRA Appendix IX Metals & 28 & 58 & 0 & 0 & 0. & 14 & 73 & 이 & 86 \\
\hline CAM (California) Metals (RCRA) & 211 & 238 & 요 & 0 & 0. & 449 & 298 & 60] & 628 \\
\hline RCRA TCLP Metals and EPTOX Met & 1852 & 2455 & 470 & 6 & 0 & 323 & 3407 & 672 & 4592 \\
\hline EPA CLP Metals (CERCLA) & 6416 & 2934 & 493 & 30 & a. & 3622 & 5601 & 651 & 9874 \\
\hline NPDES Metals (Clean Water Act) & 1060 & 274 & 0 & 0 & 0 & 1334 & 0 & 0 & 1334 \\
\hline Drinking Water Metals (SDWA) & 56 & 0 & 0 & 0 & 0] & 56 & 0 & 요 & 56 \\
\hline Metals by Other Regulatory Suite & 14815 & 559 & 0 & 0 & 0 & 3027 & 2940 & 9622 & 15481 \\
\hline Metals by Suite, Other & 0 & 2493 & 74 & 0 & 0 & 1253 & 617 & 204 & 2321 \\
\hline Metals by Metal Except Mercury & 1096 & 3808 & 2 & 0 & 0 & 954 & 3222 & 706 & 4894 \\
\hline Mercury & 2010 & 4948 & 8 & 3 & 0. & 2850 & 3599 & 243 & 6831 \\
\hline Anions/Cations-IC & 2615 & 1064 & 0 & 0 & 0 & 2382 & 104 & 1193 & 3679 \\
\hline Inorganics Wet Chemistry & 29001 & 7784 & 4250 & 59 & 0 & 11139 & 11278 & 19442 & 41476 \\
\hline Other Inorganics & 5120 & 2947 & 0 & 0 & 0 & 3682 & 2966 & 1418 [ & 8067 \\
\hline TOTAL INORGANICS & 64280 & 29561 & 5298 & 98 & O] & 31086 & 34105 & 34210] & 99319 \\
\hline \\
\hline Gross Alpha/Beta & 15325 & 11057 & 701 & 185 & 0 & 9351 & 12176 & 2361 & 25578 \\
\hline Gamma Scan & 5916 & 3369 & 194 & 74 & 0 & 2395 & 4840 & 2318 & 9553 \\
\hline Transuranic & 0 & 29 & 0 & 0 & 0] & 0 & 29 & ㅇ. & 29 \\
\hline Plutonium isotopes & 7080 & 1090 & 71 & 15 & 0. & 2393 & 4568 & 1294 & 8255 \\
\hline Uranium isotopes & 8005 & 4438 & 44 & 13 & 0 & 3417 & 7016 & 2069 & 12501 \\
\hline Curium Isotopes & 0 & 544 & 9 & 4 & o. & 176 & 135 & 246 ] & 557 \\
\hline Thorium Isotopes & 1203 & 1241 & 48 & 6 & 0 & 127 & 1164 & 1206 & 2498 \\
\hline Radium Isotopes & 1276 & 223 & 0 & 0 & o. & 928 & 350 & 220 & 1498 \\
\hline Americium-241 & 5747 & 577 & 54 & 7 & 0 & 2341 & 3947 & 98 & 6386 \\
\hline Neptunium-237 & 1203 & 758 & 0 & 0 & 0 & 43 & 497 & 1421 & 1961 \\
\hline Polonium-210 & 272 & 223 & 0 & 0 & 0 & 0 & 272 & 223 & 495 \\
\hline Tritium & 2385 & 3740 & 681 & 15 & 0. & 1875 & 2966 & 46 & 5854 \\
\hline Radon-222 & 21 & 0 & 0 & 0 & 0 & 21 & 0 & 0. & 21 \\
\hline Lead-210 & 0 & 1 & 0 & 0 & 0 & 0 & 0 & 1 & 1 \\
\hline Carbon-14 & 0 & 0 & 0 & 0 & 0 & 0 & 0 & 0 & 0 \\
\hline Nickel-63 & 0 & 0 & 0 & 0 & 0. & 0 & 0 & 0 & 0 \\
\hline Selenium-79 & 211 & 38 & 0 & 0 & 0 & 233 & 10 & 7 & 249 \\
\hline Technetium-99 & 12 & 879 & 12 & 0 & 으 & 26 & 662 & 214 & 903 \\
\hline Strontium Isotopes & 0 & 25 & 0 & 0 & o. & 0 & 11 & 14 & 25 \\
\hline Strontium-89 & 0 & 0 & 0 & 0 & 0 & 0 & 0 & 0 & 0 \\
\hline Strontium-90 & 223 & 1089 & 516 & 15 & 0 & 382 & 1440 & 24 & 1844 \\
\hline lodine-129 & 245 & 514 & 0 & 0 & 0 & 728 & 19 & 12 & 759 \\
\hline Nickel-59 & 0 & 0 & 0 & 0 & 0 & 0 & 0 & 요 & 0 \\
\hline Total Uranium & 4850 & 1722 & 0 & 0 & 이 & 413 & 546 & 5613 & 6572 \\
\hline Total Thorium & 0 & 462 & 0 & 0 & (0) & 107 & 355 & 0 & 462 \\
\hline Total Strontium & 2064 & 1 & 0 & o) & 0] & 991 & 1020 & 54 & 2065 \\
\hline Other RAD & 11143 & 4707 & 611 & 15 & 0 & 2798 & 8215 & 5462 & 16475 \\
\hline TOTAL RAD & 67180 & 36728 & 2941 & 350 & (0) & 28746 & 50237 & 22904 & 104543 \\
\hline & & & & & & $=$ & & & \\
\hline Other Wet Chemistry & 5473 & 230 & $\overline{\mathbf{0}}$ & 0 & 0 & 4790 & 913 & 0 & 5703 \\
\hline TCLP Extraction & 1865 & 3354 & 26 & 12 & (0) & 712 & 3353 & 1168 & 5245 \\
\hline Asbestos & 91 & 8678 & 0 & 0 & (0) & 0 & 6631 & 2138 & 8769 \\
\hline Industrial Hygiene, NOS & 344 & 22 & 0 & 0 & 0 & 283 & 80 & 2 & 366 \\
\hline Physical Properties & 746 & 1901 & 40 & 6 & 0 & 788 & 1126 & 780 & 2694 \\
\hline Other, Not Otherwise Specified (NO & 2459 & 320 & 0 & 0 & D. & 295 & 22 & 2460 & 2778 \\
\hline TOTAL OTHER & 10979 & 14505 & 66 & 18 & Do & 6868 & 12124 & 6550 & 25554 \\
\hline TOTAL SAMPLE ANALYSES & 167366 & 101206 & 9201 & 518 & 0] & 75573 & 121187 & 76148 & 275599 \\
\hline
\end{tabular}


Table Va: FY 93 Samples and Sample Analyses by Rad Level and Matrix Type (iv) EM-60

\begin{tabular}{|c|c|c|c|c|c|c|c|c|c|}
\hline $\begin{array}{c}\text { Rad-Matrix/ } \\
\text { Sample-Sample Analysis } \\
\end{array}$ & RO & R1 & R2 & R3 & $\overline{\mathrm{R4}}$ & M1 & $\overline{\mathrm{M} 2}$ & $\overline{\mathrm{M3}}$ & TOTAL \\
\hline CUSTOMER SAMPLES & 1968 & 680 & 9 & 11 & $0]$ & 1962 & 5421 & $6][$ & 2584 \\
\hline & & & & & & & & & \\
\hline \multicolumn{10}{|l|}{ SAMPLE ANALYSES } \\
\hline Volatiles by GC/MS & 4054 & 0 & 0 & \begin{tabular}{l|l}
0 & \\
\end{tabular} & 0 & 3032 & 1022 & 0 & 4054 \\
\hline Volatiles by GC & 1711 & 45 & 16 & 0 & 0. & 1462 & 148 & 0 & 1691 \\
\hline Semi-Volatiles & 1416 & 2 & 0 & 0 & 0 & 688 & 730 & 0 & 1418 \\
\hline Herbicides/Pesticides/PCBs & 1575 & 2 & 0 & 0 & 0 & 569 & 1008 & 0 & 1577 \\
\hline Other Organics & 858 & 149 & DI & 0 & 0 ] & 818 & 41 & 0] & 933 \\
\hline TOTAL ORGANICS & 9615 & 197 & 16 & 01 & $0]$ & 6568 & 2949 & 1 1. & 9673 \\
\hline & & & & & & & 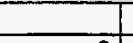 & 7 & \\
\hline RCRA Appendix IX Metals & 15 & 0 & 0 & 0 & 0 & 9 & 6 & o. & 15 \\
\hline CAM (California) Metals (RCRA) & 0 & 0 & 0 & 0 & 0 & 0 & 0 & 0 & 0 \\
\hline RCRA TCLP Metals and EPTOX Me & 1572 & 45 & 16 & 0 & 0 & 1298 & 160 & 0 & 1546 \\
\hline EPA CLP Metals (CERCLA) & 5953 & 0 & 0 & 0 & 0 & 3341 & 2607 & 6 & 5953 \\
\hline NPDES Metals (Clean Water Act) & 3 & 26 & 0 & 0 & 0 & 29 & 0 & 0 & 29 \\
\hline Drinking Water Metals (SDWA) & 411 & 0 & 0 & 0 & 0 & 411 & 0 & 0 & 411 \\
\hline Metals by Other Regulatory Suite & 5454 & 0 & 0 & 0 & 0 & 3320 & 2129 & 6 & 5455 \\
\hline Metals by Suite, Other & 0 & 148 & o & 0 & 0 & 0 & 0 & 0 & 74 \\
\hline Metals by Metal Except Mercury & 1660 & 43 & 16 & 0 & 0 & 1394 & 150 & 0 & 1632 \\
\hline Mercury & 1569 & 195 & 16 & 0 & O. & 1308 & 148 & 0 & 1618 \\
\hline Anions/Cations-IC & 24 & 3 & 0 & 0 & 0 & 25 & 2 & 0 & 27 \\
\hline Inorganics Wet Chemistry & 20666 & 2 & 0 & 0 & o] & 19092 & 1575 & 1. & 20668 \\
\hline Other Inorganics & 951 & 21 & 0 & 0 & 0. & 95 & 1 & 1[ & 97 \\
\hline TOTAL INORGANICS & $37422 !$ & 465 & 49 & 01 & 0. & 30321 & 6778 & 15 & 37525 \\
\hline & & & & & & & & & \\
\hline Gross Alpha/Beta & 10750 & 480 & 0 & D & 0 & 5613 & 5453 & 15 & 11156 \\
\hline Gamma Scan & 5524 & 160 & 0 & 0 & 0 & 2280 & 3397 & 8 & 5684 \\
\hline Transuranic & 0 & 5 & 0 & 0 & 0 & 0 & 5 & 0 & 5 \\
\hline Plutonium Isotopes & 6577 & 5 & 0 & 0 & 0 & 3295 & 3281 & 6 & 6582 \\
\hline Uranium Isotopes & 5199 & 5 & 0 & 0 & 0 & 2689 & 2509 & 6 & 5204 \\
\hline Curium lsotopes & 0 & 0 & 0 & 0 & 0 & 0 & 0 & 0 & 이 \\
\hline Thorium Isotopes & 0 & 0 & 0 & 1 & 0 & 0 & 0 & 1 & 1 \\
\hline Radium Isotopes & 2800 & 0 & 0 & 0 & 0 & 2230 & 570 & 0 & 2800 \\
\hline Americium-241 & 6054 & 0 & 0 & 0 & 0 & 3194 & 2854 & 6 & 6054 \\
\hline Neptunium-237 & 0 & 0 & 0 & 0 & 0 & 0 & 0 & 0 & ㅇ. \\
\hline Polonium-210 & 0 & 3 & 0 & 0 & 0 & 3 & 0 & 0 & 3 \\
\hline Tritium & 3769 & 0 & 0 & 0 & 0 & 2690 & 1079 & 0 & 3769 \\
\hline Radon-222 & 0 & 0 & 0 & 0 & 0 & 0 & 0 & 0 & 0 \\
\hline Lead-210 & 0 & 0 & 0 & 0 & 0 & 0 & 0 & 0 & 으 \\
\hline Carbon-14 & 0 & 0 & 0 & 0 & 0 & 0 & 0 & 0 & 의 \\
\hline Nickel-63 & 0 & 0 & 0 & 0 & 0 & 0 & 0 & 0. & 요 \\
\hline Selenium-79 & 0 & 0 & 0 & 0 & 0. & 0 & 0 & 0 & - \\
\hline Technetium - 99 & 87 & 0 & 0 & 0 & ol & 87 & 0 & 0 & 88 \\
\hline Strontium Isotopes & 0 & 0 & 0 & 0 & 0 & 0 & 0 & D. & 의 \\
\hline Strontium -89 & 0 & 0 & 0 & 0 & 0 & 0 & 0 & 0 & 의 \\
\hline Strontium-90 & 87 & 0 & 으 & 0 & 0 & 87 & 0 & 0 & 87 \\
\hline lodine-129 & 249 & 0 & 0 & 0 & 0 & 249 & 0 & 0 & 249 \\
\hline Nickel-59 & 0 & 0 & 0 & 0 & 0 & 0 & 0 & 0 & 요 \\
\hline Total Uranium & 87 & 01 & 0 & 0 & 0 & 87 & 0 & a & 87 \\
\hline Total Thorium & 0 & 0 & 0 & 0 & 0 & 0 & 0 & 0 & 0 \\
\hline Total Strontium & 3419 & 0 & 0 & 0 & 0 & 2042 & 1370 & 6 & 3419 \\
\hline Other RAD & 7629 & 43 & 16 & 0 & 0 & 4245 & 3440 & 1 & 7687 \\
\hline TOTAL RAD & 52231 & 702 & 17 & 11 & 0 & 28793 & 23957 & 52 & 52877 \\
\hline & & & & & & & & & \\
\hline Other Wet Chemistry & 9623 & 0 & 0 & 이 & 0. & 8508 & 1115 & 0 & 9624 \\
\hline TCLP Extraction & 1584 & 44 & 17 & 0) & 0 & 1296 & 174 & 0 & 1558 \\
\hline Asbestos & 53 & 0 & 0 & 0 & 0 & 2 & 51 & 0 & 53 \\
\hline Industrial Hygiene, NOS & 27 & 0 & 0 & 0 & 0 & 19 & 8 & 0 & 27 \\
\hline Physical Properties & 1742 & 0 & 0 & 0 & 0 & 385 & 1357 & 0 & 1742 \\
\hline Other, Not Otherwise Specified (NO & 0 & 20 & 0 & 0 & 0 & 0 & 0 & 0 & 10 \\
\hline TOTAL OTHER & 13030 & 64 & 17 & 0 & 0 & 10211 & 2706 & 0 & 13015 \\
\hline & & & & & 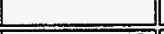 & & & $\Rightarrow$ & \\
\hline TOTAL SAMPLEANALYSES & 112298 & 1429 & 99 & 1 & 0] & 75894 & 36391 & 67 & 113090 \\
\hline
\end{tabular}


Table Va: FY 93 Samples and Sample Analyses by Rad Level and Matrix Type ( $v$ ) Fernald

\begin{tabular}{|c|c|c|c|c|c|c|c|c|c|}
\hline $\begin{array}{c}\text { Rad-Matrix } \\
\text { Sample-Sample Analysis }\end{array}$ & $\overline{\mathrm{RO}}$ & $\overline{\mathrm{RI}}$ & $\overline{\mathrm{R} 2}$ & $\overline{\mathrm{R3}}$ & $\overline{\overline{\mathrm{R4}}}$ & $\overline{\mathrm{M} 1}$ & $\overline{\mathrm{M} 2}$ & $\overline{\mathrm{M3}}$ & TOTAL \\
\hline CUSTOMEA SAMPLES & 1542 & 6481 & 350 & 201 & 0[ & 1731 & 5304 & 751 & 8089 \\
\hline & & & & & & & & & \\
\hline \multicolumn{10}{|l|}{ SAMPLE ANALYSES } \\
\hline Volatiles by $\mathrm{GC} / \mathrm{M} \overline{\mathrm{S}}$ & 2646 & 476 & 15 & 01 & 0 & 881 & 554 & 1708 & 3140 \\
\hline Volatiles by GC & 598 & 2780 & 198 & 4 & o. & 204 & 3008 & 360 & 3575 \\
\hline Semi-Volatiles & 507 & 642 & 19 & 11 & 0 & 252 & 815 & 113 & 1175 \\
\hline Herbicides/Pesticides/PCBs & 2309 & 723 & 4 & 2 & 0 & 411 & 459 & 2179 & 3043 \\
\hline Other Organics & 98 & 243 & 0 & 0 & 0. & 44 & 100 & 91 & 289 \\
\hline TOTAL ORGANICS & 6159 & 4864 & 236 & 71 & 0] & 1792 & 4935 & 4452] & 11222 \\
\hline & & & & & & & & & \\
\hline RCRA Appendix IX Metals & 8 & 8 & 0 & 0 & 0 & 5 & 12 & o] & 17 \\
\hline CAM (California) Metals (RCRA) & 44 & 99 & 0 & 0 & 0 & 143 & 124 & 25 & 218 \\
\hline RCRA TCLP Metals and EPTOX Me & 605 & 578 & 191 & 1 & 0 & 20 & 959 & 246 & 1301 \\
\hline \begin{tabular}{|l|l} 
EPA CLP Metals (CERCLA) & \\
\end{tabular} & 1150 & 489 & 49 & 4 & 0 & 863 & 612 & 217 & 1692 \\
\hline NPDES Metals (Clean Water Act) & 142 & 114 & 0 & 0 & 0 & 255 & o & 0 & 255 \\
\hline Drinking Water Metals (SDWA) & 1 & 0 & 0 & 0 & of & 1 & 0 & 0. & 1 \\
\hline Metals by Other Regulatory Suite & 4953 & 9 & 0 & 0 & 0 & 792 & 263 & 3925 & 4971 \\
\hline Metals by Suite, Other & 0 & 444 & 8 & 0 & 0 & 175 & 67 & 84 & 388 \\
\hline Metals by Metal Except Mercury & 231 & 954 & 0 & 0 & 0 & 242 & 939 & 4 & 1185 \\
\hline Mercury & 348 & 1437 & 1 & 0 & o) & 754 & 837 & 89 & 1733 \\
\hline Anions/Cations-IC & 637 & 181 & 0 & 0 & 0 & 325 & 11 & 481 & 817 \\
\hline Inorganics Wet Chemistry & 9616 & 2192 & 1728 & 8 & 0 & 2376 & 3515 & 7971 & 13703 \\
\hline Other Inorganics & 1721 & 1030 & 0 & 0 & 0 & 1122 & 1049 & 580 & 2751 \\
\hline TOTAL INORGANICS & 19454 & 7536 & 1978 & 13 & 0] & $7 \overline{074}$ & $838 \overline{8}$ & 13622 & 29032 \\
\hline \multicolumn{10}{|l|}{ 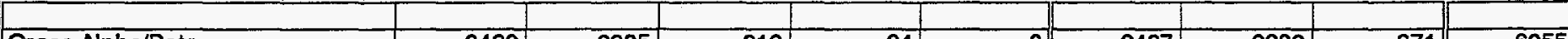 } \\
\hline Gross Alpha/Beta & 3462 & 2905 & 219 & 24 & 0 & 2427 & 2202 & 871 & 6055 \\
\hline Gamma Scan & 1335 & 752 & 24 & 10 & 0 & 420 & 834 & 866 & 2120 \\
\hline Transuranic & 0 & 0 & 0 & 0 & 0 & 0 & 0 & 0 & 0 \\
\hline Plutonium Isotopes & 1779 & 180 & 8 & 2 & 0 & 571 & 889 & 511 & 1970 \\
\hline Uranium Isotopes & 2002 & 842 & 5 & 2 & D. & 826 & 1209 & 815 & 2851 \\
\hline Curium Isotopes & 01 & 201 & 1 & 1 & D. & 63 & 38 & 102 & 203 \\
\hline Thorium lsotopes & 499 & 110 & 5 & 1 & 0 & 13 & 103 & 499 & 615 \\
\hline Radium Isotopes & 118 & 92 & 0 & 0 & 0 & 88 & 31 & 91 & 210 \\
\hline Americium-241 & 1258 & 121 & 6 & 1 & 0 & 557 & 810 & 18 & 1385 \\
\hline Neptunium - 237 & 499 & 188 & 0 & 0 & 0 & 5 & 94 & 587 & 687 \\
\hline Polonium-210 & 113 & 92 & o) & 0 & 0. & 0 & 113 & 92 & 206 \\
\hline Tritium & 270 & 1081 & 215 & 2 & 0 & 230 & 532 & 5 & 1167 \\
\hline Radon-222 & 9 & 0 & 0 & 0 & 0 & 9 & 0 & 0 & 9 \\
\hline Lead-210 & 0 & 0 & 0 & 0 & 0 & 0 & o) & - & 0 \\
\hline Carbon-14 & 0 & 0 & 0 & 0 & 0 & 0 & 0 & 0 & 0 \\
\hline Nickel-63 & 0 & 0 & 0 & 0 & 0 & 0 & 0 & 0 & 0 \\
\hline Selenium-79 & 44 & 3 & 0 & 0 & 0 & 45 & 1 & 1 . & 47 \\
\hline Technetium -99 & 0 & 95 & 1 & 0 & 0 & 2 & 5 & 89 & 96 \\
\hline Strontium Isotopes & 0 & 10 & 0 & 0 & 0 & 0 & 5 & 6. & 10 \\
\hline Strontium -89 & 0 & 0 & 0 & 0 & 0 & 0 & 0 & 0 & 0 \\
\hline Strontium-90 & 44 & 172 & 197 & 2 & 0 & 59 & 355 & 2 & 415 \\
\hline lodine-129 & 44 & 195 & 0 & o & 0 & 237 & 1 & 1 & 239 \\
\hline Nickel-59 & 0 & 0 & 0 & 0 & 0 & 0 & 0 & 0 & 0 \\
\hline Total Uranium & 1986 & 449 & 0 & 0 & 0 & 147 & 111 & 2178 & 2435 \\
\hline Total Thorium & 0 & 155 & 0 & 0 & 0 & 45 & 111 & 0 & 155 \\
\hline Total Strontium & 258 & 0 & 0 & 0 & 0 & 155 & 97 & 6 & 258 \\
\hline Other RAD & 2957 & 690 & 207 & 2 & D. & 749 & 858 & 2246 & 3854 \\
\hline TOTAL RAD & 16676 & 8335 & 888 & 45 & OI & 6648 & 8399 & 8985 & 24988 \\
\hline & & & & & & & & & \\
\hline Other Wet Chemistry & 878 & 95 & 0. & 可 & 0] & 885 & 89 & ㅁ․ & 973 \\
\hline TCLP Extraction & 627 & 832 & 4 & 2 & 0. & 130 & 906 & 428 & 1464 \\
\hline Asbestos & 0 & 90 & 0 & 0 & 0. & 0 & 90 & 의 & 90 \\
\hline Industrial Hygiene, NOS & 117 & 2 & 0 & 0 & 0. & 110 & 8 & 0. & 118 \\
\hline Physical Properties & 99 & 619 & 5 & 1 & o. & 242 & 158 & 324 & 723 \\
\hline Other, Not Otherwise Specified (NO & 1017 & 89 & 0 & 0 & o] & 79 & 71 & 1020 & 1106 \\
\hline TOTAL OTHER & 2737 & 1727 & 8 & 2 & $0]$ & 1445 & 1257 & 1772 & 4475 \\
\hline & & & & & & & & & \\
\hline TOTAL SAMPLE ANALYSES & 45026 & 22462 & 3110 & 67) & ]. & 16959 & 22979 & 28830 & $69 \overline{9717}$ \\
\hline
\end{tabular}


Table Va: FY 93 Samples and Sample Analyses by Rad Level and Matrix Type (vi) INEL

\begin{tabular}{|c|c|c|c|c|c|c|c|c|c|}
\hline $\begin{array}{c}\text { Rad-Matrix } \\
\text { Sample-Sample Analysis }\end{array}$ & Ro & $\overline{\mathrm{R} 1}$ & $\overline{R 2}$ & $\overline{\mathrm{R3}}$ & $\overline{\mathrm{R4}}$ & $\overline{\mathrm{M} 1}$ & $\overline{M 2}$ & $\overline{\mathrm{MB}}$ & TOTAL \\
\hline CUSTOMEA SAMPLES & 2123 & 8909 & 295 & 49 & 3 & 5255 & 3353 & 1078 & 10532 \\
\hline & & & & & & & & & \\
\hline \multicolumn{10}{|l|}{ SAMPLE ANALYSES } \\
\hline Volatiles by GC/MS & 531 & 402 & 17 & 0 & 0 & 308 & 529 & 112 & $\overline{950}$ \\
\hline Volatiles by GC & 725 & 242 & 29 & 9 & 0 & 531 & 373 & 52 & 980 \\
\hline Semi-Volatiles & 386 & 396 & 22 & 2 & 0 & 245 & 508 & 51 & 805 \\
\hline Herbicides/Pesticides/PCBs & 471 & 778 & 9 & 4 & 0 & 311 & 746 & 205 & 1262 \\
\hline Other Organics & 653 & 1335 & o) & 0 & 0 & 298 & 426 & 11 & 1361 \\
\hline \multirow[t]{2}{*}{ TOTAL OAGANICS } & 2767 & 3152 & 75 & $15 !$ & 0 & 1693 & 2582 & 431 & 5358 \\
\hline & & & & & & & & & \\
\hline RCRA Appendix IX Metals & 3 & 7 & 0 & 0 & 0 & 5 & 5 & 0 & 10 \\
\hline CAM (California) Metals (RCRA) & 12 & 0 & 0 & 0 & 0 & 12 & 0 & 0 & 13 \\
\hline RCRA TCLP Metals and EPTOX Me & 581 & 315 & 14 & 2 & 0 & 484 & 340 & 30 & 883 \\
\hline EPA CLP Metals (CERCLA) & 504 & 250 & 58 & 9 & 0 & 272 & 526 & 22 & 821 \\
\hline NPDES Metals (Clean Water Act) & 112 & 224 & 0 & 0 & 0 & 336 & 0 & 0 & 336 \\
\hline Drinking Water Metals (SDWA) & 131 & 0 & 0 & 0 & 0. & 131 & 0 & 0 & 131 \\
\hline Metals by Other Regulatory Suite & 608 & 141 & 0 & 0 & 0 & 285 & 319 & 165 & 759 \\
\hline Metals by Suite, Other & 0 & 1438 & 7 & 0 & - & 104 & 56 & 3 & 804 \\
\hline Metals by Metal Except Mercury & 646 & 373 & 5 & 0 & 0 & 523 & 279 & 166 & 996 \\
\hline Mercury & 745 & 1641 & 7 & 1 & 0 & 655 & 418 & 12 & 1739 \\
\hline Anions/Cations-IC & 207 & 123 & 0 & 0 & 0 & 269 & 34 & 26 & 330 \\
\hline Inorganics Wet Chemistry & 1498 & 702 & 91 & 17 & - & 1302 & 654 & 354 & 2310 \\
\hline Other Inorganics & 241 & 151 & 0 & 0 & 0 & 262 & 96 & 34 & 392 \\
\hline \multirow[t]{2}{*}{ TOTAL INORGANICS } & 5288 & 5366 & 182 & 28 & 0 & 4642 & 2729 & 814 & 9524 \\
\hline & & & & & & & & & \\
\hline Gross Alpha/Beta & 1832 & 5076 & 55 & 53 & 0 & 3850 & 1475 & 110 & 6225 \\
\hline Gamma Scan & 1000 & 1636 & 44 & 21 & 0 & 1515 & 1113 & 73 & 2701 \\
\hline Transuranic & 0 & 8 & 0 & 0 & 0 & 0 & 8 & 0 & 8 \\
\hline Plutonium Isotopes & 676 & 142 & 15 & 4 & 0 & 403 & 402 & 33 & 838 \\
\hline Uranium Isotopes & 625 & 443 & 7 & 4 & 0 & 420 & 613 & 46 & 1079 \\
\hline Curium Isotopes & 0 & 18 & 3 & 1 & 0 & 5 & 11 & 5 & 21 \\
\hline Thorium Isotopes & 17 & 185 & 6 & 8 & 0 & 13 & 178 & 24 & 215 \\
\hline Radium Isotopes & 114 & 3 & 0 & 0 & 묘 & 88 & 27 & 3 & 117 \\
\hline Americium-241 & 553 & 70 & 8 & 2 & 0 & 368 & 252 & 13 & 633 \\
\hline Neptunium-237 & 17 & 45 & 2 & 0 & 0 & 6 & 34 & 23 & 63 \\
\hline Polonium -210 & 0 & 4 & 0 & 0 & 0 & 1 & 0 & 3 & 5 \\
\hline Tritium & 493 & 195 & 35 & 4 & 0 & 400 & 290 & 8 & 713 \\
\hline Radon-222 & 0 & 0 & 0 & 0 & 0 & 0 & 0 & 0 & 의 \\
\hline Lead-210 & 0 & 3 & 0 & 0 & 0 & 0 & 0 & 3 & 3 \\
\hline Carbon-14 & 0 & 0 & 0 & 0 & 0 & 0 & 0 & 0 & 0 \\
\hline Nickel-63 & 0 & 0 & 0 & 0 & 0 & 0 & 0 & 0 & ㅇ․ \\
\hline Selenium -79 & 12 & 12 & 0 & 0 & 0 & 19 & 3 & 2 & 24 \\
\hline Technetium-99 & 28 & 152 & 1 & 0 & 요 & 29 & 147 & 5 & 181 \\
\hline Strontium Isotopes & 0 & 0 & 0 & 0 & 0 & 0 & of & 의 & 0 \\
\hline Strontium -89 & 0 & 0 & 0 & 0 & 0 & 0 & 0 & 0 & 0 \\
\hline Strontium-90 & 40 & 147 & 20 & 4 & 0 & 77 & 127 & 8 & 211 \\
\hline lodine-129 & 92 & 24 & 0 & 0 & 0 & 106 & 6 & 4] & 116 \\
\hline Nickel-59 & 0 & 0 & 0 & 0 & 0 & 0 & o & $0]$ & 0 \\
\hline Total Uranium & 100 & 120 & 0 & 0 & 0 & 37 & 32 & 151 & 219 \\
\hline Total Thorium & 0 & 0 & 0 & 0 & 0 & 0 & 0 & 0 & 0 \\
\hline Total Strontium & 169 & 3 & 0 & 0 & 0 & 72 & 93 & 7 & 172 \\
\hline Other RAD & 758 & 741 & 31 & 4 & 0 & 397 & 1029 & 99: & 1530 \\
\hline TOTALRAD & 6524 & 9028 & 226 & 107 & 0 & 7807 & 5841 & 620 & 15077 \\
\hline & & & & & & & & & \\
\hline Other Wet Chemistry & 792 & 5 & 0 & 0 & 0 & 725 & 72 & 0 & 797 \\
\hline TCLP Extraction & 556 & 406 & 20 & 3 & o & 514 & 361 & 55 & 958 \\
\hline Asbestos & 10 & 1929 & 0 & 0 & o. & 0 & 1444 & 495 & 1940 \\
\hline Industrial Hygiene, NOS & 160 & 7 & 0 & 0 & 0 & 155 & 12 & 1 & 167 \\
\hline Physical Properties & 176 & 86 & 7 & 2 & 0 & 153 & 107 & 11 & 271 \\
\hline Other, Not Otherwise Specified (NO & 34 & 16 & 0 & 0 & 0 & 9 & 0 & 34 & 46 \\
\hline TOTAL OTHER & 1729 & 2449 & 27 & 51 & 0 & 1557 & 1995 & 596 & 4179 \\
\hline & & & & & & & & & \\
\hline TOTAL SAMPLE ANALYSES & 16307 & 19996 & 5111 & 155 & 0] & 15698 & 13147 & 2462 & 34138 \\
\hline
\end{tabular}


EM-263 ASSESSMENT OF EM SAMPLING AND ANALYSIS NEEDS

Table Va: FY 93 Samples and Sample Analyses by Rad Level and Matrix Type (vii) NTS

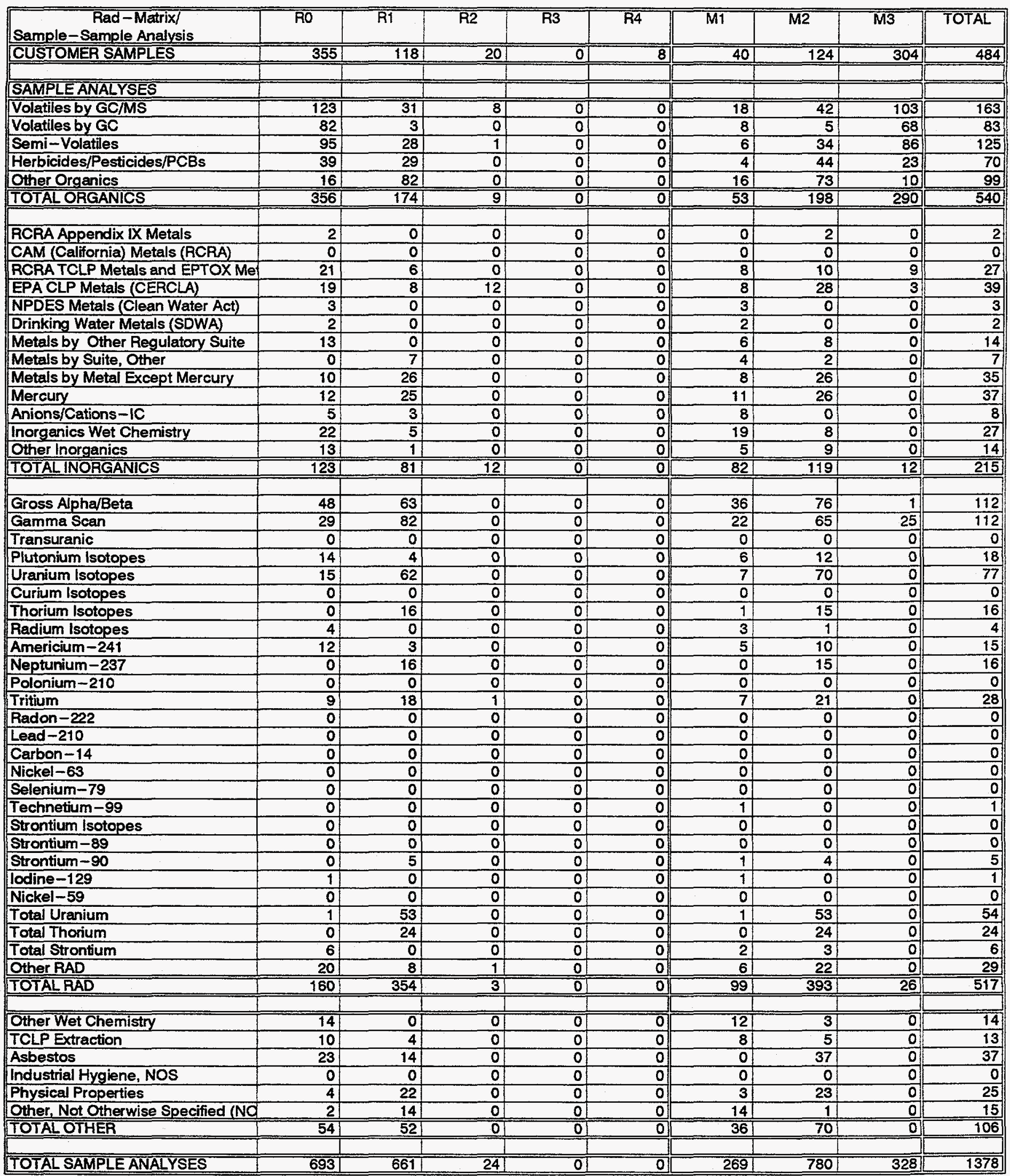


Table Va: FY 93 Samples and Sample Analyses by Rad Level and Matrix Type (viii) Oak Ridge K-25

\begin{tabular}{|c|c|c|c|c|c|c|c|c|c|}
\hline $\begin{array}{c}\text { Rad-Matrix/ } \\
\text { Sample-Sample Analysis }\end{array}$ & RO & R1 & $\overline{R 2}$ & $\overline{\mathrm{R3}}$ & $\overline{\mathrm{R} 4}$ & M1 & M2 & $\overline{\mathrm{M3}}$ & TOTAL \\
\hline CUSTOMER SAMPLES & 265 & 872 & 203 & DI & $1][$ & 373 & 341 & 450 & 1252 \\
\hline \multirow{2}{*}{\multicolumn{10}{|c|}{ SAMPLE ANALYSES }} \\
\hline & & & & & & & & & \\
\hline Volatiles by GC/MS & 179 & 466 & 114 & ot & (0) & 94 & 299 & 367 & $\overline{760}$ \\
\hline Volatiles by $\mathrm{GC}$ & 111 & 15 & 1 & 0 & 0 & 60 & 52 & 8 & 123 \\
\hline Semi-Volaties & 106 & 324 & 101 & 0 & 0 & 64 & 256 & 211 & 531 \\
\hline Herbicides/Pesticides/PCBs & 109 & 711 & 0 & 0 & 0 & 565 & 136 & 121 & 821 \\
\hline Other Organics & 54 & 21 & 12 & 0 & 0 & 43 & 28 & 13 & 86 \\
\hline TOTAL ORGANICS & 559 & 15371 & 2281 & 01 & -0] & 8261 & 771 & 721 & 2321 \\
\hline \multicolumn{10}{|l|}{ RCBA Anpendix IX Metals } \\
\hline RCRA Appendix IX Metals & 1 & 2 & 0 & 0 & 요 & 0 & 3 & 26 . & 16 \\
\hline CAM (California) Metals (RCRA) & 0 & 0 & 0 & 0 & 0 & 0 & 0 & 0 & 0 \\
\hline RCRA TCLP Metals and EPTOX Me & 66 & 199 & 198 & 0 & 0 & 54 & 22 & 406 & 473 \\
\hline EPA CLP Metals (CERCLA) & 184 & 432 & 18 & 0 & 0 & 80 & 209 & 344 & 634 \\
\hline NPDES Metals (Clean Water Act) & 41 & 1 & 0 & 0 & 0 & 41 & 0 & 0 & 41 \\
\hline Drinking Water Metals (SDWA) & 16 & 0] & 0 & 0 & 0 & 16 & 0 & 0. & 16 \\
\hline Metals by Other Regulatory Suite & 154 & 412 & 901 & 0 & 0 & 376 & 178 & 110 & 660 \\
\hline Metals by Suite, Other & 0 & 80 & 3 & 0 & 0 & 47 & 26 & 0. & 78 \\
\hline Metals by Metal Except Mercury & 72 & 943 & 91 & 0 & 0 & 471 & 41 & 87 & 853 \\
\hline Mercury & 105 & 471 & 13 & 0 & o. & 825 & 120 & 133 & 834 \\
\hline Anions/Cations-IC & 63 & 504 & 42 & 0 & o) & 90 & 3 & 101 & 401 \\
\hline Inorganics Wet Chemistry & 247 & 174 & 17 & 0 & 0 & 290 & 155 & 433 & 658 \\
\hline Other Inorganics & 57 & 108 & 01 & 0 & 0 & 56 & 20 & 89 & 165 \\
\hline TOTAL INORGANICS & 1007 & 3327 & 4711 & 01 & $0]$ & 2347 & 778 & 1730 & 4830 \\
\hline \multicolumn{10}{|l|}{0} \\
\hline Gross Alpha/Beta & 393 & 1064 & 17 & 0 & 0 & 997 & 338 & 138 & 1474 \\
\hline Gamma Scan & 207 & 162 & 15 & 0 & 0 & 67 & 180 & 136 & 383 \\
\hline Transuranic & 0 & 350 & 360 & 0 & 0 & 480 & 230 & 0 & 710 \\
\hline Plutonium Isotopes & 147 & 141 & 14 & 0 & 0. & 53 & 120 & 129 & 302 \\
\hline Uranium Isotopes & 163 & 699 & 294 & of & 0 & 667 & 254 & 234 & 1155 \\
\hline Curium Isotopes & 0 & 3 & 0 & 0 & 0 & 2 & 2 & 0. & 3 \\
\hline Thorium isotopes & 1 & 431 & 14 & 0 & 0 & 299 & 21 & 126 & 446 \\
\hline Radium /sotopes & 43 & O) & 0 & 0) & 0 & 31 & 12 & 요 & 43 \\
\hline Americium-241 & 132 & 98 & 2 & D & 0 [ & 48 & 94 & 90 & 232 \\
\hline Neptunium -237 & 1 & 283 & 242 & 0 & 0 & 251 & 148 & 127 & 526 \\
\hline Polonium-210 & 0 & 0 & 12 & 0 & 0 & 0 & 0 & 12 & 12 \\
\hline Tritium & 97 & 66 & 9 & 0 & 0. & 72 & 96 & 2 & 170 \\
\hline Radon-222 & 0 & 0 & 0 & 0 & o & 0 & 0 & 0 & 0 \\
\hline Lead-210 & 0 & 0 & of & 0. & O) & 0 & 0 & 0 & 0 \\
\hline Carbon-14 & 0 & 0 & 0 & 0 & 0 & 0 & 0 & 0 & 0 \\
\hline Nickel-63 & 0 & 0 & 0 & 0 & 0 & 0 & 0 & 0 & 으 \\
\hline Selenium-79 & 0 & 1 & 0 & 0 & 0 & 0 & 0 & 0 & 1 \\
\hline Technetium -99 & 3 & 465 & 373 & 0 & 0 & 484 & 232 & 125 & 841 \\
\hline Strontium Isotopes & 0 & 0 & 0 & 0 & 0 & 0 & 0 & 0. & - \\
\hline Strontium - 89 & 0 & 0 & 0 & 0 & 0. & 0 & 0 & 0. & 0 \\
\hline Strontium-90 & 3 & 30 & 1 & 0 & 0 & 8 & 26 & 0 & 34 \\
\hline lodine-129 & 10 & 1 & 0 & 0 & 0 & 11 & 0 & 0 & 11 \\
\hline Nickel-59 & 0 & 0 & 0 & 0 & 0 & 0 & 0 & 0. & 0 \\
\hline Total Urenium & 11 & 283 & 352 & 0 & 0 & 126 & 416 & 104 & 646 \\
\hline Total Thorium & 0 & 13 & 0 & 0 & 0 & 0 & 13 & 0 & 13 \\
\hline Total Strontium & 65 & 0 & 0 & 0 & 0 & 25 & 38 & 2 & 65 \\
\hline Other RAD & 214 & 435 & 18 & 0 & 0 & 346 & 216 & 105 & 668 \\
\hline TOTAL RAD & 1491 & 4527 & 1721 & 0 & O) & 3967 & 2436 & 1333 & 7737 \\
\hline \\
\hline Other Wet Chemistry & 155 & 0 & 01 & 0 & 0 & 122 & 33 & 0 - & 155 \\
\hline TCLP Extraction & 65 & 435 & 106 & 0 & 0. & 58 & 81 & 460 & 602 \\
\hline Asbestos & 2 & 18 & 0 & 0 & o & 0 & 20 & 0 & 20 \\
\hline Industrial Hygiene, NOS & 4 & 0 & 0 & 0 & 0 & 1 & 3 & 0 & 5 \\
\hline Physical Properties & 32 & 422 & 1 & 0 & D. & 317 & 28 & 111 & 455 \\
\hline Other, Not Otherwise Specified (NO & 4 & 11 & 12 & 0 & 0 & 10 & 1 & 15 & 26 \\
\hline TOTAL OTHER & 262 & 886 & 119 & 0 & (0) & 508 & 166 & 586 & 1263 \\
\hline & & & & & & & & & \\
\hline TOTAL SAMPLE ANALYSES & 3318 & 10278 & 2538 & $\underline{\underline{0}}$ & D.] & 7649 & 41501 & $4369]$ & 16151 \\
\hline
\end{tabular}


Table Va: FY 93 Samples and Sample Analyses by Rad Level and Matrix Type (ix) Oak Ridge X-10

\begin{tabular}{|c|c|c|c|c|c|c|c|c|c|}
\hline \begin{tabular}{|c|} 
Rad-Matrix/ \\
Sample-Sample Anaiysis \\
\end{tabular} & $\overline{\text { Ro }}$ & R1 & R2 & R3 & R4 & M1 & M2 & M3 & TOTAL \\
\hline CUSTOMER SAMPLES & 356 & 5898 & 26 & 5 & 0 & 3164 & 1373 & 678 & $\overline{5751}$ \\
\hline & & & & & & & & & \\
\hline \multicolumn{10}{|l|}{ SAMPLE ANALYSES } \\
\hline Volatiles by GC/MS & 154 & 262 & $\overline{4}$ & 0 & 요 & 118 & 285 & 17 & 420 \\
\hline Volatiles by GC & 88 & 33 & 1 & 0 & 0 & 66 & 48 & 5 & 121 \\
\hline Semi-Volatiles & 93 & 223 & 4 & 0 & 0 & 73 & 241 & 6 & 320 \\
\hline Herbicides/Pesticides/PCBs & 221 & 466 & 0 & 0 & 0 & 178 & 408 & 101 & 688 \\
\hline Other Organics & 21 & 941 & 0 & 0 & 0 & 31 & 2 & 1 & 498 \\
\hline \multirow[t]{2}{*}{ TOTAL ORGANICS } & 578 & 1925 & 10 & 0 & 0 & 467 & 984 & 129 & 2046 \\
\hline & & & & & & & & & \\
\hline RCRA Appendix IX Metals & 2 & 3 & 0 & 0 & 0. & 4 & 1 & 0 & 5 \\
\hline CAM (California) Metals (RCRA) & 44 & 0 & 0 & 0 & 요 & 45 & 0 & 0 & 45 \\
\hline RCRA TCLP Metals and EPTOX Me & 65 & 148 & 1 & 0 & 0 & 57 & 149 & 4 & 212 \\
\hline \begin{tabular}{|l|} 
EPA CLP Metals (CERCLA) \\
\end{tabular} & 145 & 68 & 12 & 0 & 0 & 72 & 147 & 6 & 224 \\
\hline NPDES Metals (Clean Water Act) & 46 & 164 & 0 & 0 & $\overline{0}$ & 210 & 0 & 0 & 210 \\
\hline Drinking Water Metals (SDWA) & 11 & 0 & 0 & 0 & 0 & 11 & 0 & 0 & 11 \\
\hline Metals by Other Regulatory Suite & 182 & 83 & 0 & 0 & 요 & 104 & 142 & 33 & 272 \\
\hline Metals by Suite, Other & 0 & 994 & 2 & 0 & 0. & 34 & 18 & 1 & 525 \\
\hline Metals by Metal Except Mercury & 132 & 205 & 0 & 0 & of & 115 & 92 & 127 & 335 \\
\hline Mercury & 191 & 1178 & 0 & 0 & 0 & 203 & 227 & 5 & 903 \\
\hline Anions/Cations-IC & 85 & 42 & 0 & 0 & 0 & 107 & 15 & 5 & 127 \\
\hline Inorganics Wet Chemistry & 696 & 191 & 9 & 0 & 0 & 620 & 228 & 50 & 897 \\
\hline Other Inorganics & 91 & 40 & 0 & 0 & 0 & 89 & 34 & 8 & 131 \\
\hline \multirow[t]{2}{*}{ TOTALINORGANICS } & 1689 & 3118 & 25 & 0 & (2) & 1669 & 1054 & 238 & 3897 \\
\hline & & & & & & & & & \\
\hline \multirow{2}{*}{$\begin{array}{l}\text { Gross Alpha/Beta } \\
\text { Gamma Scan }\end{array}$} & 724 & 3136 & 4 & 0 & 0 & 2557 & 346 & 28 & 3398 \\
\hline & 155 & 1073 & 2 & 0 & 0 & 1004 & 208 & 18 & 1230 \\
\hline \multirow{2}{*}{\begin{tabular}{|l|} 
Transuranic \\
Plutonium Isotopes \\
\end{tabular}} & 0 & 5 & 0 & 0 & 요 & 0 & 5 & 0 & 5 \\
\hline & 242 & 19 & 3 & 0 & 0 & 172 & 86 & 7 & 264 \\
\hline \multirow{2}{*}{\begin{tabular}{|l} 
Uranium Isotopes \\
Curium Isotopes
\end{tabular}} & 255 & 178 & 3 & 0 & 0 & 191 & 230 & 15 & 436 \\
\hline & 0 & 4 & 0 & 0 & 0 & 1 & 1 & 2 & 4 \\
\hline \multirow{2}{*}{\begin{tabular}{|l|} 
Thorium Isotopes \\
Radium Isotopes \\
\end{tabular}} & 2 & 128 & 1 & 5 & - & 6 & 123 & 8 & 136 \\
\hline & 30 & 0 & o & 0 & 0 & 22 & 9 & 0 & 31 \\
\hline \multirow{2}{*}{\begin{tabular}{|l} 
Americium-241 \\
Neptunium-237 \\
\end{tabular}} & 231 & 11 & 1 & 0 & o & 172 & 67 & 3 & 243 \\
\hline & 2 & 12 & 2 & 0 & 0. & 1 & 11 & 5 & 17 \\
\hline \multirow{2}{*}{\begin{tabular}{|l|} 
Polonium-210 \\
Tritium \\
\end{tabular}} & 0 & 1 & 0 & 0 & 0 & 0 & 0 & 0 & 1 \\
\hline & 185 & 46 & 9 & 0 & 0 & 168 & 65 & 2 & 237 \\
\hline \multirow{2}{*}{\begin{tabular}{|l|} 
Radon-222 \\
Lead-210
\end{tabular}} & 0 & 0 & 0 & 0 & 0 & 0 & 0 & 0 & 0 \\
\hline & 0 & 2 & 0 & 0 & 0 & 0 & 0 & 2 & 2 \\
\hline \multirow{2}{*}{\begin{tabular}{|l|} 
Carbon-14 \\
Nickel-63
\end{tabular}} & 0 & 0 & 0 & 0 & o. & 0 & 0 & 0 & 0 \\
\hline & 0 & 0 & a & 0 & 0 & 0 & 0 & 0 & 0 \\
\hline \multirow{2}{*}{\begin{tabular}{||l|} 
Selenium-79 \\
Technetium -99
\end{tabular}} & 44 & 0 & 0 & 0 & 0 . & 44 & 0 & 0 & 44 \\
\hline & 2 & 117 & 0 & 0 & 0 & 3 & 115 & 2 & 120 \\
\hline Strontium Isotopes & 0 & 0 & 0 & 0 & 0 & 0 & 0 & 0 & 0 \\
\hline Strontium -89 & 0 & 0 & 0 & 0 & 0 & 0 & 0 & 0 & 0 \\
\hline Strontium-90 & 46 & 19 & 1 & 0 & 0 & 48 & 18 & 0 & 66 \\
\hline lodine-129 & 51 & 1 & 0 & 0 & 0 & 52 & 0 & 0 & 52 \\
\hline Nickel-59 & 0 & 0 & 0 & 0 & 0 & 0 & 0 & 0 & 0 \\
\hline Total Uranium & 14 & 84 & 0 & 0 & 0 & 5 & 16 & 78 & 98 \\
\hline Total Thorium & 0 & 0 & 0 & 0 & 0. & 0 & 0 & 0 & 0 \\
\hline Total Strontium & 49 & 2 & 0 & 0 & 0 & 22 & 27 & 3 & 52 \\
\hline Other RAD & 218 & 429 & 5 & 0 & 0. & 108 & 513 & 24 & 648 \\
\hline TOTAL RAD & 2252 & 5269 & 31 & 5 & 0 & 4576 & 1840 & 198 & 7085 \\
\hline & & & & & & & & & \\
\hline Other Wet Chemistry & 353| & 2 & 0 & 0 & o. & 331 & 23 & 0 & 355 \\
\hline TCLP Extraction & 51 & 171 & 7 & 0 & 0 & 49 & 154 & 23 & 227 \\
\hline Asbestos & 0 & 1529 & 0 & 0 & 0 & 0 & 1135 & 396 & 1530 \\
\hline Industrial Hygiene, NOS & 113 & 0 & 0 & 0 & 0 & 111 & 2 & 0 & 113 \\
\hline Physical Properties & 39 & 47 & 1 & 0 & 0 & 33 & 52 & 2 & 86 \\
\hline Other, Not Otherwise Specified (NO & 5 & 4 & 0 & 0 & 0 & 4 & 0 & 5 & 9 \\
\hline TOTAL OTHER & 562 & 1753 & 8 & 0 & O) & 528 & 1366 & 425 & 2321 \\
\hline & & & & & & & & & \\
\hline TOTAL SAMPLE ANALYSES & 5081 & 12065 & 74 & 5 & 0] & 7239 & 5244 & $991]$ & 15349 \\
\hline
\end{tabular}


Table Va: FY 93 Samples and Sample Analyses by Rad Level and Matrix Type (x) Oak Ridge Y-12

\begin{tabular}{|c|c|c|c|c|c|c|c|c|c|}
\hline $\begin{array}{c}\text { Rad-Matrix } \\
\text { Sample-Sample Analysis }\end{array}$ & $\overline{\mathrm{RO}}$ & $\overline{\mathrm{R} 1}$ & $\overline{\mathrm{R} 2}$ & $\overline{\mathrm{R} 3}$ & $\overline{\mathrm{R4}}$ & $\overline{\mathrm{M} 1}$ & M2 & $\overline{\text { M3 }}$ & TOTAL \\
\hline CUSTOMER SAMPLES & 140 & 441 & 32 & 0] & 0 & 108 & 251 & $20]$ & 496 \\
\hline & & & & & & & & & \\
\hline \multicolumn{10}{|l|}{ SAMPLE ANALYSES } \\
\hline Volatiles by GC/MS & 204 & 64 & 7 & 01 & 0 & 1021 & 150 & 22 & 275 \\
\hline Volatiles by GC & 46 & 29 & 2 & 0 & 0 & 2 & 70 & 6 & 77 \\
\hline Semi-Volatiles & 104 & 59 & 7 & 0 & 0 & 63 & 102 & 5 & 170 \\
\hline Herbicides/Pesticides/PCBs & 133 & 71 & 0 & 0 & 0 & 44 & 140 & 20 & 205 \\
\hline Other Organics & of & 15 & 0 & a) & 0 & 11] & 3 & 1[ & 16 \\
\hline TOTAL ORGANICS & 487 & 238 & 16 & 0 & 0] & 223 & 465 & 55 & 742 \\
\hline \multicolumn{10}{|l|}{ RCRA Anpendix IX Metals } \\
\hline RCRA Appendix IX Metals & 0] & 3 & 0 & o. & o. & 0 & 2 & 0. & 3 \\
\hline CAM (California) Metals (RCRA) & 0 & 0 & 0 & of & 0 & 0 & 0 & o) & 0 \\
\hline RCRA TCLP Metals and EPTOX Me & 3 & 15 & 2 & 0 & 0 & 0 & 17 & 3 & 19 \\
\hline EPA CLP Metals (CERCLA) & 201 & 113 & 20 & 0 & 0 & 79 & 244 & 10 & 334 \\
\hline NPDES Metals (Clean Water Act) & 48 & 1 & 0 & 0 & o. & 49 & 0 & 0 & 49 \\
\hline Drinking Water Metals (SDWA) & 0 & 0 & 0 & 0 & of & 0 & 0 & 0 & 0 \\
\hline Metals by Other Regulatory Suite & 192 & 0 & 0 & 0 & 0 & 50 & 108 & 43 & 197 \\
\hline Metals by Suite, Other & 0 & 92 & 4 & 0 & 0 & 55 & 30 & 1 & 91 \\
\hline Metals by Metal Except Mercury & 6 & 24 & 0 & 0 & 0 & 4 & 26 & 0 & 30 \\
\hline Mercury & 49 & 21 & 0 & 0 & 0 & 49 & 21 & 1 & 70 \\
\hline Anions/Cations - IC & 76 & 37 & 0 & 0 & 0 & 106 & 3 & 5 & 113 \\
\hline Inorganics Wet Chemistry & 286 & 81 & 17 & 0 & 0 & 170 & 134 & 80 & 383 \\
\hline Other Inorganics & 65 & 29 & 0 & of & o] & 65 & 23 & $6]$ & 94 \\
\hline TOTALINORGANICS & 926 & 414 & 43 & 01 & 0. & 627 & 608 & 148 & 1383 \\
\hline \multicolumn{10}{|l|}{2} \\
\hline Gross Alpha/Beta & 394 & 146 & 7 & a) & 0 & 183 & 337 & 19 & 543 \\
\hline Gamma Scan & 143 & 46 & 3 & 0 & o & 65 & 111 & 17 & 192 \\
\hline Transuranic & 0 & 0 & 0 & 0 & 의 & 0 & 0 & o. & 0 \\
\hline Plutonium Isotopes & 145 & 30 & 2 & 0 & 0 & 42 & 127 & 8 & 177 \\
\hline Uranium Isotopes & 181 & 87 & 2 & of & 0 & 73 & 189 & 9 & 270 \\
\hline Curium Isotopes & 0 & 5 & 0 & 0 & 0] & 2 & 2 & 1 & 5 \\
\hline Thorium Isotopes & 5 & 19 & 2 & 0 & 0 & 5 & 16 & 5 & 26 \\
\hline Radium Isotopes & 46 & 1 & o) & 0 & 0. & 32 & 14 & 1 & 47 \\
\hline Americium-241 & 139 & 11 & 2 & 0 & o] & 42 & 108 & 3 & 152 \\
\hline Neptunium-237 & 5 & 14 & 0 & 0 & 0. & 1) & 12 & 6 & 19 \\
\hline Polonium-210 & 0 & 1 & 0 & 0 & 0 & 0 & 0 & 1 & 1 \\
\hline Tritium & 89 & 74 & 11 & 0 & o] & 67 & 96 & 2 & 170 \\
\hline Radon-222 & 01 & 0 & 0 & D. & 0] & 0 & 0 & 0 & 0 \\
\hline Lead-210 & 01 & 0 & 0 & O) & 0. & 0 & 0 & 0 & 0 \\
\hline Carbon-14 & 01 & 0 & o. & 0 & 0. & 0 & 0 & 0 & 0 \\
\hline Nickel-63 & 0 & 0 & 0 & 0 & o. & 0 & 0 & 0 & 0 \\
\hline Selenium-79 & 0 & 0 & 0 & 0 & 0 & 0 & 0 & 0 & 0 \\
\hline Technetium-99 & 0 & 3 & 1 & 0 & 0 & 1 & 2 & 1 & 4 \\
\hline Strontium Isotopes & 0 & 0 & 0 & 0. & 0 & 0 & o & 0. & 0 \\
\hline Strontium-89 & 0 & 0 & 0 & 0 & 0 & 0 & 0 & 0 & 0 \\
\hline Strontium -90 & D) & 30 & 2 & 0) & 0 . & 3 & 30 & 0 & 33 \\
\hline lodine-129 & 0 & 2 & 0 & 0 & 0 & 2 & 0 & 0 & 2 \\
\hline Nickel-59 & 0 & 0 & 0 & O) & 0 & 0 & 0 & 0 & 0 \\
\hline Total Uranium & 23 & 2 & 0 & D) & 0 & 4 & 1 & 20 & 25 \\
\hline Total Thorium & 0 & 0 & 0 & D) & 0 & 0 & 0 & 0 & 0 \\
\hline Total Strontium & 72 & 0 & D. & 0 & 0 & 27 & 43 & 3 & 72 \\
\hline Other RAD & 226 & 57 & 8 & 0 & 0 & 23 & 246 & 22 & 291 \\
\hline TOTALRAD & 1467 & 529 & 41 & 0 & $0]$ & 571 & 1332 & 116 & 2028 \\
\hline \\
\hline Other Wet Chemistry & 157 & 1 & 0 & (0) & 0 & 119 & 39 & 0 & 157 \\
\hline TCLP Extraction & 2 & 20 & $\overline{0}$ & 0 & 0 & 2 & 15 & 4 & 22 \\
\hline Asbestos & 0 & 13 & 0 & 0 & 0 & 0 & 13 & o) & 13 \\
\hline Industrial Hygiene, NOS & 4 & 0 & 0 & 0 & 0 & 1 & 4 & o & 4 \\
\hline Physical Properties & 21 & 11 & 1 & 0 & 0 & 12 & 18 & 3 & 33 \\
\hline Other, Not Otherwise Specified (NO & 10 & 4 & 0 & 0 & 0 & 4 & 0 & 10 & 14 \\
\hline TOTAL OTHER & 194 & $\overline{48}$ & 1 & 0. & 0 & 137 & 89 & 18 & 243 \\
\hline & & & & & & & & & \\
\hline TOTAL SAMPLE ANALYSES & 3075 & 1228 & 101 & 0 & 0 . & 1558 & 2493 & 336 & 4396 \\
\hline
\end{tabular}


Table Va: FY 93 Samples and Sample Analyses by Rad Level and Matrix Type (xi) Rocky Flats

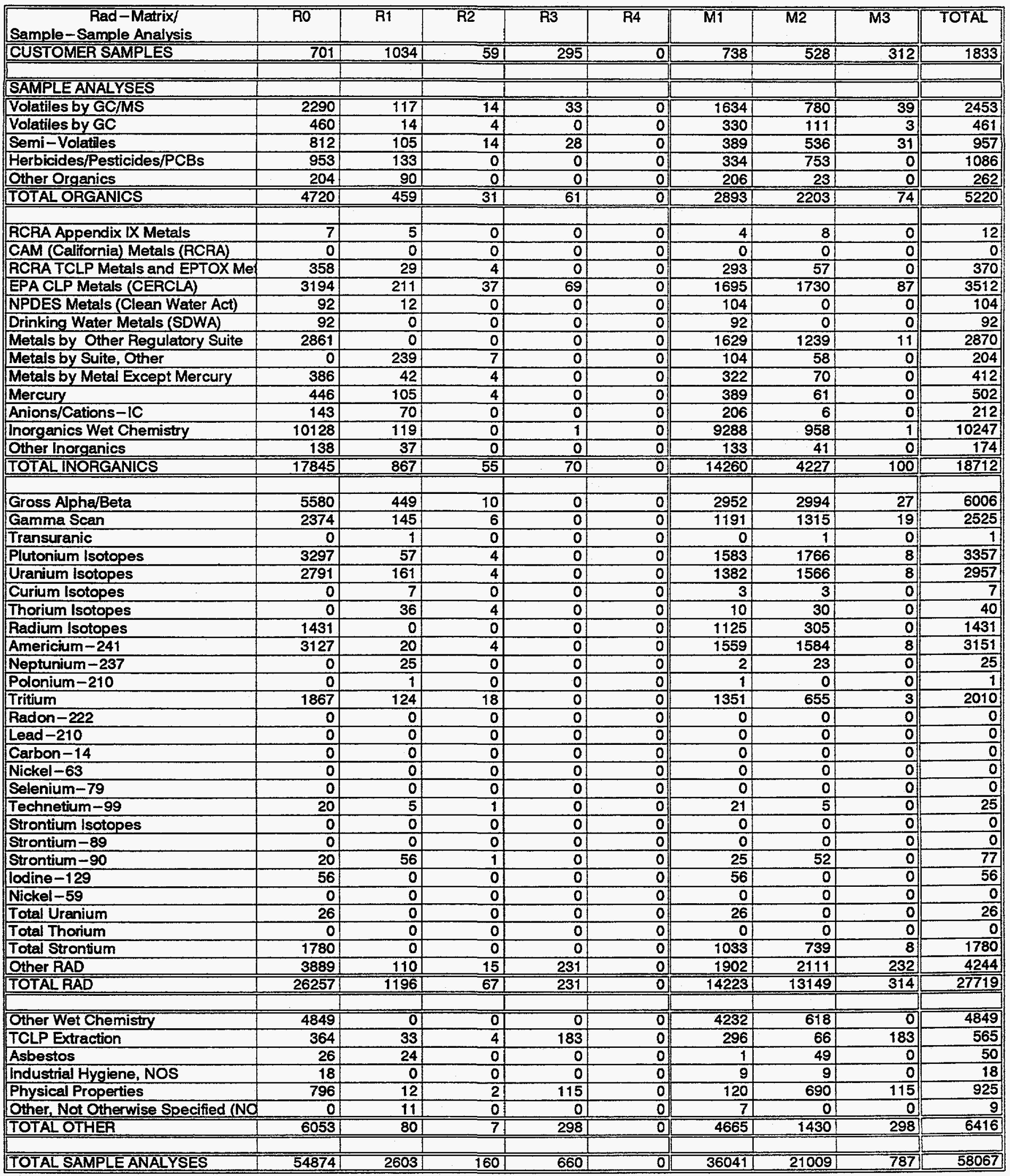


Table Va: FY 93 Samples and Sample Analyses by Rad Level and Matrix Type (xii) Savannah River

\begin{tabular}{|c|c|c|c|c|c|c|c|c|c|}
\hline $\begin{array}{c}\text { Rad-Matrix } \\
\text { Sample-Sample Analysis }\end{array}$ & $\mathrm{RO}$ & $\overline{\mathrm{R} 1}$ & R2 & $\overline{\mathrm{R3}}$ & R4 & M1 & M2 & M3 & TOTAL \\
\hline CUSTOMER SAMPLES & 10161 & 19488 & 227 & 62 & $210]$ & 21299 & 808 & 3216 & 27735 \\
\hline & & & & & & & & & \\
\hline \multicolumn{10}{|l|}{ SAMPLE ANALYSES } \\
\hline Volatiles by GC/MS & 129 & 1 & 0 & 0 & 0] & 131 & 0 & 0 & 131 \\
\hline Volatiles by GC & 87 & 137 & 15 & 8 & 0 & 139 & 102 & 7 & 247 \\
\hline Semi-Volatiles & 1278 & 78 & 8 & 2 & 0 & 1012 & 57 & 297 & 1366 \\
\hline Herbicides/Pesticides/PCBs & 507 & 94 & 8 & 4 & 0 & 527 & 75 & 11 & 612 \\
\hline Other Organics & 2001 & 4660 & 0 & 0 & $0]$ & 1737 & 1 & 280 & 4339 \\
\hline TOTAL ORGANICS & 4002 & 4972 & 31 & 13 & $0]$ & 3545 & 235 & $594]$ & 6695 \\
\hline \multirow{2}{*}{\multicolumn{10}{|c|}{\begin{tabular}{|l|} 
RCRA Appendix IX Metals \\
\end{tabular}}} \\
\hline & 11 & 9 & 0 & 0 & 0 & 19 & 0 & 1 & 20 \\
\hline CAM (California) Metals (RCRA) & 0 & 0 & 0 & 0 & 0 & 0 & 0 & 0 & 0 \\
\hline RCRA TCLP Metals and EPTOX Met & 67 & 86 & 2 & 2 & 0 & 115 & 35 & 7 & 157 \\
\hline \begin{tabular}{|l|l|} 
EPA CLP Metals (CERCLA) & \\
\end{tabular} & 66 & 32 & 15 & 8 & 0 & 66 & 55 & o. & 122 \\
\hline NPDES Metals (Clean Water Act) & 86 & 820 & 0 & 0 & 0 & 906 & 0 & 0 & 906 \\
\hline Drinking Water Metals (SDWA) & 0 & 0 & 0 & 0 & 0 & 0 & 0 & 0 & 0 \\
\hline Metals by Other Regulatory Suite & 298 & 4 & 0 & 0 & 0 & 302 & 0 & 0 & 302 \\
\hline Metais by Suite, Other & 2680 & 4644 & 0 & 0 & 0 & 1420 & 0 & 1260 & 5002 \\
\hline Metals by Metal Except Mercury & 1773 & 33 & 0 & 0 & 105 & 1003 & 27 & 807 & 1874 \\
\hline Mercury & 1973 & 4791 & 2 & 1 & 105 & 1353 & 33 & 767 & 4512 \\
\hline Anions/Cations - IC & 3825 & 83 & 0 & 0 & 210 & 3196 & 63 & 680 & 4028 \\
\hline Inorganics Wet Chemistry & 4529 & 101 & 31 & 15 & 0 & 3627 & 183 & 867 & 4676 \\
\hline Other Inorganics & 245 & 76 & 0 & 0 & 0 & 251 & 44 & 27 & 321 \\
\hline TOTALINORGANICS & 15553 & 10680 & 50 & 25 & 420 & 12257 & 439 & 4414 & 21919 \\
\hline Gross Aloha/Beta & 2221 & 15279 & 34 & 48 & 210 & 12148 & 370 & 114 & 15212 \\
\hline Gamma Scan & 64 & 4887 & 34 & 19 & 110 & 4794 & 167 & $\frac{174}{73}$ & $\frac{1021 \leq}{5074}$ \\
\hline Transuranic & 0 & 0 & 0 & 0. & 110 & 0 & 0 & 30 & 70 \\
\hline Plutonium Isotopes & 677 & 44 & 18 & 4 & 210 & 677 & 56 & 40 & 862 \\
\hline Uranium Isotopes & 679 & 55 & 9 & 3 & 210 & 678 & 60 & 38 & 866 \\
\hline Curium Isotopes & 0 & 9 & 2 & 1 & 10 & 0 & 6 & 7 & 18 \\
\hline Thorium Isotopes & 0 & 4 & 2 & 25 & 0 & 0 & 7 & 24 & 31 \\
\hline Radium Isotopes & 1 & 0 & 0 & 0 & 0 & 1 & 0 & 0 & 1 \\
\hline Americium-241 & 677 & 26 & 3 & 2 & 20 & 677 & 25 & 7 & 718 \\
\hline Neptunium-237 & 0 & 3 & 8 & 0 & 0 & 0 & 3 & 8 & 11 \\
\hline Polonium-210 & 0 & 0 & 0 & 0 & 0 & 0 & 0 & 0 & 0 \\
\hline Tritium & 590 & 27 & 18 & 4 & 0 & 592 & 40 & 7 & 639 \\
\hline Radon-222 & 0 & 0 & 0 & 0 & 0 & 0 & 0 & 0 & 0 \\
\hline Lead-210 & 0 & 12 & 0 & 0 & 0 & 0 & 0 & 12 & 12 \\
\hline Carbon-14 & 0 & 0 & 0 & 0 & 0 & 0 & 0 & 0 & 0 \\
\hline Nickel-63 & 0 & 0 & 0 & 0 & 0 & 0 & 0 & 0 & 0 \\
\hline Selenium-79 & 0 & 0 & 0 & 0 & 0 & 0 & 0 & 0 & 0 \\
\hline Technetium-99 & 0 & 8 & 0 & 0 & 0 & 0 & 0 & 8 & 8 \\
\hline Strontium Isotopes & 0 & 0 & 0 & 0 & 0 & 0 & 0 & 0 & 0 \\
\hline Strontium-89 & 0 & 0 & 0 & 0 & 0 & 0 & 0 & 0 & 0 \\
\hline Strontium -90 & 0 & 26 & 11 & 4 & 0 & 1 & 40 & 0 & 41 \\
\hline lodine-129 & 0 & 0 & 0 & 0 & 0 & O & 0 & 0 & 0 \\
\hline Nickel-59 & 0 & 0 & 0 & 0 & 0 & 0 & 0 & 0 & 0 \\
\hline Total Uranium & 0 & 0 & 0 & 0 & 210 & 0 & 0 & 30 & 120 \\
\hline Total Thorium & 0 & 0 & 0 & 0 & 0 & 0 & 0 & 0 & 0 \\
\hline Total Strontium & 15 & 12 & 0 & 0 & 0 & 19 & 0 & 8 & 27 \\
\hline Other RAD & 83 & 193 & 8 & 4 & 10 & 143 & 701 & 71 & 291 \\
\hline TOTAL RAD & 5007 & 20585 & 147 & 114 & 1100 & 19730 & $845 \mid$ & 476 & 24002 \\
\hline & & & & & 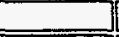 & & $=1$ & 7 & \\
\hline Other Wet Chemistry & 1219 & 7 & 0 & 0 & 30 & 1225 & 0 & 30 & 1255 \\
\hline TCLP Extraction & 1 & 85 & 38 & 3 & 0 & 67 & 47 & 13 & 127 \\
\hline Asbestos & 0 & 0 & 0 & of & (0) & 0 & 3 & 0. & 1 \\
\hline Industrial Hygiene, NOS & 552 & 0 & 0 & 0 & 0 & 552 & 0 & 0 & 552 \\
\hline Physical Properties & 12084 & 7 & 4 & 2 & 0 & 7884 & 13 & 4200 & 12097 \\
\hline Other, Not Otherwise Specified (NO & 0 & 8 & 0 & 0 & 0 & 8 & 0 & 0 & 8 \\
\hline TOTAL OTHER & 13856 & 107 & 42 & 5ा & 30 & 9737 & 621 & 4243 & 14041 \\
\hline & & & & $1=7$ & 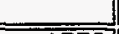 & & 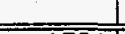 & & \\
\hline TOTAL SAMPLE ANALYSES & 38418 & 36343 & 270 & 157 & 1550 & 45267 & 1581 & 9727 & 66657 \\
\hline
\end{tabular}


Table V: FY 94 Samples and Sample Analyses by Rad Level and Matrix Type (i) Complex-wide

\begin{tabular}{|c|c|c|c|c|c|c|c|c|c|}
\hline $\begin{array}{c}\text { Rad-Matrix } \\
\text { Sample-Sample Analysis }\end{array}$ & $\overline{\mathrm{RO}}$ & $\overline{\mathrm{R1}}$ & $\overline{\mathrm{R} 2}$ & R33 & $\overline{\mathrm{R4}}$ & $\overline{\mathrm{M1}}$ & $\overline{\mathrm{M} 2}$ & $\overline{\mathrm{M3}}$ & TOTAL \\
\hline CUSTOMER SAMPLES & 56288 & 241242 & 19800 & 4426 & 607 & 118703 & 92473 & 68488 & 301013 \\
\hline & & & & & & & & & \\
\hline \multicolumn{10}{|l|}{ SAMPLE ANALYSES } \\
\hline Volatiles by $\mathrm{GC} / \mathrm{MS}$ & 38526 & 46244 & 9368 & 459 & 0 & 18173 & 35747 & 40706 & 94610 \\
\hline Volatiles by GC & 11144 & 19458 & 1384 & 45 & 0 & 4507 & 24648 & 2699 & 31942 \\
\hline Semi-Volatiles & 18654 & 34981 & 8428 & 400 & 0 & 11185 & 32623 & 18634 & 62452 \\
\hline Herbicides/Pesticides/PCBs & 30458 & 71630 & 45 & 22 & 0 & 51682 & 25683 & 24852 & 102186 \\
\hline Other Organics & 9764 & 21896 & 964 & 0 & 0 & 7632 & 3153 & 2379 & 22894 \\
\hline TOTAL ORGANICS & 108545 & 194208 & 20188 & 927 & 0 & 93178 & 121854 & 89269 & 314085 \\
\hline & & & & & & & & & \\
\hline RCRA Appendix IX Metals & 114 & 322 & 0 & 0 & 0 & 119 & 312 & 2093 & 1480 \\
\hline CAM (California) Metals (RCRA) & 557 & 135 & 0 & 0 & 0 & 692 & 169 & 34 & 794 \\
\hline RCRA TCLP Metals and EPTOX Me & 5662 & 23139 & 17126 & 9 & 요 & 3025 & 10261 & 34236 & 46728 \\
\hline EPA CLP Metals (CERCLA) & 32650 & 40559 & 2193 & 1004 & o. & 15376 & 30823 & 30207 & 76406 \\
\hline NPDES Metals (Clean Water Act) & 5467 & 4069 & 0 & 0 & a & 9536 & 0 & 0 & 9536 \\
\hline Drinking Water Metals (SDWA) & 706 & 0 & 0 & 0 & 요 & 706 & 0 & 0 & 706 \\
\hline Metals by Other Regulatory Suite & 53746 & 35229 & 7227 & 0. & 0 & 38988 & 23920 & 34472 & 96791 \\
\hline Metals by Suite, Other & 7478 & 30071 & 390 & o. & 0] & 9944 & 3221 & 4084 & 27593 \\
\hline Metals by Metal Except Mercury & 10627 & 85350 & 7255 & 0 & 293 & 40414 & 10035 & 12416 & 83195 \\
\hline Mercury & 15765 & 67933 & 1000 & 4 & 293 & 72579 & 19071 & 13526 & 95086 \\
\hline Anions/Cations-IC & 21740 & 42954 & 3373 & of & 586 & 21019 & 657 & 13151 & 51739 \\
\hline Inorganics Wet Chemistry & 129588 & 32996 & 12536 & 104 & - & 78572 & 43729 & 88768 & 193146 \\
\hline Other Inorganics & 12329 & 16362 & 0 & 0 & 0 & 12467 & 5232 & 10992 & 28691 \\
\hline TOTAL INORGANICS & 296429 & 379120 & 51100 & 1121 & 1172 & 303437 & 147428 & 243979 & 711893 \\
\hline & & & & & & & & & \\
\hline Gross Alpha/Beta & 75960 & 169474 & 2998 & 281 & 586 & 149792 & 54941 & 17462 & 235747 \\
\hline Gamma Scan & 29490 & 39801 & 1481 & 112 & 307 & 31156 & 22902 & 16910 & 71079 \\
\hline Transuranic & 0 & 28245 & 28908 & 0 & 307 & 38544 & 18609 & 84 & 57348 \\
\hline Plutonium Isotopes & 33369 & 13268 & 1264 & 22 & 586 & 13599 & 20545 & 13862 & 48258 \\
\hline Uranium Isotopes & 35121 & 64204 & 23699 & 20 & 586 & 63852 & 35820 & 23457 & 123380 \\
\hline Curium Isotopes & 0 & 1687 & 13 & 7 & 28 & 569 & 434 & 705 & 1721 \\
\hline Thorium Isotopes & 3316 & 37961 & 1189 & 107 & 0 & 24248 & 4860 & 13465 & 42573 \\
\hline Radium Isotopes & 9665 & 614 & 0 & 0 & 0 & 7187 & 2484 & 608 & 10279 \\
\hline Americium-241 & 29056 & 9007 & 234 & 11 & 56 & 13410 & 17485 & 7413 & 38337 \\
\hline Neptunium-237 & 3316 & 23664 & 19466 & 0 & 0 & 20248 & 12206 & 13992 & 46447 \\
\hline Polonium - 210 & 154 & 619 & 964 & 0 & - & 6 & 154 & 1578 & 1737 \\
\hline Tritium & 18387 & 13333 & 2359 & 22 & 0 & 14292 & 13930 & 238 & 31281 \\
\hline Radon-222 & 57 & 0 & 0 & 0 & 0 & 57 & 0 & 0 & 57 \\
\hline Lead-210 & 0 & 50 & 0 & 0 & 0 & 0 & 0 & 50 & 50 \\
\hline Carbon-14 & 0 & 0 & 0 & 0 & 의 & 0 & 0 & 0 & 0 \\
\hline Nickel-63 & 0 & 0 & 0 & 0 & 0 & 0 & 0 & 0 & 0 \\
\hline Selenium-79 & 557 & 85 & 0 & 0 & 0 & 605 & 21 & 16 & 643 \\
\hline Technetium-99 & 150 & 40876 & 29934 & 0 & 0 & 38763 & 21538 & 10659 & 70961 \\
\hline Strontium isotopes & 0 & 70 & 0 & 0 & 0 & 0 & 32 & 38 & 70 \\
\hline Strontium - 89 & 0 & 0 & 01 & 0 & 0 & 0 & 0 & 0 & 0 \\
\hline Strontium -90 & 707 & 4229 & 1396 & 22 & 0 & 1237 & 5077 & 53 & 6361 \\
\hline lodine-129 & 985 & 1389 & 0 & 0 & 0 & 2304 & 43 & 27 & 2374 \\
\hline Nickel-59 & 0 & 0 & 0 & 0 & 0. & 0 & 0 & o & 0 \\
\hline Total Uranium & 13654 & 25142 & 28265 & 0 & 586 & 10819 & 33195 & 23130 & 67396 \\
\hline Total Thorium & 0 & 673 & 0 & 0 & 0 & 61 & 612 & o) & 673 \\
\hline Total Strontium & 13613 & 50 & o) & 0 & 0 & 6504 & 6839 & 321 & 13663 \\
\hline Other RAD & 49768 & 49941 & 2931 & 3233 & 28 & 36387 & 42938 & 26504 & 105865 \\
\hline TOTAL RAD & 317327 & 524383 & 145101 & 3840 & 3069 & 473640 & 314666 & 170572 & 976299 \\
\hline Other Wet Chemistry & 38763 & & & & 84 & 33132 & 5004 & 801 & 39507 \\
\hline TCLP Extraction & $\begin{array}{r}50 / 03 \\
5089\end{array}$ & $\frac{001}{44454}$ & $\frac{0}{8640}$ & $\frac{0}{2562}$ & $\frac{84}{0}$ & \begin{tabular}{r|r|}
33432 \\
3426
\end{tabular} & $\begin{array}{r}5991 \\
14272\end{array}$ & \begin{tabular}{r|}
84 \\
42746
\end{tabular} & $\frac{39007}{60594}$ \\
\hline Asbestos & 152 & 39409 & 0 & 0 & a. & 3 & 29760 & 9808 & 39566 \\
\hline Industrial Hygiene, NOS & 2900 & 48 & 0 & 0 & 요 & 2535 & 407 & 5 & 2948 \\
\hline Physical Properties & 39090 & 37801 & 151 & 1607 & 의 & 48502 & 5845 & 24302 & 78649 \\
\hline Other, Not Otherwise Specified (Nd & 6754 & 918 & 964 & 0 & 0 & 817 & 37 & 7748 & 8620 \\
\hline TOTAL OTHER & 92748 & 123291 & 9754 & 4169 & 84 & 88716 & 56313 & 84694 & 229884 \\
\hline TOTAL SAMPLE ANALYSES & 815049 & 1221002 & 226143 & 10057 & 4325 & 958972 & 640261 & 588514 & 2232161 \\
\hline
\end{tabular}


Table Vb: FY 94 Samples and Sample Analyses by Rad Level and Matrix Type (ii) EM-30

\begin{tabular}{|c|c|c|c|c|c|c|c|c|c|}
\hline $\begin{array}{c}\text { Rad-Matrix } / \\
\text { Sample-Sample Analysis }\end{array}$ & Ro & $\overline{\mathrm{R} 1}$ & $\overline{\mathrm{R2}}$ & $\overline{\mathrm{R3}}$ & $\overline{\mathrm{R} 4}$ & $\overline{\mathrm{M} 1}$ & $\mathrm{M} 2$ & M3 & TOTAL \\
\hline CUSTOMER SAMPLES & 29609 & 81769 & 1670 & 3782 & 569 & 78695 & 4905 & 15012 & 108006 \\
\hline & & & & & & & & & \\
\hline \multicolumn{10}{|l|}{ SAMPLE ANALYSES } \\
\hline Volatiles by GC/MS & 1236 & 2215 & 567 & 401 & 0 & 590 & 829 & 3000 & 4419 \\
\hline Volatiles by GC & 1100 & 1677 & 129 & 21 & 0 & 1095 & 1604 & 177 & 2901 \\
\hline Semi-Volatiles & 3787 & 1846 & 524 & 345 & 0 & 3012 & 1220 & 2271 & 6502 \\
\hline Herbicides/Pesticides/PCBs & 3121 & 3778 & 21 & 10 & 0 & 5182 & 249 & 1500 & 6930 \\
\hline Other Organics & 5724 & 18553 & 64 & 0 & 0. & 4992 & 51 & 861 & 15122 \\
\hline TOTAL ORGANICS & 14968 & 28070 & 1304 & 777 & $0]$ & 14872 & 3951 & 7808 & 35875 \\
\hline \multicolumn{10}{|l|}{\begin{tabular}{|l|} 
RCRA Aopendix IXMetals \\
\end{tabular}} \\
\hline \begin{tabular}{|l|} 
RCRA Appendix IX Metals \\
CAM (California) Metals (RCRA)
\end{tabular} & 42 & 38 & 0 & 0 & 0 & 75 & 0 & 143 & 149 \\
\hline CAM (California) Metals (RCRA) & 249 & 2 & 0 & 0 & 0 & 251 & 2 & 0 & 253 \\
\hline RCRA TCLP Metals and EPTOX Met & 879 & 1537 & 1137 & 4 & 0 & 869 & 495 & 2273 & 3597 \\
\hline EPA CLP Metals (CERCLA) & 395 & 1975 & 42 & 859 & 0 & 388 & 172 & 2710 & 3270 \\
\hline NPDES Metals (Clean Water Act) & 340 & 3297 & 0 & 0 & 0 & 3637 & 0 & o. & 3637 \\
\hline Drinking Water Metals (SDWA) & 130 & 0 & 0 & 0 & 0 & 130 & 0 & 0 & 130 \\
\hline Metals by Other Regulatory Suite & 3008 & 2192 & 477 & 0 & 0 & 3018 & 460 & 2199 & 5677 \\
\hline Metals by Suite, Other & 7262 & 18481 & 0 & 0 & 0 & 3855 & 1 & 3451 & 16525 \\
\hline Metais by Metal Except Mercury & 5852 & 5268 & 482 & 0 & 285 & 5850 & 473 & 2657 & 10433 \\
\hline Mercury & 6586 & 21657 & 73 & 2 & 285 & 8829 & 898 & 2829 & 20579 \\
\hline Anions/Cations-IC & 10825 & 2870 & 222 & 0 & 569 & 8979 & 246 & 2576 & 13144 \\
\hline Inorganics Wet Chemistry & 19192 & 1738 & 888 & 53 & 0 & 13896 & 2310 & 8007 & 23042 \\
\hline Other Inorganics & 1216 & 1190 & 0 & 01 & o] & 1309 & 280 & 817 & 2405 \\
\hline TOTALINORGANICS & 55977 & 60245 & 3320 & 919 & 1138 & 51085 & 5336 & 27662 & 102840 \\
\hline \multicolumn{10}{|l|}{ 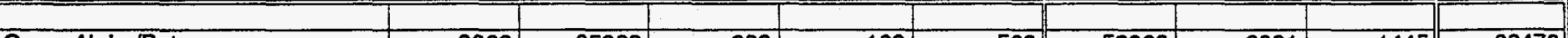 } \\
\hline Gross Alpha/Beta & 9666 & 65968 & 239 & 129 & 569 & 52929 & 2001 & 1445 & 66473 \\
\hline Gamma Scan & 1280 & 20192 & 156 & 52 & 298 & 19086 & 1393 & 1283 & 21870 \\
\hline Transuranic & 0 & 1855 & 1907 & 0 & 298 & 2542 & 1220 & 81 & 3951 \\
\hline Plutonium Isotopes & 3138 & 756 & 124 & 10 & 569 & 2824 & 283 & 1002 & 4353 \\
\hline Uranium Isotopes & 3033 & 3487 & 1580 & 9 & 569 & 5935 & 656 & 1599 & 8435 \\
\hline Curium Isotopes & 0 & 117 & 6 & 3 & 27 & 26 & 30 & 70 & 140 \\
\hline Thorium Isotopes & 216 & 2166 & 69 & 98 & 0 & 1557 & 19 & 973 & 2548 \\
\hline Radium Isotopes & 33 & 40 & 0 & 0 & 0 & 34 & 0 & 40 & 73 \\
\hline Americium-241 & 2817 & 574 & 9 & 5 & 54 & 2793 & 121 & 492 & 3433 \\
\hline Neptunium-237 & 216 & 1440 & 1313 & 0 & 0 & 1324 & 697 & 948 & 2970 \\
\hline Polonium-210 & 2 & 41 & 64 & 0 & 0 & 1 & 2 & 104 & 107 \\
\hline Tritium & 2519 & 470 & 143 & 10 & 0 & 2458 & 286 & 27 & 2956 \\
\hline Radon-222 & 4 & 0 & 0 & 0 & 0 & 4 & 0 & 0 & 4 \\
\hline Lead-210 & 0 & 47 & 0 & 0 & 0 & 0 & 0 & 47 & $4 \overline{7}$ \\
\hline Carbon-14 & 0 & 0 & 0 & 0 & 0 & 0 & 0 & 의 & 으 \\
\hline Nickel-63 & 0 & 0 & 0 & 0 & 0 & 0 & 0 & o. & 0 \\
\hline Selenium-79 & 249 & 1 & 0 & 0 & 0 & 250 & 0 & 의 & 251 \\
\hline Technetium-99 & 28 & 2522 & 1970 & 0 & 0 & 2570 & 1218 & 732 & 4520 \\
\hline Strontium Isotopes & 0 & 5 & 0 & 0 & 0 & 0 & 2 & 2 & 5 \\
\hline Strontium -89 & 0 & 0 & 0 & 0 & 0 & 0 & 0 & 의 & 은 \\
\hline Strontium -90 & 277 & 110 & 113 & 10 & 0 & 286 & 224 & 1 & 511 \\
\hline lodine-129 & 328 & 84 & 0 & 0 & 0. & 411 & 1 & 0 & 412 \\
\hline Nickel-59 & 0 & 0 & 0 & 0 & 0 & 0 & 0 & 0 & 0 \\
\hline Total Uranium & 885 & 1461 & 1864 & 0 & 569 & 705 & 2120 & 1466 & 4535 \\
\hline Total Thorium & 0 & 1 & 0 & 0 & 0. & 1 & 0 & 0 & 1 \\
\hline Total Strontium & 93 & 47 & 0 & 0 & 0 & 95 & 13 & 32 & 140 \\
\hline Other RAD & 1741 & 2991 & 172 & 2817 & 27 & 2725 & 398 & 4548 & 7709 \\
\hline TOTALRAD & 26524 & 104377 & 9728 & 3144 & 2981 & 98556 & 10683 & 14893 & 135442 \\
\hline Other Wet Chemistry & 5034 & 68 & \begin{tabular}{l|l}
0 \\
\end{tabular} & 01 & 81[ & 5102 & 0! & 81 & 5183 \\
\hline TCLP Extraction & 590 & 2855 & 706 & 2232 & 0 & 685 & 756 & 4887 & $\frac{5183}{6356}$ \\
\hline Asbestos & 1 & 2 & 0 & 0 & 0 & 0 & 13 & 요 & 8 \\
\hline Industrial Hygiene, NOS & 2196 & 1 & 0 & 0 & 0 & 2194 & 3 & 0 & 2197 \\
\hline Physical Properties & 32967 & 2360 & 11 & 1401 & 0 & 23194 & 43 & 13501 & 36739 \\
\hline Other, Not Otherwise Specified (NO & 437 & 47 & 64 & 0 & 0 & 36 & 0 & 505 & 544 \\
\hline TOTAL OTHER & 41226 & 5332 & 781 & 3633 & 81 & 31211 & 816 & 18975 & 51027 \\
\hline TOTAL SAMPLE ANALYSES & 138694 & 198023 & 15133 & 8473 & 4200 & 195723 & 20786 & 69338 & $\overline{325185}$ \\
\hline
\end{tabular}


Table Vb: FY 94 Samples and Sample Analyses by Rad Level and Matrix Type (iii) EM-40

\begin{tabular}{|c|c|c|c|c|c|c|c|c|c|}
\hline $\begin{array}{c}\text { Rad-Matrix/ } \\
\text { Sample-Sample Analysis } \\
\end{array}$ & RO & $\overline{\mathrm{R} 1}$ & R2 & $\overline{\mathrm{R3}}$ & R4 & $\overline{\mathrm{M} 1}$ & $\overline{\mathrm{M} 2}$ & $\overline{\mathrm{M3}}$ & TOTAL \\
\hline CUSTOMEA SAMPLES & 23353 & 156205 & 18069 & 530 & 20 & 35247 & 86737 & 53022 & 186592 \\
\hline & & & & & & & & & \\
\hline \multicolumn{10}{|l|}{ SAMPLE ANALYSES } \\
\hline Volatiles by GC/MS & 32230 & 43963 & $\overline{8784}$ & 46 & 0] & 13807 & 33628 & 37616 & 85037 \\
\hline Volatiles by GC & 7898 & 17675 & 1231 & 24 & 0. & 1573 & 22814 & 2516 & 26866 \\
\hline Semi-Volatiles & 13003 & 33078 & 7889 & 45 & - & 7232 & 30465 & 16296 & 54004 \\
\hline Herbicides/Pesticides/PCBs & 25297 & 67733 & 24 & 12 & 0 & 45641 & 24182 & 23304 & 93096 \\
\hline Other Organics & 2810 & 2609 & 898 & 0 & 0 & 1481 & 3050 & 1492 & 6170 \\
\hline TOTAL ORGANICS & 81237 & 165058 & 18826 & 126 & D] & 69735 & 114139 & 81224 & 265173 \\
\hline RCRA Appendix IX Metals & 53 & 282 & 0 & 0 & 0 & $\overline{31}$ & 304 & 1946 & 1308 \\
\hline CAM (California) Metals (RCRA) & 300 & 131 & 0 & 0 & o. & 432 & 164 & 33 & 530 \\
\hline RCRA TCLP Metals and EPTOX Me & 2809 & 21501 & 15935 & 5 & 0 & 527 & 9549 & 31895 & 41110 \\
\hline EPA CLP Metals (CERCLA) & 24874 & 38526 & 2150 & 120 & 0 & 10836 & 27424 & 27410 & 65670 \\
\hline NPDES Metals (Clean Water Act) & 5113 & 642 & 0 & 0 & 애 & 5755 & 0 & 0. & 5755 \\
\hline Drinking Water Metals (SDWA) & 65 & 0 & 0 & 0 & o & 65 & 0 & 0 & 65. \\
\hline Metals by Other Regulatory Suite & 43897 & 32972 & 6736 & 0 & 0] & 31766 & 20815 & 32200 & 84193 \\
\hline Metals by Suite, Other & 0 & 10858 & 390 & 0 & 애 & 5974 & 3220 & 531 & 10486 \\
\hline Metals by Metal Except Mercury & 2551 & 79870 & 6739 & 0 & 0 & 32667 & 9362 & 9681 & 70434 \\
\hline Mercury & 7044 & 45384 & 905 & 2 & - & 61863 & 17963 & 10613 & 71887 \\
\hline Anions/Cations-IC & 10565 & 39996 & 3144 & 0 & 0 & 11743 & 401 & 10499 & 38173 \\
\hline Inorganics Wet Chemistry & 84280 & 31202 & 11622 & 49 & 0. & 40655 & 39404 & 80523 & 143867 \\
\hline Other inorganics & 10939 & 15134 & 0 & 0 & 요 & 10996 & 4927 & 10150 & 26074 \\
\hline TOTAL INORGANICS & 192488 & 316499 & 47621 & 176 & 묘묘 & 213310 & 133533 & 215479 & 559554 \\
\hline Gross Alpha/Beta & 52691 & 100958 & 2752 & 148 & 0 & 88335 & 46133 & 15956 & 153487 \\
\hline Gamma Scan & 21340 & 18813 & 1320 & 59 & 0 & 8684 & 17269 & 15580 & 41533 \\
\hline Transuranic & 0 & 26329 & 26945 & 0 & o. & 35926 & 17347 & 0 & 53274 \\
\hline Plutonium isotopes & 21993 & 12483 & 1136 & 12 & 0. & 6611 & 16191 & 12823 & 35625 \\
\hline Uranium Isotopes & 25554 & 60603 & 22072 & 11 & 0 & 54406 & 32036 & 21798 & 108240 \\
\hline Curium Isotopes & 0 & 1566 & 7 & 4 & 0 & 542 & 403 & 632 & 1577 \\
\hline Thorium Isotopes & 3094 & 35731 & 1118 & 5 & 0 & 22645 & 4841 & 12462 & 39948 \\
\hline Radium Isotopes & 6171 & 573 & 0 & 0 & 0 & 4397 & 1780 & 567 & 6744 \\
\hline Americium-241 & 18657 & 8416 & 225 & 6 & 0 & 6580 & 13825 & 6899 & 27304 \\
\hline Neptunium-237 & 3094 & 22181 & 18113 & 0 & 0 & 18884 & 11489 & 13016 & 43389 \\
\hline Polonium-210 & 150 & 573 & 898 & 0 & 0 & 1 & 150 & 1471 & 1621 \\
\hline Tritium & 11137 & 12849 & 2212 & 12 & 0 & 8436 & 12302 & 210 & 23579 \\
\hline Radon-222 & 53 & 0 & 0 & 0 & 0 & 53 & 0 & 0 & 53 \\
\hline Lead-210 & 0 & 1 & 0 & 0 & 0 & 0 & 0 & 1 & 1 \\
\hline Carbon-14 & 0 & 0 & 0 & 0 & 0 & 0 & 0 & 의 & 0 \\
\hline Nickel-63 & 0 & 0 & 0 & 0 & 0 & 0 & 0 & o] & 0 \\
\hline Selenium -79 & 300 & 84 & 0 & 0 & 0 & 348 & 21 & 16 & 384 \\
\hline Technetium - 99 & 14 & 38279 & 27906 & 0 & 0 & 36009 & 20284 & 9905 & 66198 \\
\hline Strontium Isotopes & 0 & 65 & 0 & 0 & 0 & 0 & 30 & 35 & 65 \\
\hline Strontium - 89 & 0 & 0 & 0 & 0 & 0 & 0 & 0 & 0 & 0 \\
\hline Strontium -90 & 314 & 4115 & 1279 & 12 & 0 & 834 & 4847 & 53 & 5727 \\
\hline lodine-129 & 340 & 1302 & 0 & 0 & o: & 1573 & 42 & 26 & 1642 \\
\hline Nickel-59 & 0 & 0 & 0 & 0 & 0 & 0 & 0 & 0 & 0 \\
\hline Total Uranium & 12635 & 23633 & 26346 & 0 & 0 & 9985 & 31012 & 21617 & 62614 \\
\hline Total Thorium & 0 & 671 & 0 & 0 & 0. & 59 & 612 & 0 & 671 \\
\hline Total Strontium & 9291 & 1 & 0 & 0 & 0 & 3881 & 5131 & 280 & 9292 \\
\hline Other RAD & 38541 & 46808 & 2734 & 333 & o] & 28328 & 38278 & 21819 & 88421 \\
\hline TOTALAAD & 225372 & 416035 & 135063 & 602 & 에 & 336518 & 274021 & 155166 & 771389 \\
\hline Other Wet Chemistry & 21680 & 591 & 0 & 0 & का & 17657 & 4613 & 0 & 22271 \\
\hline TCLP Extraction & 2516 & 41459 & 7892 & 264 & o & 1117 & 13272 & 37714 & 52116 \\
\hline Asbestos & 85 & 39407 & 0 & 0 & o & 1 & 29683 & 9808 & 39492 \\
\hline Industrial Hygiene, NOS & 603 & 47 & 0 & 0 & 0 & 251 & 394 & 5 & 650 \\
\hline Physical Properties & 2993 & 35371 & 139 & 165 & 0 & 24143 & 4124 & 10401 & 38668 \\
\hline Other, Not Otherwise Specified (NO & 6305 & 846 & 898 & 0 & 0 & 780 & 37 & 7228 & 8047 \\
\hline TOTAL OTHER & 34181 & 117721 & 8929 & 428 & [0] & 43950 & 52124 & 65156 & 161244 \\
\hline & & & & & & & & & \\
\hline TOTAL SAMPLE ANALYSES & 533278 & 1015313 & 210438 & 1332 & 0] & 663513 & 573817 & 517026 & 1757359 \\
\hline
\end{tabular}


Table Vb: FY 94 Samples and Sample Analyses by Rad Level and Matrix Type (iv) EM-60

\begin{tabular}{|c|c|c|c|c|c|c|c|c|c|}
\hline $\begin{array}{c}\text { Rad-Matrix/ } \\
\text { Sample-Sample Analysis }\end{array}$ & RO & $\overline{\mathrm{RI}}$ & $\overline{\mathrm{A2}}$ & R3 & R4 & M1 & $\bar{M} 2$ & $\overline{\mathrm{M} 3}$ & TOTAL \\
\hline CUSTOMER SAMPLES & 2493 & 862 & 12 & $1 !$ & 0 & $2486]$ & 686 & $8]$ & 3273 \\
\hline & & & & & & & & & \\
\hline \multicolumn{10}{|l|}{ SAMPLE ANALYSES } \\
\hline Volatiles by GC/MS & 5135 & 01 & 0 & 0 & 0 & 3841 & 1295 & 0 & 5136 \\
\hline Volatiles by GC & 2167 & 57 & 20 & 0 & 요 & 1852 & 188 & 0 & 2142 \\
\hline Semi-Volatiles & 1794 & 2 & 0 & 0 & 요 & 871 & 925 & 0 & 1796 \\
\hline Herbicides/Pesticides/PCBs & 1995 & 2 & 0 & 0 & 요 & 720 & 1277 & ? & 1997 \\
\hline Other Organics & 1087 & 189 & 0 & 0 & - & 1036 & 52 & 0] & 1182 \\
\hline TOTAL ORGANICS & 12179 & 250 & 20 & 0 & ] & 8320 & 3736 & $1][$ & 12253 \\
\hline \multirow{2}{*}{\multicolumn{10}{|c|}{\begin{tabular}{|l|l|} 
RCRA Appendix IX Metals & \\
\end{tabular}}} \\
\hline & 19 & 0 & 이 & o) & ㅇ․ & 11 & 8 & 의 & 19 \\
\hline CAM (California) Metals (RCRA) & O & 0 & O. & 0 & 요 & of & 0 & 0 & 0 \\
\hline RCRA TCLP Metals and EPTOX Me & 1991 & 57 & 20 & D. & 0. & 1644 & 203 & ㅇ. & 1958 \\
\hline EPA CLP Metals (CERCLA) & 7541 & 0 & 0 & 0 & of & 4232 & 3302 & 8 & 7541 \\
\hline NPDES Metals (Clean Water Act) & 3 & 33 & 0 & O & 0 & 37 & 0 & 0 & 37 \\
\hline Drinking Water Metals (SDWA) & 520 & 0 & 0 & 0 & 0. & 520 & 0 & 0 & 520 \\
\hline Metals by Other Regulatory Suite & 6909 & 0 & 0 & 0 & 0 & 4205 & 2697 & 8 & 6909 \\
\hline Metals by Suite, Other & 0] & 188 & 0) & 0 & 0 & 01 & 0 & o) & 94 \\
\hline Metals by Metal Except Mercury & 2103 & 55 & 20 & 0 & 0 & 1766 & 190 & o. & 2067 \\
\hline Mercury & 1987 & 247 & 20 & 0 & 0 & 1657 & 188 & 0 & 2050 \\
\hline Anions/Cations $-1 \mathrm{C}$ & 30 & 3 & 0 & 0 & 0. & 31 & 2 & 0 & 34 \\
\hline Inorganics Wet Chemistry & 26177 & 3 & 0 & 0 & 0 & 24184 & 1995 & 1 & 26180 \\
\hline Other Inorganics & 120 & 3 & 0 & 0 & O) & 120 & 2 & 1 & 123 \\
\hline TOTAL INORGANICS & 47401 & 589 & 611 & (0) & 0] & 38407 & 8586 & $19]$ & 47531 \\
\hline \multicolumn{10}{|l|}{20} \\
\hline $\begin{array}{l}\text { Gross Alpha/Beta } \\
\text { Gamma Scan }\end{array}$ & $\frac{13617}{6998}$ & $\frac{608}{203}$ & $\frac{0}{0}$ & 0 & 0 & 7110 & 6907 & $\frac{19}{10}$ & $\frac{14131}{7300}$ \\
\hline Transuranic & 0 & $\frac{203}{6}$ & $\frac{0}{0}$ & \begin{tabular}{l|l}
0 \\
0
\end{tabular} & $\frac{0}{0}$ & $\frac{2888}{0}$ & $\begin{array}{r}4302 \\
6\end{array}$ & $\frac{10}{0}$ & $\frac{7200}{6}$ \\
\hline Plutonium Isotopes & 8331 & 6 & 0 & 0 & 0 & 4174 & $\begin{array}{r}0 \\
4155\end{array}$ & 8 & 8337 \\
\hline Uranium Isotopes & 6585 & 6 & 0 & 0 & o) & 3406 & 3177 & 8 & 6592 \\
\hline Curium Isotopes & 0 & 0 & 0 & 0 & 0. & 0 & 0 & o. & 의 \\
\hline Thorium Isotopes & of & O & 0 & 11 & 0. & 0 & 0 & 11 & 1 \\
\hline Radium Isotopes & 3546 & 0 & 0 & 0 & 0 & 2824 & 722 & 0 & 3546 \\
\hline Americium-241 & 7669 & 0 & 0 & 0 & 0 & 4045 & 3615 & 8 & 7669 \\
\hline Neptunium - 237 & 0 & 0 & 0 & 0 & 0 & 0 & 0 & 0 & 의 \\
\hline Polonium-210 & 0. & 4 & 0 & 0 & 0 & 4 & 0 & 0 & 4 \\
\hline Tritium & 4774 & 11 & 0 & 0 & O & 3408 & 1367 & 0 & 4775 \\
\hline Radon-222 & 0 & 0 & 01 & 0 & 인 & 0 & 0 & 0 & 의 \\
\hline Lead-210 & 0 & 0 & 0 & 0 & 0 & o & 0 & 0 & 의 \\
\hline Carbon-14 & 0 & 0 & 0 & 0 & 0 & 0 & 0 & 0 & 윽 \\
\hline Nickel-63 & of & 0 & 0 & 0 & O & O & 0 & 0 & 의 \\
\hline Selenium-79 & 0) & 0 & 0 & 0 & 0 & o) & D) & 0 & ㅇ. \\
\hline Technetium-99 & 111 & 0 & 0 & 0 & 요 & 111 & 0 & 0 & 111 \\
\hline Strontium Isotopes & 0 & 0 & 0 & 0 & 0 & 0. & O & 0 & 요 \\
\hline Strontium-89 & 0 & 0 & 0 & 0 & 0. & 0 & 0 & 0 & 의 \\
\hline Strontium-90 & 111 & 0 & 0 & 0 & 0. & 111 & 0 & 0 & 111 \\
\hline lodine-129 & 316 & 0 & 0 & 0 & 0 & 316 & 0 & 0 & 316 \\
\hline Nickel-59 & 0 & 0 & 0 & 0 & o. & 0 & 0 & 욤 & 0 \\
\hline Total Uranium & 111 & 0 & 0 & 0 & 요 & 111 & 0 & 0 & 111 \\
\hline Total Thorium & 0 & 0 & 0 & o! & 0 & 0 & 0 & 0 & 0 \\
\hline Total Strontium & 4331 & 0 & 0 & 0 & 0 & 2587 & 1736 & 8 & 4331 \\
\hline Other RAD & 9663 & 54 & 20 & 0 & 0. & 5377 & 4357 & 2 & 9737 \\
\hline TOTALRAD & 66160 & 890 & 22 & $1]$ & $0]$ & 36472 & 30346 & 65 & 66978 \\
\hline & & & & & & & & & \\
\hline Other Wet Chemistry & 12190 & 01 & 0 & 이 & 요 [ & 10777 & 1413 & 0] & 12190 \\
\hline TCLP Extraction & 2007 & 56 & 22 & 0 & 0 & 1642 & 221 & 1 & 1974 \\
\hline Asbestos & 68 & 0 & 0 & 0 & 0 & 3 & 65 & 0. & 68 \\
\hline Industrial Hygiene, NOS & 35 & 0 & of & 의 & 0 & 24 & 10 & D) & 35 \\
\hline Physical Properties & 2206 & 0 & 0 & of & 0 & 488 & 1719 & 0. & 2206 \\
\hline Other, Not Otherwise Specified (NO & 01 & 25 & 01 & of & 0 & 0 & 01 & 0 & 13 \\
\hline TOTAL OTHER & 16505 & 81 & $22 !$ & OI & On & 12934 & 3427 & 1[ & 16485 \\
\hline & & & & & & & & & \\
\hline TOTAL SAMPLE ANALYSES & 142244 & 1810 & 126 & 11 & $0]$ & 96133 & 46095 & 85 & 143247 \\
\hline
\end{tabular}


Table Vb: FY 94 Samples and Sample Analyses by Rad Level and Matrix Type (v) Fernald

\begin{tabular}{|c|c|c|c|c|c|c|c|c|c|}
\hline $\begin{array}{c}\text { Rad-Matrix/ } \\
\text { Sample-Sample Analysis }\end{array}$ & RO & $\overline{\mathrm{R} 1}$ & $\overline{\mathrm{R} 2}$ & $\mathrm{A3}$ & $\overline{\mathrm{R} 4}$ & M1 & $\overline{\mathrm{M} 2}$ & $\overline{\mathrm{M} 3}$ & TOTAL \\
\hline CUSTOMER SAMPLES & 1546 & 11603 & 440 & 1 & 0 & 2679 & 9138 & 1527 & 13467 \\
\hline \multirow{2}{*}{\multicolumn{10}{|c|}{ SAMPLE ANALYSES }} \\
\hline & & & & & & & & & \\
\hline Volatiles by GC/MS & 3803 & 653 & $\overline{44}$ & 0 & 0 & 426 & 266 & 3773 & 4463 \\
\hline Volatiles by GC & 1142 & 6100 & 422 & 0 & 0 & 451 & 6436 & 766 & 7659 \\
\hline Semi-Volatiles & 555 & 1045 & 4 & $\overline{0}$ & 0 & 137 & 1241 & 237 & 1610 \\
\hline Herbicides/Pesticides/PCBs & 4503 & 722 & 0 & 0 & 으 & 524 & 240 & 4473 & 5231 \\
\hline Other Organics & 356 & 364 & 0 & 0 & 0 & 52 & 365 & 197 & 667 \\
\hline TOTAL ORGANICS & 10360 & 8884 & 430 & 0 & 0 & 1591 & 8548 & 9447 & 19630 \\
\hline \multicolumn{10}{|l|}{\begin{tabular}{|l|} 
RCRA Appendix IX Metals \\
\end{tabular}} \\
\hline RCRA Appendix IX Metals & 8 & 8 & 0 & 0 & 0 & 8 & 8 & 이 & 17 \\
\hline CAM (California) Metals (RCRA) & 44 & 46 & 0 & 0 & 0 & 89 & 57 & 11 & 124 \\
\hline RCRA TCLP Metals and EPTOX Me & 763 & 1239 & 422 & 0 & 0 & 49 & 1754 & 552 & 2389 \\
\hline \begin{tabular}{|l|l|} 
EPA CLP Metals (CERCLA) \\
\end{tabular} & 492 & 589 & 12 & 0 & 0 & 407 & 239 & 448 & 1093 \\
\hline NPDES Metals (Clean Water Act) & 48 & 230 & 0 & 0 & 0 & 277 & 0 & 0 & 277 \\
\hline Drinking Water Metals (SDWA) & 1 & 0 & 0 & 0 & 0 & 1 & 0 & 에 & 1 \\
\hline Metals by Other Regulatory Suite & 9136 & 33 & 0 & 0 & 0 & 389 & 92 & 8687 & 9168 \\
\hline Metals by Suite, Other & 0 & 400 & 2 & 0 & 0 & 83 & 22 & 186 & 346 \\
\hline Metals by Metal Except Mercury & 439 & 1760 & 0 & 0 & 0 & 376 & 1812 & 11 & 2199 \\
\hline Mercury & 453 & 1991 & 0 & 0 & 0 & 554 & 1585 & 199 & 2391 \\
\hline Anions/Cations-IC & 1110 & 255 & 0 & 0 & 0 & 285 & 10 & 1069 & 1365 \\
\hline Inorganics Wet Chemistry & 18935 & 4509 & 3796 & 0 & 0 & 2490 & 7201 & 17697 & 27314 \\
\hline Other Inorganics & 1623 & 2224 & 0 & 0 & 0 & 1710 & 848 & 1288 & 3847 \\
\hline TOTALINORGANICS & 33053 & 13283 & 4232 & $\overline{0}$ & 0 & 6719 & 13628 & 30148 & 50531 \\
\hline \\
\hline Gross Alpha/Beta & 2856 & 5120 & 425 & 0 & 0 & 2144 & 2421 & 1899 & 7433 \\
\hline Gamma Scan & 1901 & 1344 & 2 & 0 & 요 & 268 & 1091 & 1888 & 3247 \\
\hline Transuranic & 0 & 0 & 0 & 0 & 0 & 0 & 0 & 0 & 0 \\
\hline Plutonium Isotopes & 1664 & 234 & 1 & 0 & 요 & 283 & 492 & 1125 & 1899 \\
\hline Uranium Isotopes & 1772 & 898 & 1 & 0 & 0 & 390 & 831 & 1450 & 2671 \\
\hline Curium Isotopes & 0 & 428 & 0 & 0 & O & 132 & 71 & 226 & 428 \\
\hline Thorium Isotopes & 1105 & 79 & 1 & 1 & 0 & 5 & 75 & 1106 & 1186 \\
\hline Radium Isotopes & 37 & 205 & 0 & 0 & 0 & 30 & 9 & 202 & 241 \\
\hline Americium-241 & 548 & 201 & 1 & 0 & 0 & 271 & 449 & 31 & 750 \\
\hline Neptunium-237 & 1105 & 279 & 0 & 0 & 0 & 6 & 77 & 1302 & 1385 \\
\hline Polonium-210 & 52 & 205 & 0 & 0 & 0 & 0 & 52 & 205 & 257 \\
\hline Tritium & 138 & 2049 & 428 & 0 & 0 & 94 & 638 & 4 & 1675 \\
\hline Radon-222 & 19 & 0 & 0 & 0 & 0 & 19 & 0 & 0 & 19 \\
\hline Lead-210 & 0 & 0 & 0 & 0 & 0 & 0 & 0 & 요 & 0 \\
\hline Carbon-14 & 0 & ol & 0 & 0 & 0 & 0 & 0 & o & 0 \\
\hline Nickel-63 & 0 & 0 & 0 & 0 & 0 & 0 & 0 & 0 & 0 \\
\hline Selenium-79 & 44 & 11 & 0 & 0 & 우 & 50 & 3 & 2 & 55 \\
\hline Technetium-99 & 0 & 200 & 0 & 0 & 0 & 3 & 1 & 196 & 200 \\
\hline Strontium Isotopes & a & 23 & 0 & 0 & 0 & 0 & 11 & 13 & 23 \\
\hline Strontium -89 & 0 & 0 & 0 & 0 & 0 & 0 & 0 & 0 & 0 \\
\hline Strontium-90 & 44 & 243 & 422 & 0 & 0 & 75 & 626 & 7 & 709 \\
\hline lodine-129 & 44 & 438 & 0 & 0 & 0 & 473 & 6 & 4 & 482 \\
\hline Nickel-59 & 0 & 0 & 0 & 0 & 0 & 0 & 0 & 0. & 0 \\
\hline Total Uranium & 4389 & 433 & 0 & 0 & - & 234 & 111 & 4478 & 4822 \\
\hline Total Thorium & 0 & 131 & 0 & 0 & 0 & 21 & 111 & 0 & 131 \\
\hline Total Strontium & 91 & 0 & 0 & 0 & 0 & 61 & 28 & 2 & 91 \\
\hline Other RAD & 4902 & 1222 & 425 & 0 & 0 & 748 & 818 & 4981 & 6548 \\
\hline TOTALRAD & 20710 & 13744 & 1708 & 1 & 0 & 5306 & 7919 & 19120 & 34253 \\
\hline Other Wet Chemistry & 436 & 211 & 0 & 0 & 0 & 621 & 26 & ( & 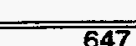 \\
\hline TCLP Extraction & 665 & 1805 & 1 & 0 & 0 & 127 & 1389 & 955 & 2471 \\
\hline Asbestos & 0 & 75 & 0 & 0 & 0 & 0 & 75 & 0 & 75 \\
\hline Industrial Hygiene, NOS & 59 & 6 & 0 & 0 & 0 & 60 & 4 & 1 & 65 \\
\hline Physical Properties & 39 & 1142 & 1 & 0 & 0 & 348 & 117 & 717 & 1182 \\
\hline Other, Not Otherwise Specified (NO & 2244 & 109 & 0 & 0 & 0 & 86 & 7 & 2261 & 2353 \\
\hline TOTAL OTHER & 3443 & 3349 & 2 & 0 & $\overline{0}$ & 1241 & 1618 & 3934 & 6793 \\
\hline & & & & & & & & & \\
\hline TOTAL SAMPLE ANALYSES & 67566 & 39260 & 6372 & 1 & 0 & 14856 & 31713 & 62648 & 111208 \\
\hline
\end{tabular}


Table Vb: FY 94 Samples and Sample Analyses by Rad Level and Matrix Type (vi) INEL

\begin{tabular}{|c|c|c|c|c|c|c|c|c|c|}
\hline $\begin{array}{c}\text { Rad-Matrix/ } \\
\text { Sample-Sample Analysis }\end{array}$ & RO & R1 & $\overline{\mathrm{R} 2}$ & $\overline{\mathrm{R} 3}$ & 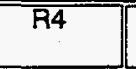 & $\overline{\mathrm{M} 1}$ & M2 & M3 & TOTAL \\
\hline CUSTOMER SAMPLES & 2349 & 9046 & 289 & 3441 & $0]$ & 5451 & 3603 & 1286[ & 11184 \\
\hline & & & & & & & & & \\
\hline \multicolumn{10}{|l|}{ SAMPLE ANALYSES } \\
\hline Volatiles by GC/MS & 506 & 404 & 13 & 33 & 0 & 307 & 528 & 120 & 956 \\
\hline Volatiles by GC & 718 & 293 & 31 & 9 & 요 & 542 & 429 & 31 & 1026 \\
\hline Semi-Volatiles & 369 & 409 & 22 & 30 & 0 & 248 & 533 & 47 & 829 \\
\hline Herbicides/Pesticides/PCBs & 480 & 810 & 9 & 4 & 0 & 330 & 750 & 224 & 1303 \\
\hline Other Organics & 812 & 1309 & 0 & 01 & 0 & 297 & 561 & 9[ & 1494 \\
\hline TOTAL ORGANICS & 2884 & 3225 & 741 & 76 & (1) & 1725 & 2801 & 430 & 5607 \\
\hline PCRA Annondix IY Motals & & & & & & & & & \\
\hline MURA AppenalX IX Metals & 3 & 7 & 0 & 이 & ㅇ.: & 5 & 5 & 0 & 10 \\
\hline CAM (California) Metais (RCRA) & 12 & 1 & 0 & 0 & 0. & 13 & 1 & 0 & 13 \\
\hline RCRA TCLP Metals and EPTOX Me & 596 & 356 & 16 & 21 & 0 & 501 & 377 & 36) & 941 \\
\hline EPA CLP Metals (CERCLA) & 506 & 253 & 54 & 78 & 0 & 275 & 523 & 93 & 890 \\
\hline NPDES Metals (Clean Water Act) & 112 & 225 & 0 & 0 & 0 & 338 & 0 & 요 & 338 \\
\hline Drinking Water Metals (SDWA) & 131 & 0 & 0 & 0 & 0 & 131 & D. & 요 & 131 \\
\hline Metals by Other Regulatory Suite & 652 & 156 & 0 & 0 & 0 & 287 & 326 & 212 & 817 \\
\hline Metals by Suite, Other & 0 & 1440 & 7 & 0 & 0 & 104 & 56 & 4 & 806 \\
\hline Metals by Metal Except Mercury & 664 & 409 & 5 & 0 & 0 & 531 & 321 & 170 & 1050 \\
\hline Mercury & 763 & 1659 & $7 \mid$ & 1 & 요 & 661 & 446 & 15 & 1776 \\
\hline Anions/Cations - IC & 212 & 133 & 0] & of & 0 & 274 & 38 & 34 & 345 \\
\hline Inorganics Wet Chemistry & 1589 & 853 & 110 & 18 & 요 & 1379 & 721 & 471 & 2570 \\
\hline Other inorganics & 247 & 183 & 0 & 01 & 이 & 280 & 106 & 44 & 430 \\
\hline TOTALINORGANICS & 5487 & 5675 & 198 & 98 & 0 & 4778 & 2919 & 1079 & 10117 \\
\hline \multicolumn{10}{|l|}{ 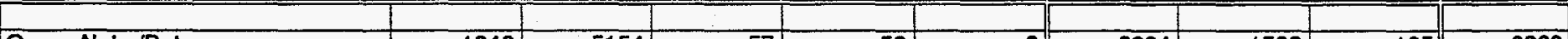 } \\
\hline Gross Alpha/Beta & 1842 & 5154 & 57 & 53 & ㅇ․ & 3884 & 1530 & 135 & 6328 \\
\hline Gamma Scan & 1007 & 1639 & 44 & 21 & 요 & 1529 & 1107 & 75 & 2711 \\
\hline Transuranic & of & 8 & 0 & 0 & 0 & 0 & 8 & 0 & 8 \\
\hline Plutonium isotopes & 685 & 158 & 15 & 4 & 요 & 412 & 409 & 41. & 862 \\
\hline Uranium Isotopes & 634 & 454 & 7 & 4 & 0. & 429 & 613 & 57 & 1099 \\
\hline Curium Isotopes & 0 & 20 & 3 & 1 & 요 & 6 & 12 & 6 & 23 \\
\hline Thorium Isotopes & 22 & 185 & 6 & 8 & o & 13 & 178 & 29 & 221. \\
\hline Radium Isotopes & 114 & 4 & 이 & 0 & 요 & 88 & 27 & 4 & 119 \\
\hline Americium-241 & 556 & 80 & 8 & 2 & 의 & 373 & 257 & 16 & 646 \\
\hline Neptunium - 237 & 22 & 49 & 2 & 0 & 요 & 8) & 36 & 30 & 73 \\
\hline Polonium-210 & 1 & 5 & 0 & 0 & 요 & 1 & 1 & 4 & 6 \\
\hline Tritium & 493 & 215 & 37 & 4 & 의 & 404 & 299 & 10 & 731 \\
\hline Radon-222 & 으 & 0 & o & 0 & 의 & o. & o. & 의 & 의 \\
\hline Lead-210 & 0 & 3 & 0 & 0 & 0 & 0 & 0 & 3 & 3 \\
\hline Carbon-14 & 0 & 0 & 0 & 0 & 0 & 0 & 0 & 요 & 0. \\
\hline Nickel-63 & 0 & 인 & 이 & 0 & 0 & 0 & 0 & 0 & 0 \\
\hline Selenium-79 & 12 & 18 & of & 0 & 0 & 22 & 4 & 3 & 30 \\
\hline Technetium-99 & 28 & 153 & 1 & 0 & 0 & 29 & 147 & 6 . & 182 \\
\hline Strontium Isotopes & 0 & 0 & 0 & 0 & 0 & 0 & 0 & o. & 0 \\
\hline Strontium - 89 & 0 & 0 & 0 & 0 & o. & 0 & 0 & 0] & 0 \\
\hline Strontium-90 & 40 & 174 & 22 & 4 & 0 & 90 & 139 & 11. & 240 \\
\hline lodine-129 & 92 & 34 & 요 & 0 & 0 & 111 & 9 & 6 & 125 \\
\hline Nickel-59 & 0 & 0 & 0 & 0) & o. & 0 & O & ㅇ. & 0 \\
\hline Total Uranium & 121 & 109 & 0 & 0 & O & 38 & 19 & 172 & 230 \\
\hline Total Thorium & 인 & 0 & 0 & 0 & 0. & 0 & 0 & 욤 & 0 \\
\hline Total Strontium & 169 & 3 & 이 & 0 & 요 & 73 & 93. & 7 & 173 \\
\hline Other RAD & 781 & 787 & 33 & 235 & o] & 420 & 1050 & 357 & 1832 \\
\hline TOTAL RAD & 6619 & 9252 & 234 & 338 & Q] & 7929 & 5939 & 973 & 15643 \\
\hline & & & & & & & $m$ & & \\
\hline Other Wet Chemistry & 795 & 6 & 0 & 0 & 요 & 728 & 72 & 요 & 801 \\
\hline TCLP Extraction & 576 & 467 & 20 & 186 & 0 & 547 & 396 & 250 & 1221 \\
\hline Asbestos & 0. & 1929 & 0 & 0 & 0. & 0 & 1435 & 495 & 1930 \\
\hline Industrial Hygiene, NOS & 160 & 10 & 0 & of & 요 & 157 & 13 & 1 . & 170 \\
\hline Physical Properties & 176 & 91 & 7 & 117 & 요 & 155 & 107 & 129 & 391 \\
\hline Other, Not Otherwise Specified (NO & 44 & 16 & 0 & 0 & 0] & 91 & 0 & 45] & 57 \\
\hline TOTAL OTHER & 1751 & 2519 & 27 & 303 & 0] & 1597 & 2022 & 920 & 4570 \\
\hline TOTAL SAMPLE ANALYSES & 16742 & 20671 & 534 & 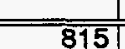 & & 16028 & & & \\
\hline
\end{tabular}


EM-263 ASSESSMENT OF EM SAMPLING AND ANALYSIS NEEDS

Table Vb: FY 94 Samples and Sample Analyses by Rad Level and Matrix Type (vii) NTS

\begin{tabular}{|c|c|c|c|c|c|c|c|c|c|}
\hline $\begin{array}{c}\text { Rad-Matrix/ } \\
\text { Sample-Sample Analysis }\end{array}$ & RO & 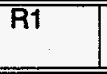 & $\mathrm{R2}$ & R3 & R4 & $\overline{\mathrm{M} 1}$ & M2 & M3 & TOTAL \\
\hline CUSTOMER SAMPLES & 996 & 2170 & 172 & 0 & $7]$ & 567 & 1235 & 293 & 2719 \\
\hline \multirow{2}{*}{\multicolumn{10}{|c|}{ SAMPLEANALYSES }} \\
\hline & & & & & & & & & \\
\hline Volatiles by GC/MS & 1464 & 407 & 44 & 0 & 0 & 833 & 981 & 103 & 1916 \\
\hline Volatiles by GC & 275 & 17 & 0 & 0 & 0 . & 8 & 219 & 65 & 292 \\
\hline Semi-Volatiles & 717 & 364 & 38 & o & 0] & 378 & 664 & 81 & 1121 \\
\hline Herbicides/Pesticides/PCBs & 766 & 443 & 0 & 0 & 0 & 271 & 925 & 19 & 1212 \\
\hline Other Organics & 19 & $134 \mid$ & 0 & 0 & $0]$ & 72 & 73 & 8. & 153 \\
\hline TOTAL ORGANICS & 3241 & 1364 & 82 & 0 & $0]$ & 1562 & 2862 & 276 & 4694 \\
\hline & & & & & & & & & \\
\hline RCRA Appendix IX Metals & 7 & 13 & 0 & 0 & 0 & 2 & 18 & 0. & 20 \\
\hline CAM (California) Metals (RCRA) & a) & 0 & 0 & D & 0 . & 0 & 0 & 요 & 0 \\
\hline RCRA TCLP Metals and EPTOX Me & 20 & 66 & 0 & 0 & 0. & 9 & 69 & 8 & 86 \\
\hline EPA CLP Metals (CERCLA) & 1610 & 586 & 112 & o & 0 & 715 & 1549 & 44 & 2308 \\
\hline NPDES Metals (Clean Water Act) & 253 & 0 & 0 & 0 & 0 & 253 & 0 & 요 & 253 \\
\hline Drinking Water Metals (SDWA) & 2 & 0 & 0 & 0 & 0 & 2 & 0 & 0. & 2 \\
\hline Metals by Other Regulatory Suite & 1308 & 0 & 0 & 0 & 0. & 559 & 778 & 23 & 1334 \\
\hline Metals by Suite, Other & 0 & 482 & 19 & 0 & 0. & 290 & 160 & 이 & 476 \\
\hline Metals by Metal Except Mercury & 36 & 164 & 0 & 0 & 0 & 25 & 174 & 애 & 199 \\
\hline Mercury & 261 & 151 & 0 & 0 & o & 261 & 151 & 요 & 412 \\
\hline Anions/Cations-IC & 378 & 190 & 0 & 0 & 0. & 553 & 15 & 이 & 569 \\
\hline Inorganics Wet Chemistry & 2969 & 328 & 0 & 0 & 0 & 2604 & 693 & ㅇ. & 3297 \\
\hline Other Inorganics & 325 & 99 & of & 0 & 0] & 307 & 118 & 0. & 425 \\
\hline \multirow[t]{2}{*}{ TOTAL INORGANICS } & 7170 & 2080 & 132 & 01 & OD & 5579 & 3725 & 75 & 9380 \\
\hline & & & & & & & & & \\
\hline Gross Alpha/Beta & 2957 & 707 & 28 & 0 & o: & 1429 & 2209 & 57 & 3694 \\
\hline Gamma Scan & 1064 & 279 & 16 & 0 & o. & 549 & 747 & 63 & 1359 \\
\hline Transuranic & 0 & 0 & 0 & 0 & ㅁ․ & 0 & 0 & 의 & 0 \\
\hline Plutonium Isotopes & 1315 & 160 & 11 & 0 & 0 & 506 & 966 & 14 & 1486 \\
\hline Uranilum Isotopes & 1406 & 552 & 11 & 0 & - & 622 & 1333 & 14 & 1969 \\
\hline Curium Isotopes & 0 & 18 & 0 & 0 & - & 9 & 9 & ㅇ. & 18 \\
\hline Thorium Isotopes & 0) & 145 & 11 & 0 & 0 & 29 & 127 & 요 & 156 \\
\hline Radium Isotopes & 510 & 0 & 0 & 0 & 0 & 378 & 132 & 으 & 510 \\
\hline Americium-241 & 1291 & 61 & 11 & 0 & 0 & 504 & 844 & 14 & 1363 \\
\hline Neptunium-237 & 0 & 116 & 0 & 0 & - & 8 & 108 & 0 & 116 \\
\hline Polonium-210 & 0 & 0 & 0 & 0 & 0 & 0 & 0 & 0 & 0 \\
\hline Tritium & 785 & 385 & 50 & 0 & o. & 586 & 624 & 9 & 1220 \\
\hline Radon-222 & 0 & 0 & 0 & 0 & 0. & 0 & 0 & ㅇ. & 0 \\
\hline Lead-210 & 0 & 0 & 0 & 0 & 0 & 0 & 0 & 0 & 0 \\
\hline Carbon-14 & 0 & 0 & 0 & 0 & 요 & 0 & 0 & ㅇ. & 0 \\
\hline Nickel-63 & 0 & 0 & 0 & 0 & ㅇ. & 0 & 0 & 0 & 0 \\
\hline Selenium-79 & 0 & 0 & 0 & 0 & 0] & 0 & 0 & 요 & 0 \\
\hline Technetium -99 & 0 & 13 & 3 & 0 & 0. & 4 & 13 & 인 & 16 \\
\hline Strontium Isotopes & 0 & 0 & 0 & 0 & 요 & 0 & 0 & ㅇ. & 0 \\
\hline Strontium -89 & 0 & 0 & 0 & 0 & o. & 0 & 0 & 0 & 0 \\
\hline Strontium-90 & 0 & 163 & 3 & 0 & 0 & 17 & 150 & 0 & 167 \\
\hline lodine-129 & 1 & 01 & 0 & 0 & (0) & 1 & 0 & 0 & 1 \\
\hline Nickel-59 & 0 & 0 & 0 & 0 & 0 & 0 & 0 & 0 & 0 \\
\hline Total Uranium & 17 & 99 & 0 & 0 & 0 & 17 & 99 & 의 & 116 \\
\hline Total Thorium & 0 & 74 & 0 & 0 & 0 & 0 & 74 & 0 & 74 \\
\hline Total Strontium & 706 & 0 & 0 & 0 & 의 & 336 & 355 & 14 & 706 \\
\hline Other RAD & 1753 & 288 & 31 & 0 & 0 & 451 & 1622 & 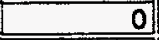 & 2073 \\
\hline TOTAL RAD & 11807 & 3061 & 175 & 01 & [0] & 5448 & 9412 & 187 & 15045 \\
\hline 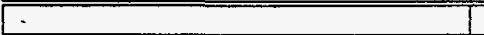 & & & & & & 7 & & & \\
\hline Other Wet Chemistry & 1711 & 0 & 0. & 0 & ㅁ] & 1398 & 314 & 0 & 1711 \\
\hline TCLP Extraction & 13 & 72 & 1 & 0 & 0 & 19 & 65 & 0 & 85 \\
\hline Asbestos & 25 & 106 & 0 & 0 & 0 & 0 & 130 & 0 & 130 \\
\hline Industrial Hygiene, NOS & 22 & 0 & 0 & 0 & 0 & 3 & 19 & o. & 22 \\
\hline Physical Properties & 249 & 98 & 6 & 0 & (0) & 62 & 291 & 0 & 353 \\
\hline Other, Not Otherwise Specified (Nd & 6 & 60 & 0 & 0 & 0 & 61 & 5 & 0 & 65 \\
\hline TOTALOTHER & 2025 & 335 & 7 & 01 & 0 & 1542 & 824 & 0 & 2367 \\
\hline & & & & $\frac{1}{01}$ & & & & & \\
\hline TOTAL SAMPLE ANALYSES & 24243 & 6840 & 396 & 이 & 0] & 14132 & 16823 & 538] & 31486 \\
\hline
\end{tabular}


Table Vb: FY 94 Samples and Sample Analyses by Rad Level and Matrix Type (viii) Oak Ridge K-25

\begin{tabular}{|c|c|c|c|c|c|c|c|c|c|}
\hline $\begin{array}{c}\text { Rad-Matrix/ } \\
\text { Sample-Sample Analysis }\end{array}$ & $\overline{\mathrm{RO}}$ & $\overline{\mathrm{R} 1}$ & $\overline{R 2}$ & R3 & R4 & M1 & M2 & $\overline{\mathrm{M3}}$ & TOTAL \\
\hline CUSTOMER SAMPLES & 309 & $22971]$ & 4948 & 01 & 11 & 5645 & 8346 & 14767 & 28493 \\
\hline & & & & & & & & & \\
\hline \multicolumn{10}{|l|}{ SAMPLE ANALYSES } \\
\hline Volatiles by $\mathrm{GC} / \mathrm{MS}$ & 179 & 12404 & 3015 & DI & 0] & 219 & 5472 & 9909 & 15599 \\
\hline Volatiles by GC & 111 & 15 & 1 & 0 & 0 & 60 & 52 & 8 & 123 \\
\hline Semi-Volatiles & 106 & 8312 & 2677 & 0 & 0 & 66 & 5332 & 5697 & 11095 \\
\hline Herbicides/Pesticides/PCBs & 109 & 19987 & 0 & o & 0 & 14743 & 1727 & 3627 & 20097 \\
\hline Other Organics & 54 & 21 & 337 & 01 & 0 & 43 & 28 & 339 & 411 \\
\hline TOTAL ORGANICS & 559 & 40739 & 6031 & 01 & 미 & 15131 & 12610 & 19580 & 47325 \\
\hline \multicolumn{10}{|l|}{ 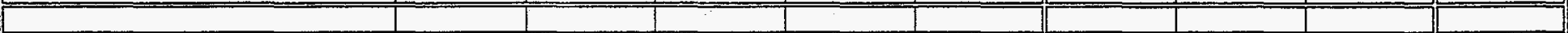 } \\
\hline RCRA Appendix IX Metals & $1)$ & 2 & 이 & 0 & 요 & 0 & 3 & 731 & 369 \\
\hline CAM (California) Metals (RCRA) & 0 & D. & 요 & 0 & ㅇ.. & 0 & o. & 0. & ㅇ. \\
\hline RCRA TCLP Metals and EPTOX Me & 110 & 5722 & 5540 & 0 & 0 & 56 & 675 & 11365 & 11734 \\
\hline EPA CLP Metals (CERCLA) & 184 & 9570 & 18 & 0 & 의 & 80 & 209 & 9482 & 9771 \\
\hline NPDES Metals (Clean Water Act) & 41 & 1 & 0 & 0 & 0 & 41 & 0 & 0 & 41 \\
\hline Drinking Water Metals (SDWA) & 16 & 0 & 0 & 0 & 0 & 16 & 0 & ㅇ. & 16 \\
\hline Metals by Other Regulatory Suite & 154 & 11940 & 2530 & D. & 0 & 9066 & 2867 & 2739 & 14649 \\
\hline Metals by Suite, Other & 0 & 129 & 3. & 0 & of & 53 & 26 & 2 & 107 \\
\hline Metals by Metal Except Mercury & 117 & 26510 & 2531 & 0 & 0 & 11643 & 378 & 3072 & 22126 \\
\hline Mercuny & 150 & 13375 & 338 & 0 & o. & 20696 & 3457 & 3758 & 20887 \\
\hline Anions/Cations-IC & 63 & 13323 & 1181 & 0 & 0 & 90 & 10 & 2798 & 8732 \\
\hline Inorganics Wet Chemistry & 291 & 3538 & 342 & 0 & 0 & 2761 & 2288 & 11521 & 10371 \\
\hline Other Inorganics & 57 & 2512 & 01 & 0 & 0 & 56 & 65 & 2448 & 2569 \\
\hline TOTALINORGANICS & 1184 & 86622 & 12483 & 01 & 요 & 44560 & 9978 & 47917 & 101372 \\
\hline \multicolumn{10}{|l|}{ Groce AloholRata } \\
\hline \begin{tabular}{|l} 
Gross Alpha/Beta \\
Gamma Scan
\end{tabular} & $\frac{393}{207}$ & $\frac{26638}{3604}$ & $\frac{342}{340}$ & 0 & 에 & $\begin{array}{r}23144 \\
80\end{array}$ & $\frac{700}{542}$ & $\frac{3530}{3528}$ & $\frac{27373}{4150}$ \\
\hline Transuranic & 0 & 9867 & 10122 & 0 & a. & 13495 & 6493 & 0 & 19989 \\
\hline Plutonium Isotopes & 147 & 3209 & 339 & 0 & 0 & 53 & 122 & 3521 & 3696 \\
\hline Uranium Isotopes & 163 & 17782 & 8211 & 0 & 0 & 16787 & 2856 & 6513 & 26156 \\
\hline Curium Isotopes & 0 & 3 & O) & 0 & 매 & 2 & 2 & 0 & 3 \\
\hline Thorium Isotopes & 1 & 12049 & 339 & 0 & 0 & 8284 & 587 & 3518 & 12389 \\
\hline Radium Isotopes & 43 & 0 & 0 & 0 & 의 & 31 & 12 & 0 & 43 \\
\hline Americium-241 & 132 & 2471 & 2 & 0 & 0 & 62 & 94 & 2449 & 2604 \\
\hline Neptunium-237 & 1 & 7434 & 6804 & 0 & 은 & 7030 & 3691 & 3518 & 14240 \\
\hline Polonium-210 & 0 & 0. & 337 & 0 & 0 & 0 & 0 & 338 & 338 \\
\hline Tritium & 97 & 66 & 9 & 0 & 은 & 72 & 96 & 2 & 170 \\
\hline Radon-222 & 0 & 0 & 0 & 0 & 요 & 0 & 0 & 0 & 0 \\
\hline Lead-210 & 0 & 0 & 0 & 0 & 0 & 0 & 0 & 0. & 요 \\
\hline Carbon-14 & 0 & 0 & 0 & 0 & a & 0 & 0 & 요 & - \\
\hline Nickel-63 & 0 & O) & 0 & 0 & 요 & 0 & 0 & ㅇ. & 은 \\
\hline Selenium-79 & 0 & 1 & 0 & 0 & 0 & 0 & 0 & 0 & 1 \\
\hline Technetium-99 & 3 & 13588 & 10459 & 0 & 0. & 13499 & 7037 & 3515 & 24051 \\
\hline Strontium Isotopes & 0 & 0 & 0 & 0 & 의 & 0 & 0 & 0. & 요 \\
\hline Strontium-89 & 0 & 0 & 0 & 0 & 므 & 0 & 0 & 요 & 0 \\
\hline Strontium-90 & 3 & 32 & 1 & 0 & 0 & 8 & 28 & 0 & 36 \\
\hline lodine-129 & 10 & 1 & 0 & 0 & 0 & 11 & 0 & 의 & 11 \\
\hline Nickel-59 & 0 & 이 & 0 & 0 & ㅇ․ & 0 & 0 & 의 & 0 \\
\hline Total Uranium & 11 & 7936 & 9897 & D) & 0. & 3380 & 11339 & 3124 & 17844 \\
\hline Total Thorium & 0 & 13 & 0 & 0 & 0] & 0 & 13 & 0 & 13 \\
\hline Total Strontium & 65 & 0 & 이 & 0 & 0] & 25 & 38 & 2 & 65 \\
\hline Other RAD & 214 & 12564 & 344 & ol & 0 : & 8318 & 1992 & 2812 & 13122 \\
\hline TOTALRAD & 1491 & 117259 & 47546 & 0 & 0 & 94281 & 35642 & 36370 & 166294 \\
\hline & & & & & & & & & \\
\hline Other Wet Chemistry & 155 & 0 & 이 & 0 & 0] & 122 & 33 & 요 & 155 \\
\hline TCLP Extraction & 109 & 12362 & 2953 & 0 & 0 & 60 & 2389 & 12968 & 15420 \\
\hline Asbestos & 2 & 7627 & 0 & 0 & 0 & 0 & 5651 & 1978 & 7629 \\
\hline Industrial Hygiene, NOS & 4 & 0 & 0 & 0 & - & 1 & 3 & 0 & 5 \\
\hline Physical Properties & 32 & 11579 & 1 & 0 & o. & 8289 & 230 & 3094 & 11612 \\
\hline Other, Not Otherwise Specified (NO & 4 & 11 & 337 & 0 & 0 & 10 & 11 & 340 & 352 \\
\hline TOTAL OTHEA & 306 & 31579 & 3291 & 0! & 0 & 8482 & 8307 & 18379 & 35172 \\
\hline & & & & & & & & & \\
\hline TOTAL SAMPLE ANALYSES & 3540 & 276198 & 69350 & 0 & 0 & 162454 & 66538 & 122246 & 350163 \\
\hline
\end{tabular}




\section{EM-263 ASSESSMENT OF EM SAMPLING AND ANALYSIS NEEDS}

Table Vb: FY 94 Samples and Sample Analyses by Rad Level and Matrix Type (ix) Oak Ridge X-10

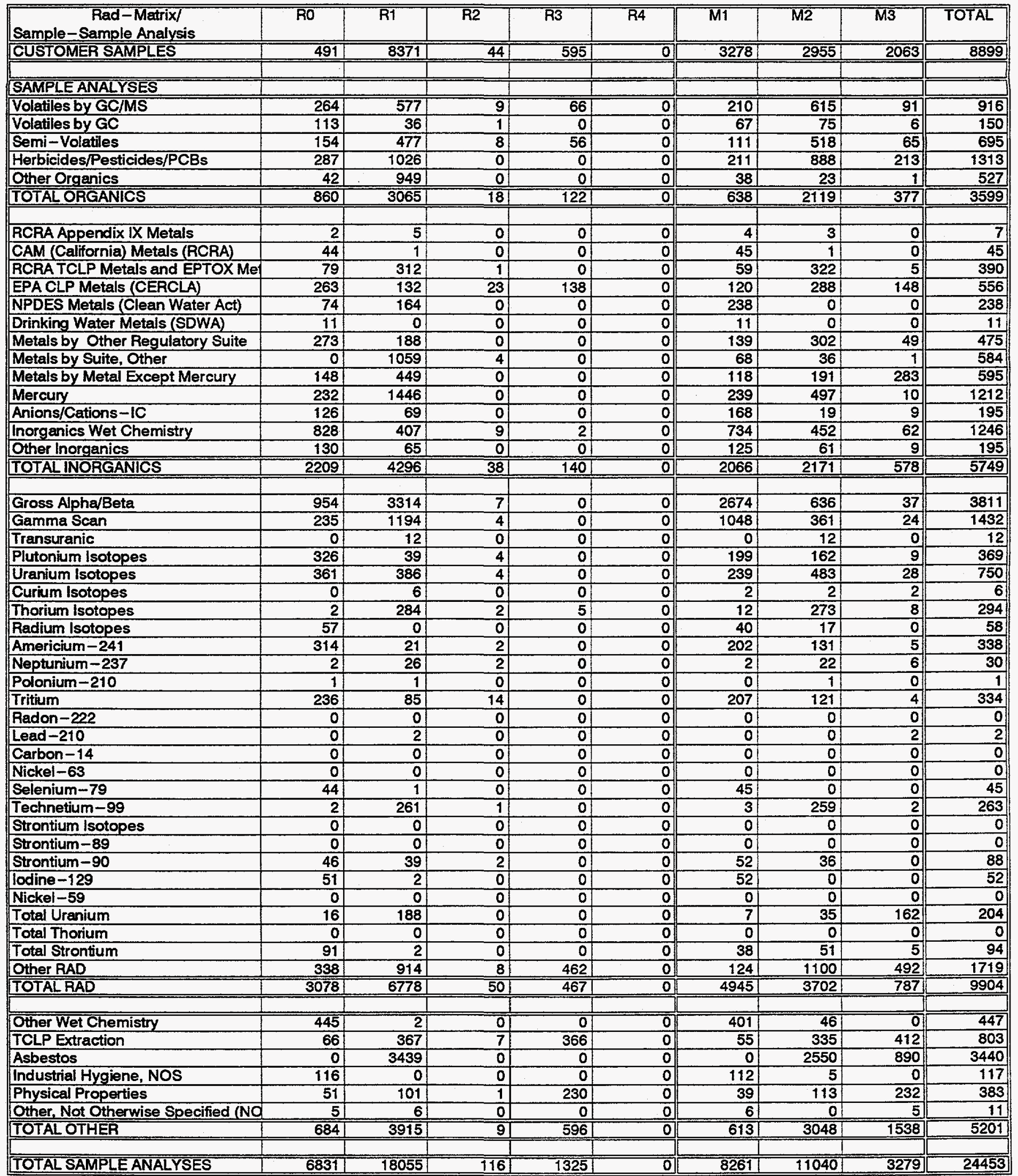


Table Vb: FY 94 Samples and Sample Analyses by Rad Level and Matrix Type (x) Oak Ridge Y-12

\begin{tabular}{|c|c|c|c|c|c|c|c|c|c|}
\hline $\begin{array}{c}\text { Rad-Matrix/ } \\
\text { Sample-Sample Analysis }\end{array}$ & Ro & $\overline{\mathrm{R1}}$ & R2 & R3 & R4 & $\overline{\mathrm{MI}}$ & $\overline{\mathrm{M} 2}$ & $\overline{\bar{M} 3}$ & TOTAL \\
\hline CUSTOMER SAMPLES & 142 & 1025 & 18 & 0 & 0 & 113 & 664 & 282[ & 1123 \\
\hline \multirow{2}{*}{\multicolumn{10}{|c|}{ SAMPLE ANALYSES }} \\
\hline & & & & & & & & & \\
\hline Volatiles by GC/MS & 123 & 153 & $4 !$ & 01 & 0 & 701 & 188 & 23 & 280 \\
\hline Volatiles by GC & 29 & 33 & 2 & 0 & o. & 3 & 53 & 6 & 63 \\
\hline Semi-Volatiles & 60 & 130 & 4 & o) & 0 & 35 & 155 & 5 & 194 \\
\hline Herbicides/Pesticides/PCBs & 99 & 236 & 0 & 0 & 0 & 47 & 233 & 58 & 337 \\
\hline Other Organics & 17 & 10 & 0 & 0 & 0 & 7 & 19 & $1]$ & 27 \\
\hline TOTAL ORGANICS & 328 & 562 & 10 & ol & 0 & 161 & 649 & 93[ & 901 \\
\hline \multicolumn{10}{|l|}{0} \\
\hline RCRA Appendix IX Metals & 2 & 1 & 0 & 0 & 0 & 0 & 3 & 0 & 3 \\
\hline CAM (California) Metals (RCRA) & 20 & 0 & 0 & 0 & 0 & 20 & 0 & 0 & 21 \\
\hline RCRA TCLP Metals and EPTOX Me & 9 & 70 & 2 & 0 & 0 & 2 & 75 & 4 & 81 \\
\hline EPA CLP Metals (CERCLA) & 111 & 62 & 11 & 0 & 0 & 45 & 132 & 6 f & 183 \\
\hline NPDES Metals (Clean Water Act) & 26 & 1 & 0 & o & 0 & 27 & 0 & of & 27 \\
\hline Drinking Water Metals (SDWA) & 0 & 0 & 0 & 0 & 0 & 0 & 0 & 0 & 0 \\
\hline Metals by Other Regulatory Suite & 124 & 36 & 0 & 0 & 0 & 31 & 91 & 46 & 164 \\
\hline Metals by Suite, Other & 0 & 54 & 2 & 0 & 0 & 30 & 16 & 1 & 52 \\
\hline Metals by Metal Except Mercury & 30 & 118 & 0| & 0 & 0 & 24 & 71 & 53 & 147 \\
\hline Mercury & 53 & 125 & 0 & 0 & 0 & 51 & 124 & 2 & 177 \\
\hline Anions/Cations-IC & 43 & 23 & 0 & 0 & 0 & 58 & 2 & 6 & 66 \\
\hline Inorganics Wet Chemistry & 298 & 120 & 17 & 0 & 0 & 208 & 144 & 85 & 436 \\
\hline Other Inorganics & 41 & 26 & 0 & 0 & 0 & 40 & 21 & 6 & 67 \\
\hline TOTAL INOAGANICS & 757 & 636 & 32 & o) & 0 & 536 & 679 & 210 & 1424 \\
\hline \multicolumn{10}{|l|}{ 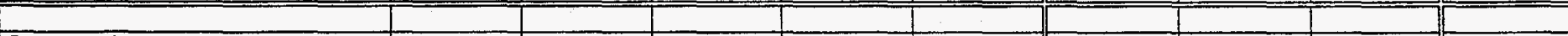 } \\
\hline Gross Alpha/Beta & 224 & 130 & 5 & 0 & 요 & 111 & 227 & 16] & 356 \\
\hline Gamma Scan & 83 & 65 & 2 & 0 & 0 & 39 & 97 & 14 & 150 \\
\hline Transuranic & 0 & 2 & 0 & 0 & o: & 0 & 2 & 0 & 2 \\
\hline Plutonium isotopes & 83 & 20 & 1 & 0 & 0 & 25 & 72 & 7 & 105 \\
\hline Uranium lsotopes & 103 & 128 & 1 & 0 & 0 & 43 & 177 & 12 & 232 \\
\hline Curium Isotopes & 0 & 4 & 0 & 0 & 0 & 1 & 1 & 1 & 4 \\
\hline Thorium Isotopes & 5 & 72 & 1 & 0 & 으. & 4 & 69 & 5 & 78 \\
\hline Radium Isotopes & 25 & 1 & 0 & 0 & - 0 & 17 & 8 & 1 & 26 \\
\hline Americium-241 & 78 & 10 & 1 & 0 & 0 & 26 & 62 & 2 & 89 \\
\hline Neptunium-237 & 5 & 23 & 0 & of & 0 & 1 & 21 & 6) & 28 \\
\hline Polonium-210 & 0 & 1 & 0 & 0 & o & 0 & 0 & 1. & 1 \\
\hline Tritium & 48 & 57 & 7 & 0 & 0 & 37 & 65 & 1 & 107 \\
\hline Radon-222 & 0 & o & 0 & 0 & 0 & 0 & 0 & 0 & 0 \\
\hline Lead-210 & 0 & 0 & 0 & 0 & o & 0 & 0 & 0 & 0 \\
\hline Carbon -14 & 0 & 0 & 0 & 0 & 0 & 0 & 0 & 0 & 0 \\
\hline Nickel-63 & 0 & 0 & 0 & 0 & - & 0 & 0 & 0 & 0 \\
\hline Selenium-79 & 20 & 7 & 0 & 0 & D & 20 & 0 & 0 & 21 \\
\hline Technetium-99 & 0 & 50 & 0 & 0 & - & 0 & 49 & 1 & 50 \\
\hline Strontium Isotopes & 0 & 0 & 0 & 0 & o & 0 & 0 & 0 & 0 \\
\hline Strontium-89 & 0 & 0 & 0 & 0 & 0 & 0 & 0 & 0 & $\overline{0}$ \\
\hline Strontium-90 & 20 & 21 & 2 & 0 & 0 & 23 & 20 & 0 & 44 \\
\hline lodine-129 & 20 & 3 & 0 & 0 & 0 & 22 & 0 & o. & 23 \\
\hline Nickel-59 & 0 & 0 & 0 & 0 & 0 & 0 & 0 & 0 & 0 \\
\hline Total Uranium & 22 & 59 & 0 & 0 & o & 3 & 29 & 48 & 80 \\
\hline Total Thorium & 0 & 22 & 0 & 0 & o. & 0 & 22 & 0 & 22 \\
\hline Total Strontium & 39 & 0 & 0 & 0 & o & 15 & 23 & 1 & 39 \\
\hline Other RAD & 153 & 191 & 5 & 0 & 0 & 37 & 287 & 25 & 349 \\
\hline TOTAL RAD & 929 & 858 & 25 & 01 & 0 & 427 & 1231 & 142 & 1806 \\
\hline & & & & & & & & 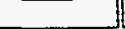 & \\
\hline Other Wet Chemistry & 86 & 1 & 0 & 01 & 0 & 66 & 21 & 에 & 87 \\
\hline TCLP Extraction & 9 & 82 & 0 & 0 & 0 & 5 & 74 & 12 & 91 \\
\hline Asbestos & 0 & 653 & 0 & 0 & 0 & 0 & 488 & 165 & 653 \\
\hline Industrial Hygiene, NOS & 3 & 0 & 0 & 0 & 0 & 1 & 2 & 0 & 3 \\
\hline Physical Properties & 12 & 44 & 1 & 0 & 0 & 7 & 46 & 3 & 56 \\
\hline Other, Not Otherwise Specified (NO & 12 & 14 & 0 & 0 & 0 & 14 & 1 & 10 & 26 \\
\hline TOTAL OTHER & 121 & 794 & 1 & o1 & 0 & 94 & 632 & 191 & 916 \\
\hline & & & & & & & & & \\
\hline TOTAL SAMPLE ANALYSES & 2134 & 2850 & 68 & 01 & 0 & 1217 & 3191 & 635 & 5047 \\
\hline
\end{tabular}


Table Vb: FY 94 Samples and Sample Analyses by Rad Level and Matrix Type (xi) Rocky Flats

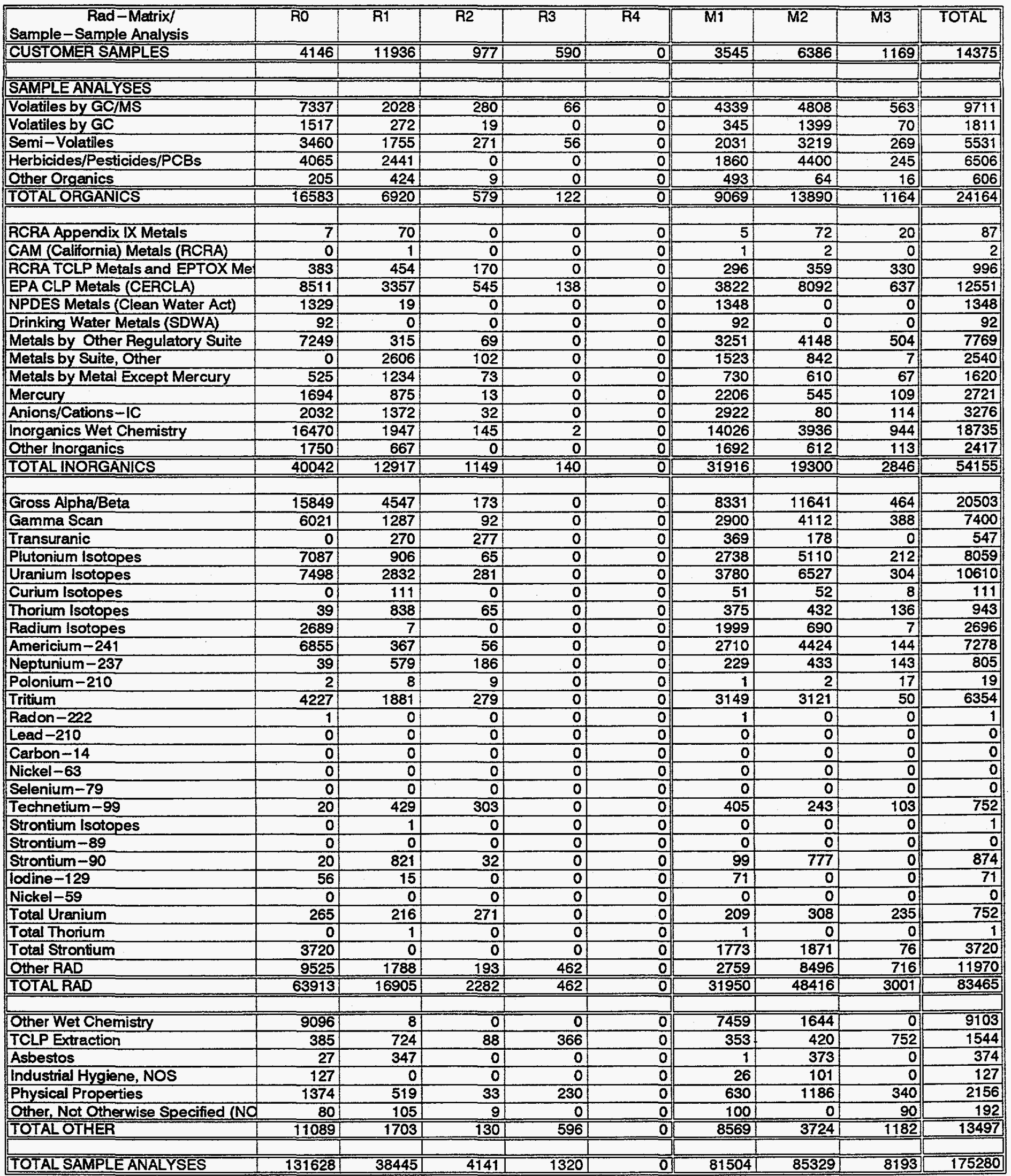


Table Vb: FY 94 Samples and Sample Analyses by Rad Level and Matrix Type (xii) Savannah Aiver

\begin{tabular}{|c|c|c|c|c|c|c|c|c|c|}
\hline $\begin{array}{c}\text { Rad-Matrix/ } \\
\text { Sample-Sample Analysis }\end{array}$ & Ro & $\mathrm{R1}$ & R2 & $\overline{\mathrm{RB}}$ & $\overline{\mathrm{R4}}$ & M1 & M2 & $\overline{\bar{M} 3}$ & TOTAL \\
\hline CUSTOMER SAMPLES & 10269 & 19656 & 233 & 62 & 211 & 21420 & 937 & 3249 & 28019 \\
\hline & & & & & & & & & \\
\hline \multicolumn{10}{|l|}{ SAMPLE ANALYSES } \\
\hline Volatiles by GC/MS & 183 & 9) & 0 & 0] & 0. & 132 & 0 & 60 & 192 \\
\hline Volatiles by GC & 104 & 234 & 22 & 8 & 0 & 145 & 203 & 19 & 367 \\
\hline Semi-Volatiles & 1289 & 93 & 8 & 2 & 0 & 1016 & 74 & 301 & 1391 \\
\hline Herbicides/Pesticides/PCBs & 647 & 100 & 8 & 4 & 0 & 603 & 75 & 80. & 759 \\
\hline Other Organics & 2008 & 4664 & 0 & O) & 0 & 1743 & 1 & 284 & 4350 \\
\hline TOTAL ORGANICS & 42311 & 5100 & 37 & 13 & 0. & 3640 & 353 & $745]$ & 7060 \\
\hline & & & & & & & & & \\
\hline RCRA Appendix IX Metals & 11 & 9 & 0 & D. & 0 & 19 & 0 & 1 & 20 \\
\hline CAM (California) Metals (RCRA) & 80 & 01 & 요 & O & 0 & 80 & 0 & 0 & 80 \\
\hline RCRA TCLP Metals and EPTOX Me & 76 & 104 & 9 & 2 & 0 & 115 & 61 & 15 & 191 \\
\hline EPA CLP Metals (CERCLA) & 67 & 41 & 15 & 8 & 0 & 67 & 57 & 7 & 131 \\
\hline NPDES Metals (Clean Water Act) & 86 & 823 & 0 & 0 & 0 & 909 & 0 & 요 & 909 \\
\hline Drinking Water Metals (SDWA) & 0 & 0 & 0 & 0 & 0 & 0 & 0 & 0. & 0 \\
\hline Metals by Other Regulatory Suite & 437 & 4 & 0 & 0 & 0 & 303 & 0 & 139 & 442 \\
\hline Metals by Suite, Other & 2690 & 4647 & 0 & of & 애 & 1426 & 0 & 1268 & 5015 \\
\hline Metals by Metal Except Mercury & 1865 & 58 & 0 & 0 & 105 & 1091 & 52 & 810 & 1990 \\
\hline Mercury & 2064 & 4815 & 2 & 1 & 105 & 1441 & 55 & 773 & 4628 \\
\hline Anions/Cations $-\mathrm{IC}$ & 3855 & 87 & 0 & 0 & 211 & 3210 & 63 & 699 & 4062 \\
\hline Inorganics Wet Chemistry & 5234 & 167 & 91 & 15 & 0 & 4061 & 295 & 1151 & 5507 \\
\hline Other Inorganics & 263 & 110 & 0 & 0 & 0 & 275 & 51 & 47 & 373 \\
\hline \multirow[t]{2}{*}{ TOTAL INORGANICS } & 16728 & 10866 & 117 & 25 & 422 & 12996 & 633 & 4909 & 23348 \\
\hline & & & & & & & & & \\
\hline Gross Alpha/Beta & 2249 & 15351 & 41 & 48 & 211 & 12165 & 399 & 144 & 15304 \\
\hline Gamma Scan & 90 & 4905 & 34 & 19 & 110 & 4794 & 182 & 103 & 5119 \\
\hline Transuranic & 0 & 0 & 0 & 0 & 110 & 0 & 0 & 30 & 70 \\
\hline Plutonium Isotopes & 695 & 46 & 18 & 4 & 211 & 677 & 58 & 58 & 883 \\
\hline Uranium Isotopes & 697 & 64 & 9 & 3 & 211 & 678 & 65 & 60 & 894 \\
\hline Curium Isotopes & 0 & 16 & 2 & 1 & 10 & 2 & 7 & 10 & 25 \\
\hline Thorium Isotopes & 18 & 4 & 2 & 25 & 0 & 0 & 7 & 42 & 48 \\
\hline Radium Isotopes & 1 & 3 & 0 & 0 & 0 & 1 & 0 & 3 & 5 \\
\hline Americium-241 & 678 & 28 & 3 & 2 & 20 & 677 & 27 & 7 & 721 \\
\hline Neptunium-237 & 18 & 6 & 8 & 0 & 0 & 0 & 3 & 29 & 32 \\
\hline Polonium-210 & 0 & 3 & 0 & 0 & 0 & 0 & 0 & 3 & 3 \\
\hline Tritium & 591 & 58 & 24 & 4 & 0 & 592 & 48 & 7 & 662 \\
\hline Radon-222 & 0 & 0 & 0 & 0 & 요 & 0 & 0 & 0 & 0 \\
\hline Lead-210 & 0 & 12 & 0 & 0 & 0 & 0 & 0 & 12 & 12 \\
\hline Carbon-14 & 0 & 0 & 0 & 0 & 0 & 0 & 0 & 0 & 0 \\
\hline Nickel-63 & 0 & 0 & 0 & 0 & 의 & 0 & 0 & 0 & 0 \\
\hline Selenium-79 & 80 & 0 & $\mathbf{0}$ & 0 & o & 80 & 0 & 0.) & 80 \\
\hline Technetium-99 & 0 & 11 & 0 & 0 & ㅇ. & 0 & 0 & 11 & 11 \\
\hline Strontium Isotopes & 0 & 0 & 0 & 0 & 0 & 0 & 0 & 0 & 0 \\
\hline Strontium-89 & 0 & 0 & 0 & 0 & o & 0 & 0 & 0 & 0 \\
\hline Strontium - 90 & 80 & 29 & 18 & 4. & o & 82 & 49 & o] & 131 \\
\hline lodine-129 & 80 & 7 & 0 & 0 & 0 & 87 & 0 & o & 87 \\
\hline Nickel-59 & 0 & 0 & 0 & 0 & of & 0 & 0 & 0 & 0 \\
\hline Total Uranium & 70 & 3 & 0 & 0 & 211 & 3 & 0 & 100 & 194 \\
\hline Total Thorium & 0 & 0 & 0 & 0 & 0 & 0 & 0 & 0 & 0 \\
\hline Total Strontium & 15 & 12 & 0 & 0 & 0 & 19] & 0 & 8 & 27 \\
\hline Other RAD & 236 & 210 & 14 & 4 & 10 & 231 & 80 & 151 & 468 \\
\hline TOTAL RAD & 5599 & 20770 & $\overline{174}$ & $11 \overline{14}$ & 1104 & 20089 & 927 & 777 & 24777 \\
\hline & & & & & 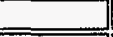 & 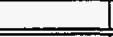 & $=$ & & \\
\hline Other Wet Chemistry & 1220 & 10 & 0 & 0 & 30 & 1230 & 0 & 30 & 1260 \\
\hline TCLP Extraction & 8 & 112 & 38 & 3 & 0 & 67 & 65 & 28 & 161 \\
\hline Asbestos & 0 & 0 & 0 & 0 & OO & 0 & 3 & 0. & 1 \\
\hline Industrial Hygiene, NOS & 552 & 0 & 0 & 0 & 요 & 552 & 0 & 요 & 552 \\
\hline Physical Properties & 12128 & 24 & 4 & 2 & 으 & 7918 & 13 & 4227 & 12157 \\
\hline Other, Not Otherwise Specified (NO & 36 & 9 & 0 & 0 & 0 & 8 & 0 & 36 & 44 \\
\hline TOTAL OTHER & 13944 & 154 & $\overline{42}$ & 5 & 30 & 9775 & $\overline{81}$ & 4321 & 14176 \\
\hline & & & & & & & & & \\
\hline TOTAL SAMPLE ANALYSES & 40501 & 36890 & 371 & 157 & 1556 & 46500 & 1994 & 10753 & 69361 \\
\hline
\end{tabular}


Table Vc: FY 95 Samples and Sample Analyses by Rad Level and Matrix Type (i) Complex-wide

\begin{tabular}{|c|c|c|c|c|c|c|c|c|c|}
\hline $\begin{array}{c}\text { Rad-Matrix } \\
\text { Sample-Sample Analysis }\end{array}$ & RO & $\overline{\mathrm{R1}}$ & R2 & $\mathrm{R3}$ & $\overline{\mathrm{R} 4}$ & M1 & M2 & M3 & TOTAL \\
\hline CUSTOMER SAMPLES & 53953 & 257423 & 19089 & 6284 & 626 & 119550 & 103326 & 81435 & 320843 \\
\hline & & & & & & & & & \\
\hline \multicolumn{10}{|l|}{ SAMPLE ANALYSES } \\
\hline Volatiles by GC/MS & 30605 & 48015 & $9 \overline{962}$ & 665 & a] & 14064 & 32372 & 42233 & 88658 \\
\hline Volatiles by GC & 9734 & 20167 & 1432 & 47 & 0 & 4718 & 23705 & 2718 & 31260 \\
\hline Semi-Volatiles & 14475 & 36044 & 8391 & 576 & o & 8567 & 31527 & 19391 & 59485 \\
\hline Herbicides/Pesticides/PCBs & 26007 & 76805 & 47 & 23 & o & 51626 & 24260 & 27044 & 102906 \\
\hline Other Organics & 10980 & 22093 & 998 & 0 & 0 & 7393 & 4048 & 2470 & 23991 \\
\hline TOTAL ORGANICS & 91800 & 203124 & 20231 & 1311 & 묘 & 86367 & 115912 & 93856 & 306300 \\
\hline \multicolumn{10}{|l|}{ 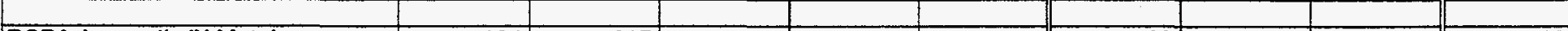 } \\
\hline RCRA Appendix IX Metals & 104 & 215 & 0 & 0 & o. & 120 & 193 & 2169 & 1400 \\
\hline CAM (California) Metals (RCRA) & 577 & 143 & 0 & 0 & o. & 721 & 179 & 36 & 828 \\
\hline ACRA TCLP Metals and EPTOX Mel & 6086 & 25442 & 17741 & 9 & 0 & 3219 & 12186 & 35511 & 50097 \\
\hline EPA CLP Metals (CERCLA) & 23764 & 36729 & 1334 & 1438 & 0] & 11888 & 20067 & 31310 & 63264 \\
\hline NPDES Metals (Clean Water Act) & 3387 & 4215 & 0 & 0 & 요 & 7602 & 0 & 0 & 7602 \\
\hline Drinking Water Metals (SDWA) & 732 & 0 & 0 & \begin{tabular}{l|l}
0 \\
\end{tabular} & 의 & 732 & 0 & a. & 732 \\
\hline Metals by Other Regulatory Suite & 47912 & 37761 & 7487 & 0 & 요 & 37772 & 20566 & 35672 & 93585 \\
\hline Metals by Suite, Other & 7747 & 26946 & 228 & 0 & 의 & 7709 & 1891 & 4236 & 24379 \\
\hline Metals by Metal Except Mercury & 10991 & 90240 & 7516 & 0 & 304 & 41756 & 10489 & 14683 & 87989 \\
\hline Mercury & 14269 & 72544 & 1036 & 5 & 304 & 73002 & 21991 & 14070 & 98611 \\
\hline Anions/Cations-IC & 19107 & 42890 & 3494 & 0 & 607 & 16798 & 581 & 13676 & 48576 \\
\hline Inorganics Wet Chemistry & 122848 & 33794 & 12979 & 113 & 0 & 72429 & 42280 & 92171 & 188307 \\
\hline Other Inorganics & 9930 & 16296 & 0 & 0 & - & 10208 & 4614 & 11403 & 26225 \\
\hline TOTAL INOAGANICS & 267452 & 387215 & 51815 & 1565 & 1214 & 283955 & 135038 & 254937 & 691595 \\
\hline \multicolumn{10}{|l|}{ 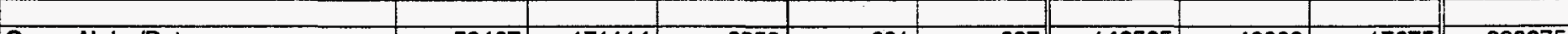 } \\
\hline Gross Alpha/Beta & 59467 & 171114 & 2850 & 291 & 607 & 146525 & 42222 & 17675 & 220375 \\
\hline Gamma Scan & 23758 & 40561 & 1392 & 117 & 318 & 29163 & 19601 & 17150 & 66030 \\
\hline Transuranic & 0 & 29338 & 29948 & 0 & 318 & 39930 & 19355 & 87 & 59488 \\
\hline Plutonium isotopes & 27392 & 12427 & 1213 & 23 & 607 & 11865 & 15020 & 14257 & 41403 \\
\hline Uranium Isotopes & 27583 & 64204 & 24455 & 21 & 607 & 62551 & 29481 & 24318 & 116610 \\
\hline Curium Isotopes & 0 & 1582 & 14 & 71 & 29 & 509 & 364 & 730 & 1618 \\
\hline Thorium Isotopes & 3433 & 39997 & 1135 & 111 & 0 & 24902 & 5821 & 13953 & 44676 \\
\hline Radium Isotopes & 7548 & 636 & 0 & 0 & 0 & 5721 & 1834 & 629 & 8184 \\
\hline Americium-241 & 22978 & 8901 & 146 & 12 & 58 & 11696 & 12772 & 7569 & 32066 \\
\hline Neptunium-237 & 3433 & 23854 & 20166 & 0 & 0 & 20921 & 12032 & 14501 & 47453 \\
\hline Polonium-210 & 163 & 641 & 998 & 0 & 0 & 6 & 163 & 1634 & 1803 \\
\hline Tritium & 14531 & 10664 & 1989 & 23 & 0 & 11388 & 9813 & 168 & 24288 \\
\hline Radon-222 & 59 & 0 & 0 & 0 & 0. & 59 & 0 & 0 & 59 \\
\hline Lead-210 & 0 & 52 & 0 & \begin{tabular}{l|l}
0 \\
\end{tabular} & 0. & 0. & 0 & 52 & 52 \\
\hline Carbon-14 & 0 & 아 & 0 & 0 & 0 & of & 0 & 0 & 0 \\
\hline Nickel-63 & 0 & 0 & 0 & 0 & ㅇ. & o. & 0 & 0] & 으 \\
\hline Selenium -79 & 577 & 116 & 0 & 0 & o. & 643 & 29 & 22 & 694 \\
\hline Technetium-99 & 156 & 43870 & 30983 & 0 & o. & 40129 & 23836 & 11042 & 75008 \\
\hline Strontium Isotopes & 0 & 72 & 0 & 0 & 0 & 0 & 33 & 39 & 72 \\
\hline Strontium - 89 & o. & 이 & 0 & 0 & 0. & ㅇ. & 이 & o. & 0 \\
\hline Strontium -90 & 733 & 3108 & 1417 & 23 & 0 & 1213 & 4003 & 73 & 5284 \\
\hline lodine-129 & 1021 & 1478 & 0 & 0 & 0 & 2404 & 58 & 36 & 2499 \\
\hline Nickel-59 & 0. & 0 & 0 & 0 & 0. & 0 & 0 & o. & - \\
\hline Total Uranium & 13983 & 27063 & 29282 & 0 & 607 & 11057 & 34434 & 24925 & 70676 \\
\hline Total Thorium & 0 & 529 & 0 & 0 & 0 & 65 & 464 & 0 & 529 \\
\hline Total Strontium & 10354 & 52 & 0 & 0 & ㅇ. & 5274 & 4925 & 207 & 10406 \\
\hline Other RAD & 41116 & 54620 & 2752 & 4680 & 29 & 36503 & 37756 & 28859 & 103158 \\
\hline \multirow[t]{2}{*}{ TOTAL RAD } & 258286 & 534877 & 148741 & 5308 & 3180 & 462520 & 274016 & 177927 & 932428 \\
\hline & & & & & & & & & \\
\hline Other Wet Chemistry & 31876 & 684 & 0 & 0 & 87 & 28306 & 4255 & 87 & 32647 \\
\hline TCLP Extraction & 5497 & 47830 & 8945 & 3708 & 0 & 3631 & 16411 & 45627 & 65824 \\
\hline Asbestos & 145 & 62055 & 0 & O & 0 & 3 & 46351 & 15857 & 62206 \\
\hline Industrial Hygiene, NOS & 2809 & 65 & 0 & 0 & 0 & 2610 & 257 & 7 & 2874 \\
\hline Physical Properties & 39359 & 39298 & 99 & 2328 & 0 & 49745 & 5502 & 25837 & 81084 \\
\hline Other, Not Otherwise Specified (NO & 6980 & 688 & 998 & 0 & 0 & 581 & 28 & 8022 & 8649 \\
\hline TOTAL OTHER & 86667 & 150620 & 10043 & 6036 & 87 & 84877 & 72804 & 95437 & 253285 \\
\hline & & & & & & & & & \\
\hline 101 AL SAMPLE ANA & 7042051 & 1275836 & 230830 & 14220 & 4480] & $917719 !$ & 5977711 & 622156 & 2183609 \\
\hline
\end{tabular}


Table Vc: FY 95 Samples and Sample Analyses by Rad Level and Matrix Type (ii) EM-30

\begin{tabular}{|c|c|c|c|c|c|c|c|c|c|}
\hline $\begin{array}{c}\text { Rad-Matrix/ } \\
\text { Sample-Sample Analysis }\end{array}$ & Ro & $\overline{\mathrm{R1}}$ & $\overline{\mathrm{R} 2}$ & R3 & R4 & $\overline{\mathrm{M1}}$ & $\mathrm{M} 2$ & $\overline{\mathrm{M3}}$ & TOTAL \\
\hline CUSTOMER SAMPLES & 29066 & 80504 & 1645 & 52961 & 561 & 77471 & 4744 & 16347 & 107817 \\
\hline & & & & & & & & & \\
\hline \multicolumn{10}{|l|}{ SAMPLEANALYSES } \\
\hline Volatiles by GC/MS & 1205 & 2181 & 559 & 571 & 0 & 575 & 815 & 3124 & 4515 \\
\hline Volatiles by GC & 1077 & 1635 & 126 & 20 & 0 & 1076 & 1561 & 172 & 2834 \\
\hline Semi-Volaties & 3725 & 1815 & 516 & 489 & 0 & 2966 & 1194 & 2385 & 6545 \\
\hline Herbicides/Pesticides/PCBs & 3066 & 3712 & 20 & 10 & 0 & 5099 & 243 & 1466 & 6808 \\
\hline Other Organics & 5596 & 18276 & 63 & 0 & 0 & 4917 & 6 & 847 & 14853 \\
\hline TOTAL ORGANICS & 14669 & 27619 & 1284 & 1090 & 0 & 14634 & 3820 & 7994 & 35555 \\
\hline \multicolumn{10}{|l|}{+2} \\
\hline RCKA APpenalx IX Metals & \begin{tabular}{r|}
42 \\
246
\end{tabular} & 38 & 0 & 0 & 요 & 74 & 으 & 141 & 147 \\
\hline RCRA TCLP Metals and EPTOX Me & 858 & $\frac{1}{1503}$ & $\frac{0}{1119}$ & $\frac{0}{4}$ & $\frac{0}{0}$ & 247 & 1 & $\frac{0}{2027}$ & 247 \\
\hline EPA CLP Metals (CERCLA) & 383 & 1944 & 41 & 1213 & 0 & $\begin{array}{l}852 \\
376 \\
\end{array}$ & $\frac{475}{169}$ & $\frac{2237}{3036}$ & $\frac{3524}{3581}$ \\
\hline NPDES Metals (Clean Water Act) & 335 & 3247 & 0 & 0 & 0 & 3582 & 0 & 0 & 3582 \\
\hline Drinking Water Metals (SDWA) & 128 & 0 & 0 & 0 & o & 128 & 0 & 0 & 128 \\
\hline Metals by Other Regulatory Suite & 2941 & 2156 & 470 & 0 & 0 & 2967 & 451 & 2149 & 5566 \\
\hline Metals by Suite, Other & 7155 & 18206 & 0 & 0 & 0. & 3797 & 1 & 3399 & 16279 \\
\hline Metals by Metal Except Mercury & 5760 & 5179 & 475 & 0 & 280 & 5761 & 453 & 2616 & 10262 \\
\hline Mercury & 6484 & 21326 & 72 & 2 & 280 & 8692 & 876 & 2786 & 20259 \\
\hline Anions/Cations-IC & 10662 & 2825 & 219 & 0 & 561 & 8845 & 242 & 2535 & 12944 \\
\hline Inorganics Wet Chemistry & 18867 & 1669 & 867 & 58 & 0. & 13660 & 2253 & 7852 & 22613 \\
\hline Other Inorganics & 1185 & 1162 & 0 & 01 & 0 & 1281 & 265 & 802 & 2348 \\
\hline TOTAL INOAGANICS & 55044 & 59256 & 3263 & 1277 & 1121 & 502611 & 5186 & 27553 & 101481 \\
\hline \\
\hline Gross Alpha/Beta & 9503 & 64962 & 234 & 127 & 561 & 52123 & 1946 & 1416 & 65436 \\
\hline Gamma Scan & 1256 & 19883 & 154 & 51 & 294 & 18796 & 1368 & 1259 & 21530 \\
\hline Transuranic & 0 & 1828 & 1878 & 0 & 294 & 2504 & 1202 & 80 & 3893 \\
\hline Plutonium Isotopes & 3080 & 741 & 122 & 10 & 561 & 2777 & 273 & 984 & 4273 \\
\hline Uranium Isotopes & 2976 & 3427 & 1557 & 9 & 561 & 5840 & 639 & 1570 & 8289 \\
\hline Curium Isotopes & 01 & 114 & 6 & 3 & 27 & 25 & 30 & 69 & 137 \\
\hline Thorium Isotopes & 211 & 2134 & 68 & 96 & 0 & 1534 & 18 & 956 & 2509 \\
\hline Radium Isotopes & 33 & 39 & 0 & 0 & 0 & 33 & 0 & 39 & 71 \\
\hline Americium-241 & 2767 & 562 & 9 & 5 & 53 & 2746 & 113 & 484 & 3370 \\
\hline Neptunium-237 & 211 & 1418 & 1294 & 0 & 0 & 1304 & 686 & 931 & 2922 \\
\hline Polonium-210 & 1 & 40 & 63 & 0 & 0 & 1 & 1 & 102 & 104 \\
\hline Tritium & 2481 & 457 & 140 & 10 & 0 & 2420 & 279 & 26 & 2906 \\
\hline Radon-222 & 4 & 0 & 0 & 0 & 0 & 4 & 0 & 0 & 4 \\
\hline Lead-210 & 0 & 47 & 0 & 0 & 0 & 0 & o. & 47 & 47 \\
\hline Carbon-14 & 0 & 0 & 0 & 0 & 0 & 0 & 0 & 0 & $\overline{0}$ \\
\hline Nickel-63 & 0 & 0 & 0 & 0 & 0 & of & 0 & 0 & 0 \\
\hline Selenium-79 & 246 & 0 & 0 & 0 & 0 & 246 & 0 & 0 & 246 \\
\hline Technetium-99 & 27 & 2485 & 1941 & 0 & 0 & 2532 & 1200 & 721 & 4453 \\
\hline Strontium Isotopes & 0 & 4 & 0 & 0 & 0 & o) & 2 & 2 & 4 \\
\hline Strontium-89 & 0 & 0 & 0 & 0 & o & 0 & 0 & 0 & $\overline{0}$ \\
\hline Strontium-90 & 273 & 101 & 111 & 10 & 0 & 278 & 217 & 0 & 495 \\
\hline lodine-129 & 323 & 80 & 0 & 0 & 요 & 403 & 0 & 0 & 403 \\
\hline Nickel-59 & 0 & 0 & 0 & 0 & D & 0 & 0 & o & $\overline{0}$ \\
\hline Total Uranium & 863 & 1437 & 1837 & 0 & 561 & 694 & 2088 & 1435 & 4457 \\
\hline Total Thorium & D) & O) & 0 & 0 & o. & o. & 0 & (0) & $\overline{0}$ \\
\hline Total Strontium & 90 & 47 & 0 & 0 & o. & 93 & 13 & 31 & 137 \\
\hline Other RAD & 1703 & 2933 & 168 & 4004 & 27 & 2674 & 386 & 5699 & 8797 \\
\hline TOTALAAD & $\overline{26046}$ & 102738 & 9581 & 4326 & 2937 & 97027 & 10460 & 15851 & 134484 \\
\hline \\
\hline Other Wet Chemistry & 4955 & 66 & $\overline{0}$ & 01 & 80 & 5021 & 0 & 80 & 5102 \\
\hline TCLP Extraction & 573 & 2796 & 696 & 3172 & 0 & 665 & 732 & 5785 & 7210 \\
\hline Astbestos & 1 & 2 & 0 & 0 & 0 & 0 & 13 & 0 & 8 \\
\hline Industrial Hygiene, NOS & 2163 & 0 & 0 & 0 & 0 & 2160 & 3 & 0 & 2163 \\
\hline Physical Properties & 32478 & 2322 & 11 & 1992 & 0 & 22849 & 43 & 13912 & 36804 \\
\hline Other, Not Otherwise Specified (NO & 426 & 46 & 63 & 01 & 0 & 35 & $\overline{0}$ & 494 & 532 \\
\hline TOTAL OTHEA & 40596 & 5232 & 770 & 5165 & 80 & 30731 & 790 & 20270 & 51817 \\
\hline & & & & & & & & & \\
\hline TOTAL SAMPLE ANALYSES & 136355 & 194845 & 14897 & 11858 & 4138 & 192654 & 20257 & 71669 & 323336 \\
\hline
\end{tabular}


Table Vc: FY 95 Samples and Sample Analyses by Rad Level and Matrix Type (iii) EM-40

\begin{tabular}{|c|c|c|c|c|c|c|c|c|c|}
\hline $\begin{array}{c}\text { Rad-Matrix } \\
\text { Sample-Sample Analysis }\end{array}$ & RO & $\overline{\mathrm{RI}}$ & $\overline{\mathrm{R} 2}$ & $\overline{R 3}$ & $\overline{\mathrm{R4}}$ & $\overline{\mathrm{M} 1}$ & M2 & 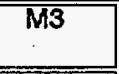 & TOTAL \\
\hline CUSTOMER SAMPLES & 19734 & 167620 & 17116 & 544 & 19 & 32808 & 96461 & 63063 & 198682 \\
\hline & & & & & & & & & \\
\hline \multicolumn{10}{|l|}{ SAMPLE ANALYSES } \\
\hline Volatiles by GC/MS & 23842 & $4 \overline{45178}$ & 8666 & 47 & 0 & 9445 & 29864 & 38445 & $\overline{77744}$ \\
\hline Volatiles by GC & 6311 & 18148 & 1262 & 24 & 0 & 1664 & 21598 & 2505 & 25757 \\
\hline Semi-Volatiles & 8537 & 33721 & 7751 & 46 & 0 & 4427 & 28995 & 16633 & 50054 \\
\hline Herbicides/Pesticides/PCBs & 20455 & 72020 & 24 & 12 & 0 & 44902 & 22470 & 25187 & 92535 \\
\hline Other Organics & 3783 & 2092 & 921 & 0 & 0 & 1012 & 3947 & 1536 & 6645 \\
\hline TOTAL ORGANICS & 62927 & 171160 & 18624 & 1301 & - & 61449 & 106873 & 84307 & 252735 \\
\hline \multicolumn{10}{|l|}{\begin{tabular}{|l|} 
BCRA Appendix IX Metals \\
\end{tabular}} \\
\hline RCRA Appendix IX Metals & 40 & 172 & 0 & 0 & o. & 29 & 183 & 1995 & 1209 \\
\hline 3) Metals (RCRA) & 308 & 138 & 0 & 0 & O. & 446 & 173 & 35 & 550 \\
\hline Metals and EPTOX Me & 3104 & 23511 & 16337 & 5 & 0 & 629 & 11342 & 32744 & 43836 \\
\hline EPA CLP Metals (CERCLA) & 15548 & 34263 & 1276 & 123 & 0 & 7113 & 16374 & 27723 & 51210 \\
\hline NPDES Metals (Clean Water Act) & 2988 & 658 & 0 & 0 & 0 & 3646 & 0 & 0 & 3646 \\
\hline Drinking Water Metals (SDWA) & 67 & 0 & 0 & 0) & o & 67 & 0 & 0 & 67 \\
\hline Metals by Other Regulatory Suite & 37335 & 35058 & 6906 & 0 & 0 & 29979 & 17172 & 32991 & 79720 \\
\hline Metals by Suite, Other & 0 & 6970 & 226 & 0 & 0 & 3560 & 1871 & 550 & 6588 \\
\hline Metals by Metal Except Mercury & 2602 & 83694 & 6908 & 0 & 0 & 33381 & 9703 & 11727 & 74007 \\
\hline Mercury & 5186 & 48680 & 928 & 2 & 0 & 61259 & 20635 & 10938 & 73815 \\
\hline Anions/Cations-1C & 7454 & 39413 & 3223 & 0 & 0 & 7115 & 313 & 10818 & 34168 \\
\hline Inorganics Wet Chemistry & 75159 & 31649 & 11914 & 50 & 0 & 32830 & 37429 & 82796 & 135914 \\
\hline Other Inorganics & 8409 & 14878 & 0 & 0 & 0 & 8602 & 4261 & 10424 & 23287 \\
\hline TOTAL INORGANICS & 158201 & 319083 & 47718 & 180 & 0] & 188656 & 119456 & 222741 & 528017 \\
\hline \multicolumn{10}{|l|}{ 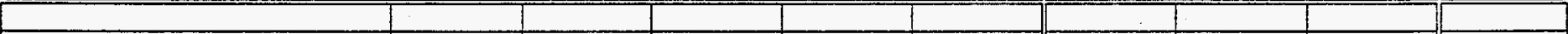 } \\
\hline Gross Alpha/Beta & 35006 & 99121 & 2569 & 152 & 0 & 82012 & 32776 & 15955 & 133795 \\
\hline Gamma Scan & 15160 & 18633 & 1213 & 61 & 요 & 5828 & 13626 & 15613 & 35067 \\
\hline Transuranic & 0 & 27068 & 27623 & 0 & 0 & 36831 & 17860 & 0 & 54691 \\
\hline Plutonium isotopes & 15452 & 11498 & 1070 & 12 & 0 & 4582 & 10404 & 13047 & 28032 \\
\hline Uranium isotopes & 17496 & 59851 & 22532 & 11 & 0 & 52221 & 25300 & 22369 & 99890 \\
\hline Curium Isotopes & 0 & 1443 & 7 & 4 & 0 & 477 & 329 & 648 & 1454 \\
\hline Thorium Isotopes & 3172 & 37294 & 1051 & 5 & 0 & 22999 & 5741 & 12782 & 41522 \\
\hline Radium Isotopes & 3888 & 587 & 0 & 0 & 0 & 2801 & 1093 & 581 & 4476 \\
\hline Americium-241 & 12082 & 8205 & 135 & 6 & 0 & 4575 & 8890 & 6963 & 20428 \\
\hline Neptunium - 237 & 3172 & 22086 & 18569 & 0 & 0 & 19305 & 11171 & 13352 & 43828 \\
\hline Polonium-210 & 158 & 588 & 921 & 0 & 은 & 1 & 158 & 1508 & 1666 \\
\hline Tritium & 6947 & 10063 & 1819 & 12 & 의 & 5267 & 8044 & 139 & 16145 \\
\hline Radon-222 & 54 & 0 & 0 & 0 & 0 & 54 & 0 & 0 & 54 \\
\hline Lead-210 & 0 & 1 & 0 & 0 & 0 & $\overline{0}$ & 0 & 1 & 1 \\
\hline Carbon -14 & 0 & 0 & O & 0 & 요 & 0 & 0 & 0 & 0 \\
\hline Nickel-63 & 0 & 0 & O) & 0 & 0 & 0 & 0 & 의 & 0 \\
\hline Selenium-79 & 308 & 115 & 0 & 0 & 0 & 373 & 29 & 22 & 423 \\
\hline Technetium-99 & 14 & 40750 & 28580 & 0 & 0 & 36887 & 22302 & 10155 & 69345 \\
\hline Strontium Isotopes & 0 & 67 & 0 & 0 & 은 & 0 & 30 & 36 & 67 \\
\hline Strontium -89 & 0 & 0 & O & 0 & 0 & 0 & 0 & 요 & 0 \\
\hline Strontium -90 & 322 & 2967 & 1284 & 12 & 0 & 791 & 3728 & 72 & 4588 \\
\hline lodine-129 & 348 & 1376 & 0 & 0 & 0 & 1631 & 58 & 36 & 1725 \\
\hline Nickel-59 & 0 & 0 & 0 & 0 & 0 & 0 & 0 & 0 & 0 \\
\hline Total Uranium & 12802 & 25236 & 27009 & 0 & 0 & 10086 & 31838 & 23124 & 65048 \\
\hline Total Thorium & 0 & 522 & 0 & 0 & 0 & 62 & 459 & 0 & 522 \\
\hline Total Strontium & 5816 & 1 & 0 & 0 & 0 & 2530 & 3124 & 163 & 5817 \\
\hline Other RAD & 29190 & 50853 & 2523 & 341 & 0 & 27869 & 32592 & 22450 & 82909 \\
\hline TOTAL RAD & 161388 & 418325 & 136904 & 617 & 0 & $\overline{317182}$ & 229551 & 159015 & 711491 \\
\hline \multicolumn{10}{|l|}{ 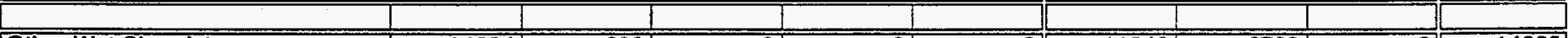 } \\
\hline Other Wet Chemistry & $\overline{14034}$ & 606 & 0 & 01 & 0 & 11842 & 2798 & 0. & 14639 \\
\hline TCLP Extraction & 2809 & 44280 & 8085 & 27.1 & 0 & 1236 & 15227 & 38953 & 55430 \\
\hline Asbestos & 75 & 61407 & 0 & 0 & 0 & 1 & 45790 & 15692 & 61482 \\
\hline Industrial Hygiene, NOS & 425 & 65 & 0 & 0 & 0 & 241 & 242 & 7 & 490 \\
\hline Physical Properties & 1944 & 36400 & 86 & 169 & 0 & 24256 & 3680 & 10662 & 38598 \\
\hline Other, Not Otherwise Specified (NO & 6451 & 607 & 921 & 0 & 0 & 537 & 28 & 7410 & 7977 \\
\hline TOTAL OTHER & 25738 & 143363 & 9092 & 439 & 0 & 38113 & 67764 & 72724 & 178617 \\
\hline & & & & & $t$ & & & & \\
\hline TOTAL SAMPLE ANALYSES & 408254 & 1051931 & 212338 & 1366 & 0 & 605401 & 523645 & 538786 & 1670860 \\
\hline
\end{tabular}


Table Vc: FY 95 Samples and Sample Analyses by Rad Level and Matrix Type (iv) EM-60

\begin{tabular}{|c|c|c|c|c|c|c|c|c|c|}
\hline $\begin{array}{c}\text { Rad-Matrix/ } \\
\text { Sample-Sample Analysis }\end{array}$ & $\overline{\mathrm{AO}}$ & $\overline{R 1}$ & R2 & R3 & $\overline{\mathrm{R4}}$ & M1 & M2 & $\overline{\mathrm{M}} \overline{3}$ & TOTAL \\
\hline CUSTOMER SAMPLES & 2196 & 759 & 10 & 1 & $\underline{0}$ & 2189 & 604 & $7]$ & 2883 \\
\hline & & & & & & & & & \\
\hline \multicolumn{10}{|l|}{ SAMPLE ANALYSES } \\
\hline Volatiles by GC/MS & 4523 & 0 & 0 & 0 & $0]$ & 3382 & 1140 & 0 ] & 4523 \\
\hline Volatiles by GC & 1909 & 50 & 18 & 0 & 0 & 1631 & 165 & o & 1886 \\
\hline Semi-Volatiles & 1580 & 2 & 0 & 0 & 0 & 767 & 814 & 0 & 1582 \\
\hline Herbicides/Pesticides/PCBs & 1757 & 2 & 0 & 0 & 0 & 634 & 1124 & 0 & 1759 \\
\hline Other Organics & 957 & 166 & 0 & 0 & 0 & 912 & 46 & 0 & 1041 \\
\hline TOTAL ORGANICS & 10726 & 220 & 18 & 01 & Q] & $7 \overline{327}$ & 3290 & $1]$ & 10791 \\
\hline & & & & & & & & 7 & \\
\hline RCRA Appendix IX Metals & 16 & 0 & 0 & 0 & 0 & 10 & 7 & 0 & 17. \\
\hline CAM (California) Metals (RCRA) & 0 & 0 & 0 & 0 & 0 & 0 & 0 & ㅇ. & 0 \\
\hline RCRA TCLP Metals and EPTOX Me: & 1754 & 50 & 18 & 0 & o. & 1448 & 179 & 0. & 1724 \\
\hline EPA CLP Metals (CERCLA) & 6641 & 0 & 0 & 0 & ㅇ. & 3727 & 2908 & 7 & 6641 \\
\hline NPDES Metals (Clean Water Act) & 3 & 29 & 0 & 0 & 0 & 32 & 0 & 0 & 32 \\
\hline Drinking Water Metals (SDWA) & 458 & D) & 0 & 0 & ㅇ. & 458 & 0 & o] & 458 \\
\hline Metals by Other Regulatory Suite & 6085 & 0 & 0 & 0 & 0 & 3703 & 2375 & 7 & 6085 \\
\hline Metals by Suite, Other & 0 & 166 & 0 & 0 & of & 0 & 0 & 0 & 83 \\
\hline Metals by Metal Except Mercury & 1852 & 48 & 18 & 0 & 0 & 1555 & 167 & 0 & 1821 \\
\hline Mercury & 1750 & 218 & 18 & 0 & 0 & 1460 & 165 & ㅇ․ & 1805 \\
\hline Anions/Cations - $1 \mathrm{C}$ & 27 & 3 & 0 & 0 & 0 & 28 & 2 & 요 & 30 \\
\hline Inorganics Wet Chemistry & 23054 & 3 & 0 & 0 & 0 & 21298 & 1757 & 1 & 23057 \\
\hline Other Inorganics & 106 & 3 & 01 & 0 & 0 & 106 & 2 & 1 & 108 \\
\hline TOTAL INORGANICS & 41746 & 519 & $54 \mid$ & 0 & [0] & 33825 & 7562 & 16 & 41861 \\
\hline & & & & & & & & & \\
\hline Gross Alpha/Beta & 11992 & 536 & 0 & 0 & 0. & 6262 & 6083 & 17 & 12445 \\
\hline Gamma Scan & 6163 & 178 & 0 & 0 & 0 & 2544 & 3789 & 8 & 6341 \\
\hline Transuranic & 0. & 5 & 0 & 0 & 0. & 0 & 5 & ㅁ․ & 5 \\
\hline Plutonium Isotopes & 7337 & 5 & 0 & 0 & 0 & 3676 & 3660 & 7 & 7343 \\
\hline Uranium lsotopes & 5799 & 5 & 0 & 0 & 0 & 3000 & 2798 & 7 & 5805 \\
\hline Curium Isotopes & 0 & 0 & 0 & 0 & 0 & 0 & 0 & 0 & 0 \\
\hline Thorium Isotopes & 0 & 0 & 0 & 1 & 0 & 0 & 0 & 1 . & 1 \\
\hline Padium Isotopes & 3123 & 0 & 0 & 0 & 0. & 2487 & 636 & 0 & 3123 \\
\hline Americium-241 & 6754 & 0 & 0 & 0 & 0 & 3563 & 3184 & 7 & 6754 \\
\hline Neptunium-237 & 0 & 0 & 0 & 0 & 0 & 0 & \begin{tabular}{l|l}
0 \\
\end{tabular} & 0 & O \\
\hline Polonium-210 & 0 & 4 & 0 & of & 0 & 4 & 0 & 0 & 4 \\
\hline Tritium & 4204 & 0 & 0 & 0 & ㅇ. & 3001 & 1204 & 0 & 4205 \\
\hline Radon-222 & 0 & 0 & 0 & 0 & o. & 0 & 0 & 0 & $\overline{0}$ \\
\hline Lead-210 & 0 & 0) & 0 & 0 & 0. & 0 & 0 & 0 & a \\
\hline Carbon-14 & 0 & 0 & 0 & 0 & 0 & 0 & 0 & 0. & ㅇ․ \\
\hline Nickel-63 & 0 & 0 & 0 & 0 & 0 & 0 & 0 & 0 & ㅇ․ \\
\hline Selenium-79 & 0 & 0 & 0 & 0 & 0 & 0 & 0 & o. & 의 \\
\hline Technetium-99 & 97 & 0 & 0 & 0 & 0 & 97 & 0 & 0 & 98 \\
\hline Strontium Isotopes & 0 & 0 & 0 & 0 & 0 & 0 & 0 & 0 & 의 \\
\hline Strontium-89 & 0 & 0 & 0 & 0 & 0 & 0 & 0 & 0 & 0 \\
\hline Strontium-90 & 97 & 0 & 0 & 0 & 0 & 97 & 0 & 0 & 97 \\
\hline lodine-129 & 278 & 0 & 0 & 0 & 0 & 278 & 0 & 0 & 278 \\
\hline Nickel-59 & 0 & 0 & 0 & 0 & 0 & 0 & 0 & 0 & 요 \\
\hline Total Uranium & 97 & 0 & 요 & 0 & 0 & 97 & 0 & 0 & 97 \\
\hline Total Thorium & 0 & 0 & 의 & 0 & 0 & 0 & 0 & 0 & 의 \\
\hline Total Strontium & 3814 & 0 & 0 & 0 & 0 & 2279 & 1529 & 7 & 3814 \\
\hline Other RAD & 8510 & 48 & 18 & 0 & 0 & 4736 & 3838 & 1 & 8575 \\
\hline TOTALAAD & 58267 & 784 & 19 & 11 & $0]$ & 32121 & 26726 & 58 & 58987 \\
\hline Other Wet Chemistry & 10736 & & & & & & 1201 & & $\overline{1072}$ \\
\hline TCLP Extraction & $\begin{array}{r}10736 \\
1767 \\
\end{array}$ & $\begin{array}{r}0 \\
49 \\
\end{array}$ & $\begin{array}{r}0 \\
19\end{array}$ & $\begin{array}{l}0 \\
0\end{array}$ & $\frac{0}{0}$ & $\frac{9492}{1446}$ & $\frac{1244}{195}$ & $\frac{0}{0}$ & $\frac{10 / 36}{1738}$ \\
\hline \begin{tabular}{|l} 
Asbestos \\
\end{tabular} & 59 & 0 & 0 & 0 & 0 & 2 & 57 & 의 & 60 \\
\hline Industrial Hygiene, NOS & 31 & 0 & 0 & 0 & 0 & 21 & 9 & 의 & 31 \\
\hline Physical Properties & 1943 & 0. & 0 & 0 & 0 & 430 & 1514 & 요 & 1943 \\
\hline Other, Not Otherwise Specified (Nd & 0 & 22 & 0 & 0 & 0.1 & 01 & 0 & 0 & 11 \\
\hline TOTAL OTHER & 14536 & 71 & 19! & $\underline{01}$ & o] & 11391 & 3019 & 미 & $1451 \overline{8}$ \\
\hline TOTAL SAMPLE ANALYSES & 125275 & 1594 & $111]$ & 11 & का & 84664 & 40596 & 75 & 126158 \\
\hline
\end{tabular}


Table Vc: FY 95 Samples and Sample Analyses by Rad Level and Matrix Type (v) Fernald

\begin{tabular}{|c|c|c|c|c|c|c|c|c|c|}
\hline $\begin{array}{c}\text { Rad-Matrix/ } \\
\text { Sample-Sample Analysis } \\
\end{array}$ & RO & R1 & R2 & R3 & R4 & $\overline{M 1}$ & M2 & M3 & TOTAL \\
\hline CUSTOMER SAMPLES & 1964 & 11579 & 428 & 11 & 0 & 2893 & 9366 & 1567 & 13899 \\
\hline & & & & & & & & & \\
\hline \multicolumn{10}{|l|}{ SAMPLE ANALYSES } \\
\hline Volatiles by GC/MS & 3722 & 590 & 1 & 0 & 0 & 381 & 164 & 3772 & 4315 \\
\hline Volatiles by GC & 1147 & 6126 & 422 & 0 & 0 & 463 & 6454 & 770 & 7691 \\
\hline Semi-Volatiles & 534 & 1000 & 1 & 0 & 0 & 115 & 1189 & 239 & 1539 \\
\hline Herbicides/Pesticides/PCBs & 4455 & 700 & 0 & 0 & 0 & 528 & 152 & 4482 & 5158 \\
\hline Other Organics & 573 & 367 & 0 & 0 & 0 & 52 & 583 & 199 & 887 \\
\hline TOTAL ORGANICS & 10430 & 8782 & 424 & 0 & 0 & 1539 & 8543 & 9462 & 19590 \\
\hline & & & & & & & & & \\
\hline RCRA Appendix IX Metals & 6 & 7 & 0 & 0 & 0 & 8 & 5 & o & 13 \\
\hline CAM (California) Metals (RCRA) & 44 & 46 & 0 & 0 & 0 & 90 & 57 & 11 & 124 \\
\hline RCRA TCLP Metals and EPTOX MeI & 786 & 1275 & 422 & 0 & 0 & 71 & 1783 & 561 & 2449 \\
\hline EPA CLP Metals (CERCLA) & 409 & 542 & 3 & 0 & o & 375 & 135 & 444 & 954 \\
\hline NPDES Metals (Clean Water Act) & 27 & 230 & 0 & 0 & - & 257 & 0 & 0. & 257 \\
\hline Drinking Water Metals (SDWA) & 1 & 0 & 0 & 0 & ㅇ. & 1 & 0 & 0 & 1 \\
\hline Metals by Other Regulatory Suite & 9072 & 54 & 0 & 0 & 0 & 369 & 54 & 8693 & 9121 \\
\hline Metals by Suite, Other & 0 & 361 & 1 & 0 & - & 59 & 9 & 186 & 308 \\
\hline Metals by Metal Except Mercury & 459 & 1758 & 0 & 0 & (0) & 383 & 1817 & 16 & 2216 \\
\hline Mercury & 455 & 1966 & 0 & 0 & 0 & 539 & 1574 & 201 & 2368 \\
\hline Anions/Cations-IC & 1080 & 252 & 0 & 0 & 0 & 245 & 13 & 1073 & 1331 \\
\hline Inorganics Wet Chemistry & 18850 & 4655 & 3796 & 0 & 0 & 2509 & 7200 & 17739 & 27375 \\
\hline Other Inorganics & 1600 & 2245 & 0 & 0 & 0. & 1699 & 853 & 1292 & 3845 \\
\hline TOTAL INORGANICS & 32788 & 13389 & 4222 & 0 & 0 & 6605 & 13502 & 30216 & 50361 \\
\hline & & & & & & & & & \\
\hline Gross Alpha/Beta & 2694 & 5168 & 423 & 0 & 0 & 2114 & 2359 & 1917 & 7337 \\
\hline Gamma Scan & 1844 & 1363 & 1 & 0 & 0 & 266 & 1050 & 1889 & 3204 \\
\hline Transuranic & 0 & 0 & 0 & 0 & 0 & 0 & 0 & 0 & 0 \\
\hline Plutonium Isotopes & 1607 & 238 & 1 & 0 & 0 & 274 & 443 & 1128 & 1845 \\
\hline Uranium Isotopes & 1700 & 852 & 1 & 0 & 0 & 369 & 729 & 1453 & 2552 \\
\hline Curium Isotopes & 0 & 427 & 0 & 0 & 0 & 131 & 70 & 226 & 427 \\
\hline Thorium Isotopes & 1105 & 51 & 0 & 1 & 0 & 2 & 49 & 1106 & 1157 \\
\hline Radium Isotopes & 17 & 205 & 0 & 0 & 0 & 17 & 2 & 202 & 221 \\
\hline Americium-241 & 491 & 206 & 0 & 0 & 0 & 258 & 407 & 33 & 698 \\
\hline Nepturium-237 & 1105 & 258 & 0 & 0 & 0 & 7 & 54 & 1303 & 1364 \\
\hline Polonium-210 & 52 & 205 & 0 & 0 & 0 & 0 & 52 & 205 & 257 \\
\hline Tritium & 101 & 2018 & 424 & 0 & 0 & 69 & 589 & 5 & 1603 \\
\hline Radon-222 & 19 & 0 & 0 & 0 & 0 & 19 & 0 & 0 & 19 \\
\hline Lead-210 & 0 & 0 & 0 & 0 & 0 & 0 & 0 & 0 & 0 \\
\hline Carbon-14 & 0 & 0 & 0 & 0 & 0 & 0 & 0 & 0 & 0 \\
\hline Nickel-63 & 0 & 0 & 0 & 0 & 0 & 0 & 0 & 0 & 0 \\
\hline Selenium-79 & 44 & 18 & 0 & 0 & 0 & 54 & 5 & 3 & 62 \\
\hline Technetium-99 & 0 & 199 & 0 & 0 & 0 & 2 & 0 & 196 & 199 \\
\hline Strontium Isotopes & 0 & 23 & 0 & 0 & 0 & 0 & 11 & 13 & 23 \\
\hline Strontium -89 & 0 & 0 & 0 & 0 & 0. & 0 & 0 & 0. & 0 \\
\hline Strontium -90 & 44 & 261 & 422 & 0 & 0 & 91 & 624 & 11 & 727 \\
\hline Jodine-129 & 44 & 448 & 0 & 0 & 0 & 478 & 9 & 6 & 492 \\
\hline Nickel-59 & 0 & 0 & 0 & 0 & 0 & 0 & 0) & 0 & 0 \\
\hline Total Uranium & 4388 & 401 & 0 & 0 & 0 & 232 & 78 & 4479 & 4789 \\
\hline Total Thorium & 0 & 99 & 0 & 0 & 0 & 21 & 78 & 0 & 99 \\
\hline Total Strontium & 60 & 0 & 0 & 0 & 0 & 50 & 10 & 1 & 61 \\
\hline Other RAD & 4815 & 1248 & 423 & 0 & 0. & 766 & 733 & 4986 & 6485 \\
\hline TOTAL RAD & 20127 & 13687 & 1694 & 1 & 0. & 5220 & 7352 & 19160 & 33620 \\
\hline & & & & & & & & & \\
\hline Other Wet Chemistry & 370 & 211 & $\overline{0}$ & OI & 0. & 572 & 9 & 0 & 581 \\
\hline TCLP Extraction & 688 & 1866 & 1 & 0 & 0 & 169 & 1422 & 964 & 2555 \\
\hline Asbestos & 0 & 52 & 0 & 0 & 0 & 0 & 52 & 0 & 52 \\
\hline Industrial Hygiene, NOS & 57 & 10 & 0 & 0 & 0 & 62 & 4 & 1 & 68 \\
\hline Physical Properties & 30 & 1111 & 0 & 0. & 0 & 343 & 81 & 717 & 1141 \\
\hline Other, Not Otherwise Specified (NO & 2241 & 90 & 0 & 0 & 0 & 66 & 5 & 2261 & 2331 \\
\hline TOTAL OTHER & 3387 & 3340 & $1]$ & 0 & 0 & 1213 & 1572 & 3943 & 6728 \\
\hline TOTAL SAMPLE ANALYSES & 66732 & 39199 & 6341 & 1 & 0 & 14577 & 30969 & 62781 & 110300 \\
\hline
\end{tabular}


Table Vc: FY 95 Samples and Sample Analyses by Rad Level and Matrix Type (vi) INEL

\begin{tabular}{|c|c|c|c|c|c|c|c|c|c|}
\hline $\begin{array}{c}\text { Rad-Matrix/ } \\
\text { Sample-Sample Analysis }\end{array}$ & Ro & R1 & $\overline{\mathrm{A} 2}$ & $\overline{\mathrm{R} \overline{3}}$ & $\overline{\overline{\mathrm{R} 4}}$ & $\overline{\overline{M 1}}$ & $\overline{\mathrm{M} 2}$ & $\overline{\mathrm{M} 3}$ & TOTAL \\
\hline CUSTOMER SAMPLES & 25841 & 10904 & 289 & 344 & 0 & 5599 & 49001 & 1936 & 13278 \\
\hline \multicolumn{10}{|l|}{ SAMPLE ANALYSES } \\
\hline Volatiles by GC/MS & 507 & $6 \overline{628}$ & 131 & 33 & 0 & 333 & 723 & 125[ & 1181 \\
\hline Volatiles by GC & 728 & 307 & 31 & 9 & 0 & 547 & 445 & 33 & 1050 \\
\hline Semi-Volatiles & 379 & 591 & 22 & 30 & 0 & 251 & 720 & 50 & 1021 \\
\hline Herbicides/Pesticides/PCBs & 480 & 1241 & 9 & 4 & 의 & 345 & 1072 & 316 & 1734 \\
\hline Other Organics & 914 & 1313 & 0 & 0 & 0 & 299 & 664 & 10 & 1600 \\
\hline TOTAL ORGANICS & 3008 & 4079 & 741 & 76 & 0 & 1776 & 3624 & $5 \overline{534]}$ & 6586 \\
\hline RCPA Annendi IX Metals & at 1 & 7 & to & 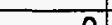 & 1 & $=1$ & $F$ & T & \\
\hline & & 1 & 0 & 0 & 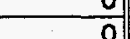 & 13 & 5 & 0 & 10 \\
\hline 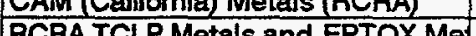 & & & & & 의 & $13 \mid$ & 1 & of & 14 \\
\hline RCRA TCLP Metals and EPTOX Me & 615 & 502 & 16 & 2 & 0 & 511 & 526 & 41 & 1106 \\
\hline EPA CLP Metals (CERCLA) & 507 & 253 & 54 & 78 & 0 & 276 & 523 & 93 & 891 \\
\hline NPDES Metals (Clean Water Act) & 112 & 225 & 0 & 0 & o. & 338 & 0 & 요 & 338 \\
\hline Drinking Water Metals (SDWA) & 131 & 0 & 0 & 0 & 0 & 131 & 0 & o. & 131 \\
\hline Metals by Other Regulatory Suite & 654 & 248 & 0 & 0 & of & 291 & 407 & 226 & 913 \\
\hline Metals by Suite, Other & 0 & 1449 & 7 & 0 & 0 & 106 & 56 & 5 ( & 812 \\
\hline Metals by Metal Except Mercury & 683 & 610 & 5 & 0 & 0 & 535 & 410 & 297 & 1270 \\
\hline Mercury & 782 & 1872 & 7 & 1 & 0 & 668 & 666 & 19 & 2007 \\
\hline Anions/Cations-IC & 212 & 143 & 0. & 0 & - & 276 & 41 & 39 & 355 \\
\hline Inorganics Wet Chemistry & 1599 & 1064 & 110 & 18 & 0 & 1427 & 870 & 496 & 2792 \\
\hline Other Inorganics & 249 & 206 & 0 & 0 & 0 & 287 & 122 & 46 & 454 \\
\hline TOTAL INORGANICS & 5559 & 6581 & 198 & 98 & 0 & 4864 & 3626 & 1261 & 11094 \\
\hline \\
\hline Gross Alpha/Beta & 1845 & 5279 & 57 & 53 & 0 & 3911 & 1642 & 146 & 6467 \\
\hline Gamma Scan & 1007 & 1733 & 44 & 21 & 0 & 1544 & 1184 & 78 & 2806 \\
\hline Transuranic & 0 & 14 & 0 & 0 & 0 & 0 & 14 & - & 14 \\
\hline Plutonium Isotopes & 686 & 168 & 15 & 4 & 0 & 416 & 413 & 44 & 873 \\
\hline Uranium Isotopes & 636 & 591 & 7 & 4 & 0 & 437 & 734 & 68 & 1238 \\
\hline Curium Isotopes & 0 & 20 & 3 & 1 & a & 6 & 12 & 6 & 23 \\
\hline Thorium Isotopes & 22 & 301 & 6 & 8 & 0 & 16 & 292 & 29 & 337 \\
\hline Radium Isotopes & 115 & 4 & 0 & 0 & 0 & 88 & 27 & 4 & 119 \\
\hline Americium-241 & 558 & 89 & 8 & 2 & 으 & 378 & 260 & 18 & 656 \\
\hline Neptunium -237 & 22 & 55 & 2 & 0 & 0 & 9 & 40 & 30 & 80 \\
\hline Polonium-210 & 1 & 5 & 0 & 0 & 0 & 1 & 1 & 4 & 6 \\
\hline Tritium & 493 & 222 & 37 & 4 & 0 & 406 & 303 & 10 & 738 \\
\hline Radon-222 & 0 & 0 & 0 & 0 & 의 & 0 & 0 & 0 & 0 \\
\hline Lead -210 & 0 & 3 & 0 & 0 & 0 & 0 & 0 & 3 & 3 \\
\hline Carbon-14 & 0 & 0 & 0 & 0 & 0 & 0 & 0 & 0 & 0 \\
\hline Nickel-63 & 0 & 0 & 0 & 0 & 0 & 0 & 0 & 은 & 0 \\
\hline Selenium-79 & 12 & 21 & 0 & 0 & 0 & 24 & 5 & 4 & 33 \\
\hline Technetium-99 & 28 & 266 & 1 & 0 & 0 & 29 & 260 & 6 & 295 \\
\hline Strontium Isotopes & 0 & 0 & 0 & 0 & 0 & 0 & 0 & 의 & $\overline{0}$ \\
\hline Strontium-89 & 0 & 0 & 0 & 0 & 0 & 0 & 0 & ㅇ. & 0 \\
\hline Strontium -90 & 40 & 190 & 22 & 4 & 0 & 98 & 146 & 13 & 257 \\
\hline lodine-129 & 92 & 38 & 0 & 0 & 0 & 113 & 10 & 7 & 130 \\
\hline Nickel-59 & 0 & 0 & 0 & 0 & 0 & 0 & 0 & 0 & 0 \\
\hline Total Uranium & 121 & 192 & 0 & 0 & 0 & 38 & 35 & 240 & 313 \\
\hline Total Thorium & 0 & 0 & 0 & 0 & 0 & 0 & 아 & 0 & 0 \\
\hline Total Strontium & 169 & 3 & 0 & 0 & 0 & 73 & 93 & 7 & 173 \\
\hline Other RAD & 782 & 1172 & 33 & 235 & 0 & 433 & 1417 & 364 & 2218 \\
\hline TOTAL RAD & 6629 & 10368 & 234 & 338 & 0 & 8020 & 6887 & 1080 & 16779 \\
\hline & \\
\hline Other Wet Chemistry & 795 & 6 & 0 & $\overline{0}$ & 0 & 729 & 72 & $\overline{0}$ & 801 \\
\hline TCLP Extraction & 596 & 645 & 20 & 186 & 0 & 568 & 552 & 271 & 1419 \\
\hline Asbestos & 0 & 3451 & 0 & 0 & 0 & 0 & 2561 & 891 & 3452 \\
\hline Industrial Hygiene, NOS & 161 & 12 & 0 & 0 & 0 & 158 & 13 & 1 & 172 \\
\hline Physical Properties & 177 & 131 & 7 & 117 & 0 & 155 & 147 & 129 & 431 \\
\hline Other, Not Otherwise Specified (NO & 44 & 16 & 0 & 0 & 0 & 9 & of & 45 & 57 \\
\hline \multirow[t]{2}{*}{ TOTAL OTHER } & 1772 & 4261 & 27 & 303 & 0 & 1620 & 3345 & 1338 & 6333 \\
\hline & & & & & & & & & \\
\hline TOTAL SAMPLE ANALYSES & 16969 & 25290 & 534 & $\overline{815}$ & $\overline{0}$ & 16281 & 17482 & 4213 & 40792 \\
\hline
\end{tabular}


Table Vc: FY 95 Samples and Sample Analyses by Rad Level and Matrix Type (vii) NTS

\begin{tabular}{|c|c|c|c|c|c|c|c|c|c|}
\hline $\begin{array}{c}\text { Rad-Matrix/ } \\
\text { Sample-Sample Analysis }\end{array}$ & $\overline{\mathrm{RO}}$ & R1 & R2 & $\overline{\mathrm{R} 3}$ & 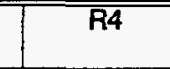 & $\overline{\mathrm{M} 1}$ & $\overline{M 2}$ & M3 & TOTAL \\
\hline CUSTOMER SAMPLES & 1028 & 9296 & 172 & $\overline{0}$ & 0 & 659 & 5784 & 2809 & 9878 \\
\hline \multicolumn{10}{|l|}{ SAMPLE ANALYSES } \\
\hline Volatiles by GC/MS & 1461 & 1272 & 44 & & $\overline{0}$ & 930 & 1727 & 123 & 2779 \\
\hline Volatiles by GC & 275 & 14 & 0 & & 0 & 8 & 219 & 65 & 290 \\
\hline Semi-Volatiles & 717 & 1045 & 38 & & 0 & 379 & 1333 & 88 & 1800 \\
\hline Herbicides/Pesticides/PCBs & 765 & 2062 & 0 & 0 & 0 & 291 & 2169 & 372 & 2830 \\
\hline Other Organics & 19 & 134 & 0 & 0 & 0 & 72 & 73 & 8 & 153 \\
\hline TOTAL OAGANICS & 3237 & 4528 & 82 & & $\overline{0}$ & 1680 & 5521 & 656. & 7852 \\
\hline RCRA Appendix IX Metals & 5 & 13 & 0 & & & & 17 & & \\
\hline CAM (California) Metals (RCRA) & 0 & 0 & 0 & & $\frac{1}{0}$ & 0 & $\frac{17}{0}$ & 0 & $\frac{10}{0}$ \\
\hline RCRA TCLP Metals and EPTOX Mef & 56 & 552 & 0 & & 0 & 10 & 586 & 11 & 608 \\
\hline EPA CLP Metals (CERCLA) & 1610 & 586 & 112 & 0 & $\overline{0}$ & 715 & 1549 & 44 & 2308 \\
\hline NPDES Metals (Clean Water Act) & 253 & 0 & 0 & 0 & $\overline{0}$ & 253 & 0 & 0 & 253 \\
\hline Drinking Water Metals (SDWA) & 2 & 0 & 0 & $\underline{0}$ & 0 & 2 & 0 & 0 & 2 \\
\hline Metals by Other Regulatory Suite & 1308 & 329 & 0 & $\overline{0}$ & $\overline{0}$ & 569 & 1085 & 66 & 1678 \\
\hline Metals by Suite, Other & 0 & 521 & 19 & & 0 & 295 & 160 & 2 & 499 \\
\hline Metals by Metal Except Mercury & 71 & 873 & 0 & 0 & $\overline{0}$ & 25 & 418 & 501 & 944 \\
\hline Mercury & 297 & 947 & 0 & $\underline{0}$ & $\underline{0}$ & 278 & 950 & 14 & 1243 \\
\hline Anions/Cations - IC & 378 & 206 & 0 & $\mathbf{0}$ & 0 & 553 & 20 & 11 & 585 \\
\hline Inorganics Wet Chemistry & 3005 & 850 & 0 & 0 & 0 & 2629 & 1206 & 20 & 3855 \\
\hline Other Inorganics & 325 & 135 & 0 & 0 & 0 & 307 & 153 & 0 & 460 \\
\hline TOTALINORGANICS & 7310 & 5012 & 132 & $\underline{\underline{0}}$ & $\underline{\underline{0}}$ & 5638 & 6144 & 668 & 12452 \\
\hline \multicolumn{10}{|l|}{ 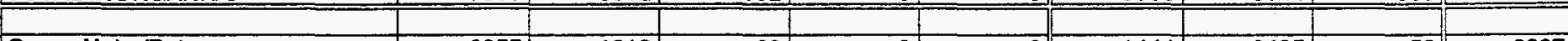 } \\
\hline Gross Alpha/Beta & 2955 & 1013 & 28 & o & 0 & 1444 & 2495 & 59 & 3997 \\
\hline Gamma Scan & 1062 & 578 & 16 & $\underline{0}$ & 0 & 559 & 1032 & 65 & 1656 \\
\hline Transuranic & 0 & 21 & 0 & 0 & 0 & 0 & 21 & 0 & 21 \\
\hline Plutonium Isotopes & 1315 & 162 & 11 & $\underline{0}$ & 0 & 506 & 966 & 16 & 1488 \\
\hline Uranium Isotopes & 1406 & 1022 & 11 & $\underline{0}$ & 0 & 632 & 1759 & 48 & 2439 \\
\hline Curium Isotopes & 0 & 18 & 0 & $\underline{0}$ & 0 & 9 & 9 & ㅇ․ & 18 \\
\hline Thorium Isotopes & 0 & 594 & 11 & 0 & 0 & 40 & 564 & 2 & 605 \\
\hline Radium Isotopes & 510 & 0 & 0 & $\overline{0}$ & 0 & 378 & 132 & 0 & 510 \\
\hline Americium - 241 & 1291 & 70 & 11 & 0 & 0 & 515 & 843 & 14 & 1372 \\
\hline Neptunium-237 & 0 & 116 & 0 & $\overline{0}$ & 0 & 8 & 106 & 2 & 116 \\
\hline Polonium-210 & 0 & 0 & 0 & $\mathbf{0}$ & 0 & 0 & 0 & 0 & 0 \\
\hline Tritium & 785 & 371 & 50 & $\underline{0}$ & 0 & 586 & 610 & 9 & 1206 \\
\hline Radon-222 & 0 & 0 & 0 & 0 & 0 & 0 & 0 & ㅇ. & 0 \\
\hline Lead-210 & 0 & 0 & 0 & 0 & 0 & 0 & 0 & 인 & 요마. \\
\hline Carbon-14 & 0 & 0 & 0 & 0 & 0 & 0 & 0 & 0 & 0 \\
\hline Nickel-63 & 0 & 0 & 0 & 0 & 0 & 0 & 0 & 0 & 0 \\
\hline Selenium-79 & 0 & 0 & 0 & 0 & 0 & o & 0 & 0 & 0 \\
\hline Technetium-99 & 0 & 467 & 3 & 0 & 0 & 4 & 467 & 0 & 471 \\
\hline Strontium Isotopes & 0 & 0 & 0 & 0 & 0 & 0 & 0 & D. & 요 \\
\hline Strontium -89 & 0 & 0 & 0 & 0 & 0 & 0 & 0 & 0 & 0 \\
\hline Strontium -90 & 0 & 162 & 3 & 0 & 0 & 16 & 150 & 으, & 166 \\
\hline lodine-129 & 1 & 0 & 0 & 0 & 0 & 1 & 0 & 은 & 1 \\
\hline Nickel-59 & 0 & 이 & 0 & 0 & 0 & 0 & 0 & 0 & 0 \\
\hline Total Uranium & 17 & 403 & 0 & 0 & 0 & 17 & 135 & 268 & 420 \\
\hline Total Thorium & 0 & 48 & 0 & $\overline{0}$ & $\overline{0}$ & 0 & 48 & 0 & 48 \\
\hline Total Strontium & 706 & 0 & 0 & 0 & $\overline{0}$ & 336 & 355 & 14 & 706 \\
\hline Other RAD & 1753 & 1722 & 31 & 0 & 0 & 451 & 3039 & 18 & 3507 \\
\hline TOTAL RAD & 11803 & 6767 & 175 & $\overline{0}$ & 0 & 5503 & 12730 & 516 & 18747 \\
\hline & & & 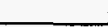 & & & 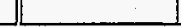 & & & \\
\hline Other Wet Chemistry & 1711 & 0 & D & $\overline{0}$ & $\overline{0}$ & 1398 & 314 & 0 & 1711 \\
\hline TCLP Extraction & 48 & 652 & 1 & 0 & 0 & 20 & 608 & 71 & 700 \\
\hline Asbestos & 25 & 6179 & 0 & $\mathbf{0}$ & $\overline{0}$ & 0 & 4621 & 1582 & 6204 \\
\hline Industrial Hygiene, NOS & 22 & 0 & 0 & $\overline{0}$ & 0 & 3 & 19 & D & 22 \\
\hline Physical Properties & 249 & 237 & 6 & 0 & 0 & 62 & 430 & 0 & 491 \\
\hline Other, Not Otherwise Specified (Nd & 4 & 45 & 0 & $\mathbf{0}$ & 0 & 46 & 3 & 0 & 49 \\
\hline TOTAL OTHER & 2059 & 7113 & 7 & $\overline{0}$ & 0 & 1529 & 5994 & 1653 & 9178 \\
\hline TOTAL SAMPLEAN & 20109 & 33419 & & $\overline{0}$ & $\overline{0}$ & 4350 & & 2003 & \\
\hline
\end{tabular}


Table Vc: FY 95 Samples and Sample Analyses by Rad Level and Matrix Type (viii) Oak Ridge K-25

\begin{tabular}{|c|c|c|c|c|c|c|c|c|c|}
\hline $\begin{array}{c}\text { Rad-Matrix } \\
\text { Sample-Sample Analysis }\end{array}$ & $\overline{\mathrm{RO}}$ & R1 & R2 & R3 & R4 & M1 & $\overline{\mathrm{M} 2}$ & $\overline{\mathrm{M3}}$ & TOTAL \\
\hline CUSTOMER SAMPLES & 282 & 22965 & 4946 & 01 & 0] & 5643 & 8338 & 14741] & 28457 \\
\hline & & & & & & & & & \\
\hline \multicolumn{10}{|l|}{ SAMPLE ANALYSES } \\
\hline Volatiles by GC/MS & 171 & 12404 & 3014 & 0 & 이 & 218 & 5471 & 9900 & 15589 \\
\hline Volatiles by GC & 105 & 15 & 1 & 0 & 0 & 60 & 52 & 3 & 117 \\
\hline Semi-Volaties & 99 & 8312 & 2677 & 0 & 0 & 66 & 5332 & 5690 & 11088 \\
\hline Herbicides/Pesticides/PCBs & 106 & 19987 & 0 & 0 & 0 & 14743 & 1727 & 3625 & 20094 \\
\hline Other Organics & 53 & 14 & 337 & OI & 0 & 42 & 22 & 338 & 403 \\
\hline TOTAL ORGANICS & 534 & 40732 & 6030 & 01 & 0 & 15130 & 12603 & 19556 & 47292 \\
\hline RCRA Appendix IX Metals & 1 & 2 & 0 & 0 & 0: & 0 & 3 & 731 & 369 \\
\hline CAM (California) Metals (RCRA) & 0 & 0 & 0 & 0 & of & 0 & 0 & 0 & 0 \\
\hline RCRA TCLP Metals and EPTOX Me & 109 & 5722 & 5540 & 0 & 0 & 56 & 675 & 11365 & 11733 \\
\hline EPA CLP Metals (CERCLA) & 184 & 9570 & 17 & 0 & 0 & 80 & 208 & 9482 & 9770 \\
\hline NPDES Metals (Clean Water Act) & 41 & 1 & 0 & 0 & 0 & 41 & 0 & 0 & 41 \\
\hline Drinking Water Metals (SDWA) & 16 & 0 & 0 & 0 & 0 . & 16 & 0 & o. & 16 \\
\hline Metals by Other Regulatory Suite & 154 & 11940 & 2530 & 0 & 0 & 9066 & 2867 & 2739 & 14649 \\
\hline Metals by Suite, Other & 0 & 129 & 3 & 0 & 0 & 53 & 26 & 2 & 107 \\
\hline Metals by Metal Except Mercury & 117 & 26510 & 2531 & 0 & 0 & 11643 & 378 & 3072 & 22126 \\
\hline Mercury & 150 & 13375 & 338 & 0 & ㅇ․ & 20696 & 3457 & 3758 & 20887 \\
\hline Anions/Cations-IC & 63 & 13323 & 1181 & 0 & o] & 90 & 10 & 2798 & 8732 \\
\hline Inorganics Wet Chemistry & 291 & 3538 & 342 & 0 & 0 & 2761 & 2288 & 11521 & 10371 \\
\hline Other Inorganics & 56 & 2512 & 01 & 0 & 0 ] & $56 \mid$ & 64 & 2448 & 2568 \\
\hline TOTAL INORGANICS & 1182 & 86622 & 12482 & 0. & 01 & 445601 & 9976 & 47916 & 101369 \\
\hline \multicolumn{10}{|l|}{\begin{tabular}{|l|l|}
+2 \\
\end{tabular}} \\
\hline Gross Alpha/Beta & 392 & 26634 & 342 & 0 & 0 & 23142 & 696 & 3530 & 27368 \\
\hline Gamma Scan & 206 & 3597 & 340 & 0 & O & 79 & 538 & 3526 & 4143 \\
\hline Transuranic & 0 & 9867 & 10122 & 0 & 0 & 13495 & 6493 & 0 & 19989 \\
\hline Plutonium Isotopes & 147 & 3209 & 339 & 0 & 0 & 53 & 122 & 3521 & 3696 \\
\hline Uranium Isotopes & 163 & 17779 & 8211 & 0 & 0 & 16787 & 2853 & 6513 & 26153 \\
\hline Curium Isotopes & 0 & 3 & 0 & 0 & 0 & 2 & 2 & o. & 3 \\
\hline Thorium Isotopes & 1 & 12049 & 339 & 0 & - & 8284 & 587 & 3518 & 12389 \\
\hline Radium Isotopes & 43 & 0 & 0 & 0 & 요 & 31 & 12 & 0 & 43 \\
\hline Americium - 241 & 132 & 2471 & 2 & 0 & 0 & 62 & 94 & 2449 & 2604 \\
\hline Neptunium-237 & 1 & 7434 & 6804 & 0 & 0 & 7030 & 3691 & 3518 & 14240 \\
\hline Polonium-210 & 0 & 0 & 337 & 0 & 0 & 0 & 0 & 338 & 338 \\
\hline Tritium & 97 & 66 & 9 & 0 & 0 & 72 & 96 & 2 & 170 \\
\hline Radon-222 & 0 & 0 & 0 & 0 & 0 & 0 & 0 & 0 & 의 \\
\hline Lead-210 & 0 & \begin{tabular}{l|l}
0 \\
\end{tabular} & 0 & 0 & 의 & 0 & 0 & 0 & 요 \\
\hline Carbon-14 & 0 & 0 & 0 & 0 & 0 & 0 & 0 & 0 & 묘 \\
\hline Nickel-63 & 0 & 0 & 0 & 0 & 의 & D & 0 & 0 & - \\
\hline Selenium-79 & \begin{tabular}{l|l}
0 \\
\end{tabular} & 1. & 0 & 0 & 0 & 0 & 0 & 0 & 1 \\
\hline Technetium-99 & 3 & 13588 & 10459 & 0 & 의 & 13499 & 7037 & 3515 & 24051 \\
\hline Strontium Isotopes & 0 & 0 & 0 & 0 & 요 & 0 & 0 & 0. & 0 \\
\hline Strontium-89 & 0 & 0 & 0 & 0 & ㅇ. & 0 & 0 & 0 & 요 \\
\hline Strontium-90 & 3 & 32 & 1 & 0 & 0 & 8 & 28 & 0 & 36 \\
\hline lodine-129 & 10 & 1 & 0 & 0 & 0 & 11 & 0 & 0. & 11 \\
\hline Nickel-59 & 0 & 0 & 0 & 이 & 0 & 0 & 아 & 0 & 의 \\
\hline Total Uranium & 11 & 7933 & 9897 & 이 & 0. & 3380 & 11337 & 3124 & 17841 \\
\hline Total Thorium & 0 & 13 & 이 & 0 & o & 0 & 13 & 0 & 13 \\
\hline Total Strontium & 65 & 0 & 0 & 0 & 0 & 25 & 38 & 2 & 65 \\
\hline Other RAD & 214 & 12564 & 344 & 0 & 0 & 8318 & 1992 & 2812 & 13122 \\
\hline TOTAL RAD & 1489 & 117243 & 47546 & 01 & -0] & 94278 & 35630 & 36368 & 166277 \\
\hline & & & & & & & & & \\
\hline Other Wet Chemistry & 155 & 0 & 이 & 0 & 0 & 122 & 33 & 에 & 155 \\
\hline TCLP Extraction & 109 & 12362 & 2953 & 0 & 으, & 60 & 2389 & 12968 & 15420 \\
\hline Asbestos & 0 & 7627 & 0 & 0 & 0 & 0 & 5649 & 1978 & 7627 \\
\hline Industrial Hygiene, NOS & 4 & 0) & 0 & 0 & 0 & 1 & 3 & 0 & 5 \\
\hline Physical Properties & 32 & 11579 & 1 & 0 & 0 & 8289 & 230 & 3094 & 11612 \\
\hline Other, Not Otherwise Specified (NO & 4 & 11 & 337 & $\overline{0}$ & 0 & 10 & 1 & 340 & 352 \\
\hline TOTALOTHEA & 304 & 31579 & 3291 & 0 & 잉 & 84821 & $8 \overline{8305}$ & 18379 & 35170 \\
\hline & & & & & & & & & \\
\hline TOTAL SAMPLE ANALYSES & 3509 & 276176 & 69349 & 0 & 0 & 162450 & $66 \overline{15}$ & 122219 & 350109 \\
\hline
\end{tabular}


Table Vc: FY 95 Samples and Sample Analyses by Rad Level and Matrix Type (ix) Oak Ridge X-10

\begin{tabular}{|c|c|c|c|c|c|c|c|c|c|}
\hline $\begin{array}{c}\text { Rad-Matrix } \\
\text { Sample-Sample Analysis }\end{array}$ & Ro & R1 & $\overline{\mathrm{R2}}$ & 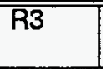 & 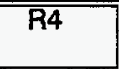 & $\overrightarrow{\mathrm{M1}}$ & M2 & M3 & TOTAL \\
\hline CUSTOMER SAMPLES & 405 & $\overline{8114}$ & 24 & 595 & 0 & 3212 & 2816 & 2058 & 8612 \\
\hline & & & & & & & & & \\
\hline \multicolumn{10}{|l|}{ SAMPLE ANALYSES } \\
\hline Volatiles by GC/MS & 142 & 537 & 4 & 66 & 0 & 144 & 516 & 89 & 748 \\
\hline Volatiles by GC & 87 & 35 & 1 & 0 & 0 & 67 & 48 & 5 & 122 \\
\hline Semi-Volatiles & 88 & 441 & 4 & 56 & 0 & 70 & 454 & 64 & 588 \\
\hline Herbicides/Pesticides/PCBs & 213 & 980 & 0 & 0 & 0 & 183 & 796 & 213 & 1193 \\
\hline Other Organics & 42 & 941 & 0 & 0 & 0 & 31 & 22 & 1 & 518 \\
\hline TOTAL ORGANICS & 571 & 2934 & 9 & 122 & 0 & 494 & 1837 & 372 & 3170 \\
\hline \multicolumn{10}{|l|}{ 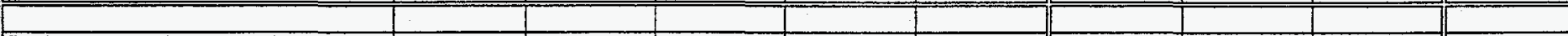 } \\
\hline RCRA Appendix IX Metals & 2 & 3 & 0 & 0 & 0 & 4 & 1 & 0 & 5 \\
\hline CAM (California) Metals (RCRA) & 44 & 1 & $\overline{0}$ & 0 & 0 & 45 & 1 & 0 & 45 \\
\hline RCRA TCLP Metals and EPTOX Me & 79 & 306 & 1 & o & 0 & 59 & 316 & 5 & 384 \\
\hline EPA CLP Metals (CERCLA) & 132 & 59 & 10 & 138 & 0 & 68 & 128 & 143 & $\overline{339}$ \\
\hline NPDES Metals (Clean Water Act) & 42 & 164 & 0 & 0 & 0 & 207 & 0 & 0 & 207 \\
\hline Drinking Water Metals (SDWA) & 11 & 0 & 0 & 0 & 은 & 11 & 0 & 0 & 11 \\
\hline Metals by Other Regulatory Suite & 173 & 188 & 0 & 0 & 0 & 106 & 231 & 46 & 372 \\
\hline Metals by Suite, Other & 0 & 999 & 2 & D) & 0 & 32 & 16 & 1 & 525 \\
\hline Metals by Metal Except Mercury & 144 & 438 & 0 & D & 0 & 116 & 179 & 283 & 580 \\
\hline Mercury & 201 & 1437 & 0 & 0 & 0 & 207 & 487 & 10 & 1171 \\
\hline Anions/Cations-IC & 79 & 46 & 0 & 0 & 0 & 99 & 17 & 9 & 125 \\
\hline Inorganics Wet Chemistry & 694 & 366 & 9 & 2 & 0 & 627 & 384 & 62 & 1072 \\
\hline Other Inorganics & 90 & 53 & 0 & 01 & 0 & 87 & 47 & 9 & 143 \\
\hline TOTAL INOAGANICS & 16911 & 4059 & 23 & 1401 & 0 & 1666 & 1808 & 570 & 4979 \\
\hline \\
\hline Gross Alpha/Beta & 701 & 3232 & 4 & (0) & o & 2557 & 421 & 30 & 3472 \\
\hline Gamma Scan & 146 & 1168 & 2 & 0 & 0 & 1006 & 291 & 19 & 1316 \\
\hline Transuranic & 0 & 12 & 0 & 0 & 으. & 0 & 12 & 0 & 12 \\
\hline Plutonium Isotopes & 235 & 20 & 3 & 0 & 0 & 171 & 79 & 7 & 257 \\
\hline Uranium Isotopes & 245 & 331 & 3 & 0 & 0 & 191 & 360 & 27 & 579 \\
\hline Curium Isotopes & 0 & 4 & 0 & 0 & 0 & 1 & 1 & 2 & 4 \\
\hline Thorium Isotopes & 2 & 272 & 1 & 5 & 0 & 9 & 263 & 8 & 280 \\
\hline Radium Isotopes & 27 & 0 & 0 & 0 & 0 & 19 & 8 & 0 & 27 \\
\hline Americium-241 & 223 & 14 & 1 & 0 & 0 & 174 & 61 & 3 & 239 \\
\hline Neptunium-237 & 2 & 17 & 2 & 0 & 0 & 1 & 14 & 6 & 21 \\
\hline Polonium-210 & 1 & 1 & 0 & 0 & 0 & 0 & 1 & 0 & 1 \\
\hline Tritium & 178 & 42 & 8 & 0 & 0 & 163 & 59 & 2 & 227 \\
\hline Radon-222 & 0 & 0 & 0 & 0 & 0 & 0 & 0 & 0 & 0 \\
\hline Lead-210 & 0 & 2 & 0 & 0 & 0 & 0 & 0 & 2 & 2 \\
\hline Carbon-14 & 0 & 0 & 0 & 0 & 0 & 0 & 0 & $\overline{0}$ & $\overline{0}$ \\
\hline Nickel-63 & 0 & 0 & 0 & of & 0 & 0 & 0 & 0 & 0 \\
\hline Selenium -79 & 44 & $\overline{1}$ & 0 & 0 & o & 45 & 0 & 0 & 45 \\
\hline Technetium-99 & 2 & 259 & 0 & 0 & 0 & 3 & 257 & 2. & 262 \\
\hline Strontium Isotopes & 0 & 0 & 0 & 0 & 은 & 0 & 0 & 0 & 0 \\
\hline Strontium -89 & 0 & 0 & 0 & 0 & 0 & 0 & 0 & 0 & 0 \\
\hline Strontium-90 & 46 & 20 & 1 & 0 & 0 & 50 & 18 & 0 & 68 \\
\hline lodine-129 & 51 & 2 & 0 & 0 & 0 & 52 & 0 & 0 & 52 \\
\hline Nickel-59 & 0 & 0 & 0 & 0 & 0 & 0 & 0 & 0 & 0 \\
\hline Total Uranium & 14 & 188 & 0 & 0 & 인 & 5 & 35 & 162 & 202 \\
\hline Total Thorium & 0 & 0 & 0 & 0 & o. & 0 & 0 & 0 & 0 \\
\hline Total Strontium & 44 & 2 & 0 & 0 & 0 & 20 & 23 & 3 & 46 \\
\hline Other RAD & 203 & 880 & 4 & 462 & o. & 110 & 941 & 492 & 1546 \\
\hline TOTAL RAD & 2165 & 6468 & 29 & 467 & 0. & 4577 & 2845 & 767. & 8659 \\
\hline \\
\hline Other Wet Chemistry & 343 & 2 & 0 & 0 & 0] & 324 & 20 & 0 & 344 \\
\hline TCLP Extraction & 66 & 359 & 7 & 366 & 0 & 53 & 328 & 412 & 795 \\
\hline Asbestos & 0 & 3431 & 0 & 0 & 0 & 0 & 2542 & 890 & 3431 \\
\hline Industrial Hygiene, NOS & 113 & 0 & 0 & 0 & 0 & 112 & 2 & 0 & $\overline{114}$ \\
\hline Physical Properties & 37 & 97 & 1 & 230 & o & 32 & 101 & 232 & 365 \\
\hline Other, Not Otherwise Specified (NO & 5 & 4 & 0 & 0 & o & 4 & 0 & 5 & 9 \\
\hline TOTAL OTHER & 565 & 3892 & 8 & 2596 & - & 525 & 2993 & 1538 & 5058 \\
\hline \multirow{2}{*}{ TOTAL SAMPLE ANALYSES } & & & t & & 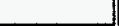 & & & & \\
\hline & 4992 & $\overline{17353}$ & 68 & 1325 & (0. & 7263 & 9484 & 3247 & 21866 \\
\hline
\end{tabular}


Table Vc: FY 95 Samples and Sample Analyses by Rad Level and Matrix Type (x) Oak Ridge Y-12

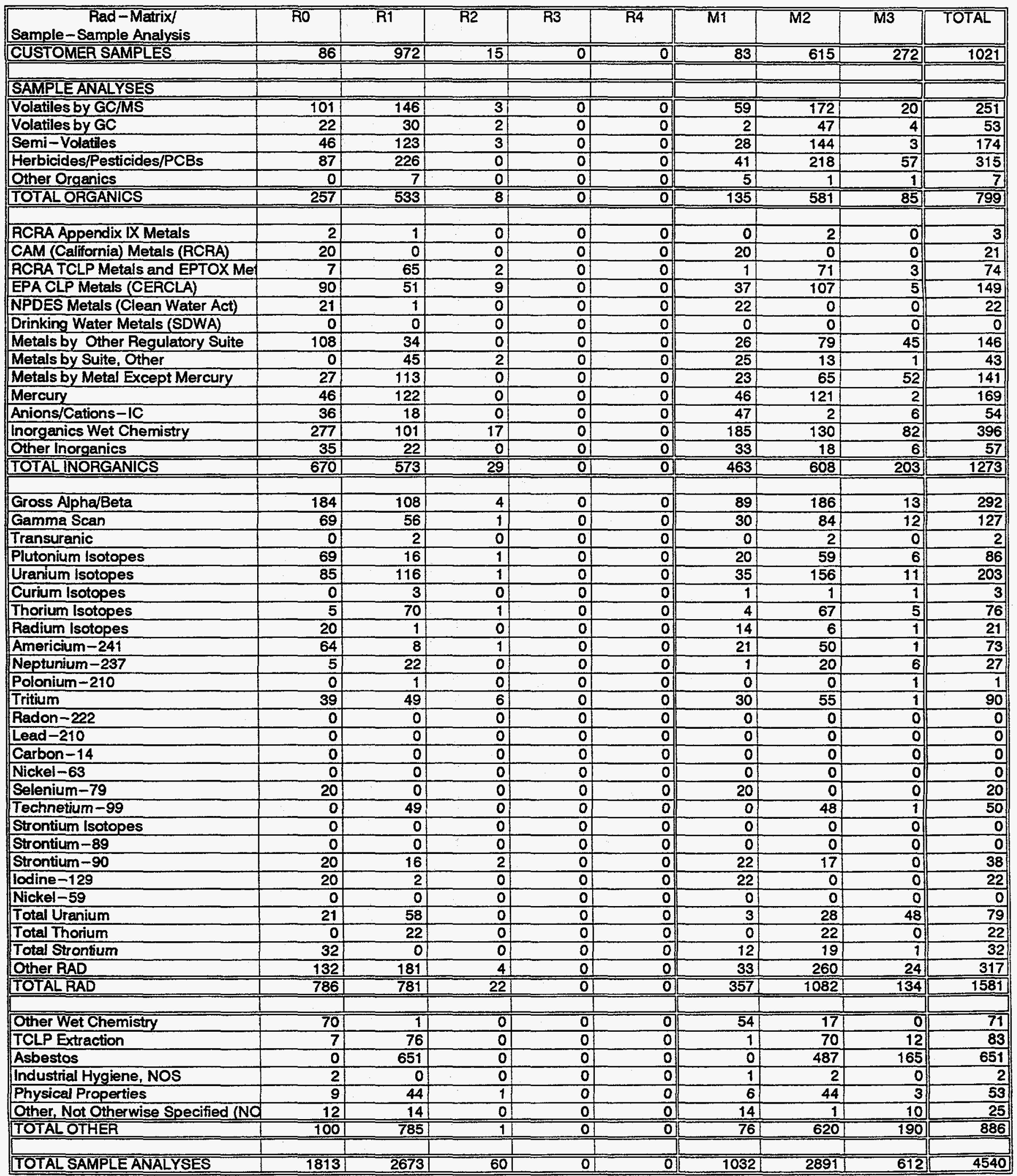


Table Vc: FY 95 Samples and Sample Analyses by Rad Level and Matrix Type (xi) Rocky Flats

\begin{tabular}{|c|c|c|c|c|c|c|c|c|c|}
\hline $\begin{array}{c}\text { Rad-Matrix } \\
\text { Sample-Sample Analysis }\end{array}$ & $\overline{\mathrm{RO}}$ & R1 & $\overline{\mathrm{R} 2}$ & $\overline{\mathrm{R} 3}$ & $\overline{\mathrm{R4}}$ & $\overline{\mathrm{MI}}$ & $\overline{\mathrm{M} 2}$ & M3 & TOTAL \\
\hline CUSTOMER SAMPLES & 2116 & 5906 & 520 & 1180 & 0 & 2003 & 3126 & 1644 & 8248 \\
\hline \multirow{2}{*}{\multicolumn{10}{|c|}{ SAMPLE ANALYSES }} \\
\hline & & & & & & & & & \\
\hline Volatiles by GCMS & 4343 & 1085 & 171 & 132 & 0 & 2686 & 2467 & 578 & 5730 \\
\hline Volatiles by GC & 912 & 247 & 19 & 0 & 0 & 345 & 768 & 46 & 1168 \\
\hline Semi-Volatiles & 1874 & 913 & 161 & 112 & (0) & 1049 & 1703 & 298 & 3055 \\
\hline Herbicides/Pesticides/PCBs & 2280 & 1372 & 0 & 0 & 0 & 1192 & 2213 & 247 & 3652 \\
\hline Other Organics & 204 & 231 & 9 & 0 & 0 & 323 & 39 & 16 & 411 \\
\hline TOTAL ORGANICS & 9613 & 3847 & 360 & 244 & 0 & 5595 & 7190 & 1185 & 14017 \\
\hline & & & & & & & & & \\
\hline RCRA Appendix IX Metals & 7 & 31 & 0 & 0 & 0] & 4 & 34 & 20 . & 48 \\
\hline CAM (California) Metals (RCRA) & 0 & 2 & 0 & 0 & 0 & 2 & 3 & 1 & 4 \\
\hline RCRA TCLP Metals and EPTOX Me & 385 & 308 & 170 & $\mathbf{0}$ & 0 & 295 & 216 & 330 & 852 \\
\hline EPA CLP Metals (CERCLA) & 5253 & 1653 & 243 & 276 & 0 & 2510 & 4262 & 653 & 7425 \\
\hline NPDES Metals (Clean Water Act) & 594 & 19 & 0 & 0 & 0 & 613 & 0 & o & 613 \\
\hline Drinking Water Metals (SDWA) & 92 & 0 & 0 & 0 & 0 & 92 & 이 & o & 92 \\
\hline Metals by Other Regulatory Suite & 4736 & 315 & 69 & 0 & 0 & 2386 & 2417 & 438 & 5181 \\
\hline Metals by Suite, Other & 0 & 1205 & 46 & 0 & 0 & 681 & 376 & 7 & 1157 \\
\hline Metals by Metal Except Mercury & 450 & 975 & 73 & 0 & 0 & 683 & 324 & 67 & 1286 \\
\hline Mercury & 960 & 657 & 13 & 0 & 0 & 1473 & 326 & 109 & 1769 \\
\hline Anions/Cations-IC & 932 & 818 & 32 & 0 & 0 & 1313 & 36 & 114 & 1622 \\
\hline Inorganics Wet Chemistry & 12718 & 995 & 145 & 4 & 0 & 10950 & 2311 & 946 & 14035 \\
\hline Other Inorganics & 831 & 378 & 0 & 0 & 0 & 802 & 294 & 113 & 1209 \\
\hline TOTALINORGANICS & 26958 & 7357 & 791 & 280 & 0 & 21805 & 10599 & 2798 & 35294 \\
\hline & & & & & & & & & \\
\hline Gross Alpha/Beta & 9630 & 2633 & 91 & 0 & 으. & 5455 & 6464 & 300 & 12286 \\
\hline Gamma Scan & 3828 & 684 & 46 & 0 & o & 1848 & 2445 & 265 & 4558 \\
\hline Transuranic & 0 & 270 & 277 & 0 & 0. & 369 & 178 & 0 & 547 \\
\hline Plutonium Isotopes & 4757 & 457 & 34 & 0 & 0 & 2002 & 3075 & 171 & 5248 \\
\hline Uranium Isotopes & 4646 & 1538 & 250 & $\overline{0}$ & 0 & 2589 & 3579 & 265 & 6434 \\
\hline Curium Isotopes & 0 & 58 & 0 & 0 & 0 & 25 & 25 & 8 & 58 \\
\hline Thorium Isotopes & 39 & 547 & 34 & 0 & 0 & 292 & 193 & 136 & 621 \\
\hline Radium Isotopes & 1888 & 7 & 0 & 0 & 0 & 1438 & 450 & 7 & 1896 \\
\hline Americium-241 & 4542 & 204 & 25 & 0 & 0 & 1977 & 2692 & 103 & 4772 \\
\hline Neptunium-237 & 39 & 374 & 186 & 0 & 0 & 209 & 249 & 143 & 600 \\
\hline Polonium-210 & 2 & 8 & 9 & 0 & 0 & 1 & 2 & 17 & 20 \\
\hline Tritium & 2763 & 878 & 133 & 0 & 0 & 2036 & 1649 & 22 & 3740 \\
\hline Radon-222 & 1 & 0 & 0 & 0 & 0 & 1 & 0 & 0 & 1 \\
\hline Lead-210 & 0 & 0 & 0 & 0 & 0 & 0 & 0 & 0 & 0 \\
\hline Carbon-14 & 0 & 0 & 0 & 0 & 0 & 0 & 0 & 0 & 0 \\
\hline Nickel-63 & 0 & 0 & 0 & 0 & 0 & 0 & 0 & 0 & 0 \\
\hline Selenium -79 & 0 & 0 & 0 & 0 & 0 & 0 & 0 & 0 & 0 \\
\hline Technetium -99 & 20 & 393 & 294 & 0 & 0 & 396 & 206 & 103 & 706 \\
\hline Strontium Isotopes & 0 & 1 & 0 & 0 & 0 & 0 & 0 & 0 & 1 \\
\hline Strontium - 89 & 0 & 0 & 0 & 0 & 0 & 0 & 0 & 0 & 0 \\
\hline Strontium - 90 & 20 & 370 & 22 & 0 & 0 & 55 & 358 & 0 & 412 \\
\hline lodine-129 & 56 & 15 & 0 & 0 & 0 & 71 & 0 & 0 & 71 \\
\hline Nickel-59 & 0 & 0 & 0 & 0 & 0 & 0 & 0 & 0 & 0 \\
\hline Total Uranium & 216 & 218 & 271 & 0 & 0 & 160 & 308 & 237 & 705 \\
\hline Total Thorium & 0 & 1 & 0 & 0 & 0. & 1 & 0 & 0 & 1 \\
\hline Total Strontium & 2503 & 0 & 0 & 0 & 0 & 1296 & 1172 & 35 & 2503 \\
\hline Other RAD & 6145 & 988 & 102 & 924 & 0 & 2332 & 4649 & 1178 & 8159 \\
\hline TOTAL RAD & 41097 & 9644 & 1774 & 924 & 0 & 22554 & 27695 & 2992 & 53339 \\
\hline & & & & & & & & & \\
\hline Other Wet Chemistry & 6404 & 8 & 0 & 0 & 0 & 5399 & 1012 & 으. & 6411 \\
\hline TCLP Extraction & 387 & 538 & 86 & 732 & 0 & 321 & 266 & 1118 & 1724 \\
\hline Asbestos & 25 & 155 & 0 & 0 & 0 & 1 & 179 & 0 & 180 \\
\hline Industrial Hygiene, NOS & 64 & 0 & 0 & 0 & 0 & 18 & 46 & 0 & 64 \\
\hline Physical Properties & 1004 & 425 & 15 & 460 & 0 & 468 & 865 & 570 & 1903 \\
\hline Other, Not Otherwise Specified (NO & 80 & 50 & 9 & 0 & 0 & 45 & 0 & 90 & 137 \\
\hline TOTAL OTHER & 7964 & 1176 & 110 & 1192 & 0 & 6251 & 2369 & 1778 & 10420 \\
\hline TOTAL SAMPLE ANALYSES & 85631 & 22023 & 3035 & 2640 & 0 & 56206 & 47853 & 8752 & 113070 \\
\hline
\end{tabular}


Table Vc: FY 95 Samples and Sample Analyses by Rad Level and Matrix Type (xii) Savannah River

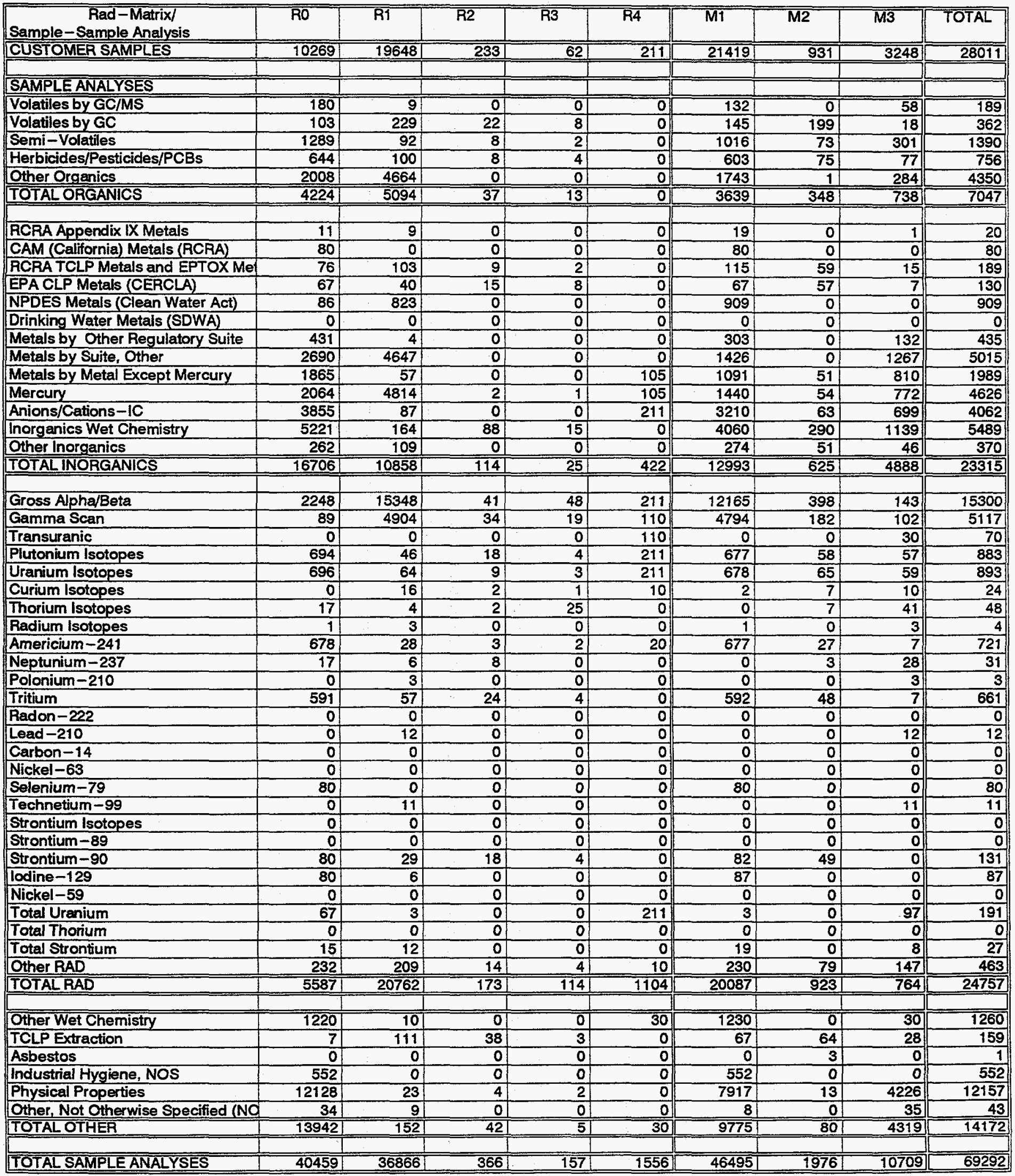


Table Vd: FY 96 Samples and Sample Analyses by Rad Level and Matrix Type (i) Complex-wide

\begin{tabular}{|c|c|c|c|c|c|c|c|c|c|}
\hline $\begin{array}{c}\text { Rad-Matrix/ } \\
\text { Sample-Sample Analysis }\end{array}$ & $\overline{\overline{\text { ROO }}}$ & R1 & $\overline{\mathrm{R} 2}$ & "R3 & R4 & $\overline{\mathrm{M1}}$ & M2 & M3 & TOTAL \\
\hline CUSTOMER SAMPLES & 52746 & 181575 & 3306 & 6198 & 624 & 98650 & 69207 & 42987 & 227646 \\
\hline & & & & & & & & & \\
\hline \multicolumn{10}{|l|}{ SAMPLE ANALYSES } \\
\hline Volatiles by GC/MS & 19747 & 13725 & 723 & 663 & 0. & 13150 & 18591 & 3138 & 34869 \\
\hline Volatiles by GC & 6409 & 2687 & 191 & 34 & 0 & 3495 & 5031 & 547 & 9197 \\
\hline Semi-Volatiles & 12642 & 11743 & 703 & 571 & 0 & 8022 & 15156 & 2485 & 25662 \\
\hline Herbicides/Pesticides/PCBs & 12704 & 23408 & 34 & 17 & 0 & 8362 & 22624 & 5223 & 36186 \\
\hline Other Organics & 11137 & 21267 & 34 & 0 & 0 & 7201 & 4196 & 952 & 22394 \\
\hline TOTAL ORGANICS & 62640 & 72830 & 1685 & 1284 & 0] & 40230 & 65598 & 12346 & 128308 \\
\hline \multicolumn{10}{|l|}{12} \\
\hline CAM (California) Metals (RCRA) & 575 & 142 & 0 & 0 & o & $\frac{105}{717}$ & 177 & $\frac{80}{35}$ & $\frac{322}{823}$ \\
\hline RCRA TCLP Metals and EPTOX Mel & 4428 & 7379 & 699 & 7 & 0 & 3206 & 7340 & 1518 & 12288 \\
\hline EPA CLP Metals (CERCLA) & 22308 & 7400 & 1184 & 1420 & 0 & 11210 & 18191 & 2911 & 32313 \\
\hline NPDES Metals (Clean Water Act) & 3084 & 3595 & 0 & 0 & 0 & 6678 & 0 & 0 & 6678 \\
\hline Drinking Water Metals (SDWA) & 729 & 0 & 0 & 0 & 0 & 729 & 0 & 0 & 729 \\
\hline Metals by Other Regulatory Suite & 21757 & 4689 & 258 & 0 & 0 & 11568 & 12903 & 3004 & 27089 \\
\hline Metals by Suite, Other & 7720 & 25650 & 205 & 0 & 0 & 7261 & 1688 & 3688 & 23106 \\
\hline Metals by Metal Except Mercury & 9927 & 12377 & 287 & 0 & 302 & 7810 & 5686 & 7437 & 21913 \\
\hline Mercury & 13023 & 32434 & 70 & 3 & 302 & 13181 & 10661 & 2810 & 36242 \\
\hline Anions/Cations - IC & 15553 & 4141 & 121 & 0 & 605 & 15501 & 558 & 2652 & 19565 \\
\hline Inorganics Wet Chemistry & 68783 & 13265 & 1021 & 87 & 0 & 59689 & 16579 & 8609 & 84017 \\
\hline Other Inorganics & 6391 & 2959 & 0 & 0 & 0 & 5462 & 3159 & 728 & 9350 \\
\hline TOTAL INORGANICS & 174382 & 114211 & 3845 & 1517 & 1210 & 143115 & 77119 & 33472 & 274436 \\
\hline \multicolumn{10}{|l|}{ 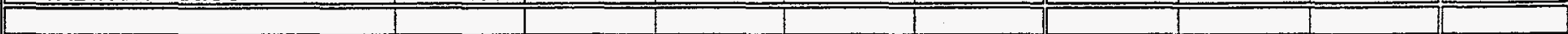 } \\
\hline Gamma Scan & 18025 & $\frac{01000}{27722}$ & 351 & $\frac{210}{84}$ & 317 & $\frac{16446}{28681}$ & $\frac{34530}{15930}$ & $\frac{2234}{1658}$ & $\frac{124082}{46384}$ \\
\hline Transuranic & 0 & 1216 & 1033 & 0 & 317 & 1378 & 872 & 86 & 2451 \\
\hline Plutonium Isotopes & 23208 & 2597 & 224 & 17 & 605 & 11516 & 13628 & 988 & 26391 \\
\hline Uranium Isotopes & 23104 & 12953 & 988 & 15 & 605 & 14289 & 20937 & 1921 & 37407 \\
\hline Curium Isotopes & 0 & 328 & 10 & 5 & 29 & 122 & 142 & 79 & 358 \\
\hline Thorium Isotopes & 248 & 6956 & 155 & 108 & 0 & 1249 & 5491 & 727 & 7468 \\
\hline Radium Isotopes & 7240 & 46 & 0 & 0 & 0 & 5500 & 1740 & 45 & 7286 \\
\hline Americium-241 & 21987 & 1339 & 128 & 8 & 58 & 11340 & 11622 & 500 & 23491 \\
\hline Neptunium - 237 & 248 & 2071 & 729 & 0 & 0 & 835 & 1503 & 710 & 3048 \\
\hline Polonium-210 & 161 & 52 & 34 & 0 & 0 & 6 & 161 & 80 & 248 \\
\hline Tritium & 13797 & 4683 & 701 & 17 & 0 & 10941 & 7754 & 159 & 19026 \\
\hline Radon-222 & 4 & 0 & 0 & 0 & 0 & 4 & 0 & 0 & 4 \\
\hline Lead-210 & 0 & 52 & 0 & 0 & 0 & 0 & 0 & 52 & 52 \\
\hline Carbon-14 & 0 & 0 & 0 & 0 & 0 & 0 & 0 & 0 & 0 \\
\hline Nickel-63 & 0 & 0 & 0 & 0 & 0 & 0 & 0 & 0 & 0 \\
\hline Selenium-79 & 575 & 122 & 0 & 0 & 0 & 644 & 31 & 23 & 697 \\
\hline Technetium-99 & 155 & 5876 & 1101 & 0 & 0 & 1566 & 5129 & 437 & 7132 \\
\hline Strontium Isotopes & 0 & 5 & 0 & 0 & 0 & 0 & 2 & 3 & 5 \\
\hline Strontium -89 & 0 & 0 & 0 & 0 & 0 & 0 & 0 & 0 & 0 \\
\hline Strontium-90 & 730 & 2452 & 178 & 17 & 0 & 1207 & 2101 & 76 & 3381 \\
\hline lodine-129 & 1017 & 270 & 0 & 0 & 0 & 1188 & 61 & 38 & 1288 \\
\hline Nickel-59 & 0 & 0 & 0 & 0 & 0 & 0 & 0 & 0 & 0 \\
\hline Total Uranium & 1318 & 4845 & 1010 & 0 & 605 & 789 & 2274 & 4196 & 7519 \\
\hline Total Thorium & 0 & 526 & 0 & 0 & 0 & 64 & 462 & 0 & 526 \\
\hline Total Strontium & 9878 & 52 & 0 & 0 & O. & 5091 & 4649 & 190 & 9930 \\
\hline Other RAD & 26689 & 19953 & 522 & 4658 & 29 & 11480 & 33739 & 6551 & 51811 \\
\hline TOTAL RAD & 200273 & 175785 & $7 \overline{743}$ & 5140 & 3169 & 184334 & 162765 & 20755 & 379982 \\
\hline \\
\hline Other Wet Chemistry & 30531 & 76 & Dा & $\overline{0}$ & 86 & 26603 & 4004 & 86 & 30693 \\
\hline TCLP Extraction & 4228 & 9505 & 502 & 3690 & o) & 3629 & 7896 & 6090 & 17770 \\
\hline Asbestos & 145 & 59009 & 0 & 0 & o & 3 & 44070 & 15092 & 59160 \\
\hline Industrial Hygiene, NOS & 2773 & 69 & 0 & 0 & D & 2598 & 236 & 8 & 2841 \\
\hline Physical Properties & 39093 & 3776 & 85 & 2317 & 에 & 25113 & 5272 & 14885 & 45271 \\
\hline Other, Not Otherwise Specified (No & 536 & 531 & 34 & 0 & 0 & 497 & 28 & 542 & 1084 \\
\hline TOTAL OTHER & 77305 & 72966 & 621 & 6007 & 86 & 58444 & 61506 & 36703 & 156819 \\
\hline & & & & & & & & & \\
\hline TOTAL SAMPLE ANALYSES & 514601 & 435792 & 13894 & 13948 & 4465 & 426124 & 366988 & 103277 & 939545 \\
\hline
\end{tabular}


Table Vd: FY 96 Samples and Sample Analyses by Rad Level and Matrix Type (ii) EM-30

\begin{tabular}{|c|c|c|c|c|c|c|c|c|c|}
\hline $\begin{array}{c}\text { Rad-Matrix } \\
\text { Sample-Sample Analysis }\end{array}$ & Ro & R1 & $\mathrm{R} 2$ & R3 & R4 & M1 & M2 & M3 & TOTAL \\
\hline CUSTOMER SAMPLES & 290611 & 79094 & 1069 & 52701 & $561]$ & 76978 & 4210 & 15291 & 105768 \\
\hline \multicolumn{8}{|l|}{ SAMPLE ANALYSES } & & \\
\hline Volatiles by GC/MS & 1200 & 1160 & 285 & 571 & 0 & 575 & 419 & 2221 & 3215 \\
\hline Volatiles by GC & 1076 & 1592 & 115 & 15 & -0 & 1075 & 1502 & 171 & 2773 \\
\hline Semi-Volatiles & 3724 & 1130 & 268 & 488 & 0 & 2965 & 776 & 1868 & 5610 \\
\hline Herbicides/Pesticides/PCBs & 3060 & 2062 & 15 & 8 & 요 & 3764 & 211 & 1170 & 5145 \\
\hline Other Organics & 5596 & 18275 & 32 & 0 & [] [ & 4917 & 6 & 816 & 14821 \\
\hline TOTAL ORGANICS & 14656 & 24219 & 715 & 1081 & (0) & 13297| & 2914 & 6247 & 31564 \\
\hline & & & & & & & & & \\
\hline RCRA Appendix IX Metals & 42 & 38 & 으 & 0 & ㅇ․ & 74 & 0 & 74. & 114 \\
\hline CAM (California) Metals (RCRA) & 246 & 1 & 0 & 0 & 요 & 247 & 1 & 0 & 247 \\
\hline RCRA TCLP Metals and EPTOX Me & 856 & 1035 & 613 & 3 & 이 & 851 & 467 & 1203 & 2514 \\
\hline EPA CLP Metals (CERCLA) & 383 & 1060 & 31 & 1208 & of & 376 & 132 & 2173 & 2682 \\
\hline NPDES Metals (Clean Water Act) & 335 & 3247 & 0 & 0 & 0] & 3582 & 0 & 0 & 3582 \\
\hline Drinking Water Metals (SDWA) & 128 & 0 & 0 & 0 & o] & 128 & 0 & 0] & 128 \\
\hline Metals by Other Regulatory Suite & 2929 & 1107 & 239 & 0 & -0] & 2148 & 233 & 1894 & 4275 \\
\hline Metals by Suite, Other & 7155 & 18206 & 0 & 0 & 0] & 3797 & 1 & 3399 & 16279 \\
\hline Metals by Metal Except Mercury & 5760 & 2852 & 244 & 0 & 280 & 4707 & 451 & 2394 & 8344 \\
\hline Mercury & 6483 & 20201 & 40 & $2 \mid$ & 280 & 6819 & 652 & 2446 & 18462 \\
\hline Anions/Cations-IC & 10661 & 1615 & 112 & 0 & 561 & 8845 & 240 & 2280 & 12156 \\
\hline Inorganics Wet Chemistry & 18841 & 1387 & 811 & 48 & 0 & 13428 & 2052 & 6784 & 21675 \\
\hline Other Inorganics & 1175 & 937 & 0 & of & 0 & 1270 & 264 & 577 & 2111 \\
\hline TOTAL INORGANICS & 54992 & 51685 & 2091 & 1261 & 1121] & 4 & 4494 & 23224 & 92570 \\
\hline Gross Alpha/Beta & & & & & & & & & \\
\hline Gamma Scan & $\frac{5000}{1253}$ & 19569 & 100 & $\begin{array}{ll}30 \\
38\end{array}$ & $\frac{501}{294}$ & $\frac{50034}{18796}$ & $\frac{1040}{1308}$ & 937 & $\frac{62805}{21148}$ \\
\hline Transuranic & 0 & 933 & 958 & 0 & 294 & 1277 & 613 & 80 & 2077 \\
\hline Plutonium Isotopes & 3079 & 422 & 86 & 8 & 561 & 2777 & 236 & 663 & 3915 \\
\hline Uranium Isotopes & 2974 & 1837 & 809 & 7 & 561 & 4320 & 407 & 980 & 5948 \\
\hline Curium Isotopes & 0 & 112 & 5 & 2 & 27[ & 25 & 25 & 69 & 132 \\
\hline Thorium Isotopes & 209 & 1090 & 36 & 96 & 요 & 782 & 14 & 635 & 1431 \\
\hline Radium Isotopes & 33 & 39 & 0 & 0 & - & 33 & 0 & 38 & 71 \\
\hline Americium-241 & 2767 & 327 & 7 & 4 & 53 & 2746 & 97 & 262 & 3131 \\
\hline Neptunium-237 & 209 & 743 & 675 & 0 & ㅁ․ & 665 & 352 & 610 & 1627 \\
\hline Polonium-210 & 1 & 40 & 32 & 0 & 요 & 1 & 1 & 71. & 73 \\
\hline Tritium & 2481 & 445 & 134 & 8 & a & 2420 & 261 & 26 & 2887 \\
\hline Radon-222 & 4 & 0 & 0 & 0 & (2) & 4 & 0 & 0 & 4 \\
\hline Lead-210 & o & 47 & 0 & 0 & a. & 0 & of & 47 & 47 \\
\hline Carbon-14 & 0 & of & 0 & 0 & 요 & 0 & 0 & 의 & 의 \\
\hline \begin{tabular}{|l|} 
Nickei-63 \\
\end{tabular} & 0 & 0 & 0 & 0 & 0. & 0 & 0 & 요 & 0 \\
\hline Selenium-79 & 246 & 의 & 0 & 0 & 0 & 246 & 요 & -0 & 246 \\
\hline Technetium-99 & 27 & 1300 & 990 & 0 & (0) & 1304 & 612 & 401 & 2317 \\
\hline Strontium Isotopes & 0 & 4 & 0 & 0 & ㅇ․ & 0 & 2 & 2 & 4 \\
\hline Strontium -89 & 0 & 0 & 0 & 0 & (0) & 0 & 0 & 0 & 요 \\
\hline Strontium-90 & 273 & 85 & 103 & 8 & 0 & 278 & 190 & 0 & 468 \\
\hline lodine-129 & 323 & 80 & 0 & 0 & 의 & 403 & 0 & ㅇ․ & 403 \\
\hline Nickel-59 & 0 & 0 & 0 & 0 & 0. & 0 & 0 & 의 & 0 \\
\hline Total Uranium & 857 & 753 & 936 & 의 & 561 & 387 & 1065 & 1175 & 2867 \\
\hline Total Thorium & 0 & 0 & 0 & 0 & 0 & 0 & 0 & 0. & ㅇ. \\
\hline Total Strontium & 90 & 47 & 0 & 0 & - & 93 & 13 & 31 & 137 \\
\hline Other RAD & 1697 & 1944 & 132 & 4002 & 27 & 1922 & 364 & 5439 & 7763 \\
\hline TOTALRAD & 26023 & 92123 & 5182 & 4266 & 2937 & 88512 & 7399 & 12559 & 119501 \\
\hline & & & & & & & & & \\
\hline Other Wet Chemistry & 4955 & 66 & o & 可 & $80]$ & 5021 & $\overline{0}$ & 80 & 5101 \\
\hline TCLP Extraction & 572 & 1725 & 424 & 3170 & (0) & 665 & 559 & 4612 & 5863 \\
\hline Asbestos & 1 & 2 & 0 & 0 & 0 & $\overline{0}$ & 13 & - & 8 \\
\hline Industrial Hygiene, NOS & 2163 & 0 & $\overline{0}$ & 0 & 0 & 2160 & 3 & 요 & 2163 \\
\hline Physical Properties & 32478 & 1283 & 8 & 1991 & 0. & 22097 & 34 & 13630 & 35760 \\
\hline Other, Not Otherwise Specified (NO & 423 & 46 & 32 & 0 & 0 & 35 & 0 & 460 & 498 \\
\hline TOTAL OTHER & 40591 & 3121 & 464 & 5162 & 80 & 29978 & 609 & 18782 & 49393 \\
\hline TOTAL SAMPLE ANALYSES & 136262 & 171148 & 8453 & 1770 & 4138 & & 5415 & & \\
\hline
\end{tabular}


Table Vd: FY 96 Samples and Sample Analyses by Rad Level and Matrix Type (iii) EM-40

\begin{tabular}{|c|c|c|c|c|c|c|c|c|c|}
\hline $\begin{array}{c}\text { Rad-Matrix/ } \\
\text { Sample-Sample Analysis }\end{array}$ & $\overline{R O}$ & $\overline{\text { R1 }}$ & R2 & $\overline{\mathrm{R} 3}$ & R4 & M1 & $\overline{\mathrm{M} 2}$ & M3 & TOTAL \\
\hline CUSTOMER SAMPLES & 18661 & 94366 & 2118 & 506 & 19 & 12949 & 63284 & 26202 & 109052 \\
\hline & & & & & & & & & \\
\hline \multicolumn{10}{|l|}{ SAMPLE ANALYSES } \\
\hline Volatiles by GC/MS & 13124 & 12343 & 411 & 47 & 0 0] & 8556 & 16657 & 734 & 25936 \\
\hline Volatiles by GC & 3034 & 903 & 45 & 17 & 0 & 465 & 3188 & 359 & 4005 \\
\hline Semi-Volatiles & 6744 & 10412 & 410 & 44 & 0 & 3902 & 13247 & 465 & 17612 \\
\hline Herbicides/Pesticides/PCBs & 7315 & 20953 & 17 & 9 & 0 & 3536 & 20890 & 3914 & 28317 \\
\hline Other Organics & 3963 & 1345 & 0 & 0 & 0 & 844 & 4095 & 70 & 5158 \\
\hline TOTAL ORGANICS & 34180 & 45956 & 883 & 116 & 0 & 17303 & 58077 & 5541 & 81028 \\
\hline \multicolumn{10}{|l|}{ 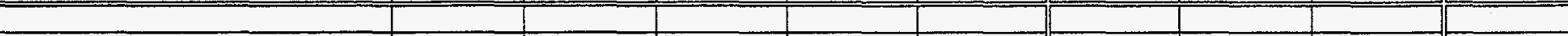 } \\
\hline RCRA Appendix IX Metals & 40 & 138 & 0 & 0 & 0] & 11 & 168 & 0 & 178 \\
\hline CAM (California) Metals (RCRA) & 307 & 137 & 0 & 0 & 0 & 444 & 171 & 34 & 546 \\
\hline RCRA TCLP Metals and EPTOX Me & 1475 & 6140 & 16 & 3 & 0 & 626 & 6558 & 218 & 7518 \\
\hline EPA CLP Metals (CERCLA) & 14135 & 6191 & 1139 & 115 & 0 & 6458 & 14569 & 552 & 21580 \\
\hline NPDES Metals (Clean Water Act) & 2689 & 57 & 0 & 0 & 0 & 2746 & 0 & 0 & 2746 \\
\hline Drinking Water Metals (SDWA) & 66 & 0 & 0 & 0 & 0 & 66 & 0 & 0 & 66 \\
\hline Metals by Other Regulatory Suite & 11501 & 3458 & 0 & 0 & 0 & 4947 & 9832 & 943 & 15340 \\
\hline Metals by Suite, Other & 0 & 5756 & 203 & 0 & 0 & 3131 & 1670 & 20 & 5390 \\
\hline Metals by Metal Except Mercury & 1578 & 9146 & 3 & of & 0 & 942 & 4954 & 4803 & 10713 \\
\hline Mercury & 3985 & 10268 & 6 & 2 & 0 & 4099 & 9664 & 169 & 14096 \\
\hline Anions/Cations-IC & 3978 & 2370 & 0 & 0 & 0 & 5864 & 294 & 190 & 6348 \\
\hline Inorganics Wet Chemistry & 21846 & 11641 & 144 & 35 & 0 & 20605 & 12225 & 1275 & 33886 \\
\hline Other Inorganics & 4924 & 1925 & 0 & 0 & 0] & 3922 & 2823 & 103 & 6848 \\
\hline \multirow[t]{2}{*}{ TOTAL INORGANICS } & 66524 & 57226 & 1511 & 156 & 0. & 53862 & 62927 & 8307 & 125256 \\
\hline & & & & & & & & & \\
\hline Gross Alpha/Beta & 27589 & 13686 & 380 & 106 & 0 & 15111 & 25313 & 1025 & 41605 \\
\hline Gamma Scan & 9518 & 6337 & 240 & 43 & 0 & 5431 & 10077 & 631 & 16139 \\
\hline Transuranic & 0 & 202 & 0 & 0 & 0 & 0 & 202 & 0 & 202 \\
\hline Plutonium Isotopes & 11353 & 2112 & 129 & 9 & 0 & 4261 & 9080 & 262 & 13603 \\
\hline Uranium Isotopes & 13099 & 10844 & 114 & 8 & 0 & 6117 & 17105 & 842 & 24064 \\
\hline Curium Isotopes & 0 & 205 & 5 & 3 & ㅁ․ & 94 & 113 & 5 & 212 \\
\hline Thorium Isotopes & 22 & 5719 & 116 & 4 & - & 401 & 5419 & 40 & 5861 \\
\hline Radium Isotopes & 3595 & 4 & 0 & 0 & o. & 2593 & 1003 & 4 & 3600 \\
\hline Americium-241 & 11139 & 975 & 119 & 4 & 0. & 4247 & 7782 & 208 & 12237 \\
\hline Neptunium-237 & 22 & 1256 & 1 & 0 & 0 & 116 & 1112 & 51 & 1279 \\
\hline Polonium-210 & 156 & 5 & 0 & 0 & 0 & 1 & 156 & 4 & 161 \\
\hline Tritium & 6246 & 4159 & 550 & 9 & 0. & 4845 & 6032 & 129 & 10985 \\
\hline Radon-222 & 0 & 0 & 0 & 0 & 0 & 0 & 0 & 0 & of \\
\hline Lead-210 & 0 & 1 & 0 & 0 & 0 & 0 & 0 & 1 & 1 \\
\hline Carbon-14 & 0 & 0 & 0 & 0 & 0 & 0 & 0 & 0 & 0 \\
\hline Nickel-63 & 0 & 0 & 0 & 0 & 0 & 0 & 0 & 0 & 0 \\
\hline Selenium-79 & 307 & 121 & 0 & 0 & 0 & 375 & 30 & 23 & 428 \\
\hline Technetium-99 & 14 & 4426 & 33 & 0 & o. & 47 & 4422 & 4 & 4473 \\
\hline Strontium Isotopes & 0 & 0 & 0 & 0 & 0 & 0 & 0 & 0 & 0 \\
\hline Strontium - 89 & 0 & 0 & 0 & 0 & 0 & 0 & 0 & 0 & 0 \\
\hline Strontium -90 & 321 & 2336 & 67 & 9 & 0 & 787 & 1877 & 76 & 2736 \\
\hline lodine-129 & 347 & 182 & 0 & 0 & 0 & 431 & 61 & 38 & 530 \\
\hline Nickel-59 & 0 & 0 & 0 & 0 & 0 & 0 & 0 & 0 & 0. \\
\hline Total Uranium & 279 & 3984 & 0 & 0 & 0 & 257 & 1114 & 2893 & 4263 \\
\hline Total Thorium & 0 & 519 & 0 & 0 & 0 & 62 & 457 & O) & 519 \\
\hline Total Strontium & 5360 & 1 & 0 & 0 & 0 & 2358 & 2856 & 147) & 5361 \\
\hline Other RAD & 14959 & 17614 & 355 & 337 & 0 & 3939 & 28655 & 674 & 33266 \\
\hline TOTAL RAD & 104328 & 74690 & 2108 & 530 & 0] & 51472 & 122867 & 7055 & 181524 \\
\hline & & & & & & & & & \\
\hline Other Wet Chemistry & 12763 & 5 & 0 & 0 & 0] & 10213 & 2554 & 0 & 12767 \\
\hline TCLP Extraction & 1563 & 7509 & 22 & 267 & 0 & 1243 & 6988 & 1101 & 9347 \\
\hline Asbestos & 75 & 58391 & 0 & 0 & 0 & 1 & 43531 & 14935 & 58466 \\
\hline Industrial Hygiene, NOS & 398 & 68 & 0 & 0 & 0 & 238 & 220 & 8 & 466 \\
\hline Physical Properties & 1809 & 2366 & 75 & 167 & 0 & 770 & 3468 & 178 & 4416 \\
\hline Other, Not Otherwise Specified (NO & 79 & 451 & 0 & 0 & 0 & 454 & 28 & 45 & 529 \\
\hline TOTALOTHER & 16687 & 68789 & 97 & 433 & 0] & 12919 & 56789 & 16267 & 85991 \\
\hline & & & & & & & & & \\
\hline TOTAL SAMPLE ANALYSES & 221718 & 246661 & 4599 & 1235 & 0 & 135556 & 300660 & 37170 & 473800 \\
\hline
\end{tabular}


Table Vd: FY 96 Samples and Sample Analyses by Rad Level and Matrix Type (iv) EM-60

\begin{tabular}{|c|c|c|c|c|c|c|c|c|c|}
\hline $\begin{array}{c}\text { Rad-Madrix/ } \\
\text { Sample-Sample Analysis }\end{array}$ & Ro & R1 & $\mathrm{R} 2$ & $\overline{\mathrm{R3}}$ & R4 & $\overline{\overline{M 1}}$ & $\overline{\mathrm{M} 2}$ & $\overline{M 3}$ & TOTTAL \\
\hline CUSTOMER SAMPLES & 2196 & 759 & 101 & 1 & $0]$ & 2189 & 604 & 7 & 2883 \\
\hline & & & & & & & & & \\
\hline \multicolumn{10}{|l|}{ SAMPLEANALYSES } \\
\hline Volatiles by GC/MS & 4523 & $\overline{0}$ & 01 & 0 & $0]$ & 3382 & 1140 & 0 & 4523 \\
\hline Volatiles by GC & 1909 & 50 & 18 & 0 & 0 & 1631 & 165 & 0 & 1886 \\
\hline Semi-Volatiles & 1580 & 2 & 0 & 01 & 0 & 767 & 814 & 0 & 1582 \\
\hline Herbicides/Pesticides/PCBs & 1757 & 2 & 0 & 0 & 0 & 634 & 1124 & 0 & 1759 \\
\hline Other Organics & 957 & 166 & 0 & 0 & 0 & 912 & 46 & 0 & 1041 \\
\hline TOTAL ORGANICS & 10726 & 220 & 181 & 01 & 0] & 7327 & 32901 & $1]$ & 10791 \\
\hline RCRA Appendix IX Metals & 16 & 0 & 0 & 0 & $0] 1$ & 10 & 7 & 0 & 47 \\
\hline CAM (California) Metals (RCRA) & 0 & 0 & o) & 0 & o & 0 & 0 & $\frac{y}{0}$ & $\frac{17}{0}$ \\
\hline RCRA TCLP Metals and EPTOX Me & 1754 & 50 & 18 & 0 & 0 & 1448 & 179 & 0 & 1724 \\
\hline EPA CLP Metals (CERCLA) & 6641 & 0 & 0 & 0 & 0 & 3727 & 2908 & 7 & 6641 \\
\hline NPDES Metals (Clean Water Act) & 3 & 29 & 0 & 0 & of & 32 & 0 & 0 & 32 \\
\hline Drinking Water Metals (SDWA) & 458 & 0 & 0 & 0 & 0 & 458 & 0 & 0 & 458 \\
\hline Metals by Other Regulatory Suite & 6085 & 0 & 0 & 0 & o: & 3703 & 2375 & 7 & 6085 \\
\hline Metals by Suite, Other & 0 & 166 & 0 & 0 & 0 & 0 & 01 & 0 & 83 \\
\hline Metals by Metal Except Mercury & 1852 & 48 & 18 & 0 & 0 & 1555 & 167 & 0 & 1821 \\
\hline Mercury & 1750 & 218 & 18 & 0 & 0 & 1460 & 165 & 0 & 1805 \\
\hline Anions/Cations-IC & 27 & 3 & 0 & 0 & 0 & 28 & 2 & 0 & 30 \\
\hline Inorganics Wet Chemistry & 23054 & 3 & 0 & 0 & 0 & 21298 & 1757 & 1 & 23057 \\
\hline Other Inorganics & 106 & 3 & 0 & 0 & 0. & 106 & 2 & 1 & 108 \\
\hline TOTAL INORGANICS & 41746 & 519 & 54 & 0 & O] & 33825 & 75621 & 16 & 41861 \\
\hline \multicolumn{10}{|l|}{20} \\
\hline Gross Alpha/Beta & 11992 & 536 & 0 & 0 & 0. & 6262 & 6083 & 17 & 12445 \\
\hline Gamma Scan & 6163 & 178 & 0 & 0 & 0 & 2544 & 3789 & 8 & 6341 \\
\hline Transuranic & 01 & 5 & 0 & 0 & 0 & 0 & 5 & 0 & 5 \\
\hline Plutonium lsotopes & 7337 & 5 & 0 & 0 & 0 & 3676 & 3660 & 7 & 7343 \\
\hline Uranium Isotopes & 5799 & 5 & 0 & 0 & 0 & 3000 & 2798 & 7 & 5805 \\
\hline Curium Isotopes & 0 & 0 & 0 & 0 & 0 & 0 & 0 & 0 & 0 \\
\hline Thorium Isotopes & 0 & 0 & 0 & 1 & 0 & 0 & 0 & 1 & 1 \\
\hline Radium Isotopes & 3123 & 0 & 0 & 0 & 0 & 2487 & 636 & 0.1 & 3123 \\
\hline Americium-241 & 6754 & 0 & 0 & 0 & 0 & 3563 & 3184 & 7 & 6754 \\
\hline Neptunium-237 & 0 & 0 & 0 & 0 & 0 & 0 & 0 & 0 & 0 \\
\hline Polonium-210 & 0 & 4 & 0 & 0 & 0 & 4 & 0 & of & 4 \\
\hline Tritium & 4204 & 0 & 0 & 0 & 0 & 3001 & 1204 & 0 & 4205 \\
\hline Radon-222 & 0 & 0 & 0 & 0 & 0 & 0 & 0 & 0 & 0. \\
\hline Lead-210 & 0 & 0 & 0 & 0 & 0 & 0 & 0 & 0 & 므 \\
\hline Carbon-14 & 0 & 0 & 0 & 0 & 0 & 0 & 0 & 0 & 의 \\
\hline Nickel-63 & 0 & 0 & 0 & 0 & 0. & 0 & 0 & 0 & 의 \\
\hline Selenium-79 & 0 & 0 & 0 & 0 & 0 & 0 & 0 & 0 & 의 \\
\hline Technetium-99 & 97 & 0 & 0 & 0 & 0 & 97 & 0 & 0 & 98 \\
\hline Strontium Isotopes & 0 & 0 & 0 & 0 & 0 & 0 & 0 & 0 & 요 \\
\hline Strontium-89 & 0 & 0 & 0 & 0 & 0 & 0 & 0 & ㅇ․ & 0 \\
\hline Strontium-90 & 97 & 0 & 0 & 0) & 0 & 97 & 0 & 0 & 97 \\
\hline lodine-129 & 278 & 0 & 0 & 0 & 0 & 278 & 0 & 0 & 278 \\
\hline Nickel-59 & 0 & 0 & 0 & 0 & 0 & 0 & 0 & 0 & 의 \\
\hline Total Uranium & 97 & 0 & 0 & 0 & 0 & 97 & o. & 0. & 97 \\
\hline Total Thorium & 0 & 0 & 0 & 0 & 0 & 0 & 0 & 0 & 의 \\
\hline Total Strontium & 3814 & 0 & 0 & 0 & 의 & 2279 & 1529 & 7 & 3814 \\
\hline Other RAD & 8510 & 48 & 18 & 0 & 0 & 4736 & 3838 & 1 & 8575 \\
\hline TOTALRAD & 58267 & 784 & 19 & 1 & 0 & 32121 & 26726 & 58 & 58987 \\
\hline & & & & & & & & & \\
\hline Other Wet Chemistry & 10736 & 0 & 0 & 0 & 0 & 9492 & 1244 & 0] & 10736 \\
\hline TCLP Extraction & 1767 & 49 & 19 & 0 & o. & 1446 & 195 & 0 & 1738 \\
\hline Asbestos & 59 & 0 & 0 & 0 & 0 & 2 & 57 & 0 & 60 \\
\hline Industrial Hygiene, NOS & 31 & 0 & 0 & 0 & - & 21 & 9 & o. & 31 \\
\hline Physical Properties & 1943 & 0 & 0 & 0 & 0 & 430 & 1514 & o. & 1943 \\
\hline Other, Not Otherwise Specified (NO & 0 & 22 & 0 & 0 & 0 & 0 & 0 & 0 & 11 \\
\hline TOTAL OTHER & 14536 & 71 & 19 & 0 & 0 & 11391 & 3019 & o. & 14518 \\
\hline & & & & & & 800 & & - & 8 - \\
\hline TOTAL SAMPLE ANALYSES & 125275 & 1594 & 111 & 1 & 0 & 84664 & 40596 & 75 & 126158 \\
\hline
\end{tabular}


Table Vd: FY 96 Samples and Sample Analyses by Rad Level and Matrix Type (v) Fernald

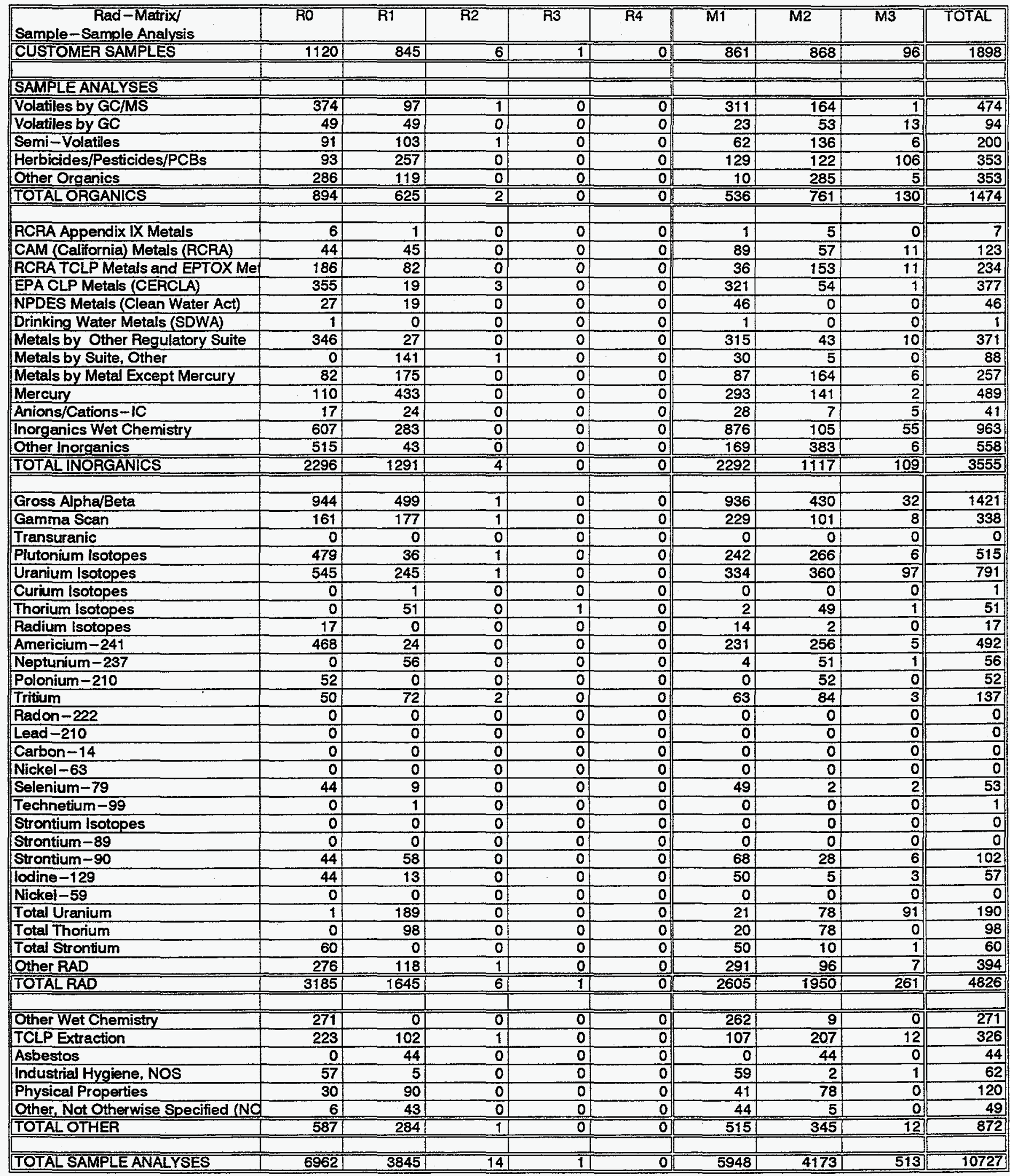


Table Vd: FY 96 Samples and Sample Analyses by Rad Level and Matrix Type (vi) INEL.

\begin{tabular}{|c|c|c|c|c|c|c|c|c|c|}
\hline $\begin{array}{c}\text { Rad-Matrix } \\
\text { Sample-Sample Analysis }\end{array}$ & Ro & R1 & $\overline{\mathrm{R} 2}$ & R3 & R4 & M1 & M2 & $\overline{\mathrm{M} 3}$ & TOTAL \\
\hline CUSTOMER SAMPLES & 3104 & 11575 & 180 & 331 & 0 & 5850 & 5631 & 2383 & 14527 \\
\hline & & & & & & & & & \\
\hline \multicolumn{10}{|l|}{ SAMPLEANALYSES } \\
\hline Volatiles by GC/MS & 220 & 674 & 2 & 33 & 0. & 193 & 613 & 123 & 928 \\
\hline Volatiles by GC & 701 & 331 & 26 & 6 & 0 & 566 & 409 & 37 & 1038 \\
\hline Semi-Volatiles & 257 & 627 & 8 & 29 & 0 & 161 & 708 & 52 & 921 \\
\hline Herbicides/Pesticides/PCBs & 306 & 1438 & 6 & 3 & 0 & 316 & 1052 & 385 & 1753 \\
\hline Other Organics & 1242 & 1306 & 0 & 0 & 0. & 288 & 992 & 14 & 1921 \\
\hline TOTAL ORGANICS & 2725 & 4375 & 42 & 71 & 0] & 1524 & 3775 & 610 & 6561 \\
\hline RCRA Appendix IX Metals & 3 & 3 & 0 & 0 & 0 & 5 & 1 & 요 & 6 \\
\hline CAM (California) Metals (RCRA) & 12 & 1 & 0 & 0 & 0 & 13 & 1 & o. & 14 \\
\hline RCRA TCLP Metals and EPTOX Met & 653 & 634 & 15 & 1 & 0 & 544 & 649 & 54 & 1276 \\
\hline EPA CLP Metals (CERCLA) & 199 & 71 & 18 & 75 & 0 & 154 & 130 & 80 & 364 \\
\hline NPDES Metals (Clean Water Act) & 39 & 225 & 0 & 0 & 0 & 264 & 0 & 0 & 264 \\
\hline Drinking Water Metals (SDWA) & 131 & 0 & 0 & 0 & 0 & 131 & 0 & D. & 131 \\
\hline Metals by Other Regulatory Suite & 418 & 330 & 0 & 0 & 0 & 216 & 302 & 238 & 752 \\
\hline Metals by Suite, Other & 0 & 1315 & 1 & 0 & 0 & 22 & 10 & 5 & 677 \\
\hline Metals by Metal Except Mercury & 714 & 755 & 5 & 0 & 0 & 544 & 492 & 382 & 1446 \\
\hline Mercury & 747 & 1996 & 6 & 1 & 0 & 604 & 813 & 24 & 2095 \\
\hline Anions/Cations-IC & 102 & 108 & 0 & 0 & o: & 121 & 43 & 46 & 211 \\
\hline Inorganics Wet Chemistry & 1291 & 1301 & 99 & 13 & 0] & 1312 & 833 & 562 & 2706 \\
\hline Other Inorganics & 151 & 226 & $\underline{0}$ & 0 & ol & 213 & 113 & 52 & 378 \\
\hline TOTAL INORGANICS & 4462 & 6966 & 146 & 90 & 0 & 4142 & 3386 & 1444 & 10318 \\
\hline Gross Alpha/Beta & 1252 & 5155 & 37 & 37 & o & 3706 & 1254 & 162 & 5802 \\
\hline Gamma Scan & 799 & 1767 & 28 & 15 & 0 & 1486 & 1050 & 72 & 2609 \\
\hline Transuranic & 0 & 17 & 0 & 0 & 0 & 0 & 17 & 0 & 17 \\
\hline Plutonium Isotopes & 472 & 138 & 9 & 3 & 0 & 365 & 210 & 47 & 622 \\
\hline Uranium Isotopes & 366 & 560 & 3 & 3 & 0 & 340 & 517 & 75 & 932 \\
\hline Curium Isotopes & 0 & 13 & 2 & 1 & a] & 3 & 7 & 6 & 16 \\
\hline Thorium Isotopes & 22 & 344 & 2 & 7 & 0 & 9 & 336 & 30 & 375 \\
\hline Radium lsotopes & 43 & 4 & 0 & 0 & 0 & 39 & 5 & 4 & 47 \\
\hline Americium-241 & 344 & 86 & 3 & 1 & 0 & 322 & 94 & 19 & 435 \\
\hline Neptunium-237 & 22 & 44 & 2 & 0 & o & 10 & 26 & 31 & 68 \\
\hline Polonium-210 & 1 & 5 & 은 & 0 & 0 & 1 & 1 & 4 & 6 \\
\hline Tritium & 357 & 139 & 20 & 3 & 0 & 309 & 162 & 10 & 500 \\
\hline Radon-222 & 0 & 0 & 0 & 0 & 0 & 0 & 0 & 0 & 0 \\
\hline Lead-210 & 0 & 3 & 0 & 0 & 0 & 0 & 0 & 3 & 3 \\
\hline Carbon-14 & 0 & 0 & 0 & 0 & 0 & 0 & 0 & 0 & 0 \\
\hline Nickel-63 & 0 & $\underline{0}$ & 0 & 0 & of & 0 & 0 & -0 & 0 \\
\hline Selenium-79 & 12 & 31 & 0 & 0 & o & 30 & 8 & 6 & 43 \\
\hline Technetium-99 & 28 & 334 & 0 & 0 & 0 & 28 & 328 & 6 & 362 \\
\hline Strontium Isotopes & 0 & 0 & 0 & 0 & ㅁ․ & 0 & 0 & o. & 0 \\
\hline Strontium -89 & 0 & 0 & 0 & 0 & 요 & 0 & 0 & 의 & 0 \\
\hline Strontium -90 & 40 & 189 & 18 & 3 & o. & 120 & 110 & 20 & 249 \\
\hline lodine-129 & 92 & 54 & 0 & 0 & 0 & 120 & 16 & 10 & 145 \\
\hline Nickel-59 & 0 & 0 & 0 & 0 & 의 & 0 & 0 & 요 & 0 \\
\hline Total Uranium & 116 & 244 & 0 & 0 & 0 & 33 & 45 & 282 & 360 \\
\hline Total Thorium & 0 & 0 & 0 & 0 & 0 & 0 & 0 & 0 & 0 \\
\hline Total Strontium & 59 & 3 & 0 & 0 & o & 32 & 27 & 3 & 62 \\
\hline Other RAD & 466 & 1392 & 21 & 234 & 0 & 441 & 1290 & 373 & 2109 \\
\hline TOTAL RAD & 4490 & 10522 & 146 & 308 & 0 & 7393 & 5504 & 1164 & 14763 \\
\hline & & & & & & & & & \\
\hline Other Wet Chemistry & 555 & $\overline{6}$ & 0 & $\overline{0}$ & (0) & 549 & $\overline{12}$ & $\overline{0}$ & 561 \\
\hline TCLP Extraction & 634 & 816 & 18 & $\overline{185}$ & 0 & 631 & 671 & 296 & 1625 \\
\hline Asbestos & 0 & 4383 & 0 & 0 & 0 & 0 & 3246 & 1138 & 4384 \\
\hline Industrial Hygiene, NOS & 154 & 18 & 0 & 0 & o. & 160 & 10 & 2 & 172 \\
\hline Physical Properties & 144 & $\overline{145}$ & 4 & 116 & o & 139 & 140 & 129 & 408 \\
\hline Other, Not Otherwise Specified (NO & 44 & 10 & 0 & $\overline{0}$ & 0 & 4 & 0 & 45 & 52 \\
\hline TOTALOTHER & $15 \overline{31}$ & 5378 & 22 & 302 & 0 & 1482 & 4079 & 1610 & 7202 \\
\hline & & & & & & & & & \\
\hline TOTAL SAMPLE ANALYSES & 13208 & $\overline{27241}$ & 356 & 771 & 0 & 14542 & 16745 & 4828 & 38845 \\
\hline
\end{tabular}


Table Vd: FY 96 Samples and Sample Analyses by Rad Level and Matrix Type (vii) NTS

\begin{tabular}{|c|c|c|c|c|c|c|c|c|c|}
\hline $\begin{array}{c}\text { Rad-Matrix/ } \\
\text { Sample-Sample Analysis }\end{array}$ & Rō & $\overline{\mathrm{R} 1}$ & $\overline{\mathrm{R} 2}$ & R3 & $\overline{\mathrm{R4}}$ & $\overline{\mathrm{M1}}$ & $\overline{\mathrm{M} 2}$ & $\overline{\text { M3 }}$ & TOTAL \\
\hline CUSTOMER SAMPLES & 1043 & 9340 & 176 & 0 & 7 & 670 & 5806 & 2810 & 9926 \\
\hline \multirow{2}{*}{\multicolumn{10}{|c|}{ SAMPLE ANALYSES }} \\
\hline & & & & & & & & & \\
\hline Volatiles by GC/MS & 1483 & 1264 & 45 & 0 & O] & 942 & 1729 & 123 & 2793 \\
\hline Volatiles by GC & 280 & 13 & 0 & 0 & 0 & 8 & 224 & 65 & 295 \\
\hline Semi-Volatiles & 730 & 1037 & 39 & 0 & 0 & 387 & 1330 & 88 & 1805 \\
\hline Herbicides/Pesticides/PCBs & 779 & 2057 & 0 & 0 & 0 & 296 & 2171 & 372 & 2838 \\
\hline Other Organics & 19 & 136 & ol & 0 & 0 & 73 & 73 & 8 & 155 \\
\hline TOTAL ORGANICS & 3292 & 4506 & $84 \mid$ & 01 & 0 & 1706 & 5527 & $657]$ & 7886 \\
\hline \multicolumn{10}{|l|}{\begin{tabular}{|l|} 
RCRA Annendix IX Metals \\
\end{tabular}} \\
\hline RCRA Appendix IX Metals & 4 & 14 & 0 & 0 & of & 1 & 16 & o. & $\overline{17}$ \\
\hline CAM (California) Metals (RCRA) & 0 & 0 & 0 & 0 & 0. & 0 & 0 & 0 & 0 \\
\hline RCRA TCLP Metals and EPTOX Me & 56 & 551 & 0 & 0 & 0. & 10 & 584 & 11 & 606 \\
\hline EPA CLP Metals (CERCLA) & 1635 & 600 & 115 & 0 & 0 & 725 & 1580 & 45 & 2350 \\
\hline NPDES Metals (Clean Water Act) & 259 & 0 & 0 & 0 & 0 & 259 & 0 & 0 & 259 \\
\hline Drinking Water Metals (SDWA) & 2 & 0 & 0 & 0 & o. & 2 & 0 & 0 & 2 \\
\hline Metals by Other Regulatory Suite & 1328 & 329 & 0 & 0 & 0 & 576 & 1099 & 66 & 1698 \\
\hline Metals by Suite, Other & 0 & 533 & 20 & 0 & - & 302 & 164 & 2 & 510 \\
\hline Metals by Metal Except Mercury & 72 & 860 & 0 & 0 & 0. & 25 & 405 & 501 & 932 \\
\hline Mercury & 303 & 934 & o) & 0 & o. & 284 & 937 & 14 & 1236 \\
\hline Anions/Cations-IC & 387 & 211 & 0 & 0 & o] & 567 & 21 & 11 & 598 \\
\hline Inorganics Wet Chemistry & 3031 & 858 & 0 & 0 & 0 & 2649 & 1219 & 20 & 3888 \\
\hline Other Inorganics & 333 & 137 & 0 & 0 & o] & 314 & 156 & $0]$ & 470 \\
\hline \multirow[t]{2}{*}{ TOTAL INORGANICS } & 7410 & 5025 & 135 & 01 & o] & 5716 & 6182 & 670 & 12569 \\
\hline & & & & & & & & & \\
\hline Gross Alpha/Beta & 3003 & 1027 & 29 & 0 & o. & 1466 & 2534 & 60 & 4060 \\
\hline Gamma Scan & 1078 & 582 & 16 & 0 & D. & 567 & 1043 & 66 & 1676 \\
\hline Transuranic & 0 & 21 & 0 & 0 & 0. & 0 & 21 & a & 21 \\
\hline Plutonium Isotopes & 1333 & 164 & 11 & 0 & 0 & 511 & 981 & 17 [ & 1509 \\
\hline Uranium Isotopes & 1429 & 1017 & 11 & 0 & of & 641 & 1767 & 48 & 2456 \\
\hline Curium Isotopes & 0 & 19 & 0 & 0 & 0 & 9 & 10 & 0 & 19 \\
\hline Thorium isotopes & 0 & 588 & 11 & 0 & 0 & 40 & 557 & 2 & 599 \\
\hline Radium Isotopes & 516 & 0 & 0 & 0 & 0 & 383 & 133 & 으 & 516 \\
\hline Americium-241 & 1309 & 70 & 11 & 0 & 0 & 520 & 856 & 15 & 1390 \\
\hline Neptunium-237 & 0 & 108 & 0 & 0 & 0 & 8 & 99 & 2 & 108 \\
\hline Polonium-210 & 0 & 0 & 0 & 0 & o. & 0 & 0 & o & 0 \\
\hline Tritium & 796 & 371 & 51 & 0 & 0 & 595 & 614 & 10 & 1219 \\
\hline Radon-222 & 0 & 0 & 0 & 0 & 0 & 0 & 0 & o & 0 \\
\hline Lead-210 & 0 & 이 & 01 & 0 & 0 & 0 & 0 & 0. & 0 \\
\hline Carbon-14 & 0 & 0 & 0 & 0 & 0. & 0 & 0 & 0] & 0 \\
\hline Nickel-63 & 0 & 0 & 0 & 0 & 0. & 0 & 0 & ㅇ. & 0 \\
\hline Selenium-79 & 0 & 0 & 0 & 0 & 0 & 0 & 0 & 0 & 0 \\
\hline Technetium-99 & 0 & 467 & 3 & 0 & 0 & 4 & 467 & 0 & 471 \\
\hline Strontium Isotopes & 0 & 0 & 0 & 0 & 0 & 0 & 0 & 0 & 0 \\
\hline Strontium -89 & 0 & 0 & 0 & 0 & 0 & 0 & 0 & 0 & 0 \\
\hline Strontium-90 & 0 & 164 & 3 & 0 & 0 & 16 & 152 & 0 & 168 \\
\hline lodine-129 & 1 & 0 & 0 & 0 & 0 & 1 & 0 & 0 & $\overline{1}$ \\
\hline Nickel-59 & 0 & 0 & 0 & $\overline{0}$ & 0 & 0 & 0 & 0 & 0 \\
\hline Total Uranium & 18 & 388 & 0 & 0 & 0 & 18 & 120 & 268 & 406 \\
\hline Total Thorium & 0 & 33 & 0 & 0 & 0 & 0 & 33 & 0 & 33 \\
\hline Total Strontium & 715 & 0 & 0 & 0 & 0 & 340 & 361 & 15 & 715 \\
\hline Other RAD & 1780 & 1726 & 32 & 0 & $0]$ & 453 & 3068 & 18 & 3538 \\
\hline TOTAL RAD & 11979 & 6748 & 179 & 0 & D] & 5572 & 12817 & $519]$ & 18907 \\
\hline & & & & & & & & & \\
\hline Other Wet Chemistry & 1731 & 0 & 0 & 可 & 0 & 1413 & 319 & 으. & 1731 \\
\hline TCLP Extraction & 48 & 652 & 1 & 0 & 0 & 21 & 608 & 71 & 700 \\
\hline Asbestos & 25 & 6173 & 0 & 0 & of & 0 & 4615 & 1582 & 6198 \\
\hline Industrial Hygiene, NOS & 23 & 0 & 0 & 0 & 0 & 3 & 19 & 0 & 23 \\
\hline Physical Properties & 251 & 224 & 6 & 0 & 0 & 63 & 419 & $\overline{0}$ & 482 \\
\hline Other, Not Otherwise Specified (NQ & 2 & 38 & 0 & 0 & 0 & 38 & 2 & o] & 40 \\
\hline TOTAL OTHER & 2081 & 7087 & 71 & 01 & $0]$ & 1538 & 5982 & 1653 & 9174 \\
\hline & & & & $\frac{1}{01}$ & $=$ & & & 250 & \\
\hline TOTA & 24762 & 366 & 4051 & OI & 0] & & & $3500]$ & 8536 \\
\hline
\end{tabular}


Table Vd: FY 96 Samples and Sample Analyses by Rad Level and Matrix Type (viii) Oak Ridge K-25

\begin{tabular}{|c|c|c|c|c|c|c|c|c|c|}
\hline $\begin{array}{c}\text { Rad-Matrix } \\
\text { Sample-Sample Analysis }\end{array}$ & $\overline{\mathrm{RO}}$ & $\overline{\mathrm{R1}}$ & $\overline{\mathrm{R} 2}$ & $\overline{\mathrm{R} 3}$ & $\overline{R 4}$ & $\overline{M 1}$ & M2 & M3 & TOTAL \\
\hline CUSTOMER SAMPLES & 203 & 9554 & 183 & 0 & - & 432 & 5907 & 3565 & 9922 \\
\hline & & & & & & & & & \\
\hline \multicolumn{10}{|l|}{ SAMPLE ANALYSES } \\
\hline Volatiles by GC/MS & 60 & 1549 & 109 & o! & - & 158 & 1180 & 381 & 1718 \\
\hline Volatiles by GC & 82 & 14 & 1 & 0 & o & 60 & 27 & 2 & 93 \\
\hline Semi-Volatiles & 39 & 1175 & 97 & 0 & 0 & 29 & 1071 & 212 & 1311 \\
\hline Herbicides/Pesticides/PCBs & 39 & 2728 & 0 & 0 & 0 & 564 & 1644 & 561 & 2768 \\
\hline Other Organics & 53 & 7 & 12 & 0 & o. & 36 & 21 & 12 & 71 \\
\hline TOTAL ORGANICS & 273 & 54721 & 219 & D) & o] & 846 & 3943 & 1168 & 5960 \\
\hline & & & & & & & & & \\
\hline RCRA Appendix IX Metals & 1 . & 1] & 0 & 0 & 으. & 0 & 1) & 26 & 15 \\
\hline CAM (California) Metals (RCRA) & 0 & 0] & 0 & 0 & 요 & 0 & 0 & 0 & 0 \\
\hline RCRA TCLP Metals and EPTOX Me & 109 & 809 & 198 & 0 & 0 & 56 & 669 & 410 & 1126 \\
\hline EPA CLP Metals (CERCLA) & 65 & 366 & 5 & 0 & 0 & 33 & 63 & 340 & 436 \\
\hline NPDES Metals (Clean Water Act) & 12 & 11 & 0 & 0 & 0 & 13 & 0 & 0 & 13 \\
\hline Drinking Water Metals (SDWA) & 16 & 0 & 0 & 0 & 0 & 16 & 0 & 0 & 16 \\
\hline Metals by Other Regulatory Suite & 64 & 823 & 90 & 0 & 0 & 359 & 498 & 160 & 997 \\
\hline Metals by Suite, Other & 0 & 75 & 1 & 0 & 0 & 21 & 8 & 2 & 53 \\
\hline Metals by Metal Except Mercury & 114 & 1852 & 91 & 0 & 0 & 469 & 367 & 713 & 1803 \\
\hline Mercury & 121 & 1490 & 13 & 0 & 0 & 819 & 1144 & 151 & 1869 \\
\hline Anions/Cations-IC & 20 & 503 & 42 & 0 & o. & 28 & 8 & 114 & 358 \\
\hline Inorganics Wet Chemistry & 171 & 790 & 17 & 0 & 0 & 224 & 736 & 458 & 1198 \\
\hline Other Inorganics & 20 & 142 & 0 & 0 & 0 & 22 & 51 & 89 & 162 \\
\hline \multirow[t]{2}{*}{ TOTALINORGANICS } & 714 & 6850 & 456 & 01 & 0] & 2062 & 3547 & 2464 & 8046 \\
\hline & & & & & & & & & \\
\hline Gross Alpha/Beta & 163 & 1370 & 14 & 0 & 0 & 910 & 502 & 134 & 1547 \\
\hline Gamma Scan & 126 & 510 & 13 & 0 & 0 & 41 & 475 & 132 & 648 \\
\hline Transuranic & 0 & 377 & 360 & 0 & 0 & 480 & 257 & 0 & 737 \\
\hline Plutonium Isotopes & 64 & 128 & 13 & 0 & 0 & 28 & 47 & 130 & 205 \\
\hline Uranium Isotopes & 59 & 1270 & 293 & 0 & 0 & 637 & 709 & 275 & 1621 \\
\hline Curium Isotopes & 0 & 1 & 0 & 0 & 0 & 1 & 1 & 요 & 1 \\
\hline Thorium Isotopes & 1 & 1001 & 13 & 0 & 0 & 309 & 578 & 129 & 1015 \\
\hline Radium Isotopes & 15 & 0 & 0 & 0 & 0 & 12 & 4 & 0 & 16 \\
\hline Americium-241 & 50 & 105 & 1 & 0 & 0. & 37 & 31 & 88 & 155 \\
\hline Neptunium-237 & 1 & 295 & 242 & 0 & o. & 251 & 159 & 129 & 538 \\
\hline Polonium-210 & 0 & 0 & 12 & 0 & 0 & 0 & 0 & 12 & 12 \\
\hline Tritium & 44 & 27 & 3 & 0 & 의 & 31 & 40 & 1 & 73 \\
\hline Radon-222 & 0 & 0 & 0. & 0 & 의 & 0 & 0 & 요 & 0 \\
\hline Lead-210 & 0 & 0 & of & 0 & 요 & 0 & 0 & ㅇ. & 0 \\
\hline Carbon-14 & 0 & 0 & 요 & 0 & 으. & 0 & 0 & 요 & 요 \\
\hline Nickel-63 & 0 & a & of & 0 & 의 & 0 & 0 & 0 & 0 \\
\hline Selenium-79 & 0 & 1 & 0 & 0 & 0 & 0 & 0 & 0 & 1 \\
\hline Technetium-99 & 3 & 1032 & 372 & 0 & 의 & 484 & 799 & 125 & 1408 \\
\hline Strontium Isotopes & 0 & 0 & of & 0 & 의 & 0 & 0 & 0. & 0 \\
\hline Strontium -89 & 0 & 0. & 0 & 0 & o. & 0 & 0 & of & 0 \\
\hline Strontium-90 & 3 & 14 & 1 & 0 & 요 & 6 & 12 & 요 & 19 \\
\hline lodine-129 & 10 & 1 & O) & 0 & 0 & 11 & $\begin{array}{lll}0 & & \\
\end{array}$ & 0 & 11. \\
\hline Nickel-59 & 0 & 0 & 0 & 0 & 0 & 0 & 01 & 0 & 0 \\
\hline Total Uranium & 10 & 693 & 352 & of & 0 & 125 & 491 & 439 & 1055 \\
\hline Total Thorium & 0 & 13 & 0 & 0 & 요 & 0 & 13 & 0 & 13 \\
\hline Total Strontium & 22 & 0 & 0 & 0 & 0 & 9 & 13 & 1 & 22 \\
\hline Other RAD & 92 & 2203 & 15 & 0 & 0 & 334 & 1848 & 128 & 2309 \\
\hline TOTAL AAD & 664 & 9044 & 1701 & O) & 0] & 3706 & 5978 & 1723 & 11408 \\
\hline & & & & & & & & & \\
\hline $\begin{array}{l}\text { Other Wet Chemistry } \\
\text { TCLP Extraction }\end{array}$ & 62 & 0 & O & O & ㅇ. & 52 & 10 & o] & 62 \\
\hline \begin{tabular}{|l} 
TCLP Extraction \\
Asbestos
\end{tabular} & 109 & 1156 & 106 & 우 & 요 & 59 & 756 & 549 & 1367 \\
\hline Asbestos & 오 & 7620 & 으 & o & 의 & 0 & 5642 & 1978 & 7620 \\
\hline Industrial Hygiene, NOS & 2 & 0 & 0 & 0 & - & 1 & 1 & o. & 2 \\
\hline Physical Properties & 20 & 620 & $\overline{0}$ & o) & 0 & 310 & 219 & 111 & 640 \\
\hline Other, Not Otherwise Specified (NO & 4 & 9 & 12 & 0 & 0 & 8 & 1 & 15] [ & 24 \\
\hline TOTAL OTHER & 196 & 9405 & 118 & 이 & ㅁ] & 430 & 6629 & $2652]$ & 9716 \\
\hline & & & & & & & & & \\
\hline TOTAL SAMPLE ANALYSES & 1848 & 30771 & 2494 & 0 & 0 & 7044 & 20096 & 8007 & 35130 \\
\hline
\end{tabular}


Table Vd: FY 96 Samples and Sample Analyses by Rad Level and Matrix Type (ix) Oak Ridge X-10

\begin{tabular}{|c|c|c|c|c|c|c|c|c|c|}
\hline $\begin{array}{c}\text { Rad-Matrix } \\
\text { Sample-Sample Analysis }\end{array}$ & Ro & R1 & R2 & $\overline{\mathrm{R} 3}$ & $\overline{\mathrm{R} 4}$ & M1 & M2 & M3 & TOTAL \\
\hline CUSTOMER SAMPLES & 378 & 5838 & 21 & 595 & 0 & 3170 & 1366 & 1271 & 6319 \\
\hline & & & & & & & & & \\
\hline \multicolumn{10}{|l|}{ SAMPLE ANALYSES } \\
\hline Volatiles by GC/MS & 120 & 250 & 3 & 66 & 0 & 100 & 256 & 82 & 439 \\
\hline Volatiles by GC & 83 & 35 & 1 & 0 & 0 & 67 & 43 & 5 & 117 \\
\hline Semi-Volatiles & 76 & 213 & 3 & 56 & 0 & 62 & 224 & 62 & 348 \\
\hline Herbicides/Pesticides/PCBs & 199 & 457 & 0 & 0 & 0 & 172 & 382 & 103 & 657 \\
\hline Other Organics & 42 & 939 & 0 & 0 & 0 & 29 & 22 & 1 & 517 \\
\hline TOTAL ORGANICS & 519 & 1895 & 7 & 122 & 0. & 431 & 928 & 253 & 2078 \\
\hline & & & & & & & 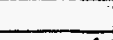 & 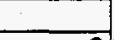 & \\
\hline RCRA Appendix IX Metals & 2 & 3 & 0 & 0 & 0 & 4 & 1 & 0 & 5 \\
\hline CAM (California) Metals (RCRA) & 44 & 1 & 0 & 0 & 0 & 45 & 1 & 0 & 45 \\
\hline RCRA TCLP Metals and EPTOX Me & 68 & 151 & 1 & 0 & 0 & 59 & 152 & 4 & 218 \\
\hline EPA CLP Metals (CERCLA) & 108 & 46 & 8 & 138 & 0 & 58 & 100 & 142 & 300 \\
\hline NPDES Metals (Clean Water Act) & 37 & 164 & of & 0 & 0 & 201 & 0 & 0 & 201 \\
\hline Drinking Water Metals (SDWA) & 11 & 0 & o. & 0 & 0 & 11 & 0 & 0 & 11 \\
\hline Metals by Other Regulatory Suite & 155 & 85 & o. & 0 & 0 & 96 & 122 & 33 & 245 \\
\hline Metals by Suite, Other & 0 & 976 & 1 & 0 & 0 & 24 & 12 & 1 & 507 \\
\hline Metals by Metal Except Mercury & 133 & 206 & 0 & 0 & 0 & 115 & 92 & 127 & 337 \\
\hline Mercury & 184 & 1178 & 이 & 0 & 0 & 196 & 227 & 5 & 896 \\
\hline Anions/Cations-IC & 71 & 36 & of & 0 & 요 & 87 & 15 & 5 & 107 \\
\hline Inorganics Wet Chemistry & 659 & 196 & 9 & 2 & 0 & 600 & 212 & 56. & 867 \\
\hline Other Inorganics & 83 & 39 & 0 & 0 & 0 & 80 & 34 & 9 & 122 \\
\hline TOTAL INORGANICS & 1554 & 3082 & 20 & 140 & 0 & 1575 & 968 & 383 & 3861 \\
\hline & & & & & & & & & \\
\hline Gross Alpha/Beta & 655 & 3122 & 3 & 0 & 0 & 2531 & 292 & 28 & 3316 \\
\hline Gamma Scan & 130 & 1069 & 1 & 0 & 의 & 995 & 188 & 17 & 1200 \\
\hline Transuranic & 0 & 5 & 0 & 0 & 0 & 0 & 5 & ㅇ. & 5 \\
\hline Plutonium Isotopes & 218 & 15 & 3 & 0 & 0 & 166 & 63 & 6 & 236 \\
\hline Uranium Isotopes & 225 & 165 & 2 & 0 & 0 & 179 & 196 & 16 & 392 \\
\hline Curium Isotopes & 0 & 4 & 01 & 0 & 0. & 1 & 1 & 2 & 4 \\
\hline Thorium isotopes & 2 & 124 & 1 & 5 & 의 & 5 & 120 & 8 & 132 \\
\hline Radium Isotopes & 21 & 0 & 0 & 0 & 0 & 16 & 6 & 0 & 22 \\
\hline Americium - 241 & 207 & 10 & 1 & 0 & 으. & 166 & 49 & 3 & 217 \\
\hline Neptunium-237 & 2 & 10 & 2 & 0 & 요 & 1 & 9 & 5 & 14 \\
\hline Polonium-210 & 1 & 1 & 0 & 0 & 으. & 0 & 1 & 0 & 1 \\
\hline Tritium & 168 & 35 & 7 & 0 & 0 & 155 & 48 & 2 & 207 \\
\hline Radon-222 & 0 & 0 & 0 & 0 & 0 & 0 & 0 & 0 & 0 \\
\hline Lead-210 & 0 & 2 & 0 & 0 & 요 & 0 & 0 & 2 & 2 \\
\hline Carbon-14 & 0 & 0 & 0 & 0 & 의 & 0 & 0 & 의 & 0 \\
\hline Nickel-63 & 0 & 0 & 0 & 0 & 요 & 0 & 0 & 은 & 0 \\
\hline Selenium -79 & 44 & 1 & 0 & 0 & 0 & 45 & 0 & 으, & 45 \\
\hline Technetium-99 & 2 & 117 & 요 & 0 & 의 & 2 & 115 & 2 & 119 \\
\hline Strontium Isotopes & 0 & 0 & 0 & 0 & 0 & 0 & 0 & 요 & 0 \\
\hline Strontium-89 & 0 & 0 & 0 & 0 & 0 & 0 & 0 & 의 & 0 \\
\hline Strontium-90 & 46 & 16 & 1 & 0 & 0 & 49 & 14 & o & 64 \\
\hline lodine-129 & 51 & 2 & o. & 0 & 0. & 52 & 0 & 0 & 52 \\
\hline Nickel-59 & 0 & 0 & 0 & 0 & 요 & 0 & 0 & 0 & 0 \\
\hline Total Uranium & 13 & 85 & 0 & 0 & 0 & 4 & 16 & 78 & 99 \\
\hline Total Thorium & 0 & 0 & 0 & 0 & 0 & 0 & 0 & 요 & 0 \\
\hline Total Strontium & 35 & 2 & 0 & 0 & 0 & 17 & 18 & 3 & 38 \\
\hline Other RAD & 179 & 424 & 4 & 462 & 0 & 108 & 468 & 486 & 1066 \\
\hline TOTALRAD & 2001 & 5209 & 25 & 467 & 0 & 4493 & 1609 & 660 & 7232 \\
\hline Other Wet Chemistry & & & & & إO & & 10 & 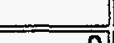 & 306 \\
\hline TCLP Extraction & $\frac{324}{55}$ & $\frac{2}{175}$ & $\frac{0}{7}$ & $\frac{0}{366}$ & $\frac{0}{0}$ & $\frac{310}{53}$ & $\begin{array}{r}16 \\
157\end{array}$ & $\frac{0}{389}$ & $\frac{326}{601}$ \\
\hline Asbestos & 0 & 1527 & 0 & 0 & 0 & 0 & 1132 & 396 & 1528 \\
\hline Industrial Hygiene, NOS & 113 & 0 & 0 & 0 & 0 & 112 & 2 & 0 & 113 \\
\hline Physical Properties & 35 & 46 & 0 & 230 & 0 & 31 & 48 & 232 & 311 \\
\hline Other, Not Otherwise Specified (Nd & 5 & 4 & 0 & 0 & 0 & 3 & 0 & 5 & 8 \\
\hline TOTAL OTHER & 532 & 1753 & 8 & 596 & 0 & 508 & 1355 & 1022 & 2887 \\
\hline TOTAL SAMPLE ANALYSES & 4606 & 11940 & 601 & 1325 & -0. & 7008 & 4860 & 2317 & 16058 \\
\hline
\end{tabular}


Table Vd: FY 96 Samples and Sample Analyses by Rad Level and Matrix Type (x) Oak Ridge Y-12

\begin{tabular}{|c|c|c|c|c|c|c|c|c|c|}
\hline $\begin{array}{c}\text { Rad-Matrix/ } \\
\text { Sample-Sample Analysis }\end{array}$ & Ro & R1 & R2 & $\overline{\mathrm{R} 3}$ & R4 & $\overline{\mathrm{M} 1}$ & M2 & M3 & TOTAL \\
\hline CUSTOMER SAMPLES & 146 & 991 & 15 & 01 & $0]$ & 116 & 657 & 278 & 1101 \\
\hline & & & & & & & & & \\
\hline \multicolumn{10}{|l|}{ SAMPLE ANALYSES } \\
\hline Volatiles by GC/MS & 101 & 146 & 3 & 0 & 0 & 59 & 172 & $20]$ & 251 \\
\hline Volatiles by GC & 25 & 34 & 2 & 0 & 0 & 3 & 51 & 5 & 60 \\
\hline Semi-Volatiles & 49 & 125 & 3 & 0 & 0 & 29 & 147 & 3 & 178 \\
\hline Herbicides/Pesticides/PCBs & 87 & 231 & 0 & 0 & 0 & 43 & 219 & 58 & 320 \\
\hline Other Organics & 27 & 8 & 0 & 0 & 0 & 61 & 28 & 1 & 35 \\
\hline TOTAL ORGANICS & 289 & 544 & 8 & 01 & $0]$ & 140 & 617 & 88 & 843 \\
\hline RCRA Appendix IX Metals & & & & 0 & & 0 & & 0 & \\
\hline CAM (California) Metals (RCRA) & 20 & 0 & o & 0 & 0 & 20 & of & $\frac{0}{0}$ & $\frac{3}{21}$ \\
\hline RCAA TCLP Metals and EPTOX Me & 10 & 71 & 2 & 0 & 0 & $\frac{2}{3}$ & 75 & 4 & 83 \\
\hline EPA CLP Metals (CERCLA) & 90 & 51 & 9 & 0 & 0 & 37 & 107 & 5 & 149 \\
\hline NPDES Metals (Clean Water Act) & 21 & 1 & 0 & 0 & 0 & 22 & 0 & 0 & 22 \\
\hline Drinking Water Metals (SDWA) & 0 & 0 & 0 & 0 & 0 & 0 & 0 & 0 & 0 \\
\hline Metals by Other Regulatory Suite & 108 & 37 & 0 & 0 & 0 & 26 & 80 & 46 & 149 \\
\hline Metals by Suite. Other & 0 & 45 & 2 & 0 & 0 & 25 & 13 & 1 & 43 \\
\hline Metals by Metal Except Mercury & 30 & 118 & 0 & 0 & 0 & 24 & 71 & 53 & 148 \\
\hline Mercury & 49 & 124 & 0 & 0 & 0 & 46 & 124 & 3 & 173 \\
\hline Anions/Cations-IC & 36 & 20 & 0 & 0 & 0. & 47 & 2 & 6 & 56 \\
\hline Inorganics Wet Chemistry & 277 & 122 & 17 & 0 & 0 & 196 & 136 & 87 & 417 \\
\hline Other Inorganics & 35 & 25 & 0 & 0 & 0 & 35 & 19 & 6 & 60 \\
\hline TOTAL INORGANICS & 678 & 615 & 291 & 0 & 0 & 481 & 6311 & 212 & 1323 \\
\hline & & & & & & & & & \\
\hline Gross Alpha/Beta & 184 & 121 & 4 & 0 & of & 94 & 197 & 16 & 308 \\
\hline Gamma Scan & 69 & 61 & 1 & 0 & 0] & 34 & 85 & 13 & 132 \\
\hline Transuranic & 0 & 2 & 0 & 0 & 0. & 0 & 2 & 0 & 2 \\
\hline Plutonium Isotopes & 69 & 18 & 1 & 0 & 요 & 21 & 60 & 7 & 88 \\
\hline Uranium Isotopes & 85 & 120 & 1 & 0 & 0] & 36 & 158 & 12 & 206 \\
\hline Curium Isotopes & 0 & 3. & 0 & 0 & (0) & 1 & 1 & 1 & 3 \\
\hline Thorium Isotopes & 5 & 70 & 11 & 0 & 0 & 4 & 67 & 5 & 76 \\
\hline Radium Isotopes & 20 & 1 & 0 & 0 & 0 & 14 & 6 & 1 & 21 \\
\hline Americium-241 & 64 & 10 & 1 & 0 & 0 & 22 & 51 & 2 & 74 \\
\hline Neptunium-237 & 5 & 22 & 0 & 0 & 0 & 1 & 20 & 6 & 27 \\
\hline Polonium -210 & 0 & 1 & 0 & 0 & 0 & 0 & 0 & 1. & 1 \\
\hline Tritium & 39 & 51 & 6 & 0 & 0 & 30 & 56 & 1 & 91 \\
\hline Radon-222 & 0 & 0 & 0 & 0 & 0 & 0 & 0 & 0 & 0 \\
\hline Lead-210 & 0 & 0 & 0 & 0 & 0 & 0 & 0 & 0 & 0 \\
\hline Carbon-14 & 0 & 0 & 0 & 0 & 0. & 0 & 0 & 0. & 0 \\
\hline Nickel-63 & 0 & o & 0 & 0 & 0. & 0 & 0 & 0 . & 0 \\
\hline Selenium-79 & 20 & 1 & 0 & 0 & 0 & 21 & 0 & 0. & 21 \\
\hline Technetium-99 & 0 & 49 & 0 & O) & 0 & 0 & 48 & 1 & 50 \\
\hline Strontium Isotopes & 0 & 0 & 0 & 이 & ㅇ․ & 0 & 0 & 0 & 0 \\
\hline Strontium -89 & 0 & 0 & 0 & of & o. & 0 & 0 & 0 & 0 \\
\hline Strontium -90 & 20 & 20 & 2 & 0 & 0 & 24 & 18 & 1. & 42 \\
\hline lodine-129 & 20 & 3 & 0 & 0 & 0 & 23 & 0 & 0 & 23 \\
\hline Nickel-59 & 0 & 0 & 0 & 0 & 0 & 0 & 0 & 0 & 0 \\
\hline Total Uranium & 21 & 58 & 0 & 0 & 요 & 3 & 28 & 48 & 79 \\
\hline Total Thorium & 0 & 22 & 0 & 0 & 0] & 0 & 22 & 0 & 22 \\
\hline Total Strontium & 32 & 0 & 0 & 0 & 0 & 12 & 19 & 1 & 32 \\
\hline Other RAD & 132 & 188 & 41 & 0 & 0 & 36 & 263 & 25 & 324 \\
\hline TOTAL RAD & 786 & 822 & $22 !$ & 0 & 에 & 376 & 1103 & 141 & 1624 \\
\hline & & & & & II & & 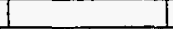 & 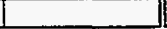 & \\
\hline Other Wet Chemistry & 70 & 1 & 0 & 0 & 의 & 54 & 17 & 0 & 71 \\
\hline TCLP Extraction & 10 & 84 & 0 & 0 & 0. & 7 & 75 & 13 & 94 \\
\hline Asbestos & 0 & 651 & 0 & 0 & 0 & 0 & 487 & 165 & 651 \\
\hline Industrial Hygiene, NOS & 2 & 0 & 0 & 0 & 0 & 1 & 2 & 요 & 3 \\
\hline Physical Properties & 9 & 44 & 1 & 0 & 0. & 6 & 44 & 3 & 53 \\
\hline Other, Not Otherwise Specified (Nd & 12 & 14 & of & 0 & 0 & 14 & 1 & 10 & 25 \\
\hline TOTALOTHEA & 1031 & 795 & 11 & 0 & 이 & 82 & 625 & 191 & 898 \\
\hline & & & & & & & & & \\
\hline TOTAL SAMPLE ANALYSES & 1857 & 2775 & 601 & 0 & 0. & 1079 & 2976 & 631 & 4689 \\
\hline
\end{tabular}


Table Vd: FY 96 Samples and Sample Analyses by Rad Level and Matrix Type (xi) Rocky Flats

\begin{tabular}{|c|c|c|c|c|c|c|c|c|c|}
\hline $\begin{array}{c}\text { Rad-Matrix/ } \\
\text { Sample-Sample Analysis }\end{array}$ & RO & $\overline{\mathrm{R} 1}$ & $\overline{\mathrm{R} 2}$ & $\overline{\mathrm{R} 3}$ & $\overline{\mathrm{R} 4}$ & $\overline{\mathrm{M} 1}$ & M2 & M3 & TOTAL \\
\hline CUSTOMER SAMPLES & 2118 & 5538 & 386 & 1180 & Q & 1859 & 3067 & 1327 & 7737 \\
\hline & & & & & & & & & \\
\hline \multicolumn{10}{|l|}{ SAMPLE ANALYSES } \\
\hline Volatiles by GC/MS & 4344 & 794 & 88 & 132 & a. & 2687 & 2365 & 308 & 5359 \\
\hline Volatiles by GC & 912 & 248 & 19 & 0 & 0 & 345 & 768 & 46 & 1169 \\
\hline Semi-Volatiles & 1874 & 726 & 88 & 112 & 0 & 1049 & 1600 & 142 & 2796 \\
\hline Herbicides/Pesticides/PCBs & 2281 & 898 & 0 & 0 & 0 & 791 & 2229 & 160 & 3179 \\
\hline Other Organics & 204 & 231 & 0 & 01 & 0 & 323 & 39 & 7 & 402 \\
\hline \multirow[t]{2}{*}{ TOTAL ORGANICS } & 9615 & 2897 & 195 & 244 & - & 5195 & 7001 & 663 & 12905 \\
\hline & & & & & & & & & \\
\hline RCRA Appendix IX Metals & 8 & 31 & 0 & 0 & 0 & 5 & 35 & o & 39 \\
\hline CAM (California) Metals (RCRA) & 0 & 2 & 0 & 0 & ㅇ. & 2 & 3 & 1 & 4 \\
\hline RCRA TCLP Metals and EPTOX Me & 385 & 172 & 19 & 0 & $\overline{0}$ & 295 & 219 & 19 & 554 \\
\hline 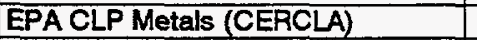 & 5253 & 1393 & 243 & 276 & 0 & 2510 & 4262 & 393 & 7166 \\
\hline NPDES Metals (Clean Water Act) & 594 & 19 & 0 & 0 & 0 & 613 & 0 & 0 & 613 \\
\hline Drinking Water Metals (SDWA) & 92 & 0 & 0 & 0 & 0 & 92 & 0 & 0 & 92 \\
\hline Metals by Other Regulatory Suite & 4736 & 0 & 0 & 0 & 0 & 2140 & 2352 & 365 & 4796 \\
\hline Metals by Suite, Other & 0 & 1205 & 46 & 0 & 0 & 681 & 376 & 7 & 1157 \\
\hline Metals by Metal Except Mercury & 450 & 290 & 4 & 0 & 0. & 366 & 338 & o. & 724 \\
\hline Mercury & 960 & 335 & 4 & 0 & 0 & 910 & 276 & 7 & 1245 \\
\hline Anions/Cations $-1 \mathrm{C}$ & 932 & 455 & 0 & 0 & 0 & 1313 & 36 & 38 & 1387 \\
\hline Inorganics Wet Chemistry & 12718 & 919 & 135 & 4 & 0 & 10881 & 2269 & 633 & 13779 \\
\hline Other Inorganics & 831 & 311 & 0 & 0 & 0 & 802 & 294 & 46 & 1142 \\
\hline \multirow[t]{2}{*}{ TOTAL INORGANICS } & 26959 & 5132 & 450 & 2801 & on & 20610 & 10459 & 1509 & 32700 \\
\hline & & & & & & & & & \\
\hline \multirow{2}{*}{$\begin{array}{l}\text { Gross Alpha/Beta } \\
\text { Gamma Scan }\end{array}$} & 9631 & 1919 & 81 & 0 & 0 & 4828 & 6466 & 204 & 11565 \\
\hline & 3830 & 598 & 37 & 0 & of & 1848 & 2448 & 169 & 4465 \\
\hline \multirow{3}{*}{\begin{tabular}{|l|} 
Transuranic \\
Plutonium Isotopes \\
Uranium Isotopes \\
\end{tabular}} & 0 & 1 & 0 & 0 & 0 & 0 & 1 & 0 & 1 \\
\hline & 4757 & 371 & 25 & 0 & 0 & 2002 & 3076 & 75 & 5153 \\
\hline & 4646 & 1087 & 25 & 0 & 0 & 2133 & 3538 & 88 & 5758 \\
\hline Curium Isotopes & 0 & 58 & 0 & 0 & 0 & 25 & 25 & 8 & 58 \\
\hline \multirow{2}{*}{\begin{tabular}{|l} 
Thorium Isotopes \\
Radium Isotopes \\
\end{tabular}} & 39 & 243 & 25 & 0 & 0 & 66 & 202 & 40 & 308 \\
\hline & 1888 & 7 & 0 & 0 & 0 & 1438 & 450 & 7 & 1896 \\
\hline \multirow{2}{*}{\begin{tabular}{|l|} 
Americium-241 \\
Neptunium-237 \\
\end{tabular}} & 4542 & 138 & 25 & 0 & 0 & 1977 & 2693 & 36 & 4706 \\
\hline & 39 & 181 & 0 & 0 & 0 & 16 & 157 & 46 & 220 \\
\hline \multirow{2}{*}{\begin{tabular}{|l|} 
Polonium-210 \\
Tritium \\
\end{tabular}} & 2 & 8 & 0 & 0 & 0 & 1 & 2 & 7 & 10 \\
\hline & 2763 & 886 & 133 & 0 & 0 & 2036 & 1657 & 22 & 3748 \\
\hline \multirow{2}{*}{$\frac{\text { Radon-222 }}{\text { Lead }-210}$} & 1 & 0 & 0 & 0 & 0 & 1 & 0 & 0 & 1 \\
\hline & 0 & 0 & 0 & 0 & 0 & 0 & 0 & 으. & 인 \\
\hline Carbon-14 & 0 & 0 & 0 & 0 & 0 & 0 & 0 & 은 & 0 \\
\hline \multirow{2}{*}{\begin{tabular}{|l|} 
Nickel-63 \\
Selenium-79
\end{tabular}} & 0 & of & 0 & 0 & 0 & 0 & 0 & 0 & 인 \\
\hline & 0 & 0 & 0 & 0 & 0 & 0 & 0 & 요 & 0 \\
\hline Technetium -99 & 20 & 37 & 7 & 0 & 0 & 27 & 29 & 7 & 64 \\
\hline Strontium Isotopes & 0 & 1 & 0 & 0 & 0 & 0 & 0 & 의 & 1 \\
\hline Strontium -89 & 0 & 0 & 0 & 0 & 0 & 0 & 0 & 요 & 0 \\
\hline Strontium -90 & 20 & 371 & 22 & 0 & 0 & 55 & 359 & 0 & 414 \\
\hline lodine-129 & 56 & 15 & 0 & 0 & 0 & 71 & 0 & 요 & 71 \\
\hline Nickel-59 & 0 & 0 & 0 & 0 & 0 & 0 & 0 & 0 & 0 \\
\hline Total Uranium & 216 & 27 & 0 & 0 & 0 & 68 & 15 & 160 & 243 \\
\hline Total Thorium & 0 & 16 & 0 & 0 & 0 & 1 & 15 & 0 & 16 \\
\hline Total Strontium & 2503 & 0 & 0 & 0 & 0 & 1296 & 1172 & 35 & 2503 \\
\hline Other RAD & 6145 & 697 & 92 & 924 & 0 & 2107 & 4652 & 1102 & 7859 \\
\hline TOTAL RAD & 41099 & 6662 & 474 & 924 & $0]$ & 19996 & 26956 & 2009 & 49060 \\
\hline & & & & & & & & & \\
\hline Other Wet Chemistry & $640 \overline{4}$ & 8 & $\overline{01}$ & 0 & 0. & 5399 & 1012 & 0 & 6411 \\
\hline TCLP Extraction & 387 & 222 & 6 & 732 & 0 & 321 & 221 & 766 & 1327 \\
\hline Asbestos & 25 & 163 & 0 & 0 & 0 & 1 & 187 & 요 & 188 \\
\hline Industrial Hygiene, NOS & 64 & 0 & 0 & 0 & 0 & 18 & 46 & 0 & 64 \\
\hline Physical Properties & 1004 & 127 & 15 & 460 & 0 & 242 & 878 & 486 & 1605 \\
\hline Other, Not Otherwise Specified (NO & 81 & 58 & 0 & 0 & $\underline{0}$ & 53 & 1 & 81 & 137 \\
\hline TOTAL OTHER & 7965 & 577 & 20 & 1192 & O] & 6033 & 2346 & 1332 & 9733 \\
\hline & & & & & & & & & \\
\hline TOTA1 & 856371 & 15268 & 1140 & 2640 & 01 & 51835 & 467611 & 5513 & 04398 \\
\hline
\end{tabular}


Table Vd: FY 96 Samples and Sample Analyses by Rad Level and Matrix Type (xii) Savannah River

\begin{tabular}{|c|c|c|c|c|c|c|c|c|c|}
\hline $\begin{array}{c}\text { Rad-Matrix } \\
\text { Sample-Sample Analysis }\end{array}$ & RO & R1 & $\overline{\mathrm{R} 2}$ & $\mathrm{R3}$ & $\overline{\mathrm{R} 4}$ & $\overline{\mathrm{M1}}$ & M2 & $\mathrm{M} \overline{3}$ & TOTAL \\
\hline CUSTOMER SAMPLES & 10267 & 19585 & 185 & 53 & 211 & 21416 & 813 & 3248 & 27888 \\
\hline \multirow{2}{*}{\multicolumn{10}{|c|}{ SAMPLE ANALYSES }} \\
\hline & & & & & & & & & \\
\hline Volatiles by GC/MS & 179 & 9 & 이 & 이 & 의 & 132 & 0 & 56 & 187 \\
\hline Volatiles by GC & 103 & 213 & 18 & 6 & 요 & 145 & 177 & 18 & 339 \\
\hline Semi-Volatiles & 1288 & 86 & 6 & 1 & - & 1016 & 65 & 301 & 1382 \\
\hline Herbicides/Pesticides/PCBs & 642 & 91 & 6 & 3 & 요마. & 603 & 63 & 75 & 741 \\
\hline Other Organics & 2008 & 4664 & O) & 0 & 마. & 1743 & 11 & 284 & 4350 \\
\hline TOTAL ORGANICS & 42201 & 50621 & 291 & 10 & ㅇ․ & 3639 & 305 & 733 & 6999 \\
\hline \multicolumn{10}{|l|}{ (2) } \\
\hline RCRA Appendix IX Metals & 11 & 9 & 0 & 0 & 의 & 19 & 0) & 1. & 20 \\
\hline CAM (California) Metals (RCRA) & 80 & 0 & 0 & 0 & o & 80 & 0 & 0) & 80 \\
\hline RCRA TCLP Metals and EPTOX Mel & 75 & 101 & 8 & 1 & 요 & 115 & 56 & 15 & 186 \\
\hline EPA CLP Metals (CERCLA) & 67 & 32 & 11 & 6 & 요 & 67 & 43 & 7 & 116 \\
\hline NPDES Metals (Clean Water Act) & 86 & 823 & 0 & 0 & 요 & 909 & 0 & o & 909 \\
\hline Drinking Water Metals (SDWA) & 0 & 0 & 0 & 0 & 0 & 0 & 0 & 0 & ㅇ. \\
\hline Metals by Other Regulatory Suite & 426 & 4 & 0 & 0 & D) & 303 & 0 & 128 & 431 \\
\hline Metals by Suite, Other & 2690 & 4647 & 0 & 0 & O. & 1426 & 0 & 1267 & 5015 \\
\hline Metals by Metal Except Mercury & 1865 & 56 & 0 & 0 & 105 & 1091 & 50 & 810 & 1988 \\
\hline Mercury & 2064 & 4812 & 1. & 1 & 105 & 1440 & 52 & 772 & 4624 \\
\hline Anions/Cations-1C & 3854 & 86 & 0 & 0 & 211 & 3210 & 62 & 698 & 4060 \\
\hline Inorganics Wet Chemistry & 5211 & 154 & 79 & 11 & 0 & 4060 & 267 & 1129 & 5456 \\
\hline Other inorganics & 258 & 108 & 0 & 0 & 0 & 269 & 50 & 45 & 365 \\
\hline TOTAL INORGANICS & 16687 & 10833 & 99 & 19 & 422 & 12987 & 581 & 4872 & 23250 \\
\hline \multicolumn{10}{|l|}{10} \\
\hline $\begin{array}{l}\text { Gross Apha/Beta } \\
\text { Gamma Scan }\end{array}$ & $\frac{2247}{88}$ & $\frac{15243}{4895}$ & $\frac{32}{36}$ & 36 & $\frac{211}{110}$ & 12164 & 358 & $\frac{142}{104}$ & $\frac{15216}{5009}$ \\
\hline Transuranic & 0 & $\frac{4000}{0}$ & 0 & $\begin{array}{r}14 \\
0\end{array}$ & \begin{tabular}{l|}
110 \\
110
\end{tabular} & $\begin{array}{r}4794 \\
0\end{array}$ & $\begin{array}{r}159 \\
0\end{array}$ & $\frac{101}{30}$ & $\begin{array}{r}5094 \\
70\end{array}$ \\
\hline Plutonium Isotopes & 694 & 35 & 16 & 3 & 211 & 677 & 44 & 57 & 868 \\
\hline Uranium Isotopes & 696 & 50 & 9 & 3 & 211 & 678 & 50 & 58 & 877 \\
\hline Curium Isotopes & 0 & 15 & 2 & 1 & 10 & 2 & 6 & 10 & 23 \\
\hline Thorium Isotopes & 16 & 3 & 1 & 25 & 0 & 0 & 5 & 40 & 45 \\
\hline Radium Isotopes & 1 & 3 & 0 & 0 & 0 & 1 & 0 & 3 & 4 \\
\hline Americium - 241 & 678 & 23 & 3 & 1 & 20 & 677 & 21 & 7) & 715 \\
\hline Neptunium - 237 & 16 & 5 & 8 & 0 & 0 & 0 & 2 & 27 & 29 \\
\hline Polonium-210 & 0 & 3 & 0 & 0 & 0 & 0 & 0 & 3 & 3 \\
\hline Tritium & 591 & 52 & 22 & 3 & 0 & 592 & 41 & 7 & 653 \\
\hline Radon-222 & 0 & 0 & 0 & 0 & 0 & 0 & 0 & 0 & 요 \\
\hline Lead-210 & 0 & 12 & 0 & 0 & 0 & 0 & 0 & 12 & 12 \\
\hline Carbon-14 & 0 & 0 & 0 & 0 & 0 & 0 & 0 & 0 & 0 \\
\hline Nickel-63 & 0 & 0 & of & \begin{tabular}{l|l}
0 \\
\end{tabular} & 0 & 0 & 0 & 0 & 0 \\
\hline Selenium-79 & 80 & 0 & 이 & 0 & 0 & 80 & 0 & 0 & 80 \\
\hline Technetium-99 & 0 & 11 & 0 & 0 & 요 & 0 & 0 & 11 & 11 \\
\hline Strontium Isotopes & 0 & 0 & 아 & 0 & 요 & 0 & 0 & 0 & 으 \\
\hline Strontium - 89 & o) & 0 & of & 0 & 0 & 0 & 0 & 0. & 0 \\
\hline Strontium-90 & 80 & 22 & 15 & 3 & 0 & 82 & 39 & 0 & 120 \\
\hline lodine-129 & 80 & 6 & 0 & 0 & 0 & 87 & 0 & 0 & 87 \\
\hline Nickel-59 & 0 & 0 & 0 & 0 & 0 & 0 & 01 & 0 & 요 \\
\hline Total Uranium & 65 & 3 & 아 & 0 & 211 & 3 & 0 & 95 & 188 \\
\hline Total Thorium & 0 & 0 & 0 & 0 & 0 & 0 & 0 & 0 & 0 \\
\hline Total Strontium & 15 & 12 & 요 & 0 & 0 & 19 & 0 & 8 & 27 \\
\hline Other RAD & 230 & 204 & 12 & 3 & 10 & 2301 & 71 & 145 & 452 \\
\hline TOTALRAD & 5578 & 20598 & 144 & 911 & 1104 & 20086 & 797 & 754 & 24577 \\
\hline & & & & & & & & & \\
\hline Other Wet Chemistry & 1220 & 10 & 0 & 0 & 30 & 1230 & 01 & $30]$ & 1260 \\
\hline TCLP Extraction & 7 & 105 & 37 & 2 & 0 & 67 & 57 & 27 & 151 \\
\hline Asbestos & 0 & of & 0 & 0 & 0 & 0 & 3 & 0. & 1 \\
\hline Industrial Hygiene, NOS & 552 & 0 & 0 & 0 & 0 & 552 & 0 & 0 & 552 \\
\hline Physical Properties & 12128 & 20 & 3 & 1 & 0 & 7917 & 10 & 4226 & 12153 \\
\hline Other, Not Otherwise Specified (ND & 33 & 9] & 0 & 0 & o. & 8) & 0 & 33 & 42 \\
\hline TOTAL OTHER & 13940 & 144 & 40 & 3 & 30 & 9774 & 70 & 4317 & 14159 \\
\hline & & & & & & & & & \\
\hline ITOTAL SAMPLE ANALYSES & 40424 & 36637 & 313 & 124 & $1556]$ & 46487 & 1752 & $10676]$ & 68984 \\
\hline
\end{tabular}


Table Ve: FY 97 Samples and Sample Analyses by Rad Level and Matrix Type (i) Complex-wide

\begin{tabular}{|c|c|c|c|c|c|c|c|c|c|}
\hline $\begin{array}{c}\text { Rad-Matrix } \\
\text { Sample-Sample Analysis }\end{array}$ & Ro & R1 & $\overline{\mathrm{R} 2}$ & $\overline{\mathrm{R} 3}$ & $\overline{\mathrm{R4}}$ & M1 & M2 & $\overline{\mathrm{M3}}$ & TOTAL \\
\hline CUSTOMER SAMPLES & 47165 & 178084 & 3607 & 7077 & 620 & 95053 & 65728 & 43064 & 220200 \\
\hline \multirow{2}{*}{\multicolumn{10}{|c|}{ SAMPLE ANALYSES }} \\
\hline & & & & & & & & & \\
\hline Volatiles by GC/MS & 19081 & 13432 & 698 & 752 & 0] & 12770 & 18012 & 3201 & 33973 \\
\hline Volatiles by GC & 6032 & 2472 & 222 & 50 & ㅇ.] & 3346 & 4686 & 495 & 8651 \\
\hline Semi-Volatiles & 12047 & 11416 & 694 & 651 & o. & 7744 & 14552 & 2516 & 24810 \\
\hline Herbicides/Pesticides/PCBs & 12316 & 22708 & 50 & 25 & 0 & 7983 & 22061 & 5099 & 35121 \\
\hline Other Organics & 8844 & 20997 & 34 & 0 & o. & 7075 & 1927 & 923 & 19900 \\
\hline TOTAL ORGANICS & 583201 & 71024 & 1698 & 1478 & 0 & 38919 & 61238 & 12234 & 122455 \\
\hline & & & & & & & & & \\
\hline RCRA Appendix IX Metals & 102 & 172 & 0 & 0 & o. & 101 & 168 & 80 & 311 \\
\hline CAM (California) Metals (RCRA) & 595 & 141 & 0 & 0 & 요 & 736 & 176 & 35 & 842 \\
\hline RCRA TCLP Metals and EPTOX Me & 4178 & 6819 & 699 & 10 & 0 & 2964 & 6879 & 1419 & 11484 \\
\hline EPA CLP Metals (CERCLA) & 21587 & 7104 & 1153 & 1623 & 0] & 10910 & 17493 & 3065 & 31467 \\
\hline NPDES Metals (Clean Water Act) & 2927 & 3570 & 0 & 0 & 0 & 6497 & 0 & 0 & 6497 \\
\hline Drinking Water Metals (SDWA) & 724 & 0 & 0 & 0 & 0 & 724 & 0 & 0] & 724 \\
\hline Metals by Other Regulatory Suite & 21174 & 4448 & 257 & 0 & ㅇ. & 11347 & 12419 & 2895 & 26270 \\
\hline Metals by Suite, Other & 7666 & 25213 & 193 & 0. & o. & 7056 & 1590 & 3662 & 22690 \\
\hline Metals by Metal Except Mercury & 9647 & 11845 & 285 & 0) & 300 & 7682 & 5107 & 7341 & 21104 \\
\hline Mercury & 12600 & 32027 & 73 & 5 & 300 & 12935 & 10250 & 2772 & 35481 \\
\hline Anions/Cations-IC & 15242 & 3889 & 120 & Of & 601 & 15052 & 508 & 2593 & 19002 \\
\hline Inorganics Wet Chemistry & 67850 & 11304 & 1079 & 122 & 0 & 58049 & 15893 & 8122 & 81210 \\
\hline Other Inorganics & 6209 & 2588 & 0 & 0 & 0. & 5157 & 2961 & 679 & 8797 \\
\hline TOTAL INORGANICS & 170501 & 109120 & 3858 & 1760 & 1201 & 139209 & 73444 & 32664 & 265879 \\
\hline & & & & & & & & & \\
\hline Gross Alpha/Beta & 50437 & 80567 & 633 & 311 & 601 & 74971 & 32865 & 1967 & 121176 \\
\hline Gamma Scan & 17514 & 27090 & 414 & 124 & 315 & 28033 & 15616 & 1579 & 45343 \\
\hline Transuranic & 0 & 1208 & 1026 & 0 & 315 & 1368 & 866 & 86 & 2434 \\
\hline Plutonium Isotopes & 22654 & 2392 & 233 & 25 & 601 & 11229 & 13231 & 929 & 25647 \\
\hline Uranium isotopes & 22449 & 12458 & 977 & 22 & 601 & 13897 & 20239 & 1857 & 36250 \\
\hline Curium Isotopes & 0 & 322 & 15 & 7 & 29 & 116 & 149 & 79 & 358 \\
\hline Thorium Isotopes & 246 & 6849 & 153 & 110 & o & 1225 & 5411 & 722 & 7358 \\
\hline Radium lsotopes & 7058 & 46 & 0 & 0 & 의 & 5371 & 1687 & 45 & 7104 \\
\hline Americium-241 & 21444 & 1215 & 128 & 12 & 57 & 11099 & 11246 & 454 & 22828 \\
\hline Neptunium - 237 & 246 & 1964 & 724 & 0 & 0 & 803 & 1431 & 700 & 2934 \\
\hline Polonium-210 & 161 & 51 & 34 & 0 & 요 & 6 & 161 & 80 & 246 \\
\hline Tritium & 13450 & 4339 & 685 & 25 & o & 10629 & 7394 & 135 & 18328 \\
\hline Radon-222 & 4 & 0 & 0 & 0 & 요 & 4 & 0 & 0. & 4 \\
\hline Lead-210 & 0 & 51 & 0 & 0 & 0 & 0 & 0 & 51 & 51 \\
\hline Carbon-14 & 0 & 0 & 0 & 0 & 의 & 0 & 0 & 0 & 0 \\
\hline Nickel-63 & 0 & 0 & 0 & 0 & 요 & 0 & 0 & 0 & 0 \\
\hline Selenium-79 & 595 & 51 & 0 & 0 & 의 & 624 & 13 & 9 & 646 \\
\hline Technetium-99 & 154 & 5829 & 1091 & 0 & 묘 & 1554 & 5086 & 434 & 7074 \\
\hline Strontium Isotopes & 0 & 5 & 0 & 0 & 으. & 0 & 2 & 3 & 5 \\
\hline Strontium -89 & 0 & 0 & 0 & 0 & 요 & 0 & of & 0. & 0 \\
\hline Strontium -90 & 749 & 2053 & 200 & 25 & 므 & 1037 & 1965 & 32 & 3030 \\
\hline lodine-129 & 1034 & 167 & 0 & 0 & 0 & 1160 & 25 & 16. & 1201 \\
\hline Nickel-59 & 0 & 0 & 0 & 0 & ㅇ. & 0 & 0 & 0 & 0 \\
\hline Total Uranium & 1300 & 4791 & 1003 & 0 & 601 & 775 & 2238 & 4168 & 7437 \\
\hline Total Thorium & 0 & 501 & 0 & 0 & 요 & 63 & 438 & 0 & 501 \\
\hline Total Strontium & 9605 & 51 & 0 & 0 & ㅇ. & 4980 & 4495 & 181 & 9656 \\
\hline Other RAD & 25946 & 19151 & 518 & 5292 & 29 & 11101 & 32634 & 7120 & 50895 \\
\hline TOTAL RAD & 195048 & 171150 & 7834 & 5954 & 3146 & 180044 & 157192 & 20647 & 370507 \\
\hline Other Wet Chemist & & & & & 86 & & & & \\
\hline TCLP Extraction & $\frac{29817}{3980}$ & $\begin{array}{r}76 \\
8734\end{array}$ & 509 & \begin{tabular}{r|}
0 \\
4192
\end{tabular} & $\frac{86}{0}$ & $\begin{array}{r}26086 \\
3157\end{array}$ & $\begin{array}{l}3866 \\
7469\end{array}$ & $\begin{array}{r}86 \\
6480\end{array}$ & $\frac{30039}{17261}$ \\
\hline Asbestos & 144 & 58552 & 0 & 0 & 0 & 3 & 43716 & 14987 & 58701 \\
\hline Industrial Hygiene, NOS & 2742 & 28 & 0 & 0 & 0 & 2556 & 219 & 3 & 2770 \\
\hline Physical Properties & 38761 & 3729 & 90 & 2632 & 0 & 24909 & 5194 & 15109 & 45212 \\
\hline Other, Not Otherwise Specified (NO & 531 & 505 & 34 & 0 & 0 & 472 & 27 & 538 & 1053 \\
\hline TOTAL OTHER & 76034 & 71624 & 633 & 6824 & 86. & 57183 & 60483 & 37204 & 155036 \\
\hline TOTAL SAMPLE ANALYSES & 499903 & 422918 & 14023 & 16016 & 4434 & $415 \overline{355}$ & 352357 & 102749 & 913877 \\
\hline
\end{tabular}


Table Ve: FY 97 Samples and Sample Analyses by Rad Level and Matrix Type (ii) EM-30

\begin{tabular}{|c|c|c|c|c|c|c|c|c|c|}
\hline $\begin{array}{c}\text { Rad-Matrix/ } \\
\text { Sample-Sample Analysis }\end{array}$ & $\overline{\mathrm{RO}}$ & $\overline{\mathrm{R1}}$ & $\overline{\mathrm{A} 2}$ & $\overline{\mathrm{R} 3}$ & $\overline{R 4}$ & $\overline{\mathrm{M1}}$ & $\overline{\bar{M} 2}$ & $\overline{\mathrm{M}} \overline{\overline{3}}$ & TOTAL \\
\hline CUSTOMER SAMPLES & 29082 & 79575 & 1450 & 6523 & 561 & 77000 & 5147 & 16468 & 107903 \\
\hline & & & & & & & & & \\
\hline \multicolumn{10}{|l|}{ SAMPLEANALYSES } \\
\hline Volatiles by $\mathrm{GC} / \mathrm{MS}$ & 1206 & 1224 & 285 & 702 & 0. [ & 581 & 487 & 2353 & 3419 \\
\hline Volatiles by GC & 1076 & 1702 & 146 & 30 & 0 & 1075 & 1652 & 171 & 2926 \\
\hline Semi-Volatiles & 3724 & 1230 & 283 & 603 & 0 & 2966 & 901 & 1980 & 5845 \\
\hline Herbicides/Pesticides/PCBs & 3061 & 2194 & 30 & 15 & 0] & 3765 & 372 & 1170 & 5304 \\
\hline Other Organics & 5596 & 18277 & 32 & 0 & 0] & $4917 !$ & 7 & 816 [ & 14823 \\
\hline TOTAL ORGANICS & 14663 & 24628 & 776 & 1351 & 0] & 13304 & 3419 & 6490 & 32316 \\
\hline & & & & & & & & & \\
\hline RCRA Appendix IX Metals & 46 & 38 & 0 & 0 & 요 & 75 & 4 & 74 & 118 \\
\hline CAM (California) Metais (RCRA) & 246 & 1 & 0 & 0 & 0 & 247 & 1 & 0. & 247 \\
\hline RCRA TCLP Metals and EPTOX Me & 858 & 1057 & 618 & 6 & 0 & 853 & 496 & 1203 & 2546 \\
\hline EPA CLP Metals (CERCLA) & 383 & 1125 & 61 & 1499 & - & 376 & 243 & 2449 & 3068 \\
\hline NPDES Metals (Clean Water Act) & 335 & 3247 & 0 & 0 & 0 & 3582 & 0 & 0 & 3582 \\
\hline Drinking Water Metals (SDWA) & 128 & 0 & 0 & 0 & 0 & 128 & 0 & o. & 128 \\
\hline Metals by Other Regulatory Suite & 2929 & 1108 & 239 & 0 & 0 & 2148 & 234 & 1894 & 4276 \\
\hline Metals by Suite, Other & 7155 & 18206 & 0 & o & 0. & 3797 & 1 & 3399 & 16279 \\
\hline Metals by Metal Except Mercury & 5760 & 2910 & 244 & 0 & 280 & 4707 & 509 & 2394 & 8402 \\
\hline Mercury & 6483 & 20267 & 43 & 3 & 280 & 6819 & 723 & 2446 & 18532 \\
\hline Anions/Cations-IC & 10661 & 1622 & 112 & 0 & 561 & 8845 & 246 & 2280 & 12162 \\
\hline Inorganics Wet Chemistry & 18841 & 1449 & 872 & 82 & 0 & 13428 & 2204 & 6788 & 21832 \\
\hline Other Inorganics & 1202 & 937 & 0 & 0 & 0. & 1297 & 264 & 577 & 2139 \\
\hline TOTAL INORGANICS & 55026 & 51965 & 2190 & 1590 & 1121: & 46302 & 4925 & 23504 & 93311 \\
\hline \multicolumn{10}{|l|}{ 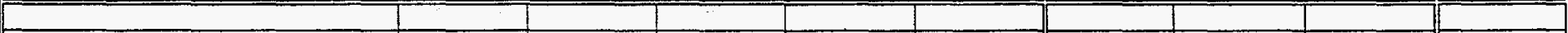 } \\
\hline Gross Alpha/Beta & 9505 & 63129 & 249 & 190 & 561 & 50036 & 2161 & 1093 & 63462 \\
\hline Gamma Scan & 1258 & 19644 & 169 & 76 & 294 & 18798 & 1493 & 937 & 21334 \\
\hline Transuranic & 0 & 933 & 958 & 0 & 294 & 1277 & 613 & 80 & 2077 \\
\hline Plutonium Isotopes & 3079 & 514 & 101 & 15 & 561 & 2777 & 349 & 663 & 4029 \\
\hline Uranium Isotopes & 2974 & 2011 & 811 & 14 & 561 & 4321 & 589 & 980 & 6130 \\
\hline Curium Isotopes & 0 & 117 & 9 & 5 & 27 & 25 & 38 & 69 & 144 \\
\hline Thorium Isotopes & 209 & 1133 & 40 & 98 & 0 & 783 & 62 & 635 & 1480 \\
\hline Radium Isotopes & 33 & 39 & 0 & 0 & of & 33 & 0 & 38 & 71 \\
\hline Americium-241 & 2767 & 369 & 14 & 8 & 53 & 2747 & 149 & 262 & 3184 \\
\hline Neptunium-237 & 209 & 784 & 675 & 0 & 0 & 666 & 393 & 610 & 1668 \\
\hline Polonium-210 & 1 & 40 & 32 & 0 & 0 & 1 & 1 & 71 & 73 \\
\hline Tritium & 2481 & 504 & 149 & 15 & 0 & 2420 & 343 & 26 & 2969 \\
\hline Radon-222 & 4 & 0 & 0 & 0 & 0 & 4 & 0 & 요 & 4 \\
\hline Lead-210 & of & 47 & 0 & 0 & 0 & 0 & 0 & 47 & 47 \\
\hline Carbon-14 & 0 & 0 & 0 & 0 & 0 & 0 & 0 & 요 & 의 \\
\hline Nickel-63 & 0 & 0 & 0 & 0 & 0 & 0 & 0 & of & 의 \\
\hline Selenium-79 & 246 & 0 & 0 & 0 & o & 246 & 0 & 0 & 246 \\
\hline Technetium -99 & 27 & 1300 & 990 & 0 & 0 & 1304 & 612 & 401 & 2317 \\
\hline Strontium Isotopes & 0 & 4 & 0 & 0 & 0 & 0 & 2 & 2 & 4 \\
\hline Strontium -89 & 0 & 0 & 0 & 0 & o. & 0 & 0 & o) & 으 \\
\hline Strontium-90 & 273 & 140 & 126 & 15 & 0 & 279 & 275 & o & 554 \\
\hline lodine-129 & 323 & 80 & 0 & 0 & 0 & 403 & 0 & 0 & 403 \\
\hline Nickel-59 & 0 & 0 & 0 & 0 & 0 & 0 & 0 & 0 & 욤 \\
\hline Total Uranium & 857 & 812 & 936 & 0 & 561 & 387 & 1123 & 1175 & 2925 \\
\hline Total Thorium & 0 & 59 & 0 & 0 & 0 & 0 & 58 & 0 & 59 \\
\hline Total Strontium & 90 & 47 & 0 & 0 & 0 & 93 & 13 & 31 & 137 \\
\hline Other RAD & 1697 & 1994 & 147 & 4931 & 27 & 1922 & 435 & 6361 & 8757 \\
\hline TOTAL RAD & 26031 & 93700 & 5405 & 5367 & 2937 & 88521 & 87091 & 13480 & 122075 \\
\hline \\
\hline Qther Wet Chemistry & 4955 & 66 & 01 & 01 & 80 & 5021 & 0 & 80 & 5101 \\
\hline TCLP Extraction & 573 & 1768 & 434 & 3906 & 0 & 666 & 619 & 5342 & 6654 \\
\hline Asbestos & 1 & 33 & 0 & 0 & 0 & of & 44 & 0 & 39 \\
\hline Industrial Hygiene, NOS & 2163 & 0 & 0 & 0 & o. & 2160 & 3 & 0. & 2163 \\
\hline Physical Properties & 32478 & 1348 & 17 & 2453 & 0 & 22097 & 111 & 14088 & 36296 \\
\hline Other, Not Otherwise Specified (NO & 427 & 78 & 32 & 01 & 0 & 68 & 4 & 460 & 534 \\
\hline TOTAL OTHER & 40597 & 3293 & 483 & 6360 & $80]$ & 30012 & 780 & 19971 & 50787 \\
\hline & & & & & & & & & \\
\hline 101 AL SAMPLE ANALYSES & 136317 & 173585 & 8853 & 14668 & 4138 & 178140 & 17832 & 63445[ & 298490 \\
\hline
\end{tabular}


Table Ve: FY 97 Samples and Sample Analyses by Rad Level and Matrix Type (iii) EM-40

\begin{tabular}{|c|c|c|c|c|c|c|c|c|c|}
\hline $\begin{array}{c}\text { Rad-Matrix / } \\
\text { Sample-Sample Analysis }\end{array}$ & Ro & R1 & $\mathrm{R2}$ & $\overline{\mathrm{R3}}$ & R4 & M1 & M2 & $\mathrm{M3}$ & TOTAL \\
\hline CUSTOMER SAMPLES & 13255 & 90345 & 2005 & 86 & 18 & 9885 & 58464 & 24964 & 99511 \\
\hline & & & & & & & & & \\
\hline \multicolumn{10}{|l|}{ SAMPLE ANALYSES } \\
\hline Volatiles by GC/MS & 12413 & 11908 & 386 & 0 & 0 & 8147 & 15909 & 668 & 24715 \\
\hline Volatiles by GC & 2664 & 581 & 45 & 17 & 0 & 337 & 2680 & 306 & 3315 \\
\hline Semi-Volatiles & 6151 & 9918 & 384 & 4 & 0 & 3627 & 12440 & 388 & 16456 \\
\hline Herbicides/Pesticides/PCBs & 6917 & 19991 & 17 & 8 & 0. & 3170 & 20028 & 3772 & 26951 \\
\hline Other Organics & 1730 & 1204 & 0 & 0 & 0 & 757 & 1834 & 48 & 2787 \\
\hline TOTAL ORGANICS & 29876 & 43602 & 831 & 29 & 0. & 16038 & 52891 & 5181 & 74224 \\
\hline & & & & & & & & & \\
\hline RCRA Appendix IX Metals & 33. & 129 & 0 & 0 & 0 & 9 & 153 & 의 & 163 \\
\hline CAM (California) Metals (RCRA) & 326 & 135 & 0 & 0 & 0 & 461 & 169 & 34 & 563 \\
\hline RCRA TCLP Metais and EPTOX Me & 1238 & 5531 & 16 & 3 & 0 & 398 & 6032 & 127 & 6673 \\
\hline EPA CLP Metals (CERCLA) & 13380 & 5795 & 1068 & 17 & 0 & 6149 & 13686 & 426 & 20261 \\
\hline NPDES Metals (Clean Water Act) & 2519 & 56 & 0 & 0 & 0 & 2575 & 0 & O. & 2575 \\
\hline Drinking Water Metals (SDWA) & 66 & 이 & 0 & 0 & 0 & 66 & of & 요 & 66 \\
\hline Metals by Other Regulatory Suite & 10916 & 3204 & 0. & 0 & 0 & 4740 & 9305 & 844 & 14504 \\
\hline Metals by Suite, Other & 0 & 5421 & 190 & 0 & 0 & 2934 & 1562 & 20 & 5063 \\
\hline Metals by Metal Except Mercury & 1349 & 8518 & 3 & 0 & ㅇ. & 857 & 4293 & 4692 & 9856 \\
\hline Mercury & 3602 & 9877 & 6 & 2 & 0. & 3890 & 9124 & 149 & 13324 \\
\hline Anions/Cations-IC & 3723 & 2110 & 0 & 0 & 0 & 5447 & 238 & 148 & 5833 \\
\hline Inorganics Wet Chemistry & 21092 & 9575 & 142 & 34 & 0] & 19111 & 11333 & 833 & 31060 \\
\hline Other Inorganics & 4690 & $15 \overline{54}$ & 0 & 0 & 0. & 3576 & 2610 & 58 & 6244 \\
\hline TOTAL INORGANICS & 62932 & 51907 & 1425 & 55] & 0 & 50212 & 58505 & 7331 & 116184 \\
\hline \multicolumn{10}{|l|}{ 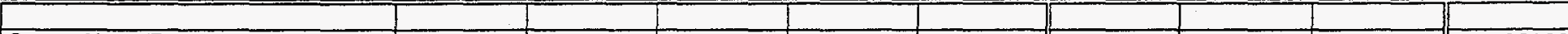 } \\
\hline Gross Alpha/Beta & 26127 & 12104 & 360 & 105 & 요 & 13970 & 23215 & 763 & 38322 \\
\hline Gamma Scan & 9001 & 5738 & 229 & 42 & 0 & 4912 & 9541 & 556 & 15010 \\
\hline Transuranic & 0 & 199 & 0 & 0 & 0 & 0 & 199 & 요 & 199 \\
\hline Plutonium Isotopes & 10808 & 1804 & 122 & 8 & 0 & 3998 & 8537 & 207 & 12742 \\
\hline Uranium Isotopes & 12429 & 10110 & 106 & 8 & 인 & 5743 & 16130 & 780 & 22653 \\
\hline Curium lsotopes & 0 & 192 & 5 & 3 & 0 & 88 & 107 & 5 & 200 \\
\hline Thorium Isotopes & 22 & 5536 & 108 & 4 & 0 & 380 & 5251 & 40 & 5670 \\
\hline Radium Isotopes & 3416 & 4 & 0 & 0 & 0 & 2467 & 949 & 4 & 3420 \\
\hline Americium-241 & 10598 & 804 & 112 & 4 & 0 & 4028 & 7328 & 163 & 11518 \\
\hline Neptunium-237 & 22 & 1104 & 1 & 0 & 0 & 88 & 992 & 46 & 1126 \\
\hline Polonium-210 & 154 & 5 & 0 & 0 & 0 & 1 & 154 & 4 & 159 \\
\hline Tritium & 5912 & 3731 & 516 & 8 & 0 & 4546 & 5559 & 105 & 10189 \\
\hline Radon-222 & 0 & 0 & 0 & 0 & 0 & 0 & 0 & 의 & 0 \\
\hline Lead-210 & 0 & 1 & 0 & 0 & 요 & o & 이 & 1. & 1 \\
\hline Carbon-14 & of & 이 & 0 & 0 & 0 & 0 & 이 & 으 & 0 \\
\hline Nickel -63 & 0 & 0 & 0 & 0 & 0 & 0 & 0 & o & 0 \\
\hline Selenium-79 & 326 & 50 & 0 & 0 & 0 & 354 & 12 & 9 & 376 \\
\hline Technetium-99 & 14 & 4357 & 31 & 0 & 0 & 45 & 4353 & 4 & 4402 \\
\hline Strontium Isotopes & 0 & 0 & 0 & 0 & 0 & 0 & 0 & 의 & 0 \\
\hline Strontium -89 & of & 0 & 0 & 0 & 은 & 0 & 0 & 0 & 0 \\
\hline Strontium-90 & 340 & 1870 & 64 & 8 & 0 & 616 & 1641 & 31 & 2285 \\
\hline lodine-129 & 366 & 80 & 0 & 0 & 0 & 405 & 25 & 16 & 446 \\
\hline Nickel-59 & 0 & 0 & 0 & 0 & 0 & 0 & 0 & 은 & 0 \\
\hline Total Uranium & 266 & 3847 & 0 & 0 & ㅇ․ & 244 & 1016 & 2853 & 4113 \\
\hline Total Thorium & D & 430 & 0 & 0 & 0 & 61 & 369 & 은 & 430 \\
\hline Total Strontium & 5084 & 1 & 0 & 0 & 0 & 2250 & 2697 & 137 & 5085 \\
\hline Other RAD & 14201 & 16662 & 334 & 8 & 0 & 3589 & 27322 & 297 & 31207 \\
\hline TOTAL RAD & 99086 & 68628 & 1986 & 199 & 0 & 47785 & 115397 & 6021 & 169551 \\
\hline & & & & & & & & & \\
\hline Other Wet Chemistry & 12150 & 5 & 0 & 0) & 0 & 9744 & 2411 & of & 12154 \\
\hline TCLP Extraction & 1325 & 6664 & 22 & 7 & 0. & 785 & 6462 & 743 & 8004 \\
\hline Asbestos & 74 & 57490 & 0 & 0 & ㅇ․ & 1 & 42839 & 14724 & 57564 \\
\hline Industrial Hygiene, NOS & 381 & 28 & 0 & 0 & D. & 211 & 194 & 3 & 409 \\
\hline Physical Properties & 1725 & 2243 & 70 & 3 & D. & 731 & 3297 & 14 & 4042 \\
\hline Other, Not Otherwise Specified (NO & 72 & 390 & 01 & 0 & 0 & 392 & 22 & 45 & 460 \\
\hline TOTAL OTHER & 15726 & 66820 & 92 & 101 & 0 & 11863 & 55226 & 15529 & 82633 \\
\hline & & & & & & & & & \\
\hline TOTAL SAMPLE ANALYSES & 207620 & 230957 & 4334 & $294 \mid$ & 0] & 125898 & 282019 & 34063 & 442593 \\
\hline
\end{tabular}


Table Ve: FY 97 Samples and Sample Analyses by Rad Level and Matrix Type (iv) EM-60

\begin{tabular}{|c|c|c|c|c|c|c|c|c|c|}
\hline $\begin{array}{c}\text { Rad-Matrix } \\
\text { Sample-Sample Analysis }\end{array}$ & Ro & RI & R2 & R3 & $\overline{\mathrm{R}} 4$ & M1 & $\overline{\mathrm{M} 2}$ & $\overline{\mathrm{M} 3}$ & TOTAL \\
\hline CUSTOMER SAMPLES & 2204 & 762 & 101 & 11 & 可 & 2198 & 607 & 7 & 2894 \\
\hline & & & & & & & & & \\
\hline \multicolumn{10}{|l|}{ SAMPLEANALYSES } \\
\hline Volatiles by GC/MS & 4541 & 0 & 01 & 0 & 0 & 3396 & 1145 & 0 & $45 \overline{41}$ \\
\hline Volatiles by GC & 1916 & 50 & 18 & 0 & 의 & 1637 & 166 & 0 & 1894 \\
\hline Semi-Volatiles & 1586 & 2 & 0 & 0 & 0 & 770 & 818 & 0 & 1588 \\
\hline Herbicides/Pesticides/PCBs & 1764 & 2 & 0 & 0 & 0 & 637 & 1129 & 0 & 1766 \\
\hline Other Organics & 961 & 167 & 0 & 0 & 0 & 916 & 46 & 0. & 1045 \\
\hline \multirow[t]{2}{*}{ TOTAL ORGANICS } & 10769 & 221 & 18 & 0 & 0. & 7356 & 3303 & 11 & 10834 \\
\hline & & & & & & & & 7 & \\
\hline RCRA Appendix IX Metals & 16 & 0 & 0 & 0 & 0 & 10 & 7 & of & 17 \\
\hline CAM (California) Metals (RCRA) & $\mathbf{0}$ & 0 & o & 0 & o. & 0 & 0 & 요 & 0. \\
\hline RCRA TCLP Metals and EPTOX Me & 1761 & 50 & 18 & 0 & 0 & 1453 & 180 & 0 & 1731 \\
\hline EPA CLP Metals (CERCLA) & 6668 & 0 & \begin{tabular}{l|l}
0 & \\
\end{tabular} & 0 & 0 & 3742 & 2919 & 7 & 6668 \\
\hline NPDES Metals (Clean Water Act) & 3 & 29 & 0 & 0 & 0 & 32 & 0 & 0 & 32 \\
\hline Drinking Water Metais (SDWA) & 460 & 0 & 0 & 0 & 0 & 460 & 0 & 0 & 460 \\
\hline Metals by Other Regulatory Suite & 6109 & 0 & 0 & 0 & 0 & 3718 & 2384 & 7 & 6109 \\
\hline Metals by Suite, Other & 0 & 166 & 0 & 0. & 0 & 0 & 0 & 0 & 83 \\
\hline Metals by Metal Except Mercury & 1859 & 48 & 18 & 0 & 0 & 1562 & 168 & 0 & 1828 \\
\hline Mercury & 1757 & 218 & 18 & 0 & o. & 1466 & 166 & 0 & 1812 \\
\hline Anions/Cations - IC & 27 & 3 & 0 & 0 & 요 & 28 & 2 & 0 & 30 \\
\hline Inorganics Wet Chemistry & 23146 & 3 & 0 & 0 & 0 & 21383 & 1764 & 1 & 23148 \\
\hline Other Inorganics & 106 & 3 & 0 & 0 & 0 & 106 & 2 & 1 & 109 \\
\hline \multirow[t]{2}{*}{ TOTALINORGANICS } & 41912 & 521 & 54 & 0 & 0 & 33960 & 7592 & 16 & 42028 \\
\hline & & & & & & & & & \\
\hline \multirow{2}{*}{\begin{tabular}{|l} 
Gross Alpha/Beta \\
Gamma Scan \\
\end{tabular}} & 12040 & 538 & 0 & 0 & 0 & 6287 & 6108 & 17. & 12494 \\
\hline & 6187 & 179 & 0 & 0 & 0 & 2554 & 3804 & 8 & 6367 \\
\hline \multirow{4}{*}{\begin{tabular}{|l|} 
Transuranic \\
Plutonium Isotopes \\
Uranium Isotopes \\
Curium Isotopes \\
\end{tabular}} & 0 & 5 & 0 & 0 & 0 & 0 & 5 & 0 & 5 \\
\hline & 7366 & 5 & 0 & 0 & 0 & 3690 & 3674 & 7 & 7372 \\
\hline & 5823 & 5 & 0 & 0 & 0 & 3012 & 2810 & 7 & 5828 \\
\hline & 0 & 0 & 0 & 0 & 0 & 0 & 0 & 0 & 0 \\
\hline \multirow{2}{*}{\begin{tabular}{|l|} 
Thorium Isotopes \\
Radium Isotopes \\
\end{tabular}} & 0 & 0 & 0 & 1 & 0 & 0 & 0 & 1 & 1 \\
\hline & 3135 & 0 & 0 & 0 & 0 & 2497 & 638 & 0 & 3135 \\
\hline \multirow{2}{*}{\begin{tabular}{|l|} 
Americium - 241 \\
Neptunium-237 \\
\end{tabular}} & 6781 & 0 & 0 & 0 & 0 & 3577 & 3197 & 7 & 6781 \\
\hline & 0 & 0 & 0 & 0 & 음 & 0 & 0 & 요 & 요 \\
\hline \multirow{2}{*}{\begin{tabular}{|l} 
Polonium-210 \\
Tritium \\
\end{tabular}} & 0 & 4 & 0 & 0 & 0 & 4 & 0 & 0 & 4 \\
\hline & 4221 & 0 & 0 & 0 & 0 & 3013 & 1208 & 0 & 4222 \\
\hline \multirow{2}{*}{\begin{tabular}{|l|} 
Radon-222 \\
Lead-210 \\
\end{tabular}} & 0 & 0 & 0 & 0 & 0 & 0 & 0 & 0 & 요 \\
\hline & 0 & 0 & 0 & 0 & 0 & 0 & 0 & 0 & 0 \\
\hline \multirow{2}{*}{\begin{tabular}{|l|} 
Carbon-14 \\
Nickel-63 \\
\end{tabular}} & 0 & 0 & 0 & 0 & 0 & 0 & 0 & 0 & 0 \\
\hline & 0 & 0 & 0 & 0 & 0 & 0 & 0 & 요 & 인 \\
\hline \multirow{2}{*}{\begin{tabular}{|l|} 
Selenium-79 \\
Technetium-99 \\
\end{tabular}} & 0 & 0 & 0 & 0 & 0 & 0 & 0 & 0 & 0 \\
\hline & 98 & 0 & 0 & 0 & 0 & 98 & 0 & 0 & 98 \\
\hline \multirow{2}{*}{\begin{tabular}{|l|} 
Strontium Isotopes \\
Strontium -89 \\
\end{tabular}} & 0 & 0 & 0 & 0 & 0 & 0 & 0 & 0 & 0 \\
\hline & 0 & 0 & 0 & 0 & 0 & 0 & 0 & 이 & 0 \\
\hline Strontium -90 & 98 & 0 & 0 & 0 & 0 & 98 & 0 & 0 & 98 \\
\hline lodine-129 & 279 & 0 & 0 & 0 & 0 & 279 & 0 & 요 & 279 \\
\hline Nickel-59 & 0 & 0 & 0 & 0 & D & 0 & 0 & 0 & 0 \\
\hline Total Uranium & 98 & 0 & 0 & 0 & 0 & 98 & 0 & 0 & 98 \\
\hline Total Thorium & 0 & 0 & 0 & 0 & 0 & 0 & 0 & 0 & 0 \\
\hline Total Strontium & 3829 & 0 & 0 & 0 & 므 & 2288 & 1535 & 7 & 3830 \\
\hline Other RAD & 8544 & 48 & 18 & 0 & 0 & 4755 & 3853 & $1]$ & 8610 \\
\hline TOTAL RAD & 58499 & 787 & 19 & 11 & 0 & 32249 & 26832 & 58 & 59222 \\
\hline & & & & & & & & & \\
\hline Other Wet Chemistry & 10778 & $\overline{01}$ & 0 & 0 & 0 & 9530 & 1249 & 0. & 10779 \\
\hline TCLP Extraction & 1774 & 49 & 19 & 0 & 0. & 1452 & 195 & 요 & 1745 \\
\hline Asbestos & 60 & 0 & 0 & 0 & 0 & 2 & 57 & 0 & 60 \\
\hline Industrial Hygiene, NOS & 31 & 0. & 0 & 0 & 0 & 22 & 9 & 0 & 31 \\
\hline Physical Properties & 1951 & 0 & 0 & 0 & 0 & 431 & 1520 & 0 & 1951 \\
\hline Other, Not Otherwise Specified (NO & 0 & 22 & 0 & 0 & 0 & 0 & 0 & 0 & 11 \\
\hline TOTAL OTHER & 14594 & 72 & 19 & 01 & 0 & 11437 & 3031 & $0]$ & 14576 \\
\hline & & & & & & & & & \\
\hline HOIAL SAMPLE ANALYSES & 125774 & 1600 & 111 & 11 & 0] & 85001 & 40758 & 76] & 126660 \\
\hline
\end{tabular}


Table Ve: FY 97 Samples and Sample Analyses by Rad Level and Matrix Type (v) Fernald

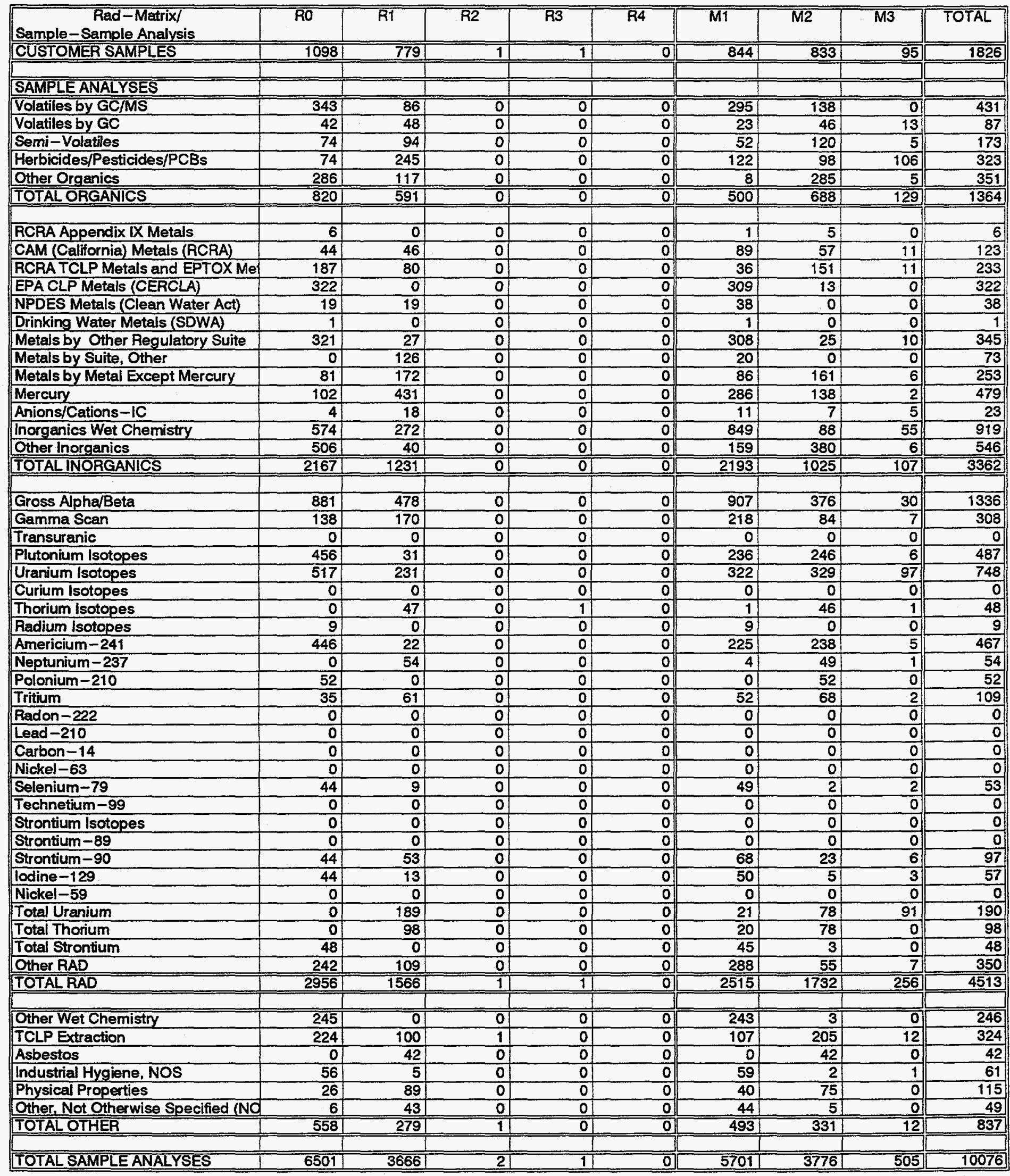


Table Ve: FY 97 Samples and Sample Analyses by Rad Level and Matrix Type (vi) INEL

\begin{tabular}{|c|c|c|c|c|c|c|c|c|c|}
\hline $\begin{array}{c}\text { Rad-Matrix } \\
\text { Sample-Sample Analysis }\end{array}$ & RO & $\overline{\mathrm{R} 1}$ & R2 & $\overline{\mathrm{R3}}$ & $\overline{R 4}$ & $\overline{\mathrm{MI}}$ & M2 & $\overline{\mathrm{M} 3}$ & TOTAL \\
\hline CUSTOMER SAMPLES & 1439 & 11030 & 180 & 6261 & 0 & 4941 & 4480 & 2526 & 12612 \\
\hline \multicolumn{10}{|l|}{ SAMPLEANALYSES } \\
\hline Volatiles by GC/MS & 220 & 674 & 2 & 66 & 0 & 193 & $\frac{1}{613}$ & 156 & 961 \\
\hline Volatiles by GC & 625 & 228 & 26 & 6 & 0 & 523 & 288 & 22 & 859 \\
\hline Semi-Volatiles & 181 & 587 & 8 & 57 & 0 & 146 & 617 & 71 & 834 \\
\hline Herbicides/Pesticides/PCBs & 306 & 1301 & 6 & 3 & 0 & 240 & 1022 & 354 & 1616 \\
\hline Other Organics & 485 & 1276 & 0 & 01 & 0 & 273 & 228 & 6 & 1134 \\
\hline TOTAL ORGANICS & 1816 & 4066 & 42 & 132 & 0 & 1375 & 2769 & 609 & 5405 \\
\hline \multicolumn{10}{|l|}{20} \\
\hline RCRA Appendix IX Metals & 3 & 3 & o & 0 & o. & 5 & 1 & 의 & 6 \\
\hline CAM (California) Metals (RCRA) & 12 & 1 & 0 & 0 & a & 13 & 1 & 마 & 14 \\
\hline RCAA TCLP Metals and EPTOX Mef & 578 & 468 & 15 & 1 & 0 & 468 & 513 & 24 & 1034 \\
\hline EPA CLP Metals (CERCLA) & 199 & 71 & 18 & 144 & 0 & 154 & 130 & 149 & 433 \\
\hline NPDES Metals (Clean Water Act) & 39 & 225 & 0 & 0 & 0 & 264 & 0 & 0 & 264 \\
\hline Drinking Water Metals (SDWA) & 131 & 0 & of & 0 & 0 & 131 & 0 & 0 & 131 \\
\hline Metals by Other Regulatory Suite & 418 & 259 & 0 & 0 & 0 & 216 & 271 & 212 & 688 \\
\hline Metals by Suite, Other & 0 & 1315 & 1 & 0 & 0 & 22 & 10 & 5 & 677 \\
\hline Metals by Metal Except Mercury & 638 & 626 & 5 & 0 & 0 & 513 & 333 & 367 & 1241 \\
\hline Mercuny & 672 & 1952 & 6 & 11 & 0 & 589 & 715 & 18 & 1976 \\
\hline Anions/Cations $-\mathrm{IC}$ & 102 & 64 & 0 & 0 & 0 & 106 & 28 & 33 & 167 \\
\hline Inorganics Wet Chemistry & 1291 & 700 & 99 & 14 & 0 & 1009 & 681 & 417 & 2106 \\
\hline Other Inorganics & 151 & 125 & 0 & 01 & 0 & 167 & 72 & 37[ & 276 \\
\hline TOTAL INORGANICS & 4234 & 5810 & 146 & 160 & 0 & 3658 & 2755 & 1261[ & 9012 \\
\hline Gross Alpha/Beta & 1252 & 4794 & 37 & 37 & 0 & 3555 & \multicolumn{2}{|c|}{ 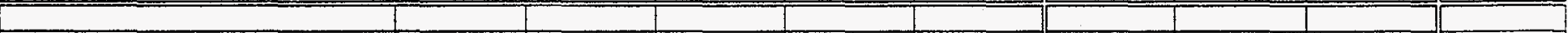 } & 5366 \\
\hline Gamma Scan & 799 & 1631 & 28 & 15 & 0 & 1395 & 1020 & 57 & 2473 \\
\hline Transuranic & 0 & 17 & 0 & 0 & 0 & 0 & 17 & 0 & 17 \\
\hline Plutonium Isotopes & 472 & 71 & 9 & 3 & 0 & 335 & 189 & 31 & 555 \\
\hline Uranium Isotopes & 366 & 471 & 3 & 3 & 0 & 309 & 473 & 60 & 843 \\
\hline Curium lsotopes & 0 & 13. & 2 & 1 & 0 & 3 & 7 & 6 & 16 \\
\hline Thorium Isotopes & 22 & 344 & 2 & 7 & 0 & 9 & 336 & 30 & 375 \\
\hline Radium Isotopes & 43 & 4 & 0 & 0 & 0] & 39 & 5 & 4 & 47 \\
\hline Americium-241 & 344 & 44 & 3 & 1 & o. & 307 & 79 & 7 & 393 \\
\hline Neptunium-237 & 22. & 27 & 2 & 0 & 0 & 3 & 19 & 30 & 51 \\
\hline Polonium-210 & 1 & 5 & 0 & 0 & 0 & 1 & 1 & 4 & 6 \\
\hline Tritum & 357 & 89 & 20 & 3 & o & 294 & 134 & 4 & 450 \\
\hline Radon-222 & 0 & 0 & 0 & 0 & 0 & 0 & 0 & - & 0 \\
\hline Lead-210 & 0 & 3 & 0 & 0 & 0 & 0 & 0 & 3 & 3 \\
\hline Carbon-14 & 0 & 0 & 0 & 0 & 0. & 01 & 0 & 0 & 0 \\
\hline Nickel-63 & 0 & 0 & 0 & 0 & 0 & 0 & 0 & 0 & 0 \\
\hline Selenium-79 & 12 & 7 & 0 & 0 & 0 & 16 & 2 & 1 & 19 \\
\hline Technetium-99 & 28 & 334 & 0 & 0 & o. & 28 & 328 & 6 & 362 \\
\hline Strontium Isotopes & 0 & 0 & 0 & 0 & 0 & 0 & of & 0 & 0 \\
\hline Strontium-89 & 0 & 0 & 0 & 0) & 0 & 0 & 0 & 0 & - \\
\hline Strontium -90 & 40 & 69 & 18 & 3 & 0 & 59 & 66 & 4 & 130 \\
\hline lodine-129 & 92 & 19 & 0 & 0 & D. & 104 & 4 & 2 & 110 \\
\hline Nickel-59 & 0 & 0 & 0 & 0 & 0 & 0 & 0 & 0. & 0 \\
\hline Total Uranium & 116 & 244 & 0 & 0 & 0 & 33 & 45 & 282 & 360 \\
\hline Total Thorium & 0 & 0 & 0 & 0 & 0 & 0 & 0 & 0 & 0 \\
\hline Total Strontium & 59 & 3 & 0 & 0 & 0 & 32 & 27 & 3. & 62 \\
\hline Other RAD & 466 & 1203 & 21 & 465 & 0] & 350 & 1207 & $589]$ & 2150 \\
\hline TOTALRAD & 4490 & 9392 & 146 & 539 & 0 ] & 6873 & 4927 & 1211 & 13789 \\
\hline & & & & & & & & & \\
\hline Other Wet Chemistry & 555 & 6 & 0 & 0 & o. & 549 & 12 & 01 & 561 \\
\hline TCLP Extraction & 558 & 574 & 18 & 368 & o. & 479 & 535 & 448 & 1490 \\
\hline Asbestos & 0 & 4383 & 0 & 0 & 0 & 0 & 3246 & 1138 & 4384 \\
\hline Industrial Hygiene, NOS & 154 & 4 & 0 & 0 & o] & 153 & 5 & 0 & 158 \\
\hline Physical Properties & 144 & 145 & 4 & 231 & 매 & 139 & 140 & 244 & 523 \\
\hline Other, Not Otherwise Specified (NC & 44 & 10 & 0 & 0 & 0 & 4 & 0 & 45. & 52 \\
\hline TOTALOTHEA & 1456 & 5122 & 22 & 600 & 0] & 1323 & 3938 & 1876 & 7169 \\
\hline TOTAL SAMPLE ANALYSES & 11996 & 24391 & 356 & 1431] & 0] & 13229 & 14389 & 4958 & 35375 \\
\hline
\end{tabular}


Table Ve: FY 97 Samples and Sample Analyses by Rad Level and Matrix Type (vii) NTS

\begin{tabular}{|c|c|c|c|c|c|c|c|c|c|}
\hline $\begin{array}{c}\text { Rad-Matrix } \\
\text { Sample-Sample Analysis }\end{array}$ & RO & $\mathrm{R1}$ & $\overline{\mathrm{R} 2}$ & R3 & $\overline{\mathrm{R4}}$ & $\overline{\mathrm{MI}}$ & $\overline{\mathrm{M} 2}$ & $\overline{\mathrm{M3}}$ & TOTAL \\
\hline CUSTOMER SAMPLES & 1043 & 9340 & 176 & 0 & 7 & 670 & 5806 & 2810 & $9 \overline{926}$ \\
\hline \multirow{2}{*}{\multicolumn{10}{|c|}{ SAMPLE ANALYSES }} \\
\hline & & & & & & & & & \\
\hline Volatiles by GC/MS & 1483 & 1264 & 45 & 0 & 0 & 942 & 1729 & 123 & 2793 \\
\hline Volatiles by GC & 280 & 13 & 0 & 0 & 0 & 8 & 224 & 65 & 295 \\
\hline Semi-Volatiles & 730 & 1037 & 39 & 0 & 0 & 387 & 1330 & 88 & 1805 \\
\hline Herbicides/Pesticides/PCBs & 779 & 2057 & 0 & 0 & 0 & 296 & 2171 & 372 & 2838 \\
\hline Other Organics & 19 & 136 & 0 & 0 & 0 & 73 & 73 & 8 & 155 \\
\hline \multirow[t]{2}{*}{ TOTAL ORGANICS } & 3292 & 4506 & 84 & 0 & 0 & 1706 & 5527 & 657[ & 7886 \\
\hline & & & & & & & & & \\
\hline RCRA Appendix IX Metals & 4 & 14 & 0 & 0 & 0 & 1 & 16 & 0 . & 17 \\
\hline CAM (California) Metals (RCRA) & 0 & 0 & 0 & 0 & D. & 0 & 0 & 0 & 0 \\
\hline RCRA TCLP Metals and EPTOX Me & 56 & 551 & 0 & 0 & 0 & 10 & 584 & 11 & 606 \\
\hline EPA CLP Metals (CERCLA) & 1635 & 600 & 115 & 0 & 0 & 725 & 1580 & 45 & 2350 \\
\hline NPDES Metals (Clean Water Act) & 259 & 0 & 0 & 0 & 0 & 259 & 0 & 0 & 259 \\
\hline Drinking Water Metals (SDWA) & 2 & 0 & 0 & 0 & 0 & 2 & 0 & 0 & 2 \\
\hline Metals by Other Regulatory Suite & 1328 & 329 & 0 & 0 & o & 576 & 1099 & 66 & 1698 \\
\hline Metals by Suite, Other & 0 & 533 & 20 & 0 & 0 & 302 & 164 & 2 & 510 \\
\hline Metals by Metal Except Mercury & 72 & 860 & 0 & 0 & of & 25 & 405 & 501 & 932 \\
\hline Mercury & 303 & 934 & 0 & 0 & 0 & 284 & 937 & 14 & 1236 \\
\hline Anions/Cations-IC & 387 & 211 & 0 & 0 & 요 & 567 & 21 & 11 & 598 \\
\hline Inorganics Wet Chemistry & 3031 & 858 & 0 & 0 & 0 & 2649 & 1219 & 20 & 3888 \\
\hline Other Inorganics & 333 & 137 & 0 & 0 & 0 & 314 & 156 & 0 & 470 \\
\hline \multirow[t]{2}{*}{ TOTALINOAGANICS } & 7410 & 5025 & 135 & 0 & 0 & 5716 & 6182 & 670 & 12569 \\
\hline & & & & & & & & & \\
\hline Gross Alpha/Beta & 3003 & 1027 & 29 & 0 & 으 & 1466 & 2534 & 60 & 4060 \\
\hline Gamma Scan & 1078 & 582 & 16 & 0 & 요 & 567 & 1043 & 66 & 1676 \\
\hline Transuranic & 0 & 21 & 0 & 0 & 0 & 0 & 21 & 0 & 21 \\
\hline Plutonium Isotopes & 1333 & 164 & 11 & 0 & 0 & 511 & 981 & 17 & 1509 \\
\hline Uranium Isotopes & 1429 & 1017 & 11 & 0 & ㅇ․ & 641 & 1767 & 48 & 2456 \\
\hline Curium Isotopes & 0 & 19 & 0 & 0 & 의 & 9 & 10 & 0 & 19 \\
\hline Thorium Isotopes & 0 & 588 & 11 & 0 & 0 & 40 & 557 & 2 & 599 \\
\hline Radium Isotopes & 516 & 0 & 0 & 0 & 의 & 383 & 133 & 0 & 516 \\
\hline Americium-241 & 1309 & 70 & 11 & 0 & 의 & 520 & 856 & 15 & 1390 \\
\hline Neptunium-237 & 0 & 108 & 0 & 0 & 0 & 8 & 99 & 2 & 108 \\
\hline Polonium-210 & 0 & 0 & 0 & 0 & 으. & 0 & 0 & 0 & 0 \\
\hline Tritium & 796 & 371 & 51 & 0. & 0 & 595 & 614 & 10 & 1219 \\
\hline Radon-222 & 0 & 0 & 0 & 0 & 요 & 0 & 0 & 0 & 0 \\
\hline Lead -210 & 0 & 0 & 0 & 0 & 의 & 0 & 0 & 0 & 0 \\
\hline Carbon-14 & 0 & 0 & 0 & 0 & 0 & 0 & 0 & 0 & 0 \\
\hline Nickel-63 & 0 & 0 & 0 & 0 & 0 & 0 & 0 & 0 & 0 \\
\hline Selenium -79 & 0 & 0 & 0 & 0 & 0 & 0 & 0 & 0 & 0 \\
\hline Technetium-99 & 0 & 467 & 3 & 0 & 으. & 4 & 467 & 0 & 471 \\
\hline Strontium Isotopes & 0 & 0 & 0 & 0 & 0. & 0 & 0 & 0 & 0 \\
\hline Strontium -89 & 0 & 0 & 0 & 0 & 요 & 0 & 0 & 0 & 0 \\
\hline Strontium -90 & 0 & 164 & 3 & 0 & 요 & 16 & 152 & 0 & 168 \\
\hline lodine-129 & 1 & 0 & 0 & 0 & 0 & 1 & 0 & 0 & 1 \\
\hline Nickel-59 & 0 & 0 & 0 & 0 & 0 & 0 & 0 & 0 & 0 \\
\hline Total Uranium & 18 & 388 & 0 & 0 & 0 & 18 & 120 & 268 & 406 \\
\hline Total Thorium & 0 & 33 & 0 & 0 & 인 & 0 & 33 & 0 & 33 \\
\hline Total Strontium & 715 & 0 & 0 & 0 & 0 & 340 & 361 & 15 & 715 \\
\hline Other RAD & 1780 & 1726 & 32 & 0 & 0 & 453 & 3068 & 18 & 3538 \\
\hline TOTAL RAD & 11979 & 6748 & 179 & 0 & 0 & 5572 & 12817 & 519 & 18907 \\
\hline & & & & & & & & & \\
\hline Other Wet Chemistry & 1731 & 0 & 0 & 0 & 0 & 1413 & 319 & 0 & 1731 \\
\hline TCLP Extraction & 48 & 652 & 1 & 0 & 0 & 21 & 608 & 71 & 700 \\
\hline Asbestos & 25 & 6173 & 0 & 0 & 0 & 0 & 4615 & 1582 & 6198 \\
\hline industrial Hygiene, NOS & 23 & 0 & 0 & 0 & 0 & 3 & 19 & 0 & 23 \\
\hline Physical Properties & 251 & 224 & 6 & 0 & 0 & 63 & 419 & 0 & 482 \\
\hline Other, Not Otherwise Specified (Nd & 2 & 38 & 0 & 0 & 0 & 38 & 2 & o. & 40 \\
\hline TOTAL OTHER & 2081 & 7087 & 7 & 0 & 0 & 1538 & 5982 & 1653 & 9174 \\
\hline & & & & & & & & & \\
\hline TOTAL SAMPLE ANALYSES & 24762 & 23366 & 405 & 0 & 0 & 14532 & 30508 & 3500 & 48536 \\
\hline
\end{tabular}


EM-263 ASSESSMENT OF EM SAMPLING AND ANALYSIS NEEDS

Table Ve: FY 97 Samples and Sample Analyses by Rad Level and Matrix Type (viii) Oak Ridge K-25

\begin{tabular}{|c|c|c|c|c|c|c|c|c|c|}
\hline $\begin{array}{c}\text { Rad-Matrix } \\
\text { Sample-Sample Analysis }\end{array}$ & $\overline{\mathrm{RO}}$ & $\overline{\mathrm{R} 1}$ & $\mathrm{R} 2$ & $\overline{\mathrm{R} 3}$ & $\overline{\mathrm{R4}}$ & M1 & $\overline{M 2}$ & M3 & TOTAL \\
\hline CUSTOMER SAMPLES & 124 & 9439 & $176 \mid$ & 0 & (0) & 381 & 5822 & 3559 & 9750 \\
\hline SAMPLE ANALYSES & & & & & & & & & \\
\hline Volatiles by GC/MS & 12 & 1533 & 107 & 0 & $0]$ & 132 & 1141 & $380][$ & $1652]$ \\
\hline Volatiles by $\mathrm{GC}$ & 69 & 10 & 1 & 0 & 0 & 59 & 14 & 1 & 77 \\
\hline Semi-Volatiles & 11 & 1160 & 95 & 0 & 0 & 13 & 1044 & 211 & 1267 \\
\hline Herbicides/Pesticides/PCBs & 10 & 2706 & 0 & 0 & 의 & 551 & 1607 & 560 & 2717 \\
\hline Other Organics & 33 & 3 & 12 & 0 & 0] & 33. & o & 12 . & 46 \\
\hline TOTAL ORGANICS & 135 & 5412 & $215 \mid$ & 0 & (0) & 786 & 3805 & 1164 & 5759 \\
\hline & & & & & & & & & \\
\hline RCRA Appendix IX Metals & $1]$ & 0 & 요 & 0. & 0. & - & 1 & 26] & 14. \\
\hline CAM (California) Metals (RCRA) & 0 & 이 & 이 & 이 & 0. & 0 & 0 & 으. & 으 \\
\hline RCRA TCLP Metals and EPTOX Me & 107 & 802 & 198 & 0 & 0. & 54 & 663 & 409 & 1117 \\
\hline EPA CLP Metals (CERCLA) & 14 & 338 & 0 & 0 & 0 & 13 & 1 & 338 & 351 \\
\hline NPDES Metals (Clean Water Act) & 0 & 1 & 0 & 0 & 0. & 1 & 0 & o.) & 1 \\
\hline Drinking Water Metals (SDWA) & 16 & 0 & 이 & 0 & 으. & 16 & 0 & 0 & 16 \\
\hline Metals by Other Regulatory Suite & 24 & 821 & 90 & 0. & -0. & 347 & 470 & 159 & 955 \\
\hline Metals by Suite, Other & 0 & 52 & 0 & O) & - & 7 & O) & 2 & 30 \\
\hline Metals by Metal Except Mercury & 111 & 1845 & 91 & 0 & ㅁ: & 468 & 358 & 713 & 1792 \\
\hline Mercury & 107 & 1485 & 13 & 0 & of & 806 & 1138 & 151 & 1850 \\
\hline Anions/Cations-IC & 2 & 492 & 42 & 0) & 0 & 1 & 7 & 114 & 329 \\
\hline Inorganics Wet Chemistry & 118 & 758 & 17 & 0 & 의 & 174 & 706 & 454 & 1113 \\
\hline Other Inorganics & 5 & 134 & 0 & 01 & 0] & 5 & 45 & 89 & 139 \\
\hline TOTALINORGANICS & 506 & 6726 & 450 & 0 & [0] & 1893 & 3388 & 2454 & 7708 \\
\hline Grose AlnhalRata & & 10001 & & & & 001 & 1101 & & 1000 \\
\hline Gamma Scan & $\frac{04}{91}$ & $\begin{array}{r}1328 \\
496\end{array}$ & $\frac{13}{12}$ & 0 & 0 & $\frac{861}{22}$ & $\frac{410}{448}$ & $\frac{130}{129}$ & $\frac{7403}{599}$ \\
\hline Transuranic & 0 & 377 & 360 & 0 & a & 480 & 257 & 0 & 737 \\
\hline Plutonium Isotopes & 28 & 119 & 12 & 0) & ㅇ. & 16 & 14 & 129 & 159 \\
\hline Uranium Isotopes & 14 & 1246 & 292 & 아 & 0. & 618 & 660 & 274 & 1552 \\
\hline Curium Isotopes & 0 & 11 & 0 & 0 & - & 0 & of & 0 & 1 \\
\hline Thorium Isotopes & 1 & 997 & 12 & 01 & ㅇ. & 307 & 574 & 129 & 1010 \\
\hline Radium Isotopes & 3 & 0 & 0 & 0) & ㅇ․ & 3 & 0 & 0 & 4 \\
\hline Americium-241 & 14 & 101 & 0 & 0 & 요 & 26 & 3 & 87 & 115 \\
\hline Neptunium-237 & 1 & 291 & 242 & 0 & Q. & 250 & 156 & 129 & 535 \\
\hline Polonium-210 & 0 & 0 & 12 & of & 요 & 0 & 0 & 12 & 12 \\
\hline Tritium & 21 & 9 & 1 & 0 & O & 14 & 15 & 0 & 30 \\
\hline Radon-222 & 0 & DI & 0 & 의 & 매 & 0 & 0 & 0 & 0 \\
\hline Lead-210 & 0 & 0 & D & 0) & 요 & 0 & 0 & 0 & -0. \\
\hline Carbon-14 & 0) & 0 & 0 & 아 & 요 & 0 & 0 & 0. & 욕 \\
\hline Nickel-63 & 아 & 0 & 0 & 0 & 의 & 0 & 0 & D. & 의 \\
\hline Selenium-79 & 0 & 0 & 0 & 0) & 요 & 0 & 01 & 0 & ㅇ․ \\
\hline Technetium-99 & 3 & 1032 & 372 & 0 & 의 & 483 & 798 & 125 & 1407 \\
\hline Strontium lsotopes & 0 & 0 & 0 & 0 & 요 & 0 & 0 & 0 & 요 \\
\hline Strontium - 89 & 아 & 0 & 0 & 0 & 의 & 0 & 0 & 0 & 요 \\
\hline Strontium -90 & 3 & 4 & 1 & 0 & 요 & 4 & 4 & 0 & 8 \\
\hline lodine-129 & 10 & 1. & 이 & 0 & D. & 10 & 0 & 0 & 10 \\
\hline Nickel-59 & 0 & 0 & 0 & 0 & 의 & 0 & 0 & 0 & 요 \\
\hline Total Uranium & 9 & 693 & 352 & 0 & 0 & 124 & 491 & 439 & 1054 \\
\hline Total Thorium & 0 & 13 & 0 & 0 & 0 & 0 & 13 & 0 & 13 \\
\hline Total Strontium & 4 & 0 & 0 & 0 & 0 & 2 & 2 & 0. & 4 \\
\hline Other RAD & 39 & 2184 & 13 & 0 & 0 & 326 & 1783 & 127 & 2237 \\
\hline TOTAL RAD & 308 & 8892 & 1693 & 01 & ㅇ] & 3548 & 5627 & 1710 & 10889 \\
\hline & & & & & & & & & \\
\hline Other Wet Chemistry & 22 & 0 & 0 & 0 & 0] & 22 & 0 & ㅁ] [ & 22 \\
\hline TCLP Extraction & 107 & 1146 & 106 & 0 & 0 & 54 & 750 & 548 & 1355 \\
\hline Asbestos & 0 & 7616 & 0 & 0 & - & 0 & 5639 & 1978 & 7616 \\
\hline industrial Hygiene, NOS & 1 & o. & 0 & 0 & 0 & 0 & 0 & 0] & 1 \\
\hline Physical Properties & 14 & 619 & 0 & 0 & - & 308 & 215 & 111 & 633 \\
\hline Other, Not Otherwise Specified (Nd & 4 & 8 & 12 & of & ]. & $7 \mid$ & 11 & 15. & 23 \\
\hline TOTAL OTHER & 148 & 9390 & 118 & 01 & [0] & 392 & 6604 & 2651 & 9651 \\
\hline & & & & & & & & & \\
\hline TOTAL SAMPLE ANALYSES & 1097 & 304201 & 2476 & 01 & ㅇ] & 6619 & 19425 & $7980 \|$ & 34008 \\
\hline
\end{tabular}


EM-263 ASSESSMENT OF EM SAMPLING AND ANALYSIS NEEDS

Table Ve: FY 97 Samples and Sample Analyses by Rad Level and Matrix Type (ix) Oak Ridge X-10

\begin{tabular}{|c|c|c|c|c|c|c|c|c|c|}
\hline $\begin{array}{c}\text { Rad-Matrix/ } \\
\text { Sample-Sample Analysis }\end{array}$ & RO & R1 & $\overline{\mathrm{R} 2}$ & R3 & R4 & $\overline{\mathrm{M} 1}$ & $\mathrm{M} 2$ & $\mathrm{M3}$ & TOTAL \\
\hline CUSTOMER SAMPLES & 361 & 5787 & 17 & 595 & 0 & 3157 & 1339 & 1270 & 6263 \\
\hline & & & & & & & & & \\
\hline \multicolumn{10}{|l|}{ SAMPLE ANALYSES } \\
\hline Volatiles by GC/MS & 96 & 242 & 2 & 66 & 0 & 87 & 237 & 82 & $\overline{406}$ \\
\hline Volatiles by GC & 78 & 35 & 1 & 0 & 0 & 67 & 38 & 5 & 112 \\
\hline Semi-Volatiles & 63 & 206 & 2 & 56 & 0 & 54 & 212 & 62 & 327 \\
\hline Herbicides/Pesticides/PCBs & 185 & 449 & 0 & 0 & 0 & 167 & 364 & 103 & 633 \\
\hline Other Organics & 42 & 938 & 0 & 0 & 0 & 28 & 22 & 1 & 515 \\
\hline TOTAL ORGANICS & 463 & 1870 & 5 & 122 & 0 & 403 & $87 \overline{3}$ & 252 & 1994 \\
\hline \multicolumn{10}{|l|}{+2} \\
\hline RCRA Appendix IX Metals & 2 & 3 & of & 0 & 0 & 4 & 1 & 0 & 5 \\
\hline CAM (California) Metals (RCAA) & 44 & 1 & 0 & o) & 0 & 45 & 1 & 0 & 45 \\
\hline RCRA TCLP Metals and EPTOX Me & 68 & 150 & 1 & 0 & 0 & 59 & 151 & 4 & 216 \\
\hline EPA CLP Metals (CERCLA) & 82 & 32 & 6 & 138 & 0 & 48 & 68 & 141 & 258 \\
\hline NPDES Metals (Clean Water Act) & 31 & 164 & 0 & 0 & 0) & 195 & 0 & 0 & 195 \\
\hline Drinking Water Metals (SDWA) & 11 & 0 & 0 & 0 & 0 & 11 & 0 & 0 & 11 \\
\hline Metals by Other Regulatory Suite & 135 & 85 & 0 & 0 & 0 & 90 & 108 & 32 & 225 \\
\hline Metals by Suite, Other & 0 & 965 & 1 & 0 & 0 & 17 & 8 & 1 & 496 \\
\hline Metals by Metal Except Mercury & 132 & 204 & 0 & 0 & 0 & 115 & 90 & 127 & 334 \\
\hline Mercury & 178 & 1176 & 0 & 0 & 0 & 190 & 226 & 5 & 888 \\
\hline Anions/Cations-IC & 62 & 32 & 0 & 0 & 요 & 73 & 14 & 5 & 93 \\
\hline Inorganics Wet Chemistry & 633 & 188 & 9 & 2 & 0 & 579 & 199 & 56 & 833 \\
\hline Other Inorganics & 75 & 37 & 01 & 0 & 0 & 72 & 31 & 9 & 112 \\
\hline \multirow[t]{2}{*}{ TOTALINORGANICS } & 1453 & 3035 & 17 & 140 & 0. & 1497 & 897 & 381 & 3711 \\
\hline & & & & & $=1$ & & & & \\
\hline Gross Alpha/Beta & 606 & 3106 & 2 & 0 & of & 2509 & 250 & 26 & 3249 \\
\hline Gamma Scan & 112 & 1064 & 1 & o) & 0 & 987 & 175 & 16 & 1178 \\
\hline Transuranic & 0 & 5 & 0 & 0 & 0 & 0 & 5 & 0 & 5 \\
\hline Plutonium Isotopes & 200 & 11 & 3 & 0 & 0 & 161 & 47 & 6 & 214 \\
\hline Uranium Isotopes & 202 & 154 & 2 & 0 & 0 & 170 & 172 & 16 & 358 \\
\hline Curium Isotopes & 0 & 3 & 0 & 0 & 0 & 1 & 1 & 2 & 3 \\
\hline Thorium Isotopes & 2 & 122 & 1 & 5 & 0 & 4 & 118 & 8 & 129 \\
\hline Radium Isotopes & 15 & 0 & 0 & 0 & 0 & 11 & 4 & 0 & 16 \\
\hline Americium - 241 & 189 & 8 & 1 & 0 & 0 & 161 & 35 & 2 & 198 \\
\hline Neptunium - 237 & 2 & 9 & 2 & 0 & ㅇ. & 1 & 7 & 5 & 13 \\
\hline Polonium-210 & 1 & 1 & 0 & 0 & 0. & o & 1 & 0 & 1 \\
\hline Tritium & 156 & 26 & 6 & 0 & 요 & 147 & 36 & 2 & 186 \\
\hline Radon-222 & 0 & 0 & 0 & 0 & 0 & 0 & 0 & 0 & 0 \\
\hline Lead-210 & 0 & 2 & 0 & 0 & 0 & 0 & 0) & 2 & 2 \\
\hline Carbon-14 & 0 & 0 & 0 & 0 & 0 & 0 & 0 & 0 & 0 \\
\hline Nickel-63 & 0 & 0 & 0 & 0 & 0 & o) & 0 & 0) & 0 \\
\hline Selenium-79 & 44 & 1 & 0 & 0 & - & 45 & 0 & 0 & 45 \\
\hline Technetium - 99 & 2 & 116 & 0 & 0) & 0 & 2 & 114 & 2 & 119 \\
\hline Strontium Isotopes & 0 & 0 & 0 & 0 & a & 0 & 0 & 0 & 0 \\
\hline Strontium - 89 & 0 & 0 & 0 & 0 & 0 & 0 & 0 & 0 & 0 \\
\hline Strontium-90 & 46 & 12 & 1 & 0 & 0 & 49 & 10 & 0 & 60 \\
\hline lodine-129 & 51 & 2 & 0 & 0 & 0 & 52 & 0 & 0 & 52 \\
\hline Nickel-59 & 0 & 0 & 0 & 0 & 0 & o) & 0 & 0 & 0 \\
\hline Total Uranium & 13 & 85 & 0 & 0 & 0 & 4 & 16 & 78 & 98 \\
\hline Total Thorium & 0 & 0 & 0 & 0 & 0 & 0 & 0 & 0 & 0 \\
\hline Total Strontum & 26 & 2 & 0 & 0 & 0 & 13 & 13 & 2 & 29 \\
\hline Other RAD & 153 & 418 & 3 & 462 & 마 & 105 & 437 & 486 & 1032 \\
\hline TOTAL RAD & 1823 & 5149 & 21 & 467 & 0 & 4421 & 1442 & 656 & 6989 \\
\hline & & & & & & & & & \\
\hline Other Wet Chemistry & 304 & 2 & 0 & 0ा & 0. & 295 & 11 & 0 & 306 \\
\hline TCLP Extraction & 55 & 174 & 7 & 366 & 0 & 52 & 155 & 389 & 599 \\
\hline Asbestos & 0 & 1525 & 0 & 0 & 0 & 0 & 1131 & 396 & 1526 \\
\hline Industrial Hygiene, NOS & 112 & 0 & 0 & 0 & 0 & 111 & 1 & 0 & 113 \\
\hline Physical Properties & 32 & 45 & 0 & 230 & 0 & 30 & 46 & 232 & 307 \\
\hline Other, Not Otherwise Specified (NO & 5 & 3 & 0 & 0 & 0 & 3 & 0 & 5 & 8 \\
\hline TOTAL OTHER & 509 & 1749 & 7 & 596 & (2) & 491 & 1344 & 1022 & 2859 \\
\hline & & & & & & & & & \\
\hline TOTAL SAMPLE ANALYSES & 4247 & 11803 & 51 & 1325 & 요 & 6813 & 4556 & 2311 & 15553 \\
\hline
\end{tabular}


Table Ve: FY 97 Samples and Sample Analyses by Rad Level and Matrix Type (x) Oak Ridge Y-12

\begin{tabular}{|c|c|c|c|c|c|c|c|c|c|}
\hline $\begin{array}{c}\text { Rad-Matrix/ } \\
\text { Sample-Sample Analysis }\end{array}$ & RO & $\overline{\mathrm{R1}}$ & $\overline{\mathrm{R} 2}$ & R3 & $\overline{\mathrm{R} 4}$ & $\overline{\mathrm{M} 1}$ & $\overline{\mathrm{M} 2}$ & $\overline{M 3}$ & TOTAL \\
\hline CUSTOMER SAMPLES & 149 & 982 & 15 & 0 & 0 & 119 & 648 & 278 & 1095 \\
\hline \multirow{2}{*}{\multicolumn{10}{|c|}{ SAMPLE ANALYSES }} \\
\hline & & & & & & & & & \\
\hline Volatiles by GC/MS & 99 & 122 & 3 & 0 & 0 & 57 & 147 & 20 & 224 \\
\hline Volatiles by $\mathrm{GC}$ & 25 & 32 & 2 & 0 & o. & 3 & 51 & 5 & 59 \\
\hline Semi-Volatiles & 49 & 103 & 3 & 0 & o. & 29 & 123 & 3 & 155 \\
\hline Herbicides/Pesticides/PCBs & 93 & 209 & 0 & 0 & 0 & 49 & 195 & 58 & 302 \\
\hline Other Organics & 27 & $8 \mid$ & $0 \mid$ & 0 & 0 & 6 & 28 & 1 & 35 \\
\hline TOTAL ORGANICS & 293 & 4741 & 81 & 0 & 0 & 144 & 544 & 88 & 775 \\
\hline PCDA Annondiv IY Matalo & & & & & & & & & \\
\hline CAM (California) Metals (RCRA) & 27 & $\frac{1}{0}$ & $\frac{0}{0}$ & $\frac{0}{0}$ & $\frac{0}{0}$ & $\frac{0}{27}$ & $\frac{1}{0}$ & $\frac{0}{0}$ & $\frac{1}{28}$ \\
\hline RCRA TCLP Metals and EPTOX Me & 10 & 66 & 2 & 0 & 0 & 3 & 71 & 4 & $\frac{28}{78}$ \\
\hline EPA CLP Metals (CERCLA) & 90 & 51 & 9 & 0 & o. & 37 & 107 & 5 & 149 \\
\hline NPDES Metals (Clean Water Act) & 21 & 1 & 0 & 0 & 0 & 22 & 0 & 0 & 22 \\
\hline Drinking Water Metals (SDWA) & 0 & 0 & 0 & 0 & 0 & 0 & 0 & 0 & 에 \\
\hline Metals by Other Regulatory Suite & 108 & 37 & 0 & 0 & 0 & 26 & 80 & 46 & 149 \\
\hline Metals by Suite, Other & 0 & 45 & 2 & 0 & 0 & 25 & 13 & 1 & 43 \\
\hline Metals by Metal Except Mercury & 37 & 96 & 0 & 0 & $\overline{0}$ & 31 & 49 & 53 & 133 \\
\hline Mercury & 56 & 102 & of & 0 & 0 & 53 & 102 & 3. & 158 \\
\hline Anions/Cations-IC & 36 & 20 & 0 & 0 & 0 & 47 & 2 & 6 & 56 \\
\hline Inorganics Wet Chemistry & 312 & 122 & 17 & 0 & ㅇ․ & 229 & 136 & 87 & 451 \\
\hline Other Inorganics & 35 & 25 & 0 & 0 & 0 & 35 & 19 & 6 & 60. \\
\hline TOTAL INORGANICS & 731 & 566 & 291 & 0 & 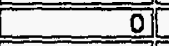 & 535 & 581 & 212 & 1327 \\
\hline \\
\hline Gross Alpha/Beta & 182 & 120 & 4 & 0 & 0 & 94 & 193 & 16 & 304 \\
\hline Gamma Scan & 68 & 59 & 1 & 0 & 0 & 33 & 82 & 13 & 127 \\
\hline Transuranic & 0 & 2 & 0 & of & 0 & 0 & 2 & 의 & 2 \\
\hline Plutonium Isotopes & 69 & 16 & 1 & 01 & 0 & 21 & 58 & 7 & 86 \\
\hline Uranium Isotopes & 85 & 95 & 1 & 0 & 0 & 36 & 134 & 12 & 182 \\
\hline Curium Isotopes & 0 & 3 & 0 & 0 & 0 & 1 & 1 & 1 & 3 \\
\hline Thorium isotopes & 5 & 57 & 1 & 0 & 0 & 3 & 54 & 5 & 63 \\
\hline Radium lsotopes & 20 & 1 & 0 & 0 & 요 & 14 & 6 & 1 & 21 \\
\hline Americium-241 & 64 & 8 & 1 & 0 & of & 21 & 50 & 2 & 73 \\
\hline Neptunium-237 & 5 & 9 & 0 & 0 & o. & 1 & 7 & 6 & 14 \\
\hline Polonium-210 & 0 & 1 & 01 & D & 0 & 0 & O. & 11 & 1. \\
\hline Tritium & 39 & 39 & 6 & 0 & 0 & 30 & 44 & 1 & 80 \\
\hline Radon-222 & 0 & 0 & 0 & 0 & 01 & 0 & 0 & 0 & 0 \\
\hline Lead-210 & 0 & 0) & 0 & 0 & o) & 0 & 0 & 0 & 0 \\
\hline Carbon-14 & 0 & 0 & 0 & 0 & 0 & 0 & 0 & 0 & 의 \\
\hline Nickel-63 & 0 & 0 & 0 & 0 & 0 & 0 & 0 & 0) & 0 \\
\hline Selenium-79 & 27 & 1 & 0 & 0 & 0 & 27 & o & 0 & 28 \\
\hline Technetium -99 & 0 & 49 & 0 & 0 & D. & 음 & 48 & 1. & 50 \\
\hline Strontium Isotopes & 0 & 0 & 0 & 0 & o) & 0 & 0 & 0 & 묘 \\
\hline Strontium - 89 & 0 & 0 & 0 & 0 & o: & 0 & 0 & 0 & 의 \\
\hline Strontium -90 & 27 & 18 & 2 & 0 & 0 & 30 & 16 & 1 & 47 \\
\hline lodine-129 & 27 & 3 & 0 & 0 & 요 & 29 & 0 & - & 30 \\
\hline Nickel-59 & 0 & 0 & 0 & 0 & 이 & 요 & 0 & 으. & 의 \\
\hline Total Uranium & 21 & 36 & 0 & 0 & o & 3 & 6 & 48 & 57 \\
\hline Total Thorium & of & 0 & of & 0 & 0 & 0 & 0 & a) & 의 \\
\hline Total Strontium & 32 & 0 & 0 & 0 & - & 12 & 19 & 1. & 32 \\
\hline Other RAD & 138 & 184 & 4 & 01 & o.] & 43 & 260 & 25 & 327 \\
\hline TOTAL RAD & 810 & 702 & 22 & 01 & o: & 4001 & 982 & 141 & 1528 \\
\hline & & & & & & & ( & & \\
\hline Other Wet Chemistry & 70 & 11 & 0 & 0 & 0 & 54 & 17 & 0. & 71 \\
\hline TCLP Extraction & 10 & 82 & 0 & 0 & 0 & 7 & 72 & 13 & 92 \\
\hline Asbestos & 0 & 640 & 0 & 0 & 0 & 0 & 475 & 165 & 640 \\
\hline Industrial Hygiene, NOS & 2 & Q) & 0 & 0 & o) & 1 & 2 & ㅇ. & 3 \\
\hline Physical Properties & 9 & 24 & 1 & 0 & D. & 6 & 25 & 3 & 34 \\
\hline Other, Not Otherwise Specified (NO & 10 & 21 & 0 & 01 & 0] & 2 & 0 & 10 & 12 \\
\hline TOTAL OTHER & 1021 & 7491 & II & 01 & 0] & 701 & 5911 & 191 & 852 \\
\hline TOTAL SAMPLE ANALYSES & & & & & & & & & \\
\hline TOTAL SAMPLE ANALYSES & 1936 & 2492 & 60 & 0 & 0] & 1148 & 2698 & 631 & 4482 \\
\hline
\end{tabular}


Table Ve: FY 97 Samples and Sample Analyses by Rad Level and Matrix Type (xi) Rocky Flats

\begin{tabular}{|c|c|c|c|c|c|c|c|c|c|}
\hline $\begin{array}{c}\text { Rad-Matrix/ } \\
\text { Sample-Sample Analysis }\end{array}$ & $\overline{\mathrm{RO}}$ & R1 & $\overline{\mathrm{R2}}$ & $\overline{\mathrm{R3}}$ & $\overline{R 4}$ & $\overline{M 1}$ & $\overline{\mathrm{M} 2}$ & $\overline{\mathrm{M3}}$ & TOTAL \\
\hline CUSTOMER SAMPLES & 2060 & 5362 & 373 & 1180 & 0 & 1815 & 2970 & 1324 & 7542 \\
\hline & & & & & & & & & \\
\hline \multicolumn{10}{|l|}{ SAMPLE ANALYSES } \\
\hline Volatiles by GC/MS & 4261 & 759 & 85 & 132 & 0 & 2642 & 2290 & 306 & 5238 \\
\hline Volatiles by GC & 894 & 246 & 19 & 0 & 0 & 345 & 750 & $\overline{46}$ & 1150 \\
\hline Semi-Volatiles & 1829 & 695 & 85 & 112 & 0 & 1021 & 1549 & 142 & 2717 \\
\hline Herbicides/Pesticides/PCBs & 2232 & 860 & 0 & 0 & 0 & 773 & 2159 & 160 & 3092 \\
\hline Other Organics & 204 & 225 & 0 & 0 & 0 & 318 & 38 & 7 & 396 \\
\hline TOTAL ORGANICS & 94211 & 2785 & 189 & 244 & 0 & 5100 & 6787 & 660 & 12593 \\
\hline \multicolumn{10}{|l|}{ 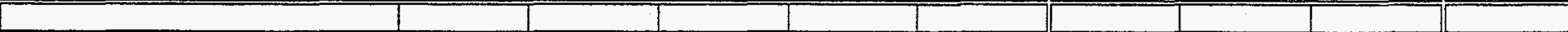 } \\
\hline RCRA Appendix IX Metals & 8 & 30 & 0 & 0 & 0 & 4 & 33 & 0 & 38 \\
\hline CAM (California) Metals (RCRA) & 2 & 2 & 0 & 0 & 0 & 4 & 3 & 1 & 5 \\
\hline RCRA TCLP Metals and EPTOX Me & 385 & 166 & 19 & o) & 0 & 295 & 213 & 19 & 548 \\
\hline EPA CLP Metals (CERCLA) & 5165 & 1344 & 234 & 276 & 0 & 2475 & 4155 & 390 & 7020 \\
\hline NPDES Metals (Clean Water Act) & 573 & 19 & 0 & $0 \mid$ & 0 & 592 & (0) & 0 & 592 \\
\hline Drinking Water Metals (SDWA) & 92 & 0 & 0 & 0 & 0 & 92 & O) & 0 & 92 \\
\hline Metals by Other Regulatory Suite & 4668 & 0 & 0 & 0 & 0 & 2118 & 2304 & 363 & 4727 \\
\hline Metals by Suite, Other & 0 & 1165 & 44 & o & 0 & 657 & 363 & 7 & 1117 \\
\hline Metals by Metal Except Mercury & 449 & 276 & 4 & O) & 0 & 366 & 323 & 0 & 709 \\
\hline Mercury & 940 & 321 & 4 & 0 & 0 & 891 & 262 & 7 & 1212 \\
\hline Anions/Cations-IC & 900 & 439 & 0 & 0 & 0 & 1266 & 35 & 38 & 1339 \\
\hline Inorganics Wet Chemistry & 12637 & 891 & 135 & 4 & 0 & 10817 & 2224 & 633 & 13670 \\
\hline Other Inorganics & 805 & 303 & 0 & 0 & 0 & 777 & 285 & 46 & 1107 \\
\hline TOTAL INORGANICS & 26623 & 4956 & 4401 & 280 & 0 & 20355 & 10198 & 1503 & 32178 \\
\hline \\
\hline Gross Alpha/Beta & 9461 & 1864 & 79 & 0 & $\overline{0}$ & 4750 & 6320 & 200 & 11336 \\
\hline Gamma Scan & 3769 & 580 & 36 & 0 & 0 & 1820 & 2400 & 166 & 4385 \\
\hline Transuranic & 0 & 1 & 0 & 0 & 0 & 0 & 1 & 0 & 1 \\
\hline Plutonium isotopes & 4696 & 358 & 24 & 0 & 0 & 1984 & 3020 & $\overline{74}$ & 5078 \\
\hline Uranium Isotopes & 4568 & 1042 & 24 & 0 & 0 & 2101 & 3447 & 87 & 5635 \\
\hline Curium Isotopes & o & 56 & 0 & 0 & 0 & 25 & 24 & 8 & 56 \\
\hline Thorium Isotopes & 39 & 230 & 24 & 0 & 0 & 64 & 190 & 40 & 294 \\
\hline Radium Isotopes & 1868 & 7 & 0 & 0 & 0 & 1424 & 444 & 7 & 1875 \\
\hline Americium-241 & 4481 & 133 & 24 & o & 0 & 1958 & 2645 & 35 & 4638 \\
\hline Neptunium-237 & 39 & 171 & 0 & D: & 0 & $\overline{16}$ & 148 & 46 & 210 \\
\hline Polonium-210 & 2 & 8 & 0 & 0 & 0 & 1 & 2 & 7 & 10 \\
\hline Tritium & 2724 & 853 & 129 & 0 & 0 & 2006 & 1611 & 21 & 3672 \\
\hline Radon-222 & 1 & 0 & 0 & 0 & 0 & 1 & 0 & 0 & 1 \\
\hline Lead-210 & 0 & 0 & 0 & 0 & - & 0 & 0 & 0 & $\overline{0}$ \\
\hline Carbon-14 & 0 & 0 & 0 & 0 & 0 & 0 & 0 & 0 & 0 \\
\hline Nickel-63 & 0 & 0 & 0 & 0 & $\overline{0}$ & 0 & 0 & 0 & 0 \\
\hline Selenium-79 & 2 & 0 & 0 & 0 & 0 & 2 & 0 & 0 & 2 \\
\hline Technetium-99 & 20 & 36 & 7 & 0 & 0 & 27 & 28 & 7 & 62 \\
\hline Strontium Isotopes & 0 & 1 & 0 & 0 & 0 & 0 & 0 & 0 & 1 \\
\hline Strontium -89 & 0 & 0 & 0 & 0 & 0 & 0 & 0 & 0 & 0 \\
\hline Strontium-90 & 21 & 357 & 22 & 0 & o & 55 & 347 & 0 & 401 \\
\hline lodine-129 & 58 & 15 & 0 & 0 & 0 & 73 & 0 & 0 & 73 \\
\hline Nickel-59 & 0 & 0 & 0 & 0 & 0 & 0 & 0 & 0 & 0 \\
\hline Total Uranium & 214 & 20 & 0 & 0 & 0 & 66 & 7 & 160 & 234 \\
\hline Total Thorium & 0 & 8 & 0 & 0 & 0 & 1 & 7 & 0 & 8 \\
\hline Total Strontium & 2471 & 0 & 0 & 0 & 0 & 1284 & 1153 & 34 & 2472 \\
\hline Other RAD & 6056 & 673 & 90 & 924 & 0 & 2099 & 4543 & 1102 & 7743 \\
\hline TOTALRAD & 40491 & 6413 & 459 & 924 & 0 & 19755 & 26339 & 1996 & 48189 \\
\hline & & & & & & & & & \\
\hline Other Wet Chemistry & 6335 & 8 & 0 & 0 & 0) & 5347 & 995 & 0 & 6342 \\
\hline TCLP Extraction & 387 & 216 & 6 & 732 & 0 & 320 & 216 & 766 & 1321 \\
\hline Asbestos & 25 & 153 & 0 & 0 & 0 & 1 & 178 & 0 & 179 \\
\hline Industrial Hygiene, NOS & 62 & 0 & 0 & 0 & 0 & 17 & 44 & 0 & 62 \\
\hline Physical Properties & 995 & 118 & 14 & 460 & 0 & 237 & 864 & 486 & 1586 \\
\hline Other, Not Otherwise Specified (Nd & 80 & 53 & 0 & 0 & o & 48 & 0 & 81 & 131 \\
\hline TOTAL OTHER & $\overline{7884}$ & 547 & 20 & 1192 & 0 & 5970 & 2297 & 1332 & 9621 \\
\hline & & & & & & & & & \\
\hline TOTAL SAMPLE ANALYSES & $\overline{84420}$ & 14701 & 1108 & 2640 & 0] & 51179 & 45622 & 5491 & 102581 \\
\hline
\end{tabular}


Table Ve: FY 97 Samples and Sample Analyses by Rad Level and Matrix Type (xii) Savannah River

\begin{tabular}{|c|c|c|c|c|c|c|c|c|c|}
\hline $\begin{array}{c}\text { Rad-Matrix } \\
\text { Sample-Sample Analysis }\end{array}$ & RO & R1 & $\overline{R 2}$ & R3 & $\overline{R 4}$ & $\overline{\mathrm{M} 1}$ & M2 & $\overline{\mathrm{M3}}$ & TOTAL \\
\hline CUSTOMER SAMPLES & 10275 & 19766 & 328 & 81 & 211 & 214241 & 1165 & 3248 [ & 28249 \\
\hline & & & & & & & & & \\
\hline \multicolumn{10}{|l|}{ SAMPLE ANALYSES } \\
\hline Volatiles by GC/MS & 181 & 33 & o1 & 0 & D) & 134 & 25 & 56 & 214 \\
\hline Volatiles by GC & 103 & 255 & 29 & 11 & o) & 145 & 233 & 18 & 397 \\
\hline Semi-Volatiles & 1288 & 124 & 11 & 3 & 0 & 1016 & 112 & 301 & 1428 \\
\hline Herbicides/Pesticides/PCBs & 642 & 140 & 11 & 6 & 0 & 603 & 124 & 75 & 801 \\
\hline Other Organics & 2008 & 4664 & 0 & 0 & 0 & 1743 & 1 & 284 & 4350 \\
\hline \multirow[t]{2}{*}{ TOTAL ORGANICS } & 4222 & 5216 & 521 & 201 & (0] & 3641 & 495 & 733 & 7190 \\
\hline & & & & & & & - & 7 & \\
\hline RCRA Appendix IX Metals & 12 & 9 & 0 & 0 & 0 & 19 & 1 & 1 & 22 \\
\hline CAM (California) Metals (RCRA) & 80 & 0 & 0 & 0 & o. & 80 & 0 & 0 & 80 \\
\hline RCRA TCLP Metals and EPTOX MeI & 76 & 110 & 10 & 2 & o) & 116 & 67 & 15 & 197 \\
\hline EPA CLP Metals (CERCLA) & 67 & 56 & 23 & 11 & 0 & 67 & 84 & 7 & 158 \\
\hline NPDES Metals (Clean Water Act) & 86 & 823 & 0 & 0 & 0 & 909 & 0 & 0. & 909 \\
\hline Drinking Water Metals (SDWA) & 0 & 0 & 0 & 0 & o) & 0 & 0 & 0 & 0 \\
\hline Metals by Other Regulatory Suite & 426 & 5 & 0 & 0 & 0 & 303 & 1 & 128 & 431 \\
\hline Metals by Suite, Other & 2690 & 4647 & 0 & 0 & D) & 1426 & 0 & 1267 & 5015 \\
\hline Metals by Metal Except Mercury & 1865 & 78 & 0 & 0 & 105 & 1091 & 72 & 810 & 2010 \\
\hline Mercury & 2064 & 4837 & 2 & 1 & 105 & 1440 & 78 & 772 & 4650 \\
\hline Anions/Cations-IC & 3854 & 88 & 0 & 0 & 211 & 3210 & 64 & 698 & 4062 \\
\hline Inorganics Wet Chemistry & 5211 & 178 & 102 & 23 & 0 & 4060 & 324 & 1129 & 5514 \\
\hline Other Inorganics & 268 & 108 & 01 & 0 & 0 & 280 & 50 & 45 & 376 \\
\hline \multirow[t]{2}{*}{ TOTAL INORGANICS } & 16699 & 10938 & 137 & 38 & 422 & 12999 & 743 & 4872 & 23424 \\
\hline & & & & & & & & & \\
\hline Gross Alpha/Beta & 2248 & 15552 & 58 & 72 & 211 & 12165 & 479 & 142 & 15463 \\
\hline Gamma Scan & 90 & 4924 & 52 & 29 & 110 & 4794 & 229 & 101 & 5164 \\
\hline Transuranic & 0 & 0 & 0 & 0 & 110 & 0 & 0 & 30 & 70 \\
\hline Plutonium Isotopes & 694 & 70 & 21 & 6 & 211 & 677 & 87 & 57 & 911 \\
\hline Uranium lsotopes & 696 & 115 & 9 & 5 & 211 & 679 & 119 & 58 & 945 \\
\hline Curium Isotopes & 0 & 17 & 3 & 2 & 10 & 2 & 10 & 10 & 27 \\
\hline Thorium Isotopes & 16 & 19 & 3 & 26 & 0 & 0 & 23 & 40. & 64 \\
\hline Radium Isotopes & 11 & 3 & 0 & 0 & 0 & 1 & 0 & 3 & 4 \\
\hline Americium-241 & 678 & 39 & 5 & 3 & 20 & 677 & 41 & 7 & 735 \\
\hline Neptunium-237 & 16 & 21 & 8 & 0 & 0 & 0 & 18 & 27 & 45 \\
\hline Polonium-210 & 0 & 3 & 0 & 0 & 0 & 0 & 0 & 3 & 3 \\
\hline Tritium & 591 & 75 & 28 & 6 & 0 & 592 & 72 & 7 & 684 \\
\hline Radon-222 & 0 & 0 & 0 & 0 & 0 & 0 & 0 & 0 & 0 \\
\hline Lead-210 & 0 & 12 & 0 & 0 & 0 & 0 & 0 & 12 & 12 \\
\hline Carbon-14 & 0 & 으 & 0 & 0 & 0 & 0 & 0 & 0 & 0 \\
\hline Nickel-63 & 0 & 0 & 0 & 0 & 0 & 0 & 0 & 0 & 0. \\
\hline Selenium-79 & 80 & 0 & 0 & 0 & 0 & 80 & 0 & 0 & 80 \\
\hline Technetium-99 & 0 & 11 & 0 & 0 & 0 & 0 & 아 & 11 & 11 \\
\hline Strontium Isotopes & 0 & 0 & 0 & 0 & a & 0 & of & 0 & 0 \\
\hline Strontium-89 & 0 & 0 & 0 & 0 & 0. & 0 & 0 & 0 & $\overline{0}$ \\
\hline Strontium-90 & 80 & 43 & 23 & 6 & o. & 82 & 71 & 0 & 153 \\
\hline lodine-129 & 80 & 6 & 0 & 0 & 0 & 87 & 0 & 0 & 87 \\
\hline Nickel-59 & 0 & 0 & 0 & 0 & 0 & 0 & 0 & o) & 0 \\
\hline Total Uranium & 65 & 25 & 0 & 0 & 211 & 3 & 22 & 95 & 210 \\
\hline Total Thorium & 0 & 22 & 0 & 0 & 0 & 0 & 22 & 0 & 22 \\
\hline Total Strontium & 15 & 12 & 0 & 0 & 0 & 19 & 0 & 8 & 27 \\
\hline Other RAD & 230 & 222 & 18 & 6 & 10 & 230 & 98 & 145 & 479 \\
\hline TOTAL RAD & 5581 & 21191 & 228 & 158 & 1104 & 20090 & 1289 & 754 & 25198 \\
\hline & & & & & & & & $=$ & \\
\hline Other Wet Chemistry & 1220 & 10 & 0 & 0 & 30 & 1230 & 0 & 30 & 1260 \\
\hline TCLP Extraction & 8 & 121 & 41 & 5 & 이 & 68 & 79 & 27 & 174 \\
\hline Asbestos & 0 & 12 & o & o & ㅇ. & 0 & 14 & 0 & 13 \\
\hline Industrial Hygiene, NOS & 552 & 0 & 01 & 0 & (0) & 552 & 0 & 0 & 552 \\
\hline Physical Properties & 12128 & 45 & 6 & 2 & 0 & 7917 & 39 & 4226 & 12182 \\
\hline Other, Not Otherwise Specified (NO & 35 & 21 & 01 & 0 & o. & 21 & 1 & 33 & 55 \\
\hline TOTAL OTHER & 13942 & 209 & 47 & 71 & 30 & 9787 & 134 & 4317 & 14236 \\
\hline & & & & 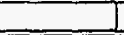 & & & & & \\
\hline TOTAL SAMPLE ANALYSES & 40445 & 37553 & 463 & 223 & 1556 & 46517 & 2661 & 10676 & 70048 \\
\hline
\end{tabular}


Table Vf: FY 98 Samples and Sample Analyses by Rad Level and Matrix Type (i) Complex-wide

\begin{tabular}{|c|c|c|c|c|c|c|c|c|c|}
\hline $\begin{array}{c}\text { Rad-Matrix/ } \\
\text { Sample-Sample Analysis }\end{array}$ & $\overline{\mathbf{R}}$ & $\overline{\mathrm{R1}}$ & $\overline{\mathrm{R} 2}$ & R3 & R4 & M1 & $\mathrm{M} 2$ & M3 & TOTAL \\
\hline CUSTOMER SAMPLES & 47637 & 185889 & 3566 & 7077 & 620 & 95527 & 71322 & 45368 & 228503 \\
\hline \multirow{2}{*}{\multicolumn{10}{|c|}{ SAMPLE ANALYSES }} \\
\hline & & & & & & & & & \\
\hline Volatiles by GC/MS & 19527 & 13927 & 635 & 752 & $\overline{0}$ & 12787 & 18384 & 3687 & 34849 \\
\hline Volatiles by GC & 6217 & 3552 & 296 & 50 & 0 & 3434 & 5813 & 630 & 9996 \\
\hline Semi-Volatiles & 12078 & 11916 & 638 & 651 & 0 & 7715 & 15105 & 2454 & 25278 \\
\hline Herbicides/Pesticides/PCBs & 13007 & 23770 & 50 & 25 & 0 & 7772 & 22987 & 6125 & 36868 \\
\hline Other Organics & 9077 & 21039 & 28 & 0 & 0 & 7079 & 2163 & 952 & 20169 \\
\hline TOTAL ORGANICS & 59907 & 74204 & 1646 & 1478 & 0 & 38787 & 64451 & 13848 & 127161 \\
\hline \multicolumn{10}{|l|}{20} \\
\hline RCRA Appendix IX Metals & 93 & 172 & 0 & 0 & $\mathbf{0}$ & 100 & 159 & 66 & 295 \\
\hline CAM (California) Metals (RCRA) & 595 & 140 & 0 & 0 & 0 & 735 & 175 & 35 & 840 \\
\hline RCRA TCLP Metals and EPTOX MeI & 4329 & 7377 & 665 & 10 & 0 & 2988 & 7632 & 1304 & 12153 \\
\hline EPA CLP Metals (CERCLA) & 21459 & 6940 & 1141 & 1623 & $\overline{0}$ & 10860 & 17350 & 2953 & 31162 \\
\hline NPDES Metals (Clean Water Act) & 2896 & 3607 & 0 & 0 & 0 & 6502 & 0 & 0 & 6502 \\
\hline Drinking Water Metals (SDWA) & 724 & 0 & 0 & 0 & 0 & 724 & 0 & 요 & 724 \\
\hline Metals by Other Regulatory Suite & 22600 & 4538 & 207 & 0 & $\overline{0}$ & 11150 & 12586 & 4410 & 27746 \\
\hline Metals by Suite, Other & 7666 & 25227 & 191 & 0 & 0 & 7030 & 1572 & 3697 & 22691 \\
\hline Metals by Metal Except Mercury & 9760 & 12181 & 236 & 0 & 300 & 7513 & 5546 & 7744 & 21640 \\
\hline Mercury & 12679 & 32666 & 66 & 5 & 300 & 12560 & 11087 & 2748 & 36056 \\
\hline Anions/Cations-IC & 15383 & 3672 & 97 & 0 & 601 & 15026 & 516 & 2740 & 19017 \\
\hline Inorganics Wet Chemistry & 70947 & 12585 & 1740 & 122 & 0 & 58262 & 17537 & 11059 & 86126 \\
\hline Other Inorganics & 6347 & 2971 & 0 & 0 & 0 & 5396 & 3061 & 861 & 9318 \\
\hline TOTAL INORGANICS & 175478 & 112075 & 4342 & 1760 & 1201 & 138846 & 77219 & 37617 & 274269 \\
\hline \\
\hline Gross Alpha/Beta & 50465 & 81150 & 697 & 311 & 601 & 74647 & 33295 & 2243 & 121704 \\
\hline Gamma Scan & 17710 & 27500 & 405 & 124 & 315 & 28024 & 15959 & 1842 & 45940 \\
\hline Transuranic & 0 & 1035 & 829 & 0 & 315 & 1105 & 759 & 86 & 2064 \\
\hline Plutonium Isotopes & 22750 & 2358 & 225 & 25 & 601 & 11208 & 13174 & 1061 & 25701 \\
\hline Uranium isotopes & 22525 & 12510 & 816 & 22 & 601 & 13538 & 20423 & 1998 & 36216 \\
\hline Curium Isotopes & 0 & 394 & 15 & 7 & 29 & 138 & 160 & 118 & 431 \\
\hline Thorium Isotopes & 440 & 6957 & 145 & 110 & 0 & 1069 & 5734 & 849 & 7652 \\
\hline Radium Isotopes & 7028 & 82 & 0 & 0 & 0 & 5351 & 1678 & 81 & 7110 \\
\hline Americium-241 & 21347 & 1203 & 127 & 12 & 57 & 11083 & 11194 & 413 & 22718 \\
\hline Neptunium-237 & 440 & 1793 & 591 & 0 & 0 & $6 \overline{66}$ & 1297 & 863 & 2825 \\
\hline Polonium-210 & 159 & 87 & 28 & 0 & 0 & 6 & 159 & 109 & 274 \\
\hline Tritium & 13402 & 4588 & 753 & 25 & 0 & 10589 & 7366 & 135 & 18429 \\
\hline Radon-222 & 8 & 0 & 0 & 0 & 0 & 8 & 0 & 0 & 8 \\
\hline Lead-210 & 0 & 51 & 0 & 0 & 0 & 0 & 0 & 51 & 51 \\
\hline Carbon-14 & 0 & 0 & 0 & 0 & 0 & 0 & 0 & 0 & 0 \\
\hline Nickel-63 & 0 & 0 & of & 0 & $\underline{0}$ & 0 & 0 & 요 & 0 \\
\hline Selenium -79 & 595 & 58 & 0 & 0 & 0 & 628 & 15 & 11 & 653 \\
\hline Technetium -99 & 154 & 6013 & 887 & 우 & 0 & 1290 & 5364 & 400 & 7054 \\
\hline Strontium Isotopes & 0 & 9 & 0 & 0 & 0 & 0 & 4 & 5 & 9 \\
\hline Strontium - 89 & 0 & 0 & 0 & 0 & 0 & of & 0 & 의 & 0 \\
\hline Strontium-90 & 749 & 2088 & 274 & 25 & 0 & 1052 & 2054 & 36 & 3139 \\
\hline lodine-129 & 1034 & 252 & 0 & 0 & 0 & 1238 & 29 & 18 & 1286 \\
\hline Nickel-59 & 0 & 0 & 0 & 0 & $\underline{0}$ & 0 & 0 & 의 & 0 \\
\hline Total Uranium & 2069 & 4855 & 810 & 0 & 601 & 743 & 1956 & 5121 & 8077 \\
\hline Total Thorium & 0 & 383 & 0 & 0 & 0 & 63 & 320 & ㅇ. & 383 \\
\hline Total Strontium & 9557 & 51 & 0 & 0 & 0 & 4961 & 4468 & 180 & 9609 \\
\hline Other RAD & 26605 & 20413 & 582 & 5292 & 29 & 11024 & 33856 & 7960 & 52880 \\
\hline TOTAL RAD & 197038 & 173830 & 7183 & 5954 & 3146 & 178431 & 159262 & 23580 & 374212 \\
\hline & & & & & & & & & \\
\hline Other Wet Chemistry & 29788 & 113 & 0 & 으 & 86 & 26059 & 3842 & 86 & 29987 \\
\hline TCLP Extraction & 4106 & 9375 & 451 & 4192 & 0 & 3203 & 8146 & 6467 & 17970 \\
\hline Asbestos & 144 & 63904 & 0 & 0 & 0 & 3 & 47659 & 16396 & 64053 \\
\hline Industrial Hygiene, NOS & 2738 & 33 & 0 & 0 & 0 & 2557 & 210 & 4 & 2771 \\
\hline Physical Properties & 38747 & 3723 & 89 & 2632 & 0 & 24793 & 5223 & 15175 & 45190 \\
\hline Other, Not Otherwise Specified (NO & 915 & 447 & 28 & 0 & 0 & 407 & 19 & 929 & 1372 \\
\hline TOTAL OTHER & 76438 & 77593 & 568 & 6824 & 86 & 57023 & 65099 & 39057 & 161343 \\
\hline TOTAL SAMPLE ANALYSES & & & & & & & & & \\
\hline
\end{tabular}


Table Vf: FY 98 Samples and Sample Analyses by Rad Level and Matrix Type (ii) EM-30

\begin{tabular}{|c|c|c|c|c|c|c|c|c|c|}
\hline $\begin{array}{c}\text { Rad-Matrix/ } \\
\text { Sample-Sample Analysis } \\
\end{array}$ & RO & $\overline{\mathrm{R1}}$ & $\overline{\mathrm{R} 2}$ & $\overline{\mathrm{R3}}$ & $\overline{\mathrm{R} 4}$ & $\mathrm{M1}$ & $\mathrm{M2}$ & M3 & TOTAL \\
\hline CUSTOMER SAMPLES & 29074 & 79282 & 1360 & 6523 & 561 & 768911 & 5065 & 16255 & 107505 \\
\hline & & & & & & & & & \\
\hline \multicolumn{10}{|l|}{ SAMPLEANALYSES } \\
\hline Volatiles by GC/MS & 1193 & 955 & 230 & 702 & ㅇ. & 575 & 340 & 2166 & 3081 \\
\hline Volatiles by GC & 1073 & 1684 & 145 & 30 & 요 & 1075 & 1639 & 169 & 2908 \\
\hline Semi-Volatiles & 3724 & 1036 & 235 & 603 & ㅇ. & 2965 & 756 & 1876 & 5598 \\
\hline Herbicides/Pesticides/PCBs & 3051 & 1811 & 30 & 15 & 은 & 3496 & 308 & 1104 & 4908 \\
\hline Other Organics & 5596 & 18276 & 26 & 0 & 0 & 4917 & 7 & 810 & 14816 \\
\hline TOTAL ORGANICS & 14637 & 23762 & 666 & 1351 & D. & 13028 & 3050 & 6125 & 31310 \\
\hline \multicolumn{10}{|l|}{0} \\
\hline RCRA Appendix IX Metals & 42 & 38 & 0 & of & 0 & 74 & 0 & 61 & 107 \\
\hline CAM (California) Metals (RCRA) & 246 & 1 & 0 & o & 0 & 247 & 1 & 0 & 247 \\
\hline RCRA TCLP Metals and EPTOX Me & 857 & 950 & 516 & 6 & 욤 & 853 & 482 & 995 & 2329 \\
\hline EPA CLP Metals (CERCLA) & 382 & 951 & 61 & 1499 & 0 & 376 & 243 & 2275 & 2894 \\
\hline NPDES Metals (Clean Water Act) & 335 & 3247 & 0 & of & 0 & 3582 & 0 & 0 & 3582 \\
\hline Drinking Water Metals (SDWA) & 128 & 0 & 0 & 우 & 0 & 128 & 0 & o. & 128 \\
\hline Metals by Other Regulatory Suite & 2911 & 898 & 193 & 0 & 0 & 1985 & 190 & 1828 & 4003 \\
\hline Metals by Suite, Other & 7155 & 18205 & 0 & O & 0 & 3797 & 1 & 3399 & 16278 \\
\hline Metals by Metal Except Mercury & 5759 & 2384 & 198 & o & 280 & 4495 & 448 & 2349 & 7957 \\
\hline Mercury & 6482 & 19982 & 37 & 3 & 280 & 6445 & 618 & 2377 & 18112 \\
\hline Anions/Cations-IC & 10659 & 1380 & 90 & 0 & 561 & 8844 & 246 & 2227 & 12003 \\
\hline Inorganics Wet Chemistry & 18805 & 1389 & 858 & 82 & 0 & 13379 & 2162 & 6544 & 21609 \\
\hline Other Inorganics & 1200 & 888 & of & 0 & 0 & 1294 & 263 & 530 & 2088 \\
\hline TOTAL INORGANICS & 54960 & 50311 & 1955 & 1590 & 1121 & 45497 & 4654 & 22586 & 91337 \\
\hline \multirow{2}{*}{\multicolumn{10}{|c|}{ Gross Alpha/Beta }} \\
\hline & 9497 & 62641 & 242 & 190 & 561 & 49615 & 2148 & 1026 & 62959 \\
\hline Gamma Scan & 1250 & 19578 & 163 & 76 & 294 & 18796 & 1481 & 869 & 21254 \\
\hline Transuranic & 0 & 753 & 773 & 0 & 294 & 1031 & 496 & 80 & 1714 \\
\hline Plutonium Isotopes & 3077 & 451 & 95 & 15 & 561 & 2777 & 345 & 596 & 3959 \\
\hline Uranium Isotopes & 2972 & 1635 & 662 & 14 & 561 & 4016 & 486 & 860 & 5603 \\
\hline Curium Isotopes & 0 & 116 & 9 & 5 & 27 & 24 & 37 & 68 & 143 \\
\hline Thorium Isotopes & 207 & 890 & 33 & 98 & 0 & 632 & 27 & 569 & 1228 \\
\hline Radium Isotopes & 33 & 38 & 0 & 0 & 0 & 33 & 0 & 38 & 71 \\
\hline Americium-241 & 2767 & 320 & 14 & 8 & 53 & 2746 & 145 & 217 & 3135 \\
\hline Neptunium-237 & 207 & 614 & 551 & 0 & 0 & 537 & 291 & 543 & 1372 \\
\hline Polonium-210 & 1 & 39 & 26 & 0 & o. & 1 & 1 & 64 & 66 \\
\hline Tritium & 2481 & 469 & 148 & 15 & 0 & 2420 & 311 & 26 & 2935 \\
\hline Padon-222 & 4 & 0 & 0 & 0 & D. & 4 & 0 & 0 & 4 \\
\hline Lead-210 & 0 & 47 & 0 & o) & 요 & 0 & 0 & 47 & 47 \\
\hline Carbon-14 & 0 & 0 & 0 & 0 & o. & o & 0 & 0 & 요 \\
\hline Nickel-63 & 0 & 0 & of & 0 & 0 & 0 & 0 & 0 & 0 \\
\hline Selenium-79 & 246 & 0 & 0 & 0 & 0 & 246 & 0 & 0 & 246 \\
\hline Technetium-99 & 27 & 1063 & 799 & 0 & 0 & 1059 & 494 & 336 & 1889 \\
\hline Strontium Isotopes & of & 4 & 0 & 0 & 0 & 0 & 2 & 2 & 4 \\
\hline Strontium -89 & 0 & 0 & 0 & 0 & 요 & 0 & 0 & ? & 0 \\
\hline Strontium -90 & 273 & 134 & 125 & 15 & 0 & 278 & 269 & 0 & 547 \\
\hline lodine-129 & 323 & 79 & 0 & 0 & 요 & 402 & 0 & 의 & 402 \\
\hline Nickel-59 & of & 0 & 0 & 0 & 0. & 0 & 0 & 0 & 0 \\
\hline Total Uranium & 848 & 616 & 756 & 0 & 561 & 325 & 860 & 1116 & 2541 \\
\hline Total Thorium & 0 & 0 & 0 & 0 & 0 & 0 & 0 & o & 0 \\
\hline Total Strontium & 90 & 47 & 0 & 0 & of & 93 & 13 & 31 & 137 \\
\hline Other RAD & 1688 & 1786 & 140 & 4931 & 27 & 1770 & 425 & 6300 & 8534 \\
\hline TOTAL RAD & 25989 & 91322 & 4537 & 5367 & 2937 & 86805 & 7833 & 12790 & 118789 \\
\hline & & & & & & & & & \\
\hline Other Wet Chemistry & 4955 & 65 & 0 & 0 & 80 & 5020 & 0 & 80 & 5101 \\
\hline TCLP Extraction & 572 & 1548 & 380 & 3906 & 0 & 666 & 580 & 5105 & 6379 \\
\hline Asbestos & 1 & 2 & 0 & 0 & o. & 0 & 13 & 0 & 8 \\
\hline Industrial Hygiene, NOS & 2163 & 0 & 0 & 01 & 0 & 2160 & 3 & 0. & 2163 \\
\hline Physical Properties & 32478 & 1088 & 17 & 2453 & a) & 21946 & 60 & 14031 & 36036 \\
\hline Other, Not Otherwise Specified (NO & 419 & 46 & 26 & 0 & $0]$ & 35 & 0 & 449 & 487 \\
\hline TOTAL OTHER & 40587 & 2749 & 423 & 63601 & $80][$ & 29828 & 655 & 19666 & 50174 \\
\hline & & & & & & & & & \\
\hline TOTAL SAMPLE ANALYSES & 136174 & 168144 & 75801 & 14668 & 4138 & 175158 & 16192 & $61166]$ & 291610 \\
\hline
\end{tabular}


Table Vf: FY 98 Samples and Sample Analyses by Rad Level and Matrix Type (iii) EM-40

\begin{tabular}{|c|c|c|c|c|c|c|c|c|c|}
\hline $\begin{array}{c}\text { Rad-Matrix/ } \\
\text { Sample-Sample Analysis }\end{array}$ & RO & $\overline{\mathrm{R} 1}$ & $\overline{\mathrm{R} 2}$ & $\overline{\mathrm{R3}}$ & $\overline{\mathrm{R4}}$ & $\overline{\mathrm{M} 1}$ & M2 & $\overline{\mathrm{M3}}$ & TOTAL \\
\hline CUSTOMER SAMPLES & 13730 & 98323 & 2060 & 86 & 18 & 10466 & 64049 & 27453 & 108093 \\
\hline & & & & & & & & & \\
\hline \multicolumn{10}{|l|}{ SAMPLE ANALYSES } \\
\hline Volatiles by $\mathrm{GC} / \mathrm{MS}$ & 12869 & 12678 & 381 & 0 & 0 & 8172 & 16429 & 1342 & 25936 \\
\hline Volatiles by GC & 2849 & 1661 & 118 & 17 & 0 & 424 & 3802 & 440 & 4655 \\
\hline Semi-Volatiles & 6182 & 10613 & 380 & 4 & 0 & 3600 & 13135 & 436 & 17175 \\
\hline Herbicides/Pesticides/PCBs & 7606 & 21440 & 17 & 8 & 0 & 3246 & 21005 & 4851 & 29087 \\
\hline Other Organics & 1960 & 1247 & 0 & 0 & 0 & 761 & 2066 & 83 & 3059 \\
\hline TOTAL ORGANICS & 31466 & 47639 & 896 & 29 & 0 & 16203 & 56437 & 7153 & 79912 \\
\hline \multicolumn{10}{|l|}{+2} \\
\hline $\begin{array}{l}\text { RCRA Appendix IX Metals } \\
\text { CAM (California) Metals (RCRA) }\end{array}$ & $\begin{array}{r}29 \\
326\end{array}$ & 129 & $\frac{0}{0}$ & 0 & 의 & 10 & 148 & 0 & 158 \\
\hline RCRA TCLP Metals and EPTOX Me & $\frac{060}{1389}$ & $\frac{134}{6192}$ & $\frac{0}{90}$ & $\frac{0}{3}$ & $\frac{0}{0}$ & $\frac{461}{423}$ & $\begin{array}{r}168 \\
6788\end{array}$ & $\frac{34}{234}$ & $\begin{array}{r}561 \\
7559\end{array}$ \\
\hline EPA CLP Metals (CERCLA) & 13258 & 5817 & 1056 & 17 & 0 & 6103 & 13546 & 498 & 20147 \\
\hline NPDES Metals (Clean Water Act) & 2488 & 93 & 0 & 0 & 0 & 2581 & 0 & 0 & 2581 \\
\hline Drinking Water Metals (SDWA) & 66 & 0 & of & 0 & 0 & 66 & 0 & o & 66 \\
\hline Metals by Other Regulatory Suite & 12338 & 3513 & 0 & 0 & - & 4721 & 9515 & 2402 & 16244 \\
\hline Metals by Suite, Other & 0 & 5436 & 188 & (0) & $\overline{0}$ & 2909 & 1543 & 54 & 5065 \\
\hline Metals by Metal Except Mercury & 1460 & 9403 & 3 & 0 & 0 & 913 & 4789 & 5135 & 10852 \\
\hline Mercury & 3680 & 10807 & 6 & 2 & 0 & 3918 & 10056 & 197 & 14333 \\
\hline Anions/Cations $-1 C$ & 3863 & 2152 & o) & 0 & 0 & 5422 & 246 & 347 & 6015 \\
\hline Inorganics Wet Chemistry & 24177 & 10897 & 806 & 34 & 0 & 19377 & 12993 & 3975 & 36129 \\
\hline Other Inorganics & 4833 & 1982 & 0) & 0 & 0. & 3815 & 2713 & 286 & 6815 \\
\hline TOTALINORGANICS & 67908 & 56555 & 2147 & 55 & 0 & 50719 & 62504 & 13163 & 126526 \\
\hline \\
\hline Gross Apha/Beta & 26170 & 13191 & 430 & 105 & 0 & 14102 & 23653 & 1105 & 39378 \\
\hline Gamma Scan & 9203 & 6210 & 227 & 42 & 0 & 4906 & 9891 & 885 & 15682 \\
\hline Transuranic & 0 & 218 & 0 & 0 & 0 & 0 & 218 & 의 & 218 \\
\hline Plutonium Isotopes & 10910 & 1835 & 120 & 8 & 0 & 3980 & 8487 & 407 & 12874 \\
\hline Uranium Isotopes & 12511 & 10558 & 105 & 8 & 0 & 5714 & 16421 & 1046 & 23182 \\
\hline Curium Isotopes & 0 & 265 & 5 & 3 & 의 & 110 & 118 & 44 & 272 \\
\hline Thorium Isotopes & 215 & 5898 & 107 & 4 & 0 & 385 & 5605 & 235 & 6224 \\
\hline Radium Isotopes & 3386 & 40 & 0 & 0 & 의 & 2447 & 939 & 39 & 3426 \\
\hline Americium-241 & 10507 & 845 & 110 & 4 & 의 & 4015 & 7283 & 169 & 11467 \\
\hline Neptunium-237 & 215 & 1115 & 1 & 0 & 0 & 89 & 967 & 275 & 1331 \\
\hline Polonium-210 & 153 & 40 & 0 & 0 & D & 1 & 153 & 40 & 194 \\
\hline Tritium & 5865 & 4013 & 583 & 8 & 의 & 4507 & 5565 & 105 & 10324 \\
\hline Radon-222 & 4 & 0 & 01 & 0 & 으. & 4 & 0 & 0 & 4 \\
\hline Lead-210 & 0 & 1 & 0 & 0 & 0 & 0 & 0 & 1 & 1 \\
\hline Carbon-14 & 0 & 0 & 0 & 0 & 요 & 0 & 0 & ㅇ. & 0 \\
\hline Nickel-63 & $\overline{0}$ & 0 & 0 & 0. & 0 & 0 & 0 & o. & 0 \\
\hline Selenium-79 & 326 & 57 & 0 & 0 & 0 & 358 & 14 & (11) & 383 \\
\hline Technetium-99 & 14 & 4788 & 30 & 0 & 0 & 45 & 4749 & 39 & 4833 \\
\hline Strontium Isotopes & 0 & 5 & 0 & 0 & 0 & 0 & 2 & 2 & 5 \\
\hline Strontium -89 & 0 & 0 & 0 & 0 & 0 & 0 & 0 & - & 0 \\
\hline Strontium-90 & 340 & 1911 & 137 & 8 & 0 & 632 & 1735 & 36 & 2400 \\
\hline lodine-129 & 366 & 164 & 0 & 0 & 0 & 484 & 28 & 18 & 530 \\
\hline Nickel-59 & 0 & 0 & 0 & 0 & 0 & 0 & 0 & - & 0 \\
\hline Total Uranium & 1031 & 4116 & 0 & 0 & 0 & 279 & 1016 & 3852 & 5147 \\
\hline Total Thorium & 0 & 375 & 0 & 0 & 0 & 61 & 314 & 0 & 375 \\
\hline Total Strontium & 5038 & 1 & 0 & 0 & 0 & 2233 & 2670 & 136 & 5039 \\
\hline Other RAD & 14861 & 18121 & 404 & 8 & 0 & 3676 & 28534 & 1186 & 33395 \\
\hline TOTAL AAD & 101115 & 73767 & 2260 & 199 & 0 & 48025 & 118364 & 9632 & 176680 \\
\hline \multirow{2}{*}{\multicolumn{10}{|c|}{ Other Wet Chemistry }} \\
\hline & 12065 & 4 & 0 & गा & 0] & 9720 & 2386 & 0] & 12106 \\
\hline TCLP Extraction & 1452 & 7526 & 22 & 7 & 0 & 831 & 7170 & 978 & 8993 \\
\hline Asbestos & 74 & 62781 & 0 & 0 & 0 & 1 & 46746 & 16109 & 62855 \\
\hline Industrial Hygiene, NOS & 377 & 32 & 0 & 0 & 0 & 213 & 193 & 4 & 410 \\
\hline Physical Properties & 1711 & 2511 & 70 & 3 & D. & 776 & 3379 & 139 & 4295 \\
\hline Other, Not Otherwise Specified (Nd & 458 & 366 & 0 & 0 & 0 & 362 & 19 & 440 & 823 \\
\hline TOTAL OTHER & 16137 & 73258 & 91 & 10 & 0 & 11903 & 59893 & 17670 & 89481 \\
\hline & & & & & & & & & \\
\hline TOTAL SAMPLE ANALYSES & 216626 & 251218 & 5395 & 294 & 0] & 126851 & 297199 & 47617 & 472600 \\
\hline
\end{tabular}


Table Vf: FY 98 Samples and Sample Analyses by Rad Level and Matrix Type (iv) EM-60

\begin{tabular}{|c|c|c|c|c|c|c|c|c|c|}
\hline $\begin{array}{c}\text { Rad-Matrix/ } \\
\text { Sample-Sample Analysis }\end{array}$ & RO & R1 & $\overline{R 2}$ & R3 & R4 & M1 & $\mathrm{M} 2$ & M3 & TOTAL \\
\hline CUSTOMER SAMPLES & 2204 & 762 & 10 & 11 & o] & 2198 & 607 & 7 & 2894 \\
\hline & & & & & & & & & \\
\hline \multicolumn{10}{|l|}{ SAMPLE ANALYSES } \\
\hline Volatiles by GCMS & 4541 & 01 & 0 & 0 & 0 & 3396 & 1145 & 0 & 4541 \\
\hline Volatiles by $\mathrm{GC}$ & 1916 & 50 & 18 & 0 & 0 & 1637 & 166 & 0 & 1894 \\
\hline Semi-Volatiles & 1586 & 2 & 0 & 0 & 0 & 770 & 818 & 0 & 1588 \\
\hline Herbicides/Pesticides/PCBs & 1764 & 2 & 0 & 0 & 0 & 637 & 1129 & 0 & 1766 \\
\hline Other Organics & 961 & 167 & 0 & 0 & 0 & 916 & 46 & 0 & 1045 \\
\hline TOTALORGANICS & 10769 & 221 & 18 & 01 & o] & 7356 & 33031 & $1]$ & 10834 \\
\hline RCRA Annendix IX Matals & 10 & & & & & & & T & 7 \\
\hline $\begin{array}{l}\text { RCRA Appendix IX Metals } \\
\text { CAM (California) Metals (RCRA) }\end{array}$ & $\frac{16}{0}$ & $\begin{array}{l}0 \\
0\end{array}$ & $\frac{0}{0}$ & $\frac{0}{0}$ & $\frac{0}{0}$ & $\begin{array}{r}10 \\
0\end{array}$ & $\frac{7}{0}$ & $\frac{0}{0}$ & $\frac{17}{0}$ \\
\hline RCRA TCLP Metals and EPTOX Me & 1761 & 50 & 18 & 0 & 0 & 1453 & 180 & of & 1731 \\
\hline EPA CLP Metals (CERCLA) & 6668 & 0 & 0 & 0 & 0 & 3742 & 2919 & 7 & 6668 \\
\hline NPDES Metals (Clean Water Act) & 3 & 29 & 0 & 0 & 0 & 32 & 0 & 0 & 32 \\
\hline Drinking Water Metals (SDWA) & 460 & 0 & 0 & 0 & 0 & 460 & 0 & 0 & 460 \\
\hline Metals by Other Regulatory Suite & 6109 & 0 & 0 & 0 & 0 & 3718 & 2384 & 7 & 6109 \\
\hline Metals by Suite, Other & 0 & 166 & 0 & 0 & 0. & 0 & o & 0 & 83 \\
\hline Metals by Metal Except Mercury & 1859 & 48 & 18 & 0 & 0 & 1562 & 168 & 0 & 1828 \\
\hline Mercury & 1757 & 218 & 18 & 0 & 0 & 1466 & 166 & 0 & 1812 \\
\hline Anions/Cations-IC & 27 & 3 & 0 & 0 & 0 & 28 & 2 & 0 & 30 \\
\hline Inorganics Wet Chemistry & 23146 & 3 & 0 & 0 & 0 & 21383 & 1764 & 1 & 23148 \\
\hline Other Inorganics & 106 & 3 & 0 & 0 & 0 & 106 & 2 & 1 & 109 \\
\hline TOTAL INORGANICS & 41912 & 521 & 54 & 01 & o] & 33960 & 7592 & 16 & 42028 \\
\hline Gross Alpha/Beta & 10010 & 5381 & & & & 0007 & 0100 & 175 & 12001 \\
\hline Gross Alpha/Beta & $\begin{array}{r}12040 \\
6187\end{array}$ & $\begin{array}{l}538 \\
179\end{array}$ & \begin{tabular}{l|l}
0 & \\
0 &
\end{tabular} & $\frac{0}{0}$ & \begin{tabular}{l|l}
0 \\
0
\end{tabular} & $\begin{array}{c}6287 \\
2554\end{array}$ & $\begin{array}{l}6108 \\
3804\end{array}$ & $\frac{17}{8}$ & $\frac{12494}{6367}$ \\
\hline Transuranic & 0 & 5 & 0 & 0 & 0 & 0 & 5 & 0 & 5 \\
\hline Plutonium Isotopes & 7366 & 5 & 0 & 0 & o. & 3690 & 3674 & 7 & 7372 \\
\hline Uranium Isotopes & 5823 & 5 & 0 & 0 & 0 & 3012 & 2810 & 7[ & 5828 \\
\hline Curium Isotopes & 0 & 0 & 0 & 0 & 0 & 0 & 0 & 0 & 요 \\
\hline Thorium Isotopes & 0 & 0 & 0 & 1 & 0 & of & 0 & 1 & 1 \\
\hline Radium Isotopes & 3135 & 0 & 0 & 0 & 0 & 2497 & 638 & 0 & 3135 \\
\hline Americium-241 & 6781 & 0 & 0 & 0 & o & 3577 & 3197 & 7 & 6781 \\
\hline Neptunium -237 & 0 & 0 & 0 & 0 & 0 & of & 0 & 0 & a \\
\hline Polonium-210 & 0 & 4 & 0 & 0 & 0 & 4 & 0 & 0 & 4 \\
\hline Tritium & 4221 & 0 & 0 & 0 & D) & 3013 & 1208 & o. & 4222 \\
\hline Radon-222 & 0 & 0 & 0 & 0 & 0 & 0 & 0 & 0 & 0 \\
\hline Lead-210 & 0 & 0 & 0 & 0 & 0 & 0 & D) & 0 & D. \\
\hline Carbon-14 & 0 & 0 & 0 & 0 & 0 & 0 & 0 & 0 & 0 \\
\hline Nickel-63 & 0 & 0 & 0 & 0 & o. & 0 & 0 & 0 & 욤 \\
\hline Selenium-79 & 0 & 0 & 0 & 0 & 0 & 0 & 0 & o. & 0 \\
\hline Technetium-99 & 98 & 0 & 0 & 0 & 0 & 98 & o & 0 & 98 \\
\hline Strontium Isotopes & 0 & 0 & 0 & 0 & D. & 0 & 0 & 0 & 요 \\
\hline Strontium - 89 & 0) & 0 & 0 & 0 & 0 & 0 & 0 & 0. & 요 \\
\hline Strontium -90 & 98 & 0 & 0 & 0 & o) & 98 & 0 & 0 & 98 \\
\hline lodine-129 & 279 & 0 & 0 & 0 & 0 & 279 & 0 & 0 & 279 \\
\hline Nickel-59 & 0 & 0 & 0 & 0 & a. & 0 & 0 & 0 & 0 \\
\hline Total Uranium & 98 & 0 & 0 & 0 & 요 & 98 & 0 & 0 & 98 \\
\hline Total Thorium & 0 & 0 & 0 & 0 & 0 & 0 & 0 & o. & 0 \\
\hline Total Strontium & 3829 & 0 & 0 & 0 & o. & 2288 & 1535 & 7 & 3830 \\
\hline Other RAD & 8544 & 48 & 18 & 0 & 0 & 4755 & 3853 & 1 & 8610 \\
\hline TOTAL RAD & 58499 & 787 & 19 & 11 & 0 & 32249 & 26832 & $5 \overline{8}]$ & 59222 \\
\hline & & & & & & & & & \\
\hline Other Wet Chemistry & 10778 & (1) & $\overline{0}$ & 0 & 0 & 9530 & 1249 & $0]$ & 10779 \\
\hline TCLP Extraction & 1774 & 49 & 19 & 0 & 0 & 1452 & 195 & 0. & 1745 \\
\hline Asbestos & 60 & 0 & 0 & 0 & 0 & 2 & 57 & o) & 60 \\
\hline Industrial Hygiene, NOS & 31 & 0 & 0 & 0 & 0 & 22 & 9 & o. & 31 \\
\hline Physical Properties & 1951 & 0 & 0 & of & 0. & 431 & 1520 & o. & 1951 \\
\hline Other, Not Otherwise Specified (No & 0 & 22 & 01 & 0 & 0 & 0 & 0 & o) & 11 \\
\hline TOTAL OTHER & 14594 & 72 & 19 & 01 & D. & 11437 & 3031 & $\overline{0}$ & 14576 \\
\hline & & & & & & & & & \\
\hline TOTAL SAMPLEANALYSES & 125774 & 1600 & 1111 & 11 & (0) & 85001 & 40758 & 76 & 126660 \\
\hline
\end{tabular}


Table Vf: FY 98 Samples and Sample Analyses by Rad Level and Matrix Type (v) Fernald

\begin{tabular}{|c|c|c|c|c|c|c|c|c|c|}
\hline $\begin{array}{c}\text { Rad-Matrix/ } \\
\text { Sample-Sample Analysis }\end{array}$ & $\overline{\mathrm{RO}}$ & $\overline{\mathrm{R1}}$ & R2 & $\overline{\mathrm{A3}}$ & R4 & M1 & M2 & $\mathrm{M3}$ & TOTAL \\
\hline CUSTOMER SAMPLES & 1253 & 1501 & 29 & $\overline{1}$ & 0 & 1028 & 1448 & 202 & 2730 \\
\hline & & & & & & & & & \\
\hline \multicolumn{10}{|l|}{ SAMPLE ANALYSES } \\
\hline Volatiles by GC/MS & 562 & 57 & 0 & 0 & 이 & 297 & 74 & 250 & 619 \\
\hline Volatiles by GC & 120 & 451 & 28 & 0 & ㅁ. & 55 & 477 & 64 & 597 \\
\hline Semi-Volatiles & 109 & 100 & 0 & 0 & o] & 56 & 134 & 21) & 210 \\
\hline Herbicides/Pesticides/PCBs & 361 & 228 & 0 & 0 & 이 & 153 & 40 & 398 & 590 \\
\hline Other Organics & 352 & 135 & 0 & 0 & 0 & 12 & 352 & 18 & 435 \\
\hline TOTAL ORGANICS & 1504 & 9711 & 28 & $\overline{0}$ & 0] & 572 & 1077 & 751 & 2451 \\
\hline \multicolumn{10}{|l|}{ 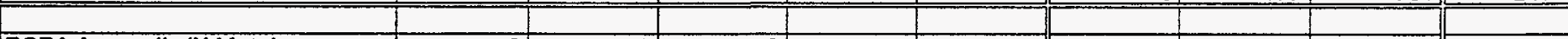 } \\
\hline RCRA Appendix IX Metals & 2 & 1 & 0 & 0 & 0 & 1 & 1 & 0 & 2 \\
\hline CAM (California) Metals (RCRA) & 44 & 46 & O & 0 & 0 & 90 & 57 & 11 & 124 \\
\hline RCRA TCLP Metals and EPTOX Me & 232 & 158 & 28 & 0 & 0. & 42 & 257 & 50 & 383 \\
\hline EPA CLP Metals (CERCLA) & 328 & 35 & 0 & 0 & 0 & 315 & 19 & 29 & 363 \\
\hline NPDES Metals (Clean Water Act) & 19 & 33 & 0 & 0 & 0. & 52 & 0 & 0 & 52 \\
\hline Drinking Water Metals (SDWA) & 1 & of & 0 & 0 & 0. & 1 & 0 & 0 & 1 \\
\hline Metals by Other Regulatory Suite & 901 & 33 & 0 & 0 & ㅁ․ & 314 & 27 & 586 & 930 \\
\hline Metals by Suite, Other & 0 & 141 & 0 & 0 & 0] & 23 & 0 & 12 & 88 \\
\hline Metals by Metal Except Mercury & 111 & 229 & 0 & 0 & 0 & 108 & 224 & 7 & 340 \\
\hline Mercury & 129 & 481 & 0 & 0 & 0. & 305 & 183 & 16 & 557 \\
\hline Anions/Cations-IC & 75 & 36 & 0 & $\overline{0}$ & 0] & 26 & 8 & 77 & 111 \\
\hline Inorganics Wet Chemistry & 1784 & 598 & 251 & 0 & 0 & 980 & 567 & 1234 & 2707 \\
\hline Other Inorganics & 582 & 192 & 0 & 0 & 에 & 264 & 417 & 92 & 774 \\
\hline TOTALINORGANICS & 4209 & 1981 & 279 & $\overline{0}$ & 0 & 2520 & 1762 & 2115 & 6433 \\
\hline \multicolumn{10}{|l|}{ (20) } \\
\hline Gross Alpha/Beta & 1000 & 805 & 28 & 0 & 0] & 998 & 514 & 159 & 1752 \\
\hline Gamma Scan & 246 & 250 & 0 & 0 & - & 225 & 139 & 132 & 497 \\
\hline Transuranic & 0 & 0 & 0 & 0 & 0. & 0 & 0 & 0 & 0 \\
\hline Plutonium Isotopes & 535 & 44 & 0 & 0 & 0 & 241 & 257 & 81. & 579 \\
\hline Uranium Isotopes & 598 & 216 & 0 & 0 & 의 & 328 & 297 & 188 & 814 \\
\hline Curium Isotopes & 0 & 28 & 0 & 0 & 요 & 9 & 5 & 15 & 28 \\
\hline Thorium Isotopes & 73 & 13) & 0 & 1 & 0 & 0 & 13 & 74 & 87 \\
\hline Radium Isotopes & 9 & 14 & 0 & 0 & 요 & 9 & 0 & 13 & 23 \\
\hline Americium-241 & 451 & 32 & 0 & 0 & 0 & 228 & 248 & 7 & 483 \\
\hline Neptunium-237 & 73 & 34 & 0 & 0 & 0 & 4 & 16 & 87 & 107 \\
\hline Polonium-210 & 52 & 14 & 0 & 0 & 0. & 0 & 52 & 14 & 66 \\
\hline Tritium & 38 & 162 & 28 & 0 & 0 & 54 & 74 & 3 & 180 \\
\hline Radon-222 & 1 & 0 & 0 & 0 & 애 & 1 & 0 & 요 & 1 \\
\hline Lead-210 & 0 & 0 & 0 & 0 & 0 & 0 & 0 & 0 & 인 \\
\hline Carbon-14 & 0 & 0 & D. & 0 & 0 & 0 & 0 & 요 & 0 \\
\hline Nickel-63 & 0 & 0 & D. & 0 & 0 & 0 & 0 & - & 은 \\
\hline Selenium-79 & 44 & 11 & 0 & 0 & of & 50 & 3 & 2 & 55 \\
\hline Technetium-99 & 0 & 13 & 0 & 0 & 0 & 0 & 0 & 13 & 14 \\
\hline Strontium Isotopes & 0 & 2 & 0 & 0 & 0 & 0 & 1 & 1 & 2 \\
\hline Strontium -89 & 0 & 0 & 0 & 0 & 0] & 0 & 0 & o. & 0 \\
\hline Strontium -90 & 44 & 68 & 28 & 0 & 0. & 72 & 60 & 7 & 140 \\
\hline lodine-129 & 44 & 44 & 0 & $\overline{0}$ & 0 & 79 & 6 & 3 & 88 \\
\hline Nickel-59 & a & 0 & 0 & 0 & 0 & 0 & 01 & 0 & 0 \\
\hline Total Uranium & 291 & 148 & 0 & $\overline{0}$ & 0 & 35 & 22 & 382 & 439 \\
\hline Total Thorium & 0 & 43 & 0. & 0 & o. & 21 & 22 & 0 & 43 \\
\hline Total Strontium & 48 & $\begin{array}{ll}0 & \\
\end{array}$ & 0 & 0 & 0 & 46 & 3 & 0 & 49 \\
\hline Other RAD & 544 & 186 ! & 28 & 0 & -] & 326 & 93 & 337 & 757 \\
\hline TOTALRAD & 4092 & 2127 & 112 & 1 & 0 & 2726 & 1825 & 1520 & 6202 \\
\hline & & & & & & & & & \\
\hline Other Wet Chemistry & 254 & 14 & 0! & 0 & 0]1 & 265 & $3 \mid$ & 0] & 268 \\
\hline TCLP Extraction & 261 & 226 & 1 & 0 & 0] & 121 & 290 & 76 & 487 \\
\hline Asbestos & 0 & 12 & O) & 0 & 0 & 0 & 12 & 0 & 12 \\
\hline Industrial Hygiene, NOS & 57 & 6 & 0 & 0 & 0 & 60 & 2 & 1 & 63 \\
\hline Physical Properties & 26 & 107 & 0 & 0 & 0. & 60 & 26 & 47 & 134 \\
\hline Other, Not Otherwise Specified (NO) & 150 & 15 & 0 & 0 & 0] & 14 & 11 & 150 & 165 \\
\hline TOTAL OTHER & 747 & 381 & 1 & 0 & 0] & 520 & 334 & 274 & 1129 \\
\hline & & & & & ד & & & & \\
\hline TOTAL SAMPLE ANALYSES & 10553 & 5460 & 420 & 1 & O] & 6338 & 4999 & 4660 & 16216 \\
\hline
\end{tabular}


Table Vf: FY 98 Samples and Sample Analyses by Rad Level and Matrix Type (vi) INEL

\begin{tabular}{|c|c|c|c|c|c|c|c|c|c|}
\hline $\begin{array}{c}\text { Rad-Matrix/ } \\
\text { Sample-Sample Analysis }\end{array}$ & RO & R1 & $\overline{\mathrm{R} 2}$ & $\mathrm{R3}$ & $\overline{\mathrm{R} 4}$ & $\overline{\mathrm{M} 1}$ & $\overline{\mathrm{M} 2}$ & $\overline{\mathrm{M3}}$ & TOTAL \\
\hline CUSTOMER SAMPLES & 1448 & 13221 & 179 & 626 & (0) & $4 \overline{964}$ & 5873 & 3307 & 14809 \\
\hline \multirow{2}{*}{\multicolumn{10}{|c|}{ SAMPLE ANALYSES }} \\
\hline & & & & & & & & & \\
\hline Volatiles by GC/MS & 206 & 951 & 2 & 66 & $\overline{0}$ & 222 & 855 & 148 & 1225 \\
\hline Volatiles by GC & 621 & 206 & 24 & 6 & 0 & 522 & 264 & 19 & 831 \\
\hline Semi-Volatiles & 180 & 805 & 8 & 57 & 0 & 146 & 832 & 72 & 1050 \\
\hline Herbicides/Pesticides/PCBs & 290 & 1814 & 6 & 3 & 0 & 244 & 1420 & 448 & 2113 \\
\hline Other Organics & 485 & 1275 & 0 & 0 & 0 & 273 & 228 & 5 & 1133 \\
\hline TOTAL ORGANICS & 1780 & 5051 & 41 & 132 & 0 & 1408 & 3599 & 692 & 6352 \\
\hline \multicolumn{10}{|l|}{ RCRA Appendix IX Metals } \\
\hline CAM (California) Metals (RCRA) & 12 & $\frac{4}{1}$ & $\frac{0}{0}$ & $\frac{0}{0}$ & $\frac{0}{0}$ & $\frac{5}{13}$ & $\frac{1}{1}$ & $\frac{0}{0}$ & $\frac{6}{13}$ \\
\hline RCAA TCLP Metals and EPTOX Me & 586 & 617 & 14 & 1 & 0 & 469 & 670 & $\frac{2}{23}$ & 1190 \\
\hline EPA CLP Metals (CERCLA) & 198 & 69 & 18 & 144 & 0 & 152 & 129 & 148 & 429 \\
\hline NPDES Metals (Clean Water Act) & 39 & 225 & 0 & 0 & 0 & 263 & 0 & 0 & 263 \\
\hline Drinking Water Metals (SDWA) & 131 & 0 & 0 & 0 & 0 & 131 & 0 & 0 & 131 \\
\hline Metals by Other Regulatory Suite & 384 & 361 & 0 & 0 & $\overline{0}$ & 218 & 367 & 193 & 762 \\
\hline Metals by Suite, Other & 0 & 1327 & 1 & 0 & 0 & 24 & 10 & 5. & 683 \\
\hline Metals by Metal Except Mercury & 648 & 850 & 5 & 0 & 0 & 512 & 412 & 523 & 1475 \\
\hline Mercury & 681 & 2202 & 6 & 1 & 0 & 593 & 968 & 21 & 2236 \\
\hline Anions/Cations-IC & 98 & 68 & 0 & 0 & 0 & 105 & 29 & 32 & 167 \\
\hline Inorganics Wet Chemistry & 1233 & 847 & 85 & 14 & 0 & 1009 & 815 & 356 & 2180 \\
\hline Other Inorganics & 145 & 128 & 0 & 0 & 0 & 161 & 80 & 32 & 273 \\
\hline TOTALINORGANICS & 4158 & 6699 & 130 & 160 & 0 & 3655 & 3482 & 1334 & 9809 \\
\hline \multicolumn{10}{|l|}{20} \\
\hline Gross Alpha/Beta & $\frac{1242}{792}$ & $\frac{4873}{1721}$ & 35 & $\frac{37}{15}$ & 음 & 3553 & 1052 & 80 & 5437 \\
\hline Transuranic & $\frac{10}{0}$ & 24 & 0 & 0 & 0 & $\frac{1398}{0}$ & 24 & $\frac{21}{0}$ & $\frac{2000}{24}$ \\
\hline Plutonium Isotopes & 466 & 71 & 9 & 3 & 0 & 334 & 188 & 28 & 550 \\
\hline Uranium Isotopes & 360 & 624 & 3 & 3 & 0 & 311 & 613 & 65 & 990 \\
\hline Curium Isotopes & 0 & 12 & 2 & 1 & 0 & 3 & 6 & 5 & 15 \\
\hline Thorium isotopes & 18 & 489 & 2 & 7 & 0 & 13 & 478 & 26 & 516 \\
\hline Radium Isotopes & 43 & 3 & 0 & 0 & 0 & 39 & 5 & 3 & 46 \\
\hline Americium-241 & 342 & 46 & 3 & 1 & 0 & 309 & 78 & 7 & 394 \\
\hline Neptunium-237 & 18 & 31 & 2 & 0 & D & 3 & 23 & 25 & 51 \\
\hline Polonium-210 & 1 & 4 & 0 & 0 & 0 & 1 & 1 & 3. & 5 \\
\hline Tritium & 357 & 81 & 19 & 3 & 0 & 294 & 132 & (4) & 445 \\
\hline Radon-222 & 0 & 0 & 0 & 0 & 0 & 0 & 0 & 0 & 0 \\
\hline Lead-210 & 0 & 3 & 0 & 0 & 0 & 0 & 0 & 3 & 3 \\
\hline Carbon-14 & o & o & 0 & 0 & 0 & 0 & 0 & 0 & 0 \\
\hline Nickel-63 & 0 & 0 & 0 & 0 & 0 & 0 & 0 & 0 & 0 \\
\hline Selenium-79 & 12 & 7 & 0 & 0 & 0 & 16 & 2 & (1) & 19 \\
\hline Technetium-99 & 28 & 475 & 0 & 0 & 0 & 28 & 470 & 5 & 503 \\
\hline Strontium Isotopes & 0 & 0 & 0 & 0 & 0 & 0 & 0 & 0 & $\overline{0}$ \\
\hline Strontium-89 & 0 & of & 0 & 0 & 0 & 0 & $\overline{0}$ & 0 & $\overline{0}$ \\
\hline Strontium-90 & 40 & 69 & 16 & 3 & 0 & 59 & 64 & 4 & 128 \\
\hline lodine-129 & 92 & 17 & 0 & 0 & 0 & 103 & 4 & 2 & 109 \\
\hline \begin{tabular}{|l|} 
Nickel-59 \\
\end{tabular} & 0 & 0 & 0 & 0 & 0 & 0 & 0 & 0 & 0 \\
\hline Total Uranium & 99 & 346 & 0 & o & 0. & 33 & 64 & 349 & 445 \\
\hline Total Thorium & 0 & 0 & 0 & 0 & 0 & 0 & 0 & 0 & $\overline{0}$ \\
\hline Total Strontium & 58 & 3 & 0 & 0 & 0 & 32 & 27 & 3 & 62 \\
\hline Other RAD & 448 & 1648 & 20 & 465 & 0 & 347 & 1649 & 576 & 2577 \\
\hline TOTALRAD & 4416 & 10551 & 140 & 539 & 0 & $\overline{6876}$ & 5986 & 1243 & $\overline{14875}$ \\
\hline \\
\hline Other Wet Chemistry & $\overline{554}$ & 5 & $\overline{0}$ & $\overline{0}$ & (0) & 547 & $\overline{12}$ & $\overline{0 .}$ & $\overline{55 \overline{9}}$ \\
\hline TCLP Extraction & 567 & 750 & 18 & 368 & 0 & 480 & 700 & 467 & $167 \overline{5}$ \\
\hline Asbestos & 0 & 6286 & 0 & 0 & $\overline{0}$ & 0 & 4654 & 1633 & 6286 \\
\hline Industrial Hygiene, NOS & 154 & 4 & 0 & 0 & 0 & 153 & 5 & 0 & 158 \\
\hline Physical Properties & 144 & 191 & 4 & 231 & 0 & 138 & 191 & 242 & 570 \\
\hline Other, Not Otherwise Specified (Nd & 36 & 10 & 0 & 0 & 0 & 4 & 0 & 36 & 43 \\
\hline TOTAL OTHEA & $\overline{1455}$ & 7246 & 22 & 2600 & 0 & 1320 & $\overline{55562}$ & 2378 & 9291 \\
\hline & & & & & & & & & \\
\hline TOTAL SAMPLE ANALYSES & 11810 & $\overline{29546}$ & 332 & $\overline{1431}$ & 0 & $\overline{13259}$ & $\overline{18629}$ & 5647 & $\overline{40327}$ \\
\hline
\end{tabular}


Table Vf: FY 98 Samples and Sample Analyses by Rad Level and Matrix Type (vii) NTS

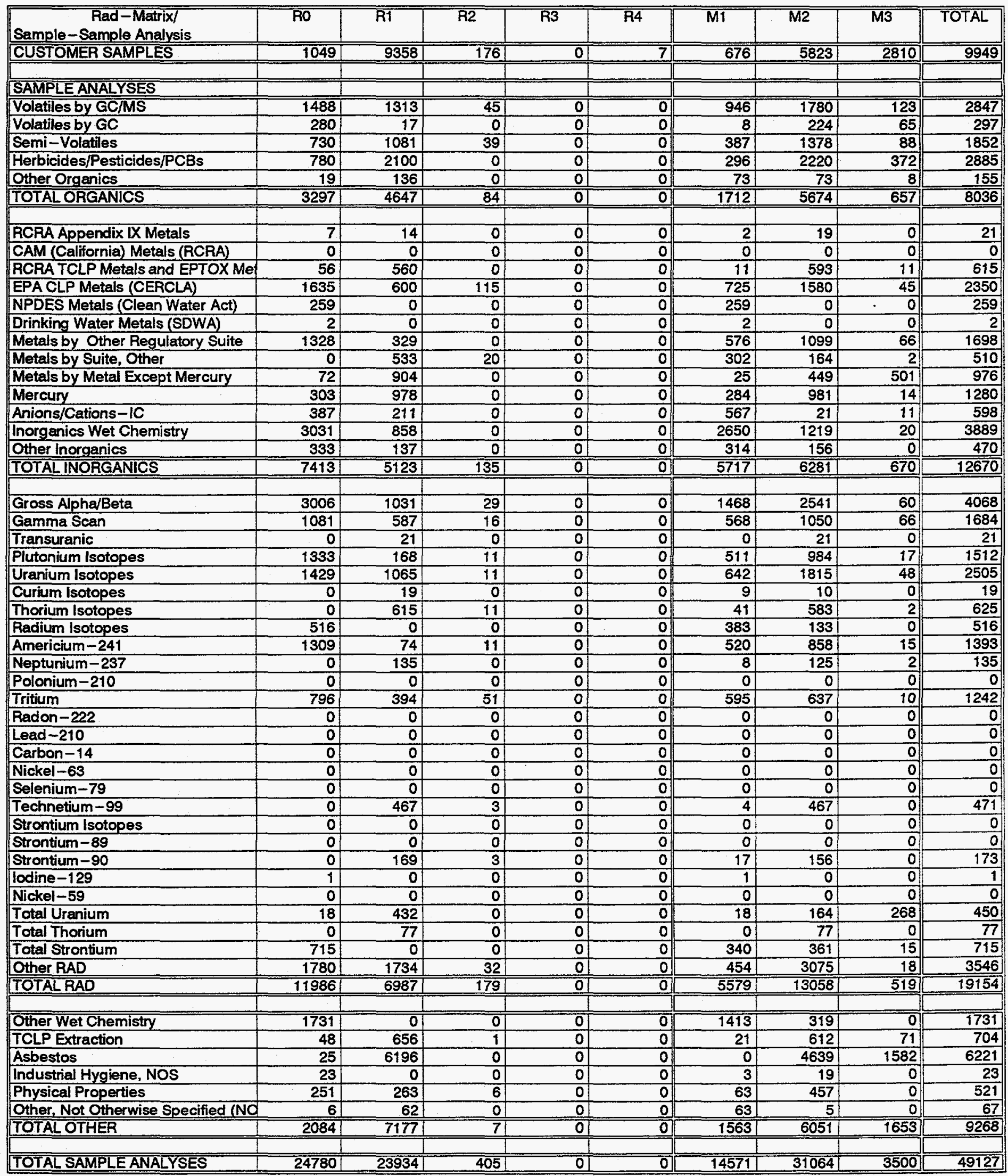


Table Vf: FY 98 Samples and Sample Analyses by Rad Level and Matrix Type (viii) Oak Ridge K-25

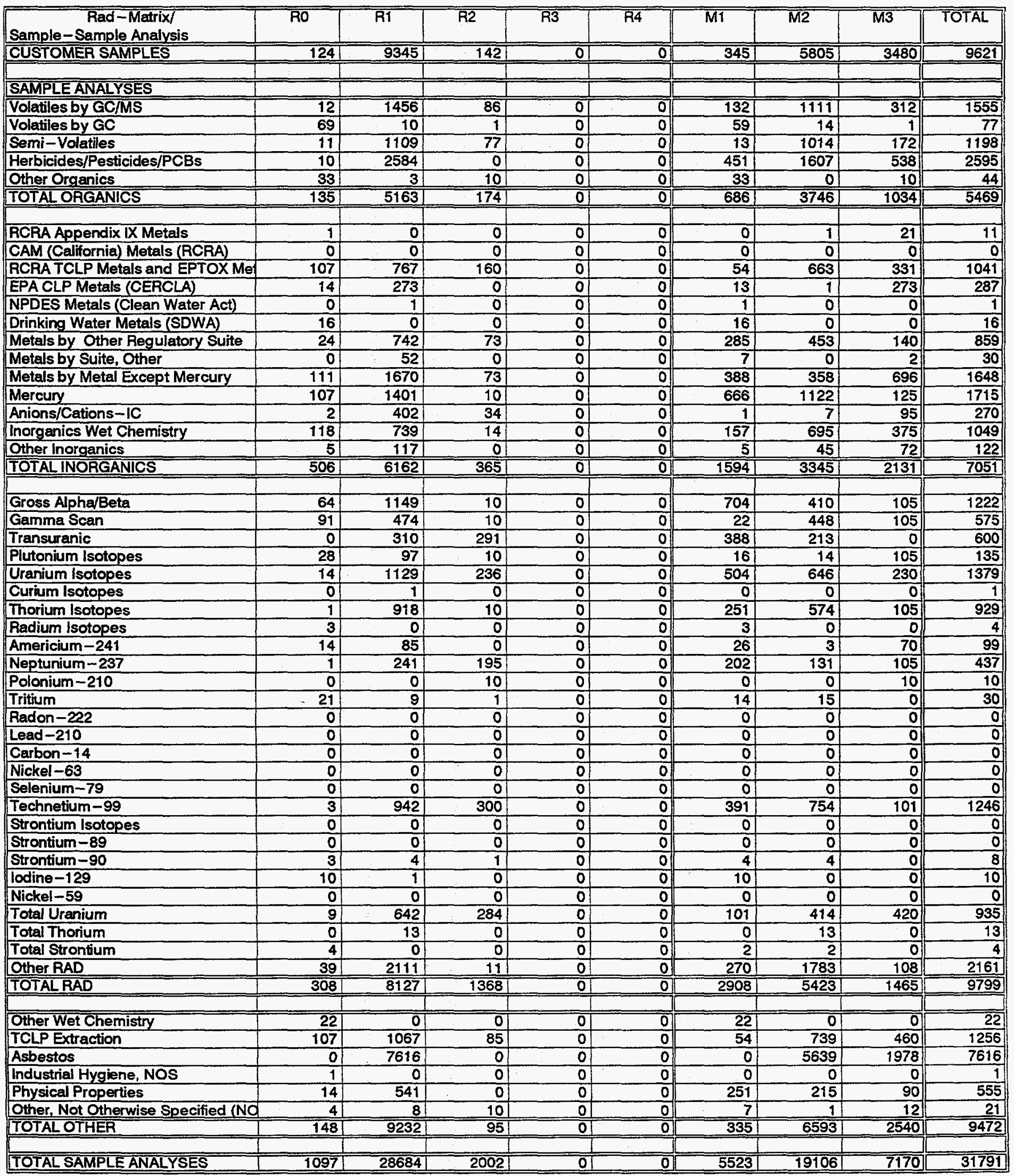


Table Vf: FY 98 Samples and Sample Analyses by Rad Level and Matrix Type (ix) Oak Ridge X-10

\begin{tabular}{|c|c|c|c|c|c|c|c|c|c|}
\hline $\begin{array}{c}\text { Rad-Matrix } \\
\text { Sample-Sample Analysis }\end{array}$ & Ro & R1 & $\overline{\mathrm{R} 2}$ & R3 & $\overline{\mathrm{R4}}$ & M1 & M2 & M3 & TOTAL \\
\hline CUSTOMER SAMPLES & 367 & 5711 & 10 & 595 & o] & 3154 & 1316 & 1272 & 6212 \\
\hline & & & & & & & & & \\
\hline \multicolumn{10}{|l|}{ SAMPLE ANALYSES } \\
\hline Volatiles by $\mathrm{GC} / \mathrm{MS}$ & 54 & 228 & 이 & 6 & o. & 64 & 203 & 81 & 348 \\
\hline Volatiles by GC & 70 & 37 & 1 & 0 & -0. & 68 & 31 & 5 & 106 \\
\hline Semi-Volatiles & 42 & 195 & 0 & 56 & 0 & 40 & 192 & 61 & 293 \\
\hline Herbicides/Pesticides/PCBs & 159 & 436 & 0 & 0 & 요 & 159 & 333 & 104 & 595 \\
\hline Other Organics & 58 & 936 & 0 & 0 & 0 & 26 & 38 & 1 & 529 \\
\hline TOTAL ORGANICS & 384 & 1831 & 2 & 122 & 0 & 357 & 797 & 252 & 1873 \\
\hline \multicolumn{10}{|l|}{202} \\
\hline RCRA Appendix IX Metals & 2 & 2 & 0 & 0 & - & 4 & 0 & 은 & 4 \\
\hline CAM (California) Metals (RCRA) & 44 & 1 & 0 & 0 & ㅇ. & 45 & 1 & 요 & 45 \\
\hline RCRA TCLP Metals and EPTOX Me & 70 & 151 & 1 & 0 & 0 & 60 & 152 & 5 & 220 \\
\hline EPA CLP Metals (CERCLA) & 37 & 7 & 1 & 138 & 0 & 30 & 14 & 139 & 183 \\
\hline NPDES Metals (Clean Water Act) & 20 & 164 & 0 & 0 & 0 & 184 & 0 & 0 & 184 \\
\hline Drinking Water Metals (SDWA) & 11 & 의 & of & 0 & 0 & 11 & 0 & 으 & 11 \\
\hline Metals by Other Regulatory Suite & 101 & 86 & 0 & 0 & 0 & 79 & 85 & 32 & 191 \\
\hline Metals by Suite, Other & 0 & 944 & 0 & 0 & - & 5 & 2 & 1. & 476 \\
\hline Metals by Metal Except Mercury & 133 & 203 & 0 & 0 & 0 & 115 & 89 & 127 & 334 \\
\hline Mercury & 169 & 1174 & 0 & 0 & 0 & 179 & 225 & 6 & 876 \\
\hline Anions/Cations-IC & 46 & 25 & 0 & $0]$ & 0 & 501 & 14 & 6 & 70 \\
\hline Inorganics Wet Chemistry & 587 & 187 & 9 & 2 & 의 & 549 & 179 & 59 & 786 \\
\hline Other Inorganics & 62 & 35 & 0 & 0 & 0 & 601 & 27 & 9 & 97 \\
\hline TOTAL INORGANICS & $1280 \mid$ & 2979 & 12 & 140 & (0) & 1371 & $786 \mid$ & 384 & 3476 \\
\hline \multicolumn{10}{|l|}{20} \\
\hline Gross Alpha/Beta & 519 & 3085 & 1 & 0 & - & 2472 & 182 & 26 & 3143 \\
\hline Gamma Scan & 82 & 1058 & 0 & 0 & 으 & 974 & 152 & 15 & 1140 \\
\hline Transuranic & 0 & 5 & 0 & 0 & - & 0 & 5 & 0 & 5 \\
\hline Plutonium Isotopes & 169 & 6 & 2 & 0 & 0. & 152 & 19 & 6 & 177 \\
\hline Uranium Isotopes & 162 & 137 & 2 & 0 & 요 & 154 & 131 & 15 & 301 \\
\hline Curium Isotopes & 0 & 2 & 0 & 0 & 으 & 0 & 0 & 2 & 2 \\
\hline Thorium Isotopes & 2 & 117 & 0 & 5 & 요 & 3 & 114 & 8 & 125 \\
\hline Radium Isotopes & 5 & 0 & 0 & 0 & 0 & 4 & 1 & 의 & 5 \\
\hline Americium-241 & 158 & 7 & 0 & 0 & 요 & 152 & 11 & 2 & 165 \\
\hline Neptunium-237 & 2 & 6 & 2 & 0 & 0] & 0 & 5 & 5 & 10 \\
\hline Polonium-210 & 11 & 1 & 0 & 0 & - & 0 & 1 & 0 & 1 \\
\hline Tritium & 136 & 13 & 3 & 0 & 묘 & 132 & 15 & 2 & 151 \\
\hline Radon-222 & 0 & 0 & 0 & 0 & 으. & 0 & 0 & 0 & 0 \\
\hline Lead-210 & 0 & 2 & 0 & 0 & 으 & 0 & 0 & 2 & 2 \\
\hline Carbon-14 & 0 & 0 & 0 & of & 의 & 0 & 0 & 욤 & 0 \\
\hline Nickel-63 & 0 & 0 & 0 & 0 & 요 & 0 & 0 & 0 & 0 \\
\hline Selenium-79 & 44 & 1 & 0 & 0 & of & 45 & 0 & 0 & 45 \\
\hline Technetium-99 & 2 & 116 & 0 & 0 & 의 & 2 & 114 & 2 & 118 \\
\hline Strontium Isotopes & 0 & 0 & 0 & of & 0 & 0 & 0 & 0 & 0 \\
\hline Strontium - 89 & 0 & 0 & 0 & 0 & 의 & 0 & 0 & 요 & 0 \\
\hline Strontium-90 & 46 & 8 & 1 & 0 & 으. & 50 & 5 & 1 & 56 \\
\hline lodine-129 & 51 & 3 & 0 & 0 & 므. & 52 & 1 & 0 & 53 \\
\hline Nickel-59 & 0 & 0 & 0 & 0 & 0 & 0 & 0 & 0 & 0 \\
\hline Total Uranium & 12 & 85 & 0 & 0 & 0 & 3 & 16 & 78 & 97 \\
\hline Total Thorium & 0 & 0 & 0 & 0 & 의 & 0 & 0 & 0 & 0 \\
\hline Total Strontium & 10 & 2 & 0 & 0 & 요 & 7 & 3 & 2 & 12 \\
\hline Other RAD & 106 & 410 & 2 & 462 & 0 & 103 & 384 & 487 & 977 \\
\hline TOTAL RAD & 1509 & 5067 & 13 & 467 & 인 & 4306 & 1160 & 653 & 6588 \\
\hline \multicolumn{10}{|l|}{ Othor WotChomiatn } \\
\hline Other Wet Chemistry & 269 & 2 & 0 & 0 & (0) & 269 & 2 & 0 & $\overline{271}$ \\
\hline TCLP Extraction & 56 & 176 & 7 & 366 & 0 & 55 & 156 & 390 & 603 \\
\hline Asbestos & 0 & 1523 & 0 & 0 & 0 & 0 & 1128 & 396 & 1523 \\
\hline Industrial Hygiene, NOS & 112 & 1 & 0 & 0 & 0 & 111 & (1) & 0 & $\overline{112}$ \\
\hline Physical Properties & 27 & 43 & 0 & 230 & 0 & 27 & 42 & 232 & 301 \\
\hline Other, Not Otherwise Specified (NO & 5 & 2 & 0 & 0 & 0 & 2 & 0 & 5 & 7 \\
\hline TOTAL OTHER & 470 & $17 \overline{47}$ & 7 & 596 & 0 & 464 & 1329 & 1022 & 2817 \\
\hline & & & & काT $12+3$ & & & & & \\
\hline TOTAL SAMPLE ANALYSES & 3642 & 11624 & 34 & 1325 & 0 & 6498 & 4072 & 2311 & 14754 \\
\hline
\end{tabular}


Table Vf: FY 98 Samples and Sample Analyses by Rad Level and Matrix Type (x) Oak Ridge Y-12

\begin{tabular}{|c|c|c|c|c|c|c|c|c|c|}
\hline $\begin{array}{c}\text { Rad-Matrix } \\
\text { Sample-Sample Analysis }\end{array}$ & Ro & $\overline{\mathrm{RI}}$ & $\mathrm{R2}$ & $\overline{\mathrm{R3}}$ & $\overline{\mathrm{R4}}$ & M1 & M2 & $\overline{\mathrm{M} 3}$ & TOTAL \\
\hline CUSTOMER SAMPLES & 149 & 9821 & 15 & 01 & $0]$ & 119 & 648 & 278 & 1095 \\
\hline & & & & & & & & & \\
\hline \multicolumn{10}{|l|}{ SAMPLEANALIYSES } \\
\hline Volatiles by GC/MS & 99 & 122 & 3 & 0 & 0] & 57 & 147 & 20 & 224 \\
\hline Volatiles by GC & 25 & 32 & 2 & 0. & 0 & 3 & 51 & 5 & 59 \\
\hline Semi-Volatiles & 49 & 103 & 3 & o) & 0 & 29 & 123 & 3 & 155 \\
\hline Herbicides/Pesticides/PCBs & 93 & 209 & 0 & 0 & 0 & 49 & 195 & 58 . & 302 \\
\hline Other Organics & 27 & $8 \mid$ & 0 & 0] & $0]$ & 6 & 28 & 1[ & 35 \\
\hline TOTAL ORGANICS & 293 & 474 & 8 & 01 & $0]$ & 144 & 544 & 88 & 775 \\
\hline \multicolumn{10}{|l|}{ RCRA Appendix IX Metals } \\
\hline RCRA Appendix IX Metals & 0 & 1 & 0 & 0. & 0 & 0 & 1 & 0 & 1 \\
\hline CAM (California) Metals (RCRA) & 27 & 0 & 0 & 0 & 0 & 27 & 0 & o) & 28 \\
\hline RCRA TCLP Metals and EPTOX Me & 10 & 66 & 2 & 0 & 0 & 3 & 71 & 4 & 78 \\
\hline EPA CLP Metals (CERCLA) & 90 & 51 & 9 & 0 & 0. & 37 & 107 & 5 & 149 \\
\hline NPDES Metals (Clean Water Act) & 21 & 1 & 0 & 0 & 0 & 22 & 0 & ㅇ. & 22 \\
\hline Drinking Water Metals (SDWA) & 0 & 0 & 0 & 0 & 0 & 0 & 0 & 0 & 0 \\
\hline Metals by Other Regulatory Suite & 108 & 37 & 0 & 0 & 0 & 26 & 80 & 46 & 149 \\
\hline Metals by Suite, Other & 0 & 45 & 2 & 0 & 0 & 25 & 13 & 1 & 43 \\
\hline Metals by Metal Except Mercury & 37 & 96 & 0 & 0 & 0 & 31 & 49 & 53 & 133 \\
\hline Mercun & 56 & 102 & 0 & Q & 0) & 53 & 102 & 3 & 158 \\
\hline Anions/Cations-IC & 36 & 20 & 0 & 0 & 0 & 47 & 2 & 6 & 56 \\
\hline Inorganics Wet Chemistry & 312 & 122 & 17 & 0 & 0 & 229 & 136 & 87 & 451 \\
\hline Other Inorganics & 35 & 25 & 0 & 01 & o] & 35 & 19 & 6 & 60 \\
\hline TOTALINOAGANICS & 7311 & 566 & 29 & 01 & $0]$ & 535 & 581 & 212 & 1327 \\
\hline \multicolumn{10}{|l|}{-10} \\
\hline Gross Alpha/Beta & 182 & 120 & 4 & 0 & 0] & 94 & 193 & 16. [ & 304 \\
\hline Gamma Scan & 68 & 59 & 1 & 0 & 0 & 33 & 82 & 13 & 127 \\
\hline Transuranic & 0 & 2 & 0 & 0 & 0 & 0 & 2 & 0. & 2 \\
\hline Plutonium Isotopes & 69 & 16 & 1 & 0 & 0 & 21 & 58 & 7 & 86 \\
\hline Uranium isotopes & 85 & 95 & 1 & 0 & 0 & 36 & 134 & 12 & 182 \\
\hline Curium Isotopes & 0 & 3 & 0 & 0 & o & 1 & 1 & 1 & 3 \\
\hline Thorium Isotopes & 5 & 57 & 1 & 0 & 0 & 3 & 54 & 5 & 63 \\
\hline Radium Isotopes & 201 & 1 & 0 & 0 & 0 & 14 & 6 & 1. & 21 \\
\hline Americium-241 & 64 & 8 & 1 & 0 & 0 & 21 & 50 & 2 & 73 \\
\hline Neptunium-237 & 5 & 9 & 0 & 0 & 0. & 1 & 7 & 6 & 14 \\
\hline Polonium-210 & 0 & 1 & 0 & D) & 0 & 0 & 0 & 1. & 1 \\
\hline Tritium & 39 & 39 & 6 & 0 & 0 & 30 & 44 & 1 & 80 \\
\hline Radon-222 & 0 & 0 & 0 & 0 & 0 & 0 & 0 & 0. & 0 \\
\hline Lead-210 & 0 & 0 & 0 & 0 & 0 & 0 & 0 & 0 & 0 \\
\hline Carbon-14 & 0 & 0 & 0 & 0 & 0 & 0 & 0 & 0 & 0 \\
\hline Nickel-63 & 요 & 0 & 0 & 0 & 0 & 0 & 0 & 0 & 0 \\
\hline Selenium-79 & 27 & 1 & 0 & 0 & 0 & 27 & 0 & 0 & 28 \\
\hline Technetium-99 & 0 & 49 & 0 & 0 & 0 & 0 & 48 & 1 & 50 \\
\hline Strontium Isotopes & 0 & 0 & 0 & 0 & 0 & 0 & 0 & 0 & 0 \\
\hline Strontium -89 & 0 & 0 & 0 & 0 & 0 & 0 & 0 & 0 & 0 \\
\hline Strontium-90 & 27 & 18 & 2 & 0 & 0 & 30 & 16 & 1 & 47 \\
\hline lodine-129 & 27 & 3 & 0 & 0 & 0 & 29 & 0 & 0 & 30 \\
\hline Nickel-59 & 0 & 0 & 0 & 0 & 0 & 0 & 0 & 0 & 0 \\
\hline Total Uranium & 21 & 36 & 0 & 0 & 0 & 3 & 6 & 48 & 57 \\
\hline Total Thorium & 0 & 0 & 0 & 0 & 0 & 0 & 0 & 0 & 0 \\
\hline Total Strontium & 32 & 0 & 0 & 0 & 0 & 12 & 19 & 1 & 32 \\
\hline Other RAD & 138 & 184 & 4 & 01 & $0]$ & 43 & 260 & 25 & 327 \\
\hline TOTAL RAD & 810 & 702 & 22 & $0 !$ & 0 & 4001 & 982 & 141 & 1528 \\
\hline & & & & & & & & & \\
\hline Other Wet Chemistry & 70 & 11 & 0 & 0 & 0 & 54 & 17 & 요 & 71 \\
\hline TCLP Extraction & 10 & 82 & 0 & 0 & 0 & 7 & 72 & 13 & 92 \\
\hline Asbestos & 0 & 640 & 0 & of & 0 & 0 & 475 & 165 & 640 \\
\hline Industrial Hygiene, NOS & 2 & D. & 0 & 0 & 0 & 1 & 2 & 0 & 3 \\
\hline Physical Properties & 9 & 24 & 1 & 0 & 0 & 6 & 25 & 3 & 34 \\
\hline Other, Not Otherwise Specified (NO & 10 & 2 & 01 & 01 & 0 & 2 & 01 & 10 & 12 \\
\hline TOTAL OTHER & 102 & 749 & 11 & OI & 0]: & 701 & 5911 & $191]$ & 852 \\
\hline & & & & & $=$ & 1 & & 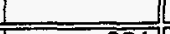 & \\
\hline TOTAL SAMPLE ANALYSES & 1936 & 2492 & 60] & 01 & $0]$ & 1148 & 2698 & $631]$ & 4482 \\
\hline
\end{tabular}


Table Vf: FY 98 Samples and Sample Analyses by Rad Level and Matrix Type (xi) Rocky Flats

\begin{tabular}{|c|c|c|c|c|c|c|c|c|c|}
\hline $\begin{array}{c}\text { Rad-Matrix/ } \\
\text { Sample-Sample Analysis }\end{array}$ & RO & R1 & $\overline{\mathrm{R} 2}$ & $\overline{\mathrm{R} 3}$ & $\overline{\mathrm{R} 4}$ & $\overline{\mathrm{M1}}$ & $\overline{\mathrm{M} 2}$ & M3 & TOTAL \\
\hline CUSTOMER SAMPLES & 2053 & 5349 & 372 & 1180 & $0]$ & 1811 & 2956 & 1323 & 7522 \\
\hline & & & & & & & & & \\
\hline \multicolumn{10}{|l|}{ SAMPLE ANALYSES } \\
\hline Volatiles by GC/MS & 4253 & 750 & 85 & 132 & 0 & 2637 & 2281 & 303 & 5221 \\
\hline Volatiles by GC & 894 & 241 & 18 & 0 & 0 & 345 & 745 & 45 & 1144 \\
\hline Semi-Volatiles & 1828 & 687 & 85 & 112 & 0 & 1021 & 1540 & 141 & 2707 \\
\hline Herbicides/Pesticides/PCBs & 2228 & 851 & 0 & 0 & 0 & 772 & 2151 & 155 & 3079 \\
\hline Other Organics & 204 & 225 & \begin{tabular}{l|l}
0 & \\
\end{tabular} & 0 & ol & 318 & 38 & 7 & 396 \\
\hline TOTAL ORGANICS & 9407 & 2754 & 189 & 244 & 0] & 5092 & 6756 & 652[ & $125 \overline{47}$ \\
\hline & & & & & & & & & \\
\hline RCRA Appendix IX Metais & 7 & 30 & 0 & 0 & 0 & 4 & 33 & o] & 37 \\
\hline CAM (California) Metals (RCRA) & 2 & 1 & 0 & 0 & 0 & 3 & 2 & 0. & 4 \\
\hline RCRA TCLP Metals and EPTOX Me & 382 & 164 & 18 & 0 & 0. & 295 & 209 & 19. & 543 \\
\hline EPA CLP Metals (CERCLA) & 5160 & 1344 & 234 & 276 & 0 & 2470 & 4155 & 390 & 7014 \\
\hline NPDES Metals (Clean Water Act) & 573 & 19 & 0 & 0 & 0 & 592 & o. & o. & 592 \\
\hline Drinking Water Metals (SDWA) & 92 & 0 & 0 & 0 & 0 & 92 & o. & 0 & 92 \\
\hline Metals by Other Regulatory Suite & 4657 & 0 & 0 & 0 & 0 & 2113 & 2304 & 357 & 4715 \\
\hline Metals by Suite, Other & 0 & 1164 & 44 & 0 & 0 & 657 & 362 & 6 & 1117 \\
\hline Metals by Metal Except Mercury & 449 & 267 & 4 & 0 & 0 & 366 & 314 & 0 & 699 \\
\hline Mercury & 940 & 309 & 4 & 0 & 0 & 887 & 253 & 7 & 1200 \\
\hline Anions/Cations-IC & 899 & 439 & 0 & 0 & 0 & 1266 & 35 & 37 & 1338 \\
\hline Inorganics Wet Chemistry & 12618 & 887 & 132 & 4 & 0 & 10809 & 2218 & 619 & 13644 \\
\hline Other Inorganics & 796 & 301 & 0 & 0 & $0]$ & 773 & 279 & 45] & 1097 \\
\hline TOTAL INORGANICS & 26575 & 4926 & 437 & 280 & 0 & 20328 & 10164 & $1481]$ & 32095 \\
\hline & & & & & & & & & \\
\hline Gross Alpha/Beta & 9446 & 1860 & 79 & 0 & 0 & 4740 & 6314 & 198 & 11319 \\
\hline Gamma Scan & 3766 & 579 & 36 & 0 & 0 & 1818 & 2397 & 164 & 4380 \\
\hline Transuranic & o) & 1 & 0 & 0 & ㅇ] & 0 & 1 & ㅇ․ & 1. \\
\hline Plutonium Isotopes & 4688 & 357 & 24 & 0 & 0 & 1980 & 3016 & 73 & 5069 \\
\hline Uranium Isotopes & 4560 & 1032 & 24 & 0 & 0 & 2096 & 3436 & 84 & 5616 \\
\hline Curium Isotopes & 0 & 56 & 0 & 0 & 0 & 24 & 24 & 8 & 56 \\
\hline Thorium Isotopes & 39 & 226 & 24 & 0 & 0 & 64 & 186 & 39 & 289 \\
\hline Radium Isotopes & 1868 & 7 & 0 & 0 & 0 & 1424 & 444 & 7 & 1875 \\
\hline Americium-241 & 4475 & 132 & 24 & 0 & 0 & 1955 & 2641 & 35 & 4631 \\
\hline Neptunium-237 & 39 & 166 & 0 & 0 & 0 & 16 & 144 & 46 & 205 \\
\hline Polonium-210 & 2 & 8 & 0 & 0 & 0 & 1 & 2 & 7 & 9 \\
\hline Tritium & 2724 & 848 & 129 & 0 & 0 & 2006 & 1607 & 21 & 3667 \\
\hline Radon-222 & 1 & 0. & 0 & 0 & 0 & 1 & 0 & o) & 1 \\
\hline Lead-210 & 0 & 0. & 0 & 0 & 0 & 0 & 0 & 0) & (0) \\
\hline Carbon-14 & 0 & 0 & 0 & 0 & 0 & 0 & O) & D & 0 \\
\hline Nickel-63 & 0 & 0 & 0 & 0 & 0 & 0 & 0 & 0 & o \\
\hline Selenium-79 & 2 & 0 & 0 & 0 & 0 & 2 & 0 & 0 & 2 \\
\hline Technetium-99 & 20 & 35 & 7 & 0 & 0 & 27 & 28 & 7 & 62 \\
\hline Strontium Isotopes & 0 & 1 & 0 & 이 & 0 & 0 & 0 & 0] & 1 \\
\hline Strontium-89 & 0 & 0 & O & 0 & 0 & 0 & 0 & o] & o. \\
\hline Strontium-90 & 21 & 357 & 22 & (0) & 0 & 55 & 346 & D & 400 \\
\hline lodine-129 & 58 & 15 & 0 & 0 & (0) & 72 & 0 & o. & 72 \\
\hline Nickel-59 & of & of & 0 & 0 & 0 & 0 & 0 & ㅁ․ & 0 \\
\hline Total Uranium & 211 & 11 & 0 & 0 & 0 & 66 & 0 & 156 & 222 \\
\hline Total Thorium & of & 1 & 0 & 0 & 0 & 1 & 0 & - & 1 \\
\hline Total Strontium & 2471 & 0 & 0 & 0 & 0 & 1283 & 1153 & 34 & 2471 \\
\hline Other RAD & 6050 & 671 & 89 & 924 & 0 & 2095 & 4541 & 1098 & 7734 \\
\hline TOTALRAD & 40437 & 63611 & \begin{tabular}{l|l|l|}
458 \\
\end{tabular} & 924 & 0 & 19726 & 26280 & 1979 & 48083 \\
\hline Other Wet Chemistry & 6331 & 7 & 01 & 0 & 01 & 5344 & 995 & 0 & 6339 \\
\hline TCLP Extraction & 384 & 214 & 6 & 732 & 0 & 319 & 212 & 765 & 1316 \\
\hline Asbestos & 25 & 149 & 0 & 0 & 0 & 1 & 174 & a & 175 \\
\hline Industrial Hygiene, NOS & 61 & 0) & 0 & 0 & 0 & 17 & 44 & o & 61 \\
\hline Physical Properties & 994 & 110 & 14 & 460 & o & 236 & 857 & 485 & 1579 \\
\hline Other, Not Otherwise Specified (Nd & 78 & 49 & 0 & 0 & o. & 43 & 0 & 79 & 125 \\
\hline TOTAL OTHEA & 7875 & 5301 & 201 & 1192 & 0 & 5960 & 2283 & 1329 & 9594 \\
\hline TOTAL SAMPLE ANALYSES & 84294 & 14570 & 1103 & 2640 & (0) & 51107 & 45482 & 5440 & 102319 \\
\hline
\end{tabular}


Table Vf: FY 98 Samples and Sample Analyses by Rad Level and Matrix Type (xii) Savannah River

\begin{tabular}{|c|c|c|c|c|c|c|c|c|c|}
\hline $\begin{array}{c}\text { Rad-Matrix/ } \\
\text { Sample-Sample Analysis }\end{array}$ & $\overline{\mathrm{RO}}$ & R1 & $\overline{\mathrm{R} 2}$ & $\overline{\mathrm{R3}}$ & R4 & $\overline{\mathrm{M} 1}$ & M2 & $\overline{\mathrm{M3}}$ & TOTAL \\
\hline CUSTOMER SAMPLES & 10272 & 19757 & 328 & 81 & 211 & 21421 & 1156 & 3248 & 28237 \\
\hline & & & & & & & & & \\
\hline \multicolumn{10}{|l|}{ SAMPLE ANALYSES } \\
\hline Volatiles by GC/MS & 179 & 9 & 0 & 0 & 0] & 132 & 0 & 56 & 187 \\
\hline Volatiles by GC & 103 & 252 & 29 & 11 & 0 & 145 & 233 & 18 & 396 \\
\hline Semi-Volatiles & 1288 & 102 & 11 & 3 & 0 & 1016 & 88 & 301 & 1405 \\
\hline Herbicides/Pesticides/PCBs & 642 & 118 & 11 & 6 & 0 & 603 & 100 & 75 & 777 \\
\hline Other Organics & 2008 & 4664 & 0 & 0 & 0 & 1743 & 1 & 284 & 4350 \\
\hline \multirow[t]{2}{*}{ TOTAL ORGANICS } & 4220 & 5146 & 521 & 201 & 0] & 3639 & 422 & 733 & 7115 \\
\hline & & & & & & & 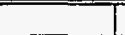 & & \\
\hline RCRA Appendix IX Metals & 11 & 9 & 0 & 0 & 0 & 19 & 0 & 1 & 20 \\
\hline CAM (California) Metals (RCRA) & 80 & 0 & 0 & 0 & 0 & 80 & 0 & 0 & 80 \\
\hline RCRA TCLP Metals and EPTOX Me & 76 & 105 & 10 & 2 & 0 & 115 & 63 & 15 & 193 \\
\hline EPA CLP Metals (CERCLA) & 67 & 56 & 23 & 11 & o & 67 & 84 & 7 & 158 \\
\hline NPDES Metals (Clean Water Act) & 86 & 823 & 0 & 0 & 0 & 909 & 0 & 0 & 909 \\
\hline Drinking Water Metals (SDWA) & 0 & 0 & 0 & 0 & o & 0 & 0 & 0 & 0 \\
\hline Metals by Other Regulatory Suite & 426 & 5 & 0 & 0 & D. & 303 & 1 & 128 & 431 \\
\hline Metals by Suite, Other & 2690 & 4647 & 0 & 0 & 0 & 1426 & 0 & 1267 & 5015 \\
\hline Metals by Metal Except Mercury & 1865 & 56 & 0 & of & 105 & 1091 & 50 & 810 & 1988 \\
\hline Mercury & 2064 & 4815 & 2 & 1 & 105 & 1440 & 56 & 772 & 4628 \\
\hline Anions/Cations-IC & 3854 & 88 & 0 & 0 & 211 & 3210 & 64 & 698 & 4062 \\
\hline Inorganics Wet Chemistry & 5211 & 177 & 102 & 23 & 0 & 4060 & 324 & 1129 & 5513 \\
\hline Other Inorganics & 268 & 108 & 0 & 0 & 0 & 280 & 50 & 45 & 376 \\
\hline \multirow[t]{2}{*}{ TOTAL INORGANICS } & 16698 & 10889 & 137 & 38 & 422 & 12998 & 693 & 4872 & 23373 \\
\hline & & & & & & & & & \\
\hline \multirow{2}{*}{$\begin{array}{l}\text { Gross Alpha/Beta } \\
\text { Gamma Scan } \\
\end{array}$} & 2247 & 15551 & 58 & 72 & 211 & 12164 & 475 & 142 & 15459 \\
\hline & 88 & 4921 & 52 & 29 & 110 & 4794 & 225 & 101 & 5160 \\
\hline \multirow{2}{*}{\begin{tabular}{|l} 
Transuranic \\
Plutonium Isotopes \\
\end{tabular}} & 0 & 0 & 0 & 0 & 110 & 0 & 0 & 30 & 70 \\
\hline & 694 & 68 & 21 & 6 & 211 & 677 & 86 & 57 & 909 \\
\hline \multirow{2}{*}{\begin{tabular}{|l|} 
Uranium Isotopes \\
Curium Isotopes
\end{tabular}} & 696 & 91 & 9 & 5 & 211 & 678 & 95 & 58 & 921 \\
\hline & 0 & 17 & 3 & 2 & 10 & 2 & 10 & 10 & 27 \\
\hline \multirow{2}{*}{\begin{tabular}{|l|} 
Thorium Isotopes \\
Radium Isotopes
\end{tabular}} & 16 & 6 & 3 & 26 & 0 & 0 & 10 & 40 & 50 \\
\hline & 1 & 3 & 0 & 0 & of & 1 & 0 & 3 & 4 \\
\hline Americium-241 & 678 & 38 & 5 & 3 & 20 & 677 & 39 & 7 & 733 \\
\hline \multirow{2}{*}{\begin{tabular}{|l|} 
Neptunium-237 \\
Polonium -210 \\
\end{tabular}} & 16 & 7 & 8 & 0 & 0 & 0 & 5 & 27 & 32 \\
\hline & 0 & 3 & 0 & 0 & 0 & 0 & 0 & 3 & 3 \\
\hline \multirow{2}{*}{\begin{tabular}{|l|} 
Tritium \\
Radon-222
\end{tabular}} & 591 & 63 & 28 & 6 & o. & 592 & 60 & 7 & 673 \\
\hline & 0 & 0 & 0 & 0 & 요 & 0 & 0 & 0 & 0 \\
\hline Lead-210 & 0 & 12 & 0 & 0 & 요 & 0 & 0 & 12 & 12 \\
\hline \multirow{2}{*}{\begin{tabular}{|l|} 
Carbon-14 \\
Nickel-63 \\
\end{tabular}} & 0 & 0 & 0 & 0 & 이 & 0 & 0 & 0 & 0 \\
\hline & o. & D. & 0 & 0 & - & 0 & 0 & 0 & 0 \\
\hline Selenium-79 & 80 & 0 & 0 & 0 & 의 & 80 & 0 & 0 & 80 \\
\hline Technetium -99 & 0 & 11 & 0 & 0 & 요 & 0 & 0 & 11 & 11 \\
\hline Strontium Isotopes & 0 & 0 & 0 & 0 & 0 & 0 & 0 & 0 & 0 \\
\hline Strontium-89 & 0 & 0 & 0 & 0 & 0 & 0 & 0 & 0 & 0 \\
\hline Strontium -90 & 80 & 41 & 23 & 6 & 0 & 82 & 69 & 0 & 150 \\
\hline lodine-129 & 80 & 6 & 0 & 0 & 0 & 87 & 0 & 0 & 87 \\
\hline Nickel-59 & 0 & 0 & 0 & 0 & 요 & 0 & 0 & 0 & 0 \\
\hline Total Uranium & 65 & 3 & 0 & 0 & 211 & 3 & 0 & 95. & 188 \\
\hline Total Thorium & 0 & 0 & 0 & 0 & 0 & 0 & 0 & 0 & 0 \\
\hline Total Strontium & 15 & 12 & 0 & 0 & 0] & 19 & 0 & 8 & 27 \\
\hline Other RAD & 230 & 219 & 18 & 6 & $10]$ & 230 & 94 & 145 & 476 \\
\hline TOTALRAD & 5578 & 21071 & 228 & 158 & 1104 & 20086 & 1169 & 754 & 25074 \\
\hline & & & & & & & + & 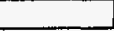 & \\
\hline Other Wet Chemistry & 1220 & 10 & 0 & 0 & 30 & 1230 & 0 & 30 & 1260 \\
\hline TCLP Extraction & 8 & 119 & 41 & 5 & o. & 68 & 77 & 27 & 172 \\
\hline Asbestos & D & 01 & ol & 0 & 0 & 0 & 3 & 0 & 1 \\
\hline Industrial Hygiene, NOS & 552 & 0 & 0 & 0 & 0 & 552 & 0 & 0 & 552 \\
\hline Physical Properties & 12128 & 26 & 6 & 2 & 0 & 7917 & 20 & 4226 & 12163 \\
\hline Other, Not Otherwise Specified (NO & 33 & 9 & 01 & 0 & 0 & 8 & 0 & 33 & 42 \\
\hline TOTAL OTHER & 13941 & 163 & 47 & 7 & 30 & 9775 & 99 & 4317 & 14189 \\
\hline & & & & & & & & & \\
\hline TOTAL SAMPLE ANALYSES & 40436 & 37269 & 463 & 223 & 1556 & 46498 & 2383 & 10676 & 69752 \\
\hline
\end{tabular}


Table Vg: FY 99 Samples and Sample Analyses by Rad Level and Matrix Type (i) Complex-wide

\begin{tabular}{|c|c|c|c|c|c|c|c|c|c|}
\hline $\begin{array}{c}\text { Rad-Matrix/ } \\
\text { Sample-Sample Analysis }\end{array}$ & $\overline{\mathrm{RO}}$ & $\overline{\mathrm{R} 1}$ & $\overline{\mathrm{R} 2}$ & $\overline{\mathrm{R3}}$ & $\overline{\mathrm{R} 4}$ & $\overline{\mathrm{M1}}$ & $\overline{\mathrm{M} 2}$ & $\overline{\mathrm{M3}}$ & TOTAL \\
\hline CUSTOMER SAMPLES & 47121 & 183127 & 3719 & 4593 & 617 & 94673 & 69843 & 42408 & 223051 \\
\hline \multirow{2}{*}{\multicolumn{10}{|c|}{ SAMPLEANALYSES }} \\
\hline & & & & & & & & & \\
\hline Volatiles by GC/MS & 18651 & 13572 & 626 & 469 & 0 & 12625 & 18019 & 2681 & 333322 \\
\hline Volatiles by GC & 5939 & 2452 & 237 & 60 & 0 & 3326 & 4656 & 484 & 8577 \\
\hline Semi-Volatiles & 11860 & 11542 & 640 & 413 & 0 & 7619 & 14651 & 2161 & 24443 \\
\hline Herbicides/Pesticides/PCBs & 12039 & 23447 & 60 & 30 & 0 & 7628 & 22685 & 5281 & 35585 \\
\hline Other Organics & 8962 & 20908 & 28 & 0 & 0] & 7030 & 2075 & 912 & 19957 \\
\hline \multirow[t]{2}{*}{ TOTAL ORGANICS } & 57450 & 71920 & 1592 & 972 & 0 & 38228 & 62087 & 11519 & 121884 \\
\hline & & & & & & & & & \\
\hline RCRA Appendix IX Metals & 82 & 168 & 0 & 0 & 0 & 97 & 147 & 65 & 279 \\
\hline CAM (California) Metals (RCRA) & 593 & 139 & 0 & 0 & 0 & 732 & 174 & 35 & 837 \\
\hline RCRA TCLP Metals and EPTOX Me & 4188 & 7090 & 587 & 12 & 0 & 2957 & 7269 & 1195 & 11649 \\
\hline EPA CLP Metals (CERCLA) & 21197 & 6767 & 1141 & 1040 & 0 & 10740 & 17140 & 2264 & 30144 \\
\hline NPDES Metals (Clean Water Act) & 2844 & 3554 & 0 & 0 & 0 & 6398 & 0 & 0 & 6398 \\
\hline Drinking Water Metals (SDWA) & 718 & 0 & 0 & 0 & 0 & 718 & 0 & 0 & 718 \\
\hline Metals by Other Regulatory Suite & 20746 & 4516 & 206 & 0 & 0 & 11054 & 12444 & 2759 & 25863 \\
\hline Metals by Suite, Other & 7639 & 25018 & 187 & 0 & 0 & 6952 & 1539 & 3649 & 22492 \\
\hline Metals by Metal Except Mercury & 9635 & 11677 & 235 & 0 & 299 & 7416 & 5049 & 7715 & 21013 \\
\hline Mercury & 12513 & 32110 & 68 & 6 & 299 & 12417 & 10626 & 2701 & 35370 \\
\hline Anions/Cations-IC & 15066 & 3587 & 96 & 0 & 599 & 14841 & 514 & 2529 & 18616 \\
\hline Inorganics Wet Chemistry & 67083 & 11688 & 1064 & 135 & 0 & 57587 & 16156 & 7684 & 80698 \\
\hline Other Inorganics & 6088 & 2528 & 0 & 0 & o] & 5057 & 2943 & 616 & 8615 \\
\hline \multirow[t]{2}{*}{ TOTAL INORGANICS } & 168390 & 108841 & 3584 & 1193 & 1197 & 136967 & 74001 & 31212 & 262693 \\
\hline & & & & & & & & & \\
\hline Gross Alpha/Beta & 49600 & 80440 & 659 & 378 & 599 & 73997 & 32712 & 1869 & 120127 \\
\hline Gamma Scan & 17185 & 27174 & 450 & 151 & 314 & 27855 & 15716 & 1474 & 45160 \\
\hline Transuranic & 0 & 1031 & 826 & 0 & 314 & 1101 & 756 & 86 & 2057 \\
\hline Plutonium isotopes & 22332 & 2335 & 233 & 30 & 599 & 11120 & 13051 & 844 & 25272 \\
\hline Uranium Isotopes & 22074 & 12193 & 812 & 27 & 599 & 13416 & 20043 & 1734 & 35449 \\
\hline Curium Isotopes & 0 & 314 & 18 & 9 & 29 & 112 & 153 & 76 & 355 \\
\hline Thorium Isotopes & 231 & 6833 & 145 & 111 & 0 & 1058 & 5624 & 639 & 7321 \\
\hline Radium Isotopes & 6962 & 43 & 0 & 0 & 0 & 5303 & 1660 & 42 & 7005 \\
\hline Americium-241 & 21144 & 1170 & 130 & 15 & 57 & 10998 & 11058 & 403 & 22488 \\
\hline Neptunium-237 & 231 & 1653 & 589 & 0 & 0 & 659 & 1199 & 615 & 2473 \\
\hline Polonium-210 & 159 & 48 & 28 & 0 & 0 & 6 & 159 & 70 & 235 \\
\hline Tritium & 13264 & 4090 & 674 & 30 & 0 & 10489 & 7119 & 133 & 17900 \\
\hline Radon-222 & 4 & 0 & 0 & 0 & 0 & 4 & 0 & 0 & 4 \\
\hline Lead-210 & 0 & 51 & 0 & 0 & 0 & 0 & 0 & 51 & 51 \\
\hline Carbon-14 & 0 & 0 & 0 & 0 & 0 & 0 & 0 & o & 0 \\
\hline Nickel-63 & 0 & 0 & 0 & 0 & 0 & 0 & 0 & 의 & 0 \\
\hline Selenium-79 & 593 & 55 & 0 & 0 & 0 & 624 & 14 & 10 & 648 \\
\hline Technetium-99 & 153 & 5953 & 883 & 0 & 0 & 1284 & 5343 & 362 & 6989 \\
\hline Strontium Isotopes & 0 & 5 & 0 & 0 & 0 & 0 & 2 & 3 & 5 \\
\hline Strontium - 89 & 01 & 0 & 0 & 0 & 0 & 0 & 0 & 으. & 0 \\
\hline Strontium -90 & 746 & 2034 & 209 & 30 & 0 & 1037 & 1954 & 35 & 3022 \\
\hline lodine-129 & 1028 & 168 & 0 & 0 & 0 & 1151 & 28 & 17 & 1196 \\
\hline Nickel-59 & 0 & 0 & 0 & 0 & 0 & 0 & 0 & ㅇ. & 0 \\
\hline Total Uranium & 1234 & 4653 & 807 & 0 & 599 & 698 & 1804 & 4278 & 7036 \\
\hline Total Thorium & 0 & 237 & 0 & 0 & 0 & 63 & 174 & 0 & 237 \\
\hline Total Strontium & 9460 & 51 & of & 0 & 0 & 4920 & 4415 & 177 & 9511 \\
\hline Other RAD & 25473 & 20079 & 506 & 3310 & 29 & 10867 & 33422 & 5028 & 49357 \\
\hline TOTAL RAD & 191874 & 170611 & 6970 & 4092 & 3135 & 176763 & 156406 & 17945 & 363898 \\
\hline & & & & & 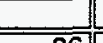 & & 0701 & & 30683 \\
\hline $\begin{array}{l}\text { Other Wet Chemi } \\
\text { TCLP Extraction }\end{array}$ & \begin{tabular}{r|}
29525 \\
3991
\end{tabular} & \begin{tabular}{r|}
73 \\
9004
\end{tabular} & \begin{tabular}{r|}
0 \\
456
\end{tabular} & \begin{tabular}{r|}
0 \\
2623
\end{tabular} & \begin{tabular}{r|}
86 \\
0
\end{tabular} & $\begin{array}{r}25803 \\
3164\end{array}$ & \begin{tabular}{l|}
3794 \\
7898
\end{tabular} & $\begin{array}{r}86 \\
4705\end{array}$ & $\frac{29683}{15921}$ \\
\hline Asbestos & 143 & 63590 & $\frac{0}{0}$ & $\frac{2020}{0}$ & 0 & $\frac{-304}{3}$ & 47402 & 16339 & 63739 \\
\hline Industrial Hygiene, NOS & 2725 & 31 & 0 & 0 & 0 & 2547 & 205 & 3 & 2756 \\
\hline Physical Properties & 38589 & 3396 & 94 & 1645 & 0 & 24638 & 5079 & 14007 & 43724 \\
\hline Other, Not Otherwise Specified (NO & 481 & 354 & 28 & 0 & 0 & 317 & 11 & 501 & 845 \\
\hline TOTAL OTHER & 754541 & 76447 & 577 & 4268 & $86]$ & 56472 & 64390 & 35641 & 156667 \\
\hline TOTAL SAMPLE ANALYSES & 493168 & 427819 & 12723 & 10525 & 4418 & 408430 & 356884 & 96318 & 905142 \\
\hline
\end{tabular}


Table Vg: FY 99 Samples and Sample Analyses by Rad Level and Matrix Type (ii) EM-30

\begin{tabular}{|c|c|c|c|c|c|c|c|c|c|}
\hline $\begin{array}{c}\text { Rad-Matrix } \\
\text { Sample-Sample Analysis }\end{array}$ & $\overline{\mathrm{RO}}$ & $\overline{\mathrm{RI}}$ & R2 & $\overline{\mathrm{R} 3}$ & R4 & $\overline{M 1}$ & $\overline{M 2}$ & $\overline{\mathrm{M3}}$ & TOTAL \\
\hline CUSTOMER SAMPLES & 29074 & 79282 & 1360 & 4169 & 561 & 76891 & 5065 & 13901 & 105151 \\
\hline \multicolumn{7}{|l|}{ SAMPLE ANALYSES } & & & \\
\hline Volatiles by GC/MS & 1193 & 955 & 230 & 439 & 需 & 575 & & 1009 & \\
\hline Volatiles by GC & 1073 & 1684 & 145 & 30 & 0 & 1075 & 1630 & 1500 & $\frac{2817}{2000}$ \\
\hline Semi-Volatiles & 3724 & 1036 & 235 & 380 & of & 2965 & 756 & $\frac{105}{1653}$ & 5374 \\
\hline Herbicides/Pesticides/PCBs & 3051 & 1811 & 30 & 15 & 0 & 3496 & 308 & 1104 & 4908 \\
\hline Other Organics & 5596 & 18276 & 26 & 0 & 0 & 4917 & 7 & 810 & 14816 \\
\hline TOTAL ORGANICS & 14637 & 23762 & 666 & 865 & 0 & 13028 & 3050 & 5638 & 30823 \\
\hline \multicolumn{10}{|l|}{20} \\
\hline RCRA Appendix IX Metals & 42 & 38 & 0 & 0 & 0 & 74 & 0 & 61 & 107 \\
\hline CAM (California) Metals (RCRA) & 246 & 1 & 0 & 0 & 0 & 247 & 1 & 0 & 247 \\
\hline RCRA TCLP Metals and EPTOX Me & 857 & 950 & 516 & 6 & 0 & 853 & 482 & 995 & 2329 \\
\hline EPA CLP Metals (CERCLA) & 382 & 951 & 61 & 948 & 0 & 376 & 243 & 1725 & 2343 \\
\hline NPDES Metals (Clean Water Act) & 335 & 3247 & 0 & 0 & 0 & 3582 & 0 & 0 & 3582 \\
\hline Drinking Water Metals (SDWA) & 128 & 0 & 0 & 0 & 0 & 128 & 0 & of & 128 \\
\hline Metals by Other Regulatory Suite & 2911 & 898 & 193 & 0 & 0 & 1985 & 190 & 1828 & 4003 \\
\hline Metals by Suite, Other & 7155 & 18205 & 0 & 0 & 0 & 3797 & 1 & 3399 & 16278 \\
\hline Metals by Metal Except Mercury & 5759 & 2384 & 198 & 0 & 280 & 4495 & 448 & 2349 & 7957 \\
\hline Mercury & 6482 & 19982 & 37 & 3 & 280 & 6445 & 618 & 2377 & 18112 \\
\hline Anions/Cations-IC & 10659 & 1380 & 90 & 0 & 561 & 8844 & 246 & 2227 & 12003 \\
\hline Inorganics Wet Chemistry & 18805 & 1389 & 858 & 74 & 0 & 13379 & 2162 & 6536 & 21601 \\
\hline Other Inorganics & 1200 & 888 & 0 & 0 & 0 & 1294 & 263 & 530 & 2088 \\
\hline TOTAL INORGANICS & 54960 & 50311 & 1955 & 1032 & 1121 & 45497 & 4654 & 22028 & 90779 \\
\hline \multicolumn{10}{|l|}{$+2+2$} \\
\hline Gross Alpha/Beta & 9497 & 62641 & 242 & 190 & 561 & 49615 & 2148 & 1026 & 62959 \\
\hline Gamma Scan & 1250 & 19578 & 163 & 76 & 294 & 18796 & 1481 & 869 & 21254 \\
\hline Transuranic & 0 & 753 & 773 & 0 & 294 & 1031 & 496 & 80 & 1714 \\
\hline Flutonium Isotopes & 3077 & 451 & 95 & 15 & 561 & 2777 & 345 & 596 & 3959 \\
\hline Uranium Isotopes & 2972 & 1635 & 662 & 14 & 561 & 4016 & 486 & 860 & 5603 \\
\hline Curium Isotopes & 0 & 116 & 9 & 5 & 27 & 24 & 37 & 68 & 143 \\
\hline Thorium Isotopes & 207 & 890 & 33 & 98 & 0 & 632 & 27 & 569 & 1228 \\
\hline Radium Isotopes & 33 & 38 & 0 & 0 & 0 & 33 & 0 & 38 & 71 \\
\hline Americium-241 & 2767 & 320 & 14 & 8 & 53 & 2746 & 145 & 217 & 3135 \\
\hline Neptunium-237 & 207 & 614 & 551 & 0 & 0 & 537 & 291 & 543 & 1372 \\
\hline Polonium-210 & 1 & 39 & 26 & 0 & 0 & 1 & 1 & 64 & 66 \\
\hline Tritium & 2481 & 469 & 148 & 15 & 0. & 2420 & 311 & 26 & 2935 \\
\hline Radon-222 & 4 & 0 & 0 & 0 & 0 & 4 & 0 & 0 ] & 4 \\
\hline Lead-210 & 0 & 47 & 0 & 0 & 0 & 0 & 0 & 47) & 47 \\
\hline Carbon-14 & 0 & 0 & 0 & 0 & 0 & 0 & 0 & 0. & 0 \\
\hline Nickel-63 & 0 & 0 & 0 & 0 & 0 & 0 & 0 & 0 & - \\
\hline Selenium-79 & 246 & 0 & 0 & 0 & 0 & 246 & 0 & 0 & 246 \\
\hline Technetium-99 & 27 & 1063 & 799 & 0 & 0 & 1059 & 494 & 336 & 1889 \\
\hline Strontium Isotopes & 0 & 4 & 0 & 0 & 0 & 0 & 2 & 2 & 4 \\
\hline Strontium - 89 & 0 & 0 & 0 & 0 & 0 & 0 & 0 & 0 & 0 \\
\hline Strontium -90 & 273 & 134 & 125 & 15 & 0 & 278 & 269 & 0 & 547 \\
\hline lodine-129 & 323 & 79 & 0 & 0 & (0) & 402 & 0 & 0 & 402 \\
\hline Nickel-59 & 0 & 0 & 0 & 0 & 0 & 0 & 0 & 0 & $\underline{0}$ \\
\hline Total Uranium & 848 & 616 & 756 & 0 & 561 & 325 & 860 & 1116 & 2541 \\
\hline Total Thorium & 0 & 0 & 0 & 0 & 의 & 0 & 0 & 0 & 0 \\
\hline Total Strontium & 90 & 47 & 0 & 0 & 의 & 93 & 13 & 31 & 137 \\
\hline Other RAD & 1688 & 1786 & 140 & 3088 & 27 & 1770 & 425 & 4457 & 6690 \\
\hline TOTAL RAD & 25989 & 91322 & 4537 & 3524 & 2937 & 86805 & 7833 & 10946 & 116946 \\
\hline & & & & & & & & & \\
\hline Other Wet Chemistry & 4955 & 65 & 0 & 0 & 80 & 5020 & 0 & 80 & 5101 \\
\hline TCLP Extraction & 572 & 1548 & 380 & 2446 & 0 & 666 & 580 & 3646 & 4919 \\
\hline Asbestos & 1 & 2 & 0 & 0 & 0 & 0 & 13 & 0 & 8 \\
\hline Industrial Hygiene, NOS & 2163 & 0 & 0 & 0 & 0 & 2160 & 3 & 0 & 2163 \\
\hline Physical Properties & 32478 & 1088 & 17 & 1536 & o. & 21946 & 60 & 13113 & 35119 \\
\hline Other, Not Otherwise Specified (NO & 419 & 46 & 26 & 0 & D. & 35 & 0 & 449 & 487 \\
\hline TOTALOTHER & 40587 & 2749 & 423 & 3982 & 80 & 29828 & 655 & 17288 & 47796 \\
\hline TOTAL SAMPLE ANALYSES & 136174 & 168144 & 7580 & 9402 & 4138 & 175158 & 16192 & 55900 & 286344 \\
\hline
\end{tabular}


Table Vg: FY 99 Samples and Sample Analyses by Rad Level and Matrix Type (iii) EM-40

\begin{tabular}{|c|c|c|c|c|c|c|c|c|c|}
\hline $\begin{array}{c}\text { Rad-Matrix/ } \\
\text { Sample-Sample Analysis }\end{array}$ & $\overline{\text { RO }}$ & $\overline{\mathrm{R1}}$ & $\overline{\mathrm{R2}}$ & $\overline{\mathrm{R} 3}$ & $\overline{\overline{R 4}}$ & $\overline{\mathrm{M} 1}$ & M2 & $\overline{\mathrm{M3}}$ & TOTAL \\
\hline CUSTOMER SAMPLES & 13338 & 95899 & 2216 & 138 & 18 & 9919 & 62614 & 27073 & 105608 \\
\hline & & & & & & & & & \\
\hline \multicolumn{10}{|l|}{ SAMPLE ANALYSES } \\
\hline Volatiles by GC/MS & 12030 & 12331 & 374 & 0 & 0 & 8028 & 16076 & 638 & 24739 \\
\hline Volatiles by GC & 2587 & 586 & 61 & 27 & 0 & 328 & 2671 & 297 & 3279 \\
\hline Semi-Volatiles & 5987 & 10249 & 383 & 7 & 0 & 3520 & 12695 & 389 & 16615 \\
\hline Herbicides/Pesticides/PCBs & 6673 & 21128 & 27 & 14 & 0 & 3120 & 20713 & 4026 & 27850 \\
\hline Other Organics & 1870 & 1186 & 0 & 0 & 0 & 735 & 1980 & 47[ & 2909 \\
\hline TOTAL ORGANICS & 29147 & 45480 & 845 & 48 & - & 15730 & 54136 & 5397 & 75392 \\
\hline RCRA Appendix IX Metals & 19 & 125 & 0 & 0 & o & 7 & 137 & oll & 144 \\
\hline CAM (California) Metals (RCRA) & 325 & 134 & 0 & 0 & 0 & 459 & 167 & 33 & 559 \\
\hline RCRA TCLP Metals and EPTOX MeI & 1260 & 5913 & 15 & 5 & (0) & 402 & 6434 & 131 & 7079 \\
\hline EPA CLP Metals (CERCLA) & 13026 & 5649 & 1057 & 27 & (0) & 6001 & 13351 & 408 & 19760 \\
\hline NPDES Metals (Clean Water Act) & 2438 & 54. & 0 & 0 & 0. & 2492 & 0 & 0 & 2492 \\
\hline Drinking Water Metals (SDWA) & 62 & $\mathbf{0}$ & o & 0 & 0 & 62 & 0 & o. & 62 \\
\hline Metals by Other Regulatory Suite & 10550 & 3494 & 0 & 0 & 0 & 4649 & 9385 & 786 & 14432 \\
\hline Metals by Suite, Other & 0 & 5298 & 184 & 0 & o. & 2846 & 1511 & 20 & 4929 \\
\hline Metals by Metal Except Mercury & 1366 & 8916 & 2 & 0 & 0 & 841 & 4303 & 5115 & 10271 \\
\hline Mercury & 3548 & 10335 & 8 & 3 & 0 & 3807 & 9605 & 160 & 13733 \\
\hline Anions/Cations-IC & 3592 & 2074 & 0 & 0 & 0 & 5272 & 245 & 148 & 5666 \\
\hline Inorganics Wet Chemistry & 20539 & 10021 & 145 & 55 & 0 & 18846 & 11650 & 692 & 30974 \\
\hline Other Inorganics & 4583 & 1549 & 0 & 0 & 0 & 3487 & 2598 & 47 & 6132 \\
\hline TOTAL INORGANICS & 61307 & 53562 & 1410 & 901 & 0 & 49171 & 59386 & 7540 & 116234 \\
\hline \multicolumn{10}{|l|}{ 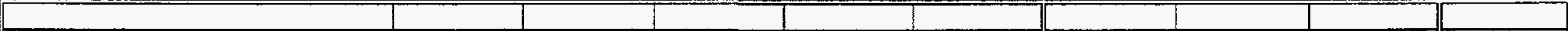 } \\
\hline $\begin{array}{l}\text { Gross Apha/Beta } \\
\text { Gamma Scan }\end{array}$ & $\frac{25402}{8716}$ & $\frac{12726}{5063}$ & $\frac{394}{271}$ & 171 & 에 & 13670 & 23110 & $\frac{742}{527}$ & $\frac{38108}{15018}$ \\
\hline Transuranic & 0 & 217 & 0 & 0 & $\frac{u}{0}$ & $\frac{4819}{0}$ & $\frac{908}{217}$ & 0 & 217 \\
\hline Plutonium Isotopes & 10538 & 1814 & 129 & 14 & 0 & 3918 & 8382 & 196 & 12495 \\
\hline Uranium Isotopes & 12101 & 10253 & 104 & 12 & 0 & 5620 & 16060 & 790 & 22470 \\
\hline Curium Isotopes & 0 & 186 & 8 & 4 & 0 & 84 & 111 & 3 & 198 \\
\hline Thorium Isotopes & 10 & 5780 & 108 & 6 & 0 & 377 & 5496 & 30 & 5903 \\
\hline Radium Isotopes & 3334 & 2 & 0 & 0 & - & 2410 & 924 & 2 & 3336 \\
\hline Americium-241 & 10345 & 814 & 113 & 7 & 0 & 3955 & 7163 & 160 & 11278 \\
\hline Neptunium-237 & 10 & 980 & 1 & 0 & o & 84 & 872 & 34 & 991 \\
\hline Polonium-210 & 153 & 2 & 0 & 0 & 0 & 0 & 153 & 2 & 155 \\
\hline Tritium & 5754 & 3526 & 506 & 14 & o. & 4429 & 5329 & 103 & 9830 \\
\hline Radon-222 & 0 & 0 & 0 & 0 & 0 & 0 & of & 0 & 요 \\
\hline Lead-210 & 0 & 1 & 0 & 0 & 0 & 0 & 0 & 1 & 1 \\
\hline Carbon-14 & 0 & 0 & 0 & 0 & 0 & 0 & 0 & 0 & (0) \\
\hline Nickel-63 & 0 & 0 & 0 & 0 & 0 & 0 & 0 & 0 & 요 \\
\hline Selenium-79 & 325 & 54 & 0 & 0 & 0 & 356 & 14 & 10 & 379 \\
\hline Technetium-99 & 13 & 4733 & 30 & 0 & ㅇ. & 43 & 4730 & 2 & 4775 \\
\hline Strontium Isotopes & 0 & 0 & 0 & 0 & 0. & 0 & 0 & 0 & 익 \\
\hline Strontium-89 & 0 & 0 & 0 & 0 & 0 & o. & 0 & 0 - & 의 \\
\hline Strontium -90 & 338 & 1858 & 75 & 14 & o. & 619 & 1638 & 34 & 2287 \\
\hline lodine-129 & 363 & 82 & 0 & 0 & 0 & 400 & 27 & 17 . & 445 \\
\hline Nickel-59 & 0 & 0 & 0 & 0 & o & 0 & 0 & 0 & 의 \\
\hline Total Uranium & 214 & 3920 & 0 & 0 & 0 & 235 & 870 & 3029 & 4134 \\
\hline Total Thorium & 0 & 231 & 0 & 0 & 0 & 60 & 171 & 0 & 231 \\
\hline Total Strontium & 4957 & 1 & 0 & 0 & $\overline{0}$ & 2201 & 2624 & 133 & 4958 \\
\hline Other RAD & 13787 & 17799 & 330 & 14 & a. & 3547 & 28122 & 263 & 31930 \\
\hline TOTAL RAD & 96359 & 70941 & 2067 & 323 & 0 & 46829 & 115684 & 6077 & 169140 \\
\hline & & & & & & & & & \\
\hline Other Wet Chemistry & 11866 & 2 & $\overline{0}$ & 0 & 0 & 9524 & 2344 & 0] & 11868 \\
\hline TCLP Extraction & 1348 & 7167 & 28 & 11 & (0) & 801 & 6929 & 798 & 8541 \\
\hline Asbestos & 74 & 62468 & 0 & 0 & D. & 1 & 46491 & 16051 & 62542 \\
\hline Industrial Hygiene, NOS & 372 & 31 & 0 & o. & 0. & 210 & 189 & 3 & 403 \\
\hline Physical Properties & 1684 & 2193 & 74 & 5 & 0 & 706 & 3244 & 7 & 3957 \\
\hline Other, Not Otherwise Specified (NO & 33 & 275 & 0 & 0 & 0 & 274 & 10 & 21 & 307 \\
\hline TOTAL OTHER & 15377 & 72137 & 103 & 16 & 0 & 11516 & 59207 & $16880]$ & 87618 \\
\hline & & & & & & & & & \\
\hline TOTAL SAMPLE ANALYSES & 202190 & 242120 & 4425 & 478 & 0 & 123246 & 288414 & 35894 & 448384 \\
\hline
\end{tabular}


Table Vg: FY 99 Samples and Sample Analyses by Rad Level and Matrix Type (iv) EM-60

\begin{tabular}{|c|c|c|c|c|c|c|c|c|c|}
\hline $\begin{array}{c}\text { Rad-Matrix/ } \\
\text { Sample-Sample Analysis }\end{array}$ & RO & $\overline{\mathrm{R} 1}$ & $R 2$ & $\overline{R 3}$ & R4 & M1 & M2 & M3 & TOTAL \\
\hline CUSTOMER SAMPLES & 2204 & 762 & 10 & 1] & 묘 & 2198 & 607 & 7 & 2894 \\
\hline & & & & & & & & & \\
\hline \multicolumn{10}{|l|}{ SAMPLEANALYSES } \\
\hline Volatiles by GC/MS & 4541 & 0 & 0 & 이 & ㅇ․ & 3396 & 1145 & 미 & 4541 \\
\hline Volatiles by GC & 1916 & 50 & 18 & 0 & 0. & 1637 & 166 & 0] & 1894 \\
\hline Semi-Volatiles & 1586 & 2 & 01 & 0 & 요 & 770 & 818 & 요 & 1588 \\
\hline Herbicides/Pesticides/PCBs & 1764 & 2 & 0 & 0 & 0. & 637 & 1129 & 0] & 1766 \\
\hline Other Organics & 961 & 167 & 0 & 0 & 0. & 916 & 46 & 0] & 1045 \\
\hline \multirow[t]{2}{*}{ TOTAL ORGANICS } & 10769 & 221 & 18 & [0] & $0]$ & 73561 & 3303 & 1 & 10834 \\
\hline & & & & & & & & 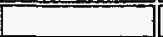 & \\
\hline RCRA Appendix IX Metals & 16 & 0 & 0 & 0 & 0 & 10 & 7 & -0 & 17 \\
\hline CAM (California) Metals (RCRA) & 0 & 0 & 0 & 0 & 0 & 0 & 0 & 요 & 으 \\
\hline RCRA TCLP Metals and EPTOX Me & 1761 & 50 & 18 & 0 & o. & 1453 & 180 & 0. & 1731 \\
\hline EPA CLP Metals (CERCLA) & 6668 & 0 & 0 & 0 & (0) & 3742 & 2919 & 7 & 6668 \\
\hline NPDES Metals (Clean Water Act) & 3 & 29 & 0 & 0 & 0. & 32 & 0 & 0 & 32 \\
\hline Drinking Water Metals (SDWA) & 460 & 0 & 0 & 0 & o & 460 & 0 & 0 & 460 \\
\hline Metals by Other Regulatory Suite & 6109 & 0 & 0 & 0 & 0 & 3718 & 2384 & 7 & 6109 \\
\hline Metals by Suite, Other & 0 & 166 & o & 0 & - & 0 & 0 & O. & 83 \\
\hline Metals by Metal Except Mercury & 1859 & 48 & 18 & 0 & 0 & 1562 & 168 & 0 & 1828 \\
\hline Mercury & 1757 & 218 & 18 & 0 & 요 & 1466 & 166 & 0 & 1812 \\
\hline Anions/Cations - IC & 27 & 3 & 0 & 0 & 0 & 28 & 2 & 0 & 30 \\
\hline Inorganics Wet Chemistry & 23146 & 3 & 01 & 0 & 요 & 21383 & 1764 & 1 & 23148 \\
\hline Other Inorganics & 106 & 3 & 0 & 0 & 0 & 106 & 2 & 1. & 109 \\
\hline \multirow[t]{2}{*}{ TOTALINORGANICS } & 41912 & 521 & 541 & D. & 0 & 339601 & 7592 & 16. & 42028 \\
\hline & & & & & & & & & \\
\hline \multirow{2}{*}{$\begin{array}{l}\text { Gross Alpha/Beta } \\
\text { Gamma Scan }\end{array}$} & 12040 & 538 & 0 & 0 & 0 & 6287 & 6108 & 17 & 12494 \\
\hline & 6187 & 179 & 0 & 0 & 0 & 2554 & 3804 & 8 & 6367 \\
\hline \multirow{2}{*}{$\begin{array}{l}\text { Transuranic } \\
\text { Plutonium Isotopes }\end{array}$} & 0 & 5 & 0 & 0 & 0 & 0 & 5 & 0 & 5 \\
\hline & \begin{tabular}{l|l|}
7366 \\
\end{tabular} & 5 & 0 & 0 & 0 & 3690 & 3674 & 7 & 7372 \\
\hline \multirow{2}{*}{\begin{tabular}{|l|} 
Uranium Isotopes \\
Curium Isotopes \\
\end{tabular}} & 5823 & 5 & 0 & 0 & 0 & 3012 & 2810 & 7 & 5828 \\
\hline & 0 & 0 & 0 & 0 & 0 & 0 & 0 & 0 & 으 \\
\hline \multirow{2}{*}{\begin{tabular}{|l} 
Thorium Isotopes \\
Radium Isotopes \\
\end{tabular}} & 0 & 0 & 0 & 1 & 의 & 0 & 0 & 1 & 1 \\
\hline & 3135 & 0 & 0 & 0 & 요 & 2497 & 638 & 0 & 3135 \\
\hline \multirow{2}{*}{\begin{tabular}{|l|} 
Americium-241 \\
Neptunium-237 \\
\end{tabular}} & 6781 & 0 & 0 & 0 & 0 & 3577 & 3197 & 7 & 6781 \\
\hline & 0 & 0 & 0 & 0 & 으 & 0 & 0 & 0 & 0 \\
\hline \multirow{2}{*}{\begin{tabular}{|l} 
Polonium-210 \\
Tritium \\
\end{tabular}} & 0 & 4 & 이 & 0 & 요 & 4 & 0 & 이 & 4 \\
\hline & 4221 & 0 & 0 & 0 & 므. & 3013 & 1208 & 0 & 4222 \\
\hline \multirow{2}{*}{$\frac{\text { Radon-222 }}{\text { Lead-210 }}$} & 0 & 0 & 01 & 0 & 요 & 0 & 0 & o) & 0 \\
\hline & 0 & 0 & 0 & 0 & ㅇ․ & 0 & 0 & 0 & 0 \\
\hline \multirow{2}{*}{\begin{tabular}{|l|} 
Carbon-14 \\
Nickel-63
\end{tabular}} & 0 & 0 & 0 & 0 & 0 & 0 & 0 & 0 & 으 \\
\hline & 0 & 0 & 0 & 0 & 의 & 0 & 0 & 0 & -0 \\
\hline \multirow{2}{*}{\begin{tabular}{|l|} 
Selenium-79 \\
Technetium -99
\end{tabular}} & 0 & 0 & 0 & 0 & 0 & 0 & 0 & 0 & 0 \\
\hline & 98 & 0 & 0 & 0 & o & 98 & 0 & 0 & 98 \\
\hline Strontium Isotopes & 0 & 0 & 0 & 0 & 0 & 0 & 0 & 0 & 0 \\
\hline Strontium-89 & 0 & 0 & 0 & 0 & 0. & 0 & 0 & 0 & 0 \\
\hline Strontium -90 & 98 & 0 & 0 & 0 & 0 & 98 & 0 & a. & 98 \\
\hline lodine -129 & 279 & 0 & 0 & 0 & 0 & 279 & 0 & 0 & 279 \\
\hline Nickel-59 & 0 & 0 & 0 & 0 & 0 & 0 & 0 & 0 & 0 \\
\hline Total Uranium & 98 & 0 & 0 & 0 & 0 & 98 & 0 & 0 & 98 \\
\hline Total Thorium & 0 & 0 & 0 & 0 & 0. & 0 & 0 & 0 & 0 \\
\hline Total Strontium & 3829 & 0 & 0 & 0 & 0 & 2288 & 1535 & 7 & 3830 \\
\hline Other RAD & 8544 & 48 & 18 & 01 & 0 & 4755 & 3853 & 1 & 8610 \\
\hline TOTAL RAD & 58499 & 787 & 19 & 11 & 0 & 32249 & 26832 & 58 & 59222 \\
\hline & & & & & & & & & \\
\hline Other Wet Chemistry & 10778 & 0 & 0 & 0 & $\overline{0}$ & 9530 & 1249 & 0] & 10779 \\
\hline TCLP Extraction & 1774 & 49 & 19 & 0 & 0 & 1452 & 195 & 0 & 1745 \\
\hline Asbestos & 60 & 0 & 0 & 0) & 으 & 2 & 57 & 0 & 60 \\
\hline Industrial Hygiene, NOS & 31 & 0 & 0 & 0 & 0 & 22 & 9 & 0 & 31 \\
\hline Physical Properties & 1951 & 0 & 0 & 0 & 0 & 431 & 1520 & 0 & 1951 \\
\hline Other, Not Otherwise Specified (NO & 0 & 22 & 0 & 01 & 0 & 01 & 0 & 요 & 11 \\
\hline TOTAL OTHER & 14594 & 72 & 19 & 01 & 0 & 11437 & 3031 & 0 & 14576 \\
\hline & & & $\square$ & $\frac{1}{11}$ & $\square$ & & & $\overline{76}$ & \\
\hline LOTAL SAMPLE ANALYSES & 125774 & 1600 & 111 & $1]$ & 0 & 85001 & 40758 & 76 & 126660 \\
\hline
\end{tabular}


Table Vg: FY 99 Samples and Sample Analyses by Rad Level and Matrix Type (v) Fernald

\begin{tabular}{|c|c|c|c|c|c|c|c|c|c|}
\hline $\begin{array}{c}\text { Rad-Matrix/ } \\
\text { Sample-Sample Analysis }\end{array}$ & RO & R1 & R2 & R3 & R4 & $\overline{\mathrm{M} 1}$ & $\overline{\mathrm{M} 2}$ & M3 & TOTAL \\
\hline CUSTOMER SAMPLES & 1239 & 804 & 1. & 1 & 0 & 916 & 914 & 109 & 1992 \\
\hline & & & & & & & & & \\
\hline \multicolumn{10}{|l|}{ SAMPLE ANALYSES } \\
\hline Volatiles by GC/MS & 341 & 24 & 0 & 0 & 0 & 292 & 74 & 므 & 365 \\
\hline Volatiles by GC & 49 & 52 & 0 & 0 & 0 & 27 & 57 & 14 & 99 \\
\hline Semi-Volatiles & 81 & 41 & 0 & 0 & 0 & 53 & 66 & 6 & 124 \\
\hline Herbicides/Pesticides/PCBs & 73 & 202 & 0 & 0 & 0 & 129 & 39 & 110 & 276 \\
\hline Other Organics & 352 & 120 & 0 & 0 & 0] & 10 & 351 & 5 & 419 \\
\hline TOTAL ORGANICS & 896 & 439 & 0 & 0 & 0 & 510 & 587 & 136 & 1284 \\
\hline \multicolumn{10}{|l|}{20} \\
\hline RCRA Appendix IX Metals & 2 & 이 & 이 & 0 & ㅇ․ & 1 & 1 & ㅇ․ & 2 \\
\hline CAM (California) Metals (RCRA) & 44 & 46 & 0 & 0 & 0 & 90 & 57 & 11 & 124 \\
\hline RCRA TCLP Metals and EPTOX Me & 195 & 83 & 0 & 0 & 0 & 41 & 153 & 14 & 243 \\
\hline EPA CLP Metals (CERCLA) & 325 & 0 & 0 & 0 & 0 & 312 & 13 & 0 & 325 \\
\hline NPDES Metals (Clean Water Act) & 19 & 19 & O) & 0 & 0 & 38 & 0 & 0 & 38 \\
\hline Drinking Water Metals (SDWA) & 1 & 0 & 0 & 0 & 0. & 1 & 0 & o & 1 \\
\hline Metals by Other Regulatory Suite & 324 & 33 & 요 & 0 & 0 & 311 & 27 & 12 & 353 \\
\hline Metals by Suite, Other & 0 & 126 & 0 & 0 & 0 & 21 & 0 & 0 & 73 \\
\hline Metals by Metal Except Mercury & 88 & 128 & 0 & 0 & 0 & 89 & 119 & 7 & 216 \\
\hline Mercury & 108 & 381 & 0 & 0 & 0 & 289 & 91 & 3 & 436 \\
\hline Anions/Cations-IC & 4 & 22 & of & 0 & 0 & 12 & 8 & 6 & 26 \\
\hline Inorganics Wet Chemistry & 577 & 324 & 0 & 0 & 0. & 879 & 101 & 68 & 975 \\
\hline Other Inorganics & 511 & 48 & 0 & 0 & 0] & 164 & 387 & 8 & 559 \\
\hline TOTAL INORGANICS & 2198 & 1210 & 0 & 0 & 0] & 2248 & 959 & $129]$ & 3372 \\
\hline \multicolumn{10}{|l|}{20} \\
\hline Gross Alpha/Beta & 884 & 505 & 0 & 0 & 0 & 924 & 394 & 37 & 1372 \\
\hline Gamme Scan & 135 & 175 & 0 & 0 & 0 & 225 & 78 & 8 & 311 \\
\hline Transuranic & 0 & 0 & 0 & 0 & 0 & 0 & 0 & 0 & 0 \\
\hline Plutonium Isotopes & 460 & 32 & 0 & 0 & 0 & 239 & 246 & 7 & 493 \\
\hline Uranium Isotopes & 522 & 178 & 0 & 0 & 0 & 327 & 274 & 99 & 700 \\
\hline Curium Isotopes & 0 & 0 & 0 & 0 & o. & 0 & 0 & 0 & 0 \\
\hline Thorium Isotopes & 0 & 13 & of & 1 & of & 0 & 13 & 1 & 14 \\
\hline Radium Isotopes & 9 & 0 & 0 & 0 & of & 9 & 0 & 0. & 9 \\
\hline Americium-241 & 450 & 21 & 0 & 0 & 0 & 227 & 238 & 6 & 471 \\
\hline Neptunium-237 & 0 & 21 & 0 & 0 & 0 & 4 & 16 & 1 & 21 \\
\hline Polonium-210 & 52 & 0. & 0 & 0 & of & 0 & 52 & O) & 52 \\
\hline Tritium & 35 & 35 & 0 & 0 & 0 & 54 & 41 & 3 & 84 \\
\hline Padon-222 & 01 & 0 & 0 & 0 & 0 & 0 & 0 & ㅇ. & 0 \\
\hline Lead-210 & 0 & 0 & 0 & 0 & 0 & 0 & 0 & 0. & 0 \\
\hline Carbon-14 & 0 & 0 & 0 & 0 & 0 & 0 & 0 & 요 & 0 \\
\hline Nickel-63 & 01 & 0 & 0 & 01 & 0 & 0 & 0 & 요 & 0 \\
\hline Selenium-79 & 44 & 11 & 0) & 0) & 0 & 50 & 3 & 2 & 55 \\
\hline Technetium-99 & 0 & 0 & 0 & 0 & 0 & 0 & 0 & 0 & 0 \\
\hline Strontium Isotopes & 0 & 0 & 0 & 0 & 0 & 0 & 0 & 0 & 0 \\
\hline Strontium -89 & 0 & 0 & 0 & 0 & 0 & 0 & 0 & ㅇ. & 0 \\
\hline Strontium-90 & 44 & 57 & 0 & 0 & 0 & 72 & 22 & 7 & 101 \\
\hline lodine-129 & 44 & 16 & 0 & 0 & 0 & 51 & 6 & 3 & 60 \\
\hline Nickel-59 & 0 & 0 & 0 & 0 & 0 & 0 & 0 & o. & 0 \\
\hline Total Uranium & 0 & 134 & 0 & 0 & 0 & 21 & 22 & 92 & 135 \\
\hline Total Thorium & 0 & 43 & 0 & 아 & 0 & 21 & 22 & 0 & 43 \\
\hline Total Strontium & 48 & 0 & 0 & 0 & 0 & 46 & 3 & 0 & 49 \\
\hline Other RAD & 244 & 116 & 0 & 0 & $0]$ & 297 & 53 & 8] & 359 \\
\hline TOTAL RAD & 2973 & 1359 & 1 & 1 & 0 & 2567 & 1483 & 274] & 4329 \\
\hline \\
\hline Other Wet Chemistry & 247 & $\overline{0 .}$ & (⿹丁口 & 0 & 0 & 245 & $\overline{3}$ & o] & 248 \\
\hline TCLP Extraction & 232 & 115 & 1 & 0 & 0 & 121 & 213 & 14 & 348 \\
\hline Asbestos & O & 12 & 0 & 0 & 0 & 0 & 12 & 0 & 12 \\
\hline Industrial Hygiene, NOS & 57 & 6 & 0 & 0 & 0 & 60 & 2 & 1 & 63 \\
\hline Physical Properties & 26 & 40 & 0 & 0 & 0 & 40 & 26 & 0 & 66 \\
\hline Other, Not Otherwise Specified (NO & $2 \mid$ & 12 & 0 & 0 & o) & 12 & 1 & 0 & 14 \\
\hline TOTAL OTHER & 564 & 185 & 11 & 0 & 0] & 478 & 257 & 15 & 750 \\
\hline \multirow{2}{*}{ TOTAL SAMPLE ANALYSES } & & & & & & & & & \\
\hline & 6631 & 3193 & $2 \mid$ & 1 & 0 ] & 5803 & 3286 & 554 & 9735 \\
\hline
\end{tabular}


Table Vg: FY 99 Samples and Sample Analyses by Rad Level and Matrix Type (vi) INEL

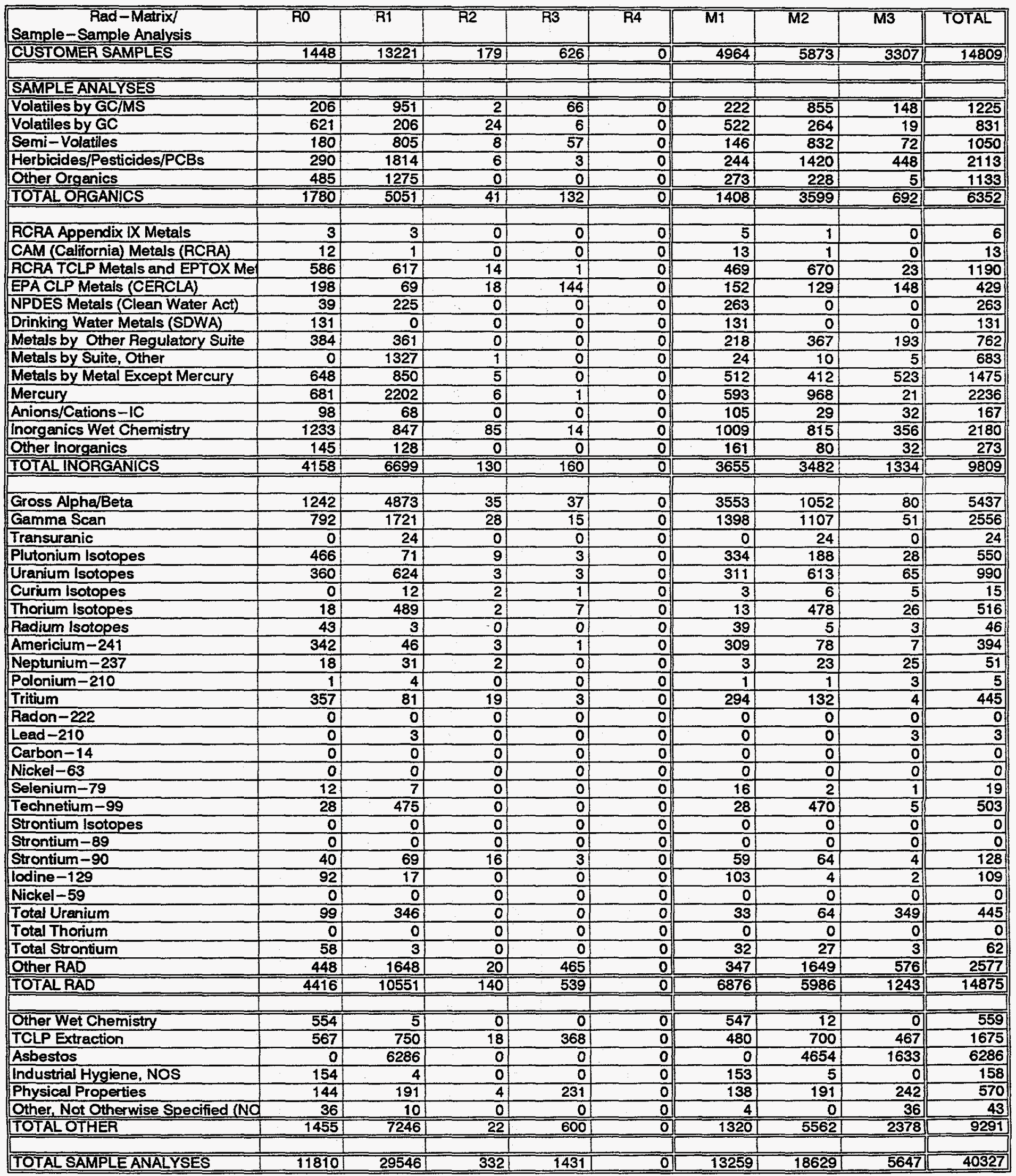


Table Vg: FY 99 Samples and Sample Analyses by Rad Level and Matrix Type (vii) NTS

\begin{tabular}{|c|c|c|c|c|c|c|c|c|c|}
\hline $\begin{array}{c}\text { Rad-Matrix } \\
\text { Sample-Sample Analysis }\end{array}$ & RO & $\overline{\mathrm{R1}}$ & R2 & R3 & $\overline{\mathrm{R} 4}$ & M1 & $\mathrm{M} 2$ & M3 & TOTAL \\
\hline CUSTOMER SAMPLES & 1042 & 9337 & 176 & 0 & 7 & 669 & 5803 & 2810 & 9922 \\
\hline & & & & & & & & & \\
\hline \multicolumn{10}{|l|}{ SAMPLE ANALYSES } \\
\hline Volatiles by GC/MS & 1483 & 1256 & 45 & 0 & 0 & 941 & 1721 & 123 & 2785 \\
\hline Volatiles by GC & 280 & 12 & 0 & 0 & 0 & 8 & 224 & 65 & 295 \\
\hline Semi-Volatiles & 730 & 1030 & 39 & 0 & 0 & 387 & 1322 & 88 & 1798 \\
\hline Herbicides/Pesticides/PCBs & 779 & 2049 & 0 & 0 & 0 & 296 & 2163 & 372 & 2830 \\
\hline Other Organics & 19 & 136 & 0 & 0 & 0 & 73 & 73 & 8 & 155 \\
\hline \multirow[t]{2}{*}{ TOTAL ORGANICS } & 3291 & 4483 & 84 & 0 & 0 & 1705 & 5503 & 657 & 7862 \\
\hline & & & & & & & & & \\
\hline RCRA Appendix IX Metals & 3 & 14 & 0 & 0 & 0 & 1 & 16 & 0 & 17 \\
\hline CAM (California) Metals (RCRA) & 0 & 0 & 0 & 0 & 0 & 0 & 0 & 0 & 0 \\
\hline RCRA TCLP Metals and EPTOX Me & 56 & 549 & 0 & 0 & $\mathbf{0}$ & 10 & 583 & 11 & 605 \\
\hline EPA CLP Metals (CERCLA) & 1635 & 600 & 115 & 0 & 0 & 725 & 1580 & 45 & 2350 \\
\hline NPDES Metals (Clean Water Act) & 259 & 0 & 0 & 0 & 0 & 259 & 0 & 0 & 259 \\
\hline Drinking Water Metals (SDWA) & 2 & 0 & 0 & 0 & 0 & 2 & 0 & 0 & 2 \\
\hline Metals by Other Regulatory Suite & 1328 & 329 & 0 & 0 & 0 & 576 & 1099 & 66 & 1698 \\
\hline Metals by Suite, Other & 0 & 533 & 20 & 0 & 0 & 302 & 164 & 2 & 510 \\
\hline Metals by Metal Except Mercury & 72 & 853 & 0 & 0 & 0 & 25 & 398 & 501 & 925 \\
\hline Mercury & 303 & 927 & 0 & 0 & 0 & 284 & 930 & 14 & 1229 \\
\hline Anions/Cations - IC & 387 & 211 & 0 & 0 & 0 & 567 & 21 & 11 & 598 \\
\hline Inorganics Wet Chemistry & 3031 & 858 & 0 & 0 & 0 & 2649 & 1219 & 20 & 3888 \\
\hline Other Inorganics & 333 & 137 & 0 & 0 & 0 & 314 & 156 & 0 & 470 \\
\hline \multirow[t]{2}{*}{ TOTAL INORGANICS } & 7410 & 5009 & 135 & 0 & 0 & 5715 & 6166 & 670 & 12552 \\
\hline & & & & & & & & & \\
\hline Gross Alpha/Beta & 3003 & 1027 & 29 & 0 & 0 & 1466 & 2533 & 60 & 4059 \\
\hline Gamma Scan & 1078 & 581 & 16 & 0 & 0 & 567 & 1042 & 66 & 1674 \\
\hline Transuranic & 0 & 21 & 0 & 0 & 0 & 0 & 21 & 0 & 21 \\
\hline Plutonium Isotopes & 1333 & 164 & 11 & 0 & 0 & 511 & 981 & 17 & 1508 \\
\hline Uranium Isotopes & 1429 & 1009 & 11 & 0 & 0 & 641 & 1759 & 48 & 2448 \\
\hline Curium Isotopes & 0 & 19 & 0 & 0 & 0 & 9 & 10 & 0 & 19 \\
\hline Thorium Isotopes & 0 & 584 & 11 & 0 & 0 & 40 & 553 & 2 & 594 \\
\hline Radium Isotopes & 516 & 0 & 0 & 0 & 0 & 383 & 133 & 0 & 516 \\
\hline Americium-241 & 1309 & 70 & 11 & 0 & 0 & 520 & 855 & 15 & 1390 \\
\hline Neptunium-237 & 0 & 104 & 0 & 0 & 0 & 7 & 95 & 2 & 104 \\
\hline Polonium-210 & 0 & 0 & 0 & 0 & 0 & 0 & 0 & 0 & 0 \\
\hline Tritium & 796 & 367 & 51 & 0 & 요 & 595 & 610 & 10 & 1215 \\
\hline Radon-222 & 0 & 0 & 0 & 0 & 0 & 0 & 0 & 0 & 0 \\
\hline Lead-210 & 0 & 0 & 0 & 0 & 0 & 0 & 0 & 0 & 0 \\
\hline Carbon-14 & 0 & 0 & 0 & 0 & 0 & 0 & 0 & 0 & 0 \\
\hline Nickel-63 & 0 & 0 & 0 & 0 & 0 & 0 & 0 & 0 & 0 \\
\hline Selenium-79 & 0 & 0 & 0 & 0 & 0 & 0 & 0 & 0 & 0 \\
\hline Technetium-99 & 0 & 467 & 3 & 0 & 0 & 4 & 467 & 0 & 471 \\
\hline Strontium Isotopes & 0 & 0 & 0 & 0 & 0 & 0 & 0 & 0 & 0 \\
\hline Strontium-89 & 0 & 0 & 0 & 0 & 0 & 0 & 0 & 0 & 0 \\
\hline Strontium-90 & 0 & 163 & 3 & 0 & 0 & 16 & 151 & 0 & 167 \\
\hline lodine-129 & 1 & 0 & 0 & 0 & 0 & 1 & 0 & 0 & 1 \\
\hline Nickel-59 & 0 & 0 & 0 & 0 & 0 & 0 & 0 & 0 & 0 \\
\hline Total Uranium & 18 & 381 & 0 & 0 & 0 & 18 & 113 & 268 & 399 \\
\hline Total Thorium & 0 & 26 & 0 & 0 & 0 & 0 & 26 & 0 & 26 \\
\hline Total Strontium & 715 & 0 & 0 & 0 & 0 & 340 & 361 & 15 & 715 \\
\hline Other RAD & 1780 & 1725 & 32 & 0 & 0 & 453 & 3066 & 18 & 3537 \\
\hline TOTAL RAD & 11978 & 6708 & 179 & 0 & 0 & 5571 & 12777 & 519 & 18866 \\
\hline & & & & & & & & & \\
\hline Other Wet Chemistry & 1731 & 0 & 0 & 0 & 0 & 1413 & 319 & 0 & 1731 \\
\hline TCLP Extraction & 48 & 651 & 1 & 0 & 0 & 21 & 607 & 71 & 699 \\
\hline Asbestos & 25 & 6169 & 0 & 0 & 0 & 0 & 4611 & 1582 & 6194 \\
\hline Industrial Hygiene, NOS & 23 & 0 & 0 & 0 & 0 & 3 & 19 & 0 & 23 \\
\hline Physical Properties & 251 & 218 & 6 & 0 & 0 & 63 & 413 & 0 & 476 \\
\hline Other, Not Otherwise Specified (No & 2 & 34 & 0 & 0 & 0 & 34 & 2 & 0 & 36 \\
\hline TOTAL OTHER & 2080 & 7072 & 7 & 0 & 0 & 1534 & 5970 & 1653 & 9159 \\
\hline & & & & & & & & & \\
\hline TOTAL SAMPLE ANALYSES & 24759 & 23272 & 405 & 0 & 0 & 14526 & 30416 & 3500 & 48439 \\
\hline
\end{tabular}


Table Vg: FY 99 Samples and Sample Analyses by Rad Level and Matrix Type (viii) Oak Ridge K-25

\begin{tabular}{|c|c|c|c|c|c|c|c|c|c|}
\hline $\begin{array}{c}\text { Rad-Matrix/ } \\
\text { Sample-Sample Analysis }\end{array}$ & $\overline{\mathrm{RO}}$ & $\overline{\mathrm{R1}}$ & R2 & $\overline{\mathrm{R} 3}$ & $\overline{\overline{R 4}}$ & $\overline{\mathrm{M} 1}$ & $\overline{\mathrm{M} 2}$ & $\overline{\overline{\mathrm{M} 3}}$ & TOTAL \\
\hline CUSTOMEA SAMPLES & 128 & 9460 & 238 & 19 & 0] & 348 & 6034 & 3480 & 9853 \\
\hline & & & & & I & & & & \\
\hline \multicolumn{10}{|l|}{ SAMPLEANALYSES } \\
\hline Volatiles by GC/MS & 12 & 1456 & 86 & $0]$ & $0]$ & 132 & 1111 & 312 & 1555 \\
\hline Volatiles by GC & 69 & 36 & 9 & 4 & o) & 59 & 51 & 1 & 115 \\
\hline Semi-Volatiles & 11 & 1120 & 81 & 1 & 0 & 13 & 1029 & 172 & 1213 \\
\hline Herbicides/Pesticides/PCBs & 10 & 2603 & 4 & 2 & 01 & 451 & 1631 & 538 & 2619 \\
\hline Other Organics & 33 & 3 & 10 & 0 & 0 & 33 & 0 & 10 & 44 \\
\hline TOTAL OAGANICS & 135 & 5219 & 189 & 7 & 0] & 686 & 3823 & 1034 & 5547 \\
\hline BCRA Anoendix IX Metals & 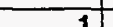 & & 07 & & & 0 & & $9[\mathrm{C}$ & \\
\hline CAM (California) Metals (RCRA) & $\div$ & $\overline{0}$ & 0 & 0 & ot & $\frac{1}{0}$ & 0 & $\frac{21}{0}$ & $\frac{17}{0}$ \\
\hline RCRA TCLP Metals and EPTOX Me & 108 & 769 & 161 & 1 & of & 54 & 667 & 331 & 1046 \\
\hline EPA CLP Metals (CERCLA) & 14 & 289 & 8 & 41 & $0)$ & 13 & 29 & 273 & 314 \\
\hline NPDES Metals (Clean Water Act) & 0 & 1 & 0 & 0 & 0 - & 1 & 0 & 0 & 1 \\
\hline Drinking Water Metais (SDWA) & 16 & o & 0 & 0 & 0 [ & 16 & 0 & o] & 16 \\
\hline Metals by Other Regulatory Suite & 24 & 742 & 73 & 0 & a. & 285 & 454 & 140 & 859 \\
\hline Metals by Suite, Other & 0 & 52 & 0 & 0 & 0 & 7 & 0 & 2 & 30 \\
\hline Metals by Metal Except Mercury & 111 & 1670 & 73 & 0 & 0 & 388 & 358 & 696 & 1648 \\
\hline Mercury & 107 & 1403 & 11 & 0 & o] & 666 & 1125 & 125 & 1718 \\
\hline Anions/Cations-IC & 2 & 403 & 34 & 0 & 0 & 1 & 8 & 95 & 271 \\
\hline Inorganics Wet Chemistry & 118 & 754 & 30 & 8 & 0 & 157 & 733 & 375 & 1088 \\
\hline Other inorganics & 12 & 117 & 0 & 0 & o. & 12 & 45 & 72 & 129 \\
\hline TOTAL INORGANICS & 513 & 62001 & 390 & 13 & 0] & 1601 & 3420 & 2131 & 7134 \\
\hline \multicolumn{10}{|l|}{20} \\
\hline Gross Alpha/Beta & 64 & 1355 & 27 & 24 & 에 & 704 & 488 & 105 & 1384 \\
\hline Gamma Scan & 91 & 492 & 27 & 10 & 0 & 22 & 491 & 105 & 619 \\
\hline Transuranic & 0 & 310 & 291 & 0 & 0 & 388 & 213 & 0 & 600 \\
\hline Plutonium Isotopes & 28 & 119 & 14 & 2 & 0 & 16 & 42 & 105 & 163 \\
\hline Uranium Isotopes & 14 & 1157 & 236 & 2 & 0 & 504 & 675 & 230 & 1408 \\
\hline Curium Isotopes & 0 & 2 & 1 & 1 & 0 & 0 & 3 & 0 & 4 \\
\hline Thorium Isotopes & 1 & 920 & 11 & 1 & 0. & 251 & 577 & 105 & 933 \\
\hline Radium Isotopes & 3 & 0 & 0 & 01 & 0 & 3 & of & 0 & 4 \\
\hline Americium-241 & 14 & 94 & 2 & 1 & 0 & 26 & 15 & 70 & 111 \\
\hline Neptunium-237 & 1 & 242 & 195 & 0 & 0 & 202 & 132 & 105 & 439 \\
\hline Polonium-210 & 0 & 0 & 10 & 0 & 0 & 0 & 0 & 10 & 10 \\
\hline Tritium & 21 & 16 & 4 & 2 & 0. & 14 & 28 & 0 & 43 \\
\hline Radon-222 & 0 & 01 & 0 & 0 & 0. & 0 & 0 & 요 & 0 \\
\hline Lead-210 & 0 & 0 & 0 & 0 & 0 & 0 & 0 & of & ? \\
\hline Carbon-14 & 0 & 0 & 0 & 0 & 0 & 0 & 0 & 의 & 인 \\
\hline Nickel-63 & 0 & 0 & 0 & 0 & o. & 0 & 0 & a. & 0 \\
\hline Selenium-79 & 0 & 0 & 0 & 0 & 0 & 0 & 0 & - & 0 \\
\hline Technetium-99 & 3 & 942 & 300 & 0 & 0 & 391 & 754 & 101 & 1246 \\
\hline Strontium Isotopes & of & 0 & 0 & 0 & 0 & 0 & 0 & o. & 0 \\
\hline Strontium -89 & 0 & 0 & 0 & 0 & 0 & 0 & 0 & - & 0 \\
\hline Strontium-90 & 3 & 16 & 6 & 2 & 0 & 4 & 24 & 0 & 28 \\
\hline lodine-129 & 10 & 1 & 0 & 0 & 0 & 10 & 0 & 0. & 10 \\
\hline Nickel-59 & 0 & 0 & 0 & 0 & 0 & 0 & 0 & 0 & 0 \\
\hline Total Uranium & 9 & 642 & 284 & 0 & 0 & 101 & 414 & 420 & 935 \\
\hline Total Thorium & 0 & 13 & 0 & 0 & ㅁ․ & 0 & 13 & 0 & 13 \\
\hline Total Strontium & 4 & 0 & 0 & 0 & 의. & 2 & 2 & o. & 4 \\
\hline Other RAD & 39 & 2121 & 15 & 21 & 0 & 270 & 1799 & 108 & 2177 \\
\hline TOTAL RAD & 308 & 8442 & 1423 & 45 & ㅁ] & 2908 & 5670 & 1465 & 10130 \\
\hline & & & & & & 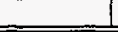 & & & \\
\hline Other Wet Chemistry & 22 & of & 0 & 0 & 0] & 22 & 0 & 0] & 22 \\
\hline TCLP Extraction & 107 & 1076 & 88 & 2 & 이 & 54 & 752 & 460 & 1269 \\
\hline Asbestos & 0 & 7616 & 0 & 0 & 0. & 0 & 5639 & 1978 & 7616 \\
\hline Industrial Hygiene, NOS & 1 & 0 & 0 & 0 & 에 & 0 & 0 & 0 & 1 \\
\hline Physical Properties & 14 & 545 & 2 & 1 & 0 & 251 & 221 & 90 & 562 \\
\hline Other, Not Otherwise Specified (NO & 41 & 8 & 10 & 01 & 0 & 7 & 11 & 12[ & 21 \\
\hline TOTAL OTHEA & 148 & 9245 & 100 & 2 & 0] & 336 & 66131 & 2540 & 9492 \\
\hline TOTAL SAMPLE ANALYSES & 1105 & & 2102 & & & & & 717010 & \\
\hline & & 29106 & 21021 & 66 & 0] & 5530 & 19526 & 7170 & 32303 \\
\hline
\end{tabular}


Table Vg: FY 99 Samples and Sample Analyses by Rad Level and Matrix Type (ix) Oak Ridge X-10

\begin{tabular}{|c|c|c|c|c|c|c|c|c|c|}
\hline $\begin{array}{c}\text { Rad-Matrix } \\
\text { Sample-Sample Analysis }\end{array}$ & RO & $\overline{\mathrm{A1}}$ & R2 & A3 & R4 & M1 & M2 & M3 & TOTAL \\
\hline CUSTOMER SAMPLES & 362 & 5710 & 10 & 595 & $\underline{0}$ & 3150 & 1314 & 1272 & 6206 \\
\hline \multirow{2}{*}{\multicolumn{10}{|c|}{ SAMPLE ANALYSES }} \\
\hline & & & & & & & & & \\
\hline Volatiles by GC/MS & 53 & 228 & 0 & 66 & 0 & 64 & 203 & 81 & 348 \\
\hline Volatiles by GC & 65 & 37 & 1 & 0 & 0 & 64 & 31 & 5 & 102 \\
\hline Semi-Volatiles & 41 & 195 & 0 & 56 & 0 & 39 & 192 & 61 & 292 \\
\hline Herbicides/Pesticides/PCBs & 159 & 436 & 0 & 0 & 0 & 159 & 333 & 104 & 595 \\
\hline Other Organics & 56 & 936 & 0 & 0 & 0 & 23 & 38 & $1]$ & 527 \\
\hline TOTAL ORGANICS & 375 & 1831 & 2 & 122 & 0 & 349 & 797 & 252 & 1864 \\
\hline RCRA Appendix IX Metals & 2 & 2 & 01 & 0 & & 4 & 0 & 0 & \\
\hline CAM (California) Metals (RCRA) & 44 & $\frac{4}{1}$ & 0 & 0 & 0 & \begin{tabular}{r|}
45 \\
45
\end{tabular} & $\frac{0}{1}$ & 0 & $\frac{4}{45}$ \\
\hline RCRA TCLP Metals and EPTOX Me & 65 & 151 & 1 & 0 & 0 & 56 & 151 & 5 & 215 \\
\hline EPA CLP Metals (CERCLA) & 36 & 7 & 1 & 138 & 0 & 29 & 13 & 139 & 182 \\
\hline NPDES Metals (Clean Water Act) & 20 & 164 & 0 & 0 & 0 & 184 & 0 & o. & 184 \\
\hline Drinking Water Metals (SDWA) & 9 & 0 & 0 & 0 & 0 & 9 & 0 & 0 & 9 \\
\hline Metals by Other Regulatory Suite & 100 & 86 & 0 & 0 & 0 & 78 & 84 & 32 & 190 \\
\hline Metals by Suite, Other & 0 & 944 & 0 & 0 & 0 & 5. & 2 & 1 & 476 \\
\hline Metals by Metal Except Mercury & 128 & 203 & 0 & 0 & 0 & 111 & 89 & 127 & 329 \\
\hline Mercury & 164 & 1174 & 0 & 0 & 0 & 176 & 224 & 6 & 872 \\
\hline Anions/Cations-IC & 45 & 25 & 0 & 0 & 0 & 50 & 14 & 6 & 70 \\
\hline Inorganics Wet Chemistry & 583 & 187 & 9 & 2 & 0 & 545 & 179 & 59 & 782 \\
\hline Other Inorganics & 61 & 35 & 01 & 0 & 0 & 60 & 27 & 9 & 96 \\
\hline TOTALINORGANICS & 1259 & 2978 & 121 & 140 & 0 & 1353 & 785 & 384 & 3455 \\
\hline \multicolumn{10}{|l|}{ Grnoo AlnhalRata } \\
\hline Gross Alpha/Beta & 515 & 3085 & 1 & 0 & 0 & 2471 & 179 & 26 & 3138 \\
\hline Gamma Scan & 76 & 1058 & 0 & 0 & 0 & 974 & 146 & 15 & 1134 \\
\hline Transuranic & 0 & 5 & 0 & 0 & 0 & 0 & 5 & 0. & 5 \\
\hline Plutonium Isotopes & 167 & 6 & 2 & 0 & 0 & 151 & 18 & 6 & 175 \\
\hline Uranium Isotopes & 162 & 137 & 2 & o & 0 & 154 & 131 & 15 & 300 \\
\hline Curium Isotopes & 0 & 2 & 0 & 0 & 0 & 0 & 0 & 2 [ & 2 \\
\hline Thorium Isotopes & 2 & 117 & 0 & 5 & 0 & 3 & 114 & 8 & 125 \\
\hline Radium Isotopes & 5 & 0 & 0 & 0 & 0 & 4 & 1 & 0 & 5 \\
\hline Americium-241 & 157 & 7 & 0 & 0 & 0 & 151 & 11 & 2 & 164 \\
\hline Neptunium-237 & 2 & 6 & 2 & 0 & 0 & 0 & 5 & 5 & 10 \\
\hline Polonium -210 & 1 & 1 & 0 & 0 & 0 & 0 & 1 & 0 & 1 \\
\hline Tritium & 135 & 13 & 3 & 0 & 0 & 131 & 15 & 2 & 149 \\
\hline Radon-222 & 0 & 0 & 0 & 0 & 0 & 0 & 0 & 0 & 0 \\
\hline Lead-210 & 0 & 2 & 0 & 0 & 0 & 0 & 0 & 2 & 2 \\
\hline Carbon-14 & 0 & 0 & 0 & 0 & 0 & 0 & 0 & 0 & 0 \\
\hline Nickel-63 & 0 & 0 & 0 & 0 & 0 & 0 & 0 & 0 & 0 \\
\hline Selenium -79 & 44 & 1 & 0 & 0 & 0 & 45 & 0 & 0 & 45 \\
\hline Technetium-99 & 2 & 116 & 0 & 0 & 0 & 2 & 114 & 2 & 118 \\
\hline Strontium Isotopes & 0 & 0 & 0 & 0 & 0 & 0 & $\overline{0}$ & 0. & 0 \\
\hline Strontium-89 & 0 & 0 & 0 & 0 & 0 & 0 & 0 & 0 & 0 \\
\hline Strontium -90 & 46 & 8 & 1 & 0 & 0 & 50 & 5 & 1 & 56 \\
\hline lodine-129 & 50 & 3 & 0 & 0 & 0 & 52 & 1 & 0 & 52 \\
\hline Nickel-59 & 0 & 01 & 0 & 0 & 0 & 0 & 0 & 0 & 0 \\
\hline Total Uranium & 12 & 85 & 0 & 0 & 0 & 3 & 16 & 78 & 97 \\
\hline Total Thorium & 0 & 0 & 0 & 0 & 0 & 0 & 0 & 0 & 0 \\
\hline Total Strontium & 10 & 2 & 0 & 0 & 0 & 7 & 3 & 2 & 12 \\
\hline Other RAD & 104 & 410 & 2 & 462 & 0 & 100 & 384 & $487]$ & 974 \\
\hline TOTALRAD & 1489 & 5067 & 13 & 467 & 0 & 4297 & 1149 & 653 & 6567 \\
\hline & & & & & & & & & \\
\hline Other Wet Chemistry & 267 & 2 & 0 & 0 & 0 & 267 & 2 & 0 & 269 \\
\hline TCLP Extraction & 52 & 176 & 7 & 366 & 0 & 51 & 156 & 390 & 599 \\
\hline Asbestos & 0 & 1523 & 0 & 0 & 0 & 0 & 1128 & 396 & 1523 \\
\hline Industrial Hygiene, NOS & 111 & 1 & 0 & 0 & 0 & 111 & 1 & 0 & 112 \\
\hline Physical Properties & 26 & 43 & 01 & 230 & 0 & 26 & 42 & 232 & 300 \\
\hline Other, Not Otherwise Specified (NO & 5 & 2 & 0 & 0 & 0 & 2 & 0 & 5 & 7 \\
\hline TOTAL OTHER & 463 & 1747 & 71 & 596 & 0 & 458 & 1328 & 1022 & 2810 \\
\hline TOTAL SAMPLE ANALYSES & 3586 & 11623 & $34]$ & 1325 & 0 & 6457 & 4059 & 2311 & 14697 \\
\hline
\end{tabular}


Table Vg: FY 99 Samples and Sample Analyses by Rad Level and Matrix Type (x) Oak Ridge Y-12

\begin{tabular}{|c|c|c|c|c|c|c|c|c|c|}
\hline $\begin{array}{c}\text { Rad-Matrix/ } \\
\text { Sample-Sample Analysis }\end{array}$ & $\mathrm{RO}$ & R1 & R2 & $\mathrm{R3}$ & R4 & $\overline{\mathrm{M} 1}$ & M2 & $\overline{\mathrm{M3}}$ & TOTTAL \\
\hline CUSTOMER SAMPLES & 48 & 8421 & $\begin{array}{ll}6 \\
6\end{array}$ & 01 & (0) & $55 !$ & 541 & 270 & 881 \\
\hline & & & & & & & & & \\
\hline \multicolumn{10}{|l|}{ SAMPLE ANALYSES } \\
\hline Volatiles by GC/MS & 42 & 103 & 1 & 01 & 0. & 26 & 100 & 19 & 146 \\
\hline Volatiles by GC & 10 & 27 & 2 & 01 & 0 & 2 & 34 & 4 & 39 \\
\hline Semi-Volatiles & 15 & 85 & 1 & 0 & 0. & 9 & 90 & 2. & 101 \\
\hline Herbicides/Pesticides/PCBs & 58 & 183 & 0 & 0 & 0 & 33 & 151 & 57 & 241 \\
\hline Other Organics & 0 & 3 & 0 & 0 & 0 & 2 & 0 & 1. & 3 \\
\hline TOTAL ORGANICS & $125 !$ & 401 & $4 \mid$ & 01 & O] & 71 & 375 & 83 & 530 \\
\hline & & & & & & & & & \\
\hline RCRA Appendix IX Metals & 01 & 0 & 0 & 우 & 으 & 0 & 0 & 0] & o \\
\hline CAM (California) Metals (RCRA) & 27 & 0 & 0 & 0 & 0 & 27 & 0 & 0. & 28 \\
\hline RCRA TCLP Metals and EPTOX Me & 7 & 58 & 2 & 0 & 0. [ & 0 & 63 & 3 & 67 \\
\hline EPA CLP Metals (CERCLA) & 28. & 17 & 3 & 0 & 0. & 13 & 32 & 3 & 47 \\
\hline NPDES Metals (Clean Water Act) & 6 & 1) & 0 & 0 & o] & 7 & 0 & 0] & 7 \\
\hline Drinking Water Metals (SDWA) & 0 & 0 & 0 & 0 & 0. & 0 & 0 & 0. & 의 \\
\hline Metals by Other Regulatory Suite & 61 & 34 & 0 & 0 & 0] & 10 & 46 & 44 & 98 \\
\hline Metals by Suite, Other & 0 & 17 & 0 & 0 & 0. & 8 & 4 & 1 & 15 \\
\hline Metals by Metal Except Mercury & 33 & 86 & 0 & 0 & 0 & 29 & 38 & 52 & 119 \\
\hline Mercury & 38 & 96 & 0 & 0 & 0 & 38 & 94 & 2 & 134 \\
\hline Anions/Cations-IC & 14 & 7 & 0 & \begin{tabular}{l|l}
0 \\
\end{tabular} & 0 & 14 & 1 & 6 & 21 \\
\hline Inorganics Wet Chemistry & 249 & 81 & 17 & 0 & 0. & 168 & 99 & 82 & 348 \\
\hline Other Inorganics & 16 & 16 & 0 & 0 & 0] & 15 & 11 & 6] [ & 32 \\
\hline TOTAL INORGANICS & 481 & 413 & 22 & $0 !$ & Q] & 330 & 389 & 199 & 917 \\
\hline & & & & & & & & & \\
\hline Gross Alpha/Beta & 63 & 68 & 3 & 0 & 0 & 34 & 82 & 10 & 130 \\
\hline Gamma Scan & 26 & 42 & 0 & 0 & 0 & 10 & 48 & 10 & 68 \\
\hline Transuranic & 0 & 2 & 0 & 0 & 0 & 0 & 2 & 0 & 2 \\
\hline Plutonium isotopes & 26 & 5 & 0 & 0 & 0 & 7 & 19 & 6 & 31 \\
\hline Uranium Isotopes & 31 & 66 & 0 & 0 & 0 & 13 & 75 & 11 & 98 \\
\hline Curium Isotopes & 0 & 2 & 0 & 0 & 0 & 1 & 1 & 1 & 2 \\
\hline Thorium Isotopes & 5 & 51 & 0 & 0 & 0 & 2 & 49 & 5 & 56 \\
\hline Radium Isotopes & 6 & 1 & 0 & 0 & 0 & 4 & 2 & 1 & 7 \\
\hline Americium - 241 & 21 & 3 & 0 & 0 & 0 & 8 & 16 & - & 25 \\
\hline Neptunium-237 & 5 & 4 & 0 & 0 & o. & 0 & 3 & 6 & 9 \\
\hline Polonium-210 & 0) & 1 & 0 & 0 & O & 0 & 0 & 1 & 1 \\
\hline Tritium & 12 & 17 & 3 & 0 & o. & 9 & 14 & ㅇ․ & 28 \\
\hline Radon-222 & 0 & 0 & 0 & 0 & 0 & 0 & 0 & 묘 & 0 \\
\hline Lead-210 & 0 & 0 & 0 & 0 & 0 & 0 & 0 & 의 & 은 \\
\hline Carbon-14 & 0 & 0 & 0 & 0 & 0 & 0 & 0 & 0. & 요 \\
\hline Nickel-63 & 0 & 0 & 0 & 0 & 0 & 0 & 0 & of & 요 \\
\hline Selenium-79 & 27 & 0 & 0 & 0 & 0 & 27 & 0 & - & 27 \\
\hline Technetium-99 & O & 49 & 0 & 0 & 0 & 0 & 48 & 1 & 49 \\
\hline Strontium lsotopes & 0 & 0 & 0 & 0 & 0 & 0 & 0 & 의 & 0 \\
\hline Strontium-89 & 0 & 0 & 0 & 0 & 0 & 0 & 0 & a) & 0 \\
\hline Strontium -90 & 27 & 5 & 2 & 0 & 0 & 27 & 6 & a & 34 \\
\hline lodine-129 & 27. & 2 & 0 & 0 & 0 & 29 & 0 & 0 & 29 \\
\hline Nickel-59 & 0 & 0 & 0 & 0 & 0 & 0 & 0 & 0 & 은 \\
\hline Total Uranium & 20 & 36 & 0 & 0 & 0 & 2 & 6 & 48 & 56 \\
\hline Total Thorium & 0 & 0 & 0 & 0 & 0 & 0 & 0 & o. & 의 \\
\hline Total Strontium & 10 & O) & 0 & 0 & 0. & 4 & 5 & 0 & 10 \\
\hline Other RAD & 75 & 161 & 3 & 0 & of & 33 & 182 & 24 & 239 \\
\hline TOTAL RAD & 382 & 516 & 12 & 01 & o] & 209 & 560 & 124 & 902 \\
\hline & & & & & & $\frac{1}{101}$ & & & \\
\hline Other Wet Chemistry & 22 & 1 & 0 & 01 & 으, & 18 & 5 & 0 & 23 \\
\hline TCLP Extraction & 7 & 70 & 0 & 0 & 0 & 1 & 64 & 12 & 77 \\
\hline Asbestos & 0 & 636 & 0 & 0 & 0 & 0 & 471 & 165 & 636 \\
\hline Industrial Hygiene, NOS & 1 & 0 & of & 0 & 0 & 0 & 0 & 0 & 1 \\
\hline Physical Properties & 3 & 22 & 0 & 0] & 0 & 3 & 19 & 3 & 25 \\
\hline Other, Not Otherwise Specified (NO & 10 & 1 & 0. & 0 & 0. & 11 & 0 & 10 & 11 \\
\hline TOTAL OTHER & 43 & 730 & of & 01 & O] & 23 & 560 & 190 & 773 \\
\hline TOTAL SAMPLE ANALYSES & 1030 & 2060 & 38 & 0 & 0 & $\frac{1}{633}$ & 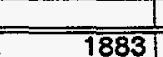 & 596 & 3121 \\
\hline
\end{tabular}


Table Vg: FY 99 Samples and Sample Analyses by Rad Level and Matrix Type (xi) Rocky Flats

\begin{tabular}{|c|c|c|c|c|c|c|c|c|c|}
\hline $\begin{array}{c}\text { Rad-Matrix/ } \\
\text { Sample-Sample Analysis }\end{array}$ & Ro & A1 & R2 & R3 & R4 & M1 & M2 & M3 & TOTAL \\
\hline CUSTOMER SAMPLES & 2053 & 5349 & 372 & 295 & 0 & 1811 & 2956 & 438 & 6637 \\
\hline & & & & & & & & & \\
\hline \multicolumn{10}{|l|}{ SAMPLE ANALYSES } \\
\hline Volatiles by GC/MS & 4253 & 750 & 85 & 33 & 0 & 2637 & 2281 & 204 & 5122 \\
\hline Volatiles by GC & 894 & 241 & 18 & 0 & 0 & 345 & 745 & 45 & 1144 \\
\hline Semi-Volatiles & 1828 & 687 & 85 & 28 & D & 1021 & 1540 & 57 & 2623 \\
\hline Herbicides/Pesticides/PCBs & 2228 & 851 & 0 & 0 & 0 & 772 & 2151 & 155 & 3079 \\
\hline Other Organics & 204 & 225 & 0 & 0 & 0 & 318 & 38 & 7 & 396 \\
\hline TOTAL ORGANICS & 9407 & 2754 & 189 & 61 & 0 & 5092 & 6756 & 469 & 12364 \\
\hline \multicolumn{10}{|l|}{ 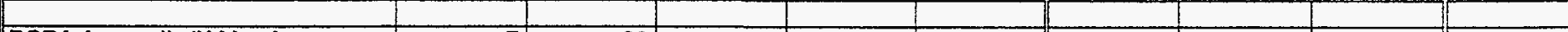 } \\
\hline RCRA Appendix IX Metals & 7 & 30 & 0 & 0 & 0 & 4 & 33 & 0 & 37 \\
\hline CAM (California) Metals (RCRA) & 2 & 1 & 0 & 0 & 0 & 3 & 2 & 0 & 4 \\
\hline RCRA TCLP Metals and EPTOX MeI & 382 & 164 & 18 & 0 & O & 295 & 209 & 19 & 543 \\
\hline EPA CLP Metals (CERCLA) & 5160 & 1344 & 234 & 69 & 0 & 2470 & 4155 & 183 & 6807 \\
\hline NPDES Metals (Clean Water Act) & 573 & 19 & 0 & 0 & 0 & 592 & 0 & 0 & 592 \\
\hline Drinking Water Metals (SDWA) & 92 & o & 0 & 0 & D. & 92 & 0 & 0 & 92 \\
\hline Metals by Other Regulatory Suite & 4657 & 이 & 0 & 0 & 0 & 2113 & 2304 & 357 & 4715 \\
\hline Metals by Suite, Other & 0 & 1164 & 44 & 0 & 0 & 657 & 362 & 6 & 1117 \\
\hline Metals by Metal Except Mercury & 449 & 267 & 4 & 0 & 요 & 366 & 314 & 요 & 699 \\
\hline Mercury & 940 & 309 & 4 & 0 & D. & 887 & 253 & 7 & 1200 \\
\hline Anions/Cations-IC & 899 & 439 & 0 & 0 & 0 & 1266 & 35 & 37 & 1338 \\
\hline Inorganics Wet Chemistry & 12618 & 887 & 132 & 1 & 0 & 10809 & 2218 & 616 & 13641 \\
\hline Other Inorganics & 796 & 301 & 0 & 0 & 0 & 773 & 279 & 45 & 1097 \\
\hline \multirow[t]{2}{*}{ TOTAL INORGANICS } & 26575 & 4926 & 437 & 70 & 0 & 20328 & 10164 & 1271 & 31885 \\
\hline & & & & & & & & & \\
\hline Gross Alpha/Beta & 9446 & 1860 & 79 & 0 & (0) & 4740 & 6314 & 198 & 11319 \\
\hline Gamma Scan & 3766 & 579 & 36 & 0 & 0 & 1818 & 2397 & 164 & 4380 \\
\hline Transuranic & 0 & 1 & 0 & 0 & 0 & 0 & 1 & 0 & 1 \\
\hline Plutonium Isotopes & 4688 & 357 & 24 & 0 & 0 & 1980 & 3016 & 73 & 5069 \\
\hline Uranium Isotopes & 4560 & 1032 & 24 & 0 & 용. & 2096 & 3436 & 84 & 5616 \\
\hline Curium Isotopes & 0 & 56 & 0 & 0 & 0 & 24 & 24 & 8 & 56 \\
\hline Thorium Isotopes & 39 & 226 & 24 & 0 & 0 & 64 & 186 & 39 & 289 \\
\hline Radium Isotopes & 1868 & 7 & 0 & 0 & 0 & 1424 & 444 & 7 & 1875 \\
\hline Americium-241 & 4475 & 132 & 24 & 0 & 0 & 1955 & 2641 & 35 & 4631 \\
\hline Neptunium-237 & 39 & 166 & 0 & 0 & 0 & 16 & 144 & 46 & 205 \\
\hline Polonium-210 & 2 & 8 & 0 & 0 & 의 & 1 & 2 & 7 & 9 \\
\hline Tritium & 2724 & 848 & 129 & 0 & ㅇ. & 2006 & 1607 & 21 & 3667 \\
\hline Radon-222 & 1 & 0 & 0 & 0 & 0 & 1 & 0 & 0 & 1 \\
\hline Lead-210 & 0 & 0 & 0 & 0 & 0 & 0 & 0 & 의 & 0 \\
\hline Carbon-14 & 0 & 0 & 0 & 0 & 요 & 0 & 0 & 의 & 0 \\
\hline Nickel-63 & 0 & D) & 0 & $\mathbf{0}$ & 0 & 0 & 0 & (0) & 0 \\
\hline Selenium-79 & 2 & 0 & 0 & 0 & 0 & 2 & 0 & 의 & 2 \\
\hline Technetium-99 & 20 & 35 & 7 & 0 & - & 27 & 28 & 7 & 62 \\
\hline Strontium Isotopes & 0 & 1 & 0 & 0 & 0. & 0 & 0 & 0 & 1 \\
\hline Strontium -89 & 0 & 0 & 0 & 0 & 0 & 0 & 0 & 의 & 0 \\
\hline Strontium-90 & 21 & 357 & 22 & 0 & 0 & 55 & 346 & 0 & 400 \\
\hline lodine-129 & 58 & 15 & 0 & 0 & 0. & 72 & 0 & ㅇ․ & 72 \\
\hline Nickel-59 & 0 & of & 0 & 0 & - & 0 & 0 & 의 & 0 \\
\hline Total Uranium & 211 & 11 & 0 & 0 & 0 & 66 & 0 & 156 & 222 \\
\hline Total Thorium & 0 & 1 & 0 & 0 & 0 & 1 & 0 & 0 & 1 \\
\hline Total Strontium & 2471 & 0 & 0 & 0 & 0 & 1283 & 1153 & 34 & 2471 \\
\hline Other RAD & 6050 & 671 & 89 & 231 & 0 & 2095 & 4541 & 405 & 7041 \\
\hline TOTAL $\overline{R A D}$ & 40437 & 6361 & 4581 & 231 & 0 & 19726 & 26280 & 1286 & 47390 \\
\hline & & & & & & & & & \\
\hline Other Wet Chemistry & \begin{tabular}{l|l|}
6331 \\
\end{tabular} & $\overline{71}$ & 0 & 0 & वा & 5344 & 995 & 0 & "6339 \\
\hline TCLP Extraction & 384 & 214 & 6 & 183 & 0 & 319 & 212 & 216 & 767 \\
\hline Asbestos & 25 & 149 & 0 & 0 & 0 & 1 & 174 & 0 & 175 \\
\hline Industrial Hygiene, NOS & 61 & 0 & 0 & 0 & 0 & 17 & 44 & 0 & 61 \\
\hline Physical Properties & 994 & 110 & 14 & 115 & 0 & 236 & 857 & 140 & 1234 \\
\hline Other, Not Otherwise Specified (NQ & 78 & 49 & 0 & 0 & 0 & 43 & 0 & 79 & 125 \\
\hline TOTAL OTHER & 7875 & 530 & 20 & 298 & 0 & 5960 & 2283 & 435 & 8700 \\
\hline & & & & & & & & & \\
\hline TOTAL SAMPLE ANALYSES & 84294 & 14570 & 1103 & 660 & 0 & 51107 & 45482 & 3460 & 100339 \\
\hline
\end{tabular}


Table Vg: FY 99 Samples and Sample Analyses by Rad Level and Matrix Type (xii) Savannah Aiver

\begin{tabular}{|c|c|c|c|c|c|c|c|c|c|}
\hline $\begin{array}{c}\text { Rad-Matrix } \\
\text { Sample-Sample Analysis }\end{array}$ & RO & R1 & R2 & R3 & $\overline{\mathrm{R} 4}$ & M1 & $\overline{M 2}$ & $\overline{\mathrm{MB}}$ & TOTAL \\
\hline CUSTOMER SAMPLES & 10272 & 19757 & 328 & 81 & 211 & 21421 & 1156 & 3248 & 28237 \\
\hline & & & & & & & & & \\
\hline \multicolumn{10}{|l|}{ SAMPLE ANALYSES } \\
\hline Volatiles by GC/MS & 179 & 9 & 0 & 0 & 0 & 132 & $\overline{\overline{0}}$ & 56 & 187 \\
\hline Volatiles by GC & 103 & 252 & 29 & 11 & 0 & 145 & 233 & 18 & 396 \\
\hline Semi-Volatiles & 1288 & 102 & 11 & 3 & 0 & 1016 & 88 & 301 & 1405 \\
\hline Herbicides/Pesticides/PCBs & 642 & 118 & 11 & 6 & 0 & 603 & 100 & 75 & 777 \\
\hline Other Organics & 2008 & 4664 & 0 & 0 & 0 & 1743 & 1 & 284 & 4350 \\
\hline TOTAL ORGANICS & 4220 & 5146 & 52 & 201 & 0 & 3639 & 422 & 733 & 7115 \\
\hline \multicolumn{10}{|l|}{20} \\
\hline RCRA Appendix IX Metals & 11 & 9 & 0 & 0 & 0 & 19 & 0 & 1 & 20 \\
\hline CAM (California) Metals (RCRA) & 80 & 0 & 0 & 0 & 0 & 80 & 0 & 0 & 80 \\
\hline RCRA TCLP Metals and EPTOX Me & 76 & 105 & 10 & 2 & 0 & 115 & 63 & 15 & 193 \\
\hline EPA CLP Metals (CERCLA) & 67 & 56 & 23 & 11 & 0 & 67 & 84 & 7 & 158 \\
\hline NPDES Metals (Clean Water Act) & 86 & 823 & $\overline{0}$ & 0 & 0 & 909 & 0 & 0 & 909 \\
\hline Drinking Water Metals (SDWA) & 0 & 0 & 0 & 0 & 0 & 0 & 0 & 0 & 0 \\
\hline Metals by Other Regulatory Suite & 426 & 5 & 0 & 0 & 0 & 303 & 1 & 128 & 431 \\
\hline Metals by Suite, Other & 2690 & 4647 & 0 & 0 & 0 & 1426 & 0 & 1267 & 5015 \\
\hline Metals by Metal Except Mercury & 1865 & 56 & 0 & 0 & 105 & 1091 & 50 & 810 & 1988 \\
\hline Mercury & 2064 & 4815 & 2 & 1 & 105 & 1440 & 56 & 772 & 4628 \\
\hline Anions/Cations - IC & 3854 & 88 & 0 & 0 & 211 & 3210 & 64 & 698 & 4062 \\
\hline Inorganics Wet Chemistry & 5211 & 177 & 102 & 23 & 0 & 4060 & 324 & 1129 & 5513 \\
\hline Other Inorganics & 268 & 108 & 0 & 0 & 0 & 280 & 50 & 45 & 376 \\
\hline TOTAL INORGANICS & 16698 & 10889 & 137 & 38 & 422 & 12998 & 693 & 4872 & 23373 \\
\hline \multicolumn{10}{|l|}{20} \\
\hline Gross Alpha/Beta & 2247 & 15551 & 58 & 72 & 211 & 12164 & 475 & 142 & 15459 \\
\hline Gamma Scan & 88 & 4921 & 52 & 29 & 110 & 4794 & 225 & 101 & 5160 \\
\hline Transuranic & 0 & 0 & 0 & 0 & 110 & 0 & 0 & 30 & 70 \\
\hline Plutonium Isotopes & 694 & 68 & 21 & 6 & 211 & 677 & 86 & 57 & 909 \\
\hline Uranium Isotopes & 696 & 91 & 9 & 5 & 211 & 678 & 95 & 58 & 921 \\
\hline Curium lsotopes & 0 & 17 & 3 & 2 & 10 & 2 & 10 & 10 & 27 \\
\hline Thorium Isotopes & 16 & 6 & 3 & 26 & 0 & 0 & 10 & 40 & 50 \\
\hline Radium Isotopes & 1 & 3 & 0 & 0 & 0 & 1 & 0 & 3 & 4 \\
\hline Americium-241 & 678 & 38 & 5 & 3 & 20 & 677 & 39 & 7 & 733 \\
\hline Neptunium-237 & 16 & 7 & 8 & 0 & 0 & 0 & 5 & 27. & 32 \\
\hline Polonium-210 & 0 & 3 & 0 & 0 & 0 & 0 & 0 & 3 & 3 \\
\hline Tritium & 591 & 63 & 28 & 6 & 0 & 592 & 60 & 7 & 673 \\
\hline Radon-222 & 0 & 0 & 0 & D) & 0 & 0 & 0 & 0 & 의 \\
\hline Lead-210 & 0 & 12 & 0 & 01 & 0 & 0 & 0 & 12 & 12 \\
\hline Carbon-14 & 0 & 0 & 0 & 0 & 0 & 0 & 0 & 0 & 0 \\
\hline Nickel-63 & 0 & 0 & 0 & 0 & 0 & 0 & 0 & 0 & 0 \\
\hline Selenium-79 & 80 & of & 0 & 0 & 0 & 80 & 0 & 0 & 80 \\
\hline Technetium-99 & 0 & 11 & 0 & 0 & 0 & 0 & 0 & 11 & 11 \\
\hline Strontium Isotopes & 0 & 0 & 0 & 0 & 0 & 0 & 0 & 0 & 0 \\
\hline Strontium -89 & 0 & 0 & 0 & 0 & 0 & 0 & 0 & 0 & 0 \\
\hline Strontium-90 & 80 & 41 & 23 & 6 & 0 & 82 & 69 & 0 & 150 \\
\hline lodine-129 & 80 & 6 & $\overline{0}$ & 0 & $\overline{0}$ & 87 & 0 & 0 & 87 \\
\hline Nickel-59 & 0 & 0 & 0 & 0 & 0 & 0 & 0 & 0 & 0 \\
\hline Total Uranium & 65 & 3 & 0 & 0 & 211 & 3 & 0 & 95 & 188 \\
\hline Total Thorium & 0 & O) & 0 & 0 & 0 & 0 & 0 & 0 & 0 \\
\hline Total Strontium & 15 & 12 & O & 0 & 0 & 19 & 0 & 8 & 27 \\
\hline Other RAD & 230 & 219 & 18 & 6 & 10 & 230 & 94 & 145 & 476 \\
\hline TOTAL RAD & 5578 & 21071 & 228 & 158 & 1104 & 20086 & 1169 & 754 & 25074 \\
\hline & & & & & & & & & \\
\hline Other Wet Chemistry & 1220 & 101 & 0 & 0 & 30 & 1230 & 0 & 30 & 1260 \\
\hline TCLP Extraction & 8 & 119 & 41 & 5 & 0 & 68 & 77 & 27 & 172 \\
\hline Asbestos & 0 & 0 & 0 & 0 & 0 & 0 & 3 & 0 & 1 \\
\hline Industrial Hygiene, NOS & 552 & O) & $\overline{0}$ & o) & 0 & 552 & 0 & 0 & 552 \\
\hline Physical Properties & 12128 & 26 & 6 & 2 & 0 & 7917 & 20 & 4226 & 12163 \\
\hline Other, Not Otherwise Specified (NO & 33 & 9 & 0 & of & 0 & 8 & 0 & 33 & 42 \\
\hline TOTAL OTHER & 13941 & 163 & 47 & 7 & 30 & 9775 & $9 \overline{9}$ & 4317 & 14189 \\
\hline & & & & & & & & & \\
\hline TOTAL SAMPLE ANALYSES & $404 \overline{36}$ & 37269 & 463 & 223 & 1556 & 46498 & 2383 & 10676 & 69752 \\
\hline
\end{tabular}


Table V: Total Sample Analyses by Fiscal Year

\begin{tabular}{|c|c|c|c|c|c|c|c|}
\hline $\begin{array}{l}\text { Samp Anall } \\
\text { Fiscal Year }\end{array}$ & $\overline{93}$ & 94 & 95 & 96 & 97 & 98 & 99 \\
\hline & & & & & & & \\
\hline SAMPIES TAKEN & & & & & & & \\
\hline CUSTOMER SAMPLES & 146050 & 301013 & 320843 & 227646 & 220200 & 228503 & 223051 \\
\hline \multicolumn{8}{|l|}{ SAMPLE ANALYSSES REQUESTED } \\
\hline \multicolumn{8}{|l|}{ ORGANICS } \\
\hline Volatiles by GC/MS & 21558 & 94610 & 88658 & 34869 & 33973 & 34849 & 33322 \\
\hline Volatiles by GC & 14734 & 31942 & 31260 & 9197 & 8651 & 9996 & 8577 \\
\hline Semi-Volatiles & 14169 & 62452 & 59485 & 25662 & 24810 & 25278 & 24443 \\
\hline Herbicides/Pesticides/PCBs & 20248 & 102186 & 102906 & 36186 & 35121 & 36868 & 35585 \\
\hline Other Organies & 18067 & 22894 & 23991 & 22394 & 19900 & 20169 & 19957 \\
\hline \multicolumn{8}{|l|}{ INORGANICS } \\
\hline RCRA Appendix IX Metals & 221 & 1480 & 1400 & 322 & 311 & 295 & 279 \\
\hline CAM (California) Metais (RCRA) & 715 & 794 & 828 & 823 & 842 & 840 & 837 \\
\hline RCRA TCLP Metals and EPTOX Me & 8949 & 46728 & 50097 & 12288 & 11484 & 12153 & 11649 \\
\hline EPA CLP Metals (CERCLA) & 19178 & 76406 & 63264 & 32313 & 31467 & 31162 & 30144 \\
\hline NPDES Metals (Clean Water Act) & 4953 & 9536 & 7602 & 6678 & 6497 & 6502 & 6398 \\
\hline Drinking Water Metals (SDWA) & 660 & 706 & 732 & 729 & 724 & 724 & 718 \\
\hline Metals by Other Regulatory Suite & 26117 & 96791 & 93585 & 27089 & 26270 & 27746 & 25863 \\
\hline Metals by Suite, Other & 18457 & 27593 & 24379 & 23106 & 22690 & 22691 & 22492 \\
\hline Metals by Metal Except Mercuny & 14873 & 83195 & 87989 & 21913 & 21104 & 21640 & 21013 \\
\hline Mercury & 26859 & 95086 & 98611 & 36242 & 35481 & 36056 & $\mathbf{3 5 3 7 0}$ \\
\hline Anions/Cations - IC & 15698 & 51739 & 48576 & 19565 & 19002 & 19017 & 18616 \\
\hline Inorganics Wet Chemistry & 85543 & 193146 & 188307 & 84017 & 81210 & 86126 & 80698 \\
\hline Other Inorganics & 10509 & 28691 & 26225 & 9350 & 8797 & 9318 & 8615 \\
\hline \multicolumn{8}{|l|}{ RADIOACTIVES } \\
\hline Gross Alpha/Beta & 101462 & 235747 & 220375 & 124082 & 121176 & 121704 & 120127 \\
\hline Gamma Scan & 37279 & 71079 & 66030 & 46384 & 45343 & 45940 & 45160 \\
\hline Transuranic & 2067 & 57348 & 59488 & 2451 & 2434 & 2064 & 2057 \\
\hline Plutonium Isotopes & 20249 & 48258 & 41403 & 26391 & 25647 & 25701 & 25272 \\
\hline Uranium Isotopes & 25194 & 123380 & 116610 & 37407 & 36250 & 36216 & 35449 \\
\hline Curium isotopes & 681 & 1721 & 1618 & 358 & 358 & 431 & 355 \\
\hline Thorium Isotopes & 3965 & 42573 & 44676 & 7468 & 7358 & 7652 & 7321 \\
\hline Radium Isolopes & 4901 & 10279 & 8184 & 7286 & 7104 & 7110 & 7005 \\
\hline Americium-241 & 16975 & 38337 & 32066 & 23491 & 22828 & 22718 & 22488 \\
\hline Neptunium-237 & .3546 & 46447 & 47453 & 3048 & 2934 & 2825 & 2473 \\
\hline Polonium-210 & 585 & 1737 & 1803 & 248 & 246 & 274 & 235 \\
\hline Tritium & 13347 & 31281 & 24288 & 19026 & 18328 & 18429 & 17900 \\
\hline Radon-222 & 23 & 57 & 59 & 4 & 4 & 8 & 4 \\
\hline Lead-210 & 47 & 50 & 52 & 52 & 51 & 51 & 51 \\
\hline Carbon-14 & 0 & 0 & 0 & 0 & 0 & 0 & 0 \\
\hline Nickel-63 & 0 & 0 & 0 & 0 & 0 & 0 & $\overline{0}$ \\
\hline Selenium -79 & 302 & 643 & 694 & 697 & 646 & 653 & 648 \\
\hline Technetium-99 & 3318 & 70961 & 75008 & 7132 & 7074 & 7054 & 6989 \\
\hline Strontium Isotopes & 29 & 70 & 72 & 5 & 5 & 9 & 5 \\
\hline Strontium -89 & 0 & 0 & 0 & 0 & 0 & 0 & 0 \\
\hline Strontium-90 & 2297 & 6361 & 5284 & 3381 & 3030 & 3139 & 3022 \\
\hline lodine-129 & 1240 & 2374 & 2499 & 1288 & 1201 & 1286 & 1196 \\
\hline Nickel-59 & 0 & 0 & 0 & 0 & 0 & 0 & 0 \\
\hline Total Uranium & 9422 & 67396 & 70676 & 7519 & 7437 & 8077 & 7036 \\
\hline Total Thorium & 502 & 673 & 529 & 526 & 501 & 383 & 237 \\
\hline Total Strontium & 6323 & 13663 & 10406 & 9930 & 9656 & 9609 & 9511 \\
\hline Other RAD & 30043 & 105865 & 103158 & 51811 & 50895 & 52880 & 49357 \\
\hline \multicolumn{8}{|l|}{ OTHER } \\
\hline Other Wet Chemistry & 22248 & 39507 & 32647 & 30693 & 30039 & 29987 & 29683 \\
\hline TCLP Extraction & 10343 & 60594 & 65824 & 17770 & 17261 & 17970 & 15921 \\
\hline Asbestos & 9568 & 39566 & 62206 & 59160 & 58701 & 64053 & 63739 \\
\hline Industrial Hygiene, NOS & 2543 & 2948 & 2874 & 2841 & 2770 & 2771 & 2756 \\
\hline Physical Properties & 38002 & 78649 & 81084 & 45271 & 45212 & 45190 & 43724 \\
\hline Other, Not Otherwise Specified (NO) & 3208 & 8620 & 8649 & 1084 & 1053 & 1372 & 845 \\
\hline & & & & & & & \\
\hline TOTAL SAMPLE ANALYSES & 691218 & 2232161 & 2183609 & 939545 & 913877 & 936985 & 905142 \\
\hline
\end{tabular}

\begin{tabular}{|l|r|r|r|r|r|r|r|}
\hline SA ClaSS/FY & \multicolumn{1}{|c|}{93} & 94 & 95 & 96 & 97 & 98 & 99 \\
\hline ORGANICS & 88776 & 314085 & 306300 & 128308 & 122455 & 127161 & 121884 \\
\hline INORGANICS & 232733 & 711893 & 691595 & 274436 & 265879 & 274269 & 262693 \\
\hline RADIOACTIVES & 283798 & 976299 & 932428 & 379982 & 370507 & 374212 & 363898 \\
\hline OTHER & 85912 & 229884 & 253285 & 156819 & 155036 & 161343 & 156667 \\
\hline \hline & $13 \%$ & $14 \%$ & $14 \%$ & $14 \%$ & $13 \%$ & $14 \%$ & $13 \%$ \\
\hline ORGANICS & $34 \%$ & $32 \%$ & $32 \%$ & $29 \%$ & $29 \%$ & $29 \%$ & $29 \%$ \\
\hline INORGANICS & $41 \%$ & $44 \%$ & $43 \%$ & $40 \%$ & $41 \%$ & $40 \%$ & $40 \%$ \\
\hline RADIOACTIVES & $12 \%$ & $10 \%$ & $12 \%$ & $17 \%$ & $17 \%$ & $17 \%$ & $17 \%$ \\
\hline OTHER & & & & &
\end{tabular}




\begin{tabular}{|l|l|}
\hline Activity: & PA/SI \\
\hline Unit: & OU \\
\hline Regulatory Driver: & \multicolumn{1}{c|}{0} \\
\hline Filename: & SUM2NEEA.WK3 \\
\hline Date: & September 21, 1994 \\
\hline
\end{tabular}

\begin{tabular}{|l|c|}
\hline Average S\&A Duration per Unit (yrs): \\
\hline Total Samples/Unit-yr: & 232.5 \\
\hline Total Samp Ans/Unit-yr: & 512.0 \\
\hline Average Batch Size: & \\
\hline \# of Unit-yrs in FY 93: & 5.2 \\
\hline
\end{tabular}

\begin{tabular}{|c|c|c|c|c|c|c|c|c|c|}
\hline & Ro & $\overline{\text { R1 }}$ & $\overline{\mathrm{R} 2}$ & $\overline{\mathrm{R3}}$ & R4 & $\overline{\mathrm{Mt}}$ & M2 & M3 & $\bar{C}$ \\
\hline SAMPLES TAKEN & & & & & & & & & \\
\hline CUSTOMER SAMPLES & 3.4 & 114.5 & 95.4 & 19.1 & 0.0 & 3.41 & 229.1 & 0.0 & 0.0 \\
\hline SAMPLE ANALYSES REQUESTED & & & & 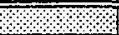 & & \% & & & \\
\hline ORGANICS & & & & & & & & & \\
\hline Volatiles by GC/MS & 0.0 & 0.0 & 0.0 & 0.0 & 0.0 & 0.0 & 0.0 & 0.0 & 0.0 \\
\hline Volatiles by GC & 0.0 & 26.2 & 7.6 & 3.8 & 0.0 & 0.0 & 37.6 & 0.0 & 0.0 \\
\hline Semi-Volatiles & 0.0 & 10.7 & 3.8 & 1.0 & 0.0 & 0.0 & 15.5 & 0.0 & 0.0 \\
\hline Herbicides/Pesticides/PCBs & 0.0 & 18.5 & 3.8 & 1.9 & 0.0 & 0.0 & 24.2 & 0.0 & 0.0 \\
\hline Other Organics & 0.0 & 0.4 & 0.0 & 0.0 & 0.0 & 0.0 & 0.4 & 0.0 & 0.0 \\
\hline INORGANICS & & & & & & & & & \\
\hline RCRA Appendix IX Metals & 0.0 & 0.0 & 0.0 & 0.0 & 0.0 & 0.0 & 0.0 & 0.0 & 0.0 \\
\hline CAM (California) Metals (RCRA) & 0.0 & 0.0 & 0.0 & 0.0 & 0.0 & 0.0 & 0.0 & 0.0 & 0.0 \\
\hline RCRA TCLP Metals and EPTOX Me & 0.4 & 2.5 & 1.1 & 0.8 & 0.0 & 0.4 & 4.4 & 0.0 & 0.0 \\
\hline EPA CLP Metals (CERCLA) & 0.0 & 16.2 & 7.6 & 3.8 & 0.0 & 0.0 & 27.7 & 0.0 & 0.0 \\
\hline NPDES Metals (Clean Water Act) & 0.0 & 0.0 & 0.0 & 0.0 & 0.0 & 0.0 & 0.0 & 0.0 & 0.0 \\
\hline Drinking Water Metals (SDWA) & 0.0 & 0.0 & 0.0 & 0.0 & 0.0 & 0.0 & 0.0 & 0.0 & 0.0 \\
\hline Metals by Other Regulatory Suite & 0.0 & 0.2 & 0.0 & 0.0 & 0.0 & 0.0 & 0.2 & 0.0 & 0.0 \\
\hline Metals by Suite, Other & 0.0 & 0.0 & 0.0 & 0.0 & 0.0 & 0.0 & 0.0 & 0.0 & 0.0 \\
\hline Metals by Metal Except Mercury & 0.0 & 0.0 & 0.0 & 0.0 & 0.0 & 0.0 & 0.0 & 0.0 & 0.0 \\
\hline Mercury & 0.0 & 1.9 & 0.8 & 0.4 & 0.0 & 0.0 & 3.1 & 0.0 & 0.0 \\
\hline Anions/Cations-IC & 0.0 & 1.5 & 0.0 & 0.0 & 0.0 & 0.0 & 1.5 & 0.0 & 0.0 \\
\hline Inorganics Wet Chemistry & 0.0 & 15.3 & 15.3 & 7.6 & 0.0 & 0.0 & 38.2 & 0.0 & 0.0 \\
\hline Other Inorganics & 6.9 & 0.0 & 0.0 & 0.0 & 0.0 & 6.9 & 0.0 & 0.0 & 0.0 \\
\hline RADIOACTIVES & 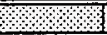 & & 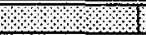 & & & $\%$ & & h & \\
\hline Gross Alpha/Beta & 0.0 & 205.2 & 17.2 & 23.9 & 0.0 & 0.01 & 78.3 & 0.0 & 0.0 \\
\hline Gamma Scan & 0.0 & 17.2 & 17.2 & 9.5 & 0.0 & 0.0 & 43.9 & 0.0 & 0.0 \\
\hline Transuranic & 0.0 & 0.0 & 0.0 & 0.0 & 0.0 & 0.0 & 0.01 & 0.0 & 0.0 \\
\hline Plutonium Isotopes & 0.0 & 21.8 & 3.8 & 1.9 & 0.0 & 0.0 & 27.5 & 0.0 & 0.0 \\
\hline Uranium Isotopes & 0.0 & 27.5 & 0.4 & 1.7 & 0.0 & 0.0 & 29.6 & 0.0 & 0.0 \\
\hline Curium Isotopes & 0.0 & 1.3 & 1.1 & 0.6 & 0.0 & 0.0 & 3.1 & 0.0 & 0.0 \\
\hline Thorium isotopes & 0.0 & 1.9 & 1.0 & 0.6 & 0.0 & 0.0 & 3.4 & 0.0 & 0.0 \\
\hline Radium Isotopes & 0.0 & 0.0 & 0.0 & 0.0 & 0.0 & 0.0 & 0.0 & 0.0 & 0.0 \\
\hline Americium-241 & 0.0 & 9.5 & 1.7 & 1.0 & 0.0 & 0.0 & 12.2 & 0.0 & 0.0 \\
\hline Neptunium -237 & 0.0 & 1.5 & 0.0 & 0.0 & 0.0 & 0.0 & 1.5 & 0.0 & 0.0 \\
\hline Polonium-210 & 0.0 & 0.0 & 0.0 & 0.0 & 0.0 & 0.0 & 0.0 & 0.0 & 0.0 \\
\hline Tritium & 0.0 & 7.1 & 3.8 & 1.9 & 0.0 & 0.0 & 12.8 & 0.0 & 0.0 \\
\hline Radon-222 & 0.0 & 0.0 & 0.0 & 0.0 & 0.0 & 0.0 & 0.0 & 0.0 & 0.0 \\
\hline Lead-210 & 0.0 & 0.0 & 0.0 & 0.0 & 0.0 & 0.0 & 0.0 & 0.0 & 0.0 \\
\hline Carbon-14 & 0.0 & 0.0 & 0.0 & 0.0 & 0.0 & 0.0 & 0.0 & 0.0 & 0.0 \\
\hline Nickel-63 & 0.0 & 0.0 & 0.0 & 0.0 & 0.0 & 0.0 & 0.0 & 0.0 & 0.0 \\
\hline Selenium-79 & 0.0 & 0.0 & 0.0 & 0.0 & 0.0 & 0.0 & 0.0 & 0.0 & 0.0 \\
\hline Technetium-99 & 0.0 & 0.0 & 0.0 & 0.0 & 0.0 & 0.0 & 0.0 & 0.0 & 0.0 \\
\hline Strontium Isotopes & 0.0 & 0.0 & 0.0 & 0.0 & 0.0 & 0.0 & 0.0 & 0.0 & 0.0 \\
\hline Strontium -89 & 0.0 & 0.0 & 0.0 & 0.0 & 0.0 & 0.0 & 0.0 & 0.0 & 0.0 \\
\hline Strontium-90 & 0.0 & 12.4 & 5.7 & 1.9 & 0.0 & 0.0 & 20.0 & 0.0 & 0.0 \\
\hline lodine-129 & 0.0 & 0.0 & 0.0 & 0.0 & 0.0 & 0.0 & 0.0 & 0.0 & 0.0 \\
\hline Nickel-59 & 0.0 & 0.0 & 0.0 & 0.0 & 0.0 & 0.0 & 0.0 & 0.0 & 0.0 \\
\hline Total Uranium & 0.0 & 0.0 & 0.0 & 0.0 & 0.0 & 0.0 & 0.0 & 0.0 & 0.0 \\
\hline Total Thorium & 0.0 & 0.0 & 0.0 & 0.0 & 0.0 & 0.0 & 0.0 & 0.0 & 0.0 \\
\hline Total Strontium & 0.0 & 0.0 & 0.0 & 0.0 & 0.0 & 0.0 & 0.0 & 0.0 & 0.0 \\
\hline Other RAD & 0.0 & 9.7 & 3.8 & 1.9 & 0.0 & 0.0 & 15.5 & 0.0 & 0.0 \\
\hline OTHER & & & & & & & & & \\
\hline Other Wet Chemistry & 0.0 & 0.0 & 0.0 & 0.0 & 0.0 & 0.01 & 0.01 & 0.0 & 0.0 \\
\hline TCLP Extraction & 0.4 & 9.4 & 2.5 & 1.5 & 0.0 & 0.4 & 13.4 & 0.0 & 0.0 \\
\hline Asbestos & 0.0 & 0.0 & 0.0 & 0.0 & 0.0 & 0.0 & 0.0 & 0.0 & 0.0 \\
\hline Industrial Hygiene, NOS & 0.0 & 0.0 & 0.0 & 0.0 & 0.0 & 0.0 & 0.0 & 0.0 & 0.0 \\
\hline Physical Properties & 0.0 & 3.6 & 2.1 & 0.8 & 0.0 & 0.0 & 6.5 & 0.0 & 0.0 \\
\hline Other, Not Otherwise Specified (NO & 0.0 & 0.0 & 0.0 & 0.0 & 0.0 & 0.0 & 0.0 & 0.0 & 0.0 \\
\hline
\end{tabular}




\begin{tabular}{|l|l|}
\hline Activity: & RI/FS \\
\hline Unit: & OU \\
\hline Regulatory Driver: & 0 \\
\hline Filename: & SUM2NEED.WK3 \\
\hline Date: & September 21, 1994 \\
\hline
\end{tabular}

\begin{tabular}{|l|c|}
\hline Average S\&A Duration per Unit (yrs): \\
\hline Total Samples/Unit-yr: & 224.1 \\
\hline Total Samp Ans/Unit-yr: & 2020.9 \\
\hline Average Batch Size: & \\
\hline \# of Unit-yrs in FY 93: & 16.4 \\
\hline
\end{tabular}

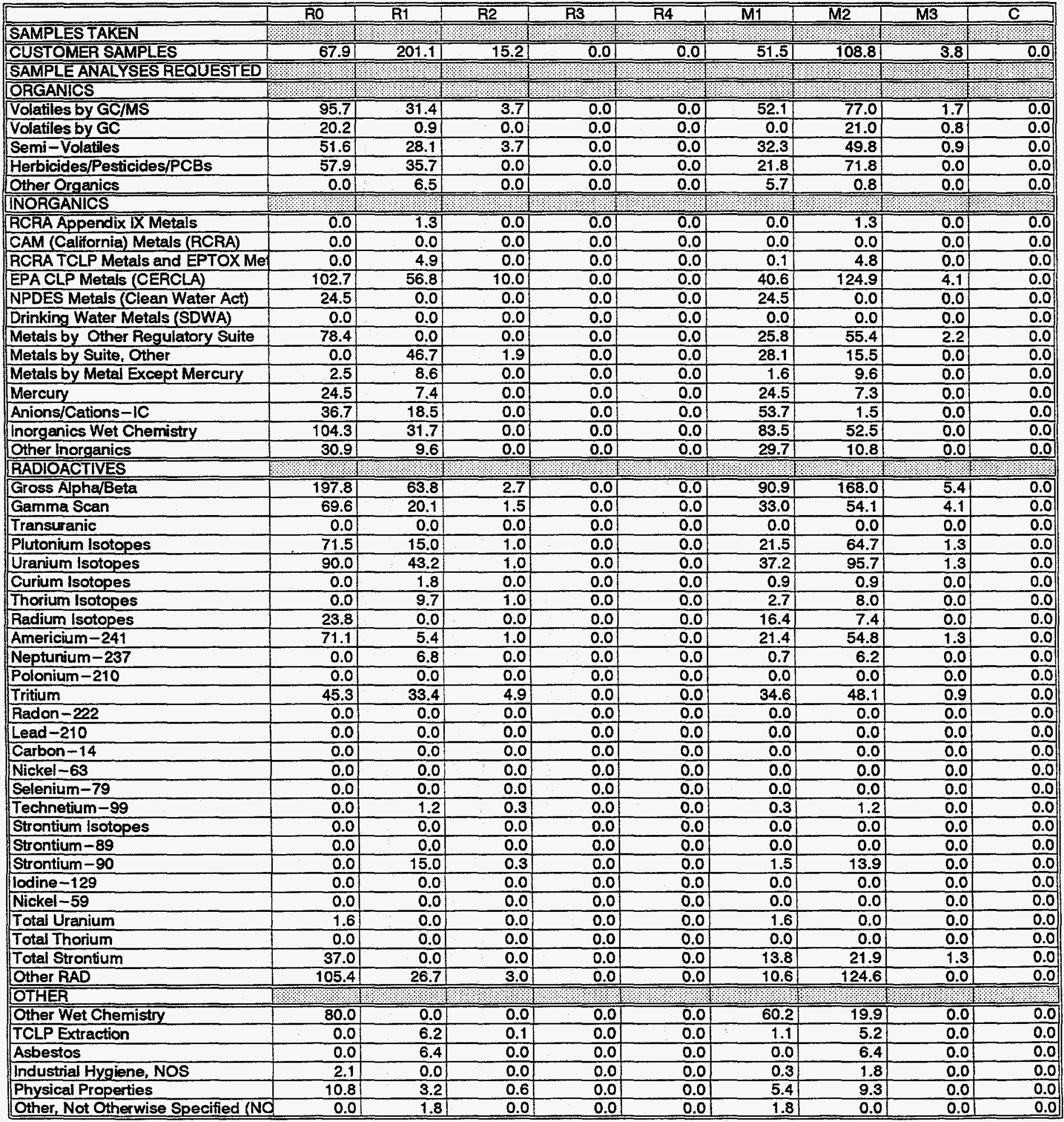




\begin{tabular}{|l|l|}
\hline Activity: & RD/RA \\
\hline Unit: & OU \\
\hline Regulatory Driver: & \multicolumn{1}{c|}{0} \\
\hline Filename: & SUM2NEEC.WK3 \\
\hline Date: & September 21.1994 \\
\hline
\end{tabular}

\begin{tabular}{|l|l|}
\hline Average S\&A Duration per Unit (yrs): \\
\hline Total Samples/Unit-yr: & 239.0 \\
\hline Total Samp Ans/Unit-yr: & 446.5 \\
\hline Average Batch Size: & \\
\hline \# of Unit-yrs in FY 93: & 6.1 \\
\hline
\end{tabular}

\begin{tabular}{|c|c|c|c|c|c|c|c|c|c|}
\hline \multirow{2}{*}{ SAMPLES TAKEN } & \multirow[t]{2}{*}{ RO } & \multirow[t]{2}{*}{ R1 } & \multirow[t]{2}{*}{$\mathrm{R2}$} & \multirow[t]{2}{*}{ A3 } & \multirow[t]{2}{*}{$\overline{\mathrm{R} 4}$} & \multirow[t]{2}{*}{ M1 } & \multirow[t]{2}{*}{ M2 } & \multirow[t]{2}{*}{$\mathrm{M} 3$} & \multirow[t]{2}{*}{$\mathrm{C}$} \\
\hline & & & & & & & & & \\
\hline CUSTOMER SAMPLES & 180.0 & 58.91 & 0.0 & 0.01 & 0.0 & 98.2 & 124.4 & 16.4 & 0.0 \\
\hline SAMPLE ANALYSES REQUESTED & & & & & & & & & 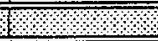 \\
\hline ORGANICS & & & & & & & & & \\
\hline Volatiles by GC/MS & 0.0 & 0.0 & 0.0 & 0.0 & 0.0 & 0.0 & 0.0 & 0.0 & 0.0 \\
\hline Volatiles by GC & 8.2 & 11.1 & 0.0 & 0.0 & 0.0 & 4.6 & 13.1 & 1.6 & 0.0 \\
\hline Semi-Volatiles & 8.2 & 4.3 & 0.0 & 0.0 & 0.0 & 1.6 & 9.8 & 1.0 & 0.0 \\
\hline Herbicides/Pesticides/PCBs & 0.0 & 14.7 & 0.0 & 0.0 & 0.0 & 8.2 & 3.3 & 3.3 & 0.0 \\
\hline Other Organics & 81.8 & 3.3 & 0.0 & 0.0 & 0.0 & 1.6 & 82.7 & 0.8 & 0.0 \\
\hline INORGANICS & & & & & & & & & \\
\hline RCRA Appendix IX Metals & 0.0 & 0.0 & 0.0 & 0.0 & 0.01 & 0.0 & 0.0 & 0.0 & 0.0 \\
\hline CAM (California) Metals (RCRA) & 0.0 & 0.0 & 0.0 & 0.0 & 0.0 & 0.0 & 0.0 & 0.0 & 0.0 \\
\hline RCRA TCLP Metals and EPTOX Mel & 8.2 & 18.0 & 0.0 & 0.0 & 0.0 & 8.2 & 14.7 & 3.3 & 0.0 \\
\hline EPA CLP Metals (CERCLA) & 0.0 & 0.0 & 0.0 & 0.0 & 0.0 & 0.0 & 0.0 & 0.0 & 0.0 \\
\hline NPDES Metals (Clean Water Act) & 0.0 & 0.0 & 0.0 & 0.0 & 0.0 & 0.0 & 0.0 & 0.0 & 0.0 \\
\hline Drinking Water Metals (SDWA) & 0.0 & 0.0 & 0.0 & 0.0 & 0.0 & 0.0 & 0.0 & 0.0 & 0.0 \\
\hline Metals by Other Regulatory Suite & 0.0 & 7.7 & 0.0 & 0.0 & 0.0 & 0.0 & 3.3 & 2.8 & 1.6 \\
\hline Metals by Suite, Other & 0.0 & 0.0 & 0.0 & 0.0 & 0.0 & 0.0 & 0.0 & 0.0 & 0.0 \\
\hline Metals by Metal Except Mercury & 8.2 & 13.9 & 0.0 & 0.0 & 0.0 & 3.3 & 17.2 & 1.6 & 0.0 \\
\hline Mercury & 8.2 & 4.7 & 0.0 & 0.0 & 0.0 & 1.6 & 10.6 & 0.7 & 0.0 \\
\hline Anions/Cations-IC & 0.0 & 4.7 & 0.0 & 0.0 & 0.0 & 1.6 & 1.6 & 1.5 & 0.0 \\
\hline Inorganics Wet Chemistry & 0.0 & 65.0 & 0.0 & 0.0 & 0.0 & 32.7 & 16.4 & 15.9 & 0.0 \\
\hline Other Inorganics & 0.0 & 11.0 & 0.0 & 0.0 & 0.0 & 4.9 & 4.4 & 1.6 & 0.0 \\
\hline RADIOACTIVES & & & & & & & & & \\
\hline Gross Alpha/Beta & 0.0 & 39.0 & 0.0 & 0.0 & 0.0 & 16.4 & 30.8 & 8.2 & 0.0 \\
\hline Gamma Scan & 0.0 & 14.7 & 0.0 & 0.0 & 0.0 & 9.8 & 3.3 & 1.6 & 0.0 \\
\hline Transuranic & 0.0 & 0.0 & 0.0 & 0.0 & 0.0 & 0.0 & 0.0 & 0.0 & 0.0 \\
\hline Plutonium isotopes & 0.0 & 7.2 & 0.0 & 0.0 & 0.0 & 3.3 & 2.3 & 1.6 & 0.0 \\
\hline Uranium Isotopes & 0.0 & 9.7 & 0.0 & 0.0 & 0.0 & 3.3 & 4.7 & 1.6 & 0.0 \\
\hline Curium Isotopes & 0.0 & 0.0 & 0.0 & 0.0 & 0.0 & 0.0 & 0.0 & 0.0 & 0.0 \\
\hline Thorium Isotopes & 0.0 & 0.0 & 0.0 & 0.0 & 0.0 & 0.0 & 0.0 & 0.0 & 0.0 \\
\hline Radium Isotopes & 0.0 & 0.0 & 0.0 & 0.0 & 0.0 & 0.0 & 0.0 & 0.0 & 0.0 \\
\hline Americium-241 & 0.0 & 4.6 & 0.0 & 0.0 & 0.0 & 1.6 & 1.6 & 1.3 & 0.0 \\
\hline Neptunium - 237 & 0.0 & 1.8 & 0.0 & 0.0 & 0.0 & 0.8 & 0.8 & 0.2 & 0.0 \\
\hline Polonium-210 & 0.0 & 0.0 & 0.0 & 0.0 & 0.0 & 0.0 & 0.0 & 0.0 & 0.0 \\
\hline Tritium & 0.0 & 5.4 & 0.0 & 0.0 & 0.0 & 1.6 & 3.1 & 0.7 & 0.0 \\
\hline Radon-222 & 0.0 & 0.0 & 0.0 & 0.0 & 0.0 & 0.0 & 0.0 & 0.0 & 0.0 \\
\hline Lead -210 & 0.0 & 0.0 & 0.0 & 0.0 & 0.0 & 0.0 & 0.0 & 0.0 & 0.0 \\
\hline Carbon-14 & 0.0 & 0.0 & 0.0 & 0.0 & 0.0 & 0.0 & 0.0 & 0.0 & 0.0 \\
\hline Nickel-63 & 0.0 & 0.0 & 0.0 & 0.0 & 0.0 & 0.0 & 0.0 & 0.0 & 0.0 \\
\hline Selenium-79 & 0.0 & 2.6 & 0.0 & 0.0 & 0.0 & 1.5 & 0.7 & 0.5 & 0.0 \\
\hline Technetium-99 & 0.0 & 0.0 & 0.0 & 0.0 & 0.0 & 0.0 & 0.0 & 0.0 & 0.0 \\
\hline Strontium Isotopes & 0.0 & 0.0 & 0.0 & 0.0 & 0.0 & 0.0 & 0.0 & 0.0 & 0.0 \\
\hline Strontium -89 & 0.0 & 0.0 & 0.0 & 0.0 & 0.0 & 0.0 & 0.0 & 0.0 & 0.0 \\
\hline Strontium-90 & 0.0 & 12.9 & 0.0 & 0.0 & 0.0 & 6.5 & 4.7 & 1.6 & 0.0 \\
\hline lodine-129 & 0.0 & 3.8 & 0.0 & 0.0 & 0.0 & 1.6 & 1.3 & 0.8 & 0.0 \\
\hline Nickel-59 & 0.0 & 0.0 & 0.0 & 0.0 & 0.0 & 0.0 & 0.0 & 0.0 & 0.0 \\
\hline Total Uranium & 0.0 & 0.0 & 0.0 & 0.0 & 0.0 & 0.0 & 0.0 & 0.0 & 0.0 \\
\hline Total Thorium & 0.0 & 0.0 & 0.0 & 0.0 & 0.0 & 0.0 & 0.0 & 0.0 & 0.0 \\
\hline Total Strontium & 0.0 & 0.0 & 0.0 & 0.0 & 0.0 & 0.0 & 0.0 & 0.0 & 0.0 \\
\hline Other RAD & 0.0 & 20.5 & 0.0 & 0.0 & 0.0 & 9.8 & 9.0 & 1.6 & 0.0 \\
\hline OTHER & & & & 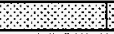 & $\%$ & & & & \\
\hline Other Wet Chemistry & 0.0 & 0.0 & 0.0 & 0.0 & 0.0 & 0.0 & 0.0 & 0.0 & 0.0 \\
\hline TCLP Extraction & 8.2 & 26.2 & 0.0 & 0.0 & 0.0 & 16.4 & 14.7 & 3.3 & 0.0 \\
\hline Asbestos & 0.0 & 0.0 & 0.0 & 0.0 & 0.0 & 0.0 & 0.0 & 0.0 & 0.0 \\
\hline Industrial Hygiene, NOS & 0.0 & 1.5 & 0.0 & 0.0 & 0.0 & 0.8 & 0.5 & 0.2 & 0.0 \\
\hline Physical Properties & 0.0 & 0.0 & 0.0 & 0.0 & 0.0 & 0.0 & 0.0 & 0.0 & 0.0 \\
\hline Other, Not Otherwise Specified (NO & 0.0 & 0.0 & 0.0 & 0.0 & 0.0 & 0.0 & 0.0 & 0.0 & 0.0 \\
\hline
\end{tabular}




\begin{tabular}{|l|l|}
\hline Activity: & RFC \\
\hline Unit: & OU \\
\hline Regulatory Driver: & O \\
\hline Filename: & SUM2NEDD.WK3 \\
\hline Date: & September 21, 1994 \\
\hline
\end{tabular}

\begin{tabular}{|l|c|}
\hline Average S\&A Duration per Unit (yrs): \\
\hline Total Samples/Unit-yr: & 11.5 \\
\hline Total Samp Ans/Unit-yr: & 295.2 \\
\hline Average Batch Size: & \\
\hline \# of Unit-yrs in FY 93: & 6.8 \\
\hline
\end{tabular}

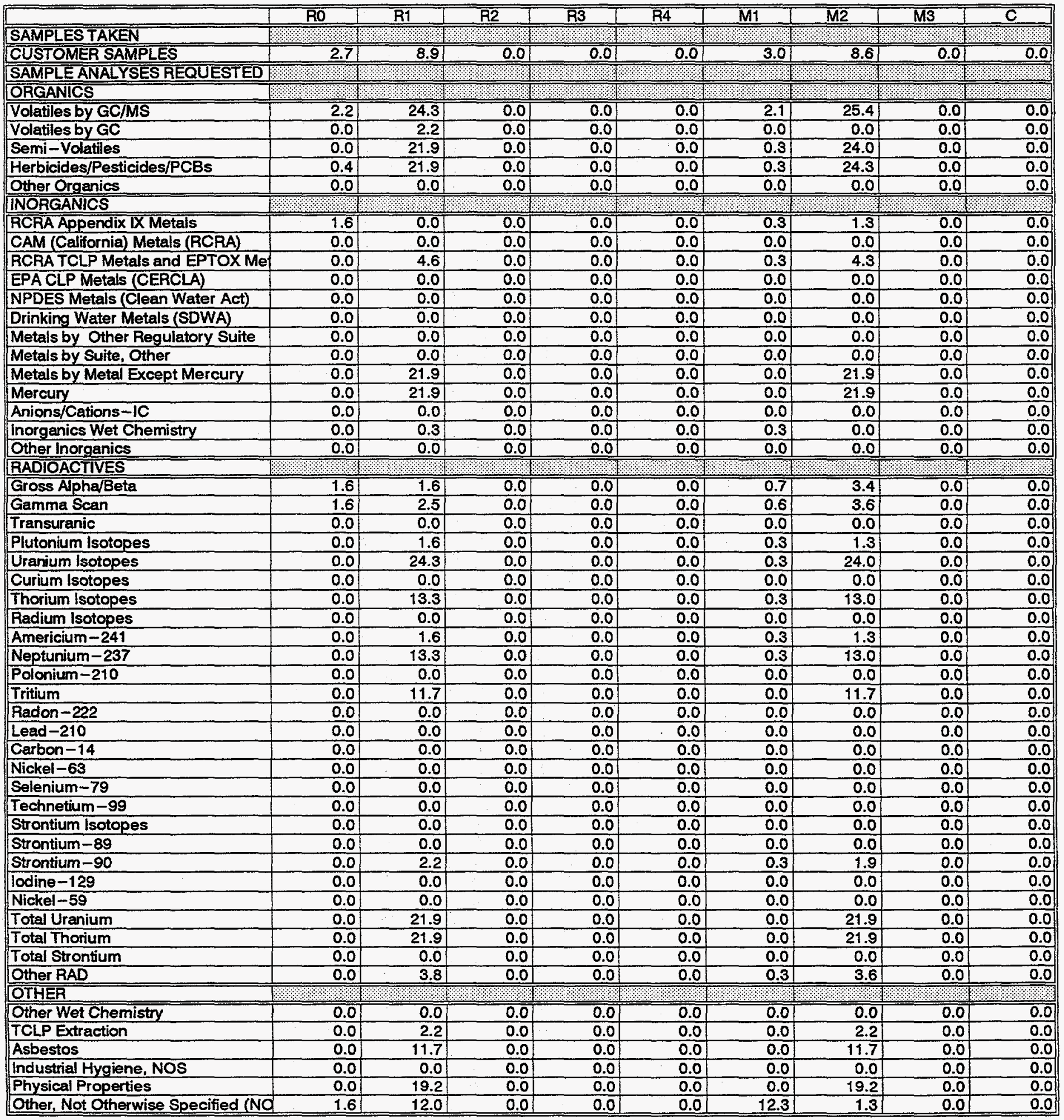




\begin{tabular}{|l|l|}
\hline Activity: & D\&D \\
\hline Unit: & Facility \\
\hline Regulatory Driver: & 0 \\
\hline Filename: & SUM2NEEE.WK3 \\
\hline Date: & September 21, 1994 \\
\hline
\end{tabular}

\begin{tabular}{|l|c|}
\hline Average S\&A Duration per Unit (yrs): \\
\hline Total Samples/Unit-yr: & 4482.8 \\
\hline Total Samp Ans/Unit-yr: & 10685.9 \\
\hline Average Batch Size: & \\
\hline$\#$ of Unit-yrs in FY 93: & 0.9 \\
\hline
\end{tabular}

\begin{tabular}{|c|c|c|c|c|c|c|c|c|c|}
\hline & $\overline{R O}$ & $\overline{\mathrm{R1}}$ & $\overline{\mathrm{R} 2}$ & $\overline{\mathrm{R3}}$ & $\overline{\mathrm{R} 4}$ & $\overline{\mathrm{M} 1}$ & $\overline{\mathrm{M} 2}$ & $\overline{\mathrm{M} 3}$ & $\mathrm{C}$ \\
\hline SAMPLESTAKEN & & & & & & & & & \\
\hline CUSTOMER SAMPLES & 22.2 & 4460.6 & 0.0 & 0.0 & 0.01 & 59.9 & 2850.2 & 1572.7 & 0.0 \\
\hline SAMPLE ANALYSES REQUESTED & & & & & & & & & \\
\hline ORGANICS & & & & & & & & & \\
\hline Volatiles by GC/MS & 0.0 & 559.4 & 0.0 & 0.0 & 0.0 & 62.2 & 485.0 & 12.2 & 0.0 \\
\hline Volatiles by GC & 0.0 & 0.0 & 0.0 & 0.0 & 0.0 & 0.0 & 0.0 & 0.0 & 0.0 \\
\hline Semi-Volatiles & 0.0 & 441.7 & 0.0 & 0.0 & 0.0 & 1.1 & 436.2 & 4.4 & 0.0 \\
\hline Herbicides/Pesticides/PCBs & 0.0 & 1028.9 & 0.0 & 0.0 & 0.0 & 12.2 & 795.8 & 220.9 & 0.0 \\
\hline Other Organics & 0.0 & 0.0 & 0.0 & 0.0 & 0.0 & 0.0 & 0.0 & 0.0 & 0.0 \\
\hline INORGANICS & & & & & & & & & \\
\hline RCRA Appendix IX Metals & 0.0 & 0.0 & 0.0 & 0.0 & 0.0 & 0.0 & 0.0 & 0.0 & 0.0 \\
\hline CAM (California) Metals (RCRA) & 0.0 & 0.0 & 0.0 & 0.0 & 0.0 & 0.0 & 0.0 & 0.0 & 0.0 \\
\hline RCRA TCLP Metals and EPTOX Me & 22.2 & 307.4 & 0.0 & 0.0 & 0.0 & 1.1 & 326.3 & 2.2 & 0.0 \\
\hline EPA CLP Metals (CERCLA) & 0.0 & 0.0 & 0.0 & 0.0 & 0.0 & 0.0 & 0.0 & 0.0 & 0.0 \\
\hline NPDES Metais (Clean Water Act) & 0.0 & 0.0 & 0.0 & 0.0 & 0.0 & 0.0 & 0.0 & 0.0 & 0.0 \\
\hline Drinking Water Metals (SDWA) & 0.0 & 0.0 & 0.0 & 0.0 & 0.0 & 0.0 & 0.0 & 0.0 & 0.0 \\
\hline Metals by Other Regulatory Suite & 0.0 & 205.3 & 0.0 & 0.0 & 0.0 & 6.7 & 192.0 & 26.6 & 0.0 \\
\hline Metals by Suite, Other & 0.0 & 24.4 & 0.0 & 0.0 & 0.0 & 3.3 & 0.0 & 1.1 & 0.0 \\
\hline Metals by Metal Except Mercury & 22.2 & 459.5 & 0.0 & 0.0 & 0.0 & 0.0 & 168.7 & 313.0 & 0.0 \\
\hline Mercury & 22.2 & 513.9 & 0.0 & 0.0 & 0.0 & 11.1 & 516.1 & 8.9 & 0.0 \\
\hline Anions/Cations-IC & 0.0 & 10.0 & 0.0 & 0.0 & 0.0 & 0.0 & 3.3 & 6.7 & 0.0 \\
\hline Inorganics Wet Chemistry & 22.2 & 326.3 & 0.0 & 0.0 & 0.0 & 15.5 & 320.8 & 12.2 & 0.0 \\
\hline Other Inorganics & 0.0 & 22.2 & 0.0 & 0.0 & 0.0 & 0.0 & 22.2 & 0.0 & 0.0 \\
\hline RADIOACTIVES & 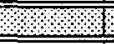 & & & & & & & & \\
\hline Gross Alpha/Beta & 0.0 & 192.0 & 0.0 & 0.0 & 0.01 & 10.0 & 180.9 & 1.1 & 0.0 \\
\hline Gamma Scan & 0.0 & 188.7 & 0.0 & 0.0 & 0.0 & 6.7 & 180.9 & 1.1 & 0.0 \\
\hline Transuranic & 0.0 & 13.3 & 0.0 & 0.0 & 0.0 & 0.0 & 13.3 & 0.0 & 0.0 \\
\hline Plutonium Isotopes & 0.0 & 2.2 & 0.0 & 0.0 & 0.0 & 0.0 & 1.1 & 1.1 & 0.0 \\
\hline Uranium Isotopes & 0.0 & 311.9 & 0.0 & 0.0 & 0.0 & 6.7 & 284.1 & 21.1 & 0.0 \\
\hline Curium Isotopes & 0.0 & 0.0 & 0.0 & 0.0 & 0.0 & 0.0 & 0.0 & 0.0 & 0.0 \\
\hline Thorium Isotopes & 0.0 & 290.8 & 0.0 & 0.0 & 0.0 & 6.7 & 283.0 & 1.1 & 0.0 \\
\hline Radium Isotopes & 0.0 & 0.0 & 0.0 & 0.0 & 0.0 & 0.0 & 0.0 & 0.0 & 0.0 \\
\hline Americium-241 & 0.0 & 6.7 & 0.0 & 0.0 & 0.0 & 6.7 & 0.0 & 0.0 & 0.0 \\
\hline Neptunium-237 & 0.0 & 10.0 & 0.0 & 0.0 & 0.0 & 0.0 & 8.9 & 1.1 & 0.0 \\
\hline Polonium-210 & 0.0 & 0.0 & 0.0 & 0.0 & 0.0 & 0.0 & 0.0 & 0.0 & 0.0 \\
\hline Tritium & 0.0 & 0.0 & 0.0 & 0.0 & 0.0 & 0.0 & 0.0 & 0.0 & 0.0 \\
\hline Radon-222 & 0.0 & 0.0 & 0.0 & 0.0 & 0.0 & 0.0 & 0.0 & 0.0 & 0.0 \\
\hline Lead-210 & 0.0 & 0.0 & 0.0 & 0.0 & 0.0 & 0.0 & 0.0 & 0.0 & 0.0 \\
\hline Carbon-14 & 0.0 & 0.0 & 0.0 & 0.0 & 0.0 & 0.0 & 0.0 & 0.0 & 0.0 \\
\hline Nickel-63 & 0.0 & 0.0 & 0.0 & 0.0 & 0.0 & 0.0 & 0.0 & 0.0 & 0.0 \\
\hline Selenium-79 & 0.0 & 0.0 & 0.0 & 0.0 & 0.0 & 0.0 & 0.0 & 0.0 & 0.0 \\
\hline Technetium-99 & 0.0 & 284.1 & 0.0 & 0.0 & 0.0 & 0.0 & 284.1 & 0.0 & 0.0 \\
\hline Strontium Isotopes & 0.0 & 0.0 & 0.0 & 0.0 & 0.0 & 0.0 & 0.0 & 0.0 & 0.0 \\
\hline Strontium -89 & 0.0 & 0.0 & 0.0 & 0.0 & 0.0 & 0.0 & 0.0 & 0.0 & 0.0 \\
\hline Strontium-90 & 0.0 & 1.1 & 0.0 & 0.0 & 0.0 & 0.0 & 1.1 & 0.0 & 0.0 \\
\hline lodine -129 & 0.0 & 0.0 & 0.0 & 0.0 & 0.0 & 0.0 & 0.0 & 0.0 & 0.0 \\
\hline Nickel-59 & 0.0 & 0.0 & 0.0 & 0.0 & 0.0 & 0.0 & 0.0 & 0.0 & 0.0 \\
\hline Total Uranium & 0.0 & 206.4 & 0.0 & 0.0 & 0.0 & 0.0 & 38.8 & 167.6 & 0.0 \\
\hline Total Thorium & 0.0 & 0.0 & 0.0 & 0.0 & 0.0 & 0.0 & 0.0 & 0.0 & 0.0 \\
\hline Total Strontium & 0.0 & 0.0 & 0.0 & 0.0 & 0.0 & 0.0 & 0.0 & 0.0 & 0.0 \\
\hline Other RAD & 0.0 & 899.0 & 0.0 & 0.0 & 0.0 & 0.0 & 887.9 & 11.1 & 0.0 \\
\hline OTHER & 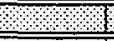 & 10 & 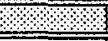 & 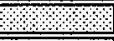 & 8 & + & 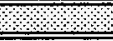 & & \\
\hline Other Wet Chemistry & 0.0 & 0.0 & 0.0 & 0.0 & 0.0 & 0.0 & 0.0 & 0.0 & 0.0 \\
\hline TCLP Extraction & 22.2 & 364.0 & 0.0 & 0.0 & 0.0 & 1.1 & 340.7 & 44.4 & 0.0 \\
\hline Asbestos & 0.0 & 3804.7 & 0.0 & 0.0 & 0.0 & 0.0 & 2815.8 & 988.9 & 0.0 \\
\hline Industrial Hygiene, NOS & 0.0 & 0.0 & 0.0 & 0.0 & 0.0 & 0.0 & 0.0 & 0.0 & 0.0 \\
\hline Physical Properties & 0.0 & 101.0 & 0.0 & 0.0 & 0.0 & 0.0 & 101.0 & 0.0 & 0.0 \\
\hline Other, Not Otherwise Specified (NO & 0.0 & 0.0 & 0.0 & 0.0 & 0.0 & 0.0 & 0.0 & 0.0 & 0.0 \\
\hline
\end{tabular}




\begin{tabular}{|l|l|}
\hline Activity: & GW MON \\
\hline Unit: & Well \\
\hline Regulatory Driver: & \multicolumn{1}{|c|}{ 0 } \\
\hline Filename: & SUM2NEEF.WK3 \\
\hline Date: & September 21, 1994 \\
\hline
\end{tabular}

\begin{tabular}{|c|c|}
\hline Average S\&A Duration per Unit (yrs & \\
\hline Total Samples/Unit-yr: & 0.4 \\
\hline Total Samp Ans/Unit-yr: & 5.2 \\
\hline Average Batch Size: & \\
\hline \# of Unit-yrs in FY 93: & 249.0 \\
\hline
\end{tabular}

\begin{tabular}{|c|c|c|c|c|c|c|c|c|c|}
\hline & $\overline{\mathrm{RO}}$ & R1 & R2 & $\overline{\mathrm{R} 3}$ & $\overline{\mathrm{R} 4}$ & M1 & M2 & $\overline{\mathrm{M} 3}$ & $\mathrm{C}$ \\
\hline SAMPLES TAKEN & & & & & & & & & \\
\hline CUSTOMER SAMPLES & 0.4 & 0.0 & 0.01 & 0.01 & 0.0 & 0.4 & 0.0 & 0.0 & 0.0 \\
\hline SAMPLE ANALYSES REQUESTED & & & & & & & & & \\
\hline OAGANICS & & & & & & & & & \\
\hline Volatiles by $\mathrm{GC} / \mathrm{MS}$ & 0.0 & 0.0 & 0.0 & 0.0 & 0.0 & 0.0 & 0.0 & 0.0 & 0.0 \\
\hline Volatiles by $\mathrm{GC}$ & 0.0 & 0.0 & 0.0 & 0.0 & 0.0 & 0.0 & 0.0 & 0.0 & 0.0 \\
\hline Semi-Volatiles & 0.0 & 0.0 & 0.0 & 0.0 & 0.0 & 0.0 & 0.0 & 0.0 & 0.0 \\
\hline Herbicides/Pesticides/PCBs & 0.4 & 0.0 & 0.0 & 0.0 & 0.0 & 0.4 & 0.0 & 0.0 & 0.0 \\
\hline Other Organics & 0.0 & 0.0 & 0.0 & 0.0 & 0.0 & 0.0 & 0.0 & 0.0 & 0.0 \\
\hline \multicolumn{10}{|l|}{ INORGANICS } \\
\hline RCRA Appendix X Metals & 0.0 & 0.0 & 0.0 & 0.0 & 0.0 & 0.01 & 0.0 & 0.0 & 0.0 \\
\hline CAM (California) Metals (RCRA) & 0.4 & 0.0 & 0.0 & 0.0 & 0.0 & 0.4 & 0.0 & 0.0 & 0.0 \\
\hline RCRA TCLP Metals and EPTOX MeI & 0.0 & 0.0 & 0.0 & 0.0 & 0.0 & 0.0 & 0.0 & 0.0 & 0.0 \\
\hline EPA CLP Metals (CERCLA) & 0.0 & 0.0 & 0.0 & 0.0 & 0.0 & 0.0 & 0.0 & 0.0 & 0.0 \\
\hline NPDES Metals (Clean Water Act) & 0.0 & 0.0 & 0.0 & 0.0 & 0.0 & 0.0 & 0.0 & 0.0 & 0.0 \\
\hline Drinking Water Metals (SDWA) & 0.0 & 0.0 & 0.0 & 0.0 & 0.0 & 0.0 & 0.0 & 0.0 & 0.0 \\
\hline Metals by Other Regulatory Suite & 0.0 & 0.0 & 0.0 & 0.0 & 0.0 & 0.0 & 0.0 & 0.0 & 0.0 \\
\hline Metals by Suite, Other & 0.0 & 0.0 & 0.0 & 0.0 & 0.0 & 0.0 & 0.0 & 0.0 & 0.0 \\
\hline Metals by Metal Except Mercury & 0.4 & 0.0 & 0.0 & 0.0 & 0.0 & 0.4 & 0.0 & 0.0 & 0.0 \\
\hline Mercury & 0.4 & 0.0 & 0.0 & 0.0 & 0.0 & 0.4[ & 0.0 & 0.0 & 0.0 \\
\hline Anions/Cations-IC & 0.0 & 0.0 & 0.0 & 0.0 & 0.0 & 0.0 & 0.0 & 0.0 & 0.0 \\
\hline Inorganics Wet Chemistry & 2.0 & 0.0 & 0.0 & 0.0 & 0.0 & 2.0 & 0.0 & 0.0 & 0.0 \\
\hline Other Inorganics & 0.0 & 0.0 & 0.0 & 0.0 & 0.0 & 0.0 & 0.0 & 0.0 & 0.0 \\
\hline \multicolumn{10}{|l|}{ AADIOACTIVES } \\
\hline Gross Alpha/Beta & 0.0 & 0.0 & 0.0 & 0.0 & 0.0 & 0.0 & 0.01 & 0.01 & 0.0 \\
\hline Gamma Scan & 0.0 & 0.0 & 0.0 & 0.0 & 0.0 & 0.0 & 0.0 & 0.0 & 0.0 \\
\hline Transuranic & 0.0 & 0.0 & 0.0 & 0.0 & 0.0 & 0.0 & 0.0 & 0.0 & 0.0 \\
\hline Plutonium Isotopes & 0.0 & 0.0 & 0.0 & 0.0 & 0.0 & 0.0 & 0.0 & 0.0 & 0.0 \\
\hline Uranium Isotopes & 0.0 & 0.0 & 0.0 & 0.0 & 0.0 & 0.0 & 0.0 & 0.0 & 0.0 \\
\hline Curium lsotopes & 0.0 & 0.0 & 0.0 & 0.0 & 0.0 & 0.0 & 0.0 & 0.0 & 0.0 \\
\hline Thorium Isotopes & 0.0 & 0.0 & 0.0 & 0.0 & 0.0 & 0.0 & 0.0 & 0.0 & 0.0 \\
\hline Radium Isotopes & 0.0 & 0.0 & 0.0 & 0.0 & 0.0 & 0.0 & 0.0 & 0.0 & 0.0 \\
\hline Americium-241 & 0.0 & 0.0 & 0.0 & 0.0 & 0.0 & 0.0 & 0.0 & 0.0 & 0.0 \\
\hline Neptunium-237 & 0.0 & 0.0 & 0.0 & 0.0 & 0.0 & 0.0 & 0.0 & 0.0 & 0.0 \\
\hline Polonium-210 & 0.0 & 0.0 & 0.0 & 0.0 & 0.0 & 0.0 & 0.0 & 0.0 & 0.0 \\
\hline Tritium & 0.0 & 0.0 & 0.0 & 0.0 & 0.0 & 0.0 & 0.0 & 0.0 & 0.0 \\
\hline Radon-222 & 0.0 & 0.0 & 0.0 & 0.0 & 0.0 & 0.0 & 0.0 & 0.0 & 0.0 \\
\hline Lead-210 & 0.0 & 0.0 & 0.0 & 0.01 & 0.0 & 0.0 & 0.0 & 0.0 & 0.0 \\
\hline Carbon-14 & 0.0 & 0.0 & 0.0 & 0.0 & 0.0 & 0.0 & 0.0 & 0.0 & 0.0 \\
\hline Nickel-63 & 0.0 & 0.0 & 0.0 & 0.0 & 0.0 & 0.0 & 0.0 & 0.0 & 0.0 \\
\hline Selenium - 79 & 0.4 & 0.0 & 0.0 & 0.0 & 0.0 & 0.4 & 0.0 & 0.0 & 0.0 \\
\hline Technetium -99 & 0.0 & 0.0 & 0.0 & 0.0 & 0.0 & 0.0 & 0.0 & 0.0 & 0.0 \\
\hline Strontium Isotopes & 0.0 & 0.0 & 0.0 & 0.0 & 0.0 & 0.0 & 0.0 & 0.0 & 0.0 \\
\hline Strontium-89 & 0.0 & 0.0 & 0.0 & 0.0 & 0.0 & 0.0 & 0.0 & 0.0 & 0.0 \\
\hline Strontium -90 & 0.4 & 0.0 & 0.0 & 0.0 & 0.0 & 0.4 & 0.0 & 0.0 & 0.0 \\
\hline lodine-129 & 0.4 & 0.0 & 0.0 & 0.0 & 0.0 & 0.4 & 0.0 & 0.0 & 0.0 \\
\hline Nickel-59 & 0.0 & 0.0 & 0.0 & 0.0 & 0.0 & 0.0 & 0.0 & 0.0 & 0.0 \\
\hline Total Uranium & 0.0 & 0.0 & 0.0 & 0.0 & 0.0 & 0.0 & 0.0 & 0.0 & 0.0 \\
\hline Total Thorium & 0.0 & 0.0 & 0.0 & 0.0 & 0.0 & 0.0 & 0.0 & 0.0 & 0.0 \\
\hline Total Strontium & 0.0 & 0.0 & 0.0 & 0.0 & 0.0 & 0.0 & 0.0 & 0.0 & 0.0 \\
\hline Other RAD & 0.4 & 0.0 & 0.0 & 0.0 & 0.0 & 0.4 & 0.0 & 0.0 & 0.0 \\
\hline \multicolumn{10}{|l|}{ OTHER } \\
\hline Other Wet Chemistry & 0.0 & 0.0 & 0.0 & 0.0 & 0.0 & 0.0 & 0.0 & 0.0 & 0.0 \\
\hline TCLP Extraction & 0.0 & 0.0 & 0.0 & 0.0 & 0.0 & 0.0 & 0.0 & 0.0 & 0.0 \\
\hline Asbestos & 0.0 & 0.0 & 0.0 & 0.0 & 0.0 & 0.0 & 0.0 & 0.0 & 0.0 \\
\hline Industrial Hygiene, NOS & 0.0 & 0.0 & 0.0 & 0.0 & 0.0 & 0.0 & 0.0 & 0.0 & 0.0 \\
\hline Physical Properties & 0.0 & 0.0 & 0.0 & 0.0 & 0.0 & 0.0 & 0.0 & 0.0 & 0.0 \\
\hline Other, Not Otherwise Specified (NQ & 0.0 & 0.0 & 0.0 & 0.0 & 0.0 & 0.0 & 0.0 & 0.0 & 0.0 \\
\hline
\end{tabular}




\begin{tabular}{|l|l|}
\hline Activity: & EFF MON \\
\hline Unit: & Station \\
\hline Regulatory Driver: & 0 \\
\hline Flename: & SUM2NEEG.WK3 \\
\hline Date: & September 21, 1994 \\
\hline
\end{tabular}

\begin{tabular}{|c|c|c|c|c|c|c|c|c|c|}
\hline \multirow{2}{*}{ SAMPLES TAKEN } & Ro & $\overline{\mathrm{R} 1}$ & R2 & $\mathbf{R 3}$ & R4 & M1 & $\mathrm{M2}$ & M3 & $\mathrm{C}$ \\
\hline & & & & & & & & & \\
\hline CUSTOMER SAMPLES & 0.5 & 10.9 & 0.0 & 0.0 & 0.01 & 8.5 & 0.2 & 0.1 & 0.0 \\
\hline SAMPLE ANALYSES REQUESTED & & & & & $\%$ & 11 & \ে: & & \\
\hline ORGANICS & & & & & 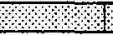 & & & & \\
\hline Volatiles by GC/MS & 0.1 & 0.0 & 0.0 & 0.0 & 0.0 & 0.1 & 0.0 & 0.0 & 0.0 \\
\hline Volatiles by GC & 0.0 & 0.0 & 0.0 & 0.0 & 0.0 & 0.1 & 0.0 & 0.0 & 0.0 \\
\hline Semi-Volatiles & 0.1 & 0.0 & 0.0 & 0.0 & 0.0 & 0.1 & 0.0 & 0.0 & 0.0 \\
\hline Herbicides/Pesticides/PCBs & 0.3 & 0.0 & 0.0 & 0.0 & 0.0 & 0.3 & 0.0 & 0.0 & 0.0 \\
\hline Other Organics & 0.0 & 2.6 & 0.0 & 0.0 & 0.0 & 0.01 & 0.0 & 0.0 & 0.0 \\
\hline INORGANICS & & & & & & & & & \\
\hline RCRA Appendix IX Metals & 0.0 & 0.0 & 0.0 & 0.0 & 0.01 & 0.0 & 0.0 & 0.0 & 0.0 \\
\hline CAM (California) Metals (RCRA) & 0.0 & 0.0 & 0.0 & 0.0 & 0.0 & 0.0 & 0.0 & 0.0 & 0.0 \\
\hline RCRA TCLP Metals and EPTOX Me & 0.0 & 0.0 & 0.0 & 0.0 & 0.0 & 0.1 & 0.0 & 0.0 & 0.0 \\
\hline EPA CLP Metals (CERCLA) & 0.0 & 0.0 & 0.0 & 0.0 & 0.0 & 0.0 & 0.0 & 0.0 & 0.0 \\
\hline NPDES Metals (Clean Water Act) & 0.0 & 0.5 & 0.0 & 0.0 & 0.0 & 0.5 & 0.0 & 0.0 & 0.0 \\
\hline Drinking Water Metals (SDWA) & 0.0 & 0.0 & 0.0 & 0.0 & 0.0 & 0.0 & 0.0 & 0.0 & 0.0 \\
\hline Metals by Other Regulatory Suite & 0.2 & 0.0 & 0.0 & 0.0 & 0.0 & 0.2 & 0.0 & 0.0 & 0.0 \\
\hline Metals by Suite, Other & 0.0 & 2.6 & 0.0 & 0.0 & 0.0 & 0.0 & 0.0 & 0.0 & 0.0 \\
\hline Metals by Metal Except Mercury & 0.1 & 0.0 & 0.0 & 0.0 & 0.0 & 0.1 & 0.0 & 0.0 & 0.0 \\
\hline Mercury & 0.2 & 2.7 & 0.0 & 0.0 & 0.0 & 0.3 & 0.0 & 0.0 & 0.0 \\
\hline Anions/Cations $-1 \mathrm{C}$ & 0.1 & 0.0 & 0.0 & 0.0 & 0.0 & 0.1 & 0.0 & 0.0 & 0.0 \\
\hline Inorganics Wet Chemistry & 0.8 & 0.0 & 0.0 & 0.0 & 0.0 & 0.7 & 0.1 & 0.0 & 0.0 \\
\hline Other Inorganics & 0.1 & 0.0 & 0.0 & 0.0 & 0.01 & 0.11 & 0.0 & 0.0 & 0.0 \\
\hline RADIOACTIVES & & & & & & & & & \\
\hline Gross Alpha/Beta & 1.3 & 8.4 & 0.0 & 0.0 & 0.0 & 6.9 & 0.1 & 0.0 & 0.0 \\
\hline Gamma Scan & 0.0 & 2.8 & 0.0 & 0.0 & 0.0 & 2.7 & 0.0 & 0.0 & 0.0 \\
\hline Transuranic & 0.0 & 0.0 & 0.0 & 0.0 & 0.0 & 0.0 & 0.0 & 0.0 & 0.0 \\
\hline Plutonium lsotopes & 0.4 & 0.0 & 0.0 & 0.0 & 0.0 & 0.4 & 0.0 & 0.0 & 0.0 \\
\hline Uranium Isotopes & 0.4 & 0.0 & 0.0 & 0.0 & 0.0 & 0.4 & 0.0 & 0.0 & 0.0 \\
\hline Curium Isotopes & 0.0 & 0.0 & 0.0 & 0.0 & 0.0 & 0.0 & 0.0 & 0.0 & 0.0 \\
\hline Thorium Isotopes & 0.0 & 0.0 & 0.0 & 0.0 & 0.0 & 0.0 & 0.0 & 0.0 & 0.0 \\
\hline Radium isotopes & 0.0 & 0.0 & 0.0 & 0.0 & 0.0 & 0.0 & 0.0 & 0.0 & 0.0 \\
\hline Americium-241 & 0.4 & 0.0 & 0.0 & 0.0 & 0.0 & 0.4 & 0.0 & 0.0 & 0.0 \\
\hline Neptunium-237 & 0.0 & 0.0 & 0.0 & 0.0 & 0.0 & 0.0 & 0.0 & 0.0 & 0.0 \\
\hline Polonium-210 & 0.0 & 0.0 & 0.0 & 0.0 & 0.0 & 0.0 & 0.0 & 0.0 & 0.0 \\
\hline Tritium & 0.3 & 0.0 & 0.0 & 0.0 & 0.0 & 0.3 & 0.0 & 0.0 & 0.0 \\
\hline Radon-222 & 0.0 & 0.0 & 0.0 & 0.0 & 0.0 & 0.0 & 0.0 & 0.0 & 0.0 \\
\hline Lead-210 & 0.0 & 0.0 & 0.0 & 0.0 & 0.0 & 0.0 & 0.0 & 0.0 & 0.0 \\
\hline Carbon-14 & 0.0 & 0.0 & 0.0 & 0.0 & 0.0 & 0.0 & 0.0 & 0.0 & 0.0 \\
\hline Nickel-63 & 0.0 & 0.0 & 0.0 & 0.0 & 0.0 & 0.0 & 0.0 & 0.0 & 0.0 \\
\hline Selenium-79 & 0.0 & 0.0 & 0.0 & 0.0 & 0.0 & 0.0 & 0.0 & 0.0 & 0.0 \\
\hline Technetium - 99 & 0.0 & 0.0 & 0.0 & 0.0 & 0.0 & 0.0 & 0.0 & 0.0 & 0.0 \\
\hline Strontium Isotopes & 0.0 & 0.0 & 0.0 & 0.0 & 0.0 & 0.0 & 0.0 & 0.0 & 0.0 \\
\hline Strontium-89 & 0.0 & 0.0 & 0.0 & 0.0 & 0.0 & 0.0 & 0.0 & 0.0 & 0.0 \\
\hline Strontium-90 & 0.0 & 0.0 & 0.0 & 0.0 & 0.0 & 0.0 & 0.0 & 0.0 & 0.0 \\
\hline lodine-129 & 0.0 & 0.0 & 0.0 & 0.0 & 0.0 & 0.0 & 0.0 & 0.0 & 0.0 \\
\hline Nickel-59 & 0.0 & 0.0 & 0.0 & 0.0 & 0.0 & 0.0 & 0.0 & 0.0 & 0.0 \\
\hline Total Uranium & 0.0 & 0.0 & 0.0 & 0.0 & 0.0 & 0.0 & 0.0 & 0.0 & 0.0 \\
\hline Total Thorium & 0.0 & 0.0 & 0.0 & 0.0 & 0.0 & 0.0 & 0.0 & 0.0 & 0.0 \\
\hline Total Strontium & 0.0 & 0.0 & 0.0 & 0.0 & 0.0 & 0.0 & 0.0 & 0.0 & 0.0 \\
\hline Other RAD & 0.0 & 0.1 & 0.0 & 0.0 & 0.0 & 0.1 & 0.0 & 0.0 & 0.0 \\
\hline OTHER & & & & & & & 17: & & \\
\hline Other Wet Chemistry & 0.7 & 0.0 & 0.0 & 0.0 & 0.0 & 0.7 & 0.0 & 0.0 & 0.0 \\
\hline TCLP Extraction & 0.0 & 0.0 & 0.0 & 0.0 & 0.0 & 0.0 & 0.0 & 0.0 & 0.0 \\
\hline Asbestos & 0.0 & 0.0 & 0.0 & 0.0 & 0.0 & 0.0 & 0.0 & 0.0 & 0.0 \\
\hline Industrial Hygiene, NOS & 0.3 & 0.0 & 0.0 & 0.0 & 0.0 & 0.3 & 0.0 & 0.0 & 0.0 \\
\hline Physical Properties & 0.0 & 0.0 & 0.0 & 0.0 & 0.0 & 0.0 & 0.0 & 0.0 & 0.0 \\
\hline Other, Not Otherwise Specified (NO & 0.0 & 0.0 & 0.0 & 0.0 & 0.0 & 0.0 & 0.0 & 0.0 & 0.0 \\
\hline
\end{tabular}

\begin{tabular}{|l|c|}
\hline Average S\&A Duration per Unit (yrs): \\
\hline Total Samples/Unit-yr: & 10.1 \\
\hline Total Samp Ans/Unit-yr: & 20.9 \\
\hline Average Batch Size: & \\
\hline \# of Unit-yrs in FY 93: & 2653.0 \\
\hline
\end{tabular}




\begin{tabular}{|c|c|c|c|c|c|c|c|c|c|}
\hline Activity: & TRU & & & Average S\& & A Duration $p$ & Der Unit (yrs) & & & \\
\hline Unit: & Drum & & & Total Samp & les/Unit-yr: & & 1.5 & & \\
\hline Regulatory Driver: & 0 & & & Total Samp & Ans/Unit-yr & & 3.3 & & \\
\hline Filename: & SUM2NEEH. & WK3 & & Average Ba & teh Size: & & & & \\
\hline Date: & September 2 & 21,1994 & & \# of Unit -y & rs in FY 93: & & 200.0 & & \\
\hline & $\overline{\mathrm{AO}}$ & $\mathrm{R1}$ & R2 & $\overline{\mathrm{R3}}$ & $\overline{\mathrm{R4}}$ & M1 & M2 & $\overline{\mathrm{MB}}$ & $\bar{C}$ \\
\hline SAMPLES TAKEN & & & & & & & & & \\
\hline CUSTOMER SAMPLES & 0.0 & 0.0 & 0.0 & 1.5 & 0.0 & 0.0 & 0.0 & 1.5 & 0.0 \\
\hline SAMPLE ANALYSES AEQUESTED & & & & & & & & & \\
\hline OAGANICS & & $\underline{1+1}$ & & 3 & & & & 3 & \\
\hline Volatiles by GC/MS & 0.01 & 0.0 & 0.0 & 0.2 & 0.0 & 0.0 & 0.01 & 0.2 & 0.0 \\
\hline Volatiles by GC & 0.0 & 0.0 & 0.0 & 0.0 & 0.0 & 0.0 & 0.0 & 0.0 & 0.0 \\
\hline Semi-Volaties & 0.0 & 0.0 & 0.0 & 0.1 & 0.0 & 0.0 & 0.0 & 0.1 & 0.0 \\
\hline Herbicides/Pesticides/PCBs & 0.0 & 0.0 & 0.0 & 0.0 & 0.0 & 0.0 & 0.0 & 0.0 & 0.0 \\
\hline Other Organics & 0.0 & 0.0 & 0.0 & 0.0 & 0.0 & 0.0 & 0.0 & 0.0 & 0.0 \\
\hline INORGANICS & & & & & & & & & \\
\hline RCRA Appendix IX Metals & 0.0 & 0.0 & 0.0 & 0.0 & 0.0 & 0.0 & 0.0 & 0.0 & 0.0 \\
\hline CAM (California) Metals (RCRA) & 0.0 & 0.0 & 0.0 & 0.0 & 0.0 & 0.0 & 0.0 & 0.0 & 0.0 \\
\hline RCRA TCLP Metals and EPTOX MeI & 0.0 & 0.0 & 0.0 & 0.0 & 0.0 & 0.0 & 0.0 & 0.0 & 0.0 \\
\hline EPA CLP Metals (CERCLA) & 0.0 & 0.0 & 0.0 & 0.3 & 0.0 & 0.0 & 0.0 & 0.3 & 0.0 \\
\hline NPDES Metals (Clean Water Act) & 0.0 & 0.0 & 0.0 & 0.0 & 0.0 & 0.0 & 0.0 & 0.0 & 0.0 \\
\hline Drinking Water Metals (SDWA) & 0.0 & 0.0 & 0.0 & 0.0 & 0.0 & 0.0 & 0.0 & 0.0 & 0.0 \\
\hline Metals by Other Regulatory Suite & 0.0 & 0.0 & 0.0 & 0.0 & 0.0 & 0.0 & 0.0 & 0.0 & 0.0 \\
\hline Metals by Suite, Other & 0.0 & 0.0 & 0.0 & 0.0 & 0.0 & 0.0 & 0.0 & 0.0 & 0.0 \\
\hline Metals by Metal Except Mercury & 0.0 & 0.0 & 0.0 & 0.0 & 0.0 & 0.0 & 0.0 & 0.0 & 0.0 \\
\hline Mercury & 0.0 & 0.0 & 0.0 & 0.0 & 0.0 & 0.0 & 0.0 & 0.0 & 0.0 \\
\hline Anions/Cations - IC & 0.0 & 0.0 & 0.0 & 0.0 & 0.0 & 0.0 & 0.0 & 0.0 & 0.0 \\
\hline Inorganics Wet Chemistry & 0.0 & 0.0 & 0.0 & 0.0 & 0.0 & 0.0 & 0.0 & 0.0 & 0.0 \\
\hline Other Inorganics & 0.0 & 0.0 & 0.0 & 0.0 & 0.0 & 0.0 & 0.0 & 0.0 & 0.0 \\
\hline RADIOACTIVES & 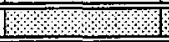 & 3 & 3 & 16 & & אै & एक & & 3 \\
\hline Gross Alpha/Beta & 0.0 & 0.0 & 0.0 & 0.0 & 0.0 & 0.0 & 0.0 & 0.0 & 0.0 \\
\hline Gamma Scan & 0.0 & 0.0 & 0.0 & 0.0 & 0.0 & 0.0 & 0.0 & 0.0 & 0.0 \\
\hline Transuranic & 0.0 & 0.0 & 0.0 & 0.0 & 0.0 & 0.0 & $0 . \overline{0}$ & 0.0 & 0.0 \\
\hline Plutonium Isotopes & 0.0 & 0.0 & 0.0 & 0.0 & 0.0 & 0.0 & 0.0 & 0.0 & 0.0 \\
\hline Uranium lsotopes & 0.0 & 0.0 & 0.0 & 0.0 & 0.0 & 0.0 & 0.0 & 0.0 & 0.0 \\
\hline Curium Isotopes & 0.0 & 0.0 & 0.0 & 0.0 & 0.0 & 0.0 & 0.0 & 0.0 & 0.0 \\
\hline Thorium Isotopes & 0.0 & 0.0 & 0.0 & 0.0 & 0.0 & 0.0 & 0.0 & 0.0 & 0.0 \\
\hline Radium isotopes & 0.0 & 0.0 & 0.0 & 0.0 & 0.0 & 0.0 & 0.0 & 0.0 & 0.0 \\
\hline Americium-241 & 0.0 & 0.0 & 0.0 & 0.0 & 0.0 & 0.0 & 0.0 & 0.0 & 0.0 \\
\hline Neptunium-237 & 0.0 & 0.0 & 0.0 & 0.0 & 0.0 & 0.0 & 0.0 & 0.0 & 0.0 \\
\hline Polonium-210 & 0.0 & 0.0 & 0.0 & 0.0 & 0.0 & 0.0 & 0.0 & 0.0 & 0.0 \\
\hline Tritium & 0.0 & 0.0 & 0.0 & 0.0 & 0.0 & 0.0 & 0.0 & 0.0 & 0.0 \\
\hline Radon-222 & 0.0 & 0.0 & 0.0 & 0.0 & 0.0 & 0.0 & 0.0 & 0.0 & 0.0 \\
\hline Lead-210 & 0.0 & 0.0 & 0.0 & 0.0 & 0.0 & 0.0 & 0.0 & 0.0 & 0.0 \\
\hline Carbon-14 & 0.0 & 0.0 & 0.0 & 0.0 & 0.0 & 0.0 & 0.0 & 0.0 & 0.0 \\
\hline Nickel-63 & 0.0 & 0.0 & 0.0 & 0.0 & 0.0 & 0.0 & 0.0 & 0.0 & 0.0 \\
\hline Selenium-79 & 0.0 & 0.0 & 0.0 & 0.0 & 0.0 & 0.0 & 0.0 & 0.0 & 0.0 \\
\hline Technetium-99 & 0.0 & 0.0 & 0.0 & 0.0 & 0.0 & 0.0 & 0.0 & 0.0 & 0.0 \\
\hline Strontium Isotopes & 0.0 & 0.0 & 0.0 & 0.0 & 0.0 & 0.0 & 0.0 & 0.0 & 0.0 \\
\hline Strontium -89 & 0.0 & 0.0 & 0.0 & 0.0 & 0.0 & 0.0 & 0.0 & 0.0 & 0.0 \\
\hline Strontium-90 & 0.0 & 0.0 & 0.0 & 0.0 & 0.0 & 0.0 & 0.0 & 0.0 & 0.0 \\
\hline lodine-129 & 0.0 & 0.0 & 0.0 & 0.0 & 0.0 & 0.0 & 0.0 & 0.0 & 0.0 \\
\hline Nickel-59 & 0.0 & 0.0 & 0.0 & 0.0 & 0.0 & 0.0 & 0.0 & 0.0 & 0.0 \\
\hline Total Uranium & 0.0 & 0.0 & 0.0 & 0.0 & 0.0 & 0.0 & 0.0 & 0.0 & 0.0 \\
\hline Total Thorium & 0.0 & 0.0 & 0.0 & 0.0 & 0.0 & 0.0 & 0.0 & 0.0 & 0.0 \\
\hline Total Strontium & 0.0 & 0.0 & 0.0 & 0.0 & 0.0 & 0.0 & 0.0 & 0.0 & 0.0 \\
\hline Other RAD & 0.0 & 0.0 & 0.0 & 1.2 & 0.0 & 0.0 & 0.0 & 1.2 & 0.0 \\
\hline OTHEA & 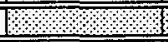 & Pि & & +1 & ?. & 2 & $=$ & & 8 \\
\hline Other Wet Chemistry & 0.0 & 0.0 & 0.0 & 0.0 & 0.0 & 0.0 & 0.0 & 0.0 & 0.0 \\
\hline TCLP Extraction & 0.0 & 0.0 & 0.0 & 0.9 & 0.0 & 0.0 & 0.0 & 0.9 & 0.0 \\
\hline Asbestos & 0.0 & 0.0 & 0.0 & 0.0 & 0.0 & 0.0 & 0.0 & 0.0 & 0.0 \\
\hline Industrial Hygiene, NOS & 0.0 & 0.0 & 0.0 & 0.0 & 0.0 & 0.0 & 0.0 & 0.0 & 0.0 \\
\hline Physical Properties & 0.0 & 0.0 & 0.0 & 0.6 & 0.0 & 0.0 & 0.0 & 0.6 & 0.0 \\
\hline Other, Not Otherwise Specified (NO & 0.0 & 0.0 & 0.0 & 0.0 & 0.0 & 0.0 & 0.0 & 0.0 & 0.0 \\
\hline
\end{tabular}




\begin{tabular}{|l|l|}
\hline Activity: & HAZ \\
\hline Unit: & Drum \\
\hline Regulatory Driver: & \multicolumn{1}{|c|}{0} \\
\hline Filename: & SUM2NEEI.WK3 \\
\hline Date: & September 21,1994 \\
\hline
\end{tabular}

\begin{tabular}{|l|l|}
\hline Average S\&A Duration per Unit (yrs): \\
\hline Total Samples/Unit-yr: & 1.3 \\
\hline Total Samp Ans/Unit-yr: & 22.2 \\
\hline Average Batch Size: & \\
\hline \# of Unit-yrs in FY 93: & 520.0 \\
\hline
\end{tabular}

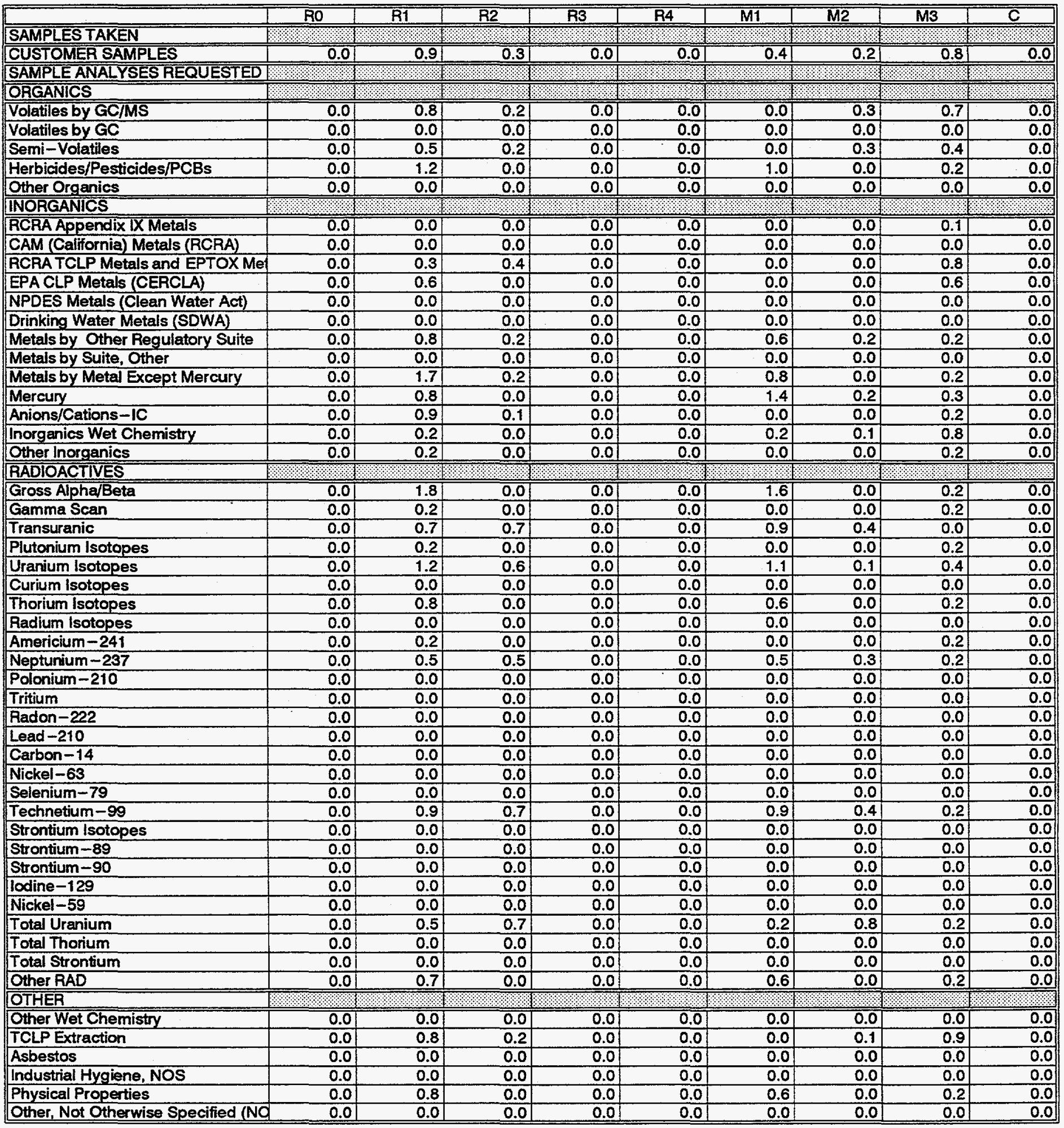




\begin{tabular}{|c|c|c|c|c|c|c|c|c|c|}
\hline Activity: & MIX/LLW & & & Average S\& & uration $p$ & Unit (yrs) & & & \\
\hline Unit: & Drum & & & Total Sampl & Unit-yr: & & 0.2 & & \\
\hline Regulatory Driver: & 0 & & & Total Samp & s/Unit-yr & & 1.5 & & \\
\hline Filename: & SUM2NEEJ. & & & Average Bat & Size: & & & & \\
\hline Date: & September 2 & 1994 & & \# of Unit - y & nFY93: & & 31530.0 & & \\
\hline & Ro & R1 & $\overline{\mathrm{R2}}$ & $\overline{\mathrm{R} 3}$ & $\mathrm{R4}$ & M1 & M2 & $\mathrm{M} \overline{3}$ & $\bar{C}$ \\
\hline SAMPLES TAKEN & & & & & & & & & \\
\hline CUSTOMEA SAMPLES & 0.01 & 0.2 & 0.0 & 0.01 & 0.0 & 0.0 & 0.11 & 0.0 & 0.0 \\
\hline SAMPLE ANALYSES REQUESTED & & & & & & & & & \\
\hline ORGANICS & & & & & & & & & \\
\hline Volatiles by GC/MS & 0.1 & 0.0 & 0.0 & 0.0 & 0.0 & 0.0 & 0.0 & 0.1 & 0.0 \\
\hline Volatiles by $G C$ & 0.0 & 0.1 & 0.0 & 0.0 & 0.0 & 0.0 & 0.1 & 0.0 & 0.0 \\
\hline Semi-Volaties & 0.0 & 0.0 & 0.0 & 0.0 & 0.0 & 0.0 & 0.0 & 0.0 & 0.0 \\
\hline Herbicides/Pesticides/PCBs & 0.1 & 0.0 & 0.0 & 0.0 & 0.0 & 0.0 & 0.0 & 0.1 & 0.0 \\
\hline Other Organics & 0.0 & 0.0 & 0.0 & 0.0 & 0.0 & 0.0 & 0.0 & 0.0 & 0.0 \\
\hline INORGANICS & & & & & & & & & \\
\hline RCRA Appendix IX Metals & 0.0 & 0.0 & 0.0 & 0.0 & 0.0 & 0.0 & 0.0 & 0.0 & 0.0 \\
\hline CAM (California) Metals (RCRA) & 0.0 & 0.0 & 0.0 & 0.0 & 0.0 & 0.0 & 0.0 & 0.0 & 0.0 \\
\hline RCRA TCLP Metals and EPTOX Me & 0.0 & 0.0 & 0.0 & 0.0 & 0.0 & 0.0 & 0.0 & 0.0 & 0.0 \\
\hline EPA CLP Metals (CERCLA) & 0.0 & 0.0 & 0.0 & 0.0 & 0.0 & 0.0 & 0.0 & 0.0 & 0.0 \\
\hline NPDES Metals (Clean Water Act) & 0.0 & 0.0 & 0.0 & 0.0 & 0.0 & 0.0 & 0.0 & 0.0 & 0.0 \\
\hline Drinking Water Metals (SDWA) & 0.0 & 0.0 & 0.0 & 0.0 & 0.0 & 0.0 & 0.0 & 0.0 & 0.0 \\
\hline Metals by Other Regulatory Suite & 0.1 & 0.0 & 0.0 & 0.0 & 0.0 & 0.0 & 0.0 & 0.1 & 0.0 \\
\hline Metals by Suite, Other & 0.0 & 0.0 & 0.0 & 0.0 & 0.0 & 0.0 & 0.0 & 0.0 & 0.0 \\
\hline Metals by Metal Except Mercury & 0.0 & 0.0 & 0.0 & 0.0 & 0.0 & 0.0 & 0.0 & 0.0 & 0.0 \\
\hline Mercury & 0.0 & 0.0 & 0.0 & 0.0 & 0.0 & 0.0 & 0.0 & 0.0 & 0.0 \\
\hline Anions/Cations - IC & 0.0 & 0.0 & 0.0 & 0.0 & 0.0 & 0.0 & 0.0 & 0.0 & 0.0 \\
\hline Inorganics Wet Chemistry & 0.3 & 0.1 & 0.1 & 0.0 & 0.0 & 0.0 & 0.1 & 0.3 & 0.0 \\
\hline Other Inorganics & 0.0 & 0.0 & 0.0 & 0.0 & 0.0 & 0.0 & 0.0 & 0.0 & 0.0 \\
\hline RADIOACTIVES & & & & & & & & & \\
\hline Gross Apha/Beta & 0.0 & 0.1 & 0.0 & 0.0 & 0.0 & 0.0 & 0.0 & 0.0 & 0.0 \\
\hline Gamma Scan & 0.0 & 0.0 & 0.0 & 0.0 & 0.0 & 0.0 & 0.0 & 0.0 & 0.0 \\
\hline Transuranic & 0.0 & 0.0 & 0.0 & 0.0 & 0.0 & 0.0 & 0.0 & 0.0 & 0.0 \\
\hline Plutonium Isotopes & 0.0 & 0.0 & 0.0 & 0.0 & 0.0 & 0.0 & 0.0 & 0.0 & 0.0 \\
\hline Uranium Isotopes & 0.0 & 0.0 & 0.0 & 0.0 & 0.0 & 0.0 & 0.0 & 0.0 & 0.0 \\
\hline Curium Isotopes & 0.0 & 0.0 & 0.0 & 0.0 & 0.0 & 0.0 & 0.0 & 0.0 & 0.0 \\
\hline Thorium Isotopes & 0.0 & 0.0 & 0.0 & 0.0 & 0.0 & 0.0 & 0.0 & 0.0 & 0.0 \\
\hline Radium Isotopes & 0.0 & 0.0 & 0.0 & 0.0 & 0.0 & 0.0 & 0.0 & 0.0 & 0.0 \\
\hline Americium-241 & 0.0 & 0.0 & 0.0 & 0.0 & 0.0 & 0.0 & 0.0 & 0.0 & 0.0 \\
\hline Neptunium - 237 & 0.0 & 0.0 & 0.0 & 0.0 & 0.0 & 0.0 & 0.0 & 0.0 & 0.0 \\
\hline Polonium - 210 & 0.0 & 0.0 & 0.0 & 0.0 & 0.0 & 0.0 & 0.0 & 0.0 & 0.0 \\
\hline Tritium & 0.0 & 0.0 & 0.0 & 0.0 & 0.0 & 0.0 & 0.0 & 0.0 & 0.0 \\
\hline Radon-222 & 0.0 & 0.0 & 0.0 & 0.0 & 0.0 & 0.0 & 0.0 & 0.0 & 0.0 \\
\hline Lead-210 & 0.0 & 0.0 & 0.0 & 0.0 & 0.0 & 0.0 & 0.0 & 0.0 & 0.0 \\
\hline Carbon-14 & 0.0 & 0.0 & 0.0 & 0.0 & 0.0 & 0.0 & 0.0 & 0.0 & 0.0 \\
\hline Nickel-63 & 0.0 & 0.0 & 0.0 & 0.0 & 0.0 & 0.0 & 0.0 & 0.0 & 0.0 \\
\hline Selenium -79 & 0.0 & 0.0 & 0.0 & 0.0 & 0.0 & 0.0 & 0.0 & 0.0 & 0.0 \\
\hline Technetium -99 & 0.0 & 0.0 & 0.0 & 0.0 & 0.0 & 0.0 & 0.0 & 0.0 & 0.0 \\
\hline Strontium Isotopes & 0.0 & 0.0 & 0.0 & 0.0 & 0.0 & 0.0 & 0.0 & 0.0 & 0.0 \\
\hline Strontium -89 & 0.0 & 0.0 & 0.0 & 0.0 & 0.0 & 0.0 & 0.0 & 0.0 & 0.0 \\
\hline Strontium -90 & 0.0 & 0.0 & 0.0 & 0.0 & 0.0 & 0.0 & 0.0 & 0.0 & 0.0 \\
\hline lodine-129 & 0.0 & 0.0 & 0.0 & 0.0 & 0.0 & 0.0 & 0.0 & 0.0 & 0.0 \\
\hline Nickel-59 & 0.0 & 0.0 & 0.0 & 0.0 & 0.0 & 0.0 & 0.0 & 0.0 & 0.0 \\
\hline Total Uranium & 0.1 & 0.0 & 0.0 & 0.0 & 0.0 & 0.0 & 0.0 & 0.1 & 0.0 \\
\hline Total Thorium & 0.0 & 0.0 & 0.0 & 0.0 & 0.0 & 0.0 & 0.0 & 0.0 & 0.0 \\
\hline Total Strontium & 0.0 & 0.0 & 0.0 & 0.0 & 0.0 & 0.0 & 0.0 & 0.0 & 0.0 \\
\hline Other RAD & 0.1 & 0.0 & 0.0 & 0.0 & 0.0 & 0.0 & 0.0 & 0.1 & 0.0 \\
\hline OTHEA & & & & & & & & & \\
\hline Other Wet Chemistry & 0.0 & 0.0 & 0.0 & 0.0 & 0.0 & 0.0 & 0.0 & 0.0 & 0.0 \\
\hline TCLP Extraction & 0.0 & 0.0 & 0.0 & 0.0 & 0.0 & 0.0 & 0.0 & 0.0 & 0.0 \\
\hline Asbestos & 0.0 & 0.0 & 0.0 & 0.0 & 0.0 & 0.0 & 0.0 & 0.0 & 0.0 \\
\hline Industrial Hygiene, NOS & 0.0 & 0.0 & 0.0 & 0.0 & 0.0 & 0.0 & 0.0 & 0.0 & 0.0 \\
\hline Physical Properties & 0.0 & 0.0 & 0.0 & 0.0 & 0.0 & 0.0 & 0.0 & 0.0 & 0.0 \\
\hline Other. Not Otherwise Specified (NO & 0.0 & 0.0 & 0.0 & 0.0 & 0.0 & 0.0 & 0.0 & 0.0 & 0.0 \\
\hline
\end{tabular}




\begin{tabular}{|l|l|}
\hline Activity: & HLW \\
\hline Unit: & Sample \\
\hline Regulatory Driver: & 0 \\
\hline Filename: & SUM2NEEKWK3 \\
\hline Date: & September 21, 1994 \\
\hline
\end{tabular}

\begin{tabular}{|l|l|}
\hline Average S\&A Duration per Unit (yrs): \\
\hline Total Samples/Unit-yr: & 1.0 \\
\hline Total Samp Ans/Unit-y: & 3.0 \\
\hline Average Batch Size: & \\
\hline$\#$ of Unit-yrs in FY 93: & 9560.0 \\
\hline
\end{tabular}

\begin{tabular}{|c|c|c|c|c|c|c|c|c|c|}
\hline & $\overline{\mathrm{RO}}$ & $\overline{\mathrm{R1}}$ & R2 & $\overline{R 3}$ & R4 & $\overline{\mathrm{M} 1}$ & $\overline{\mathrm{M2}}$ & $\overline{\mathrm{M3}}$ & $\bar{c}$ \\
\hline SAMPLES TAKEN & & & & & & & & & \\
\hline CUSTOMER SAMPLES & 1.0 & 0.0 & 0.01 & 0.01 & 0.01 & 0.7 & 0.0 & 0.31 & 0.0 \\
\hline SAMPLE ANALYSES REQUESTED & & & $1 \%$ & 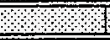 & $\$$ & $\$$ & ? & & \\
\hline ORGANICS & & & & & & & & & \\
\hline Volatiles by GC/MS & 0.0 & 0.0 & 0.0 & 0.0 & 0.0 & 0.0 & 0.0 & 0.0 & 0.0 \\
\hline Volatiles by GC & 0.0 & 0.0 & 0.0 & 0.0 & 0.0 & 0.0 & 0.0 & 0.0 & 0.0 \\
\hline Semi-Volatiles & 0.1 & 0.0 & 0.0 & 0.0 & 0.0 & 0.1 & 0.0 & 0.0 & 0.0 \\
\hline Herbicides/Pesticides/PCBs & 0.0 & 0.0 & 0.0 & 0.0 & 0.0 & 0.0 & 0.0 & 0.0 & 0.0 \\
\hline Other Organics & 0.2 & 0.0 & 0.0 & 0.0 & 0.0 & 0.2 & 0.0 & 0.0 & 0.0 \\
\hline INORGANICS & & & & & & & & & \\
\hline RCRA Appendix IX Metals & 0.0 & 0.0 & 0.01 & 0.0 & 0.0 & 0.0 & 0.0 & 0.01 & 0.0 \\
\hline CAM (California) Metals (RCRA) & 0.0 & 0.0 & 0.0 & 0.0 & 0.0 & 0.0 & 0.0 & 0.0 & 0.0 \\
\hline RCRA TCLP Metals and EPTOX Me: & 0.0 & 0.0 & 0.0 & 0.0 & 0.0 & 0.0 & 0.0 & 0.0 & 0.0 \\
\hline EPA CLP Metals (CERCLA) & 0.0 & 0.0 & 0.0 & 0.0 & 0.0 & 0.0 & 0.0 & 0.0 & 0.0 \\
\hline NPDES Metals (Clean Water Act) & 0.0 & 0.0 & 0.0 & 0.0 & 0.0 & 0.0 & 0.0 & 0.0 & 0.0 \\
\hline Drinking Water Metals (SDWA) & 0.0 & 0.0 & 0.0 & 0.0 & 0.0 & 0.0 & 0.0 & 0.0 & 0.0 \\
\hline Metals by Other Regulatory Suite & 0.0 & 0.0 & 0.0 & 0.0 & 0.0 & 0.0 & 0.0 & 0.0 & 0.0 \\
\hline Metals by Suite, Other & 0.3 & 0.0 & 0.0 & 0.0 & 0.0 & 0.1 & 0.0 & 0.1 & 0.0 \\
\hline Metals by Metal Except Mercury & 0.2 & 0.0 & 0.0 & 0.0 & 0.0 & 0.1 & 0.0 & 0.1 & 0.0 \\
\hline Mercury & 0.2 & 0.0 & 0.0 & 0.0 & 0.0 & 0.1 & 0.0 & 0.1 & 0.0 \\
\hline Anions/Cations-IC & 0.4 & 0.0 & 0.0 & 0.0 & 0.0 & 0.3 & 0.0 & 0.1 & 0.0 \\
\hline Inorganics Wet Chemistry & 0.3 & 0.0 & 0.0 & 0.0 & 0.0 & 0.2 & 0.0 & 0.1 & 0.0 \\
\hline Other Inorganics & 0.0 & 0.0 & 0.0 & 0.0 & 0.0 & 0.0 & 0.0 & 0.0 & 0.0 \\
\hline RADIOACTIVES & & & & & & 10 & & 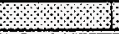 & \\
\hline Gross Alpha/Beta & 0.0 & 0.0 & 0.0 & 0.0 & 0.0 & 0.0 & 0.0 & 0.0 & 0.0 \\
\hline Gamma Scan & 0.0 & 0.0 & 0.0 & 0.0 & 0.0 & 0.0 & 0.0 & 0.0 & 0.0 \\
\hline Transuranic & 0.0 & 0.0 & 0.0 & 0.0 & 0.0 & 0.0 & 0.0 & 0.0 & 0.0 \\
\hline Plutonium Isotopes & 0.0 & 0.0 & 0.0 & 0.0 & 0.0 & 0.0 & 0.0 & 0.0 & 0.0 \\
\hline Uranium Isotopes & 0.0 & 0.0 & 0.0 & 0.0 & 0.0 & 0.0 & 0.0 & 0.0 & 0.0 \\
\hline Curium Isotopes & 0.0 & 0.0 & 0.0 & 0.0 & 0.0 & 0.0 & 0.0 & 0.0 & 0.0 \\
\hline Thorium Isotopes & 0.0 & 0.0 & 0.0 & 0.0 & 0.0 & 0.0 & 0.0 & 0.0 & 0.0 \\
\hline Radium Isotopes & 0.0 & 0.0 & 0.0 & 0.0 & 0.0 & 0.0 & 0.0 & 0.0 & 0.0 \\
\hline Americium-241 & 0.0 & 0.0 & 0.0 & 0.0 & 0.0 & 0.0 & 0.0 & 0.0 & 0.0 \\
\hline Neptunium - 237 & 0.0 & 0.0 & 0.0 & 0.0 & 0.0 & 0.0 & 0.0 & 0.0 & 0.0 \\
\hline Polonium-210 & 0.0 & 0.0 & 0.0 & 0.0 & 0.0 & 0.0 & 0.0 & 0.0 & 0.0 \\
\hline Tritium & 0.0 & 0.0 & 0.0 & 0.0 & 0.0 & 0.0 & 0.0 & 0.0 & 0.0 \\
\hline Radon-222 & 0.0 & 0.0 & 0.0 & 0.0 & 0.0 & 0.0 & 0.0 & 0.0 & 0.0 \\
\hline Lead -210 & 0.0 & 0.0 & 0.0 & 0.0 & 0.0 & 0.0 & 0.0 & 0.0 & 0.0 \\
\hline Carbon-14 & 0.0 & 0.0 & 0.0 & 0.0 & 0.0 & 0.0 & 0.0 & 0.0 & 0.0 \\
\hline Nickel-63 & 0.0 & 0.0 & 0.0 & 0.0 & 0.0 & 0.0 & 0.0 & 0.0 & 0.0 \\
\hline Selenium-79 & 0.0 & 0.0 & 0.0 & 0.0 & 0.0 & 0.0 & 0.0 & 0.0 & 0.0 \\
\hline Technetium-99 & 0.0 & 0.0 & 0.0 & 0.0 & 0.0 & 0.0 & 0.0 & 0.0 & 0.0 \\
\hline Strontium Isotopes & 0.0 & 0.0 & 0.0 & 0.0 & 0.0 & 0.0 & 0.0 & 0.0 & 0.0 \\
\hline Strontium - 89 & 0.0 & 0.0 & 0.0 & 0.0 & 0.0 & 0.0 & 0.0 & 0.0 & 0.0 \\
\hline Strontium -90 & 0.0 & 0.0 & 0.0 & 0.0 & 0.0 & 0.0 & 0.0 & 0.0 & 0.0 \\
\hline lodine-129 & 0.0 & 0.0 & 0.0 & 0.0 & 0.0 & 0.0 & 0.0 & 0.0 & 0.0 \\
\hline Nickel-59 & 0.0 & 0.0 & 0.0 & 0.0 & 0.0 & 0.0 & 0.0 & 0.0 & 0.0 \\
\hline Total Uranium & 0.0 & 0.0 & 0.0 & 0.0 & 0.0 & 0.0 & 0.0 & 0.0 & 0.0 \\
\hline Total Thorium & 0.0 & 0.0 & 0.0 & 0.0 & 0.0 & 0.0 & 0.0 & 0.0 & 0.0 \\
\hline Total Strontium & 0.0 & 0.0 & 0.0 & 0.0 & 0.0 & 0.0 & 0.0 & 0.0 & 0.0 \\
\hline Other RAD & 0.0 & 0.0 & 0.0 & 0.01 & 0.0 & 0.0 & 0.0 & 0.0 & 0.0 \\
\hline OTHER & 4 & & \% & 1 & 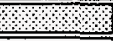 & 9 & 8 & & \\
\hline Other Wet Chemistry & 0.0 & 0.0 & 0.0 & 0.0 & 0.0 & 0.0 & 0.0 & 0.0 & 0.0 \\
\hline TCLP Extraction & 0.0 & 0.0 & 0.0 & 0.0 & 0.0 & 0.0 & 0.0 & 0.0 & 0.0 \\
\hline Asbestos & 0.0 & 0.0 & 0.0 & 0.0 & 0.0 & 0.0 & 0.0 & 0.0 & 0.0 \\
\hline Industrial Hygiene, NOS & 0.0 & 0.0 & 0.0 & 0.0 & 0.0 & 0.0 & 0.0 & 0.0 & 0.0 \\
\hline Physical Properties & 1.3 & 0.0 & 0.0 & 0.0 & 0.0 & 0.8 & 0.0 & 0.4 & 0.0 \\
\hline Other, Not Otherwise Specified (NQ & 0.0 & 0.0 & 0.0 & 0.0 & 0.0 & 0.0 & 0.0 & 0.0 & 0.0 \\
\hline
\end{tabular}




\begin{tabular}{|l|l|}
\hline Activity: & ENV MON \\
\hline Unit: & Location \\
\hline Regulatory Driver: & 0 \\
\hline Filename: & SUM2NEEL.WK3 \\
\hline Date: & September 21.1994 \\
\hline
\end{tabular}

\begin{tabular}{|c|c|c|c|c|c|c|c|c|c|}
\hline \multirow{2}{*}{ SAMPLES TAKEN } & RO & R1: & R2 & $\overline{\mathrm{R3}}$ & $\overline{\mathrm{R4}}$ & \multirow[t]{2}{*}{ M1 } & \multirow[t]{2}{*}{$\mathrm{M2}$} & \multirow[t]{2}{*}{ M3 } & \multirow{2}{*}{$\overline{\mathrm{C}}$} \\
\hline & & & & & & & & & \\
\hline CUSTOMER SAMPLES & 5.5 & 0.21 & 0.01 & 0.01 & 0.0 & 4.21 & 1.51 & 0.0 & 0.0 \\
\hline SAMPLE ANALYSES REQUESTED & & $\$ 1$ & 1 & 1 & 10 & ? & 6 & & \\
\hline ORGANICS & & मा & & 1 & & 1 & & & \\
\hline Volatiles by GC/MS & 0.5 & 0.0 & 0.0 & 0.0 & 0.0 & 0.4 & 0.1 & 0.0 & 0.0 \\
\hline Volatiles by GC & 4.9 & 0.1 & 0.0 & 0.0 & 0.0 & 4.2 & 0.4 & 0.0 & 0.0 \\
\hline Semi-Volatiles & 0.8 & 0.0 & 0.0 & 0.0 & 0.0 & 0.7 & 0.1 & 0.0 & 0.0 \\
\hline Herbicides/Pesticides/PCBs & 0.3 & 0.0 & 0.0 & 0.0 & 0.0 & 0.3 & 0.1 & 0.0 & 0.0 \\
\hline Other Organics & 2.3 & 0.0 & 0.0 & $0.0]$ & 0.01 & 2.3 & 0.01 & 0.01 & 0.0 \\
\hline INORGANICS & & & & . & & & $\widehat{15}$ & $\$$ & \\
\hline RCRA Appendix IX Metals & 0.0 & 0.0 & 0.0 & 0.0 & 0.0 & 0.0 & 0.0 & 0.0 & 0.0 \\
\hline CAM (California) Metals (RCRA) & 0.0 & 0.0 & 0.0 & 0.0 & 0.0 & 0.0 & 0.0 & 0.0 & 0.0 \\
\hline RCRA TCLP Metals and EPTOX Mel & 4.4 & 0.1 & 0.0 & 0.0 & 0.0 & 3.7 & 0.4 & 0.0 & 0.0 \\
\hline EPA CLP Metals (CERCLA) & 1.0 & 0.0 & 0.0 & 0.0 & 0.0 & 0.9 & 0.1 & 0.0 & 0.0 \\
\hline NPDES Metals (Clean Water Act) & 0.0 & 0.0 & 0.0 & 0.0 & 0.0 & 0.0 & 0.0 & 0.0 & 0.0 \\
\hline Drinking Water Metals (SDWA) & 1.2 & 0.0 & 0.0 & 0.0 & 0.0 & 1.2 & 0.0 & 0.0 & 0.0 \\
\hline Metals by Other Regulatory Suite & 1.0 & 0.0 & 0.0 & 0.0 & 0.0 & 0.9 & 0.1 & 0.0 & 0.0 \\
\hline Metals by Suite, Other & 0.0 & 0.0 & 0.0 & 0.0 & 0.0 & 0.0 & 0.0 & 0.0 & 0.0 \\
\hline Metals by Metal Except Mercury & 4.7 & 0.1 & 0.0 & 0.0 & 0.0 & 3.9 & 0.4 & 0.0 & 0.0 \\
\hline Mercury & 4.4. & 0.1 & 0.0 & 0.0 & 0.0 & 3.7 & 0.4 & 0.0 & 0.0 \\
\hline Anions/Cations-IC & 0.1 & 0.0 & 0.0 & 0.0 & 0.0 & 0.1 & 0.0 & 0.0 & 0.0 \\
\hline Inorganics Wet Chemistry & 3.7 & 0.0 & 0.0 & 0.0 & 0.0 & 3.6 & 0.1 & 0.0 & 0.0 \\
\hline Other Inorganics & 0.2 & 0.0 & 0.0 & 0.0 & 0.0 & 0.2 & 0.0 & 0.0 & 0.0 \\
\hline RADIOACTIVES & & & & \% & & & 6 & & \\
\hline Gross Alpha/Beta & 4.3 & 0.0 & 0.0 & 0.0 & 0.0 & 1.2 & 3.1 & 0.0 & 0.0 \\
\hline Gamma Scan & 6.3 & 0.0 & 0.0 & 0.0 & 0.0 & 0.4 & 5.9 & 0.0 & 0.0 \\
\hline Transuranic & 0.0 & 0.0 & 0.0 & 0.0 & 0.0 & 0.0 & 0.0 & 0.0 & 0.0 \\
\hline Plutonium Isotopes & 1.9 & 0.0 & 0.0 & 0.0 & 0.0 & 1.1 & 0.8 & 0.0 & 0.0 \\
\hline Uranium Isotopes & 0.8 & 0.0 & 0.0 & 0.0 & 0.0 & 0.7 & 0.1 & 0.0 & 0.0 \\
\hline Curium Isotopes & 0.0 & 0.0 & 0.0 & 0.0 & 0.0 & 0.0 & 0.0 & 0.0 & 0.0 \\
\hline Thorium Isotopes & 0.0 & 0.0 & 0.0 & 0.0 & 0.0 & 0.0 & 0.0 & 0.0 & 0.0 \\
\hline Radium Isotopes & 0.2 & 0.0 & 0.0 & 0.0 & 0.0 & 0.2 & 0.0 & 0.0 & 0.0 \\
\hline Americium-241 & 1.0 & 0.0 & 0.0 & 0.0 & 0.0 & 0.8 & 0.1 & 0.0 & 0.0 \\
\hline Neptunium - 237 & 0.0 & 0.0 & 0.0 & 0.0 & 0.0 & 0.0 & 0.0 & 0.0 & 0.0 \\
\hline Polonium-210 & 0.0 & 0.0 & 0.0 & 0.0 & 0.0 & 0.0 & 0.0 & 0.0 & 0.0 \\
\hline Tritium & 1.5 & 0.0 & 0.0 & 0.0 & 0.0 & 1.0 & 0.5 & 0.0 & 0.0 \\
\hline Radon-222 & 0.0 & 0.0 & 0.0 & 0.0 & 0.0 & 0.0 & 0.0 & 0.0 & 0.0 \\
\hline Lead-210 & 0.0 & 0.0 & 0.0 & 0.0 & 0.0 & 0.0 & 0.0 & 0.0 & 0.0 \\
\hline Carbon-14 & 0.0 & 0.0 & 0.0 & 0.0 & 0.0 & 0.0 & 0.0 & 0.0 & 0.0 \\
\hline Nickel-63 & 0.0 & 0.0 & 0.0 & 0.0 & 0.0 & 0.0 & 0.0 & 0.0 & 0.0 \\
\hline Selenium -79 & 0.0 & 0.0 & 0.0 & 0.0 & 0.0 & 0.0 & 0.0 & 0.0 & 0.0 \\
\hline Technetium-99 & 0.2 & 0.0 & 0.0 & 0.0 & 0.0 & 0.2 & 0.0 & 0.0 & 0.0 \\
\hline Strontium Isotopes & 0.0 & 0.0 & 0.0 & 0.0 & 0.0 & 0.0 & 0.0 & 0.0 & 0.0 \\
\hline Strontium-89 & 0.0 & 0.0 & 0.0 & 0.0 & 0.0 & 0.0 & 0.0 & 0.0 & 0.0 \\
\hline Strontium -90 & 0.2 & 0.0 & 0.0 & 0.0 & 0.0 & 0.2 & 0.0 & 0.0 & 0.0 \\
\hline lodine-129 & 0.7 & 0.0 & 0.0 & 0.0 & 0.0 & 0.7 & 0.0 & 0.0 & 0.0 \\
\hline Nickel-59 & 0.0 & 0.0 & 0.0 & 0.0 & 0.0 & 0.0 & 0.0 & 0.0 & 0.0 \\
\hline Total Uranium & 0.2 & 0.0 & 0.0 & 0.0 & 0.0 & 0.2 & 0.0 & 0.0 & 0.0 \\
\hline Total Thorium & 0.0 & 0.0 & 0.0 & 0.0 & 0.0 & 0.0 & 0.0 & 0.0 & 0.0 \\
\hline Total Strontium & 0.3 & 0.0 & 0.0 & 0.0 & 0.0 & 0.2 & 0.1 & 0.0 & 0.0 \\
\hline Other RAD & 2.4 & 0.1 & 0.0 & 0.0 & 0.0 & 2.3 & 0.3 & 0.0 & 0.0 \\
\hline OTHER & & & & & & & & & \\
\hline Other Wet Chemistry & 1.5 & 0.0 & 0.0 & 0.01 & 0.0 & 1.5 & 0.0 & 0.0 & 0.0 \\
\hline TCLP Extraction & 4.4 & 0.1 & 0.0 & 0.0 & 0.0 & 3.7 & 0.4 & 0.0 & 0.0 \\
\hline Asbestos & 0.0 & 0.0 & 0.0 & 0.0 & 0.0 & 0.0 & 0.0 & 0.0 & 0.0 \\
\hline Industrial Hygiene, NOS & 0.0 & 0.0 & 0.0 & 0.0 & 0.0 & 0.0 & 0.0 & 0.0 & 0.0 \\
\hline Physical Properties & 1.0 & 0.0 & 0.0 & 0.0 & 0.0 & 0.9 & 0.1 & 0.0 & 0.0 \\
\hline Other, Not Otherwise Specified (NO & 0.0 & 0.1 & 0.0 & 0.01 & 0.0 & 0.0 & 0.01 & 0.0 & 0.0 \\
\hline
\end{tabular}

\begin{tabular}{|c|c|}
\hline \multicolumn{2}{|c|}{ Average S\&A Duration per Unit (yrs): } \\
\hline Total Samples/Unit-yr: & 5.7 \\
\hline Total Samp Ans/Unit-yr: & 56.4 \\
\hline Average Batch Size: & \\
\hline \# of Unit-yrs in FY 93: & 217.0 \\
\hline
\end{tabular}


ACTIVITY SAMPLING AND ANALYSIS PROFILE (NSTS/NEEDS)

FOR ONE UNIT - YEAR

\begin{tabular}{|l|l|}
\hline Activity: & UST \\
\hline Unit: & Tank \\
\hline Regulatory Driver: & \multicolumn{1}{c|}{0} \\
\hline Filename: & SUM2NEEM.WK3 \\
\hline Date: & September 21, 1994 \\
\hline
\end{tabular}

\begin{tabular}{|l|l|}
\hline Average S\&A Duration per Unit (yrs): \\
\hline Total Samples/Unit-yr: & 35.7 \\
\hline Total Samp Ans/Unit-yr: & 54.3 \\
\hline Average Batch Size: & \\
\hline \# of Unit-yrs in FY 93: & 18.4 \\
\hline
\end{tabular}

\begin{tabular}{|c|c|c|c|c|c|c|c|c|c|}
\hline & $\overline{\mathrm{RO}}$ & R1 & $\overline{\mathrm{R} 2}$ & $\overline{\mathrm{A3}}$ & R4 & M1 & $\mathrm{M2}$ & $\mathrm{M3}$ & $\bar{C}$ \\
\hline SAMPLES TAKEN & & & & & & & & & \\
\hline CUSTOMER SAMPLES & 27.6 & 6.6 & 1.5 & 0.0 & 0.7 & 1.7 & 8.0 & 25.3 & 0.0 \\
\hline SAMPLE ANALYSES REQUESTED & & & & & & & 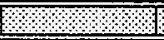 & & \\
\hline ORGANICS & $\$$ & 8 & & & & & 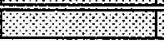 & & \\
\hline Volatiles by GC/MS & 8.9 & 0.0 & 0.7 & 0.0 & 0.0 & 0.7 & 0.3 & 8.6 & 0.0 \\
\hline Volatiles by GC & 5.8 & 0.0 & 0.0 & 0.0 & 0.0 & 0.0 & 0.1 & 5.6 & 0.0 \\
\hline Semi-Volatiles & 7.2 & 0.0 & 0.0 & 0.0 & 0.0 & 0.0 & 0.0 & 7.2 & 0.0 \\
\hline Herbicides/Pesticides/PCBs & 2.5 & 0.0 & 0.01 & 0.0 & 0.0 & 0.0 & 0.6 & 1.9 & 0.0 \\
\hline Other Organics & 1.0 & 6.8 & 0.0 & 0.0 & 0.0 & 0.9 & 6.1 & 0.8 & 0.0 \\
\hline \multicolumn{10}{|l|}{ INORGANICS } \\
\hline RCAA Appendix IX Metals & 0.0 & 0.0 & 0.01 & 0.0 & 0.0 & 0.0 & 0.0 & 0.0 & 0.0 \\
\hline CAM (California) Metals (RCAA) & 0.0 & 0.0 & 0.0 & 0.0 & 0.0 & 0.0 & 0.0 & 0.0 & 0.0 \\
\hline RCRA TCLP Metals and EPTOX Me & 1.0 & 0.0 & 0.0 & 0.0 & 0.0 & 0.0 & 0.3 & 0.8 & 0.0 \\
\hline EPA CLP Metals (CERCLA) & 0.2 & 0.0 & 0.9 & 0.0 & 0.0 & 0.0 & 0.9 & 0.2 & 0.0 \\
\hline NPDES Metals (Clean Water Act) & 0.0 & 0.0 & 0.01 & 0.0 & 0.0 & 0.0 & 0.0 & 0.0 & 0.0 \\
\hline Drinking Water Metals (SDWA) & 0.0 & 0.0 & 0.0 & 0.0 & 0.0 & 0.0 & 0.0 & 0.0 & 0.0 \\
\hline Metals by Other Regulatory Suite & 0.0 & 0.0 & 0.0 & 0.0 & 0.0 & 0.0 & 0.0 & 0.0 & 0.0 \\
\hline Metals by Suite, Other & 0.0 & 0.0 & 0.0 & 0.0 & 0.0 & 0.0 & 0.0 & 0.0 & 0.0 \\
\hline Metals by Metal Except Mercury & 0.0 & 0.0 & 0.0 & 0.0 & 0.0 & 0.0 & 0.0 & 0.0 & 0.0 \\
\hline Mercury & 0.0 & 0.0 & 0.0 & 0.0 & 0.0 & 0.0 & 0.0 & 0.0 & 0.0 \\
\hline Anions/Cations-IC & 0.0 & 0.0 & 0.0 & 0.0 & 0.0 & 0.0 & 0.0 & 0.0 & 0.0 \\
\hline Inorganics Wet Chemistry & 0.0 & 0.0 & 0.0 & 0.0 & 0.0 & 0.0 & 0.0 & 0.0 & 0.0 \\
\hline Other Inorganics & 0.7 & 0.0 & 0.01 & 0.0 & 0.0 & 0.0 & 0.7 & 0.0 & 0.0 \\
\hline \multicolumn{10}{|l|}{ AADIOACTIVES } \\
\hline Gross Alpha/Beta & 0.8 & 4.3 & 0.0 & 0.0 & 0.0 & 1.6 & 3.5 & 0.0 & 0.0 \\
\hline Gamma Scan & 0.4 & 6.4 & 0.01 & 0.0 & 0.0 & 1.3 & 3.5 & 2.0 & 0.0 \\
\hline Transuranic & 0.0 & 0.0 & 0.0 & 0.0 & 0.0 & 0.0 & 0.0 & 0.0 & 0.0 \\
\hline Plutonium Isotopes & 0.0 & 0.0 & 0.0 & 0.0 & 0.0 & 0.0 & 0.0 & 0.0 & 0.0 \\
\hline Uranium Isotopes & 0.0 & 2.4 & 0.0 & 0.0 & 0.0 & 0.0 & 2.4 & 0.0 & 0.0 \\
\hline Curium Isotopes & 0.0 & 0.0 & 0.0 & 0.0 & 0.0 & 0.0 & 0.0 & 0.0 & 0.0 \\
\hline Thorium isotopes & 0.0 & 0.0 & 0.0 & 0.0 & 0.0 & 0.0 & 0.0 & 0.0 & 0.0 \\
\hline Radium Isotopes & 0.0 & 0.0 & 0.0 & 0.0 & 0.0 & 0.0 & 0.0 & 0.0 & 0.0 \\
\hline Americium-241 & 0.0 & 0.0 & 0.0 & 0.0 & 0.0 & 0.0 & 0.0 & 0.0 & 0.0 \\
\hline Neptunium-237 & 0.0 & 0.0 & 0.0 & 0.0 & 0.0 & 0.0 & 0.0 & 0.0 & 0.0 \\
\hline Polonium-210 & 0.0 & 0.0 & 0.0 & 0.0 & 0.0 & 0.0 & 0.0 & 0.0 & 0.0 \\
\hline Tritium & 0.0 & 0.0 & 0.0 & 0.0 & 0.0 & 0.0 & 0.0 & 0.0 & 0.0 \\
\hline Radon-222 & 0.0 & 0.0 & 0.0 & 0.0 & 0.0 & 0.0 & 0.0 & 0.0 & 0.0 \\
\hline Lead-210 & 0.0 & 0.0 & 0.0 & 0.0 & 0.0 & 0.0 & 0.0 & 0.0 & 0.0 \\
\hline Carbon-14 & 0.0 & 0.0 & 0.0 & 0.0 & 0.0 & 0.0 & 0.0 & 0.0 & 0.0 \\
\hline Nickel-63 & 0.0 & 0.0 & 0.0 & 0.0 & 0.0 & 0.0 & 0.0 & 0.0 & 0.0 \\
\hline Selenium-79 & 0.0 & 0.0 & 0.0 & 0.0 & 0.0 & 0.0 & 0.0 & 0.0 & 0.0 \\
\hline Technetium-99 & 0.0 & 0.0 & 0.0 & 0.0 & 0.0 & 0.0 & 0.0 & 0.0 & 0.0 \\
\hline Strontium Isotopes & 0.0 & 0.0 & 0.0 & 0.0 & 0.0 & 0.0 & 0.0 & 0.0 & 0.0 \\
\hline Strontium -89 & 0.0 & 0.0 & 0.0 & 0.0 & 0.0 & 0.0 & 0.0 & 0.0 & 0.0 \\
\hline Strontium -90 & 0.0 & 0.0 & 0.0 & 0.0 & 0.0 & 0.0 & 0.0 & 0.0 & 0.0 \\
\hline lodine-129 & 0.0 & 0.0 & 0.0 & 0.0 & 0.0 & 0.0 & 0.0 & 0.0 & 0.0 \\
\hline Nickel-59 & 0.0 & 0.0 & 0.0 & 0.0 & 0.0 & 0.0 & 0.0 & 0.0 & 0.0 \\
\hline Total Uranium & 0.0 & 2.4 & 0.0 & 0.0 & 0.0 & 0.0 & 2.4 & 0.0 & 0.0 \\
\hline Total Thorium & 0.0 & 0.0 & 0.0 & 0.0 & 0.0 & 0.0 & 0.0 & 0.0 & 0.0 \\
\hline Total Strontium & 0.0 & 0.0 & 0.0 & 0.0 & 0.0 & 0.0 & 0.0 & 0.0 & 0.0 \\
\hline Other RAD & 0.0 & 0.0 & 0.0 & 0.0 & 0.0 & 0.0 & 0.0 & 0.0 & 0.0 \\
\hline \multicolumn{10}{|l|}{ OTHER } \\
\hline Other Wet Chemistry & 0.0 & 0.0 & 0.0 & 0.0 & 0.0 & 0.0 & 0.0 & 0.0 & 0.0 \\
\hline TCLP Extraction & 0.1 & 0.0 & 0.0 & 0.0 & 0.0 & 0.0 & 0.1 & 0.0 & 0.0 \\
\hline Asbestos & 2.0 & 0.0 & 0.0 & 0.0 & 0.0 & 0.0 & 2.0 & 0.0 & 0.0 \\
\hline Industrial Hygiene, NOS & 0.0 & 0.0 & 0.0 & 0.0 & 0.0 & 0.0 & 0.0 & 0.0 & 0.0 \\
\hline Physical Properties & 0.0 & 0.0 & 0.0 & 0.0 & 0.0 & 0.0 & 0.0 & 0.0 & 0.0 \\
\hline Other, Not Otherwise Specified (NO & 0.0 & 0.0 & 0.0 & 0.0 & 0.0 & 0.0 & 0.0 & 0.0 & 0.0 \\
\hline
\end{tabular}




\begin{tabular}{|l|l|}
\hline Activity: & MISC R/C \\
\hline Unit: & Sample \\
\hline Regulatory Driver: & 0 \\
\hline Filename: & SUM2NEEN.WK3 \\
\hline Date: & September 21, 1994 \\
\hline
\end{tabular}

\begin{tabular}{|l|c|}
\hline Average S\&A Duration per Unit (yrs): \\
\hline Total Samples/Unit-yr: & 0.0 \\
\hline Total Samp Ans/Unit-yr: & 20.0 \\
\hline Average Batch Size: & \\
\hline$\#$ of Unit-yrs in FY 93: & 2250.0 \\
\hline
\end{tabular}

\begin{tabular}{|c|c|c|c|c|c|c|c|c|c|}
\hline & $\overline{\mathrm{RO}}$ & R1 & R2 & $\mathrm{R} 3$ & $\overline{\mathrm{A4} 4}$ & M1 & $\mathrm{M2}$ & M3 & $\bar{c}$ \\
\hline SAMPLESTAKEN & & & & & & & & & \\
\hline CUSTOMEA SAMPLES & 0.0 & 0.0 & 0.0 & 0.0 & 0.0 & 0.0 & 0.0 & 0.0 & 0.0 \\
\hline SAMPLE ANALYSES REQUESTED & & & 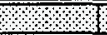 & & & & & & \\
\hline ORGANICS & 8 & . & 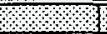 & । & $\%$ & 8 & & & \\
\hline Volatiles by GC/MS & 0.8 & 0.0 & 0.0 & 0.0 & 0.01 & 0.6 & 0.2 & 0.0 & 0.0 \\
\hline Volatiles by GC & 0.0 & 0.0 & 0.0 & 0.0 & 0.0 & 0.0 & 0.0 & 0.0 & 0.0 \\
\hline Semi-Volatiles & 0.2 & 0.0 & 0.0 & 0.0 & 0.0 & 0.1 & 0.2 & 0.0 & 0.0 \\
\hline Herbicides/Pesticides/PCBs & 0.3 & 0.0 & 0.0 & 0.0 & 0.0 & 0.1 & 0.2 & 0.0 & 0.0 \\
\hline Other Organics & 0.0 & 0.0 & 0.0 & 0.0 & 0.0 & 0.0 & 0.0 & 0.0 & 0.0 \\
\hline INORGANICS & & & $\$$ & & & & & & \\
\hline RCRA Appendix IX Metals & 0.0 & 0.0 & 0.0 & 0.0 & 0.0 & 0.0 & 0.0 & 0.0 & 0.0 \\
\hline CAM (California) Metals (RCRA) & 0.0 & 0.0 & 0.0 & 0.0 & 0.0 & 0.0 & 0.0 & 0.0 & 0.0 \\
\hline RCRA TCLP Metals and EPTOX Me & 0.0 & 0.0 & 0.0 & 0.0 & 0.0 & 0.0 & 0.0 & 0.0 & 0.0 \\
\hline EPA CLP Metals (CERCLA) & 1.2 & 0.0 & 0.0 & 0.0 & 0.0 & 0.7 & 0.6 & 0.0 & 0.0 \\
\hline NPDES Metals (Clean Water Act) & 0.0 & 0.0 & 0.0 & 0.0 & 0.0 & 0.0 & 0.0 & 0.0 & 0.0 \\
\hline Drinking Water Metais (SDWA) & 0.0 & 0.0 & 0.0 & 0.0 & 0.0 & 0.0 & 0.0 & 0.0 & 0.0 \\
\hline Metals by Other Regulatory Suite & 1.1 & 0.0 & 0.0 & 0.0 & 0.0 & 0.6 & 0.5 & 0.0 & 0.0 \\
\hline Metals by Suite, Other & 0.0 & 0.0 & 0.0 & 0.0 & 0.0 & 0.0 & 0.0 & 0.0 & 0.0 \\
\hline Metals by Metal Except Mercury & 0.0 & 0.0 & 0.0 & 0.0 & 0.0 & 0.0 & 0.0 & 0.0 & 0.0 \\
\hline Mercury & 0.0 & 0.0 & 0.0 & 0.0 & 0.0 & 0.0 & 0.0 & 0.0 & 0.0 \\
\hline Anions/Cations-IC & 0.0 & 0.0 & 0.0 & 0.0 & 0.0 & 0.0 & 0.0 & 0.0 & 0.0 \\
\hline Inorganics Wet Chemistry & 4.2 & 0.0 & 0.0 & 0.0 & 0.0 & 3.9 & 0.3 & 0.0 & 0.0 \\
\hline Other Inorganics & 0.0 & 0.0 & 0.0 & 0.0 & 0.0 & 0.0 & 0.0 & 0.0 & 0.0 \\
\hline RADIOACTIVES & & & & & 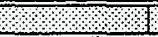 & & & 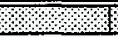 & 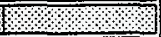 \\
\hline Gross Alpha/Beta & 2.0 & 0.0 & 0.01 & 0.0 & 0.0 & 1.0 & 0.9 & 0.0 & 0.0 \\
\hline Gamma Scan & 0.7 & 0.0 & 0.0 & 0.0 & 0.0 & 0.4 & 0.3 & 0.0 & 0.0 \\
\hline Transuranic & 0.0 & 0.0 & 0.0 & 0.0 & 0.0 & 0.0 & 0.0 & 0.0 & 0.0 \\
\hline Plutonium Isotopes & 1.3 & 0.0 & 0.0 & 0.0 & 0.0 & 0.6 & 0.7 & 0.0 & 0.0 \\
\hline Uranium Isotopes & 1.1 & 0.0 & 0.0 & 0.0 & 0.0 & 0.5 & 0.5 & 0.0 & 0.0 \\
\hline Curium Isotopes & 0.0 & 0.0 & 0.0 & 0.0 & 0.0 & 0.0 & 0.0 & 0.0 & 0.0 \\
\hline Thorium Isotopes & 0.0 & 0.0 & 0.0 & 0.0 & 0.0 & 0.0 & 0.0 & 0.0 & 0.0 \\
\hline Radium Isotopes & 0.6 & 0.0 & 0.0 & 0.0 & 0.0 & 0.5 & 0.1 & 0.0 & 0.0 \\
\hline Americium-241 & 1.2 & 0.0 & 0.0 & 0.0 & 0.0 & 0.6 & 0.6 & 0.0 & 0.0 \\
\hline Neptunium - 237 & 0.0 & 0.0 & 0.0 & 0.0 & 0.0 & 0.0 & 0.0 & 0.0 & 0.0 \\
\hline Polonium-210 & 0.0 & 0.0 & 0.0 & 0.0 & 0.0 & 0.0 & 0.0 & 0.0 & 0.0 \\
\hline Tritium & 0.7 & 0.0 & 0.0 & 0.0 & 0.0 & 0.5 & 0.2 & 0.0 & 0.0 \\
\hline Radon-222 & 0.0 & 0.0 & 0.0 & 0.0 & 0.0 & 0.0 & 0.0 & 0.0 & 0.0 \\
\hline Lead-210 & 0.0 & 0.0 & 0.0 & 0.0 & 0.0 & 0.0 & 0.0 & 0.0 & 0.0 \\
\hline Carbon-14 & 0.0 & 0.0 & 0.0 & 0.0 & 0.0 & 0.0 & 0.0 & 0.0 & 0.0 \\
\hline Nickel-63 & 0.0 & 0.0 & 0.0 & 0.0 & 0.0 & 0.0 & 0.0 & 0.0 & 0.0 \\
\hline Selenium-79 & 0.0 & 0.0 & 0.0 & 0.0 & 0.0 & 0.0 & 0.0 & 0.0 & 0.0 \\
\hline Technetium-99 & 0.0 & 0.0 & 0.0 & 0.0 & 0.0 & 0.0 & 0.0 & 0.0 & 0.0 \\
\hline Strontium Isotopes & 0.0 & 0.0 & 0.0 & 0.0 & 0.0 & 0.0 & 0.0 & 0.0 & 0.0 \\
\hline Strontium - 89 & 0.0 & 0.0 & 0.0 & 0.0 & 0.0 & 0.0 & 0.0 & 0.0 & 0.0 \\
\hline Strontium -90 & 0.0 & 0.0 & 0.0 & 0.0 & 0.0 & 0.0 & 0.0 & 0.0 & 0.0 \\
\hline lodine-129 & 0.0 & 0.0 & 0.0 & 0.0 & 0.0 & 0.0 & 0.0 & 0.0 & 0.0 \\
\hline Nickel-59 & 0.0 & 0.0 & 0.0 & 0.0 & 0.0 & 0.0 & 0.0 & 0.0 & 0.0 \\
\hline Total Uranium & 0.0 & 0.0 & 0.0 & 0.0 & 0.0 & 0.0 & 0.0 & 0.0 & 0.0 \\
\hline Total Thorium & 0.0 & 0.0 & 0.0 & 0.0 & 0.0 & 0.0 & 0.0 & 0.0 & 0.0 \\
\hline Total Strontium & 0.7 & 0.0 & 0.0 & 0.0 & 0.0 & 0.4 & 0.3 & 0.0 & 0.0 \\
\hline Other RAD & 1.5 & 0.0 & 0.0 & 0.0 & 0.0 & 0.7 & 0.7 & 0.0 & 0.0 \\
\hline OTHER & & & & & & & & & \\
\hline Other Wet Chemistry & 2.0 & 0.0 & 0.0 & 0.0 & 0.0 & 1.7 & 0.2 & 0.0 & 0.0 \\
\hline TCLP Extraction & 0.0 & 0.0 & 0.0 & 0.0 & 0.0 & 0.0 & 0.0 & 0.0 & 0.0 \\
\hline Asbestos & 0.0 & 0.0 & 0.0 & 0.0 & 0.0 & 0.0 & 0.0 & 0.0 & 0.0 \\
\hline Industrial Hygiene, NOS & 0.0 & 0.0 & 0.0 & 0.0 & 0.0 & 0.0 & 0.0 & 0.0 & 0.0 \\
\hline Physical Properties & 0.3 & 0.0 & 0.0 & 0.0 & 0.0 & 0.0 & 0.3 & 0.0 & 0.0 \\
\hline Other, Not Otherwise Specified (NO & 0.0 & 0.0 & 0.0 & 0.0 & 0.0 & 0.0 & 0.0 & 0.0 & 0.0 \\
\hline
\end{tabular}


Budget Figures by Site and Fiscal Year (M\$)

\begin{tabular}{|c|c|c|c|c|c|c|c|}
\hline $\begin{array}{l}\text { Fiscal Year/ } \\
\text { EM Off-Ops Off-Site }\end{array}$ & 93 & $\overline{94}$ & 95 & 96 & $\overline{97}$ & $\overline{98}$ & $\overline{\overline{99}}$ \\
\hline & & & & & & & \\
\hline \multicolumn{8}{|l|}{ Fernald } \\
\hline$E M-30$ & 0 & 0 & 0 & 0 & $\overline{0}$ & 0 & $\overline{0}$ \\
\hline$E M-40$ & 292 & 302 & 294 & 308 & 321 & 339 & 356 \\
\hline$E M-60$ & 0 & 0 & 0 & 0 & 0 & 0 & 0 \\
\hline TOTAL Fernald & 294 & 304 & 294 & 308 & 321 & 339 & 356 \\
\hline \multirow{2}{*}{\multicolumn{8}{|c|}{ INEL }} \\
\hline & & & & & & & \\
\hline$E M-30$ & 189 & 125 & 122 & 128.2034 & 134.4068 & 142.3826 & 150.063 \\
\hline$E M-40$ & 0 & 0 & 0 & 0 & 0 & 0 & 0 \\
\hline$E M-60$ & 0 & 44 & 54 & 56.39241 & 58.78481 & 62.20253 & 65.27848 \\
\hline TOTAL INEL & 207 & 194 & 175 & 183.7368 & 192.2088 & 203.3283 & 214.1831 \\
\hline & & & & & & & \\
\hline \multicolumn{8}{|l|}{ Nevada } \\
\hline$E M-30$ & 25 & 22 & 23 & 24 & 25 & 27 & $\overline{28}$ \\
\hline$E M-40$ & 45 & 39 & 41 & 42 & 44 & 47 & 49 \\
\hline$E M-60$ & 0 & 0 & 0 & 0 & 0 & 0 & 0 \\
\hline TOTAL Nevada & 70 & 61 & 64 & 67 & 70 & $74 !$ & 77 \\
\hline & & & & & & & \\
\hline \multicolumn{8}{|l|}{ Oak Ridge Res } \\
\hline$E M-30$ & 263 & 253 & 274 & 287.8484 & 300.7076 & 318.5126 & 335.3285 \\
\hline$E M-40$ & 215 & 130 & 132 & 144.9467 & 163.8038 & 175.3433 & 189.1343 \\
\hline$E M-60$ & 2 & 0 & 0 & 0 & 0 & 0 & 0 \\
\hline TOTAL OR Res & 480 & 531 & 557 & 592.8335 & 637.6254 & 672.4049 & 711.4002 \\
\hline & & & & & & & \\
\hline \multicolumn{8}{|l|}{ Rocky Flats } \\
\hline$E M-30$ & 118 & 111 & 122 & 128 & $13 \overline{3}$ & 141 & $14 \overline{8}$ \\
\hline$E M-40$ & 156 & 148 & 161 & 169 & 176 & 186 & 195 \\
\hline$E M-60$ & 5 & 209 & 241 & 253 & 264 & 279 & 293 \\
\hline TOTALRF & 291 & 477 & 524 & 549 & 573 & 605 & $\overline{636}$ \\
\hline & & & & & & & \\
\hline \multicolumn{8}{|l|}{ Savannah River } \\
\hline$E M-30$ & 700 & 644 & 669 & 702 & 732 & 773 & 812 \\
\hline$E M-40$ & 63 & 70 & 65 & 68 & 71 & 75 & $\overline{78}$ \\
\hline $\mathrm{EM}-60$ & 1 & 0 & 11 & 1 & 1 & 1 & 1 \\
\hline TOTAL SR & 779 & 757 & 735 & 770 & 803 & 848 & 891 \\
\hline & & & & & & & \\
\hline \multicolumn{8}{|l|}{ EM } \\
\hline$E M-30$ & 3397 & 3122 & 3215 & 3374 & 3524 & 3726 & 3915 \\
\hline$E M-40$ & 1852 & 1914 & 1976 & 2077 & 2172 & 2299 & 2418 \\
\hline$E M-60$ & 18 & 721 & 743 & 780 & 815 & 862 & 906 \\
\hline TOTALEM & 5524 & 6462 & 6764 & 7091 & 7400 & 7814 & 8205 \\
\hline \multicolumn{8}{|l|}{ ALL 9 SITES } \\
\hline$E M-30$ & 1295 & 1155 & 1210 & 1270 & 1325 & 1402 & $\overline{1473}$ \\
\hline$E M-40$ & 771 & 689 & 693 & 732 & 776 & 822 & 867 \\
\hline$E M-60$ & 8 & 253 & 296 & 310 & 324 & 342 & 359 \\
\hline TOTAL ALL 9 SITES & 2121 & 2324 & 2349 & 2471 & $25 \overline{97}$ & 2742 & 2886 \\
\hline \multicolumn{8}{|l|}{ FACTOR EM/9 SITES } \\
\hline$E M-30$ & 2.62 & 2.70 & 2.66 & 2.66 & $2 . \overline{2.66}$ & 2.66 & 2.66 \\
\hline$E M-40$ & 2.40 & 2.78 & 2.85 & 2.84 & 2.80 & 2.80 & 2.79 \\
\hline$E M-60$ & 2.25 & 2.85 & 2.51 & 2.51 & 2.52 & 2.52 & 2.52 \\
\hline TOTALEM & 2.60 & 2.78 & 2.88 & 2.87 & $2 . \overline{85}$ & 2.85 & 2.84 \\
\hline
\end{tabular}




\begin{tabular}{|l|l|}
\hline Activity: & OTHER \\
\hline Unit: & Sample \\
\hline Regulatory Driver: & 0 \\
\hline Filename: & SUM2NEEO.WK3 \\
\hline Date: & September 21, 1994 \\
\hline
\end{tabular}

\begin{tabular}{|l|l|}
\hline Average S\&A Duration per Unit (yrs): \\
\hline Total Samples/Unit-yr: & 0.0 \\
\hline Total Samp Ans/Unit-yr: & 0.5 \\
\hline Average Batch Size: & \\
\hline \# of Unit-yrs in FY 93: & 29112.0 \\
\hline
\end{tabular}

\begin{tabular}{|c|c|c|c|c|c|c|c|c|c|}
\hline & Ro & R1 & R2 & $\mathrm{R3}$ & R4 & $\overline{~ M 1}$ & M2 & M3 & $\bar{C}$ \\
\hline SAMPLES TAKEN & & & & & & & & & \\
\hline CUSTOMER SAMPLES & 0.0 & 0.0 & 0.01 & 0.0 & 0.0 & 0.0 & 0.0 & 0.01 & 0.0 \\
\hline SAMPLE ANALYSES REQUESTED & & & & & & & & & \\
\hline ORGANICS & & & & & & & & & \\
\hline Volatiles by GC/MS & 0.0 & 0.0 & 0.0 & 0.0 & 0.0 & 0.0 & 0.0 & 0.0 & 0.0 \\
\hline Volatiles by GC & 0.0 & 0.0 & 0.0 & 0.0 & 0.0 & 0.0 & 0.0 & 0.0 & 0.0 \\
\hline Semi-Volatiles & 0.0 & 0.0 & 0.0 & 0.0 & 0.0 & 0.0 & 0.0 & 0.0 & 0.0 \\
\hline Herbicides/Pesticides/PCBs & 0.0 & 0.0 & 0.0 & 0.0 & 0.0 & 0.0 & 0.0 & 0.0 & 0.0 \\
\hline Other Organics & 0.0 & 0.0 & 0.01 & 0.0 & 0.0 & 0.0 & 0.0 & 0.01 & 0.0 \\
\hline INORGANICS & & & & & & & & & \\
\hline RCRA Appendix IX Metals & 0.0 & 0.0 & 0.01 & 0.0 & 0.0 & 0.0 & 0.0 & 0.01 & 0.0 \\
\hline CAM (California) Metals (RCRA) & 0.0 & 0.0 & 0.0 & 0.0 & 0.0 & 0.0 & 0.0 & 0.0 & 0.0 \\
\hline RCRA TCLP Metais and EPTOX MeI & 0.0 & 0.0 & 0.0 & 0.0 & 0.0 & 0.0 & 0.0 & 0.0 & 0.0 \\
\hline EPA CLP Metals (CERCLA) & 0.0 & 0.0 & 0.0 & 0.0 & 0.0 & 0.0 & 0.0 & 0.0 & 0.0 \\
\hline NPDES Metals (Clean Water Act) & 0.0 & 0.0 & 0.0 & 0.0 & 0.0 & 0.0 & 0.0 & 0.0 & 0.0 \\
\hline Drinking Water Metals (SDWA) & 0.0 & 0.0 & 0.0 & 0.0 & 0.0 & 0.0 & 0.0 & 0.0 & 0.0 \\
\hline Metals by Other Regulatory Suite & 0.0 & 0.0 & 0.0 & 0.0 & 0.0 & 0.0 & 0.0 & 0.0 & 0.0 \\
\hline Metals by Suite, Other & 0.0 & 0.0 & 0.0 & 0.0 & 0.0 & 0.0 & 0.0 & 0.0 & 0.0 \\
\hline Metals by Metal Except Mercury & 0.0 & 0.0 & 0.0 & 0.0 & 0.0 & 0.0 & 0.0 & 0.0 & 0.0 \\
\hline Mercury & 0.0 & 0.0 & 0.0 & 0.0 & 0.0 & 0.0 & 0.0 & 0.0 & 0.0 \\
\hline Anions/Cations-IC & 0.0 & 0.0 & 0.0 & 0.0 & 0.0 & 0.0 & 0.0 & 0.0 & 0.0 \\
\hline Inorganics Wet Chemistry & 0.0 & 0.0 & 0.0 & 0.0 & 0.0 & 0.0 & 0.0 & 0.0 & 0.0 \\
\hline Other Inorganics & 0.0 & 0.0 & 0.0 & 0.0 & 0.0 & 0.0 & 0.0 & 0.0 & 0.0 \\
\hline RADIOACTIVES & & & & 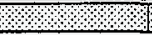 & 7 & & & & \\
\hline Gross Alpha/Beta & 0.1 & 0.0 & 0.0 & 0.0 & 0.0 & 0.0 & 0.0 & 0.0 & 0.0 \\
\hline Gamma Scan & 0.0 & 0.0 & 0.0 & 0.0 & 0.0 & 0.0 & 0.0 & 0.0 & 0.0 \\
\hline Transuranic & 0.0 & 0.0 & 0.0 & 0.0 & 0.0 & 0.0 & 0.0 & 0.0 & 0.0 \\
\hline Plutonium Isotopes & 0.0 & 0.0 & 0.0 & 0.0 & 0.0 & 0.0 & 0.0 & 0.0 & 0.0 \\
\hline Uranium Isotopes & 0.0 & 0.0 & 0.0 & 0.0 & 0.0 & 0.0 & 0.0 & 0.0 & 0.0 \\
\hline Curium Isotopes & 0.0 & 0.0 & 0.0 & 0.0 & 0.0 & 0.0 & 0.0 & 0.0 & 0.0 \\
\hline Thorium Isotopes & 0.0 & 0.0 & 0.0 & 0.0 & 0.0 & 0.0 & 0.0 & 0.0 & 0.0 \\
\hline Radium Isotopes & 0.0 & 0.0 & 0.0 & 0.0 & 0.0 & 0.0 & 0.0 & 0.0 & 0.0 \\
\hline Americium-241 & 0.0 & 0.0 & 0.0 & 0.0 & 0.0 & 0.0 & 0.0 & 0.0 & 0.0 \\
\hline Neptunium - 237 & 0.0 & 0.0 & 0.0 & 0.0 & 0.0 & 0.0 & 0.0 & 0.0 & 0.0 \\
\hline Polonium-210 & 0.0 & 0.0 & 0.0 & 0.0 & 0.0 & 0.0 & 0.0 & 0.0 & 0.0 \\
\hline Tritium & 0.0 & 0.0 & 0.0 & 0.0 & 0.0 & 0.0 & 0.0 & 0.0 & 0.0 \\
\hline Radon-222 & 0.0 & 0.0 & 0.0 & 0.0 & 0.0 & 0.0 & 0.0 & 0.0 & 0.0 \\
\hline Lead-210 & 0.0 & 0.0 & 0.0 & 0.0 & 0.0 & 0.0 & 0.0 & 0.0 & 0.0 \\
\hline Carbon-14 & 0.0 & 0.0 & 0.0 & 0.0 & 0.0 & 0.0 & 0.0 & 0.0 & 0.0 \\
\hline Nickel-63 & 0.0 & 0.0 & 0.0 & 0.0 & 0.0 & 0.0 & 0.0 & 0.0 & 0.0 \\
\hline Selenium-79 & 0.0 & 0.0 & 0.0 & 0.0 & 0.0 & 0.0 & 0.0 & 0.0 & 0.0 \\
\hline Technetium-99 & 0.0 & 0.0 & 0.0 & 0.0 & 0.0 & 0.0 & 0.0 & 0.0 & 0.0 \\
\hline Strontium Isotopes & 0.0 & 0.0 & 0.0 & 0.0 & 0.0 & 0.0 & 0.0 & 0.0 & 0.0 \\
\hline Strontium -89 & 0.0 & 0.0 & 0.0 & 0.0 & 0.0 & 0.0 & 0.0 & 0.0 & 0.0 \\
\hline Strontium -90 & 0.0 & 0.0 & 0.0 & 0.0 & 0.0 & 0.0 & 0.0 & 0.0 & 0.0 \\
\hline lodine-129 & 0.0 & 0.0 & 0.0 & 0.0 & 0.0 & 0.0 & 0.0 & 0.0 & 0.0 \\
\hline Nickel-59 & 0.0 & 0.0 & 0.0 & 0.0 & 0.0 & 0.0 & 0.0 & 0.0 & 0.0 \\
\hline Total Uranium & 0.0 & 0.0 & 0.0 & 0.0 & 0.0 & 0.0 & 0.0 & 0.0 & 0.0 \\
\hline Total Thorium & 0.0 & 0.0 & 0.0 & 0.0 & 0.0 & 0.0 & 0.0 & 0.0 & 0.0 \\
\hline Total Strontium & 0.0 & 0.0 & 0.0 & 0.0 & 0.0 & 0.0 & 0.0 & 0.0 & 0.0 \\
\hline Other RAD & 0.0 & 0.0 & 0.0 & 0.0 & 0.0 & 0.0 & 0.0 & 0.0 & 0.0 \\
\hline OTHER & & 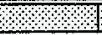 & 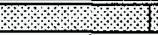 & . & 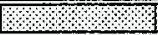 & 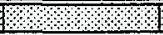 & 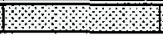 & 8 & \\
\hline Other Wet Chemistry & 0.0 & 0.0 & 0.0 & 0.0 & 0.0 & 0.0 & 0.0 & 0.0 & 0.0 \\
\hline TCLP Extraction & 0.0 & 0.0 & 0.0 & 0.0 & 0.0 & 0.0 & 0.0 & 0.0 & 0.0 \\
\hline Asbestos & 0.0 & 0.0 & 0.0 & 0.0 & 0.0 & 0.0 & 0.0 & 0.0 & 0.0 \\
\hline Industrial Hygiene, NOS & 0.0 & 0.0 & 0.0 & 0.0 & 0.0 & 0.0 & 0.0 & 0.0 & 0.0 \\
\hline Physical Properties & 0.0 & 0.0 & 0.0 & 0.0 & 0.0 & 0.0 & 0.0 & 0.0 & 0.0 \\
\hline Other, Not Otherwise Speci & 0.0 & 0.0 & 0.0 & 0.0 & 0.0 & 0.0 & 0.0 & 0.0 & 0.0 \\
\hline
\end{tabular}

